\title{
Non-communicable disease epidemic: epidemiology in action (EuroEpi 2013 and NordicEpi 2013)
}

\author{
Aarhus, Denmark from 11 August to 14 August 2013
}

(C) Springer Science+Business Media Dordrecht 2013

Jørn Olsen, Ellen Aagaard Nфhr, Reimar Wernich Thomsen, Henrik Støvring

Modern societies are increasingly challenged by the frequency of noncommunicable diseases (NCDs) such as mental disorders, obesity, diabetes, cardiovascular and cerebrovascular disease, cancer, COPD, and arthritis. These conditions affect large proportions of the population and lead to premature mortality and loss of healthy life, which later will lead to less productive societies and loss of European socio-economic development. Both incidence and prevalence of these NCDs increase with age, which translates into the challenge only mounting over the coming decades. At the same time injuries and infections remain important, because their risk is increased with NCDs.

While this has been a European reality for at least three decades, similar patterns are beginning to appear in many other parts of the world. Type 2 diabetes for example is a disease reaching near-Western prevalences in China and India. It is therefore reasonable to ask, what epidemiologists on a global scale can learn from the European experiences when trying to identify causes and risk factors for NCDs. Also when searching for costeffective interventions for NCD prevention and treatment at an individual and at a societal level. To address this challenge, many different and crossdisciplinary viewpoints needs to be embraced. There is urgent need for input from risk factor epidemiology, clinical sciences,- - omics research, psychology and behavioral research, consumer studies, and statistics, while maintaining a public health perspective to identify credible action strategies. Proper use of European experiences might mean that something good could come out of past failures and mistakes.

With this setting in mind, the EuroEpi 2013 and NordicEpi 2013 are held in Aarhus, Denmark, August 11-14, 2013, with a program with a dual focus on cutting edge methodological developments for the study of causal effects and effect modifiers, and with corresponding presentations of current applications and challenges when using these techniques. As an integrated part of the congress the NordicEpi focus on register-based epidemiologic research in Nordic countries. On the first day of the congress six pre-congress courses are offered with a very distinguished panel of senior epidemiologists. During the congress nine special symposia are held, of which one is dedicated to the overall theme of the congress. All parts of the congress are open to all interested. The congress had received 668 abstracts at the end of the deadline from 561 presenters. Interestingly more than 100 abstracts came from outside Europe (Table 1).

153 abstracts were selected for oral presentation in one of the 18 contributed sessions, while 491 were accepted for poster presentation. Topics of the submitted abstracts were broad, ranging from disease specific themes over exposures to methods and public health (Table 2).
Table 1 Geographical distribution of submitted abstracts, EuroEpi and NordicEpi 2013

\begin{tabular}{lc}
\hline Region & $\mathrm{n}(\%)$ \\
\hline Scandinavia & $226(33.8)$ \\
Western Europe & $293(43.9)$ \\
Eastern Europe & $25(3.7)$ \\
US, Canada, Australia, New Zealand & $33(4.9)$ \\
South America & $44(6.6)$ \\
Others & $47(7.0)$ \\
Total & $668(100.0)$ \\
\hline
\end{tabular}

Table 2 Author-chosen topics of submitted abstracts, EuroEpi and NordicEpi 2013

\begin{tabular}{lc}
\hline Topic & $\mathrm{n}(\%)$ \\
\hline Diseases—COPD & $7(1.1)$ \\
Diseases_CVD/diabetes & $69(10.3)$ \\
Diseases—cancer & $61(9.1)$ \\
Diseases—infections & $38(5.69)$ \\
Diseases-others & $52(7.8)$ \\
Diseases-psychiatry & $45(6.7)$ \\
Diseases—other & $1(0.2)$ \\
Exposure-enviromental factors & $56(8.4)$ \\
Exposure-lifestyle factors & $104(15.6)$ \\
Exposure-other & $31(4.7)$ \\
Methods-data analysis & $34(5.1)$ \\
Methods-data collection & $20(3.0)$ \\
Methods-interpretation & $12(1.8)$ \\
Methods—others & $9(1.4)$ \\
NordicEpi-nordic studies & $4(0.6)$ \\
NordicEpi-others & $1(0.2)$ \\
NordicEpi-register-based epidemiology & $53(7.9)$ \\
Other-health promotion & $36(5.4)$ \\
Other-others & $25(3.7)$ \\
Other-politics & $10(1.5)$ \\
Total & $668(100.0)$ \\
\end{tabular}




\section{Reviewers}

The Scientific and Organizing Committees would like to thank the following reviewers for having evaluated the abstracts submitted to the Congress:

Anne-Marie Nybo Andersen

Ingelise Andersen

Niels Trolle Andersen

Bodil Hammer Bech

Christine Stabell Benn

Gabriele Berg-Beckhoff

Bo Martin Bibby

Anders Borglykke

Charlotte Cerqueira

Christian Christiansen

Kaare Christensen

Adele Seniori Costantini

Deirdre Cronin-Fenton

Bianca DeStavola

Vera Ehrenstein

Anders Ekbom

Morten Frydenberg

Mika Gissler

Andrej Gribovskij

Morten Grønbæk

Cecilia Ramlau Hansen

Lotte Husemoen

Marianne Uhre Jakobsen

Peter Jepsen

Bernard Jeune

Søren Johnsen

Svend Juul

Torben Jørgensen

Katrine Strandberg Larsen

Jennifer Leigh Lund

Rikke Lund

Elsebeth Lynge

Mia Madsen
Ellen Aagaard Nøhr

Mette Nørgaard

Kirstine Magtengaard Robinson

Mads Melbye

Juan Merlo

Carsten Obel

Jørn Olsen

Inge Petersen

Jan P. Vandenbroucke

Grete Skøtt Pedersen

Charlotta Pisinger

Naja Hulvej Rod

Rodolfo Saracci

Henrik Støvring

Henrik Toft Sørensen

Elisabeth Svensson

Mette Søgaard

Mikael Thinggaard

Reimar Wernich Thomsen

Betina Thuesen

Ulla Toft

Mogens Vestergaard

Sonja Vestergaard

Chunsen Wu

Mette Aadahl

\section{Organization/Committees}
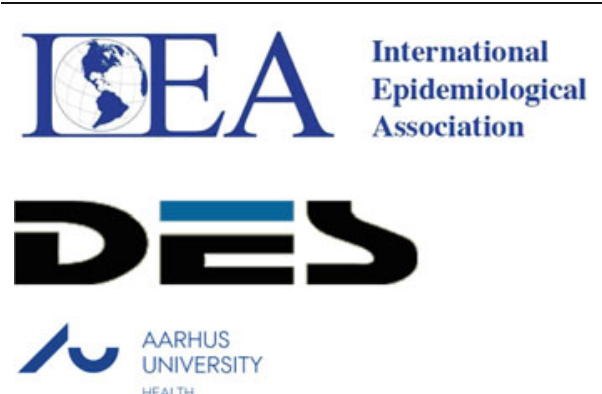

International Epidemiological Association

- European Epidemiology Federation

http://www.iea-europe.org

Danish Epidemiological Society

http://www.dansk-epidemiologisk-

selskab.dk

Health, Aarhus University

http://health.au.dk 


\section{International Scientific Committee}

Anne-Marie Nybo Andersen, Copenhagen University, Denmark Henrique Barros, University of Porto, Portugal

Raj Bhopal, University of Edinburgh, Scotland

Kaare Christensen, University of Southern Denmark, Denmark

Adele Seniori Costantini, ISPO, Italy

Bianca DeStavola, London School of Hygiene and Tropical Medicine, UK

Anders Ekbom, Karolinska Institutet, Sweden

Mika Gissler, National Institute for Health and Welfare, Finland

Morten Grønbæk, University of Southern Denmark, Denmark

Sigrid Haraldsdóttir, Directorate of Health, Iceland

Marjolein M Iversen, Høgskolen I Bergen, Norway

Inger Kristin Larsen, Cancer Registry, Norway

Rolv Terje Lie, Bergen University, Norway

Elsebeth Lynge, Copenhagen University, Denmark

Ellen Aagaard Nøhr, Aarhus University, Denmark

Torben Jørgensen, Glostrup University Hospital, Denmark

Manolis Kogevinas, Centre for Research in Environmental Epidemiology, Spain

Mia Madsen, University of Southern Denmark, Denmark

Mads Melbye, Statens Serum Institut, Denmark

Juan Merlo, Skåne University Hospital, Sweden

Jørn Olsen, Aarhus University, Denmark

Anna Oudin, Umeå University, Sweden

Charlotta Pisinger, Glostrup University Hospital, Denmark

Eero Pukkala, Finnish Cancer Registry, Finland

Naja Hulvej Rod, Copenhagen University, Denmark

Rodolfo Saracci, IARC, France

Henrik Støvring, Aarhus University, Denmark

Thorkil IA Sørensen, Copenhagen University, Denmark

Henrik Toft Sørensen, Aarhus University Hospital, Denmark

Reimar Wernich Thomsen, Aarhus University Hospital, Denmark

Lau Caspar Thygesen, Copenhagen University, Denmark

Laufey Tryggvadóttir, Cancer Registry, Iceland

Jan P. Vandenbroucke, Leiden University, The Netherlands

\section{Local Organizing Committee}

Mia Madsen, Institute of Public Health, University of Southern Denmark, Denmark

Ellen Aagaard Nøhr, Department of Public Health, Aarhus University, Denmark

Charlotta Pisinger, Forskningscenter for Forebyggelse og Sundhed, Glostrup University Hospital, Denmark

Naja Hulvej Rod, Department of Public Health, Copenhagen University, Denmark

Henrik Støvring, Department of Public Health, Aarhus University, Denmark

Reimar Wernich Thomsen, Department of Clinical Epidemiology, Aarhus University Hospital, Denmark

\section{National Steering Committee}

Jørn Olsen, Department of Public Health, Aarhus University, Denmark

Mia Madsen, Institute of Public Health, University of Southern Denmark, Denmark
Ellen Aagaard Nøhr, Department of Public Health, Aarhus University, Denmark

Charlotta Pisinger, Forskningscenter for Forebyggelse og Sundhed, Glostrup University Hospital, Denmark

Naja Hulvej Rod, Department of Public Health, Copenhagen University, Denmark

Henrik Støvring, Department of Public Health, Aarhus University, Denmark

Reimar Wernich Thomsen, Department of Clinical Epidemiology, Aarhus University Hospital, Denmark

\section{Congress Secretariat}

KongresKompagniet A/S

Address: Pakhus 13, Nordhavnsgade 4, DK-8000 Aarhus C, Denmark Phone: +45-86296960

Fax: $+45-86296980$

E-mail: epi2013@kongreskompagniet.dk

Web: www.kongreskompagniet.dk

\section{International epidemiological association: European epidemiology federation}

International Epidemiological Association Council (2011-2014)

President: Cesar Victora, Brazil

Treasurer: Ahmed Mandil, Egypt

President-Elect: Patricia Buffler, USA

Past President: Neil Pearce, New Zealand

\section{IEA-EEF board}

Adele Seniori Costantini, International Epidemiological Association (IEA), European Councillor

Representatives of Societies

Henrik Støvring, Danish Epidemiological Society (DES), Denmark Ester Villalonga Olives, European Young Epidemiologists, Europe

Tapio Luostarinen, Finnish Epidemiological Society. Finland

Beatrice Blondel, ADELF, France

Thierry Lang, ADELF, France

Eva Grill, German Association of Epidemiology (DGepi), Germany Laufey Tryggvadóttir, Icelandic Society for Epidemiology and Biostatistics, Iceland

Alessio Petrelli, Italian Association of Epidemiology, Italy

Biljana Danilovska, Macedonian Epidemiological Association, Macedonia

Maurice Zeegers, The Netherlands Epidemiological Society, Netherlands

Andrej Grjibovski, Norwegian Epidemiological Association (NOFE), Norway

Katarzyna Szamotulska, Polish Epidemiological Society, Poland Henrique Barros, Portuguese Epidemiological Association, Portugal

Biljana Kocic, Serbian Epidemiological Society, Serbia \& Montenegro

Alberto Ruano, Spanish Epidemiological Society, Spain

Magnus Stenbeck, Swedish Association of Epidemiology, Sweden

Christoph Junker, Swiss Society of Public Health, Switzerland

David Batty, Society for Social Medicine, United Kingdom 
Detailed scientific programme-per 24 June 2013

Sunday 11 August-EuroEpi

\subsection{0-12.30 Pre-congress courses I}

How to write and get published in Epidemiology, Henrik Toft Sorensen, Aarhus University

Causal Inference in Epidemiology, Miguel Hernan, Harvard

Modelling repeatedly measured data in epidemiological studies, Debbie Lawlor, Bristol University

12.30-13.15 Lunch

13.15-16.15 Pre-congress courses II

Reproductive Epidemiology, Jørn Olsen, Ellen Aagaard Nøhr, Aarhus University

Mediation Analysis, Tyler VanderWeele, Harvard

How to get research ideas and prune them into feasible studies, Jan P. Vandenbroucke, Leiden University

\subsection{0-17.15 Official opening}

17.15-18.15 Plenary session

2nd Sir Richard Peto lecture, Valerie Beral, Oxford University

Pemberton lecture, Neil Pearce, LSHTM, London

18.30-20.00 Welcome Reception at Aarhus City Hall

Monday 12 August-EuroEpi

08.30-10.30 Plenary session-understanding interactions and effect measure modification

Modern developments in assessing interaction, Tyler Vanderweele, Harvard

What can we learn from an example of geneenvironment interaction, Jan P Vandenbroucke, Leiden University

10.30-11.00 Coffee

11.00-12.30 Parallel oral sessions-contributed abstracts

Diabetes and metabolic syndrome (abstract $\mathrm{O}-001-O$ 009)

Cancer incidence and risk factors (abstract $\mathrm{O}-010-\mathrm{O}$ 018)

Infections (abstract O-019-O-026)

Risk factors during pregnancy and child health (abstract O-027-O-035)

12.30-13.30 Lunch and poster presentation

13.30-14.00 Invited lecture

Epidemiological data and methods as health care policy tools, Harold S. Luft, Palo Alto Medical Foundation

14.00-15.00 Parallel oral session-contributed abstracts

Mental health: risk factors and outcomes (abstract O-036-O-041)
13.30-15.00 Parallel oral sessions—contributed abstracts

Cardiovascular risk (abstract O-042-O-050)

Cancer: genes, prognosis and mortality (abstract $O$ 051-O-058)

Others (abstract O-059-O-067)

15.00-15.30 Coffee

\subsection{0-17.00 Special Symposia}

The use of cancer screening data in epidemiological research, Piret Veerus

Confounding in epidemiological studies, is Selfcontrolled case series the answer? Irene Petersen

E-Epi: Expanding the Horizons, John Gallacher

Problems in interpreting the excess fraction as an etiological fraction-ethical considerations and societal consequences, Henrik Kolstad

Health economics and social epidemiology, Esben Agerbo

17.00-18.30 IEA/EEF assembly

Young Epidemiologists Meeting

19.00 - Congress dinner at Varna Mansion

Tuesday 13 August-EuroEpi

08.30-10.30 Plenary session-Confounding and adjustments

Time-varying confounding in randomized and observational studies, Miguel Hernan, Harvard

Confounder control in reproductive epidemiology, Debbie Lawlor, Bristol University

10.30-11.00 Coffee

11.00-12.30 Parallel oral sessions-contributed abstracts

Health policy and societal effects (abstract $\mathrm{O}-068-O$ 076)

Epidemiological methods and psychiatric disease (abstract O-077-O-085)

Cohort outcome studies and repeated measures (abstract O-086-O-094)

Population incidence and mortality (abstract $\mathrm{O}-095-\mathrm{O}$ 103)

12.30-13.30 Lunch and poster presentation

13.30-15.00 Parallel oral sessions-contributed abstracts

Exposure-lifestyle factors (abstract O-104-O-112)

Socioeconomic and lifestyle factors (abstract $O-113$ $O-121)$

Risk factor epidemiology (abstract $O-122-O-130$ )

13.30-15.00 Special symposium

Epidemiologists' role in working towards global health goals

15.00-15.30 Coffee 


\subsection{0-16.30 Editor's Choice session}

Best posters, Shah Ebrahim, Int J Epidemiology, Henrik Toft Sørensen, Clin Epidemiology, Peter Allebeck, Eur J Public Health

15.30-16.30 Special symposia

Greenland-health transition, Anders Koch

Migrant reproductive health, Anne-Marie Nybo Andersen

Why do screening and life style interventions not work in prevention of chronic diseases and why do we still think they work? Charlotta Pisinger

16.35-17.15 Plenary session

The future of epidemiology - where do we go from here? Timothy Lash, Emory University

17.45-18.30 Guided architectural tour of Aarhus University Campus

18.30-19.15 Reception at The Museum of Ancient Art at Aarhus University

19.30 Social dinner at Aarhus University

Wednesday 14 August-NordicEpi

08.30-09.50 Plenary session

Use of Nordic registers in concert: Opportunities and challenges, Juni Palmgren, Sweden

Nordic cancer registries: Making the most of the differences, Eero Pukkala, Finland

09.50-10.10 Coffee

10.10-11.30 Plenary session

NorPEN: Pharmaco-epidemiology using Nordic registers, Anders Ekbom, Sweden

Research opportunities for population-based biobanks, Mads Melbye, Denmark

11.30-12.30 Lunch and poster presentation

12.30-13.45 Parallel oral sessions-contributed abstracts

Reproductive epidemiology (abstract $O-131-O-137$ )

Register based epidemiology (abstract $O-138-O-144$ )

Register based epidemiology (abstract $O-145-O-151$ )

12.30-13.45 Special symposium

Applied epidemiology: The Danish perspective, Anders Green

13.45-14.00 Coffee

14.00-15.10 Plenary session

Nordic Council of Ministers (NORDFORSK): Their opportunities to support Nordic epidemiology, Riita Mustonen

Translational epidemiology in public health: Challenges and good examples, Camilla Stoltenberg, Norway

15.10-15.30 Official closing and adjournment
Diabetes and metabolic syndrome

12.08.2013 11:00-12:30

0-001

The association between dietary energy density and type 2 diabetes in Europe: results from the EPIC-InterAct Study

Presenter: Saskia van den Berg

Saskia van den Berg 1)

(1) National Institute for Public Health and the Environment (RIVM)

Background: Observational studies implicate higher dietary energy density (DED) as a potential risk factor for weight gain and obesity. It has been hypothesized that DED may also be associated with risk of type 2 diabetes (T2D), but limited evidence exists.

Objectives: Aim of this study was to investigate the association between DED and risk of T2D in a large prospective study with heterogeneity of dietary intake.

Methods: A case-cohort study was nested within the European Prospective Investigation into Cancer (EPIC) study of 340,234 participants contributing 3.99 million person years of follow-up, identifying 12,403 incident diabetes cases and a random subcohort of 16,835 individuals from eight European countries. DED was calculated as energy ( $\mathrm{kcal}$ ) from foods (except beverages) divided by the weight (gram) of foods estimated from dietary questionnaires. Prentice-weighted Cox proportional hazard regression models were fitted by country. Risk estimates were pooled by random effects metaanalysis and heterogeneity was evaluated.

Results: Estimated mean (SD) DED was 1.5 (0.3) kcal/g among cases and subcohort members, varying across countries (range 1.4-1.7 kcal/ g). After adjustment for age, sex, smoking, physical activity, alcohol intake, energy intake from beverages and misreporting of dietary intake, no association was observed between DED and T2D (HR 1.02 (95\% CI 0.93-1.13), which was consistent across countries $\left(\mathrm{I}^{2}=2.9 \%\right)$.

Conclusions: In this large European case-cohort study no association between DED of solid and semi-solid foods and risk of T2D was observed. However, despite the fact that there currently is no conclusive evidence for an association between DED and T2DM risk, choosing low energy dense foods should be promoted as they support current WHO recommendations to prevent chronic diseases.

\section{0-002}

Relationship of HbA1c variability, absolute changes in HbA1c, and mortality in type 2 diabetes: a Danish population-based observational study

Presenter: Mette Vinther Skriver

Mette Vinther Skriver 1); Annelli Sandbæk 1); Jette Kolding Kristensen 1); Henrik Støvring 1)

1) Dept. of Public Health, Aarhus University, Denmark

Background: As prevalence of type 2 diabetes continues to increase worldwide, diabetes-related morbidity and mortality increase as well. A widely used criterion for optimizing diabetes care is to aim at reducing the hemoglobin $\mathrm{A} 1 \mathrm{C}$ (HbA1c) level below a given limit. However, evidence is scarce on how variability in $\mathrm{HbAlc}$ or absolute changes in individual HbA1c level affect mortality. 
Objectives: To assesses the relationship of mortality with HbAlc variability and absolute change in $\mathrm{HbA} 1 \mathrm{c}$ in persons with type 2 diabetes.

Methods: The study population consisted of 11,205 Danish individuals identified with type 2 diabetes during 2001 to 2006, who had at least three $\mathrm{HbA} 1 \mathrm{c}$ measurements: one index measure, one closing measure 22-26 month later, and at least one measurement inbetween. Absolute change in $\mathrm{HbAlc}$ was defined as the difference between closing and index value. HbAlc variability was defined as the mean absolute residual around the line connecting index value with closing value. Information on morbidity prior to inclusion and mortality during follow-up was obtained from nationwide Danish Registers. Analyses were stratified on index $\mathrm{HbA} 1 \mathrm{c}$ and used Cox proportional hazard models with restricted cubic splines.

Results: An HbA1c variability between 0 and 0.5 was not associated with mortality in any index $\mathrm{HbA1c}$ groups. HbA1c variability above 0.5 was associated with increased mortality for index $\mathrm{HbA} 1 \mathrm{c} \leq 8.0 \%$. For index $\mathrm{HbA} 1 \mathrm{c}>8.0 \%$, no such association could be identified. For an index HbA1c $\leq 8.0 \%$, mortality increased when HbAlc subsequently declined, but was stable when HbAlc rose. For index $\mathrm{HbA} 1 \mathrm{c}>8.0 \%$, a linear relationship between change in $\mathrm{HbA} 1 \mathrm{c}$ and mortality was identified with lowest mortality for greatest decline.

Conclusions: For individuals with an index HbA1c below $8.0 \%$ both high $\mathrm{HbA} 1 \mathrm{c}$ variability and a decline in $\mathrm{HbA} 1 \mathrm{c}$ were independent risk factors for death. For individuals with an index HbAlc above $8.0 \%$, a decline in HbA1c was associated with lower mortality, whereas variability was not.

\section{O-003}

Adult overweight and metabolic syndrome in relation to body mass index at the age of 5 years

\section{Presenter: Lise Graversen}

Lise Graversen 1); Thorkild IA Sørensen 2), 9); Liselotte Petersen 3); Ulla Sovio 4), 10); Marika Kaakinen 5); Jaana Laitinen 6); Anja Taanila 7); Annelli Sandbæk 1); Marjo-Riitta Jarvelin 5), 8); Carsten Obel 1)

1) Section of General Medical Practice, Department of Public Health, Aarhus University, Denmark; 2) Institute of Preventive Medicine, Bispebjerg and Frederiksberg University Hospital, The Capital Region, Copenhagen, Denmark; 3) National Centre for RegisterBased Research, Faculty of Social Sciences, Aarhus University, Denmark; 4) Department of Non-communicable Disease Epidemiology, London School of Hygiene and Tropical Medicine, UK; 5) Institute of Health Sciences, University of Oulu, Finland; 6) Finnish Institute of Occupational Health, Finland; 7) Institute of Health Sciences, University of Oulu, Finland; 8) Oulu University Hospital, Unit of Primary Care, Oulu Finland; 9) Novo Nordisk Foundation Center for Basic Metabolic Research, Faculty of Health and Medical Sciences, University of Copenhagen, Denmark; 10) Department of Epidemiology and Biostatistics, Imperial College, London, UK

Background: Metabolic health is partly determined early in life as intrauterine factors program the development of overweight and metabolic changes in adulthood. We have previously found that BMI at the age of 5 years is strongly associated with overweight in adolescence, and this association has been shown to be stable over the development of the obesity epidemic. To be able to establish deprogramming interventions we need to develop tools to identify children at risk of developing metabolic problems. The aim of this study is to test the association between body mass index at the age of 5 years and central adiposity and metabolic changes at the age of 31 years, taking into account routinely collected growth data up to the age of 5 years.
Methods: The Northern Finland Birth Cohort 1966 (NFBC1966; $\mathrm{N}=4111$ ) is a population based cohort followed from birth to the age of 31 years, where participants were invited to a clinical examination including height, weight, waist circumference, blood pressure, fasting glucose, HDL and triglycerides. We tested the association between body mass index at the age of 5 years and these metabolic measures at the age of 31 years.

Results: We found body mass index at the age of 5 years above the 90th percentile to have a relative risk of overweight, obesity, central adiposity and metabolic syndrome at the age of 31 years among women of 3.2 (2.6-4.0), 5.8 (3.5-9.7), $3.2(2.3-4.4)$ and $4.4(2.5-7.7)$, respectively, and 2.0 (1.7-2.4), 6.2 (3.6-10.9), 4.5 (2.7-7.3) and 3.4 (1.7-6.5), respectively, among men.

Conclusions: A tool to identify children with the highest risk of developing metabolic syndrome is the first step when interventions to prevent early metabolic problems are to be developed.

\section{O-004}

Lifestyle, diabetes and dementia: chronology of diseases and synergistic associations with physical inactivity and obesity

Presenter: Kirsten Mehlig

Kirsten Mehlig 1); Ingmar Skoog 2); Junmei Miao Jonasson 1); Margda Waern 2); Cecilia Björckelund 1); Svante Östling 2); Lauren Lissner 1)

1) Dept. of Public Health and Community Medicine, Sahlgrenska Academy at Göteborg University, Göteborg Sweden; 2) Inst. for Neuroscience and Physiology, Neuropsychiatric Epidemiology Unit, Sahlgrenska Academy at Göteborg University, Göteborg Sweden

Background: There is mixed evidence on whether type 2 diabetes mellitus is a causal risk factor for dementia or if both diseases are comorbidities with common risk factors.

Objectives: We aim to compare selected mid-life risk factors for diabetes and dementia, as well as the association and relative chronology between the diseases in a prospective setting.

Methods: A community-based sample of 1,448 Swedish women aged 38-60 years and free of diabetes and dementia in 1968 was followed by means of up to five examinations spread over 34 years. $9.6 \%$ of all women developed diabetes and $11.4 \%$ developed dementia, with over 42,000 person years of follow-up for each disease. Cumulative incidence and cause-specific hazard functions were calculated to assess the influence of selected risk factors and their interactions on dementia and diabetes, and the chronology of the two diseases.

Results: Comparing risk factors for incident diabetes and dementia, both diseases showed a synergistic adverse association with obesity combined with physical inactivity (hazard ratio (HR) for interaction $=2.6,95 \%$ confidence interval $(\mathrm{CI})=1.1,6.2$ for diabetes; and 3.7, $95 \% \mathrm{CI}=1.2,11.5$ for dementia). Development of diabetes doubled the risk for subsequent dementia $(\mathrm{HR}=2.0,95 \% \mathrm{CI}=1.0$, 4.0), with a tendency of a stronger association with a quasi-simultaneous diagnosis of diabetes ( $\mathrm{HR}=4.6,95 \% \mathrm{CI}=0.9,23.7)$ than with diabetes earlier in life $(\mathrm{HR}=1.7,95 \% \mathrm{CI}=0.8,3.7)$, compared to no diabetes.

Conclusions: Shared lifestyle-related risk factors and parallel occurrences of diagnoses suggest a similar aetiology of diabetes and dementia.

\section{0-005}

Longitudinal association of C-reactive protein and Hemoglobin A1C over 13 years: European Prospective Investigation into Cancer Norfolk study

Presenter: Sara Ahmadi Abhari 
Sara Ahmadi Abhari 1); Robert Luben 1); Nicholas Wareham 2); Kay-Tee Khaw 1)

1) Department of Public Health and Primary Care, University of Cambridge, Cambridge, UK; 2) MRC Epidemiology Unit, Institute of Metabolic Sciences, Cambridge, UK

Background: Type-2 diabetes is associated with systemic inflammation and higher C-reactive protein (CRP) levels. However, the longitudinal association of CRP and hemoglobin A1c (HbA1C) in large prospective studies is not well known. Understanding such associations may shed light on the role of systemic inflammation in development of type-2 diabetes.

Objectives: We aimed to investigate the cross-sectional and longitudinal association of CRP and HbA1C.

Methods: EPIC-Norfolk is a cohort study of men and women aged 40-79 years recruited in 1993-1997. CRP was measured using a high-sensitivity assay at baseline and at 13 year follow-up. HbA1C was measured at baseline, 4 , and 13 years. Participants were excluded if they had diagnosed diabetes or were taking diabetes medication. Data on Serum CRP and HbA1C was available for 7,695 participants ( $30 \%$ of the cohort) at baseline assessment who were entered in the present analysis. Cross-sectional association of CRP and HbA1C was assessed by multiple linear regression analysis and longitudinal associations were examined using multivariable linear mixed models adjusted for age, sex, body mass index, waist circumference, smoking, physical activity, alcohol intake, corticosteroid medication, cancer, myocardial infarction and stroke and for women only, menopausal status and postmenopausal hormone replacement therapy. Rate of longitudinal change of HbA1C across categories of baseline CRP was calculated in mixed models from the interaction term for CRP*time.

Results: Median (inter-quartile range) CRP at baseline and 13 years was $1.4(0.7-3.0)$ and $1.9(1.2-3.2)$ respectively. Mean [standard deviation (SD)] $\mathrm{HbA} 1 \mathrm{C}$ at baseline, 4, and 13 years were 5.3 (8.4), 5.4 (0.7), and 5.8 (0.6) respectively. In the cross-sectional analysis, loge-CRP was positively associated with $\mathrm{HbA} 1 \mathrm{C}$ with a 1-SD higher baseline loge-CRP (about three-fold higher CRP) associated with 0.060 (95\% CI 0.046, 0.074) higher HbA1C. In categorical analysis, compared to participants with $\mathrm{CRP}<1 \mathrm{mg} / \mathrm{l}$, those with CRP $>10 \mathrm{mg} / \mathrm{l}$ had 0.261 (95\% CI 0.184, 0.338) higher HbA1C. Longitudinal change in CRP over 13 years was to a similar extent positively associated with increase in $\mathrm{HbA} 1 \mathrm{C}$ over the same period with a 1-SD higher longitudinal change in loge-CRP associated with 0.054 (95\% CI $0.041,0.067)$ increase in HbA1C. HbA1C increased by about 0.04 annually, however rate of change of $\mathrm{HbA1C}$ over time was not dependent on baseline CRP levels.

Conclusion: In this study we found longitudinal observational evidence suggesting that increase in systemic inflammation is associated with an increase in $\mathrm{HbA} 1 \mathrm{C}$ and thus systemic inflammation may have a role in development of type- 2 diabetes.

\section{O-006}

Dietary intake of carbohydrates and risk of type 2 diabetes: European prospective investigation into cancer Norfolk study

Presenter: Sara Ahmadi Abhari

Sara Ahmadi Abhari 1); Robert Luben 1); Natasha Powell 1); Amit Bhaniani 1); Nicholas Wareham 2); Nita Forouhi 2); Kay-Tee Khaw 1)

1) Department of Public Health and Primary Care, University of Cambridge, Cambridge, UK; 2) MRC Epidemiology Unit, Institute of Metabolic Sciences, Cambridge, UK
Background and objectives: To better understand the role of dietary patterns in development of type 2 diabetes we investigated the risk of type 2 diabetes in association with dietary intake of carbohydrates and the effect of substitution of carbohydrates for other energy producing nutrients in a large prospective study.

Methods: A total of 25,639 men and women aged 40-79 were recruited in the European Prospective investigation into Cancer in Norfolk study. Among participants free of known diabetes, cancer, myocardial infarction and stroke at baseline, incident cases of diabetes $(\mathrm{N}=749)$ were identified over a median follow up of 6 years, and were compared with a randomly selected sub-cohort of 3,496 participants. Seven-day food diary administered at baseline was used for dietary assessment. We performed modified Cox-proportional hazards regression analyses and compared results from the different methods of adjustment for total energy intake. Multivariable models were adjusted for age, sex, body mass index, family history of diabetes, physical activity, smoking, units of alcohol intake per week, and level of education.

Results: Dietary intakes of sucrose, starch, lactose, maltose, or total carbohydrates were not significantly related to diabetes risk after adjustment for confounders. However, in the residual method for energy adjustment, intakes of fructose and glucose were inversely related to diabetes risk. The multivariable adjusted hazard ratio $(95 \%$ CI) of diabetes comparing extreme quintiles of intake was 0.79 $(0.59-1.07, P$-value for trend 0.03$)$ for glucose and $0.62(95 \% \mathrm{CI}$ $0.46-0.83 ; P$ for trend 0.01 ) for fructose. In the nutrient density method, only fructose was inversely related with diabetes (HR 0.65 , $95 \%$ CI 0.48-0.88). Exchanging $5 \%$ energy intake from fructose for saturated fatty acids was associated with $30 \%$ lower diabetes risk (HR 0.69, $95 \%$ CI 0.50-0.96). Results of the standard and energy partition method were similar to the residual method.

Conclusions: These prospective findings suggest that dietary intakes of starch and sucrose are not associated with diabetes risk. However, replacing other sources of energy (specifically saturated fatty acids) with fructose and glucose is inversely associated with diabetes risk. Whether the inverse associations with fructose and glucose merely reflect the effect of substitution of these carbohydrate sub-types for other nutrients, the effect of their net higher intake, or the effect of other nutrients associated with their intake, remains to be established through further investigation.

\section{0-007}

Do socioeconomic and early life-factors influence the risk of becoming overweight or obese in children of Swedishand foreign-born parents?

\section{Presenter: Amal Khanolkar}

Amal Khanolkar 1), 2); Ulla Sovio 3); Jonathan Bartlett 3); Thomas Wallby 4); Ilona Koupil 1)

1) Centre for Health Equity Studies (CHESS), Karolinska Institutet/ Stockholm University; 2) Institute for Environmental Medicine, Karolinska Institutet; 3) Faculty of Epidemiology and Population Health, London School of Hygiene and Tropical Medicine; 4) Central Child Healthcare Unit, Uppsala Akademiska Hospital

Background: Ethnic minorities/immigrants have differential health compared to natives. It is not clear if immigrants/ethnic minorities and lower socioeconomic groups have equally benefitted from the 'levelling-off' recently observed in the child overweight and obesity (OW/OB) epidemic in Sweden.

Objectives: To investigate (i) if non-ethnic Swedish children are at increased risk for being OW/OB compared to ethnic Swedish children, and (ii) if this association can be explained by differences in 
parental socioeconomic position (SEP) and/or health behaviours and early life factors (breastfeeding, maternal smoking, maternal BMI and birth weight).

Methods: We analysed data on 10,628 singleton children (51\% boys, mean age 4.8 years, born 2000-2004), residing in Uppsala County. We used IOTF's sex- and age-specific cut-offs to define OW/OB. Ethnicity was based on maternal nativity. Parental SEP was based on education, disposable income and cohabitation status. Ethnicity-OW/ $\mathrm{OB}$ associations were analysed using multivariable logistic regression with adjustment for potential confounders. An additional model with father's nativity was fitted in conjunction with mother's nativity.

Results: $19 \%$ of girls and $14 \%$ of boys were OW/OB. Children of North African, South American and Turkish ethnicity had increased odds for being OW/OB compared to ethnic Swedish children [OR $1.96,95 \%$ CI (1.20-3.17), 2.53 (1.60-4.02), and 2.31 (1.41-3.80), respectively and adjusted for age, sex and family clustering]. Adjustment for SEP did not change the OR materially for these three groups. Adjustment for breastfeeding, smoking and birth weight increased OR by $20-40 \%$ and OR in Iranian children became statistically significant $(1.64,1.00-2.70)$. Adjustment for maternal BMI further increased OR in North African and Turkish groups. OR after adjustment for all covariates were 2.60 (95\% CI 1.57-4.27), 1.68 (1.03-2.72), 3.00 (1.86-4.80), and 2.91 (1.73-4.87) for the North African, Iranian, South American and Turkish groups respectively. The model including both maternal and paternal ethnicity showed evidence of an independent association with $\mathrm{OW} / \mathrm{OB}$ for maternal $(p<0.0001)$, but not for paternal nativity $(p=0.70)$. Formal tests for interaction with gender were statistically non-significant.

Conclusions: Certain ethnic groups are at increased risk for OW/OB in early childhood. While parental SEP did not explain the observed differences, certain risk factors like maternal smoking, BMI and breast feeding could be targeted to help reducing ethnic disparities in $\mathrm{OW} / \mathrm{OB}$ to some extent. This is important given that OW/OB often tracks into adulthood.

\section{O-008}

Inequalities in childhood thinness: how do they compare to overweight and obesity? Findings from the UK Millennium Cohort Study

Presenter: Anna Pearce

\section{Anna Pearce 1); Russell Viner 2); Lee Hudson 2);} Catherine Law 1)

1) MRC Centre of Epidemiology for Child Health, UCL Institute of Child Health; 2) General and Adolescent Paediatrics Unit, UCL Institute of Child Health

Background: Childhood overweight and obesity has increased dramatically in developed countries. Research effort has responded accordingly, and it is now well established that children from disadvantaged socio-economic circumstances (SECs) are more likely to be overweight or obese. However, there is limited contemporary research examining the prevalence and social distribution of thinness, despite its serious consequences for children's health and development and mortality in adulthood.

Objectives: To examine inequalities in thinness, overweight and obesity in 16,715 Millennium Cohort Study children with objective body mass index (BMI, $\mathrm{kg} / \mathrm{m}^{2}$ ) at ages 3, 5 and 7 years.

Methods: Weight status was classified with International Obesity Task-Force cut-offs: very thin (equivalent adult BMI $<17$ ), thin (BMI 17-18.4), normal weight, overweight (BMI 25-29.9), or obese (BMI 30+). We used multinomial logistic regression to estimate relative risk ratios (RRRs) and $95 \%$ confidence intervals for weight status (baseline, normal BMI), by several SECs measures: social class, mother's education, equivalised income, area deprivation. Associations did not vary with age, so we pooled data and adjusted for repeated measures. Ethnic minority children were more likely to be thin and to live in disadvantaged SECs; therefore we repeated analyses in White children only.

Results: As many children were thin $(5.2 \%$, of which $1 \%$ were very thin) as obese $(5.7 \%)$. The risk of obesity increased incrementally with disadvantage, whereas for thinness, only the most disadvantaged groups were at an elevated risk. For example, compared to children whose mothers had degrees, children whose mothers had no educational qualifications were more than twice as likely to be obese $(\mathrm{RRR}=2.32[1.90-2.85])$, followed by those whose mothers who had general (1.82 [1.51-2.19]) or advanced (1.36 [1.09-1.70]) secondary-school qualifications. In contrast, only children whose mothers had no educational qualifications were more likely to be thin (1.45 [1.19-1.75]) or extremely thin (1.64 [1.13, 2.39]); RRRs were $<1$ and non-significant for children whose mothers had general or advanced secondary-school qualifications. Inequalities in overweight were small and frequently non-significant. These same patterns were observed for all other SECs measures. When analyses were restricted to White children, inequality in obesity was unaffected. Inequality in thinness was reduced, but often remained significant.

Conclusions: Thinness is as prevalent as obesity in early childhood. In contrast to a strong social gradient in obesity, a threshold effect was observed for thinness, with only the most disadvantaged children at an elevated risk. Researchers and policymakers should focus on the environmental determinants of thinness as well as obesity.

\section{0-009}

Associations between glycaemic deterioration and aortic stiffness in screen-detected non-diabetic individuals: the ADDITION-PRO Study

Presenter: Nanna B Johansen

Nanna B Johansen 1), 2); Signe S Rasmussen 3); Niels Wiinberg 4); Erling B Pedersen 5); Torsten Lauritzen 6); Annelli Sandbæk 6); Daniel R Witte 7)

1) Department of Clinical Pharmacology, Bispebjerg Hospital, University of Copenhagen, Copenhagen, Denmark; 2) Steno Diabetes Center A/S, Gentofte, Denmark; 3) Department of EndocrinologyGastroenterology, Bispebjerg Hospital, University of Copenhagen, Copenhagen, Denmark; 4) Department of Clinical Physiology, Frederiksberg Hospital, Frederiksberg, Denmark; 5) Department of Medical Research, Holstebro Hospital and University of Aarhus, Holstebro, Denmark; 6) Department of Public Health, Section of General Practice, Faculty of Health Sciences, Aarhus University, Aarhus, Denmark; 7) Centre de Recherche Public de la Santé, Strassen, Luxembourg

Background: Besides identifying individuals with unknown diabetes, screening programmes for diabetes also identify individuals without diabetes but at increasing levels of diabetes risk. However, little evidence exists regarding the cardiovascular risk among individuals with normoglycaemia despite a high diabetes risk. Furthermore, screening programmes for diabetes include regular reassessments of glycaemia in high risk individuals. Quantification of the impact of changes in glycaemia over and above the current glycaemic level is therefore essential in the cardiovascular risk stratification.

Objectives: We examined levels of aortic stiffness, an early marker of cardiovascular disease, among individuals with different levels of diabetes risk and examined the impact of glycaemic changes on aortic stiffness. 
Methods: A Danish population-based stepwise screening program for diabetes identified groups of individuals at increasing levels of diabetes risk. After 7.8 years (IQR 6.5;8.8) of follow-up, 2,048 individuals underwent assessment of aortic stiffness by carotid-femoral pulse wave velocity (aPWV). We compared differences in aPWV at follow-up between the diabetes risk groups defined at screening. Furthermore, we examined the impact of glycated haemoglobin A1c (HbA1c) at screening and change in HbA1c during follow-up on aPWV at follow-up. The analyses were adjusted for age, sex, changes in waist circumference, heart rate, mean arterial pressure, lipid lowering and antihypertensive medication, and incident diabetes during follow-up.

Results: At screening, median age was 59.0 years (IQR 54.2;63.7) and median HbA1c was $5.7 \%$ (IQR 5.4;6.0). Compared to individuals with high diabetes risk but normal glucose tolerance, individuals with low diabetes risk had similar levels of aortic stiffness, whereas individuals with impaired glucose regulation had higher levels of aortic stiffness. Both $\mathrm{HbAlc}$ at screening and change in $\mathrm{HbA1c}$ during follow-up were positively associated with aPWV at follow-up in the basic model. After adjusting for confounders, aPWV was $0.23 \mathrm{~m} / \mathrm{s}$ (95\% CI $0.00 ; 0.46)$ higher per $1 \%$-point $\mathrm{HbA} 1 \mathrm{c}$ at screening, and $0.08 \mathrm{~m} / \mathrm{s}(95 \% \mathrm{CI}-0.07 ; 0.23)$ higher per $0.1 \%$-point annual increase in $\mathrm{HbA} 1 \mathrm{c}$.

Conclusions: After 7.8 years of follow-up, aortic stiffness was similar across groups of individuals with high risk of diabetes but with normoglycaemia and individuals with low diabetes risk, indicating that individuals with normoglycaemia despite high risk of diabetes may be regarded as having low cardiovascular risk. Furthermore, we found an indication that $\mathrm{HbAlc}$ and increases in $\mathrm{HbAlc}$ over time were associated with aortic stiffness, suggesting that regular reassessment of glycaemia may have an added value in cardiovascular risk assessment in individuals at high risk of developing diabetes.

\section{Cancer incidence and risk factors}

12.08.2013 11:00-12:30

\section{O-010}

International trends in the incidence of cutaneous malignant melanoma 1953-2008: signs of a levelling off in young generations?

\section{Presenter: Friederike Erdmann}

Friederike Erdmann 1); Joannie Lortet-Tieulent 2); Joachim Schüz 1); Hajo Zeeb 3); Rüdiger Greinert 4); Eckhard W. Breitbart 4); Freddie Bray 2)

1) Section of Environment and Radiation, International Agency for Research on Cancer (IARC); 2) Section of Cancer Information, International Agency for Research on Cancer; 3) Department of Prevention and Evaluation, BIPS - Institute for Epidemiology and Prevention Research; 4) Association of Dermatological Prevention (ADP) e. V

Background: The incidence of malignant melanoma has steeply increased over the past decades in predominately fair-skinned populations. Meanwhile the increase is reported to have levelled off in several Northern and Western European countries, North America and in Australia and New Zealand.

Objectives: For a better understanding of the global evolution of melanoma occurrence, we studied patterns and trends of melanoma incidence worldwide, with a focus on time trends analyses in different age groups and trends in successive birth cohorts.
Methods: We analysed incidence data from 84 high quality cancer registries (regional and national) in 39 countries worldwide, calculating the cumulative risk and age-standardised (world) incidence rates of melanoma, the estimated annual percentage change in the last decade available (with $95 \%$ confidence intervals) alongside observed rates versus period and birth cohort for each age group and incidence rate ratios obtained on fitting age-period-cohort models.

Results: The highest rates of melanoma are by far those observed in New Zealand and Australia, with a lifetime risk of $3.6 \%$, compared with $1.9 \%$ in the US. Whites, $1.1 \%$ in Canada and ranging from 0.3 to $1.6 \%$ in European countries. In Europe, rates are elevated in the Nordic populations, Switzerland, the Netherlands, and Czech Republic. Trends in Australia, New Zealand, Canada, the US, Israel, and Norway (and recently in Iceland and France) suggest some stabilisation in incidence, most notably among younger age groups. In some contrast, in most other European populations, incidence rates continue to rise in all ages.

Where rates are high and have become stable or declined-in Australia, New Zealand, the US, Canada and Norway, uniform increases in rates are seen in successive birth cohorts born up to the late-1940s, followed in more recent generations by a stabilisation or decline. As well as cohort effects, a period-related influence on the trends was also detected, in certain populations.

Conclusion: The recent observed trends in melanoma appear to be more related to birth cohort than calendar period effects, while the time lag between changing UV exposure and the resulting stabilisation in incidence might be too short to be the direct result of skin cancer prevention activities. Incidence rates of melanoma continue to rise in most European countries, although the reduction in the incidence of melanoma observed in several high-risk countries among younger people and recent birth cohorts is encouraging. The temporal patterns highlight the importance of targeted UV radiation awareness campaigns.

\section{O-011}

Familial risk of colorectal cancer, when high risk families with known genetic predisposition are excluded: a Danish cohort study

Presenter: Charlotte K. Lautrup

Charlotte K. Lautrup 1), 2); Ellen M. Mikkelsen 2); Timothy L. Lash 2), 3); Niels Katballe 4); Lone Sunde 1)

1) Department of Clinical Genetics, Aarhus University Hospital; 2) Department of Clinical Epidemiology, Aarhus University Hospital; 3) Department of Epidemiology, Emory University, USA; 4) Department of Cardiothoracic Surgery, Aarhus University Hospital

Purpose: The risk of colorectal cancer (CRC) has been estimated to be increased twofold in individuals with at least one first degree relative (FDR) diagnosed with CRC, rising to a 3-4 fold if more than one relative is affected or if the relative with CRC was diagnosed young. However, most studies estimating CRC risk in FDRs have not excluded patients with high risk monogenetic syndromes such as Lynch Syndrome. Although these syndromes only account for a small part of all CRC cases, including the syndromes may overestimate the risk of CRC for FDRs whose families do not have the syndrome.

Objective: We aimed to investigate familial risk of CRC after excluding high risk families with Lynch syndrome/HNPCC or FAP. Methodology: We conducted a prospective cohort study including FDRs to 1,196 probands diagnosed with primary CRC in 1995-1998. FDRs were identified using the Danish Civil Registration System (DCRS) in combination with church books, censuses and population registry cards. Information on CRC incidence among all the FDRs 
was sourced from The Danish Cancer Registry and the Danish National Registry of Patients. Status on CRC incidence in each family was made at the date of the proband's diagnosis and high risk families were excluded. FDRs living without CRC at this date were included in the follow-up cohort. Using the DCRS we established a comparison cohort by matching the FDRs on age and gender with 10 individuals from the background population. We followed both cohorts to either event of CRC, death or December 312010 and calculated standardized incidence ratios (SIR) comparing the risk in the FDRs to the comparison cohort.

Results: After excluding 26 high risk families, 4,185 FDRs from 1,061 families were included. We found an overall increased risk of CRC in FDRs to CRC patients; SIR 1.77 (95 \% CI 1.45-2.15). For FDRs to patients diagnosed before the age of 50 the SIR was; 2.23 (95\% CI 0.92-5.40), whereas the risk estimates for FDRs who had two or more relatives with CRC did not differ from the overall estimate; SIR 1.79 (95\% CI 1.22-2.60).

Conclusion: Once excluding high risk syndromes, we find the overall risk in FDRs to CRC patient to be comparable with results of previous studies. The risk of CRC in individuals with two or more FDRs with $\mathrm{CRC}$ or one FDR diagnosed young may, however, be lower than reported in previous studies.

\section{0-012}

Previous pulmonary disease and lung cancer risk in a multi-national consortium of case-control studies

\section{Presenter: Rachel Denholm}

Rachel Denholm 1); Ann Olsson 1), 2); Desh Deepak 3); Isabelle Stücker 4), 5); Karl-Heinz Jöckel 6); Kurt Straif 1); Joachim Schüz 1)

1) International Agency of Research on Cancer, Lyon, France; 2) The Institute of Environmental Medicine, Karolinska Institutet,

Stockholm, Sweden; 3) Dr. R.M.L. Hospital, New Delhi, India; 4)

Inserm, Centre for Research in Epidemiology and Population Health, Villejuif, France; 5) Université Paris-Sud, Villejuif, France; 6) Institute for Medical Informatics, Biometry and Epidemiology, University of Duisburg-Essen, Essen, Germany; 7) on behalf of the SYNERGY-INCA study group

Background: Recent evidence suggests previous pulmonary diseases (PPD), such as chronic bronchitis, emphysema, tuberculosis, pneumonia and asthma, are associated with an increased risk of lung cancer. Few studies have investigated multiple conditions simultaneously, or the relationship between PPD and age of lung cancer diagnosis.

Objectives: Assess the risk of lung cancer associated with bronchitis, emphysema, tuberculosis, pneumonia and asthma by sex and age of lung cancer diagnoses.

Methods: Data from four studies (10 centers) in the SYNERGY project, a consortium of case-control studies from Europe and North America, had pooled information on all 5 PPD for 8,601 lung cancer cases and 9,680 age- and sex-matched controls (1988-2005). All PPD were included in multivariate logistic regression models simultaneously, which were stratified by sex, and adjusted for age, center, high-risk occupation, level of education, smoking status, cigarette pack-years and time-since quitting smoking. Multinomial logistic regression models investigated the relationship between PPD and age of lung cancer diagnoses; $<55,55-62.9,63-68.9$ and $\geq 69$ years.

Results: Of participants with one PPD diagnosis, between 35 and $82 \%$ reported another PPD, depending on the disease. Lung cancer was positively associated with pneumonia in men and women (odds ratio $(\mathrm{OR})=1.24 ; 95 \%$ confidence interval $(\mathrm{CI}): 1.13,1.37$,
$\mathrm{OR}=1.26$; CI 1.05,1.52, respectively); and among men with bronchitis and emphysema ( $\mathrm{OR}=1.34$; CI 1.20,1.48, OR $=1.50 ; \mathrm{CI}$ $1.20,1.87$, respectively). Asthma was inversely associated with lung cancer; this was statistically significant for women $(\mathrm{OR}=0.72$; CI $0.53,0.98)$. Relationships remained significant when excluding PPD diagnosed $<2,5$ and 10 years prior to lung cancer diagnoses/interview (amongst studies/participants with information). Only for pneumonia in males did restriction to PPD diagnosed $\geq 2$ years prior to lung cancer/interview yield a non-significant association. Amongst men, all PPD were significantly associated with older age of lung cancer diagnoses. In men and women, emphysema was inversely associated with lung cancer diagnosed at age $<55$ year (though numbers were small), but positively associated at older ages (63-68.9 and $\geq 69$ year), compared to controls. There was little difference in risk when stratified by smoking status, though results were difficult to interpret in never smokers due to small numbers.

Conclusions: There was a high level of co-occurrence amongst PPD, with bronchitis, emphysema and pneumonia positively associated with lung cancer. In contrast, asthma was inversely related to lung cancer, possibly reflecting differences in exposure to known risk factors such as smoking status. We found no evidence that PPD's were associated with early age of lung cancer diagnoses.

\section{O-013}

Mortality and incidence of new primary cancers in men with prostate cancer: a danish population-based cohort study

\section{Presenter: Deirdre Cronin-Fenton}

Deirdre Cronin-Fenton 1); Sussie Antonsen 1); Michael Borre 2); Timothy Lash 3)

1) Department of Clinical Epidemiology, Aarhus University; 2) Department of Urology, Aarhus University Hospital, Skejby, Aarhus, Denmark; 3) Department of Epidemiology, Rollins School of Public Health, Emory University, Atlanta, GA, USA

Background: Prostate cancer (PC) survivors may have an increased risk of new primary cancers (NPCs) due to shared risk factors or PCdirected treatments.

Objectives: To examine mortality and incidence of NPCs in men diagnosed with PC compared with a matched comparison cohort of men without PC.

Methods: Using Danish registries, we conducted a cohort study of men with $(\mathrm{n}=30,220)$ and without $\mathrm{PC}(\mathrm{n}=151,100)$ (comparators), matched 1:5 on age and PC diagnosis/index date. We computed incidence rates of NPCs per 10,000 person years (PY) and associated $95 \%$ confidence intervals (CI), and used Cox proportional hazards regression to compute hazard ratios (HRs) and $95 \% \mathrm{CI}$, adjusting for comorbidities. In order to obviate any impact of shorter survival among PC patients, we censored comparator patients when the matched PC patient died or was censored.

Results: Follow-up spanned 113,487 PY and 462,982 PY in the PC and comparison cohorts, respectively. $65 \%$ of the cohorts were aged $>70$ years at diagnosis. Among PC patients, $51 \%$ had distant/ unspecified stage, and $63 \%$ had surgery as primary treatment. The PC cohort had lower incidence of NPCs than their comparators. The adjusted HR of NPC among men with PC versus the comparators was $0.84(95 \% \mathrm{CI}=0.80,0.88)$. Lowest HRs were among older men, those with distant stage, and were particularly evident for cancers of the brain, liver, pancreas, respiratory, upper gastrointestinal, and urinary systems.

Conclusions: We find no evidence of an increased risk of NPCs among men with PC. The deficit of NPCs among men with PC may be a true effect but is more likely due to lower levels of risk factors 
(e.g., smoking) in PC patients versus comparators, clinical consideration of cancers at new organs as metastases rather than new primaries, or under-recording/under-reporting of NPCs among PC patients.

\section{0-014}

\section{Sarcoidosis and cancer risk: a Danish nationwide cohort study}

\section{Presenter: Mette Norgaard}

Mette Nørgaard 1); Reimar Wernich Thomsen 1); Claus Sværke 1); Kistine Kobberøe Søgaard 1); Henrik Toft Sørensen 1)

1) Department of Clinical Epidemiology, Institute of Clinical Medicine, Aarhus University Hospital, 8200 Aarhus N, Denmark

Background: The inflammation caused by sarcoidosis may increase the risk of cancer. Previous studies have found an increased risk of cancer overall and haematological cancers and lung cancer in particular. Yet, data are inconsistent.

Objectives: To examine the incidence of various cancers after a firsttime hospital contact with sarcoidosis

Methods: We linked nationwide data from the Danish National Registry of Patients covering all Danish hospitals and the Danish Cancer Registry. We compared observed versus expected incidences of cancer in patients who had a first-time hospital contact with sarcoidosis based on age-, sex-, and anatomical site-specific incidence rates in the Danish general population.

Results: Among 10,604 patients with a diagnosis of sarcoidosis, we identified 935 cancers (median follow-up 8.9 years), corresponding to a standardized incidence ratio (SIR) of 1.38 ( $95 \%$ confidence interval (CI): 1.29-1.47). In the first year after the sarcoidosis diagnosis, the overall SIR for cancer was 3.99 (95\% CI 3.45-4.59). In the second and subsequent years of follow-up, the SIR for any cancer was 1.17 (95\% CI 1.09-1.26). We found particular high SIRs for Hodgkin's disease, SIRs $=31.77(95 \%$ CI 14.55-60.35) for the first year of follow up and 1.94 (95\% CI 0.63-4.53) for the second and following years, for non-Hodgkin lymphoma, SIRs $=20.09 \quad(95 \%$ CI 13.00-29.66) for the first year and 1.95 (95 \% CI 1.33-2.75) for the following years, cancer of the tonsils, SIRs $=19.54(95 \%$ CI 12.24-29.59) for the first year and 1.70 (95\% CI 1.05-2.60) for the following years, and myeloid leukemia, SIRs $=6.73(95 \% \mathrm{CI}$ 1.39-19.67) for the first year and 1.74 (95 \% CI 0.80-3.30) for the following years. For lung cancer we found an increased risk in the first year of follow-up (SIR $=7.32$ (95\% CI 5.21-10.01) but not in the second and following years (SIR $=0.73$ (95\% CI 0.54-0.96).

Conclusions: In the first year after a hospital presentation with sarcoidosis, we found a four-fold increase in the risk of any cancer, largely dependent on the increased risk of haematological cancers. In subsequent years, a $17 \%$ increase in overall cancer risk persisted. These findings could, however, be explained by detection bias because patients with sarcoidosis are monitored by regular clinical examinations and chest X-rays.

\section{0-015}

Risk of hepatocellular carcinoma in relation to the intakes of natural fruit juices and sweetened non-alcoholic beverages within the EPIC cohort

Presenter: Magdalena Stepien

Magdalena Stepien); Talita Duarte-Salles 1); Veronika Fedirko 2); Antonia Trichopoulou 3); Christina Bamia 3); Isabelle Romieu 1); Mazda Jenab 1)
1) International Agency for Research on Cancer (IARC-WHO), Lyon, France; 2) Rollins School of Public Health, Emory University, Atlanta, USA; 3) Hellenic Health Foundation, Athens, Greece

Background: Hepatocellular carcinoma (HCC) represents the majority of primary liver cancers. It has poor prognosis and high mortality. In western countries, HCC incidence is increasing, possibly due to increasing prevalence of obesity, diabetes mellitus and nonalcoholic fatty liver disease. Increasing intakes of high sugar nonalcoholic beverages have been linked to the incidence of these HCCassociated diseases, but few prospective studies have investigated their direct relationship with HCC.

Objectives: To study the association between intakes of natural fruit and vegetable juices (referred to as "juices") and sweetened nonalcoholic beverages (including soft drinks and sweetened juices; referred to as "drinks") and the risk of HCC.

Methods: Within a large cohort of over 520,000 participants from ten Western European Countries, 191 HCC cases were identified after a mean of 11 years of follow up. Dietary intakes were assessed by country-specific dietary questionnaires.

Both crude (adjusted for total energy intake) and multivariable (additionally adjusted for relevant confounders) Cox regression models were used to estimate hazard ratios (HR) and $95 \%$ confidence intervals $(\mathrm{CI})$ for HCC risk, comparing non-consumers to tertiles of consumers (T1, T2, T3). Risks were also assessed with intake as portions equivalent to $200 \mathrm{~mL}$ for "juices" and $330 \mathrm{~mL}$ for "drinks". Results: The median consumption of "juices" and "drinks" among the consumers was 42.9 and $50 \mathrm{~g} / \mathrm{day}$, respectively. Highest intakes of "juices" were observed for Germany (median $112 \mathrm{~g} / \mathrm{d}$ ), while the Netherlands represented the highest consumption of "drinks" (median $92 \mathrm{~g} / \mathrm{d}$ ). For "juices", the lowest tertile of consumers was significantly associated with reduced risk of HCC after adjustment for confounders $(\mathrm{T} 1: \mathrm{HR}=0.52,95 \% \mathrm{CI} 0.32-0.84 ; \mathrm{T} 2: \mathrm{HR}=0.63$, $95 \%$ CI 0.39-1.02; T3: HR $=0.84,95 \%$ CI 0.53-1.33). Considering intake as portions, a similar pattern was observed with consumption of less than a glass a week $(200 \mathrm{ml})$ associated with reduced $\mathrm{HCC}$ risk $(\mathrm{HR}=0.60,95 \%$ CI 0.38-0.95). For "drinks", compared to non-consumers the highest tertile of consumers showed an increased risk of HCC after adjustment for confounders (T1: $\mathrm{HR}=0.82,95 \%$ CI $0.52-1.3 ; \mathrm{T} 2: \mathrm{HR}=0.94,95 \%$ CI $0.60-1.48$; T3: HR $=1.46,95 \%$ CI 0.99-2.16). Accordingly, consumption of more than six cans per week $(330 \mathrm{ml})$ of drinks was significantly associated with increased risk of HCC, as compared to non-consumers $(\mathrm{HR}=1.83,95 \%$ CI 1.11-3.02); no significant associations were observed for lower intakes.

Conclusion: Daily intake of sweetened non-alcoholic beverages, but not natural juices, may contribute to HCC development; some intake of natural juices may be beneficial.

\section{O-016}

\section{Cancer occurrence in offspring of long-lived siblings}

Presenter: Jacob Krabbe Pedersen

\section{Jacob Krabbe Pedersen 1); Axel Skytthe 1); Kaare Christensen 1 )}

1) Department of Epidemiology, University of Southern Denmark

Background: Longevity is of interest as it is generally a marker of successful ageing, e.g. previous Danish studies have shown that increasing longevity is associated with less hospitalization earlier in life. While it is well documented that there is a familial component to longevity the underlying mechanisms are largely unknown. The availability of unique high quality registry data on off-spring of longlived families and cancer incidence makes it possible to compare the 
incidence of different types of cancer in offspring to that in the entire Danish population.

Objectives: We hypothesize that cancer incidence in offspring of long-lived families is lower than in the general population.

Methods: In 2004, the Danish Civil Registration System and parish birth records were combined to identify 3,638 long-lived families with at least two living siblings having reached the age of 88 years. Through interviews the complete pedigree of 646 families were established. By linking each of the identified offspring to the Danish Cancer Registry, we studied cancer incidence after April 2nd, 1968. We compared overall cancer incidence as well as breast cancer, colon cancer, lung cancer and tobacco-related cancer. By calculating standardized incidence rates (SIRs), observed cancers in offspring to long-lived siblings were compared to expected numbers based on gender-, calendar period-, and age-specific incidence rates in the general population. Analyses were based on Poisson regression, using robust standard deviation estimates to adjust for familial clusters.

Results: A total of 5,343 offspring from 646 families were included in the analyses. Compared to the background Danish population, cancer incidence among offspring was lower for overall, lung and tobacco-related cancer, while for breast and colon cancer the incidence in offspring was similar to or moderately lower. The main SIR (95\% confidence interval) results were: $0.75(0.69-0.81)$ for overall cancer; $0.88(0.74-1.05)$ for breast cancer; $0.87(0.65-1.18)$ for colon cancer; $0.35(0.26-0.49)$ for lung cancer; $0.65(0.57-0.74)$ for tobacco-related cancer.

Conclusions: Offspring of long-lived siblings have lower cancer incidence, in particular lung cancer, suggesting that avoidance or postponement of cancer partially explains clustering of long lives in these families. The low lung cancer occurrence in offspring suggests environmental factors as key to longevity in these families.

\section{0-017}

Genetic influence on lung cancer based on the nordic twin study of cancer (NorTwinCan) cohorts

Presenter: Jacob Hjelmborg, Axel Skytthe

Jacob Hjelmborg 1); Tellervo Korhonen 2); Lorelei Mucci 3); Thomas Scheike 4); Axel Skytthe 1); Jaakko Kaprio 5)

1) Biostatistics and Epidemiology, Institute of Public Health, University of Southern Denmark, Odense, Denmark; 2) Department of Public Health, Hjelt Institute, University of Helsinki, Finland; 3) Cancer Epidemiology, Harvard School of Public Health, Boston, USA; 4) Department of Biostatistics, University of Copenhagen, Denmark; 5) Department of Public Health, Hjelt Institute, University of Helsinki, Finland and National Institute for Health and Welfare, Helsinki, Finland and Institute for Molecular Medicine (FIMM), Helsinki, Finland

We study the genetic and environmental influences on lung cancer using the Nordic twin cohorts. The combined Nordic cohorts of twins consists of more than 154,000 twin pairs of which 133 are concordant for lung cancer and 2,690 are discordant at follow-up of twins born since 1870. The cumulative incidence in the twins agrees well with that of the background population when taking censoring and the competing risk of death into account. The lifetime risk of lung cancer in a twin is 3.6 ( $\mathrm{SE}=0.6)$ times higher if the co-twin has lung cancer and the pair is monozygotic. For dizygotic pairs this relative risk is estimated at $2.4(0.3)$. This is indicating familial and further genetic influences to lung cancer. We consider how the concordance varies over time depending on zygocity and smoking status giving insight to the action of genetic and environmental causes.

\section{O-018}

Cancer risk in parous women following fertility treatment: utilising the medical birth registry and the cancer registry of Norway

Presenter: Marte Myhre Reigstad

Marte Myhre Reigstad 1); Inger Kristin Larsen 2); Trude Eid Robshamn 2); Nan Oldereid 3); Siri Vangen 1); Ritsa Storeng 1)

1) Norwegian Resource Centre for Women's Health, Women and Children's Division, Oslo University hospital, Rikshospitalet; 2)

Cancer Registry of Norway; 3) Section for Reproductive Medicine, Women and Children's Division, Oslo University hospital, Rikshospitalet

Background: Although numerous studies have sought to explore the effects of hormones used in assisted reproductive technology (ART) on cancer risk, the conclusions are ambiguous as to whether or not an increased risk may be due to the fertility treatment.

Objectives: The purpose of this study is to investigate the cancer risk for parous women who gave birth following ART, either in vitro fertilisation (IVF) or intracytoplasmic sperm injection (ICSI), and to compare it to the cancer risk in women who gave birth after natural conception.

Methods: Data from the Medical Birth Registry of Norway (MBRN) was linked to the Cancer Registry of Norway (CRN) through a unique personal identification number. All 812,986 women who gave birth in Norway between January 1st 1984 and December 31st 2010 constituted the study cohort. The cancer risk in women exposed to fertility treatment was compared to the risk in unexposed women who conceived naturally. Incidence rate ratios (IR) and hazard rate ratios (HR) were calculated and Cox proportional hazards model was used, adjusting for possible confounders such as parity, age, time period and region of residence. Statistical analyses are being conducted for overall cancer, and separately for: breast cancer, gynaecologic cancers, malignant melanoma, thyroid cancer, and colorectal cancer.

Results: The 809,018 women eligible for the study, generated $12,317,721$ person years follow up, mean follow up time 15.2 years. Registry linkage gave 24,822 women with at least one cancer diagnosis between 1953 and the end of 2010. In total, 16,704 women gave birth to at least one child after fertility treatment during the period, and of these, 457 women were diagnosed with cancer at least once. Of these, 118 were diagnosed with cancer prior to fertility treatment $(3$ breast, 6 ovarian, 13 cervical, 16 thyroid, 13 other endocrine cancers, 13 cancers of the central nervous system, and 54 various other cancers). Three hundred and forty-six (346) women were diagnosed with cancer after fertility treatment ( 7 of these had a cancer diagnosis prior to ART) out of which 134 were breast cancers, 16 ovarian cancers, 25 cervical cancers, 4 uterine cancers, 14 thyroid cancers, 8 other endocrine cancers, 28 cancers of the central nervous system and 117 various other cancers.

Conclusion: Statistical analyses are in progress, but by the abstract submission deadline they are not finalised.

All results and conclusions from the study will be presented at the conference. 


\section{Infections}

12.08.2013 11:00-12:30

\section{O-019}

Measles-mumps-rubella-vaccination reduces hospital admissions due to non-targeted infections: a Danish Nationwide register based study

\section{Presenter: Signe Sørup}

Signe Sørup 1); Christine S. Benn 1), 2); Anja Poulsen 3); Tyra Krause 4); Peter Aaby 1), 2); Henrik Ravn 1), 2)

1) Research Center for Vitamins and Vaccines (CVIVA), Bandim Health Project, Statens Serum Institut, Copenhagen; 2) Bandim Health Project, Indepth Network, Bissau, Guinea-Bissau; 3) The Child \& Adolescent Clinic, Rigshospitalet, Copenhagen, Denmark; 4) Department of Infectious Disease Epidemiology, Statens Serum Institut, Copenhagen, Denmark

Background: In low-income countries, live measles vaccine reduces mortality from causes other than measles infection.

Objective: To examine whether MMR-vaccine against measles, mumps, and rubella reduces the risk of infectious disease hospital admissions in a high-income setting.

Methods: 506,461 Danish children born 1997-2006 were followed from 11 months to 2 years of age in Danish registers for vaccinations and hospital admissions; these services are free-of-charge in Denmark. We used Cox regression to estimate the admission incidence rate ratios (IRRs) with age as the underlying timescale. The model was adjusted for sex, birth weight, gestational age, caesarean section, chronic diseases, number of infectious disease admissions before 11 months of age, admitted to hospital for any cause within the last 30 days, maternal age at birth of the child, parental origin, adult composition of the household, other children in the household, and population density; furthermore the model was stratified by date of birth to control completely for any effect of age, season, and year.

Results: The study included 46,244 infectious disease hospital admissions during 445,379 person-years (rate 10.4/100 person-years). MMR was associated with an adjusted IRR of 0.86 (95\% confidence interval (CI), 0.84 to 0.88 ) compared to the third DTaPPHib-vaccination against diphtheria, tetanus, pertussis, polio, and haemophilus influenzae type $\mathrm{b}$ for the 485,876 children who followed the recommended schedule and received MMR after DTaPPHib3. The protective effect was strongest for lower respiratory infections (IRR, 0.79; $95 \%$ CI 0.76-0.83) and for admissions lasting more than 2 days (IRR, 0.80; $95 \%$ CI 0.76-0.84). 20,585 children reversed the schedule receiving DTaPPHib3 after MMR and this transition was associated with an increase in admissions [IRR, 1.56 (95 \% CI 1.25-1.95)]. Changes in IRR only appeared from 15 days after vaccination.

Conclusion: MMR-vaccine may protect against infectious disease admissions compared with DTaPPHib3-vaccine, irrespective of the sequence of the two vaccines. Hence, a beneficial immune training by MMR and not selection bias is likely to be the main explanation for the beneficial effect. The protective effect of MMR was particularly strong for lower respiratory infections and admissions lasting more than 2 days.

\section{O-020}

Are COPD and diabetes mellitus risk factors for herpes zoster?
Harriet Forbes 1); Krishnan Bhaskaran 1); Sara Thomas 1); Liam Smeeth 1); Sinead Langan 1)

1) London School of Hygiene and Tropical Medicine, London, UK

Background: The impact of severe immunosuppression on risk of herpes zoster is well established but the roles of more common conditions such as diabetes mellitus and chronic obstructive pulmonary disease (COPD), which may be moderately immunosuppressive, are poorly understood. Identifying any increase in herpes zoster risk associated with these morbidities would help to establish the potential benefits and risks of herpes zoster vaccination in affected patients.

Objectives: To investigate the risk of herpes zoster associated with COPD and diabetes mellitus.

Methods: A population based case control study was carried out using the UK Clinical Practice Research Datalink (CPRD) and linked hospital episodes statistics (HES) data. The cases comprised of 145,341 individuals with herpes zoster, aged $\geq 18$ years, with a first ever episode of herpes zoster between 2000 and 2011 in CPRD or linked HES. Cases were matched for age, sex, general practice and observation period to up to 10 controls. Controls had no history of herpes zoster at the index date (date of first zoster) of their matched case, however could develop herpes zoster later during the study period (concurrent sampling). Conditional logistic regression was used to account for the matched nature of the study population and to adjust for relevant confounders.

Results: The crude odds ratio (OR) for the association between COPD and herpes zoster was 1.50 (95\% CI 1.46-1.54), reducing to 1.15 (95\% CI 1.12-1.19) after adjusting for known immunosuppressive factors (HIV, leukaemia, lymphoma, myeloma, organ/tissue transplant and immunosuppressive therapy 3 months prior to the index date), autoimmune conditions (rheumatoid arthritis, systemic lupus erythematosus, Wegener's granulomatosis and inflammatory bowel disease), chronic kidney disease, smoking status, alcohol use and body mass index. The crude OR for the association between diabetes and herpes zoster was 1.29 (95\% CI 1.26-1.31), reducing to 1.02 (95\% CI 0.99-1.04) after adjusting for confounders.

Conclusions: We found no evidence of an association between diabetes and herpes zoster after adjusting for confounding. COPD appeared to be associated with some increased risk of herpes zoster. This may have implications for targeting use of the herpes zoster vaccination among COPD patients.

0-021

Trajectories of injecting behaviour in the Amsterdam Cohort Study among drug users

Presenter: Rafael Mikolajczyk

Rafael Mikolajczyk 1), 2); Maria Prins 3), 4); Lucas Wiesing 5); Mirjam Kretzschmar 6), 7)

1) Department of Epidemiology, Helmholtz Centre for Infection Research, Braunschweig, Germany; 2) Hannover Medical School, Hannover, Germany; 3) Department of Internal Medicine, Academic Medical Centre, CINIMA, Amsterdam, The Netherlands; 4) Public Health Service Amsterdam, Cluster Infectious Diseases, Department of research, Amsterdam, The Netherlands; 5) European Monitoring Centre for Drugs and Drug Addiction (EMCDDA), Lisbon, Portugal; 6) Julius Centre for Health Sciences and Primary Care, University Medical Centre Utrecht, Utrecht, The Netherlands; 7) Centre for Infectious Disease Control, RIVM, Bilthoven, The Netherlands

Background: Injecting behaviour among injecting drug users can change over time along distinct trajectories. The resulting risk changes are likely to reflect in changed incidence of HIV and HCV 
infections or other drug-related risks (e.g. overdose death) over time. We aimed to assess patterns of longitudinal changes in injecting behaviour, their predictors and their association with the incidence of HIV and HCV.

Methods: We analysed data from the Amsterdam Cohort Study among Drug Users. We selected participants who were recruited between April 1985 and November 2005 and who started injecting drugs before cohort entry and for whom records in at least three different 6 months intervals were available $(\mathrm{N}=740)$. A finite mixture model was used to identify distinct trajectories describing changes in injecting frequency and changes in the probability of current injecting over time in the cohort. Multinomial regression was applied to identify variables associated with specific patterns and Kaplan-Meier analysis for the estimation of the cumulative HIV and $\mathrm{HCV}$ incidence across these patterns.

Results: Five distinct patterns for injecting frequency and for probability of having injected in the last 6 months were identified. Although there was on average a decrease in injecting over time, most IDU displayed stable patterns of injecting. Male (OR 2.48 (1.34-4.58) for "constant daily injecting" vs. "rare to no injecting") and younger IDU (1.06 (1.01-1.21) per year), those with longer duration of injecting at cohort entry $(1.07(1.02-1.12)$ per year) and those who entering the cohort in earlier years $(1.30(1.17-1.45)$ per year) tended to have more risky drug use patterns. The cumulative HIV incidence and its changes over calendar time mirrored the patterns of change in injecting. The results for $\mathrm{HCV}$ were similar, but due to a higher seropositivity at cohort entry, the sample was too small for a rigorous analysis.

Conclusions: Individual longitudinal patterns of changes in injecting behaviour are related to socio-demographic and drug use variables and are reflected in the incidence of HIV infections. Understanding these patterns might provide valuable information for targeting interventions to improve prevention effectiveness.

\section{O-022}

Patient-important benefits of clearing the hepatitis $\mathrm{C}$ virus through treatment: a simulation study

Presenter: Hamish Innes

Hamish Innes 1), 2); David Goldberg 2), 3); Sharon Hutchinson 2), 3)

1) Health Protection Scotland; 2) University of Strathclyde; 3) Glasgow Caledonian University

Background: Persons with chronic hepatitis $\mathrm{C}$ virus (HCV) infection face a dilemma apropos whether to accept or forego a course of therapy that on one hand can permanently clear their viral infection (i.e. the optimal treatment outcome), but on the other, can induce a multitude of quality-of-life impairing adverse effects.

Objectives: To inform this decision point we explored (via a mathematical simulation) the clinical benefit of treatment-induced viral clearance according to patient age (30,45 and 60 years) and initial fibrosis stage (mild, moderate and severe liver fibrosis).

Methods: The HCV Individualised Treatment-decision model (the HIT-model) was created to simulate the lifetime course of liver disease, on a per patient basis, henceforth from two distinct scenarios. These being:

(i) Treatment-induced viral clearance attained, and

(ii) Treatment-induced viral clearance not attained.

Then, for each model subject, the course of liver disease under these alternative scenarios was compared. These comparisons focused on patient-important outcomes; in particular: (a) Whether viral clearance conferred additional total life years, and (b) Whether viral clearance conferred additional life years spent in asymptomatic health states.

Results: The probability of benefiting from treatment-induced viral clearance varied starkly according to individualised patient factors. It was lowest among patients at 60 years of age with initially mild fibrosis. For such patients the probability of gaining additional life years was $1.6 \%$ (95 \% CI 0.8-2.7), whilst the probability of gaining additional years in asymptomatic health states was $2.9 \%(95 \% \mathrm{CI}$ 1.5-4.7). In contrast, it was highest among patients with initially compensated cirrhosis aged 30 years. Here the probability of gaining additional life years was $57.9 \%$ (95\% CI 46.0-69.0), and the probability of incurring extra years in asymptomatic health states was $67.1 \%$ (95\% CI 54.1-78.2).

Conclusions: It is often fallaciously assumed that clearing $\mathrm{HCV}$ through treatment is a beneficial outcome in itself (the SVR is a SVR heuristic). In contrast, these data show that for older patients with less advanced liver fibrosis, viral clearance is unlikely to confer a tangible health benefit to the patient. This perspective is not conveyed in clinical guidelines or in information made available to patients. These data have far-reaching implications. Foremost, it may prove a deciding factor vis-à-vis the contemporary dilemma of immediate treatment with existing therapies (that have poor adverse effect profiles) versus awaiting future regimens that promise better tolerability.

\section{O-023}

Congenital cerebral palsy and prenatal exposure to self-reported maternal infections, fever or smoking

Presenter: Elani Streja

Elani Streja 1); Jessica Miller 1); Bodil Hammer Bech 2); Lars Henning Pedersen 2), 3); Marshalyn Yeargin-Allsopp 4); Kim Van Naarden Braun 4); Diana Schendel 4); Deborah Christensen 4); Peter Uldall 5); Jørn Olsen 1), 2)

1) Department of Epidemiology, School of Public Health, University of California, Los Angeles, California; 2) Section of Epidemiology, Department of Public Health, Aarhus University, Aarhus, Denmark; 3) Department of Obstetrics and Gynecology, Institute of Clinical Medicine, Aarhus University Hospital, Aarhus, Denmark; 4) National Center on Birth Defects and Developmental Disabilities, Centers for Disease Control and Prevention, Atlanta, Georgia; 5) The Danish Cerebral Registry, National Institute of Public Health, University of Southern Denmark; 6) Pediatric Department, Rigshospitalet, University of Copenhagen, Denmark

Background: Congenital cerebral palsy (CP) is the 2nd most common physical developmental disability in children. Maternal fever, maternal infections, and lifestyle factors such as maternal smoking have been associated with an increased risk of CP irrespective of gestational age. However, these previous studies were from hospital records and therefore subject to recall bias and additionally could not distinguish between infection types, particularly vaginal and urinary infections, nor adjust for a large number of potential confounders.

Objective: To investigate the association between maternal selfreported infections, fever and smoking in the prenatal period and subsequent risk for $\mathrm{CP}$.

Methods: We included the 81,066 mothers of singletons born between 1996 and 2003, who participated in the Danish National Birth Cohort. Children were followed through December 2008. Information on maternal infections, fever, smoking and other demographic and lifestyle factors during pregnancy were reported by mothers in computer-assisted telephone interviews in early and midgestation. We identified $139 \mathrm{CP}$ cases including 121 cases of spastic 
$\mathrm{CP}(\mathrm{sCP})$ as confirmed by the Danish National Cerebral Palsy Register. Cox proportional hazards regression models were used to estimate adjusted hazard ratios (aHR) and $95 \%$ confidence intervals. Results: Self-reported vaginal infections were associated with an increased risk of $\mathrm{CP}$ and SCP (aHR: 1.52; $95 \%$ CI 1.04-2.24 and aHR: 1.73 ; $95 \%$ CI $1.16-2.60$, respectively) and particularly untreated vaginal infections were associated with an increased risk of sCP (aHR: 1.95; $95 \%$ CI 1.16-3.26). Fever was associated with the risk of CP (aHR: 1.53; $95 \%$ CI 1.06-2.21). Smoking 10 or more cigarettes per day during pregnancy was also associated with $\mathrm{SCP}$ (aHR: 1.80; $95 \%$ CI 1.10-2.94). After excluding preterm births, the associations for $\mathrm{sCP}$ with vaginal infections and heavy smoking and for $\mathrm{CP}$ with fever were also significant. No other self-reported infections were significantly associated with $\mathrm{CP}$.

Conclusions: Self-reported vaginal infections, fever and smoking 10 or more cigarettes per day during pregnancy were associated with a higher risk of overall $\mathrm{CP}$ and/or sCP.

\section{0-024}

Risk factors for diarrhea in children under-2 years old in Madagascar: a case-control study

\section{Presenter: Rindra Randremanana}

Rindra Randremanana 1); Frédérique Randrianirina 1); Rita Affa 1); Richter Razafindratsimandresy 1); Charles-Emile Ramarokoto 1); Antoine Talarmin 2); Vincent Richard 3)

1) Institut Pasteur de Madagascar, BP 1274, Ambohitrakely, Antananarivo (101), Madagascar; 2) Institut Pasteur de la Guadeloupe, Morne Jolivière, 97183 Les Abymes cedex; 3) Institut Pasteur de Dakar, 36 Avenue Pasteur, B.P. 220 - Sénégal, DAKAR

Background and objectives: Diarrhea continues to be an important health problem in developing countries, especially among young children. In Madagascar, there is a lack of data about childhood diarrhea, little is known about the risk factors of this common disease. The objective of this study was to determine the risk factors for diarrhea in children below the age of 2 in Madagascar.

Methods: We conducted a community-based matched case-control study in children less than 2 years old in 14 districts of Madagascar from 2008 to 2009. Cases were diarrheal children, and controls were non diarrheal children, with the same age, gender and villages with cases. A standard questionnaire was administered to parents/guardians of cases and controls, questions included environmental exposures (household exposures, sanitation conditions, animals), characteristics of mother and the child. Stools specimen were collected and examined for presence of intestinal parasites, virus and bacteria. Multivariable conditional logistic regression models were used to assess the association between exposures and case-control status and were applied with factors selected based on results from bivariate analyses.

Results: We enrolled 182 cases and 182 matched-controls; $52.2 \%$ $(190 / 364)$ of children were female, and the mean age was 13.6 months $( \pm 5.7)$. A potential pathogen was detected in $45.6 \%$ (166/364) of children: $46.7 \%(85 / 182)$ of cases and $44.5 \%(81 / 182)$ of controls. Parasites were identified in $26.9 \%$ of cases and in $18.1 \%$ of controls. In the bivariate analysis, five variables were associated with diarrhea including the age of the child's mother, the household size, the number of children under-5 years old in the household, the presence of intestinal parasites and giardiasis. In the main model, the major diarrhea determinant was the presence of intestinal parasites such as Giardia lamblia ( $\mathrm{OR}=1.6$; CI $95 \%$ 1.4-17.4). Neither socio-economic status nor poor sanitation conditions were found to be associated with diarrhea.
Conclusions: Our results underline the role of intestinal parasite in the onset of diarrhea in Madagascar. It is concluded that measures about the community education on hygiene must be improved.

\section{O-025}

Reduced mortality after general measles vaccination campaign in rural Guinea-Bissau

Presenter: Ane Fisker

Ane Fisker 1); Amabelia Rodrigues 2); Cesario Martins 2); Stine Byberg 2); Sanne Thysen 2); Marie Pedersen 2); Manuel Fernandes 2); Henrik Ravn 1); Christine S. Benn 1); Peter Aaby 2)

1) Research Center for Vitamins and Vaccines (CVIVA), Bandim Health Project, Statens Serum Institut, 2300 Copenhagen S, Denmark; 2) Bandim Health Project, INDEPTH Network, Apartado 861, Bissau, Guinea-Bissau

Background: Measles vaccine (MV) protects against non-measles deaths in randomised trials. MV-campaigns are conducted to eliminate measles infection; no study has assessed the effect on overall mortality.

Objective: To evaluate the effect of an MV-campaign on child mortality.

Methods: Bandim Health Project follows children under 5 years of age in villages in rural Guinea-Bissau. The 2006-MV-campaign targeted children aged 6 months- 15 years. We compared mortality after the campaign among children eligible for the 2006-MV-campaign with mortality in the same age groups and time periods during the two previous years using Cox proportional-hazards models with age as underlying time-scale.

Results: A total of 8,160 children aged 6-59 months were under surveillance during the 2006-campaign and 8,004 and 8,111 at the same time the previous years. Among children eligible for the 2006-campaign, 165 died during 1 year of follow-up compared with 208 and 206 among their peers in 2004 and 2005, the mortality rate ratio (MRR) being $0.79(0.66-0.94)$; $0.85(0.62-1.16)$ for children aged $6-11$ months and $0.77(0.62-0.94)$ for children aged $1-4$ years. The mortality reduction tended to be more marked for girls (MRR = $0.72(0.56-0.91))$ than boys $(\mathrm{MRR}=0.85(0.64-1.13))$, and was stronger in the first 6 months after the campaign MRR $=0.76(0.61-$ 0.94) than 6-12 months after the campaign MRR $=0.86(0.58$ 1.27).

There were no measles deaths between 2005 and 2007 but 15 deaths in 2004 were classified as due to measles. Censoring measles deaths, the MRR was $0.82(0.68-0.97)$.

Conclusion: Mortality levels were stable during 2004 and 2005 but mortality dropped following the 2006-MV-campaign and the reduction was not explained by prevention of measles infection. The mortality reduction was strongest during the first 6 months after the campaign, more pronounced for children aged 1-4 years (who would not receive routine MV in the previous years) and it was more pronounced for girls. These facts support that the reduction was related to MV rather than a generally decline in mortality. If MV-campaigns reduce mortality unrelated to prevention of measles infection, the elimination of measles and phasing out of MV and campaigns may increase mortality.

\section{O-026}

Effectiveness of BCG against tuberculosis in adults: Ean historical cohort study

Presenter: Patrick Nguipdop Djomo 
Patrick Nguipdop Djomo 1); Punam Mangtani 1); Laura Rodrigues 1); Karin Ronning 2); Inger Cappelen 2); Einar Heldal 2); Ibrahim Abubakar 1)

1) London School of Hygiene and Tropical Medicine; 2) Norwegian Institute of Public Health

Background: Tuberculosis represents an occupational hazard for healthcare workers. The Bacillus Calmette-Guerin (BCG) vaccine is widely used to protect against tuberculosis. Its efficacy is likely similar on drug sensitive and drug resistant tuberculosis, which gives the vaccine an obvious appeal in HCWs' occupational health, in a context of resurgence of tuberculosis and emergence of multidrug resistant tuberculosis. Unfortunately there is little evidence on the effectiveness of the vaccine in adults to inform robust policy formulations on the place of BCG in occupational health.

Objectives: Assess BCG effectiveness against tuberculosis when administered to tuberculin-negative adults aged $30+$ years.

Methods: This was a population-based historical cohort study using the Norwegian-born population that underwent mass screening for tuberculosis and were offered BCG vaccination from 1962 to 1975. Only tuberculin negative subjects were included. Cox regression was used to compare rate of tuberculosis up till 2011 between vaccinated and unvaccinated subjects.

Results: 56,874 BCG vaccinated individuals and 42,239 unvaccinated were followed from 1962 up till 2011, for respective total follow-up of 2,097,150 and 1,470,960 person-years. The proportion of female was similar in groups, respectively 68 and $66 \%$. The median age at entry of vaccination was 40 years (IQR: 35-45 years). After controlling for current age and secular trends in tuberculosis rate, crude BCG effectiveness was $51 \%$ (95\% CI 24-69\%). This remained similar after adjusting for sex, occupation of the head of household at entry, type of municipality of residence (urban or rural) and household overcrowding (VE $53 \%, 95 \%$ CI 25-70 \%).

Conclusion: There is strong evidence that BCG can confer protection when administered to tuberculin negative adults. These results could be relevant to occupational health and travel medicine.

\section{Risk factors during pregnancy and child health}

12.08.2013 11:00-12:30

\section{O-027}

Obstetrician versus midwife supervision in low-risk deliveries: does it matter for newborns' health?

Presenter: Reyn Van Ewijk

\section{Reyn Van Ewijk 1); Meltem Daysal 2); Mircea Trandafir 3)}

1) Institute for Medical Biostatistics, Epidemiology and Informatics, University Medical Center Mainz, Germany; 2) Tilburg University, The Netherlands; 3) Universite de Sherbrooke, Canada

Background: Medical expenditures increased tremendously over the last few decades throughout the developed world. In response, ways are sought to decrease medical costs without harming health. Letting midwives take over tasks of obstetricians during low-risk deliveries might be one example for this. In The Netherlands, after a strict risk selection, half of all women are classified as low-risk. Their deliveries are entirely supervised by a midwife and no obstetrician is present, unless a complication arises during delivery.

Objectives: To assess whether midwife and obstetrician supervision are equally safe for low-risk deliveries.
Methods: We use the subsample of all 790,000 deliveries that were classified as low-risk until the onset of delivery in the 2000-2008 Dutch Perinatal Registry. Our analyses use the fact that in The Netherlands, these women generally deliver under supervision of a midwife, without the presence of an obstetrician. However, if their delivery starts before 37 completed weeks of gestation, it has to be supervised by an obstetrician; had their delivery started only slightly later, it would have been supervised by a midwife. This creates a socalled fuzzy regression "discontinuity design" that allows us to compare very similar deliveries that are supervised by different types of medical professionals. We estimate the effect of midwife versus obstetrician supervision on mortality and apgar scores of low-risk newborns using the econometric technique of Instrumental Variables. Results: We find no benefits of obstetrician supervision on the health outcomes of low-risk newborns with gestational ages around 37 completed weeks. Our results are robust to a large number of robustness checks.

Conclusion: This study suggests that supervision of deliveries by midwives, without the presence of an obstetrician is safe for low-risk deliveries with gestational ages close to 37 completed weeks.

\section{O-028}

Birth weight, head circumference and exposure to ambient air pollution during pregnancy: A European cohort study (Escape)

Presenter: Marie Pedersen

Marie Pedersen 1); Lise Giorgis-Allemand 2);

Mark J. Nieuwenhuijsen 3); Göran Pershagen 4); Ulrike Gehring 5); Bert Brunekreef 6); Manolis Kogevinas 7); Rémy Slama 9); on behalf of the ESCAPE birth outcome working group (EU Contract no. 211250). 10)

1) Centre for Research in Environmental Epidemiology (CREAL), Barcelona, Spain, INSERM U823, Team of Environmental Epidemiology Applied to Reproduction and Respiratory Health, Grenoble, France, CIBER Epidemiología y Salud Pública (CIBERESP), Barcelona, Spain; 2) INSERM U823, Team of Environmental Epidemiology Applied to Reproduction and Respiratory Health, Grenoble, France; 3) Centre for Research in Environmental Epidemiology (CREAL), Barcelona, Spain, CIBER Epidemiología y Salud Pública (CIBERESP), Barcelona, Spain; 4) Department of Occupational and Environmental Health, Stockholm, Sweden, Institute of Environmental Medicine, Karolinska Institutet, Stockholm, Sweden; 5) Institute for Risk Assessment Sciences, Utrecht University, Utrecht, The Netherlands; 6) Institute for Risk Assessment Sciences, Utrecht University, Utrecht, The Netherlands, Julius Center for Health Sciences and Primary Care, University Medical Center Utrecht, Utrecht, The Netherlands; 7) Julius Center for Health Sciences and Primary Care, University Medical Center Utrecht, Utrecht, The Netherlands; 8) Centre for Research in Environmental Epidemiology (CREAL), Barcelona, Spain, IMIM, Hospital del Mar Medicine Research Institute, Barcelona, Spain, CIBER Epidemiología y Salud Pública (CIBERESP), Barcelona, Spain, National School of Public Health, Athens, Greece; 9) INSERM U823, Team of Environmental Epidemiology Applied to Reproduction and Respiratory Health, Grenoble, France, University Joseph Fourier, Grenoble, France; 10) www.escapeproject.eu/

Background: Adverse effects of ambient air pollution on foetal growth have been reported, but findings are inconsistent.

Objectives: We evaluated the impacts of exposure to traffic-related air pollution during pregnancy on newborn size using standardized exposure assessment taking into account fine-scale spatial and temporal contrasts. 
Methods: We harmonised and pooled data on 74,000 singleton births (1994-2011) from 14 cohorts in 12 European countries, and applied the same protocol within the European Study of Cohorts for Air Pollution Effects (ESCAPE). Ambient nitrogen dioxide $\left(\mathrm{NO}_{2}\right)$, nitrogen oxides (NOX), particulate matter (PM10, PM2.5), PM2.5-10 and PM2.5 absorbance (soot) concentrations at the home addresses during pregnancy were estimated using temporally adjusted land-use regression models. Linear and logistic regressions were used to estimate associations.

Results: Pregnancy mean exposure $\left(\mu \mathrm{g} / \mathrm{m}^{3}\right)$ was 26.2 for $\mathrm{NO}_{2}(5-95$ th percentiles: 11.7-45.8), $25.4(11.6-39.1)$ for PM10 and 16.5 (8.8-24.9) for PM2.5. Pregnancy mean exposure to PM2.5 was associated with an increased odds of $1.18(1.06,1.33)$ for term low birth weight $(<2,500 \mathrm{~g}$ among births after 37 weeks of gestation), a decrease by $-10(-19,-1) \mathrm{g}$ in mean birth weight, and -0.08 $(-0.12,-0.03) \mathrm{cm}$ in mean head circumference per $5 \mu \mathrm{g} / \mathrm{m}^{3}$ increment in PM2.5 after adjustment for individual information on potential confounders. A $10-\mu \mathrm{g} / \mathrm{m}^{3}$ increase in $\mathrm{NO}_{2}$ and PM10 were associated with an increased risk of term low birth weight [1.09 (1.00, $1.19)$ and $1.16(1.00,1.35)]$. Reductions in head circumference were also observed for the other pollutants.

Conclusions: In this large European cohort study using standardized exposure assessment maternal exposure to ambient air pollution from traffic emissions during pregnancy was associated with reduced foetal growth. Risk estimates are higher than those of previous meta-analyses based on different or less harmonized exposure metric.

\section{O-029}

In utero Ramadan exposure and children's academic performance

Presenter: Reyn Van Ewijk

Reyn Van Ewijk 1); Bhashkar Mazumder 2); Douglas Almond 3)

1) IMBEI, University Medical Center, Mainz, Germany; 2) Chicago Federal Reserve, Chicago, USA; 3) Columbia University, New York, USA

Background: There is increasing evidence that maternal nutrition during pregnancy can affect the child's health child throughout its life course $(1,2)$. Exposure in utero can have particularly pronounced effects on fetal organs going through critical growth phases (3). For the brain, especially the first half of pregnancy seems a vulnerable period (4). It is unknown whether maternal intermittent fasting during Ramadan affects the offspring's cognitive development.

Objective: To assess how prenatal exposure to Ramadan affects 7-year-old children's cognitive performance.

Methods: All children in English state schools receive Key Stage 1 assessments in math, reading and writing when they are approximately 7 years old. We use school registry data containing all around 222,000 Pakistani and Bangladeshi students receiving KS1 assessments in England between 1998 and 2007. These students are virtually all Muslims. We compared test scores of Muslims who had, versus had not been in utero during a Ramadan. We used information on overlap between Ramadan and gestation, but were uninformed about whether mothers had actually fasted. This "intent-to-treat" approach implicitly assumes that all Muslims who were pregnant during a Ramadan, did fast. This avoids several types of bias, but underestimates the true Ramadan effect to the extent that not all mothers fasted (5).

Children's test scores vary strongly according to birth month particularly because August-born children enter school and are tested almost 1 year earlier than September-born children. Since our exposure categorization is based on birth dates, it is essential to separate season-of-birth from Ramadan effects. We use seasonal patterns among other non-Muslim ethnic groups-who have the same seasonof-birth patterns, but cannot have been exposed to Ramadan-to do so in a "difference-in-differences" estimation.

Results: Test scores at age 7 are lower for Pakistani/Bangladeshi students who had experienced Ramadan in early pregnancy than for unexposed Pakistani/Bangladeshi. Results are the same irrespective of which non-Muslim ethnic group we use in difference-in-differences estimations and we also find the same results when estimating our regressions on Muslims alone, i.e. without difference-in-differences. Furthermore, no similar pattern is found among non-Muslims, which confirms the robustness of our results.

Concusions: Ramadan fasting during the first trimester of pregnancy may negatively affect the cognitive development of the offspring.

\section{References}

1. Lumey et al. Annu Rev Public Health 2011 32:237-262.

2. Koletzko et al. Am J Clin Nutr 2011:94(6):2036S-2043S.

3. Barker, British Medical Bulletin 1997:53(1)96-108.

4. Gluckman \& Hanson MA, ISBN: 0521542359.

5. Van Ewijk, J Health Econ 2011 30(6):1246-1260.

\section{O-030}

Acetaminophen use during pregnancy, behavioral problems and hyperkinetic disorders in Danish children

Presenter: Zeyan Liew

Beate Ritz 1); Cristina Rebordosa 2); Pei-chen Lee 1); Jørn Olsen 3); Zeyan Liew 1)

1) Department of Epidemiology, Fielding School of Public Health, University of California at Los Angeles; 2) Centre for Research in environmental epidemiology (CREAL), Municipal Institute of Medical Research, Barcelona, Spain; 3) Department of Epidemiology, Institute of Public Health, University of Aarhus

Background: Acetaminophen, or paracetamol, is one of the most common pain and fever medications used by pregnant women. In animal studies, acetaminophen has been shown to be acting as a strong hormonal disruptor, and there is some human evidence indicating that hormone levels during pregnancy may influence behavioral dysfunction in early childhood. Here, we investigate for the first time whether the use of acetaminophen during pregnancy increases the risk of behavioral problem or Hyperkinetic Disorders (HKD) in children.

Method: We studied 64,322 liveborn singleton children and mothers first enrolled in the Danish National Birth Cohort (DNBC) during 1997-2003. Use of acetaminophen during pregnancy was assessed in 3 computer-assisted telephone interviews and in 1 self-administered baseline questionnaire between 6 and 12 weeks of pregnancy and 6 months of age of the child. Three different data sources were used to ascertain the outcome. First, we assessed behavioral problems of children at 7 years of age as reported by parents in the Strength and Difficulties Questionnaire (SDQ). Second, prior to end of follow-up in 2011, 834 children received a HKD diagnosis as reported in the Danish National Hospital Registry or the Danish Psychiatric Central Registry. Third, we used information about HKD prescriptions for the children in the DNBC recorded in the national prescription database. Results: Children born to mothers who used acetaminophen during pregnancy were at higher risks of receiving a hospital diagnosis of HKD (HR $=1.3795 \%$ CI 1.19-1.59), receiving HKD medications $(\mathrm{HR}=1.2995 \% \mathrm{CI} 1.15-1.44)$, or having behavioral problems at 7 years of age $(\mathrm{RR}=1.1395 \%$ CI 1.01-1.27). Stronger effects for acetaminophen were estimated when mothers used acetaminophen in 
more than one pregnancy trimesters, and a dose response was detected with increasing frequency of acetaminophen use during gestation and HKD diagnosis or medication ( $p$-trend $<0.0001$ ) and behavioral problems $(p$-trend $=0.0006)$.

Conclusion: Maternal intake of acetaminophen during pregnancy may increase the risk of Hyperkinetic Disorders and behavioral problems in children by age seven.

\section{O-031}

Risk of miscarriage in relation to history of eating disorder and pre-pregnant BMI status

Presenter: Anne-Marie Nybo Andersen

Anne-Marie Nybo Andersen 1); Emilie Helligsø de Leon 1); Pernille Stemann Larsen 1); Nadia Micali 2)

1) Section of Social Medicine, Department of Public Health, University of Copenhagen. Denmark; 2) Institute of Child Health, University College of London, UK

Background: Many women develop an eating disorder during their teenage years. Persisting active eating disorder disturb fertility seriously, however, many women recover and get pregnant later in life. Pregnancies in these women are likely to be complicated, and previous evidence has shown that women with bulimia nervosa have more miscarriages in their history compared to women without eating disorders. The association has not been investigated in a prospective and adequately powered study.

Objectives: To estimate the relative risk of miscarriages in women with a history of eating disorder and to assess how pre-pregnant weight status affects this risk.

Methods: The Danish National Birth Cohort (DNBC), established in the period from 1996 to 2002, encompassed 100,418 women who were recruited early in pregnancy and followed-up with respect to outcome of pregnancy. In total, 92,626 women provided information on pre-pregnant health through an interview scheduled in pregnancy week 12 or, for 2,552 women, after an early miscarriage that happened to occur before the scheduled interview. Information on previous eating disorder and pre-pregnant weight and height were self-reported, and pregnancy outcomes were obtained from the National Discharge register. The relative risk of miscarriage (fetal death before 22 weeks of gestation) was calculated as Hazard Ratios (HR) using Cox Proportional Hazard model with entry at gestational age at enrollment in the study. The risks were estimated according to type of eating disorder (anorexia, bulimia or both), to pre-pregnant BMI-group (under-, normal- and overweight/obese) adjusted for the potential confounders: maternal age, parity and occupational class in model 1, and furthermore for five potential mediators: maternal alcohol use, smoking, physical exercise, coffee drinking, and heavy lifting during pregnancy in model 2.

Results: The crude and the confounder-adjusted HRs of miscarriage for women with an eating disorder were 1.42 (95\% CI 1.23-1.63), and adjustment for possible mediators deflated the HR to 1.32 (95\% CI 1.15-1.53). The miscarriage risk was not increased in women who reported to have suffered from bulimia [HR $1.09 \quad(95 \%$ CI 0.87-1.38)], while women who reported to have suffered from anorexia had a relative risk of 1.50 (95\% CI 1.24-1.82). The risk of miscarriage was also substantially elevated for underweight women compared to normal weight women [HR 1.23 (95 \% CI 1.05-1.44)], while overweight/obese women had no increased risk of miscarriage [HR 1.05 (95\% CI 0.97-1.14)]. Women with anorexia had a substantially increased risk of miscarriage, whatever their pre-pregnant BMI status was, underweight, normal weight, or overweight.
Conclusion: Anorexia and pre-pregnant underweight are strong risk factors for miscarriages, independently of each other.

\section{0-032}

Maternal antibodies to microbial and tissue antigens during pregnancy and childhood epilepsy

Presenter: Yuelian Sun

Yuelian Sun 1); Jakob Christensenb 2); Jørn Olsen 1), 3)

1) Section for Epidemiology, Department of Public Health, Aarhus University, Aarhus, 8000 C, Denmark; 2) Department of Neurology and Department of Clinical Pharmacology, Aarhus University Hospital, Aarhus, 8000 C, Denmark; 3) Department of Epidemiology, School of Public Health, University of California at Los Angeles, Los Angeles, California, USA

Background: An increasing number of epidemiologic studies show associations between infections during pregnancy and an increased risk for mental and neurological disorders in the offspring. Molecular mimicry has been suggested to play a role and several infections have been indicated as potential causal candidates for psychiatric as well as neurological disease with an onset in childhood or later.

Objectives: To examine the association between maternal serum IgG antibodies to microbial and tissue antigens during pregnancy and the risk of epilepsy in offspring.

Methods: We used a case-cohort design within the Danish National Birth Cohort (DNBC). We included 275 children with a diagnosis of epilepsy before 10 years of age and a sample of 282 children randomly identified from the DNBC. We tested 25 microbial and tissue antigens in the maternal blood sample taken during the second trimester. We used a Prentice-weighted Cox regression model to estimate the hazard ratio (HR) and $95 \%$ confidence interval (CI) for epilepsy.

Results: Higher levels of maternal antibodies against herpes simples virus type 1 (HSV1) were associated with a higher risk of childhood epilepsy, but higher levels of maternal antibodies against pneumoccocal polysaccharide 18 (PnPS18) were associated with a lower risk of childhood epilepsy. The significantly higher risk associated with HSV1 antibodies was seen for childhood absence epilepsy (HR for trend $=2.08,95 \%$ CI 1.12-3.85) and for epileptic encephalopathies (HR for trend $=1.49,95 \%$ CI 1.01-2.22). The significantly lower risk associated with PnPS18 antibodies was seen for infantile spasms (HR for trend $=0.47,95 \%$ CI 0.26-0.85). None of the other 23 antigen-specific antibodies were significantly associated with the risk of epilepsy.

Conclusions: Maternal antibodies during pregnancy against HSV1and PnPS18 may be associated with the risk of specific types of epilepsy in offspring but further studies are needed to replicate these findings and to see if they also affect other outcomes.

\section{O-033}

Risks of major congenital anomaly in the children of women with clinically diagnosed depression: A United Kingdom population-based cohort study

Presenter: Lu Ban

Lu Ban 1); Jack Edward Gibson 1); Joe West 1); Linda Fiaschi 1); Rachel Sokal 1); Liam Smeeth 2); Pat Doyle 2);

Richard Hubbard 1); Laila Jal Tata 1) 
1) Division of Epidemiology \& Public Health, University of Nottingham; 2) Department of Non-communical Disease, London School of Hygiene \& Tropical Medicine

Background: Although there is concern over use of selective serotonin reuptake inhibitors (SSRIs) in pregnant women and the risk of major congenital anomalies in their children, inconsistency remains about the impact of individual drugs and there are few studies of their relative harm to tricyclic antidepressants (TCAs).

Objectives: To compare the prevalence of major congenital anomalies in the children of mothers with and without diagnoses of depression, and to investigate possible adverse impact of SSRIs and TCAs on the risk of major congenital anomalies.

Methods: We identified all singleton live births for women aged 15-45 years between 1990 and 2009 from a large United Kingdom primary care database. Children were grouped according to whether their mothers had depression but were un-medicated during the first trimester of pregnancy or whether they had been exposed to SSRIs or TCAs. We calculated absolute risks of any major congenital anomaly and system-specific anomalies and used logistic regression with a generalised estimating equation to compare risks, adjusting for maternal age at birth, year of childbirth, socioeconomic deprivation, smoking, body mass index and comorbid illnesses.

Results: The prevalence of major congenital anomaly in 13,432 children born to women with un-medicated depression was $2.8 \%$, compared with $2.7 \%$ in 7,683 children exposed to SSRIs early antenatally, $3.1 \%$ in 2,428 children exposed to TCAs, $2.8 \%$ in 290 children exposed to both drugs, and $2.7 \%$ in 325,294 children born to women without depression. Compared with children whose mothers did not have depression during pregnancy or in the year before pregnancy, the adjusted odds ratios for major congenital anomalies were 1.07 (95\% confidence interval [95\% CI] 0.96-1.18) for maternal depression which was un-medicated during the first trimester, 1.01 (95\% CI 0.88-1.17) for antenatal SSRI exposure and 1.09 (95\% CI 0.87-1.38) for TCAs exposure. System-specific anomalies did not show associations with specific drug classes or individual SSRIs apart from an increase of congenital heart anomalies overall in children exposed to paroxetine (adjusted odds ratio $=1.78$, $95 \%$ CI 1.09-2.88). There was no evidence that paroxetine was related to a specific heart defect diagnosis.

Conclusions: The prevalence of major malformation was similar for the children of mothers with, and without, un-medicated depression in pregnancy. We found no overall association between the use of SSRIs or TCAs during early pregnancy and the risk of major congenital anomaly and system-specific groups in offspring. However, we did find an increased risk of congenital heart anomaly in children exposed to paroxetine.

\section{O-034}

Antenatal exposure to anti-anxiety and hypnotic drugs and the risks of major congenital anomalies: a United Kingdom population-based cohort study

Presenter: Lu Ban

Lu Ban 1); Joe West 1); Jack Edward Gibson 1); Linda Fiaschi 1); Rachel Sokal 1); Laila Jal Tata 1)

1) Division of Epidemiology \& Public Health, University of Nottingham

Background: Few population-based studies have been able to examine the effects of individual hypnotic and anxiolytic drugs on the risk of major congenital anomalies (MCAs) with consideration of women's underlying mental illnesses and associated drug treatments.
Objectives: To estimate risks of MCAs in children exposed to specific benzodiazepines and non-benzodiazepine hypnotics taken with and without antidepressants in the first trimester of pregnancy compared with unexposed children.

Methods: We identified 387,065 singleton live births for women aged 15-45 between 1990 and 2010 from a large United Kingdom primary care database, excluding women with serious mental illness or epilepsy. Diagnoses of MCAs were identified in children's medical records and classified into system-specific groups according to the European Surveillance of Congenital Anomalies. First-trimester exposure to all specific benzodiazepines and non-benzodiazepine hypnotics were identified from the mothers' medical records. Only 342 out of 3,287 children were exposed to drugs other than diazepam, temazepam or zopiclone, therefore we measured the effect of these three drugs alone and excluded others from the study population. Children were grouped according to their first trimester hypnotic or anxiolytic drug exposures with/without antidepressants, or the presence/absence of maternal diagnoses of depression and/or anxiety during or in the year before pregnancy. We calculated absolute risks of MCAs for children in each group and used logistic regression with a generalised estimating equation to compare risks with adjustment for year of childbirth, maternal age, socioeconomic status, and maternal smoking. In recognition of the large number of comparisons, $99 \%$ confidence intervals (99\% CIs) were calculated for adjusted odds ratios (AORs).

Results: Overall MCA prevalence was $2.7 \%$ in 1,169 children exposed to diazepam alone, $2.9 \%$ in 381 children with temazepam alone, $2.5 \%$ in 407 children with zopiclone alone and $2.7 \%$ in 29,492 children with maternal depression and/or anxiety alone, compared with $2.7 \%$ in 354,286 children with no such exposures $(\mathrm{AORs}=1.01[99 \% \mathrm{CI}$ 0.63-1.62] for diazepam, $1.06[99 \% \mathrm{CI}$ 0.48-2.35] for temazepam, 0.96 [99\% CI 0.42-2.19] for zopiclone, and 1.02 [99\% CI 0.63-1.62] for depression/anxiety alone). Risks of overall and system-specific MCAs were generally higher when children were also exposed to first trimester antidepressants, however, the only statistically significant association was a threefold increase of congenital heart anomalies, mainly septal defects, in children exposed to both temazepam and antidepressants (AOR $=3.29,99 \% \mathrm{CI}$ 1.11-9.77) based on six heart anomalies in 239 such dual drug exposures.

Conclusions: There is little evidence of an increase of MCAs in children exposed to diazepam, temazepam and zopiclone in the first trimester.

\section{O-035}

Pre- and postnatal stress and variation in the glucocorticoid regulating enzyme 11- -HSD2 in children who later develop schizophrenia

Presenter: Liselotte Petersen

Jean-Christophe Philippe Goldtsche Debost 1), 4); Liselotte Petersen 1), 4); Jakob Grove 2), 4); Mette Nyegaard 2), 4); Tine Brink Henriksen 3); Preben Bo Mortensen 1), 4)

1) National Center for Register based Research, Department of Economics and Business, Faculty of Business and Social Sciences, Aarhus University, Denmark; 2) Institute for Biomedicine, Human Genetics, Aarhus University, Denmark; 3) Institute for Clinical Medicine, Department of Pediatrics, Aarhus University Hospital, Denmark; 4) The Lundbeck Foundation Initiative for Integrative Psychiatric Research, iPSYCH, Denmark

Introduction: Studies have associated maternal stress during pregnancy with CNS disorders including schizophrenia. In Danish register 
data we showed that the loss of a close relative during and after pregnancy, as an objective proxy measure for stress, was associated with offspring schizophrenia risk. It has been speculated that stressful life events enhance production of glucocorticoids that adversely affect neurodevelopment in the immature brain, increasing the risk of mental illness in adolescence or adult life. Functional variation of the glucocorticoid regulating enzyme 11-hydroxysteroid-dehydrogenase type 2 because of single nucleotide polymorphisms (SNPs) in the gene encoding it (HSD11B2) has been associated with a stress syndrome called apparent mineralocorticoid excess with overproduction of glucocorticoids. The enzyme is found in high concentration in the placenta where it protects the fetus from glucocorticoid overflow, and the authors hypothesized that the effect of stress might be mediated via cortisol, and in part be explained by variation in the gene HSD11B2.

Method: A matched case-control study with 736 schizophrenia cases and 799 controls, matched on sex, exact date of birth, born in Denmark, alive and with no history of schizophrenia on the date of first diagnosis of schizophrenia of the matched case, and also including all their first-degree relatives. We used information from national registers and GWAS data from the Danish neonatal biobank, to determine whether the effect of loss of a relative could be explained by variation in HSD11B2. SNPs were imputed into the locus of HSD11B2, and data was analyzed with conditional logistic regression.

Results: To ensure estimable rate ratios only SNPs with a minor allele frequency of more than $8 \%$ were chosen. We found a main effect of loss of a close relative after pregnancy and a main effect of four out five SNPs. For some of the SNPs we also found an interaction with loss of a close relative, independent of psychiatric family history and place of birth, which are known to influence the risk.

Conclusions: Our results suggest that there is an interaction between loss of a close relative during fetal or early life and SNPs in HSD11B2 in offspring who later develop schizophrenia. This might suggest involvement of abnormal glucocorticoid metabolism as a mediating factor between maternal stress and offspring mental disorders.

\section{Mental health: risk factors and outcomes}

\subsubsection{3:30-14:30}

\section{O-036}

Sleep apnea and risk of permanent exclusion from the labor market and cause-specific mortality: a nationwide registry linkage study

Presenter: Naja Rod

Naja Rod 1); Linnea Kjeldgård 2); Torbjörn Åkerstedt 3); Paula Salo 4); Kristina Alexanderson 2 )

1) Department of Public Health, University of Copenhagen, Copenhagen, Denmark; 2) Department of Insurance Medicine, Karolinska Institute, Sweden; 3) Department of Clinical Neuroscience, Karolinska Institute, Sweden; 4) Finnish Institute of Occupational Health, Helsinki, Finland

Background: Sleep apnea is a common and highly under-recognized public health issue. Sleep apnea results in poor and fragmented sleep, which is likely to have a major impact on daily functioning and labor market participation. Smaller and mostly clinic-based studies have also suggested a higher mortality risk among those with sleep apnea, but the relationship remains to be settled in large population-based samples.

Objectives: The study aims to evaluate the effect of sleep apnea on permanent exclusion from the labor market and cause-specific mortality in a large population-based study including all diagnosed sleep apnea cases in Sweden between 2000 and 2009. We also aim to distinguish between inpatient and outpatient cases as they are likely to differ in severity of sleep apnea.

Methods: Between 2000 and 2009, we identified a total of 74,543 individuals of working age with a sleep apnea diagnosis $(60,125$ outpatient and 14,418 inpatient cases) in the Swedish National Hospital Register. Each case was frequency matched by sex to five noncases. Sleep apnea cases and non-cases were followed up in nationwide registers until 2010, with less than $1 \%$ loss to follow-up. Permanent exclusion from the labor market was defined as being granted disability pension or being on a sick leave spell lasting more than 2 years. All analyses were adjusted for age, education, country of birth, family situation, and area of residence.

Results: During a mean 5.1 year follow-up period, inpatient sleep apnea was associated with a three- to fourfold higher risk of permanent exclusion from the labor market in both men $(\mathrm{HR}=3.34 ; 95 \%$ : $3.13-3.57)$ and women $(4.51 ; 4.04-5.03)$. This corresponds to $13 \%$ of men and $21 \%$ of women with an inpatient diagnosis of sleep apnea becoming permanently excluded from the labor market during followup. In terms of mortality risk, inpatient sleep apnea was associated with a higher risk of all-cause mortality in both men $(\mathrm{HR}=1.71$; $95 \%$ CI $1.59-1.84)$ and women $(\mathrm{HR}=2.33 ; 2.04-2.67)$, while no associations were found between outpatient sleep apnea and total mortality. Inpatient sleep apnea was found to be most strongly associated with deaths due to ischemic heart disease, respiratory disorders, and suicide, while there were no associations with cancer mortality.

Conclusions: An inpatient diagnosis of sleep apnea is related to a markedly higher risk of permanent exclusion from the labor market and higher mortality risk in a large population sample.

0-037

Common mental disorders predict sickness absence and disability pension award

Presenter: Ann Kristin Knudsen

Ann Kristin Knudsen 1); Samuel B Harvey 2); Arnstein Mykletun 3); Matthew Hotopf 4); Helene Aakvaag 5); Simon Øverland 6)

1) Department of Global Public Health and Primary Care, University of Bergen; 2) School of Psychiatry, University of New South Wales; 3) Department of Genetics, Environment and Mental Health, Norwegian Institute of Public Health; 4) Institute of Psychiatry, King's College London; 5) Norwegian Centre for Violence and Traumatic Stress Studies; 6) Department of Health Promotion and Development, University of Bergen

Background: The common mental disorders anxiety and depression will affect 1 in 4 adults at least once during their lifetime. These disorders are also increasingly prevalent within the official benefits statistics. The majority of studies examining the contribution from common mental disorders on sickness absence and disability pension award have either relied on the diagnoses stated on sick-leave notes or disability pension application, or have employed a cross-sectional design. Little is known about the role of common mental disorders as risk factors for sickness absence and disability pension award.

Objectives: By linking information from an epidemiological health survey with data from official registries over sickness and disability benefits, we aimed to examine the long-term consequences of 
common mental disorders on sickness absence and disability pension award in the general population.

Methods: Baseline information on common mental disorders and potential important covariates (socio-economic status, health behaviour, physical health) was taken from the Hordaland Health Study (HUSK) $(\mathrm{N}=18,565)$, conducted in the period 1997-1999 in western Norway. This information was then linked with official information on sickness benefits and disability pension award in the period 1997-2004, allowing for up to 7 years follow-up. Associations were examined with Cox regression models and multi-nominal logistic regression models. The burden of sub case-level versus caselevel symptom loads of common mental disorders on disability pension awards in the population was investigated by calculating population attributable fractions (PAF).

Results: Common mental disorders were found to be long-lasting risk factors for both sickness absence and disability pension award. Common mental disorders were particular strong risk factors for very long duration and frequent recurrence of sickness absence. Comorbid anxiety and depression showed the strongest associations with the different outcomes, and gave a five-fold increased risk for disability pension award in general, and tripled the risk for disability pension award for non-mental medico-legal diagnoses. The risk of disability pension award was lower for sub case-level symptom loads, but due much higher prevalence of these conditions, the proportion of disability pensions attributable to sub case-level symptom loads was comparable to the proportion of disability pension awards attributable to case-level anxiety and depression symptom loads.

Conclusions: The findings indicate that common mental disorders are more important contributors to sickness absence and disability pension award than acknowledged in official benefit statistics.

\section{O-038}

Suicide-related events in young people following prescription of SSRIs and other antidepressants: a self-controlled case series analysis

Presenter: Linda Wijlaars

Linda Wijlaars 1); Irwin Nazareth 1); Heather Whitaker 2); Stephen Evans 3); Irene Petersen 1)

1) Department of Primary Care and Population Health, UCL; 2) Department of Mathematics and Statistics, Open University; 3) Department of Medical Statistics, LSHTM

The treatment of young people with selective serotonin reuptake inhibitors (SSRIs) other than fluoxetine has been discouraged by the UK drug authority since 2003 as concerns have been raised about a possible association between antidepressants and suicide-related behaviour in this population. The evidence, however, for the risk of suicide-related behaviour is inconsistent.

We examined the temporal association between SSRI and tricyclic antidepressant (TCA) prescriptions and suicidal behaviour in children.

We used a self-controlled case series design among 81 young people aged 10-18 years with a record of completed suicide, 1,496 with a recorded suicide attempt, 1,178 with suicidal ideation and 2,361 with intentional self-harm. We used electronic health records from 479 general practices in The Health Improvement Network (THIN) UK primary care database from 1995 to 2009 and compared Incidence Rate Ratios (IRRs) before, during and after an antidepressant prescription was recorded.

For attempted suicide, suicidal ideation or intentional self-harm, IRRs were similar for the time the person was prescribed either SSRIs or TCAs: IRRs increased during pre-exposure, peaked on prescription day, were stable up to the fourth prescription-week, and decreased after prescriptions stopped. For both types of antidepressants, IRRs were lower or similar to pre-exposure levels during the period of prescription. For SSRIs, there was an increase in the IRR for completed suicide on the day of prescription $(\mathrm{N}=5$; IRR $=42.5,95 \%$ CI 4.5-403.4), and during the fourth week of SSRI prescription $(\mathrm{N}=2 ; \mathrm{IRR}=11.3,95 \%$ CI 1.1-115.6).

Overall, there are no systematic differences between the association of TCAs and SSRIs with incidence risk ratios for attempted suicide, suicidal ideation or intentional self-harm. Moreover, apart from the day of prescription, rates were not statistically significantly different from pre-exposure levels. The pattern of death from suicide for SSRIs was similar to that found in non-fatal suicide-related behaviour.

\section{O-039}

Lifecourse predictors of psychological well-being age 49-51 years in the Newcastle thousand families study

Presenter: Kay Mann

Kay Mann 1); Morven Brown 1); John Wildman 1); Louise Parker 2); Mark Pearce 1)

1) Institute of Health \& Society, Newcastle University, UK; 2) Departments of Medicine and Paediatrics, Dalhousie University, Canada

Background: Early growth has been associated with psychological health in a number of longitudinal studies. However the relative importance of early life associations is often overlooked and not quantified, nor are mediating pathways of lifecourse data explored.

Objectives: To investigate lifecourse predictors of psychological well-being, assessed using the 28 -item General Health Questionnaire (GHQ), in the Newcastle Thousand Families Study at age 49-51 years. Path analysis was used to investigate the relative contribution of predictors and mediating pathways of factors from across the lifecourse

Methods: Detailed information was collected prospectively during childhood, including birth weight, duration breast fed, socioeconomic status and childhood IQ. At age 49-51 years, 574 study members returned questionnaires including the GHQ-28 and one on health and lifestyle. The four domains of the GHQ-28 were collated into a total GHQ score. A total cut off score of 24 was used to identify 'caseness'. Scores and explanatory variables were analysed using linear and logistic regression. Linear regression models, estimating direct effects, for total GHQ score were reconstructed as path diagrams and potential mediating factors added to estimate indirect associations, pathways and relative importance.

Results: After adjustment for all other significant variables, total GHQ score in females was positively associated with standardised birth weight, divorced or separated marital status, long-term illness and negatively associated with sports activity at age 49-51 years. Total GHQ score in males was negatively associated with childhood IQ, overall physical activity at 49-51 years and positively associated with long-term illness. Of highest relative importance, long-term illness mediated the effects of alcohol consumption and smoking. Indirect effects from social class at birth had a moderate importance in males which were mediated through childhood IQ, achieved education and social class at age 49-51 years, whereas in females the importance of social class at birth was small.

Conclusions: Standardised birth weight was significantly associated with psychological well-being in this cohort, albeit in the opposite direction to that expected and only in females. Mediating effects showed some influence of early life on later psychological well-being however the relative importance of these effects were small in 
comparison to physical activity and marital status at age 49-51 years and long term illness. Our findings suggest that both ends of the birth weight spectrum may be of interest, sex differences should be further investigated and that mediating effects of predictive factors have the potential to explain causal pathways.

\section{O-040}

Social determinants for mental health: a Finnish nationwide follow-up study on mental disorders

\section{Presenter: Mika Gissler}

\section{Reija Paananen 1); Tiina Ristikari 1); Marko Merikukka 1); Mika Gissler 2)}

1) Finnish National Institute for Health and Welfare, Department of Children, Young People and Families; 2) Finnish National Institute for Health and Welfare, Information Department

Background: Mental health in childhood and adolescence is an increasing public health concern, since most adulthood mental disorders begin in childhood and adolescence. Risk factors are pre- and perinatal, genetic as well as environmental and family-related. Mental disorders, marginalisation and inequalities in health, income and other opportunities in life are interwoven. Research evidence is still insufficient in providing basis for understanding life-course development of mental disorders and intergenerational transmission of inequalities in well-being.

Methods: The 1987 Finnish Birth Cohort covers all children born in Finland in 1987 surviving perinatal period $(\mathrm{N}=59,476)$. The study covers detailed health, social welfare and socio-demographic data of cohort members and their parents from various Finnish registers.

Results: Altogether 7,578 (12.7\%) of the cohort members had had a diagnosed mental disorder during the follow-up 1987-2008. Several perinatal, childhood and adolescence risk factors for mental disorders were found and the likelihood for a mental disorder varied also according to sex. Main familial risk factors for mental disorders were having a teenage parent OR 1.96 (1.76-2.20), parents' divorce OR 1.68 (1.60-1.76), death of a parent OR 1.82 (1.68-1.97), parents having basic education only OR 1.91 (1.74-2.10), childhood family receiving social assistance OR 2.16 (2.06-2.27) or having a parent treated as inpatient at psychiatric ward OR 1.79 (1.70-1.89). Parental education together with social assistance receipt was the strongest interaction studied. The risk for children's mental disorders was higher if parents had had social assistance and had low education. Perinatal problems, such as prematurity had only minor effect on mental disorders OR 1.19 (1.09-1.30). The risk increased if the adolescent had no education after comprehensive school OR 4.75 (4.47-5.04) or had received social assistance OR 3.86 (3.67-4.05). Conclusions: Mental disorders had many social risk factors. Financial problems, low education and psychiatric disorders pass on through generations and are interlinked. Although the family difficulties increased children's risk for mental disorders, that was clearly determined by adolescent's own low education and financial hardship. Families with social adversities and with parental mental health problems should be supported to secure children's development and to prevent marginalisation. Links and pathways of social factors, inequalities and mental well-being need further clarification.

\section{O-041}

Increased use of antidepressants after diagnosis of breast cancer: a nationwide and population-based cohort study in Denmark 1998-2006

Presenter: Nis Palm Suppli
Nis Palm Suppli 1); Christoffer Johansen 1), 2); Jane Christensen 1); Susanne Oksbjerg Dalton 1)

1) Danish Cancer Society Research Center, Copenhagen, Denmark; 2) Rigshospitalet, University of Copenhagen, Denmark

Background: Prevalence of depressive symptoms is reported to be 20-30\% among breast cancer patients. With detrimental effect on psychological wellbeing, adverse effects on areas such as work performance and marital functioning, and negative impact on survival in cancer patients, depression related to breast cancer is an issue that calls for attention. Numerous studies have assessed depression among breast cancer patients with questionnaires and interviews, but investigations of the occurrence of depression recognized and treated in the clinical setting are sparse.

Objectives: The primary aim of this study is to compare the occurrence of medically treated depression among breast cancer patients with the general population. Secondary it is investigated if sociodemographic, cancer or treatment related factors are associated with medically treated depression among breast cancer patients.

Methods: During the study period 1998-2006 1,490,427 Danish women free of cancer at study entry were followed for medically treated depression, defined as first prescription of antidepressants redeemed. Women diagnosed with breast cancer during the study period were considered exposed from date of diagnosis. To distinguish between recurrent and first episode of depression, the total population was classified as 'not prior medically treated' and 'prior medically treated' according to use of antidepressants and/or hospital contacts for depression prior to study entry. All statistical analyses were conducted separately for the cohorts of women not prior and prior medically treated. To investigate risk of use of antidepressants after breast cancer standardized incidence ratios (SIR) and excess absolute risk were calculated. Multiplicative Poisson regression models were used to investigate associations of socio-demographic and clinical factors with use of antidepressants.

Results: Among 23,465 women diagnosed with breast cancer a fivefold increase in risk for use of antidepressants was found in the first year after diagnosis among both women not prior and prior medically treated for depression (SIR 5.45, $95 \%$ CI 5.22-5.69; SIR $4.82,95 \%$ CI 4.54-5.12, respectively). In women not prior treated the risk remained significantly elevated more than 6 years after diagnosis. Regardless of treatment of depression prior to diagnosis of breast cancer comorbidity, high number of tumor positive axillary lymph nodes, and being premenopausal yielded the highest relative risk of use of antidepressants.

Conclusions: Breast cancer patients have an increased risk of medically treated depression both in the immediate course and long term survivorship. It is suggested that socio-demographic and clinical factors associated with risk of use of antidepressants could prove useful in early clinical screening for depression.

\section{Cardiovascular risk}

12.08.2013 13:30-15:00

\section{O-042}

Age specific trends of disease, mortality and case-fatality from cardiovascular disease, myocardial infarction and stroke among 85+ in Sweden

Presenter: Karin Modig

Karin Modig 1); Tomas Andersson 1); Sven Drefahl 2); Anders Ahlbom 1) 
1) Institute of environmental medicine, Karolinska Institutet;

2) Department of Sociology, Demography unit, Stockholm University

Background: Despite an ageing population it is not clear how the age specific trends for cardiovascular disease (CVD) look like for those 85 years and above.

Objectives: The main aim of this study were to describe the age specific trends in total CVD, as well as in myocardial infarction (MI) and stroke separately among the oldest (85-99 years of age) in the population, with respect to incidence (first events), 28 days case fatality, and overall mortality for the period 1994-2010. A further aim was to compare the trends among those below 85 years of age to those above 85 years of age to see whether the improvements observed among the younger population the past decades have extended to the oldest old.

Methods: The registry of the total Swedish population was used to identify the population at risk. Information about CVD, MI and stroke were extracted from national registries of hospital admissions and causes of deaths. All analyses were based on 1-year age- and sexspecific figures.

Results: The risk for CVD increased with every age above 85 years although the rate of increase was leveling off with age. Over time, the risk for CVD and MI decreased for all ages and for stroke for ages up to 89 years. However, the risk of MI increased until around 2001 in all age groups and both sexes but decreased after that. The overall mortality improved for all outcomes over the period 1994-2010, so did the survival within 28 days from the event. The average annual decline in mortality over all ages 85 and above was $3 \%$ for MI and $2 \%$ for stroke and CVD. Corresponding figures for ages 60-84 was $4 \%$ for each of MI, stroke and CVD. The results were very similar for men and women.

Conclusions: Improvements in CVD risks observed among ages up to 85 years appear to have extended also to ages above 85 years, even if the rate of improvement was leveling off with age. The improvements in 28 days survival for all ages up to 99 years give no support to the hypothesis that more fragile individuals reach higher ages. Additional research is needed to find out if improvement in survival can be seen also for the second and third event of CVD, stroke and MI.

\section{0-043}

Preconception cardiovascular determinants of gestational hypertension: Cohort Norway and the Medical Birth Registry of Norway

\section{Presenter: Grace Egeland}

Grace Egeland 1), 2); Kari Klungsøyr 1), 2); Marta Ebbing 1); Grethe Tell 1), 2); Nina Øyen 2); Rolv Skjærven 1), 2); Øyvind Næss 1)

1) Norwegian Institute of Public Health, Bergen and Oslo, Norway; 2) Dept. of Global Public Health and Primary Care, University of Bergen, Bergen, Norway

Background: Women with a history of gestational hypertension, particularly preeclampsia, are at increased risk for cardiovascular mortality. However, few studies have evaluated cardiovascular risk factors prior to pregnancy for their ability to predict pregnancyrelated complications.

Objectives: To evaluate the preconception determinants of gestational hypertension.

Methods: A total of 16,429 women were identified as participating in the Cohort Norway (CONOR) 1994-2003 health survey population prior to a pregnancy registered in the Medical Birth Registry of Norway. Women with diabetes and hypertension prior to pregnancy (as noted in CONOR and Medical Birth Registry data) were excluded
( $\mathrm{n}=483$ ), leaving 15,946 women for analyses. Non-fasting total cholesterol, HDL-cholesterol, and triglycerides were assessed by an enzymatic method and time since last meal (an average of $2 \mathrm{~h}$ ) was entered as a covariate. Logistic regression evaluated unadjusted and multivariable adjusted odds ratios (OR) and $95 \%$ confidence intervals (CI) for novel and established risk factors for gestational hypertension.

Results: In the CONOR baseline preconception data, the average age of participants was 28.3 years $(\mathrm{SD}=4.4)$, and $24 \%$ reported current smoking, $32 \%$ were either overweight or obese, $22.7 \%$ had an enlarged waist circumference $(>80 \mathrm{~cm})$, and $46 \%$ reported regular light physical activity ( 3 or more times per week). The Medical Birth Registry identified $28 \%$ as nulliparous. A total of 775 women developed gestational hypertension of whom 534 had preeclampsia. In unadjusted analyses, preconception predictors of gestational hypertension included a total cholesterol/HDL-cholesterol ratio $>5$ $(\mathrm{OR}=1.5 ; 95 \%$ CI 1.1-1.9), and triglycerides (3rd quartile $\mathrm{OR}=1.3 ; 95 \% \mathrm{CI} 1.1-1.7$ and 4 th quartile $\mathrm{OR}=1.7 ; 95 \% \mathrm{CI}$ 1.4-2.1 compared to the lowest quartile). In addition, regular light physical activity when compared to activity less than 3 times per week was protective $(\mathrm{OR}=0.8 ; 95 \% \mathrm{CI} 0.6-0.9)$. As expected, overweight and obesity, enlarged waist circumference, and elevated blood pressure were associated with a significantly greater risk while parity and smoking were inversely related to risk for gestational hypertension.

Conclusions: Cardiovascular risk factors prior to pregnancy are important predictors of gestational hypertension. Life-course epidemiology provides evidence of the importance of life-time behavioral modifications to reduce the burden of cardiovascular disease in adulthood.

\section{0-044}

Association between reported baseline physical activity and hospital admission for Venus thromboembolism in a prospective cohort study

\section{Presenter: Miranda Armstrong}

Miranda Armstrong 1); Benjamin J. Cairns 1); Jane Green 1); Gillian Reeves 1); Valerie Beral 1); the Million Women Study Collaborators)

\section{1) Cancer Epidemiology Unit, University of Oxford}

Background: While long periods of inactivity are known to be associated with an increased risk of venous thromboembolism (VTE), there is limited evidence on the association between regular physical activity and the risk of VTE.

Objectives: To examine the associations between usual physical activity and hospital admissions for VTE in a large prospective study of middle-aged UK women.

Methods: 1.1 million Women completed a baseline questionnaire at recruitment, answering questions on anthropometric, lifestyle, and reproductive factors. Approximately 3 years later, they reported on the hours spent per week doing a range of specific activities. Women were followed by linkage to the National Health Service cause-specific hospital records with an average follow-up period of 9.2 years. Cox regression was used to calculate adjusted relative risks for VTE. Results: During follow-up, 13,040 women were admitted to hospital or died from VTE. VTE risk decreased with strenuous activity reported at baseline: compared with inactive women, relative risks (RRs) were 0.85 [95\% confidence interval (CI) 0.81-0.88)] for strenuous activity up to once per week and 0.82 (95\% CI 0.78-0.86) for strenuous activity more than once per week. The RR for VTE risk was 0.76 (95\% CI $0.70-0.83$ ) for walking more than $5 \mathrm{~h}$ per week compared to walking up to $1 \mathrm{~h}$ per week. Greater than an estimated 20 
excess MET-hours per week for an aggregate of various activities (walking, cycling, gardening, housework, and strenuous activity) was also associated with a reduced risk of incident VTE when compared to those reporting 20 or fewer excess MET-hours per week. Housework had a borderline effect on the risk of VTE, the RR associated with more than $10 \mathrm{~h}$ of housework versus less than $2 \mathrm{~h}$ of housework per week was 0.88 (95\% CI 0.79-0.99). There were similar associations with strenuous and any physical activity separately for pulmonary embolism and for venous thrombosis without pulmonary embolism.

Conclusions: Overall measures of physical activity and various specific activities were associated with a reduction in the risk of incident VTE in UK women with the greatest difference in risk between the inactive and active women.

\section{0-045}

Variants in the NOS1 and NOS3 genes interact with long-term vehicle air pollution exposure on risk of acute myocardial infarction

\section{Presenter: Anna Levinsson}

Anna Levinsson 1); Anna-Carin Olin 1); Lars Modig 2); Hanne Krage Carlsen 1); Lena Björck 3); Annika Rosengren 3); Fredrik Nyberg 1), 4)

1) Occupational and Environmental Medicine, Department of Social Medicine and Public Health, Institute of Medicine, Gothenburg University, Sweden; 2) Department of Public Health and Clinical Medicine, Occupational and Environmental Medicine, University of Umeå, Sweden; 3) Department of Molecular and Clinical Medicine, Institute of Medicine, Gothenburg University, Sweden;

4) AstraZeneca R\&D, Mölndal, Sweden

Background: Experimental and epidemiological studies have reported associations between air pollution exposure, in particular related to traffic-related air pollution, and cardiovascular disease. A potential pathophysiological pathway is that air pollution exposure induces pulmonary oxidative stress, with secondary systemic inflammation. It has been suggested that polymorphisms in genes implicated in oxidative stress contribute to determining individual susceptibility to air pollution as a promoter of coronary vulnerability. Polymorphisms in the nitric oxide synthase (NOS) genes have previously been associated with risk of cardiovascular disease.

Objectives: We aimed to investigate effects of long-term trafficrelated air pollution exposure on risk of acute myocardial infarction (AMI), as well as whether the air pollution effects are modified by variants in the NOS1, NOS2 and NOS3 genes.

Methods: AMI cases from 2 hospitals $(\mathrm{n}=118)$ and randomly selected population controls $(n=1304)$ from the INTERGENE/ ADONIX study were genotyped at 58 single nucleotide polymorphisms (SNPs) in the NOS genes, of which the six most strongly associated with AMI were selected for interaction analyses.

Air pollution levels were modeled as annual means of $\mathrm{NO}_{2}$ (as marker of vehicle exhaust pollution) using central monitoring data and dispersion models, and linked to individuals' home addresses using a geographic information system to estimate individual exposure. Main effects of air pollution as well as interactions between air pollution and genotype were estimated using logistic regression. Models were adjusted for age, age squared, sex and residential area.

Results: Air pollution was associated with risk of AMI: OR 1.71 (95\% CI $1.00-2.93$ ) per $10 \mu \mathrm{g} / \mathrm{m}^{3}$ of long-term $\mathrm{NO}_{2}$ exposure. Two SNPs stand out in the interaction analyses, for both of which we see a large difference between the genotype strata in the relative risk of later AMI event associated with air pollution exposure. For NOS1 rs9658253, the OR was $2.03(1.14-3.62)$ per $10 \mu \mathrm{g} / \mathrm{m}^{3}$ increase of NO2 for CC genotype and $0.92(0.39-2.19)$ for combined genotypes CT \& TT. For NOS3 rs3918226, the ORs were $1.53(0.87-2.71)$ for the CC genotype and $2.98(1.12-7.92)$ for CT \& TT.

Conclusion: $\mathrm{NO}_{2}$, a marker for vehicle exhaust air pollution, is significantly associated with an increased risk of AMI. In addition, variants in the NOS genes may modify the effect of $\mathrm{NO}_{2}$ on the risk of AMI.

\section{O-046}

Socioeconomic factors from childhood to adulthood as risk factors for stroke

Presenter: Nicholas Henschke

Nicholas Henschke 1); Frederick Palm 2); Anton Safer 1); Florian Buggle 2); Caspar Grond-Ginsbach 3); Armin Grau 2); Heiko Becher 1)

1) Institute of Public Health, University of Heidelberg, Heidelberg, Germany; 2) Department of Neurology, Klinikum der Stadt Ludwigshafen am Rhein, Ludwigshafen am Rhein, Germany; 3) Department of Neurology, University of Heidelberg, Heidelberg, Germany

Stroke is the third leading cause of death and the leading cause of early disability in Western countries. Several studies have indicated that poor socioeconomic conditions in childhood and during prenatal life are associated with an increased risk of stroke in adulthood. While socioeconomic conditions can change during an individual's lifetime, there is some evidence that the influence of socioeconomic determinants on health is cumulative over an individual's lifespan.

The main objective of this study was to determine the level to which adverse socioeconomic factors, particularly those of childhood, remain associated with stroke after adjustment for major risk factors.

A population-based case-control study was performed, recruiting all patients aged between 18 and 80 years who suffered an initial ischaemic stroke between 1st October 2007 and 31st March 2012 in Ludwigshafen, Germany. Age-and sex-matched control subjects were enrolled from the general population of the same city. For all participants a detailed standardised history was recorded, including basic anthropometric and demographic data, vascular risk factors and preexisting conditions, current medication, smoking and alcohol consumption, physical activity, and family history. Socioeconomic data pertaining to childhood (e.g. occupation of father, employment status of mother, number of siblings, presence of a car), youth (e.g. highest school degree, initial profession), and adulthood (e.g. profession, periods of unemployment, income) were collected for all participants. In multivariate models the association of individual factors from childhood and stroke risk were evaluated. The individual factors were also combined into scores for childhood, youth, and adulthood to allow an analysis of the relationship between stroke risk and the aggregation of adverse conditions.

This study included 470 (40\% female) patients with a single stroke and 809 (42\% female) control subjects. The mean ( \pm standard deviation) age was $65.8( \pm 10.9)$ for cases and $66.8( \pm 10.1)$ for controls. After adjusting for comorbid diagnoses and smoking, a lower socioeconomic status in childhood (odds ratio 1.24; $95 \% \mathrm{CI}$ $0.80-1.94$ ) or youth (odds ratio 1.16 ; $95 \%$ CI $0.79-1.70$ ) was not significantly related to stroke risk. However, those with a lower socioeconomic status in adulthood had a higher risk of stroke (odds ratio 5.04; $95 \%$ CI 3.23-7.87). Over the whole life course, a significantly greater risk of stroke was seen for those in the lowest (odds ratio 4.12 ; $95 \%$ CI 2.92-5.82) compared to the highest socioeconomic group. 
Contrary to previous suggestions, we found socioeconomic status in childhood not to be independently related to stroke risk, however socioeconomic status in adulthood is.

\section{0-047}

Dietary fibre and risk of haemorrhagic or ischaemic stroke in the UK Women's Cohort Study

\section{Presenter: Diane Threapleton}

Diane Threapleton 1); Victoria Burley 1); Darren Greenwood 2); Maryam Aldwairji 1); Janet Cade 1)

1) Nutritional Epidemiology, University of Leeds;

2) Division of Biostatistics, University of Leeds

Background: Stroke is a leading cause of mortality worldwide and a major source of disability. Dietary fibre may influence risk of stroke events by modifying one or more risk factor such as overweight, hypercholesterolaemia or hypertension. Few prospective studies in the UK have reported the relationship between dietary fibre and haemorrhagic or ischaemic stroke separately.

Objectives: Examine associations between dietary fibre consumption and risk of haemorrhagic, ischaemic and total stroke in the United Kingdom Women's Cohort Study.

Methods: 27,754 middle-aged women, with no prior history of cardiovascular disease, were recruited in the mid 1990s. Diet was assessed with a food-frequency questionnaire, along with other health and lifestyle information. Intakes of total dietary fibre were estimated both as non-starch polysaccharide (NSP) and using Association of Official Analytical Chemist (AOAC) values. Insoluble fibre, soluble fibre and fibre (NSP) from various food sources were also examined.

Stroke deaths were identified through the National Health Service Information Centre and incident cases using inpatient hospital episode statistics. Survival analysis was undertaken using Cox proportional hazards regression. Adjustments included age, body mass index, energy intake, alcohol, physical activity, smoking and socio-economic status. Hazard ratios are presented for increments of approximately one standard deviation of fibre intake.

Results: After a median of 14.3 years, 133 haemorrhagic and 173 ischaemic cases were observed. A further 135 strokes cases were reported without the type of stroke being specified in the records. For any type of stroke, cereal fibre intake was associated with reduced risk: hazard ratio (HR) per $3 \mathrm{~g} /$ day intake was $0.93(0.86,1.00)$.

Fibre intake was not specifically associated with haemorrhagic or ischaemic stroke risk. Several fibre exposures were associated with reduced risk of unspecified-type strokes, per $6 \mathrm{~g} /$ day increase in total NSP fibre, HR $=0.77$ (95\% confidence interval 0.65, 0.92). The HR was $0.75(0.60,0.94)$ per $3 \mathrm{~g} /$ day increase in soluble fibre and 0.83 $(0.71,0.98)$ per $4 \mathrm{~g} /$ day increase in insoluble fibre. For every $2 \mathrm{~g} /$ day increase in fibre from vegetable sources, $\operatorname{HR}=0.79(0.68,0.91)$ and per $0.2 \mathrm{~g} /$ day increase in fibre from nuts/seeds, $\mathrm{HR}=0.91(0.83,1.00)$. Conclusions: Results suggest that fibre from certain food sources and not others may be more beneficial for prevention of stroke, possibly because different foods contain fibre fractions in varying quantities. The nature of stroke that resists classification, or the characteristics of the women that experience them may potentially explain why protective associations were only observed for unclassified and not with haemorrhagic or ischaemic strokes.

\section{O-048}

Body mass index in relation to incidence of stroke subtypes among UK women: Cohort study

Presenter: Mary Kroll
Mary Kroll 1); Gillian Reeves 1); Jane Green 1); Valerie Beral 1); Cathie Sudlow 2)

1) University of Oxford; 2) University of Edinburgh

Background: Stroke is a major cause of disability and death worldwide. Although increasing body mass index (BMI) is a known risk factor for stroke in general, the evidence is weighted by findings for ischaemic stroke, the most common pathological type. Prospective studies to date have generally accrued too few stroke outcomes with information on pathological type to estimate reliably the separate associations of BMI with haemorrhagic as well as ischaemic stroke types.

Objectives: We aimed to clarify these associations by examining data from the UK Million Women Study.

Methods: During 1996-2001, 1.3 million middle-aged women were recruited through the UK national breast-cancer screening program. Participants returned a baseline health and lifestyle questionnaire, and were followed by linkage to electronic National Health Service death registers and hospital records, which are coded according to the 10th edition of the International Classification of Diseases. Incident stroke was defined as first hospital admission mentioning stroke, or death due to stroke. We estimated relative risk using Cox regression, adjusted for socioeconomic status, region, smoking, alcohol and physical activity.

Results: 13,754 incident strokes (5,994 ischaemic, 1914 intracerebral haemorrhage, 2,440 subarachnoid haemorrhage, 3,406 uncertain pathological type) occurred during an average follow-up of 9.2 years. Increasing BMI was associated with increased risk of ischaemic stroke [relative risk 1.21 (95\% confidence interval 1.17-1.25) per $5 \mathrm{~kg} / \mathrm{m}^{2}$ increase in BMI] but decreased risk of haemorrhagic stroke [intracerebral or subarachnoid, relative risk $0.90(0.86-0.93)$ per $5 \mathrm{~kg} /$ $\mathrm{m}^{2}$ increase in BMI; $P<0.00001$ for heterogeneity]. There was no significant difference between intracerebral and subarachnoid haemorrhage in the association with BMI ( $P=0.4$ for heterogeneity). In women who smoked or reported being treated for hypertension at recruitment, the increasing trend for ischaemic stroke was weaker, and the decreasing trend for haemorrhagic stroke was stronger.

Conclusions: In middle-aged UK women, increasing BMI was associated with increased risk of incident ischaemic stroke, but decreased risk of both intracerebral and subarachnoid haemorrhage.

\section{O-049}

Congenital heart disease and microcephaly at birth: a population based study of 2467 children

Presenter: Niels Matthiesen

Niels Matthiesen 1); Tine Brink Henriksen 2); James William Gaynor 3); Vibeke Hjortdal 4); Peter Agergaard 1); John Rosendahl Østergaard 1)

1) Department of Pediatrics, Aarhus University Hospital, Denmark; 2) Perinatal Epidemiology Research Unit and Department of Pediatrics, Aarhus University Hospital, Denmark; 3) Division of Cardiothoracic Surgery, The Children's Hospital of Philadelphia, USA; 4)

Department of Cardiothoracic Surgery, Aarhus University Hospital, Denmark

Background: It is recognized that neurodevelopmental disorders are frequent in children with congenital heart disease (CHD) and these disorders are largely attributed to prenatal factors. Reduced head circumference at birth, a marker of reduced fetal cerebral growth, has been demonstrated in children with CHD, and the relation to impaired neurodevelopment has been shown. However, existing studies, due to small sample sizes and highly selected populations, have largely failed to identify risk factors for impaired cerebral growth. Moreover, 
genetic syndromes were not sufficiently accounted for in these studies and the focus was on complex CHDs, and the impact of more common CHDs has yet to be evaluated.

Objectives: The objective of the present study was to investigate the correlates of microcephaly at birth in a population based study of children with all types of CHD.

Methods: 2,947 Danish children born 2000-2008 were eligible for validation. A total of 2,467 were eligible for genetic testing of the two most common genetic syndromes in CHD: trisomy 21 and $22 \mathrm{q} 11$ deletion syndrome. Further genetic, newborn, and maternal characteristics were identified in national registries. We present preliminary results of a matched cohort study. Each child was randomly matched to three children from the general population according to gender and gestational age. The risk of microcephaly $(<-2 \mathrm{SD})$ in non-syndromic children with $\mathrm{CHD}(\mathrm{n}=1,535)$ and syndromic children with CHD $(n=241)$ was compared to healthy children by means of conditional logistic regression. The results were adjusted for birth weight.

Results: Unadjusted measures of head circumference were smaller in both the non-syndromic $(-0.2 \mathrm{~cm} 95 \% \mathrm{CI}-0.3 ;-0.1)$ and the syndromic newborns with CHD $(-1.3 \mathrm{~cm} 95 \%$ CI $-1.3 ;-1.0)$ compared to the general population. The risk of microcephaly was higher in both the non-syndromic (OR $=1.795 \% \mathrm{CI} 1.3 ; 2.2)$ and the syndromic newborns with $\mathrm{CHD}(\mathrm{OR}=4.195 \%$ CI $2.4 ; 7.2)$. When birth weight was adjusted for, the difference virtually disappeared in the non-syndromic newborns $(\mathrm{OR}=1.195 \% \mathrm{CI} 0.7 ; 1.6)$, whereas the risk remained considerable in the syndromic newborns $(\mathrm{OR}=2.495 \% \mathrm{CI} 1.2 ; 5.0)$. Further results will be presented at the meeting.

Conclusions: Preliminary results from a subset of the CHD cohort support the hypothesis of reduced head circumference and increased risk of microcephaly in children with both syndromic and nonsyndromic CHD. It is further suggested that microcephaly in nonsyndromic CHD may largely be explained by a general fetal growth restriction, whereas brain growth in syndromic children with CHD seems even more compromised than overall growth during the fetal period. Further analyses will evaluate the association between head circumference and specific CHDs, specific genetic disorders, maternal anthropometrics, parity, smoking status, maternal medical diseases, sociodemographic factors and placental weight.

\section{O-050}

Blood pressure in Germany 1998 and 2008-2011: comparison of methods and key findings from two national health surveys

Presenter: Hannelore Neuhauser

Hannelore Neuhauser 1); Carolin Adler 1); Michael Thamm 1); Ute Ellert 1)

1) Robert Koch Institute, Berlin, Germany

Background: High blood pressure is one of the biggest global risk factors for disease and its distribution as well as changes over time in populations are therefore of great public health importance.

Objectives: The aim of this study is to provide updated epidemiological data on the distribution of high blood pressure in Germany and to evaluate time trends from 1998 to 2008-2011. A concurrent separate calibration study aimed at estimating the effect of a change in methods (devices and cuffs) and providing a calibration formula. Methods: Systolic and diastolic blood pressure (SBP, DBP) were measured in national health surveys in Germany in 1998 and 2008-2011, each with more than 7,000 participants aged 18-79 years according to standardized measurement protocols which were identical except for the device (standard mercury and Datascope Accutorr
Plus) and the manufacturer-provided cuffs. The mean of the second and third measurement was used for analysis. A separate calibration study evaluated the agreement of the old and the new protocol, i.e. of measurements taken with the two devices and respective set of three cuffs (455 measurement pairs, study protocol based on the International Protocol for the validation of BP measuring devices in adults of the European Society of Hypertension).

Results: In 2008-2011 mean SBP/DBP in $\mathrm{mmHg}$ were $120.8 / 71.2$ in women and $127.4 / 75.3$ in men. Blood pressure was hypertensive $(\geq 140 / 90 \mathrm{mmHg})$ in $12.7 \%$ of women and $18.1 \%$ of men. Hypertension defined as hypertensive blood pressure or taking antihypertensive medication in known cases of hypertension was found in $29.9 \%$ of women and $33.3 \%$ of men. Standard mercury/old cuffs measurements as used in 1998 were shown to be systematically higher than Datascope/new cuffs measurements and a formula was derived through linear regression modeling to predict calibrated 1998-Datascope values. After calibration of 1998 data and weighting of both surveys with a factor to correct deviations in the sample from the population as of 31 Dec 2010 in Germany, Germany, mean SBP appears to have dropped from 1998 to $2008-2011$ by $3.0 \mathrm{mmHg}$ in men and 5.4 in women (DBP by 3.4 and 5.0). Mean differences were most pronounced in treated hypertensives but a significant decrease of mean SBP and DBP was noted also in those without hypertension, particularly in women. The prevalence of hypertension, which includes controlled hypertension, did not markedly change in women and even slightly increased in men.

Conclusions: These findings suggest successful blood pressure decrease in Germany through treatment but also through primary prevention. However, one in three adults in Germany have hypertension and high blood pressure remains a primary public health concern

\section{Cancer: genes, prognosis and mortality}

12.08.2013 13:30-15:00

\section{O-051}

Psychological distress and cancer mortality risk: individual participant meta-analysis of 148,748 people from sixteen prospective cohort studies

Presenter: David Batty

David Batty 1), 2); Tom Russ 3); Manos Stamatakis 1); Mika Kivimaki 1)

1) Department of Epidemiology and Public Health, University College, London, UK; 2) Centre for Cognitive Ageing \& Cognitive Epidemiology, University of Edinburgh, UK; 3) Alzheimer Scotland Dementia Research Centre, University of Edinburgh, UK

Background: People with psychological distress (anxiety, depression) are more likely to smoke, drink heavily, have a poor diet, and be sedentary relative to their distress-free counterparts. With these health behaviours being established risk factors for selected cancers, there is a strong primae facie case for a distress-cancer relationship. There is, however, a paucity of such studies. This individual participant metaanalysis is the largest study to date to examine the link between distress and cancer.

Objective: To examine the role of psychological distress as a risk factor for cancer.

Methods: We pooled raw data from 16 country-wide, independent, prospective cohort studies comprising the Health Survey for England 
$(\mathrm{N}=13)$ and the Scottish Health Survey $(\mathrm{N}=3)$. Between 1994 and 2008 , a total of 148,748 people aged 16 years or older participated in baseline surveys. Psychological distress was ascertained using the 12 item General Health Questionnaire (score range 0 [no distress symptoms] to 12). Health behaviours, height, weight, and socioeconomic status were quantified using standard protocols. Study members were linked to national mortality registers.

Results: A mean follow-up of 10 years gave rise to 4,331 cancer deaths. In age- and sex-adjusted analyses, highly distressed individuals (GHQ score: 7-12) experienced a 1.50-fold increased rate of total cancer mortality relative to persons with no distress (GHQ score: 0 ). All sixteen cancer sites under study were related to high distress (hazard ratio range 1.75-4.14). Of the five most commonly occurring carcinomas, there were positive relationships for malignancy of the lung (hazard ratio; confidence interval: $1.81 ; 1.38,2.36$ ), colorectum $(1.86 ; 1.29,2.67)$, breast $(1.82 ; 1.31,2.53)$, prostate $(2.08 ; 1.27,3.39)$, and pancreas $(1.82 ; 1.09,3.02)$. Controlling for the afore described health behaviors, plus body mass index and socio-economic status, had little impact on the strength of these relationships. For selected cancer sites, there was also evidence of elevated risk at moderate levels of distress.

Conclusions: In addition to being a pernicious condition in its own right, in the present study, psychological distress was associated with the occurrence of several major malignancies.

\section{O-052}

High procedure volume is strongly associated with improved survival after lung cancer surgery

\section{Presenter: Henrik Moller}

Henrik Møller 1), 2)

1) King's College London; 2) Aarhus University

Background: A number of studies have reported an association between high hospital volume and increased survival for non-small cell lung cancer (NSCLC) surgery.

Objective: The present paper explores the association between hospital volume and lung cancer survival in England, accounting for case-mix and the propensity to resect.

Methods: We analysed data on 134,293 patients with NSCLC diagnosed in England between 2004 and 2008 of whom 12,862 (9.6\%) underwent surgical resection. Hospital volume was defined according to the number of patients with resected lung cancer in each hospital in each year of diagnosis and divided into five groups; <70; 70-99; 100-129; 130-149 and 150+ patients/year. We calculated hazard ratios for death in three pre-defined periods [early post-operative (0-30 days post-surgery), intermediate (31-365 days) and long-term (>365 days)] according to hospital volume, sex, age, socioeconomic deprivation, comorbidity and the propensity to resect.

Results: There was a strong association with increased survival in hospitals performing more than 150 surgical resections compared with those carrying out less than 70 [HR 0.78 (95\% CI 0.67-0.90), ptrend $<0.01]$. The association between hospital volume and survival was present in all three periods of follow-up, but the magnitude of the association for hospital volume was greatest in the early post-operative period [HR 150+ vs. <70: $0.58(0.38-0.89)]$, and smallest in the long-term [HR $0.84(0.71-0.99)]$. The difference in cumulative survival reached a maximum at around 2 years post-surgery.

Conclusions: This study shows that high volume hospitals $(>70$ resections per year) have higher resection rates, operate on patients who are older, have lower socioeconomic status, more comorbidities and despite that they achieve better survival, most notably in the early post-operative period.
O-053

Gene-environment interaction between 41 newly identified breast cancer susceptibility loci and established environmental risk factors

Presenter: Anja Rudolph

Anja Rudolph 1); Anja Schoeps 1); Thérèse Truong 2); Julia Knight 3), 4); Stefan Nickels 1); Marjanka Schmidt 5); Doug Easton 6), 7); Montserrat Garcia-Closas 8), 9); Roger Milne 10); Jenny Chang-Claude 1)

1) Division of Cancer Epidemiology, German Cancer Research Center (DKFZ), Heidelberg, Germany; 2) Inserm (National Institute of Health and Medical Research), CESP (Center for Research in Epidemiology and Population Health), U1018, Environmental Epidemiology of Cancer, Villejuif, France; 3) Samuel Lunenfeld Research Institute, Mount Sinai Hospital, Toronto, Ontario, Canada; 4) Division of Epidemiology, Dalla Lana School of Public Health, University of Toronto, Toronto, Ontario, Canada; 5) Netherlands Cancer Institute, Antoni van Leeuwenhoek Hospital, Amsterdam, The Netherlands; 6) Centre for Cancer Genetic Epidemiology, Department of Public Health and Primary Care, University; 7) Centre for Cancer Genetic Epidemiology, Department of Oncology, University of Cambridge, Cambridge, UK; 8) Division of Cancer Epidemiology and Genetics, National Cancer Institute, Rockville, Maryland, United States of America; 9) Sections of Epidemiology and Genetics, Institute of Cancer Research and Breakthrough Breast Cancer Research Centre, London, United Kingdom; 10) Genetic and Molecular Epidemiology Group, Human Cancer Genetics Program, Spanish National Cancer Research Centre [CNIO]

Background: A recently conducted large-scale genotyping project identified 41 new breast cancer susceptibility loci. Breast cancer risk associated with these newly identified susceptibility loci may differ according to exposure levels of established environmental risk factors for breast cancer.

Objectives: To evaluate potential gene-environment interactions between 41 common single nucleotide polymorphisms and eight risk factors in women of European ancestry.

Methods: We included 23 studies from the Breast Cancer Association Consortium. The environmental risk factors considered were age at menarche, ever parous, number of full-term pregnancies, adult height, postmenopausal body mass index, current use of menopausal estrogen-progestagen therapy, current use of menopausal estrogen monotherapy and cumulative lifetime alcohol consumption. The analysis comprised up to 27,272 invasive breast cancer cases and 29,192 unaffected controls. We utilized logistic regression models adjusted for study, genetic principal components and age and included a multiplicative interaction term to evaluate gene-environment interaction by means of a likelihood ratio test.

Results: We observed five gene-environment interactions with Pinteraction $<0.01$. The per-allele odds ratio (OR) (95\% confidence interval) for breast cancer associated with rs13329835 in CDYL2 was 1.32 (1.11-1.57) in postmenopausal women using estrogen-progestagen therapy compared to $1.00(0.92-1.08)$ in non-users (Pinteraction $=0.003)$. A similar more than multiplicative interaction was observed between rs13329835 and increasing duration of current use of estrogen-progestagen therapy (Pinteraction $=0.007$ ), with non-user as reference group. The association of rs4808801 in ELL with breast cancer risk was modified by number of fullterm pregnancies (Pinteraction $=0.003$ ); the per-allele $\mathrm{OR}$ varied from $0.97(0.91-1.03)$ in women with one full-term pregnancy to 0.83 $(0.76-0.91)$ in women with four or more pregnancies. For rs11780156 located close to MYC, the per-allele OR also varied by number of full-term pregnancies, from $1.03(0.95-1.11)$ in women with one full-term pregnancy to $1.25(1.12-1.40)$ in women with four or more pregnancies (Pinteraction $=0.009)$. Furthermore, the association of rs17356907 in 
NTN4 differed in parous and nulliparous women (Pinteraction $=0.005)$ with a per-allele OR of $0.82(0.77-0.88)$ in nulliparous women and 0.92 (0.89-0.94) in parous women.

Conclusion: The associated effects on breast cancer risk of recently discovered susceptibility loci do not seem to be strongly modified by environmental risk factors. The observed potential gene-environment interactions warrant replication in further studies.

\section{0-054}

Regional variations in German mesothelioma mortality rates: 2000-2010

\section{Presenter: Sara J. Schonfeld}

\section{Sara J. Schonfeld 1); Valerie McCormack 1); Joachim Schüz 1)}

1) Section of Environment and Radiation, International Agency for Research on Cancer

Background: Mesothelioma is a fatal malignancy of the mesothelium that is almost entirely attributable to asbestos exposure. Previous studies have described higher mesothelioma mortality rates in Western than Eastern Europe. Germany presents a unique country in which to further examine geographical heterogeneity. Political division and contrasting trade routes between the Federal Republic of Germany (West Germany) and German Democratic Republic (East Germany) led to differences with respect to the quantity, sources and types of asbestos fibres used prior to the 1990 reunification. This period also represents the relevant exposure period (1960s-1970s) for mesothelioma deaths occurring early in the twenty-first century, given the 30-40 year latency period. In contrast, these formerly separate countries are now served by the same health system and mortality reporting, and bans on all types of asbestos were introduced in 1993. Objectives: We investigated mesothelioma mortality rates by gender and region across Germany during 2000-2010.

Methods: Mesothelioma mortality (ICD 10: C45) and population data were obtained from the Federal Statistical Bureau of Germany, stratified by age, sex, and federal state. The 16 federal states were collapsed into West Germany and East Germany, with Berlin as separate unit. We calculated truncated age-standardized mesothelioma mortality rates per 100,000 persons $(40+$ years using standard European population, denoted as ASRs40+). Age- and calendar year-adjusted mortality rate ratios (MRRs) and $95 \%$ confidence intervals (CIs) for region and sex were estimated from Poisson regression models.

Results: There were 12,855 mesothelioma deaths at ages $40+$ in Germany during 2000-2010. In both males and females, ASRs40+ were highest in West Germany (males 4.4; females 0.8), intermediate in Berlin (males 2.7; females 0.7) and lowest in East Germany (males 1.7; females 0.6). MRRs for West versus East Germany were 2.7 $(95 \%$ CI 2.5, 2.9) among males and 1.4 (95\% CI 1.3, 1.6) among females. The MRR for males versus females was 5.3 (95\% CI 5.1, 5.6) in West Germany, 4.0 (95 \% CI 3.1, 5.1) in Berlin and $2.9(95 \%$ CI 2.6, 3.3) in East Germany.

Conclusions: This initial investigation of mesothelioma mortality rates showed marked regional differences across Germany. Rates were significantly higher in the West than East, similar to patterns previously reported on a broader scale across Europe. Mesothelioma mortality rates were higher among males than females across all regions. Further analyses will be conducted to investigate regional patterns at more detailed geographic level such as federal state.

\section{O-055}

Biologic interaction between prostate cancer and comorbidities and its impact on the rate of venous thromboembolism

Presenter: Mary Nguyen-Nielsen
Mary Nguyen-Nielsen 1); Erzsébet Horváth-Puhó 1); Timothy L. Lash 1), 2); Michael Borre 3); Vera Ehrenstein 1); Henrik Toft Sørensen 1)

1) Department of Clinical Epidemiology, Aarhus University Hospital, Aarhus, Denmark; 2) Department of Epidemiology, Rollins School of Public Health, Emory University, Atlanta, Georgia, USA; 3) Department of Urology, Aarhus University Hospital, Aarhus, Denmark

Background: Venous thromboembolism (VTE) is associated with increased morbidity and mortality and its incidence increases with cancer disease. Given that prostate cancer (PC) is the most common non-skin cancer among men worldwide (in Denmark: $\sim 4,000$ new PC cases per year; age-standardized annual incidence of 142 per 100,000 men), understanding the rate of VTE among PC patients is clinically important.

Objectives: We aimed to: (1) estimate the incidence of VTE associated with PC; and (2) estimate the impact of biologic (causal) interaction between PC and comorbidities on VTE rate, i.e. by estimating the Interaction Contrast (IC) by comorbidity level. The IC is the "departure from the risk difference, contrasting from what would be expected if no interaction were present."

Methods: We conducted a nationwide historical matched cohort study in Denmark (population 6.9 million during study period). PC patients were identified from the Danish Cancer Registry and the Danish National Reg istry of Patients (DNRP) in 1995-2010. For each man with PC, we selected up to five cancer-free men matched by age and comorbidity level (Charlson Comorbidity Index [CCI]: CCI $0=$ none; CCI $1=$ low; CCI $2-3=$ medium; CCI $\geq 4=$ high). Follow-up was from date of PC diagnosis, or matching, until date of VTE diagnosis in the DNRP (defined as deep venous thromboembolism [DVT] and/or pulmonary embolism [PE]), death, emigration, or 31-Dec-2011. We reported descriptive characteristics of the PC and matched cohorts, including CCI level, treatments received, prostate-specific antigen (PSA) values at diagnosis, Gleason score, and clinical staging. We used the Kaplan-Meier method to estimate 5-year cumulative incidence of VTE in the PC and matched cohorts. Cox proportional-hazards regression was used to estimate adjusted Incidence Rate Ratios (IRR). Finally, we used age-standardized rates to calculate the IC of VTE occurrence by comorbidity level, and then estimated the proportion of the 5-year cumulative incidence of VTE that could be attributed to biologic interaction between PC and comorbidity.

Results: We identified 43,084 PC patients and 208,555 men in the matched cohort. Median age at PC diagnosis was 72 years (interquartile range 66-79). The 5-year cumulative incidence of VTE was $3.0 \%$ for the PC men and $1.5 \%$ for the matched cohort. The agestandardized VTE rate per 1,000 person-years was 6.0 (95\% CI 5.6, 6.4) for the PC cohort and 3.1 (95\% CI 2.9, 3.2) for the matched cohort. The adjusted IRR for VTE was 2.0 (95\% CI 1.9, 2.2) overall, $2.3(95 \%$ CI $2.1,2.6)$ for DVT, and 1.7 (95\% CI 1.5, 1.9) for PE. The interaction between prostate cancer and low, medium, and high comorbidity levels accounted for $-5,5$, and $5 \%$, respectively, of the 5 -year cumulative incidence of VTE.

Conclusion: Men with PC have a two-fold excess rate of VTE compared with men of the same age and comorbidity level. Approximately $5 \%$ of the 5-year cumulative incidence of VTE is attributabl e to biologic interaction between prostate cancer and comorbidity. Although the excess rate of VTE cannot be explained entirely by the independent effects of cancer or comorbidity, the biologic interaction is minimal.

O-056

Enterolactone levels and prognosis after invasive postmenopausal breast cancer: potential effect modifiers

Presenter: Jenny Chang-Claude 
Petra Seibold 1); Alina Vrieling 1), 2); Theron Johnson 1); Katharina Buck 1), 3); Rudolf Kaaks 1); Jakob Linseisen 4); Judith Heinz 5); Nadia Obi 5); Dieter Flesch-Janys 5); Jenny Chang-Claude 1)

1) German Cancer Research Center (DKFZ), Heidelberg, Germany; 2) Radboud University Medical Centre, Nijmegen, The Netherlands; 3) National Center for Tumor Diseases (NCT), Heidelberg, Germany; 4) Helmholtz Center Munich, Neuherberg, Germany; 5) University Cancer Center Hamburg, University Medical Center HamburgEppendorf, Germany

Background: We previously reported that high levels of post-diagnostic serum enterolactone, the main metabolite of lignans, a class of phytoestrogens, are associated with lower mortality in 1,140 postmenopausal breast cancer patients. Two subsequent studies confirmed this finding. In order to provide more insight into this association, we investigated whether enterolactone concentrations are correlated with other lifestyle factors and tumor characteristics and whether the association might be restricted to subgroups defined by these factors. Methods: We additionally measured enterolactone concentrations in patients of the Hamburg study center of the two-center (Hamburg and Rhine-Neckar-Karlsruhe regions) MARIEplus study. The 2,048 postmenopausal breast cancer patients, aged 50-74 years and diagnosed between 2001 and 2005 in Germany, were prospectively followed up until end of 2009. Vital status was assessed via local population registries, deaths were verified by death certificates. Information on the clinical course and treatment was collected and verified by clinical records. Hazard ratios (HR) and $95 \%$ confidence intervals $(\mathrm{CI})$ for post-diagnostic enterolactone levels in relation to overall survival (OS) were estimated using delayed-entry Cox proportional hazards models stratified by age at diagnosis and study center and adjusted for prognostic factors. Potential differential effects by tumor subtype, and lifestyle factors were assessed.

Results: Overall, 267 patients died after a median follow-up time of 5.5 years. Median enterolactone levels for deceased and non-deceased patients were 17.7 and $22.6 \mathrm{nmol} / \mathrm{L}$, respectively. Patients with higher compared to lower enterolactone levels were more likely to have tumors of smaller size, lower grade, and positive hormone receptor status and to be HRT users and have normal weight, and less likely to be current smokers. High enterolactone levels were associated with better OS (highest vs. lowest quartile, HR 0.59, $95 \%$ CI $0.40-0.87$ ). Per $10 \mathrm{nmol} / \mathrm{L}$ increment, HR was 0.94 (95\% CI 0.90-0.98) overall and not different for luminal A, luminal B, HER2 positive and triple negative tumors (HR 0.89, 0.97, 0.68, and 0.94, respectively). Associations were stronger in normal weight women than overweight women $(p$ het $=0.046)$, in more active than less active women $(p$ het $>0.05)$ and in current smokers than never/former smokers ( $p$ het $>0.05$ ). In patients who had a healthy lifestyle (normal weight, active, non-current smoker), an inverse association with OS was still apparent (per $10 \mathrm{nmol} / \mathrm{L}$ HR 0.92, 0.86-0.99).

Conclusion: The inverse association of high enterolactone levels with overall mortality was not differential by tumor subtype and cannot be entirely explained by a healthy lifestyle.

\section{O-057}

Diabetes in elderly breast cancer patients in relation to all-cause mortality and recurrence risk

Presenter: Esther Bastiaannet

Esther Bastiaannet 1), 2); Mandy Kiderlen 1), 2); Nienke de Glas 1); Charla Engels 1); Willemien van de Water 1), 2); Ton de Craen 2); Johanneke Portielje 3); Cornelis van de Velde 1); Gerrit-Jan Liefers 1)
1) Department of Surgery, Leiden University Medical Center, Leiden, The Netherlands; 2) Department of Gerontology \& Geriatrics, Leiden University Medical Center, Leiden, The Netherlands; 3) Department of Medical Oncology, Haga Hospital, The Hague, The Netherlands

Background: In developed countries, $40 \%$ of the breast cancer patients are older than 65 years of age at diagnosis, of whom $16 \%$ additionally suffer from diabetes.

Objectives: The aim of this study was to assess the impact of diabetes on relapse free period and overall mortality in elderly breast cancer patients.

Methods: Patients were selected from the retrospective FOCUS cohort, which contains detailed information of elderly breast cancer patients. Relapse free period was calculated using Fine \& Gray competing risk regression models for diabetics versus non-diabetic breast cancer patients. For overall survival analyses, patients were divided into four groups: no comorbidity, solely diabetes, diabetes and other comorbidity or other comorbidity without diabetes and analysed using multivariable Cox regression models.

Results: Overall, 3,124 patients with non-metastasized breast cancer were included. Relapse free period was better for patients with diabetes compared to non-diabetics (multivariable $\mathrm{HR} 0.77,95 \% \mathrm{CI}$ 0.59-1.01), irrespective of other comorbidity, especially in patients aged 75 years and older (HR 0.67, $95 \%$ CI 0.45-0.98). In overall survival analyses, patients with solely diabetes had a similar outcome as patients without comorbidity (HR $0.86,95 \%$ CI $0.55-1.33$ ), while patients with diabetes and other comorbidity had the worst overall survival (HR 1.70, 95 \% CI 1.44-2.01).

Conclusions: When taking competing mortality into account, relapse free period was better in elderly breast cancer patients with diabetes compared to non-diabetics. Moreover, patients with diabetes without other comorbidity had a similar overall survival as patients without any comorbidity. Possibly, unfavourable effects of (complications of) diabetes on overall survival are counterbalanced by beneficial effects of metformin on the occurrence of breast cancer recurrences.

\section{O-058}

Primary malignant tumors of the central nervous system in the Arkhangelsk region, Russia in 2000-2011

Presenter: A Dyachenko, Andrej Grjibovski

A Dyachenko 1); Anna Subbotina 1), 2); Timur Izmailov 3); Sergey Asakhin 1); Natalia Glukhareva 1); Dmitri Korotov 1); Andrey Krasilnikov 1); Dinara Ospanova 4); Andrej Grjibovski 5), 6); Mikhail Valkov 1)

1) Northern State Medical University, Arkhangelsk, Russia; 2) Institute of Biomedicine, Sahlgrenska Academy at Gothenburg University, Gothenburg, Sweden; 3) Russian Scientific Center of Radiology and Radiotherapy, Moscow, Russia; 4) Institute of Postgraduate Medical Education (AGIUV), Almaty, Kazakhstan; 5) International School of Public Health, Northern State Medical University, Arkhangelsk, Russia; 6) Department of International Public Health, Norwegian Institute of Public Health, Oslo, Norway

Background: The incidence of and mortality from primary tumors of the central nervous system (CNS-PT) varies widely around the world. An increase in the incidence of CNS-PT has been reported in many Western countries. However, little is known about the epidemiology of CNS-PT in Russia. Even less is known about the situation in Northwest Russia.

Objective: To describe epidemiology of malignant CNS-PT in the Arkhangelsk region (AR), Russia, for the period from 2000 to 2011. Methods: Data on all cases of CNS-PT (ICD-10 codes: C70-C72) that occurred in the AR in 2000-2011 were extracted from the 
Arkhangelsk Regional Cancer Registry. Size of the population of the AR was obtained from the Regional Bureau of Statistics. Ageadjusted incidence and mortality rates were calculated per 100,000 using the standard world population. Proportions of cases by sex, location and histological types were also studied. Only malignant tumors were included in this study.

Results: Altogether, 836 cases of the CNS-PT were recorded in the AR during the studied period. The median age of patients at the moment of setting the diagnosis was 52 years. Only malignant tumors were recorded. Nine percent of all CNS-PT were recorded among children aged $0-18$ years. Men constituted $52 \%$ of the sample. Seventy-two percent of all cases occurred among urban residents. The most common were tumors of cerebral hemispheres $(54 \%)$ by location and of neuroepithelial structure $(74 \%)$ by histological type. The incidences of CNS-PT of the meninges were the greatest in the agegroup of 60-69 years, while the incidence of neuroepithelial tumors was the highest in the age-group of 50-59 years. The standardized (world standard) incidence of CNS-PT in AR among men increased from 5.61 per 100,000 in 2000 to 6.20 per 100,000 in 2011 . The corresponding numbers for women were 4.48 and 4.78. Standardized mortality rates for CNS-PT were 3.40 and 3.22 per 100,000 for men and women, respectively.

Conclusion: The epidemiology of the CNS in the AR in 2000-2011 is similar to what has been reported from most of the other industrialized countries. The registration of not only malignant, but also benign CNS-PT is warranted in Russia.

\section{Others}

12.08.2013 13:30-15:00

\section{0-059}

Cross-sectional and prospective associations between serum vitamin $D$ and body composition in a German cohort of older adults

Presenter: Susanne Vogt

Susanne Vogt 1); Astrid Zierer 1); Michael Laxy 2); Wolfgang Koenig 3); Birgit Linkohr 1); Annette Peters 1); Barbara Thorand 1)

1) Institute of Epidemiology II, Helmholtz Zentrum München, German Research Center for Environmental Health, Neuherberg, Germany; 2) Institute of Health Economics and Health Care Management, Helmholtz Zentrum München, German Research Center for Environmental Health, Neuherberg, Germany; 3) Department of Internal Medicine II-Cardiology, University of Ulm Medical Center, Ulm, Germany

Background: Low levels of serum 25-hydroxyvitamin D3 (25(OH)D) have been found to be related to obesity in adult populations but there is limited information about the nature of this association in older people. Furthermore, very few studies have examined the prospective association of the two conditions in this age group and the results are ambiguous.

Objectives: We sought to investigate the cross-sectional and prospective association of baseline serum 25(OH)D levels and body composition (body mass index (BMI), waist hip ratio (WHR) and body fat) in a cohort of older adults.

Methods: We used data from the population-based KORA (Cooperative Health Research in the Region of Augsburg)-Age cohort (2008-2012). This study includes subjects born in 1943 or earlier who participated in previous MONICA/KORA studies. Serum 25(OH)D levels were measured at baseline using an enhanced chemiluminescence immunoassay (Roche, Mannheim). A total of 1,008 participants with baseline examination (weight, height, waist/hip circumference, body fat) were included in the cross-sectional analysis. 801 participants who also took part in the follow-up examination were included in the prospective analysis. Multivariable linear models were used for the cross-sectional analyses and general estimation equation (GEE) models for the longitudinal analyses.

Preliminary results: The median follow-up time was 2.7 years $(\mathrm{SD}=0.1) .52 \%$ of the participants were vitamin $\mathrm{D}$ deficient [25(OH)D concentration $<20 \mathrm{ng} / \mathrm{ml}]$ and another $27 \%$ had insufficient vitamin D levels [25(OH)D concentration: $20-29.9 \mathrm{ng} / \mathrm{ml}]$. The mean $( \pm \mathrm{SD})$ change in BMI per year of follow-up was $-0.14( \pm 0.5)$ $\mathrm{kg} / \mathrm{m}^{2}$. In the cross-sectional analysis, in the adjusted models (accounting for age, sex, smoking status, outdoor walking, alcohol consumption and season of blood sample collection), serum levels of $25(\mathrm{OH}) \mathrm{D}$ were significantly associated with BMI and WHR. Every $10 \mathrm{ng} / \mathrm{ml}$ increase of serum $25(\mathrm{OH}) \mathrm{D}$ levels was associated with a 0.8 unit decrease in BMI $(p<0.0001)$ and a 0.005 point decrease in WHR $(p<0.0017)$. In the adjusted GEE model, baseline serum levels of $25(\mathrm{OH}) \mathrm{D}$ were associated with BMI change over the followup period. Every $10 \mathrm{ng} / \mathrm{ml}$ increase of serum $25(\mathrm{OH}) \mathrm{D}$ levels was associated with an average 0.8 unit decrease in BMI $(p<0.0001)$.

Preliminary conclusions: Our results demonstrate an inverse crosssectional relationship between serum $25(\mathrm{OH}) \mathrm{D}$ levels and both BMI and WHR, as well as an inverse prospective association between baseline serum $25(\mathrm{OH}) \mathrm{D}$ and BMI change in an aged population. This association is independent of lifestyle factors.

\section{0-060}

Maternal pre-pregnancy obesity and intelligence quotient (IQ) in the children

Presenter: Mette Bliddal

Mette Bliddal 1); Henrik Stoevring 2); Hanne-Lise Eriksen 3); Ulrik Kesmodel 4); Thorkild Soerensen 5); Jørn Olsen 6); Ellen Noehr 6)

1) Odense University Hospital, The Research Unit of Gynaecology and Obstetrics, Denmark; 2) Department of Public Health, Biostatistics, University of Aarhus, Denmark; 3) Center of Functionally Integrative Neuroscience, Institute for Clinical Medicine, University of Aarhus, Denmark; 4) Aarhus University Hospital, Unit of Gynaecology and Obstetrics, Denmark; 5) Novo Nordisk Foundation Center for Basic Metabolic Research, University of Copenhagen, Denmark; 6) Department of Public Health - Institute of Epidemiology and Social Medicine, University of Aarhus, Denmark

Background: Obesity is related to several adverse pregnancy outcomes and may have a negative effect on fetal brain development, perhaps related to the hormone production in fat tissue or the contaminants of fat tissue by fat soluble neuro-toxins. Obesity has been related to a low IQ in adults, but whether this association is causal is not known.

Objectives: We hypothesized that pre-pregnancy body mass index (BMI) is inversely correlated with IQ in children at age 5, even after adjustment for social factors and maternal IQ. We further expected that child IQ is not correlated with paternal BMI, since his fat tissue has no direct influence on the intrauterine environment. We further expected that child IQ is not correlated with paternal BMI, since his fat tissue has no direct influence on the intrauterine environment. Should such an association exist it would suggest that the association 
between maternal BMI and child IQ are through causal pathways unrelated to intrauterine effects of body fat.

Methods: We had information on maternal pre-pregnancy BMI, paternal BMI, and child IQ in 1,782 children from the Danish National Birth Cohort, who participated in an extensive 3-h assessment of cognitive ability including IQ tests of both the mother and the child. Multiple linear regression analyses were used to estimate the association between parental BMI and child IQ adjusted for a comprehensive set of potential confounders.

Results: The crude association between maternal BMI and child IQ was in the direction as predicted as a higher BMI was associated with a lower IQ with a regression coefficient of $-0.40(p=0.001)$. This association was attenuated after adjustments for social factors and maternal IQ, but remained statistically significant with a value of $-0.27(p=0.03)$. When parental BMI was adjusted for together with all other factors except maternal IQ, the association between paternal BMI and child IQ yielded a regression coefficient of -0.26 $(p=0.12)$ which was comparable to that seen for maternal BMI $(-0.20 ; p=0.11)$

Conclusion: Although maternal pre-pregnancy BMI is inversely associated with the IQ of her child, this study suggests that this association is not a specific pregnancy-based effect, because the paternal BMI shows the same association with the child IQ

\section{0-061}

Comprehensive cardiac rehabilitation improves return to work rate on a short term basis but not on a long term basis

Presenter: Lau Thygesen

Lau Thygesen 1); Anne Soja 2), 3); Shan Alpin 1); Ann-Dorthe Zwisler 1), 2), 4)

1) National Institute of Public Health, University of Southern Denmark; 2) Department of Cardiology, Bispebjerg Hospital, Copenhagen, Denmark; 3) Department of Cardiology S, Herlev University Hospital, Herlev, Denmark; 4) Copenhagen Trial Unit, Rigshopsitalet, Denmark

Background: Coronary artery disease (CAD) incidence is high and growing worldwide. Among CAD patients $25-30 \%$ is taking active part in labor market when hospitalized. Keeping patients affiliated to the labor market has been an important goal in the treatment of CAD. Cardiac rehabilitation may have a positive effect on return to work and labor market participation on a short and long term basis.

Objective: To determine the short- and long term effects of comprehensive cardiac rehabilitation including vocational guidance on labour market affiliation, sick leave and welfare payment compared to usual care. Methods: The study was conducted in an open, single centre centrally concealed randomized trial including 770 patients eligible to cardiac rehabilitation called the DANREHAB trial. The trial compared comprehensive cardiac rehabilitation versus usual care among patients with congestive heart failure, $\mathrm{CAD}$ or being at high risk of developing CAD. The comprehensive rehabilitation program over a 12 weeks period contained elements of patient education, supervised physical training, dietary counselling, cooking lessons, smoking cessation, psycho-social support, pharmacological therapy, and risk factor control. 211 patients were affiliated to the labour market at the time of randomization. Labour market participation, sick leave, welfare payment and personal income were ascertained from nationwide registers. Patients were followed from inclusion in 2000-2003 to 2010. To differentiate between shortterm and long-term effect, follow-up was divided into 1,3,5 and 7 years from randomization.

Results: The return to work rate was higher in the cardiac rehabilitation group looking at 1 year after randomization when compared to the usual care group $(p=0.03)$ but this difference was not retrieved on a long term basis after 3,5 and 7 years. The usual care group had more sick leave days after 3-7 years compared to the cardiac rehabilitation group although these differences were not significant.

Conclusions: In a randomized setting within comprehensive cardiac rehabilitation, the rate of patients returning to work was significantly higher 1 year after inclusion but not on the long run.

\section{0-062}

Transnet-Phelophepa as a source of primary health care data in rural South Africa

\section{Presenter: Ilse Truter}

\section{Ilse Truter 1)}

1) Drug Utilization Research Unit (DURU), Nelson Mandela Metropolitan University

Epidemiological and pharmacoepidemiological data for rural South Africa are not readily available. Large information gaps exist about health access, and data on disease prevalence and medicine utilisation in rural areas are often lacking. The aim of the study was to illustrate how existing data can aid in providing an overview of rural health care. Transnet-Phelophepa is the only primary health care train in the world. The 18-coach train is operational for 35 weeks annually, and is stationed for 1-2 weeks at a railway station in a rural area or area that has been identified as underserviced in terms of health infrastructure. Transnet-Phelophepa started in 1994 and reaches approximately 180,000 individuals a year. Apart from its permanent staff, TransnetPhelophepa uses students in health disciplines (pharmacy, nursing, dental, optometry and psychology) to administer the services, whilst overseen by qualified practitioners, thereby providing students with a unique learning experience. The data gathered by Transnet-Phelophepa include demographic information, diagnoses and medicines/ treatment prescribed. Although the focus is on acute primary health care conditions, it is a valuable source of epidemiological data for each area visited. Between its inception in 1994 and 2009, a total of $2,455,044$ individuals have been reached, and 322,468 prescriptions have been dispensed. In 2009 a total of 26,249 prescriptions were dispensed. In Caledon, a farming community in the Western Cape Province, 737 prescriptions (2,745 items) were dispensed in 1 week during 2009. Patients were suffering primarily from musculoskeletal ailments, urinary tract infections (females) and ophthalmic conditions (dry eyes). There was an increase in the number of respiratory diseases/infections since its previous visit. The highest number of patients seen was in 2012 at Swartkops in the Eastern Cape, where approximately 2,000 patients visited the train. Swartkops is not a true ruraI area, indicating that health services in the area are not functioning properly. In January 2013, the train visited the Northern Cape, a scarcely populated area with mostly farming communities. Examples of common conditions reported were also musculoskeletal system disorders (especially arthritis) and dry eyes as a result of a heat wave while the train was stationed there. A second train started operating in 2012 allowing Transnet-Phelophepa to double its reach. Although health statistics for rural areas are not readily available, there are information sources such as Transnet-Phelophepa that can assist in identifying the epidemiological profile of an area, and also assist in identifying areas that are severely underserviced.

\section{O-063}

Maternal exposure to a heatwave increases the risk of preterm birth

Presenter: Shilu Tong 
Shilu Tong 1); Jiajia Wang 1); Gail williams 2); Paul colditz 3); Jorn Olsen 4)

1) School of Public Health and Institute of Health and Biomedical Innovation, Queensland University of Technology, Brisbane, Australia; 2) School of Population Health, University of Queensland, Brisbane, Australia; 3) Perinatal Research Centre, University of Queensland, Brisbane, Australia; 4) Department of Public Health, Aarhus University, Denmark

Background: A few studies have assessed the relation between exposure to ambient temperatures during pregnancy and the risk of preterm birth (PTB) and only little is known about the impact of maternal exposure to a heatwave on the occurrence of PTB.

Aims: To examine the relation between maternal exposure to a heatwave during pregnancy and the risk of PTB (birth before 37 weeks of gestation) in Brisbane, Australia, during 2000-2010.

Methods: We obtained the daily data, from relevant government agencies, on sociodemographic factors, air pollutants and meteorological factors and births for Brisbane, Australia, during 2000-2010. Logistic regression model was used to examine the association between maternal exposure to a heatwave and the risk of PTB, after adjusting for maternal age, parity, indigenous status and infants' gender, socioeconomic index for area, relative humidity, air pressure and air pollution.

Results: There was a statistically significant association between maternal exposure to a heatwave and the risk of PTB. The relative risk for PTB was 1.25 (95\% confidence interval (CI): 1.15-1.35), 1.15 (95 \% CI 1.07-1.24), and 1.28 (95\% CI 1.18-1.39) for maternal exposure, in the 1st trimester, to a heatwave defined as daily maximum temperatures $>$ the 90th percentile for 2,3 or 4 days, respectively. However, no apparent association was observed for maternal exposure to a heatwave in the 2 nd or 3 rd trimester. Similar results were found when we changed the definition of heatwave (e.g., maximum temperatures $>$ the 95 th percentile for 2 or more consecutive days).

Conclusions: Maternal exposure to a heatwave during early pregnancy is a significant risk for PTB. Further research is necessary to detail the likely important implications of this finding for the improvement of prenatal care.

\section{O-064}

Caffeine intake during pregnancy and risk of cerebral palsy in offspring: a joint study in three large Nordic birth cohorts

Presenter: Katrine Strandberg-Larsen

Katrine Strandberg-Larsen 1); Mette Tollånes 2); Bodil Hammer Bech 3); Dag Moster 2); Tine Brink Henriksen 4); Kacey Eichelberger 5); Allen Wilcox 6)

1) Section of Social Medicine, Department of Public Health, University of Copenhagen; 2) Department of Public Health and Primary Health Care, University of Bergen; 3) Department of Public Health, Institute of Epidemiology and Social Medicine, Aarhus University, Denmark; 4) Perinatal Epidemiological Research Unit, Aarhus University Hospital, Aarhus, Denmark; 5) Department of Obstetrics and Gynecology, University of North Carolina at Chapel Hill School of Medicine, North Carolina, USA; 6) Epidemiology Branch, National Institute of Environmental Health Sciences, Durham, North Carolina, USA

Background: The aetiology of cerebral palsy (CP) is largely unknown. CP causes physical disability in childhood in around two of 1,000 liveborn. Caffeine citrate is routinely used to prevent episodes of apnea in premature infants, and treated infants have decreased risk of $\mathrm{CP}$.
Objective: We examined if intake of caffeinated beverages during pregnancy was associated with $\mathrm{CP}$.

Methods: Information on intake of caffeine were obtained from three birth cohorts-the Danish National Birth Cohort, the Aarhus Birth Cohort and the Norwegian Mother and Child Cohort Study, comprising a total of 165,320 pregnancies and $307 \mathrm{CP}$ cases. The national $\mathrm{CP}$ registry (Denmark) and maternal report (Norway) provided information on $\mathrm{CP}$. Associations were investigated nation-wise in Cox regression models, with adjustment for maternal age, sociooccupational status, assisted reproduction and maternal smoking.

Results: In Denmark, every cup increase in the daily number of cups of coffee in first half of pregnancy increased the risk of CP with 1.06 (95\% confidence interval (CI) 1.00-1.12). Increase in the daily consumption of cola and overall intake of caffeine in first half of pregnancy also increased the risk of $\mathrm{CP}, p$ for trend 0.02 and 0.01 , respectively. In Norway, daily intake of $6+$ cups of coffee in late pregnancy compared to no intake was associated with excess risk of $\mathrm{CP}$ in offspring (RR 3.4, $95 \% \mathrm{CI} 1.3-9.0$ ), whereas intake before pregnancy or earlier in pregnancy was not. Exposure to 2+ servings of cola daily in mid-pregnancy resulted in a RR of CP of 3.4 (1.6-7.3). Conclusion: These findings suggest that intrauterine exposure to caffeine may increase the risk of CP. We will, before presenting this work, have more results on the importance of intake of caffeinated beverages at different periods of pregnancy.

\section{O-065}

Alcohol consumption and time to pregnancy: a danish prospective cohort study

Presenter: Ellen M. Mikkelsen

Ellen M. Mikkelsen 1); Anders H. Riis 1); Lauren A. Wise 2); Elisabeth E. Hatch 3); Kenneth J. Rothman 3), 4); Henrik Toft Sørensen 1), 3)

1) Department of Clinical Epidemiology, Aarhus University Hospital, Denmark; 2) Slone Epidemiology Center, Boston University, MA, USA; 3) Department of Epidemiology, School of Public Health, Boston University, MA, USA; 4) RTI Health Solutuions, NC, USA

Background: Some studies have found alcohol consumption to be associated with decreased fecundability whereas others have found no such association or even indicate a positive association between moderate alcohol intake and fecundability.

Objectives: We evaluated the association between consumption during the preconception period of any alcohol, as well as specific categories of alcohol-containing beverages (beer, wine and spirits), and time to pregnancy (TTP).

Methods: We examined these relations in a prospective cohort study of 3,895 Danish female pregnancy planners. Data were self-reported via internet-based questionnaires at baseline and updated every 2 months for a year or until conception occurred. Participants were asked to consider their alcohol intake during the past month and report their average weekly consumption in standard servings [bottles of beer $(330 \mathrm{ml})$, glasses of red or white wine $(120 \mathrm{ml})$, dessert wine $(50 \mathrm{ml})$, and spirits $(20 \mathrm{ml})]$. We used a proportional probabilities regression model to estimate fecundability ratios (FRs) and $95 \%$ confidence intervals (CIs).

Results: Compared with no alcohol consumption, the FRs for consumption of $1-3,4-7,8-13$ or $\geq 14$ servings per week were 0.95 $(95 \% \mathrm{CI}=0.88-1.03), 1.01 \quad(95 \% \mathrm{CI}=0.91-1.12), 1.01 \quad(95 \%$ $\mathrm{CI}=0.84-1.20)$ and $0.77(95 \% \mathrm{CI}=0.52-1.12)$, respectively, after adjustment for demographic, socioeconomic and lifestyle factors. We observed no relation between TTP and category of alcohol-containing beverage consumed after mutual adjustment for alcohol types. 
Conclusions: Our data indicate that pre-conception alcohol consumption of less than 14 servings per week is not materially associated with TTP. Greater consumption, however, may be associated with a slight delay in TTP.

\section{O-066}

Digital literacy can improve cognition and reduce health inequalities: findings from the English Longitudinal Study of Ageing (ELSA)

Presenter: Andre Xavier

Andre Xavier 1); Eleonora Dorsi 2); Cesar Oliveira 3); Martin Orrel 4); Panayotes Demakakos 3); Jane Biddulph 3); Michael Marmot 3)

1) University of South of Santa Catarina, Florianópolis, Santa Catarina, Brazil; 2) Department of Public Health, Federal University of Santa Catarina, Florianópolis, Santa Catarina, Brazil; 3) Department of Epidemiology and Public Health, University College London, London, UK; 4) Mental Health Sciences Unit, Faculty of Brain Sciences, University College London, London, UK

Background: Cognitive decline is a major risk factor for disability, dementia and death. Digital Literacy (DL) is a pervasive social phenomenon that requires new skills and supports many activities. Previous studies indicate that stimulating mental activities may lead to a slower rate of cognitive decline. This work aims to study whether DL can reduce cognitive decline and inequalities in cognitive health. Methods: The study included 6,442 participants aged 50-89 years followed up for 8 years from the English Longitudinal Study of Ageing (ELSA). DL (current user of internet/email, no user, new user or stopped using) was investigated as a predictor of decline in immediate recall, delayed recall and verbal fluency. Unadjusted and adjusted beta regression coefficients and their $95 \%$ confidence intervals were calculated through Generalized Estimating Equations, controlling for age, gender, wealth, education, physical disability, diabetes, cardiovascular diseases, baseline cognitive function, depressive symptoms and time.

Findings: Participants who were current users of internet/email during the follow-up had better performance in all three cognitive outcomes relative to those who were not users. Becoming a new user had a stronger effect in delayed recall among those with lower income especially in the older (65-89) age group reducing this health inequality. Age, diabetes, physical disability, lower previous cognitive function and lower educational level worsened cognitive outcomes.

Conclusions: The implementation of Digital Literacy as a public health policy tool is likely to reduce cognitive decline and also inequalities in cognitive health.

\section{O-067}

\section{Causes of infertility and the risk of preterm birth}

Presenter: Carmen Messerlian

Carmen Messerlian 1); Robert Platt 1); Baris Ata 2); Seang-Lin Tan 2); Robert Gagnon 2); Olga Basso 1), 2)

1) Department of Epidemiology \& Biostatistics, McGill University; 2) Department of Obstetrics \& Gynecology, McGill University

Background: Singleton pregnancies conceived through assisted reproductive technology (ART) are at higher risk of preterm birth. Babies born to couples conceiving without treatment after a long time to pregnancy also appear to be at elevated risk. Few studies, however, examined the underlying causes of infertility as determinants of preterm birth.

Objective: We assessed whether specific causes of infertility are associated with an increased risk of preterm birth among pregnancies conceived after infertility investigation compared with pregnancies with no known infertility.

Methods: We assembled a hospital-based cohort of women giving birth at a large tertiary care hospital in Montreal (Canada) between April 2001 and September 2007 using the hospital's obstetrical and neonatal database. We included only women who resided within the city and who were not referred for a high-risk pregnancy. Diagnostic and treatment information was abstracted from the medical charts of women evaluated for infertility at the hospital's reproductive centre, and linked to the hospital's maternal and neonatal database. We restricted this analysis to singletons.

Results: Our study population consisted of 18,147 pregnancies: 16,712 in the reference group (i.e., with no documented infertility) and 1,435 in the infertile group ( $43 \%$ conceived without any reported treatment, and $57 \%$ conceived through medically assisted reproduction). The prevalence of preterm birth ( $\leq 37$ weeks) was $7.2 \%$ in the reference group, and 8.6 and $11.4 \%$ in the untreated and treated infertile group, respectively. The examined causes of infertility were: male factor, ovulatory dysfunction, peritoneal factors (tubal disease/ endometriosis), uterine abnormalities (e.g. fibroids, cysts, malformations), and unexplained and unspecified infertility. We included treatment level (no treatment, ovulation induction and/or artificial insemination, and treatment involving gamete manipulation), parity, maternal age and educational level as covariates. Compared with the reference group, women with multiple causes, diagnosed with at least two among ovulatory dysfunction, peritoneal factors, and uterine abnormalities, had a significantly higher risk of preterm birth: 2.52 (95\% Confidence Interval: 1.40-4.57). This association remained after restricting the analysis to women who conceived without assisted reproduction. Male factor infertility (1.57: 0.96-2.60) and uterine abnormalities (1.75: 0.97-3.22) individually may also be associated with preterm birth.

Conclusion: Women presenting with more than one female cause of infertility may be at particular risk for preterm birth independent of medically assisted reproduction. The presence of male factor infertility and uterine abnormalities individually may also confer an increased risk.

\section{Health policy and societal effects}

\subsubsection{1:00-12:30}

\section{O-068}

Fast food, body mass index (BMI) and the role of market deregulation: a cross-national time series analysis

Presenter: Roberto De Vogli

Roberto De Vogli 1); Anne Kouvonen 2); David Gimeno 3)

1) University of California Davis; 2) University of Belfast; 3) University of Texas at San Antonio

Objective: Previous studies have suggested that fast food consumption contributes to weight gain and obesity. We investigated the effect of fast food on population-wide increases in body mass index (BMI) and the role of market deregulation as a determinant of fast food and BMI. 
Methods: Multivariate panel regression models were used to analyze the within-country association between fast food consumption and age-standardized mean BMI in 25 high-income OECD countries between 1999 and 2008. Models adjusted for income, urbanization, trade openness, lifestyle indicators and other covariates. We also tested soft drinks, animal fats and total calories intake as possible mediators for the association between fast food and BMI. Additionally, we used two-stage least squares regression models with economic freedom (market deregulation) as an instrumental variable for the effect of fast food on BMI.

Findings: After adjustment for covariates, each 10 units increase in annual fast food transactions per capita was associated with $0.33 \mathrm{~kg} /$ $\mathrm{m}^{2}$ higher age-standardized BMI $(95 \%$ CI 0.13, 0.52; $p<0.001)$. Neither animal fats, nor total calories, but soft drinks mediated the observed association $(=0.030 ; 95 \%$ CI $0.010,0.050 ; p<0.01)$. Economic freedom was an independent predictor of fast food $(=0.27$; $95 \%$ CI $0.16,0.37 ; p<0.001)$. When economic freedom was used as an instrument for the effect of fast food on BMI, the magnitude of the association weakened, but remained significant $(=0.023 ; 95 \% \mathrm{CI}$ 0.001, 0.045; $p<0.05)$.

Conclusion: Increasing fast food consumption is an independent predictor of rising population BMI. Market regulations might be able to mitigate the obesity epidemic by hindering the spread of fast food consumption.

\section{O-069}

The association of economic difficulties with subsequent disability retirement

\section{Presenter: Ossi Rahkonen}

\section{Ossi Rahkonen 1); Elina Mauramo 1); Eero Lahelma 1);} Tea Lallukka 1), 2)

1) Hjelt Institute, Department of Public Health, University of Helsinki, Finland; 2) Finnish Institute for Occupational Health, Helsinki, Finland

Background: Disability retirement is socioeconomically patterned. However, previous studies have focused on conventional socioeconomic indicators such as education, income and occupational class. Less is known about the association of economic difficulties with disability retirement.

Objectives: Our aim was to examine whether current economic difficulties were associated with subsequent disability retirement due to all cause, mental disorders and musculoskeletal diseases among public sector employees. The contribution of childhood economic difficulties, conventional socioeconomic measures, health behaviours, body-mass index, working conditions and work-to-family interface on the association was also examined.

Methods: Helsinki Health Study (2000-2002) mail survey data among 40-60 year old City of Helsinki employees were linked with register data on disability retirement and diagnoses (ICD-10) from the Finnish Centre for Pensions were used. Disability retirement events until the end of 2010 were linked with the baseline data on economic difficulties (difficulties in buying food and clothes and paying bills). Cox regression analysis was used to calculate hazard ratios (HR) and their $95 \%$ confidence intervals (CI) for disability retirement.

Results: One fifth of women and men reported frequent economic difficulties. Age adjusted frequent economic difficulties were associated with subsequent all-cause disability retirement among women (HR 1.85, $95 \%$ CI 1.48-2.32) and men (HR 2.45, $95 \%$ CI 1.60-3.47). In addition, frequent economic difficulties were associated with disability retirement due to mental disorders (HR 2.54, $95 \%$ CI 1.75-3.68) and musculoskeletal diseases (HR $1.8095 \%$ CI
1.35-2.41). After adjusting for conventional socioeconomic factors and work- and family-related factors the associations attenuated but frequent economic difficulties remained associated with disability retirement due to all causes and mental disorders but disappeared with disability retirement due to musculoskeletal diseases.

Conclusions: Economic difficulties are associated with subsequent disability retirement. Past and present economic difficulties are different dimensions of socioeconomic measures and also capture other aspects of socioeconomic circumstances than conventional socioeconomic measures. Preventing disability retirement and reducing socioeconomic differences in disability retirement also economic difficulties among employees should be considered.

\section{O-070}

Socioeconomic inequality in psychological complaints among Nordic adolescents: how to get the full picture?

Presenter: Line Nielsen

\section{Line Nielsen 1); Mogens Trab Damsgaard 1); Charlotte Meilstrup 1); Bjørn Holstein 1)}

1) National Institute of Public Health, University of Southern Denmark

Background: Psychological complaints are widespread among adolescents and seem to be socially patterned, but studies among Nordic adolescents show different results. Socioeconomic differences have mainly been explored in relative terms with no mentioning of absolute differences. The existing studies are difficult to compare due to differences in study population and measurement of psychological complaints and socioeconomic position.

Objectives: The objective of this study is to examine both absolute and relative socioeconomic differences in psychological complaints among Nordic adolescents using standardised data from five countries. More specific, we want to examine (1) if psychological complaints among adolescents are associated with socioeconomic position measured by family affluence, (2) if socioeconomic differences in psychological complaints are consistent across the Nordic countries, and (3) if the study of absolute and relative socioeconomic inequality provide different conclusions.

Methods: The Health Behaviour in School-aged Children (HBSC) international study from 2005/2006 provided data on 29.642 adolescents aged 11-15 from nationally random samples in Denmark, Finland, Iceland, Norway and Sweden. The outcome measure was daily psychological complaints (feeling low, irritability or bad temper and feeling nervous). Socioeconomic position was measured by the Family Affluence Scale (FAS). We developed a graphical presentation which shows the prevalence of psychological complaints and the size of the FAS groups in each country. Further, we applied four measures of inequality: prevalence difference, odds ratio, Slope Index of Inequality and Relative Index of Inequality.

Results: The overall country level prevalence of psychological complaints ranged from $8.1 \%$ in Denmark to $13.2 \%$ in Iceland. There were large variations in the size of the low FAS group, which ranged from $2 \%$ in Iceland to $12 \%$ in Finland. Psychological complaints among adolescents were associated with socioeconomic position in each of the Nordic countries. The largest inequality was found in Iceland for all measures of inequality. The smallest absolute and relative inequality was found in Finland for girls and in Denmark for boys.

Conclusions: This study finds both absolute and relative inequality in psychological complaints among Nordic adolescents. From a public health perspective the prevalence of an outcome is essential information. Unfortunately, the prevalence is hidden in summary measures 
of inequality. We recommend the use of a graphical presentation which shows the entire picture: the prevalence of the outcome, the size of the socioeconomic groups, and the regression line representing the summary indices of inequality.

\section{0-071}

Using the "4Ps" marketing approach to evaluate healthy food policies: a rapid scoping review

Presenter: Lois Orton

Lois Orton 1); Ffion Lloyd-Williams 1); Helen Bromley 1); Corinna Hawkes 2); David Taylor-Robinson 1); Martin O'Flaherty 1); May Moonan 1); Mike Rayner 3); Simon Capewell 1)

1) Department of Public Health \& Policy, University of Liverpool, UK; 2) World Cancer Research Fund International, London, UK; 3) Department of Public Health, University of Oxford, UK

Background: Population-wide policy actions to promote a healthy diet offer potentially large benefits in terms of reducing the noncommunicable disease burden. However, not all interventions are equally effective.

Objectives: In order to make sense of the wide range of policy options, we conducted a rapid scoping review using a simple conceptual framework based on the marketing "4Ps" approach.

Methods: We searched for evaluations of food policies in six electronic databases. Reference lists were screened. Key informants identified additional evidence. Items were systematically assessed for inclusion. Extracted data were categorised with a modified version of the 4Ps framework: Price, Product, Place and Promotion. Interventions that involve a combination of these approaches were included under a 5th category "Multi-component interventions". In stage one, we sought systematic reviews. Where there were none, we next sought primary studies and then modelling studies. Included studies were too heterogeneous to combine quantitatively. Instead they were synthesised as a narrative review.

Results: The marketing 4Ps framework reveals support for the causal links between certain food policies, dietary behaviours and health outcomes. Overall, the evidence is most convincing for comprehensive, multi-component and multi-nutrient interventions as well as those targeted at decreasing salt and transfats or, conversely, increasing fruit and vegetable consumption. Price interventions are potentially powerful, with greater effect likely in vulnerable (low income) populations who are usually more price sensitive (1 systematic review; 3 econometric modelling studies). Product interventions are mostly confined to reformulation. All interventions are not equally effective, and formal evaluations are few ( 2 modelling studies; 2 primary evaluations). The evidence for Promotion suggests modest benefits for national media campaigns (such as " 5 a day"), but less convincing evidence for health education, food labelling and marketing and dietary advice ( 4 systematic reviews; 4 primary evalu ations; 1 modelling study). In terms of where to target interventions, there has been some success in schools and workplaces, whilst more research is needed to elucidate the most effective way to intervene in the local community and neighbourhood environments ( 5 systematic reviews; 10 primary evaluations). Empirical and modelling evidence supports the assumption that interventions (across the 4Ps) are more effective when mandatory and enforced.

Conclusions: The marketing 4Ps framework offers a useful approach to making sense of the evidence for the effectiveness of a wide range of healthy food policies. Future research must consider the effects of population-level $4 \mathrm{P}$ interventions in vulnerable populations in order to avoid intervention-generated inequalities.
O-072

The impact of common health conditions on disability in Europe Presenter: Gabriela Barbaglia

Gabriela Barbaglia 1); Núria D. Adroher 2); Gemma Vilagut 3); Jordi Alonso 4)

1) IMIM-Institut Hospital del Mar d'Investigacions Mèdiques, Barcelona, Spain; 2) CIBER Epidemiología y Salud Pública, Spain; 3) Pompeu Fabra University

Background: Disability is an increasing component of the burden of disease worldwide of which common health conditions are substantial contributors. Currently, there are no comparable data on disability associated to common health conditions in Europe.

Objectives: To examine the effect of common health conditions on disability across different regions of Europe.

Methods: Household interviews of representative samples of the adult general population $(n=37,289)$ of 10 European countries of the World Mental Health (WMH) Surveys Initiative, grouped into three regions: Central-East (CE) (Bulgaria, Romania), Central-West (CW) (Belgium, France, Germany, the Netherlands, Northern Ireland), and South (Italy, Portugal, Spain). Mental disorders were assessed with the Composite International Diagnostic Interview (CIDI 3.0) and physical conditions with standard checklists. Number of days with full and with partial role limitation (WHO Disability Assessment Schedule 2) was analyzed at the individual level (among sufferers of disorders) and at the societal level (among the whole sample) using the population risk attributable proportion (PARP). Two-part models were adjusted to estimate the joint predictability of mental and physical conditions when using the number of days with full and partial role limitation, controlling for age, sex, employment status, and number of disorders.

Results: At individual level, a higher number of days with either full (28.2 days per year $\mathrm{SE}=2.7$ ) or partial role limitation $(25.8$ $\mathrm{SE}=2.4$ ) were associated to (any) mental disorder than to (any) physical condition (16.8 SE $=1.4$ and 18.0 $\mathrm{SE}=1.3$, respectively). For any (physical or mental) disorder, interregional differences were found: $\mathrm{CW}$ and South regions reported similar number of days with full (25.8 $\mathrm{SE}=2.8$ and $21.1 \mathrm{SE}=2.3$, respectively) and partial role limitation $(23.1 \mathrm{SE}=2.8$ and $22.4 \mathrm{SE}=2.1)$, while in the $\mathrm{CE}$ region were much lower $(13.7 \mathrm{SE}=2.5$ and $16.3 \mathrm{SE}=2.0)$. At societal level, two-third of all days per year with full role limitation $(63 \%$ $\mathrm{SE}=3.0)$ and almost half of all days with partial limitation $(47.5 \%$ $\mathrm{SE}=2.7$ ) would be reduced if all common health conditions considered here were removed. Pain disorders contribute to the highest proportion of population risk in both kinds of role limitation (full: $20.8 \% \mathrm{SE}=3.0$; partial: $16.3 \% \mathrm{SE}=2.4$ ).

Conclusions: Common health conditions are causing a great deal of burden in Europe. At individual level, despite of differences across regions on the number of days with role limitation, mental disorders were the most impacting conditions. At societal level, pain disorders accounted for the highest role limitation. Regional differences in the impact of common health conditions deserve further research.

\section{O-073}

Associations between neighborhood characteristics and recreational and transportation physical activity: results from the RECORD Cohort study

\section{Presenter: Noëlla Karusisi}

\section{Noëlla Karusisi 1); Basile Chaix 1)}

1) UMR-S 707 (Inserm - UPMC) Faculté de médecine Saint-Antoine 27 rue Chaligny 75012 Paris 
Background: Physical activity is considered as a major component of a healthy lifestyle. Regular physical activity is known to prevent chronic diseases and built environment features are increasingly recognized as important determinants of physical activity.

Objectives: We examined the associations between environmental characteristics and informal recreational activity (jogging), formal recreational activities (requiring facilities), and transportation activity (commuting) to investigate the physical activity behavior in the Paris metropolitan area.

Methods: We conducted cross-sectional analyses with the RECORD Cohort Study (7,290 participants, 30-79 years, Paris region). Multiple environmental characteristics were measured and spatial accessibility to sport facilities was assessed with two approaches that take into account the street network (distance and count of facilities). Active commuting was studied by geocoding the place of residence, the workplace and the supermarket.

Markov chain Monte Carlo approaches were used to estimate multilevel models investigating variations in the outcomes.

Results: After controlling for individual characteristics, a high individual education was associated with a higher probability of jogging and with the practice of racket sports, swimming, and fitness over the previous 7 days. The presence and high quality of green and open spaces was associated both with a greater probability of jogging and with the practice of jogging within rather than outside the neighborhood. Spatial accessibility to swimming pools was associated with swimming. The spatial accessibility to facilities was not related to the practice of other sports. High neighborhood income was associated with the practice of a racket sport and fitness. Moreover, a high social cohesion was associated with a higher probability of jogging while the presence of a lake or waterway in the residential neighborhood increased the probability of jogging inside the neighborhood. Neighborhood affective and relational experiences were also related to jogging.

After determining the shortest path between the residence and the workplace and the supermarket with a Geographic Information System, we found that geographic characteristics of the neighborhood and of these paths were associated with the reported walking time to work and to shop.

Conclusions: Our results suggest that physical activity is a multidimensional concept that integrates geographical, financial, and environmental aspects. Our work supports the evidence that strategies to increase participation in sport activities should include paying attention to the attractiveness of the neighborhood, social relationships among neighbors and improve the spatial/financial access to specific facilities. These findings should be taken into account in public health prevention actions against obesity and cardiovascular diseases.

\section{0-074}

Variance components of different grouping strategies of psychosocial work exposures

Presenter: Morten Vejs Willert

Morten Vejs Willert 1); Ioannis Basinas 2); Vivi Schlünssen 2); Matias Grynderup 1); Zara A. Stokholm 1); Henrik A. Kolstad 1); and the PRISME study group)

1) Department of Occupational Medicine, Danish Ramazzini Centre, Aarhus University Hospital; 2) Department of Public Health, Institute of Environmental and Occupational Medicine, Aarhus University

Background: Psychosocial exposures show considerable variability over time and therefore attenuation bias in dose-response relations is expected if individual exposure measures are used. Furthermore, epidemiological studies relying on self-reported exposures analysed on the individual level may be prone to reporting bias. Group-based exposure assessment may circumvent both types of bias. In occupational epidemiology grouping strategies have been investigated for chemical, dust and musculoskeletal exposures. However, which grouping strategy is better suited for psycho-social exposures remains uncertain.

Objectives: To investigate the implications of different grouping strategies for exposure assessment of the psychosocial work environment.

Methods: In 2007, 4,291 public employees from Aarhus, Denmark were enrolled in a longitudinal study of the psychosocial work environment and mental health. The participants were approached again in 2009 and 2011. We retrieved the following grouping variables from the employer's payroll register: department, work unit, and collective agreement. Exposures of interest were psychological demands and decision latitude. In order to assess variance components we fitted linear mixed-effect models using maximum likelihood estimation with exposure as outcome and grouping variables as random effects. Results are reported as the between-group variance, the within-group variance and as the contrast in mean exposure levels between the groups, using the formula described by Kromhout and Heederik: contrast $=$ between-group variance/(between-group variance + within-group variance).

Results: At present, only a subset of the data is ready for analysis, comprising 2,042 workers grouped within 15 departments, 209 work units, and 14 collective agreements. In assessment of exposure to psychological demands grouping by department yilded betweengroup variance $<0.001$, within-group variance $=0.084$ and contrast $<0.001$. Grouping by work units gave between-group variance $=$ 0.034 , within-group variance $=0.063$, contrast $=0.347$. Grouping by collective agreement resulted in between-group variance $=$ 0.038 , within-group variance $=0.063$, contrast $=0.380$. In assessment of exposure to decision latitude grouping by department yielded between-group variance $=0.031$, within-group variance $=0.103$, contrast $=0.233$. Grouping by work units gave between-group variance $=0.042$, within-group variance $=0.085$, contrast $=0.333$. Finally, grouping by collective agreement resulted in betweengroup variance $=0.054$, within-group variance $=0.080$, contrast $=$ 0.403 .

Conclusions: For both exposures grouping by department yielded the lowest contrast, while grouping by collective agreement gave the highest contrast, closely followed by grouping by work unit. The magnitudes of the contrasts are comparable to contrasts found for grouping in other occupational exposures, i.e. wood dust. The results will be used to decide on the final exposure assessment strategy used in the full cohort.

\section{O-075}

Interaction between education and household wealth on the risk of obesity in women in Egypt

Presenter: Amina Aitsi-Selmi

Amina Aitsi-Selmi 1); Tarani Chandola 1); Sharon Friel 1); Reza Nouraei 2); Martin Shipley 1); Michael Marmot 1)

1) University College London; 2) Imperial College, London

Background: Obesity has been traditionally considered a disease of affluent societies yet it is a growing problem in lower income countries particularly among women. Egypt has one of the highest levels of female obesity in the world which is unexpected relative to its level of economic development. There are few studies exploring the separate and joint effects of socioeconomic status indicators on obesity in 
depth in these countries. Therefore, this study examines the interaction of education and wealth in relation to obesity, hypothesising that education protects against the obesogenic effect of wealth.

Methods: Four datasets of women of reproductive age from the Egyptian Demographic and Health Surveys spanning the period 1992-2008 were used to examine two distinct time periods: 1992/95 $(\mathrm{N}=11657)$ and $2005 / 08(\mathrm{~N}=16,138)$. Changes in the prevalence of obesity by socioeconomic group were estimated taking the survey design into account, and a multivariate logistic regression model that included an interaction term was used to estimate the separate and joint effects of education level and household wealth in relation to the odds of being obese. Estimates were adjusted for age group, area of residence and number of children.

Results: An interaction was found between education and wealth on obesity in both time periods $(P$-value for interaction $<0.001)$. For women with the lowest education level, moving up one wealth quintile was associated with a $81 \%$ increase in the odds of obesity in 1992/95 (OR; $95 \%$ CI 1.81; 1.70,1.91) and a $38 \%$ increase in 2005/08 (OR; $95 \%$ CI 1.38;1.31,1.45). For women with the highest level of education, there was little evidence of an association between wealth and obesity (OR; $95 \%$ CI 1.09; 0.82,1.49 in 1992/95 and 1.02; $0.91,1.15$ in 2005/08). Obesity levels increased most in women who were in the no/primary education, poorest wealth quintile and rural groups (absolute difference in prevalence percentage points between the two time periods: $10.9 ; 13.4$; and 14.6 respectively $(P$ for difference $<0.001$ for all group differences).

Conclusion: This study shows that wealth appears to be a risk factor for obesity in women with lower education levels, while women with higher education are relatively protected. The findings also support the observation that the social gradient of obesity is reversing and that this can be explained by the large increase in obesity levels in lower socioeconomic groups (low education/poor/rural dwelling) over the time period investigated.

\section{O-076}

\section{Unexpected effects of health interventions in real life}

\section{Presenter: Christine S. Benn}

Christine S. Benn 1), 2); Ane B. Fisker 1), 2); Henrik Ravn 1), 2); Peter Aaby 1), 2)

1) Research Center for Vitamins and Vaccines, Statens Serum Institut; 2) Bandim Health Project, Guinea-Bissau

Background: It is assumed that if a health intervention prevents a health problem such as an infectious disease or a micronutrient deficiency, then the intervention reduces overall mortality proportionally to the number of deaths due to that particular health problem. However, the real life effects of health interventions are sometimes quite different from the assumed effects.

Objective: To discuss whether there are lessons to be learned from unexpected real-life effects of health interventions.

Methods: Review of unexpected outcomes of interventions and trials, based on the authors' own experience.

Results: Surprisingly often the real life effect of health interventions is quite different from the assumed effect. For instance BCG and measles vaccine seem to reduce overall mortality more than can be ascribed to prevention of tuberculosis and measles infection, whereas the diphtheria-tetanus-pertussis vaccine, though protective against the target diseases, may increase mortality from other pathogens. Furthermore, many micronutrient intervention trials show that in spite of preventing deficiency, micronutrient supplementation may have no effect or even negative effects on overall health. Also, though it is generally not acknowledged that males and females may respond differently to interventions, this is often the case.
Conclusions: These observations are often dismissed as post hoc observations or due to uncontrolled confounding because they do not fit the current assumptions. However, there are important lessons to be learned from pursuing the unexpected "unbelievable" observations. How can we make sure that we do not over-report spurious findings-but at the same time keep our eyes open for unexpected effects? Health and demographic surveillance systems in low income countries and population based registers in high income countries offer the possibility of testing whether the unexpected observation spurious findings or biology.

\section{Epidemiological methods and psychiatric disease}

\subsubsection{1:00-12:30}

O-077

Does dementia risk begin in early life? Examining the relationship between height and dementia mortality in an individual participant meta-analysis

Presenter: David Batty

Tom Russ 1); Mika Kivimaki 2); Manos Stamatakis 2); John Starr 1); David Batty 2), 3)

1) Alzheimer Scotland Dementia Research Centre, University of Edinburgh, UK; 2) Department of Epidemiology and Public Health, University College, London, UK; 3) Centre for Cognitive Ageing \& Cognitive Epidemiology, University of Edinburgh, UK

Background: Risk factors measured in middle age do not fully explain the occurrence of future dementia risk. This raises the possibility that pre-adult risk factors may be implicated in its aetiology. Height is a proxy for early life circumstances (early life illness, adversity, nutritional privation, or psychosocial stress). Lower physical stature is associated with elevated cardiovascular disease (CVD) rates. Since CVD and dementia have a shared aetiology, we examined the little-explored association between height and later-life dementia. This individual participant meta-analysis is the largest study to date to examine this relationship.

Objective: To examine the relationship between height and future dementia risk.

Methods: We pooled raw data from 18 country-wide, independent, prospective cohort studies comprising the Health Survey for England $(\mathrm{N}=15)$ and the Scottish Health Survey $(\mathrm{N}=3)$. Between 1994 and 2008 , a total of 181,800 people aged 16 years or older participated in baseline surveys. Height was measured to the nearest millimetre with shoes removed with the study member stretching to their maximum height. Covariate data, including health behaviours, weight, selfreported general health, and socioeconomic status were quantified using standard protocols. Cause-specific mortality was ascertained through linkage to national mortality registers.

Results: A mean follow-up of 10 years gave rise to 426 dementia deaths in men and 667 in women. There was a dose-response association between shorter stature and risk of dementia death that was stronger for men (age- and sex-adjusted hazard ratio per standard deviation decrease in height; $95 \%$ confidence interval: $1.26 ; 1.14$, $1.40)$ than women $(1.10 ; 1.01,1.19)$. Incorporating measures of adulthood socioeconomic status, health, and health behaviours into the multivariable model had a negligible effect on the strength of these associations.

Conclusions: Early life circumstances, as indexed by adult height, may influence later dementia risk. These findings suggest that improved early life conditions may decrease dementia risk. 


\section{O-078}

Socioeconomic inequality in cause-specific mortality: differential exposures and differential vulnerability of behavioral risk factors

Presenter: Helene Nordahl

Helene Nordahl 1); Theis Lange 1); Birgitte Frederiksen 2); Merete Osler 2); Ingelise Andersen 1); Finn Diderichsen 1); Naja Hulvej Rod 1)

1) Department of Public Health, University of Copenhagen, Copenhagen, Denmark; 2) Research Center for Prevention and Health, Glostrup University Hospital, Glostrup, Denmark

Background: Approaching the hypothesis of differential exposure and at the same time differential vulnerability by assessment of mediation in the presence of interaction between socioeconomic exposures and modifiable behavioral mediators of common causes of death has not been comprehensively addressed.

Objective: To decompose total effects of education on mortality into direct effects, indirect effects, and interactive effects, to make clearer the role of education-by-smoking interaction when questions of mediation and pathways through smoking is of interest.

Method: We applied a straightforward and intuitive three-way decomposition of the absolute educational inequality in deaths from all-causes, cardiovascular, cancer, and respiratory diseases to address mediation by smoking among 76,000 women and men in the Danish multicenter Social Inequality in Cancer cohort study from 1980 to 2009.

Results: Less favorable health behavior was concentrated among both women and men with short education, and having a short or medium education was associated with higher risk of deaths from all-causes, CVD, cancer, and respiratory diseases compared to long education. Absolute educational inequalities in mortality peaked at 1,270 (95\% confidence interval $=1,056,1,484)$ deaths from all-causes per 100,000 person years (PY) in men and 725 (95 \% CI 576, 875) deaths per 100,000 PY in women aged 65 years and older. Although a substantial portion of the effect of education on mortality was attributable to the "pure indirect" effect through smoking, a considerable part of the mediated effect could be ascribed to the interaction education-by-smoking. This finding was most pronounced in older men, where $28 \%(95 \%$ CI 13,44$)$ of the $152(95 \%$ CI 96, 208) additional deaths from respiratory diseases per 100,000 PY due to educational differences could be ascribed to the mediated interaction education-by-smoking, and $20 \%(95 \%$ CI 7,43$)$ was attributable to the "pure" indirect effect through smoking, corresponding to an overall proportion mediated of $48 \%$. In general, the additional numbers of deaths due to educational difference were most pronounced among older men. However, the highest overall proportion mediated through smoking was found in deaths from cancer among women $(62 \%)$.

Conclusion: The results indicated that the pathways underlying educational inequalities in mortality were partly a result of differential exposure to smoking, but at the same time persons with short education were more vulnerable to the effect of smoking on deaths from all-causes, CVD, cancer, and respiratory diseases than persons with long education, supporting the hypothesis of differential vulnerability.

\section{O-079}

Comparative effectiveness and safety of SSRI use in combination with NSAIDs, selective COX inhibitors or paracetamol

Presenter: Ole Köhler

Ole Köhler 1); Liselotte Petersen 2); Ole Mors 1); Christiane Gasse 2)
1) Aarhus University Hospital Risskov, Department P, Reserach Unit; 2) National Centre for Register-Based Research, Aarhus University

Background: Concomitant use of selective serotonin reuptake inhibitors (SSRIs) and non-steroidal anti-inflammatory drugs (NSAIDs) is associated with increased risk of gastrointestinal bleeding. However, findings regarding their synergistic antidepressant effect are inconsistent.

Objectives: To compare effectiveness and safety of SSRI use with and without concomitant NSAID or paracetamol use, we performed a register-based follow-up study.

Methods: Within a $25 \%$ random sample of the Danish population, we identified all incident SSRI users between 1997 and 2006. We compared rates of all-cause and cause-specific mortality, any psychiatric hospitalization, hospitalization with depression or somatic events during periods of SSRI use only with rates during periods of combined SSRI and NSAID or paracetamol use. We applied COX regression and competing risk analyses with adjustment for confounders and report adjusted hazard rate ratios (HRR) with $95 \%$ confidence intervals (CI).

Results: We identified 124,465 incident SSRI users, 24,284 (19.5\%) using NSAIDs and 13,286 (10.7\%) paracetamol concomitantly. Comparing SSRIs in combination with NSAIDs to SSRI monotherapy was associated with a decreased risk for any psychiatric hospitalization [HRR $(95 \% \mathrm{CI}): 0.75(0.67 ; 0.85)]$ and hospitalization with depression $[0.80(0.67 ; 0.94)]$ and no increased mortality [1.10 $(0.98$; 1.24)]. Paracetamol in combination with SSRIs revealed an increased mortality-risk $[2.72(2.46 ; 3.00)]$, especially cardiovascular [2.98 $(2.40 ; 3.70)]$. No associations with gastrointestinal adverse events were observed. Concomitant use of low-dose acetylsalicylic acid (ASA) reduced the risk for psychiatric hospitalizations [0.44 (0.33; $0.58]$ and due to depression $[0.38(0.27 ; 0.52)]$ and death $[0.86(0.73$; 0.99)]. Ibuprofen showed synergistic and safe treatment effects, whereas diclofenac $[1.88(1.39 ; 2.54)]$ and the group of selective COX-2 inhibitors $[1.79(1.35 ; 2.36)]$ were associated with increased mortality.

Conclusions: Effectiveness and safety outcomes varied across combinations of SSRIs with individual NSAIDs. Especially low-dose ASA may represent a beneficial adjunctive antidepressant treatment option. The increased mortality risk of concomitant use of paracetamol should be investigated further.

\section{O-080}

Geographic disparity in participation in clinical research studies: uptake of atrial fibrillation screening aiming at stroke prevention

Presenter: Ulf Strömberg

Ulf Strömberg 1); Anders Holmén 1); Mårten Rosenqvist 2); Johan Engdahl 3)

1) Department of Research, Development and Education, Region Halland, Sweden; 2) Department of Clinical Sciences and Education, Karolinska Institute, Sweden; 3) Department of Medicine, Halland Hospital, Sweden

Background: Possible disparities in access to clinical research studies need be examined by consideration to the target population. It is important to understand factors that distinguish participants from non-participants of a clinical study. Such factors may be related to clinical profile, socio-economy and residence area.

Objectives: In a screening study for silent atrial fibrillation (AF), which is a frequent source of cardiac emboli with ischemic stroke, the proportion of non-participants was considerable and their clinical profile differed from the participants' profile. We intended to geo-map the target population and non-participation in an attempt to 
understand factors related to screening uptake and, thereby, obtain useful information needed to intervene for improved uptake.

Methods: In the municipality of Halmstad, Sweden, all residents born in 1934-1935 were invited to the screening study during April 2010 to February 2012. The total study group included 848 participants and 367 non-participants from 12 parishes. Geo-maps displaying participation, along with target-population-based geo-maps displaying proportion of immigrants and ischemic stroke incidence, were used. Results: Smoothed non-participation ratios (SmNPR) varied from 0.81 to 1.24 across different parishes $(\mathrm{SmNRP}=1$ corresponds to the expected participation based on the total study group). Among high risk individuals, the geographical variation was more pronounced (SmNPR range 0.75-1.51). Two parishes with higher share of immigrants and elevated population-based ischemic stroke incidence showed markedly lower participation, particularly among high-risk individuals.

Conclusions: We applied a novel approach, using geo-maps, to analyze participation in a community-wide screening program for AF and its geographic relation to cardiovascular risk and socioeconomic factors. Geo-mapping of target population and non-participation yielded useful information needed to intervene for improved screening uptake.

\section{O-081}

Adolescent conduct problems and premature mortality: evidence from the MRC national survey of health and development

Presenter: Mai Stafford

\section{Mai Stafford 1); Barbara Maughan 1); Imran Shah 1); Diana Kuh 1)}

1) MRC Unit for Lifelong Health and Ageing; 2) Institute of Psychiatry, King's College London

Background: From high risk samples, we know that antisocial behaviour in youth is a risk factor for premature mortality. Representative samples are beginning to suggest there may be a graded association between premature mortality and adolescent conduct problems across a spectrum.

Objectives: We set out to assess the prospective association between conduct problems measured in adolescence and premature mortality in a population-based sample of men and women followed to age 65 . Methods: Teachers assessed conduct problems at ages 13 and 15 years of age for 4158 members of the Medical Research Council National Survey of Health and Development (the British 1946 birth cohort). Follow-up to age 65 via the UK National Health Service Central Register provided data on date and cause of death. Cox proportional hazards models were used to examine associations between conduct problems and all-cause mortality and deaths from cancer, coronary heart disease, and unnatural causes, separately for men and women.

Results: Higher teacher-rated adolescent conduct problems were associated with increased hazards of death from coronary heart disease by age 65 in men, and with all-cause and cancer mortality in women. Compared with women at the 5th centile of conduct problems, those at the 95th centile had a hazard ratio of $2.10(95 \% \mathrm{CI}$ $1.43,3.08$ ), adjusted for childhood socioeconomic position and childhood cognition. Adolescent conduct problems were not associated with increased risks of unnatural/substance-related deaths in men or women in this representative sample.

Conclusions: Earlier studies, typically in samples of high risk men, found greater likelihood of unnatural and alcohol or substance abuserelated deaths in early adulthood. We found marked differences in mortality risk from other causes emerging later in the life course among women as well as men.
0-082

Assessing sex/gender sensitivity of epidemiological publications: development of a screening tool

Presenter: Ingeborg Jahn

\section{Ingeborg Jahn 1); Johann Frick 1); Dirk Gansefort 1)}

1) Leibniz-Institut for Prevention Research and Epidemiology - BIPS $\mathrm{GmbH}$

Background: There is an established gender bias in epidemiological literature, notably a lack of sex/gender based analysis and a limitation of analyses to a simplified broken down by "sex"-approach. Existing critiques-frequently from a gender research point of view-often focus on the absence of the investigation of gender relations and health. Differentiated reviews of the consideration of sex/gender aspects in publications regarding research where gender relations are not the main question are missing.

Objectives: We aimed to develop and test a literature screening tool to assess sex/gender aspects in abstracts of published epidemiologic research articles.

Methods: Taking existing literature and tools into account, an abstract screening instrument was developed. The final instrument includes specific questions on sex/gender aspects in abstracts of research publications: Was the aim of the study to investigate gender relations and/or sex/gender differences? Were sex/gender based methods of analyses (stratification, adjustment, etc.) addressed? Were sex/gender differences and/or similarities reported in the results section? Papers were eventually classified as "gender research", "gender differences/similarities reported with or without an explicit aim to identify them", or as "no sex/gender aspects considered".

To test the instrument, we applied it to all abstracts of original research published from January 2006 to March 2011 in the Journal of Epidemiology and Community Health that contained the words "sex and/or gender" in the PubMed-field title/abstract.

Results: 248 papers were identified. After exclusion of methodological papers, research papers focusing on one sex only and papers without an abstract $(\mathrm{N}=28), 220$ were reviewed by two scientists. Using the screening tool we developed, 19 papers $(9 \%)$ were classified as gender research. 115 abstracts $(52 \%)$ reported sex/gender differences and/or similarities, with 34 of them explicitly aiming to investigate these. 86 (39\%) abstracts did not give sex/gender based information on the issues assessed.

Conclusion: It is evident that substantial information about sex/gender based analysis in studies is also reported in short abstracts. The results support the assumption that sex/gender differences/similarities are differently edited, although the studies don't focus on gender relations in their objective. This is also demonstrated by the fact that studies report both differences and similarities. A selection bias must be considered because of the social-epidemiological focus in the journal JECH. Further steps include (1) examination of the results on the basis of full texts and (2) application of the screening tool to other epidemiological journals not specially focusing on social-epidemiological topics.

\section{O-083}

Ethnic inequalities in adverse events in Dutch hospital care? A record review study

Presenter: Floor van Rosse

Floor van Rosse 1), 2); Martine de Bruijne 2); Karien Stronks 1); Marie-Louise Essink-Bot 1); Cordula Wagner 2), 3)

1) Department of Public Health, Academic Medical Center, University of Amsterdam, the Netherlands; 2) Department of Public 
\& Occupational health, EMGO Institute, VU University Medical Center, Amsterdam, The Netherlands; 3) NIVEL, Netherlands Institute for Health Services Research, Utrecht, the Netherlands

Background: Although US studies showed that ethnic minority patients are at increased risk of adverse events (AEs) in hospital care, ethnic inequalities in patient safety were never investigated empirically in Europe.

Objective: To analyse incidence, type, nature, impact and preventability of AEs by ethnic origin.

Methods: We conducted a prospective cohort study in four Dutch urban hospitals. Inclusion criteria: Dutch or non-western ethnic origin, age range 45-75 and admission to hospital for at least one night. Eligible patients completed a questionnaire on ethnic origin (based on country of birth criteria) and on potential determinants of ethnicityrelated risks of AEs (e.g. Dutch language proficiency and health literacy) at hospital admission and signed a consent form for record review.

After discharge, a two-stage medical record review study was conducted. The method of determining AEs was comparable with those of other international studies. We compare incidence, type (e.g. diagnostic AEs), impact, and nature of AEs between ethnic Dutch patients and patients of non-western ethnic origin, while adjusting for case mix (age, sex and primary diagnosis). Subsequently, we analyse the contribution of patient related determinants to the risk of AEs using multilevel logistic regression techniques.

We finalized our data collection with focus group discussions with record reviewers.

Results: 1,390 patients who met the inclusion criteria were willing to participate (790 Dutch, 590 of non-western origin). Actual participation rate was around $80 \%$. Record review was finished in March 2013 and data analysis is currently going on.

Focus group discussions with record reviewers provided hypothetical directions for the results: We expect no ethnic inequalities in overall $\mathrm{AE}$ rates, but we do expect ethnic inequalities in type and nature of AEs, e.g. more diagnostic AEs in non-western patients. Also, ethnic inequalities in medical decision making regarding specific domains such as end-of-life care were mentioned. Complete results will be available in summer 2013 and will be presented at the conference.

Conclusions: This study is the first to quantify ethnic inequalities in AEs in hospital care in Europe. The results provide insight in patientrelated determinants of patient safety.

\section{0-084}

\section{A survey on the occupational risks in veterinary practices}

Presenter: João Rodrigo Mesquita

Carla Costa 1), 2); Cármen Nóbrega 1); Helena Vala 1), 3); Carla Santos 1); Fernando Esteves 1); Rita Cruz 1); Cristina Mega 1); Luís Montenegro 2); Maria São José Nacimento 4), 5); João Rodrigo Mesquita 1), 5)

1) Escola Superior Agrária, Instituto Politécnico de Viseu. Quinta da Alagoa - Estrada de Nelas, Ranhados, 3500-606 Viseu, Portugal; 2) Hospital Referência Veterinária Montenegro, Rua da Póvoa, 34, 4000 - 395 Porto, Portugal; 3) Centro de Estudos em Educação, Tecnologias e Saúde. CI\&DETS. Escola Superior Agrária, Instituto Politécnico de Viseu, Viseu, Portugal; 4) Laboratório de Microbiologia, Departamento de Ciências Biológicas, Faculdade de Farmácia, Universidade do Porto. Rua de Jorge Viterbo Ferreira, 228, 4050-313 Porto, Portugal; 5) CIBIO/UP, Centro de Investigação em Biodiversidade e Recursos Genéticos/Universidade do Porto, Campus Agrário de Vairão, Vairão, Portugal

Background: Needlestick and sharps injuries (NSI) are considered an important occupational health \& safety hazard by acting not only as a source for physical trauma, but also as a potential route for exposure to-and transmission of blood-borne infectious diseases among healthcare workers. Reports of diseases acquired by NSI during the veterinary practice are scarce and probably for this reason bloodborne transmission of zoonotic pathogens have generally been considered a minor hazard. However, underreporting might be possible and may help explain the lack of information. Furthermore, to date, studies on needle and sharps handling practices, risk factors and NSI reporting practices and rates are manifestly lacking.

Objectives: Therefore, this study attempted to fill this knowledge gap by assessing the occurrence of NSI in veterinary professionals. Behaviours that could be potentially associated to NSI occurrence were also studied.

Methods: Veterinary doctor and nursing staff from veterinary practices registered in the official Veterinary Profession Associations in Portugal were asked to complete an online questionnaire-based needlestick and sharps injuries survey. Additionally, participants of a 2013 Veterinary Meeting in Portugal were asked to complete the same questionnaire. A needlestick/sharps injury was defined as a puncture or laceration with a needle or a sharp. Univariate and multivariate logistic regression models were performed for risk factor analysis using "Epicalc" package in the R software (R 2.13.1).

Results: A total of 159 questionnaires were returned. Ages ranged from 20 to 54 years-old and $28.3 \%$ were male. Over $45 \%$ had low experience (less than 1 year of experience), $12.6 \%$ between 1 and 2 years of experience, $21.4 \%$ between 2 and 5 years of experience and $18.9 \%$ over 5 years of experience. Regarding needlesticks, working mixed-shifts (OR: 3.28, $95 \%$ CI 1.34,8.01), and experiencing vertigo/dizziness once a day (OR: $2.42,95 \%$ CI $0.04,7.23$ ) and once a month (OR: 1.28, $95 \%$ CI $0.01,5.71)$ were found to be risk factors. Regarding sharps injuries, working prolonged week schedules $(>50 \mathrm{~h} /$ week) was a risk factor (OR: 3.33, $95 \% \mathrm{CI}$ 1,11.07)

Conclusions: The exposure rate of NSI in veterinary professionals in Portugal is high and clearly is a major occupational health problem. Health and safety refresher courses could have a positive effect on safety culture change and gradually reduce work-related injuries in the future.

\section{O-085}

Evidence appraisal: a standardised approach based on critical appraisal tools (CATs)

Presenter: Laura Martino

Laura Martino 1); Elisa Aiassa 1); Laura Ciccolallo 1); Saghir Bashir 1)

\section{1) EFSA}

The mission of the European Food Safety Authority (EFSA) is to performing risk assessment in all fields which have a direct or indirect impact on food and feed safety. In this activity there is a need for transparently and objectively evaluating the scientific evidence used to inform the risk assessment process. Such evaluation consists in critically assessing a study in terms of its internal validity (closeness to the truth) precision (level of reliability) and external validity (general applicability) and weighing up these elements to derive an indication on the overall methodological quality.

We developed a standardised approach for appraising the scientific evidence. Such approach is meant to harmonise and structure the study quality evaluation process and ensure openness, transparency and objectivity. Although the main concepts and principles behind this setting stem from the scientific literature on appraising the methodological quality and the quality of reporting of a study, their 
application to the food/feed domain is not trivial and has requested some adaptations.

As part of this approach we set up:

1. a standardised appraisal procedure;

2. a standardised appraisal report;

3. Critical Appraisal Tools (CATs), tailored for different study designs (e.g. randomised controlled trials in nutrition, systematic review of interventions etc.).

The evaluation procedure requires:

1. at least two reviewers appraising each study and finding an agreement on the judgement;

2. the use of a standardised report and CAT. The CATs take the form of a checklist and are meant to guide the reviewer in the evaluation process through a list of items to be addressed in a systematic way. Ideally the tools should be used by a multidisciplinary team of experts that shall include methodologists and domain experts.

Each CAT includes:

a. a list of 'appraisal questions';

b. a summary of the information retrieved in the study and its narrative appraisal;

c. a summarised appraisal in the form of answer to the 'appraisal question';

d. a proposed action that can include asking clarification to the authors.

Nowadays the approach foresees that the overall quality of a study is evaluated on the basis of a qualitative summary of the single elements considered in the appraisal. This step of the process is partially subjective. A methodology to perform a quantitative synthesis might be established in the future.

The approach and the developed report and CATs have started to be pilot tested by an internal group of EFSA scientists.

\section{Cohort outcome studies and repeated measures}

\subsubsection{1:00-12:30}

\section{O-086}

Growth in early life as a predictor of kidney function at age 63-64 years: the Newcastle Thousand Families study

\section{Presenter: Mark Pearce}

\section{Mark Pearce 1); Stephanie Harrison 1); Kay Mann 1)}

1) Institute of Health \& Society, Newcastle University, UK

Background: It is suggested that impaired fetal growth can affect kidney development, resulting in fewer glomeruli being formed and reduced kidney function later in life. However, the majority of previous studies were conducted in children or young adults, and the oldest mean age for any of these studies was 49 years. The age at which these studies were conducted is important, as the effect of early life influences on kidney function may not become apparent until later in adult life. Further, little research has been done to investigate the impact of other early life influences in relation to adult kidney function such as socio-economic status (SES) and breastfeeding, or to simultaneously take into account risk factors in later life.

Objectives: The aim of this study was to investigate early life variables in relation to adult kidney function, and compare these to the influence of later life variables, all within the Newcastle Thousand Families Birth Cohort.
Methods: The Newcastle Thousand Families study began as a prospective study of all 1,142 children born in May and June 1947 to mothers resident in Newcastle upon Tyne, in northern England. Detailed information has been collected prospectively since that time. At the age of 63-64 years, 335 participants had serum creatinine successfully measured and completed a lifestyle questionnaire. These measurements were used to calculate their estimated glomerular filtration rate (eGFR). This was analysed in relation to a range of early life factors, as well as potential confounders and adult risk factors for poor kidney function.

Results: Body mass index (BMI) and being female were significantly negatively associated with eGFR. Birth weight was significantly positively associated with eGFR. In sex-specific analyses, the variables significant at the univariate level (those above, plus cigarette smoking and alcohol consumption) remained significant for males $(n=154)$, whereas none of the variables remained significant for females $(\mathrm{n}=181)$. Upon analysis for males exclusively, BMI (R $6.0 \%$ ) had a twofold increased contribution to eGFR in comparison to the contribution from birth weight (R $3.1 \%$ ).

Conclusions: The findings suggest that sex, size at birth and BMI may be important variables influencing adult kidney function. However, as only a small amount of variance in eGFR was accounted for by the variables investigated in this study, further studies comparing early and later life factors would be beneficial to determine different factors which may influence kidney function and which are the most important for focussing intervention studies.

\section{O-087}

Low income and the risk of heart failure: mediation by physiological, behavioral and psychological factors

Presenter: Ingelise Andersen

Theis Lange 2); Finn Diderichsen 1), 5); Eva Prescott 3), 4); Naja Hulvej Rod 1), 5); Ingelise Andersen 6)

1) Department of Social Medicine, Institute of Public Health, University of Copenhagen, Copenhagen, Denmark; 2) Department of Biostatistics, Institute of Public Health, University of Copenhagen, Copenhagen, Denmark; 3) Department of Cardiology, Bispebjerg University Hospital, Copenhagen, Denmark; 4) The Copenhagen City Heart Study, Bispebjerg University Hospital, Copenhagen, Denmark; 5) Copenhagen Stress Research Center, Copenhagen, Denmark; 6) Copenhagen University/Institute of Public Health Science

Introduction: Low socioeconomic status is associated with risk of heart failure. We aimed to investigate the association between low equivalent income and first-time heart failure hospitalization and to calculate the expected mediated proportion of the physiological (fibrinogen, hypertension, hypercholesterolemia, diabetes and BMI), the behavioral (tobacco, alcohol, physical inactivity) and the psychological pathways (vital exhaustion), respectively.

Materials and methods: Our analyses are based on 7,102 persons, aged 40-90 years, $57 \%$ women, from the Copenhagen City Heart Study (Denmark) 3rd wave taking place in 1991-1993. Information on income and hospitalization was obtained from nationwide registries. During 18 years of follow-up 964 persons experienced first-time hospitalization with heart failure. The approach to measure the mediated proportion of the three potential pathways, were based on counterfactuals to calculate natural direct, indirect and total effects by means of additive hazard models.

Results: The total effect of being exposed to low income compared to high income increased the incidence of heart failure to 30.9 cases/ 10,000 person years (CI $95 \%$ : 8.5-53). Of these could 3.8 cases/ 
10,000 person years $(3.6-11.2)$ or $12 \%$ be attributed to a pathway through biomarkers. The mediated proportion was $13 \%$ for behavioral and $12 \%$ for psychological pathways. Analyses of the single covariates in the pathways indicate that physical inactivity, $\mathrm{BMI} \geq 30 \mathrm{~kg} / \mathrm{m}^{2}$ and vital exhaustion have the strongest direct effect on heart failure.

Conclusion: The results indicate that all three pathways contribute to the association between low income and the risk of heart failure. Additive hazards models calculate the actual number of cases caused by the different pathways, which might be intuitively easy to understand.

\section{O-088}

A cohort analysis to measure the effect of orlistat or bariatric surgery on weight and body mass index in a general UK population sample

\section{Presenter: Ian Douglas}

\section{Ian Douglas 1); Krishnan Bhaskaran 1); Liam Smeeth 1)}

\section{1) London School of Hygiene \& Tropical Medicine}

Background: The efficacy of currently available treatments for obesity has been well characterised in randomised clinical trials, but their effectiveness in the general population is less well defined.

Objective: To measure the change in weight and body mass index (BMI) after receiving either bariatric surgery or treatment with orlistat, in a general population sample.

Methods: We used electronic healthcare records from the United Kingdom Clinical Practice Research Datalink (CPRD). All patients with a record of bariatric surgery or any prescription for orlistat, and with at least 12 months prior research quality registration were included. Weight and height measures were extracted and in patients with an available height measure, BMI was calculated at all time points when weight was recorded. For patients who received orlistat and later had bariatric surgery, follow up in the orlistat analysis was censored at the date of surgery. Patients receiving orlistat post-surgery were only analysed in the surgery group, with no censoring at first orlistat. Multilevel models were developed using repeated measures of weight and BMI after treatment, to characterise changes in these parameters over time following each intervention.

Results: We identified 2,474 patients undergoing bariatric surgery (median age 45 years, $82 \%$ female), and 94,464 receiving orlistat (median age 46, $77 \%$ female). Mean BMI before the intervention was $44.5 \mathrm{~kg} / \mathrm{m}^{2}$ (standard deviation $(\mathrm{SD})=8.7$ ) for bariatric surgery and $37.2(\mathrm{SD}=6.4)$ for orlistat. Subsequent measures of BMI were available for $80 \%$ bariatric surgery patients and $89 \%$ orlistat users. Mean follow time post intervention was 2.3 years for bariatric surgery patients and 4.3 years for patients receiving orlistat. The mean first BMI measure following each intervention was 40.7 for surgery patients and 36.8 for orlistat patients. Further results will be presented to quantify the change in weight and BMI following either bariatric surgery or treatment with orlistat.

Conclusions: Our results will help to clarify whether the effects of orlistat treatment and bariatric surgery that have been demonstrated in clinical trials are carried over in broad clinical practice.

\section{O-089}

Self-reported health following percutaneous coronary intervention: results from a cohort followed for 3 years with multiple measurements

Presenter: Karin Biering
Karin Biering 1); Morten Frydenberg 2), 3); Niels Henrik Hjøllund 4)

1) Department of Occupational Medicine, Regional Hospital West Jutland; 2) VestKronik, Regional Hospital West Jutland; 3)

Department of Clinical Epidemiology, Aarhus University Hospital; 4) Section of Biostatistics, Department of Public Health, Aarhus University

Objective: Improvements in the treatment of coronary heart disease have increased the number of patients living with a chronic heart disease. Patient-reported outcomes are required to adequately describe prognosis. We here report self-rated health in a populationbased cohort of patients with coronary heart disease treated with percutaneous coronary intervention (PCI).

Study design and setting: Over 3 years, we followed 1,726 patients treated with PCI with eight repetitive questionnaires. With the use of multiple imputation, we described the course of self-rated health using SF-12 mental component summary (MCS) and physical component summary (PCS) and analysed adjusted differences by gender, age, educational level, indication for PCI, and left ventricular ejection fraction along with an analysis of decrease in health status.

Results: MCS scores increased during follow-up, while PCS scores were stable over time. Men rated higher in MCS and PCS than women and older patients rated higher in MCS than younger. Other differences were negligible. Low age was identified as a risk factor for marked decrease in mental health over time.

Conclusion: In a complete population-based cohort of PCI patients with multiple measurements, we found improvements in mental, but not physical health over time. Demographic differences in health were larger than were disease-related differences.

\section{O-090}

Factors associated with repeated work-related injuries in an Italian region

Presenter: Anteo Di Napoli

Anteo Di Napoli 1); Enrica Lapucci 1); Aurora Marchetti 1); Domenica Di Lallo 1); Gabriella Guasticchi 1)

1) Agency for Public Health of Lazio Region

Background: Factors associated with risk of repeated work-related injuries have not been sufficiently studied.

Objectives: To evaluate incidence rates of experiencing repeated injuries in an Italian region, taking into account occupation and individual characteristics of workers.

Methods: We analysed 275,556 work-related injuries, occurred to workers of industries, services, craftsmanship, aged 25-55, with a permanent disability level $\leq 35 \%$ to exclude severe injuries, in Lazio region (Italy) in 2000-2010, gathered by Italian Workers' Compensation Authority (INAIL) database. We selected a cohort of 157,339 workers with a "first" injury (previous 2 years injury-free), evaluating the occurrence of further accidents within 2 years in the same occupation, taking into account occupational sector and individual factors (age, gender, nationality, disability level, severity of first accident). Incidence rates of repeated work-related injuries were calculated through Poisson regression models.

Results: Mean age of the 157,339 workers at first injury was 38.8 (SD 8.6); $70.5 \%$ were men, $9.8 \%$ were foreigners; mean disability level was $0.8 \%$ (SD 2.6), mean number of absence-work days were 31.5 (SD 45.1). We observed 10,943 repeated work-related injuries in the same occupation. Compared with workers of Utilities-Services, we found higher Incidence Rate Ratio (IRR) of repeated injuries among Health Services workers (IRR $=1.26$; $95 \%$ CI 1.18-1.35) and lower 
IRR for workers of: Engineering (IRR $=0.91 ; 95 \%$ CI 0.83-0.98), Wooden Industry (IRR $=0.80 ; 95 \%$ CI $0.66-0.97$ ), Chemical Industry $\quad(\mathrm{IRR}=0.70 ; \quad 95 \% \quad \mathrm{CI} \quad 0.59-0.82), \quad$ Textile Industry $(\mathrm{IRR}=0.68 ; 95 \%$ CI $0.50-0.92)$, Trade $(\mathrm{IRR}=0.67 ; 95 \% \mathrm{CI}$ 0.62-0.73), Energy-Water (IRR $=0.66$; $95 \%$ CI 0.53-0.83), Construction (IRR $=0.61 ; 95 \%$ CI $0.57-0.65)$, Mining $($ IRR $=0.56$; $95 \%$ CI 0.36-0.88), Electrics (IRR $=0.51 ; 95 \%$ CI 0.39-0.67). We found a lower IRR also for: women (IRR $=0.74 ; 95 \%$ CI $0.71-0.78$ ), 1 year increase of age at first injury (IRR $=0.98 ; 95 \%$ CI 0.98-0.99), foreign workers compare d with Italians (IRR $=0.71$; $95 \%$ CI 0.66-0.77), $1 \%$ disability level increase (IRR $=0.63 ; 95 \%$ CI $0.62-0.64$ ), for 1 absence-work day decrease (IRR $=0.98 ; 95 \%$ CI 0.98-0.98).

Conclusions: We compared economic activity sectors with UtilitiesServices, because $40 \%$ of injuries occurred in this sector. The finding of a not expected lower IRR of repeated work-related injuries in industrial sectors (including Construction, Mining), could be explained by higher propensity to change occupation after first injury in industrial sectors; higher probability of injury charges underreporting in industrial sectors, because smaller companies' size and different propensity to look to health care. The hypothesis of underreporting is supported by finding that health services workers had the highest IRR of repeated work-related injuries. Furthermore, we also found a lower IRR of repeated work-related injuries among foreign workers, a group with a risk of underreporting probably more frequent than Italians. As expected we found a lower IRR for women, probably because employed in less dangerous occupation, and for older workers, probably because more experienced.

\section{0-091}

Repeated measurements of modifiable risk factors account for more of the socioeconomic gradient in cardiovascular mortality than using baseline only

\section{Presenter: Inger Ariansen}

Inger Ariansen 1); Sidsel Graff-Iversen 1), 2); Hein Stigum 1), 3); Bjørn Heine Strand 1); Øyvind Næss 1), 4)

1) Division of Epidemiology, Norwegian Institute of Public Health, Oslo, Norway; 2) Institute of Community Medicine, University of Troms $\emptyset$, Norway; 3 ) Institute of Preventive Medicine and Epidemiology, University of Oslo, Norway; 4) Institute of Health and Society, University of Oslo, Norway

Background: Conventional modifiable risk factors for cardiovascular disease (CVD) account for the majority of CVD mortality, but do not account for the excess CVD mortality by lower socioeconomic position. Repeated assessments of modifiable CVD risk factors capture changes in population lifestyle over time, and might better explain the social gradient in CVD mortality.

Objectives: To examine whether repeated measures of the conventional modifiable risk factors explain more of the socioeconomic gradient in CVD mortality than if they are measured only once at baseline.

Methods: The Norwegian Counties Health Examination Studies was performed in 1974-1978, 1977-1983 and 1985-1988, and includes 91,920 men and women from the general population aged $20-49$ years. Analyses are based on 34,895 persons participating in all three health examinations with complete modifiable CVD risk factors followed up for mortality trough year 2009. Education as measure of socioeconomic position was classified as basic ( 9 years), secondary (12 years) and tertiary (13 years or longer). The following modifiable CVD risk factors assessed at all three health examinations were investigated; daily smoking, physical inactivity, body mass index, systolic blood pressure and non-fasting serum total cholesterol. A Cox proportional hazards regression model was fitted first including gender, age and length of education. Then the modifiable risk factors were included first with measurements from baseline and then with repeated measurements.

Results: 7,692 persons died during follow up; 2,747 died from CVD. Those with basic education had more than twice the CVD hazard rate (95\% confidence interval) than those with tertiary education $(2.37$ (1.97-2.86)). This association was attenuated by $57 \%$ when adjusted for health behaviours at baseline corresponding to 1.59 (1.32-1.92) times higher rate of CVD death for those with basic education than for those with tertiary education, and by $65 \%$ when adjusted for health behaviours assessed at three time points corresponding to 1.48 (1.23-1.79) times higher rate of CVD death for those with basic education than for those with tertiary education.

Conclusions: Compared with one measurement only, repeated measurements during midlife of modifiable risk factors accounted for more of the social gradient in CVD mortality.

\section{0-092}

Changes in lung function between age 50 and 61 years and fibrinogen levels in the Newcastle Thousand Families Study

Presenter: Mark Pearce

\section{Kay Mann 1); John Gibson 2); Mark Pearce 1)}

1) Institute of Health \& Society, Newcastle University, UK; 2) Institute of Cellular Medicine, Newcastle University, UK

Background: Reduced respiratory function is associated with an increased risk of adverse cardiovascular events, suggesting that impaired lung function and cardiovascular disease may share some aetiological factors. A key feature of cardiovascular disease is increased concentration of inflammatory proteins such as fibrinogen which has been shown to be associated with lung function, independently of cigarette smoking. There are however queries as to whether this association is symptomatic or causative.

Objectives: This study investigated changes in fibrinogen levels between the ages of 50 and 61 years in the Newcastle Thousand Families Study in relation to changes in lung function over the same period adjusting for potential confounders, including smoking.

Methods: The Newcastle Thousand Families birth cohort began in 1947 in Newcastle upon Tyne, UK. During clinical assessments at age 50 and 61 years spirometric testing determined lung function through FEV1 measurement and blood samples were assayed for Clauss fibrinogen levels.

Results: Percentage change in FEV1] between age 50 and 61 years was associated with percentage change in fibrinogen over the same time. With a one percent increase in fibrinogen level between age 50 and 61 years, lung function declined by $0.07 \%$ (95\% CI -0.14 , $0.001 p=0.054)$. However, this association was heavily influenced by an outlying observation which yielded insignificant results when excluded from analyses. Regardless of whether influential observations were included or excluded, the association with fibrinogen became negligible when the change in body mass index (BMI) between age 50 and 61 years was adjusted for. A one unit increase in BMI from age 50 to 61 years resulted in a $1.13 \%(95 \% \mathrm{CI}-1.75$, $-0.64, p<0.001)$ decline in lung function over the 11 year period. Along with BMI, continuous and new smokers between age 50 and 61 years had $7 \%$ decline in lung function whereas smokers at age 50 years, that stopped before age 61 years, had a $3 \%$ reduction in lung function, both in comparison to none smokers $(p=0.012)$.

Conclusions: The association between change in fibrinogen level and change in lung function was not independent of change in BMI or 
smoking status in this cohort. Targets to reduce smoking and promote a healthy body size may be more important in maintaining lung function

\section{0-093}

Modelling temporal dependencies in associations with time-varying exposures

Presenter: Antonio Gasparrini

\section{Antonio Gasparrini 1)}

1) London School of Hygiene and Tropical Medicine

Background: In epidemiological research, a health effect is frequently associated with protracted exposures of varying intensity, with the risk changing depending on the specific exposure pattern sustained in the past. This phenomenon is common to different types of exposures, such as environmental stressors, drugs or carcinogenic substances, amongst others. Although the issue is common, a general conceptual and analytical approach is lacking. Terminology differs across research fields, with the terms latency and delayed or lagged effects, for example, employed in cancer and environmental epidemiology, respectively. Similarly, the analytical methods developed so far are limited and subject-specific, and usually restricted to linear exposure-response associations.

Objectives: In this contribution, I illustrate a unified conceptual and statistical framework for modelling linear or non-linear relationships with protracted time-varying exposures, generally referred here as exposure-lag-response associations.

Methods: The main complexity of modelling and interpreting exposure-lag-responses lies in the additional temporal dimension needed to express the association, as the risk depends on both intensity and timing of past exposures. The problem is therefore inherently bi-dimensional. Here I propose a methodology which extends distributed lag-non-linear models (DLNMs), a modelling class originally developed in time series analysis, to provide a general approach for different study designs and regression models. The method is based on the definition of a cross-basis, a combination of two independent functions for modelling the shape of the exposureresponse and the lag structure, respectively. The framework is fully implemented in the R package dlnm.

Results: A series of examples of application will be illustrated, covering different research fields, exposures and study designs. In particular, I will show applications of DLNMs for investigating the health effects of environmental stressors (e.g. temperature, air pollution, pesticides) and carcinogens (e.g. smoking, radon and asbestos) in time series, case-control, cohort and other longitudinal studies. Results from different models, ranging from standard approaches to complex bi-dimensional DLNMs, will be compared and critically evaluated. Advantages and limitations, together with assumptions and interpretational issues, will be discussed.

Conclusions: The DLNM framework is based on a general conceptual and statistical development, and represents a promising analytical tool for epidemiological studies. The methodology is potentially applicable to different research areas, to study associations with exposures over periods spanning from few hours to decades.

\section{0-094}

Weight loss predicts mortality independent of baseline BMI among patients with coronary heart disease

Presenter: Aziza Azimi
Aziza Azimi 1); Christian Torp-Pedersen 3); Gunnar H. Gislason 1); Peter Riis Hansen 1); Leif Thuesen 4); Per Føge Jensen 5); Per Thayssen 2); Hans-Henrik Tilsted 3); Mette Mette Gitz Charlot 1)

1) Department of Cardiology, Copenhagen University Hospital Gentofte, Hellerup, Denmark; 2) Department of Cardiology, Odense University Hospital, University of southern Denmark, Denmark;

3) Department of Cardiology, Aalborg University, Aalborg, Denmark; 4) Department of Cardiology, Aarhus University Hospital Skejby, Aarhus, Denmark; 5) The Heart Centre, Copenhagen University Hospital Rigshospitalet, Copenhagen, Denmark

Background: Overweight and obesity are risk factors for developing cardiovascular disease and they also increase risk of death. Recent studies show that obesity is paradoxically associated with better survival, but weight loss is detrimental for patients with cardiovascular disease.

Objectives: There exists an unresolved paradox in relation to obesity and improved prognosis in cardiovascular disease. We examined the association between weight changes and survival among patients with established coronary artery disease.

Methods: This cohort study included 5,021 Danish patients (mean age 64 (SD 12) years) with coronary artery disease from 2005 to 2011. Information about weight changes $>6$ months was based on anesthesiology records and records at the time of coronary angiography. Weight change was stratified into three groups (stable weight, weight loss, weight gain) and further divided into four subgroups according to baseline body mass index (BMI).

Results: During the 6-year follow up, 600 (11.9 \%) patients died. The adjusted Cox regression analysis showed that overweight-stable weight and obese-weight gain had lower mortality (hazard ratio (HR) 0.65 ; $95 \%$ CI $0.45-0.92$, HR 0.59; $95 \%$ CI 0.38-93) compared with a reference group of normal weight patients without weight change, while weight loss had increased mortality independent of (BMI).

Conclusions: Overweight and obese patients with coronary artery disease with stable weight had improved survival compared with normal weight patients.

But weight loss was associated with higher mortality independent of body mass index.

Increased focus on weight changes in patients with coronary artery disease is warranted.

\section{Population incidence and mortality}

\subsubsection{1:00-12:30}

\section{O-095}

Secular trends in physical activity and cardiovascular risk factors in a 40-42-year-old rural population in Norway 1975-2010

Presenter: Ane Solbraa

Ane Solbraa 1); Geir Kåre Resaland 1); Eivind Aadland 2); Sidsel Graff-Iversen 3); Ingar Morten Holme 4); Sigmund Alfred Anderssen 4)

1) Sogn og Fjordane University College, Faculty of Teacher Education and Sport, Sogndal, Norway; 2) Sogn og Fjordane University College, Faculty of Health Studies, Førde, Norway; 3) Norwegian Institute of Public Health, Division of Epidemiology, Oslo, Norway; 4) Norwegian School of Sport Sciences, Department of Sports Medicine, Oslo, Norway

Background: Substantial geographical differences in physical activity (PA) level and other risk factors for cardiovascular disease 
(CVD) have been shown in Norway. Compared to other Norwegian counties, long-term beneficial health status has been documented for the population in Sogn og Fjordane, a rural county in the west of Norway.

Objectives: To investigate secular trends in PA and other CVD risk factors over a 35 -year period in a 40-42-year-old healthy rural population in the county of Sogn og Fjordane, and compare them with national trends.

Methods: Data from The National Health Screening Services screening program and The Physical Activity among Adults and Older People Study were used. In total 21,361 40-42-year-olds from Sogn og Fjordane attended the eight cohort-studies from 1975 to 2010. To compare trends from Sogn og Fjordane with national trends, national data from 354,310 40-42-year-olds attending the same cohort-studies were used. Data were obtained from questionnaires and physical examination and were analyzed using a mixed model. Results are reported as changes (continuous variables) or odds ratios (dichotomous variables) per year (smoking was analyzed using a categorical time variable due to a non-linear trend).

Results: On average per year, PA level categorized by sedentary behavior, walking and moderate intensity PA decreased [Odds ratio, $95 \%$ confidence interval $(\mathrm{CI})][0.978,0.973$ to $0.983, p \leq 0.001$ ], $[0.962,0.959$ to $0.965, p \leq 0.001]$ and $[0.963,0.959$ to 0.967 , $p \leq 0.001]$, respectively, whereas high intensity PA increased [1.023, 1.007 to $1.038, p \leq 0.004]$. On average per year, systolic blood pressure (SBP), diastolic blood pressure (DBP), total cholesterol (TC) and high-density cholesterol (HDL) decreased [regression coefficient, $95 \% \mathrm{CI}][-0.25,-0.28$ to $-0.23 \mathrm{mmHg}, p \leq 0.001],[-0.40,-0.42$ to $-0.38 \mathrm{mmHg}, p \leq 0.001],[-0.027,-0.028$ to $-0.025 \mathrm{mmol} / \mathrm{L}$, $p \leq 0.001]$ and $[-0.003,-0.004$ to $-0.002 \mathrm{mmol} / \mathrm{L}, p \leq 0.001]$, respectively, whereas body mass index (BMI) increased [0.043, 0.037 to $\left.0.049 \mathrm{~kg} / \mathrm{m}^{2}, p \leq 0.001\right]$. The odds ratio for being a current smoker in 2010 compared to 1975 was [0.35, 0.25-0.47, $p \leq 0.001$ ]. Compared to national trends, BMI and high intensity PA have increased less, sedentary PA, walking, moderate intensity PA, smoking and HDL have decreased less, whereas SBP, DBP and TC have decreased more.

Conclusions: Trends in PA and other CVD risk factors in Sogn og Fjordane were similar to trends found elsewhere. Compared to national trends, Sogn og Fjordane has maintained a beneficial health status for some variables and to a lesser extent for others. These findings might have public health implications.

\section{O-096}

Estimating salt intake in a Caucasian population: can spot urine substitute 24-hour urine samples?

Presenter: Ulla Toft

Ulla Toft 1); Charlotte Cerqueira 1); Anne Helms Andreasen 1); Betina Heinsbæk Thuesen 1); Peter Laurberg 2); Lars Ovesen 3); Hans Perrild 4); Torben Jørgensen 1)

1) Research Centre for Prevention and Health, Glostrup University Hospital, Building 84/85, DK-2600 Glostrup, Denmark; 2) Department of Clinical Medicine, Aalborg Hospital, Aalborg, Denmark; 3) Department of Gastroenterology, Slagelse Hospital, Slagelse, Denmark; 4) Endocrine Unit, Department of Internal Medicine, Bispebjerg University Hospital, Bispebjerg, Denmark

Background: The gold standard for estimating sodium intake is (repeated) 24-h urine collection. However, this method is cumbersome, and a simple and valid alternative for 24-h urine collection to estimate populational 24-h urinary sodium excretion would be desirable for example for monitoring sodium intake in populations.
Aim: To assess the validity of the predicted 24-h urinary sodium excretion using spot urine and two different prediction methods in a Danish population.

Methods: Overall 473 Danish individuals provided a PABA-validated complete 24-h urine collection and a spot urine sample. Data were collected in the DanThyr study (248 women aged 25-30 years and 60-65 years) and the Inter99 study (102 men and 113 women aged $30-60$ years), respectively. The measured $24-\mathrm{h}$ urine sodium excretion was compared with the predicted 24-h sodium excretion from a causal urine specimen, using both the Tanaka prediction method and a prediction model developed in a Danish population.

Results: The measured 24-h sodium excretion (median [5 and $95 \%$ ]) was: men: 195 [110; 360]; women: 139 [61; 258] whereas the predicted 24-h sodium excretion was: men: 171 [117; 222]; women: 153 [92; 228] for the Tanaka model and: men: 207 [146; 258]; women: 134 [103; 163] for the Danish Model. The correlation (Spearman) between predicted and measured 24-h sodium excretion was 0.39 and 0.49 for the Tanaka and the Danish model, respectively. For both prediction models the proportion of individuals classified in the same or adjacent quintile was $74 \%$ for men; $64 \%$ for women.

Conclusion: Both prediction models gave a reasonable classification of individuals according to their sodium excretion. However, the median daily sodium intake was estimated more precisely by the Danish model, especially among men.

\section{0-097}

The development in body mass index, overweight and obesity in three regions in Denmark from 2006/2007 to 2010

\section{Presenter: Ulla Toft}

Ulla Toft 1); Anker Lund Vinding 2); Finn Breinholt 3); Michael Falk Hvidbergb 2); Kirstine Magtengaard Robinson 1); Charlotte Glümer 1)

1) Research Centre for Prevention and Health, Glostrup University Hospital, Building 84/85, DK-2600 Glostrup, Denmark; 2) Department for health, The North Denmark Region; 3) Department for health promotion and prevention, The Central Denmark Region

The aim of this study was:

- to assess the changes in body mass index (BMI), overweight and obesity from 2006/2007 to 2010 in three regions in Denmark.

- to investigate if the changes in BMI differs significantly between regions

Methods: The study was based on data from two cross-sectional surveys in three regions in Denmark (The Capital Region of Denmark; The Central Denmark Region; The North Denmark Region) in 2006/2007 and 2010. A random sample of citizens aged 25-79 from each municipality in the three regions received a questionnaire and an invitation to participate in the study. The overall response rate was $57.5 \%(\mathrm{~N}=143114)$. All participants completed the questionnaire "How are you?" with similar questions in 2006/2007 and 2010 on weight, height, smoking habits and alcohol intake. Information on demographic and socioeconomic characteristics was obtained from central registers.

Comparisons within and between regions were analysed by multiple logistic regression analyses using the proc survey logistic procedure and linear regression analyses, using the proc surveyreg procedure.

Results: BMI increased significantly from 2006/2007 to 2010 in all three regions. However, the average BMI increased significantly more in The North Denmark Region compared with both the Capital Region of Denmark $(P=0.003)$ and the Central Denmark Region $(P=0.046)$. These differences remained after adjustments for 
socioeconomic status and ethnicity. In the North Denmark Region there was a $17 \%$ greater risk of developing overweight from 2006/2007 to 2010 compared with the Capital Region of Denmark (OR: 1.17 [1.08-1.26]). Compared with the Central Denmark Region the risk was $12 \%$ higher in the North Denmark Region (OR: 1.12 [1.04-1.22]). No significant difference was found between the Central Denmark Region and the Capital Region of Denmark (OR 1.04 [0.98-1.10]). Similar, the odds ratio of developing obesity in the North Denmark Region was 1.25 [1.13-1.39] compared with the Capital Region of Denmark and 1.20 [1.08-1.35] compared with the Central Denmark Region. No significant difference was found between the Central Denmark Region and the Capital Region of Denmark (OR 1.04 [0.96-1.13]).

Conclusion: The results showed a significantly higher increase in BMI and a greater risk of developing overweight/obesity in The North Denmark Region compared with the Capital Region of Denmark and the Central Region Denmark.

\section{0-098}

Paediatric sleep disorders: trends, treatment and association with adolescent depression

Presenter: Linda Wijlaars

Linda Wijlaars 1); Irwin Nazareth 1); Irene Petersen 1)

1) Department of Primary Care and Population Health, UCL

Little is known on the epidemiology of paediatric sleep disorders, their pharmalogical treatment and association with adolescent depression.

The aim of the study was to study trends in paediatric sleep disorders \& hypnotic prescriptions, and their association with adolescent depression.

We used The Health Improvement Network (THIN) UK primary care database to identify $1,224,100$ children aged $5-18$ years who were registered with a general practice for at least 1 year between 1995 and 2011. We identified children diagnosed with sleep disorders and assessed time, age and prescription trends, adjusting for deprivation and sex. We used Cox regression to assess the association between sleep disorders and adolescent depression.

Overall, 19,518 (2\%) children were diagnosed with a sleep disorder and hypnotics were prescribed to 9,816 (1\%) children. Sleep disruptive behaviours (12,567 children, $42 \%)$ and insomnia $(12,273$ children, $41 \%$ ) were the most common disorders. Sleep disorder diagnoses were stable over time at 4.3 (95\% CI 4.2-4.3) per 1,000 person-years at risk (PYAR).

Between 1995 and 2010 prescription rates for hypnotics increased from 7.8 (95\% CI 6.0-10.0) to 19.8 (95\% CI 18.6-21.0) per 10,000 PYAR in 2010. This increase was mainly caused by increase in prescribing of melatonin, which increased from no prescriptions in 2000 to 9.3 (95\% CI 8.5-10.0) per 10,000 PYAR in 2010, and the newer non-benzodiazepine hypnotics, which increased from 2.1 (95\% CI 1.2-3.4) in 1995 to 8.0 (95\% CI 7.2-8.8) per 10,000 PYAR in 2010. Children aged $<12$ years who prescribed hypnotics were more likely to be diagnosed with a developmental or behavioural disorder.

Paediatric sleep disorders were associated with later adolescent depression both for children aged 5-12 years (HR: 4.42; $95 \%$ CI 3.78-5.18) and children aged 13-18 years (HR: 2.11; $95 \% \mathrm{CI}$ 2.03-2.20).

Paediatric sleep disorders are recognised and treated in primary care. Although the association could be bidirectional, childhood sleep disorders appear to increase the chance of developing depression in adolescence.
O-099

Evolutions in fetal and neonatal mortality rates in European countries: results from the Euro-Peristat project

Presenter: Ashna Mohangoo

\section{Ashna Mohangoo 1); Jennifer Zeitlin 2)}

1) TNO Netherlands Organization for Applied Scientific Research, Department Child Health, Leiden, The Netherlands;

2) Epidemiological Research Unit on Perinatal and Women's and Children's Health, INSERM UMRS 953, Paris, France

Background: 2004 data from the Euro-Peristat project documented wide variations in fetal and neonatal mortality rates in Europe.

Objectives: We sought to evaluate whether mortality rates had declined in 2010 and whether these declines reduced inequalities between countries.

Methods: Aggregated data on live births, fetal and neonatal deaths by gestational age from 25 participating countries in 2004 and 2010 were used to compute fetal and neonatal mortality rates using inclusion thresholds of $\geq 28$ weeks of gestation for fetal deaths and $\geq 24$ weeks of gestation for neonatal deaths.

Results: In most countries, fetal and neonatal mortality rates declined, but decreases differed in magnitude. Neonatal mortality rates declined between 9 and $51 \%$ (average $29 \%$ ) and fetal mortality rates between 3 and $39 \%$ (average $15 \%$ ). Reductions tended to be more pronounced for countries with higher mortality rates in 2004, but some countries with lower mortality rates achieved significant continued improvements in outcomes. Wide variations in mortality rates persisted in 2010, with highest mortality rates being three times higher than lowest mortality rates (range $1.5-4.3$ per 1,000 total births for fetal deaths and 1.2-3.3 per 1,000 live births for neonatal deaths). Conclusions: Compared with 2004, fetal and neonatal mortality rates have declined in most European countries in 2010 but disparities have persisted. Investigation of health care policies and practices in high performing countries could provide insight into effective strategies for improving perinatal health outcomes.

\section{0-100}

Projected age- and sex-specific prevalence of cardiovascular disease

Presenter: Danja Sarink

Danja Sarink 1); Lee Nedkoff 2); Tom Briffa 2); Dianna Magliano 1); Jonathan Shaw 1); Anna Peeters 1)

1) Baker IDI Heart and Diabetes Institute; 2) University of Western Australia

Introduction: Primary prevention and improved treatment have substantially reduced the prevalence of smoking, hypertension and other risk factors for cardiovascular disease (CVD) over the last decades. Yet CVD remains one of the main causes of death and burden of disease worldwide, and the favourable trends in CVD mortality and morbidity may eventually be counterbalanced by increasing obesity and diabetes trends. Inevitably, a substantial lagtime exists before these known risk factors will impact CVD prevalence at a population level. We therefore hypothesized that the slowing in the decline in CVD incidence and mortality at younger ages that has been shown previously will lead to an increase in the prevalence of CVD at older ages in the coming decades.

Objectives: To predict age- and sex-specific future prevalence of CVD from 2010 to 2030 based on current incidence and mortality in Australia. 
Methods: Sex- and age-specific 5-year transition probabilities (2005-2009) of incident CVD and mortality were calculated from the Western Australian Data Linkage System (WADLS). A cohort multistate life table and a series of linked multi-state life tables were set up using these 5-year transition probabilities, starting in 2010 and ending 2030. These were used to calculate prevalence, life expectancy, and lifetime risk of CVD.

Results: Based on the event rates observed between 2005 and 2009, CVD prevalence in the Western Australian adult population is estimated to increase by approximately $30 \%$ between 2010 and 2030 . Greater relative increases were seen in men and women under 60 years for whom prevalence increased by one-fifth and two-fifth respectively. Western Australian adults aged 25-29 years in 2010 are estimated to have a lifetime risk for CVD of $49 \%$. Of these, men and women can expect to live approximately 18 and $11 \%$ of their remaining life expectancy with CVD.

Conclusions: Assuming that the incidence and mortality rates observed between January 12005 and December 312009 remain constant, we predict that between 2010 and 2030 the age- and sexspecific prevalence of CVD will increase in Western Australian adults. This is consistent with retrospective reports of a halting decline or even increasing incidence of CVD at younger ages and continuing improvements in mortality at older ages, building up to greater prevalence in older age groups over the next decades. These findings need to be confirmed by studies in which prevalence is consistently and empirically measured and monitored over time.

\section{O-101}

Estimating the prevalence of female genital mutilation among child-bearing women in Europe

\section{Presenter: Alison Macfarlane}

\section{Alison Macfarlane 1), 3); Efua Dorkenoo 2); The Euro-Peristat Group 3) \\ 1) City University London; 2) Equality Now; 3) The Euro-Peristat Group}

Background: It is suspected that prevalence of female genital mutilation (FGM) has been increasing in Europe, mainly due to migration from countries where it is practised, but there are no reliable data on this. Data needed to inform appropriate care for affected women and policies to protect their daughters from the practice. In England and Wales, Belgium and the Netherlands, data from surveys in the mothers' countries of birth have been used to estimate prevalence among women who have migrated. The Euro-Peristat collaboration includes all EU member states plus Switzerland, Norway and Iceland and aims to use aggregated data from each participant to compile perinatal indicators on a comparable basis. In order to construct its indicator of the percentage of births to foreign born women, it compiles data about numbers of women delivering, tabulated by their country of birth.

Objectives: To estimate for countries and regions which submitted appropriate data to Euro-Peristat the numbers and percentages of deliveries which were to women who had undergone FGM.

Methods: The typology of FGM, I, II and III was based on that used in WHO FGM prevalence surveys in the 29 identified practising countries. To estimate the numbers and proportions of women who had undergone FGM, prevalence rates derived from these surveys were applied to registered numbers of women who were born in those countries and who gave birth in 2010 in countries and regions participating in Euro-Peristat.

Results: Some Euro-Peristat participants record mothers' nationalities or ethnicity rather than their countries of birth and others group countries together. Data about mothers' countries of birth were available for the Brussels, Flanders and Wallonia regions of Belgium, Switzerland, Cyprus, Czech Republic, Spain, Finland, France, Ireland, Malta, Norway, Portugal, and England and Wales, Scotland and Northern Ireland in the UK. Over $1 \%$ of women giving birth in Brussels, Malta, France, Norway and England and Wales were estimated to have undergone FGM compared with under $0.5 \%$ in Cyprus, the Czech Republic, Spain, Finland, the Irish Republic and Northern Ireland.

Discussion: These estimates give a broad picture but they are subject to a number of biases and prevalence is likely to vary considerably within countries. Better data are needed but direct data collection poses many challenges.

Conclusions: Despite their limitations, these estimates suggest that in some parts of Europe there considerable numbers of births to women with FGM.

\section{0-102}

Evidence of increasing mortality with longer diagnostic intervals for five common cancers: a cohort study in primary care

\section{Presenter: Marie Louise Torring} Marie Louise Tørring 1); Morten Frydenberg 2); Rikke Pilegaard
Hansen 1); Frede Olesen 1); Peter Vedsted 1)

1) Research Centre for Cancer Diagnosis in Primary Care (CaP), Research Unit for General Practice in Aarhus; 2) Department of Public Health - Section for Biostatistics, Aarhus University, Denmark

Background: Numerous observational studies of time to cancer diagnosis have reported counterintuitive results showing that cancer patients with short diagnostic intervals suffer higher mortality than the rest. From a primary care perspective, the diagnostic interval can be viewed as a selection process in which general practitioners (GPs) move people on the continuum from cancer suspicion to confirmation. This process implies assessing risk based on the interpretation of symptoms and tests. As patients are selected for different paces of diagnosis based on clinical indication, the mortality estimates become confounded by indication.

Objectives: To assess the association between the length of the diagnostic interval and the 5-year mortality for the five most common cancers in Denmark while addressing the problem of confounding by indication. Methods: A total of 1,128 patients with colorectal, lung, melanoma skin, breast or prostate cancer were included in a prospective, population-based study in a Danish county. The diagnostic interval was defined as the time from the first presentation of symptoms in primary care till the date of diagnosis. Each type of cancer was analysed separately and combined. To take into account confounding-by-indication, all analyses were stratified according to the GP's interpretation of the presenting symptoms. To further display confounding by indication, we used conditional logistic regression to estimate 5-year mortality odds ratios as a function of the diagnostic interval using restricted cubic splines and adjusting for comorbidity, age, sex and type of cancer.

Results: We found increasing mortality with longer diagnostic intervals among the approximately $40 \%$ of the patients who presented in primary care with symptoms suggestive of cancer or any other serious illness. In the same group, very short diagnostic intervals were also associated with increased mortality. Patients presenting with vague symptoms not directly related to cancer or a serious illness had longer diagnostic intervals and the same survival probability as those who presented with cancer suspicious/serious symptoms. For the former, we found no statistically significant association between the length of the diagnostic interval and mortality.

Conclusion: In full coherence with clinical logic, the healthcare system instigates prompt investigation of seriously ill patients. This 
likely explains the counter-intuitive findings of high mortality with short diagnostic intervals; but it does not explain the increasing mortality with longer diagnostic intervals. Thus, the study provides further evidence for the hypothesis that the length of the diagnostic interval affects mortality negatively.

\section{O-103}

Is increasing incidence or decreasing mortality the driving force of increasing prevalence of diabetes in Denmark?

\section{Presenter: Bendix Carstensen}

\section{Bendix Carstensen 1)}

\section{1) Steno Diabetes Center}

Background: Since inception of the Danish National Diabetes Register (NDR) in 1995, mortality among diabetes patients has decreased, and incidence of diabetes has increased, but it is not known how much each of these contribute to the increasing prevalence of diabetes.

Objectives: Describe the diabetes prevalence in Denmark as it is now, and show how much can be attributed to decreasing mortality and how much to increasing incidence over the last 15 years. Describe the simulation model needed for this and how to use it to tease out the relevant quantities.

Methods: Mortality rates were computed for the diabetic and the nondiabetic part of the population, based on total mortality counts from Statistics Denmark and mortality counts from the NDR. Incidence rates of diabetes were derived from the NDR. All rates were modelled by sex, age and calendar time (APC-model), using 1-year classified data and natural splines for the age period and cohort effects. Prevalence of diabetes at 1.1.2010 were simulated from the prevalence at 1.1.1995 and the estimated mortality and incidence rates, by predicting prevalences in steps of 1/10 year from a competing risk model with diabetes and death as outcome. This was compared to the actually observed prevalences in 2010. By replacing the actual trends in incidence and mortality rates by rates constant since 1995 we could simulate what the diabetes prevalence in 2010 would have been under different scenarios: 1: as observed, 2: fixed mortality at 1995 level, 3: fixed incidence at 1995 level, 4: both mortality and incidence fixed at 1995 level.

Results: The model used with estimated rates did accurately predict the observed prevalences in 2010. Using the differences between the 4 scenarios we found that $7 \%$ of the prevalent cases in 2010 could be attributed to decreasing mortality and $25 \%$ to increasing incidence rates, $25 \%$ could be attributed to non-equilibrium in 1995; the remaining $43 \%$ were the number of cases corresponding to the agespecific prevalences in 1995.

Conclusion: The major determinant of the increasing number of diabetes patients is the increasing incidence rates and the existing disequilibrium between mortality and incidence. The study shows that careful exploitation of standard probability theory approximating the continuous time scenario is feasible, but also revealed that usual approximations using 1 or 5 year age and time classes are far too inaccurate.

\section{Exposure: lifestyle factors}

13.08.2013 13:30-15:00

\section{0-104}

Poor health may be a reason for lifetime abstention: implications for the protective effects of moderate alcohol consumption

Presenter: Linda $\mathrm{Ng} \mathrm{Fat}$
Linda Ng Fat 1); Noriko Cable 1); Nicola Shelton 1)

1) Public Health and Epidemiology, UCL, London, UK

Background: Non-drinkers have been repeatedly shown to have worse health than moderate drinkers in later life in conditions such as coronary heart disease, leading some researchers to suggest that moderate alcohol consumption has beneficial effects on health. However the causal direction between non-drinking and worse health has been contested. Whether poor health is associated with continued non-drinking from early adulthood will be investigated.

Objectives: To explore whether poor health is a reason why some never ever take up drinking. Adolescent health status of non-drinkers in early adulthood is examined. Secondly whether continued poor health is associated with remaining a non-drinker across different stages of the life course is assessed.

Methods: Using two prospective British birth cohort studies established in 1958 (NCDS) and in 1970 (BCS) the adolescent health status of non-drinkers in early adulthood is explored using a medical examination carried out in adolescence.

Participants who reported 'never' or 'never had an alcoholic drink' to drinking status questions in successive waves from age 23 and 26 in NCDS/BCS were derived as 'Lifetime abstainers'.

Logistic regression on the odds of being a lifetime abstainer was carried out on changes in limiting longstanding illness (LLSI) in the NCDS and longstanding illness (LSI) in the BCS, adjusting for sex, education, and poor mental health, marital and parental status.

Results: Non-drinkers at age 23 have higher rates of medical conditions at age 16 as assessed by a medical officer such as having a mental condition $(p<0.001)$ a physical condition and heart and haematological condition $(p<0.01)$.

Participants with a LLSI in consecutive waves since 23 years had 4.50 times the odds of someone who did not have a LLSI of being a lifetime abstainer at 33 years (95\% CI 1.99-10.18) and 7.02 times the odds at 42 years (2.39-20.66) after adjusting for all factors. Similarly in the BCS having a LSI in consecutive waves resulted in higher odds of being a lifetime abstainer at 30 years (OR 2.80, 1.88-4.18) and 34 years (OR 3.33, 2.01-5.53)

Conclusion: Persistent longstanding illness was associated with remaining a non-drinker across adulthood. Even after excluding exdrinkers from non-drinkers, poor health may be a reason for lifetime abstention. Studies comparing the health outcomes of moderate drinkers to lifetime abstainers that do not account for pre-existing poor health may overestimate the better health outcomes from moderate alcohol consumption.

\section{0-105}

Internet-based intervention and telephone counselling for smoking cessation: results from a 4-arm randomised controlled trial

Presenter: Lise Skrubbeltrang Skov-Ettrup

Lise Skrubbeltrang Skov-Ettrup); Peter Dalum); Janne Schurmann Tolstrup)

1) National Institute of Public Health, University of Southern Denmark; 2) Danish Cancer Society; 3) National Institute of Public Health, University of Southern Denmark

Background: Ideally smoking cessation interventions should have high efficacy, high reach and low cost. Smoking cessation aid delivered through internet and as telephone counselling represent two different approaches that may come closer to reaching this ideal than for instance face-to face counselling.

Objectives: To compare the efficacy of (1) a tailored smoking cessation program delivered through internet and mobile phone, (2) five 
proactively initiated telephone counselling sessions, (3) reactive telephone counselling sessions and (4) a written self-help booklet.

Methods: The study was based on a four-arm randomised controlled trial. Participants were 1,809 daily cigarette smokers who were at least 16 years old and had a valid e-mail address and mobile phone. After randomisation there was a 2-month intervention period. Followup data was collected 1, 6 and 12 months after the intervention period. Data was collected by internet-based questionnaire, textmessages and telephone interviews. The response rates were 78,82 and $80 \%$ respectively. The primary outcome was 30-day point prevalence abstinence measured self-reportedly at 12 months followup.

Results: In preliminary analysis using the intention-to-treat principle (non-responders counted as smokers) the 30-day point prevalence abstinence at 12 months follow-up was $18.8 \%$ in the proactive telephone counselling group, $15.5 \%$ in the internet group, $14.6 \%$ in the reactive counselling group and $17.5 \%$ in the booklet group. Repeated 30-day point prevalence abstinence at 1, 6, and 12 months follow-up was $8.2 \%$ in the proactive counselling group, $6.0 \%$ in the internet group, $1.8 \%$ in the reactive counselling group and $4.0 \%$ in the booklet group. The repeated 30-day point prevalence abstinence was significantly higher in the group receiving proactive telephone counselling than in the group receiving a self-help booklet $(p=0.01)$.

Conclusions: The repeated abstinence was highest in the group receiving proactive telephone counselling. This indicates that proactive telephone counselling is effective in attaining long-term abstinence.

\section{0-106}

\section{Drunkenness among adolescents and parental alcohol norms}

Presenter: Mathilde Vinther-Larsen

\section{Mathilde Vinther-Larsen 1); Rikke Krølner 1); Mogens Trab Damsgaard 1)}

1) National Institute of Public Health, University of Southern Denmark, Copenhagen

Background: Risky drinking among young people is an issue of public concern in western countries. Adolescents' drinking behavior is influenced by the social arenas around the adolescents such as behavior of peers and parents. Further, prior research has found that a strict parental attitude towards drinking may postpone the drinking onset and lower the alcohol intake of the adolescent. However, it is unknown if this association also can be found in a liberal alcohol culture. Furthermore, potential interactions between parental attitude towards drinking and parents' own alcohol behavior have not been investigated in depth.

Objectives: The aim of this study was to clarify how adolescents' drunkenness is associated with their parents' alcohol norms (behavior and attitude).

Methods: Cross-sectional data from the school-based Danish Youth Cohort was used $(\mathrm{N}=12.498$, mean age: 13.4) The determinant parental alcohol norms was constructed by combining two variables from the student questionnaire: 'parental attitude towards the adolescent's drinking' and 'if the adolescents had seen their parents been drunk'. This combined variable had four categories: (1) Had experienced their parents drunk and lenient parental attitude, (2) Had not experienced parents drunk and lenient parental attitude, (3) Had seen parents drunk and strict parental attitude, (4) Had not seen parents drunk and strict parental attitude. The outcome variable measured if the adolescents had ever been drunk. Multilevel logistic regression analyses included 8,615 adolescents and were restricted to adolescents of Danish ethnicity. The analysis was adjusted for family structure and family affluence and was stratified by sex.

Results: Parental alcohol norms were significantly associated with drunkenness of the adolescent. Using the adolescents who had not seen their parents drunk and who had a strict drinking attitude as a reference group, the adolescent who had seen their parents drunk and the parent were lenient the risk of drunkenness was ORGirls $=3.6$ (CI $95 \%$ 2.6-4.6) and ORBoys $=2.7$ (CI $95 \% 2.1-3.3$ ). If the adolescents had parents with a lenient drinking attitude and they had not seen their parents drunk the risk of drunkenness was ORGirls $=3.6(\mathrm{CI} 95 \% 2.6-4.8)$ and ORBoys $=3.8(\mathrm{CI} 95 \% 2.9-4.9)$. If the adolescents had seen their parents been drunk and had parents with a lenient drinking attitude they have an excessive higher risk of drunkenness OR for girls $=7.9$ (CI $95 \%$ 5.5-10.5) and OR for boys $=7.7$ (CI $95 \% 5.8-10.3$ ).

Conclusions: Parental alcohol norms do matter in relation to the adolescents' drunkenness in a liberal alcohol culture. Adolescents who both have experienced their parents drunk and whose parents have a lenient attitude towards drinking are especially at risk for drunkenness.

\section{0-107}

Does smoking reduction in midlife reduce mortality risk? Results from 2 prospective cohort studies of men and women in Scotland

Presenter: Carole Hart

Carole Hart 1); Laurence Gruer 1); Linda Bauld 2)

1) University of Glasgow; 2) University of Stirling

Background: A long-term cohort study of working males in Israel reported smokers who reduced their cigarette consumption between 2 screenings 2 years apart had lower subsequent mortality rates than those who maintained their amount smoked.

Objectives: We investigated whether this effect could be seen in other populations by conducting comparable analyses on male and female smokers screened twice in 2 long-term prospective cohort studies in Scotland.

Methods: The Collaborative study screened a working population in 1970-1973 and 1977 ( $\mathrm{n}=1,524$ smokers aged 40-65) and the Renfrew \& Paisley study screened a general population in 1972-1976 and 1977-1979 ( $\mathrm{n}=3,730$ smokers aged 45-64). Mortality followup was to the end of 2010. Smoking categories were defined as $1-10$, $11-20$ and $\geq 21$ cigarettes/day at each screening and none at 2 nd screening. 4 groups were defined: increased, maintained, reduced and quit. The reduced group was also subdivided into reducing from $\geq 21$ cigarettes/day (heavy) or from $\leq 20$ cigarettes/day (lighter). Cox proportional hazards models were used to calculate hazard ratios of mortality for each smoking intensity category, with the maintained group as the baseline category.

Results: In the follow-up period, 1252 Collaborative participants (82\%) and 3,187 Renfrew \& Paisley participants (85\%) died (median follow-up 21.1 and 18.7 years, maximum 33.9 and 33.8 years in the 2 studies respectively). There was no evidence of lower mortality in all reducers compared to maintainers: multivariate adjusted hazard ratios of mortality were 0.91 (95\% confidence interval (CI): $0.75,1.10)$ in the Collaborative and 1.08 (95\% CI $0.97,1.20)$ in the Renfrew \& Paisley studies, but clear evidence of lower mortality in quitters (hazard ratios $0.66(95 \%$ CI $0.56,0.78)$ and 0.75 (95\% CI 0.67,0.84) respectively). A lower multivariate adjusted hazard ratio of mortality was observed in heavier smoker reducers in the Collaborative study only $(0.67(95 \%$ CI $0.50,0.90))$ which was similar to the quitters. Overall results were similar for men and women when analysed separately and when both cohorts were combined. 
Conclusions: In these cohort studies, we were unable to detect significant overall long-term survival benefit among smokers who reported reducing their daily consumption of cigarettes between 2 screenings a few years apart so could not reproduce the findings of the Israeli study. These inconclusive results support the view that reducing cigarette consumption should not be promoted as a means of reducing mortality although it may have a valuable role as a stepping stone to smoking cessation.

\section{O-108}

Dose-response modelling for variables with a "spike at zero" theory and applications in epidemiology

Presenter: Eva Lorenz

Eva Lorenz 1); Carolin Jenkner 2); Willi Sauerbrei 2); Heiko Becher 1)

1) Epidemiology and Biostatistics Unit, Medical Faculty, University of Heidelberg, Germany; 2) IMBI, Freiburg University Medical Center, Germany

Background: A common goal in epidemiology is to estimate the effect of variables on an outcome quantitatively using appropriate regression models. Such variables often have a proportion of individuals with zero exposure while the distribution is continuous in those exposed (i.e. variables with a spike at zero). Examples are cigarette smoking, alcohol consumption or duration of breastfeeding. Often these variables are analysed by ignoring the spike, or by categorization where the unexposed are represented by a separate category.

Objectives: To develop strategies to estimate the effect of these variables with a spike at zero on an outcome using the complete information available.

Methods: We investigated the theoretical shape of the dose-response curve for variables with a spike at zero under various distributional assumptions in the univariate case. The fractional polynomial (FP) approach has been extended to model the corresponding doseresponse function in univariate situations. However, several variables often need to be considered simultaneously in a multivariable model. Therefore we considered the bivariate normal and log normal distributions with a bivariate spike as well as single spikes in either one or the other variable. We developed conditions for the occurrence of confounding and interaction based on the covariance matrix in diseased and non-diseased.

Results: We present the correct functional forms for the specific distributions mentioned above. We illustrate the modified method with an example of a population-based case-control study on laryngeal cancer in south-west Germany with 257 cases and 769 population controls. The primary aim of the study was to investigate additional effects of occupational risk factors on laryngeal cancer. We consider alcohol consumption and smoking as continuous risk factors with a spike at zero. We compare findings from models using the FP-spike procedure to those from previous analyses.

Conclusions: The extended FP procedure is useful to analyse spike at zero situations which occur frequently in epidemiology and clinical research. Further research to expand the procedure to multivariable scenarios and application to other datasets is ongoing.

\section{0-109}

Alcohol-attributable and alcohol-preventable mortality in Denmark: an analysis of the impact of different intake levels on mortality

Presenter: Marie Eliasen
Marie Eliasen 1); Ulrik Becker 1), 2); Morten Grønbæk 1); Knud Juel 1); Janne S. Tolstrup 1)

1) National Institute of Public Health, University of Southern Denmark, Copenhagen, Denmark; 2) Department of Medical Gastroenterology, Hvidovre Hospital, Copenhagen University Hospital, Denmark

Background: Alcohol consumption has both detrimental and beneficial effects on health. These must be taken into consideration in the discussion of the disease burden of alcohol consumption including the setting of recommendations for average alcohol consumption.

Objectives: To quantify alcohol-attributable and -preventable mortality, totally and stratified on alcohol consumption in Denmark 2010, and to estimate alcohol-related mortality assuming different scenarios of changes in alcohol distribution in the population.

Methods: Quantification of alcohol-related mortality was based on alcohol-attributable fractions and alcohol-preventable fractions for conditions causally related to alcohol and number of deaths due to the alcohol-related conditions. The alcohol-attributable and -preventable fractions were estimated using information on relative risk functions from meta-analyses on alcohol and the alcohol-related conditions and information on alcohol consumption in Denmark obtained from 14,458 participants in the Danish National Health Survey 2010 and corrected for adult per capita consumption. Cause-specific mortality was obtained from the Danish Register of Causes of Death.

Results: 1373 deaths among women (5.0\% of all deaths) and 2,522 deaths among men (9.5\% of all deaths) were attributable to alcohol, while $765(2.8 \%)$ and $583(2.2 \%)$ deaths were prevented by alcohol in Denmark 2010. Of the alcohol-attributable deaths, 73 and $81 \%$ occurred within the high alcohol consumption group ( $>14 / 21$ drinks/ week for women/men). A reduction of $50 \%$ in the alcohol consumption was associated with a decrease of 1,406 partly alcoholattributable deaths (46\%) and 37 alcohol-preventable deaths $(3 \%)$. Total compliance with sensible drinking guidelines with a low risk limit $(<7 / 14$ drinks/week for women/men) and high risk limit $(<14 / 21$ drinks/week for women/men) was associated with a reduction of $2,379(75 \%)$ and $1,976(63 \%)$ partly alcohol-attributable deaths, respectively.

Conclusions: $5.0 \%$ of deaths among women and $9.5 \%$ among men were attributable to alcohol in Denmark 2010. The minority of Danish women and men had high alcohol consumption (16 and $26 \%$ ). However, the majority of all alcohol-attributable deaths among women and men were caused by high consumption (73 and $81 \%$ ).

\section{0-110}

Development of a UK online 24-hour dietary recall assessment tool: 'MyFood24'

Presenter: Janet Cade

Michelle Carter 1); Petra Wark 2); Nisreen Alwan 1); Charlotte Evans 1); Salwa Albar 1); Darren Greenwood 3); Neil Hancock 1); Gary Frost 4); Janet Cade 1)

1) Nutritional Epidemiology Group, School of Food Science \& Nutrition, University of Leeds, UK; 2) Department of Epidemiology and Biostatistics, School of Public Health, Imperial College London, UK; 3) Division of Biostatistics, Leeds Institute of Genetics, Health and Therapeutics, University of Leeds, UK; 4) Nutrition and Dietetic Research Group, Department of Investigative Medicine, Hammersmith Hospital, Imperial College London, UK

Background: Accurate and reliable dietary assessment is essential in order to identify potential dietary determinants of disease and monitor the effectiveness of public health interventions. Traditional paper- 
based methods are often time consuming and costly as they require trained nutritionists and extensive nutrient coding. An online 24-hr dietary recall tool could be a convenient way to assess dietary exposures in a large sample and substantially reduce researcher burden. Whilst existing tools such as DIETDAY and ASA24 have been developed in the USA there is currently no British online 24-h dietary recall tool.

Objective: To develop an online 24-hr recall tool suitable for the British population to assess usual long term dietary intakes.

Methods: The development of the tool has been guided by data collected from focus groups. Ten focus groups have been conducted in three age groups; adolescents $(n=28)$, adults $(n=24)$ and older people $(\mathrm{n}=8)$. A large food and drink database is being constructed by 'mapping' items from the British McCance and Widowson food composition tables to 'back of pack' nutrient data collected from food manufacturers and supermarkets. This will give an extensive branded and generic food and drink database (providing both macronutrient and micronutrient output) which will be incorporated into the tool.

Results: Focus groups described a desire for a system that is quick and easy to use with minimal pop-ups and probes. They feared 'endless list of foods' returned by the database search and required portion size pictures to aid portion estimation. A protocol has been developed for mapping food and drink items from McCance and Widowson tables to 'back of pack' with 38,000 food and drink items mapped to date and a further 35,000 items to be mapped by the end of May 2013. The tool is called 'MyFood24' and a beta version should be completed by June 2013. Usability testing is planned to further refine the beta version. The tool will then be validated against repeated interviewer-administered dietary recalls, using independent nutrient biomarkers including urinary nitrogen, potassium, sucrose and fructose. It will be piloted for feasibility in subgroups of two epidemiological cohorts; the UK Women's Cohort Study, their children and grandchildren; and the UK Clinical Biobank.

Conclusions: The tool should be ready for dissemination and use in the UK by 2015, free of charge to researchers, and with further plans to optimise it to function on smartphones.

\section{0-111}

Dietary protein and subsequent 6-year change in fat and fat free mass

Presenter: Mikkel Z Ankarfeldt

Kristian Gottliebsen 1); Mikkel Z Ankarfeldt 1); Berit L Heitmann 1); Lars Ängquist 1); Arne Astrup 2); Thorkild IA Sørensen 1)

1) Institut of Preventive Medicine, Bispebjerg \& Frederiksberg Hospitaler, Region H; 2) Department of Nutrition, Exercise and Sports (NEXS), Faculty of Science, University of Copenhagen

Background: A high protein intake may increase satiation, thermogenic response and preserve lean mass during weight loss. While a number of trials show that high protein diets promote fat loss and prevent weight regain after weight loss, observational studies suggest that high protein is associated with weight gain. The discrepant findings may relate to underlying changes in fat mass (FM) and fatfree mass (FFM).

Objectives: This study examines associations between protein intake and subsequent 6-year change in weight, FM and FFM.

Methods: In the Danish MONICA-study protein intake was measured by diet history interview (DHI) and from 24-h urinary nitrogen collections. FM and FFM where measured by impedance at baseline and 6 years later. Of 552 participants invited, complete information on body composition, diet intake was measured on 381 participants with
DHI, and 229 with validated 24-h urine collections. Energy-substitution models were used to study association between protein and change in body composition.

Results: When protein intake was estimated from the DHI, FM increased 40 gram/year with every $1 \mathrm{E} \%$ protein substituting fat $(p=0.002)$. When estimating protein intake from 24-h urinary nitrogen FM increased 58 gram/year with every $1 \mathrm{E} \%$ protein substituting other macronutrients $(p<0.001)$, and FFM increased by 20 gram/year $(p=0.002)$.

Conclusions: Increases in protein by substitution for other nutrients were followed by gain in both FM and FFM, with strongest association to FM. These findings are in contrast to expectations based on physiologic and clinical trials. A better understanding of the longterm mechanisms that can explain the discrepancy between observational studies and randomized clinical trials is warranted.

\section{0-112}

Misperceptions of peer drinking behaviour and personal alcohol consumption among students in Central and Eastern Europe

\section{Presenter: Rafael T Mikolajczyk}

Stefanie Maria Helmer 1); Rene Sebena 2); John McAlaney 3); Janina Petkeviciene 4); Robert Urbàn 5); Rafael T Mikolajczyk 6)

1) Department Prevention and Evaluation, Leibniz Institute for Prevention Research and Epidemiology, Bremen, Germany;

2) Department of Psychology, Faculty of Arts, PJ Safarik University in Kosice, Kosice, Slovak Republic; 3) Division of Psychology, University of Bradford, Bradford, UK; 4) Preventive Medicine Department, Institute for Biomedical Research, Kaunas University of Medicine, Kaunas, Lithuania; 5) Department of Personality alth Psychology, Eötvös Loránd University, Budapest, Hungary;

6) Helmholtz Centre for Infection Research, Braunschweig, Germany

Background: Recent research has demonstrated that the perceptions the students hold about the alcohol consumption of their peers are often inaccurate. Students tend to overestimate the number of drinks at a typical occasion as well as the binge drinking of their peers. Furthermore, misperceptions exaggerating the consumption of others have been identified to be a predictor of higher personal alcohol consumption. To date, the vast majority of research in this field was conducted in North-America. In Europe, comparatively less is known about perceptions of peers' alcohol use.

Objectives: The objective of this study was to assess perceptions on peer drinking and to examine associations between overestimations and personal alcohol consumption among university students in Central and Eastern Europe.

Methods: This study examined alcohol use and perceptions about peers' alcohol use among a sample of 2,690 Eastern European university freshman students. Data came from wave I of a web-based international longitudinal study Student Life Cohort in Europe (SLiCE) conducted in universities in Hungary, Lithuania, and Slovak Republic. Students were asked about the frequency of alcohol use and heavy drinking occasions ( 6 and more drinks per occasion) in the last month and about opinions concerning the drinking behaviour of a typical student.

Results: The majority of female and male students have been involved in heavy drinking occasions never or once in the past month ( $55.8 \%$ for male and $86.4 \%$ for female students). At the same time, at least half of the sample in all countries overestimated the frequency of peer heavy drinking occasions (68.4 of male and $66.3 \%$ of female students). Incorrect perceptions existed in all three countries in both, female and male students with no significant differences between genders. Overestimations of peer heavy drinking differed significantly 
between countries. In Slovak Republic $81.3 \%$ of students overestimated the frequency of heavy drinking of their peers, whereas in Lithuania this happened in $70.1 \%$ and in Hungary in $53.4 \%$. An overestimation of peers heavy drinking was significantly correlated with higher own personal heavy drinking.

Coclusion: In agreement with previous US based studies, this study gives evidence that students in Central and Eastern Europe typically overestimate the alcohol use of peers. Given the association between misperceptions of peer alcohol use and individual drinking behaviour, future research should investigate if targeting misperceptions in students would have an impact on unhealthy alcohol consumption.

\section{Socioeconomic and liftestyle factors}

13.08.2013 13:30-15:00

\section{0-113}

Stressful social relations and mortality: is the effect modified by socio-economic status and gender?

Presenter: Rikke Lund

Rikke Lund 1), 2); Charlotte Juul Nilsson 1); Margit Kriegbaum 1); Ulla Christensen 1); Naja Hulvej Rod 1)

1) Section of Social Medicine, Dept. Public Health, University of Copenhagen; 2) Center for Healthy Aging, Faculty of Health Sciences, University of Copenhagen

Background: Few studies have addressed the relationship between stressful aspects of social relations and all cause mortality and they have primarily been focused on the closest confidant.

Objectives: We aim to address the association between conflicts with and worries/demands from partner, children, other family, friends and neighbors and all-cause mortality and to investigate the possible interacting effects with socio-economic status (SES) and gender.

Methods: The 8,708 participants in Danish Longitudinal Study on Work, Unemployment and Health aged 36-52 years responded to a questionnaire in 2000 and were linked to the Danish Cause of Death Registry for all-cause mortality until 31st December 2011.

Results: Those who 'always' (Hazard Ratio $=1.93 ; 95 \%$ CI $1.02-3.65)$ or 'often' $(\mathrm{HR}=1.81 ; 1.23-2.67)$ experienced worries/ demands from their partner had a higher mortality risk after adjustment for age, gender, SES, cohabitation status and prior hospitalization compared to those who did not. Frequent worries/ demands from children were also associated with higher mortality risk $(\mathrm{HR}=1.55 ; 1.08-2.20)$. The experience of conflicts with any type of social relations was associated with higher mortality e.g. conflicts with partner $(\mathrm{HR}=2.19 ; 1.49-3.21)$, friends $(\mathrm{HR}=2.63$; $1.16-5.93)$ or even neighbors $(\mathrm{HR}=3.07 ; 1.49-6.32)$. Further adjustment for depressive symptoms did not change the overall conclusions. There appeared to be an interaction between SES and strain from partner or friends. For example the joint exposure to both low SES and conflicts with partner was associated with a markedly higher mortality risk $(\mathrm{HR}=4.52 ; 2.87-7.12)$ than expected from their individual effects. Interaction between gender and strain from partner, friends or neighbors was also suggested, with men exposed to high levels being in markedly increased risk of premature death.

Conclusion: Stressful social interactions are associated with higher mortality risk across several social roles, and appear to interact with SES and gender.

\section{0-114}

Association of socioeconomic status with allostatic load in the Swiss population-based CoLaus study

Presenter: Silvia Stringhini

Edouard Nicod 1); Silvia Stringhini 1); Pedro Marques-Vidal 1); Fred Paccaud 1); Gerard Waeber 2); Karine Lamiraud 3); Peter Vollenweider 2); Murielle Bochud 1)

1) IUMSP (University Institute of Social and Preventive Medicine), Community Prevention Unit; 2) Department of Medicine, Internal Medicine, Lausanne University Hospital; 3) ESSEC Business School, Paris

Background: Allostatic load reflects cumulative exposure to stressors throughout lifetime and has been associated with cardiovascular disease morbidity and mortality as well as with poorer cognitive and physical functioning. It is hypothesized that people with low socioeconomic status are exposed to higher chronic stress and have therefore greater levels of allostatic load.

Objective: We analyzed the association of two indicators of socioeconomic status (receiving social transfers and education) with chronic stress, as expressed by allostatic load.

Methods: We selected participants aged over 35 years and under the age of retirement from the population-based CoLaus study (Lausanne, Switzerland, 2003-2006). We computed an allostatic load index aggregating cardiovascular, metabolic, dyslipidemic and inflammatory markers.

Results: 3,589 participants $(1,812$ women) were included in the present study, of whom $16 \%$ reported to receive social transfers and $56 \%$ had a low educational attainment. Men receiving social transfers and those with low education were more likely to have higher levels of allostatic load than those not social transfers or having high education, respectively $(\mathrm{OR}=1.93,95 \% \mathrm{CI}=1.45 ; 2.55$ for receiving social transfers and $\mathrm{OR}=3.12,95 \% \mathrm{CI}=2.34 ; 4.17$ for low education). The same patterns were observed among women $(\mathrm{OR}=1.45,95 \% \mathrm{CI}=1.13 ; 1.87$ for social transfers and $\mathrm{OR}=$ $2.88,95 \% \mathrm{CI}=2.14 ; 3.88$ for low education). Associations persisted after controlling for smoking, alcohol consumption, physical activity and marital status.

Conclusions: We found strong and robust associations between socioeconomic status and allostatic load in adult men and women from a Swiss population-based study. The two indicators of socioeconomic status examined, receiving social transfers and having a low educational attainment, independently and cumulatively predicted high allostatic load and dysregulation of several homeostatic systems.

\section{0-115}

Association of lifecourse socioeconomic status with chronic inflammation and type 2 diabetes risk: evidence from the Whitehall II study

Presenter: Silvia Stringhini

Silvia Stringhini 1); David Batty 2); Pascal Bovet 1); Martin Shipley 2); Micahel Marmot 2); Meena Kumari 2); Adam Tabak 2); Mika Kivimaki 2)

1) IUMSP (University Institute of Social and Preventive Medicine), Community Prevention Unit; 2) University College London, Department of Epidemiology and Public Health

Background: Socioeconomic adversity in early life has been hypothesized to 'program' a vulnerable phenotype with exaggerated 
inflammatory responses, so increasing the risk of developing type 2 diabetes in adulthood. For the first time in a study of humans, we examine if the association between lifecourse socioeconomic status and type 2 diabetes incidence is explained by chronic inflammation. Methods and findings: We use data from the British Whitehall II study, a prospective cohort of adults established in 1985. The inflammatory markers C-reactive protein and Interleukin-6 were measured repeatedly and type 2 diabetes incidence (new cases) was monitored over an 18-year follow-up. Our analytical sample consisted of 6387 non-diabetic participants (1,813 women), of whom 731 developed type 2 diabetes over the follow-up. Cumulative exposure to low socioeconomic status from childhood to middle age was associated with a doubling (95\% confidence intervals: 1.5-2.7) of the risk of developing type 2 diabetes in adulthood. Up to one third of this excess risk was attributable to chronically elevated inflammation (95\% confidence intervals 16-62\%).

Conclusions: In the present study, inflammation was an important contributing factor to the link between lifecourse socioeconomic disadvantage and type 2 diabetes. Further work in humans is required to confirm these observational findings.

\section{0-116}

Physical activity and recurrence and mortality among breast cancer patients

\section{Presenter: Martina Schmidt}

Martina Schmidt 1); Jenny Chang-Claude 1); Alina Vrieling 1); Petra Seibold 1); Judith Heinz 2); Nadia Obi 2); Dieter Flesch-Janys 2); Karen Steindorf 1)

\section{1) German Cancer Research Center (DKFZ); 2) University Cancer Center Hamburg (UCCH)}

Background: Primary-preventive effects of physical activity (PA) against breast cancer are well-known. More recently, evidence is emerging that PA may also improve overall survival after breast cancer. However, the effects of PA on cancer recurrence and diseasespecific mortality are little investigated.

Objectives: To evaluate the association of pre-diagnosis PA with breast cancer recurrence, breast cancer-specific mortality as well as deaths due to other causes, and to investigate the amount of PA needed to improve prognosis and survival.

Methods: 3,393 non-metastatic breast cancer patients from a German population-based case-control study, who were diagnosed with invasive breast cancer between 2001 and 2005 and had no previous other tumors, were followed prospectively (MARIEplus study). Patients were 50-74 years of age at diagnosis. Vital status and causes of death were verified through end of 2009. Pre-diagnosis PA was assessed using a detailed interviewer-administered questionnaire from which recreational PA in MET-hours per week was derived. We investigated the associations between PA and recurrence, overall mortality, breast cancer-specific mortality, and mortality due to other causes using multiple Cox proportional hazards models with delayed entry, stratified by age at diagnosis and adjusted for prognostic and potential confounding factors such as TNM status, hormone receptor status, histological grading, BMI and smoking. Dose-response relationships were explored by multiple fractional polynomial analyses. Results: During a median follow-up of 5.6 years, 367 patients died. Overall mortality was significantly inversely associated with prediagnosis recreational PA, but this was mainly attributable to deaths unrelated to breast cancer. Multiple fractional polynomial analyses yielded a non-linear association with markedly increased non-breast cancer mortality for women who did not engage in any cycling or sports in the years before breast cancer diagnosis (hazard ratio HR (none vs. any PA) $=1.71,95 \%$ confidence interval: $(1.16,2.52)$ ). Risks did not decrease further with increasing PA levels. The association with breast cancer-specific mortality was less pronounced with $\mathrm{HR}$ (none vs. any PA) $=1.22(0.91,1.64)$.

Cancer recurrence, in contrast, showed a linear dose-response with increasing PA level, yet, this association was restricted to hormone receptor-negative cases ( $p$-interaction $=0.033$ ) with HR (high vs. no $\mathrm{PA})=0.53(0.24,1.16), p$-trend $=0.0045$.

Conclusions: Although breast cancer mortality has decreased over the past decades, patients with a physically inactive pre-diagnostic lifestyle are at increased risk of premature death due to causes other than their breast cancer. Higher levels of recreational PA were associated with a reduced risk of cancer recurrence among patients with hormone receptor negative tumors.

\section{0-117}

Prospective study of marital status and ischaemic heart disease risk in women

Presenter: Sarah Floud

Sarah Floud 1); Angela Balkwill 1); Benjamin, J. Cairns 1); Jane Green 1); Gillian, K. Reeves 1); Valerie Beral 1)

1) Cancer Epidemiology Unit, University of Oxford, Oxford, UK

Background: Being married is associated with a lower risk of ischaemic heart disease mortality for men, but it is not clear if marriage confers a similar benefit for women. In addition, few studies have examined associations between marital status and ischaemic heart disease incidence and mortality in the same population.

Objectives: Our aim was to examine the relation for women between being married and ischaemic heart disease incidence and mortality in a large cohort of UK women.

Methods: A total of 747,532 women (mean age: 59.8, SD 4.9), without previous heart disease, stroke or cancer, were followed prospectively for an average of 6.1 years for hospital admissions and deaths. We used Cox regression models to calculate relative risks for the first ischaemic heart disease (IHD) event and for IHD deaths comparing married women to unmarried women, adjusting for age, region of residence, socio-economic status, health-related behaviours, social support, depression and self-rated health. We also calculated relative risk of subsequent death in participants whose first IHD event was a hospital admission (mean follow-up 2.7 years).

Results: At baseline, in 2000-2003, $81 \%$ of the women were married or living with a partner. Over follow-up, there were 20,458 first IHD events (19,572 non-fatal and 886 fatal), and among those whose first event was a hospital admission there were 407 deaths subsequently. Married women had lower rates of smoking and physical inactivity, were less deprived, and less likely to rate their health as poor. Married women had a $34 \%$ lower risk of IHD death as a first event than unmarried women $(p<0.001)$, but did not have a significantly reduced risk of a hospital admission for IHD. Among those whose first IHD event was a hospital admission, married women did not have a significantly reduced risk of subsequent mortality.

Conclusions: Being married or living with a partner appears to reduce the risk of death, but not of hospital admission, for IHD in women, which is not explained by health-related behaviours or by socio-economic status.

\section{0-118}

Does the Parkinson's disease caffeine relationship differ by sex and reproductive factors?

Presenter: Naomi Greene 


\section{Naomi Greene 1); Christina Funch Lassen 2); Beate Ritz 1)}

1) UCLA School of Public Health, Department of Epidemiology, Los Angeles, CA, USA; 2) The Research Center, Danish Cancer Society

Background: Epidemiologic studies have found that caffeine is negatively associated with PD in men but not women while one research group suggests the caffeine-PD relationship in women depends on hormone replacement status. Recent work suggests a negative relation between caffeine use and PD in women carrying either of two polymorphisms thought to be associated with lower circulating estrogen levels. This led the authors to propose that caffeine use infers a lower PD risk in conditions of relatively lower levels of estrogen (for example, being male).

Objective: In a large case-control study, examine sex-differences in the caffeine-PD relationship and influences of other reproductive factors on the caffeine-PD association.

Methods: The PASIDA (Parkinson's disease in Denmark) Study has enrolled 1,813 cases of idiopathic PD (1,070 men and 743 women) diagnosed between 1996 and 2009 as well as 1,887 age- and sexmatched controls identified from the general Danish Central Person Registry (1122 men and 765 women). Covariate information was collected in computer-assisted telephone interviews covering an extensive array of topics including reproductive and lifestyle factors. Results: Ever-use of caffeine use was negatively associated with PD in both men and women (men: odds ratio $(\mathrm{OR})=0.41,95 \% \mathrm{CL}$ $0.24,0.70$, women: $\mathrm{OR}=0.55,95 \% \mathrm{CL} 0.34,0.89$ ) with statistically significant $p$-values for trend with increasing numbers of cups of coffee/day ( $p$-values for men and women both $<0.001$ ). In women, among never-users of hormone replacement (HRT) (72\% of the women), the caffeine-PD OR was 0.54 (95\% CL 0.31,0.95) while in women reporting HRT ever-use the OR was 1.06 (95 \% CL 0.38,2.96). In the $8 \%$ of women with menarche at $\leq$ age 11 there was no caffeine-PD association ( $\mathrm{OR}=1.03,95 \% \mathrm{CL} 0.17,6.45)$. Among neverusers of oral contraceptives (47\%), the caffeine-PD association was $0.40(95 \%$ CL 018,0.89) and among ever-users, it was 0.79 (95\% CL $0.41,1.52)$. The trend $p$-values with increasing coffee consumption for ever-users of HRT and those with menarche at $\leq 11$ were non-significant whereas the trend $p$-values for never-users of HRT and those with menarche $>11$ were statistically significant (all $<0.003$ ).

Conclusions: Our results are consistent with the notion that the caffeine-PD association differs across levels of female reproductive factors and support the observation that caffeine appears protective only under conditions of relatively low estrogen.

\section{0-119}

\section{What is important for a 93 year old to reach 100 years of age?}

Presenter: Mikael Thinggaard

Mikael Thinggaard 1); Matt McGue 2); Rune Lindahl-Jacobsen 1); Bernard Jeune 1); Kaare Christensen 3)

1) The Danish Aging Research Center, Institute of Public Health, University of Southern Denmark, Odense C, Denmark; 2) Department of Psychology, University of Minnesota, Minneapolis, MN 55455, USA; 3) The Danish Aging Research Center, Institute of Public Health, University of Southern Denmark, Odense C, Denmark and Department of Clinical Genetics, Odense University Hospital, Odense C, Denmark and Department of Clinical Biochemistry and Pharmacology, Odense University Hospital, Odense C, Denmark

Background: Only a few studies have analyzed what predicts mortality for the oldest old and these studies have used complete case analysis. Hence, the studies have only analyzed a selective group of the oldest old, namely the individuals with the most robust health.
Objectives: We analyzed the association between reaching 100 years of age for people aged 93 with measures of activity of daily living (ADL), physical and cognitive abilities, lifestyle factors, diseases, and the two most promising longevity genes, ApoE and FOXO3.

Methods: We used a population sample from the Danish 1905 Cohort. In 1998 all Danes born in 1905 and still living in Denmark, which comprised 3,600 persons, were invited for the first time to participate in the Danish 1905 Cohort Survey. A total of 2,262 (63\%) participated, $1,814(80.2 \%)$ in person and $448(19.8 \%)$ via a proxyparticipant. We used multiple imputation analyses to perform the analyses, since this made it possible to analyze the total sample of 2,262 persons.

Results: In the univariate analyses having good ADL, high physical and cognitive abilities were important for a 93 years old person to reach 100 years of age. But also a body mass index (BMI) above 22, low depression symptomatology, good self-rated health, frequent social contact, few numbers of drugs, absence of angina pectoris and stroke, and variants of $\mathrm{ApoE}$ and $\mathrm{FOXO} 3$ were associated with increased chance of reaching 100 years of age. However, socioeconomic status, education, smoking and cancer had no association with turning 100 years old. In a multivariable analysis only ADL ( $P$-value $<0.0001)$, physical ability $(P$-value $=0.0128)$, cognitive ability $(P$-value $<0.0001)$, BMI $(P$-value $=0.0007)$, angina pectoris and stroke $(P$-value $=0.0014)$, and ApoE and FOXO3 variants $(P$-value $=0.0024)$ were significantly associated with reaching 100 years of age (area under the receiver operating characteristic curve equals 0.81 in the logistic regression).

Conclusions: Physical condition, cognitive ability, not being too slim, no prior or present occurrence of angina pectoris or stroke, and specific gene variants increased the chance of making it from 93 to 100 years of age. Hence, the chance of reaching 100 years old as a nonagenarian is not a stochastic process but a number of phenotypes which are associated with mortality in the young elderly have lost their importance.

\section{0-120}

Socio-economic inequalities in the quality of life of older Europeans across the life course: does the welfare state matter?

Presenter: Claire Niedzwiedz

Claire Niedzwiedz 1); Srinivasa Katikireddi 2); Jill Pell 1); Richard Mitchell 1)

1) Institute of Health and Wellbeing, University of Glasgow;2) Medical Research Council/Chief Scientist Office Social and Public Health Sciences Unit, Glasgow

Background: Positive dimensions of wellbeing are considered key components of health by the World Health Organisation but remain under-researched. How individuals evaluate their lives, respond to their present environment, and look to the future can be considered an outcome of individual life courses, which are influenced by the welfare state.

Objectives: To investigate the extent of socio-economic inequality in the quality of life of Europeans aged 50-75 years using different indicators of socio-economic position from across the life course and the potential role of the welfare state regime in determining these associations.

Methods: Data from 13 countries were derived from Waves 2 and 3 of the Survey of Health, Ageing and Retirement in Europe $(\mathrm{N}=15,625)$. Slope indices of inequality (SIIs) were calculated for the association between multiple indicators of socio-economic position (including father's occupation, education level and household wealth) and CASP-12, a measure of positive quality of life. Multi- 
level linear regression was used to assess the overall relationship between socio-economic position and quality of life, and interaction terms between socio-economic position and welfare state regime (classified into Southern, Scandinavian, Post-communist and Bismarckian) were included to investigate the influence of the type of welfare state regime on the relationship.

Results: Socio-economic inequalities in quality of life were present in all welfare state regimes and across different dimensions of socioeconomic position from both childhood and adulthood. Effect modification by welfare state regime was apparent. Compared to the Scandinavian welfare state regime where no significant educational inequalities in quality of life were found in men (SII $=0.15,95 \% \mathrm{CI}$ $-0.97,1.27)$ or women $(\mathrm{SII}=0.97,95 \% \mathrm{CI}-0.10,2.05)$, the quality of life gap between the least and most educated was significantly wider in Southern and Post-communist regimes in both genders, and in the Bismarckian regime among men. More proximal measures of socio-economic position were associated with larger SIIs, with wealth displaying the strongest overall association among both men (SII: $3.85,95 \%$ CI 3.45, 4.26) and women (SII $=3.96,95 \% \mathrm{CI}$ $3.56,4.36)$.

Conclusions: Socio-economic inequalities in the quality of life of older Europeans are apparent across a number of dimensions of socioeconomic position in all welfare regimes. Scandinavian and Bismarckian welfare state regimes displayed the narrowest socio-economic inequalities in quality of life. Reducing social inequalities at all stages of the life course, perhaps through more generous welfare state provision, is likely to decrease inequality in the quality of life of older people.

\section{0-121}

Changes of body mass index in relation to mortality: results of a cohort of 42,099 adults

\section{Presenter: Gabriele Nagel}

Jochen Klenk 1), 2); Kilian Rapp 2); Hanno Ulmer 3); Hans Concin 4); Gabriele Nagel 1), 4)

1) Institute of Epidemiology and Medical Biometry, Ulm University, Ulm, Germany; 2) Clinic for Geriatric Rehabilitation, Robert-Bosch Hospital, Stuttgart, Germany; 3) Department of Medical Statistics, Informatics and Health Economics, Medical University, Innsbruck, Austria; 4) Agency for Preventive and Social Medicine, Bregenz, Austria

Background: Obesity is associated with increased all-cause mortality, but little is known about the effect of short- and long-term (BodyMass-Index) BMI change on mortality.

Objectives: The aim of the study was to determine how long-term BMI chance affects mortality.

Methods: Within a population-based prospective cohort of 42,099 Austrian men and women with at least three BMI measurements we investigated the relationship of BMI at baseline and two subsequent BMI change intervals of 5 years each with all-cause mortality using Cox proportional Hazard models.

Results: During median follow-up of 12 years 4,119 deaths were identified. The lowest mortalities were found in persons with normal weight or overweight at baseline and stable BMI over 10 years. Weight gain $(\geq 0.10 \mathrm{~kg} / \mathrm{month} /$ year $)$ during the first 5 years was associated with increased mortality in overweight and obese people. For weight gain during both time intervals mortality risk remained significantly increased only in overweight [1.39 (95\% confidence interval: $1.01 ; 1.92)]$ and obese women $[1.85$ (95\% confidence interval: $1.18 ; 2.89)]$. Weight loss $(<-0.10 \mathrm{~kg} / \mathrm{month} /$ year $)$ increased all-cause mortality in men and women consistently.
BMI change over time by accepted WHO BMI categories showed no increased mortality risk for people who remained in the normal or overweight category for all three measurements. In contrast, HRRs for stable obese men and women were $1.57(95 \%$ CI $1.31 ; 1.87)$ and 1.46 (95\% CI 1.25; 1.71), respectively.

Conclusion: Our findings highlight the importance of weight stability and obesity avoidance in prevention strategy.

\section{Risk factor epidemiology}

13.08.2013 13:30-15:00

\section{0-122}

Metabolic syndrome and hepatocellular carcinoma risk

Presenter: Maurizio Montella

Federica Turati 2); Renato Talamini 3); Claudio Pelucchi 2); Jerry Polesel 3); Silvia Franceschi 4); Anna Crispo 1); Francesco Izzo 1); Paolo Boffetta 5); Carlo La Vecchia 2); Maurizio Montella 1)

1) National Cancer institute Fondazione Pascale Napoli; 2) Mario Negri Institute Milano; 3) CRO Aviano; 4) International Agency for Research on Cancer Lyon; 5) International Prevention Research Institute Lyon

Background: Hepatocellular carcinoma (HCC) has been associated to diabetes and obesity, but a possible association with the metabolic syndrome (MetS) and its potential interaction with hepatitis is open to discussion.

Objectives: Explored the association between MetS and HCC among subgroups of hepatitis-free and affected patients.

Methods: Between 1999 and 2002, a case-control study on HCC was carried out in the province of Pordenone (North-eastern Italy) and in the city of Naples (southern Italy). Cases were subjects younger than 85 years with incident HCC, admitted to teaching and general hospitals in Pordenone and Naples. Odds ratios (ORs) and $95 \%$ confidence intervals $(\mathrm{CI})$ were computed from unconditional logistic regression models. The indicator of MetS was defined according to the criteria agreed in the 2009 joint interim statement, adapted to our data, as the presence of at least three components among the following: (1) abdominal obesity, (2) history of a clinical diagnosis or drug-treated hypercholesterolaemia (as a proxy indicator of increased low-density lipoprotein/reduced HDL cholesterol levels), (3) history of drug-treated hypertension (as an alternate indicator of elevated blood pressure), and (4) history of diabetes. We also considered various components of the MetS separately.

Results: Among the MetS components, diabetes and obesity (i.e., body mass index (BMI) $>30 \mathrm{~kg} \mathrm{~m}^{-2}$ ) were positively associated to HCC risk, with OR, of 4.33 (95\% Cl 1.89-9.86) and 1.97 (95\% C1 1.03-3.79), respectively. The ORs, for the MetS were $4.06(95 \% \mathrm{Cl}$ $1.33-12.38)$ defining obesity as $\mathrm{BMI}>25$, and $1.92(95 \% \mathrm{Cl}$ 0.38-9.76) defining it as BMI $>30$. The risk increased with the number of MetS components, up to an almost four-fold excess risk among subjects with $>2$ MetS factors. Among subjects without chronic infection with hepatitis B and/or C the OR for those with $>2$ MetS components was over six fold elevated. There was no consistent association in subjects with serological evidence of hepatitis B and/or C infection.

Conclusions: Our results are in broad agreement with previous data on the issue, which showed a positive association of MetS with liver cancer. When the single MetS components were analysed separately, 
overweight/obesity and high blood glucose revealed the strongest associations with liver cancer. Of specific interest, we found an association between the number of MetS components and HCC risk only among subjects without markers of chronic infection with $\mathrm{HBV}$ and/or HCV.

\section{O-123}

Occupational prestige and inflammation in postmenopausal women: evidence from the women's health initative

\section{Presenter: Jolene Masters Pedersen}

Jolene Masters Pedersen 1); Rikke Lund 1); Naja Hulvej Rod 1); Igor Burstyn 2); Candyce Kroenke 4); Lorena Garcia 5); AJ DeRoos 2); Yuko You 3); Simin Liu 3); Yvonne Michael 2)

1) University of Copenhagen, Institute of Public Health; 2) Drexel University School of Public Health, Philadelphia, PA 19102; 3) UCLA Department of Epidemiology, Los Angeles, CA 90095; 4) 3Kaiser Permanente Division of Research Oakland, CA 94612; 5) UC Davis Department of Public Health Science Davis, CA 95616

Background: While the link between socioeconomic position and health is well established, the mechanisms are less clear. Biomarkers of inflammation are associated with the development and progression of a host of diseases including atherosclerotic disease, coronary heart disease, stroke, peripheral arterial disease and type 2 diabetes.

Objectives: This study estimated the association between occupational prestige and inflammation in women aged 50-79 years.

Methods: We analyzed data from the controls in a nested case-control study of the Women's Health Initiative. Six markers of inflammation, C-reactive protein (CRP), interleukin-6 (IL-6), tumor necrosis factor (TNF-), soluble intercellular adhesion molecule-1 (sICAM-1), vascular cell adhesion molecule-1 (VCAM-1), and E-selectin were measured in 2,198 randomly selected women free from diabetes and cardiovascular disease. Women reported up to three jobs held longest since age 18, which were coded using the 2010 Standard Occupational Classification (SOC). Occupational prestige was assessed for each SOC code using the Occupational Information Network social status item, a standardized and occupation-specific descriptor ranging from 0 to 100 . Multivariable linear regression was used to test the association of occupational prestige from the longest held job and inflammation adjusting for age, marital status and education.

Results: A ten unit increase in prestige score was associated with decreased inflammation; CRP $(-0.31 ; 95 \%$ confidence interval $(\mathrm{CI})$ $-0.48,-0.13)$, IL-6 (-0.10;-0.23,0.03), TNF- (-29.78;-53.93, $-5.62)$, sICAM-1 (-3.95;-6.82,-1.08), VCAM-1 (-4.10; $-11.65,3.61)$, and E-selectin $(-0.76 ;-1.39,-0.12)$.

Conclusion: Occupational prestige merits further investigation as a plausible mechanism linking socioeconomic position and adverse health outcomes.

\section{0-124}

Increased risk of venous thrombosis after arterial thrombosis: causal or explained by common risk factors?

\section{Presenter: Linda Flinterman}

Linda Flinterman 1), 2); Caroline Lind 3); Marianne Severinsen 4); Suzanne Cannegieter 1); John-Bjarne Hansen 3); Kim Overvad 2)

1) Department of Clinical Epidemiology, Leiden University Medical Center, Leiden, The Netherlands; 2) Department of Public Health,
Aarhus University, Aarhus, Denmark; 3) Department of Clinical Medicine, University of Troms $\varnothing$, Troms $\varnothing$, Norway; 4) Department of Clinical Epidemiology, Clinical Institute, Aarhus University Hospital, Aarhus, Denmark

Background: Venous and arterial thrombosis (VT and AT) are traditionally considered as two different diseases. However, recently associations between the two entities have been described.

Objectives: We aimed to investigate explanations for the increased risk of VT after an AT.

Methods: The study was performed in two large cohorts from Denmark and Norway, i.e. the Diet, Cancer and Health cohort (DCH) and the Troms $\varnothing$ study. The DCH cohort included individuals recruited between 1993 and 1997 aged 50-64 years and without prior cancer at time of recruitment. The Troms $\emptyset$ study is a population-based cohort conducted between 1994 and 1995. Both cohorts were regularly linked to their national registries by means of the civil registration number. Incidence rates of VT were calculated for the overall cohorts and for subjects with AT, starting from the date of the arterial event, to study the overall risk and the effect of time on the incidence. The ratios between the two events were calculated using a time-dependent Cox regression model. To investigate the mechanism we adjusted for the following confounders: sex, age, Body Mass Index, smoking, alcohol intake, hypertension, diabetes, and education level.

Results: Out of 6,200 patients with an arterial thrombosis, 108 developed a venous thrombosis, leading to an overall incidence of VT of 4.0 (CI95 3.3-4.8) per 1,000 person years in this group, compared to an incidence of 1.2 (CI95 1.1-1.3) per 1,000 person years in subjects without AT. This led to an overall 2.7-fold (CI95 2.2-3.3) higher risk of VT in patients with AT. When we studied the timetrend between the two events we found an incidence rate for VT of 39.8 per 1,000 person years (CI95 24.7-64.0) in the first 2 months after arterial thrombosis compared with an incidence of 1.1 per 1,000 person years (CI95 1.0-1.2) in those without AT. This incidence rate decreased over time to 1.9 per 1,000 person years. After adjustment the overall hazard ratio for common risk factors, attenuated from 2.7 to 1.5 (CI95 1.2-1.9), indicating that common risk factors partly explained the increased risk. Patients in whom the VT occurred within 2 months after AT were less likely to have common risk factors for arterial and venous thrombosis than those who had a VT more than 2 months after an AT.

Conclusions: In the first 2 months after an arterial thrombosis, the risk of a venous event was increased. This risk decreased over time but remained higher. The long-term association was partly explained by common risk factors. However, in the high-risk period shortly after AT, the role of common risk factors seemed to be limited.

\section{0-125}

Does bereavement cause stress? A two year follow-up study of salivary cortisol level

Presenter: Henrik A. Kolstad

Henrik A. Kolstad 1); Sigurd Mikkelsen 2); Matias B. Grynderup 1); Jens Peter Bonde 2); Reiner Rugulies 3); Linda Kærlev 4); Henriette Buttenschøn 5); Jane F. Thomsen 2); Johan H. Andersen 6); Åse Marie Hansen 7)

1) Department of Occupational Medicine, Danish Ramazzini Centre, Aarhus University Hospital; 2) Department of Occupational and Environmental Medicine, Bispebjerg University Hospital; 3) National Research Centre for the Working Environment; 4) Center for National Clinical Databases South, Odense University Hospital; 5) Centre for Psychiatric Research, Aarhus University Hospital; 6) Department of 
Occupational Medicine, Danish Ramazzini Centre, Regional Hospital Herning; 7) Department of Public Health, University of Copenhagen

Background: The death of a close relative or friend causes grief and distress in almost everyone and bereavement has consistently been associated with increased mortality and morbidity. Sustained stressactivation of the hypothalamus-pituitary-adrenal (HPA) axis has been hypothesised as the mechanism responsible for this mind-body transition.

Objectives: We examined if cortisol level, a key feature of HPA axis activity, was increased following the grief-associated death of a close relative or friend.

Methods: We analysed 2,725 public employees from Aarhus, Denmark, who participated at baseline in 2007 and again at follow up in 2009 in the PRISME study of occupational risk factors of mental disorders. At both rounds, they provided 2,135 morning salivary samples about half-an-hour after awakening and 2,618 evening samples about $8 \mathrm{pm}$ (a total of 9,506 salivary samples). They also reported whether a close relative or friend recently had died and if the death was associated with grief. The salivary samples were analysed for cortisol concentration with competitive radioimmunoassay. We analysed the cross-sectional relation between bereavement status (bereavement with grief, bereavement without grief, and no bereavement) and cortisol level as well as the longitudinal change in cortisol level by change in bereavement status in regression models that accounted for the repeated measurements.

Results: During the last 6 months, $20 \%$ of the participants (11 percent at baseline and 11 percent at follow up) reported bereavement with grief. Ten percent of the participants (748 salivary samples) changed status from no bereavement at baseline to bereavement with grief at follow up. For them we observed a $5 \%$ increase in cortisol level when analysed longitudinally, but this finding was not of statistical significance (95\% CI -5 to $14 \%)$. Contrary to this, we observed a $5 \%$ lower cortisol level $(95 \% \mathrm{CI}$ -10 to $-1 \%)$ for bereavement with grief $(1,011$ salivary samples) compared with no bereavement (7,512 salivary samples), when analysed cross-sectionally. Sex, age, sampling time, awakening time, study round, smoking, BMI, or change in these factors from baseline to follow up had little impact on the results.

Conclusion: These data do not provide consistent evidence that griefassociated bereavement is associated with sustained stress activation of the HPA axis.

\section{0-126}

Five-year prognosis in cardiothoracic surgical patients with acute kidney injury: a cohort study

Presenter: Malene Karslund Hansen

Malene Kærslund Hansen 1); Henrik Gammelager 1); Martin Mikkelsen 2); Vibeke Hjortdal 3); Bradley Layton 4); Søren Johnsen 1); Christian Christiansen 1)

1) Department of Clinical Epidemiology, Aarhus University Hospital, Denmark; 2) Department of Dermato- and Venerolgy, Aarhus University Hospital, Denmark; 3) Department of Cardiothoracic and Vascular Surgery, Aarhus University Hospital, Denmark; 4) Department of Epidemiology, University of North Carolina at Chapel Hill, USA

Objectives: Acute kidney injury (AKI) is associated with early mortality after cardiothoracic surgery, but its prognostic impact on long-term clinical outcomes remain controversial. We examined the 5 -year risk of myocardial infarction (MI), stroke and death after elective cardiothoracic surgery complicated by AKI.
Methods: We conducted a cohort study among 1,030 adult elective cardiothoracic surgical patients between April 1, 2005 and October 8, 2007 without severe chronic kidney disease (serum creatinine $>200 \mu \mathrm{mol} / \mathrm{L}$ ) and/or previous heart or renal transplant surgery. AKI was defined by the Acute Kidney Injury Network (AKIN) criteria as more than $50 \%$ increase in serum creatinine from baseline level, acute rise of $\geq 26.5 \mu \mathrm{mol} / \mathrm{L}$ within $48 \mathrm{~h}$, and/or initiation of renal replacement therapy within 4 days after surgery. We followed patients from fifth post-operative day until MI, stroke or death for up to 5 years. Data were obtained through linkage to the Danish National Registry of Patients and the Danish Civil Registration System. Absolute risks were computed using a cumulative incidence method and hazard ratios using a Cox proportional hazards regression model adjusted for propensity score, besides a propensity score matched analyses.

Results: A total of 287 (27.9 \%) of the 1,030 patients developed AKI. AKI patients were older, had higher level of comorbidity and higher baseline serum creatinine values. Five-year risk of MI was $5.0 \%$ (95\% CI 2.9-8.1) among AKI patients and 3.3\% (95\% CI 2.1-4.8) among non-AKI patients, of stroke; $5.0 \%$ (95\% CI 2.8-7.9) among AKI patients and $4.2 \%$ (95\% CI 2.9-5.8) among non-AKI patients. Adjusted HRs were 1.5 (95\% CI 0.7-3.2) and 0.9 (95\% CI 0.5-1.8), respectively. Five-year risk of death was $26.5 \%$ (95\% CI 21.2-32.0) among AKI patients and $12.1 \%$ (95\% CI 10.0-14.7) among nonAKI patients. Adjusted HR was 1.6 (95\% CI 1.1-2.2).

Conclusion: AKI following elective cardiothoracic surgery was related to long-term mortality. The risk increased with increasing AKI stage. In contrast, no clear association was found between AKI and the risk of MI and stroke.

\section{0-127}

Does disturbed sleep mediate the longitudinal relationship between work stress and depression?

Presenter: Holendro Singh Chungkham

Linda Magnusson Hanson 1); Holendro Singh Chungkham 1); Torbjörn Ảkerstedt 1); Hugo Westerlund 1); Mika Kivimaki 2)

1) Stress Research Institute, Stockholm University; 2) Department of Epidemiology \& Public Health, University College London, UK

Background: Previous research has established that there is a close connection between stress and sleep and we have observed that the psychological demands at work component of the demand-controlsupport model is prospectively linked to sleep problems, although some reciprocal patterns were also indicated. Sleep also seems to play a critical role in the development of depression. However, the role of sleep problems in the pathway from work stress to long term development of depression is not clearly elucidated.

Objectives: To investigate whether sleep disturbances is a mediator in the longitudinal relationship between work stress (measured by psychological demands) and depressive symptoms.

Methods: Analyses were based on questionnaire data from 2,017 working participants of the Swedish Longitudinal Occupational Survey of Health (SLOSH) 2006, 2008, 2010 and 2012 a nationally representative study of work life, social situation and health. The three factors of interest were work stress measured by psychological demands (4 items) based on the Demand Control Questionnaire, sleep disturbances (4 items) based on the Karolinska Sleep Questionnaire and depressive symptoms assessed by a brief subscale (6 items) from the Hopkins Symptom Checklist. We applied autoregressive longitudinal mediation models using structural equation modelling. The significance of the mediated effects was tested by calculating confidence intervals (CIs) using Monte Carlo simulation. 
Results: The longitudinal autoregressive models supported a partial mediating role of sleep disturbances in the pathway from psychological demands to depressive symptoms. A basic model showed a total indirect effect of 0.089 (CI $0.060 ; 0.120$ ) for psychological demands which corresponds to $27 \%$ of the total effect. Further models were also fitted with additional paths justified theoretically including wave-skipping autoregressive, cross-lagged, and additional direct paths, which showed more or less similar indirect effects. The modified model showed that $37 \%$ of the total effect was mediated by sleep disturbances. The fitted models also showed significant timespecific indirect effects in the path of demands in 2006 to depression in 2012). However, generally very small cross-lagged relationships over time were evident.

Conclusions: Our findings support a partial mediating role of sleep disturbances in the relationship between work stress, as measured by psychological demands at work, and depressive symptoms. This indicates that stress at work might cause an increase in depressive symptoms in part by increasing sleep disturbances, which was not likely explained by reverse causation. Measures to improve sleep may thus help to prevent depression among people with work stress.

\section{O-128}

Does full-shift occupational noise exposure have acute or sustained effects on blood pressure? A 24-hour observational study of industrial workers

Presenter: Zara Ann Stokholm

Zara Ann Stokholm 1); Kent Lodberg Christensen 2); Thomas Whinter Frederiksen 1); Jesper Meedom Vestergaard 1); Åse Marie Hansen 3); Jens Peter Bonde 4); Jesper Kristiansen 5); Søren Peter Luund 5); Henriette Buttenschön 6); Henrik Albert Kolstad 1)

1) Department of Occupational Medicine, Danish Ramazzini Centre, Aarhus University Hospital; 2) Department of Internal Medicine and Cardiology A, Aarhus University Hospital; 3) Faculty of Health, University of Copenhagen; 4) Department of Occupational and Environmental Medicine, Bispebjerg Hospital; 5) National Research Centre for the Working Environment, Copenhagen; 6) Institute for Clinical Medicine, Aarhus

Background: Sudden high-level noise exposure is followed by an acute increase in blood pressure. Long-term environmental noise exposure has repeatedly been related to increased risk of cardiovascular disease at exposure levels as low as $35 \mathrm{~dB}(\mathrm{~A})$. Occupational exposure levels are orders of magnitude the environmental levels. Objectives: We examined if blood pressure was increased during and subsequent to occupational noise exposure in blue-collar workers.

Methods: We analysed 390 blue-collar industrial workers selected as a random sample of 10 industrial trades in the former Aarhus County, Denmark between 2009 and 2010. Noise exposure levels were recorded every $5 \mathrm{~s}$ by personal dosimeters for $24 \mathrm{~h}$ and we calculated the LAeq value for work hours. For 242 workers who kept a hearing protection devices (HPD) diary, we subtracted $10 \mathrm{~dB}$ from every noise recording obtained during HPD use and estimated the LAEq value at the ear. Ambulatory blood pressure was measured every $20 \mathrm{~min}$ for $24 \mathrm{~h}$ and mean values for work, leisure and night-time were calculated.

Results: Full-shift occupational noise exposure levels ranged between 59 and $97 \mathrm{~dB}(\mathrm{~A})$. Preliminary results of linear regression models that adjusted for sex, age, income, body mass index, alcohol, tobacco, salt intake, family history of hypertension, suggest no statistically significant relation between occupational noise exposure level and blood pressure levels during work, leisure or night time. This was neither the case in analyses taking account of HPD use.

Conclusion: These preliminary results suggest that full-shift 59-97 $\mathrm{dB}(\mathrm{A})$ noise exposure levels had neither an acute nor a sustained effect on blood pressure. This does not corroborate that median-level occupational or low-level environmental noise exposure affects health by increasing blood pressure.

\section{0-129}

Pneumonia and subsequent risk of cancer: a Danish nationwide cohort study

Presenter: Kirstine Kobberøe Søgaard

Kirstine Kobberøe Søgaard 1); Dóra Körmendiné Farkas 1); Reimar Wernich Thomsen 1); Henrik Toft Sørensen 1)

1) Department of Clinical Epidemiology, Aarhus University Hospital, Denmark

Background: Pneumonia is a very frequent cause of hospitalization and may precede undiagnosed occult cancer. The previous literature on pneumonia and subsequent cancer risk is limited and conflicting regarding this association.

Objectives: We assessed the risk of a cancer diagnosis following first-time hospitalization with pneumonia. Methods: We conducted a nationwide population-based cohort study by linking Danish medical registries during 1995-2010. We quantified the excess risk of various cancers in hospitalized pneumonia patients versus expected national incidences, using relative (standardized incidence ratios, SIRs) and absolute (excess absolute risk per 10,000 person-years at risk, EAR) risk calculations.

Results: We observed a total of 31,352 cancers versus 19,196 expected among 363,552 patients hospitalized for first-time pneumonia (mean follow-up of 4.7 years). Within the first 6 months after pneumonia, the SIR of any cancer was highly elevated at $4.79(95 \%$ CI 4.70-4.89), mainly due to a ninefold increased risk of tobaccorelated and immune-related cancers, including a 17 -fold increased risk of airway and respiratory system cancers. Between 6 and 12 months of follow-up the cancer SIR remained increased at 1.41 (95\% CI 1.35-1.46). With more than 1 year of follow up the SIR was 1.22 (95\% CI 1.21-1.24). The risk of lung cancer was markedly increased; the SIR was 18.07 (95\% CI 17.53-18.62) within 6 months, 2.60 (95\% CI 2.38-2.83) for 6-12 months, and 1.96 (1.89-2.03) after the first year, respectively. For children and young adults, the SIR for the first 6 months after pneumonia was eightfold increased, mainly due to a highly increased risk of hematological cancers.

Conclusions: Hospital presentation with pneumonia is a substantial clinical marker of prevalent cancer, particularly lung cancer, but also for other tobacco-related, immune-related and hematological cancers. In addition, pneumonia predicts increased long-term cancer risk.

\section{0-130}

Implication of multiple risk factors on the risk of ischemic stroke in patients with and without atrial fibrillation

Presenter: Christine Benn Christiansen

Christine Benn Christiansen 1); Gunnar Gislason 1); Christian Torp-Pedersen 2); Morten Lamberts 1); Soeren Lund Kristensen 1); Jonas Bjerring Olesen 1) 
1) Gentofte University Hospital, Department of Cardiology; 2) Aalborg University Hospital, Institute of Health, Science and Technology

Background: The majority of ischemic strokes occur in patients without atrial fibrillation.

Objectives: We aimed to identify patients without atrial fibrillation with high risk of ischemic stroke based on multiple risk factors, secondly we compared stroke risk in patients with and without atrial fibrillation.

Methods: From Danish nationwide registries we retrieved information about discharge diagnoses and redeemed prescriptions 2000-2010. All Danish citizens were included in the analyses and Poisson regression was used for identifying factors associated with ischemic stroke, and stroke rates in relation to the number of present risk factors were calculated.

Results: We included 4,198,119 persons without prior stroke or transient ischemic attack between 18 and 90 years of age. Of these $161,651(3.85 \%)$ persons had atrial fibrillation. The following risk factors for stroke in patients without atrial fibrillation were significant and thus included in the additive model: Heart failure, chronic systemic inflammation, hypertension, prior myocardial infarction, renal disease, diabetes, carotid stenosis, retinal vascular occlusion, peripheral artery disease, prior venous thromboembolism, migraine, epilepsy and excessive alcohol consumption. Patients with 0 risk factors and no atrial fibrillation had a stroke rate of $0.32(95 \%$ confidence interval $0.31-0.32$ ) per 100 person-years versus 2.55 (2.47-2.64) in patients with atrial fibrillation. With 1-2 risk factors present the stroke rate was 1.77 (1.76-1.78) (no atrial fibrillation) and 5.69 (5.61-5.78) (with atrial fibrillation). For patients with 3-4 risk factors corresponding stroke rates were 4.88 (4.80-4.96) versus 6.96 (6.79-7.14) and finally in the presence of 5 or more risk factors stroke rates were $7.27(6.82-7.74)$ versus $8.00(7.34-8.71)$ per 100 personyears for patients without and with atrial fibrillation, respectively. Conclusion: Patients with multiple risk factors have a high risk of stroke regardless of the presence of atrial fibrillation. Further knowledge on implication of multiple risk factors in patients without atrial fibrillation is warranted and might influence future strategies in stroke prevention.

\section{Reproductive epidemiology}

\subsubsection{2:30-13:45}

\section{O-131}

Caesarean delivery and subsequent birth interval, ectopic pregnancy, miscarriage or stillbirth-a Danish register-based study

Presenter: Sinead M O Neill

Sinead M O Neill 1); Esben Agerbo 2); Louise c Kenny 3); Tine B Henriksen 4); Patricia M Kearney 5); Richard A Greene 1); Preben Bo Mortensen 2); Ali S Khashan 3)

1) National Perinatal Epidemiology Centre, University College Cork, Ireland; 2) National Centre for Register-based Research, Arrhus University, Denmark; 3) Anu Research Centre, University College Cork, Ireland; 4) Perinatal Epidemiological Research Unit, Aarhus University Hospital, Denmark; 5) Department of Epidemiology \& Public Health, University College Cork, Ireland

Background: Despite high Caesarean delivery rates worldwide, limited data are available on the effect of Caesarean delivery on subsequent fertility.
Aim: To estimate the time to next birth and the risk of miscarriage, ectopic pregnancy and stillbirth in women with a prior Caesarean delivery compared to women with a prior vaginal delivery.

Methods: Using Danish registry data (Medical Birth Register, National Hospital Register), we identified a cohort of women giving birth between 1982 and $2010(\mathrm{n}=833,162)$. The cohort was followed from the index birth until the next birth or censoring by death, emigration or study end (31/12/2010). Women with an index Caesarean were compared to women with an index vaginal birth using Cox proportional hazards regression models stratified by mode of delivery (spontaneous vaginal delivery, operative vaginal delivery, elective Caesarean section, emergency Caesarean section, and maternally-requested Caesarean section).

Results: Women with an index Caesarean section were less likely to have a subsequent delivery and had an increased time to next birth [Hazard Ratio (HR) 0.83, $95 \%$ CI 0.82, 0.84]. Sub-group analyses by smoking status, history of access to fertility services, maternal BMI, preterm birth \& low birth weight did not modify the results. Analyses for ectopic pregnancy [HR 1.02, $95 \%$ CI $0.95,1.09$ ] and miscarriage [HR 0.92, $95 \%$ CI 0.90, 0.95] showed no increased risk among women with a prior Caesarean section delivery, however a significantly increased risk for subsequent stillbirth was reported [HR 1.16, $95 \%$ CI 1.04, 1.28].

Conclusion: Prior Caesarean delivery, particularly elective \& maternally requested Caesareans were associated with an increased time to next birth. An increased hazard ratio of stillbirth in subsequent deliveries but not ectopic pregnancy or miscarriage among women with an index Caesarean was also found.

\section{0-132}

Effect of interpregancy interval on the subsequent perinatal outcomes in Northern Tanzania: a registry-based study

\section{Presenter: Michael Johnson Mahande}

Michael Johnson Mahande 1); Anne Kjersti Daltveit 2); Rachel Manongi 1); Blandina Theophil Mmbaga 3); Gileard Masenga 5); Oystein Aziansen Haaland 4); Joseph Obure 5); Rolv Terje Lie 2)

1) Kilimanjaro Christian Medical College, Moshi, Tanzania; 2) Department of Public Health and Primary Health Care, University of Bergen, Norway; 3) Centre for International Health, University of Bergen, Norway; 4) Norwegian Institute of Public Health, Norway; 5) Department of Obstetrics and Gynaecology, Kilimanjaro Christian Medical Centre, Moshi

Background: Both short and long interpregancy intervals have been associated with increased risk of adverse pregnancy outcomes. There is limited information about effect of interpregnancy interval on the subsequent risk of perinatal outcomes in Tanzania.

Objectives: We aimed to assess the effect of interpregancy interval on subsequent adverse perinatal outcomes among women who had at least two consecutive singleton pregnancies.

Methods: This was a prospective cohort study using maternallylinked data from Kilimanjaro Christian Medical Centre (KCMC) birth registry. A total of 17,030 births from women who had at least two consecutive singleton births at KCMC from 2000 to 2010 were studied. A unique maternal hospital identification number was used to link mothers with their respective siblings in subsequent births. All women with multiple gestations, referals from rural areas for various medical reasons and unmatched pairs according to criteria were excluded. Perinatal outcomes included preterm birth, low birthweight and perinatal death. We used log-binomial regression models to determine the association between interpregnancy interval and adverse perinatal outcomes, while controlling for confounders. 
Results: Compared to the reference group (intervals of 2-3 years), short interpregnancy intervals ( $<2$ years) was associated with an increased risks of preterm birth 1.5 (95\% CI 1.3-1.7); low birth weight $1.6(95 \% \mathrm{CI} 1.3-1.7)$ and perinatal death, $1.6(95 \% \mathrm{CI}$ 1.2-1.9) after controlling for confounding factors in the first pregnancy. Consequently, long interpregancy interval ( $\geq 7$ years) was associated with an increased risks of preterm birth, 1.1 (95\% CI $1.0-1.2$ ) and low birth weight, 1.1 (95\% CI 1.0-1.2), while the risk of perinatal death declined overtime.

Conclusions: Short and long interpregnancy intervals were independently associated adverse perinatal outcomes in the subsequent pregnancy. Health care providers should identify high risk women and provide counselling about the effect of interpregancy intervals on next pregnancy to improve fetal outcomes.

\section{O-133}

Maternal thyroid disease and risk of seizure in the child: a Danish nationwide cohort study

Presenter: Stine Linding Andersen

Stine Linding Andersen 1); Peter Laurberg 1); Chun Sen Wu 2); Jørn Olsen 2)

1) Department of Endocrinology, Aalborg University Hospital, Aalborg, Denmark; 2) Section for Epidemiology, Department of Public Health, Aarhus University, Aarhus, Denmark

Background: Thyroid hormones are essential for fetal brain development and required for normal myelination, the regulation of cell migration in the cerebral cortex, hippocampus and cerebellum, the differentiation of neurons, oligodendrocytes, astrocytes and microglia and synaptogenesis. Thyroid disorders are common in women of reproductive age, and several studies have shown that maternal thyroid disease affects child neurocognitive development. Some types of seizures may also depend upon early exposure of the central nervous system, and we hypothesized that maternal thyroid disease could increase the risk of seizure in the child.

Objectives: To study the association between maternal hyper- and hypothyroidism diagnosed before or after birth of the child and the risk of neonatal seizure, febrile seizure and epilepsy in the child.

Methods: In a population-based cohort study using Danish nationwide registers we identified all singleton live births in Denmark between January 1, 1978 and November 30, 2006. Information on maternal diagnosis of hyper- or hypothyroidism and neonatal seizure, febrile seizure and epilepsy in the child was obtained from the Danish National Hospital Register. Cox proportional hazards model was used to estimate adjusted hazard ratio (HR) with $95 \%$ confidence interval $(95 \% \mathrm{CI})$ for the risk of neonatal seizure, febrile seizure and epilepsy in the child.

Results: Maternal diagnosis of thyroid dysfunction before or after birth of child was registered in 34,582 $(2.0 \%)$ of the singleton births $(\mathrm{n}=1,699,693)$. Maternal hyperthyroidism (adjusted HR $1.22(95 \%$ CI 1.10-1.35)) and hypothyroidism (1.21 (1.04-1.41)) first time diagnosed after birth of child were associated with increased risk of epilepsy in the child. Moreover, hypothyroidism after birth of child was associated with increased risk of neonatal seizure (1.83 (1.27-2.65)) and febrile seizure (1.29 (1.16-1.43)). No association with maternal thyroid disease diagnosed prior to birth of the child or with paternal thyroid disease was observed.

Conclusions: Both maternal hyper- and hypothyroidism first time diagnosed in a Danish hospital after birth of child were associated with increased risk of epilepsy in the child, and maternal hypothyroidism with an increased risk of neonatal and febrile seizure. We are aware of immortal time bias, but find it unlikely to explain our findings, and lack of an association with paternal thyroid disease contradicts a genetic component to some extent. Undiagnosed subclinical thyroid disease might have been present during the pregnancy in mothers diagnosed after birth of child, and this untreated condition may present a neurotoxic risk.

\section{0-134}

Impact of parental death on school performance: a national register-based cohort study

Presenter: Lisa Olsson

\section{Lisa Olsson 1); Mikael Rostila 1); Anders Hjern 1), 2)}

1) Centre for Health Equity Studies, Stockholm University/Karolinska Institutet, Stockholm, Sweden; 2) Clinical Epidemiology, Department of Medicine, Karolinska Institutet, Stockholm, Sweden

Background: The death of a parent during childhood constitutes a presumably traumatic life event that may affect the child in different ways, potentially including an impact on school performance. Primary school performance may predict educational achievement and adult socioeconomic position, and has been associated with different adolescent and adult health behaviors and outcomes, such as alcohol and drug abuse and mental health problems. Poor school performance may thus have implications over the individual's entire life course. Objective: To investigate the impact of parental death during childhood on school performance in the ninth and final compulsory school year (at age 15-16), taking into account a range of potentially confounding factors, including the socioeconomic status of the childhood household and adverse childhood experiences (indicated by parental alcohol or drug abuse, mental health problems and criminality).

Methods: This study was performed using data from Swedish national registers. The study population consisted of 690,129 individuals born in Sweden in 1973-1981. School performance was analysed as (1) mean grade points, (2) low grades (i.e. mean grade point $<-1 \mathrm{SD}$ ), and (3) school failure (i.e. having graduated without complete grades). Data were analysed using linear and logistic regression models.

Results: The proportion with low grades among children who had experienced the death of a parent was $26.4 \%$, compared to $16.7 \%$ among those who had not lost a parent (reference group). Corresponding numbers for school failure were 6.3 versus $3.5 \%$. Parental death was associated with lower mean grades $(-0.21$ [95\% CI $0.21,-0.19]$ ) in linear models, although the associations were weakened after inclusion of household socioeconomic status $(-0.15$ $[-0.16,-0.14])$, and indicators of adverse childhood experiences ($0.10[-0.11,-0.09])$ in the models. The fully adjusted effect of parental death from external causes was similar to the unadjusted effect of parental death from natural causes. In fully adjusted models, OR of low grades was $1.41(1.34-1.48)$ and OR of school failure was 1.33 (1.25-1.41) among children who had lost a parent. Similar results were seen for boys and girls and no differences were seen regarding the child's age at the time of the death.

Conclusions: Parental death during childhood was associated with lower mean grades and school failure in primary school. Much of this effect for parental deaths by external causes was associated with other adverse childhood exposures.

\section{O-135}

Topical pharmacological treatment of hemorrhoids during pregnancy and congenital malformations: a nation-wide cohort study

Presenter: Espen Jimenez-Solem 
Espen Jimenez-Solem 1); Jon Traerup Andersen 1); Kasper Meidahl Petersen 1); Christian Torp-Pedersen 2); Henrik Enghusen Poulsen 1)

1) Dept. Clinical Pharmacology, Bispebjerg Hospital, Copenhagen, Denmark; 2) Dept. Cardiology, Gentofte Hospital, Copenhagen, Denmark

Background: Hemorrhoids are common during pregnancy. In spite of this, safety of their pharmacological treatment has not been assessed. Objectives: The aim of this study is to determine the association between exposure to antihemorrhoidal agents during the first trimester and congenital malformations.

Methods: We identified all pregnancies resulting in a live birth between 1997 and 2009 through the Danish Medical Birth Registry. Redeemed prescriptions were identified through the Danish Register of Medicinal Product Statistics, and diagnoses of major congenital malformations through the Danish National Hospital Register. The risk of congenital malformations was calculated using multivariable logistic regression models adjusted for potential confounding variables.

Results: We identified 844,194 pregnancies; of which 7,210 (0.85\%) redeemed a prescription for an antihemorrhoidal agent during the first trimester. All redeemed drugs contained corticosteroids; either hydrocortisone $(n=4,553)$ or fluocortolone $(n=2,433)$. The risk of major congenital malformations for any antihemorrhoidal agent was; adjusted OR 1.03 (95\% CI 0.91-1.17). We found no elevated risk of specific major malformations. Stratifying analyses for different corticosteroids gave no elevated risk of major congenital malformations. Conclusions: We found no association between redeeming a prescription for an antihemorrhoidal agent during the first trimester and congenital malformations. To our knowledge, this is the first study to analyze this relation.

\section{O-136}

Prenatal exposure to bereavement and type-2 diabetes: a Danish longitudinal population based study

Presenter: Jasveer Virk

Jasveer Virk 1); Jiong Li 2); Mogens Vestergaard 3); Carsten Obel 4); Jette Kolding Kristensen 5); Jørn Olsen 6)

1) UCLA; 2) Aarhus University; 3) Aarhus University; 4) Aarhus University; 5) Aarhus University; 6) Aarhus University

Background: The etiology of type-2 diabetes is only partly known, and a possible role of prenatal stress in programming offspring for insulin resistance has been suggested by animal models. Previously, we found an association between prenatal stress and type-1 diabetes. Here we examine the association between prenatal exposure to maternal bereavement during preconception and pregnancy and development of type- 2 diabetes in the off-spring.

Methods: We utilized data from the Danish Civil Registration System to identify singleton births in Denmark born January 1st 1979 through December 31st 2008 ( $N=1,878,246)$, and linked them to their parents, grandparents, and siblings. We categorized children as exposed to bereavement during prenatal life if their mothers lost an elder child, husband or parent during the period from 1 year before conception to the child's birth. We identified 45,302 children exposed to maternal bereavement; the remaining children were included in the unexposed cohort. The outcome of interest was diagnosis of type-2 diabetes. We estimated incidence rate ratios (IRRs) from birth using log-linear poisson regression models and used person-years as the offset variable. All models were adjusted for maternal residence, income, education, marital status, sibling order, calendar year, sex, and parents' history of diabetes at the time of pregnancy.
Results: We found children exposed to bereavement during their prenatal life were more likely to have a type- 2 diabetes diagnosis later in life (aIRR: 1.31, 1.01-1.69). These findings were most pronounced when bereavement was caused by death of an elder child (aIRR: 1.51, 0.94-2.44). Results also indicated the second trimester of pregnancy to be the most sensitive period of bereavement exposure (aIRR:2.08, 1.15-3.76).

Conclusions: Our data suggests that fetal exposure to maternal bereavement during preconception and the prenatal period may increase the risk for developing type- 2 diabetes in childhood and young adulthood.

\section{0-137}

Socio-demographic characteristics of women sustaining injuries during pregnancy: a study from the Danish national birth cohort

Presenter: Jasveer Virk

\section{Jasveer Virk 1); Paul Hsu 1); Jørn Olsen 2)}

1) UCLA; 2) Aarhus University

Objectives: To describe adverse birth outcomes associated with hospital treated injuries that took place among women in the Danish National Birth Cohort (DNBC).

Design: Longitudinal Cohort Study

Setting: Denmark

Participants: 90,452 women and their off-spring selected from the DNBC

Primary and secondary outcome measures: To determine if injured women were more likely to deliver an infant preterm, with low birth weight, stillborn or have a spontaneous abortion we estimated hazard ratios. Odds ratios were generated to assess APGAR scores and infants born small for gestational age. Models were adjusted for maternal smoking and drinking during pregnancy, household socioeconomic status, eclampsia/pre-eclampsia or gestational diabetes status during pregnancy and maternal age at birth; estimates for preterm birth were also adjusted for prior history of preterm birth.

Results: In our cohort of 90,452 pregnant women, 3,561 (3.9\%) received medical treatment for an injury during pregnancy. Injured pregnant women were more likely to deliver infants that were stillborn or have pregnancies terminated by spontaneous abortion. We did not detect an adverse effect between injuries sustained during pregnancy and delivery of preterm, low birth weight or small for gestational age infants, or infants with an APGAR score of less than 7 .

Conclusion: Our study shows that injuries occurring among women from an unselected population may not have an adverse effect on birth weight, gestational age, APGAR score, or small for gestational age status but may adversely affect the risk of stillbirth and spontaneous abortions in some situations.

\section{Registerbased epidemiology}

14.08.2013 12:30-13:45

\section{0-138}

Mental disorders increase the risk of accidental death: a Swedish nationwide cohort study

Presenter: Casey Crump 
Casey Crump 1); Kristina Sundquist 2); Marilyn Winkleby 3); Jan Sundquist 2)

1) Department of Medicine, Stanford University, Stanford, California, USA; 2) Center for Primary Health Care Research, Lund University, Malmö, Sweden; 3) Stanford Prevention Research Center, Stanford University, Stanford, California, USA

Background: Mental disorders are well-known risk factors for suicide, but little is known about their association with accidental death, which is substantially more common. People with mental disorders may be at increased risk of accidental death for multiple reasons, including common psychiatric symptoms, medication side effects, comorbid substance use, or increased risk-taking behavior. Previous studies of this issue have been limited by the use of only hospitalized psychiatric cases or insufficient sample sizes. A more comprehensive understanding of the link between mental disorders and accidental death is needed to enable better risk management.

Objectives: To examine the associations between mental disorders and accidental death in the largest and most comprehensive study to date.

Methods: We conducted a national cohort study of all Swedish adults $(\mathrm{N}=6,908,922)$ who were followed up for 8 years (2001-2008) for mental disorders identified using all outpatient and inpatient diagnoses nationwide, and accidental deaths identified using the Swedish Death Registry. Cox regression was used to compute hazard ratios for the associations between mental disorders and accidental death (overall and by specific types), while adjusting for sociodemographic confounders and examining the potential mediating effect of comorbid substance use.

Results: There were $22,419(0.3 \%)$ accidental deaths in the entire population, including 5,933 (0.9\%) accidental deaths compared with $3,731(0.6 \%)$ suicides among psychiatric patients. $26.0 \%$ of people who died from accidents had been diagnosed with any mental disorder, compared with $9.4 \%$ in the general population. Any mental disorder was associated with a 5.3-fold (95\% CI 5.0-5.6) risk of accidental death among women and a 6.6-fold (95\% CI 6.4-6.9) risk among men, relative to those without mental disorders. Strong associations were found irrespective of age or socioeconomic status, and for different types of accidental death (especially poisoning or falls). Although risk of accidental death was highest among those with substance use disorders ( $\sim 8$ - to 14-fold), the risk also was increased among those with personality disorders $(\sim 4-$ to 7 -fold), and those with schizophrenia, bipolar disorder, depression, or anxiety disorders ( $\sim 2$ - to 4-fold), which did not seem fully explained by comorbid substance use. Sociodemographic risk factors included male sex, older age, unmarried status, and low socioeconomic status.

Conclusions: In this large cohort study, all mental disorders were strong independent risk factors for accidental death, which was substantially more common than suicide. Interventions to reduce premature mortality among people with mental disorders should address common determinants of both suicide and accidental death.

\section{0-139}

Are women with gestational hypertension or pre-eclampsia at an increased long term risk of kidney function impairment?

Presenter: Sohinee Bhattacharya

Sohinee Bhattacharya 1); Dolapo Ayansine 2); Corri Black 1); Cairns Smith 1)

1) Institute of Applied Health Sciences, University of Aberdeen;

2) Medical Statistics Team, Institute of health Sciences, University of Aberdeen
Background: Preeclampsia is known to cause impairment of kidney function in pregnancy, manifest as proteinuria. Previous studies have found an association between preeclampsia and kidney disease but were restricted in their numbers or had short follow up times.

Objectives: To assess the long term effects of hypertensive disorders of pregnancy on kidney function in later life.

Methods: The Aberdeen Maternity and Neonatal Databank (AMND) records and stores all pregnancy related events occurring in women living in the Grampian region of Scotland. From the AMND, we identified the first singleton pregnancy of all women with date of birth on or before 30th June 1969. These women were linked by means of their identifying information to the local renal biochemistry database (GRBD). A cohort study design was used where exposure was gestational hypertension $(\mathrm{GH})$ or preeclampsia (PE) defined according to Davey and MacGillivray's classification of hypertensive disorders of pregnancy. Outcome assessed was Chronic Kidney Disease (CKD) stages 1-5 based on the NICE modified Kidney Disease Outcomes Quality Initiative definition, occurring at least 1 year following delivery. Those with GH and PE were compared to normotensive women using multivariate logistic regression to adjust for potential confounders. Survival analysis was used to assess whether there was a difference in the timing of onset of CKD in the different exposure groups.

Results: A total of 14,675 women who had been linked to the GRBD and had complete information regarding age, socio-economic class, smoking and body mass index (BMI) were included in a multivariate model. CKD was diagnosed in $13.3 \%$ of women who previously had GH or PE in contrast to $12.8 \%$ in normotensive women. The unadjusted odds ratio (95\% confidence interval) of having CKD in preeclamptic women was $2.04(1.53,2.71)$ and that in women with gestational hypertension was $1.37(1.15,1.65)$, while the adjusted odds ratio (95\% confidence interval) of CKD was $1.93(1.44,2.57)$ and $1.36(1.13,1.63)$ in preeclamptic women and women with gestational hypertension respectively compared to women who were normotensive in their first pregnancy. Kaplan-Meier curves of survival time to development of chronic kidney disease revealed that women with PE were susceptible to kidney function impairment earliest, followed by those with GH.

Conclusions: There was an increased subsequent risk of CKD associated with hypertensive disorders of pregnancy. Women with GH and PE were also found to have CKD earlier than normotensive women.

\section{0-140}

Partial sick leave and subsequent work participation: a register based study of working-aged Finnish women and men

Presenter: Johanna Kausto

Johanna Kausto 1); Svetlana Solovieva 1); Lauri J Virta 2); Eira Viikari-Juntura 1)

1) Finnish Institute of Occupational Health, Helsinki, Finland; 2) Social Insurance Institution, Research Department, Turku, Finland

Background: Increasing work participation of working aged people is currently a matter of concern in Finland as in many other Western countries. Partial social security benefits have been promoted as part of active labour market policy with anticipated positive influence on work participation. In Finland, partial sickness benefit was introduced in 2007 and so far $74 \%$ of the users have been women.

Objectives: The aim of the study was to examine the effect of partial sick leave in comparison with full sick leave on future work participation in working population with long term sickness absence. 
Additionally, it will be examined whether the effects differ by gender, age, socioeconomic status or diagnostic category.

Methods: Data were derived from the national sickness insurance register of the Social Insurance Institution of Finland and the pension and earnings registers of the Finnish Centre for Pensions. Two study cohorts were drawn and analyzed separately. Individuals who received partial sickness benefit in 2007 and a comparison group of those on full sickness benefit at the same time $(n=1,047$ and $\mathrm{n}=28,380$, respectively) constituted the first study cohort. The second cohort included receivers of partial or full sickness benefit in $2008(\mathrm{n}=1,738$ and $\mathrm{n}=56,754)$. The analyses were focused on four most prevalent diagnostic groups-musculoskeletal and mental disorders, traumas and tumours. We obtained longitudinal register data on employment periods as well as information on temporary and permanent, full and partial health related benefits (sickness and rehabilitation benefits, and disability pensions), and old-age pensions. To remove confounding due to both observed and unobserved factors, we opted for contrafactual approach using quasi-experimental study design with propensity score and difference-in-differences methods. Results: The propensity score analyses indicated that partial sickness benefit reduced the risk (change in absolute risk) of full disability pension by $4 \%$ in women and by $10 \%$ in men. The risk of partial disability pension was increased by 9 and $5 \%$, respectively. There were no large differences in the effects between the largest diagnostic categories of musculoskeletal and mental disorders. With differencein-difference analyses we will further examine in a more comprehensive dataset (second cohort) the effect of the use of the partial sickness benefit on work participation.

Conclusions: The results from the first cohort suggest that partial sick leave in comparison with full sick leave may increase work retention at population level. The findings encourage use of the benefit among men.

\section{0-141}

Individual and area level socio-demographic factors as predictors of mortality among Swedish men and women: a longitudinal study

\section{Presenter: Ailiana Santosa}

\section{Ailiana Santosa 1); Joacim Rocklöv 1); Ulf Högberg 2); Peter Byass 1)}

1) Department of Public Health and Clinical Medicine, Division of Epidemiology and Global Health, Umeå Centre for Global Health Research, Umeå University, Umeå, Sweden; 2) Department of Women's and Children's Health, Uppsala University, Uppsala, Sweden

Background: Health and social inequalities in Sweden still exist although a significant reduction on mortality and morbidity in both men and women has been demonstrated in recent decades. To date, few studies have examined how individual and area level characteristics contribute to the mortality among men and women in Swedish context.

Objectives: This study aimed to assess mortality trends over time among men and women, to examine whether mortality changes were independently related to socio-demographic factors, as well as measuring associations between community characteristics and mortality rates and how much mortality among total Swedish population was attributable to social and gender factors.

Methods: Data were extracted from the Linnaeus Database 1991-2005 that links individual data on socio-demographics from the National Board of Health Welfare to the national cause-of-death register. Temporal trends in the standardized mortality rates were evaluated for men and women separately by using Poisson regression models adjusting for the total population under exposure by stratifying per 5-year periods. The mortality incidence rate ratio's in relation to socio-demographic factors were studied separately for men and women and stratified by 5-year periods using multilevel Poisson regression models adjusting for area level by random intercepts.

Results: Total observations cover 8,743,637 individuals over $113,152,127$ person-years with mean age 47.5 years $(S D=19.9)$. A significant reduction in overall and CVD mortality occurred among men and women in Sweden during 1991-2005, with excess male: female mortality persisting (incidence rates for overall mortality 8.63 vs. 7.55 per 1,000 population). Age, marital status, employment status and migration status showed consistent associations with overall mortality among men after adjustment, and for women except for migration status. Education level and pensioner were consistently protective factors for male mortality, but less so for women after controlling for other factors. The effect of area socioeconomic effects on overall mortality, after adjustment for individual socio-demographic characteristics, markedly increased over study period. The county and municipality levels played an important part in understanding individual differences for CVD mortality, but less so for overall mortality. A significant variation in CVD mortality across counties in Sweden during 15 years follow up, and to a lesser across municipalities within counties was found.

Conclusions: The relative contribution of area (contextual) factors, as compared with individual factors, to socioeconomic gradients in overall and CVD mortality strengthened over the period. Contextual epidemiology is essential for understanding social disparities in overall and CVD mortality.

\section{0-142}

Suicide and other unnatural deaths in a national cohort of people diagnosed with diabetes

Presenter: Roger Webb

Roger Webb 1); Paul Lichtenstein 2); Marie Dahlin 2); Navneet Kapur 1); Jonas Ludvigsson 2); Bo Runeson 2)

1) University of Manchester, UK; 2) Karolinska Institutet, Sweden

Background: Elevated risk of suicide is associated with an array of major physical illnesses including diabetes mellitus. However, detailed investigations of premature death in diabetes have to date focused almost entirely on natural causes. Therefore the specific mechanisms that explain higher suicide risk in these patients are largely unknown. Examination of the full range of unnatural causes is also needed.

Objectives: To estimate relative risk for unnatural causes of death in a national cohort of people in Sweden diagnosed with diabetes. To determine what factors may explain the mechanisms of elevated risk in this population.

Methods: Using the Swedish Hospital Discharge Register and the National Diabetes Register, between 1st January 1996 and 31st December 2010 we identified a cohort of 419,023 affected individuals. These subjects were matched on age and gender to five unexposed individuals from the general population without a diagnosis of diabetes. Cause of death was extracted from the national registers and linked completely to the study cohort. Relative risk was estimated as a risk ratio (RR) via fixed effects Poisson regression models.

Results: Unnatural deaths were a small minority of all deaths in the diabetes cohort, but risk was elevated versus the general population (RR 2.4, CI 2.3-2.5). Each specific unnatural cause also had higher risk: suicide (RR 3.7, CI 3.3-4.2); accident (RR 2.1, CI 2.0-2.2); homicide (RR 3.5, CI 1.8-6.9); medical or surgical complications 
(RR 2.6, CI 2.0-3.4). Especially raised risk was seen with poisoning deaths irrespective of coronial verdict. When we examined all poisoning deaths combined, to minimise potential misclassification and to maximise statistical power, we found elevated risk in relation to each main poisoning type: alcohol (RR 3.8, CI 2.6-5.5); psychotropic medication (RR 4.3, CI 3.0-6.1); narcotics \& hallucinogens (RR 5.2, CI 2.9-9.2); carbon monoxide \& other gases (RR 7.5, CI 3.6-15.6) and unspecified medication (RR 9.2, CI 6.7-12.7).

Conclusions: Patients with diabetes are at higher risk of dying by suicide and by each of the other main unnatural causes: accident, homicide, and medical or surgical complication. The particularly large elevation in risk for fatal poisoning by an unspecified form of medication may be partially due to insulin overdoses. However, our results indicate that multiple types of accidental or deliberate selfpoisoning occur far more frequently in this population. Therefore comprehensive and well-coordinated preventive strategies are needed if the large elevation in unnatural mortality risk is to be reduced among people diagnosed with diabetes.

\section{O-143}

\section{Subacromial impingement syndrome: a register study of genetic aspects of aetiology}

\section{Presenter: Susanne Wulff Svendsen}

Susanne Wulff Svendsen 1); Berit Lindum Waltoft 2); Preben Bo Mortensen 2); Poul Frost 3)

1) Danish Ramazzini Centre, University Department of Occupational Medicine, Herning Regional Hospital, Denmark; 2) National Centre for Register-Based Research, Aarhus University, Denmark; 3) Danish Ramazzini Centre, Department of Occupational Medicine, Aarhus Hospital, Aarhus University Hospital, Denmark

Background: Subacromial impingement syndrome (SIS) is an important cause of shoulder complaints and occurs with a prevalence of $2-8 \%$. In $2008,0.1-0.2 \%$ of the Danish working-age population underwent surgery for SIS. Genetic influences have been suggested based on familial aggregation. However, a propensity for or against surgery may also aggregate in families, which may mimic genetic effects.

Objectives: The aim of this study was to determine (1) if the risk of surgery for SIS is increased in persons with siblings who have undergone surgery for SIS, when compared to persons without such siblings, and (2) if full-siblings, who have undergone surgery for SIS, convey a larger risk than corresponding half-siblings.

Methods: For individuals born in 1950 or later, the Danish Civil Registration System contains personal identifiers of parents. We established a cohort of all individuals born in Denmark 1950-1995 with registered identity of both parents; half- and full-siblings were identified through the links to their parents. Information on surgery for SIS was obtained from the Danish National Patient Register, 1996-2010. Other registers provided information on birth and death dates, diabetes, region of residence, and work histories. We used Poisson regression analyses. When a first-time event of surgery for SIS occurred, the proband was censored from follow up, and any siblings stopped contributing to person-years at risk in the unexposed group and started contributing in the exposed group. Occupational biomechanical exposures to the shoulder, which may be similar within families, will be entered into the models later.

Results: During 27,236,994 person-years of follow up, 19,840 firsttime events of surgery for SIS occurred, and 530 patients had siblings who had undergone surgery for SIS. Preliminary analyses showed a rate ratio (RR) of 1.90 (95\% confidence interval (CI) 1.74-2.07) for patients with siblings who had undergone surgery for SIS, when compared to patients without such siblings. Among women, the RR was 2.40 (95\% CI 2.12-2.71) in case of full-siblings, who had undergone surgery for SIS, whereas the corresponding RR for halfsiblings was 1.43 (95\% CI 1.01-2.00). Among men, the RRs were $1.70(95 \%$ CI $1.48-2.00)$ and $1.18(0.81-1.73)$ for full- and halfsiblings, respectively. The gender difference was significant regarding full-siblings $(p<0.001)$.

Conclusions: The risk of surgery for SIS was higher among persons with siblings who had undergone surgery for SIS when compared to persons with siblings who had not undergone such surgery. Fullsiblings conveyed a higher risk than half-siblings, which favours genetic explanations.

\section{O-144}

Low stress resilience in adolescence and increased mortality risk in adulthood: National cohort study of Swedish men

Presenter: Ruzan Udumyan

Ruzan Udumyan 1); Scott Montgomery 1), 2); Unnur Valdimarsdóttir 3), 4); Fang Fang 5); Katja Fall 1)

1) Clinical Epidemiology and Biostatistics, Örebro University Hospital \& School of Health and Medical Sciences, Örebro University, Sweden; 2) Clinical Epidemiology Unit, Karolinska Institutet, Sweden; 3) Center of Public Health Sciences, University of Iceland, Iceland; 4) Department of Epidemiology, Harvard School of Public Health, Boston, MA; 5) Karolinska Institutet, Sweden

Background: Previous studies suggest adverse affects of psychosocial stress on morbidity and mortality risk, but the explanatory role of pre-existing stress resilience level in late adolescence with subsequent mortality risk is unclear.

Objectives: To investigate the association between stress resilience in adolescence and mortality risk in adulthood and the possible modifying role of cognitive function, low levels of which have been linked with higher mortality.

Methods: A cohort of 244,907 male conscripts born between 1952 and 1956 was followed to an average age of 54 years using information from Swedish registers. Stress susceptibility was measured through a semi-structured interview with a psychologist assessing conscripts' potential ability to cope with warfare. The cognitive function score is an aggregate of four tests reflecting verbal, logical, spatial and technical abilities. Stress resilience and cognitive function were measured at conscription (typically at ages 18-19 years), with higher values indicating better functioning (range 1-9). Cox regression estimated the association of stress resilience with mortality, with attained age as the underlying time-scale and adjusting for parental socioeconomic index and household crowding in 1960, region of residence, and from conscription: height, body mass index, systolic and diastolic blood pressure, disease severity, physical capacity and cognitive function scores.

Results: Lowest stress resilience (21.9\% with scores 1-3) compared with highest (23.8\% with scores $7-9$ ) is associated with an elevated mortality risk, producing adjusted hazard ratios (HR) with $95 \%$ confidence intervals of $1.6(1.5,1.7)$ for all-cause mortality, $1.3(1.2$, 1.6) for cardiovascular mortality and $2.1(1.8,2.4)$ for suicide.

The association of stress resilience with all-cause mortality and suicide was modified by cognitive function ( $p$ for interactions $\leq 0.02$ ). Comparing low with high stress resilience among men with the highest cognitive function (scores 7-9) rendered HRs of $1.7(1.4,1.9)$ for all-cause mortality and $2.7(2.0,3.6)$ for suicide. Among men with the lowest cognitive function (scores 1-3), the corresponding figures were $1.5(1.3,1.7)$ and $1.7(1.2,2.4)$. 
Conclusions: Low stress resilience in adolescence is associated with increased all-cause mortality. The underlying mechanisms may involve behavioural factors such as smoking and biological components such as altered sympathetic signalling.

Although higher cognitive function is associated with reduced mortality, the greatest mortality, particularly suicide, risk was in men with the combination of high cognitive function and low stress resilience. The mechanisms linking cognition and stress susceptibility with mortality are complex. This may assist in identification of intervention targets.

\section{0-145}

Paternal exposure to lead and schizophrenia in offspring

Presenter: Markku Sallmén

Markku Sallmén 1); Mark G.A Opler 2); Jaana Suvisaari 3); Marja-Liisa Lindbohm 1); Anzalee Khan 4); Dolores Malaspina 2)

1) Finnish Institute of Occupational Health, Centre of Expertise for Health and Work Ability, Helsinki, Finland; 2) New York University, School of Medicine, Department of Psychiatry, New York, NY; 3) National Institute for Health and Welfare, Department of Mental Health and Substance Abuse Services, Helsinki, Finland; 4) Nathan Kline Institute, Department of Psychiatric Research, New York, NY

Background: Schizophrenia is a complex, multifactorial disorder caused by a combination of genetic and environmental risk factors, with heritability varying from 65 to $80 \%$. The lifetime prevalence of schizophrenia in Finland is $1 \%$. Schizophrenia is a neurodevelopmental disease, in which the illness is the end state of abnormal neurodevelopmental processes beginning during prenatal development. The role of paternal lead exposure has not been investigated as a risk factor for schizophrenia in offspring. De novo mutations or epigenetic mechanisms are both potential mechanisms.

Objectives: The aim was to investigate whether father's occupational exposure to inorganic lead increases the risk of schizophrenia in offspring.

Methods: This register-based study was conducted using a cohort study design. We identified exposed men from blood lead measurements taken at the Finnish Institute of Occupational Health in 1973-1983. We obtained data on their children born in 1972-1984 and the mothers from the national Population Information System. From this register, we selected four population referent children matched on date of birth, gender and area. Paternal preconceptual exposure to lead was assessed using biological measurements and employment histories obtained from Finnish Centre for Pensions. Referent fathers were assumed to be unexposed. We identified schizophrenia cases from The Finnish Hospital Discharge Register. We compared the incidence of schizophrenia in children of exposed fathers to that of unexposed fathers. Data on schizophrenia were analysed using proportional hazards regression. In conditional multivariable analyses we considered parental history of schizophrenia and psychosis, language of the child, age of parents at conception, father's employment, and father's work as self-employed person.

Results: There were no differences in the incidence of schizophrenia between children of exposed $(82 / 11,863 ; 0.69 \%)$ and unexposed fathers $(318 / 47,445 ; 0.67 \%)$ after follow-up of $26-38$ years. This was also true for higher occupational lead exposure: adjusted hazard ratios (aHR) of schizophrenia were 1.07; $95 \%$ CI 0.52-2.19 and 1.29; $95 \%$ CI $0.47-3.59$ for paternal blood lead of $1.0-1.4 \mu \mathrm{mol} / \mathrm{L}$ and $>1.5 \mu \mathrm{mol} / \mathrm{L}$, respectively. Schizophrenia of the mother (aHR 7.84; $95 \%$ CI 3.25-18.89) and the father (aHR 6.65; $95 \%$ CI 1.85-23.84) were related to increased risk of schizophrenia in the offspring.
Swedish speaking children were less likely to develop schizophrenia $(0.17 \%)$ than Finnish speaking children $(0.70 \%)$ : aHR $0.27 ; 95 \%$ CI $0.11-0.68$.

Conclusions: Our findings suggest that paternal exposure to lead does not increase the risk of schizophrenia in offspring.

\section{O-146}

Parental inflammatory bowel disease and risk of autism spectrum disorders in offspring: a nationwide cohort study in Denmark

Presenter: Ane Birgitte Telén Andersen

Ane Birgitte Telén Andersen 1); Vera Ehrenstein 1); Rune Erichsen 1); Trine Frøslev 1); Henrik Toft Sørensen 1)

1) Department of Clinical Epidemiology, Aarhus University Hospital, Aarhus, Denmark

Background: Autism spectrum disorders (ASD) and inflammatory bowel disease (IBD) may share genetic and enviromental risk factors. Furthermore, increases in ASD and IBD prevalence have coincided in time.

Objective: To examine if parental IBD is associated with an increased risk of ASD in offspring.

Methods: We conducted a nationwide registry-based cohort study including all children born alive in Denmark from 1 January 1994 to 31 December 2009, with follow-up throughout 2010. The children and their mothers were identified through the Danish Medical Birth Registry (DMBR) and fathers were identified through the DMBR and the Danish Civil Registration System. IBD in parents and ASD in offspring were identified using in- and out-patient hospital diagnoses recorded in the Danish National Registry of Patients and the Danish Psychiatric Central Register, respectively. We computed the 5-year risk of ASD and used Cox proportional-hazards regression to compute incidence rate ratios (IRR) with $95 \%$ confidence intervals (CI), adjusting for year of birth, child's sex, mother's and father's age at delivery, maternal smoking during pregnancy, and multiple births. We evaluated risk of ASD according to maternal and paternal IBD, Crohn's disease (CD), and ulcerative colitis (UC). Children without a history of parental IBD were used as reference.

Results: The cohort consisted of 1,005,330 children. A total of 11,888 $(1.2 \%)$ had a parent with IBD and a total of $8,087(0.8 \%)$ were identified with ASD during up to 17 years of follow-up (median follow-up time was 8.9 years). Of those children with parental IBD, $62(0.5 \%)$ had an ASD diagnosis. The 5-year risk of ASD was $0.2 \%$ among both offspring of parents with and without IBD. The adjusted IRR for ASD associated with parental IBD was 0.8 (95 \% CI 0.6-1.0) and results were similar regardless of parent of IBD origin or parental CD or UC.

Conclusion: We found no evidence of an association between parental IBD and ASD in offspring.

\section{0-147}

Prenatal maternal bereavement and congenital heart defects in offspring: a registry-based study

Presenter: Jin Liang Zhu

Jin Liang Zhu 1); Jørn Olsen 2); Henrik Sørensen 3); Jiong Li 2); Ellen Nohr 2); Carsten Obel 1); Mogens Vestergaard 4); Morten Olsen 3)

1) Research Program for Children's Mental Health, Department of Public Health, Aarhus University; 2) Section of Epidemiology, 
Department of Public Health, Aarhus University; 3) Department of Clinical Epidemiology, Aarhus University Hospital; 4) Section of General Practice, Department of Public Health, Aarhus University

Background: Congenital heart defects (CHD) are one of the leading causes of perinatal and infant mortality in many industrialized countries. Although the etiology of CHD is largely unknown, some studies have suggested maternal emotional stress during cardiogenesis as a risk factor.

Objectives: We examined this association using bereavement around the time of conception as an indicator of maternal exposure to stress in a large registry-based study in Denmark.

Methods: Using the Medical Birth Registry and the Civil Registration System we identified $1,770,878$ singletons born in Denmark from 1 January 1978 to 31 December 2008 and their close relatives (parents, siblings, mother's siblings, and mother's parents). Of these, 44,820 children were exposed prenatally to maternal bereavement, i.e., their mothers had lost a first-degree relative during the time period from 1 year before their last menstrual period until delivery of the index child (6,080 mothers lost an older child or partner and 38,740 mothers lost a parent or sibling). CHD diagnoses in children were identified from the Danish Registry of Congenital Heart Disease. We used logistic regression models to calculate prevalence odds ratios (ORs) of CHD for exposed children compared with unexposed children.

Results: Overall, 14,507 (0.82 \%) children had CHD, with $26.5 \%$ diagnosed soon after birth and $72.9 \%$ within their first year of life. We found that the exposed children had a higher prevalence of CHD than the unexposed children ( 0.94 vs. $0.82 \%$; adjusted $\mathrm{OR}=1.11$, $95 \%$ confidence interval 1.00-1.22). The association was most marked for children of mothers who had lost a child or partner (1.15 vs. $0.82 \%$; adjusted $\mathrm{OR}=1.32,1.04-1.67$ ). Furthermore, maternal exposure to bereavement during the period from 6 months before the last menstrual period through the second trimester of pregnancy tended to be associated with an increased prevalence of CHD.

Conclusions: Our findings support the hypothesis that prenatal exposure to severe stress is associated with an increased prevalence of CHD in children. Health professionals should be prepared to help and support the bereaved mother to cope with this serious life event.

\section{0-148}

Understanding the contextual effect of ethnicity: a multilevel analysis on ethnic differences in asthma treatment among Swedish adolescents

\section{Presenter: Lourdes Cantarero Arévalo}

Lourdes Cantarero Arévalo 1); Raquel Perez-Vicente 2); Sol Pía Juárez 2); Juan Merlo 3)

1) Faculty of Health and Medical Sciences, Department of Pharmacy, Section for Social and Clinical Pharmacy, University of Copenhagen, Denmark; 2) Faculty of Medicine, Unit for Social Epidemiology, Lund University, Malmö, Sweden; 3) Centre for Economic Demography, Lund University, Lund, Sweden

Background: Asthma is a common chronic respiratory disease in adolescents for which pharmacological treatment with anti-asthmatics (AAD) is of fundamental relevance. It is known that in the USA ethnic minority adolescents are at a higher risk of under-treatment and that these inequalities are related to economic conditions such as lack of insurance and inability to afford the cost of medicines. However, there are many knowledge gaps concerning the use of AAD among ethnic minority Swedish adolescents. In a health system such as the Swedish one, which is characterized by access to health care on equal terms and according to needs, economic barriers should not be a major determinant of AAD use. This circumstance should allow us to investigate whether, over and above individual needs and economic difficulties, AAD use is conditioned by ethnic background per se or in interaction with society (i.e., social injustice).

Objectives: Adopting a multilevel approach we aim to quantify to which degree the country of origin of the mother conditions adolescent differences in AAD use, over and above individual socioeconomic factors and medical needs.

Methods: Using the Medical Birth Register, which is linked to several other Swedish registries, we identified all 782,857 children born between 1988 and 1991 who were residing in the country until the year in which they turned 17, and whose mother's country of birth could be identified. From the Swedish National Prescription Register, which contains information on out-patient prescription for all residents in Sweden, we identified use of AAD distinguishing between relief (short-acting) and preventive medication.

We applied a two level multilevel logistic regression analysis with 781,632 adolescents nested within 36 maternal countries of birth. We compute a risk score for AAD use including variables related to socioeconomic and medical needs. We distinguished between measures of association (odds ratios, (OR)) and measures of variance (Intra-class correlation (ICC)).

Results: Comparing with adolescents born from Swedish mothers and adjusting for the risk score, ethnic minority Swedish adolescents had an underutilization of preventive AAD (OR $=0.49,95 \% \mathrm{CI}$ 0.38-0.63). The ICC for mother's country of birth was $2.62 \% 95 \%$ CI $1.17-4.96 \%$.

Conclusions: Mother's country of birth was associated to adolescent use of AAD suggesting the existence of inequalities in access to health care affecting ethnic minority Swedish adolescents. However, the small ICC indicates that the predictive accuracy of maternal country of birth to identify adolescent use of AAD is very low.

\section{0-149}

Prenatal prescription corticosteroids and offspring diabetes

Presenter: Naomi Greene

Naomi Greene 1); Lars H. Pedersen 2), 3); Simin Liu 1), 4); Jørn Olsen 1), 3)

1) UCLA School of Public Health, Department of Epidemiology, Los Angeles, CA, USA; 2) Department of Obstetrics and Gynecology, Institute of Clinical Medicine, Aarhus University, Aarhus, Denmark; 3) Department of Public Health, Epidemiology Section, Aarhus University, Aarhus, Denmark; 4) UCLA School of Medicine, Department of Obstetrics and Gynecology, Los Angeles, CA, USA

Background: Prenatal exposure to supraphysiologic levels of glucocorticoids has been linked to altered structure and function of multiple systems, including the HPA axis, the immune system, as well as glucose-insulin homeostasis. These changes may be induced by epigenetic mechanisms and persist after birth, possibly leading to an increased risk of chronic diseases such as diabetes.

Objective: Using linked information from 4 Danish administrative registers, we sought to examine the association between prenatal exposure to maternal prescription glucocorticoids and diabetes in the offspring.

Methods: We constructed a nation-wide birth cohort made up of children from singleton pregnancies born in Denmark between 1 January 1997 and 31 December 2004 and obtained follow-up information through 31 Dec 2008. We extracted linked information from 4 Danish administrative registers on specific exposures, outcomes and covariates of interest among 505,386 children born alive to 360,484 women. We calculated Type 1 and Type 2/elevated blood glucose hazard rates using Cox proportional hazard rates comparing children 
exposed prenatally to maternal prescription glucocorticoids (defined as $\geq 1$ prescription corticosteroid redeemed during pregnancy) with those unexposed. We performed sensitivity analyses in which (1) exposure was redefined as redemption of $>1$ prescription corticosteroid during pregnancy, (2) the cohort was restricted to only the firstborn child to each mother, and (3) the cohort was restricted to children born at term.

Results: Prenatal exposure to prescription corticosteroids was associated with a small increase in offspring type 1 diabetes incidence rate $[\mathrm{HR}=1.20,95 \%$ confidence limits $=0.94,1.53]$ and with an increase in type 2 diabetes/elevated blood glucose hazard ratio when comparing children exposed prenatally to prescription corticosteroids with those unexposed $[\mathrm{HR}=1.51,95 \%$ confidence limits $=0.69$, 3.31]. The data were consistent with a monotonic increase in overall diabetes hazard ratios with increasing strength of the corticosteroid. Sensitivity analyses did not alter the direction/magnitude of associations.

Conclusion: There may be an association between prenatal exposure to maternal prescription corticosteroids and diabetes in the offspring. Further studies are warranted to better characterize the exposure and identify critical exposure windows. Leaving women untreated for conditions requiring steroids during pregnancy may carry a greater risk for their offspring than being treated even if the associations we present are causal. Nonetheless, there is a paucity of research into the long-term effects in offspring of prenatal exposure to glucocorticoids and a potential association between prescription corticosteroids and offspring diabetes could have significant public health implications.

\section{0-150}

\section{Association between parental socioeconomic status and prevalence of asthma, atopic eczema and hay fever in children}

\section{Presenter: Lene Hammer-Helmich}

Lene Hammer-Helmich 1); Allan Linneberg 1); Simon Francis Thomsen 2); Charlotte Glümer 1), 3)

1) Research Centre for Prevention and Health, Capital Region of Denmark, Nordre Ringvej 57, DK-2600 Glostrup, Denmark; 2) Department of Dermatology, Bispebjerg Hospital, Bispebjerg Bakke DK-2400 Copenhagen NV, Denmark; 3) Department of Health Science and Technology, University of Aalborg, Denmark

Background: The prevalence of allergic diseases is increasing worldwide. Consistent evidence links higher parental socioeconomic status (SES) with better child health. Evidence of the relationship between SES and allergic diseases in children, however, has been inconsistent.

Objective: To determine the prevalence of asthma, atopic eczema and hay fever among children in different age groups and to examine the associations between allergic diseases and socioeconomic indicators of the primary caregiver and the household.

Methods: A cross sectional health survey of 4 complete birth cohorts in the municipality of Copenhagen was conducted. Children aged 11 and 15 years and parents of children aged 3 and 6 years completed written questionnaires on general health, symptoms and various diseases. Data were linked to information from national registers on demographics, education, employment status and income. In total 9,720 children/parents responded (50.5\%). Logistic regression analyses were performed.

Results: The prevalence of asthma and hay fever increased with increasing age, whereas the prevalence of atopic eczema was similar in all age groups. The prevalence of asthma was for children aged 3 years: $3.2 \%$; 6 years: $4.7 \%$; 11 years: $11.2 \%$; and 15 years:
$15.4 \%$. The prevalence of hay fever was for children aged 3 years: $3.1 \%$; 6 years: $5.9 \%$; 11 years: $13.1 \%$; and 15 years: $21.3 \%$. The prevalence of atopic eczema was for children aged 3 years: $17.8 \%$; 6 years: $16.7 \%$; 11 years: $16.4 \%$; and 15 years: $15.6 \%$.

Low educational level was significantly associated with increased risk of asthma and hay fever but decreased risk of atopic eczema and eczema symptoms. Odds Ratios for children from households with the lowest versus highest educational level were $1.56 \quad(95 \%$ $\mathrm{CI}=1.22-1.99)$ for asthma; $1.66(95 \% \mathrm{CI}=1.33-2.07)$ for hay fever; and 0.69 (95\% CI $=0.57-0.84)$ for atopic eczema. Unemployment was significantly associated with a decreased risk of atopic eczema and eczema symptoms. There was no independent association between household income and any of the allergic outcomes when adjusted for educational level and employment status.

Conclusion: The prevalence of allergic diseases in children of Copenhagen is relatively high, especially among the oldest children. Atopic eczema is still the affluent children's disease, whereas both asthma and hay fever, in an urban context with a health care system free of charge, are associated with poor SES. These results support a hypothesis of a translational shift in the burden of allergic diseases towards becoming the deprived children's diseases.

\section{$0-151$}

Is offspring sex composition related to parental risk factors, cancer and cause specific mortality?

Presenter: Øyvind Naess

\section{Øyvind Næss 1); George Davey Smith 2)}

Background and objective: It has been suggested that there is a trade off between reproduction and somatic maintenance as both are energetically costly. This may depend on sex of the offspring and differ between men and women because boys are heavier and larger at birth and require more energy during gestation and lactation. There is also some evidence that carriers of BRCA mutations are more likely to have female offspring. Here we investigated if offspring sex composition is related to parental risk factors, cancer and cause specific mortality.

Methods: The population was as all men and women born in Norway in the period 1925-1954 having survived to the age of 50. Identity of offspring was available from the Norwegian multigenerational database. The data was organized into trios with mother, father and offspring. This gave 920,177 offspring with 484,122 mothers and 475,703 fathers. These were linked to their offspring and further to the Cause of Death Registry, the Cancer Registry, data from Statistics Norway and from the Cohort of Norway. Two strategies were used in the analysis: Estimating effect of number of boys and girls, and then sex of first, second and third offspring conditioning on having at least two or three offspring.

Main results: In women, there was a graded increase in risk of all cause death and cardiovascular causes that was stronger for number of boys than girls. Both number of boys and girls were inversely related to risk of incident cases of breast and ovarian cancer. In men, there was similar increased risk of all cause death and cardiovascular for both number of boys and girls. In stratified analysis of combinations of the first offspring sexes there was an increasing effect for cardiovascular causes that was stronger in women compared to men and inverse effect for breast cancer in women. Restricting to those with two offspring only gave similar results. Risk factors increased with both number of boys and girls in both men and women. Triglycerides and Cholesterol were increased in both men and women if first offspring sexes were boys.

Conclusions: For both men and women, there is increased risk of cardiovascular mortality with number of boys, which is more 
pronounced in women, which could be mediated by risk factors. Risk of breast cancer is reduced in women who have more offspring boys which is consistent with BRCA mutation carriers being more likely to give female offspring.

\section{Poster presentation}

P-001

Impact of comorbidity on risk of venous thrombosis in Danish breast cancer patients

Topic: Reviewer's choice

Presenter: Deirdre Cronin-Fenton

Anne Ording 1); Erzsébet Puhó 1); Jens Peter Garne 2); Petra Nyström 3); Henrik Sørensen 1); Timothy Lash 1), 4)

1) Department of Clinical Epidemiology, Aarhus University Hospital, Denmark; 2) Breast Clinic, Aalborg Hospital, Denmark; 3) Department of Oncology, Uppsala University Hospital, Sweden; 4) Department of Epidemiology, Rollins School of Public Health, Emory University, Atlanta, Georgia, USA

Background: Venous thromboembolism (VTE) is a serious complication of cancer and cancer therapy, and associated with poor survival. Whether comorbidity plays a role in the association between breast cancer and VTE risk has not been studied in detail.

Objectives: To examine biological interaction between breast cancer and comorbidity on the risk of VTE. This interaction is equal to the excess rate of VTE beyond that expected from the individual effects of comorbidity and breast cancer acting alone.

Methods: We used nationwide medical registries to conduct a population-based cohort study encompassing all Danish breast cancer patients diagnosed between 1995 and 2010 and a comparison cohort of women free of breast cancer, matched to the breast cancer patients on specific comorbidities. We computed rate and rate ratios of VTE for different comorbidity levels using the Charlson Comorbidity Index (CCI) and studied the interaction between comorbidity and breast cancer on the rate of VTE.

Results: The study included 62,461 breast cancer patients and 305,595 comparison women from the general population. After 1 year of follow-up, the rate of VTE was 7.7 (95\% confidence interval (CI): $7.0,8.5)$ in the breast cancer cohort and 2.0 (95\% CI 1.9, 2.2) per 1,000 person-years (PY) in the comparison cohort. The interaction between breast cancer and the CCI score accounted for 3.1 cases of VTE among breast cancer patients with a CCI score of $1,0.4$ cases of VTE among breast cancer patients with a CCI score of $2-3$, and -3.7 cases of VTE among breast cancer patients with a CCI score of $\geq 4$ per 1,000 PY, representing 27, 3.8, and $-28 \%$ of the total VTE rates among breast cancer patients, respectively. For VTE diagnosed within 3 months after surgery, the interaction accounted for 3.1 (47\%), 0.2 $(4.4 \%)$, and $1.5(20 \%)$ cases of VTE per 1,000 PY, for a CCI score of $1,2-3$ and $\geq 4$ respectively.

Conclusion: There was a clear interaction between breast cancer and comorbidity measured with the CCI in the first year after breast cancer, in particular when results were restricted to post-surgery VTE, suggesting that higher comorbidity burden may increase the rate of VTE in breast cancer patients receiving surgery.

\section{P-002}

Alcohol intake and prognosis of incident atrial fibrillation patients

Topic: Reviewer's choice
Presenter: Thure Filskov Overvad

Thure Filskov Overvad 1), 4); Lars Hvilsted Rasmussen 1), 3); Flemming Skjøth 1); Kim Overvad 1), 2); Ida Ehlers Albertsen 1), 4); Deirdre Lane 4); Gregory Lip 3), 4); Torben Bjerregaard Larsen 1), 3)

1) Department of Cardiology, Cardiovascular Research Centre, Aalborg University Hospital, Aalborg, Denmark; 2) Section for Epidemiology, Department of Public Health, Aarhus University, Aarhus, Denmark; 3) Thrombosis Research Unit, Faculty of Medicine, Aalborg University, Aalborg, Denmark; 4) University of Birmingham Centre for Cardiovascular Sciences, City Hospital, Birmingham, United Kingdom

Background: Alcohol is associated with the development of atrial fibrillation, but the prognostic impact of alcohol intake among atrial fibrillation patients is sparsely investigated. Reported alcohol intake is at present not included in risk stratification schemes for thromboembolism and death among atrial fibrillation patients.

Objectives: To assess reported alcohol intake as a risk factor for thromboembolic events and death among patients with incident atrial fibrillation.

Methods: The prospective Danish Diet, Cancer and Health study included 57,053 participants (27,178 men and 29,875 women) aged between 50 and 64 years. The study population for this study included the 3,107 participants (1,999 men and 1,108 women) who developed incident atrial fibrillation during follow-up.

Results: During a median follow-up of 4.9 years 621 deaths and 218 thromboembolic events occurred. Among those who developed atrial fibrillation, $690(35 \%)$ men and $233(21 \%)$ women had a high intake of alcohol $(>20$ drinks/week for men and $>13$ drinks/week for women). After adjustment for use of oral anticoagulation and components of the risk stratification tool CHA2DS2-VASc (congestive heart failure, hypertension, age, diabetes mellitus, previous stroke and vascular disease) men with an intake of $>27$ drinks/week had a higher risk for 'thromboembolism or death' than men with an intake of $<14$ drinks/week (hazard ratio (HR) 1.33, $95 \%$ confidence interval (CI) 1.08-1.63). Women with an intake of $>20$ drinks/week also had a higher risk (HR 1.23, 0.78-1.96) than women in the low intake category. The higher risk among men was primarily driven by mortality (HR 1.51, 1.20-1.89), whereas the risk among women was driven by thromboembolism (HR 1.71, 0.81-3.60).

Conclusions: High alcohol intake is associated with 'thromboembolism or death', even after adjustment for established clinical risk factors, and may help identify 'high-risk' atrial fibrillation patients, who could benefit from stroke and cardiovascular prevention strategies.

\section{P-003}

Multisite musculoskeletal pain and sickness absence trajectories: a 7-year follow-up among occupationally active Finns

Topic: Reviewer's choice Presenter: Eija Haukka

Eija Haukka 1); Leena Kaila-Kangas 1); Anneli Ojajärvi 1); Helena Miranda 2); Jaro Karppinen 1); Eira Viikari-Juntura 1); Markku Heliövaara 3); Päivi Leino-Arjas 1)

1) Finnish Institute of Occupational Health, Helsinki, Finland; 2) Occupational Health Services, OP-Pohjola Group, Helsinki, Finland; 3) National Institute for Health and Welfare, Helsinki, Finland

Background: Concurrent pain in several body sites (multisite pain) is more common than pain in a single site, as based on several European general population studies. Little is known on its effects on sickness absence in working populations. 
Objectives: We studied the number of musculoskeletal pain sites as a predictor of sickness absences during a 7-year follow-up.

Methods: A nationally representative sample (the Health 2000 survey) comprised of 3,420 occupationally active Finns aged 30-55 years (52\% female). Baseline data (questionnaire, interview, clinical examination by a physician) were gathered in 2000-2001 and linked with information from national registers on annual compensated sickness absence periods ( $>10$ workdays) covering the years 2002-2008. Pain during the preceding month in 18 body locations was inquired and combined into four sites (neck, upper limbs, low back, lower limbs). Demographic factors, body mass index, smoking, leisure-time physical activity, sleep disorders, physical and psychosocial workload, and chronic diseases were assessed. Trajectory analysis and multinomial logistic regression were used.

Results: Four distinct sickness absence trajectories emerged, labelled as Low (no sickness absences of at least 10 workdays, $59 \%$ of the subjects), Ascending (ascending pattern of sickness absence, $21 \%$ ), Mixed (first ascending then descending pattern, $11 \%$ ), and High (high absence level persistently, $9 \%$ ). The odds ratios (ORs) for belonging to the High versus the Low trajectory increased with the number of pain sites, being 2.1 for single-site pain, 2.6 for two, 2.9 for three, and 4.1 for four pain sites, after adjustment for chronic diseases, demographic and lifestyle factors, and workload. The confidence intervals of the ORs did not include unity. The adjusted ORs for belonging to the Ascending trajectory were 1.1, 1.3, 1.7, and 1.7, respectively.

Conclusions: As the number of pain sites was a strong independent predictor of work absenteeism, early screening of workers with multisite pain and interventions to support work ability seem warranted.

\section{P-004}

Occupational COPD among Danes aged 45 to 84 years: a population based study

Topic: Reviewer's choice

Presenter: Else Toft Würtz

Else Toft Würtz 1); Tine Malling 1); Vivi Schlünssen 2); Jens Georg Hansen 3); Charlotte Brasch Andersen 4); Øyvind Omland 1)

1) Occupational Medicine, Aalborg University Hospital, Aalborg, Denmark; 2) Institute of Environmental and Occupational Medicine, Public Health, University of Aarhus, Aarhus, Denmark; 3) Clinical Epidemiology, Aarhus University Hospital, Aarhus, Denmark; 4) Human Genetics, University of Southern Denmark, Odense, Denmark

Background: Smoking is the major risk factor for Chronic Obstructive Pulmonary Disease (COPD), but growing evidence suggests that about $15 \%$ of COPD could be attributable to an occupational exposure.

Objectives: The aim of this study was to analyse for occupational COPD among 4.747 subjects aged 45-84 years in a cross-sectional population based study.

Methods: The subjects were recruited from 155 general practices from two Danish counties in 2004-2006. Stratified sampling through the Danish Civil Registration System recruited 3,111 males and 1,636 females to the study. COPD was defined when measured forced expiratory volume per second (FEV1) and forced vital capacity (FVC) ratio (FEV1/FVC) z-score was below 2 standard deviations based on the lower limit of normal (LLN) criteria. From questionnaire a smoking and occupational history was obtained. The Danish version of The International Standard Classification of Occupations (ISCO) from 1988, was used (DISCO-88) together with expert derived assessment to define occupational exposure in jobs where vapour, gas, dust (organic and inorganic), and fume (VGDF) were risk factors. Low exposure was defined as 0-4 years in a job, medium 5-14 years, and high $\geq 15$ years.

Results: Of 372 DISCO- 88 codes 72 were identified with high exposure to VGDF; 34 for organic dust, 20 for inorganic dust, 7 for both inorganic dust and fume, 5 for vapour, 3 for fume, 2 for both organic- and inorganic dust, 1 for inorganic dust, fume, and vapour. $28 \%$ of the participants had a high exposure for VGDF. Adjusted for smoking status (never, former or current), increased risk of COPD was found among workers in jobs with DISCO-88 codes with high exposure for organic dust OR 1.79 (95 \% CI 1.35-2.36). However, for workers in jobs with DISCO-88 codes with high exposure for inorganic dust OR 0.75 (95\% CI 0.45-1.36), fume OR $1.20(95 \% \mathrm{CI}$ 0.70-2.04), and vapour OR 0.76 (95\% CI 0.23-2.46) no increased risk were observed. We observed a dose-response relationship in organic dust exposure.

Conclusions: Controlled for smoking status increased risk for COPD was observed among subjects in jobs with DISCO- 88 codes with high exposure for organic dust in a dose response fashion.

\section{P-005}

Risk of permanently reduced work capacity after haematological malignancies: a register-based cohort study

Topic: Reviewer's choice

Presenter: Trine Allerslev Horsb $\phi$

Trine Allerslev Horsbøl 1); Claus Vinther Nielsen 2), 3); Niels Trolle Andersen 4); Bendt Nielsen 1); Annette Ladefoged de Thurah 5)

1) Department of Haematology, Aarhus University Hospital, Denmark; 2) Public Health and Quality Improvement, Central Denmark Region, Aarhus, Denmark; 3) Section for Clinical Social Medicine and Rehabilitation, Department of Public Health, Aarhus University, Denmark; 4) Section for Biostatistics, Department of Public Health, Aarhus University, Denmark; 5) Department of Public Health \& Department of Clinical Medicine, Aarhus University, Denmark

Background: Patients recovered from haematological malignancies seem to have an increased risk of work-related problems compared to the general population. However, studies are sparse and documentation concerning factors possibly associated with increased risk of permanently reduced work capacity (PRCW) among patients with haematological malignancies is limited.

Objectives: 1) To determine the risk of PRWC among patients diagnosed with a haematological malignancy between 2000 and 2007 compared to an age- and gender-matched reference cohort without a history of these malignancies.

2) To evaluate the influence of socio-economic and demographic factors, type of haematological malignancy, comorbidity, and use of antidepressants or anxiolytics following the diagnosis (used as a surrogate marker of anxiety and depression) on PRWC. 3) To investigate if these associations differ between the reference cohort and the patient cohort.

Methods: Using data from the Danish Cancer Registry, we conducted a cohort study including all patients aged $19-55$ years with a firsttime diagnosis of a haematological malignancy from 2000 to 2007. For each cancer case 10 age- and gender-matched reference individuals without a history of haematological malignancies were sampled from the Danish Civil Registration System.

A total of 3,194 patients and 28,627 reference individuals were included in the study. 
The two cohorts were followed until PRWC, emigration, old age pension, death or 26 February 2012, whichever came first.

Information on PRWC was retrieved from the Danish Register for Evaluation of Marginalisation (DREAM) containing week-by-week registrations of welfare benefit payments for all Danish citizens. Individuals granted either wage subsidized employment or disability pension were defined as having PRWC.

Results: In all, $805(25 \%)$ patients and 2,174 (8\%) reference individuals became permanently work disabled during the follow-up period.

Analyses are ongoing. However, preliminary results suggest that type of haematological malignancy is associated with PRWC; the highest proportion of PRWC was found among patients with multiple myeloma $(58 \%)$, and the lowest among patients with Hodgkin lymphoma (13\%).

Preliminary data also suggest that comorbidity and anxiety or depression following diagnosis is associated with PRWC.

Conclusion: The final results are expected to be ready for presentation at the conference.

\section{P-006}

Reproduction of young males who have survived a cancer diagnosis-preliminary results from a national cohort study in Norway

Topic: Reviewer's choice

Presenter: Maria Winther Gunnes

Maria Winther Gunnes 1); Dag Moster 1); Tone Bjørge 1); Sara Ghaderi 1); Rolv Terje Lie 1)

1) Dept of Global Public Health and Primary Care, University of Bergen, Norway

Background: The number of survivors of childhood, adolescent and young adult cancer has shown a steady increase over the past 30 years, and with this follows an increasing population of adults in need of special care and counseling, especially during special life events such as pregnancy. A substantial number of publications regarding reproductive outcomes of female survivors exists, but less information is available regarding male survivors.

Objectives: The objective of this study was to study specific reproductive issues in a national cohort of men surviving a cancer diagnosis before the age of 25 and compare with the general population in Norway.

Methods: A total of 542,691 men born in Norway 1967-1985, still alive and living I Norway at age 15, were followed through 2011 by linking the Norwegian Cancer Registry, the Medical Birth Registry and the Central Population Registry. Fertility, use of assisted reproductive technologies (ART), congenital anomalies, preterm birth and birth weight of offspring were assessed in logistic regression models comparing cancer and non-cancer survivors.

Results: A total of 2,431 men in the cohort were diagnosed with cancer before the age of 25 , of which 978 had fathered at least one child. Fertility was significantly reduced in the male cancer group (OR of fathering at least one child $=0.48,95 \%$ CI $0.44-0.52$ ) compared to the non-cancer group. An increased proportion of offspring of cancer survivors were conceived by assisted reproductive technologies (OR $=3.62,95 \%$ CI 3.08-4.93), especially for survivors of Hodgkin lymphoma and testicular cancer. We did not find any increased risk for preterm birth or low birth weight. Neither did we find an increased risk of congenital malformations.

Conclusions: Further in-depth analyses on large cohorts of male cancer survivors are needed in order to clarify fertility and outcomes of pregnancy in their partners. Our results suggest reduced fertility of the male survivors, but no increased risk for congenital malformations, preterm birth or low birth weight in their offspring.

\section{P-007}

Acute maternal infection and risk of pre-eclampsia: a populationbased case-control study

Topic: Reviewer's choice

Presenter: Caroline Minassian

Caroline Minassian 1); Sara L Thomas 1); David J Williams 2); Oona Campbell 1); Liam Smeeth 1)

1) Faculty of Epidemiology and Population Health, London School of Hygiene and Tropical Medicine; 2) Institute for Women's Health, University College London Hospital

Background: Infection in pregnancy may be involved in the aetiology of pre-eclampsia, possibly by enhancing the maternal inflammatory response. However, a clear association between acute maternal infection and pre-eclampsia has not been established. Previous studies assessing the role of maternal urinary infection in preeclampsia failed to account for renal disease and other known confounders, and data on the effects of other acute infections are lacking. Objectives: To assess whether acute urinary tract infection, respiratory tract infection, and antibiotic drug prescriptions in pregnancy (a likely proxy for maternal infection) are associated with an increased risk of pre-eclampsia.

Methods: We used a matched nested case-control design and data from the UK General Practice Research Database to examine the association between maternal infection and pre-eclampsia. Primiparous women aged at least 13 years and registered with a participating practice between January 1987 and October 2007 were eligible for inclusion. We selected all cases of pre-eclampsia and a random sample of pregnant women without pre-eclampsia (controls). Cases were individually matched with up to ten controls on practice and year of delivery. We calculated odds ratios and $95 \%$ confidence intervals for pre-eclampsia comparing women exposed and unexposed to infection using multivariable conditional logistic regression. Results: 1,533 cases and 14,236 controls met the eligibility, inclusion and matching criteria. After adjusting for maternal age, pre-gestational hypertension, diabetes, renal disease and multifetal gestation, the odds of pre-eclampsia were increased in women prescribed antibiotic drugs (adjusted odds ratio $1.28 ; 1.14-1.44$ ) and in women with urinary tract infection (adjusted odds ratio $1.22 ; 1.03-1.45$ ). We found no association with maternal respiratory tract infection (adjusted odds ratio $0.91 ; 0.72-1.16$ ). Further adjustment for maternal smoking and pre-pregnancy body mass index made no difference to our findings. Conclusions: Primiparous women who acquire a urinary tract infection during pregnancy, but not those who have a respiratory tract infection, are at an increased risk of pre-eclampsia. Maternal antibiotic drug prescriptions are also associated with an increased risk. Further research is required to elucidate the underlying mechanism of this association and to determine whether, among women who acquire infections in pregnancy, prompt treatment or prophylaxis against infection might reduce the risk of pre-eclampsia.

\section{P-008}

Somatic complaints are associated with incident type 2 diabetes: results from the MONICA/KORA Augsburg cohort study 1984-2002

Topic: Reviewer's choice 


\section{Presenter: Jens Baumert}

Jens Baumert 1); Karoline Lukaschek 1); Johannes Kruse 2); Rebecca Thwing Emeny 1); Ina-Maria Rückert 1); Christa Meisinger 1); Karl-Heinz Ladwig 1)

1) Institute of Epidemiology II, Helmholtz Zentrum München, Neuherberg, Germany; 2) Department of Psychosomatic Medicine and Psychotherapy, University of Gießen and Marburg, Gießen, Germany

Background: Somatic complaints such as vertigo, palpitation or numbness are reported frequently by subjects but can often not be assigned to a specific underlying disease. However, several studies indicate associations of somatic complaints with severe outcomes which have led to a growing interest on the role of somatic complaints in risk prediction.

Objectives: The aim of the present study was to assess the impact of somatic complaints on the incidence of type 2 diabetes.

Methods: The study population consisted of 10,511 participants aged 25-74 years drawn from the population-based MONICA/KORA Augsburg Cohort conducted in 1984-2002 in the Augsburg region (Southern Germany). Somatic complaints were assessed by a selfadministrated questionnaire using the von Zerssen symptom checklist and calculating a score by the sum of the nine single items faintness, feelings of heaviness in the legs, vertigo, eye fibrillation, dyspnea, feelings of suffocation, palpitation, numbness and strong perspiration. Cox regression using fractional polynomials (FP) transformations was applied to estimate the risk of the somatic complaints score on the incidence of type 2 diabetes within a mean follow-up time of 12 years.

Results: The somatic complaints score were higher in women than in men, in older age groups, obese subjects and in subjects with hypertension $(p<0.001)$. Cox regression adjusted for age, sex and survey revealed a significant linear increase of type 2 diabetes risk by somatic complaints with a hazard ratio (HR) of $1.052(95 \% \mathrm{CI}$ $1.046-1.058, p<0.001)$ per one unit increase of the somatic symptom score. Adjusting additionally for metabolic risk factors these estimates were attenuated $(\mathrm{HR}=1.029)$ but remained significant $(<0.001)$. No significant modification of these effects by age, sex or any of the metabolic risk factors was observed.

Conclusions: Somatic complaints were significantly associated with incident type 2 diabetes, even after adjusting for metabolic risk factors. Further research is needed to assess the underlying pathophysiological mechanism and potential causal relationships between somatic complaints and diabetes.

\section{P-009}

Ischaemic atherosclerotic disease and chest pain prior to acute myocardial infarction and subsequent coronary mortality

Topic: Reviewer's choice

Presenter: Emily Herrett

Emily Herrett 1); Krishnan Bhaskaran 1); Adam Timmis 2); Spiros Denaxas 3); Harry Hemingway 3); Liam Smeeth 1)

1) London School of Hygiene and Tropical Medicine; 2) Barts and the London School of Medicine and Dentistry; 3) University College London

Background: Chest pain, angina and ischaemia in different arterial territories occurring shortly before myocardial infarction may be clinical correlates of ischaemic preconditioning. However, no studies have evaluated prospectively collected information on ischaemia and their effects on short and long term coronary mortality.
Objective: To compare patients with and without pre-infarction new ischemic presentations in terms of subsequent infarct characteristics and coronary mortality.

Methods: As part of the 'CArdiovascular disease Research using LInked Bespoke studies and Electronic health Records' (CALIBER) programme we linked data from primary care, hospital admission, an acute coronary syndrome register and cause specific mortality to identify patients with first acute myocardial infarction $(\mathrm{N}=16429)$. We analyzed time from myocardial infarction to coronary heart disease mortality ( $\mathrm{n}=5,282$ deaths) using Cox regression over a median of 2.6 years follow up, comparing patients with and without a recent new ischaemic presentation, defined as a primary care consultation for chest pain or new atherosclerotic disease in the 90 days before myocardial infarction.

Results: Patients with new ischaemic presentations in the 90 days before myocardial infarction experienced lower coronary heart disease mortality in the first 7 days after myocardial infarction compared to those with no prior ischaemic presentations, after adjusting for age, sex, smoking, diabetes, blood pressure and cardiovascular medications [hazard ratio 0.68 (95\% CI $0.58-0.79) \quad P<0.001$ ], but subsequent mortality was higher [hazard ratio 1.46 (1.17-1. 82) $P=0.001]$. Patients with ischaemic presentations closer in time to their infarct had the lowest 7 day mortality $(p$-trend $=0.016)$. For patients in whom myocardial infarction type was recorded, those with previous ischaemia were more likely to have non ST-elevation type (62.8\% of myocardial infarctions, compared with $42.5 \%$ among those with no prior ischaemic presentations).

Conclusion: In the first large prospective study of ischaemic presentations prior to myocardial infarction, we have shown that those occurring closest to the infarct are associated with lower short term but higher long-term coronary mortality following myocardial infarction. These observations could represent a natural ischaemic preconditioning effect, observed in a clinical setting.

\section{P-010}

Epidemiology of occupational accidents with severe outcome in 2002-2011 in the Arkhangelsk region, Northwest Russia

Topic: Reviewer's choice

Presenter:

Zhanna Varakina 1), 2); Alexander Vyazmin 1); Anatoliy Sannikov 1); Andrej Grjibovski 2), 3)

1) Department of Public Health, Health Care and Social Work, Northern State Medical University, Arkhangelsk, Russia; 2) International School of Public Health, Northern State Medical University, Arkhangelsk, Russia; 3) Department of International Public Health, Norwegian Institute of Public Health, Oslo, Norway

Background: Incidence of occupational accidents with severe outcome (OASO) is one of most important indicator of occupational safety. In Russia, around 130 workers per day experience OASO, degree of disability is estimated for 20 of them mainly in mining industry, agriculture, construction and transport.

Objectives: To describe the occurrence and causes of OASO in the Arkhangelsk region, Northwest Russia, in 2002-2011.

Methods: A descriptive study. Data on all OASO reported in the Arkhangelsk region in 2002-2011 were obtained from the State Labor Inspection. The incidence of OASO was calculated by age, gender and branches per 100000 employed. The age was categorized into 10-year groups except the group of 16-29-year olds and those who were 60 years of age and older. Data on the number of employed were collected at the Regional Federal State Statistics Service. Ninety-five 
percent confidence intervals CI were calculated for all incidence rates using the Wilson's method.

Results: The overall incidence of OASO for the study period was 19.9 (95\% CI 16.8-23.6) per 100,000. Altogether, 1,282 cases occurred during the study period, $1,088(84.9 \%)$ of them occurred to men. The incidence of OASO decreased from 44.9 (95\% CI 38.4-52.6) in 2002 to 20.9 (95\% CI 16.5-26.4) in 2011 among men and from 11.3 (95\% CI 8.2-15.6) in 2002 to 4.5 (95\% CI 2.7-7.5) in 2011 among women. The incidence of OASO decreased over time in all age-groups, the highest was recorded in the 60 years and above [25.5 (95\% CI 18.8-33.0)] and 50-59 years old [23.2 (95\% CI 20.0-25.6)]. Agriculture, hunting and forestry sector has the highest incidence [47.5 (95\% CI 43.1-54.2)] followed by construction [39.7 (95\% CI 38.0-40.1] and manufacturing and mining sector [39.2 (95\% CI 35.9-42.7)]. In manufacturing, the largest proportions of OASO were in timber processing and pulp-and-paper industry. The decrease in overall incidence of OASO might be associated with the computerization of different industries and probably also with the improvement in occupational safety in the region. At the same time, we found that OASO incidence for the study period has increased in the economic sector of operations with real estate, rent and rendering of services (by 5.3 times). The most important causes for OASO where unsatisfactory labour organization $(29.4 \%)$, infringement of the accident prevention $(13.3 \%)$ and unsatisfactory organization and maintenance of the territory, workplaces $(10.3 \%)$.

Conclusions: The incidence of OASO in the Arkhangelsk region is high although it has decreased over the last decade and shows different trends by gender, branches and age groups.

\section{P-011}

The sex prevalence of major congenital anomalies (CA) in a United Kingdom (UK) primary care population

Topic: Reviewer's choice

Presenter: Rachel Sokal

\section{Rachel Sokal 1); Laila Tata 1); Linda Fiaschi 1); Kate Fleming 1)}

1) Division of Epidemiology and Public Health, University of Nottingham, Nottingham, United Kingdom

Background: Sex differences in several specific congenital anomalies (CA) have been frequently documented. However, few studies have assessed differences across the entire spectrum of CAs and there is a lack of studies conducted at a population level that have been able to adjust for socio-demographic and maternal factors.

Objectives: To assess sex differences in major congenital anomaly (MCA) diagnoses within a large population-based data source representative of the whole of the United Kingdom (UK) and to examine the effects of socio-demographic and maternal factors.

Methods: We included all children born between 1990 and 2009 who had records in a large UK primary care research database (The Health Improvement Network) and classified their diagnoses of MCA according to the European Surveillance of Congenital Anomalies. Prevalence was calculated in males and females for any and 60 individual MCA diagnoses per 10,000 live births. Prevalence ratios (PR) with $95 \%$ confidence intervals were calculated to compare the MCA risk in males to females. In a sub-population with linked maternal records, we assessed the effects of socioeconomic status (Townsend deprivation index), geographical area, year of birth and maternal age, smoking, body mass index, diabetes and epilepsy status. Results: We identified 794,169 children of whom $51.4 \%$ were male. The prevalence of any MCA was 307 per 10,000 (95\% CI 302-313) in males and 243 per 10,000 in females (95\% CI 238-248) resulting in a $26 \%$ greater overall risk in males (PR 1.26, $95 \%$ CI 1.23-1.30).
PRs indicated important differences between males and females across six of 15 system-specific MCA subgroups. MCA risks were greater in males in five systems: genital $(\mathrm{PR}=16.6,95 \% \mathrm{CI}$ 14.2-19.4), urinary (1.53, $95 \%$ CI 1.39-1.67), musculoskeletal (1.36, $95 \%$ CI 1.20-1.54) digestive (1.29, $95 \%$ CI 1.12-1.49), and orofacial clefts $(1.20,95 \%$ CI 1.07-1.34) but lower in males for limb defects (PR 0.71, $95 \%$ 0.67-0.75). System-level PRs, however, masked considerable sex variation in individual diagnoses. For example within limb defects there was a female preponderance of hip dislocation (PR 0.26, $95 \%$ CI 0.23-0.29) whereas clubfoot and polydactyl were more common in males. Socio-demographic and maternal factors had little effect on sex ratios.

Conclusions: Overall the risk of MCA is greater in males than females although this masks substantial sex variation for individual diagnoses. Socio-demographic and maternal factors do not appear to affect these relationships.

\section{P-012}

Congenital cerebral palsy, child sex and parent cardiovascular risk

Topic: Reviewer's choice

Presenter: Elani Streja

Elani Streja 1); Chunsen Wu 2); Peder Uldall 3), 4); Jakob Grove 5); Onyebuchi Arah 1); Jørn Olsen 1), 2)

1) Department of Epidemiology, School of Public Health, University of California, Los Angeles, California; 2) Section for Epidemiology, Department of Public Health, Aarhus University, Aarhus, Denmark; 3) The Danish Cerebral Registry, National Institute of Public Health, Southern University, Denmark; 4) Pediatric Department,

Rigshospitalet, University of Copenhagen, Denmark; 5) Institute of Biomedicine and Bioinformatics Research Centre, University of Aarhus, Aarhus, Denmark

Background: Genes associated with cardiovascular disease may be risk factors for congenital cerebral palsy $(\mathrm{CP})$ and this association may be modified by sex since there is an increased risk of $\mathrm{CP}$ in male children.

Objective: We investigated the association between $\mathrm{CP}$ of the child with cardiovascular disease in parents, taking sex of the child in consideration.

Methods: All parents of non-adopted singletons born in Denmark between 1973 and 2003 were included. Parents who had a child with congenital cerebral palsy (CP) confirmed by the Danish National CP registry were considered exposed. Cox proportional hazards regressions were used to model risk of cardiovascular outcomes for exposed parents compared to all other parents beginning at child's 10th birthday. Results: We identified 733,896 mothers and 666,945 fathers among whom 1,591 and 1,483 , respectively, had a child with CP. The mean age for mothers at end of follow up was $50 \pm 8$ years. After adjustment for maternal age, maternal education, child's sex, child's residence, child being small for gestational age and maternal hypertensive disorder during pregnancy, mothers of $\mathrm{CP}$ male children had excess cardiovascular disease (HR: 1.48, $95 \%$ CI 1.13-1.95), attributable mostly to an increased incidence of hypertension and cerebrovascular disease. The association became nonsignificant for cardiovascular disease and hypertension but remained significant for cerebrovascular disease after adjustment for preterm birth (HR: 2.75, $95 \%$ CI 1.41-5.48). There was no increased risk of cardiovascular events in mothers of female $\mathrm{CP}$ children or fathers of $\mathrm{CP}$ children of any sex.

Conclusions: Women that have a male child with $\mathrm{CP}$ are at increased risk for premature cardiovascular disease. Preterm delivery was an important confounder or mediator for this association. 


\section{P-013}

Female reproductive factors and risk of Parkinson's disease

Topic: Reviewer's choice

Presenter: Naomi Greene

\section{Naomi Greene 1); Christina Funch Lassen 2); Beate Ritz 1)}

1) UCLA School of Public Health, Department of Epidemiology, Los Angeles, CA, USA; 2) The Research Center, Danish Cancer Society

Background: Parkinson's disease is the second most common neurodegenerative disorder, causing disability and loss of quality of life in more than $1 \%$ of all people over age 60 . Most epidemiologic studies have found that PD is more common in men than in women at an approximate ratio of 1.5:1. To date, there is no consensus as to whether female reproductive factors including hormone use affect Parkinson's disease risk. Additionally and less-well studied, women may be at lower risk of Parkinson's disease due to lower systemic and brain iron before menopause because of menstruation. Childhood overweight, an early source of estrogens, has not been evaluated in studies to date.

Objective: Our objective was to examine the relationship between Parkinson's disease and female reproductive factors in the largest population-based Parkinson's disease case-control study to date.

Methods: 743 female Parkinson's disease cases diagnosed between 1996 and 2009 were selected from the Danish National Hospital Register, diagnoses confirmed by medical record review, and the cases matched by birth-year to 765 female controls randomly selected from the Danish Civil Registration System. Covariate information was collected in computer-assisted telephone interviews covering an extensive array of topics including reproductive and lifestyle factors. Results: Early menarche (first period at $\leq 11$ years), ever-use of oral contraceptives, high parity ( $\geq 4$ children), and bilateral ovariectomy were negatively associated with Parkinson's disease risk; the adjusted odds ratios $[\mathrm{aOR}]$ and $95 \%$ confidence limits [CL] were respectively 0.68 (CL 0.41,1.12) for early menarche, 0.90 (CL 0.67,1.20) for use of contraceptives, 0.70 (CL 0.46,1.06) for high parity, and 0.61 (CL $0.38,0.98)$ for bilateral ovariectomy. The aOR for ever-use of postmenopausal hormones was 0.88 (CL $0.64,1.20$ ). We found no support for associations between Parkinson's disease and short fertile life length ( $<36$ years between menarche and menopause), or age at menopause. The aOR for use of iron supplementation for more than a year was 1.87 (CL 1.35,2.60) and for childhood overweight, 1.31 (CL 0.89,1.92).

Conclusions: Female reproductive factors occurring relatively early in women's reproductive lives, such as age at menarche, oral contraceptives use, high parity, and surgical menopause appear to be predictive of subsequent Parkinson's disease risk.

\section{P-014}

Smoking and risk of provoked and unprovoked venous thromboembolism: a Danish cohort study

Topic: Reviewer's choice

Presenter: Henrik Solli

Henrik Solli 1); Morten Schmidt 1); Morten Olsen 1); Rikke Bech Nielsen 1); Finn Breinholt Larsen 2); Henrik Toft Sørensen 1)

1) Department of Clinical Epidemiology, Aarhus University Hospital, Aarhus, Denmark; 2) Public Health and Quality Improvement, Central Denmark Region, Aarhus, Denmark

Background: Smoking is a strong risk factor for myocardial infarction and stroke, but it is less clear if smoking also is a risk factor for venous thromboembolism (VTE).
Objectives: To examine if smoking is a risk factor for VTE. Methods: We performed a cohort study based on self-reported questionnaire data from the Danish survey How Are You?. We categorized smoking status into never smokers (never smoked on a daily basis), former smokers (previously smoked on a daily basis, but not currently), and current smokers (smoked on a daily basis at the time of the questionnaire). Using the Danish National Registry of Patients, covering all Danish hospitals, we identified all inpatient and outpatient diagnoses of VTE. We subdivided VTE into provoked VTE (diagnosis of fracture, surgery, trauma, or pregnancy within 90 days before, or a diagnosis of malignancy prior to or within 90 days after the VTE diagnosis) and unprovoked VTE (remaining cases). We followed participants from return of questionnaire in spring 2006 until VTE, emigration, death, or 31 December 2011, whichever came first. We used Cox regression with person age as the underlying time-scale to compute hazard ratio (HR) with $95 \%$ confidence interval adjusting for sex.

Results: Our study cohort consisted of 21,439 individuals, among which $41.7 \%$ were never smokers, $27.7 \%$ were former smokers, and $25.6 \%$ were current smokers (4.9\% had missing data). A total of $53.2 \%$ were female and the average age was 51 years. Compared with never smokers, the adjusted HR for VTE overall was $0.76(95 \%$ CI 0.50-1.16) for former smokers and 1.47 (95\% CI 1.01-2.16) for current smokers. The adjusted HR for provoked VTE was $0.90(95 \%$ CI 0.46-1.76) for former smokers and 1.74 (95\% CI 0.94-3.22) for current smokers. For unprovoked VTE, the adjusted HR was 0.69 (95\% CI 0.40-1.19) for former smokers and $1.37(95 \% \mathrm{CI}$ 0.84-2.22) for current smokers.

Conclusions: Current smoking was associated with an increased risk of both provoked and unprovoked VTE, whereas former smoking was not.

\section{P-015}

Trends in breast cancer stage distribution in Norway before, during, and after introduction of a screening program

Topic: Reviewer's choice

Presenter: Mette Lise Kronborg

Mette Lise Kronborg 1); Ivar Sønbø Kristiansen 2); Bjørn Møller 3); Henrik Støvring 1)

1) Department of Public Health, Biostatistics, Aarhus University, Aarhus, Denmark; 2) Department of Health Management and Health Economics, Oslo University, Oslo, Norway; 3) The Cancer Registry of Norway, Oslo, Norway

Background: Screening is intended to detect cancer at a less advanced stage thereby shifting the stage distribution towards more locally confined stages.

Objectives: Estimate the trends in stage-specific breast cancer incidence, before, during, and after the gradual introduction of a national screening program.

Methods: From the Norwegian Cancer Registry we retrieved information on all women aged 20 or older diagnosed with a first-time breast cancer (ICD-10 code: C50) during the period 1987-2010 in Norway (approximate population size: 4.9 million). Data included date of diagnosis, stage at diagnosis and age at diagnosis. Data on size of population at risk were drawn from Statistics Norway. We defined three calendar-time periods: before (1987-1995), during (1996-2004), and after (2005-2010) the introduction of screening; and three age groups: women eligible for screening (50-69), younger (20-49), or older (70+). Poisson regression was used to estimate the incidence of localized stage (I) or more advanced stages (II+) in each of the three time periods in each age group. 
Results: In total 48,076 women were diagnosed with breast cancer during the study period with reported stage. The incidence of stage I breast cancer among women aged 50-69 more than doubled from 63.4 per 100,000 per year before the introduction of screening to 140.3 per 100,000 afterwards, corresponding to an increase of $121 \%$ (95\% confidence interval: $110 \% ; 133 \%$ ). The incidence of stage II+ increased from 86.9 to 117.3 per 100,000 afterwards, corresponding to a $35 \%(29 \% ; 42 \%)$ increase. The incidence in the younger age group was 16.6 per 100,000 per year before increasing with $12 \%$ ( $3 \% ; 21 \%$ ) to 18.6 afterwards of stage I, and 25.5 before increasing with $32 \%(24 \%$; $40 \%)$ to 33.6 afterwards of stage II+ breast cancers. The change in incidence of localized stage was thus significantly higher in the screened age group, whereas the changes for more advanced stages were similar for the two.

Conclusions: The absolute incidence of earlier stage cancers increased among women aged 50-69 after the introduction of the screening programme, while the incidence of more advanced cancers was not reduced in the same time period, also when compared to the corresponding development in the younger age group.

\section{P-016}

Reproductive history \& mortality in postmenopausal women with breast cancer in the European prospective investigation into cancer and nutrition (EPIC)

Topic: Reviewer's choice

Presenter: Mai Kadi

Mai Kadi 1); Elio Riboli 1); Petra Wark 1); Marc Gunter 1); Teresa Norat 1); Petra Peeters 1), 2); Anne Tjønneland 3), 4); Pernille Envold Bidstrup 4), 5); Laure Dossus 6); on behalf of the EPIC investigators)

1) Imperial college London, Department of Epidemiology and Biostatistics, School of Public Health; 2) Department of Epidemiology, Julius Center for Health Sciences and Primary Care, University Medical Center Utrecht; 3) Faculty of Health Sciences, University of Copenhagen; 4) Danish Cancer Society Research Center; 5) Department of Psychosocial Cancer Research; 6) CESP Equipe Nutrition, Hormones et Santé des Femmes

Background: It has been hypothesised that factors influencing breast cancer incidence could also represent risk factors for prognosis and survival among breast cancer patients. However, while there is substantial evidence supporting an association of reproductive and hormonal factors with risk of developing breast cancer, data relating reproductive factors to survival among breast cancer survivors are more limited and generally inconclusive.

Objective: To investigate associations between reproductive factors and all-cause and breast cancer-specific mortality among postmenopausal women diagnosed with breast cancer in the EPIC study.

Methods: We used data from 5,872 breast cancer cases that were identified among 145,362 women who were postmenopausal at study entry in the European Prospective Investigation into Cancer and Nutrition (EPIC). During an average 8.6 years of follow up, 852 deaths occurred. Cox proportional hazards modelling was used to evaluate associations between reproductive factors (parity, age at menarche and menopause, use of exogenous hormones) and all-cause and breast cancer-specific mortality.

Results: Having ever been pregnant was associated with a 25 and $33 \%$ reduced risk of all-cause and breast cancer-specific mortality in age- and centre-adjusted models compared to being nulliparous (hazard ratio [HR]: $0.75,95 \%$ confidence interval [CI]: $0.61-0.93$ and HR: $0.67,95 \% \mathrm{CI} 0.50-0.91$, respectively). The multivariate model adjusted for BMI, physical activity, smoking status, alcohol drinking, education level, breast cancer stage and HT use showed a significant $41 \%$ reduced risk of breast cancer-specific mortality in women who have ever been pregnant compared to being nulliparous (HR: 0.59 , $95 \%$ CI 0.40-0.88). Giving birth to three children was associated with a significantly reduced risk of breast cancer-specific mortality in the multivariate model compared to being nulliparous (breast cancer-specific: 3 births vs. nulliparous, HR: 0.56, $95 \% \mathrm{CI}$ $0.34-0.92 ; P$-trend $=0.01$ ).

Conclusion: Our results show that certain reproductive factors that influence breast cancer incidence in postmenopausal women may also influence survival among breast cancer patients.

\section{P-017}

Internet use and cancer preventive behaviours in the English Longitudinal Study of Ageing

Topic: Reviewer's choice

Presenter: Andre Xavier

Andre Xavier 1); Eleonora Dorsi 2); Jane Wardel 3); Samuel Smith 3); Christian Von Wagner 3)

1) University of South of Santa Catarina, Florianópolis, Santa Catarina, Brazil; 2) Department of Public Health, Federal University of Santa Catarina, Florianópolis, Santa Catarina, Brazil; 3) Department of Epidemiology and Public Health, University College London, London, UK

Background: Internet use has been shown to mitigate physical, mental and socioeconomic restrictions associated with old age. Less is known about its association with health behaviors. This study examined associations between extent of internet use over 8 years and subsequent primary and secondary cancer preventive behaviors in older adults.

Method: Data were from the English Longitudinal Study of Ageing (ELSA); which is a biennial, longitudinal, panel study of men and women living in England aged 50 years or over. Data were from Waves 2-5 collected in 2004-2005, 2006-2007, 2008-2009, and 2010-2011. A self-report measure of internet use was completed at each wave. Health behavior outcomes assessed at wave 5 were early detection (breast and colorectal cancer (CRC) screening) and lifestyle (daily intake of at least 5 portions of fruits/vegetables, weekly moderate/vigorous physical activity, current smoking). All analyses controlled for age, sex, wealth, education, health literacy, cognitive function and limitations in activities of daily living.

Results: We included individuals with data available for waves $2-5$ $(\mathrm{n}=5,625)$. Just over a quarter $(27 \%)$ reported using the internet at all 4 waves ('consistent users'), $30 \%$ reported internet use in 1-3 waves ('intermittent users') and $43 \%$ never reported internet use ('never users'). Each of the health behaviors was significantly associated with levels of internet use: CRC screening (never users: $53 \%$, intermittent users: $62 \%$, consistent users: $70 \%$ ), breast screening $(83,95,97 \%)$, physical activity $(60,78,88 \%)$, fruit and vegetable intake $(53,58,62 \%)$ and current smoking $(13,11,7 \%)$. A multivariable analysis confirmed that compared with never users, those who used the internet in all waves were more likely to report CRC screening $(\mathrm{OR}=1.90 ; 95 \% \mathrm{CI}=1.37-2.64)$, physical activity $(\mathrm{OR}=1.71 ; 1.38-2.12)$ and fruit and vegetable consumption $(\mathrm{OR}=1.28 ; 1.08-1.51)$ and less likely to report current smoking $(\mathrm{OR}=0.59 ; 0.44-0.79)$. Women in the intermittent user group were more likely to report breast screening $(\mathrm{OR}=1.62 ; 1.00-2.63)$.

Conclusions: Older adults in the ELSA cohort who reported using the internet were more adherent to primary and secondary cancer prevention behaviors independent of socio-demographic, cognitive and 
health factors. Promoting internet use may help to enhance healthy lifestyles.

\section{P-018}

Cancer mortality and recognized risk factors among 180 countries, by using spatial analysis and 3D modeling

Topic: Diseases - COPD

Presenter: Dimitra Sifaki-Pistolla

\section{Dimitra Sifaki-Pistolla 1); Georgia Pistolla 2)}

1) Epidemiologist, PhD student, School of Medicine, University of Crete; 2) Mathematician, PhD, School of Medicine, University of Crete

Background: Cancer is one of the major causes of mortality worldwide. Several studies have been conducted in order to monitor and capture the disease's dynamics and future risk. Contrary to this, little research has been done with recognizing the four three of epidemiology; place, time, person.

Objectives: This study aims to monitor and describe the impact of several behavioral, environmental, clinical and demographic risk factors on cancer mortality rates by using the spatial statistics and dynamic modeling analysis as an alternative to conventional statistical methods. Finally, it will capture the diseases dynamics by the use of $3 \mathrm{D}$ modeling and will propose future preventive measures through new technologies.

Methods: Data were obtained from the World Health Organization database (WHO) in 2010 from 180 different countries globally and referred to cancer deaths per 100,000 people and several risk factors. Spatial statistics were applied in order to test cancer mortality and the associated risk factors. Specifically, spatial clustering (Moran's I) and spatial Ordinary Least Squares (OLS) regression were performed for the detection of spatial autocorrelation and high risk areas in the Arcmap 10. Finally, 3D maps were constructed in the ArcScene 10. Results: Positive spatial autocorrelation was found in 10 out of 14 risk factors and in cancer deaths (Moran's $\mathrm{I}=0.12 ; p$ value $=0.02$; $95 \% \mathrm{CI}$ ). High spatial heterogeneity and variance was observed. Through OLS, high risk areas were estimated and the strong correlation and effect of the 10 risk factors (such as tobacco, alcohol, outdoor pollution exposure and BMI) was proved. USA, Canada, Chile, Hungary, Greece, Turkey, Sweden, Russia, UK, France, Spain, Germany, Italy, China and other countries were identified as risk areas (std.Dev. $=1.09-2.54 ; p$ value $<0.001$ ).

Conclusions: The present study introduces an interesting approach for research of cancer mortality and the main basic risk factors at an international level, while it suggests an alternative analysis of cancer statistics. An analysis at the national level remains to be tested in order to provide additional insights on cancer determinants. Finally, several preventive measures are suggested.

\section{P-019}

Cold temperatures and daily counts of ambulance calls for asthma exacerbations in Astana, Kazakhstan in 2006-2010

Topic: Diseases - COPD

Presenter: Andrej Grjibovski

Andrej Grjibovski 1), 2); Bibigul Adilbekova 3); Bayan Aynabekova 3); Gulmira Kozhakhmetova 3); Nassikhat Nurgaliyeva 3), 4); Aliya Kosbayeva 4); Bettina Menne 5)

1) Department of International Public Health, Norwegian Institute of Public Health, Oslo, Norway; 2) International School of Public
Health, Northern State Medical University, Arkhangelsk, Russia; 3) Medical University of Astana, Astana, Kazakhstan; 4) WHO office in Kazakhstan, Astana, Kazakhstan; 5) WHO European Centre for Environment and Health, Bonn Office, Bonn, Germany

Background: Associations between cold temperatures and mortality from respiratory diseases have been observed in many countries. Less is known about the effects of temperature on acute morbidity.

Objectives: To study associations between minimum apparent temperature and the number of ambulance calls for asthma in Astana, Kazakhstan during the cold season.

Methods: Daily counts of ambulance calls for asthma (ICD-10 code J45) for the population of Astana, Kazakhstan, for the cold seasons (1 October-31 March) during 2006-2010 were obtained from the Municipal Ambulance Station. Associations between the number of calls and minimum apparent temperature (average for lags 0-15) were studied using Poisson regression controlling for wind speed and effects of month, year, weekends and holidays. Analyses were performed separately for children and adults aged 18-44, 45-59 and 60 years and older.

Results: Altogether, there were 7,372 ambulance calls for asthma during cold seasons of 2006-2010. An inverse association between minimum apparent temperature and absolute counts of calls was observed for the age-group 60 years and older ( 3,110 calls): decrease of temperature was associated with an increase in the number of calls by $1.7 \%$ (95\% CI $0.1-3.3 \%$ ) across the whole temperature spectrum. No associations between the temperature and the number of calls were found among other age-groups.

Conclusions: The results suggest an inverse association between the minimum apparent temperature and the number of ambulance calls for asthma during the cold season in Astana, Kazakhstan, but this is limited to the oldest age-group. Further research is needed to study sensitivity and adaptive capacity of this vulnerable group to develop strategies to mitigate the health risks related to climate change.

\section{P-020}

Longitudinal association of C-reactive protein and lung function over 13 years: European prospective investigation into cancer Norfolk study

Topic: Diseases - COPD

Presenter: Sara Ahmadi Abhari

Sara Ahmadi Abhari 1); Stephen Kaptoge 1); Robert Luben 1); Nicholas Wareham 2); Kay-Tee Khaw 1)

1) Department of Public Health and Primary Care, University of Cambridge, Cambridge, UK; 2) MRC Epidemiology Unit, Institute of Metabolic Sciences, Cambridge, UK

Background: Chronic obstructive pulmonary disease and impaired lung function are known to be associated with systemic inflammation. However, the longitudinal association of C-reactive protein (CRP) and lung function, especially among never-smokers, has not been thoroughly investigated in large prospective studies.

Objectives: We aimed to investigate the cross-sectional and longitudinal association of CRP and lung function and the association of temporal change in CRP and change in lung function over time.

Methods: In the EPIC-Norfolk cohort study of men and women aged 40-79 years at baseline, lung function was assessed by measurement of forced vital capacity (FVC) and forced expiratory volume in one second (FEV1) at baseline, 4, and 13 years. Serum CRP was measured using a high-sensitivity assay at baseline and at 13 year followup. Cross-sectional and longitudinal associations of loge-CRP and lung function were examined using multivariable linear mixed models adjusted for age, sex, height, body mass index, smoking, physical 
activity, alcohol intake, corticosteroid medication, and for women only, menopausal status and postmenopausal hormone replacement therapy. Rate of longitudinal change of FEV1 or FVC across categories of baseline CRP was calculated in mixed models from the interaction term for CRP*time.

Results: Among participants that attended and had provided sufficient data for analysis at the baseline $(\mathrm{N}=18,110)$ and follow-up health examinations, median (inter-quartile range) CRP (mg/l) was 1.3 $(0.7-2.6)$ at baseline and $2.0(1.3-3.5)$ at 13 years. Mean (standard deviation (SD)) FEV1 (ml) at the baseline, 4, and 13 years were 2,699 (701), 2,610 (701), and 2,448 (712) respectively. The corresponding figures for FVC (ml) were 3,286 (911), 3,114 (878), and 3,000 (888). In the cross-sectional analysis, serum loge CRP level was negatively associated with lung function. A 1-SD higher baseline loge-CRP (about three-fold higher CRP) was associated with $-86.3 \mathrm{ml}(95 \%$ CI -93.9, -78.6) lower FEV1. Longitudinal change in CRP over 13 years was also negatively associated with decline in lung function over the same period, although to a lesser extent than that found in the cross-sectional analyses, with a 1-SD higher longitudinal change in loge-CRP associated with $-64.0 \mathrm{ml}(95 \% \mathrm{CI}-72.1,-55.8)$ decline in FEV1. The associations were similar for FVC and persisted among lifetime never-smokers. Baseline CRP levels were not predictive of rate of change of FEV1 or FVC over time.

Conclusion: In this study we found longitudinal observational evidence suggesting that increase in systemic inflammation is associated with a decline in lung function.

\section{P-021}

COPD as an indicator of urban metabolism and an upshot of people and place

Topic: Diseases - COPD

Presenter: Sara Velho

\section{Sara Velho 1); Ana Monteiro 1), 2); Mário Almeida 1);} Vânia Carvalho 1)

\section{1) ISPUP; CITTA; 2) Department Geography Porto University}

Background: COPD is a disease state that affect more than 210 million people in the world and that cause the death of more than 3 million/year (WHO, 2010). It is characterized by the presence of airflow limitation that can be accompanied by airway hyper reactivity (American Thoracic Society 1995). It depends on genetics deficiency in alpha-1-antitrypsin (Pauwels et al. 2001; Wood and Stockley 2006) and the high prevalence among siblings (Hemminki et al. 2008) and the higher reactivity and severity observed in blacks compared with other races (Eisner et al. 2010) or the high incidence in Caucasian men and the high mortality rates among African-Americans (Dransfield et al. 2006), underlines the importance of heredity. It is associated with smoking, body mass index and occupational exposures. The socio-economic and environmental conditions like temperature and air quality ( $\mathrm{PM}<10)$, as well as lifestyle may be considered important aggravating factors. So, evidences of relationships between COPD aggravation and urban lifestyle is a win-win process as it make easier the implementation of sustainable planning policies and it may help to decrease the disease aggravation.

Objectives: Our purpose is the evaluation of the cause-effect relationship among temperature and PM10 concentration at Porto and the number of admissions with COPD at the 4 main hospitals in the area. Methods: We create a GIS with the location of people with some important origin COPD factors-bronchitis and asthma and tuberculosis - and with several socio-economic aggravation factors like the presence of elderly people, low incomes from unemployment and from other social benefits, buildings with more than 40 years, $\mathrm{N}$ and $\mathrm{E}$ exposures, population density, literacy, lower percentages of green areas and road density. We used a multi criteria hierarchical analysis to weight each of these variables and we compare the distribution of COPD admissions with the most vulnerable areas. Then, we collect the daily temperature and the COPD admissions at Porto 4 main hospitals. Afterwards we select the 95th percentile of COPD monthly admissions and we compare with the temperature and the PM10 concentration.

Results: The COPD admissions coincide with people living in urban areas where the origin and aggravating factors are higher-Campanhã and historical city center. The higher number of COPD admissions occurred during all cold spells events and in days with minimum temperatures below $6{ }^{\circ} \mathrm{C}$ and with thermal amplitudes above $10{ }^{\circ} \mathrm{C}$. There were any expressive relationship with higher PM10.

Conclusions: Urban planning is a complex negotiation process between lifestyle improvement expectancy paradigms and local ecosystem capacity. The implementation of sustainable programs needs to convince people to modify their wellbeing goals, which is one of the mains constraints. The demonstration of health benefits is seen as a consensus good that can easy motivate a lot of citizen's attitudes change. Among all diseases, COPD seems to be a good example.

\section{P-023}

Factors distorting cardiovascular mortality of working population in Russia

Topic: Diseases - CVD/diabetes

Presenter: Tamara Sabgayda

\section{Galina Evdokushkina 1); Viktoria Semyonova 1); Tamara Sabgayda 1); Nataly Gavrilova 2); Svetlana Nikitina 3)}

1) Federal Research Institute for Health Organization and Informatics of Ministry of Health of Russian Federation; 2) The Center of Demography and Economy of Ageing, the University of Chicago, USA; 3) Federal State Statistics Service, Moscow, Russia

There are solid reasons to suppose that growth of cardio-vascular mortality in population of young ages could be doubtful in Russia. At the same time deaths from external causes could be higher than it comes from official data of Federal State Statistics Service.

The aim of the study is to define reasons of distortion of cardiovascular mortality.

Methods: Analysis of factors distorting death causes was conducted on the base of official mortality database compiled according to full list of diagnosis including sex of deceased person, dates of birth and death, place of residence, death caused according to ICD-10 as well as attributes of person who established death and source of information about death causes.

Results: Cardiovascular pathology is increasing by priority rates in young ages when genesis and chronization of cardiovascular diseases are not physiologically conditioned. Losses from cardio-vascular diseases maximally increased during 1989-2010 in males 15-29 years (by $78 \%$ ), rates of mortality increase became lower with age: in ages $30-44$ years mortality growth estimated $58 \%$, in $45-59$ years-31.6 \%. In females maximal growth (by $88.3 \%$ ) was characteristic to ages 30-44 years, with a sharp decline of mortality growth in age group 45-59 years (only $8.8 \%$ ). The maximal rates of growth were not observed to ischemic heart diseases or Cerebrovascular diseases, but to Other forms of heart disease. Mortality from the last pathologies increased among young population approximately threefold; among middle population (30-44 years old) by about 6 times and among population aged $45-59$ years by around 5 times. Currently in population aged 15-29 years more than one half of cardio-vascular mortality is determined by "Other forms of heart 
diseases" (56.7 and $52.4 \%$ in males and females correspondingly); in 30-44 years olds-more than $40 \%$; and only in older working ages 45-59 years the share of such diseases occurred to be 18.3 and $18.9 \%$ correspondingly. At that relevance of "Other forms of heart diseases" increased in comparison with 1989 multiply in all age groups.

Existing legal framework (absence of obligation to test alcohol level in blood during autopsy for pathologists; absence of obligation to indicate alcoholic anamnesis in death certificate) promotes underestimation of mortality from accidental alcoholic poisonings through cardio-vascular diseases. The possibility of accounting of mortality from accidental alcohol poisoning as cardio-vascular diseases leads to overestimation of mortality from the last causes. The growth of mortality from ischemic heart diseases and cerebrovascular diseases in the ages of 15-44 years also forces to suppose the existence of alcoholic factor.

Conclusion: So, the epidemic scales of cardio-vascular diseases in Russia especially in young ages is not that great as it is shown in official statistics. Some diagnoses of death from cardiovascular diseases are partly hidden in the alcoholic poisonings.

\section{P-024}

Multiple mediation effects of fetuin-A and body mass index on the association between AHSG polymorphism and type 2 diabetes

Topic: Diseases - CVD/diabetes

Presenter: Ammarin Thakkinstian

Ammarin Thakkinstian 1); Laor Chailurkit 2); Suwannee Chanprasertyothin 2); Wipa Ratanachaiwong 3); Sukit Yamwong 2); Piyamitr Sritara 2); Boonsong Ongphiphadhanakul 2)

1) Section for Clinical Epidemiology and Biostatistics, Faculty of Medicine, Ramathibodi Hospital, Mahidol University, Thailand.; 2) Department of Medicine, Faculty of Medicine, Ramathibodi Hospital, Mahidol University, Thailand.; 3) Medical and Health Office, Electricity Generating Authority of Thailand, Nonthaburi, Thailand

Background: Alpha 2 Hereman-Schmid glycoprotein (ASHG) is associated with type 2 diabetes (T2D), but we wanted to determine if the effect was directed, or mediated through fetuin-A and BMI pathways.

Objective: To assess average causal direct (DE) and mediation effects (ME) of ASHG on T2D.

Methods: We used cross-sectional data of an Electricity Generating Authority of Thailand cohort. ASHG at rs2248690 was genotyped on 1,743 subjects for measures of fetuin and confounding data. Fetuin-A level was categorized as $>620.2$ versus $\leq 620.2 \mathrm{~g} / \mathrm{ml}$ according to Yuden index. Data for TT and AT were combined due to small numbers of TT in T2D. Three mediation and outcome models were constructed in three steps. First, the fetuin-A group was regressed on AHSG gene (path a1). Second, the BMI group ( $\geq 25 \mathrm{vs.}<25 \mathrm{~kg} / \mathrm{m}^{2}$ ) was fitted on AHSG gene and fetuin-A (path a2d). Finally, the T2D was fitted on 2 mediators (fetuin-A and BMI) and AHSG gene (path a1db2). These three models were adjusted for age, gender, and cholesterol. A bootstrap with 1,000 replications was applied to estimate $95 \%$ confidence interval of effects. All analyses were performed using STATA 12.0

Results: The mean age of 1,743 subjects was 39.9 (SD $=6.6), 72 \%$ were males, and mean BMI and fetuin-A levels were $23.9(\mathrm{SD}=3.8)$ and $92.6(\mathrm{SD}=22.4)$, respectively. The frequency of AA, AT, and TT genotypes were 1,260, 430, and 50, complying with HardyWeinberg equilibrium $(p=0.077)$. The prevalence of diabetes was 3.8. The effect of AHSG on T2D through fetuin-A (a1b1) bypassing
BMI was significant with OR of 4.29 (95\% CI 1.75, 10.52). The second model carrying the effect of AA genotype (a2b2) on T2D through BMI bypassing fetuin-A was non-significant $(\mathrm{OR}=1.17$, $95 \%$ CI $0.90,1.51)$. Finally, effect of AA genotype on T2D through fetuin-A and BMI (a1db2) was 1.91 (95\% CI 0.92, 3.96). Total ACME was 9.58 (95\% CI 2.93, 31.28), i.e., carrying AA genotype of the AHSG gene was 9.58 times higher odds of T2D through fetuin-A and BMI than TA/TT genotypes. The estimated ACDE was non significant 0.78 (95\% CI $0.39,1.57)$. The effects were explained through fetuin-A, BMI, and fetuin-BMI pathways of 58.2, 6.2, and $25.8 \%$ respectively; whereas $9.7 \%$ was by direct effects.

Conclusions: Our study suggested a causal effect of AHSG on T2D through fetuin-A and BMI pathways with proportions mediated 58.2 and $25.8 \%$ respectively. Carrying the AA genotype increases fetuin$\mathrm{A}$ and $\mathrm{BMI}$, so increasing the risk of diabetes.

\section{P-025}

Investigating the causal effect of vitamin $D$ on serum adiponectin using a Mendelian randomization approach

Topic: Diseases - CVD/diabetes

Presenter: Lotte Husemoen

Lotte Husemoen 1); Tea Skaaby 1); Torben Martinussen 2); Torben Jørgensen 1), 3); Betina Thuesen 1); Caroline Kistorp 4); Jørgen Jeppesen 5), 3); Jacob Thyssen 6); Allan Linneberg 1)

1) Research Centre for Prevention and Health, Copenhagen University Hospital Glostrup, Glostrup, Denmark.; 2) Department of Biostatistics, University of Copenhagen, Copenhagen, Denmark.; 3) Faculty of Health Science, University of Copenhagen, Copenhagen, Denmark.; 4) Department of Cardiology and Endocrinology, Copenhagen University Hospital Frederiksberg, frederiksberg, Denmark.; 5) Department of Medicine M, Copenhagen University Hospital Glostrup, Glostrup, Denmark.; 6) National Allergy Research Centre, Department of Dermato Allergology, Copenhagen University Hospital Gentofte, Hellerup, Denmark

Background: Adiponectin is a protein involved in a number of metabolic processes, including glucose regulation, and is associated with obesity and type 2 diabetes. Recent cross-sectional studies suggest an association between blood concentrations of vitamin D and adiponectin. This raises the hypothesis that vitamin $\mathrm{D}$ may be causally related to adiponectin concentrations, and provides a potential mechanism whereby vitamin D may affect the development of diabetes.

Objective: The aim was to examine the causal effect of vitamin D on serum adiponectin using a multiple instrument Mendelian randomization approach.

Methods: Serum 25-hydroxyvitamin D (25(OH)D) and serum total or high molecular weight adiponectin were measured in two Danish population based studies: the Inter99 study (6,405 adults, 30-65 years) conducted in 1999-2001, and the MONICA10 study (2,656 adults, 41-71 years) conducted in 1993-1994. We used rs2282679 located in the GC gene and filaggrin gene (FLG) loss-offunction mutations as instrumental variables known to influence blood $25(\mathrm{OH}) \mathrm{D}$ concentrations, and applied the two-stage least square multiple instrument approach to estimate the causal effect of vitamin $\mathrm{D}$ on adiponectin.

Results: In the Inter99 study, 25(OH)D were positively associated with total adiponectin (the effect estimate in \% per doubling of $25(\mathrm{OH}) \mathrm{D}$ was $4.78,95 \% \mathrm{CI} 1.96,7.68, P<0.001)$. Using mutations in the vitamin $\mathrm{D}$ binding protein gene and the filaggrin gene as instrumental variables, the causal effect in $\%$ was estimated to 61.46 , $95 \%$ CI $17.51,120.28, P=0.003$ higher adiponectin per doubling of 
25(OH)D. In the MONICA10 cohort, no significant association was observed between the serum concentrations of 25(OH)D and HMW adiponectin (the effect estimate in $\%$ per doubling of $25(\mathrm{OH}) \mathrm{D}$ was $1.51,95 \% \mathrm{CI}-5.80,2.98, P=0.50$ ), although the instrumental variables analysis to some extent supported a positive causal association (the effect estimate in $\%$ per doubling of $25(\mathrm{OH}) \mathrm{D}$ was 37.13 , $95 \% \mathrm{CI}-3.67,95.20, P=0.080$ ).

Conclusion: The results support a causal association between serum $25(\mathrm{OH}) \mathrm{D}$ and total adiponectin.

\section{P-026}

In the Australian aboriginal population, is lower birth weight associated with higher fasting glucose and insulin in adolescence?

Topic: Diseases - CVD/diabetes

Presenter: Mark Pearce

Mark Pearce 1); Susan Sayers 2); Susan Mott 3); Kay Mann 1); Gurmeet Singh 2)

1) Institute of Health \& Society, Newcastle University, UK;

2) Menzies School of Health Research, Charles Darwin University, Australia; 3) Centre for Chronic Disease, University of Queensland, Australia

Background: The highest risk for type 2 diabetes in relation to fetal growth is reported when LBW/FGR occurs in conjunction with later overweight/obesity. Despite recent improvements, Australian Aboriginal low birth weight (LBW) rates remain double those of the non-Aboriginal population and type 2 diabetes rates are $10-30 \%$. LBW may be a contributor to the current high rates of type 2 diabetes in the Aboriginal population.

Objectives: Using data from a prospective life course Aboriginal birth cohort based in the Australian Northern Territory, our aim was to examine the relationships of glucose and insulin metabolism with birth and current adolescence size.

Methods: This study was within the longitudinal prospective study of the Aboriginal Birth Cohort. 686 Aboriginal babies born at the Royal Darwin Hospital between January 1987 and March 1990 were followed-up between December 2006 and January 2008. Undernutrition, overweight and obese were defined using BMI $<18.5 \mathrm{~kg} /$ $\mathrm{m}^{2}$, BMI $>25 \mathrm{~kg} / \mathrm{m}^{2}$ and BMI $>30 \mathrm{~kg} / \mathrm{m}^{2}$ respectively. The relationships between birth weight and later anthropometric markers and fasting insulin and glucose and HOMA-IR, were examined using regression models adjusted for sex, gestational age, current age and urban/rural residence.

Results: The combination of LBW/FGR with later overweight/ obesity was rare in adolescence. There was a significant positive relationships of birth weight with glucose $(p=0.002)$, but not other measures. However, current weight was significantly and positively related to fasting measures of insulin, glucose and HOMA-IR. There were significant interactions between birth weight and height for insulin $(p=0.006)$ and HOMA-IR $(p=0.015)$. The relative contributions to explaining variation in fasting glucose and insulin, calculated as the difference in R2 between fully adjusted models with and without the variable of interest, were around $2 \%$ for birth weight on HOMA-IR and insulin, but $7 \%$ on glucose. In contrast, current weight explained around $30 \%$ of the variation in HOMA-IR and insulin and $8 \%$ in glucose.

Conclusions: In this young Aboriginal population, current weight, not birth weight, is related to the biomarkers of type 2 diabetes. Our findings confirm that fasting concentrations of insulin and glucose are not determined solely by fetal life and implies the current high rates of type 2 diabetes in the Aboriginal population may be decreased by life style interventions in childhood and adolescence.

\section{P-027}

Creation of a scale to assess severity in acute decompensated heart failure

Topic: Diseases - CVD/diabetes

Presenter: Jose Maria Quintana

Susana Garcia-Gutierrez 1); Maria Soledad Gallardo 2); Esther Pulido 2); Anette Unzurrunzaga 1); Iratxe Lafuente 1); Antonio Escobar 3); Elena Zubillaga 4); Mikel Sanchez 2); Nerea Gonzalez 1); Jose Maria Quintana 1)

1) Research Unit. Hospital Galdakao-Usansolo.Red de Investigación en Servicios de Salud en Enfermedades Crónicas (REDISSEC); 2) Emergency Department. Hospital Galdakao-Usansolo; 3) Research Unit. Hospital de Basurto. Red de Investigación en Servicios de Salud en Enfremedades Crónicas (REDISSEC); 4) Internal Medicine. Hospital Donostia; 10) Research Unit. Hospital GaldakaoUsansolo.Red de Investigación en Servicios de Salud en Enfermedades Crónicas (REDISSEC)

Background: Scales to assess severity in acute decompensated heart failure were created in hospitalized patients or in stable ones or based on large databases and result difficult to use in acute setting.

Objective: To create a scale to assess severity in acute decompensated heart failure.

Design: Prospective cohort study. Patients attending emergency departments of three emergency departments in Basque Country with acute decompensated of heart failure were included (de novo and chronic decompensated heart failure). Clinical variables at arrival to ED were collected as well as information about admission in the case of hospitalized patients and at 7 days after discharge from ED in the discharged home patients from ED. Sociodemographical variables, clinical signs and symptoms, gasometrical and laboratory parameters, basal echocardiographic parameters as well as basal and due to decompensation treatments and the response to them were collected. Main outcome was mortality during 7 days after index episode. Statistical analysis: We performed logistic regression model being dependent variable mortality after 7 days after index episode and independent variables those clinical, gasometrical and laboratory parameters at the arrival to ED. C statistic was calculated to assess the predictive ability of the models was performed and the HosmerLemeshow (HL) to evaluate the calibration of the models. All analyzes were conducted in SAS v9.2 for Windows.

Results: We recruited 878 patients for the study, the $51.47 \%$ of them were females, and mean age was 79,54 (9,51 years). The $80.25 \%$ presented hypertension, 5,147 dyslipemia and $25 \%$ of them were exsmokers. The $53 \%$ of them presented basal functional situation II/IV measured by NYHA scale and more than $90 \%$ presented level III-IV/ IV at the arrival to ED. $4 \%$ of them died during admission or at 7 days after their visit to ED. Creatinine, systolic blood pressure and hemodinamic instability were related to mortality. AUC for this model reached 0,78 with good calibration. (Hosmer-Lemeshow $>0.05$ ).

Conclusions: We have identified several easily collected variables which could predict fatal evolution of ADHF. External validation of the scale is needed.

\section{P-028}

Nonfasting triglycerides predict incident acute myocardial infarction: cohort Norway and CVDNOR: 11.5 year follow-up

Topic: Diseases - CVD/diabetes

Presenter: Grace Egeland 
Grace Egeland 1), 2); Jannicke Igland 2); Gerhard Sulo 2); Ottar Nygård 3); Marta Ebbing 1); Grethe Tell 2)

1) Cardiovascular Disease Registry, Norwegian Institute of Public Health, Bergen, Norway; 2) Department of Global Public Health and Primary Care, University of Bergen, Bergen, Norway; 3) Department of Clinical Science, University of Bergen \& Department of Heart Disease, Haukeland University Hospital, Bergen, Norway

Background: The importance of triglycerides as a predictor of coronary heart disease (CHD) is controversial given that its' association with CHD disappears or is greatly attenuated after adjustment for conventional risk factors.

Objectives: To prospectively evaluate the risk of incident acute myocardial infarction (AMI) associated with nonfasting triglyceride levels in men and women in multivariable models testing for potential effect modification by sex, high waist circumference, and low HDL-C.

Methods: A population-based Cohort Norway (CONOR) health survey population of 173,243 men and women assessed between 1994 and 2003 and followed through Dec 2009 via record linkages to the Cause of Death Registry and hospital discharge diagnoses available through the Cardiovascular Disease in Norway (CVDNOR) Study, 1994-2009. Hazard ratios for AMI by triglyceride deciles and quartiles were evaluated in Cox Proportional Hazard analyses which tested for interactions by sex, an enlarged waist circumference $(>102 \mathrm{~cm}$ for men and $>88 \mathrm{~cm}$ for women), and at-risk HDL-C $(<1.0 \mathrm{mmol} / \mathrm{L}$ for men and $<1.3 \mathrm{mmol} / \mathrm{L}$ for women). Multivariable models included baseline age, diabetes, systolic blood pressure, smoking, nonHDL-C, alcohol intake, and time since last meal.

Results: After excluding participants with prevalent cardiovascular disease at baseline, $3,219(4.8 \%)$ men and 1,434 (1.9\%) women developed an AMI during follow-up. In age-adjusted analyses, women had a steeper gradient in AMI risk with increasing triglyceride decile than men (interaction term, $P<0.001$ ). Further, a significant at-risk HDL-C by triglyceride interaction term was observed in men and women separately evaluated in $(P \mathrm{~s}<0.001)$. Multivariable analyses were therefore stratified by at-risk HDL-C and separately evaluated for men and women and will be presented. The presence of an enlarged waist circumference, however, did not modify triglyceride's association with MI in age-adjusted or multivariable models. Conclusions: Nonfasting triglyceride levels may help identify a subset of individuals at risk for CHD not identified through conventional risk factors.

\section{P-029}

Body fat, body fat distribution, lean body mass and atrial fibrillation: a Danish cohort study

Topic: Diseases - CVD/diabetes

Presenter: Lars Frost

Lars Frost 1); Emelia J Benjamin 2); Morten Fenger-Grøn 3); Asger Pedersen 4); Anne Tjønneland 5); Kim Overvad 6)

1) Department of Medicine, Silkeborg Hospital \& Institute of Clinical Medicine, Aarhus University Hospital, Aarhus, Denmark; 2) Department of Medicine, School of Medicine and Department of Epidemiology, School of Public Health, Boston University, Boston, MA, USA; 3) Research Unit for General Practice, Aarhus University, Aarhus, Denmark; 4) Hammel Neurorehabilitation and Research Centre, Hammel, Denmark; 5) Institute of Cancer Epidemiology, Danish Cancer Society, Copenhagen, Denmark; 6) Section for Epidemiology, Department of Public Health, Aarhus University, Aarhus, Denmark, and Department of Cardiology, Center for Cardiovascular Research, Aalborg University Hospital, Aalborg, Denmark
Background: It is well recognized that body height and weight are positively associated with risk of atrial fibrillation (AF) but it is unclear whether risk of $\mathrm{AF}$ is related to body fat, body fat location, or lean body mass.

Objective: We investigated the associations between bioelectrical impedance derived measures of body composition and combinations of anthropometric measures of body fat distribution and risk of AF. Methods and Results: We studied the Danish population-based prospective cohort Diet, Cancer and Health conducted from 1993 to 2009 among 55,273 men and women 50-64 years of age at recruitment and without AF at baseline. Participants were followed in the Danish National Registry of Patients and in the Danish Civil Registration System.

Results: During follow-up (median 13.5 years) AF developed in 1,669 men and 912 women. Higher body fat at any location was associated with a higher risk of AF. The adjusted hazard ratio (HR) (95\% confidence interval) per 1 sex-specific standard deviation (SD) increment in body fat mass was 1.29 (95\% confidence interval [CI] 1.24-1.33). In addition, higher lean body mass was associated with an elevated risk of AF. The adjusted HR for 1 sex-specific SD increment was 1.40 (95\% CI 1.35-1.45).

Conclusion: Higher body fat as well as higher lean body mass were associated with a higher risk of $\mathrm{AF}$ in men and women.

\section{P-030}

Trends in risk for a recurrence after the first acute myocardial infarction hospitalization in Norway 2001-2008: a CVDNOR project

Topic: Diseases - CVD/diabetes

Presenter: Gerhard Sulo

Gerhard Sulo 1); Stein Emil Vollset 1), 4); Ottar Nygård 2), 3); Jannicke Igland 1); Grace M. Egeland 1), 5); Marta Ebbing 5); Grethe S. Tell 1), 4)

1) Department of Global Public Health and Primary Care, University of Bergen, Norway; 2) Section for Cardiology, Institute of Medicine, University of Bergen, Norway; 3) Department of Heart Disease, Haukeland University Hospital, Bergen, Norway; 4) Norwegian Institute of Public Health, Bergen, Norway; 5) Cardiovascular Registry, Norwegian Institute of Public Health, Bergen, Norway

Background: Aging of the population and changes in type, severity, acute and long-term treatment of incident acute myocardial infarction (AMI) has influenced survival and the risk for a recurrent AMI.

Objectives: To study trends in 6-months, 1- and 3-year risk of having a second AMI (recurrence) among patients hospitalized for an incident AMI during 2001-2008.

Methods: All AMI hospitalizations (ICD9 410; ICD10 I21, I22) in Norway in individuals $>25$ years were obtained during 1994-2009. Of these, persons with incident hospitalizations (defined as an AMI hospitalization with no prior hospitalization for AMI during the previous 7 years) during 2001-2008 were identified and followed for a recurrence (defined as a second AMI event-hospitalization or outof-hospital AMI deaths-more than 28 days from the incident AMI) within 6 months, 1 and 3 years from their incident event. Time trends in risk of recurrences were explored using competing risk regression analyses with year of incident AMI as the independent variable (trend analysis), adjusted for age at the first AMI. Sub-hazard ratios (SHR) for year as a continuous variable were interpreted as average annual change in risk, and reported for men and women separately. Deaths from other causes than AMI were considered as competing events in the models. Three separate analyses were conducted; with 6 months, $1-$, and 3-years recurrence as outcomes. 
Results: 102326 patients (39.4\% women) were hospitalized for an incident AMI during 2001-2008. Mean age (SD) at hospitalization was 72.5 (13.7) years. $6.8 \%$ had a recurrent AMI event within 6 months and $9.6 \%$ within 1 year. Of 76762 patients hospitalized for an incident AMI during 2001-2006, $15.8 \%$ had a recurrent event within 3 years.

During 2001-2008, 6 months risk of a recurrent event was reduced by $28 \%(\mathrm{SHR}=0.72 ; 95 \%$ CI $0.66-0.79)$ in men and $31 \%$ (SHR $=0.69 ; 95 \%$ CI $0.62-0.77)$ in women. Reductions of $29 \%$ $(\mathrm{SHR}=0.71 ; 95 \% \mathrm{CI} 0.66-0.77)$ and $31 \%(\mathrm{SHR}=0.69 ; 95 \% \mathrm{CI}$ 0.63-0.76) were observed for 1-year risk for a recurrence in men and women. During 2001-2006, 3-year risk for a recurrence was reduced by $25 \%$ (SHR $=0.75 ; 95 \%$ CI $0.70-0.81)$ among men and $26 \%$ $(\mathrm{SHR}=0.74 ; 95 \%$ CI $0.68-0.80)$ among women.

Conclusions: During 2001-2008, a consistent decline in the risk of recurrent AMI events was observed among patients hospitalized for their first AMI in Norway. The reduction was similar for short and long term risk and among both men and women.

\section{P-031}

Differences in acute myocardial infarction incidence in Norway according to education level: a CVDNOR-project

Topic: Diseases - CVD/diabetes

Presenter: Jannicke Igland

Jannicke Igland 1); Stein E. Vollset 1); Ottar Nygård 2); Gerhard Sulo 1); Marta Ebbing 3); Grethe S. Tell 1)

1) Department of Global Public Health and Primary Care, University of Bergen, Bergen, Norway; 2) Department of Clinical Science, University of Bergen, Bergen, Norway; 3) Norwegian Institute of Public Health, Bergen, Norway

Background: Increasing differences in cardiovascular disease (CVD) mortality across levels of education have been reported in Norway. Because of lack of national incidence data on CVD up to 2012 when the Norwegian Cardiovascular Disease Registry was established, we do not know whether these increasing differences is also present for incidence rates or if it is mainly caused by difference in survival.

Objectives: To compare trends in acute myocardial infarction (AMI) incidence across levels of education during 2001-2009 using data from the Cardiovascular Disease in Norway (CVDNOR) project.

Methods: Information on all hospitalizations and out-of-hospital deaths due to CVD in Norway between 1994 and 2009 was obtained through patient administrative systems at the hospitals and the Cause of Death Registry and linked to the National Education Database. Incident AMI was defined as a hospitalization with an AMI (ICD-9 code 410 , or ICD-10 codes I21-I22) as main or secondary diagnosis or a death with AMI as the underlying cause of death in individuals with no AMI-events the previous 7 years. Education was categorized in three groups: Basic education or less, upper secondary education and tertiary education (college or university). In addition to age-standardized incidence rates per calendar year we calculated incidence rate ratios (IRR) for the different education groups using Poisson regression with tertiary education as the reference. All calculations where done separately in four gender and age strata: Men and women aged 30-64 and 65-100 years.

Results: A total of 131,332 incident AMIs, of which 113,816 were hospitalized and 17,516 were deaths occurring out-of-hospital, were identified and classified according to education level. Incidence rates decreased during the period 2001-2009 in all education groups for men and women aged 65-100 years. For the age group 30-64 years, a decrease was found only among men with upper secondary education. There was a statistically significant trend in IRRs across education levels in all gender and age strata. The largest ratio in incidence was found among women aged 30-64, with IRR (95\% CI) for basic versus tertiary education equal to 3.33 (3.09-3.60). Tests for interaction between education level and calendar year did not reveal any significant changes in educational differences in AMI incidence during 2001-2009 in any of the four gender and age strata.

Conclusions: There are substantial differences in AMI incidence across educational levels in Norway, especially for women between 30 and 64 years of age.

\section{P-032}

Risk of incident diabetes associated with statin therapy in a cohort enrolled through administrative databases

Topic: Diseases - CVD/diabetes

Presenter: Valeria Fano

Valeria Fano 1); Alessandra Blasi 1); Katia Bontempi 1); Alessandra Berti 2); Lucia Gualandi 2); Marco Filogna 1); Maria Miceli 1); Eugenia Pagnozzi 1); Alessandra Rossi 1); Roberta Di Turi 1)

1) Local Health Authority RMD, Rome, Italy;

2) Cineca Interuniversity Consortium Bologna Italy

Background: Statin therapy has been shown to be effective for reduction of cardiovascular events and is generally recognised as being safe and well tolerated. However, randomised trials reported conflicting findings on the risk of development of diabetes mellitus in non diabetic patients taking statins.

Objectives: To estimate the risk of incident diabetes associated with statin therapy in a cohort of residents enrolled through administrative health databases.

Methods: Adults $(35+; \mathrm{n}=331,923)$ residents in 2007 in the Local Health Authority 'Roma D' (southern part of Rome) were included in the study population (prevalence of diabetes in $2007=8.3 \%$ ). Statins prescribed during the period 2007-2011 were retrieved from a database collecting information on all drugs prescribed by GPs and public ambulatories. Patients were classified according to their statin prescriptions as 'not statin users', 'continous users' (over $80 \%$ of total days per year) and 'discontinuous users' ( $<80 \%$ days per year). New cases of diabetes were ascertained through record linkage with administrative databases of years 2008-2011. Relative Risks (RR) of developing diabetes were computed across categories of statin users using Poisson models adjusted for socio-demographic variables.

Results: Among non diabetic patients $(\mathrm{n}=308,281)$ the incidence of diabetes in 2008-2011 was $4.2 \%(\mathrm{n}=12,869)$. RR were significantly higher for 'continuous users' compared to 'non-users' ( $\mathrm{RR}=4.9 ; p$-value $<0.05)$ and for 'continuous users' with respect to 'discontinuous users' $(\mathrm{RR}=1.7 ; p$-value $<0.05)$. Among elderly over 65 , RR were still statistically significant but less marked (continuous users vs. non-users $\mathrm{RR}=3.1$; continuous vs. discontinuous users $\mathrm{RR}=1.7$ ). When restricting the analyses to people with previous cardiovascular hospital admissions, lower RR were observed (continuous users vs. non-users $\mathrm{RR}=2.5$; continuous vs. discontinuous users $\mathrm{RR}=1.7$; $p$-value $<0.05$ ).

Conclusions: Among adults residents in the southern part of Rome statin therapy is associated with the risk of developing diabetes. Continous users show the highest relative risks, both among adults $35+$ and among elderly $65+$, in agreement with the results of previous meta-analyses estimating diabetes incidence among high doses statin group compared with moderate doses. 
P-033

Utilising a novel framework to assess public health nutrition actions across 30 European countries (EuroHeart II Project)

Topic: Diseases - CVD/diabetes

Presenter: Lois Orton

Helen Bromley 1); Ffion Lloyd Williams 1); Lois Orton 1); Rory McGill 1); Elspeth Anwar 1); David Taylor Robinson 1); Martin O'Flaherty 1); Maria Guzman Castillo 1); Mike Rayner 2); Simon Capewell 1)

1) British Heart Foundation Health Promotion Research Group, University of Oxford; 2) Department of Public Health and Policy, University of Liverpool

EuroHeart II is a European programme designed to inform cardiovascular health strategies across Europe. Our project examines the role of food policies in cardiovascular disease prevention, including the development and piloting of a novel conceptual framework for categorising public health nutrition policies.

Objective: A mapping exercise to identify and categorise public health nutrition policy actions across 30 European countries using a novel framework.

Design: The framework was based on the traditional marketing "4Ps" approach: Price, Product, Promotion and Place (the "marketing mix"). A database was created to summarise public health nutrition policy for 30 European countries (EU 27 plus Iceland, Norway and Switzerland). National policies were classified according to Price (taxes, subsidises, other); Product (reformulation, new healthier products); Place (schools, workplaces, community settings); and Promotion (advertising, food labelling and health education initiatives).

Results: Dialogue, recommendations and nutrition guidelines are now widespread across Europe.

Information and education campaigns are also widespread. These include campaigns covering the general population, and campaigns targeting schools, the workplace or communities.

Subsidies for fruit in schools are almost universal through the EU School Fruit Scheme, but implementation differs across the 30 countries.

New EU legislation supports limited, back of pack food labelling. Some countries have also implemented national legislation requiring more detailed label information about the nutritional value of foods (e.g. Finland). However, the presentation and information vary widely.

Voluntary reformulation of foods is common, especially for salt, sugar and total fat (e.g. salt reduction in the UK). However, mandatory reformulation of products to reduce saturated fat and salt are still limited to trans fat bans in Austria, Denmark Iceland and Switzerland.

Legislation/regulation affecting salt, sugar, fat and fruit and vegetable consumption is uncommon, although several countries have legal requirements regarding the maximum salt content in certain foods (Belgium, Bulgaria, Finland, Greece, Latvia, Lithuania, Netherlands, Portugal, Romania, Slovak Republic, Slovenia and Wales).

Taxes to promote healthy nutrition are currently used infrequently. However, Finland, France, Hungary and Portugal have implemented 'sugar taxes' on sugary foods and sugar-sweetened beverages. Hungary and Portugal also tax salty products.

Conclusions: The diverse public health nutrition activities across 30 European countries might initially appear complex and bewildering. However, the "4Ps" framework offers a potentially structured and comprehensive categorisation of these diverse interventions. National food policies in Europe are currently at very different stages of development and implementation. However, exemplar countries might include Denmark, Finland, Hungary, Iceland, the UK and Portugal.
P-034

Assessing the potential effect of healthy eating policy interventions on socioeconomic inequalities: systematic review

Topic: Diseases - CVD/diabetes

Presenter: Lois Orton

Rory McGill 1); Elspeth Anwar 1); Lois Orton 1); David Taylor-Robinson 1); Helen Bromley 1); Ffion Lloyd-Williams 1); Martin O'Flaherty 1); Mark Petticrew 2); Margaret Whitehead 1); Simon Capewell 1)

1) University of Liverpool, Department of Public Health \& Policy; 2) London School of Hygiene \& Tropical Medicine, Department of Social \& Environmental Health Research

Background: Cardiovascular disease (CVD) is one of the main contributors to health inequalities. And CVD primary prevention includes potentially powerful interventions to promote healthy eating. However, might some dietary interventions actually widen the heath gap between rich and poor, thus leading to intervention-generated inequalities?

Objective: To systematically review the evidence for differential socio-economic effects associated with healthy eating policy interventions.

Methods: We initially searched two bibliographic databases (MEDLINE \& Psycinfo) using a piloted search strategy. Results from further databases and additional sources will be reported in the final review. Search results were screened independently by two reviewers. We included evaluations of policy interventions to promote healthier diets, (defined as the reduced intake of salt, sugar, trans fats, saturated fat, total fat, or total calories, or increased consumption of fruit and vegetables). Studies were only included if quantitative results were presented by socio-economic group (SEG), defined by income and education level, ethnicity, or occupational status. Extracted data were categorised with a modified version of the 4Ps marketing framework: Price, Product, Place and Promotion, with a 5th "P" labelled "Personal", relating to person-based health education.

Results: We identified 14,449 studies in the initial search. Following screening, only 14 articles $(0.1 \%)$ met the inclusion criteria. Preliminary results analysed using the "5 P's" framework suggest that some food policy interventions may generate socio-economic differentials. Price interventions showed the greatest potential to reduce health inequalities ( 3 of 5 studies). Conversely, Personal interventions tended to widen inequalities ( 3 of 4 studies showed preferential outcomes in higher SEGs). Results relating to Place interventions were mixed, with 2 school-based studies reporting a preferential outcome in higher SEGs, and 1 work-based study reporting a preferential outcome in lower SEGs. Evidence for Product and Promotion interventions appears sparse, with only 1 study found for each category. However, both reporting preferential outcomes in higher SEGs. Conclusions: Interventions categorised by the " 5 P's" show differential effects on healthy eating outcomes by SEG, with interventions categorised as Personal appearing the most likely to increase health inequalities. However the vast majority of studies retrieved did not explore differential effects by socio-economic group. Future policies aimed at improving population health should be routinely evaluated for their potential impact on health inequalities.

\section{P-035}

Plasma variations of oxidized LDL-cholesterol and chronic inflammation biomarkers among participants of the Malmö Diet and Cancer cohort

Topic: Diseases - CVD/diabetes 


\section{Presenter: Joana Dias}

Joana Dias 1); Sophie Hellstrand 1); Ulrika Ericson 1); Bo Gullberg 1); Jan Nilsson 1); Ragnar Alm 1); Margaretha Persson 1); Gunnar Engström 1); Gunilla Fredrikson 1); Bo Hedblad 1)

\section{1) Department of Clinical Sciences in Malmö, Lund University}

Background: Oxidized-LDL and certain pro-inflammatory cytokines such as IL-1, IL-6, IL-8 and TNF-assume an important role as mediators of inflammation process and can serve as markers of chronic inflammation. Oxidative stress and chronic inflammation are believed to play a key role in the development of diseases such as type 2 diabetes mellitus, cardiovascular diseases and certain types of cancer. The Malmö Diet and Cancer (MDC) cohort has unique dietary data and a well maintained bio-bank of non-fasting blood samples, which will enable future investigation regarding dietary factors and processes of chronic inflammation.

Objectives: The reliability of biomarkers in both fasting and nonfasting blood samples was examined in a sub-sample from the MDC by estimating the intraclass correlation coefficients (ICC) of biomarkers. Also, associations between biomarkers and selected nutrient intakes were examined.

Methods: In 2010-2011, a sub-sample from the MDC was recruited (50 men and 45 women, 66-76 years) to donate 3 fasting and 3 nonfasting blood samples during a 6-8 week period. Dietary data was collected with 4 day food records using web-based data entry. A brief questionnaire assessed socioeconomic and lifestyle variables. ICCs were estimated for all 5 biomarkers separately for men and women; in fasting and non-fasting blood samples. Partial correlation coefficients were calculated between selected nutrients and the biomarkers, adjusting for possible confounders.

Results: In men, the lowest ICCs were seen for IL-6, ICC $=0.61$ (95\% CI 0.46-0.74); and IL-8, ICC $=0.49$ (95\% CI 0.32-0.64) from non-fasting samples while the other ICCs were above 0.65 . For women, IL-1 in fasting samples had the lowest value of ICC $=0.66$ (95\% CI $0.52-0.78$ ). Dietary cholesterol and MUFAs were positively correlated to IL-6 in non-fasting samples; similar associations were seen for MUFAs and total fat with IL-8. Additionally, inverse associations were seen for -6-PUFA, -carotene and magnesium with IL-1beta; and for monosaccharaides with IL-6 and IL-8.

Conclusions: The observed ICCs suggest that single measurements of these biomarkers allow meaningful analysis in epidemiological studies. The observed nutrient intake-biomarker correlations are similar to those of previous studies.

\section{P-036}

Ambient air temperature and daily mortality from cardiovascular diseases during warm season in Astana, Kazakhstan

Topic: Diseases - CVD/diabetes

Presenter: Andrej Grjibovski

Andrej Grjibovski 1), 2); Nassikhat Nurgaliyeva 3), 4); Altay Sharbakov 3); Telman Seysembekov 3); Aliya Kosbayeva 4); Bettina Menne 5)

1) Department of International Public Health, Norwegian Institute of Public Health, Oslo, Norway; 2) International School of Public Health, Northern State Medical University, Arkhangelsk, Russia; 3) Medical University of Astana, Astana, Kazakhstan; 4) WHO office in Kazakhstan, Astana, Kazakhstan; 5) WHO European Centre for Environment and Health, Bonn Office, Bonn, Germany

Background: Associations between high temperatures and both the overall mortality and mortality from diseases of circulatory system have been observed in many cities in Europe and North America.
However, the evidence from rapidly developing settings with newly built infrastructure is scarce.

Objectives: To study associations between maximum apparent air temperature and daily mortality from cardiovascular and cerebrovascular causes in a rapidly developing city with a continental climate-Astana, Kazakhstan.

Methods: Daily counts of deaths from hypertensive diseases (ICD-10 codes: I10-I15), ischemic heart disease (I20-I25) and cerebrovascular diseases (I60-I69) among adults 18 years and older in Astana, Kazakhstan during warm periods (1 April-30 September) in 2000-2001 and 2006-2010, were obtained from the City Registry Office. Associations between mortality and maximum apparent temperature (average for lags 0-3) during the warm period were studied using first order autoregressive Poisson regression models controlling for wind speed and effects of month, year, weekends and holidays. Analyses were stratified by gender.

Results: An inverse association between the temperature and deaths from hypertensive diseases was found among women, but not men: an increase in temperature by $1{ }^{\circ} \mathrm{C}$ was associated with a decrease in the daily number of deaths by $3.1 \%$ (95\% CI $0.2-6.1$ ). No associations between temperature and deaths from ischemic health disease for were found. Deaths from cerebrovascular diseases were positively associated with the temperature only among women: an increase in temperature by $1{ }^{\circ} \mathrm{C}$ was associated with an increase in the daily number of deaths by $1.9 \%$ (95\% CI 0.4-3.5).

Conclusions: The effects of temperature were limited to women. While higher temperatures were associated with higher mortality for cerebrovascular diseases, an inverse association was observed for hypertensive diseases. Factors behind this association warrant further research with further going aim to develop strategies to mitigate the effects on climate change on population health.

\section{P-037}

Prognosis after ischemic heart disease according to body size: a cohort study

Topic: Diseases - CVD/diabetes Presenter: Christina C Dahm

Christina C Dahm 1); Anders Helles Carlsen 2); Anne Tjønneland 3); Thorkild IA Sørensen 4); Kim Overvad 15)

1) Section for Epidemiology, Department of Public Health, Aarhus University; 2) Section for Biostatistics, Department of Public Health, Aarhus University; 3) Institute of Cancer Epidemiology, Danish Cancer Society, Copenhagen, Denmark; 4) Institute of Preventive Medicine, Copenhagen University Hospitals, Copenhagen, Denmark; 5) Department of Cardiology, Center for Cardiovascular Research, Aalborg Hospital, Aalborg, Denmark

Background: Among incident cases of ischemic heart disease, overweight or mildly obese patients experience lower mortality than patients of normal or low weight or those who are very obese. This may reflect differing associations of lean and fat mass with mortality. Here, we investigate associations between pre-diagnostic body mass index (BMI), waist circumference (WC), fat and lean body mass indices and mortality among incident cases of ischemic heart disease. Methods: The Danish cohort study Diet, Cancer and Health consists of 57,053 men and women aged 50-64 at recruitment (1993-1997), when data on anthropometry, bioelectrical impedance and potential confounders were collected in study clinics. Incident ischemic heart disease status, obtained by linkage to national registers, was designated as entry time to this study, and failure as death or censoring. Centred restricted cubic splines of body size and composition measures of cases were related to mortality using Cox proportional hazards models adjusted for potential confounders. 
Results: 2,356 incident cases were included, recruited 4 months12 years prior. Hazards of death were greater for cases of $\mathrm{BMI}<23 \mathrm{~kg} / \mathrm{m}^{2}$ and $>31 \mathrm{~kg} / \mathrm{m}^{2}$ compared to cases of median BMI $\left(26.9 \mathrm{~kg} / \mathrm{m}^{2}\right)$, and did not level off at still greater body mass indices. Greater hazards of death were observed for those of low BMI for given WC or of low fat mass index, compared to median measures. However, cases of either very low or very high lean mass indices experienced greater hazards of death, compared to the median. Large, but not small, WC were associated with greater hazard of dying, compared to the median. Nevertheless, when WC was adjusted for BMI, the association was linear and positive (hazard ratio $=1.03$, $95 \%$ confidence interval $1.01 ; 1.04$ per $\mathrm{cm}$ ).

This study indicates that among ischemic heart disease cases, having a trim waist and average muscle mass prior to incident disease are related to the best chance of survival afterwards.

\section{P-038}

Female breast cancer in Central Serbia during the period 1999-2009

Topic: Diseases - CVD/diabetes

Presenter: Isidora Vujcic

\section{Isidora Vujcic 1); Sandra Sipetic 1)}

1) Insitute of Epidemiology, Medical Faculty, University of Belgrade

Background: Breast cancer is the most common cancer in women, both in the developed and the developing world. Nearly 1.38 million new breast cancer cases and 458,000 million cancer deaths occurred in 2008 worldwide

Objective: The aim of this descriptive epidemiologic study was to analyze trend incidence and mortality from breast cancer in women of Central Serbia during the period 1999-2009.

Methods: Cancer incidence and mortality was collected from the cancer registries of Central Serbia from 1999 to 2009. In data analysis age specific and standardised incidence and mortality rates were used. Standardised rates were calculated using WHO world standard population by method of direct standardisation. In order to analyze trend mortality from breast cancer we used linear trend.

Results: Breast cancer is leading cancer in serbian women. During the period 1999-2009. the average incidence rate was $61.0 / 100,000$ and average mortality rate 20.4/100,000. In Central Serbia breast cancer accounted for about $24.2 \%$ of all malignant tumors cases and $18.9 \%$ of malignant tumors deaths. In the same period in Central Serbia 2,800 women got and 1,068 died from breast cancer on the average each year. Incidence and mortality rates rose with age and the highest rates were in age group 70 and more years. During the observed period we noticed increase in trend incidence, and decrease in trend mortality which were non significant. Significant increase in incidence from breast cancer was noticed in age group 60-69 years. Significant increase in mortality was noticed in age groups 69-69 and over 70, while in age group 40-49 we noticed significant decrease in mortality. The highest average incidence and mortality rates were noticed in Belgrade district.

Conclusion: In order to reduce mortality from brest cancer it is necessary to preform adequate primary (elimination of risk factors) and seccondary prevention measures (screening and adequate therapy).

\section{P-039}

The associations between smoking and adverse outcomes in patients with atrial fibrillation: the diet, cancer and health study

Topic: Diseases - CVD/diabetes
Presenter: Ida Ehlers Albertsen

Ida Ehlers Albertsen 1), 2); Lars Hvilsted Rasmussen 3); Deirdre Lane 2); Thure Filskov Overvad 1), 2); Flemming Skjøth 1); Kim Overvad 4); Gregory Lip 2), 3); Torben Bjerregaard Larsen 1)

1) Department of Cardiology, Aalborg AF Study Group, Aalborg University Hospital, Aalborg, Denmark; 2) University of Birmingham Centre for Cardiovascular Sciences, City Hospital, Birmingham, UK; 3) Thrombosis Research Centre, Aalborg University, Aalborg, Denmark; 4) Section for Epidemiology, Department of Public Health, Aarhus University, Aarhus, Denmark

Background: Smoking and atrial fibrillation are major health problems worldwide and responsible for many health care costs but smoking has not yet been formerly assessed as a risk factor for adverse outcomes among AF patients.

Objectives: Our aim was to investigate whether smoking impacts on the risk of stroke and death in patients with atrial fibrillation. To test this hypothesis, we analysed data from a large Danish prospective cohort, the Diet, Cancer and Health study.

Methods: Prospective cohort study of 57,053 persons aged 50-64 years. The risk of thromboembolism or death according to smoking habits among 3,161 patients with incident atrial fibrillation (mean age 66.9 years; 2,032 men, 1,129 women) was assessed using Cox proportional hazard models.

Results: During the 17,391 person-years of follow-up (median 4.9 years $) 619(19.6 \%)$ patients died and $215(6.8 \%)$ had an ischemic stroke or arterial thromboembolism. Of the subjects with atrial fibrillation $34 \%$ were current smokers and $37 \%$ former. After adjustment for vitamin $\mathrm{K}$ antagonist treatment with never smokers as the reference, the hazard ratios of thromboembolism or death was $3.13(1.72-6.37)$ and $2.73(2.02-3.70)$ among heavy ( $>25 \mathrm{~g}$ of tobacco/d) current female and male smokers, respectively. The associations remained after adjustment for well-established risk factors used in risk stratification schemes (vitamin $\mathrm{K}$ antagonisttreatment, congestive heart failure, hypertension, diabetes, prior stroke/thromboembolism/transient ischemic attack, vascular disease and age) with hazard ratios of 3.64 (1.88-7.07) and 2.17 (1.59-2.95) among women and men, respectively. Smoking was still highly associated with thromboembolism or death after excluding people with a cancer diagnosis during follow-up in a sensitivity analysis.

Conclusions: Smoking is associated with a higher risk of thromboembolism or death in patients with atrial fibrillation even after adjusting for well-recognised risk factors used in stroke risk stratification schemes. Patients with AF who smoke represent a 'high-risk' population, which necessitates proactive management of all cardiovascular risk factors. Based on the association found, oral anticoagulation should be considered to possibly reduce the risk of 'thromboembolism and death' in these patients.

\section{P-040}

Rapid response systems and in-hospital delay in acute coronary syndrome

Topic: Diseases - CVD/diabetes

Presenter:

António Madureira Dias 1); Carlos Pereira 1); Maria João Monteiro 2); Célia Santos 3); Nuno Santos 4); Madalena Cunha 1); Regina Célia Saraiva 1)

1) CI\&DETS and FCT (PEst-OE/CED/UI4016/2011) - Superior Health School, Polytechnic Institute of Viseu, Portugal; 2) University of Tras-os-Montes and Alto Douro - Superior Nursing School, Portugal; 3) ICBAS-University of Porto, Superior Nursing School of 
Porto, Portugal; 4) Department of Emergency, Hospital Center Tondela-Viseu, Portugal

Background: Coronary acute disease is, because of its prevalence, morbidity and mortality, an emergency medical situation that justifies a planned intervention of Emergency Medicine. Coronary Green Way, name usually used in Portugal to nominate Rapid Response Systems (RRS), is an organized strategy that aims to improve patients' accessibility in the acute phase to more suitable medical cares, diagnosis and treatment. The time factor is critical in Acute Coronary Syndrome (ACS), since half of the deaths occur in the first hours after onset of symptoms. The sooner you start the treatment, the better the chances of survival.

Objective: To evaluate the effectiveness of the RRS in classifying ACS patients and its effect on in-hospital delay (IHD).

Methods: It is a quantitative cross-sectional study. We retrospectively evaluated all the patients with ACS admitted to the Coronary Care Unit through the Emergency Department (ER) of Hospital Center Tondela-Viseu in the first 9 months of 2010. Patients were divided into two groups according to RRS (Group 1: referenced by RRS; Group 2: not referenced by RRS). We compared the groups in terms of clinical and demographic characteristics, triage time (TT), door-to-ECG time (DET), medical-ECG time (MET), and door-toballoon time (DBT); We studied 204 patients with a mean age of $69.8 \pm 12.7$ years, $143(70.1 \%)$ were male. Of the patients referred by RRS: Type of ACS: $35.4 \%$ of patients had ST-elevation myocardial infarction (STEMI), $44.9 \%$ had AMI without ST elevation, $19.7 \%$ with unstable angina; As for the accomplished treatment, $60.3 \%$ was primary-Percutaneous Coronary Intervention and $39.7 \%$ underwent fibrinolysis.

Results: Group 1 was consisted of 147 patients (72.1\%) and Group 2 of $57(27.9 \%)$. No significant differences were observed between the two groups regarding clinical and demographic characteristics. Patients in Group 1 had average times lower than those in Group 2, as the TT $(p=0.005)$, DET $(p=0.000)$, MET $(p=0.000)$ and DBT $(p=0.000)$.

Conclusion: It is intended, through the RRS, to promote the improvement of access to the patient with chest pain to quick diagnostic confirmation and institution of better therapy, more appropriate to the ACS. In addition to increasing public awareness about the need to seek a doctor immediately after the onset of ACS, using an ambulance as a direct transportation to the acute hospital is fundamental a collaborative model between the ER and Cardiology/Cardiac catheterization. Only in this way it is possible to translate 'time is tissue' and 'time is life'.

\section{P-041}

Use of medicine claims data to investigate cardiovascular disease prevalence in South African patients with metabolic syndrome

Topic: Diseases - CVD/diabetes

Presenter: Johanita Burger

\section{Johanita Burger 1); Martie Lubbe 1)}

1) MUSA, North-West University (Potchefstroom campus), Potchefstroom, South Africa

Background: There is controversy over the clinical impact of metabolic syndrome, with inconsistent findings from various studies. However, several studies strongly suggest that the syndrome is an important risk factor for cardiovascular disease (CVD). Depending on the defining criteria for the syndrome, the relative risk of CVD associated with the metabolic syndrome is higher in women compared with men.
Objectives: The aim of the study was to determine the prevalence of cardiovascular conditions and association with gender in patients meeting the metabolic syndrome criteria, by using South African private health care medicine claims data. In addition, we sought to identify other chronic conditions that may influence the strength of this association.

Methods: A cross-sectional study was conducted on paid prescription claims for 17,866 patients, meeting AHA/NHBLI metabolic syndrome diagnostic criteria. Data were obtained from a pharmaceutical benefit management company, for the period January, 1, 2008 through December, 31, 2008. Cardiovascular conditions (cardiac failure, cardiomyopathy, coronary artery disease and dysrhythmia) were identified using ICD-10 codes specified for reimbursement purposes. Associations between cardiovascular conditions and gender in metabolic syndrome patients were investigated using logistic regression analysis, age-adjusted odds ratios (aORs), and $95 \%$ confidence intervals (CIs).

Results: Females [mean age 65.1 (SD 12.3) years] comprised $46.1 \%$ of the study population. Males were slightly younger at 62.6 (SD 11.6) years $(\mathrm{d}=0.2)$. Cardiac failure, cardiomyopathy, coronary artery disease and dysrhythmia were present in 4.2, 1.5, 5.4 and $1.8 \%$ of patients, respectively. In age-adjusted analysis, female gender was negatively associated with prevalent cardiac failure $(\mathrm{aOR}=0.78$, $95 \%$ CI $0.68-0.91, P=0.0013)$, cardiomyopathy $(\mathrm{aOR}=0.80$, $95 \%$ CI $0.63-1.03, P=0.0867)$, coronary artery disease $(\mathrm{aOR}=$ $0.60, \quad 95 \%$ CI $0.52-0.69, \quad P<0.0001)$ and dysrhythmia $(\mathrm{aOR}=0.54,95 \% \mathrm{CI} 0.42-0.68, P<0.0001)$. In the copresence of hypothyroidism, however, the age-adjusted odds for females was 4.65 [95\% CI 3.05-7.07] for cardiac failure, 3.71 [95 \% CI 2.0-6.91] for cardiomyopathy, and 3.16 [95\% CI 2.12-4.70] for coronary artery disease (all trends $P<0.0001$ ), respectively. The age-adjusted odds for females having copresent dysrhythmia and hypothyroidism were 1.44 (95\% CI 2.12-4.70, $P=0.0330$ ).

Conclusion: The association between cardiovascular conditions and metabolic syndrome was particularly significant for males. Females had strong increased associations only in the copresence of hypothyroidism. The presence of hypothyroidism in metabolic syndrome is therefore an important discriminating factor for cardiovascular disease in females.

\section{P-042}

Epidemiological study of chronic kidney disease progression: a large-scale population-based cohort study

Topic: Diseases - CVD/diabetes

Presenter: Phisitt Vejakama, Atiporn Ingsathit, Ammarin Thakkinstian

Phisitt Vejakama 1); Atiporn Ingsathit 1); Ammarin Thakkinstian 1)

1) Section for Clinical Epidemiology and Biostatistics, Ramathibodi Hospital, Mahidol University, Thailand

Background: Chronic kidney disease (CKD) is recognized as one of the leading health problems. However, information about the disease progression is still limited.

Objectives: To estimate the time of changing stage and probability of end-stage renal disease (ESRD) at 2, 5, and 10 years in CKD with/ without diabetes.

Methods: A retrospective cohort was conducted by retrieving CKD data from the annual health screening and community-hospital databases in Ubon Ratchathani, Thailand, from 1997 to 2011. Diabetes was identified from the ICD10 codes or receiving oral hypoglycemic drugs or insulin. CKD was determined by having urine abnormalities or 
decreased level of kidney function for 3 months or longer. KaplanMeier method was applied to estimate the median of changing stage and probability of ESRD. A sub-distribution competing risk model was applied to determine the ESRD risk treating death as a competing risk. Results: Among 29,078 non-diabetic CKDs, 4,732, 6,092, 8,956, 6,076, 3,222 were respectively diagnosed as CKD stage 1, 2, 3a, 3b, and 4 at enrollment. The median follow-up was 5.9 (range 1.0-4.2) years. The mean age was 62.4 years and $56 \%$ were females. Among 22,099 diabetic CKDs, 2,998, 4,174, 8,249, 4,692, 1,985, and 1 patients were respectively stage $1,2,3 \mathrm{a}, 3 \mathrm{~b}, 4$, and 5 at enrollment. The median follow-up was 6.3 (range 1.0-14.1) years. The mean age was 61.3 years and $72 \%$ were females.

The median CKD progression from stage 1 to 2,2 to $3 \mathrm{a}, 3 \mathrm{a}$ to $3 \mathrm{~b}$, $3 \mathrm{~b}$ to 4 , and 4 to 5 were respectively $12.1,13.3,7.4,8.3$, and 8.3 years for non-diabetic patients. The CKD progressed more rapidly in diabetic patients with the corresponding median of 3.8, 4.3, 3.8, 4.6, and 5.5 years, respectively.

The overall ESRD rates were 0.9/100/year $(95 \%$ CI $0.8,1.0)$ and $2.2 / 100 /$ year $(95 \%$ CI 2.1, 2.3) in non-diabetes and diabetes, respectively. The ESRD probability at 2, 5, and 10 years were respectively $1.0 \%$ (95\% CI 0.9, $1.2 \%), 4.3 \%$ (95\% CI 4.0, $4.6 \%$ ), $10.5 \%(95 \%$ CI $9.8,11.2 \%)$ for non-diabetes and $1.6 \%(95 \% \mathrm{CI}$ $1.5,1.8 \%), 9.0 \%$ (95\% CI 8.6, $9.4 \%$ ), $25.3 \%$ (95\% CI 24.2, $26.4 \%$ ) for diabetes. The ESRD risk was 2.2 times (95 \% CI 2.1, 2.3) higher in diabetes than non-diabetes.

Conclusions: This study describes the natural history of CKD in an Asian population with ordinary practice. Diabetes reveal significantly rapid decline in GFR category than non-diabetes. Knowing prognostic factors may help in delaying CKD progression and increase patients' survival.

\section{P-043}

Data mining procedures establish an accurate predictive model for incident hypertension

Topic: Diseases - CVD/diabetes

Presenter:

Glenn Fung 2); Till Ittermann 1); Sebastian Baumeister 1); Marcus Dörr 1); Allan Linneberg 3); Torben Jørgensen 3); Heyo Kroemer 1)

1) University Greifswald; 2) Siemens Healthcare; 3) University of Copenhagen, RCPH Glostrup

Background: Data mining represents an alternative approach to identify new predictors of multifactorial diseases.

Objective: This work aimed at building an accurate predictive model for incident hypertension using data mining procedures.

Methods: The primary study population consisted of 1,605 normotensive individuals aged 20-79 years with 5-year follow-up from the population-based SHIP. The initial set was randomly split into a training and a testing set. We used a probabilistic graphical model applying a Bayesian network to create a predictive model for incident hypertension and compared the predictive performance with the established Framingham risk score for hypertension. Finally, the model was validated in 2,887 subjects from INTER99, a Danish community-based intervention study.

Results: In the training set of SHIP data, the Bayesian network used a small subset of relevant baseline features including age, mean arterial pressure, rs16998073, serum glucose und urinary albumin concentrations. Furthermore, we detected relevant interactions between age and serum glucose as well as between rs16998073 and urinary albumin concentrations (AUC 0.76). The model was confirmed in the SHIP validation set (AUC 0.78) and externally replicated in INTER99
(AUC 0.80). Compared to the established Framingham risk score for hypertension, the predictive performance of the new model was similar in the SHIP validation set and even better in INTER99.

Conclusions: Data mining procedures identified a predictive model for incident hypertension, which included innovative and easy-tomeasure variables. The findings promise great applicability in screening settings and clinical practice.

\section{P-044}

Channeling of statin use towards low-risk population and patients with diabetes

Topic: Diseases - CVD/diabetes

Presenter: Maria Rikala

Maria Rikala 1); Risto Huupponen 1), 2); Arja Helin-Salmivaara 1), 3); Maarit Jaana Korhonen 1), 4)

1) Department of Pharmacology, Drug Development and Therapeutics, University of Turku, Turku, Finland; 2) Unit of Clinical Pharmacology, Turku University Hospital, Turku, Finland; 3) Unit of Primary Health Care, Hospital District of Helsinki and Uusimaa, Helsinki, Finland; 4) Department of Public Health, University of Turku, Turku, Finland

Background: Statins are part of the standard therapy for individuals with established cardiovascular disease (CVD) or diabetes. In lowrisk individuals, however, statin use remains a subject of ongoing debate. Over the past decades, statin use has increased dramatically in European countries, including Finland. There is a suggestion that this increase may be attributed to expansion of statin prescribing to lowrisk individuals.

Objectives: This study aimed to analyze trends in characteristics of statin users in the whole community-dwelling population of Finland between 1999 and 2008.

Methods: Individuals purchasing statins in 1999-2008 were identified from the nationwide Prescription Register. For each calendar year, incident users were defined as those who purchased statins for the first time ever, while ongoing users refer to those who had earlier statin purchases, including both continuous users and re-initiators. Trends in demographics, comorbidities and co-medications were analyzed using data from nationwide comprehensive health registers. Results: Over the study period, the annual number of incident users increased 1.6-fold from 50,125 to 78,058 and that of ongoing users increased 4.6-fold from 114,091 to 521,218. The proportion of incident users without cardiovascular disease (CVD) or diabetes increased from 23.6 to $27.8 \%$ while the proportion of those with diabetes increased from 15.7 to $19.5 \%$. An increasing proportion of ongoing users had diabetes (from 13.8 to $22.8 \%$ ). The proportion of ongoing users without CVD or diabetes remained below one-fifth; however, their number increased fivefold. Over the study period, there was a clear shift toward prescribing of higher statin doses both among incident and ongoing users.

Conclusions: Statin use is expanding to individuals with low cardiovascular risk despite the fact that clinical guidelines emphasize interventions other than pharmacotherapy for this population. At the same time, statin use is increasingly targeted to patients with diabetes, a high-risk group that is likely to benefit from it.

\section{P-045}

Trends in case-fatality of myocardial infarction and stroke, by social class in Sweden 1987-2007

Topic: Diseases - CVD/diabetes 


\section{Presenter: Ninoa Malki}

Ninoa Malki 1); Alex Ploner 1); Ilona Koupil 2); Paul Lambert 3); Sanna Tiikkaja 1); Sara Hägg 1); Par Sparen 1)

1) Department of Medical Epidemiology and Biostatistics, Karolinska Institutet; 2) Centre for Health Equity Studies, CHESS, SU; 3)

Department of Health Sciences, University of Leicester

Background: In Europe, men between 20 and 64 years of age in manual occupations run a three times higher risk of premature death from cardiovascular diseases (CVD) compared to those in non-manual occupations. Health inequalities are substantial, and amount to between 5 and 10 years difference in average life expectancy at birth. There are concerns about the persistent significant socioeconomic differences in incidence and case-fatality of CVD in Sweden.

Objectives: In this study we analyze temporal trends in survival after myocardial infarction (MI) and stroke for different socioeconomic groups in Swedish men and women aged $30-75$ years.

Method: We used a national matched cohort study, based on all Swedish residents born 1932-1960; the Swedish Hospital Discharge Register and Cause of death Register were used to identify incident cases of MI and stroke. The socioeconomic status was retrieved from 1980 and 1990 Population and Housing Censuses. Socioeconomic groups were classified as Low Manual, High Manual, Self-Employed, Low Non-Manual and High Non-Manual. In total 142,223 MI cases and 77,652 stroke cases where identified using ICD-9 and ICD-10 codes. Logistic regression will be used to analyze 1 day case-fatality and flexible parametric survival models were used to analyze the long term case-fatality ( 28 days, 3 month and 1 year). We provide estimates of both the absolute case-fatality rates as well as the casefatality rate ratios comparing the different socioeconomic groups with respect to their survival in MI and stroke on different time scales. More detailed result will be available for presentation at the conference.

Preliminary results: Among MI cases totally 32,831 died, 24,609 died the first day and 8,178 death occurred within 1 year. For stroke, of totally 77,652 cases; 9,715 died (3,422 died first day, 6,293 within 1 year). There were substantial socioeconomic differences in case fatality in MI and stroke. The case-fatality rate has generally decreased over time for all socioeconomic groups. Self-employee men had highest case-fatality rate at years (1987-2010) followed by low manual group. In year (2007-2010) the self-employee men decreased the case-fatality rate and have lower case-fatality than low manual and high manual.

Preliminary conclusions: We observed a general reduction in MI case-fatality rate over time with; the largest reduction seen in selfemployed men.

\section{P-046}

Health condition of the elderly with diabetes mellitus in São Paulo-Brazil, in 2003 and 2008: a population-based study

Topic: Diseases - CVD/diabetes

Presenter: Sheila Rizzato Stopa

Sheila Rizzato Stopa 1); Chester Luiz Galvão Cesar 1); Neuber José Segri 2); Moisés Goldbaum 3); Vanessa Valente Guimarães 4); Erika Laide Nigro 1); Marilisa Berti de Azevedo Barros 5); Maria Cecília Goi Porto Alves 6); Luana Carandina 7)

1) School of Public Health, University of São Paulo.; 2) Federal University of Mato Grosso; 3) Medical School, University of São Paulo; 4) School of Arts, Sciences and Humanities, University of São Paulo; 5) Faculty of Medical Sciences, University of Campinas; 6)
Health Institute, Secretary for Health of Sao Paulo State; 7) Botucatu Medical School, São Paulo State University

Background: Diabetes Mellitus has been considered a worldwide epidemic affecting mainly middle-aged and elderly people.

Objective: Describe the health status of the elderly with diabetes in the city of São Paulo-Brazil, in two different periods (2003 and 2008), with its association to demographic, socioeconomic, lifestyle characteristics.

Methods: Data from two cross-sectional population-based health surveys (ISA-Capital 2003 and ISA-Capital 2008). Data analysis was carried out by comparison of prevalences at two different times and their $95 \%$ confidence intervals. The difference between these two periods was considered statistically significant when there was no overlap between the confidence intervals.

Results: In 2003, the prevalence of diabetes among the elderly was $17.56 \%$ (95\% CI 14.91-20.56) and in $200820.08 \%$ (95\% CI 17.32-23.15), which was statistically significant. Furthermore, the prevalence of diabetes was higher in elderly with education and income being lower. Among the adopted means to control diabetes, the oral medication routine had the highest prevalence, occurring an increase of $10.87 \%$ during the period, with a statistically significant difference. There was also an increase in the perception of such habit as important for disease control, from $49.00 \%$ in 2003 to $63.31 \%$ in 2008 , which was also statistically significant. With regard to the use of health services for diabetes control by the elderly population, there were significant differences related to access and use of public service, which was higher in the elderly with the lowest income level and lowest degree of education.

Conclusions: Diabetes is a complex and challenging disease, both for the elder and the society and for the public health systems. Still, actions are needed to encourage health promotion practices, since they had lower prevalence in comparison to oral medication routine. It proves investments in public health policies are necessary, particularly for those elderly targeted at low income and low education. The results of this study strengthen the importance that these changes are essential for improving the health conditions of elderly diabetics.

Keywords: Elderly Health, Chronic Diseases, Diabetes Mellitus, Lifestyle, Health Surveys, Cross-sectional Study

\section{P-047}

Clinical decision making in chronic kidney disease: a novel record linkage cohort study to develop tools to support patient care

Topic: Diseases - CVD/diabetes

Presenter: Corri Black

Corri Black 1), 2); Angharad Marks 1), 2); William Simpson 2); Gordon Prescott 1); Nick Fluck 2); Lynn Robertson); WCS Smith)

1) University of Aberdeen; 2) NHS Grampian

Background: Chronic kidney disease (CKD) affects $8-10 \%$ of the adult population, rising to more than $50 \%$ of those over 85 years of age. The vast majority of individuals have moderate CKD (stage 3) and current practice in the UK aims to register all such individuals onto CKD registers in primary care. Less than $1 \%$ of these people will progress to end stage kidney disease over the next 5 years, but many will be at increased risk of cardiovascular disease and mortality. The opportunity then, is to identify patients who would benefit most from close management and to target their care to match need. Across the healthcare system we gather a great deal of information about patients. This study explores ways to use healthcare information to help plan care. 
Objectives: To determine if routine healthcare data could be used to identify different risk groups among patients with CKD and whether that information could be translated into tools to support patient care. Methods: A large population based cohort (GLOMMS II) was constructed using data linkage of patients' laboratory data to hospital episode and registry data. A cohort with CKD and a comparison group without CKD was established based on their kidney function in 2003. Data linkage allowed follow up to July 2009 for death, myocardial infarction, renal replacement therapy (RRT), admissions to hospital and worsening kidney function. Incidence rate ratios (IRR) were estimated for each outcome and to explore potential predictors. This information was used to develop prediction tools.

Results: GLOMMS II comprised 70,780 people, of which approximately 19,000 had CKD. Adjusted IRR for RRT among people with CKD was 436.9 (60.3-3165.2) compared to those with normal renal function. The risk of dying with CKD was 1.22 times, and myocardial infarction 1.95 times, that of people with normal kidney function. Being male, worse kidney function and proteinuria were associated with increased risk of poor outcomes. A simple clinical tool was constructed to describe outcomes at 5 years. A logistic regression prediction model for RRT performed well (c statistic 0.9581, sensitivity 0.735 , specificity 0.979 ) classifying $98 \%$ of patients correctly. A planning tool was developed as a spreadsheet.

Conclusion: In the UK, the interface between patient self-care, primary care and secondary care is critical to successful management of patients with chronic conditions. We have shown that linking together information from across the healthcare system can help inform patient care and service planning.

\section{P-048}

Distribution and determinants of $\mathrm{C}$-reactive protein in the older adult population: European prospective investigation into cancer Norfolk study

Topic: Diseases - CVD/diabetes

Presenter: Sara Ahmadi Abhari

\section{Sara Ahmadi Abhari 1); Robert Luben 1); Nicholas Wareham 2);} Kay-Tee Khaw 1)

1) Department of Public Health and Primary Care, University of Cambridge, Cambridge, UK; 2) MRC Epidemiology Unit, Institute of Metabolic Sciences, Cambridge, UK

Background: C-reactive protein (CRP) is a well documented predictor of cardiovascular diseases and mortality.

Objectives: We aimed to better understand the distribution and determinants of CRP in the population.

Methods: Study participants were men and women aged 40-79 in the UK based EPIC-Norfolk population based cohort study. CRP was measured in 18,586 available serum samples $(8,334$ men and 10,252 women) using a high-sensitivity assay. General multiple regression analysis with logarithmic-transformed CRP as outcome was performed separately for men and women. Geometric-mean (95\% confidence interval) CRP was calculated by back transforming (exponentiating) adjusted mean loge-CRP values across categories of exposure.

Results: In cross-sectional analyses, the range of serum CRP (mg/l) was $0.1-188.3$ and the median (inter-quartile range) was 1.5 (0.8-3.3). A third of the population had CRP levels above 3 and $5 \%$ had CRP levels above $10 \mathrm{mg} / \mathrm{l}$. CRP levels were comparable in men and women who were not taking postmenopausal hormone replacement therapy (HRT). Women who were taking HRT had double CRP levels compared to HRT non-users [adjusted geometric mean CRP $2.25 \mathrm{mg} / \mathrm{l}$ (95\% CI 2.08-2.29) in postmenopausal HRT users vs.
$1.32 \mathrm{mg} / \mathrm{l}$ (1.28-1.36) among HRT non-users]. Smoking was also strongly related to CRP [adjusted geometric mean CRP comparing current vs. never smokers were $2.56(2.40-2.72)$ vs. $1.29(1.24-1.35)$ for men and $1.57(1.40-1.76)$ vs. $1.25(1.13-1.38)$ for women respectively]. Serum CRP was positively and independently associated with age, body mass index, and waist circumference and inversely with height (multivariable adjusted (standard error) regression coefficients (corresponding to percent difference in CRP $(\mathrm{mg} / \mathrm{l})$ values $)$ were $0.10(0.01)$ in men and $0.09(0.01)$ in women per 5 years age, $0.06(0.03)$ in men and $0.22(0.02)$ in women per $4 \mathrm{~kg} / \mathrm{m}^{2}$ (1-SD) higher BMI, $0.26(0.02)$ in men and $0.20(0.02)$ in women per $10 \mathrm{~cm}(1-\mathrm{SD})$ higher waist, and $-0.16(0.02)$ in men and -0.08 $(0.02)$ in women per $10 \mathrm{~cm}(1-\mathrm{SD})$ higher height; all $P$-values $<0.01)$. Social class and alcohol intake were not independently related to CRP but there was a strong inverse association with educational status [geometric mean CRP (95 \% CI) 1.17 (1.05-1.31) amongst women with university education vs. 1.34 (1.21-1.49) among women with below O-level or no education, $P$-value $<0.001$, similar results for men].

Conclusions: The distribution of serum CRP in the population is similar in men and women after taking into account smoking and HRT use. Anthropometric factors as well as educational status are strongly related to serum CRP.

\section{P-049}

Medicine possession rate of antihypertensive medicine in the private healthcare market of South Africa and the cost implication thereof

Topic: Diseases - CVD/diabetes

Presenter: Martie Lubbe

\section{Rianda Joubert 1); Martie Lubbe 1); Jan Serfontein 1)}

1) Medicine usage in South Africa, Faculty of Health Sciences, North-West University, Potchefstroom Campus, Potchefstroom, South Africa

Background: Hypertension is one of the top chronic conditions in South Africa affecting approximately 105 cases per 1,000 people, accounting for $7 \%$ of all deaths in persons $>60$, whilst persons $>55$ years have a $90 \%$ residual lifetime risk of developing hypertension.

Objectives: To investigate the medicine possession rate (MPR) of hypertensive patients, in a section of the private healthcare market of South Africa.

Methods: A retrospective drug utilization study was conducted on patients $(\mathrm{N}=376$ 280) receiving antihypertensive treatment (including MIMS categories 7.3-hypertensive medicine items and 16.1 - diuretics) more than once during the study period (1 January 2005-31 December 2011). The study population was stratified according to gender, and three age groups. Age group (AG) 1 included patients $\leq 18$ years, AG2 included patients $>18$ and $\leq 45$ years, and AG3 included patients $>45$ years. The MPR was calculated per trade name by using the following equation: MPR $=$ (total number of days of antihypertensive medicine items supplied - days supplied at the last refill)/(date last claimed - date first claimed) $\times 100 \%$. The MPR was divided into three categories: $\mathrm{C} 1$ indicating unacceptable low MPR $(\leq 80 \%), \mathrm{C} 3$ indicating unacceptable high MPR $(\geq 110 \%)$, and $\mathrm{C} 2$ indicating acceptable MPR $(80 \%<$ MPR $\leq 110 \%)$. (RSA Rand(R)/\$US $=$ R8.12 on 31 Dec. 2011).

Results: The average MPR for all antihypertensive medicine items were $82.6 \%$ [95 \% CL 82.4-82.9] ( $\mathrm{N}=925$ 075), however only $48 \%$ of all these items showed acceptable MPR levels, and accounted 
for $51 \%$ of the total cost $(\mathrm{N}=\mathrm{R} 933967$ 808.62). Approximately $42 \%$ antihypertensive medicine items showed unacceptable low MPR with an associated cost of almost $44 \%$. Male patients showed a statistically significant higher average MPR (83.9\% [95\% CL 83.6-84.4] than their female counterparts $(81.5 \%$ [95\% CI $81.2-81.9]) \quad(p<0.0001 ; \mathrm{d}$ value $=0.02)$. However only $49 \%$ female patients and $48 \%$ male patients had acceptable MPR levels, accounting for $39 \%$ (Nfemale = R273 368 548.66; Nmale = R243 563 013.30) of the total cost. Patients in AG1, AG2 and AG3 showed 65, 60 and $51 \%$ unacceptable high and low MPR levels combined, respectively, accounting for $60 \%$ (AG1), $60 \%$ (AG2) and $61 \%$ (AG3) of the total cost.

Conclusion: Antihypertensive medicine with unacceptable MPR levels had a fairly large cost and thus a large financial impact on members and medical schemes. Improvement in MPR will lead to less wastage of funds and improved therapeutic benefits. Younger patients showed higher levels of unacceptable antihypertensive MPR whilst older patients had higher levels of acceptable MPR.

\section{P-050}

Obesity and risk of provoked and unprovoked venous thromboembolism: a Danish cohort study

Topic: Diseases - CVD/diabetes

Presenter: Henrik Solli

Henrik Solli 1); Morten Schmidt 1); Morten Olsen 1); Rikke Bech Nielsen 1); Finn Breinholt Larsen 2); Henrik Toft Sørensen 1)

1) Department of Clinical Epidemiology, Aarhus University Hospital, Aarhus, Denmark; 2) Public Health and Quality Improvement, Central Denmark Region, Aarhus, Denmark

Background: Increasing evidence support obesity as a risk factor for venous thromboembolism (VTE), but the precise relation remain uncertain and may be different for provoked and unprovoked VTE.

Objectives: To examine the association between obesity and provoked and unprovoked VTE.

Methods: We performed a cohort study based on self-reported questionnaire data from the Danish survey How Are You? We defined four categories of body mass index (BMI): underweight (BMI < 18.5), normal weight $(18.5 \leq \mathrm{BMI}<25)$, overweight $(25 \leq \mathrm{BMI}<30)$, and obesity (BMI $\geq 30)$. Using the Danish National Registry of Patients, covering all Danish hospitals, we identified all inpatient and outpatient diagnoses of VTE. We subdivided VTE into provoked VTE (diagnosis of fracture, surgery, trauma, or pregnancy within 90 days before, or a diagnosis of malignancy prior to or within 90 days after the VTE diagnosis) and unprovoked VTE (remaining cases). We followed participants from return of questionnaire in spring 2006 until VTE, emigration, death, or 31 December 2011, whichever came first. We used Cox regression with person age as the underlying time-scale to compute hazard ratio (HR) with $95 \%$ confidence interval adjusting for sex.

Results: Our study cohort consisted of 21,439 individuals, among which $47.0 \%$ were of normal weight, $3.8 \%$ underweight, $35.7 \%$ overweight and $13.6 \%$ obese. A total of $53.2 \%$ were female and the average age was 51 years. Compared with persons of normal weight, the adjusted HR for VTE overall was 1.36 (95\% CI 0.65-2.86) for underweight, 1.11 (95\% CI 0.77-1.59) for overweight, and 1.99 (95\% CI 1.33-3.00) for obese individuals. For provoked VTE the adjusted HR was 1.27 (95\% CI 0.38-1.4.26) for underweight, 1.13 (95\% CI 0.64-2.01) for overweight, and 1.93 (95\% CI 1.00-3.71) for obese individuals. Lastly, the assessment for unprovoked VTE yielded an adjusted HR of 1.39 (95\% CI 0.54-3.58) for underweight,
1.06 (95\% CI 0.66-1.70) for overweight and 1.99 (95\% CI 1.18-3.35) for obese individuals.

Conclusions: Obesity is a strong risk factor for both provoked and unprovoked VTE.

P-051

The association between plasma homocysteine and coronary heart disease is modified by the MTHFR $677 \mathrm{C}>\mathrm{T}$ polymorphism

Topic: Diseases - CVD/diabetes

Presenter: Kirsten Mehlig

Kirsten Mehlig 1); Karin Leander 2); Fredrik Nyberg 1), 3); Christina Berg 4); Lena Björck 5); Henrik Zetterberg 6); Gianluca Tognon 1); Elisabeth Strandhagen 1); Lauren Lissner 1); Dag Thelle 1), 7)

1) Dept Public Health and Community Medicine, Institute of Medicine, Sahlgrenska Academy, University of Gothenburg, Sweden; 2) Unit of Cardiovascular Epidemiology, Institute of Environmental Medicine, Karolinska Institutet, Stockholm, Sweden; 3) AstraZeneca R\&D, Mölndal, Sweden; 4) Dept of Food and Nutrition, and Sport Science, University of Gothenburg, Sweden; 5) Dept of Molecular and Clinical Medicine, Institute of Medicine, Sahlgrenska Academy, University of Gothenburg, Sweden; 6) Dept Psychiatry and

Neurochemistry, Institute of Neuroscience and Physiology, Sahlgrenska Academy, University of Gothenburg, Sweden; 7) Dept of Biostatistics, Institute of Basic Medical Science, University of Oslo, Norway

Background: An elevated level of total plasma homocysteine (tHcy) has been associated with risk of coronary heart disease (CHD). The level of tHcy is affected by lifestyle, e.g. smoking, physical activity, weight and diet, in addition to genetic predisposition. The MTHFR $677 \mathrm{C}>\mathrm{T}$ polymorphism (rs1801133) is among the strongest genetic predictors of tHcy.

Objectives: We aimed to examine whether the association between tHcy and CHD is modified by the MTHFR 677C $>$ T polymorphism. Methods: Data from two case-control studies of CHD were analyzed in parallel. The Stockholm Heart Epidemiology Programme (SHEEP) was based on first-time myocardial infarction (MI) cases and controls recruited in Stockholm between 1992 and 1994. The INTERGENE study was based on cases of MI and unstable angina and controls recruited in Gothenburg between 2001 and 2004. Total plasma homocysteine was determined in 885 patients and 1159 controls from SHEEP as well as in 294 patients and 624 control subjects from INTERGENE. Multiple logistic regression analysis was used to investigate the association between tHcy and CHD in the two studies, and the modification of this association by genotype.

Results: High tHcy was confirmed to be a risk factor for CHD in both studies despite differences in the prevalence of risk factors for tHcy and CHD, e.g. higher prevalence of obesity and lower prevalence of smoking and physical inactivity in the later study. In SHEEP, the association between tHcy and MI was seen in MTHFR 677 C-homozygotes $(\mathrm{OR}=1.5,95 \% \mathrm{CI} 1.3,1.8$, for increase by 1 standard deviation) and heterozygotes $(\mathrm{OR}=1.4,95 \% \mathrm{CI} 1.2,1.6)$ but not in T-homozygotes, a significant effect modification that was only slightly reduced when adjusted for waist-to-hip ratio, smoking and physical activity. An effect modification of similar magnitude was observed but not statistically significant in the smaller INTERGENE study. A pooled analysis of both studies confirmed the reduction of risk due to tHcy in T-homozygotes compared to carriers of the C-allele (OR for interaction $=0.71,95 \%$ CI 0.56, 0.90).

Conclusions: Two Swedish case-control studies showed a similar modification by MTHFR 677 genotype of the effect of tHcy on CHD, 
despite secular differences in the prevalence of risk factors for high tHcy and CHD.

\section{P-052}

Mortality from endocrine, nutritional and metabolic diseases in Brazil: the impact of diabetes and the influence of social inequalities

Topic: Diseases - CVD/diabetes

Presenter: Paulo Cesar Ramos

\section{Maria Célia Formiga 1); Paulo Cesar Ramos 1); Nilma Costa 2 ) \\ 1) Department of Demography and Actuarial Science/UFRN; \\ 2) Department of Collective Health/UFRN}

Background: Nutritional and metabolic endocrine diseases-DENM (ICD-10 chapter IV) represented the fifth main group of causes of death in the Brazilian population, in the year 2010. Most of these deaths occurred due to diabetes mellitus, which, in turn, are mostly associated with obesity conditions of the population. According to data from the Ministry of Health (2011) almost half of the Brazilian adult population ( $48.5 \%$ ) is overweight and $15.8 \%$ are obese, which configures a worrying picture in the scenery of the nutritional transition..

Objectives: To analyze the data of deaths due to DENM in Brazil in the year 2010, highlighting the impact of diabetes, in the context of its Federated Units (UF), revealing the possible associations with social inequalities identified regionally in the country.

Methods: The source of mortality and socioeconomic data was the Ministry of Health-Department of Informatics of the Unified Health System (DATASUS), for the year of 2010. The UF was used as units of analysis and a descriptive study of proportional mortality by DENM (MpDENM) and diabetes (MpD) was conducted, contextualizing the regional inequalities by the socioeconomic indicators. A multiple linear regression model (MRLM) was adjusted, taking the logits of the $\mathrm{MpD}$ as the response variable and indicators of literacy, employment and income, as explanatory variables, using a $5 \%$ level of significance.

Results: The results showed that deaths by DENM in Brazil, in the year 2010, occupied the fifth position, representing $6.6 \%(70,276$ deaths), being the UF in the Northeast region, those with higher relative percentage, reaching $10 \%$ at some UF. The female population is the most affected (55\% of the cases, on average), and the deaths from diabetes mellitus the main cause of DENM, representing $78 \%$ of the cases, although, reaching more than $80 \%$ in several UF, especially those from the Northeast, socioeconomically more vulnerable. The MRLM showed that education (illiteracy rate) is the variable with greater explanatory power for the $\mathrm{MpD}(p$-value $<0.05)$. On the other hand, the proportion of persons living with less than half of minimum wage income, also showed to be statistically significant on the explanation of deaths from diabetes ( $p$-value $<5 \%$ ), when adjusted, with the unemployment rate of the population.

Conclusions: Given the strong influence of the education variable, it can be concluded that investments in improving the educational level of the population should be privileged as a strategy to reduce the deaths from diabetes in Brazil.

\section{P-053}

Pre-gestational diabetes and the risks of fetal and infant death: a population-based study

Topic: Diseases - CVD/diabetes

Presenter: Peter Tennant
Peter Tennant 1); Svetlana Glinianaia 1); Rudy Bilous 2); Judith Rankin 1), 3); Ruth Bell 1), 3)

1) Institute of Health \& Society, Newcastle University, Newcastle upon Tyne, UK; 2) James Cook University Hospital, South Tees NHS Trust, Middlesbrough, UK; 3) Regional Maternity Survey Office, Newcastle upon Tyne, UK

Background: Pre-gestational diabetes is associated with a range of adverse pregnancy outcomes including an increased risk of stillbirth, but few studies have described the association in contemporary populations, particularly excluding the known effect of congenital anomalies.

Objectives: This study used data from a long-standing populationbased survey of women with pre-gestational diabetes to investigate the association between diabetes and the risks of fetal and infant death, and to explore and quantify the contribution of glycaemic control.

Methods: All normally-formed singleton deliveries occurring in women with pre-gestational diabetes in the North of England during 1996-2008 were identified from the Northern Diabetes in Pregnancy Survey. Relative risks (RRs) of fetal death ( $\geq 20$ completed weeks of gestation) and infant death were estimated by comparing with prevalence data from the population-based Northern Perinatal Mortality and Morbidity Survey. Predictors of fetal and infant death in women with pre-gestational diabetes were examined by logistic regression. Glycated haemoglobin concentrations (HbAlc) within 3 months periconception and in the third trimester (as markers of blood glucose concentration) were analysed by piecewise regression due to curvilinear associations.

Results: There were 46 fetal deaths and 10 infant deaths among 1,548 deliveries in women with pre-gestational diabetes. The prevalence of fetal death in women with diabetes was four-and-a-half times greater than among those without $(\mathrm{RR}=4.54,95 \%$ confidence interval, CI $3.41-6.05, p<0.0001$ ), while for infant death it was nearly two times greater $(\mathrm{RR}=1.86,95 \% \mathrm{CI} 1.00-3.46, p=0.046)$. There was no evidence of that the relative risk of a stillbirth or infant death had changed over time $(p=0.95)$.

Increasing peri-conception HbA1c above $45 \mathrm{mmol} / \mathrm{mol}$ (adjusted odds ratio, $\mathrm{aOR}=1.02,95 \% \mathrm{CI} 1.01-1.03, p=0.006$ ), retinopathy diagnosed pre-pregnancy $\quad(\mathrm{aOR}=2.02,95 \%$ CI $1.05-3.89$, $p=0.03$ ), and lack of pre-pregnancy folic acid supplement use (aOR $=2.44,95 \%$ CI 1.12-5.26, $p=0.03$ ) were all independently associated with increased risks of fetal and infant death. After 28 weeks, increasing third-trimester $\mathrm{HbAlc}$ above $36 \mathrm{mmol} / \mathrm{mol}$ was associated with an increased risk of fetal or infant death $(\mathrm{aOR}=1.05$, $95 \%$ CI $1.02-1.08, p=0.001$ ), with no apparent additional contribution from peri-conception HbA1c.

Conclusions: Pre-gestational diabetes is associated with a substantially increased risk of fetal and infant death in normally-formed offspring, the effect of which is largely moderated by glycaemic control. Greater vigilance in the control of blood glucose, both before and throughout pregnancy, is needed if the risks of serious adverse pregnancy outcomes in women with pre-gestational diabetes are to be reduced towards the same levels as those without.

\section{P-054}

Congenital heart disease prevalence and trends in the UK: a multicentre, register study

Topic: Diseases - CVD/diabetes

Presenter: Kate Best

Kate Best 1); Elizabeth Draper 2); Jenny Kurinczuk 3); Rosie Thompson 4); David Tucker 5); Diana Wellesley 6); Judith Rankin 1) 
1) Institute of Health \& Society, Newcastle University, Newcastle upon Tyne, UK; 2) Department of Epidemiology and Public Health, University of Leicester, Leicester, UK; 3) National Perinatal Epidemiology Unit, University of Oxford, Oxford, UK; 4) Institute of Child Life and Health, St Michael's Hospital, Bristol, UK; 5) Swansea NHS Trust, Congenital Anomaly Register \& Information Service for Wales, UK; 6) Faculty of Medicine, University of Southampton and Wessex Clinical Genetics Service, Southampton, UK

Background: Congenital heart diseases (CHDs) are the largest group of congenital anomalies, encompassing subtypes that are diverse in terms of severity, morbidity and mortality. More severe CHDs require highly specialised health care, which may involve multiple complex and often life-saving surgeries.

Objective: To describe the prevalence and temporal trends of CHD in the UK, according to CHD severity.

Methods: All cases of CHD notified to six British Isles Network of Congenital Anomaly Registers (BINOCARs) between 1991 and 2010 formed this population-based case-series. Isolated cases were categorised into groups of very severe, severe and least severe. Very severe included single ventricle, hypoplastic left heart, hypoplastic right heart, Ebstein's anomaly and tricuspid atresia; severe included pulmonary valve atresia, common arterial truncus, atrioventricular septal defects, aortic valve atresia/stenosis, transposition of great vessels, tetralogy of Fallot (ToF), total anomalous pulmonary venous return and coarctation of aorta $(\mathrm{CoA})$; and least severe included ventricular septal defect, atrial septal defect, pulmonary valve stenosis. Prevalence and $95 \%$ confidence intervals (95\% CIs) were calculated as the number of cases (occurring in live births, fetal deaths ( $>20$ weeks gestation) or terminations for fetal anomaly (any gestation)) per 10,000 live and stillbirths. Relative risks (RRs) of CHD corresponding to year of delivery were estimated using a multilevel Poisson model with a random intercept.

Results: There were 19,146 cases of CHD among a population of 2,952,209, giving a total prevalence of 65.0 (95\% CI 64.1-66.0) per 10,000 births. $3,464(18.1 \%)$ cases occurred with chromosomal anomalies or genetic syndromes and 6,401 (33.4\%) cases with additional major structural anomalies. There were 9,281 isolated cases of CHD (prevalence: 31.4 (95\% CI 30.8-32.1) per 10,000 births) of which $652(7.0 \%)$ were very severe, $1,761(18.0 \%)$ were severe and 5,493 $(59.2 \%)$ were least severe. The total prevalence of most and least severe CHD remained stable over time $(\mathrm{RR}=1.01$, $95 \%$ CI $0.99-1.02 ; p=0.370$ and $\mathrm{RR}=1.00,95 \%$ CI $0.99-1.00$; $p=0.447$, respectively) with no significant trends in the separate subtypes. There was a significant decreasing trend in severe CHD $(\mathrm{RR}=0.99,95 \% \mathrm{CI} 0.98-1.00 ; p=0.028)$. Specifically, there were significant decreasing trends in aortic valve atresia/stenosis $(\mathrm{n}=194)$ $(\mathrm{RR}=0.96,95 \%$ CI $0.93-0.99 ; p=0.002)$ and $\mathrm{CoA}(\mathrm{RR}=0.95$, $95 \%$ CI $0.93-0.98 ; p<0.001)$. Conversely, there was an increasing trend in $\mathrm{ToF}(\mathrm{RR}=1.03,95 \% \mathrm{CI} 1.01-1.05 ; p=0.012)$.

Conclusion: The prevalence of very and least severe CHD has remained stable over time, but a decreased in severe CHD. However, the yearly decrease in risk was small $(\sim 1 \%)$ suggesting this may be due to differences in ascertainment.

\section{$\mathbf{P - 0 5 5}$}

Socio-economic position and appropriate antiplatelet therapy after percutaneous coronary intervention: a population-based cohort study in Rome, Italy

Topic: Diseases - CVD/diabetes

Presenter: Flavia Mayer
Flavia Mayer 1); Anna Maria Bargagli 1); Valeria Belleudi 1); Nera Agabiti 1); Danilo Fusco 1); Luigi Pinnarelli 1); Ursula Kirchmayer 1); Mirko Di Martino 1); Silvia Cascini 1); Marina Davoli 1)

1) Department of Epidemiology of Lazio Regional Health Service

Background: Socio-economic differences in health-care systems are an important area to be monitored to improve health care quality. According to scientific guidelines, Appropriate Antiplatelet Therapy (AAT) for patients who underwent Percutaneous Coronary Intervention (PCI) consists of Clopidogrel, for a minimum of 1 month up to 12 months after discharge, with Acetylsalicylic Acid (ASA), indefinitely (dual antiplatelet therapy). Few studies have investigated whether adherence to AAT is associated to socio-economic position (SEP).

Objectives: To evaluate if SEP is associated with adherence and continuity to AAT during the year after discharge, in a cohort of PCI patients.

Methods: Patients resident in Rome, who underwent their first PCI during 2006-2007 were selected from the Hospital Information System. Drug claims for the 1-year after discharge were retrieved from Regional Drug Dispense Registry. Drug use was measured with Proportion of Days Covered (PDC) dividing the total number of dispensed Defined Daily Dose by each patient's follow-up time. Dual antiplatelet therapy with PDC $\geq 75 \%$ and single therapy based on Clopidogrel with PDC $\geq 75 \%$ were considered as AAT. We used a composite area-based index of socio-economic position (SEP) by census block of residence built using the 2001 census of Rome, assuming 5 levels (from $1=$ High SEP to $5=$ Low SEP). Proportions of patients treated with AAT by SEP was measured for the overall year and by semester. The association between SEP and adherence to AAT was estimated through logistic regression models. Results: The study population included 5901 patients; $76 \%$ were men and $63 \%$ belonged to medium-low SEP. In the 1-year follow-up, the proportion of patients adherent to AAT was $65 \%$; SEP was associated with AAT (OR high vs. low SEP 1.26, I.C. $95 \%$ $1.05-1.51, p$ trend $=0.002$ ) and an interaction between SEP and presence of Acute Myocardial Infarction (AMI) diagnosis at admission was found (PCI not in AMI: OR high vs. low SEP $=1.12$, I.C. $95 \%$ 0.85-1.47; PCI in AMI: OR high vs. low SEP $=1.35$, I.C. $95 \%$ 1.07-1.70). The adherence to AAT decreased from $72 \%$ in the first semester to $58 \%$ in the second semester with the highest reduction among low SEP patients ( -21 vs. $-15 \%$ in the high SEP patients).

Conclusions: The adherence to AAT of PCI patients was unsatisfactory and decreased overtime more in medium-low SEP patients than in high SEP patients. This effect was higher in presence of AMI. Strategies to improve adherence to AAT among PCI patients need to be identified, in particular for low SEP patients.

\section{P-056}

Maternal vitamin B12 and folate status during pregnancy and offspring insulin resistance and body composition at 12 years

Topic: Diseases - CVD/diabetes

Presenter: Tom Bond

Tom Bond 1); Charu Joglekar 2); Ella Marley-Zagar 1); Himangi Lubree 2); Kalyanaraman Kumaran 2); Chittaranjan Yajnik 2); Caroline Fall 1)

1) MRC Lifecourse Epidemiology Unit, Southampton General Hospital, Southampton, SO16 6YD; 2) Diabetes Unit, KEM Hospital and Research Centre, Pune, Maharashtra, India 
Maternal nutrition during pregnancy may influence offspring risk of developing type 2 diabetes mellitus and cardiovascular disease in later life. Previous studies have shown that low maternal vitamin B12 and high folate concentrations during pregnancy were associated with higher insulin resistance in the offspring at 6 years. As both B12 and folate are regulators of 1-carbon (1-C) metabolism, this suggested a role for 1-C metabolism in the development of type 2 diabetes mellitus. The current study aimed to test the hypothesis that low maternal vitamin B12 concentration and high maternal folate concentration during pregnancy are associated with higher insulin resistance and adiposity in the offspring at 12 years. Data was from the Pune Maternal Nutrition Study, a community based prospective study of a rural Indian birth cohort. Plasma vitamin B12 and erythrocyte folate (exposures) were measured at 18 and 28 weeks gestation in 638 women in six villages near Pune, India, along with anthropometry and dietary assessment $(24 \mathrm{~h}$ intake and food frequency questionnaire). Outcomes measured in the children at 12 years included fasting plasma glucose and insulin, vitamin B12, total homocysteine (tHcy), serum folate and lipid concentrations, blood pressure and body composition (anthropometry and dual X-ray absorptiometry scans (DXA)). Insulin resistance was estimated using the HOMA model. Relationships were analysed using multiple linear regression, adjusting for important covariates and potential confounders. Maternal erythrocyte folate concentration at 18 and 28 weeks gestation was positively related to offspring weight, BMI, fat and lean mass and triceps skinfold ( $p<0.05$ for all), but not to insulin resistance. Maternal vitamin B12 concentration was not related to offspring insulin resistance or adiposity measures. Higher maternal vitamin B12 concentration was related to higher offspring B12 concentration and lower offspring tHcy concentration ( $p<0.01$ for both). All reported associations were adjusted for offspring age, sex, socioeconomic status and serum folate concentration at 12 years. Associations with maternal folate as the exposure were also adjusted for offspring serum vitamin B12 concentration at 12 years. In this population, higher maternal folate concentration during pregnancy was related to higher offspring adiposity measures at 12 years while the relationship between lower maternal B12 and higher offspring insulin resistance observed at 6 years had disappeared. This may be due to metabolic alterations occurring during puberty and requires further investigation.

\section{P-057}

Detection of a decrease in vagal regulation of cardiac activity in patients with hypertension

Topic: Diseases - CVD/diabetes

Presenter: Liliya Poskotinova

Liliya Poskotinova 1), 2); Denis Demin 1), 2); Elena Krivonogova 1), 2); Andrej Grjibovski 3), 4)

1) Institute of Biomedical Research of the Northern Arctic Federal University, Arkhangelsk, Russia; 2) Institute of Environmental Physiology, Russian Acad. Sci., Ural Branch, Arkhangelsk, Russia; 3) Department of International Public Health, Norwegian Institute of Public Health, Oslo, Norway; 4) International School of Public Health, Northern State Medical University, Arkhangelsk, Russia

Background: Reduction of total heart rate variability (HRV) has been shown to be associated with increased risk of sudden cardiac death and other complications of hypertension. Early detection of disturbances of heart rate vagal regulation in patients with high blood pressure (BP) may contribute to more effective prevention of complications in hypertensive patients. Studies of biofeedback have a potential for assessment of heart rate variability, although only a few studies have been studied.
Methods: We studied biofeedback parameters of HRV in 33 healthy individuals (11 men and 22 women) with normal blood pressure (below $140 / 90 \mathrm{~mm} \mathrm{Hg}$, group I) and 20 people (10 men and 10 women) with increased baseline hypertension (140/90-160/100 mm $\mathrm{Hg}$, group II). HRV registration was performed using "Varicard" device (Russia) while blood pressure (BP) was registered using the A\&D equipment (Japan). We recorded BP at baseline (5 min), during the biofeedback session $(5 \mathrm{~min}$ ) in order to increase the total power spectrum of HRV (TP) and after the biofeedback session ( 5 min). Stress index (SI) was also assessed. Biofeedback techniques were used as specified in national patent 2317771 RU (Russia). Paired samples were compared using Wilcoxon tests while Mann-Whitney tests were used for unpaired data.

Results: There were no statistically significant differences between the groups in baseline heart rate (HR) and HRV. During the biofeedback session, a decrease in systolic BP $(p=0.008)$ and HR $(p=0.025)$ was observed among women in group I; these indices remained below the baseline after the biofeedback session $(p=0.032$ and $p=0.004$, respectively). TP increased significantly in the biofeedback session in men $(p=0.005)$ and in women $(p<0.001)$ in group I. Moreover, during the session, we observed a decrease in SI among men $(p=0.041)$, but not among women $(p=0.072)$. No differences between the baseline and at subsequent time points in the group with normal blood pressure were observed.

Conclusions: An ability to increase the total power HRV spectrum by biofeedback technique during standard time $(5 \mathrm{~min})$ may be regarded as a test of vagal autonomic regulation of cardiac function in patients with elevated blood pressure. More details on biofeedback technique as well as on the factors behind the observed results will be presented.

\section{P-058}

\section{Epidemiology of cancer in Niger}

Topic: Diseases - Cancer

Presenter: Hinde Hami

Salamatou Mamoudou Garba 1); Hinde Hami 1); Harouna Mahamadou Zaki 2); Abdelmajid Soulaymani 1); Hassan Nouhou 2); Ali Quyou 1)

1) Laboratory of Genetics and Biometry, Faculty of Sciences, Ibn Tofail University, Kenitra, Morocco; 2) Laboratory of Pathological Anatomy and Cytology, Faculty of Health Sciences, Abdou Moumouni University, Niamey, Niger

Background: Cancer is predicted to be an increasingly important cause of morbidity and mortality in the next few decades, in all regions of the world.

Objectives: The aim of this study is to determine the main epidemiological characteristics of cancer in Niger.

Methods: A descriptive retrospective study of cancer cases, reported between 1992 and 2009 to the National Cancer Registry of Niger, was conducted.

Results: During the period of study, 7,031 cases of cancer have been diagnosed. Of these, $4,108(58.4 \%)$ were females. The average age at diagnosis was 43 years and $7 \%$ were children under the age of 15 years. According to available data, $70.3 \%$ came from the Niamey region. Breast cancer was the most common cancer diagnosed in females $(27.4 \%)$, followed by cervical and ovarian cancer (13.4 and $8.8 \%$, respectively). In males, the most commonly diagnosed cancers were liver $(19 \%)$, skin $(8 \%)$, bladder $(4.9 \%)$ and prostate cancer $(4.5 \%)$. Carcinoma was the most common type of cancer $(27 \%)$ and Burkitt's Lymphoma was the most common malignant lymphoma in children $(15.1 \%)$. Among the 6,984 cases for whom the evolution is known, $899(12.9 \%)$ of them died during the study period. 
Conclusions: The rise of cancer and related deaths can be reversed, and gains can be achieved rapidly if appropriate action is taken such as the development of specific strategies to prevent cancer.

\section{P-059}

Tumor-infiltrating lymphocytes predict cutaneous melanoma survival

Topic: Diseases - Cancer

Presenter: Cristina Fortes

Cristina Fortes 1); Simona Mastroeni 1); Thomas

Manooranparanpampil 1); Francesca Passarelli 1); Alba Zappalà 1); Claudia Marino 2); Paola Michelozzi 2); Marina Davoli 2);

Tulio Faraggiana 1)

1) IDI-IRCCS; 2) Dipartimento di Epidemiologia Regionale Lazio

Introduction: Tumor-infiltrating lymphocytes (TILs) is considered a manifestation of the host immune response to tumor, but the role of TILs on melanoma mortality is controversial. The aim of this study was to investigate the role of TILs on melanoma mortality, controlling for all known histological prognostic parameters.

Methods: We conducted a 10-year cohort study among 4,143 patients from the same geographic area (Lazio) with primary cutaneous melanoma diagnosed between January 1998 and December 2008. Survival probability was determined by Kaplan-Meier estimates, and prognostic factors were evaluated by multivariate analysis (Cox proportional hazards model).

Results: Survival decreased with increasing age $(P$ for trend $<0.001)$ and Breslow thickness $(P$ for trend $<0.001)$. In the multivariate Cox model, the presence of high levels of tumour infiltrating immune cells in primary invasive melanomas was associated with lower risk of melanoma death (RR: 0.32 ; $95 \%$ CI $0.13-0.82, P$ for trend $<0.001$ ), after controlling for sex, age, breslow thickness, histological type, mitotic rate and ulceration.

Conclusion: These results suggest that immune microenvironment affects melanoma survival. Understanding differences in survival across distinct subgroups of melanoma patients may help choosing types of therapy.

\section{P-060}

How much smoking, alcohol consumption and occupational exposure contribute to the association between educational inequalities and laryngeal cancer?

Topic: Diseases - Cancer

Presenter: Irene Santi

Irene Santi 1); Lars Eric Kroll 2); Andreas Dietz 3); Heiko Becher 1); Heribert Ramroth 1)

1) Institute of Public Health, University of Heidelberg, Im Neuenheimer Feld 324, 69120 Heidelberg, Germany; 2) Robert KochInstitute, Postfach 6502 61, D-13302 Berlin, Germany; 3) Department of Otorhinolaryngology, Head and Neck Surgery, University of Leipzig, Liebigstrasse 10-14, 04103 Leipzig, Germany

Background: Previous studies tried to measure the extent to which the association between socioeconomic status (SES) and laryngeal cancer is mediated by smoking, alcohol consumption and occupational exposure. These analyses were based on the comparison of ORs in models with different control variables.

Objectives: To estimate the proportion of social inequalities explained by lifestyle and occupational risk factors using the method proposed by Karlson, Holm and Breen (KHB).
Materials and methods: A population-based case-control study on laryngeal cancer conducted in South-West Germany in 1998-2000 included 236 male cases and 702 controls. Information on occupational history, smoking, alcohol consumption and education was collected through face-to-face interviews. Jobs were coded according to ISCO-68. A recently developed job-classification index covering physical and psychosocial dimensions was used to account for the occupational burden. A sub-index was extracted to focus on jobs involving potentially carcinogenic agents (CAI) for the upper aero digestive tract. Odd ratios across nested logistic regression were used to estimate the effect of lifestyle and occupational risk factors on educational inequalities applying the KHB method.

Results: When adjusted for smoking and alcohol consumption, higher odds ratios (ORs) were found for lower education (OR for less than 9 years vs. more than 10 years $=4.0,95 \%$ confidence interval $(\mathrm{CI})$ : 2.2-7.4). This OR decreased after further adjustment using the occupational job indices starting with the overall job index (OJI: $\mathrm{OR}=2.8,95 \% \mathrm{CI} 1.4-5.5)$ to the more specific CAI $(\mathrm{OR}=2.6$, $95 \%$ CI 1.3-5.2). Applying the KHB method, $26.2 \%$ of the reduction in the ORs was due to the occupational index CAI, while smoking and alcohol consumption contributed for 21.3 and $5.0 \%$ respectively.

Conclusions: Occupational aspects, in particular, the exposure to carcinogenic agents explained a large portion of the association between low educational level and laryngeal cancer risk. The occupational effect was similar to the smoking effect. Together with alcohol consumption, the occupational and lifestyle factors contribute for $52.5 \%$ of the observed educational inequalities.

\section{P-061}

Breast cancer data quality at the population-based cancer registry of Säo Paulo: implications for public health planning

Topic: Diseases - Cancer

Presenter: Daniele Silveira, Elizabeth Artmann

\section{Daniele Silveira 1), 2); Elizabeth Artmann 2)}

1) Braxilian Federal Regulatory Agency for Health insurance and Plans - ANS; 2) National School of Public Health/Oswaldo Cruz Foundation - ENSP/FIOCRUZ

Background: Information based on population data, such as Cancer Registries, are essential to endorse health planning and provision of cancer care services. In Brazil, This paper aims to present a data quality evaluation and coverage of breast cancer incidence data from the Population Based Cancer Registry of the Municipality of São Paulo, Brazil. The evaluation was based on international quality standards adopted by international agencies worldwide.

Methods: The study included 46,305 new breast cancer cases recorded during a two 5-year periods (1997-2001 and 2002-2006). Population-based cohort study was led focusing on variables such as: date and age at diagnosis, method of diagnosis, clinical stage and topography. Data collected by other Registries, in other countries, were used as a data proxy for coverage comparisons with São Paulo Cancer Registry.

Results: The study has shown that some variables considered in the literature as essential to the validity and comparability of Registries, such as the diagnosis by histopathology exam and age of the patients, has a good information, while than for other critical variables, such as clinical staging, there are problems of data completeness. Diagnostic criteria and classification based on International Classification of Disease (ICD-10) presented $100 \%$ of completeness. However, the completeness of death registration was around $20 \%$, less than the expected for a cancer registry. 
Conclusion: Record linkage methodologies can be applied to improve data quality in population-based registries and comparisons with others population-based information systems can be a good method of accounting for missing information and amplifying the usability of population-based cancer registries.

\section{P-062}

Cancer incidence pattern a large migrant population in Germany in comparison to the host population: a cohort study

Topic: Diseases - Cancer

Presenter: Heiko Becher

Heiko Becher 1); Volker Winkler 1); Bernd Holleczek 2); Christa Stegmaier 2)

1) University of Heidelberg, Institute of Public Health; 2) Saarland Cancer Registry

Background: Migrants from the Former Soviet Union (so-called "Aussiedler") are the second biggest migrant group in Germany with more than two million individuals migrated after the fall of the iron curtain. They settled approximately uniformly all over Germany. While mortality patterns have been studied before, there is no knowledge on their cancer incidence. Since the mortality rates in the country of origin are much higher than in Germany, we hypothesized that many common diseases were more frequent in this migrant group as well.

Objectives: To investigate cancer incidence in this migrant population and to analyse time trends.

Methods: We set up a register based cohort of the Aussiedler who migrated from 1990 to 2005 and first settled in the federal state of Saarland, South-West Germany $(\mathrm{n}=18,619)$. Incident cancer cases and vital status were ascertained through record linkage with the Saarland Cancer Registry and local population registries. A comparison with the autochthonous Saarland population was performed by calculating the standardized incidence ratio (SIR). Poisson regression was used to investigate time trends in cancer incidence rates. The yearly age-standardized rates for the most frequent cancer diagnoses (stomach cancer, colorectal cancer, lung cancer, prostate, and breast cancer) and all cancers combined.

Results: During the follow up period from 1990 to 2009 we observed 638 incident diagnoses of malignant neoplasms (except non melanoma skin cancer). Overall, cancer incidence in Aussiedler was very similar to that in the German population with an overall SIR of 0.98 (95\% confidence interval 0.92-1.04). However, site-specific SIRs revealed large differences. Stomach cancer incidence was significantly higher among Aussiedler (SIR $=2.8$ ). Lung cancer was elevated for males, but lower among females. Additionally, colorectal cancer among males occured significantly lower. Age-standardized rates (ASR) over time show further differences to the cancer pattern in the autochthonous Saarland population, although smaller numbers somewhat limit the interpretation. ASR for overall cancer incidence of Aussiedler show an average yearly decrease of about one percent $(p=0.06)$ whereas Saarland rates remain stable. Lung cancer and prostate cancer incidence rates show increasing disparity from Saarland rates. Female breast cancer incidence develops in parallel. Stomach cancer rates are declining towards the rates of the Saarland population.

Conclusions: In contrast to prior hypothesis, cancer incidence rates of Aussiedler are generally much closer to the rates in the host country than in the country of origin. Furthermore, some patterns indicate differences in health care utilisation of Aussiedler compared to the Saarland population.

\section{P-063}

Testicular cancer incidence to increase by $23 \%$ by 2025 in Europe: age-period-cohort model predictions based on cancer registry data

Topic: Diseases - Cancer

Presenter: Charlotte Le Cornet

Charlotte Le Cornet 1), 2); Joannie Lortet-Tieulent 1);

David Forman 1); Rémi Beranger 1), 2); Béatrice Fervers 2); Joachim Schuz 1); Freddie Bray 1)

1) International Agency for Research on Cancer (IARC), Lyon, France; 2) Léon Bérard Centre, Lyon, France

Purpose: Over 18,000 new cases of testicular cancer were estimated in Europe in 2008. It is the most common malignancy among young adult men aged 15-34 years and highest incidences are found in western population. Despite a good prognosis, testicular cancer is associated with a heavy co-morbidity. Incidence rates vary between neighboring countries in Europe, with rates in Denmark and Norway suggesting recent declines, while in other European countries large increases are observed. The aim of this study was to predict the future testicular cancer burden in the 40 countries of Europe in 2025.

Methods: 23 countries matched the inclusion requirement (15 consecutive years of data and compilation in the last volume of the CI5 series). The projection population up to 2025 was obtained from the World Population Prospects (2010 Revision). Current trends stratified by 5-year period of diagnosis and 5-year age groups were projected into future years using an age-period-cohort model developed in the Nordic countries (Moller et al. 2003).

Results: Wide variations in the incidence burden are predicted between European countries despite the varying but commonly increasing trends. The burden in the UK and France will tend to level off possibly decline in Norway and Denmark. Large increases in the burden are foreseen in Southern countries; respectively 15.2 and 13.2 cases for 100,000 men in Slovenia and in Croatia. The number of new cases of testicular cancer will have risen by $23 \%$ in Europe by 2,025 compared with 2005 , reaching more than 22,000 new cases.

Conclusion: This is the first study to predict the future testicular cancer burden across Europe. Testicular cancer incidence is expected to increase on the continent over the next decades. The changes in incidence are mainly due changes in risk with the demographic changes having a lesser impact. Testicular cancer risk factors remain largely unknown and this study emphasizes the need to further develop epidemiological research hypotheses to prevent the escalating burden of the disease.

\section{P-064}

Survival from childhood acute lymphoblastic leukaemia in Germany: does socio-demographic background matter?

Topic: Diseases - Cancer

Presenter: Friederike Erdmann

Friederike Erdmann 1); Peter Kaatsch 2); Eve Roman 3); Hajo Zeeb 4); Tracy Lightfoot 3); Joachim Schüz 1)

1) Section of Environment and Radiation, International Agency for Research on Cancer (IARC); 2) German Childhood Cancer Registry, Institute for Medical Biostatistics, Epidemiology and Informatics (IMBEI); 3) Epidemiology \& Cancer Statistics Group, Department of Health Sciences; 4) Department of Prevention and Evaluation, BIPS Institute for Epidemiology and Prevention Research 
Background: Survival from acute lymphoblastic leukaemia (ALL) has considerably improved over the past decades, with 5-year survival exceeding $80 \%$ in developed countries. It is well accepted that sex and age, as well as immunophenotype and white blood cell count at diagnosis are important predictors for survival. Although the general association between social inequality and differences in cancer morbidity and mortality is well-known, there are little data about the relationship between socio-economic factors and survival from ALL in childhood.

Objectives: We explored whether there are differences in ALL survival by socio-demographic background in Germany.

Methods: ALL cases were taken from a nationwide population-based German case-control study, diagnosed between 1992 and 1994 $(\mathrm{N}=788)$. The children were followed for up to 10 years after diagnosis, using survival data from the German Childhood Cancer Registry. Two primary outcomes were defined for these analyses: overall survival with death from all causes as endpoint and event-free survival with the first, if any, of relapse, second malignant neoplasm and death from all causes defined as events. Cox proportional hazards models to estimate hazard ratios (HR) were calculated to assess the impact of selected demographic and socio-economic (family net income and parental school education) characteristics on overall and event-free survival probabilities.

Results: The overall survival of all cases was $82.5 \%$, with girls more likely to survive than boys ( 85 vs. $81 \%$ ). We found a statistically significant and non-linear relationship between age at diagnosis and survival, with poorer survival in infants and children aged $>5$ years. There was no association between socio-economic factors and survival or risk of relapse. For 5 levels of increasing family income, all HRs were close to 1 . We also did not see an association with maternal or paternal educational level.

Conclusion: In contrast to other international studies, socio-economic factors did not impact on ALL survival in German children in this analysis. However, dissimilarities in social welfare systems, access to health care (including both treatment and clinical care), lifestyle and differences in treatment protocols make an international comparison challenging. Our observation of no social inequalities in paediatric ALL survival in Germany is encouraging, although the finding should be interpreted with caution and further research is needed.

\section{P-065}

Prognostic factors for small cell neuroendocrine carcinoma of the uterine cervix: an institutional experience

Topic: Diseases - Cancer

Presenter: Suthida Intaraphet

Suthida Intaraphet 1); Nongyao Kasatpibal 2); Sumalee

Siriaunkgul 3); Anchalee Chandacham 4); Kornkanok Sukpan 3); Jayanton Patumanond 5)

1) Boromarajonnani College of Nursing, Khon Kaen, Khon Kaen, Thailand.; 2) Faculty of Nursing, Chiang Mai University, Chiang Mai, Thailand.; 3) Department of Pathology, Faculty of Medicine, Chiang Mai University, Chiang Mai, Thailand.; 4) Department of Gynecology and Obstetrics, Nakornping Hospital, Chiang Mai,

Thailand.; 5) Clinical Epidemiology Unit, Department of Community Medicine, Faculty of Medicine, Chiang Mai University, Chiang Mai, Thailand

Background: Small cell neuroendocrine carcinoma of the uterine cervix (SNEC) is a rare and highly aggressive tumor. The prognostic factors of patients with SNEC remain undetermined. Previous studies had rather small sample sizes of SNEC patients were limited by the variation in treatment policy or the lack of a central pathology review.
Objective: To determine the clinicopathologic characteristics that affected the survival in patients with SNEC.

Materials and methods: All patients with SNEC treated at Chiang Mai University Hospital between January 1995 and October 2011 were retrospectively reviewed with histological confirmation of SNEC diagnosis. The prognostic factors for survival were assessed using competing-risks regression analysis concerning the probabilities of competing events.

Results: One hundred and thirty histologically confirmed SNEC patients met the study criteria. The median overall survival and median cancer-specific survival (CSS) for entire group were 47.8 and 58.1 months, respectively. Five-year CSS for patients with early stage was $62.6 \%$ and advanced stage was $18.1 \%(P<0.001)$. Among the surgically treated early stage patients, those with adjuvant chemotherapy had a better 5-year survival rate than those with surgery alone, those with adjuvant radiotherapy and those with adjuvant chemoradiation therapy $(P=0.041)$. In multivariable analyses, decreased survival in early stage patients was associated with age at diagnosis $>60$ year (hazard ratio [HR] 4.9; $95 \%$ CI 1.5-15.8) and deep stromal invasion (HR, 2.9; $95 \%$ CI 1.3-6.9). Among the advanced stage patients, decreased survival was associated with age at diagnosis ( $>60$ years, HR 9.9; $95 \%$ CI $2.8-35.4$, and $<45$ years, HR, 3.4; $95 \%$ CI 1.1-10.5) and FIGO stage IV (HR, 7.4; $95 \% \mathrm{CI}$ $1.3-42.1)$

Conclusion: FIGO stage, age at diagnosis, and deep stromal invasion were important prognostic factors for cancer-specific survival in patients with SNEC. Adjuvant chemotherapy may provide survival benefits in surgically treated early stage SNEC patients.

\section{P-066}

Body mass index and risk of postmenopausal endometrial carcinomas by histological subtypes

Topic: Diseases - Cancer

Presenter: TienYu Owen Yang

TienYu Owen Yang 1); Benjamin J. Cairns 1); Gillian K. Reeves 1); Valerie Beral 1)

1) On Behalf of the Million Women Study Collaborators; Cancer Epidemiology Unit, University of Oxford

Background: The strong association between body mass index and endometrioid-type carcinomas of the endometrium in postmenopausal women has been proposed to be linked to adiposity-related oestrogen excess. The association between body mass index and other endometrial carcinomas in postmenopausal women is less clear, and potential modification effects of other hormonal factors are underinvestigated.

Objectives: We investigated the association between body mass index and risk of postmenopausal endometrial carcinomas by histological subtypes in a large cohort of postmenopausal women.

Methods: Approximately 1.3 million middle-aged women completed a questionnaire about their body size, reproductive history, as well as many lifestyle factors in the Million Women Study and were followed up for 11 years on average. Cancer incidence was obtained from the National Cancer Registry, and endometrial carcinomas were classified by histology using International Classification of Diseases for Oncology: Morphology of Neoplasms, Edition 3 (ICD-O3) for endometrioid and non-endometrioid carcinomas. We use Cox proportional hazard regression to estimate cancer risk by body mass index.

Result: During $7 \mathrm{~m}$ person-years of follow up, we identified 4,191 cases of endometrioid carcinomas and 330 cases of non-endometrioid carcinomas. Higher body mass index was associated with risk of both 
endometrioid and non-endometrioid carcinomas, although the relative risk was significantly smaller for non-endometrioid carcinomas $(p<0.001)$. The results were not sensitive to adjustment for various body size, hormonal and behavioural factors, nor were they changed when we restricted the analyses to serous carcinomas.

Conclusion: In this large cohort of postmenopausal women, higher body mass index was associated with risk of non-endometrioid carcinomas of the endometrium. Although the association was weaker than that with risk of endometrioid carcinomas, adiposity-related hormonal effects might still be involved in aetiology of non-endometrioid carcinomas.

\section{P-067}

Parameters related to short-term changes in quality of life in colorectal cancer patients

Topic: Diseases - Cancer

Presenter: José M. Quintana

José M. Quintana 1); Urko Aguirre 1); Nerea González 1); Santiago Lazaro 2); Cristina Sarasqueta 3); Eduardo Briones 4); Antonio Escobar 5); Juan Antonio Blasco 6); Marisa Baré 7)

1) Hospital Galdakao-Usansolo. Unidad de Investigación. Red de Investigación en Servicios Sanitarios y Enfermedades Crónicas (REDISSEC); 2) Hospital Galdakao-Usansolo. S ${ }^{\circ}$ Cirugía General.; 3) Hospital Donostia. Unidad de Investigación.; 4) Distrito Sanitario de Sevilla. Unidad de Epidemiología.; 5) Hospital Universitario Basurto. Unidad de Investigación. Red de Investigación en Servicios Sanitarios y Enfermedades Crónicas (REDISSEC); 6) Agencia Lain Entralgo. Red de Investigación en Servicios Sanitarios y Enfermedades Crónicas (REDISSEC); 7) Corporació Parc Taulí. Unidad de Epidemiología Clínica. Red de Investigación en Servicios Sanitarios y Enfermedades Crónicas (REDISSEC)

Background: Little information is available on the variables that may influence the changes in health-related quality of life (HRQoL) in patients with colon-rectal cancer in a short-term evolution.

Objectives: To develop statistical predictive models which included some simple and easily recorded variables from the patient medical record and some patient reported outcomes measures (PROMs), related to the general health and psychological status of the patient, and to relate them to short term change in health related quality of life (as measured by the EuroQol-5D questionnaire) in patients undergoing curative surgery by colorectal cancer.

Methods: Prospective cohort study of patients diagnosed of colorectal cancer that underwent curative surgery in any of the 16 participant hospitals. Patients were followed from the moment they got in touch with the index hospital through diagnostic and, if necessary, non surgical treatment pre-intervention and, them, at admission, intervention and in the follow-up at 30 days after discharge. Patient sociodemographic (age, gender), clinical (Charlson comorbidity index, TNM stage, location of tumour, complications at admission) variables were retrieved from the patient medical record. Additionally, patients fulfilled the Hospital Anxiety and Depression (HAD) and EuroQol-5D questionnaires before the surgical intervention and at 30 days after discharge. For the statistical analysis we developed a multivariate linear model where all the statistically significant variables from the univariate analysis were included as well as age, the location of the tumour (colon vs. rectum) and the tumour stage (based on the TNM classification of malignant tumours).

Results: These preliminary results were based on a sample of 640 patients included in 16 participant hospitals. Based on the multivariate model, the number of comorbidities, as measured by the Charlson index, the ASA risk class, the depression HAD domain score (nor the anxiety HAD domain) and the basal EuroQol-5D score were correlated with the changes in the EuroQol-5D scores from before the intervention up to 30 days after discharge. Neither age, TNM stage, location of cancer nor the hospital variable were related to the EuroQol-5D changes.

Conclusions: We identify some clinical (basically, presence of comorbidities and ASA risk stage) and HRQoL variables that were, homogeneously in all participant hospitals, related to changes in the quality of life of these patients. From all of them, it can be outlined the important role of the psychological status (presence of depression before surgery) of the patient which may help to outline psychological intervention with these patients in these early stages of their treatment.

\section{P-068}

Follow up of women with atypical Pap screening in Santiago de Chile

Topic: Diseases - Cancer

Presenter: Fanny López.

Fanny López 1); Estela Arcos 1); Orlando Quezada 2); Juan Carlos Oyanedel 1)

1) Universidad Andres Bello; 2) Barros Luco Hospital

Background: Atypical Pap smears are a potential risk for lesions. For a definitive diagnosis, clinical practices of repetition are established for the Pap smear or more complex procedures.

Objective: To determine the magnitude and characteristics of the lesions after the results of an initial atypical Pap screening in Primary Care Health Services of Santiago, Chile.

Methods: Cyto-histological follow-up study by type of atypia for a 3 year period of women with atypical Pap reports in 2008-2009. By applying the selection criteria on 765 atypical Paps, 4 cohorts of women were formulated: (a) Atypical squamous cells-undetermined significance (ASC-US) ( $\mathrm{n}=255$ women); (b) cannot exclude high grade squamous intraepithelial lesion (ASC-H) ( $\mathrm{n}=59$ women); (c) Atypical Glandular Cells-undetermined significance $(\mathrm{n}=17$ women); (d) Atypical Glandular Cells favor neoplastic $(n=5$ women). Information was obtained of the basis Cito-Expert of the Barros Luco Hospital laboratory (central hospital). in Santiago de Chile. The collected data were analyzed electronically through Microsoft Excel (version 2007) and the "Conditional Probabilities Tree Diagram" which allowed showing the number, type and outcome between tests of patients.

Results: The cyto-histological follow-up was evaluated with the high grade lesion for each woman, obtaining an ASC-US cohort of 20 women with low grade lesions and 34 with high grade lesions. For the ASC-H cohort: 15 women with low grade lesions and 31 with high grade lesions. In the undetermined Atypical Glandular Cells cohort: 4 women with low grade lesions and 2 with high grade lesions. And in the Atypical Glandular Cells favor neoplastic: 1 woman with low grade lesions and 2 with high grade lesions. On percentages: $21.2 \%$ of squamous atypia for ASC-US and $77.09 \%$ for ASC-H. For glandular atypia: $35.3 \%$ for undetermined Atypical Glandular Cells and $60 \%$ for Atypical Glandular Cells favor neoplastic .

Conclusions: A specific type of atypia correlates to the magnitude of the lesions. The undetermined atypia had a lower proportion of cervical lesions, since the reports included undetermined atypia that, in general evolves to normal. In contrast, defined atypia have a higher correlation with cervical lesions, a situation that coincides with the literature. 


\section{P-069}

Waiting time to surgical intervention and its relationship with other parameters and mortality in colorectal cancer patients

Topic: Diseases - Cancer

Presenter: José M. Quintana

José M. Quintana 1); Nerea González 1); Urko Aguirre 1); Susana Garcia-Gutierrez 1); Marisa Baré 2); Maximino Redondo 3); Nerea Fernandez-de-Larrea 4)

1) Hospital Galdakao-Usansolo. Unidad de Investigación. Red de Investigación en Servicios Sanitarios y Enfermedades Crónicas (REDISSEC); 2) Corporació Parc Taulí. Unidad de Epidemiología Clínica. Red de Investigación en Servicios Sanitarios y Enfermedades Crónicas (REDISSEC); 3) Hospital Costa del Sol. Unidad de Investigación. Red de Investigación en Servicios Sanitarios y Enfermedades Crónicas (REDISSEC); 4) Departamento de Salud

Comunidad de Madrid. Red de Investigación en Servicios Sanitarios y Enfermedades Crónicas (REDISSEC)

Background: Processes of care of patients are an important part of the quality of health care in any pathology but in cancer patients this should be even more important due to the severity of the disease evolution of these patients. Undesirable variability and equity problems should be detected and avoided.

Objectives: To determine the relationship of waiting time from cancer diagnosis before surgery to the intervention time in patients undergoing curative interventions by colorectal cancer, and its relationship with patient sociodemographic, clinical characteristics, participant hospital and mortality at 30 days after discharge.

Methods: Prospective cohort study of patients diagnosed of colorectal cancer who underwent curative surgery in any of the 16 participant hospitals. Patients were followed from the moment they got in touch with the index hospital through diagnostic and non surgical treatment pre-intervention and, them, at admission for surgery, and until 30 days after hospital discharge. Waiting time from diagnostic colonoscopy to surgery was recorded. Also, patient sociodemographic (age, gender, level of studies, socioeconomic, living and marital status and social support) and clinical (TNM stage, cancer location, ASA risk class, complications at admission) variables were retrieved from the patient medical record. Death until 30 days after hospital discharge was recorded. For the statistical analysis we performed univariate analysis and developed multivariate analysis where all the statistically significant variables were included.

Results: Preliminary results of this study with 1,321 patients show important differences in the waiting times from colonoscopy to surgery between the 16 participant hospitals. Among the different sociodemographic and clinical variables, there were found differences in waiting time from colonoscopy to surgery in the level of studies, age and location of the tumour. There was found an association between waiting time to surgery and mortality at 30 days in the univariate analysis that disappeared when adjusting by main clinical and sociodemographic characteristics (location of tumour, ASA risk, complications at admission, comorbidities, age and participant hospital).

Conclusions: We identify some important differences among hospitals in waiting time from diagnosis to surgery, and, more importantly, a relationship with some sociodemographic parameters as age and level of studied that pointed out possible equity problems. But, those differences had no relationship to mortality at 30 days after proper adjustment. Nevertheless, the process of care should be unified in these hospitals belonging to the same National Health Service to increase the quality of care and equity.

\section{P-070}

Follow-up protocol in women with atypical squamous cells of undetermined significance (ASC-US) Pap, in 2008-2009

Topic: Diseases - Cancer

Presenter: Estela Arcos

Estela Arcos 1); Orlando Quezada 2); Fanny López 1)

1) Universidad Andres Bello; 2) Barros Luco Hospital

Background: The National Program for Research and Control of Cervical Cancer in Chile has two types of algorithms based on the 2001 Bethesda System: (i) Algorithm of derivation to specialist of 2001 and (ii) algorithm to diagnostic confirmation, to detect evolutionary changes. The clinical conduct for the follow up the for first ASC-US result is the taking of a Pap on a 6 months period and if the result is normal, a second Pap is taken 3 years later.

Objective: Evaluate the fulfillment of the clinical protocol of the follow up derivatives and diagnostically algorithms on woman with atypical squamous cells of undetermined significance (ASC-US) Pap, between 2008 and 2009

Methods: A retrospective epidemiological, longitudinal and observational study of a cohort of woman who have received their first atypical Pap results, 2008-2009. A cohort of 195 women was formulated by applying the following selection criteria: normal cytology history previous to the first ASC-US result. Information was obtained from the diagnosis archives of the Cito-Expert system of the South Health Care Service in Santiago de Chile. The follow up time was a 3 year period (measured in months). Descriptive statistics of position, dispersion and measure of association were calculated.

Results: The ages of women were between 18 and 87 years, with a median age of 38 year and a mode of 26 year. In $17.9 \%$ of the cases there was no follow up. Only $42.5 \%$ of the cohort fulfills the norm of re-taking the Pap in the following 6 months. The age of the women wasn't associated to the fulfillment of the norm, $p \geq 0.05$. The $74.8 \%$ took 2 cervical cytological smears, however, the period of time and frequency for the Pap collects wasn't applying for the clinic staff in accordance with algorithm criteria.

Conclusion: In the studied cohort, the epidemic follow up doesn't fulfill the pre-established norms on the algorithms. This demonstrates an unequal opportunity to the epidemiological surveillance for some women and when it happens, it fulfills only partially the established standards in frequency and temporality.

\section{P-071}

Developing and validating risk prediction model for screening breast cancer in Thai women

Topic: Diseases - Cancer

Presenter: Thunyarat Anothaisintawee

Thunyarat Anothaisintawee 1), 2); Ammarin Thakkinstian 1); Cholatip Wiratkapun 3); Yot Teerawattananon 4);

Panuwat Lerdsitthichai 5); Vijj Kasamesup 6);

Sansanee Wongwaisayawan 7); Jiraporn Srinakarin 8); Siriporn Hirunpat 9); Piyanoot Woodtichartpreecha 9)

1) Section for Clinical Epidemiology and Biostatistics, Faculty of Medicine, Ramathibodi Hospital, Bangkok, Thailand; 2) Department of Family Medicine, Faculty of Medicine, Ramathibodi Hospital, Bangkok, Thailand; 3) Department of Radiology, Faculty of Medicine, Ramathibodi Hospital, Mahidol University, Bangkok, 
Thailand; 4) Health Intervention and Technology Assessment program, Ministry of Public Health, Thailand; 5) Department of Surgery, Faculty of Medicine, Ramathibodi Hospital, Mahidol University, Bangkok, Thailand; 6) Department of Community Medicine, Faculty of Medicine, Ramathibodi Hospital, Mahidol University, Bangkok, Thailand; 7) Department of Pathology, Faculty of Medicine, Ramathibodi Hospital, Mahidol University, Bangkok, Thailand; 8) Department of Radiology, Faculty of Medicine, Srinagarind Hospital, Khon Kaen University, Khon Kaen, Thailand; 9) Department of Radiology, Faculty of Medicine, Prince of Songkla University, Songkla, Thailand

Background: Breast cancer risk prediction models are widely used in clinical practice. They also should be useful for prioritizing high risk women for breast cancer screening in limited-resource countries. Our systematic review showed poor discriminative performance of previous models in both derived and validated settings.

Objectives: To develop and validate a breast cancer risk prediction model in Thai women

Methods: A cross-sectional study consisting of derived and validation phases was conducted during September 2011 to January 2013. Data collected from Ramathibodi Hospital and other two tertiary hospitals were used for derived and external validated the prediction model, respectively. Adult women without any cancer or ductal carcinoma in situ were eligible. 17 predictive factors were considered in the model. Missing data were imputed using multiple-chain equation with 10 imputations. Logistic regression was applied to derive the model. Calibration and discriminative performances were assessed using observed per expected $(\mathrm{O} / \mathrm{E})$ ratio and concordance statistic $(\mathrm{C}$ statistic), respectively. The model was internally validated by bootstrap with 200 repetitions. The received operating characteristic curve (ROC) was used to compare our and Gail model's performance.

Results: For the derived phase, 15,718 women were eligible with breast cancer prevalence of $0.68 \%$ (95\% CI $0.56 \%,-0.82 \%)$. 3/17 predictors (i.e. menopausal status, body mass index (BMI), and use of oral contraceptives $(\mathrm{OC})$ ) significantly associated with breast cancer and thus were kept in the model. The $\mathrm{O} / \mathrm{E}$ ratio and the $\mathrm{C}$-statistic were 1.01 (95\% CI 0.82, 1.21) and 0.643 (95\% CI 0.588, 0.698), respectively. The coefficients of included variables were used to create scoring scheme (ranged from 0 to 2.65). The scores were classified into very low, low-intermediate, intermediate, and high risk with likelihood ratio positive of 1, 2.19 (95 \% CI 1.66, 2.88), 3.55 (95\% CI 2.12, 5.95), and 5.19 (95\% CI 2.74, 9.81), respectively.

Internal validation shows good performance with a bias of -0.003 (95\% CI $-0.011,0.005)$ and C-statistics of 0.645 (95\% CI 0.641 , $0.649)$; the same as external validation with $\mathrm{O} / \mathrm{E}$ ratio of $0.97(95 \%$ CI $0.68,1.35$ ) and C-statistic of 0.625 (95\% CI $0.534,0.717$ ). $\mathrm{C}$-statistic of our model was significantly higher than C-statistic of the Gail model (0.413 (95\% CI 0.362, 0.464), $p<0.001$ ).

Conclusion: A simplified risk prediction score was created. Although discriminative performance of our model was fair, it was much better than the Gail model. The model will aid to prioritize high risk women to receive a nation-wide screening program in limited-resource settings.

\section{P-073}

Associations between body mass index and a range of cancers: a population-based study within the UK Clinical Practice Research Datalink

Topic: Diseases - Cancer

Presenter: Krishnan Bhaskaran

Krishnan Bhaskaran 1); Ian Douglas 1); Harriet Forbes 1); Liam Smeeth 1)
1) Department of Non-Communicable Diseases Epidemiology, London School of Hygiene and Tropical Medicine, London, UK

Background: Associations between body mass index (BMI) and a number of cancers have been reported, but there have been few studies of large individual data sources in which BMI-cancer associations for a wide range of site-specific malignancies have been compared within a consistent framework.

Objectives: To investigate the association between BMI and a wide range of specific cancers using routinely collected data from a large UK primary care database.

Methods: We used routinely collected primary care data from the UK Clinical Practice Research Datalink (CPRD). All individuals with at least one BMI entered on their record were included. Follow-up started at the latest of: date the patient came under follow-up within a CPRD practice, date the practice data was deemed by CPRD to be up to research quality standard, date of the first available BMI in the patient's record. We used Cox models with age as the underlying timescale to investigate the association between BMI (based on the first BMI recorded in the patient's follow-up) and each of the 20 most commonly diagnosed cancers in the UK, and gallbladder cancer because of previous evidence linking this outcome with BMI. The 12-month period following the BMI record defining exposure was excluded from analysis to guard against reverse causality. All models were adjusted for potential confounders including smoking, alcohol, diabetes and (for non-gender-specific cancers) sex.

Results: 6.2 million patients were included. Increased BMI was associated with higher risk of a number of cancers. The largest associations were for cancers of the uterus, gallbladder, kidneys, liver and cervix $(p<0.0001$ in all cases). For each of these cancers we estimated a $>10 \%$ increase in risk per $5 \mathrm{~kg} / \mathrm{m}^{2}$ increase in BMI. The largest association was for cancer of the uterus (hazard ratio per $5 \mathrm{~kg}$ / $\mathrm{m}^{2}$ increase in BMI $=1.29,95 \%$ CI $\left.1.27-1.30, p<0.001\right)$. For lung cancer and oral cancers, higher BMI appeared to be associated with reduced cancer risk (hazard ratio $=0.83,0.82-0.85$ and 0.83 , $0.78-0.88$ respectively), as observed in other studies.

Conclusions: BMI-cancer associations appear to vary by cancer site, with higher BMI associated with a substantial increase in risk of a number of cancers, including of the uterus, gallbladder, kidneys, liver and cervix. Though all analyses were adjusted for smoking, inverse associations between BMI and strongly smoking-related cancers may be explained by BMI acting as a proxy for amount of smoking. Ongoing work will include examination of potential non-linear BMIcancer associations.

\section{P-074}

Understanding cancer risks due to radiation exposures from paediatric CT

Topic: Diseases - Cancer

Presenter: Mark Pearce

Mark Pearce 1); Sarah Baatout 2); Elisabeth Cardis 3); Andreas Jahnen 4); Michael Hauptmann 5); Magnus Kaijser 6); Carlo Maccia 7); Isabelle Thierry-Chef 8); Ausra Kesminiene)

1) Institute of Health \& Society, Newcastle University, Newcastle upon Tyne, United Kingdom; 2) University of Ghent, Belgium; 3) Centre for Research in Environmental Epidemiology, Spain; 4) Henri Tudor Centre, Luxembourg; 5) The Netherlands Cancer Institute, Amsterdam, The Netherlands; 6) Karolinska University Hospital, Sweden; 7) Centre d'Assurance de qualité des Applications Technologiques dans le domaine de la Santé, France; 8) International Agency for Research on cancer (IARC), Lyon, France 
Background: Although computed tomography (CT) has great clinical utility, serious concerns have been raised about the potential cancer risks from the associated ionising radiation, particularly for children, who are more radiosensitive.

Objective: To summarise the findings of the UK CT Scan Study, the epidemiological protocol of the European EPI-CT project and the ongoing worldwide research in this area.

Methods: Within the UK CT Scan Study, the cohort comprised patients without previous cancer diagnoses, aged $<22$ years at first CT, scanned in Great Britain between 1985 and 2002 and followed up to 2008. The absorbed brain and red bone marrow doses were estimated per scan in milligray (mGy) and analysed in relation to cancer outcomes using Poisson relative risk models.

The EPI-CT study was designed as a multinational cohort study of paediatric and young adults who have received substantial doses of ionising radiation from CT scanning for non-oncological purposes, using a similar design to the original UK study. It has partners in 9 European countries and aims to recruit over 1,000,000 patients. As well as detailed epidemiology research, it also includes work packages on dosimetry, radiation biology and dose optimisation.

Results: The initial results of the UK CT Scan Study were published in the Lancet in 2012. They showed significantly increased risks of both leukaemia and brain tumours with increasing red bone marrow and brain dose, respectively. Compared to less than $5 \mathrm{mGy}$, the RR for a cumulative dose of $30+$ mGy (mean $=50 \mathrm{mGy}$ ) was 3.18 (95\% CI 1.46-6.94) for leukaemia and for 50-74 mGy (mean $=60 \mathrm{mGy}$ ) the RR was $2.82(95 \%$ CI 1.34-6.03) for brain tumours. For perspective, in children currently 5-10 head CTs gives approximately $50 \mathrm{mGy}$ cumulative red bone marrow dose and 2-3 head CTs approximately $60 \mathrm{mGy}$ cumulative brain dose. Within the EPI-CT study, about two-thirds of the target patient population has been accrued and data linkage, dosimetry and collection of data on potential confounders are already underway. Similar studies are also underway in Canada and Australia.

Conclusions: The results of the initial UK CT study suggest that there are increased risks of leukaemia and brain tumours with increasing radiation doses. However, the cumulative absolute risks are small. So far, the evidence suggests that if CT is clinically justified, then the benefits should outweigh the small absolute risks. The EPI-CT study will allow rarer cancer types to be investigated and will allow better dose estimation.

\section{P-075}

Methods and rationale used in a matched cohort study of the incidence of new primary cancers following prostate cancer

Topic: Diseases - Cancer

Presenter: Deirdre Cronin-Fenton

\section{Deirdre Cronin-Fenton 1); Sussie Antonsen 1); Timothy Lash 1), 2)}

1) Department of Clinical Epidemiology, Aarhus University Hospital, Aarhus, Denmark; 2) Department of Epidemiology, Rollins School of Public Health, Emory University, Atlanta, GA, USA

Background: We conducted a population-based cohort study to examine the rates of new primary cancers in men with and without prostate cancer (PC).

Objectives: Here, we describe the epidemiological methods and rationale we used to compare the rates of new primary cancers in a Danish population-based matched cohort study comparing rates of new primary cancers in men with and without PC.

Methods: We matched 30,220 men with PC to 151,100 men without PC (comparators) on age ( \pm 2 years) and PC diagnosis/index date. We focused on the following methodologic issues: survival differences between the cohorts, diagnostic bias, and multiple comparisons. To address survival differences, we compared rates with and without censoring comparators on the date that their matched PC patient died or was censored. To address diagnostic bias, we excluded men with a history of cancer from the comparator cohort and we graphed the hazard of new primary cancer in both cohorts, with and without prostate cancer as an outcome. Lastly, we used empirical Bayes methods to explore the effect of adjusting for multiple comparisons. Results: After 18 months of follow-up, cumulative person-time was lower in the PC cohort than the comparators due to higher mortality among PC patients. Terminating person-time in comparators at the time of the matched PC patient's death or loss to follow-up resulted in comparable person-time up to 30 months of follow-up and lower person-time in the comparison cohort thereafter (because follow-up of PC patients was not terminated when their comparators died). The hazard of new primary cancer was lower among men with PC than that in comparators throughout follow-up. However, there was little difference in rates beyond the first 4 years of follow-up after removing PC as an outcome. Empirical Bayes adjustment for multiple comparisons had little effect on the estimates.

Conclusions: Addressing the issues of competing risks, treatment interference or diagnostic bias, and multiple comparisons lowered the deficit rate of new primary cancers among men with a history of PC compared with those without PC. However, we cannot exclude the possibility that risk factor differences between the two cohorts, which were unavailable, may also explain the differing rates of new primary cancers between the two cohorts.

\section{P-076}

Metabolism and transport of tamoxifen in relation to its effectiveness: new perspectives on an ongoing controversy

Topic: Diseases - Cancer

Presenter: Deirdre Cronin-Fenton

Deirdre Cronin-Fenton 1); Per Damkier 2); Timothy Lash 1), 3)

1) Department of Clinical Epidemiology, Aarhus University, Aarhus, Denmark; 2) Department of Epidemiology, Rollins School of Public Health, Emory University, Atlanta, USA.; 3) Department of Clinical Chemistry and Pharmacology, Odense University Hospital, Denmark

Background: Adjuvant tamoxifen treatment of estrogen receptor positive breast cancer reduces the rate of recurrence by about onehalf. Genetic or drug-induced inhibition of cytochrome P450 2D6 (CYP2D6) reduces the serum concentration of tamoxifen's most active metabolites. Some have advocated for the use of CYP2D6 genetic testing in clinical practice, but no guideline recommends CYP2D6 genotyping.

Objectives: To conduct a qualitative review and quantitative metaanalysis of the clinical epidemiology studies investigating the association of CYP2D6 inhibition and breast cancer recurrence.

Methods: We searched for the terms "tamoxifen" and "CYP2D6" in PubMed, including all papers and abstracts through 31 January 2013 on the association of CYP2D6 gene variants or drug-drug interactions and the risk of breast cancer recurrence or mortality. We included 30 and 12 papers on genetic or drug-induced inhibition, respectively. We used random-effects meta-analytic models to generate summary effect estimates (EE) and associated $95 \%$ confidence intervals $(95 \%$ $\mathrm{CI}$ ) and funnel plots to assess publication bias. We generated 4 metaanalytic models: (1) weak CYP2D6 inhibitors such as citalopram, (2) strong CYP2D6 inhibitors such as paroxetine, (3) any reduced function allele, and (4) two reduced function alleles. 
Results: The summary EE associating breast cancer recurrence with concomitant use of tamoxifen and a weak CYP2D6 inhibitor was $1.05,95 \% \mathrm{CI}=0.91,1.22$; that of a strong CYP2D6 inhibitor was $1.03,95 \% \mathrm{CI}=0.86,1.23$. The summary $\mathrm{EE}$ associating recurrence with inheritance of any reduced function allele was $1.38,95 \%$ $\mathrm{CI}=1.10,1.73$; that of two reduced function alleles was $2.08,95 \%$ $\mathrm{CI}=1.40,3.10$. Individual results were widely heterogeneous and the heterogeneity is not easily explained. Important design flaws were identified for some positive studies.

Conclusions: Weakly positive summary associations between CYP2D6 inhibition and breast cancer recurrence are dominated by poorly designed studies.

\section{P-077}

MnSOD polymorphism and breast cancer recurrence in patients treated with cyclophosphamide-based chemotherapy: casecontrol study and meta-analysis

Topic: Diseases - Cancer

Presenter: Deirdre Cronin-Fenton

Deirdre Cronin-Fenton 1); Mariann Christensen 2); Timothy Lash 1), 3); Thomas Ahern 4); Lars Pedersen 1); JensPeter Garne 5), 6); Marianne Ewertz 5), 7); Herman Autrup 8); Henrik Toft Sørensen 1); Stephen Hamilton-Dutoit 2)

1) Department of Clinical Epidemiology, Aarhus University, Denmark; 2) Institute of Pathology, Aarhus University, Denmark; 3) Department of Epidemiology, Rollins School of Public Health, Emory University, Atlanta, USA; 4) Channing Division of Network Medicine, Brigham and Women's Hospital and Harvard Medical School, Boston, USA; 5) Danish Breast Cancer Cooperative Group, Copenhagen, Denmark; 6) Department of Breast Surgery, Aalborg University Hospital, Aalborg, Denmark; 7) Department of Oncology, Odense University Hospital, Institute of Clinical Research, University of Southern Denmark, Denmark; 8) Institute of Public Health, Aarhus University, Denmark

Background: Manganese superoxide dismutase (MnSOD) inhibits oxidative damage thereby modulating the effectiveness of cancer therapy. A polymorphism in its encoding gene (SOD2: Val16Ala) results in increased MnSOD activity. This polymorphism may confer poorer breast cancer survival, but research is inconsistent. We examined the association of SOD2 genotype and breast cancer recurrence among patients treated with cyclophosphamide-epirubicin5-fluorouracil (CEF) chemotherapy. We compared our findings with those of published studies using meta-analytic models.

Methods: We conducted a case-control study of breast cancer recurrence nested in the population of female residents of Jutland, Denmark who were diagnosed with non-metastatic breast cancer from 1991 to 2001, received adjuvant CEF, and were registered in the Danish Breast Cancer Cooperative Group. We identified 118 cases of recurrent breast cancer and 213 matched controls. We genotyped SOD2 and used conditional logistic regression to compute the odds ratio (OR) and associated $95 \%$ confidence intervals (95\% CI) of breast cancer recurrence. We used random-effects meta-analytic models to assess the evidence regarding the association of SOD2 polymorphisms and breast cancer recurrence.

Results: The frequency of the SOD2 Ala allele was $70 \%$ in cases and $71 \%$ in controls; 40 versus $44 \%$ were heterozygotes, while 30 and $25 \%$ were homozygotes, respectively. Heterozygote and homozygote carriers of the Ala allele had no evidence of an increased rate of breast cancer recurrence $(\mathrm{OR}=1.1,95 \% \mathrm{CI}=0.65,2.0$, and $\mathrm{OR}=0.87$, $95 \% \mathrm{CI}=0.47,1.6$, respectively). Six studies informed the metaanalytic models yielding summary effect estimates associating breast cancer recurrence for homozygote, heterozygote or any inheritance of the variant Ala allele were $1.12(95 \% \mathrm{CI}=0.70,1.80), 1.07(95 \%$ $\mathrm{CI}=0.81,1.40)$, and $1.06,(95 \% \mathrm{CI}=0.79,1.43)$, respectively. Conclusion: Our study does not indicate that increased MnSOD enzymatic activity impacts on rates of breast cancer recurrence among patients treated with CEF.

\section{P-078}

Validity of the cohort of crete in the seven countries study: a timeseries study applied to the cancer mortality trend between 1960 and 2011

Topic: Diseases - Cancer

Presenter: Dimitra Sifaki-Pistolla

Dimitra Sifaki-Pistolla 1); Christos Hatzis 1); Christopher Papandreou 1); Gregory Chlouverakis 2); Anthony Kafatos 1); Nikolaos Tzanakis 3), 4)

1) Department of Social Medicine, Preventive Medicine and Nutrition Clinic; 2) Laboratory of Biostatistics, Department of Social Medicine; 3) Department of Thoracic Medicine, University General Hospital; 4) Department of Epidemiology, Social Medicine, Medical School of the University of Crete, Heraklion, Greece

Background: One of the challenges facing prospective research designs is the recruitment of representative samples to enhance the external validity of the results. The majority of the larger famous cohorts assessing risk factors have been criticized as suffering from a non-representative studied subpopulation leading to marked sampling bias. Although the Seven countries study (SCS) has widely recognized results, it has also been criticized by some researchers, as suffering from sampling bias. Thus it is important to investigate those doubts.

Purpose: This study aimed to examine whether the Cretan cohort of the SCS is representative for the entire population in the island using cancer mortality registries and by comparing secular trends of major cancer mortality figures. It also compares the variance of cancer mortality rates of the SCS Cretan cohort during 51 years of follow-up with those of the general population of Crete during the same period. Methods: The analysis was carried out on the Cretan cohort of the SCS cancer mortality data and a similar cancer registry for the general population during a 51-year follow-up (1960-2011). Information was obtained from official death certificates and classified according to the ICD 10. Two time series models of mortalities from cancer, using data from the Cretan cohort and the Hellenic Statistical Office (EL.STAT), were developed using Matlab software. The existence of long-term memory in the data was tested by rescaled range analysis (Hurst Mandelbrot). State-space reconstruction was applied to identify the simplest system that was able to re-create the present timeseries.

Results: In the cohort, cancer mortalities accounted for $18.9 \%$ of total mortalities. The EL.STAT time-series analysis generated mean $\mathrm{V}$ statistics (95\% CI) of $0.69815(0.398-0.999)$ and 0.677143 (0.301-0.897) for the general population and the seven countries cohort, respectively. The embedding dimension for the EL.STAT data was equal to 1 for the general population and for the Cretan cohort $(\mathrm{m}=1)$. The exponent $\mathrm{H}$ values for the two time-series were almost equal. In the two time-series the proposed time delay of cancer mortalities was 2. The Cretan cohort of the SCS and the entire population of the island followed similar time patterns of cancer mortality.

Conclusions: The present study revealed that the two time series for cancer mortality were of the same behavior, pattern, nature and reconstruction, with the Cretan cohort having a slightly lower number 
of mortalities per year. These observations support the statement that the SCS' Study Cretan cohort is representative of the island's entire population.

\section{P-079}

Competing risk analysis of metabolic factors and risk of prostate cancer

Topic: Diseases - Cancer

Presenter: Christel Häggström

Christel Häggström 1); Tanja Stocks 1), 2); Tone Bjørge 3), 4); Gabriele Nagel 5), 6); Hanno Ulmer 7); Jonas Manjer 8); Håkan Jonsson 9); Pär Stattin 1), 10)

1) Department of Surgical and perioperative sciences, Urology and andrology, Umeå University, Umeå, Sweden; 2) Institute of Preventive Medicine, Copenhagen University Hospital, Copenhagen, Denmark; 3) Department of Public Health and Primary Health Care, University of Bergen, Bergen, Norway; 4) Norwegian Institute of Public Health, Bergen, Norway; 5) Institute of Epidemiology and Medical Biometry, Ulm University, Ulm, Germany; 6) Agency for Preventive and Social Medicine, Bregenz, Austria; 7) Department of Medical Statistics, Informatics and Health Economics, Innsbruck Medical University, Innsbruck, Austria; 8) Department of Surgery, Skåne University Hospital, Lund University, Malmö, Sweden; 9) Department of Radiation Sciences, Oncology, Umeå University, Umeå, Sweden; 10) Department of Surgery, Urology Service, Memorial Sloan-Kettering Cancer Center, New York, NY, USA

Background: Previous studies of metabolic factors and prostate cancer risk have mainly used conventional survival analysis. However, these methods do not account for competing events that may influence the risk of disease. For example men at risk of prostate cancer also are at risk of death due to high age and men with high levels of metabolic factors also at risk of death mainly due to cardiovascular diseases.

Objectives: The aim of this study was to assess probabilities of diagnosis and death from prostate cancer for high versus low levels of body mass index (BMI), blood pressure, glucose, cholesterol, and triglycerides, taking all-cause and other causes of death into account in a competing risk analysis.

Methods: In the Metabolic syndrome and Cancer project (Me-Can), data on body mass index (BMI), blood pressure, and blood levels of glucose, cholesterol, and triglycerides were collected for 289,866 men. Cumulative incidence of diagnosis and death from prostate cancer and from their competing events was calculated by high and low levels of metabolic exposures. In a sub analysis only men diagnosed with prostate cancer were analysed and probabilities of death from prostate cancer and other causes were calculated.

Results: During a mean follow-up of 12 years, 6,122 men were diagnosed with prostate cancer, 1,016 died from prostate cancer and 27,048 men died from other causes. No differences between high and low levels of BMI, blood pressure, glucose, cholesterol and triglycerides were found for probability of prostate cancer diagnosis and death. However, men with high levels of metabolic factors had a higher probability of death from all and other, and therefore shorter overall survival. Among men diagnosed with prostate cancer, we found that men with low levels of metabolic factors had lower probability for death from prostate cancer and other causes and therefor highest probability for overall survival.

Conclusions: Men with high levels of metabolic factors do not have a higher probability of prostate cancer diagnosis or death, but higher probability of death from other causes, and therefore shorter overall survival.

\section{P-080}

\section{Evaluation of cervical cytological abnormalities in Albanian population}

Topic: Diseases - Cancer

Presenter: Anila Xhani, Kozeta Filipi

Anila Xhani 1); Kozeta Filipi 2)

1) 2. Maternity Geraldina Queen “, Tirana, Albania”;2) 1. Institute of Public Health, Department of Epidemiology and Health System,

Tirana, Albania

Introduction: Cervical cancer is one of the most common female malignancies with high mortality rates in developing countries. Our purpose was to determine the prevalence of cervical cytologic abnormalities in Albanian population and the detection rate of epithelial abnormalities by cervical cytology (CC).

Materials and methods: A total of 5532 conventional pap smear tests collected between January 2010 and January 2013 from hospitals and private clinic, and are retrospectively analyzed.

Results: Total of $258(4.8 \%)$ cases had epithelial abnormalities. The numbers and rates of epithelial abnormalities were as the followings: Atypical squamous cell of undetermined significance (ASCUS; $\mathrm{n}=159[61.6 \%])$; atypical glandular cell of undetermined significance (AGUS; $\mathrm{n}=15$ [5.8\%]); low-grade squamous intraepitelial lesion (LSIL; $\mathrm{n}=71[27.5 \%]$ ); high-grade squamous intraepithelial lesion (HSIL; $\mathrm{n}=10$ [3.8\%]); and squamous cell carcinoma (SCC; $\mathrm{n}=3[1.1 \%])$

Conclusion: The prevalence of cervical cytological abnormality in our study was $4.8 \%$. Recently, some conflicting results from the same population were published. More prospective studies with larger numbers are needed.

\section{P-081}

Offspring birth weight in the most recent pregnancy and mortality in premenopausal breast cancer

Topic: Diseases - Cancer

Presenter: Mohammadhossein Hajiebrahimi

Mohammadhossein Hajiebrahimi 1); Shahram Bahmanyar 1), 2); Mats Lambe 3), 4); Jan Adolfsson 5); Tommy Fornander 6); Fredrik Wärnberg 7); Sven Cnattingius 1)

1) Clinical Epidemiology Unit, Department of Medicine, Karolinska Institutet, Sweden; 2) Center for pharmacoepidemiology, Department of Medicine, Karolinska Institutet, Sweden; 3) Department of Medical Epidemiology and Biostatistics, Karolinska Institutet, Sweden; 4) Regional Cancer Center, Uppsala, Sweden; 5) Department of Clinical Science, Intervention and Technology, Karolinska Institutet, Sweden; 6) Department of Oncology-Pathology, Karolinska Institutet, Sweden; 7) Department of Surgical Science, Uppsala University, Sweden

Background: Estrogens are well-established risk factors for breast cancer and may also influence breast cancer prognosis. Mortality rates are higher among women diagnosed with breast cancer up to 10 years following delivery, and the prognosis is especially poor in women with breast cancer diagnosed during or shortly after pregnancy. In a recent study we found an increased mortality risk in premenopausal breast cancer associated with higher placental weight-as indirect markers of pregnancy hormone exposures-which was most pronounced among estrogen receptor (ER) negative and progesterone receptor $(\mathrm{PR})$ negative tumors. Birth weight is another indicator for pregnancy hormone exposure. 
Objective: To study the influence of offspring birth weight on mothers' risk of premenopausal breast cancer mortality, taking tumor characteristics into account. We also investigated if the association is modified by age at childbirth, time since childbirth, parity, and age at diagnosis.

Methods: We followed 6,129 women with premenopausal breast cancer diagnosed from 1992 to 2008 from the date of diagnosis with breast cancer until emigration, death or until December 31st, 2009, whichever occurred first. We used Cox proportional hazard regression models, adjusted for gestational age, parity, age at diagnosis, and education level, to estimate the association between offspring birth weight and mothers mortality risk. Birth weight was considered both as a continuous and a categorized variable $(<3,000,3,000-3,499$, $3,500-3,999$ and $\geq 4,000 \mathrm{~g}$ ). In stratified analyses, mortality risks were estimated by tumor stage, estrogen receptor or progesterone receptor status.

Results: There was no association between offspring birth weight (as a continuous or a categorical variable) and risk of premenopausal breast cancer mortality: hazard ratio for each $100 \mathrm{~g}$ increase in birth weight was $1.00,95 \%$ confidence interval $0.99-1.01$. We did also not find any associations in analyses stratified by gestational age, time difference between last pregnancy and date of diagnosis, tumor stage, and receptor status. We also performed the stratified analyses for joint receptor status $(\mathrm{ER}+\mathrm{PR}+, \mathrm{ER}-\mathrm{PR}+, \mathrm{ER}+\mathrm{PR}-, \mathrm{ER}-\mathrm{PR}-)$ and found no association. There was no association between risk of mortality and other proxies of hormone exposures, including birth length and head circumference.

Conclusion: Premenopausal breast cancer mortality is not associated with offspring birth weight in the most recent pregnancy before the diagnosis. The association is not modified by tumor characteristics.

\section{P-082}

Male germ-cell cancer in car manufacturing workers: results of a nested case-control study

Topic: Diseases - Cancer

Presenter:

Wolfgang Ahrens 1); Ingo Langner 1); Nils Schmeißer 1); Birte Mester 1); Thomas Behrens 2)

1) Leibniz Institute for Prevention and Epidemiology - BIPS GmbH; 2) Institute for Prevention and Occupational Medicine of the German Social Accident Insurance, Institute of the Ruhr-University-Bochum (IPA)

Keywords: Testicular cancer, Etiological factors, Endocrine disrupting agents, Cutting fluids, Car industry, Occupational exposures Introduction: This case-control study nested in a cohort of car manufacturing workers investigated occupational and non-occupational factors to explain the excess incidence previously observed in this cohort. Particular attention was paid to pesticides in avocational farm work and to electromagnetic fields (EMF)/endocrine disrupting chemicals (EDC) within the industry.

Methods and materials: Personal interviews of 205 cases and 1,091 controls matched by age ( \pm 2 years) covered medical and personal characteristics, dietary habits, occupational history and environmental factors. Specific job tasks were assessed by 37 job-specific-questionnaires. An expert panel developed a job-exposure-matrix (JEM) and assessed exposure for each individual with possible exposure according to the JEM. Odds ratios (ORs) and $95 \%$ confidence intervals (CI) were calculated by conditional logistic regression.

Results: The prevalence of farming and forestry working was below expectation $(5.3 \%$ in cases and $6.3 \%$ in controls) and related exposure to pesticides, fertilisers, or disinfectants was not associated with GCC. Metal-cutting and non-cutting jobs yielded ORs of 1.87 (CI 1.31-2.67) and 1.24 (CI 0.68-2.28), respectively, amongst machine fitters, machine assemblers and precision instrument makers. Exposure to oil-based cutting fluids showed an excess risk of nonseminoma after more than 5,000 exposure-hours $(\mathrm{OR}=4.72 ; \mathrm{CI}$ 1.48-15.09). Ever-exposure to bisphenol A (OR $=1.39$; CI $0.93-2.06)$, epoxy resins (OR $=1.41$; CI $0.95-2.09)$ and the glycolether $\operatorname{EGBE}(\mathrm{OR}=1.3$; CI $0.93-1.83)$ was associated nonsignificantly with GCT. An elevated risk due to dimethylformamide was indicated after 3.5 to 8.5 years $(\mathrm{OR}=3.48$; CI $0.98-12.34)$. EMF exposure showed no risk elevation.

Discussion: Exposures in farming activities did not explain the excess risk in the cohort. Our data indicate a possible risk of GCC related to exposure to metal-cutting fluids. EGBE belongs to the agent exposures that warrant further attention in this regard. Exposure misclassification may disguise associations for this and other EDCs that showed moderately elevated risks.

\section{P-083}

Breast cancer and barriers to accessing primary and specialist health care among New Zealand women

Topic: Diseases - Cancer

Presenter: Fiona McKenzie

Fiona McKenzie 1), 2); Lis Ellison-Loschmann 2);

Ridvan Firestone 2); Lucy Aquilina 3); Michelle Gray 2); Mona Jeffreys 3)

1) International Agency for Research on Cancer; 2) Centre for Public Health Research, Massey University; 3) School of Social and Community Medicine, University of Bristol

Background: Unequal access to high quality care contributes to disparities in cancer outcomes.

Objective: To examine the ethnic disparity in barriers to accessing primary and specialist health care experienced by New Zealand women with breast cancer.

Methods: Women registered with a primary invasive breast cancer between April 2005 and April 2007 were eligible. Participants completed a questionnaire, in which twelve potential barriers were listed. For each barrier, women could answer yes or no separately for accessing primary and specialist health care. Barriers were classified into three broad domains for analysis: personal, practical and health care process factors. Logistic regression was used to estimate odds ratios (OR) for the association between ethnicity and each of the three barrier domain groupings, and adjusted for age and deprivation quintile.

Results: Of 1,799 respondents, 302 were Maori (indigenous New Zealanders), 70 were Pacific and 1,427 were non-Maori/non-Pacific women. The prevalence of reporting barriers to primary care was highest in Pacific women (over $10 \%$ for three specific barriers), the most common being cost, not being able to get an appointment at a suitable time, preferring not to know (or fear of) their condition, and not trusting a health professional. For Maori women, the most commonly reported barriers were preferring not to know, cost, and not wanting to make a fuss. For non-Maori/non-Pacific women, the most common barriers were cost and not wanting to make a fuss. The frequency of reporting cost and not being able to get an appointment soon enough as barriers to specialist care were reported by all ethnic groups. Maori and Pacific women were more likely to face delays in seeing a specialist than non-Maori/non-Pacific women. These delays were not explained by the reported barriers. Women with diabetes reported an over two-fold higher risk of facing barriers to care compared to those without diabetes (age- and ethnicity-adjusted OR: 
2.06, $95 \%$ CI 1.20-3.57). The effect of having a comorbidity on reported barriers to care did not appear to differ by ethnic group. Conclusions: Our results underline the importance of ensuring that the health system is fully accessible and meets the needs of women with breast cancer, and that the various needs of women from different ethnic groups must be recognised.

\section{P-084}

A retrospective cohort study on cancer incidence and survival of HIV seropositive patients

Topic: Diseases - Cancer

Presenter: Elena Raffetti

Elena Raffetti 1); Laura Albini 2); Daria Gotti 2); Eugenia Quiros-Roldan 2); Francesco Donato 1)

1) Unit of Hygiene, Epidemiology and Public Health, University of Brescia, Italy; 2) Clinic of Infectious and Tropical Diseases, University of Brescia, Italy

Background: The use of combination antiretroviral therapy (cART) has dramatically improved the survival in HIV-infected people. However, these patients are at higher cancer risk compared to the general population.

Objectives: To assess cancer incidence, 5-year survival and related demographic and clinical factors (covariates) among HIV-infected patients by type of cancer [AIDS-defining cancers (ADCs) or nonAIDS-defining cancers (NADCs)] and by year of cancer diagnosis [ $<1998$ (pre-cART era) or $\geq 1998$ (post-cART era)].

Methods: HIV-infected patients included in the MASTER Italian Cohort (January 1986-September 2012, http://www.mastercohort.it), with at least one CD4+ T-cell and HIVRNA test, were enrolled. Cancers were classified in ADCs or NADCs. The ratio of the observed to expected cases (standardized incidence ratio [SIR] with $95 \%$ confidence interval [CI]), stratified before and after 1998, was calculated. The expected numbers of cancer cases were computed on the basis of the incidence rates reported by Italian Cancer Registries. Poisson regression analysis was used to evaluate the multivariate associations between covariates and cancer incidence. Survival in cancer patients was estimated according to Kaplan-Meier method and the association with covariates was tested by the log-rank test. Subsequently, statistically significant variables were entered in a multivariate Cox regression model.

Results: Among 16,305 patients, contributing 134,291 person-years, $11,208(74 \%)$ were males, with a median age of 32 years at entry (interquartile range 27-39). During a median of 6.2 years of followup, 1,195 cancers were diagnosed: 700 ADCs and 495 NADCs. Agestandardized incidence rates in ADCs and NADCs increased after 1998 (from 13.2 to 19.6 per 1,000 person-years and from 2.7 to 9.6 per 1,000 person-years, respectively). However, the SIR for ADCs decreased from 66.6 (95 \% CI 62.2-71.2) in pre-cART era to 30.8 (28.7-33.0) in post-cART era. For NADCs, the SIR remained stable [0.9 (0.6-1.2) before 1998 and $0.9(0.8-1.0)$ after 1998]. 5-year survival ( \pm standard error) in ADCs was $37.3 \%( \pm 3.4)$ before 1998 versus $68.8( \pm 2.4)$ after 1998 , and $49.2( \pm 8.0)$ versus $59.2( \pm 2.7)$ for NADCs. On multivariate analysis, survival was associated with CD4+ T-cell count: the Hazard ratio (HR) for CD4+ T-cell counts $<200$ versus $>200$ decreased from pre-cART to post-cART era in both ADCs (from $\mathrm{HR}=3.7$ to $\mathrm{HR}=2.3$ ) and NADCs (from $\mathrm{HR}=2.7$ to $\mathrm{HR}=1.5$ ).

Conclusions: HIV-positive subjects showed the same risk of NADC occurrence of the Italian general population, independently from cART introduction. We detected an improvement of survival in postcART era for both types of cancer.

\section{P-085}

GSTM1 and GSTT1 null genotypes and gastric cancer: a casecontrol study of gene-environment interactions in Turkish population

Topic: Diseases - Cancer

Presenter: Gul Ergor

Ozgul Vupa 1); Elcin Bora 2); Ayfer Ulgenalp 2); Seymen Bora 2); Gul Ergor 2)

1) Dokuz Eylul University Faculty of Sciences; 2) Dokuz Eylul University Faculty of Medicine

Background: Stomach cancer is a major cause of cancer mortality worldwide. It is the fourth most common cancer in men and third in women in Turkey. There are several environmental and genetic factors playing a role in the development of gastric cancer. Glutathione S-transferases (GSTs) appear to play a critical role in the protection from the effects of carcinogens. The contribution of GSTM1 and GSTT1 genotypes to susceptibility to the risk of SC and their interaction with cigarette smoking are unclear in Turkish population.

Objective: The aim of this study was to determine whether there was any association between genetic polymorphisms of GSTM1 and GSTT1 and stomach cancer as well as any interaction between polymorphisms and smoking.

Methods: A case-control study was carried out in Izmir, Turkey. The data were collected by a questionnaire from 127 cases and 101 healthy controls. Polymerase chain reaction (PCR) method was used to detect the presence or absence of the GSTM1 and GSTT1 genotypes. Genotype data were obtained for 79 cases and for 99 controls. The relationships between stomach cancer and smoking and interaction with GSTs were assessed using ORs and $95 \%$ CI's derived from univariate, stratified and multivariate analyses.

Results: Prevalence of GSTM1 and GSTT1 null genotypes were 58.2 and $22.8 \%$ in cases, 46.5 and $22.2 \%$ in controls, respectively. This study revealed that, GSTM1 polymorphism and smoking has a potential role for interaction in stomach cancer. Our data suggested an increased risk for GSTM1 null genotype although a significant association was not found. There was no significant association for GSTT1 genotype. Smoking prevalence were 55.9 and $43.6 \%$ in cases and controls, respectively $(p=0.064)$. However when this variable was investigated as pack-years of smoking, there was significant difference in pack-years between cases and controls ( $p$-value $=$ 0.001). Using cigarette more than 20 pack-years was increasing the risk 3.95 times for stomach cancer.

Conclusions: The effect of smoking on stomach cancer was present after adjusting for age, gender, GSTM1\&GSTT1 polymorphisms. GSTM1 polymorphism in stomach cancer has a potential role for interaction between this polymorphism and smoking. The combined effect (OR) was 2.60 (1.1-5.9) higher than a multiplicative effect 1.22 $(1.07 * 1.14)$ and this indicated the presence of interaction between GSTM1 genotype and smoking status in stomach cancer.

\section{P-086}

Epidemiology of stomach cancer and influence of inhabitance place on survival in the Arkhangelsk region, Russia in 2000-2010

Topic: Diseases - Cancer

Presenter: Natalia Glukhareva, Andrej Grjibovski

Natalia Glukhareva 1); Anna Subbotina 1), 2); Dinara Ospanova 3); Mikhail Valkov 1); Andrej Grjibovski 4), 5)

1) Northern State Medical University, Arkhangelsk, Russia; 2) University of Tromso, Norway; 3) Institute of Postgraduate Medical 
Education (AGIUV), Almaty, Kazakhstan; 4) International School of Public Health, Northern State Medical University, Arkhangelsk, Russia; 5) Department of International Public Health, Norwegian Institute of Public Health, Oslo, Norway

Background: The incidence of stomach cancer (SC) has been decreasing worldwide and in Russia. The Arkhangelsk Regional Cancer Register (ARCR) collects the data on all cancers since 1997. There are no publications about epidemiology of SC in Arkhangelsk region and connection of survival with place of inhabitance of patients.

Objectives: To assess the incidence and mortality for both genders from SC in the Arkhangelsk region, to study influence of place of inhabitance of patient on survival from SC.

Methods: All new cases of SC in the Arkhangelsk region in 2000-2010 were extracted from ARCR. Population size was obtained from the Regional Bureau of Statistics. Incidence and mortality were calculated per 100,000 standardized to world population for both genders. Survival was assessed by Kaplan-Meier method and Cox Regression. Three region of Arkhangelsk region (with not favorable ecological environment) Arkhangelsk, Novodvinsk, Korazhma were compared with others parts of region.

Results: During the period from 2000 to 2010 in the Arkhangelsk region There were 6,086 new cases of SC in the Arkhangelsk region during the period from 2000 to 2010 . Incidence of SC decreased for males (from 58.8 to 38.9) and females (from 21.6 to 17.0) during study period. Mortality from SC increased for males (from 24.5 to 36.4) and females (from 8.7 to 15.7). There was no difference in survival of patients from SC in regions with not favorable ecological environment in comparison with other parts of region (Log Rank 0.80 in Kaplan-Meier analysis and $p=0.81$ in Cox Regression model).

Conclusions: The incidence of SC decreased for both genders, the mortality from SC increased for both cases in Arkhangelsk region of Russia during study period. Not favorable ecological environment was not influence on survival of patients with SC.

\section{P-087}

Epidemiology of and mortality from soft tissue sarcomas in Arkhangelsk region, Russia: a registry-based study

Topic: Diseases - Cancer

Presenter: Lyudmila Valkova, Andrej Grjibovski

Lyudmila Valkova 1); Anna Subbotina 2), 3); Sergey Asakhin 4); Andrey Krasilnikov 4); Dinara Ospanova 5); Andrej Grjibovski 2), 6); Mikhail Valkov 1)

1) Northern State Medical University, Arkhangelsk, Russia; 2) International School of Public Health, Northern State Medical University, Arkhangelsk, Russia; 3) University of Tromso, Tromso, Norway; 4) Arkhangelsk Clinical Oncology Hospital, Arkhangelsk, Russia; 5) Institute of Post-graduate Medical Education (AGIUV), Almaty, Kazakhstan; 6) Department of International Public Health, Norwegian Institute of Public Health, Oslo, Norway

Background: Soft tissue sarcomas (STS) represent rare and nonhomogenous group of malignant tumors with very different clinical presentation and prognosis. There are variations in both incidence of and mortality from STS worldwide. Epidemiology of STSs in Northwest Russia remains unknown since no studies on this issue have been published yet.

Objective: To describe the incidence, mortality and structure of STS in Arkhangelsk Region, Northwest Russia in 2000-2010.

Methods: Data on all cases of STS diagnosed in the resion between 2000 and 2011 were extracted from the Arkhangelsk Regional Cancer Registry (ARCR). Depersonalized database included information on date of birth, place of residence, gender, age, ICDO-3 histological code, and location of tumor, dates of diagnosis and death or the last checkup. Information on the population size of the region was obtained from the Arkhangelsk regional bureau of statistics. Incidence and mortality data were standardized using world standard population.

Results: Altogether, 451 cases of STS occurred in the region and were registered in the ARCR. Median age at diagnosis was 55.3 years. Male to female ratio was 1 to1.8. Smooth muscle sarcomas, fibroblastic/myofibroblastic tumors, adipocytic, fibrohistiocytic, skeletal muscle, vascular tumours, and tumours of uncertain differentiation occurred in $30,12,12,8,6,3$, and $11 \%$ of all SRS, respectively. Sarcomas, not otherwise specified, were diagnosed in $18 \%$ of cases. Primary tumors were located on the extremities (30\%), body (35\%), head and neck (5\%), in the gastrointestinal tract $(8 \%)$, gynecological $(15 \%)$, and not specified by location (7\%). The overall incidence of STS remained unchanged over the period, being 2.1 per 100000 in 2000 and 1.9 per 100,000 in 2010 among men and 2.6 per 100,000 in 2000 and 3.6 per 100000 in 2010 among women. The peak incidences were registered in the age 75-79 among women and in the age $85+$ among men, i.e. the incidence was 13.8 per 100,000 and 53.1 per 100,000 , respectively. The mortality from STS was on average 1.1 per 100,000 in males and 1.5 per 100,000 in females.

Conclusions: The incidence of STS in Arkhangelsk Region, Northwest Russia is slightly below the rates reported from other countries and male to female ratio is inverted comparatively to that was reported worldwide. Potential factors that may explain this discrepancy will be discussed.

\section{P-088}

Epidemiology of colon cancer in the Arkhangelsk region, Russia in 2000-2010

Topic: Diseases - Cancer

Presenter: Ludmila Lebedeva, Andrej Grjibovski

Ludmila Lebedeva 1); Natalia Glukhareva 1); Andrey

Krasilnikov 2); Dinara Ospanova 3); Andrej Grjibovski 4), 5); Mikhail Valkov 1)

1) Northern State Medical University, Arkhangelsk, Russia;

2) Arkhangelsk Clinical Oncology Hospital, Arkhangelsk, Russia;

3) Institute of Post-graduate Medical Education (AGIUV), Almaty,

Kazakhstan; 4) International School of Public Health, Northern State Medical University, Arkhangelsk, Russia; 5) Department of International Public Health, Norwegian Institute of Public Health, Oslo, Norway

Background: The incidence of colon cancer (CC) has been increasing worldwide and in Russia, while in the developed countries the trend to its leveling and even decrease during past two decades is observed. The data on CC incidence in Arkhangelsk region, Russia has not been published yet.

Objectives: To evaluate the incidence of $\mathrm{CC}$ in the Arkhangelsk region during the period from 2000 to 2010 .

Methods: All new cases of $\mathrm{CC}$ in the Arkhangelsk region in 2000-2010 and their characteristics were extracted from Arkhangelsk Regional Cancer Register (ARCR). Population size was obtained from the Regional Bureau of Statistics. Incidence was calculated per 100,000 and standardized to world population (WHO 2002).

Results: There were 3,225 cases of CC in the Arkhangelsk region during the period from 2000 to2010. Females and males constituted 62.5 and $37.5 \%$ of cases, respectively. The average age was 68.5 years. Cancer of the sigmoid colon, caecum, ascending colon, 
transverse colon, descending colon, and others were 43.0, 12.5, 10.1, $8.8,7.9$ and $17.7 \%$, respectively. Stage I, II, III, IV was registered in $6.0,23.4,35.3$ and $29.5 \%$ cases, respectively, unknown stage in $5.8 \%$. The proportion of urban population (Arkhangelsk, Severodvinsk, Novodvinsk, Kotlas, Koryazhma, Mirny) is $66.4 \%$ of all diagnosed cases of $\mathrm{CC}$ in the Arkhangelsk region. During the period the standardized incidence rates of CC have increased from 14.0 to 17.8 per 100,000 . The crude incidence among men and women have increased from13.6 per 100,000 in 2000 to 22.1 per 100,000 in 2010 and from 21.0 per 100,000 in 2000 to 28.8 in 2010 , respectively. The incidence among the urban population has risen from 15.5 per 100,000 in 2000 to 24.4 per 100,000 in 2010 . The incidence in rural areas was slightly higher and ranged from 23.3 per 100,000 in 2000 to 29.2 per 100,000 in 2010 .

Conclusions: The incidence and the mortality of $\mathrm{CC}$ in the Arkhangelsk region of Russia increased during the period 2000-2010, resembling incidence pattern in countries in transition. Most of the patients are females. The incidence is higher among rural population.

\section{P-089}

\section{Impact of diabetes mellitus on long-term survival of hepatocellular carcinoma}

Topic: Diseases - Cancer

Presenter: Elena Raffetti, Francesco Donato

Elena Raffetti 1); Giovanni Caccamo 1); Rossella Lamera 1); Sarah Molfino 2); Andrea Celotti 2); Rosa Maria Limina 1); Arianna Coniglio 2); Nazario Portolani 2); Francesco Donato 1)

1) Unit of Hygiene, Epidemiology and Public Health, University of Brescia, Italy; 2) Surgical Clinic, University of Brescia, Italy

Background: The influence of diabetes mellitus (DM) on hepatocellular carcinoma (HCC) incidence remains obscure and it is not clear whether it may affect the overall survival.

Objective: Evaluation of DM influence on HCC survival.

Methods: We prospectively enrolled 329 patients, with first diagnosis of HCC from 1995 to 2001, in Brescia, Italy, Etiology was assessed by interviewing patients regarding their history of alcohol intake and by testing sera for hepatitis B surface antigen and anti-hepatitis $\mathrm{C}$ virus (HCV) antibodies and HCV RNA. Patients was considered to be diabetics in presence of hospital discharge DM code. Survival was determined from the date of HCC diagnosis to the end of follow-up, which was December 31, 2012. Cumulative survival curves were modeled by using the Kaplan-Meier method. The association of each variable with patient survival was tested by univariate analysis using the log-rank test. The same variables were tested by multivariate analysis using Cox proportional hazard models.

Results: Among 329 patients with HCC $271(82.4 \%)$ were males and $98(29.8 \%)$ had DM. Heavy alcohol intake ( $>40 \mathrm{~g}$ in men and $>20 \mathrm{~g}$ in women of ethanol per day for at least 1 decade) was found in $36.5 \%$ of cases, hepatitis virus infection in $15.5 \%$, alcohol and hepatitis virus infection in $41.9 \%$ and other factor in $6.1 \%$. Thirteen patients $(4.0 \%)$ were alive at the end of follow-up, with a median survival of 19.8 months (IC95 \% 17.1-22.5). Overall survival at 1, 5 and 10 years was $61.3,22.9$ and $7.6 \%$ respectively. On multivariate analysis, survival was associated with serum ALT $>100 \mathrm{U} / 1$ (hazard ratio $[\mathrm{HR}]=1.4, p=0.018)$, stage $\mathrm{pT}(\mathrm{HR}=1.2 ; p=0.023)$, portal vein invasion $(\mathrm{HR}=1.9 ; p<0.001)$, cirrhosis $(\mathrm{HR}=1.66$, $p=0.001)$, metastasis $(\mathrm{HR}=2.7, p=0.001)$, treatment (radical treatment vs. palliative treatments/no treatment $\mathrm{HR}=3.0, p<$ 0.001 ), Child classification (A vs. B HR $=1.38, p=0.032$; A vs. $\mathrm{C} \mathrm{HR}=1.82, p=0.003$ ). The presence of DM was negatively associated with survival (HR 1.3, $p=0.07$ ).
Conclusions: In this study a higher prevalence of DM was found in HCC patients as compared to italian general population in the same age group $(12.5 \%)$ and negative influence of DM on HCC survival was observed.

\section{P-090}

Socioeconomic inequalities in cancer survival for the most common cancer sites in the North Region of Portugal, 2000-2006

Topic: Diseases - Cancer

Presenter: Denisa Mendonça

Luis Antunes 1); Bernard Rachet 2), 3); Maria Jose Bento 1); Denisa Mendonça 4), 5)

1) Registo Oncológico Regional do Norte (RORENO), Instituto Português de Oncologia do Porto; 2) Cancer Survival Group, London School of Hygiene and Tropical Medicine (LSHTM); 3) Centre for Cancer Control and Statistics, Osaka Medical Centre for Cancer and Cardiovascular diseases (OMCC); 4) Instituto Ciências Biomédicas Abel Salazar (ICBAS); 5) Instituto Saúde Pública da Universidade do Porto (ISPUP)

Background: Socioeconomic inequalities in cancer survival have been consistently reported in different countries for most of the adult cancers. However, this has not yet been confirmed for Portuguese cancer patients.

Objectives: To describe the survival of patients diagnosed in the North Region of Portugal with one of the most common cancers (stomach, colorectum, lung, breast, bladder) during the period 2000-2006 and to study the influence of socioeconomic conditions in survival.

Materials and methods: Data consisted in cancer patients resident in the North Region of Portugal and registered by the corresponding population-based cancer registry (RORENO). All malignant, invasive, primary tumours of breast, colorectum, stomach, lung and bladder diagnosed among adult in 2000-2006, were considered for analysis. Socioeconomic conditions were assigned to each patient using ecological variables defined at parish level, namely, level of education, illiteracy, unemployment, index of rurality and index of accessibility to goods and services. The levels for each geographical area and each year of diagnosis were estimated based on the information of two different population census (2001 and 2011). Relative survival was calculated using Ederer II method. Excess hazards ratios (EHR) were estimated using a flexible modelling approach enabling to model the effects of sex, age and socio-economic condition.

Results: A total of 40,768 patients were diagnosed in the period of interest (breast-27\%; colorectal-29\%; stomach-19\%; lung-16\%; bladder-9 \%). Five-year relative survival was lower for lung $(10.5 \%)$ and stomach cancer $(33.8 \%)$ and higher for colorectal $(59.7 \%)$, bladder $(73.7 \%)$ and breast cancer $(87.0 \%)$. For stomach, lung and bladder cancer, women had a better survival than men (EHR adjusted for age: $0.81,0.79$ and 0.84 , respectively) while for colorectal, no differences were found EHR $=0.98$ ). Preliminary results have shown that stomach and bladder cancer patients coming from areas with a higher index of rurality or lower level of education have lower survival, while for colorectal, lung and breast cancers the survival rates are similar across socioeconomic levels.

Discussion: Socioeconomic inequalities in cancer survival were found more significant for bladder and stomach cancers. The median number of individuals by parish is relatively small (745) but some urban areas reach more than forty thousand inhabitants, what can lead to an underestimation of the socioeconomic gap in survival. The proportion of missing information on stage of disease at diagnosis was higher than fifty percent, precluding the inclusion of this variable in the analysis. 


\section{P-091}

Trends in incidence of ovarian cancer in the elderly (aged 65+ years) in England, 1971-2010

Topic: Diseases - Cancer

Presenter: Anjum Memon

\section{Pascale Avery 1); Anjum Memon 1)}

1) Brighton and Sussex Medical School, UK

Background: Ovarian cancer is the 5th most common cancer in females in the UK. Over the past four decades, incidence rates of ovarian cancer have increased by around $50 \%$. The highest rates are observed in the elderly (age 65+ years), with post-menopausal women accounting for over $80 \%$ of cases.

Objectives: To determine trends in incidence of ovarian cancer in the elderly in England during the period 1971-2010.

Methods: Population-based national cancer registration data were analyzed to determine the incidence of ovarian cancer (ICD-9:183; ICD-10:C56) by age (65-69, 70-74, 75-79, 80-84, and 85+) ethnicity, laterality, and morphological sub-type. Microsoft Excel and SPSS software were used for analysis.

Results: During the 40 year period, a total of 97,291 cases of ovarian cancer in the elderly were registered in England. Overall, the number of cases increased by $86 \%$-from 7,848 in 1971-1975 (1,570 cases/ year) to 14,628 in $2006-2010$ (2,926 cases/year). There was a $57 \%$ increase in incidence rate (from 39.8/100,000 in 1971-1975 to 62.5 in 2006-2010). The largest increase $(111 \%)$ was observed in women aged $85+$ years. Laterality was established in $45 \%$ of the cases $(16 \%$ right, $15 \%$ left, $14 \%$ bilateral). Adenocarcinoma was the most common morphological subtype $(20 \%)$. Proportion of women surviving $>5$ years following the diagnosis of ovarian cancer increased from 10.2 to $30.4 \%$.

Conclusions: During the past four decades, there has been a substantial increase in incidence of ovarian cancer among the elderly women in England. These findings are also relevant for the planning of oncology services, resource allocation, screening programmes for early diagnosis, and public health education for primary prevention.

\section{P-092}

Epidemological survey of thalassemia in Hyderabad, sindh during year 2012

Topic: Diseases - Others

Presenter: Faryal Ahmed

\section{Faryal Ahmed 1)}

1) Department of Microbiology, University of Sindh, Jmashoro.Pakistan

The thalassemia is a diverse group of genetic blood diseases. It is the most common inherited single gene disorder in the world. There are an estimated 60-80 million people in the world carrying the beta thalassemia trait alone. This is a very rough estimate; the actual number of thalassemia major patients is unknown due to the prevalence of thalassemia in less developed countries. Countries such as India and Pakistan are seeing a large increase of thalassemia patients due to lack of genetic counseling and screening. There is growing concern that thalassemia may become a very serious problem in the next 50 years, one that will burden the world's blood bank supplies and the health system in general. There are an estimated 1,000 people living with thalassemia major in the United States and an unknown number of carriers. In Pakistan Health experts claim that approximately 5,000 children are diagnosed with thalassemia-major, every year in Pakistan. Thalassemia is a major health concern in Pakistan and is the most prevalent genetically transmitted blood disorder, with a carrier rate of 5-8\%. One of the possible solutions to combat the health hazard is public awareness. Experts pointed out that media can help Pakistan reduce the preventable disease by carrier detection and avoiding in-family marriages. The survey was conducted by the BSIV students of the Department of Microbiology, University of Sindh, Jamshoro on the topic Epidemiological survey on thalassemia in Hyderabad Sindh during the year 2012. In order to achieve the survey different hospitals, blood banks and laboratories of Hyderabad were visited and a Questionnaire containing sufficient questions for the informative survey filled by the 100 patients of thalassemia and result shows that Girls are more affected with thalassemia.

\section{P-093}

The impact of liver disease on 30-day mortality after diverticular disease surgery: a nationwide follow-up study

Topic: Diseases - Others

Presenter: Jonathan Montomoli

Jonathan Montomoli 1); Rune Erichsen 1); Lisa Lynn Strate 2); Lars Pedersen 1); Tove Nilsson 1); Henrik Toft Sørensen 1)

1) Department of Clinical Epidemiology, Aarhus University Hospital, Denmark; 2) Division of Gastroenterology, Department of Medicine, Harborview Medical Center, University of Washington Medical School, Seattle, Washington, USA

Background: The prevalence of diverticular disease (DD) is increasing in Western countries with an increase in the number of surgical procedures performed for complicated DD. The coexistence of liver disease in patients undergoing DD surgery may pose a serious challenge, since liver diseases are becoming more prevalent too. However, knowledge on postoperative mortality after DD surgery in patients with liver disease is limited.

Objectives: To investigate postoperative mortality risk and frequency of reoperation after colorectal surgery because of DD in patients with liver disease compared to those without liver disease.

Methods: Using individual-level linkage of Danish medical databases, we conducted a nationwide cohort study of all patients undergoing colorectal surgery for DD in Denmark from 1977 through 2011. We further identified patients diagnosed with any liver disease before or at the time of DD surgery and categorized patients into three cohorts: patients with non-cirrhotic liver disease, those with liver cirrhosis, and patients with no history of liver disease (comparison cohort). Using the Kaplan-Meier method, we computed 30-day postoperative mortality in each of these cohorts overall and stratified by elective and nonelective surgery. We used a Cox regression model to compute hazard ratios as measures of the relative risk (RR) of death controlling for potential confounders including other comorbidities. In addition, we assessed the frequency of reoperation within 30 days and we identified main causes of death in each cohort.

Results: A total of 14,408 patients underwent DD surgery: 233 $(1.6 \%)$ had non-cirrhotic liver disease and $91(0.6 \%)$ had liver cirrhosis. Thirty-day mortality was $9.9 \%$ (elective surgery $2.3 \%$ vs. nonelective surgery $15 \%$ ) in patients without liver disease and $15 \%$ (elective $2.3 \%$ vs. nonelective $22 \%$ ) in patients with non-cirrhotic liver disease corresponding to an adjusted RR of 1.64 (95\% confidence interval (CI): 1.16-2.31). Among patients with liver cirrhosis, mortality was $24 \%$ (elective $3.5 \%$ vs. nonelective $34 \%$ ), corresponding to an adjusted RR of 2.70 (95\% CI 1.73-4.22). Frequency of reoperation was approximately $10 \%$ in each cohort. Peritonitis was responsible for one third of death among both patients with and without liver disease. 
Conclusion: Pre-existing liver cirrhosis was associated with markedly increased 30-day mortality following DD surgery.

\section{P-094}

Pressure ulcer prevention: performance of the Braden scale in ICU and NCU

Topic: Diseases - Others

Presenter: Thomas Petzold

Thomas Petzold 1); Maria Eberlein-Gonska 2); Jochen Schmitt 1)

1) Center for Evidence-based Healthcare; 2) Department of Quality and medical Risk Management

Background: Pressure ulcer prevention is a relevant public health goal due to the substantial clinical and economic burden of pressure ulcers. Therefore, the Braden Scale is a frequently applied scale for measuring the risk to develop a pressure ulcer.

Objectives: Evaluation of the pressure ulcer documentation in the university hospital Carl Gustav Carus Dresden, Germany (UKD). Comparison of the performance of the Braden Scale in intensive care units (ICU) and normal care units (NCU).

Methods: Longitudinal study including all inpatients treated at the UKD between 2007 and $2011(\mathrm{n}=246,162 ; 48.4 \%$ female and $41.6 \%$ male; mean age 49.6 years). Documentation of pressure ulcer risk by means of the Braden scale and clinical signs of pressure ulcers at admission, weekly follow-up examinations, and at discharge by trained staff. Primary outcome was incident pressure ulcer during inpatient treatment. ROC curve analysis was applied to evaluate the performance of the Braden Scale in NCU versus ICU. The area under curve (AUC) is equal to the probability that a classifier will rank a randomly chosen positive instance higher than a randomly chosen negative one.

Results: The overall incidence of pressure ulcers during inpatient treatment was $0.78 \%$. As expected, a higher rate of pressure ulcers was observed at ICU versus NCU (4.77 vs. $0.59 \%)$. At the proposed cutoff of 19, the sensitivity, specificity, positive predictive value (PPV) and negative predictive value (NPV) of the Braden scale at NCU were 44.57, 89.95, 3.14 and $99.69 \%$. In contrast, at ICU, sensitivity, specificity, PPV, and NPV of the Braden scale were 96.61, 28.46, 5.86 and $99.24 \%$.

The area under the ROC curve as an indicator for the overall performance of the Braden Scale was $84.89 \%$ at NCU versus $69.00 \%$ at ICU.

Conclusions: This validation study based on data of a very large prospective study indicates that the Braden score is an appropriate instrument to determine the risk for incident pressure ulcer in normal care units, whereas its performance in intensive care units is not as good. Therefore, alternative instruments need to be developed to determine the risk for incident pressure ulcers in the ICU setting.

\section{P-095}

Sagittal standing posture and quality of life among adults from the general population: a sex-specific association

Topic: Diseases - Others

Presenter: Fábio Miguel Azevedo Araújo

Fábio Miguel Azevedo Araújo 1), 2); Raquel Lucas 1), 2); Nuno Alegrete 3), 4); Ana Azevedo 1), 2); Henrique Barros 1), 2)

1) Department of Clinical Epidemiology, Predictive Medicine and Public Health, University of Porto Medical School, Porto, Portugal;
2) Institute of Public Health - University of Porto (ISPUP), Porto, Portugal; 3) Centro Hospitalar São João, Porto, Portugal; 4) Department of Surgery, University of Porto Medical School, Porto, Portugal

Background: Clinical studies have shown the association of sagittal standing posture with pain and reduced quality of life, but this relation has not been assessed in the general adult population.

Objectives: To analyze the relation of sagittal standing posture with back pain severity and health-related quality of life among men and women from the general population.

Methods: As part of the EPIPorto population-based study of adults, 178 men and 311 women were evaluated during 2005-2008. Age, education and body mass index were recorded. Radiographic data collection consisted of 36-inch sagittal radiographs obtained in freestanding posture. Sex-specific thirds of individual spino-pelvic parameters were computed and 1 of 4 sagittal types of postural patterns attributed to each participant (Roussouly classification). Back pain prevalence and severity were assessed based on self-reported data and health-related quality of life using 2 main components of the Short Form 36 (SF-36).

Results: In men, only pelvic-tilt/pelvic-incidence ratio was significantly associated with back pain severity. Women in the 1st and especially in the 3rd third of pelvic incidence and sacral slope presented higher prevalence of and more severe back pain. In women, sagittal vertical axis, pelvic tilt and pelvic-tilt/pelvic-incidence ratio were directly associated with back pain severity, also showing an inverse dose-response relation with the physical component of SF-36 (highest z-score mean difference of 8.8 between the 1st and 3rd thirds of sagittal vertical axis; $p<0.001$ ).

Conclusions: Sagittal standing posture was not consistently associated with pain and quality of life among men. Pelvic incidence and sacral slope outside neutral ranges, increased sagittal balance, pelvic tilt and pelvic retroversion may be involved in causing severe back pain and consequently decreased quality of life among women.

\section{P-096}

Association between musculoskeletal symptoms and work accidents in Botucatu, Brazil

Topic: Diseases - Others

Presenter: Adriano Dias

\section{Maila Brito 1); Adriano Dias 1)}

1) Post-grade Program in Publich Health, Botucatu Medical Schoold, UNESP

Introduction: Work accidents (WA) are "those that occur by carrying out the work, causing injury or functional disorder that lead to death, loss or reduction, permanent or temporary on work capacity". They are one of the most important public health problems and responsible for the high demand of complaints for medical care in urgency and emergency services (UES). Inadequacies on ergonomic work environment can lead to discomforts in workers, like pain or fatigue, and are associated with the occurrence of WA.

Objective: To investigate the relationship between musculoskeletal symptoms and the occurrence of WA in Botucatu/SP, Brazil.

Methods: Case-control study. It was used as protocol the Nordic Questionnaire, that assesses musculoskeletal symptoms in neck, shoulder, upper back, elbow, hand/wrist, lower back, hips/thighs, knees, ankles/feet, by four questions (yes or no): 1st) you had problems (pain, tingling) in some part of the body in the last 12 months; 2nd) you were unable to perform activities (work, leisure) in the last 12 months; 3 rd) you had an appointment with a health professional in the past 12 months; 4th) you have had problems in the last 7 days 
before the accident. Conducted with 82 patients (41 CA/41 CO) who were assessed at Botucatu Medical School UES. They were evaluated by sex, age group (16-60 years), presence or absence of pain.

Results: Were observed that women suffer WA less than men $(p=0.007)$. The average age of the cases was $34.85(17-56)$ and 36.9 of controls (16-57). Regarding the 1st question, no significant difference between cases and controls. For the 2nd, it was observed a significant difference in the prevalence of problems in the wrist/hands between cases and controls $(p=0.03)$. This implies a lower incidence of WA, preventable by absence from work. For the other evaluated body parts and in the 3rd question there was no statistical significant difference between cases and controls. For the 4th question, it was observed that patients with problems on the upper back $(p=0.029)$ and hip/thigh $(p=0.017)$ were more prone to WA. For the other parts of the body examined there was no statistical significant difference.

Conclusion: Despite the frequent complaint of pain, the demand for health services remains low, even though this attitude can be an important contributory factor to reducing the accident rates in workers. Thus, it is necessary awareness of employers and workers in the proper valuation of musculoskeletal symptoms, since their importance in reducing the productive capacity of the worker and economic and social costs entailed by WA.

\section{P-097}

Dose response for predicted FEV1: a preliminary meta-analysis

Topic: Diseases - Others

Presenter: Frank van Boven

\section{Frank van Boven 1)}

1) on personal title

Background: Allergic asthma is the most common childhood disease in western countries. Avoidance of airborne allergens should be included for management of asthma. However, the effectiveness of mite allergen control is debatable and dominated by a meta-analysis (Gøtzsche, P.C. and H.K. Johansen. Cochrane Database of Systematic Reviews 2008, Issue 2. No. CD1187).

Objectives: We revisited this meta-analysis and studied the relationship between mite allergen load from mattresses and predicted forced expiratory volume in one second (FEV1) percentages at baseline.

Methods: We started with a subgroup of 26 trials reporting on bedding control from the existing meta-analysis. Data was extracted on mite allergen load and predicted FEV1 percentages at baseline, type of patient, use of steroids and the judgement on allocation by Gøtzsche. Treatment and control groups were combined. A recent trial on bedding control (El-Ghitany, Environ Health Prev Med, 2012) and a trial on ventilation were also found positive for data extraction. Data on mite load were converted to the natural logarithmic scale. The data on FEV1 were deducted with a constant of 10 to create proportions. Because of the explanatory variables, the dose response for FEV1 was examined with a mixed effect model with the package Metafor in $\mathrm{R}$ (version 2.15.2).

Results: Eleven trials included 813 patients, of which 271 were children (4 trials). In 8 trials patients used steroids. The risk of bias judged about allocation concealment by Gøtzsche included 2 trials at high risk, 7 of unclear risk and 2 at low risk. The proportion of FEV1 was only related to the logarithmic mite allergen load $(b=-0.031$; se $=0.015 ; p=0.036$ ). Other factors resulted negative for reduced omnibus tests.

Conclusions: A meta-regression regarding the dose response between mite allergen load from mattresses and predicted FEV1 indicates that
FEV1 is slightly influenced by the mite allergen load. The point estimator for every one degree reduction of the logarithmic load results in an increase of 3 percent in the predicted FEV1.

\section{P-098}

Dietary N-6 polyunsaturated fat, carbohydrate-to-protein ratio, and change in body weight and waist circumference: a follow-up study

Topic: Diseases - Others

Presenter: Marianne U Jakobsen

Marianne U Jakobsen 1); Lise Madsen 2); Claus Dethlefsen 3); Karen M Due 3); Jytte Halkjær 4); Thorkild IA Sørensen 5); Kristiansen K 2); Overvad K 1)

1) Department of Public Health, Section for Epidemiology, Aarhus University, Aarhus, Denmark; 2) Department of Biology, University of Copenhagen, Copenhagen, Denmark; 3) Department of Cardiology, Center for Cardiovascular Research, Aalborg Hospital, Aarhus University Hospital, Aalborg, Denmark; 4) The Danish Cancer Society Research Center, Copenhagen, Denmark; 5) Institute of Preventive Medicine, Bispebjerg and Frederiksberg University Hospitals, Copenhagen, the Capital Region, Denmark

Background: The balance between carbohydrate and protein in the diet may determine the effect of intake of $n-6$ polyunsaturated fatty acids (PUFA) on body fat mass; n-6 PUFA may decrease fat mass at a low carbohydrate-to-protein ratio, but increase fat mass at a high ratio.

Objectives: We investigated the association between the intake of n- 6 PUFA and subsequent change in body weight and waist circumference at different levels of the carbohydrate-to-protein ratio.

Methods: Women and men $(\mathrm{n}=29,152)$ were followed for a median of 5.3 years. We applied linear regression models with a 5 -y change in weight or waist circumference as the outcome, including a two-way interaction term between n-6 PUFA and carbohydrate intakes, lowerorder terms, protein intake, long-chain n-3 PUFA intake, and other potential confounders. Due to adjustment for intake of protein, levels of carbohydrate may indirectly reflect levels of the carbohydrate-toprotein ratio.

Results: For high intake of n-6 PUFA (6.9 \% energy) versus low intake of n-6 PUFA (3.4\% energy), the difference in 5-year weight change was $-189.7 \mathrm{~g}(95 \%$ confidence interval (CI): $-636.8,257.4)$ at a low carbohydrate-to-protein ratio and $-86.7 \mathrm{~g}(95 \% \mathrm{CI}-502.9$, 329.6) at a high carbohydrate-to-protein ratio; the differences in 5 -year waist circumference change were $0.26 \mathrm{~cm}(95 \% \mathrm{CI}-0.47$, $0.98)$ and $-0.52 \mathrm{~cm}(95 \% \mathrm{CI}-1.19,0.15)$, respectively. Inclusion of the dietary glycemic index did not change the results.

Conclusions: No consistent associations between the intake of n-6 PUFA and change in body weight or waist circumference at different levels of the carbohydrate-to-protein ratio were observed.

\section{P-099}

Risks of major congenital anomalies in children born to women with inflammatory bowel disease: a United Kingdom populationbased cohort study

Topic: Diseases - Others

Presenter: Lu Ban

Lu Ban 1); Laila Jal Tata 1); Card Tim 1)

1) University of Nottingham 
Background: Inflammatory bowel disease (IBD) affects women at their most fertile period of age. However, previous studies of pregnant women with IBD on the risk of major congenital anomalies have inconsistent results due to diverse study population and small sample size.

Objectives: To estimate the risk of any major congenital anomaly and system-specific anomalies in children born to women with IBD compared with children born to women without IBD in the United Kingdom population.

Methods: We identified all singleton live births to women aged 15-45 between 1990 and 2010 from a large UK primary care database. We grouped children according to whether their mothers had IBD before childbirth or not and whether if so this was Crohn's disease (CD) or ulcerative colitis (UC). For children born to women with IBD, we also extracted records of prescriptions of 5-aminosalicylic acid, steroids and azathioprine in the first trimester of pregnancy. We calculated absolute risks of any major congenital anomaly and system-specific anomalies, and used logistic regression with a generalised estimating equation to compare risks. In women with IBD, we repeated the analyses to estimate the risks in children exposed or not exposed to medication. We adjusted the results for maternal age, year of childbirth, socioeconomic deprivation and maternal smoking.

Results: Of 1,703 children born to women with IBD and 384,811 children born to women without IBD, 2.7 and $2.8 \%$ had records of any major congenital anomaly respectively. The risks of major congenital anomaly for $\mathrm{CD}$ and UC were 3.7 and $1.9 \%$ respectively. The adjusted odds ratio (AOR) of IBD with any major congenital anomaly was 0.98 (95\% confidence interval [95\% CI] 0.73-1.31). In children of women with IBD, $32.4 \%$ were exposed to 5-aminosalicylic acid in the first trimester and 12.3 and $8.7 \%$ to steroids and azathioprine respectively. There was no statistically significant increase in the risk of major congenital anomaly in children exposed to 5-aminosalicylic acid (AOR $=0.82,95 \%$ CI 0.42-1.61), steroids (AOR $=0.48,95 \%$ CI $0.15-1.50$ ) or azathioprine (AOR $=1.27,95 \%$ CI $0.48-3.39$ ) in the first trimester compared with those unexposed. For system-specific anomalies, no increased risks in heart, limb or genital system were found.

Conclusions: There are similar risks of major congenital anomalies in children born to women with and without IBD. No evidence of potential teratogenic effects of 5-aminosalicylic acid, steroids or azathioprine was found in this study. Previous guidance that women may be advised to continue these medications remains appropriate.

\section{P-100}

Self-reported health after stroke: a follow-up study with multiple measurements over a 2 years period

Topic: Diseases - Others

Presenter: Louise Pape

Louise Pape 1); Karin Biering 2); Søren Paaske Johnsen 3); Grethe Andersen 4); Niels Henrik Hjøllund 5)

1) Department of health Science, University of Aarhus, Denmark; VestKronik, Regional Hospital Herning; Denmark; 2) Department of Occupational Medicine, Regional Hospital Herning; Denmark; 3) Department of Clinical Epidemiology, Aarhus University Hospital, Aarhus N, Denmark; 4) Department of Neurology, Aarhus University Hospital, Aarhus C, Denmark; 5) VestKronik, Regional Hospital Herning; Denmark; Department of Clinical Epidemiology, Aarhus University Hospital, Aarhus N Denmark

Background: Self-rated health is an important indicator of quality of life as well as a good predictor of future health.
Aim: The aim of the study was to describe the course of self-rated mental and physical health in a population of stroke patients and analyse whether gender, age, education level, diagnose, stroke severity and comorbidity at time of admission affected the health course.

Methods: A cohort of patients admitted with first time stroke in Region Central Denmark $(n=2,090)$ was followed over a 2 year period with 5 repetitive questionnaires.

The course of self-rated health was described using SF-12 Physical Component Summary (PCS) and Mental Component Summary (MCS).

Data was analysed in regression models with the use of multiple imputation.

Results: MCS increased slightly throughout the observation period $(41.69 ; 42.17 ; 42.05 ; 42.0 ; 41.99)$ while PCS did not change over time $(45.75 ; 46.85 ; 47.06 ; 47.25 ; 47.10)$.

Neither the course of MCS nor PCS was affected by any of the investigated covariates.

The level of PCS strongly depended on stroke severity and comorbidity and to a minor degree also on gender, age and educational level, while the level of MCS only depended minor on gender and age.

Conclusion: In a population-based cohort of stroke patients with multiple measurements we found minor improvements in mental, but not in physical health over a 2 year period.

\section{P-101}

Risk of acute liver failure leading to transplantation after NSAID or paracetamol exposure: study of acute liver transplant (SALT)

Topic: Diseases - Others

Presenter: Ezgi Gulmez

\section{Ezgi Gulmez 1)}

1) Pharmacology, Université Bordeaux Segalen, INSERM CICP0005, Bordeaux, France; 2) France; 3) United Kingdom; 4) Netherlands; 5) Ireland; 6) Italy; 7) Greece; 8) Portugal; 9) Belgium; 10) Spain

Background: Most drugs are common causes of ALF leading to liver transplantation (ALFT). NSAIDs and paracetamol are often involved. Objectives: To estimate population event rates of ALFT in patients without identified clinical etiology (ICE) and exposed to NSAIDs and paracetamol.

Methods: Multicentre, case-population study performed in France, Greece, Ireland, Italy, Netherlands, Portugal, and UK between 2005 and 2007 in adults. Patients were identified through national/local LT registries. Demographic, clinical, and drug use data were collected for ALFT cases. Exposure to NSAID or paracetamol was determined within 30 days prior to initial symptoms of liver disease (index date, ID). Rate per million treatment-years (tt-yrs) was calculated using sales data from IMS.

Results: Of the 62 LT centers, 57 were eligible, and 52 contributed data. Among the 9,479 patients identified from LT lists for the 3-year period, $600(6 \%)$ were ALF: 219 (36\% of all-cause ALF) had an ICE, 18 ( $3 \%$ of all-cause ALF) had unavailable medical files, 62 (11\%) were not drug-exposed and had no ICE, and $301(52 \%)$ were drug-exposed without ICE. Among the latter, 40 (7\% of analyzable ALF) were exposed to NSAIDs, mean age 43.9 years, 29 (72\%) female. Event rates per million tt-yrs were 1.59 (95 \% CI 1.14-2.17) for all NSAIDs pooled, $2.28(1.21-3.90)$ for ibuprofen, 1.88 (0.81-3.70) for nimesulide, $1.55(0.57-3.38)$ for diclofenac, 1.55 $(0.32-4.52)$ for ketoprofen, and $1.63(0.20-5.89)$ for naproxen. Event rate for non-overdose paracetamol was 3.31 (2.63-4.11), and 7.84 
(6.77-9.04) when intentional or non-intentional overdoses were included. Event rates for all NSAID pooled were almost four $(95 \%$ CI 1.2-12.5) times higher in Ireland than all countries pooled.

Conclusions: The risk of ALFT was rare and showed no differences among the most used NSAIDs. Non-overdose paracetamol-exposed ALFT was twice more common than NSAID-exposed ALF.

\section{P-102}

Causality of drugs involved in acute liver failure leading to transplantation: Results from the Study of Acute Liver Transplant) (SALT)

Topic: Diseases - Others

Presenter: Ezgi Gulmez,

\section{Ezgi Gulmez 1)}

1) Pharmacology, Université Bordeaux Segalen, INSERM CICP0005, Bordeaux, France; 2) United Kingdom; 3) Belgium; 4) Netherlands; 5) Italy

Background: To assess causality of all drug-exposed cases of acute liver failure leading to transplantation (ALFT) in SALT study.

Methods: SALT is a multi-centre, retrospective, case-population study in France, Greece, Ireland, Italy, Netherlands, Portugal, and the UK over 3-year period (2005-2007) in NSAID-exposed adult cases of ALFT within 30 days prior to onset of liver disease (index date). All drug-exposed ALFT were assessed for causality by the Case Adjudication Committee (CAC) experts, in accordance with the consensus results of the Salome pilot study; having full data on drugs including INN and doses except for NSAIDs (kept blinded as "NSAID"), and using WHO causality scale.

Results: Fifty-two liver transplant centres contributed data. Among the 9,479 patients identified, $600(6.3 \%)$ were cases of ALFT. Among the drug-exposed without identified clinical cause ALFT cases $(\mathrm{n}=301,51.7 \%), 114(19.6 \%)$ were acute drug overdose. Among the 301 ALFT cases without identified clinical cause, 40 $(6.9 \%)$ were NSAID-exposed ALFT cases. Thirty-one cases with a total of 33 NSAID exposures had causality scores possible $(n=32)$ or probable $(n=1)$. Sixteen of the 31 NSAID-related cases were also exposed to paracetamol, 13 of which had paracetamol causality scores possible, probable or highly probable. Paracetamol was attributed to $111(97.3 \%)$ of 114 acute drug overdose ALFT cases. There were 18 ALFT cases for which information was not sufficient to determine whether or not they had been exposed to drugs and therefore were not considered by the CAC.

Conclusions: In drug-exposed ALFT cases without other clinical causes, the main differentiating characteristic for drugs used was the previous knowledge of possible hepatotoxicity, which is not helpful when one is trying to discover new signals. Purely epidemiological approaches, such as the case-population approach used in the SALT study, seem to give more relevant results than a case-based approach using individual causality assessment of cases.

\section{P-103}

Drug-associated acute liver failure leading to registration for transplantation in France from the study of acute liver transplant (SALT)

Topic: Diseases - Others

Presenter: Ezgi Gulmez

\section{Ezgi Gulmez 1)}

1) Pharmacology, Université Bordeaux Segalen, INSERM CICP0005, Bordeaux, France
Background: Drug-associated acute liver injury is a common concern in drug safety, especially acute liver failure leading to registration for transplantation (ALFT).

Objectives: SALT was designed to explore drug-associated ALFT. Methods: French ALFT cases exposed to drugs within 30 days of first symptoms were compared to drug utilisation data from the 1/97 sample of the French National Healthcare database (EGB). Event rates were computed per billion DDD dispensed over the period and per million users, compared with the average number of DDD dispensed per user over 3 years. Chronic liver disease, documented clinical causes and drug overdoses were excluded.

Results: The 65 cases of ALFT identified in France (2005-2007) had been exposed to 235 different drugs. The drug classes most found were paracetamol (47 cases), anxiolytics $(\mathrm{n}=13)$, antiepileptic drugs $(\mathrm{n}=11)$, NSAIDs $(\mathrm{n}=10)$, H1 antihistamines $(\mathrm{n}=8)$, proton pump inhibitors $(n=7)$, and antidepressants $(n=6)$. Other classes were associated with 5 or fewer cases. Rates ranged from 1.9 (bromazepam) to 372 cases per billion DDD (prazepam). Per user rates ranged from 0.19 (pantoprazole) to 56 per million (phenytoin). For NSAIDs, PPI, and some $\mathrm{H} 1$ antagonists, event rates decreased with increasing average number of DDD dispensed. In these classes the event rate per user was below 1 per million users. For other drug classes such as antiepileptic drugs, the event rates per billion DDD were similar, and rates per million users increased with increasing average number of DDD dispensed per subject. Drugs fell into three main categories: event rates below one per million users (NSAIDs, PPI, most antihistamines, some benzodiazepines), from 1 to 10 per million users (paracetamol, benzodiazepines, antiepileptic drugs), and above 10 per million users. Antidepressants were around one case per million users. Two antiepileptic drugs had event rates at or above 10 per million users. Overall, drugs with longer duration of use tended to have higher per user event rates.

Conclusion: These results are still tentative because of the small number of cases for individual drugs. SALT should be extended.

\section{P-104}

Leisure-time physical activity over the life course and cognitive functioning in late mid-adult years: a cohort-based investigation

Topic: Diseases - Others

Presenter: Alex Dregan

\section{Alex Dregan 1); Martin Gulliford 1)}

1) Kings College London, NIHR Biomedical Research Centre based at Guy's and St Thomas'; NHS Foundation Trust and King's College London

Background: The objective of the present study was to estimate the association between different leisure-time physical activity (LTPA) parameters from 11 to 50 years and cognitive functioning in late midadulthood.

Method: The study used a prospective birth cohort study including participants in the UK National Child Development Study (NCDS) from age 11 to 50 years. Standardized z scores for cognitive, memory and executive functioning at age 50 represented the primary outcome measures. Exposures included self-reported LTPA at ages 11, 16, 33, 42, 46 and 50 years. Analyses were adjusted for important confounders including educational attainment and long-standing illness. Results: The adjusted difference in cognition score between women who reported LTPA for at least 4 days/week in five surveys or more and those who never reported LTPA for at least 4 days/week was 0.28 [95\% confidence interval (CI) 0.20-0.35], 0.10 (95\% CI 0.01-0.19) for memory score and 0.30 (95\% CI $0.23-0.38)$ for executive functioning score. For men, the equivalent differences were: 
cognition 0.12 (95\% CI $0.05-0.18)$, memory $0.06 \quad(95 \%$ CI $-0.02-0.14$ ) and executive functioning 0.16 (95\% CI 0.10-0.23).

Conclusions: This study provides novel evidence about the lifelong association between LTPA and memory and executive functioning in mid-adult years. Participation in low-frequency and low-intensity LTPA was positively associated with cognitive functioning in late mid-adult years for men and women. The greatest benefit emerged from participating in lifelong intensive LTPA.

\section{P-105}

Risk factors for hip fracture and mortality risk of hip fracture: a Danish nationwide cohort study 1996-2009

Topic: Diseases - Others

Presenter: Terese Sara Hфj Jфrgensen

Terese Sara Høj Jørgensen 1); Annette Højmann 2); Gunnar Gislason 3); Christian Torp-Pedersen 4); Ellen Holm 1)

1) Department of geriatric medicine, Nykøbing-Falster Hospital, Denmark; 2) Department of geriatric medicine, Slagelse Hospital, Denmark; 3) Department of Cardiology, Gentofte Hospital, Denmark; 4) Institute of Health, Science and Technology, Aalborg University, Denmark

Background: Comorbidity increases mortality among patients with hip fracture; however it remains unclear whether the risk of hip fracture increases with common chronic diseases. Furthermore, newer research on the relationship between hip fracture and all-cause mortality is needed. Our objectives are therefore to investigate (1) the relationships between chronic obstructive pulmonary disease, dementia, depression, diabetes, heart failure, ischaemic heart disease, osteoporosis, Parkinson disease, stroke, and hip fracture and (2) whether hip fracture increases all-cause mortality.

Methods: In the present study, data from the Danish National Patient Registry and the National Population Registry are used to identify individuals aged 52 years or above and information on gender, age, household size, mortality, admissions with hip fracture and occurrence of the above mentioned chronic conditions.

A multivariable Poisson regression model was applied to estimate incidence rate ratio (IRR) for hip fracture adjusted for age, gender, household size, calendar year and comorbidity. A multiple logistic regression model was applied to identify the odds ratio (OR) for allcause mortality among individuals with hip fracture adjusted for age, gender, household size and comorbidity.

Results: The study cohort included 1,369,348 individuals aged 52 years or above (44.6\% males, mean age 65.6 [SD 9.6] years). During the period 1996-2009 9.5\% $(130,336)$ had chronic obstructive pulmonary disease, $5.2 \%(70,741)$ had dementia, $2.5 \%(40,880)$ had depression, $8.9 \%(121,660)$ had diabetes, $10.2 \%(139,117)$ had heart failure, $7.2 \%(98,065)$ had ischaemic heart disease, $6.3 \%(85,723)$ had osteoporosis, $1.2 \%(15,952)$ had Parkinson disease and $12.8 \%$ $(174,667)$ had stroke. 153,384 patients $(11.2 \%)$ had a hip fracture and 579,592 individuals $(42.3 \%$ ) died during the follow-up period.

The adjusted Poisson regression analysis revealed increased risk of hip fracture among patients with chronic obstructive pulmonary disease $(\mathrm{IRR}=1.03$, confidence interval $(\mathrm{CI})$ : 1.01-1.05), dementia $($ IRR $=1.12$, CI 1.10-1.14), depression $($ IRR $=1.12$, CI 1.10-1.15), osteoporosis (IRR $=1.14$, CI 1.12-1.16) and Parkinson disease (IRR $=1.74$, CI 1.67-1.80), whereas the risk decreased with ischaemic heart disease (IRR $=0.65$, CI 0.64-0.67), heart failure $(\mathrm{IRR}=0.72$, CI $0.70-0.73)$, and stroke (IRR $=0.82$, CI 0.80-0.83). No association was found for diabetes (IRR $=1.00$, CI $0.98-1.02$ ). Finally, when adjusted for potential confounders, all-cause mortality increased considerably with hip fracture $(\mathrm{OR}=2.24$, CI 2.21-2.27).
Conclusions: The study provides evidence that (1) chronic obstructive pulmonary disease, dementia, depression, osteoporosis and Parkinson disease are significant risk factors for hip fracture and that (2) hip fracture is associated with an increased odds ratio of all-cause mortality when adjusted for comorbidity.

\section{P-106}

Associations between high summer temperatures and cerebrovascular mortality in Astana, Kazakhstan: a timeseries analysis

Topic: Diseases - Others

Presenter: Andrej Grjibovski

Andrej Grjibovski 1), 2); Nassikhat Nurgaliyeva 3); Gulmira Kozhakhmetova 3), 4); Bibigul Adilbekova 3); Aliya Kosbayeva 4); Bettina Menne 5); Telman Seysembekov 3); Altay Sharbakov 3)

1) Department of International Public Health, Norwegian Institute of Public Health, Oslo, Norway; 2) International School of Public Health, Northern State Medical University, Arkhangelsk, Russia; 3) Medical University of Astana, Astana, Kazakhstan; 4) WHO office in Kazakhstan, Astana, Kazakhstan; 5) WHO European Centre for Environment and Health, Bonn Office, Bonn, Germany

Background: Associations between high temperatures and cerebrovascular mortality have been observed in many European settings. However, the evidence from rapidly developing settings particularly with arid and semiarid climate is almost non-existent.

Objectives: To study associations between maximum apparent air temperature and daily counts of deaths from cerebrovascular causes in Astana-a rapidly developing capital of Kazakhstan.

Methods: Daily counts of deaths cerebrovascular diseases (I60-I69) among adults 18 years and older in Astana, Kazakhstan during warm periods (1 April-30 September) in 2000-2001 and 2006-2010, were obtained from the City Registry Office. Associations between cerebrovascular mortality and maximum apparent temperature (average for lags 0-3) during the warm period were studied using first order autoregressive Poisson regression models controlling for wind speed and effects of month, year, weekends and holidays. Analyses were stratified by gender.

Results: Daily counts of deaths from cerebrovascular diseases were significantly associated with the maximum apparent air temperature among women: an increase in temperature by $1{ }^{\circ} \mathrm{C}$ was associated with an increase in the daily number of deaths by $1.9 \%(95 \% \mathrm{CI}$ 0.4-3.5). No significant associations were observed among men. Conclusions: The effects of temperature on cerebrovascular mortality in Astana, Kazakhstan, was limited to women. Higher temperatures were associated with higher mortality from cerebrovascular diseases. Factors behind this association warrant further research with a further going aim to develop strategies to mitigate the effects on climate change on population health.

\section{P-107}

Prevalence of dermatological disorders among patients in an urban area in Namibia

Topic: Diseases - Others

Presenter: Martie S Lubbe

Ronja King 1); Martie S Lubbe 1); Jan J Gerber 1)

1) Medicine Usage in South Africa 
Background: The burden of skin diseases has been increasingly emphasised over the past few years. Dermatological disorders and diseases are common in developing countries due to poor hygiene, underlying causes such as HIV/AIDS, overcrowding in some areas and poverty. Skin diseases such as scabies, superficial mycoses, pyoderma, pediculosis, eczema, dermatitis, pigmentary anomalies, acne and HIV/AIDS related skin disease are most common in developing countries.

Objectives: To establish the prevalence of skin diseases among patients in an urban area in Namibia.

Methods: A cross-sectional study was done on 302 dermatological prescriptions obtained over a 3 month period, from two community pharmacies, located in the Northern and Southern parts of Windhoek in Namibia. These pharmacies were strategically chosen to ensure a broad spectrum of prescriptions from various ethnic groups. Only general practitioners' prescriptions were included, Prescriptions from dermatologists were excluded. Written consent to participate in the study was obtained from every second patient with a dermatological prescription. The following prescription details were recorded: Patient gender, age, race, the diagnosis as obtained from the patient or general practitioner (telephonically); medical treatment and treatment duration, smoking, pregnancy, HIV status and allergy information. Association between dermatological disorders and gender, age group, race, and HIV-status were investigated using the Chi square test. $P$-values less than 0.05 was considered statistical significant.

Results: Approximately $57.3 \%$ of patients with dermatological conditions were black, $50.7 \%$ were male, and $72.2 \%$ were in the age group older than 12 and younger than 60 years. Urticaria was the most common skin disease identified, affecting $11.9 \%$ of all patients. Other conditions included contact dermatitis (9.3\%), eczema $(9.3 \%)$, acne vulgaris $(8.3 \%)$, Herpes Simplex $(7.3 \%)$, cellulitis $(6.3 \%)$ and folliculitis $(6.3 \%)$. Patients received on average 1.69 [95\% CI 1.59-1.77] dermatological items per prescription, with $49 \%$ of patients receiving only one. The most common dermatological items prescribed were corticosteroids $(25.5 \%)$, antibiotics $(12.4 \%)$, and fungicides $(11.6 \%)$. Statistical significant association were found between the type of dermatological disorder and/gender $(p=0.004)$, age group $(p<0.0001)$ and HIV-status $(p=0.0002)$. 51 patients $(16.9 \%)$ were HIV-positive and experienced mostly problems with urticaria, melasma, and warts

Conclusions: The results reveal the prevalence of dermatological disorders and the treatment thereof in the private health system of Namibia. It will make health professionals in Namibia more aware of the significance of dermatological diseases in their region.

\section{P-109}

The influence of early growth and socio-economic status on hearing at age 61-63 years: Newcastle thousand families study

Topic: Diseases - Others

Presenter: Mark Pearce

Mark Pearce 1); Kay Mann 1); Fiona Pearson 1); Adrian Davis 2); Adrian Rees 3)

1) Institute of Health \& Society, Newcastle University, UK; 2) The Ear Institute, University College London, UK; 3) Institute of Neuroscience, Newcastle University, UK

Background: Almost three million people are affected by hearing loss worldwide. In recent years there have been reports that early growth may influence later hearing status, although it may be that the effects of the early socio-economic environment may be more important.
Objectives: This study investigated whether associations exist between factors in very early life, such as gestation, birth weight, housing conditions and socio-economic status and hearing function at age 61-63 years in the Newcastle Thousand Families Study, after adjusting for potential confounders, including exposure to workplace dust and noise.

Methods: The Newcastle Thousand Families study is a birth cohort of all 1,142 children born in May and June 1947 to mothers resident in Newcastle upon Tyne, UK. The health, growth and development of the cohort were followed in great detail through childhood, with further detailed follow-ups at various points in adulthood. During the most recent follow-up, study members underwent audiological testing as part of a thorough clinical assessment. A trained research nurse measured air conduction audiograms bilaterally with measurements taken at seven frequencies between $250 \mathrm{~Hz}$ and $8 \mathrm{kHz}$ in octave steps and at 3 and $6 \mathrm{kHz}$ following British Society of Audiology guidelines. Results: Complete data were available for 346 study members (157 males, 189 females). There were significant differences between males and females across the higher frequencies at 3, 4, 6 and $8 \mathrm{kHz}$ $(p<0.001$, all frequencies). Across these frequencies females had between 6 and $11 \mathrm{~dB}$ better hearing function than males, depending on the frequency. No significant associations between gestational age, birth weight, standardised birth weight and hearing function at any frequency were found. Social class at birth was significantly associated with hearing function at frequencies above and including $1 \mathrm{kHz}$. Hearing function was better for those in more advantaged classes and worse for those in the least advantaged classes, compared to those from the middle class. These associations remained after the adjustment for sex, hearing aid use, ever experiencing workplace dust and workplace loud noise. Good housing conditions at birth were significantly associated with the higher hearing function frequencies at 2, 6 and $8 \mathrm{kHz}$.

Conclusions: Our findings do not support the hypothesis that early growth is related to later hearing function. However, early housing conditions and socio-economic status were associated with hearing, suggesting that some of the antecedents of impaired hearing may lie early in life.

\section{P-110}

Gestational age patterns in cesarean section rates: an international comparison

Topic: Diseases - Others

Presenter: Marie Delnord

Marie Delnord 1); Béatrice Blondel 1); Nicolas Drewniak 1); Ashna Mohangoo 2); Jennifer Zeitlin 1); The Euro-Peristat Preterm Birth Study Group 3)

1) INSERM, UMRS 953 Epidemiological Research Unit on Perinatal and Women's and Children's Health, Paris, France; 2) Department Child Health, TNO Netherlands Organization for Applied Scientific Research, Leiden, The Netherlands; 3) Euro-Peristat Preterm Birth Study Group

Objective: While international variations in overall cesarean section rates are well documented, less information is available for specific clinical sub-groups. We studied differences in the use of cesarean section by multiplicity and gestational age.

Methods: This study used routine aggregate data from 17 European countries and the United States on the number of singleton and multiple live births with cesarean versus vaginal delivery by week of gestation in 2008. Overall and gestation-specific cesarean section rates were analyzed. We computed rate differences and rate ratios to 
compare practices for selected gestational age groups (very preterm, preterm, near term, term and post-term).

Results: Cesarean rates for singletons varied from $15 \%$ (the Netherlands and Slovenia) to over $30 \%$ (US and Germany). Everywhere, rates were highest for very preterm deliveries and declined to lows at 40 weeks of gestation $(8.0 \%$ for Sweden and Norway to $22.5 \%$ in the US). However, patterns of change differed; the average rate difference between very preterm and term births was 42 points, but this ranged from 15 to $57 \%$. High variations in rate differences and ratios were also observed for near term versus term births. For multiples, rates declined by gestational age in some countries, whereas in others rates were similar across all weeks of gestation.

Conclusions: Gestational age patterns in the use of cesarean section were highly heterogeneous across countries; these differences highlight areas where consensus on best practices is lacking and could inform strategies to promote the reduction of cesarean section rates.

\section{P-111}

C-reactive protein is associated with fracture risk: European prospective investigation into cancer Norfolk Study

Topic: Diseases - Others

Presenter: Sara Ahmadi Abhari

\section{Sara Ahmadi Abhari 1); Robert Luben 1); Nicholas Wareham 2);} Kay-Tee Khaw 1)

1) Department of Public Health and Primary Care, University of Cambridge, Cambridge, UK; 2) MRC Epidemiology Unit, Institute of Metabolic Sciences, Cambridge, UK

Background: Serum C-reactive protein (CRP) is a marker of general systemic inflammation and is suggested to be a marker of the agerelated chronic inflammatory state. The association of CRP and fracture risk has not been studied thoroughly in large cohort studies. Investigating the association of CRP and fracture risk, in addition to risk prediction purposes, helps in better understanding the underlying pathophysiology of osteoporosis and fractures.

Objectives: We aimed to investigate the association of CRP and risk of all-fractures and hip fractures in a large prospective study.

Methods: Men and women aged 40-79 were recruited, from 1993 to 1997, to the European Prospective Investigation into Cancer in Norfolk (EPIC-Norfolk) study. Among 18,560 men and women with available serum CRP measurement at baseline, 792 incident fractures (291 hip fractures) were observed after 261,563 person-years of follow up (mean follow up was 14 years). The association of log-CRP (continuous analysis) and fracture risk (all-types and hip fractures) was tested using fractional polynomial Cox-regression analysis. Association of CRP (categorized using clinical cut-points) and fracture risk (all-types and hip fractures) was tested using Coxproportional hazards regression analyses. All multivariable models were adjusted for age, body mass index, height, smoking, alcohol intake, past history of fracture, Steroid medication use, and a composite variable representing sex, menopausal status and hormone replacement therapy.

Results: In fractional polynomial Cox-regression models, a U-shaped association was observed between CRP and fracture risk (a quadratic term for log-CRP in the model for all-fractures was statistically significant). The lowest risk of fracture was observed for men and women in the CRP category 1.1-2 $\mathrm{mg} / \mathrm{L}$. Hazard ratios of all-types of fracture for participants with CRP levels $0.1-0.5 ; 0.6-1 ; 1.1-2 ; 2.1-3$; $3.1-10$; and above $10 \mathrm{mg} / \mathrm{L}$ were 1.29 (1.01-1.64); $1.20(0.97-1.50)$; 1.00 (referent category); 1.06 (0.83-1.36); 1.24 (1.01-1.53); 1.34 (0.95-1.88). Corresponding hazard ratios for hip fractures were 1.10
(0.74-1.64); $0.87 \quad(0.61-1.26) ; \quad 1.00 \quad$ (referent category); 0.89 (0.60-1.34); 1.14 (0.82-1.58); 1.45 (0.86-2.44).

Conclusion: Above $1 \mathrm{mg} / \mathrm{L}, \mathrm{CRP}$ is positively associated with fractures of all-types and hip fractures. The increased risk of fracture observed at lower levels of CRP require further exploration, confirmation in other populations, and investigation into potential biological mechanisms.

\section{P-112}

Epidemiology of congenital diaphragmatic hernia in Europe: a register-based study

Topic: Diseases - Others

Presenter:

Mark McGivern 1); Kate Best 1); EUROCAT Working Group 2); Judith Rankin 1)

1) Institute of Health \& Society, Newcastle University, UK; 2) EUROCAT Central Registry, Univesity of Ulster, Northern Ireland, UK

Background: Congenital diaphragmatic hernia (CDH) is a hole in the diaphragm, which occurs when the diaphragm fails to close during gestation. The hole allows the abdominal viscera to protrude into the chest cavity. $\mathrm{CDH}$ can lead to serious morbidity and is associated with a high risk of mortality.

Objectives: The aim of this study was to describe the epidemiology of $\mathrm{CDH}$ in Europe, including associated anomalies, pregnancy outcomes, prevalence and temporal trends.

Methods: All cases of CDH delivered between January 1980December 2009 notified to 31 European Surveillance of Congenital Anomaly Registers formed the population-based case-series. Cases occurring in multiple pregnancies, with unknown plurality, with chromosomal anomalies or genetic syndromes were excluded. Prevalence and $95 \%$ confidence intervals (95\% CIs) were calculated as the number of cases (occurring in live births, fetal deaths ( $>20$ weeks gestation) or terminations of pregnancy for fetal anomaly (TOPFA; any gestation)) per 10,000 live and stillbirths. Relative risks (RRs) and $95 \%$ credible intervals (CrIs) of CDH corresponding to year of delivery were estimated using a multilevel Poisson model with a random intercept.

Results: There were 3,373 CDH cases reported among 12,155,491 registered total births. $139(4.1 \%)$ cases occurred in multiple pregnancies, $103(3.1 \%)$ with unknown plurality and $353(10.5 \%)$ were associated with a chromosomal anomaly or genetic syndrome. There were 2,776 singleton cases remaining, of which $784(28.2 \%)$ occurred with additional major structural congenital anomalies and $1992(71.8 \%)$ were isolated cases. Of the 2,776 cases, 2,317 (83.4\%) occurred in live births, $360(13.0 \%)$ in TOPFAs and $99(3.6 \%)$ in fetal deaths. Of the isolated cases, $1,767(88.7 \%)$ occurred in live births, $177(8.9 \%)$ in TOPFAs and $48(2.4 \%)$ in fetal deaths. Total prevalence was 2.3 (95\% CI 2.2-2.4) and 1.6 (95\% CI 1.6-1.7) per 10,000 births for total and isolated $\mathrm{CDH}$, respectively. There was a small but significant increase in the prevalence of total $\mathrm{CDH}$ over time $(\mathrm{RR}=1.01,95 \% \mathrm{CrI}: 1.00-1.01 ; p=0.030)$ but there was no significant increase in isolated cases over time $(\mathrm{RR}=1.00,95 \% \mathrm{CrI}$ : $0.99-1.01 ; p=0.359)$. There was significant variation in total and isolated $\mathrm{CDH}$ prevalence between registers $(p<0.001$ for both) but there was no significant association with maternal age. Of the live born cases, $69.3 \%$ total cases and $72.7 \%$ isolated cases survived to 1 week.

Conclusion: This large population-based study found a small increase in total $\mathrm{CDH}$ prevalence over time. $\mathrm{CDH}$ prevalence varied significantly by geographical location but not by maternal age. 


\section{P-113}

Predictors of work incapacity among patients examined with nerve conduction studies on suspicion of ulnar neuropathy: a prognostic cohort study

Topic: Diseases - Others

Presenter: Poul Frost

Poul Frost 1); Birger Johnsen 2); Anders Fuglsang-Frederiksen 2); Susanne Wulff Svendsen 3)

1) Danish Ramazzini Centre, Department of Occupational Medicine, Aarhus University Hospital, Aarhus, Denmark; 2) Department of Neurophysiology, Aarhus University Hospital, Aarhus, Denmark; 3) 3Danish Ramazzini Centre, University Department of Occupational Medicine, Herning Regional Hospital, Herning, Denmark

Background: Ulnar neuropathy is the second most common peripheral neuropathy after carpal tunnel syndrome. We have shown that around $50 \%$ of patients undergoing nerve conduction studies due to suspected ulnar neuropathy still have symptoms and experience disability of the arm, shoulder and hand 1-7 years after the examination. Occupational exposure to forceful work predicted worse prognosis with respect to severity of symptoms and disability.

Objectives: To evaluate the ability of occupational mechanical exposures, lifestyle factors, abnormal nerve conduction as opposed to normal nerve conduction, and affliction of the dominant arm to predict work incapacity among patients undergoing nerve conduction studies on suspicion of ulnar neuropathy.

Methods: A prognostic cohort study of working men and women undergoing nerve conduction studies at a university hospital 2001-2007 on suspicion of ulnar neuropathy. We used a job exposure matrix for assessing exposure to forceful work, nonneutral postures and repetitive movements of the arm. The Danish National Register on Public Transfer Payments provided information on health related transfer payments for the year following the date of the nerve conduction studies. Information on lifestyle factors was obtained by questionnaire. Information was also available for a sample of age and gender matched referents. We applied logistic regression to analyze the odds ratio of work incapacity, in terms of receiving health related transfer payment at any time during 52 weeks of follow up (yes/no). Results: 526 of 720 patients provided information for the analyses. 219 had abnormal nerve conduction according to the department's clinical practice and 307 had normal nerve conduction. The mean time of receiving health related transfer payment during the year following the nerve conduction studies was 9.3 and 10.6 weeks respectively. Among 1,401 referents the mean time was 2.2 weeks. Compared with low exposure to forceful work, the adjusted odds ratio for work incapacity was 2.0 (95\% CI 1.04-3.69) for those with medium and 2.8 (95\% CI 1.56-5.03) for those with high exposure to forceful work. Previous fracture of the affected arm and affliction of the dominant arm were also predictors, while abnormal nerve conduction, body mass index, current smoking, and other mechanical exposures were not.

Conclusions: patients undergoing nerve conduction studies for suspected ulnar neuropathy received health related transfer payments for longer time than matched controls. Among patients, exposure to forceful work, previous fracture, and affliction of the dominant arm predicted work incapacity while abnormal nerve conduction did not.

\section{P-115}

Abortion in young women: an epidemiological analysis in a city of Northeast of Brazil, 2008 and 2009

Topic: Diseases - Others
Presenter: Nilma Dias Leão Costa

Alice da Costa Uchoa 1); Mário Guimarães de Amorim 1); Kallil Monteiro Fernandes 1); Ricardo Luiz Oliveira Alves 1); Wesley Bruno Nunes Izídio 1); Nilma Dias Leão Costa 1); Lidiane de Araújo Torres 1); Michelle Rafaelle Andrade Gurgel 1); Maria Helena Constantino Spyrides 1); Maria Célia de Carvalho Formiga 1)

\section{1) Universidade Federal do Rio Grande do Norte}

Background: Abortion is a serious public health problem worldwide, especially in underdeveloped countries. In Brazil, abortion is an illegal practice, except for specific conditions. Because of its importance, health care in abortion has been included among the Millenium Development Goals by United Nations.

Objectives: Analyze the sociodemographic, epidemiologic, reproductive and obstetric profiles of women in the age groups of adolescent and young women undergoing abortion.

Methods: Descriptive, cross-sectional study conducted in a referral maternity for obstetric care in Natal, Rio Grande do Norte, Brazil. Data collection was conducted from September 2011 to March 2012. The population totaled 1,129 records of women treated in the years 2008 and 2009, according to the criteria: age up to 24 years, living in Rio Grande do Norte, gestational age up to 23 weeks, diagnosis of abortion. We investigated sociodemographic, clinical, gynecological and reproductive variables and the hospital assistance. Patients were categorized by age in early adolescent, late adolescent and young adult. Descriptive statistics analysis was performed using Pearson's Chi squared test at a $5 \%$ significance level.

Results: It was observed a predominance of young adults $(60 \%)$, in marital union $(53.9 \%)$, from the capital and metropolitan area $(75.6 \%)$, with highschool education or ongoing $(53.5 \%)$, nonremunerated occupations $(64.19 \%)$, without history of alcoholism $(85.7 \%)$ or smoking $(91.4 \%)$. The average age of onset of sexual activity was 15.8 years, most women referred use of contraceptive methods $(76.4 \%)$, being the oral ones the most widely used $(59 \%)$. Most of them were primigravid (58.6\%), in early abortion $(63.8 \%)$. The procedure most widely used was curettage $(55.2 \%)$, with no intercurrences $(97.6 \%$ ); average hospital stay was 1.2 days. There was statistical significance $(p<0.05)$ in the association between the age group and the following variables: marital status, number of partners, use of contraceptives, reason for seeking care and therapeutic procedure performed.

Conclusions: Despite the illegality, there is a large demand for abortion care. The high prevalence of abortion among adolescents $(40 \%)$ and single women $(46.1 \%)$ highlights the necessity of more effective family planning policies. The low rate of intercurrences may be related to the usage of Misoprostol, both in-hospital, as in induction (illegal abortion), as shown in other papers in Brazil. The study shows the presence of effective hospital care to women in abortion; however, confirms the major use of curettage in Brazilian public hospitals, instead of manual vacuum aspiration, an effective technique, more humanized, less expensive and recommended by Brazilian Ministry of Health.

\section{P-116}

The potential of general practice data for congenital anomaly research

Topic: Diseases - Others

Presenter: Rachel Sokal

Rachel Sokal 1); Kate Fleming 1); Linda Fiaschi 1); Laila Tata 1)

1) Division of Epidemiology and Public Health, University of Nottingham, Nottingham, United Kingdom 
Background: Congenital anomalies (CAs) are a leading cause of infant mortality and morbidity in high income countries. Of all children born in the United Kingdom (UK) $2.2 \%$ have a major CA, and one in five of these children are stillborn. Despite an increasing number of children with CAs surviving longer into childhood and adulthood, relatively little is known about the causes and consequences of most CAs. General practice data provide large populationbased cohorts of individuals with prospectively collected medical information with promising potential for studying risk factors and the long-term outcomes for children with CAs. We sought to validate these data through comparison with congenital anomaly registries.

Methods: The Health Improvement Network (THIN) is a computerised database of anonymised patient primary care records in theUK. Our study population was 794,209 children in THIN, born between 1990 and 2009 with a median follow-up to 6.7 years of age. We compared the birth prevalence of any major congenital anomaly and system-specific subgroups by year of birth with the European Surveillance of Congenital Anomalies (EUROCAT) UK registries.

Results: The birth prevalence of any major CA for children in THIN, diagnosed before 1 year of age, was 198 per 10,000 (95\% CI 195-201) slightly higher than that reported by EUROCAT, 167 per 10,000 (risk ratio $1.18,95 \%$ confidence interval 1.16-1.20). Absolute differences in prevalence between THIN and EUROCAT were small across 16 system-specific anomaly groups. The pattern of prevalence by year of birth in THIN was closely consistent with EUROCAT registry data. The majority of children in THIN with major CAs had diagnoses recorded before 1 year of age $(72 \%)$, but including children diagnosed after age 1 increased the overall prevalence to 277 per 10,000 births.

Conclusions: The prevalence of congenital anomalies in THIN was consistent with EUROCAT for early diagnoses, demonstrating THIN to be a valuable source for investigating congenital anomalies. Age of diagnosis is an important factor in explaining a higher overall prevalence in THIN; the inclusion of diagnoses made after 1 year of age substantially improves capture of diagnoses indicating that THIN may have more complete diagnostic information than registry data for some children. These data provide an opportunity to conduct further study of CAs in areas where epidemiological research has been limited including examining the prospectively collected maternal data to assess risk factors and the description of long-term outcomes in children with CAs within a population-based cohort.

\section{P-117}

Chronic liver disease and 30-day mortality after hip fracture: a population-based cohort study

Topic: Diseases - Others

Presenter: Mary Nguyen-Nielsen

Mary Nguyen-Nielsen 1); Rune Erichsen 1); Maja Tarp 1); Lea Kjær Andersen 1); Henrik Gammelager 1); Henrik Toft Sørensen 1)

1) Department of Clinical Epidemiology, Aarhus University Hospital, Aarhus, Denmark

Background: Patients with chronic liver disease (CLD) are at high risk of surgical complications and death. Since hip fracture (HFx) is a common and serious condition often requiring major surgery, it is clinically relevant to understand the mortality rate among HFx patients with CLD. OBJECTIVES We examined the 30-day cumulative and relative mortality among HFx patients with CLD compared with HFx patients without CLD.

Methods: We conducted a nationwide cohort study in Denmark (population 5.5 million) based on medical databases. We included all first-time HFx patients registered from 1996 to 2010 in the Danish National Registry of Patients (DNRP), covering all Danish hospitals. First-time HFx was defined as HFx diagnosis, with no registration of HFx up to 15 years prior to the incident HFx. We also used the DNRP to obtain information on CLD diagnoses registered at any time prior to the incident HFx, Charlson Comorbidity Index (CCI) scores (CCI 0 ; CCI 1-2; CCI $\geq 3$ ), atrial fibrillation, and osteoporosis diagnoses registered up to 10 years prior to the incident HFx. CLD was categorized into 3 types: liver cirrhosis (LC), non-cirrhotic alcoholic liver disease (NC-ALD), and non-cirrhotic non-alcoholic liver disease (NC-NALD). Among the NC-NALD patients, we also identified patients with non-alcoholic steatohepatitis (NASH) and non-alcoholic fatty liver disease (NAFLD). We followed patients from HFx diagnosis until the end of 30-day follow-up, death, or emigration. The Kaplan-Meier method was used to compute 30-day cumulative mortality for HFx patients with and without CLD. The Cox proportional-hazards model was used to compute crude and adjusted mortality rate ratios (MRR), overall and by CLD-type. In the adjusted model, we controlled for age, gender, CCI score, calendar period of HFx diagnosis, and history of atrial fibrillation and/or osteoporosis.

Results: We identified $139,858 \mathrm{HFx}$ patients. Among these, 2,615 $(1.9 \%)$ had a history of CLD (CLD cohort) and 137,243 had no history of CLD (comparison cohort). When comparing the CLD cohort with the comparison cohort, CLD patients were younger (53 vs. $77 \% \geq 70$ years), there was a higher proportion of males (39 vs. $32 \%$ ), and there were higher proportions of CCI 1-2 (39 vs. $32 \%$ ) and CCI $\geq 3$ (17 vs. $10 \%$ ). Among the CLD cohort, 759 patients were recorded with LC; 463 with NC-ALD; and 1,393 with NCNALD. Within the NC-NALD subset, 94 patients had NASH and 82 had NAFLD. The overall 30-day mortality was $11.0 \%$ for CLD patients and $9.2 \%$ for patients without CLD. The 30-day mortality by CLD-type was $13.4 \%$ for LC, $9.0 \%$ for NC-ALD, and $11.0 \%$ for NC-NALD. The adjusted MRR for CLD was $1.39(95 \% \mathrm{CI}$ 1.23-1.57) compared to those without CLD. By CLD-type, the adjusted MRR for LC was 2.00 (95\% CI 1.63-2.45), 1.11 (95\% CI 0.78-1.57) for NC-ALD, and 1.31 (95\% CI 1.10-1.55) for NCNALD. For the subset with NASH and/or NAFLD, the adjusted MRR was 1.39 (95\% CI 1.23-1.57).

Conclusion: CLD patients have an increased 30-day risk of death after HFx. The relative increase in mortality among HFx patients with CLD was greatest among patients with LC, with a two-fold higher relative mortality compared with HFx patients without CLD.

\section{P-119}

Cervical cancer survival in the Arkhangelsk region, Russia in 2000-2012: a registry study

Topic: Diseases - Others

Presenter: Anna Subbotina, Andrej Grjibovski

Anna Subbotina 1), 2); Lyudmila Valkova 1); Natalia Glukhareva 1); Andrey Krasilnikov 3); Andrej Grjibovski 4), 5); Mikhail Valkov 1)

1) Northern State Medical University, Arkhangelsk, Russia; 2) International School of Public Health, Northern State Medical University, Arkhangelsk, Russia; 3) University of Tromso, Tromso, Norway; 4) Arkhangelsk Clinical Oncology Hospital, Arkhangelsk, Russia; 5) Institute of Post-graduate Medical Education (AGIUV), Almaty, Kazakhstan; 6) Department of International Public Health, Norwegian Institute of Public Health, Oslo, Norway

Background: Cervical cancer is a slow-growing cancer, which is mostly curable when diagnosed at the early stage. Data on cervical cancer survival are needed for estimating the effectiveness of 
detection and treatment. Little is known on survival from cervical cancer in Northwest Russia.

Objectives: To estimate survival from cervical cancer in the Arkhangelsk region, Northwest Russia, during the period from 2000 to 2012 and to assess factors associated with the survival.

Methods: Data were extracted from the Arkhangelsk regional cancer registry, a population-based registry with compulsory reporting of all cases of malignant neoplasms. One and 5-year cervical cancer specific survival rates were calculated using Kaplan-Meyer method. Cox proportional regression model was used for estimating hazard ratios. Results: Altogether, 1,457 cases of cervical cancer were registered in 2000-2012 in the Arkhangelsk region. During the observation period, a total number of 558 deaths from cervical cancer occurred. Age at diagnosis ranged from 20 to 91 years (Mean 50.1, standard deviation 16.1 years). One-year cervical cancer specific survival was $79 \%$ (95\% CI 76-81\%), 5-years survival $60 \%$ (95\% CI 57-63\%). Survival rates decreased substantially with patients' age. Thus, 5 -years survival was $71 \%$ for those younger than $40,60 \%$ for $40-59$ years, and $50 \%$ for those who were 60 and older. Survival differed significantly by stage. One-year survival for patients with stage I was $98 \%$, stage II $89 \%$, stage III $60 \%$ and stage IV $15 \%$ (95\% CI 10-22). Five-years survival for stage I was $93 \%$, stage II $61 \%$, stage III $33 \%$, and stage IV $6 \%$. Five-years survival differed by tumour morphology, being $63 \%$ for squamous cell carcinoma, $44 \%$ for adenocarcinoma, $45 \%$ for unspecified, and $25 \%$ when morphology was unknown. The final proportional Cox regression model included the following significant predictors: age, morphology, stage and stage and age interaction term. Adjusted HR were: age 40-59 years $\mathrm{HR}=1.41 \quad(95 \%$ CI $0.90-2.20), 60$ and older $\mathrm{HR}=2.66$ (1.16-6.07) (reference group 20-39 years); HR = 1.47 $(1.09-1.98)$ for adenocarcinomas, $\mathrm{HR}=2.69 \quad(1.85-3.91)$ for unspecified morphology, HR $=2.39(1.47-3.87)$ for missing morphology as compared to squamous cell carcinoma; stage II HR $=7.26$ (4.84-10.87), stage III HR = 22.79 (14.33-36.25), stage IV HR $=141.54$ (76.70-261.20) as compared to stage I.

Conclusions: Survival from cervical cancer in the Arkhangelsk region is below the European average probably due to lower survival on more advanced stages. The significant predictors of poorer survival are stage, morphology and age at diagnosis.

\section{P-120}

Dental caries as a public health problem among pre-school children in urban areas of Northwest Russia

Topic: Diseases - Others

Presenter: Mikhail Pastbin, Andrej Grjibovski

Mikhail Pastbin 1), 2); Irina Pastbina 3); Maria Gorbatova 1), 2); Lyubov Gorbatova 4); Andrej Grjibovski 1), 5)

1) Department of pediatric dentistry, Northern State Medical Unvieristy, Arkhangelsk, Russia; 2) International School of Public Health, Northern State Medical University, Arkhangelsk, Russia; 3) Ministry of health of the Arkhangelsk region, Russia; 4) Northern State Medical University, Arkhangelsk, Russia; 5) Department of International Public Health, Norwegian Institute of Public Health, Oslo, Norway

Background: Recent dental epidemiological surveys revealed high caries prevalence and experience in permanent teeth among schoolchildren in Russia. Moreover, caries experience in primary dentition among preschool children is known to be a risk factor for caries in their permanent dentition. However, it remains unknown how caries prevalence and experience are and where is a difference between urban settings in Russia.
Aim: To assess caries experience among 3-4 year-olds in urban settings in Northwest Russia in 2009-2011.

Methods: A cross-sectional study was performed in 7 towns, namely, Arkhangelsk $(\mathrm{n}=147)$, Severodvinsk $(\mathrm{n}=276)$, Novodvinsk $(\mathrm{n}=94)$, Onega $(\mathrm{n}=158)$, Mirny $(\mathrm{n}=93)$, Kotlas $(\mathrm{n}=184)$ and Koryazhma $(n=93)$. Caries experience was assessed at D3 level by a single calibrated examiner. Caries experience was estimated using a sum of decayed, missing, filled teeth (dmft index) and presented as means (M) with standard errors (m). Given non-equal variances, the means were compared using Welch tests with Games-Howell post hoc tests

Results: The prevalence of dental caries varied from $49 \%(95 \% \mathrm{CI}$ 39-60) in Mirny to 79 \% (95 \% CI 70-87) in Novodvinsk. Mean dmft index varied from $3.0 \pm 0.4$ in Mirny to $5.0 \pm 0.4$ in Novodvinsk. Mean $\mathrm{dt}$, mean $\mathrm{mt}$, mean $\mathrm{ft}$ for the whole sample were $2.9 \pm 0.1$, $0.8 \pm 0.1,1.2 \pm 0.4$, respectively. We observed significant variations in total dmft index $(p<0.001)$ and all components: $\mathrm{dt}(p=0.042)$, $\mathrm{mt}(p=0.042)$ and $\mathrm{ft}(p<0.001)$, respectively. Post-host tests revealed significant differences between the mean $\mathrm{ft}$ between the regional centre of Arkhangelsk and all other locations while highest mean of dt was observed in Novodvinsk.

Conclusions: Significant urban-urban variations were observed among 3-4 year-old children. Our findings may reflect better availability and accessibility of dental health services in the regional centre. Dental caries in primary dentition could be a possible risk factor of dental caries in permanent dentition among school children in Russia.

\section{P-121}

\section{Cervical cancer screening in the East Kazakhstan region in 2008-2012}

Topic: Diseases - Others

Presenter: Gulnar Shalgumbayeva

Gulnar Shalgumbayeva 1); Yuliia Semenova 1); Aigul Baibussinova 1); Marzhan Dauletyarova 1); Aidos Tlemisov 1)

\section{1) Semey State Medical University, Kazakhstan}

Background: Cervical cancer is the second most common cancer among women. Cervical cancer screening program was initiated in Kazakhstan in 2008. The National Cervical Screening Program in Kazakhstan currently recommends that sexually active women between 30 and 60 years undergo routine screening every 5 years. Objectives: To assess the results of the screening program in East Kazakhstan region from 2008 to 2012.

Methods: Altogether, 128,168 women aged 30-60 years underwent screening in the East Kazakhstan during the study period. All dichotomous data were presented as proportions. We present the data on the age-adjusted prevalence of cervical cancer using the world population.

Results: Mean age of women who were screened during the study period was 47.5 years old. Altogether, $3.5 \%$ of tests were positive. Among those tests which were positive, $72.3 \%$ were CIN1 (cervical intraepithelial neoplasia), 24.5-CIN2, $1.7 \%$-CIN3, $0.2 \%$ adenocarcinoma in situ (AIS), and $1.2 \%$-invasive cancers.

Age-adjusted prevalence for CINI during study period was higher than for other types of cervical dysplasia and was markedly higher in women of 60 years old (5.94). ASIR for CIN2, CIN3 also was higher in women of 60 years old and corresponded to $1.85,0.14$. Opposite to this finding ASIR for AIS was higher in women of 45 years old (0.14). ASIR for invasive cancers was higher in women of 40 years old (0.06)

Conclusions: Screening for cervical cancer in East Kazakhstan revealed $3.5 \%$ of tests to be positive. According to the data, $72.3 \%$ 
of cervical dysplasia in teh region belonged to CIN1 and was markedly higher in women of 60 years old. ASIR for AIS and invasive cancers was higher in young women aged 40-45 years old.

\section{P-122}

Overweight in relation to respiratory and allergic symptoms and objective disease markers in children: results from ISAAC phase two

Topic: Diseases - Others

Presenter: Gabriele Nagel

Gudrun Weinmayr 1); Francesco Forastiere 2); Gisela Büchele 1); Andrea Kleiner 1); David Strachan 3); Gabriele Nagel 1); ISAAC Phase Two Study group 4)

1) Institute of Epidemiology and Medical Biometry, Ulm University, Ulm, Germany; 2) Department of Epidemiology, Local Health Authority Rome/E, Rome, Italy; 3) Division of Community Health Sciences, St. Georges's, University of London, London, United Kingdom; 4) ISAAC

Background: Both obesity and asthma prevalence rise worldwide. Little is known whether symptoms and objective makers of asthma and allergic disease differ in overweight and obese children by region., in particular between affluent and non-affluent countries.

Objective: To investigate the association of disease symptoms and objective disease markers with obesity in an international context.

Methods: Cross-sectional studies of stratified random samples of 8-12-year-old children $(\mathrm{n}=10652)$ used the standardized methodology of Phase Two of the International Study of Asthma and Allergies in Childhood (ISAAC). Symptoms of asthma, rhinitis, eczema and cough and phlegm were ascertained by parental questionnaires. Skin examination, hypertonic saline bronchial challenge, six aeroallergen skin prick tests were performed and serum total $\mathrm{IgE}$ was measured. Height and weight were measured, and overweight and obesity were defined according to international age and sex-specific percentile curves for Body mass index (BMI). Prevalences and odds ratios (ORs) were calculated.

Results: Overweight and obesity were related to increased reported wheeze prevalence. This relation was stronger in affluent compared to non-affluent centres and in North-Central-Europe compared to Southern Europe where it was not statistically significant. Similar results were found for cough and phlegm, rhinitis and eczema but seemed to be driven mostly by children with wheeze, less though for cough and phlegm. Except for a decline in FEV1/FVC, results for the other objective markers were null or not conclusive.

Conclusions: Respiratory symptoms related to overweight and obesity differed by affluence status worldwide and residence. We found little evidence for an association with atopy.

\section{P-123}

Factors related to the increase in the negative impact of oral health on quality of life among Brazilian elders

Topic: Diseases - Others

Presenter: Fabiola Bof de Andrade

Fabiola Bof de Andrade 1); Maria Lúcia Lebrão 2); Yeda Duarte 3); Jair Santos 4)

1) René Rachou Research Institute, Oswaldo Cruz Foundation (FIOCRUZ), Belo Horizonte, Brazil; 2) School of Public Health -
University of São Paulo; 3) Nursing School - University of São Paulo; 4) Ribeirão Preto School of Medicine - University Of São Paulo

Objective: To evaluate the factors associated with the increase in the negative impact of oral health on quality of life among elderly individuals.

Methods: This was a longitudinal study with a sample of non-institutionalized older adults who participated in the second (2006) and third (2010) waves of the Health, Wellbeing and Aging cohort study (Saúde, Bem-Estar e Envelhecimento-SABE). The impact of oral health on quality of life was assessed by the Geriatric Oral Health Assessment Index (GOHAI). Changes in GOHAI scores were calculated by subtracting the total score at 2006 from the total score at follow-up (2011). The higher the GOHAI score the better the evaluation of oral health, thus, a negative result of this equation represents an increase in the negative impact, a positive result means reducing the impact and a score of zero demonstrates no changes. The outcome variable was the increase in the negative impact categorized as yes and no. The independent variables were related to socioeconomic conditions, general health and clinical oral health measures. All variables with a $p$-value $<0.20$ in the bivariate analysis were included in the multiple logistic regression model. Stata 11.0 was used for the analyses, and a correction for the design effect was made using the survey command to analyze data originating from a complex sample. Results: The final sample consisted of 628 individuals, representing 549,280 adults aged 60 and older, with complete information for GOHAI in 2006 and 2011. After 5 years it was found that $57.47 \%$ had an increase in the negative impact of oral health on quality of life. From the multiple logistic regression analysis it was found that the chance of an increase in the negative impact of oral health on quality of life was higher among the 70-79 (OR 2.23) and 80+ year old group (OR 2.36); individuals without depression in 2006 that developed the disease in 2011 (OR 6.47); the ones with self-reported health as poor in both waves of the study (OR 2.02) and the older adults with need for dental treatment in 2006 and 2011 (OR 2.09).

Conclusion: The increase in the negative impact of oral health on quality of life is associated with age and general health conditions and need for dental treatment.

\section{P-124}

Nutritional status of schizophrenic patients attending outpatient department of psychiatry in Dr.Hasan Sadikin Hospital

Topic: Diseases - Psychiatry

Presenter: Tiara Aulia Maisyarah

Tiara Aulia Maisyarah 1); Gaga Irawan Nugraha 2); Lynna Lydiana 3)

1) Faculty of Medicine, Universitas Padjajaran; 2) Department of Nutritional Sciences, Dr. Hasan Sadikin Hospital; 3) Department of Psychiatry, Dr. Hasan Sadikin Hospital

Background: Nowadays, schizophrenia is associated with many health problems due to weight changes caused by lifestyle changes and consumption of antipsychotic drugs (APDs). Nutritional status assessment is needed in order to lower the comorbidity by early detection of risk factors.

Methods: This descriptive type of cross-sectional study involved 94 schizophrenic patients selected using consecutive sampling on October 2012. An anthropometric measurement was verified (body mass index and waist circumference), physical activity level, type of antipsychotic drugs (APDs) used and duration of treatment acquired from medical records. 
Results: Among the subjects, $29.8 \%$ were aged $28-37$ years old, with $69.1 \%$ were male and $30.9 \%$ female. Most of them were paranoid schizophrenia $(71.3 \%)$. Body Mass Index of $46.8 \%$ subjects were normal, $45.74 \%$ were overweight, whereas $7.45 \%$ were underweight. Male subjects mostly had lower waist circumference result $(78 \%)$ compared to female which were dominantly above normal (52\%). Physical activity levels were mostly sedentary $(76 \%)$. Single typical APDs were dominantly prescribed $(46 \%)$. Subjects with normal Body Mass Index were mostly found among single typical APDs prescribers (53.5\%), whereas the majority of atypical APDs users were overweight $(61.9 \%)$. A total of $63 \%$ subjects had been prescribed APDs for 1-5 years, dominated by subjects with normal Body Mass Index (78 \%).

Conclusion: The majority of study subjects $(46.8 \%)$ were found normal in Body Mass Index. Male subject mostly had normal waist circumference $(78 \%)$ while females were higher in risk whose measurement above normal were dominant $(52 \%)$.

\section{P-125}

Neuropsychological assessment in the BREATHE project: preliminary results

Topic: Diseases - Psychiatry

Presenter: Monica López-Vicente

Joan Forns 1); Monica López-Vicente 1); Mikel Esnaola 1); Judith González 1); Mar Álvarez-Pedrerol 1); Núria SebastianGalles 2); Jordi Sunyer 1)

1) Centre for Research in Environmental Epidemiology (CREAL), Barcelona; Hospital del Mar Research Institute (IMIM), Barcelona, Spain; Spanish Consortium for Research on Epidemiology and Public Health (CIBERESP), Barcelona, Spain; 2) Center for Brain and Cognition, Department of Technology, Universitat Pompeu Fabra (UPF), Barcelona, Spain

Background: The BREATHE (brain development and air pollution ultrafine particles in school children) project aims to discover the effects of urban air pollution on neuropsychological development.

Aims: We aimed to study the relationships between the different neuropsychological measures of the BREATHE project.

Methods: A follow-up with four repeated evaluations of the cognitive performance in children aged from 7 to 9 years at baseline has being conducted during the period from January 2012 to February 2013. We evaluated 2.735 children using two different computerized paradigms: n-back task (to assess working memory) and attentional network task (ANT) (to assess attentional networks). Attention Deficit and Hyperactivity Disorder (ADHD) symptomatology (DSM-IV) and school achievement of children were reported by teachers. Parents filled in the Strenghts and Difficulties Questionnaire (SDQ). Parental sociodemographic variables were also collected by questionnaire. We analyzed the bivariate associations between outcomes and ADHD, SDQ and some covariates. Furthermore, the relationships between all the neuropsychological variables were studied using Bayesian Networks.

Results: We observed that those children with higher ADHD symptomatology, higher behavioral problems (SDQ) and lower school achievement performed significantly worse the n-back task. We also observed four main paths in the DAG analyzing the interdependencies between variables: ADHD, behavioral problems (SDQ) and two paths for n-back scores. All of these paths showed dependence with maternal education and school achievement subsequently.

Conclusions: These preliminary results indicate that n-back scores seem to be more sensitive than ANT scores to some important variables such as child's age, maternal education, ADHD symptomatology and behavioral problems. However, n-back score distributions are independent from those variables assessing ADHD symptomatology and behavioral problems.

\section{P-126}

Depression and anxiety among patrents of children with disabilities

Topic: Diseases - Psychiatry

Presenter: M. Naeem Mohsin, M. Nadeem Mohsin

\section{Naeem Mohsin 1); M. Nadeem Mohsin 1)}

1) G.C University Faisalabad

Key words: Depression, Anxiety, Disability, Intellectual disabilities

The current study aimed to find out the relationship between depression and anxiety among parents of children with disabilities and to compare the parent's depression and anxiety with the type of disability among children. Sample was comprised of 80 parents having one or more disabled children with the age range of 5-20 years. Purposive sampling technique was used. Depression, Anxiety and Stress Scale, DASS (Lovibond and Lovibond 1995) with 42 items was used.

The results of this study showed that a highly significant positive correlation was found between depression and anxiety with $(\mathrm{r}=.833$, $p<.01)$ A significant difference was found on depression subscale of DASS among parents with disabled boys than disabled girls with $\mathrm{t}(158)=2.301, p<0.01$. The major findings of this study revealed that parents of children with intellectual disability showed high level of depression and anxiety than parents of visual, audio and physical disability. Mothers of disabled children were more depressed and stressed than fathers of disabled children.

\section{$\mathbf{P - 1 2 7}$}

Identifying a "at risk" population for exclusive breastfeeding cessation: Psychological factors

Topic: Diseases - Psychiatry

Presenter: Cristina Fortes

Juliana Lindau Fortes 1); Simona Mastroeni 2); Andrea Gadini 3); Domenico Di Lallo 3); Martina Patanè 1); Paolo Giradi 1); Cristina Fortes 2)

1) NESMOS Department (Neurosciences, Mental Health and Sensory Functions), Sapienza University, 2nd Medical School, Sant'Andrea Hospital, Rome, Italy; 2) Clinical Epidemiology Unit, Istituto Dermopatico dell'Immacolata, IDI-IRCCS, Rome; 3) Public Health Agency, Lazio Regium, Rome, Italy

Background: The health benefits of breast feeding is well documented for both children and mothers.

Objective: The aim of this study was to identify a at risk population" for exclusive breastfeeding cessation within a population of mothers that intended to exclusively breastfeed.

Methods: This study was embedded in a prospective cohort study of 605 children. Information on psycho-socio demographic characteristics, intention to exclusively breastfeed, parity, type of delivery, planned pregnancy, breastfeeding practice, duration and exclusiveness and smoking were obtained by questionnaires. Clinical data was obtained by reviewing clinical records. Logistic regression was the method used to estimated odds ratio and $95 \%$ confidence intervals. Results: This study showed a strong association between a psychological risk profile and exclusive breastfeeding for at least 4 months. 
After adjustment for age, education, smoking, antenatal education, type of delivery and pacifier use, women with a psychological "at risk" profile (previous depression episode or insomnia and traumatic perception of birth) were three times more likely to interrupt exclusive breastfeeding (OR 3.08; CI $95 \%$ CI 1.05-9.08), after controlling for age, education, smoking, attendance to prenatal course, type of deliver and pacifier use.

Conclusion: Psychological factors are important predictors of exclusive breastfeeding and if a population "at risk" for breastfeeding cessation could be identified, prevention may be target to this group.

\section{P-128}

Prevalence, risk factors and possible under-diagnosis of depression in older adults in Tripolis, Greece

Topic: Diseases - Psychiatry

Presenter: Eleni Jelastopulu

Konstantinos Argyropoulos 1), 2); Christos Bartsokas 1); Philippas Gourzis 3); Eleni Jelastopulu 1)

1) Department of Public Health, Medical School, University of Patras, Greece; 2) Department of Psychiatry, Panarcadian General Hospital of Tripolis, Greece; 3) Department of Psychiatry, University Hospital of Patras, Greece

Background: With a rapidly aging society, geriatric depression is emerging as important public health concern. Symptoms like sadness, loss of energy and sleeping disorders are prevalent among older adults and affect negatively their quality of life. Older adults with depression often seek help in primary care. However, approximately half of all patients are not recognized as having depressive symptoms by their physician.

Objectives: The aim of this study was to estimate the prevalence and possible under-diagnosis of depression in elderly of a semi-urban area as well as investigate the association of depressive symptoms with various aggravating or protective factors.

Methods: The sample consisted of 139 subjects, aged $>65$ years, members of daycare center for older people" (KAPI) in the municipality of Tripolis, Peloponnese, Greece. A questionnaire was developed to collect basic demographic and socioeconomic data, including three questions from the "European Health Interview Survey" (EHIS), regarding self-reported and/or by a physician diagnosed depression. Moreover, to all participants the Greek validated version of the Geriatric Depression Scale (GDS-15) was applied, to screen the elderly for depressive symptoms. The scores of the GDS were (a) compared to the corresponding answers of the EHIS questions and (b) associated to the various recorded basic parameters. Statistical analyses were performed using the SPSS v. 17.0.

Results: According to GDS-15, $53.3 \%$ of the studied population screened positive for depressive symptoms ( $43.2 \%$ moderate, $10.1 \%$ severe type), while having ever been affected with chronic depression reported $16.5 \%$, of them $10.1 \%$ being diagnosed by a medical doctor. In 106 subjects who reported never have been affected by a depression and in $10(7.2 \%)$ of individuals who reported not to know if they have depression, depressive symptoms were observed in 43 $(44.7 \%)$ and 9 (90\%), respectively, applying the GDS-15. Depressive symptoms were more frequent in women (62.9 vs. $45.5 \%$, $p=0.012$ ), in not married, including divorced and widowed (85.6 vs. $38.9 \%, p<0.001)$ and in subjects without children (91.7 vs. $49.2 \%$, $p<0.001)$. Depressive symptoms were common in elderly with chronic diseases compared to elderly without co-morbidity (62.4 vs. $4.5 \%, p<0.001$ ), in participants with low monthly income (63.7 vs. $25.8 \%, p<0.001)$ and in older adults suffering from insomnia $(88.6$ vs. $6.7 \% . p<0.001)$.
Conclusions: High prevalence of depression in elderly population is evident in this study, unfortunately the majority of depressed elders, remain under diagnosed and untreated. The systematic use of GDS-15 in Primary Care may increase detection rates of depression among the elderly.

P-129

Vitamin D status during pregnancy and the risk of subsequent postpartum depression

Topic: Diseases - Psychiatry

Presenter: Nina Odgaard Nielsen

Nina Odgaard Nielsen 1), 2); Marin Strøm 1); Heather Boyd 1), Elisabeth W. Andersen 1); Jan Wohlfahrt 1); Marika Lundqvist 3); Arieh Cohen 3); David M. Hougaard 3); Mads Melbye 1)

1) Department of Epidemiology Research, Statens Serum Institut, Denmark; 2) National Institute of Public Health, University of Southern Denmark; 3) Department of Clinical Biochemistry and Immunology, Statens Serum Institut, Denmark

Background: Epidemiological studies have provided evidence of an association between vitamin D insufficiency and depression and other mood disorders, and a role for vitamin D in various brain functions has been suggested. We hypothesized that low vitamin D during pregnancy might increase the risk of postpartum depression (PPD). Objective: To investigate the relationship between vitamin D status during pregnancy and risk of subsequent PPD.

Methods: We performed a case-control study nested in the Danish National Birth Cohort established in 1996-2002, and included 605 women with PPD and 875 controls frequency matched for maternal age at delivery and year of delivery. We used register data on antidepressant use as a measure of PPD. The concentration of vitamin D $(25[\mathrm{OH}] \mathrm{D} 3)$ was measured in serum using a highly sensitive liquid chromatography tandem mass spectrometry method. We calculated odds ratios for PPD for six levels of vitamin D.

Results: Overall, we found no association between vitamin D levels and risk of PPD $(p=0.08)$. Compared with women with vitamin D levels between 50 and $79 \mathrm{nmol} / \mathrm{L}$, the adjusted odds ratios for PPD were 1.35 (95\% CI 0.6-2.9), 0.83 (CI 0.5-1.4) and 1.13 (CI 0.8-1.5) among women with vitamin D levels $<15,15-24$ and $25-49 \mathrm{nmol} / \mathrm{L}$, respectively, and 1.53 (CI 1.04-2.3) and 1.89 (CI 1.06-3.37) among women with vitamin D levels of $80-99$ and $\geq 100 \mathrm{nmol} / \mathrm{L}$, respectively. In a post hoc analysis among women with sufficient vitamin D ( $\geq 50 \mathrm{nmol} / \mathrm{L}$ ), we observed a significant positive association between vitamin D levels and PPD.

Conclusions: Our results did not support an association between low maternal vitamin D levels during pregnancy and risk of PPD. Instead, an increased risk of PPD was found among women with the highest vitamin D levels.

\section{P-130}

Prevalence of anxiety and depression in undergraduate students: a cross-sectional study at the University of Patras, Greece

Topic: Diseases - Psychiatry

Presenter: Eleni Jelastopulu

Maria Dimopoulou 1); Dimitra Giannopoulou 1);

Christos Bartsokas 1); Ilektra Savoglidou 1);

Konstantinos Argyropoulos 1); Eleni Jelastopulu 1)

1) Department of Public Health, Medical School, University of Patras, Greece 
Background: Depression seems to be the most common mental health disorder in general population. However, research indicates an increase in the prevalence of depression and anxiety in university students, reducing their capability of coping with daily life's demands.

Objectives: The aim of the present study was to assess the prevalence of and investigate risk factors for anxiety and depression in undergraduate students at the University of Patras, Greece.

Methods: A cross-sectional study was conducted on a representative sample of 1.233 undergraduate students, from October to December 2012. The instrument used to assess the anxiety and depression levels was the Hospital Anxiety and Depression Scale (HADS), with scores from 8 to 10 identifying possible and 11 or more probable cases of clinically meaningful anxiety or depression. Furthermore, questions regarding socioeconomic and educational characteristics were included in the study. Statistical analyses were performed using the SPSS v.17.0.

Results: Anxiety was present in $423(34.2 \%)$ and depression in 215 $(17.4 \%)$ students. Both were found concomitantly in $152(12.3 \%)$ students. Higher age $(p=0.014)$, female gender, higher year of study, school and city being not the first choice, reduction in income and ignorance of support services available to students were significantly associated with anxiety $(p<0.001)$. Depression was significantly associated $(p<0.001)$ with higher age and year of study, school and city being not the first choice, growing up with one parent, having more than three siblings, reduction in income and ignorance of support services available to students. Additionally, depression and anxiety in males were significantly associated with low educational level of father ( $p=0.000$ and $p=0.029$, respectively), whereas in females anxiety was associated with higher educational level of mother $(p=0.026)$.

Overall mean HADS scores were 6.30 (males 5.63, females 6.85) for anxiety and 4.74 (males 4.81, females 4.69 ) for depression. Among schools, mean anxiety scores ranged between 3.50 and 8.61 and mean depression scores between 3.89 and 6.90, with highest depression scores being observed in the school of engineering and school of natural sciences. Prevalence of depression ranged between $5.0 \%$ (business administration) and $35.5 \%$ (architecture) among the departments.

Conclusions: This study indicates that students in the University of Patras experience anxiety and depression which are associated with various sociodemographic and educational factors. The prevalence of both depression and anxiety varies significantly among the schools, thus further studies are needed to investigate the reasons. However, the study findings highlight the need of counseling and support center available to vulnerable students.

\section{P-131}

Treatment-resistant schizophrenia and clozapine treatment: a population-based pharmacoepidemiological study

Topic: Diseases - Psychiatry

Presenter: Theresa Wimberley

Theresa Wimberley 1); Henrik Støvring 2); Christiane Gasse 1)

1) National Centre for Register-based Research, Aarhus University, Denmark; 2) Department of Public Health, Biostatistics, Aarhus University, Denmark

Background: Approximately $30 \%$ of patients with schizophrenia (SZ) are considered to be treatment-resistant, i.e. not responding to first-line antipsychotic (AP) treatment. Clozapine (CLZ) is recommended in treatment-resistant schizophrenia (TRS), but is considered to be underused. However, little is known about predictors that could identify patients with TRS eligible for CLZ.
Objectives: To determine base-line TRS predictors and to investigate the association between known risk factors of SZ and TRS

Methods: Population-based register study using Danish register data on patients diagnosed with SZ between 1995 and 2006 and information on AP prescriptions from 1995 to 2010. TRS is defined from prescription data as CLZ eligibility defined according to treatment guidelines as redemption of CLZ prescription or a fourth, different AP prescription, whatever comes first. The event of TRS within 5 years after first SZ diagnosis is analyzed using multivariate logistic regression analysis to identify factors predictive of TRS.

Results: Of all SZ patients $(n=12,087), 12.4 \%$ redeemed a CLZ prescription and $21.4 \%$ redeemed a fourth, different AP prescription (not CLZ), resulting in $31.4 \%$ with TRS during follow-up (median $=9.3$ years; IQR: 6.4-11.3). Female gender was associated with an increased odds ratio (OR) of TRS within 5 years (OR 1.56; $95 \%$ CI 1.41-1.72), as were psychiatric hospitalization and younger age at first SZ diagnosis. A family history (parents or siblings) of SZ was not significantly associated with TRS (OR 0.95; $95 \%$ CI 0.78-1.15). The individual predicted risk of TRS within 5 years ranged from 7.1 to $47.5 \%$, mean $20.4 \%$ (95\% CI; $95 \%$ CI 18.5-22.4\%). Applying the stricter TRS definition, i.e. redemption of at least one CLZ prescription, gave similar results and the additional finding that the paranoid subtype at first SZ diagnosis was associated with increased CLZ treatment. Calendar year of first SZ diagnosis was associated with increased odds of TRS per year, and conversely, decreased odds of CLZ treatment per year.

Conclusions: Analyses using Danish prescription data indicate that CLZ is potentially underused. Several factors observable at the first SZ diagnosis were significantly associated with TRS, in terms of eligibility for CLZ treatment as well as CLZ treatment. No significant association was found between a family history of SZ and TRS.

Funding: The present research has received funding from the European Community's Seventh Framework Programme (FP7/20072013) under grant agreement no 279227.

\section{P-132}

\section{Maternal age at childbirth and risk for ADHD in offspring}

Topic: Diseases - Psychiatry

Presenter: Zheng Chang

Zheng Chang 1); Paul Lichtenstein 1); Niklas Långström 1); Brian D'Onofrio 2); Henrik Larsson 1)

1) Department of Medical Epidemiology and Biostatistics, Karolinska Institutet, Stockholm, Sweden; 2) Department of Psychological and Brian Sciences, Indiana University, Bloomington, IN, USA

Background: Women who give birth at younger ages (e.g., teenage mothers) are more likely to have children who exhibit behaviors problems, in particular antisocial behaviors. However, the link between early maternal age and offspring ADHD has not been established. In addition, it is not clear whether early maternal age at childbearing is causally associated with poor offspring outcomes or confounded by familial factors.

Method: The association between maternal age at childbirth and offspring's ADHD was studied using a large, nationally representative sample with quasi-experimental designs. We used children born in Sweden between 1992 and $1998(\mathrm{~N}=720,764)$ and their mother identified from the Multi-generation Registry. Offspring ADHD $(n=13,963)$ were identified from the National Patient Registry and the Prescribed Drug Registry. We also compared differentially exposed siblings to account for unmeasured familial factors that could account for the association. Further, children of siblings and children 
of twins comparisons were conducted to identify the extent to which the association was confounded by genetic or environmental factors. Result: Results showed that early maternal age at childbearing (maternal age $<20$ ) was significantly associated with the risk of ADHD in offspring (Hazard ratio $=2.22,95 \%$ CI 2.06-2.38). Comparison of differentially exposed siblings indicated no withinfamily association. We further explored the association between maternal age at first birth (MAFB) and offspring ADHD using children of siblings and children of twins comparison, and the association was robust.

Conclusion: The results provided support for a causal association between MAFB and offspring ADHD, and the lack of within-family association could be explained by carry-over effect of MAFB on all siblings within a family.

\section{P-133}

Changes in lifetime prevalence of suicidal ideation and suicidal thoughts among Norwegian doctors from 2000 to 2010: a longitudinal study

Topic: Diseases - Psychiatry

Presenter: Judith Rosta

\section{Judith Rosta 1); Olaf G. Aasland 1), 2)}

1) Institute for Studies of the Medical Profession, Oslo, Norway; 2) Institute of Health Management and Health Economics, University of Oslo, Norway

Background: International studies suggest high mortality for suicide in doctors. Since suicidal ideation and suicidal thoughts are important determinants of the multifactorial background of suicide risk, to monitor the prevalence of suicidal ideation and suicidal thoughts is useful to describe changes in the prevalence of suicidal risk.

Objectives: The objectives of the present study were to describe the changes in the lifetime prevalence of suicidal ideation and suicidal thoughts from 2000 to 2010, and to describe the predictors of serious suicidal thoughts in 2010 among Norwegian doctors.

Methods: The study population consisted of a representative sample of approximately 1,600 Norwegian doctors, surveyed in 2000 and 2010. The number of doctors who answered all items of the Suicidal Feelings Questionnaire of Paykel was 1,253 in 2000 and 962 in 2010. 715 doctors responded at both points in time (cohort). Changes were tested with $95 \%$ CI. Simultaneous effects were analysed through logistic regression models.

Results: From 2000 to 2010, the lifetime prevalence of suicidal ideation (SI) and suicidal thoughts (ST) decreased in all doctors (SI: 27.1 vs. $23.2 \%$; ST: 30.8 vs. $25.4 \%$ ) as well as in the cohort (SI: 26 vs. $23 \%$; ST: 29 vs. $24 \%$ ).

The predictors of serious suicidal thoughts in 2010 were similar in all doctors and the cohort. In the cohort, poor or average self-rated health (OR 2.96; 1.42-6.16), high psychosocial work stress (OR 2.16; $95 \%$ CI 1.09-4.31) and subjective well-being (OR 0.70; $95 \%$ CI $0.51-0.97)$ were all independent significant predictors of serious suicidal thoughts in multivariate logistic model. Age, gender, specialty and job satisfaction had no effect on the model.

Conclusion: The observed decrease in lifetime prevalence of suicidal ideation and suicidal thoughts indicates a reduction in suicide risk among Norwegian doctors. Individual and work-related factors were associated with serious suicidal thoughts. Compared with other professionals in Norway and doctors in Germany, Norwegian doctors showed no higher risk of suicidal thoughts.

\section{P-134}

National study of suicide method in violent criminal offenders

Topic: Diseases - Psychiatry

Presenter: Roger Webb

Roger Webb 1); Ping Qin 2); Hanne Stevens 3); Jenny Shaw 3); Louis Appleby 3); Preben Mortensen 3)

1) University of Manchester, UK; 2) University of Oslo, Norway; 3) University of Aarhus, Denmark

Background: Aggressive or violent individuals have a higher risk of suicidal behaviour. Genetic mechanisms may partly determine choice of suicide method among people who are violent, but this decision may also be strongly driven by prevailing culture, urbanicity and availability of particular means.

Objectives: We compared suicide method profiles between violent offenders, other offender types and non-offenders. We hypothesised that violent offenders would have a higher relative risk for suicide by a violent method than by a nonviolent one. We reasoned that violent offenders would be more prone to killing themselves using methods that are often particularly painful or disfiguring. For example, they may more frequently use guns and knives, as many of these individuals possess or have ready access to such weapons. We also believed that they would have a greater propensity for taking their lives impulsively without foresight or planning, using especially violent means such as jumping from a height or in front a train, or deliberately crashing a motor vehicle.

Methods: Using interlinked national Danish registers we constructed a nested case-control study dataset of all adult suicides, 1994-2006: $\mathrm{N}=9708$ cases and $\mathrm{N}=188,134$ age and sex matched living controls. Completely ascertained ICD-10 cause-specific mortality codes were examined, along with all criminal charges and convictions since 1980, and covariate information on secondary care psychiatric treatment and socio-demographics. Self-poisonings were classified as 'nonviolent' suicide; all other methods as being 'violent'.

Results: Among male and female violent offenders, relative risk for nonviolent suicide was greater than that for violent suicide versus the general population. Risk was especially high for self-poisoning with narcotics \& hallucinogens. We could only examine the full range of suicide methods in male violent offenders as our national investigation had insufficient statistical power for undertaking a detailed profiling of specific suicide methods among female violent offenders. In these men, hanging was the most frequently used method, although risk was markedly and significantly elevated across virtually the entire range of regularly used suicide methods.

Conclusions: Gaining a greater knowledge of the methods by which violent offenders die by suicide can inform tailored preventive strategies. Our findings indicate that violent offenders do not have a greater propensity for selecting a violent suicide method. Their high relative risk for self-poisoning by illicit drugs underlines the importance of access to means and of prevailing subculture. Comprehensive and broadly-based preventive approaches are needed for tackling the markedly raised suicide risk in this population.

\section{P-135}

Adverse working conditions are associated with increased risk for suicide mortality: findings from the MONICA/KORA Augsburg Cohort Study

Topic: Diseases - Psychiatry 


\section{Presenter: Jens Baumert}

Jens Baumert 1); Barbara Schneider 2); Karoline Lukaschek 1); Rebecca Thwing Emeny 1); Christa Meisinger 1); Natalia Erazo 3); Karl-Heinz Ladwig 1), 3)

1) Institute of Epidemiology II, Helmholtz Zentrum München, Neuherberg, Germany; 2) LVR-Klinik Köln, Cologne, Germany; 3) Department of Psychosomatic Medicine, Medical Psychology and Psychotherapy, Klinikum rechts der Isar, Technische Universität München, Munich, Germany

Background: Several studies have shown associations of negative occupational attributes such as work-related stress or adverse working conditions with specific disease outcomes. Recently, the impact of work-related stress and adverse working conditions on suicidality have gained increased attention.

Objectives: The aim of the present study was therefore to evaluate the impact of work-related stress and working conditions on suicide mortality in a longitudinal, population-based German study.

Methods: The study population consisted of 6,721 men and women who were employed at baseline examination and drawn from the MONICA/KORA Augsburg surveys conducted in 1984-1995 and followed up on average for 12.0 years. Work-related stress and working conditions were assessed by a self-administered questionnaire. Work-related stress was defined by the control-demand model developed by Karasek. Working conditions comprised nine items about chronobiological (such as shift, night or overtime work) and physical (such as noise, dangerous work or pollutants) components. Cox regression was used to assess the suicide mortality risk.

Results: A total of 28 subjects died by suicide within the follow-up period. High adverse working conditions increased significantly the risk for suicide mortality (Hazard ratio 2.48, $95 \%$ CI 1.15-5.35) compared to low or intermediate adverse working conditions in an age-, sex- and survey-adjusted model ( $p$ value 0.021). Additional adjustment for depressed mood, smoking, high alcohol consumption, obesity and family status did not alter these findings substantially ( $p$ value 0.035 ). Work-related stress, in contrast, had no impact on subsequent suicide mortality risk.

Conclusions: Adverse working conditions such as shift, night or overtime work as well as noise or pollutants in the work environment had a high impact on suicide mortality risk in this population-based, longitudinal study, even after controlling for known suicide-related risk factors. Surprisingly, work-related stress was not associated with suicide mortality. Therefore, interventions aimed to directly improve conditions of the work environment might be a suitable approach to prevent employed subjects to commit suicide. However, future studies with shorter follow-up periods are needed to assess direct consequences of impaired working conditions on suicide mortality.

\section{P-136}

A pharmacoepidemiological study of Alzheimer's disease in a South African patient population

Topic: Diseases - Psychiatry

Presenter: Ilse Truter

\section{Ilse Truter 1)}

1) Drug Utilization Research Unit (DURU), Nelson Mandela Metropolitan University

Alzheimer's disease was the sixth leading cause of death in 2011 in the United States of America and the only cause of death among the top 10 that could not be prevented or cured. While death rates are declining for most major diseases, deaths from Alzheimer's disease are increasing. Few pharmacoepidemiological studies on Alzheimer's disease have been conducted in Africa. The aim of the study was to investigate the prescribing patterns of medicine to patients who received products for Alzheimer's disease in a South African patient population. A retrospective, cross-sectional pharmacoepidemiological study was conducted on claims data for 2011 of a medical aid administrator. The database contained 2298312 records for medicines, medical devices and procedures. Data for all patients who received one or more products for Alzheimer's disease during 2011 were extracted for analysis. A total of only 33 patients received medication for Alzheimer's disease (51.52\% males). The average age of patients was $73.24(\mathrm{SD}=9.94)$ years. A total of 147 products for Alzheimer's disease were dispensed at an average amount claimed of R456.22 per product. Patients received on average $4.45(\mathrm{SD}=3.64)$ products for Alzheimer's disease over the year, with males receiving on average 5.18 products. Donepezil accounted for $64.63 \%$ of prescriptions, followed by memantine $(23.81 \%)$ and galantamine $(11.56 \%)$. Differences were observed between females and males with respect to the prescribing frequency of the different active ingredients $\left(\chi^{2}=12.979 ; d f=2 ; p<0.01\right)$. Most patients $(87.88 \%)$ received only one active ingredient during the year. Donepezil in combination with memantine was the only combination prescribed, with the average age of these patients $71.75(\mathrm{SD}=2.50)$ years. Two-thirds of patients $(78.79 \%)$ received other central nervous system (CNS) medication, and $21.21 \%$ received antipsychotic products despite the black box" warnings regarding antipsychotic prescribing to dementia patients. The most often prescribed drug classes (excluding products for Alzheimer's disease and other CNS products) were cardiovascular agents, vitamins, tonics, minerals and electrolytes, and urinary system agents. A limitation of this study was the small patient sample. Yet, the results were similar to those of previous studies and confirmed that these products are generally not used on a continuous basis, that it is mostly older patients receiving these products and that other CNS medicines are often co-prescribed. More comprehensive studies on Alzheimer's disease are needed including qualitative studies.

\section{P-137}

Alterations in family structure and ADHD-related symptoms among the affected children

Topic: Diseases - Psychiatry

Presenter: Anita Tønder Nielsen

Anita Tønder Nielsen 1); Jin Liang Zhu 2); Jørn Olsen 2); Carsten Obel 1)

1) Aarhus University, Department of public health; 2) Aarhus University, Department of epidemiology

Background: Attention-deficit/hyperactivity disorder (ADHD) is probably defined in a way that makes it the most common childhood psychiatric disorder worldwide with an estimated prevalence of 3-5 \%. The incidence of ADHD in many countries has been rising the last decades. ADHD has substantial impact on the affected children, their families, peers, and society. The disorder is characterized by pervasive symptoms of inattention, hyperactivity, and impulsivity. ADHD most often co-occurs with other psychiatric disorders. The etiology of ADHD is not well established, but both genetic and environmental factors like negative life events and stress in the social environment play a role. Parental divorce is a common and known stressor to the affected children; Alterations in family structure following the divorce has been associated with social adjustment problems in the affected children.

Objectives: We aimed to study if children in Denmark who had changed their family structure following a divorce show a higher level of ADHD-related symptoms compared to children living with both their parents. 
Methods: Data on family structure and psychosocial symptoms were obtained from the 7-year follow up in The Danish National Birth Cohort. This cohort consists of about 100,000 Danish children born between 1996 and 2003. We used data from the parent-reported Strengths and Difficulties Questionnaire (SDQ), a standardized measure of emotional, behavioral, and social functioning in children and adolescents. We calculated mean with standard deviation of ADHD-related symptoms, differentiating according to whom the child lives with most of time.

Results: Children living with one of their parents, one of their parents and a new partner, or equally with both parents had reports of more conduct problems, hyperactivity and peer problems than children living with both their parents. Children living with one parent and a new partner showed the highest level of conduct problems and hyperactivity compared to other family structure categories; the mean score of hyperactivity was $3.8(\mathrm{SD}=2.8)$ for children living with their father and his new partner, $3.2(\mathrm{SD}=2.5)$ for children living with their mother and her new partner, compared to 2.3 (SD 2.1) for children living with both their parents. The mean score of conduct problems showed a similar pattern.

Conclusions: In children who had divorced parents there were reported higher levels of ADHD-related symptoms compared with children living with both of their parents. This finding may be due to shared genetic factors in dysfunctional families or social stress exposure.

\section{P-138}

Use of ADHD drugs during pregnancy from 1995 to 2010: a Danish register-based study

Topic: Diseases - Psychiatry

Presenter: Katrine Strandberg-Larsen

Katia Buch Hærvig 1); Laust Hvas Mortensen 1); Anne Vinkel Hansen 1); Katrine Strandberg-Larsen 1)

\section{1) Faculty of Health Sciences}

Background: The use of ADHD drugs among young women has grown rapidly in the last decade. This potentially implies that more pregnant women are now at risk of using ADHD drugs, particularly before the pregnancy is recognized. Pregnant women are advised not to use ADHD drugs as this may be harmful to the fetus, but the knowledge on use of ADHD drugs and their effects during pregnancy is still lacking.

Objectives: The purpose of this study was to describe the trends in use of ADHD drugs among pregnant women in Denmark from 1995 to 2010. Moreover we explored characteristics of pregnant women who used ADHD drugs and examined whether these women had increased risk of adverse pregnancy outcomes.

Method: A linkage between various national health registries was used to identify all women who were pregnant between 1995 and 2010 in Denmark. Use of ADHD drugs was obtained from the Danish National Prescription Registry, and defined as a redeemed prescription on methylphenidate, modafinil and atomoxetine from 28 days before the first day of the last menstrual period until the end of pregnancy.

Results: Of the $1,399,874$ women who were pregnant in the period from 1995 to 2010, a total of 567 women used ADHD drugs during pregnancy. Use of ADHD drugs among pregnant women has increased from 5 per 100,000 person year in 2003 to 305 in 2009. Compared with unexposed pregnant women, women who used ADHD drugs during pregnancy were likely to be younger, single, lower educated, receiving social security benefits, and more likely also to use anxiety medication and SSRI. Women who used ADHD drugs during pregnancy had higher risk than unexposed women of induced abortions on maternal request (odds ratio 4.96, $95 \% \mathrm{CI}$ 4.08-6.08), induced abortion on medical indications (odds ratio 3.06, $95 \% \mathrm{CI} 1.43-6.53$ ), and miscarriage (odds ratio $2.38,95 \% \mathrm{CI}$ 1.81-3.12) compared to unexposed.

Conclusions: This study indicates that ADHD drugs are used during pregnancy, and there has been an increase in use among pregnant women from 2003 to 2009. Use of ADHD drugs was associated with increased risk of induced abortions and miscarriage, but whether this increased risk is attributable to the drugs per se or by confounding by indication or lifestyle is unclear. Thus, it is important to follow whether the use will continue to increase in the years to come.

\section{P-139}

Health effects of overeducation: a Swedish cohort study

Topic: Diseases - Psychiatry

Presenter: Hanna Hultin

Hanna Hultin 1); Andreas Lundin 2); Michael Lundberg 1); Cecilia Magnusson 1)

1) Karolinska Institutet, Department of Public Health Sciences; 2) Karolinska Institutet, Institute of Environmental Medicine

Background: Previous studies have indicated that the increasing educational level in many post-industrial nations may imply that subgroups of the population work in occupations for which they are overeducated. Considering the increasing numbers of university students in many European countries, surprisingly few studies of the health effects of over education have been made on recent populationbased European data.

Objectives: The aim is to investigate whether university-educated individuals who work in occupations requiring less education, have increased risks of future adverse self-rated health or psychological distress, compared to individuals in occupations which matches their education.

Methods: The study is based on data from the Stockholm Public Health Cohort, a Swedish population-based cohort of inhabitants of Stockholm County. The analytical sample consisted of 21,159 employed individuals aged 25-64 years. Individuals who reported having a university education and working in occupations which required less than university education in 2006 or 2007, were considered as overeducated. The outcomes self-rated health and psychological distress were measured in a questionnaire in 2010. The data was analyzed with logistic regression and the results are presented as odds ratios (OR) with $95 \%$ confidence intervals (CI).

Results: In the study group, $6 \%$ of men and $7 \%$ of women were overeducated at baseline. The prevalence of over education differed by type of education, with over education being most common among those with a university education in arts or humanities, and least common among those with a pedagogical education.

After adjustment for socio-demographic factors, health status, and health behavior at baseline, overeducated individuals had an increased odds of adverse self-rated health in 2010 (OR $1.3695 \%$ CI 1.06-1.73) compared to highly educated individuals working in matching occupations. Stratified analyses indicated increased risks of adverse self-rated health among overeducated women aged 25-34 and 35-54 years old. No increased risk of psychological distress was found for the overeducated group.

However, we also performed analyses in which the overeducated group was compared with less educated individuals working in similar occupations. Preliminary results from these analyses indicate no increased risks of either outcome for the overeducated group.

Conclusions: Our study shows that individuals who are overeducated for the work tasks they perform, have increased risks of future adverse 
self-rated health, compared to other highly educated individuals. However, the fact that the overeducated do not have increased health risks compared to lower educated individuals in similar occupations, may imply that the association is largely due to differences in working conditions.

\section{P-140}

Food selectivity in autism spectrum disorders: a systematic review

Topic: Diseases - Psychiatry

Presenter: Maria M. Morales-Suárez-Varela

Maria M. Morales-Suárez-Varela 1); Salvador Mari-Bauset 2); Agustin LLopis-Gonzalez 3)

1) Unit of Public Health and Environmental Care, Department of Preventive Medicine, University of Valencia, Valencia, Spain; 2) CIBER Epidemiology and Public Health (CIBERESP), Spain; 3) Center for Public Health Research (CSISP), Valencia, Spain

Background: Autism spectrum disorders (ASDs) are characterised by difficulties with reciprocal social interactions, deficiency in verbal and non-verbal communication, and restricted, repetitive and stereotyped patterns of behaviour and interest; one of these characteristic behaviours is food selectivity. It is estimated that prevalence is 11.3 cases per 1,000 people, and is more common in males (ratio of 4:1). Food selectivity is not exclusive to children with ASDs. Approximately one quarter of all children have eating problems during the first years of life, although this rate may be as high as $80 \%$ in children with developmental disabilities. It should be underlined that, although this situation does not disappear completely, it tends to improve with age.

Objectives: For these reasons, we considered that a review to assess the state of food selectivity knowledge would be useful. The resulting description of the studies conducted to date intends to serve as a summary of the available scientific evidence for both the research community and the professionals involved in the diagnosis, assessment and implementation of interventions in people with ASDs.

Methods: We conducted a systematic review of the scientific literature on food selectivity. During the search, the following key words were used: feeding assessment, selective eating, nutrition, growth, BMI, autism, ASD, autism spectrum disorders, feeding disorders, mealtime problems and review. Specifically, the identification of information sources was based on using the Medline Database. This paper complies with the methodological requirements established for the publication of systematic reviews and the PRISMA recommendations.

Results: All the reviewed articles referred to dietary selectivity in children with ASDs. However, almost all of them had validity issues for various reasons: lack of a control group, analysis based on single individuals or anecdotal data, heterogeneous groups in age terms, small sample sizes, and no statement of inclusion criteria, and failure to consider phenotypic variation between either individuals or alternative explanations.

Conclusions: Accordingly, we conclude that behavior at meal times should be monitored as part of the routine follow-up of patients with ASDs. In addition, sensory motor skills should be assessed systematically, as should the presence of possible GI disorders.

Moreover, if we consider that all the reviewed articles were unanimous that food selectivity is associated with ASDs, pediatricians, parents and other careers of people with ASDs, especially those with dietary restrictions, must be aware of the potential seriousness of deficiencies. Indeed, prevention of deficiencies should be a highpriority nutritional objective to encourage children with ASDs to eat a varied, balanced and moderate diet. Nevertheless, further research is required which should particularly include blind, randomized, controlled trials with larger sample sizes.

\section{P-141}

Daily suicide counts and ambient air temperature in Astana, Kazakhstan in 2006-2010

Topic: Diseases - Psychiatry

Presenter: Andrej Grjibovski

Andrej Grjibovski 1), 2); Gulmira Kozhakhmetova 3); Nassikhat Nurgaliyeva 3), 4); Bibigul Adilbekova 3); Aliya Kosbayeva 4); Bettina Menne 5)

1) Department of International Public Health, Norwegian Institute of Public Health, Oslo, Norway; 2) International School of Public Health, Northern State Medical University, Arkhangelsk, Russia; 3) Medical University of Astana, Astana, Kazakhstan; 4) WHO office in Kazakhstan, Astana, Kazakhstan; 5) WHO European Centre for Environment and Health, Bonn Office, Bonn, Germany

Background: Seasonal variations in the incidence of suicide have been observed in many industrialized countries. Moreover, it is known that the incidence of suicide increase during both economic crises and periods of rapid economic growth. At the same time, the evidence on the associations between air temperature and suicide in rapidly developing settings is scarce.

Objectives: To study associations between four different indicators of air temperature and suicide daily counts in Astana-the fast growing capital of Kazakhstan.

Methods: Daily counts of suicides (ICD-10 codes: X60-X84) for the population of Astana, Kazakhstan, during 2006-2010 were obtained from the City Registry Office. Associations between the number of calls and mean, mean apparent, maximum and maximum apparent temperatures were studied using negative binomial regression models controlling for effects of month, year, weekends and holidays. In addition, we assessed associations between suicide counts and barometric pressure and humidity.

Results: Altogether, there were 685 suicides in Astana during the study period. A clear seasonal pattern with the peak during the spring and summer moths was observed. In crude analyses, significant associations between suicide counts and all temperatures as well as barometric pressure and humidity were observed. After adjustment for potential confounders, only air temperatures remained significantly associated with the outcome. An increase in mean temperature by $1{ }^{\circ} \mathrm{C}$ was associated with an increase by $1.53 \%$ in suicide counts (95\% CI 0.15-2.92). Similar results were obtained for mean apparent temperature $(2.01 \%, 95 \%$ CI 0.39-3.62) and maximum apparent temperature $(1.11 \%, 95 \%$ CI $0.03-2.19)$.

Conclusions: The results suggest a linear relationship between temperature and daily suicide counts across the whole spectrum of temperatures with no thresholds in the city of Astana, Kazakhstan. Factors behind this association warrant further research with further going aim to develop mitigation strategies in the period of climate change.

\section{P-142}

Epidemiology of suicide in Western Iran, 2004-2011

Topic: Diseases - Psychiatry

Presenter: Farid Najafi

Farid Najafi 1); Mehdi Moradinazar 1) 
1) School of Public Health, Kermanshah University of Medical Sciences, Kermanshah, Iran

Background: Suicide is a complex phenomenon being affected by several factors. In fact, such factors are mostly dependent to cultural and social characteristics in each society.

Objectives: The current study aimed to investigate the epidemiology of suicide in Kermanshah province which has one of the highest incidence rates in Iran.

Method: For the purpose of this study, we collected all successful suicide (fatal) cases in Kermanshah province during 2004-2011 from the forensic medicine files. To calculate the incidence of suicide, total number of cases was divided by total population in Kermanshah province in each year. Using world standard population, we directly standardized incidence rates. Trends were investigated using Poisson regression.

Results: Between 2004 and 2011 (8 years), 2015 people died of suicide, of which $1,168(58 \%)$ were men and $847(47 \%)$ were women. The average annual mortality rate of suicide during 8 years was 12.9 per 100,000 people $(11.35,14.45$ for women and men, respectively). From total, $25 \%$ were people aged less than 25 years old. There was a significant relationship between gender and suicide methods; self-immolation (57 \%) and hanging (52\%) were the most common successful suicide methods among women and men, respectively. Suicide by hanging increased while the death of selfimmolation increased since 2008 and decreased after that. Trend of other suicide methods showed no significant changes.

Conclusion: Suicide in Kermanshah is high compared to other provinces in Iran. In order to reduce the burden of suicide we need to control the social and cultural factors contributing to suicide. In addition the prevention services at different levels of society, particularly for high-risk groups can have a significant role in reducing the number of suicide.

\section{P-143}

\section{The Danish national reaction-to-severe-stress cohort}

Topic: Diseases - Psychiatry

Presenter: Elisabeth Svensson

Jaimie Gradus 2); Imre Bozi 1); Elisabeth Svensson 1); Timothy Lash 1); Patricia Resick 2); Jens Georg Hansen 1); Henrik Toft Sørensen 1)

1) Department of Clinical Epidemiology, Aarhus University, Aarhus, Denmark; 2) Department of Psychiatry, Boston University School of Medicine, USA

Background: Large-scale population-based studies of patients who receive a diagnosis of a Reaction-to-Severe-Stress (RSS) disorder are scarce, with the exception of PTSD. Such studies are warranted to better understand the clinical course of disorders associated with stress and trauma.

Objectives: To create a nationwide longitudinal cohort of all Danishborn residents of Denmark who have received an International Classification of Diseases-10th Ed (ICD-10) RSS diagnosis between 1995 and 2011

Methods: We identified all persons with an ICD-10 F43.x diagnosis in the Psychiatric Central Research Registry, creating a national cohort of all Danish-born citizens with first ICD-10 acute stress reaction (ICD-10 code: F43.0); posttraumatic stress disorder (ICD-10 code: F43.1); adjustment disorder (ICD-10 code: F43.2); other reaction to severe stress (ICD-10 code: F43.8) or reaction to severe stress, unspecified (ICD-10 code: F43.9) diagnoses from 1995 through 2011. Results: In total, 96,736 Danish-born adults and children received an ICD-10 RSS diagnosis between 1995 and 2011, corresponding to an incidence of 115 per 100000 person years at risk (pyr). The most common diagnosis was adjustment disorder, with an incidence of 74 per 100000 pyr. More women than men received an RSS diagnosis (60 vs. $40 \%$ ). There was a $50 \%$ increase in unspecified reaction to severe stress and acute stress reaction diagnosis in 2007.

Conclusions: Based on Danish medical registers, it was possible to create a cohort of over 96000 patients with a RSS disorder. Research projects based on this cohort can elucidate various aspects of stress and trauma by examining longitudinal outcomes and correlates of these disorders.

\section{P-144}

Risk factors for suicide and violent offending in a national cohort of people diagnosed with bipolar disorder

Topic: Diseases - Psychiatry

Presenter: Roger Webb

\section{Roger Webb 1); Paul Lichtenstein 2); Mikael Landen 2);} Seena Fazel 3)

1) University of Manchester, UK; 2) Karolinska Institutet, Sweden; 3) University of Oxford, UK

Background: Across the full array of psychiatric diagnoses, previous systematic reviews have indicated a particularly highly raised suicide risk with bipolar disorder, and externalized violence is also known to occur more commonly in this population.

Objectives: To estimate relative risk for suicide and violent criminal offending in a national cohort of people diagnosed with bipolar disorder, and to make a etiological comparisons between these two adverse outcomes.

Methods: Using national Swedish registers we created a historic cohort study that consisted of all people diagnosed with bipolar disorder in the course of at least two separate secondary care treatment episodes (inpatient and/or outpatient) during 1973-2010. We examined $\mathrm{N}=15,114$ such patients matched for age and gender to $\mathrm{N}=302,262$ unaffected living individuals selected randomly from the general population. From several interlinked registers we obtained data on demographic and clinical factors that pertained to individuals and also to their parents.

Results: Compared with the general population the bipolar disorder cohort had relative risk for suicide of 19.2 (CI 16.3-22.7) and of 4.8 (CI 4.4-5.3) for violent offending. Within the bipolar disorder cohort, male gender predicted higher risk of both suicide and violence toward others. Several other demographic factors were linked with externalised violence only, including low income, being an immigrant, and, in male conscripts, low intelligence quotient (IQ). Being unmarried was strongly protective against suicide. Having more severe bipolar disorder and history of self harm predicted both adverse outcomes, as did various measures indicating histories of substance misuse and criminal offending. Parental risk factors were predictive of violent crime risk, but not suicide, although these weak associations were explained by the individual-level risk factors for violence in multiple regression models.

Conclusions: Our findings indicate markedly raised risk for suicide and violent offending in this national cohort of people diagnosed with bipolar disorder, with the elevation in risk for suicide being particularly high. We found several shared risk factors for both adverse outcomes, and some factors that were predictive or protective of one outcome only. Clinicians ought to be particularly vigilant when assessing male bipolar patients with histories of substance misuse disorders and criminal offending, and they should carefully assess the risks for both suicide and interpersonal violence in this patient group. 


\section{P-145}

\section{Socioeconomic worsening and mental health}

Topic: Diseases - Psychiatry

Presenter: Gabriela Barbaglia

Gabriela Barbaglia 1); Margreet ten Have 2); Saskia van Dorsselaer 2); Gemma Vilagut 1); Jordi Alonso 1); Ron de Graaf 2)

1) Health Services Research Group, IMIM-Institut Hospital del Mar d'Investigacions Mèdiques, Barcelona, Spain. CIBER Epidemiología y Salud Pública, Spain. Pompeu Fabra University, Barcelona, Spain; 2) Netherlands Institute of Mental Health and Addiction, Utrecht, The Netherlands

Background: The link between socioeconomic disadvantage and mental disorders is well established. However, negative changes in socioeconomic circumstances are less clear as predictors of the development of mental disorders

Objective: To investigate whether socioeconomic worsening-i.e. loosing job, income or both- is associated with the development of mental disorders

Methods: The Netherlands Mental Health Survey and Incidence Study-2 is a longitudinal study collecting data on mental disorders in a representative sample of the Dutch population. Face-to-face interviews were carried out at baseline (2007-2009) and 3 years later at follow-up (2010-2012). DSM-IV mental disorders were assessed using the Composite International Diagnostic Interview (CIDI 3.0). Workers (individuals working 12 or more hours per week) at baseline were selected for this analysis $(\mathrm{n}=3,676)$. The outcome variable was any incident or recurrent mental disorder during follow-up. Categories of disorders included any mood, any anxiety and any substance use disorders. Logistic regression models were used to estimate the association between mental disorders in individuals who lost their job, who had a substantial loss of their household income or both in comparison to those who did not, adjusted by age, sex and time between waves

Results: $11.0 \%(\mathrm{n}=331)$ of the respondents developed an incident or recurrent disorder during 3-year follow-up. For any mood disorder, this figure was $3.6 \%(\mathrm{n}=218)$, for any anxiety disorder $5.4 \%$ $(\mathrm{n}=164)$ and for any substance use disorders $3.8 \%(\mathrm{n}=114)$. During follow-up, $3.0 \%(\mathrm{n}=92)$ lost their job, $7.8 \%(\mathrm{n}=294)$ substantially lost their household income and $3.2 \%(\mathrm{n}=106)$ lost both. Those who lost job, lost substantial income and both had a higher risk of developing any mental disorder (lost job OR 1.8, $95 \%$ CI 0.8-4.0; lost income OR 1.20, $95 \%$ CI 0.7-2.1; lost both OR 3.5, $95 \%$ CI 1.6-7.4) than those who did not. Differences among categories of disorders were found. Worsening in socioeconomics was more strongly associated with developing a mood disorder (job OR 2.9, $95 \%$ CI 1.3-6.7; income OR 2.1, $95 \%$ CI 1.4-3.3; both OR 4.9, $95 \%$ CI 2.6-9.4) than an anxiety disorder (job OR 2.0, $95 \%$ CI $0.8-4.9$; income OR $1.2,95 \%$ CI $0.7-2.3$; both OR $1.9,95 \%$ CI 0.6-6.2) or a substance use disorder (job OR 1.7, $95 \%$ CI 0.8-4.0; income OR 1.1, $95 \%$ CI 0.5-2.3; both OR 1.5, $95 \%$ CI 0.4-5.1)

Conclusions: Worsening in socioeconomic conditions importantly increases the likelihood of developing mental disorders. Overall, such an increase tends to be additive, i.e., the more worsened conditions, the higher the risk. Research on preventive interventions on these individuals is needed

\section{P-146}

The effect of N-3 fatty acids on psychological distress in the Inuit population of northern Canada

Topic: Diseases - Psychiatry

\section{Presenter: Hans-Ragnar Skogli Skogli}

Hans-Ragnar Skogli Skogli 1); Dominique Geoffroy 2); Grace M. Egeland 1); Laurence J. Kirmayer 2)

1) National Institute of Public Health, University of Bergen, Department of Global Public Health and Primary Care; 2) Division of Social \& Transcultural Psychiatry, McGill University

Background: Individuals suffering from psychological distress may have unique nutritional needs. In addition, suboptimal nutritional status may contribute to psychological sequelae. Among Indigenous Peoples' undergoing rapid cultural changes, traditional food access and utilization are important for social cohesion and well-being.

Objectives: To evaluate a biomarker of traditional food intake and exposure to long-chain n-3 fatty acids for its' association with psychological distress among Canadian Inuit.

Methods: The International Polar Year Inuit Health Survey was conducted in 2007 and 2008. Researchers visited 36 communities in the 3 regions of the northern Canadian Arctic. Clinical tests were conducted and information about living standards, food consumption, community life and physical and mental health was gathered from a total of 2,595 participants. Red blood cell (RBC) long-chain n-3 fatty acids (FA) was assessed as a biomarker of traditional food intake and specifically of food of marine origin. Psychological distress was assessed by the Kessler 6-item questionnaire. The Kessler scale, ranges from 0 to 24, where a score of 13 and above indicates severe psychological distress which has been validated as predictive of respondents with a high likelihood of having a serious and diagnosable mental disorder. RBC long-chain n-3 FAs were evaluated as tertiles. Univariate and multivariable logistic regression models were constructed to evaluate the association of RBC n-3 FA with severe psychological distress.

Results: In unadjusted analyses, increasing tertiles of RBC n-3 FA were associated with a decreasing prevalence of severe psychological distress $(15,10,7 \%$, respectively, from lowest to highest tertile of RBC n-3 FA; X2 Ps < 0.001). In preliminary multivariable analyses (Model 1) adjusting for important determinants of psychological distress, the inverse association of RBC n-3 FA remained significant $(P<0.01)$

Conclusions: In this study we found that n-3 FAs are inversely related to SPD. The cross sectional nature of the survey does not allow causal interpretations of the results, but it opens up for further analyses on the relationship between biomarkers and mental health.

\section{P-147}

Risk of schizophrenia and factors predicting urban birth: a register-based propensity score analysis

Topic: Diseases - Psychiatry

Presenter: Esben Agerbo

Esben Agerbo 1); John McGrath 2); Evangelos Vassos 3); Carsten Bøcker Pedersen 1)

1) CIRRAU - Centre for Integrated Register-based Research at Aarhus University, Denmark; 2) Queensland Centre for Mental Health Research, The Park Centre for Mental Health Locked Bag 500 Sumner Park BC, QLD 4074 Australia; 3) King's College London, Institute of Psychiatry MRC SGDP Centre, Box P082 De Crespigny Park London, SE5 8AF United Kingdom

Background: Urban birth is one of most consistently replicated risk factors for schizophrenia. Putative exposures underlying the urban birth risk factor have included e.g. parental psychiatric illness, socioeconomic position, divorce, education, age, immigration, genetic factors, substance misuse, subject's birth order, age at onset, toxins, 
psychiatric service utilization, household crowding, and infectious agents such as influenza. However, the hypotheses proposed to explain the urban birth risk factor have been unsatisfying

Objectives: Mindful that an assumption of no unmeasured confounders is required, we aim to construct a comprehensive model for the propensity of urban birth and to estimate the risk of schizophrenia while adjusting for this propensity.

Methods: Data were obtained by linking three Danish populationbased registers: (1) the Danish Civil Registration System which contains since 1968 information on birthplace, gender, date of birth, deaths, immigration and emigrations, (2) the Psychiatric Central Research Register which includes admission dates and diagnoses to psychiatric inpatient facilities, (3) the Integrated Database for Longitudinal Labour Market Research contains annual information on e.g. gross income, labour market affiliation, educational attainment and marital status from administrative register.

The Kaplan-Meier estimator and Cox regression were used to estimate crude associations with schizophrenia. Weighted Cox regression model was used to estimate the parameters of marginal structural models. The weights (i.e. inverse probability and standardized-mortality-ratio) were based on a logistic regression model with explanatory variables containing information on both parents own birthplace, psychiatric history, gross income, labour market affiliation, educational attainment, marital status, age and the offspring's parity.

Results: A total of 1609 subjects with schizophrenia were identified during the 3.84 million years of follow-up corresponding to an incidence rate of 42 per 100000 years at risk. The rate ratio of schizophrenia associated with urban birth was $1.85(1.59-2.15)$.

The propensity of being born in the capital was 0.67 and 0.05 for subject's whose father was born in the capital and a rural area respectively corresponding to a ratio of 14.7 (14.3-15.0). The propensity was also strongly associated parental psychiatric history, gross income, labour market affiliation, educational attainment, marital status, age and the offspring's parity. The propensity ranged between 0.001 and 0.997 .

For instance, the rate ratio of schizophrenia decreased to 1.44 (1.14-1.82) in a propensity score stratified analysis.

Conclusions: The increased risk of schizophrenia remained significant across the three causal models, which suggests the existent of an independent effect of urban birth.

\section{P-148}

Modelling the contribution of family history and variation in single nucleotide polymorphisms to risk of schizophrenia

Topic: Diseases - Psychiatry

Presenter: Esben Agerbo

Esben Agerbo 1); Preben Bo Mortensen 1); Carsten Wiuf 3); Michael S. Pedersen 1); John McGrath 2); Mads V. Hollegaard 4); Bent Nørgaard-Pedersen 4); David M. Hougaard 4); Ole Mors 5); Carsten Bøcker Pedersen 1)

1) $\mathrm{iPSYCH}$ - the Initiative for Integrative Psychiatric Research, CIRRAU - Centre for Integrated Register-based Research at Aarhus University, Denmark; 2) Queensland Centre for Mental Health Research, The Park Centre for Mental Health Locked Bag 500 Sumner Park BC, QLD 4074, Australia; 3) Department of Mathematical Sciences, University of Copenhagen, Denmark; 4) Section of Neonatal Screening and Hormones, Statens Serum Institut, Copenhagen, Denmark; 5) Centre for Psychiatric Research, Aarhus University Hospital, Psychiatric Hospital, Denmark
Background: Epidemiological studies indicate that having any family member with schizophrenia increases the risk of schizophrenia in the probands. However, genome-wide association studies (GWAS) have accounted for little of this variation.

Objectives: The aim of this study was to use a population-based sample to explore the influence of single-nucleotide polymorphisms (SNP) on the excess schizophrenia risk in offspring of parents with a psychotic, bipolar affective or other psychiatric disorder.

Methods: A nested case-control study with 739 cases with schizophrenia and 800 controls. Their parents and siblings. Information from national health registers and GWAS data from the national neonatal biobank.

Results: Offspring schizophrenia risk was elevated in those whose mother, father or siblings had been diagnosed with schizophrenia or related psychosis, bipolar affective disorder or any other psychiatric disorder. The rate ratio was $9.31(3.85 ; 22.44)$ in offspring whose $1 \mathrm{st}$ degree relative was diagnosed with schizophrenia. This rate ranged between 8.31 and 11.34 when adjusted for each SNP individually and shrank to $8.23(3.13 ; 21.64)$ when adjusted for $25 \%$ of the SNPvariation in candidate genes. The percentage of the excess risk associated with a family history of schizophrenia mediated through genome-wide SNP-variation ranged between $-6.1 \%(-17.0 ; 2.6 \%)$ and $4.1 \%(-3.9 ; 15.2 \%)$. Analogous results were seen for each parent and for histories of bipolar affective and other psychiatric diagnoses.

Conclusions: The excess risk of schizophrenia in offspring of parents who have a psychotic, bipolar affective or other psychiatric disorder is not currently explained by the SNP variation included in this study in accordance with findings from published genetic studies.

\section{P-149}

Obesity and physical inactivity are associated with ADHD symptoms in adolescents

Topic: Diseases - Psychiatry

Presenter: Natasha Khalife

Natasha Khalife 1); Marko Kantomaa 1), 2); Vivette Glover 3); Tuija Tammelin 2); Jaana Laitinen 4); Marjo-Riitta Järvelin 1), 5); Alina Rodriguez 1), 6)

1) Department of Epidemiology and Biostatistics, Imperial College, London, United Kingdom; 2) LIKES - Research Center for Sport and Health Sciences, Jyväskylä, Finland; 3) Institute of Reproductive and Developmental Biology, Imperial College, London, United Kingdom; 4) Finnish Institute for Occupational Health, Helsinki, Finland; 5) Institute of Health Sciences, University of Oulu, Oulu, Finland; 6) Department of Social Sciences - Psychology, Mid Sweden University, Östersund, Sweden

Background: Recent evidence suggests that obesity and related factors, including physical inactivity, are co-morbid with attentiondeficit hyperactivity disorder (ADHD) symptoms. It is unknown whether specific subtype dimension(s) of ADHD contribute to the associations in the general population. We focus on the period of adolescence because it is a stage where adulthood health and lifestyle patterns are forming, and so is a key target for early intervention to prevent adverse health trajectories.

Objectives: By examining a large population-sample of adolescents (16 years) we aim to:

Investigate bi-directional associations between obesity (body mass index $[\mathrm{BMI}]$ and waist-hip ratio [WHR])/physical inactivity and ADHD symptoms. 
Investigate the relative associations of specific ADHD symptom subtypes (inattention, hyperactivity-impulsivity and combined ADHD) with obesity/physical inactivity.

Methods: Data were obtained from the Northern Finland Birth Cohort (NFBC) $1986(n=6856)$. Parents provided information on adolescent behaviour (using the Strengths and Weaknesses of ADHD symptoms and Normal behaviour [SWAN] scale), maternal education and family conditions. Data on BMI and WHR were obtained from clinical examinations. Adolescents self-reported their physical activity levels, which were then transformed to metabolic equivalent of task (MET) hours per week. We assessed the associations between obesity/physical inactivity (categorical) and ADHD symptoms (continuous) at 16 years using multiple regression analyses, controlling for gender, maternal education and change in family structure.

Results: We found significant bi-directional associations between obesity/physical inactivity and ADHD symptoms at 16 years. Obesity (BMI and WHR) predicted inattention symptoms (for BMI: $=.03$, $p=.01$; for WHR: $=.04, p=.01$ ); likewise, inattention symptoms predicted obesity (for BMI: OR 1.02, $95 \%$ CI 1.00-1.04; for WHR: OR 1.02, $95 \%$ CI 1.01-1.04). Physical inactivity predicted all ADHD symptoms (for combined ADHD symptoms: $=.10, p<.001$ ); likewise all ADHD symptoms predicted physical inactivity (for combined ADHD symptoms: OR 1.01, $95 \%$ CI 1.01-1.02).

Conclusions: Our work supports the concept of co-morbidity between obesity/physical inactivity and ADHD symptoms in the general population of youth. Inattention symptoms in particular were associated with obesity, whilst all ADHD symptoms were linked with physical inactivity. This work highlights the importance of monitoring the eating patterns/behaviour and physical activity levels of youth with either ADHD or obesity, in order to enhance the diagnosis and treatment of both disorders. Ongoing work includes examining possible common genetic variations underlying obesity and ADHD, as well as longitudinal analyses, to investigate the directionality of these associations.

\section{P-151}

Incidence of ICD-10 mental health disorders with onset in early childhood (0-3 years): a nationwide registerbased study

Topic: Diseases - Psychiatry

Presenter: Susanne V. Koch

Susanne V. Koch 1); Carolina Hvelplund 2); Mikael Andersson 3); Anne Mette Skovgaard 4)

1) Child- and Adolescent Center, Copenhagen Region; 2) Pediatric Clinic, Herlev Hospital; 3) Statens Serum Institut, Epidemiological Research, Copenhagen; 4) Child- and Adolescent Psychiatric Center, Copenhagen Region, Institute of Health Sciences, University of Copenhagen

Background: Mental health disorders with onset in early childhood ( $0-3$ years) is an area of increasing interest as accumulating evidence point to an early origin of mental health disorders. Prevalence of mental health disorders in this age group has been investigated in clinical and community-based studies, but to our knowledge no population-based incidence study of mental health disorders using a whole population as cohort has been carried out.

Objectives: To estimate the incidence of ICD-10 mental health disorders with onset in early childhood ( $0-3$ years of age) in the Danish population.

Methods: All children born in Denmark in the period 1.1.1997-31.12.10 were identified in the Danish Civil Registration System. From the Medical Birth Register and the Danish National
Patient Register data on pregnancy and birth characteristics and psychiatric diagnoses according to ICD-10 (all F-diagnosis, R41.8, R62, R62.0, R62.9) were obtained for the same period. The incidence rates (IR) of first diagnoses of mental health disorders in children aged 0-3 years were calculated by log-linear Poisson regression analyses.

Results: The cohort included 918.310 children aged $0-3$ years of which a total of $16.080(1.75 \%)$ were diagnosed with a mental health disorder. IR for any ICD-10 mental health diagnosis was 525 per 100.000 (95\% confidence interval (CI) 517-533) with a male:female ratio 1.4:1. The most frequent diagnoses were general developmental disorders (IR 313 per 100.000, $95 \%$ CI 306-319), specific and pervasive developmental disorders (IR 95 per 100.000, $95 \%$ CI 92-99), eating disorders (IR 60 per 100.000, $95 \%$ CI 58-63), and adjustment reactions (IR 55 per $100.000,95 \%$ CI 52-57).

Conclusions: This study confirms that mental health disorders among children aged 0-3 years in Denmark are of a significant magnitude. Neuro-developmental disorders are the most frequent, but also eating-, emotional-, behavioral- and relational-disorders are present to a degree that claims further investigation and intervention.

\section{P-152}

Using register-based data to study changes in the gender gap in substance use disorder

Topic: Diseases - Psychiatry

Presenter: Steinn Steingrimsson

Steinn Steingrimsson 1), 3); Hanne Krage Carlsen 2); Martin Ingi Sigurdsson 2); Sigmundur Sigfusson 4); Andres Magnusson 1), 2)

1) Mental health services, Landspitali the National University Hospital of Iceland; 2) Faculty of Medicine, University of Iceland; 3) Department of Psychiatry, Sahlgrenska University Hospital; 4) Department of Psychiatry, Akureyri Hospital

Introduction: In recent years, there has been a change in the gender gap of substance use disorders (SUDs), especially in societies with higher gender equality. The aim of this study was to use registerbased data to evaluate changes over a 25 -year period in the gender gap in discharge diagnoses of alcohol use disorder (AUD) and other SUDs in psychiatric inpatients.

Materials and methods: This is a register-based study of all admissions to psychiatric hospitals in Iceland between January 1983 and December 2007. The annual rate of admissions to psychiatric hospitals, adjusted for changes in the size of the population, was studied using the discharge diagnosis of SUD as a main outcome. Furthermore, gender-specific analysis of changes in discharge diagnoses of AUD solely and other SUD (including AUD with other SUDs). The changes over time were correlated using Pearson correlation coefficient.

Results: During the study period, the proportion of patients with a discharge diagnosis of any SUD in all psychiatric admissions increased considerably. This increase was most pronounced in SUDs other than solely AUD. AUD increased for women and decreased for men. The male to female ratio of AUD alone decreased from 4.2 to $1.5(p<0.001)$. There was no significant change in the gender gap for other SUDs $(p=0.96)$.

Discussion: There has been a marked convergence of the gender gap in discharge diagnosis of alcohol use disorder among psychiatric inpatients in Iceland over the last decades. For other substance use disorders, the change was not as pronounced. Our results emphasize the importance of monitoring changes in substance use disorder diagnosis as this may uncover different treatment needs in this group of vulnerable individuals. Furthermore, this study shows that the use of register data can provide excellent information to compliment traditional cross-sectional surveys. 


\section{P-153}

Intravenous use of methylphenidate in Iceland

Topic: Diseases - Psychiatry

Presenter: Steinn Steingrimsson

Gudrun Dora Bjarnadottir 1), 3); Steinn Steingrimsson 2); Magnus Haraldsson 1), 3); Bjarni Ossurarson Rafnar 1), 3); Engilbert Sigurdsson 1), 3); Helena Bragadottir 1); Magnus Johannsson 3); Andres Magnusson 1)

1) Mental health services, Landspitali the National University Hospital of Iceland; 2) Psychiatric department, Sahlgrenska University Hospital; 3) Faculty of Medicine, University of Iceland

Background: Prescription and sale of methylphenidate (MPH) has increased sharply in Iceland, as well as worldwide, in recent years. MPH is known to have abuse potential but significant intravenous use of prescription stimulants has not been previously. However, clinical experience in Iceland has indicated such use of MPH. The aim of the study was to characterize the prevalence and pattern of intravenous MPH use among treatment seeking patients with substance use disorder in a nationwide cohort.

Method: This is a cross-sectional study using a semi-structured interview designed for this study. All treatment seeking patients admitted to one of three inpatient alcohol and drug treatment programs in Iceland who reported use of any intravenous substance in the past 30 days were offered to participate. The aim was to include around 100-120 individuals representing approximately of active intravenous substance users in Iceland.

Results: A total of 102 patients were interviewed. The average age was 33 years $(\mathrm{SD}=10.3$ years $)$ and $59(58 \%)$ were men. $87 \%$ of subjects admitted to having used MPH intravenously in the last 30 days. MPH was the most commonly used substance intravenously (66 \%) and $65 \%$ reported MPH as the substance of preference in the last 30 days. For the majority of the sample, stimulants were the first drugs used intravenously ( $89 \%$ ) with $29 \%$ reporting MPH as their first intravenous drug ever used.

Conclusion: To the authors' knowledge, extensive intravenous use of MPH is reported for the first time. MPH is the most commonly reported intravenous drug used in Iceland and almost 2/3 report it as their preferred substance. It is important to further verify and characterize the pattern of intravenous MPH use. The results have implications for other countries where prescription of MPH has increased dramatically in recent years and close monitoring of both the use and misuse of MPH is recommended.

\section{P-154}

Co-occurrence of chronic musculoskeletal diseases and common mental disorders as a predictor of work disability in Finland

Topic: Diseases - Psychiatry

Presenter: Päivi Leino-Arjas

Leena Kaila-Kangas 1); Eija Haukka 1); Helena Miranda 2); Teija Kivekäs 1); Kirsi Ahola 1); Ritva Luukkonen 1); Rahman Shiri 1); Sanna Kääriä 1); Markku Heliövaara 3); Päivi Leino-Arjas 1)

1) Finnish Institute of Occupational Health, Helsinki, Finland; 2) Occupational Health Services, OP-Pohjola Group, Helsinki, Finland; 3) National Institute for Health and Welfare, Helsinki, Finland

Background: Musculoskeletal and mental disorders are the main causes of work disability pensions in Finland. Strong associations exist between musculoskeletal pain and symptoms of depression or anxiety, but little is known about the effect of the co-occurrence of clinically diagnosed musculoskeletal and mental disorders on work disability.

Objectives: We studied the independent and joint effects of chronic musculoskeletal and common mental disorders as predictors of disability pension awards in Finland.

Methods: A nationally representative sample (the Health 2000 survey) comprised 3,799 occupationally active Finns aged between 30 and 63 years. Chronic musculoskeletal diseases were assessed in a clinical examination by a physician according to a predetermined protocol, and depressive and anxiety disorders (common mental disorders) using the Composite International Diagnostic Interview. Information on disability pension awards during 2000-2009 from national pension records was linked to the data. Cox proportional hazards regression was used to estimate the risk of disability pension allowing for several potential confounders with censoring for death, other pension or the age of 65 years.

Results: The prevalence of chronic musculoskeletal disorders at baseline was $27.3 \%$, of common mental disorders $6.2 \%$, and of their co-occurrence $3.3 \%$. The hazard ratio of subsequent disability pension was 2.3 (95\% CI 1.9-2.9) for musculoskeletal disorders, 2.7 (1.8-4.0) for common mental disorders, and 5.2 (3.6-7.3) for their cooccurrence, allowing for age, gender, marital status (other) chronic diseases, BMI, smoking, leisure-time physical activity, job demands, job control, social support at work, number of physically loading work factors, occupational status, education, and household income. In gender-stratified analyses, the hazard ratio for the co-occurrence was 4.4 (2.6-7.7) for men, and 5.8 (3.6-9.2) for women, when all covariates were allowed for. Substantial modification of the hazard ratio by the covariates was found in men but not in women.

Conclusions: Workers with both chronic musculoskeletal and common mental disorders had a very high risk to be prematurely pensioned. It is important to identify subjects with both of these disorders and support their physical as well as mental work ability.

\section{P-155}

Prevalence and burden of depressive symptoms and diagnosed depression in Germany

Topic: Diseases - Psychiatry

Presenter: Markus Busch

Markus Busch 1); Ulrike Maske 1); Beate Gaertner 1); Livia Ryl 1); Ulfert Hapke 1)

1) Robert Koch Institute

Background: Data on the prevalence and burden of depression in the population provide the basis for assessing disease burden, estimating health care needs and planning preventive measures.

Objectives: To determine the prevalence and burden of depressive symptoms and diagnosed depression in the general population in Germany.

Methods: Data came from the first wave of the German Health Interview and Examination Survey (DEGS1), a population-based cross-sectional study that collected health data from a representative sample $(\mathrm{n}=7988)$ of German adults aged 18-79 years in 2009-2011. Current depressive symptoms were assessed with the Patient Health Questionnaire (PHQ-9) and diagnosis of depression within the last 12 months was assessed by standardized computerassisted physician interview. Associations between these indicators of depression and a range of health-related outcomes were analysed using multivariable regression analyses adjusted for sociodemographic characteristics and other relevant confounders. 
Results: The overall prevalence of current depressive symptoms (PHQ-9 $\geq 10$ ) is $8.1 \%$ (women $10.2 \%$, men $6.1 \%$ ). Prevalence is highest among 18- to 29-year-olds and gradually declines with increasing age. The 12-month prevalence of diagnosed depression is $6.0 \%$ (women $8.1 \%$, men $3.8 \%$ ) and is highest among 50- to 59 -year-olds. Prevalences of current depressive symptoms and diagnosed depression decrease with increasing socioeconomic status (SES). After adjusting for age, sex and SES, adults living in small towns are less likely to report current depressive symptoms or diagnosed depression, compared to larger towns, cities and rural areas. In analyses adjusted for sociodemographic factors and other relevant confounders, current depressive symptoms and diagnosed depression are associated with adverse health behaviours, higher prevalences of chronic diseases, lower self-rated health and health-related quality of life, reduced physical functioning and higher health service use.

Conclusions: Current depressive symptoms and diagnosed depression are wide-spread among adults in Germany and associated with a wide range of adverse health characteristics. The associations with health behaviours suggest the need for a multidimensional, integrated approach to prevention and care of depression.

\section{P-156}

Neurophysiological status of adolescents in different latitudes of the Russian North

Topic: Diseases - Psychiatry

Presenter: Denis Demin

Denis Demin 1), 2); Liliya Poskotinova 1), 2); Elena Krivonogova 1), 2); Andrej Grjibovski 3), 4)

1) Institute of Biomedical Research of the Northern Arctic Federal University, Arkhangelsk, Russia; 2) Institute of Environmental Physiology, Russian Acad. Sci., Ural Branch, Arkhangelsk, Russia; 3) Department of International Public Health, Norwegian Institute of Public Health, Oslo, Norway; 4) International School of Public Health, Northern State Medical University, Arkhangelsk, Russia

Background: Climate in the Russian North varies considerably by latitude from the Arctic in the Polar Regions to temperate in more Southern regions. Several studies have shown associations between indicators of the physiology of the nervous system in adolescents and climatic factors, but only a few studies from Russia have been published.

Objectives: To study functional brain activity in adolescents from two locations in the European North of Russia at two different geographical latitudes with different climatic conditions.

Methods: A cross-sectional study was conducted in two districts of the Russian European North-one in the Subpolar region $\left(64^{\circ} 30^{\prime} \mathrm{N}\right)$ and one in the Polar region $\left(67^{\circ} 40^{\prime} \mathrm{N}\right)$. Two random samples of 250 adolescents aged 14-17 years were studied. Electroencephalogram (EEG) was recorded using Encephalan-131-03" device ("Medicom MTD", Russia) from 16 standard monopolar leads. Amplitude, index and absolute spectral power in three frequency bands $(4-7,8-12$, and 13-25 Hz) were recorded for each group and compared using MannWhitney tests for continuous data and Chi squared tests for categorical data.

Results: We identified age-related decrease (from 14 to 17 years) of background values of the EEG parameters in all frequency bands (all $p<0.05)$ in adolescents from both regions. In adolescents in the Subpolar region we found intensified age optimization of neural processes and the formation of the amplitude-frequency relationship with the gradual dominance of the alpha rhythm. In adolescents of the Polar region, we revealed higher activity of diencephalic subcortical brain structures: increased levels of theta-activity $(p<0.001)$, especially in the frontal zones, marked diffuse photic driving reaction (43 vs. $17 \%, p<0.001$ ), as well as paroxysmal brain activity.

Conclusions: Our results suggest that the rate of development of cerebral bioelectric activity in adolescents may vary by between settings at different latitudes. The mechanisms behind the differences observed in the study will be discussed.

\section{P-157}

Assessment of heart rate variability biofeedback in people with the different individual psychodynamic properties

Topic: Diseases - Psychiatry

Presenter: Elena Krivonogova

Elena Krivonogova 1), 2); Liliya Poskotinova 1), 2); Denis Demin 1), 2); Andrej Grjibovski 3), 4)

1) Institute of Biomedical Research of the Northern Arctic Federal University, Arkhangelsk, Russia; 2) Institute of Environmental Physiology, Russian Acad. Sci., Ural Branch, Arkhangelsk, Russia; 3) Department of International Public Health, Norwegian Institute of Public Health, Oslo, Norway; 4) International School of Public Health, Northern State Medical University, Arkhangelsk, Russia

Background: For a more successful and efficient realization of the heart rate variability (HRV) biofeedback technology requires assessment of individual personality properties that will allow for a shorter period of time to achieve the positive effect.

Methods: A random sample of 250 healthy young persons-volunteers in Archangelsk area (age 15-19 years) were examined. HRV biofeedback sessions was carried out by the patent 2317771 RU. It was necessary with the help of breathing, muscle relaxation, positive emotional attitude to increase the total power of spectrum HRV (TP). HRV parameters were determined with the use of the device Varikard" (Russia) in the sitting position (5 min) and the biofeedback session $(5 \mathrm{~min})$. There were carry out 10 sessions of biofeedback training. There were diagnosed to individual psychodynamic characteristics on the test V.M. Rusalov (assessment of temperament) before the training - endurance, flexibility (the ease of switching from one activity to another object), tempo, emotions. There were evaluated the marks on the temperament scales-a high level (9-12 marks), the middle (4-9 marks) and lowest (0-3 marks).

Results: The people who have the high endurance temperament, flexibility and tempo, and the middle emotion, noted a persistent increase in TP (in comparison with baseline, $p=0.024$ ) from the first session and the next 10 sessions. Thus, these people have the high level of coordination of all sub-systems of the mental processes and body regulation. In individuals with the middle endurance, plasticity, tempo and emotion observed a significant increase in TP (in comparison with baseline, $p=0.031$ ) after $4-5$ sessions biofeedback training. In individuals with low values for each of the properties mentioned unstable temperament increase TP from session to session. This indicates that these peoples need more time to achieve effectiveness of biofeedback training.

Conclusions: People with such parameters as high temperament endurance, plasticity, and the middle rate of emotion have a high potential for increasing the total power spectrum of HRV by biofeedback technology through the first session of training.

\section{P-158}

Time trend of registered diagnoses of autism spectrum disorder, hyperkinetic disorder, obsessive compulsive disorder, and tourette's syndrome: a multi-national comparison

Topic: Diseases - Psychiatry 


\section{Presenter: Hjördis Osk Atladottir}

\section{Hjördis Osk Atladottir)}

Background: In the last two decades, the prevalence of registered Autism Spectrum Disorder (ASD) diagnoses has displayed an over sixfold increase. In a previous Danish study, we studied the time trend of ASD, hyperkinetic disorder (HKD), Tourette's syndrome (TS) and obsessive compulsive disorder (OCD), and concluded that the increase in cumulative incidence of registered neuropsychiatric diagnoses is a shared epidemiologic phenomenon across different neurodevelopmental disorders in Denmark. It is unknown whether the time trends observed in Denmark are replicable in other geographic settings.

Objectives: The main aim of this study is to compare the time trend of ASD, HKD, TS, and OCD across different geographical regions; Denmark, Finland, Sweden and (for ASD) Western Australia, after adding 71 years of follow-up to the original Danish study. We coordinate the study period, diagnostic codes used to define the conditions, and statistical analyses across countries.

Methods: In each country, the cumulative incidence of each disorder was analyzed using time-to-event analysis methods (Kaplan-Meier). To summarize and facilitate the interpretation of results, the analytic birth cohorts for each condition were stratified by year of birth into 3 -year intervals. Within each analytic birth cohort, follow-up time begins for all children at time of birth and continues until end of follow-up on December 31, 2011. We performed a test for trend using a Cox regression model using the birth cohort groups as a continuous variable.

Results: We observe a statistically significant increase in cumulative incidence for registered diagnoses of all four disorders across birthyear cohorts in all countries. The prevalence of the disorders varied slightly between countries. The highest prevalence of ASD, TS and OCD were observed in Denmark. Finland displayed the highest prevalence of HKD.

Conclusions: We suggest that the observed pattern of increase among different childhood psychiatric conditions could be attributable to shared factors. These shared factors are probably non-aetiological factors; such as broadening of the diagnostic concepts, diagnostic switching from other developmental disabilities to another, improved service availability, and increased awareness of neurodevelopmental problems in both the lay and professional community. A true increase in registered prevalence can, however, not be ruled out.

\section{P-159}

\section{A meta-analysis of Chronic Fatigue Syndrome/Myalgic Encephalomyelitis prevalence}

Topic: Methods - Data analysis Presenter: Samantha Johnston

\section{Samantha Johnston 1); Ekua Brenu 1); Don Staines 2); Sonya Marshall-Gradisnik 1)}

1) Griffith Health Institute, National Centre for Neuroimmunology and Emerging Diseases, Griffith University, Parklands, QLD, Australia; 2) Gold Coast Public Health Unit, Queensland Health, Robina, QLD, Australia

Background: Different methods are used to report the prevalence of Chronic Fatigue Syndrome or Myalgic Encephalomyelitis (CFS/ME). This may be a significant source of variability found in prevalence estimates.

Objectives: To perform a meta-analysis to examine variability in prevalence estimates of CFS/ME, according to method of assessment. Methods: Databases were systematically searched for studies using specific inclusion criteria. Studies primary outcome were to determine
CFS/ME prevalence in adults according to the 1994 Centers for Disease Control and Prevention (CDC) case definitions. Estimates were categorized according to their method for assessing cases: selfreporting of symptoms versus clinical verification of symptoms. Prevalence estimates were pooled according to assessment and stratified by sample setting (community or primary care) by random effects modelling. Sensitivity analysis was used to test the influence of possible outliers and heterogeneity was examined using the I2 statistic.

Results: Of two hundred and sixteen records found, prevalence estimates from fourteen studies were considered suitable for inclusion. The pooled prevalence for self-reporting assessment was $3.28 \%$ (95\% CI 2.24-4.33) and $0.76 \%$ (95\% CI 0.23-1.29) for clinical assessment. High variability was observed among self-reported estimates, while greater consistency was found among studies that involved clinical assessment in their design.

Conclusion: This study summarised CFS/ME prevalence according to the 1994 CDC case definition, which appeared to be the most widely used tool for assessment of cases. The heterogeneity observed in reported prevalence may be due to different methods used to fulfil the clinical definition of CFS/ME. Stakeholders should be cautious of prevalence based on the self-reporting of symptoms alone. Improved clinical definitions and their international adoption will also allow better comparisons of findings and inform health systems about the true burden of CFS/ME

\section{P-162}

Late response analysis on the association of household income and health complaints

Topic: Methods - Data analysis

Presenter: Gabriele Berg-Beckhoff

Gabriele Berg-Beckhoff 1); Juergen Breckkamp 2); Bernd Kowal 3); Brigitte Schlehofer 4); Maria Blettner 5)

1) University of Southern Denmark; 2) University of Bielefeld, Germany; 3) German Diabetes Center, Heinrich Heine University, Düsseldorf, Germany; 4) German Cancer Institute, Heidelberg; 5) University of Mainy, Germany

Response bias distorts prevalence estimations in cross-sectional analyses. The question should be asked what happens with association estimated with distorted prevalence's. The aim of the present analysis is to consider a late response analysis to compare prevalences of and associations between household income and health complaints. Late response analysis looks at different periods participants are answering the questionnaire.

Method: A population based cross-sectional study was carried out, embedded in a large panel survey. 30047 (response rate $58.6 \%$ ) persons took part in the nationwide study. A self-administered list of 38 health complaints revealing a higher score with more complaints was filled in; household income was assessed as an indicator of socioeconomic situation. Respondents were categorized in the three tertiles of early, middle, and late responders corresponding to when they sent the questionnaire back to the study secretary. A multiple linear regression model was fitted to estimate the association between household income and self-reported health complaints. This analysis was stratified by tertile of response time, and adjusted for age, sex, and study region.

Results: Mean health score was 51.2 (stddv: 12.4) and it did not vary between early, middle and late-responders, when considering the means, standard deviations and missing values. However, early responders had more often lower income and late responders more often higher income compared to the other groups. Overall, multiple 
linear regression showed that household income is negatively associated with health complaints. The corresponding betas increased from medium, low and very low household income compared to high household incomes from 1.0 (95\% CI: 0.6-1.4), 1.8 (95\% CI: 1.4-2.2), to 3.7 (95 \% CI: 3.1-4.2), respectively. This clear trend was less pronounced in early responders but more pronounced in late responders. This was shown in the stratified analysis for the three responder tertiles comparing the beta for very low to high income: early responder: 2.9; middle responder: 3.9; late responder: 4.6.

Conclusions: The results showed the well-known association of household income with health complaints. The association was more pronounced in late responders. Adjustment for a response bias is limited in the analysis of association, but it allows discussing different selection procedures due to response rates. Considering the fact that late responders correspond better to non-responders then the other respondents, would lead to the conclusion that the overall association of household income and heath complains might be underestimated in the present cross-sectional study design due to response bias.

\section{P-164}

Estimating prevalence of polypharmacy using data from a health administrative database: A comparison of results obtained with different algorithms

Topic: Methods - Data analysis

Presenter: Valeria Fano

Valeria Fano 1); Francesco Chini 2); Patrizio Pezzotti 2); Katia Bontempi 1); Alessandra Rossi 1)

1) Local Health Unit Roma D, Rome, Italy; 2) Agency for Public Health of Lazio Region, Rome, Italy

Background: To date only few studies have been carried out to estimate prevalence of polypharmacy in the general population using administrative databases. Different methods and definitions were proposed, but no comparisons were provided.

Objectives: To estimate prevalence of polypharmacy with different algorithms using a prescription database.

Methods: Adults $(35+; \mathrm{n}=331,923)$ residents in 2008 in the Local Health Authority 'Roma D' (southern part of Rome) were included in the study population. Prescriptions (years 2009-2010) were retrieved from a database collecting information on all drugs prescribed by GPs and public ambulatories, including patients' demographics characteristics, code of the Anatomical Therapeutic Chemical Classification System (ATC), number of packages, date of prescription and days of therapy. Polypharmacy was defined as minor (2-3 different drugs), moderate (4-5), major ( $>5)$. We initially calculated the number of different drugs (ATC codes) prescribed for at least 60 days of therapy per year (Algorithm 1). We then considered the number of drugs prescribed for at least 60 days per quarter per year (i.e., fixed time windows from January to March, and so on; Algorithm 2). Finally, a 90 days-mobilewindow was used (i.e., from January to March, from February to April, and so on; Algorithm 3). All analyses were stratified by gender and age class (above/below 65 years) and all algorithms were tested considering up to 4 and 6 digits of ATC code.

Results: Estimates of polypharmacy for year 2009 (4-digits-ATC) showed substantial differences among algorithms. Algorithm 1 detected more subjects that received $\geq 2$ drugs $(+19$ and $+13 \%$ vs. Algorithm 2 and 3, respectively). Moreover, Algorithm 1 showed a higher prevalence of $>5$ drugs $(8,5$, and $6 \%$ with Algorithm 1,2 and 3 , respectively; $p<0.001$ for each comparison). When stratifying by gender and age, differences between the algorithms in females and in older subjects were more marked. Similar results were obtained for year 2010, and using 6-digits-ATC.

Conclusions: Our study yields similar results to those reported by Veheof et al. (2000) where a prevalence of $5 \%$ patients using $>5$ drug was reported, using the same method as Algorithm 2 with 4-digits-ATC. As expected, Algorithm 1 provided higher estimates of the prevalence of polypharmacy, as single drugs used in different periods of the year are considered as overlapping with this method. The other two algorithms seem more appropriate to identify overlapping drugs; however, Algorithm 3 using a mobile time window is more sensitive compared to Algorithm 2.

\section{P-166}

Comparison of In-Hospital and 30-Day Mortality after Hospitalization for Acute Cardiovascular Events

Topic: Methods - Data analysis Presenter: Martin Berg Johansen

Martin Berg Johansen 1); Christian Fynbo Christiansen 1); Henrik Toft Sørensen 1); Lars Pedersen 1)

1) Department of Clinical Epidemiology, Aarhus University Hospital, Aarhus, Denmark

Background: Short-term outcome of acute cardiovascular events is reported as either in-hospital mortality or mortality at 1 month from admission. In many studies, only in-hospital mortality data are available. Data on the correlation between the two measures are few. Objectives: We examined the difference between in-hospital and 30-day mortality in patients with common acute cardiovascular events.

Methods: We conducted this nationwide cohort study in Denmark among patients with an acute hospital admission for cardiovascular events in 2006 through 2010. We included all patients with ruptured abdominal aorta aneurysm, pulmonary embolism, myocardial infarction, subarachnoidal hemorrhage, ischemic stroke, or congestive heart failure.

We computed in-hospital and 30-day mortality risk as the proportion of patients dying according to each measure. We also computed the ratio of these two types of estimates.

We examined the impact of the choice of outcome measures by computing the unadjusted risk ratio (RR), using log-binomial regression, to compare patients aged over versus below 70 years as an example of an exposure.

Results: 115,274 patients were included in the analysis. In-hospital mortality risk ranged from $8.5 \%$ (30-day mortality risk: $9.3 \%$ ) among patients with ischemic stroke to $56.8 \%$ (30-day mortality risk: $55.3 \%$ ) among patients suffering from ruptured abdominal aorta aneurysm.

As expected, in-hospital mortality was considerably lower than 30-day mortality for patients suffering from congestive heart failure (in-hospital/30-day mortality ratio: 0.79 (95\% CI 0.78-0.81)), but also slightly lower for patients suffering from pulmonary embolism, myocardial infarction, or ischemic stroke (in-hospital/30-day mortality ratio around 0.9 ). Ruptured abdominal aorta aneurysm and subarachnoidal hemorrhage had an in-hospital/30-day mortality ratio slightly above 1 .

The RRs based on 30-day mortality were larger than the estimates based on in-hospital mortality for all diseases except pulmonary embolism. The largest difference was seen for the ischemic stroke (inhospital RR: 2.94 (95\% CI 2.68-3.22), 30-day RR: 3.43 (95\% CI 3.13-3.77)).

Conclusions: We found that the two types of outcome measures were different for several types of acute cardiovascular events. 


\section{P-167}

Length of the look-back period and prevalence of captured comorbidity in the Danish National Registry of Patients

Topic: Methods - Data analysis

Presenter: Morten Madsen

Morten Madsen 1); Vera Ehrenstein 1); Henrik Toft Sørensen 1)

1) Department of Clinical Epidemiology

Background: Charlson Comorbidity Index (CCI) is an important tool for describing and modelling morbidity in epidemiologic studies. The Danish National Registry of Patients (DNRP) contains data on visits to Danish hospitals since 1977, including renewed registration of diagnoses in case of readmission. The DNRP is frequently used to compute CCI in epidemiologic studies. When using the DNRP for measuring comorbid diseases, the length of the look-back period may affect the completeness of the ascertainment. However, there is little quantitative evidence about the relation between the length of the look-back period and the completeness of comorbidity ascertainment. Objectives: We examined the association between the length of the look-back period and the sensitivity of capturing the presence of the 10 most prevalent chronic comorbid diseases from CCI in the DNRP. Methods: We included all patients registered in the DNRP with firsttime hospitalisation for myocardial infarction (MI) in 2010. For these patients, we identified all inpatient, outpatient, and emergency-room registrations with diagnoses of the 10 comorbid diseases from 1977 until the MI diagnosis in the DNRP. To determine the sensitivity of registration in 1-, 5- and 10-year look-back periods, we compared proportion of patients with registration in each of the periods with the registrations during the maximum available ascertainment period (since 1977, up to 34 years). The maximum period was assumed to have the most complete measure of comorbidity.

Results: Among the 8,704 patients, $61 \%$ were men. The age range at MI was 0-104 years, median 70 years (quartiles, 59-80). The proportion of patients identified with at least one comorbid disease was $20 \%$ for a 1 year look-back period prior to the MI, $36 \%$ for a 5 year look-back period, $43 \%$ for a 10 year look-back period compared with $49 \%$ for a maximum look-back period. Thereby only $40 \%$ of the total patients with comorbidities ascertained over 33 years were identified with a 1 year look-back period. The proportion of the underascertained events varied by disease type. For the 1-year look-back period, sensitivity ranged from $15 \%$ (for ulcer) to $50 \%$ (for moderate to severe kidney disease). For the 5-year look-back period, sensitivity ranged from $38 \%$ (for ulcer) to $79 \%$ (for moderate to severe kidney disease).

Conclusions: The proportion of patients with 10 most prevalent chronic diseases in the Charlson Comorbidity Index was directly related to the length of the look-back period, and varied by disease type.

\section{P-168}

\section{Incidence percentile}

Topic: Methods - Data analysis

Presenter: Nicola Orsini

\section{Nicola Orsini 1); Matteo Bottai 1) \\ 1) Unit of NutritiInstitute of Environmental Medicine, Karolinska Institutet}

Background: The distribution of the time to some event of interest (e.g. death, myocardial infarction, relapse) is commonly summarized in terms of incidence rates or cumulative incidence, but percentiles of the time to event, or simply incidence percentiles (IPs), are being used in an increasing number of prospective epidemiological studies.

Objectives: Our aim is to show the relation between IPs and traditional incidence measures and to present the potential advantages offered by IPs.

Methods: The $p$-th IP (e.g. incidence median) is the time point $t$ that splits the distribution of time to the event of interest in two parts: $p$ percent $(50 \%)$ of the population experience the event no later than time $t$ and the other $(1-p)$ percent $(50 \%)$ of the population experience it after time t. The $p$-th IP, conditionally on exposure and covariates, can be estimated using Laplace regression.

Results: We consider a large population based cohort of 72,522 Swedish middle-aged and elderly men and women. We analyze the time from entry into the study (January 1, 1998) until death or end of the follow-up (December 31, 2010) whichever occurred first. Censoring occurred only at the end of the study period. During 13 years of follow-up 11,681 (16\%) participants died. The 16th IP is 13 years. At baseline, a person randomly selected from this population had a $16 \%$ probability of dying within 13 years or, equivalently, an $84 \%$ probability of surviving 13 years. The rate of death within the first 13 years of the study period $(11,681$ deaths divided by 882,544 person-years) can be approximated by the 16th IP simply as $\mathrm{p} /(\mathrm{p}$-th IP) $=0.16 / 13=0.01,1$ death every 100 person-years. We can estimate any IP below the 16th IP and characterize survival over the whole follow-up interval or in any subinterval, but no data are available to estimate higher IPs (17th IP to 99th IP).

Conclusions: The IP makes explicit the link between an incidence proportion and an incidence time. Traditional measures of disease frequency such as incidence rates can be derived from IPs. More importantly, the IP can sometimes facilitate the interpretation and communication of research findings to the general public.

\section{P-169}

Geo-epidemiology of tobacco smoking in relation to twelve tobacco control measures. Are there any hot spots among the European countries?

Topic: Methods - Data analysis

Presenter: Dimitra Sifaki-Pistolla

Dimitra Sifaki-Pistolla 1); Georgia Pistolla 2)

1) Epidemiologist, PhD student, School of Medicine, University of Crete; 2) Mathematician, $\mathrm{PhD}$, School of Medicine, University of Crete

Background: Tobacco use is one of the major premature causes of death worldwide.

Objectives: Purpose of the present study is to examine the main control measures and indentify their preventive or risky effects, in order to determine the various risk areas for any daily tobacco use among the European countries.

Methods: Data were selected from the World Health Organization for the years 2009. Daily smoking of any tobacco product (DSTP) and other 12 factors referring to tobacco control were used as well. The final sample consisted of 45 European countries. Spatial statistics were performed in Arcmap 10 (GIS). Moran's I and OLS spatial regression model were applied for the detection of spatial correlations and the high risk areas.

Results: High spatial heterogeneity was demonstrated through the SDTP distribution. Greece and Austria have the highest rates (38-49 \%), Bulgaria, Albania, Bosnia Herzegovina, and Russia follow with $30-37 \%$. Three out of the twelve factors examined were found to be random. The rest of them were used in the regression model, highlighting the high risk areas. Greece presented the highest 
risk (Exp B $=2.9 ; 95 \%$ CI 2.183-3.326), Bulgaria, Turkey, Serbia and other countries followed (Exp B $=2.7 ; 95 \%$ CI 1.937-3.013), whereas Finland, Norway and UK proved to be of no present risk (Exp B $=0.76$; $95 \%$ CI 0.258-0.936).

Conclusions: Geo-epidemiology of tobacco monitors the phenomenon's dynamics, effectively. It offered an insight about applying measures, co-examining for the first time, the effects of twelve measures of tobacco control as possible preventive or risk factors.

\section{P-170}

Clinical and pharmacovigilance approaches for exploring an electronic medical record dataset of Type II diabetics and thiazolidinedione drug safety

Topic: Methods - Data analysis

Presenter: Melissa Davidson

Melissa Davidson 1); Douglas McNair 2); Donald Mattison 3), 4); Daniel Krewski 1), 3), 4), 5)

1) Institute of Population Health, University of Ottawa, Ottawa, Canada; 2) Cerner Corporation, Kansas City, USA; 3) McLaughlin Centre for Population Health Risk Assessment, Ottawa, Canada; 4) Risk Sciences International, Ottawa, Canada; 5) Faculty of Medicine, University of Ottawa, Canada

Background: Patient data collected through HealthFacts electronic medical records (EMR) provide a means for exploring patient characteristics, drug use and effectiveness of treatment. Such datasets also provide an advantage over passive datasets in investigating adverse drug reactions where other studies have shown conflicting results. For example, thiazolidinediones (TZDs), a class of drugs used alone, or in combination with other medications, to treat Type II diabetes (TIIDM), have been associated with cardiovascular health risks in some studies but not others and warrants further investigation.

Objectives: To determine characteristics of, and treatment regimes for, TIIDM patients in a large longitudinal dataset and investigate associations between TZDs and adverse cardiovascular events.

Methods: A retrospective cohort study was conducted using an American EMR dataset of TIIDM patients. Characteristics of patients were quantified including biochemical markers (glycosylated hemoglobin $[\mathrm{HbA} 1 \mathrm{c}]$, glucose measurements), comorbidities and drug treatments (alone or in combination). Effectiveness of treatment was measured by examining mean change in biochemical markers preand post-treatment. Hazard ratios (HR) and $95 \%$ Confidence Intervals $(\mathrm{CI})$ were calculated for heart failure (HF) and myocardial infarction (MI) for patients taking TZD drugs using Cox regression. Results: Among the TIIDM patients, 111,646 were treated with one drug and 104,937 with combination therapy. Mean age of patients was approximately 65 years, half were male and the majority were Caucasian. Combination therapy patients had a greater length of follow-up (1,024 vs. 825 days), though number of comorbidities were similar in both groups. Most patients were treated with insulin alone and number of patients decreased as number of drugs in a treatment regime increased. Greatest mean change in glucose and $\mathrm{HbAlc}$ levels were observed in patients taking insulin alone $(42.62 \mathrm{mg} / \mathrm{dL}$ and $0.23 \%$ ). For patients taking TZD drugs an increased risk was observed for HF (HR 1.52; $95 \%$ CI 1.48, 1.57) and MI (HR 1.78; $95 \%$ CI 1.39, 2.27).

Conclusions: The use of a large longitudinal dataset provided a detailed overview of patient characteristics and drug use that can help inform benefits and risks in a clinical setting and demonstrated increased cardiovascular risks for patients taking TZD drugs.
P-172

Work-unit measures of effort-reward imbalance and risk of depression-a 2-year cohort study

Topic: Methods - Data analysis

Presenter: Matias B. Grynderup

Matias B. Grynderup 1); Ole Mors 2); Åse M. Hansen 3); Jens P. Bonde 4); Anette Kærgaard 5); Linda Kærlev 6); Sigurd Mikkelsen 4); Reiner Rugulies 7); Jane F. Thomsen 4); Henrik A. Kolstad 1)

1) Department of Occupational Medicine, Danish Ramazzini Centre, Aarhus University Hospital; 2) Centre for Psychiatric Research, Aarhus University Hospital; 3) Department of Public Health, University of Copenhagen; 4) Department of Occupational and Environmental Medicine, Bispebjerg University Hospital; 5) Department of Occupational Medicine, Danish Ramazzini Centre, Regional Hospital Herning; 6) Center for Clinical Epidemiology, Odense University Hospital; 7) National Research Centre for the Working Environment

Background: The effort-reward imbalance model describes when work-related efforts are not compensated by adequate rewards in term of esteem, job security, financial gains, and career opportunities. High levels of effort combined with low levels of reward have been suggested as a risk factor of depression.

Objectives: The aim of this study is to analyse if effort-reward imbalance at work, analysed as aggregated workplace means, increases the risk of depression.

Methods: A total of 4,237 non-depressed Danish public employees within 378 different work units were enrolled in 2007. Mean levels of effort, reward, and effort-reward imbalance (effort score divided by reward score) were computed for each work unit to obtain exposure measures that were robust to reporting bias which may have biased earlier studies relying on individual reports. Two years later in 2009, $3,047(72 \%)$ participated at follow-up and those reporting high levels of depressive, burnout or stress symptoms went through a psychiatric interview by which 58 cases of new onset depression were diagnosed. Results: Depression odds ratios by work unit level of effort, reward, and effort-reward imbalance were estimated by logistic regression taking established risk factors into account. Scoring in the lowest tertile of reward (adjusted odds ratios of 4.38, $95 \%$ CI 1.74-11.04) predicted the onset of depression. Scoring in the highest tertiles of effort $(0.65,0.30-1.39)$ and effort-reward imbalance (1.79, 0.80-3.99) did not predict depression.

Conclusion: Our results indicate that a work environment characterized by low reward is a risk factor for depression, but that high effort and an imbalance between effort and rewards are not associated to depression.

\section{P-173}

The crossing database of AIDS: challenges for a system of quality information

Topic: Methods - Data analysis

Presenter:

Cláudia Helena Soares de Morais Freitas 1); Maria Helena

Rodrigues Galvão 1); Lucélia Lourenço Cassemiro 1); Mailza Gomes de Oliveira 2); Ivoneide Lucena Pereira 2)

1) Paraiba Federal University; 2) Department of Public Health of Paraiba 
Background: In Brazil, the AIDS is a harm of compulsory notification. All cases should be duly notified in Information System for Notifiable Diseases (SINAN), that controls the registration and processing such data in Nationwide, providing information for analysis of the morbidity and contributing thereby, for decision-making at the level municipal, state and federal. Besides SINAN other systems offer subsidies for the analysis and epidemiological characterization of AIDS in Brazil, namely the Information System on Mortality (SIM) and the Information Logistics Control System of Drugs (SICLOM). The existence of failures in the confluence of these banks results in bad quality of available information.

Objectives: The study aimed to describe the challenges found in the process of qualification of information about AIDS in the Paraíba state of Northeast of Brazil.

Methods: Were fulfilled a cross of banks SINAN and SICLOM, provided by the Department of Computing the SUS - Datasus, the Ministry of Health verifying patients enrolled in antiretroviral therapy referral service for treatment of AIDS in the state of Paraíba and reported cases AIDS obtained in SINAN in the state of Paraíba. To perform the cross were used the following user data in both databases: patient's full name, mother's full name and date of birth.

Results: Among the 2520 registered users on antiretroviral therapy in reference service $549(21.7 \%)$ were not reported in SINAN, $26(1 \%)$ the users found themselves to register with the release of the drug doubled in SICLOM and $27(1 \%)$ users were notified twice for the offense in SINAN AIDS.

Conclusions: Epidemiological vigilance should seek to improve the quality of information with the use of all available data sources. There was duplication of information on banks, and underreporting, pointing to the need for the Health Department of the State of Paraíba, Northeast of Brazil endeavor to implement a policy of information management and training of its professionals, and encourage professional working with diagnosis and treatment of disease carry the notification of all confirmed cases. The qualification system information on AIDS may contribute to the reduction of underreporting, allowing a better understanding of the magnitude of the AIDS epidemic and thus public policy toward the problem may bring greater benefits to the population.

\section{P-174}

Comparison of intervention and cohort study of dietary protein in body weight regulation: reconciling contrasting results?

Topic: Methods - Data analysis Presenter: Mikkel Z Ankarfeld

Mikkel Z Ankarfeldt 1); Lars Ängquist 1); Tanja Stocks 1); Marianne U Jakobsen 2); Kim Overvad 2); Wim HM Saris 3); Arne Astrup 4); Thorkild IA Sørensen 1)

1) Institut of Preventive Medicine, Bispebjerg and Frederiksberg Hospitaler, Region H; 2) Section for Epidemiology, Department of Public Health, Aarhus University; 3) NUTRIM School for Nutrition, Toxicology and Metabolism, University of Maastrich; 4) Department of Nutrition, Exercise and Sports (NEXS), Faculty of Science, University of Copenhagen

Background: Physiological evidence indicates that high protein diet may increase satiety, thermogenic response and decrease subsequent caloric intake. Thus diet high in protein might prevent weight gain and weight-regain following weight loss. Clinical trials, including the Diet, Obesity and Genes trial (DiOGenes), show such effect. Largescale, long-term, observational cohort studies suggest the opposite effect i.e. higher protein diets may increase body weight. Discrepancy between trials and cohort studies could be that individuals recruited for trials are a subset of the population in cohort studies important for the effect of protein.

Objectives: The aim of this study is to investigate explanations of the discrepancy by analyzing different subsets on participants in a cohort study.

Methods: Subsets $(\mathrm{n}=1872)$ of the Diet, Cancer and Health (DCH) cohort $(n=57.055)$ comparable with the DiOGenes trial $(n=548)$ was selected based on inclusion criteria of DIOGenes, matching DCH-participants with DiOGenes-participants on exposure and different possible effect-modifiers and finally analyzed similar to the DiOGenes trial.

Results: When matching on intake of protein, carbohydrates, glycemic index and on body fat $\%$ a high protein intake was associated with better weight maintenance compared with low protein intake indicating similar effect as the DiOGenes trial. However, the association of protein on weight change did depend on matching criteria and the statistical analysis.

Conclusions: The association between dietary protein intake and subsequent body weight changes appears to depend on the selection characteristics of the participants and the statistical analysis, which may reconcile the otherwise contrasting evidence from physiological and clinical human experiments and large-scale cohort studies.

\section{P-175}

Exploring multiple imputation with time-dependent covariates in survival analysis: Body Mass Index and total knee replacement

Topic: Methods - Data analysis

Presenter: David Culliford

\section{David Culliford 1)}

1) Faculty of Medicine, University of Southampton

Background: Total knee replacement (TKR) is an effective intervention in patients with end-stage knee osteoarthritis. The proportion of obese patients undergoing TKR in the UK is increasing. Time to event models for knee replacement failure often incorporate body mass index (BMI) as explanatory data, but its use as a time-dependent covariate within large clinical databases of routinely collected data has not been widely explored.

Objectives: We describe a pragmatic application of multiple imputation using incomplete BMI data with long follow-up from population-level UK general practice data. We seek to show that it is possible to use such data as a time-dependent covariate, to multiply impute this data and to estimate its effect on time to event in an extended Cox regression model of time to knee revision surgery. We compare point and interval estimates of BMI as an annualised, imputed, time-dependent covariate with fixed covariate estimates.

Methods: We use data from the UK General Practice Research Database (GPRD), identifying all subjects $(\mathrm{N}=46957)$ undergoing TKR between 1991 and 2011, with pre-operative BMI. Post-operative BMI values may also be recorded. For each subject, we take the BMI recorded on the nearest date to the annual anniversary after TKR and then perform multiple imputation on these "nearest regularised" BMI variables. We use PROC MI in SAS with extended Cox regression as our substantive model. We also use alternative methods to make use of the time-dependent BMI data: (1) Joint modelling of longitudinal and survival elements (package JM in R) (2) Twofold Fully Conditional Specification (FCS) multiple imputation in Stata.

Results: Using multiply imputed annualised value (nearest observed within 6 months of the anniversary of a subject's TKR operation), BMI had an estimated hazard ratio (HR) of $0.997(95 \% \mathrm{CI}$ 0.969-1.025) after adjusting for age (HR 0.970, $95 \%$ CI 0.956-0.984) and gender (HR 0.602, $95 \%$ CI $0.451-0.804$ ). This 
contrasts with a previous analysis without imputation which used only observed, pre-operative BMI (HR 1.017, 95 \% CI 1.005-1.029). Joint modelling attempts using time-dependent BMI with exact dates as originally recorded (i.e. no imputation nor "annualisation") were unsuccessful due to computational difficulties. Twofold FCS multiple imputation was more successful, but early results require more verification.

Conclusions: This exploratory work has no theoretical backing, but early results generate interesting questions and highlight difficulties in imputing, fitting and interpreting routinely, irregularly recorded timedependent data within medical research databases which clinicians consider potentially important in explaining time-to-event outcomes.

\section{P-176}

\section{Matched survival data in discordant family designs}

Topic: Methods - Data analysis

Presenter: Mia Madsen

\section{Mia Madsen 1); Per Kragh Andersen 2); Mette Gerster 3)}

1) Unit of Epidemiolgy, Institute of Public Health, University of Southern Denmark; 2) Section of Biostatistics, Instistute of Public Health, University of Copenhagen; 3) Unit of Biostatistics, Insitute of Public Health, University of Southern Denmark

Background: Twin and sibling data have increasingly been used to address research questions in social- and life course epidemiology where the influence from early life factors on exposure-outcome relationships can be complicated. The discordant family design assumes that twins or siblings, who have been reared together, are matched on early environment and partly or fully on genetic setup. This implies that a number of family-level factors are held constant within pairs, thus providing less biased estimates of effect.

Many analyses have focused on binary or continuous outcomes, using models such as conditional logistic regression and fixed- or random-effects linear regression models. However, when dealing with matched survival data the recommended course of action seems less clear in the literature.

One approach is the stratified Cox regression model where factors shared by twins/siblings are incorporated in a pair-specific baseline hazard that is allowed to vary freely between pairs without any distributional assumptions. However, only pairs who are discordant on exposure and with the shortest waiting time being non-censored are informative.

As an alternative, the shared frailty model has the advantage of being able also to make use of information from concordant pairs. Shared factors are included in a shared frailty which is assumed to follow a gamma or log-normal distribution. An additional assumption of the model is that exposure is independent of the frailty.

Objectives: To compare the validity of a stratified Cox model and a shared frailty Cox model by means of a simulation study.

Methods: In all simulated scenarios, data were generated from the model hij(tl i) $=h 0(t) i \exp \left({ }^{\prime} z i j\right), i=1, \ldots, n ; j=1,2$ where the $i$ were latent (frailty) variables shared within twin pairs, thereby generating dependence of the waiting times for the twins. Simulation scenarios were defined according to: Population size, frailty variables drawn from a log-normal or gamma distribution, dependence or independence between the exposure and latent variable.

Results: The stratified Cox model yielded unbiased results in all scenarios, but had larger standard errors than the shared frailty model. When the exposure variable and the frailty variable were correlated (i.e. familial confounding), estimates were substantially biased in the shared frailty model.

Conclusions: The assumption of independence between the latent variable and exposure seems to be crucial in the shared frailty model, and estimates were severely biased when violated. Since discordant family designs are often applied to address potential familial confounding, the stratified Cox model seems preferable when analyzing matched survival data.

\section{P-177}

\section{Measures of discriminatory accuracy in multilevel analysis}

Topic: Methods - Data analysis

Presenter: Philippe Wagner

\section{Philippe Wagner 1); Juan Merlo 1)}

1) Unit for Social Epidemiology, Faculty of Medicine, Lund University, Skåne University Hospital

Background: During the last years we have been promoting the use of measures of variance and clustering, such as the median odds ratio (MOR) and the intra-class correlation (ICC), for the estimation of "general contextual effects" (GCE) in multilevel analyses. Knowledge on GCE is necessary for the interpretation of measures of association between specific contextual variables and individual outcomes; that is, specific contextual effects (SCE). These ideas appear to be similar to the use of measures of discriminatory accuracy versus the odds ratio in gauging the performance of new risk factors and biomarkers in epidemiology that are currently promoted by Pepe et al. and others. In fact, the ICC measure we are using in social epidemiology is, under certain conditions, equivalent to a measure of discriminatory accuracy.

Objectives: We aim to produce measures of discriminatory accuracy (e.g., ROC curve, area under the curve (AUC)) for multilevel random effects (second level variance) and thereby develop new measures of GCE for contextual units (e.g. neighborhoods, hospitals). These measures will have an interpretation similar to those used for gauging discriminatory accuracy in studies of new risk factors and biomarkers. Methods: We examine the distributions of the random effects among cases and non-cases in different scenario by means of theoretical investigation, simulation studies and in an empirical multilevel analysis on hospital differences in mortality following incident breast cancer diagnoses (BCS) in Sweden 2005-2012. The distributions are subsequently used to produce ROC curves and corresponding AUC measures. We also illustrate a mathematical connection between the AUC and ICC of a random effect under certain conditions.

Results: We found several scenarios were the ICC can be directly translated into a ROC and AUC measure. For others an empirical estimate could be created. In the case of mortality after a BCS in Sweden, the hospital GCE was rather high $(\mathrm{ICC}=0.30$, AUC $=0.75$ ). Conclusions: Measures of discriminatory accuracy can easily be applied to quantify contextual variance and estimate GCE. They provide a nice alternative to the MOR and ICC used in social epidemiology. Concerning mortality after breast cancer in Sweden, we found a rather high hospital GCE, possibly due to patient mix. That is, a fair amount of the individual variation was at the hospital level so the treating hospital might be a relevant determinant of survival after a breast cancer in Sweden.

\section{P-178}

Using multi-state models in the evaluation of a peritoneal dialysis program

Topic: Methods - Data analysis

Presenter: Denisa Mendonça

Laetitia Teixeira 1); Anabela Rodrigues 2); Denisa Mendonça 3) 
1) PDMA - Institute of Biomedical Sciences Abel Salazar and Faculty of Sciences, University of Porto; 2) Nephrology Department (CHPHGSA) and Institute of Biomedical Sciences Abel Salazar, University of Porto; 3) Population Studies Department - Institute of Biomedical Sciences Abel Salazar and Public Health Institute, University of Porto

Background: Competing risks situations are sometimes considered the rule rather than the exception in epidemiological and clinical follow-up studies. It is generally held that better understanding of a disease process will lead to more appropriate treatment and disease management and multi-state statistical models are particularly useful for this analysis. Multi-state models can be used in competing risk studies, when subjects are observed over time and can experience several events. The states can describe conditions like healthy, diseased, diseased with complication and dead. A change of state is called a transition or an event. States out of which transitions are modeled are called transient states and absorbing states are states out of which transitions are not possible (e.g. death).

Objectives: The main objective of this study is to discuss multi-state models and to estimate the effect of prognostic factors on the transition rates using an example in the area of a peritoneal dialysis. Methods: The sample comprises patients, included in a university hospital peritoneal dialysis program, between October 1985 and June 2011 ( $\mathrm{n}=444)$. A multi-state model, with peritonitis as transient state and death, transfer to haemodialysis and renal transplantation as absorbing states, was performed to analyze the effect of prognostic factors (gender, age and diabetes) on the transition rates.

Results: Seven transitions were considered in this multi-state model: peritonitis, death with and without peritonitis, transfer to haemodialysis with and without peritonitis and renal transplantation with and without peritonitis. Analyzing the effect of prognostic factors on the transition rates in multi-state model, the most important findings were: older age was associated with higher rates of peritonitis and death (before and after peritonitis); diabetic patients presented significantly higher rates of death, having or not experienced a peritonitis episode. All others effects were found not to be statistically significant.

Conclusions: A correct statistical analysis using advanced statistical methods and a clear interpretation of epidemiological and clinical data will help to improve diseases surveillance and quality control of therapy strategies. Multi-state modeling offers a flexible tool for the study of covariate effects on the various transition rates. These models describe the disease/recovery process of patients in more details, thus yielding more insight into the disease management. This methodology provides an intuitive way of examining the disease process from a dynamic perspective and can be beneficial in the analysis of relevant predictors.

\section{P-179}

\section{Adjusting for a collider can be a sin worth committing: an example from perinatal epidemiology}

Topic: Methods - Data analysis

Presenter: Olga Basso

\section{Olga Basso 1); Allen J Wilcox 2); Clarice R Weinberg 3)}

1) Dept. of Obstetrics and Gynecology \& Dept of Epidemiol, Biostats, Occ Hlth, McGill University, Montreal, QC (Canada); 2)

Epidemiology Branch NIEHS (NIH. DHHS), Durham, NC (USA); 3) Biostatistics Branch NIEHS (NIH. DHHS), Durham, NC (USA)

Background: It has been shown that, when attempting to estimate the direct effect of a prenatal exposure on neonatal death, adjustment for length of gestation can cause serious "collider" bias if strong unmeasured factors influence gestational length and account for a large fraction of mortality. When analyzing birth weight, investigators routinely adjust for gestational length, to remove the strong effect of early delivery on weight at birth.

Objectives: To examine the extent to which inclusion of gestational length in linear regression models (in the presence of unmeasured factors) biases the estimated effect of a given exposure on birth weight.

Methods: We simulated populations of 500,000 babies, each having a target birth weight and a target duration of gestation, representing the birth weight and length of gestation that a baby would achieve if no other factor intervened. Both are assigned based on independent normal distributions (mean birth weight: $3,500 \mathrm{~g}( \pm 450)$ at 40 weeks; mean gestational length: 280 days $( \pm 10)$ ). Three factors $(E, U 1$, and $\mathrm{U} 2$ ), dichotomous and independent of one another, decrease both target birth weight and gestational length. E, a measured exposure, affects $2.5 \%$ of babies and reduces gestational length by 20 days $( \pm 7)$ and birth weight by $270 \mathrm{~g}( \pm 30)$. U1 and $\mathrm{U} 2$, the "unmeasured" factors, decrease gestational length by $50( \pm 15)$ and $35( \pm 10)$ days, respectively and birth weight by variable amounts. We applied Gardosi's proportionality formula (1995) to derive, for each simulated baby, the birth weight achieved at the attained gestation after including the effects of the above factors. We then estimated the effect of $\mathrm{E}$ on birth weight through linear regression, comparing a "full" model (including a cubic function of gestational, E, U1, U2, and their cross-products with gestational age) with a model ignoring $\mathrm{U} 1$ and U2. For each scenario, we averaged the estimates from 10 iterations.

Results: Even with rather extreme effects of U1 and U2 (e.g., both having a frequency of $4 \%$, and reductions in birth weight of 1,000 and $600 \mathrm{~g}$, respectively), the estimate adjusted for gestational length omitting $\mathrm{U} 1$ and $\mathrm{U} 2$ resulted in modest bias (10\% or less) compared with the (unbiased) estimate of the effect of $E$ from the full model. Conclusions: When continuous birth weight is the outcome, adjustment for gestational length does not appear to cause substantial bias, even when factors having a strong effect on both duration of gestation and birth weight are omitted from the model.

\section{P-180}

Household income as a measure of wealth: an appraisal of the benefits of equivalization in epidemiological research

Topic: Methods - Data analysis Presenter: Kate Timmins

Kate Timmins 1); Claire Hulme 2); Janet Cade 1)

1) Nutrition Epidemiology Group, School of Food Science \& Nutrition, University of Leeds; 2) Academic Unit of Health Economics, Leeds Institute of Health Sciences, University of Leeds

Background: Including an indicator of socioeconomic status (SES) in epidemiological studies is crucial for considering health inequalities. Composite measures of SES attempt to account for the multiple facets of SES, such as education and occupation; however these variables are not always available and are often not generalizable. Household income is commonly used as a representation of SES as it gives an indication of wealth, yet it is not comparable across different household compositions. Equivalization accounts for these household differences, and is commonplace in poverty research and national figures, but seldom employed in nutritional epidemiology.

Objectives: This study aimed to establish the potential benefits of equivalization over household income, using adult data from a representative UK national survey, the National Diet and Nutrition Survey (NDNS). This exploration was framed in the context of an investigation into income differences in body mass index (BMI). 
Methods: Household income category was obtained by interview. Equivalized income was derived from the midpoint of each category using the modified OECD scale, then categorised to match household income classifications. Cross-tabulation identified the impact of equivalization. Univariate analyses examined differences in BMI and obesity prevalence between income groups. For each income variable, adjusted regression examined its association with BMI, and logistic regression its relationship with obesity. Underweight participants were excluded.

Results: Following equivalization, 376 participants (42\%) were categorised differently. Mean BMI was $27.7 \mathrm{~kg} / \mathrm{m}^{2}(\mathrm{SD} 5.4, \mathrm{n}=834)$ and differed between equivalized $(p=0.019)$ but not household $(p=0.065)$ income categories. The proportion of the sample obese was $28 \%$; this differed significantly between both equivalized $(p=0.003)$ and household $(p=0.025)$ income groups. Linear regression revealed no association. Logistic regression found no overall association; however, the odds of being obese were significantly lower in the highest equivalized category compared to the lowest (OR $0.53,95 \%$ CI $0.29,0.97, p<0.05$ ). Odds ratios of extreme household income categories, on the other hand, were not appreciably different.

Conclusions: In this study, equivalizing income clarified the contrast between income groups in a univariate comparison of BMI, and in logistic regression. In other analyses equivalization did not confer additional insight. The reasons for this are unclear, but may be due to a possible non-linear relationship: previously published studies, for example, have reported a non-linear relationship between equivalized income and self-reported health. Further investigation into income and BMI using non-linear methods may be informative. Equivalized income presents an easily measured wealth metric where composite measures of socioeconomic status are unavailable.

\section{P-181}

Using multiple imputation to deal with missing data and attrition in longitudinal studies with repeated measures of patient-reported outcomes

Topic: Methods - Data analysis

Presenter: Morten Frydenberg

\section{Karin Biering 1), 2); Niels Henrik Hjøllund 3), 4); Morten} Frydenberg 5)

1) Department of Occupational Medicine, Regional Hospital West Jutland, Denmark; 2) Danish Ramazzini Centre; 3) VestKronik, Regional Hospital West Jutland, Denmark; 4) Department of Clinical Epidemiology, Aarhus University Hospital,Denmark; 5) Section of Biostatistics, Department of Public Health, Aarhus University, Denmark

Background: Missing data is a ubiquitous problem in epidemiology, decreasing sample sizes and causing possible bias. In longitudinal studies, special problems relate to attrition and death during follow-up. Objective:Toillustrate how multiple imputation (MI)combined with sensitivity analysis can be applied to deal with these challenges. MI and sensitivity analysis have been used for several years in randomized trials, but rarely applied in observational studies. In these studies the problem with missing data is to often ignored.

Methods: In a cohort of patients treated with PCI followed with use of repetitive questionnaires and information from national registers over 3 years, only 417 out of 1726 patients had complete data on all measure points and covariates. We suggest strategies for the use of MI and different methods for dealing with death along with sensitivity analysis of deviations from the assumption of missing-at-random (MAR), all with the use of standard statistical software. The Mental Component Score from SF-12 was used as an example.
Results: It is nowadays possible to do multiple imputation in longitudinal study and perform quantitative sensitivity analysis only using standard software.

Conclusion: Ignoring missing data may cause bias of unknown size and direction in longitudinal studies. Usually this is ignored, but with MI and use of external data, it is possible to address these problems. How to handle dropout due to death is still open for debate. MI is a useful tool to deal with the challenges of missing data, including attrition and non-response, and should in general be applied in combination with sensitivity analysis in the analysis of longitudinal studies.

\section{P-182}

Agreement between myocardial infarction patients and their spouses on reporting of exposure to cardiovascular risk factors

Topic: Methods - Data analysis Presenter: Hedley Quintana

Hedley Quintana 1); Tomas Andersson 1), 2); Max Vikström 1); Johan Hallqvist 3); Karin Leander 1)

1) Cardiovascular Epidemiology Unit, Enviromental Medicine Institute, Karolinska Institute; 2) Stockholm County Council; 3) Department of Public Health and Caring Sciences, Family Medicine and Preventive Medicine, Uppsala University

Background: Etiological studies of diseases with rapidly progressive outcome, such as myocardial infarction $[\mathrm{MI}]$, may require the collection of proxy information. Few earlier studies, considering only a limited number of exposures, have analyzed the agreement between information collected from MI cases and their spouses.

Objective: To assess the validity of data collected from spouses or common-law spouses to MI cases identified within the Stockholm Heart Epidemiology Program [SHEEP], a population-based case-control study designed to study risk factors of MI in men and in women.

Methods: Eligible cases in this sub-study of SHEEP were non-fatal cases, defined as 28-day survivors, identified between 1993-04-01 and 1993-12-31, aged 45-70 years, residing in Stockholm County and reporting they lived together with a spouse or a common-law spouse. All eligible cases and their spouses who agreed to participate were included. Controls, individually matched to cases on sex, age and residential area, are included as well. Data were collected using mailed questionnaires. Using conditional logistic regression we calculated for each of a large number of exposure variables the odds ratio [OR] based on information collected from cases and their controls [ORcase] and the OR based on information collected from spouses and the same matched controls [ORproxy]. We measured the agreement between results obtained from using the two different sources of data by calculating the ratio between ORcase and ORproxy. A $95 \%$ confidence interval [CI] of this ratio was calculated using resampling bootstrap with 5000 iterations, taking into account the variance of each OR point estimate. Calculations were done using SAS version 9.2.

Results: Among the eligible subjects, 296 case-spouse pairs answered the questionnaire. For the vast majority of the exposures considered, including diet, smoking, education, work-related stress, previous personal cardiovascular disease and psychosocial exposures, the agreement between MI cases and their spouses was fairly high. However, physical inactivity was overestimated by the spouses and the history of cardiovascular disease [CVD] in the father before he turned 65 years was under-reported by spouses as compared to cases. Some questions related to work environment showed disagreement and among questions related to quality of sleep, 3 out of 11 items also showed clear disagreement.

Conclusion: Our results suggest spouses share information on everyday matters in the current private domain, but not necessarily on matters related to work environment or family history of disease. 


\section{P-183}

Factor structure of parent-reported Strengths and Difficulties Questionnaire in a pre-school age community sample from Born in Bradford

Topic: Methods - Data analysis

Presenter: Stephanie Prady

Stephanie Prady 1); Tim Croudace 1), 2); Emily Petherick 3); John Wright 3); Kate Pickett 1)

1) Department of Health Sciences, University of York; 2) Hull York Medical School, University of York; 3) Bradford Institute for Health Research, Bradford Teaching Hospitals NHS Foundation Trust

Background: Goodman's Strengths and Difficulties Questionnaire (SDQ) is a short, multi-dimensional screening instrument for detecting psychiatric disorders in children and adolescents. A 5-subscale structure (4 problem oriented; Emotional symptoms, Peer problems, Hyperactivity/inattention, Conduct problems, and 1 positive; Prosocial behaviour) is typical and has been empirically explored in several studies of primary school age children. We aimed to expand upon its psychometric properties using categorical data factor analysis procedures (multidimensional item response theory in Mplus), applied to data from parent ratings of pre-schoolers. The structure may be influenced by; (1) treatment of ratings as ordinal, (2) different properties of items comprising the 5 factors, and (3) the contribution of 5 positively worded questions in the otherwise negatively worded problem subscales (accommodated here by an uncorrelated methods factor).

Objectives: To examine the latent structure of parent reported SDQ ratings in children aged 3, from a subsample of Born in Bradford, a community birth cohort study in an economically deprived city in northern England.

Methods: We analysed data compiled on singleton children from 943 questionnaires completed in English and 232 in Urdu using confirmatory factor analysis with a weighted least squares estimator. We tested 7 models: (1) 3 factors comprising Internalising, Externalising and Prosocial subscales; (2) 4 factors comprising Emotional, Peer, Conduct and Hyperactivity subscales; (3) 5 factors comprising Emotional, Peer, Conduct, Hyperactivity and Prosocial subscales; (4) 6 factors comprising model 3 plus a methods factor; (5) a 2nd-order model comprising Emotional, Peer, Conduct, Hyperactivity and Prosocial subscales forming a Total difficulties scale; (6) a bifactor model of the 5 subscales and (7) a bifactor model of the 5 subscales plus methods factor. Model fit was assessed using acceptable thresholds for CFI, TLI and RMSEA statistics.

Results: Models 1-4 had poor fit. For the English cases, marginally acceptable fit was observed with the methods factor (model 5). The 2 bifactor models had good fit with best fit observed for model 7 (bifactor analysis of 5 subscales plus methods factor). Questionnaires in Urdu demonstrated less good fit. Regressing English bifactor coefficients on sex, adjusted for age and parent-rated difficulties, there were associations between boys and worse total $(p=0.03)$, Hyperactivity/ inattention $(p=0.001)$ and Conduct $(p=0.009)$ scores.

Conclusion: In this pre-school population, bifactor analysis suggests that the SDQ may usefully characterize a general severity continuum as well as a multi-dimensional screening profile of scores, after accounting for the variance in the underlying methods factor.

\section{P-184}

Application of honey in the treatment of burns

Topic: Methods - Data analysis

Presenter: Madalena Cunha, Eduardo Santos
Madalena Cunha 1); Eduardo Santos 1);

1) Fundação Aurélio Amaro Diniz (Serviço de Medicina)

Introduction: Since ancient times, honey has always occupied a prominent place in the treatment of wounds and throughout history have been gradually emerging research that showed its importance especially in the treatment of burns. However, this continues even today to be a matter of debate due to lack of evidence and lack of consensus in the scientific community that occur in clinical praxis different approaches.

In this context, this work aims to gather and systematize the results of studies on the effectiveness of the application of honey in treatment of burns by performing a systematic literature review and meta-analysis to document the 'state of the art.'

Methods: We followed the principles proposed by the Cochrane Handbook. The review was conducted by two researchers separately and statistical analysis using the Review Manager 5.1.7.

Results: We included 10 randomized controlled trials (RCT) involving a total of 1754 users. Honey is effective in the treatment and shortening of healing of burns, 1. And 2. Degree (WMD =4.19 days; $95 \%$ CI $-6.74,-1.63$ days, $p=0.001$ ).

Conclusion: The honey is an effective measure in the treatment of burns 1. And 2. Degree reducing the healing time compared with traditional therapeutic options (silver sulfadiazine, opsite ${ }^{\circledR}$, amniotic membrane, gauze fat soframicina...).

\section{P-185}

The UGT1A1*28 allele and extreme neonatal hyperbilirubinaemia, a National Danish case-control study

Topic: Methods - Data collection

Presenter: Jesper Padkar Petersen

Jesper Padkær Petersen 1), 2); Tine Brink Henriksen 2); Mads Vilhelm Hollegaard 3); Pernille Kure Vandborg 1); Ole Thorlacius-Ussing 4); David Hougaard 3); Finn Ebbesen 1)

1) Pediatric Department, Aalborg University Hospital; 2) Pediatric Department, Aarhus University Hospital; 3) Section of Screening and Hormones, Statens Serum Institut; 4) Department of Surgery, Aalborg University Hopsital

Background: Extreme neonatal hyperbilirubinaemia (serum bilirubin $>420 \mu \mathrm{mol} / \mathrm{L}$ ) is an important risk factor of severe bilirubin encephalopathy. In a considerable number of patients the reason of extreme hyperbilirubinaemia is never established. UGT1A1 is the rate limiting enzyme in bilirubins metabolism. The UGT1A1*28 allele causes a markedly reduced activity of this enzyme, but the influence on hyperbilirubinaemia is unsettled and the risk of extreme hyperbilirubinaemia not studied.

Objective: We aimed to test whether the UGT1A $1 * 28$ allele is associated with extreme hyperbilirubinaemia.

Methods: The UGT1A1*28 allele was assessed in a case-control study of all 257 incident extreme hyperbilirubinaemia cases of Caucasian descent in Denmark 2000-2007 and 514 Caucasian controls. Case status was obtained from The Danish Extreme Hyperbilirubinaemia Database and genotypes obtained through The Danish Newborn Screening Biobank.

Results: We observed no association between UGT1A1*28 allele genotype and risk of extreme hyperbilirubinaemia in 207 cases (50 pending analysis): compared to controls, newborns with extreme hyperbilirubinaemia had an odds ratio of 0.9 (95\% CI 0.5-1.7) for being UGT1A $1 * 28$ homozygous. For a subgroup of blood type AB0 incompatible newborns with extreme hyperbilirubinaemia, we found an odds ratio of 1.6 (95 \% CI 0.6-3.7) for being UGT1A1*28 allele homozygous. 
Conclusions: This is the first study to explore whether the UGT1A $1 * 28$ allele is a clinically important risk factor for developing hazardous serum levels of bilirubin. This was not found in Caucasian infants born in Denmark, but may be the case in newborns with $\mathrm{AB} 0$ incompatibility.

\section{P-186}

Development of a novel method for recruitment of cases of druginduced cholestatic liver injury to a genetic epidemiology cohort

Topic: Methods - Data collection

Presenter: Kevin Wing

Kevin Wing 1); Ian Douglas 1); Krishnan Bhaskaran 1); Liam Smeeth 1); Tjeerd-Pieter van Staa 2)

1) London School of Hygiene and Tropical Medicine, London, UK; 2) Clinical Practice Research Datalink, MHRA, London, UK

Background: Idiosyncratic drug-induced liver injury (DILI) contributes significantly to hospital admissions in Europe and the US. The importance of a genetic susceptibility is becoming clearer. The Phenotype Standardization Project (PSP) has provided a standardized DILI phenotype definition, in order to facilitate recruitment to genetic epidemiology studies. This abstract provides an overview of how patients with drug-induced cholestatic liver injury could be recruited by applying these standard definitions to a database of stored electronic health records (the UK Clinical Practice Research Datalink (CPRD)).

Objectives: The development and testing of a method that (1) allows individuals with drug-induced cholestatic liver injury to be identified in a database of stored routinely collected electronic health records (the CPRD) for recruitment into a genetic epidemiology cohort and (2) could be applied to monthly updates of the CPRD database.

Methods: The following steps have been completed:

1. A systematic review of studies of drug-induced cholestatic liver injury (allowing preparation of CPRD diagnostic codelists for identifying potential cases and exclusions)

2. Design of an algorithm for:

a. Selecting potential cholestatic liver injury cases and applying appropriate exclusions using CPRD diagnostic codes

b. Identifying those who have CPRD liver test result records characteristic of cholestatic liver injury (using PSP DILI criteria)

c. Selection of those exposed to a drug of interest within a specified risk period for record review against the PSP DILI criteria

3. Implementation of the algorithm in a (STATA) program that could facilitate monthly recruitment

4. Preparation of a questionnaire to be sent to General Practitioners of suspected cases

Results: Within the 3 months prior to injury, people identified by the algorithm had 3.22 (95\% CI 2.63-3.94) times the odds of being exposed to flucloxacillin compared with age and sex matched controls, 3.96 (1.89-8.31) times the odds of being exposed to chlorpromazine and 5.62 (4.53-6.98) times the odds of being exposed to amoxicillin-clavulanate. There was minimal difference in the odds of injury within users of oxytetracycline (OR 1.21, $95 \%$ CI $0.80-1.84)$.

Conclusions: Those identified by our algorithm were more likely to have been exposed to risk factors for cholestatic injury (flucloxacillin, amoxicillin-clavulanate and chlorpromazime) but not to oxytetracycline (not considered a risk factor for cholestatic injury). Further work aims to allow monthly recruitment of cases of flucloxacillin-induced cholestatic liver injury to a genetic epidemiology cohort.

\section{P-187}

Interviewer variability in response rates in the Belgian Health Interview Survey

Topic: Methods - Data collection

Presenter: Johan Van der Heyden

Johan Van der Heyden 1); Stefaan Demarest 1); Jean Tafforeau 1)

1) Scientific Institute of Public Health

Background: In the Belgian Health Interview Survey (HIS) information is gathered on the health status, life style and medical consumption of a representative sample of the population through a face to face interview and a self-administered questionnaire. Interviewers are recruited all over the country and have to pass through a standard training program.

Objectives: The aim of this study was to verify if household's nonresponse varies significantly among interviewers and if there is a link between interviewer's characteristics and non-response. This was investigated both for contact failure and refusal rate.

Methods: Data were used from the HIS 2004, in which 10.013 households were selected for participation and 264 interviewers carried out the fieldwork. Information on age, sex and nationality of the household's reference person, household composition and place of residence was obtained from the national population registry. Information on the housing type and characteristics of the residential area was collected during the fieldwork. The household database was merged with data on the interviewer's gender, age and educational attainment. Non-reponse was explored by using multilevel logistic regression models with interviewer's characteristics as dependent variables, household characteristics as covariates and household's neighbourhood and interviewer as random effects.

Results: After adjustment for household characteristics and neighbourhood the proportion of non participating households is lower among interviewers belonging to the older age groups. Compared to the reference group of youngest interviewers $(<45$ years) the odds ratio for refusal is 0.67 (95\% CI $0.60-0.75$ ) for interviewers between 45 and 64 years and 0.46 (95\% CI 0.35-0.61) for interviewers of $65+$ years. The odds ratios for contact failure are respectively 0.69 (95\% CI 0.59-0.81) and 0.62 (95\% CI 0.41-0.92). Interviewers with tertiary education have significantly lower refusal rates than interviewers with only secondary education (OR 0.88; $95 \% \mathrm{CI}$ 0.79-0.99). The variation of contact failure and refusal rates by interviewer remains significant after adjustment for household and interviewer characteristics.

Conclusions: The response rate in the Belgian HIS could probably be improved by putting more effort in the recruitment, training and follow up of interviewers. Efforts to improve the field work of young interviewers and interviewers with a lower education level may yield better participation rates. Focusing the recruitment of interviewers on higher educated persons in the age groups above 45 years may also be a strategy to improve the participation rate.

\section{P-188}

Development of a tool for the characterization of the health status and living conditions of elderly populations

Topic: Methods - Data collection 


\section{Presenter: Monica Machon}

Monica Machon 1); Isabel Larrañaga 2); Miren Dorronsoro 3); Nerea Larrañaga 2); Kalliopi Vrotsou 1); Bakarne Aguirre 1); Itziar Vergara 1)

1) Primary Care Research Unit Gipuzkoa, Osakidetza; 2) Public Health Department Gipuzkoa, Basque Government; 3) Health Department, Basque Government

Background: The assessment of the health status and the living conditions of elderly populations is a frequently tackled task, and the convenience of a comprehensive approach of these two aspects is being increasingly recognized, both in the social as well as in the health domain. Appropriate tools that allow and facilitate this integrated assessment are needed.

Objectives: To develop a specific tool that allows for a comprehensive assessment of the health status and living conditions of elderly populations.

Method: A collaborative working process was planned in order to fulfill the following tasks: (1) a literature review was performed, in order to describe the state of the art of the issue of interest; (2) a draft of the tool was proposed, defining the main domains and variables; (3) the draft was reviewed and changes were suggested by a multidisciplinary team including professionals of the health system, social services, statistical institutes and elderly people; (4) the improved version was piloted in a sample of 50 subjects and changes were included regarding the results of the pilot study; (6) the resulting version was used in a sample of 1.000 elderly individuals (over 65 years old) in order to validate the tool; (7) after this test and with an additional collaborative review, the definitive version of the tool will be settled (the project is currently at this phase); (8) once the tools is finished, it will be presented and its implementation facilitated among the health, social and statistical agencies of interest.

Results: The proposed tool is composed by 145 items and takes around $36 \mathrm{~min}$ to be fulfilled. The main domains included in the tool are: socioeconomic characteristics (age, sex, civil status, academic level, income, working situation); health status (self-perceived health, health related quality of life, multi-morbidity, drug prescriptions, mental health status, falls, senses); autonomy; healthy habits (physical activity, healthy eating, smoking, drinking habits, sleep); social networks (living alone, performed and received help, family and friends network); use of health and social services; characteristics of the home (equipment, barriers, access); characteristics of the community (services accessibility, elderly friendly community). The tool includes mostly, variables that could be obtained from various secondary sources as well as validated scales.

Conclusions: This tool could become a useful resource for agencies and institutions trying to obtain comprehensive and integrated information about the health status and living conditions of elderly populations. In addition to its comprehensiveness, this tool is able to recall information regarding the community context in which the individuals are developing their daily lives.

\section{P-189}

Charlson scores derived from administrative data and case-note review compared favourably in a population-based cohort

Topic: Methods - Data collection

Presenter: Marjorie Johnston

Marjorie Johnston 1), 2); Angharad Marks 1); Mike Crilly 1), 2); Gordon Prescott 1); Lynn Robertson 1); Corri Black 1), 2)

1) Division of Applied Health Sciences, University of Aberdeen; 2) NHS Grampian, Aberdeen
Background: Comorbidity describes the burden of disease coexisting with a particular disease of interest. It can affect the course and outcome of disease or illness and is an important confounding factor as well as being predictive of outcomes. Consequently, the accurate assessment of its impact is important clinically, for example for case management and health-care planning, as well as in research. The Charlson index is a widely used measure of comorbidity and was originally developed using case-note review data, often considered to be the gold-standard method. However, the case-note review process is resource intensive and as a result the Charlson index has been adapted for use with routine administrative datasets. Despite this, there remains uncertainty about the appropriateness of its application to administrative data.

Objectives: The objective was to compare Charlson index scores calculated using administrative data to those calculated using casenote review in relation to all-cause mortality and initiation of renal replacement therapy (RRT) in a Scottish population based chronic kidney disease cohort.

Methods: Modified Charlson scores were calculated for each individual from both case-note review and administrative data in the Grampian Laboratory Outcomes Morbidity and Mortality Study (GLOMMS-1) cohort. Agreement between scores was assessed using the weighted kappa. The association with outcomes was assessed using Poisson regression and the performance of each was compared using net reclassification improvement.

Results: Of 3,382 individuals, median age 78.5 years, $56 \%$ female, there was moderate agreement between scores derived from the two data sources (weighted kappa 0.41). Both scores were associated with mortality independent of a number of confounding factors. Administrative data Charlson scores were superior to case-note review scores at classifying the risk of death using net reclassification improvement. Neither score was associated with commencing RRT. Conclusions: Despite only moderate agreement, Charlson scores from both data sources were associated with mortality. Neither was associated with commencing RRT. Administrative data compared favourably, and may be superior, to case-note review when used in the Charlson index to predict mortality.

\section{P-190}

Registry-based monitoring of maternal mortality in rural southwest Ethiopia

Topic: Methods - Data collection

Presenter: Yaliso Yaya

Yaliso Yaya 1), 2); Tadesse Data 3); Bernt Lindtjørn 1)

1) Centre for International Health, University of Begen, Norway; 2) Arba Minch College of Health Sciences, Arba Minch, Ethiopia; 3) Gamo Gofa zone Health Department, Arba Minch, Ethiopia

Background: In developing countries there are few communitybased registries to track empirical information on births and birthoutcomes. Information is often provided by modelled proxy data that cannot show local trends, and cannot be used to monitor progress in work to reduce maternal and child deaths.

Objective: We aimed to monitor maternal deaths through community-based birth registries of over 500,000 rural people in south-west Ethiopia.

Methods: Ethiopian health extension workers (HEWs) prospectively noted births and birth-outcomes in their villages during their routine house-to-house visits. These community health workers have 1 year health education, and are responsible for primary health care services in the local communities. In January 2010 we established birthregistries in four woredas (provinces) in south-west Ethiopia, and this 
registration work was linked to work on reducing maternal deaths, mainly by making comprehensive obstetric care services available for rural population. The primary outcome was maternal mortality, and the secondary outcome was institutional delivery rates with skilled attendants.

Results: We registered 39,698 births: $88.1 \%$ were at home, $5.2 \%$ in health posts (served by HEWs), $2.8 \%$ in health centres, and $3.5 \%$ in hospitals. Most (42\%) deliveries were attended by traditional birth attendants (TBAs), $32 \%$ by family members, $19.2 \%$ by HEWs, and $6.3 \%$ by midwives, nurses, and doctors. There were 148 maternal deaths (maternal mortality ratio (MMR) 376 per 100,000 live births (LB). The highest MMR was in Demba Gofa province (510) followed by Arba Minch Zuria (401). During the registration, we validated the registration by doing a survey among 5,000 households. The survey data and birth registration were comparable. From January 2010 to December 2012, the maternal mortality ratio declined from 478 to 291 per 100,000 LB (linear trend, $p=0.02$ ). During the same period, the proportion of deliveries with skilled attendants (HEW, nurses, midwives and doctors) increased from 20 to $35 \%(p<0.01)$, and the rate of deliveries attended by TBAs declined from 44 to $36 \%$ $(p<0.001)$. Villages without car roads experienced higher MMR compared to villages with good roads (401 vs. 270 per 100,000 LB.); $\mathrm{OR}=1.48(95 \%$ CI 1.02-2.24).

Conclusion: It is possible to establish valid community-based registries in rural Ethiopia. The registries can be an important tool to monitor births and birth-outcomes in resource-limited rural communities.

\section{P-191}

Factors influencing willingness for participation in a randomized exercise intervention study in breast cancer patients during adjuvant radiotherapy

Topic: Methods - Data collection

Presenter: Karen Steindorf

Karen Steindorf 1); Sandra Gollhofer 1); Joachim Wiskemann 1); Martina Schmidt 1); Oliver Klassen 1); Cornelia Ulrich 1); Karin Potthoff 2)

1) Division of Preventive Oncology, German Cancer Research Center, Heidelberg, Germany; 2) Department of Radiation Oncology, University of Heidelberg Medical Center, Heidelberg, Germany;

Background: In general, only a small proportion of all cancer patients participate in randomized controlled trials. These low rates result from strict study protocols together with patient and physician related factors. This fact might compromise the generalizability of results of clinical trials including those on exercise intervention studies. To our knowledge, the factors influencing willingness for participation of cancer patients in exercise intervention trials have not yet been investigated.

Objectives: We investigated cancer related and motivational factors influencing participation and nonparticipation in a randomized exercise intervention trial (BEST-study) for breast-cancer patients undergoing adjuvant radiotherapy.

Methods: From July until December 2011121 breast cancer patients eligible for recruitment into the BEST-study were screened for study inclusion. Socio demographic characteristics (age, marital status, living status, travel distance), clinical data (body mass index (BMI), tumor stage, tumor size and lymph node status, comorbidities, chemotherapy), fatigue, and physical activity level were assessed. In addition, motivational and general reasons for participation or refusal were recorded. Characteristics of participants and nonparticipants were compared using Student's $t$ test (metric data), Mann-Whitney-
$U$ test (ordinal data), or $\chi^{2}$-test (categorical data). Multiple logistic regression analyses were performed to analyze the associations between the assessed characteristics and nonparticipation. $P$ values below 0.05 were considered as statistically significant.

Results: 117 patients (52 participants, 65 nonparticipants) were evaluable for analysis. Nonparticipants included a higher proportion of the oldest ( $\geq 70$ years) and the youngest patients ( $<40$ years) $(p=.02)$, lived more often alone $(p=.01)$, had a longer travel distance $(p=.02)$, were rather not affected by fatigue $(p=.01)$, and had more advanced disease characteristics (all $p<.05)$ than participants. Multiple regression analyses revealed significant higher odds for nonparticipation among elderly, those living alone, with higher travel distance, free of fatigue, with more advanced disease, more comorbidities, or previous chemotherapy. No associations were found with BMI and physical activity.

Conclusions: Willingness of breast cancer patients to participate in a randomized exercise intervention study differed by age and health status. However, no selection by current physical activity level or BMI was observed. Reduction of personal inconveniences, e.g. by decentralized training facilities and flexible training schedules, might be most promising to enhance the participation rate in exercise intervention trials.

\section{P-192}

What's mode got to do with it? The effect of mode of collection on individual response to sensitive topics

Topic: Methods - Data collection

Presenter: Martin P. Davoren

Martin P. Davoren 1); Frances Shiely 1); Michael Byrne 2); Ivan J. Perry 1)

1) Department of Epidemiology and Public Health, University College Cork, Ireland; 2) Student Health Department, University College Cork, Ireland

For the past number of decades web-based questionnaires have become a common method for collecting student based health behaviour surveys. Compared to traditional 'pen and paper' methods it provides a fast, cost efficient approach to surveillance. Previous research examining the affect of mode of collection on sensitive behaviours such as smoking, alcohol and drug use have provided conflicting results. The aim of this study was to examine the optimum mode for collecting student behavioural information among a third level student population by comparing web-based and face-to-face lecture theatre based data collection.

Degree programmes at one Irish university were randomly selected to participate in the study. A total of 2,275 self-completed questionnaires addressing lifestyle, substance misuse and well-being, were obtained over a two-week period in March 2012 by distribution at lecture theatres. Well-being was assessed using the Warwick Edinburgh Mental Well-being Scale. A response rate of $51 \%$ was achieved for the face-to-face survey. The response rate for those in attendance was $84 \%$. The web-based questionnaire yielded a response rate of $2.4 \%$ for the undergraduate population, with a total of 333 undergraduate students responding. Mantel-Haenszal tests, independent samples $t$ tests and multivariate logistic regression were utilised to investigate the impact of mode of collection on response.

The sample was predominantly female $(60.8 \%)$, in first year $(36.7 \%)$ and living in their parents' house $(41.6 \%)$ or in a rented house or flat $(42.7 \%)$. The median age of participants was 20 years. Demographic information such as faculty, year in college and gender of the web based sample differed significantly from the overall institution sampled. In analysis adjusted for age and gender, those 
who completed the web-based survey were $60 \%$ less likely to report high levels of physical activity but twice as likely to have taken recreational drugs in the previous 12 months. Alcohol consumption and mental health and well-being were higher among the web based sample sample.

In this comparison of web-based and face-to-face questionnaires among a third level student sample, significant differences in reporting of sensitive data were observed. Although web-based survey methods are efficient and relatively inexpensive, they cannot be relied upon to monitor trends in attitudes and behaviours in this setting. Thus, face-to-face lecture based sampling strategies as opposed to a web-based strategies are advocated for behaviour and attitude surveys among third level students.

\section{P-193}

\section{Assessment of monitoring system of food-borne illnesses} in Morocco

Topic: Methods - Data collection

Presenter: Fatine Hadrya

Fatine Hadrya 1); Hinde Hami 1); Sanae Benlarabi 2); Doha Ben Ali 1); Rachida Soulaymani-Bencheikh 2), 3); Abdelmajid Soulaymani 1)

1) Laboratory of Genetics and Biometry, Faculty of Sciences, Ibn Tofail University, Kenitra (Morocco); 2) Moroccan Poison Control Center, Rabat (Morocco); 3) Faculty of Medicine and Pharmacy, Mohammed V University, Rabat (Morocco)

Background: Besides the sufferings they cause, the food-borne illnesses have a peculiarly severe impact on the economy of developing countries. Morocco is particularly affected by this problem. This is why the monitoring of these diseases is crucial and necessary.

Objectives: This work consists in determining the annual and geographic distributions of food-borne illnesses in Morocco, in order to reduce the morbidity and mortality which they are associated.

Methods: A retrospective study was carried out on a series of food poisoning cases reported to the Moroccan Poison Control Center between 1994 and 2008, through two information systems: Toxicovigilance (for treatment of mails) and Toxicological Information (for treatment of calls). The mapping of health indicators was made with ArcGIS 9.3 software

Results: The Center received 16591 declarations, including 45 deaths: 14620 were collected by Toxicovigilance system and 1971 by Toxicological Information system. The study of health indicators showed that the annual incidence is 4 cases per 100000 inhabitants and the annual mortality is 1 case per 10 million inhabitants. The year $2003 \mathrm{had}$ recorded most cases of poisoning, and that most deaths had occurred in 2006. Moreover, all the regions of Morocco were affected by these intoxications, but not in the same way. Laayoune-Boujdour-Sakia El Hamra region registered the highest incidence, which knew a quick decrease through the years; the lowest incidence was observed in Oued Ed-Dahab-Lagouira region. Mortality is constantly relocated.

Conclusions: Over the last 15 years, the poisoning surveillance system has proven itself: the number of notifications has increased considerably and this in the whole country except the Saharan region of Oued EdDahab-Lagouira. However, our results should be taken with caution because of under-reporting still observed for this type of poisoning.

\section{P-194}

An innovative questionnaire assessing physical activity patterns

Topic: Methods - Data collection

Presenter: Boris Brühmann
Boris Brühmann 1), 2); Martina Schmidt 2); Birgit Teucher 3), 5); Angelika Wientzek 4); Matthäus Vigl 4); Heiner Boeing 4); Rudolf Kaaks 3); Karen Steindorf 1), 2)

1) Division of Preventive Oncology, National Center for Tumor Diseases (NCT), Heidelberg, Germany; 2) Unit of Physical Activity and Cancer, Division of Environmental Epidemiology, German Cancer Research Center (DKFZ), Heidelberg, Germany; 3) Division of Cancer Epidemiology, German Cancer Research Center (DKFZ), Heidelberg, Germany; 4) Department of Epidemiology, German Institute of Human Nutrition Potsdam-Rehbrücke (DIfE), Germany; 5) Department of Translational Pneumology, Heidelberg University Hospital, Translational Lung Research Center (TLRC), Heidelberg, Germany

Background: Even today it is still unclear which type, intensity, and temporal pattern of activity is most beneficial to health. The effect of irregular or acute periods of exhaustive activity may differ from the effect of constant exercise or an overall active lifestyle. With the type and level of information collected using existing questionnaires, it still remains difficult to give concrete public health recommendations. Objectives: Our aim was to develop and test an innovative questionnaire that determines and describes different physical activity patterns in various settings, a topic still difficult to approach, even in the era of accelerometers.

Methods: The questionnaire assesses occupation, household, locomotion, leisure-time physical activity, sports, climbing stairs, and sitting over the previous 12 months. For feasibility, reliability, and validity testing, the questionnaire has been included in an ongoing substudy of the European Prospective Investigation into Cancer and Nutrition (EPIC) project. Reliability was tested in $n=119$ participants, who filled out the questionnaire twice within a 6-8 week interval, by comparing individual settings and the overall physical activity level (PAL). Validity was tested in $\mathrm{n}=935$ participants, who also wore a combined heart rate and movement sensor (Actiheart), by comparing PAL values. Analyses included Bland-Altman-Plots and analyses of correlation (Pearson).

Results: Analyses showed distinct setting-specific activity patterns and also enabled the visualisation of activity patterns (regular/irregular activity or "weekend warrior") that resulted in the same total amount of energy expenditure (EE). A distinction between types of exercise and training methods was also possible and it required only 15 min (median) to complete the current questionnaire. Reliability testing showed good correlation for most of the settings and the overall PAL value (varying from 0.4 to 0.9 ). Validity testing showed good and unbiased agreement between PAL estimates from the questionnaires and the accelerometers. The correlation of $r=0.29$ is comparable to other internationally used instruments (e.g. RPAQ, IPAQ). Individual PAL differences revealed that $81 \%$ of the participants had a difference of less than 0.2 , representing a good and unbiased agreement, and $92 \%$ had a difference of less than 0.3 , i.e. acceptable agreement.

Conclusions: Our innovative questionnaire can be used to assess activity/inactivity in all relevant settings, identify setting-specific activity patterns, and differentiate between participants with similar EE but different activity patterns. The data on distinct activity patterns may finally enable researchers/investigators to analyse activity more specifically in terms of health benefits and to provide public health recommendations not solely based on EE.

\section{P-195}

Access to hospital among three birth cohort defined by gestational age: a 3 year follow-up study in a Italian region

Topic: Methods - Data collection 
Presenter: Enrica Lapucci

\section{Enrica Lapucci 1); Carlo Corchia 2); Domenico Di Lallo 1)}

1) Agency for Publich Health; 2) ICBD - Alessandra Lisi

International Centre on Birth Defects and Prematurity

Background: Preterm births, even after the neonatal period, are associated with a higher use of hospital services.

Objectives: To evaluate the emergency department access (EDA) and the inpatient hospital admission (IHA) for a regional preterm birth cohort during 36 months since discharge home from birth hospitalization.

Methods: The study was carried out in Lazio region, central Italy. The birth cohort was analysed by Gestational Age (GA), comparing very preterm ( $\leq 31$ weeks) and late preterm (32-36 weeks) to at term (37-41 weeks) babies. We used deterministic record linkage procedures linking three health administrative databases. From the Birth database we selected all 2007-2008 births home discharged. A longitudinal dataset was built up by linking birth cohort with EDA and IHA databases from 2007 to 2011 (36 months of follow-up). KaplanMeier analysis was used to calculate the cumulative incidence by GA groups. The Hazard Ratio (HR) for first hospital access in three different periods after home discharge $(<90 ; 90-365 ;>365$ days after $)$ was estimated by Cox regression. A multinomial regression model was performed to compare infants with no hospital access with infants who experienced 1 hospital access and those with 2 or more access. Results: A total of 90,545 infants were analysed: $0.7 \%$ with GA $\leq 1$ weeks, $6.8 \%$ at 32-36 weeks and $92.5 \%$ at 37-41 weeks. Babies who had at least one EDA ranging from $60 \%$ for vey preterm to $55.9 \%$ for at term. The same values stands at 35.2 and $18.3 \%$ for hospital admissions. In the three GA groups, the 3-year cumulative incidence of first EDA or IHA shows a faster increase during the months immediately after birth home discharge then it continues to growth at a slow pace. In the first 3 months after birth home discharge, a statistically significant higher risk for EDA and IHA was observed among the very preterm (HREDA 1.50; HRIHA 3.24) and late preterm (HREDA 1.28; HRIHA 1.71) compared to at term babies; for the other two periods, conversely, only the risks for IHA remain significantly higher, although estimated values stepwise decreased. The result of multinomial model indicates that GA was a strong predictor for IHA (Relative Risk [RR] $=1.99$ for very preterm; $\mathrm{RR}=1.35$ for late preterm) but not of EDA when only one event was considered; otherwise, when considering 2 or more events, GA was a strong predictor for both IHA and EAD.

Conclusions: Our study showed that GA at birth had a strong impact on the subsequent use of the hospital in term of emergency department access and hospitalization. Among very and late preterm babies the risk for EDA and IHA is higher during the first month after birth home discharge. This study proved the feasibility of record-linkage procedures among health administrative database to carry out followup studies.

\section{P-196}

Evaluation of matching algorithms using identifiable patient information

Topic: Methods - Data collection

Presenter: Xinran LI, Lemlih Ouchchane

Xinran LI 1), 2); Aline Guttmann 1), 2); Sébastien Cipière 3), 4); Lydia Maigne 3), 4); Jean-Yves Boire 1), 2); Lemlih Ouchchane 1), 2)

1) UMR 6284 CNRS Institut des Sciences de l'Image pour les Techniques interventionnelles, Clermont-Ferrand, France; 2) Service de Biostatistiques, Faculté de Médecine, Université d'Auvergne, Clermont-Ferrand, France; 3) UMR 6533 LPC, CNRS/IN2P3, Aubière, France; 4) Université Blaise Pascal, BP 10448, ClermontFerrand, France

Background: To create a grid network infrastructure dedicated to e-health and epidemiology for a French administrative region, the linking of patient data recorded in distributed databases is required. Without planned interoperability (by common identification key), identifiable patient information such as last name, first name, gender, date and place of birth can be used for matching these distributed data. The matching algorithm produces a similarity score for any pair of records and their linkage is based upon the position of that score regarding one (match/no match) or two thresholds (match/uncertainty/ no match).

Objectives: The aim of this study is to assess the robustness of our matching algorithm regarding the stability of the threshold(s), the collision risk (wrongly matching different patients) and the duplication risk (wrongly separating different records from the same patient) when identifiable patient information is subjected to degradation such as transcription errors.

Methods: Pairs of databases sharing common patients are simulated and randomly disturbed according to determined error types and rates. The disturbances involve, for instance, omitting, substituting, adding and reversing one or more characters among the identifiable patient information. The matching algorithm provides as many scores of similarity as possible pairs of patients (Cartesian product of databases). In order to evaluate their informational value, these scores of similarity are compared with the real matching of patients. We first aimed to assess the most (unique) discriminating threshold. Subsequently, we assessed two thresholds framing an interval. Outside this interval, neither collision nor duplication should occur. Within this interval, a manual decision shall be required. The estimation of these decision thresholds is based upon a ROC analysis and their stability is assessed by cross-validation.

Results: The values of the areas under the ROC curves are very close to 1 . The single decision threshold only varies slightly no matter what type or rate disturbance are applied to the datasets. For the uncertainty interval, the proportion of pairs of patients to check manually is very low. The thresholds obtained on the learning data provide very satisfactory results in cross-validation.

Conclusions: For the range of types and rates of transcription errors we studied, our results are consistent with the stability of the decision thresholds applied to the scores of similarity.

\section{P-197}

Non-participation in a health survey of the population 65 years and older: Who is missed and why?

Topic: Methods - Data collection

Presenter: Markus Busch

Beate Gaertner 1); Markus Busch 1); Martin Holzhausen 1), 2); Ina Seitz 1); Judith Fuchs 1); Peter Martus 3); Christa ScheidtNave)

1) Department of Epidemiology and Health Monitoring, RobertKoch-Institute Berlin, Germany; 2) Institute of Biometry and Clinical Epidemiology, Charité University Medicine Berlin, Germany; 3) Department of Clinicial Epidemiology and Applied Biometry, Eberhard Karls Universität Tübingen, UKT, Germany

Objectives: To compare participants and non-participants of a health examination survey with respect to demographics and health status, and to assess reasons for non-participation. 
Method: An age-and-sex-stratified random sample of 1481 community residents 65 years and older from inner city population registries was invited for a health survey. Information for all individuals included age, sex, nursing home residence, non-German nationality and areal deprivation score. Non-participants were asked to complete a short questionnaire covering health status. Multiple reasons for nonparticipation were assessed during recruitment or 1 year later. Four groups were distinguished: participants $(\mathrm{P})$, non-participants who declined with short questionnaire (SQ), non-participants who declined without short questionnaire (D), and non-respondents who never responded (NR). Descriptives, logistic and multinomial logistic regressions were calculated. To take into account the correlation of participants within geographical areas, confidence intervals were determined using survey procedures with Taylor linearized variance estimation in Stata 12.

Results: Of the net sample, $\mathrm{n}=299$ were $\mathrm{P}, \mathrm{n}=384 \mathrm{SQ}, \mathrm{n}=324$ $\mathrm{D}$, and $\mathrm{n}=301 \mathrm{NR}$. Compared to participants, non-participants were more likely to be older ( $\mathrm{MP}=75.0$ vs. $\mathrm{MSQ}=78.1, \mathrm{MD}=77.7$ ), women (P:45.8 \% vs. SQ:54.7\%), of non-German nationality (P:2.7 \% vs. D:7.4\%, NR:19.6\%), to reside in nursing homes (P:0.7 \% vs. SQ:6.0 \%, D:4.0 \%), to live in socially deprived areas (P:28.1 \% vs. D:37.0 \%), and to report worse health (P vs. SQ). For $87 \%$ of all non-participants, reasons for non-participation could be assessed. The two most frequent reasons were general refusal to participate in scientific studies $(42.1 \%)$ and being too ill $(30.5 \%)$.

Discussion: Reasons for non-participation can be assessed for the majority of non-participants. Disadvantaged groups likely to have a higher burden of disease are not.

\section{P-198}

Systematic clinical application of Patient Reported Outcome (PRO). A new potential in clinical epidemiology.

Topic: Methods - Data collection

Presenter: Niels Henrik Hjollund

Niels Henrik Hjollund 1), 2); Liv Marit Schougaard 1); Louise Pape Larsen 1)

1) VestKronik, Regional Hospital West Jutland; 2) Department of Clinical Epidemiology, Aarhus University Hospital

Background: PRO's were initially developed for use in clinical research and in recent years they have been used to assess and improve health care quality e.g. by inclusion of PRO data in clinical databases. Systematic use of PROs in daily clinical practice is still uncommon and largely restricted to isolated projects with limited impact.

Objectives: Clinical epidemiological research depends on welldefined cohorts of patients with relevant outcome measures, including PRO. We aimed to describe potentials of regional cohorts based on outpatient created for clinical use of PRO.

Methods: A PRO system was developed and introduced for use in all three neurological departments in Central Denmark Region. In March 2012 the system was integrated with the electronic health record system, where PRO-based overviews are displayed. The system is used for clinical decision support, flagging important symptoms and reallocating of resources by cancelling unnecessary appointments. The PRO questionnaires consists of items developed to support clinical decisions (cancelling or not) as well as disease specific and generic health measures. The PRO's were collected by an integrated system allowing both web- and paper based forms. The set-up allows easy inclusion of extra items when appropriate. Results presented here are based on data from the main regional neurological department at
Aarhus University Hospital, where a number of 3000 epilepsy outpatients are permanently referred to.

Results: Until March 2013, a number of 2,039 (68.1\%) of all patients with epilepsy (DG401-DG409) were referred to the system and additional 90-120 patients are referred monthly. The response-rate in the first questionnaire was 93.9 and $98.2 \%$ in the following questionnaires.

Conclusions: Application of clinical cohorts for research purpose is promising, not at least due to very high response rates. PRO from cohorts of patients have a number of applications but are normally difficult and expensive to collect, but this data-collection is already implemented and run by the hospitals. Based on the experiences it has been decided to include all new patients with epilepsy in the system which then will constitute a complete cohort. The Central Denmark Region has decided to support further implementation of the system (AmbuFlex) in other patient outpatient populations and the first RCT based on the system is planned.

\section{P-199}

Implementing best practice in data governance to enable health improvement through high quality data linkage research

Topic: Methods - Data collection

Presenter: Corri Black

Corri Black 1); Jillian Evans 2); Pam Lowbrigde 2); Joanne Rodger 2); Sharon Gordon 1); Stephannie Hall 1); Katie Wilde 1); Phil Hannaford 1)

1) University of Aberdeen; 2) NHS Grampian

Background: With the growth in electronic data capture within health and social care, there is huge potential for population health benefits through the use of linked clinical datasets for health surveillance, improved care planning and epidemiological research. Scotland has a world class reputation for rich, routinely collected population data, particularly in relation to health. The recent Scottish Health Informatics Programme Blueprint for Health Records Research in Scotland sets out the key principles to which those undertaking and facilitating research using linked datasets should aspire. Key institutional challenges are to support both data custodians and researchers to meet their legal and regulatory obligations, to maintain patient confidentiality and to ensure best practice in research governance; whilst enabling important health and population research.

Aims: To ensure best practice in data governance for health records research within a single geographical administrative region, through the development of a collaborative academic and health service data safe haven.

Methods: A multidisciplinary steering group was established. Operational and technical subgroups undertook a mapping exercise, wide consultation with local researchers and review of safe haven initiatives nationally and internationally. Processes and procedures for the acquisition, storage and use of linked datasets were developed.

Results: In May 2012, the Grampian Data Safe Haven (DaSH) was established as a collaboration between health scientists, NHS Grampian Health Intelligence, NHS Grampian Research and Development Team and the University of Aberdeen College of Life Science and Medicine Data Management Team. DaSH provides support to researchers undertaking data linkage and a secure environment to facilitate data linkage research. Processes and procedures for the acquisition, storage and use of linked datasets were established. Individual projects are supported to develop a specific data and risk disclosure management plan for approval by the appropriate 
authorising officials including the Data Custodians, Caldicott Guardians and Ethics committees.

Conclusion: To date, DaSH has worked with researchers on 27 projects as part of piloting the processes. DaSH operates within the developing Scottish Safe Haven Network to facilitate data linkage and minimize risk of data disclosure, providing a mechanism to tailor the Data Management Plan to provide proportionate security measures appropriate for the project specific risks. DaSH provides the necessary support and secure environment to ensure that high quality research to improve health continues while maintaining best practice in data governance for data linkage.

\section{P-200}

\section{Assessment of breast cancer risk by use of the Danish Working Hour Database}

Topic: Methods - Data collection Presenter: Helene Tilma Vistisen

Helene Tilma Vistisen 1); Anne Helene Garde 2); Johnni Hansen 3); Åse Marie Hansen 2), 4); Peer Christiansen 5); Henrik Kolstad 1)

1) Department of Occupational Medicine, Danish Ramazzini Centre, Aarhus University Hospital, Denmark; 2) National Reasearch Centre for the Working Environment, Denmark; 3) Insitute of Cancer Epidemiology, Danish Cancer Society, Denmark; 4) Department of Public Health, University of Copenhagen, Denmark; 5) Surgical Department P, Aarhus University Hospital, Denmark

Background: In 2007, the International Agency for Research on Cancer classified nightshift work that involves circadian disruption as probably carcinogenic to humans, based partly on an increased risk of breast cancer among long-term nightshifts workers. Since then, several epidemiologic studies have examined this relation. Despite these efforts, inadequate information on nightshift work is an Achilles heel in the studies leading to persisting uncertainty regarding causality and there is a need for prospectively collected, objective, and detailed data that allow analyses based on exposure metrics that reflect relevant effects on the circadian rhythm.

Objectives: To study the association between night-work and risk of breast cancer by use of the newly established Danish Working Hour Database (DWHD). DWHD is a large, national cohort of public health care professionals with a high prevalence of night shift work. Methods: A great amount of the Danish health care professionals are employed by the Danish Regions. For every employee, the Danish Regions keep information regarding the personal identification number, sex, date of birth, position, absence and reasons therefore, e.g. vacation, sickness and maternity leave, and seniority, in addition to date, hour, and minute for the beginning and end of every work duty. DWHD was established in 2012 and encompasses data as of 2007. The data are updated on an annual basis. Data from DWHD will be linked with national cancer registers.

Results: The database encompasses for 2007102.487 female employees of which 36.419 work nightshifts. During the 6 year follow-up period 2007-2012, we expect a total of 300 breast cancer cases among women working nightshifts. The age group from 50 to 59 is expected to account for nearly $50 \%$ of those cases. This group encompasses 7.274 nightshift workers with a mean of 2.5 nightshifts per month.

Conclusions: The Danish Working Hour Database provides highquality data on work hours that in combination with national health register information will form a unique resource for assessing potential health effects of nightshift work.

\section{P-201}

GIS, an essential tool to gather the needed information to decide at the primary health care level: a case study at Porto, Portugal

Topic: Methods - Data collection

Presenter: Luis Fonseca

Luis Fonseca 1); Ana Monteiro 2); José Leitão 3); José Sousa 3); Sara Velho 1)

1) ISPUP; CITTA; 2) Department of Geography; ISPUP; CITTA;

3) ACES Espinho Gaia

Background: The primary health care goals of promoting health and prevent diseases implies the availability of accurate information about the geographical and health profile context of each user. The efficient and effective management of each health centres group (ACES), requires a close proximity to the geographic area, a huge closeness to people and a commitment with home care. The hospitalization reduction implies a tighter relationship between families and health actors to disseminate healthy lifestyles and raise awareness of risk factors. GIS has been seen as an important tool since, for instance, Snow (1849) that used it to show and explain the deaths with cholera in London.

Objectives: Our ambition is to understand the ideal architecture of a multi-purpose system of data storage able to be constantly updated and flexible enough to provide the needed information to decide in the several domains of health promotion and disease prevention. To achieve this objective we conceive a GIS project in partnership with all local health actors prepared to offer better conditions of data record-keeping and transmission within ACES and able to generate quickly the required information for decision-programs, projects or actions-really adapted to the target people.

Methods: We start defining a query suggested by health actors: how to apply the family nurses by geographical area, a legal obligation not yet implemented mainly because it should ensure equity among personnel and answer to a extensive list of criteria inevitable to follow the previewed goals. Afterwards, we conceive and create a WebSIG anchored in the Porto University central server to guarantee the obligatory requirements of confidentiality implied in almost all the data that must be gathered. We choose the software ArcGis to produce our project. Thereafter, we collect all physical and human geographical data and socioeconomic statistics at micro scale level and produce the adequate cartography. Then, we collect all the individual data of ACES sub-unit with 17000 users-name, address, age, diseases etc. Finally, the ACES users were geocoded. Lastly, we create and validate some examples of homogeneous areas that fit the requested legal criteria.

Results: The GIS project construction showed a considerable number of problems due to inadequate registration procedures and data storage software that do not interrelate with each other. The GIS start suggesting a list of simple improvements essential to turn easier the access and reduce errors. The ACES sub-unit 17000 users were geocoded with a success ratio of $97.5 \%$. The automatic and manual homogenous area design procedures are interactive and show clearly the multidimensional profile of the users endorsed to each nurse.

Conclusions: Considering all the geographical and health criteria, this dedicated GIS project delivers so many homogeneous areas as desired family nurses. Each family nurse geographical areas produced allows a continuous ballot by all involved actors. 


\section{P-202}

Interpreting chronic fatigue syndrome/myalgic encephalomyelitis prevalence: differences in clinical definitions

Topic: Methods - Interpretation

Presenter: Samantha Johnston

\section{Samantha Johnston 1); Ekua Brenu 1); Don Staines 2); Sonya Marshall-Gradisnik 1)}

1) Griffith Health Institute, National Centre for Neuroimmunology and Emerging Diseases, Griffith University, Parklands, QLD, Australia; 2) Gold Coast Population Health Unit, Queensland Health, Robina, QLD, Australia

Background: Several case definitions have been proposed for Chronic Fatigue Syndrome or Myalgic Encephalomyelitis (CFS/ME). These definitions differ significantly in the symptoms they emphasise, as well as inclusion criteria for comorbid disorders. This can contribute to the inconsistency found in reported prevalence.

Objectives: To determine how case definitions have been adopted internationally in prevalence research.

Methods: A systematic review of studies on CFS/ME prevalence during the past 25 years was conducted as part of an investigation on methodological differences in assessment of prevalence. Studies must have fulfilled inclusion criteria including primary research that used prospective survey methods to estimate prevalence, and recruitment of patients in a community or primary care setting. Reported prevalences were summarised and examined according to the clinical definitions used. Differences in their specificity and selectivity of cases are discussed.

Results: Thirty-one studies were considered suitable prevalence studies for review. Five different case definitions have been used to report prevalence. Additional studies were also found to apply their own case definitions or approximate versions of published case definitions. Early estimates were based on the 1988 Centres for Disease Control and Prevention (CDC) definition, Australian and Oxford definitions. More recent estimates are based on the 1994 CDC definition. Only one study has reported prevalence according to the 2003 Canadian definition. Furthermore, all studies of prevalence in paediatrics are based on the 1994 CDC definition for adults. The latest 2011 International Consensus definition has yet to be assessed in prevalence studies.

Conclusion: Based on these findings, new developments in clinical case definitions have received little attention from prevalence research on CFS/ME. It is proposed that future assessment of prevalence adopt the latest International Consensus definition. If applied systematically, it will enable international comparisons to be made among estimates as well as detect more specific cases of CFS.

\section{P-203}

\section{Calibration plot joining Multiple Imputation Datasets}

Topic: Methods - Interpretation

Presenter: Ana Royuela

Ana Royuela 1); Francisco M. Kovacs 3); Alfonso Muriel 2); Víctor Abraira 2)

1) CIBER Epidemiología y Salud Pública (CIBERESP). Spain; Unidad de Bioestadística Clínica. Hospital Ramón y Cajal. IRICYS. Madrid. Spain; Spanish Back Pain Research Network; 2) Unidad de Bioestadística Clínica. Hospital Ramón y Cajal. IRICYS. Madrid. Spain; CIBER Epidemiología y Salud Pública (CIBERESP). Spain; Spanish Back Pain Research Network; 3) Fundación Kovacs, Palma de Mallorca, Spain; Spanish Back Pain Research Network.
Background: Multiple imputation provides a useful strategy for dealing with data sets with missing values. Rubin's (1987) multiple imputation procedure replaces each missing value with a set of plausible values that represent the uncertainty about the right value to impute. These multiply imputed data sets are then analyzed by using standard procedures for complete data and combining the results from these analyses.

Validation is an essential issue in predictive regression models. Calibration and discrimination are frequently studied as performance measures, especially for binary outcomes. A calibration plot represents in an easy way the actual probability versus the predicted probability, both on average and over the whole range of predictions. Harrell describes the plot showing the Apparent curve" (representing predicted probability using the model against actual probability), the "Ideal curve" (representing perfect calibration) and the "Bias-corrected curve" (representing the overfitting-corrected calibration curve, obtained by bootstrap resampling).

Objectives: To develop a global calibration measure that takes into account the multiple imputation procedure.

Methods: Data from a 7,976 patients registry were used to perform a logistic regression model in order to predict spinal pain (SP) improvement. Multiple imputation was carried out and 10 imputed datasets were obtained. Calibration was assessed by means of the calibration plot described by Harrell. Curves were drawn using the lowess function of Stata, which provides locally weighted scatterplot smoothing. Discrimination was assessed through the Dxy index.

Results: At discharge, a clinically relevant SP improvement was experienced by $75 \%$ of patients. Coefficients from the model were obtained in order to predict the SP improvement. Discrimination of the model was moderated (corrected Dxy index equal to 0.241).

In order to draw the global "Apparent curve", we estimated the slope and the intercept in each one of the imputed datasets by 10 logistic regression models with the linear predictor as the only predictor variable (log odds(improvement in SP) $=0+1 *$ linear predictor) and then averaged to obtain a global 0 (equal to 0.120 ) and a global 1 (equal to 0.890). The "Bias-corrected curve" was obtained by 500 bootstrap resamples.

Conclusions: It is feasible to draw a calibration plot which provides bias-corrected indexes and takes into account the multiple datasets obtained after a multiple imputation procedure.

\section{P-204}

Applying discriminative measures to revisit the determinants of small-for-gestational-age

Topic: Methods - Interpretation

Presenter: Sol Juárez.

\section{Sol Juárez 1); Philippe Wagner 2); Juan Merlo 2)}

1) Centre for Economic Demography, Lund University; 2) Faculty of Medicine, Department of Clinical Sciences in Malmö, Unit for Social Epidemiology, Lund University

Background: Small-for-gestational-age (SGA) is considered an indicator of intrauterine growth restriction and multiple maternal and new-born risk factors have been identified so far (i.e., maternal age, smoking, hypertension, gestational age, birth order.). However, the identification of these as risk factors" has been exclusively based on measures of statistical association (i.e., odds ratios) rather than on measures of discriminatory accuracy. That is, the capacity of a risk factor to discriminate disease cases from non-cases. This information is fundamental in order to deliver potential clinical and/or preventive treatment based on the presence/absence of the risk factor.

Objectives: We aim (1) to replicate the association between traditional risk factors and SGA, (2) to revisit the clinical/public health 
relevance of these risk factors by applying measures of discriminatory accuracy, and (3) to identify which combination of risk factors provides the best discriminatory accuracy.

Methods: Using the Swedish Medical birth Register, we investigated 731,989 full-term babies born during 1987-1993. We performed logistic regression models to estimate the association (OR and $95 \%$ confidence intervals) between SGA and a number of maternal and child characteristics considered in the literature as determinants of SGA (i.e., birth order, new-born's sex, maternal age, smoking during pregnancy, maternal complications -diabetes, hypertension and urinary infections, marital status, maternal education, and country of origin). We also calculate measures of discriminatory accuracy like ROC-curves, area under the ROC curve (AU-ROC), net reclassification improvement (NRI), integrated discrimination improvement (IDI) and true positive fraction for a $5 \%$ false positive fraction (TPFFPF-5 \%).

Results: We were able to replicate all the expected associations between SGA and its traditional risk factors. The strongest observed association was for smoking ( $\mathrm{OR}=2.21$ for light and 2.78 for heavy smokers) and for hypertension $(\mathrm{OR}=2.92)$. However, overall, the discriminatory accuracy was rather low. The AU-ROC was 0.60 for smoking, and 0.51 for hypertension. The AU-ROC for a model including both smoking and hypertension was 0.60 and the inclusion of the rest of variables to the smoking-hypertension model rendered a minor improvement (AU-ROC $=0.66$ ).

Conclusions: Despite being conclusively associated to SGA, none of the traditional risk factors had a substantial capacity for discriminating SGA babies from those with a normal size. Our finding questions the public health relevance of current risk factors for SGA. The public health repercussions of using risk factors with a low discriminative accuracy need to be discussed.

\section{P-205}

Epidemiology in Public Health intervention research: expectations, promises and challenges

Topic: Methods - Interpretation

Presenter: Maria Rosaria Galanti

Maria Rosaria Galanti 1), 2); Yvonne Forsell 1), 2);

Jette Möller 1); Karin Engström 1), 2); Anna Månsdotter 1), 3)

1) Department of Public Health Sciences, Karolinska Institutet; 2) Centre for Epidemiology and Community Medicine, Stockholm Health Care District; 3) The Swedish National Board of Health and Welfare

Background: There is an increasing demand on epidemiologists to be involved in the evaluation of complex public health interventions, beyond the traditional field of clinical and disease-related research. Through case discussion, we will illustrate the challenges deriving from the application of epidemiological methods to intervention research, particularly concerning the level of causal inference.

Methods: Utilization of experimental, quasi-experimental, observational and mixed-designs for the purpose of evaluation and implementation of local health policies, health promotion interventions and preventive programs. Two examples will illustrate these applications: an evaluation of impact of health care investments in underprivileged areas on health outcomes, and an evaluation of effectiveness of a telephone-based counseling in reducing risky alcohol drinking.

Results: Experimental and quasi-experimental designs applied for the evaluation of public health interventions often imply randomization or other methods for assignment of comparable conditions of organizational clusters, such as schools, municipalities, or health districts.
Correct inference on effects requires minimization of bias both at the cluster and individual level. Constraints due to unfeasibility of a pure experimental design, such as lack of blindness or of placebo condition, and intervention fidelity often make these studies less recommendable. Observational studies (e.g. cohort studies, interrupted time series, natural experiments) can be combined to answer questions of effectiveness while overcoming the inherent potential for bias in each respective design. In addition, consistency of results at the different levels of adequacy, plausibility or probability overcomes the limitations of single hypothesis-testing and conventional statistical significance.

Conclusions: Epidemiology needs to undergo a rapid methodological development in order to keep in pace with the expectations from the Public Health sector.

The following processes seems crucial for this development:

1. Connection with non-medical disciplines, such as sociology, political sciences, economy and management, anthropology and ethics

2. Use of mixed design, allowing different levels of inference within the same study

3. Application of statistical methods outside the traditional toolbox for analyses of epidemiological studies, such as time series analysis, analysis of contextual variables.

4. Combine quantitative and qualitative data in the phase of participant recruitment as well as in the interpretation of results

\section{P-206}

Discriminatory accuracy and population attributable fractions: the case of traditional risk factors and novel biomarkers for coronary heart disease

Topic: Methods - Interpretation

Presenter: Juan Merlo

\section{Juan Merlo 1); Philippe Wagner 1); Bo Hedblad 2)}

1) Faculty of Medicine, Dept Clini Sci, Unit for Social Epidemiology, Lund University, Malmö, Sweden; 2) Faculty of Medicine, Dept Clini Sci, Unit for Cardiovascular Epidemiology, Lund University, Malmö, Sweden

Background: Traditional risk factors and novel biomarkers are intended to improve our capacity to discriminate the individuals who will develop the disease from those who will not. From a population level perspective, we also use risk factors to calculate the population attributable fraction (PAF). The PAF tries to estimate the share of the disease burden in a population that is attributable to a certain risk factor and that is, thereby, potentially preventable. For those purposes, we normally use simple measures of association like the odds ratio (OR) or hazard ratios (HR). Nevertheless, measures of association alone are unsuitable for discriminatory purposes. From a clinical and public health perspective what matters most is the capacity of the exposure (whatever it is a traditional risk factor or a novel biomarker) to discriminate disease cases from non-cases as this information determines how accurately we can deliver a potential preventive treatment.

Objectives: Focusing on coronary heart disease, we aim (1) to quantify to what extend the discriminatory accuracy of simple demographic variables like age and sex is improved by adding traditional cardiovascular risk factors and novel biomarkers, (2) to analyse the relation between measures of PAF and the discriminatory accuracy of the risk factors used for the PAF computation.

Methods: We investigated 6,103 men and women who participate in the baseline (1991-1996) of the cardiovascular cohort of the Malmö 
Diet and Cancer study. We followed these individuals until to January 1,2009 , death or coronary heart disease event.

We performed logistic regression to analyse models including (A) age and sex, (B) traditional risk factors (blood pressure, Cholesterol, diabetes, smoking) (C) biomarkers (CRP, NTBNP, Cystatin C, LpPLA2 activity) and combinations of A, B, C. We calculate measures of discriminatory accuracy (ROC curves, AU-ROC, TPFFPF $5 \%$, NRI, IDI, and pseudo-R2) and risk assessment plots" including PAF and variance explained values for different risk factors thresholds and prevalences of a risk factor defined using the risk scores from the model $\mathrm{A}+\mathrm{B}+\mathrm{C}$.

Results: Comparing with the model A (AUROC $=0.68)$, model A+B improved the discriminatory accuracy by 0.07 units and model $\mathrm{A}+\mathrm{B}+\mathrm{C}$ by 0.08 units. For $\mathrm{A}+\mathrm{B}+\mathrm{C}$ the TPFFPF $5 \%=29 \%$, the NRI and $49.5 \%$ and the IDI $=0.044$. For a risk threshold of $9 \%$ the $\mathrm{PAF}=53 \%$, and the false positive fraction $(\mathrm{FPF})=28 \%$. For a risk factor prevalence $=90 \%$ the PAF $=92 \%$ but the FPF $=90 \%$. Conclusions: Neither traditional risk factors nor biomarkers substantially improved the discriminatory accuracy obtained by just considering age and sex. The size of the FPF needs be considered in the interpretation of the PAF measure.

\section{P-207}

The tyranny of the averages and the indiscriminate use of risk factors in public health: a call for revolution

Topic: Methods - Interpretation

Presenter: Juan Merlo

\section{Juan Merlo 1); Philippe Wagner 1)}

1) Faculty of Medicine, Dept Clini Sci, Unit for Social Epidemiology, Lund University, Malmö, Sweden

Background: Both public health practitioners and ordinary people are overwhelmed by a plethora of both established and novel risk factors and biomarkers for diseases. There are also countless socioeconomic, ethnical and geographical disparities in health that are frequently highlighted by the media. Most of this information is obtained by calculating differences between group averages (i.e., exposed and unexposed groups) and presented as measures association (e.g., odds ratio). However, even when the association is apparently strong, the meaning on many of those risk factors and health disparities needs be critically questioned. In fact, recent research demonstrates that measures of association are insufficient for gauging the ability of risk factors to discriminate the individuals who will develop the disease from those who will not. An indiscriminate use of average associations might shadows the identification of truly relevant health determinants and might, in the long way, harm the scientific credibility of modern public health epidemiology. Nevertheless, a new methodological and conceptual approach based on measures of discriminative accuracy (DA) like the AU-ROC, TPFFPF $5 \%$, NRI, IDI, and risk assessment plots" might help us to identify which risk factors are more relevant than others and, thereby, to develop better strategies of prevention.

Objectives: Focusing on cardiovascular diseases and the investigation of neighbourhood effects on health, we aim to apply the new DA perspective, to critically question previous knowledge concerning novel biomarkers, established risk factors and traditional socioeconomic, ethnic and geographic disparities in health.

Methods: Unstructured critical review of the literature and detailed discussion of empirical examples.

Results: DA measures are being increasingly applied for quantifying the value added of novel biomarkers in relation to traditional risk factors. However, an analogous approach is necessary but almost absent when it comes to the investigation of socioeconomic, ethnical and geographical disparities in health. It appears that the DA of most socioeconomic, ethnical and geographical variables is rather low.

Conclusions: The normal investigation of average causal effects (based on differences between group means), is an idealization that needs be urgently questioned. We need to differentiate natural heterogeneity from statistical uncertainty and integrate measures of discriminative accuracy when understanding biomedical as well as socioeconomic, ethnic and geographical differences in health. It is also essential to develop epidemiological methods of analysis that like the "n-of-1 trials", case-cross over, and sibling analysis allow us investigating the heterogeneity of individual effects in the population. What are the ethical repercussions of using risk factors with low discriminative accuracy? Are there problems of inefficiency, medicalization and stigmatization?

\section{P-208}

You don't know what you don't know: The issue of non-responders in long-term follow-up

Topic: Methods - Interpretation

Presenter: Sarah Seaton

Sarah Seaton 1); Elaine Boyle 1); Elizabeth Draper 1); Samantha Johnson 1); David Field 1); Bradley Manktelow 1)

1) Department of Health Sciences, University of Leicester

Background: Follow-up of high-risk neonatal cohorts suffers from high levels of dropout and poor response rates. Interpreting results is problematic as the characteristics of those who respond often differ from those who do not. Studies have examined these issues in specific gestational age based cohorts. This work examines the issue of nonresponse across all gestational ages.

Objective: To investigate if differences exist between responders and non-responders to questionnaire-based follow-up of child development at 2 years of age.

Methods: Data were obtained from two studies which recruited mothers of babies born in the East Midlands and Yorkshire, UK: Preterm AND After (PANDA) study recruited babies born at $<31$ weeks (very preterm: VPT); Late And Moderate preterm Birth Study (LAMBS) recruited babies born at 32-36 weeks (late and moderate preterm: LMPT) and 37 + weeks (term). Children were followed up at 2 years using a postal questionnaire to assess health and development. Responders were compared to the non-responders for mother's age, previous preterm birth, socioeconomic status, birthweight below 10th percentile, ethnicity and multiplicity of birth. Results: Mothers who responded were older than those who did not: VPT 30.5 versus 28.0 years; LMPT 30.4 versus 28.2 years; term 31.1 versus 28.4 years $(p<0.001)$. Women in the least deprived quintile were more likely to respond: VPT 68 versus $34 \%$; LMPT 81 versus $44 \%$; term 77 versus $44 \%(p<0.001)$ and women of white ethnic origin were more likely to respond than non-white: VPT 52 versus $37 \%$; LMPT 62 versus $45 \%$; term 65 versus $45 \%(p<0.01)$.

Differences were seen for multiple birth compared to singletons by gestational age group: VPT $57 \%$ (multiples) versus $46 \%$ (singletons) $p=0.008$; LMPT 54 versus $58 \% p=0.292$; term 52 versus $63 \% p=0.002$. A difference was only seen in the VPT group for women with a previous preterm birth compared to those without: VPT $37 \%$ (previous) versus $51 \%$ (no previous) $p=0.028$; LMPT 52 versus $52 \% p=0.99$; term 57 versus $57 \% p=0.99$.

For mothers of babies born below the tenth centile for birth weight there was no statistically significant difference compared to other mothers: VPT $44 \%$ ( $\leq 10$ th centile) versus $49 \%$ ( $>10$ th centile); LMPT 53 versus $58 \%$; term 52 versus $61 \%(p \geq 0.05)$. 
Conclusions: Characteristics of non-responders are typically associated with poor infant developmental outcomes and thus may cause bias in the interpretation of study results. As the characteristics of non-responders are similar across all gestational ages, similar strategies could possibly be used to improve response rates.

\section{P-209}

Lifestyle-related risk factors and adherence to stations: the finish public sector study

Topic: Methods - Interpretation

Presenter: Maarit Jaana Korhonen

Maarit Jaana Korhonen 1); Heli Halava 2), 3); Risto Huupponen 1), 4); Jaana Pentti 3); Mika Kivimäki 5); Jussi Vahtera 2), 3)

1) Department of Pharmacology, Drug Development and Therapeutics, University of Turku, Turku, Finland; 2) Department of Public Health, University of Turku, Turku, Finland; 3) Finnish Institute of Occupational Health, Turku, Turku, Finland; 4) Turku University Hospital, Turku, Finland; 5) Department of Epidemiology and Public Health, University College London, London, UK

Background: Previous research suggests that observational studies on statin effects may be distorted by so called healthy-adherer effect. The assumption underlying this bias is that patients adhering to preventive medications are likely to have a healthier lifestyle than do non-adherers.

Objectives: We aimed to reveal potential mechanisms underlying the healthy-adherer effect by investigating the associations of selected lifestyle-related risk factors and their combinations with statin adherence in a large sample of Finnish public sector employees. We hypothesized that normal weight, never-smoking, moderate alcohol consumption, physical activity, and good adherence to statins are all markers of a healthy lifestyle, so that those patients with unhealthier levels of these risk factors would be more likely to be non-adherent. We evaluated the above associations separately for those with and without pre-existing cardiovascular disease (CVD) or diabetes.

Methods: Using data from a prospective cohort study with recordlinkage with national health registers (Finnish Public Sector Study), we identified 9,285 public sector employees (mean age 56 years, $76 \%$ female) initiating stating therapy in 1998-2010 after responding to a survey measuring lifestyle. Adherence was measured as the proportion of days covered (PDC) and defined as good for those participants with $\mathrm{PDC} \geq 80 \%$, moderate for those with PDC $40-79 \%$ and poor for those with PDC $<40 \%$ during the first year. The fourth group, discontinuers, included participants who redeemed only one prescription during the first year.

Results: During the first treatment year, $53 \%$ of the initiators had good adherence while $12 \%$ discontinued the therapy. Among initiators with CVD or diabetes, high versus moderate alcohol consumption and having 3-4 risk factors at the unhealthiest level versus none were associated with non-adherence (adjusting for age, gender and initiation year, odds ratios [OR] for moderate versus good adherence; $1.56,95 \%$ confidence interval [CI] 1.15-2.13 and 1.61, $95 \%$ CI 1.09-2.39, respectively). Among initiators without CVD and diabetes, obesity and overweight predicted better adherence in comparison with normal weight as did former smoking comparing with never-smoking (ORs for discontinuation vs. good adherence; 0.76, $95 \%$ CI $0.61-0.95 ; 0.73,95 \%$ CI $0.62-0.87$; and $0.76,95 \% \mathrm{CI}$ $0.64-0.90$, respectively). We observed no associations between physical activity and the level of adherence.

Conclusions: Unhealthy levels of the four risk factors studied were not consistently associated with non-adherence to statins. Our findings provide little support for healthy lifestyle as a potential mechanism of the healthy-adherer effect.

\section{P-210}

Socioeconomic inequality measurement in self assessed health: Turkish case

Topic: Methods - Interpretation

Presenter: Nesrin Cilingiroglu

\section{Nesrin Cilingiroglu 1); Mahmut Sadi Yardim 1)}

1) Hacettepe University Faculty of Medicine Department of Public Health

Background: The use of self assessed health (SAH) is among the common measures of perceived current health status in empirical research in spite of its proneness to inaccuracies due to reporting and it's sensitiveness to variations in socioeconomic conditions. Examining the effect of socioeconomic inequalities in health with SAH is not new, there is little empirical evidence of this association in Turkey, and Survey of Income and Living Conditions (SILC) data is never used for this purpose.

Objective: This study aims to determine health-related socioeconomic factors' effect in SAH in order to see inequalities in health between 2006 and 2010 .

Methods: In this study, 2006 and 2010 country representative data from SILC-Turkey was used. SILC is a natural source of data for this measurement work, given its provenance, frequency and comparability. Turkish Statistical Institute has conducted SILC since 2006 as a part of the EU-SILC. The target population is all adults aged 16 and over. Self-assessed health was used as outcome measure while the independent variables representing the inequalities are gender and age, income, education and urban-rural residence. Decomposition of Concentration Index (CI) was employed in order to disentangle the main contributors of inequalities in SAH to allow clear understanding of how factors affect inequality.

Results: According to preliminary findings, CI for SAH was -0.170 for 2006. Decomposition of CI indicated: $73.1 \%$ of inequality in SAH was generated by income, $53.9 \%$ by education and, $0.8 \%$ by rural residency. The effect of gender-age groups was "-25.8 \%". For the year 2010, CI was "-0.167". Decomposition of CI for 2010 indicated: $77.2 \%$ of inequality was generated by income, $45.3 \%$ by education and, $1.7 \%$ by rural residency. The effect of gender-age groups was " $-27.9 \%$ ". The analysis showed a gradient effect of income for good assessed-health.

Conclusion: Research on health inequalities has been substantial, although more needs to be done to inform policy in a better way. This study provided empirical evidence that income negatively influences health: the lower the income group, the poorer the perception of health in Turkey. Besides, lower level of education had a further negative effect on health perception.

Understanding and acting on socioeconomic health inequalities has proven to be a difficult task. Despite the increase in life expectancy and the continuing improvements in overall population health in Turkey, the findings presents evidence on persistence of social health inequalities. To conclude, policies and programs should include all key sectors of society not just the health sector in order to decrease inequalities.

\section{P-211}

Is low birth weight prevalence still a valid indicator for comparisons between populations?

Topic: Methods - Interpretation

Presenter: Katarzyna Szamotulska

Katarzyna Szamotulska 1); Ashna Mohangoo 2); Jennifer Zeitlin 3) 
1) National Research Institute of Mother and Child, Department of Epidemiology, Warsaw, Poland; 2) TNO Netherlands Organization for Applied Scientific Research, Department Child Health, Leiden, The Netherlands; 3) INSERM, UMR S953, Epidemiological Research Unit on Perinatal Health and Women's and Children's Health, Paris, France

Background: The prevalence of low birth weight (birth weight less than 2,500 g among live births, LBW \%) has been used as an indicator of wellbeing of newborn babies in different populations for many years. It is a heterogeneous measure and reflects LBW \% in etiologically distinct and numerically different subgroups of children: multiples, premature singletons and term singletons. In population studies, LBW \% is often interpreted as a proxy of intrauterine growth restriction (IUGR) and a measure reflecting maternal under nutrition and unhealthy lifestyle. However, in European countries, due to increasing proportions of deliveries which are induced before term and increasing proportions of multiple pregnancies, LBW \% may poorly reflect factors proven to be related to IUGR.

Objectives: To assess the correlation of LBW \% and mean birth weight among singleton live births born at 40 weeks of gestational age with selected risk factors for IUGR in European countries.

Methods: National 2010 data on birth weight, multiplicity (singletons, multiples), maternal smoking during pregnancy and body mass index (BMI) from 24 European countries and regions participating in the EURO-PERISTAT project were used. Ecological correlations were calculated with Spearman's rho.

Results: Total LBW \% ranged from 3.4 to $9.8 \%$. On average, multiples constituted $29 \%$ of low birth weight babies (range 21-36\%), preterm singletons were $44 \%(33-58 \%)$ and term singletons were $26 \%(16-38 \%)$. In countries for which data were available on maternal prepregnancy BMI less than $18.5(\mathrm{n}=11)$ or maternal smoking $(n=9)$, there was in general weaker or less reliable association of these variables with total LBW \% and LBW \% among preterm and multiple births than with LBW \% among term singletons. There was a marginally significant positive association for total LBW \% $(\rho=0.600 p=0.088)$ and term singletons LBW \% ( $\rho=0.633$, and $p=0.067$ ) with maternal smoking.

In these countries, mean birth weight among singleton live births born at 40 weeks of gestational age was strongly and significantly associated with both low maternal prepregnancy BMI and smoking in the 3rd trimester $(\rho=-0.791, p=0.004$ and $\rho=-0.717, p=$ 0.030 ), respectively.

Conclusions: In contrast to mean birth weight among singleton live births born at 40 weeks of gestational age, LBW \% which is commonly used as a measure of infant health outcomes related to IUGR is poorly associated with two principal risk factors for inadequate fetal growth.

\section{P-212}

Initial non-participation and loss to follow-up in a Danish youth cohort: implications for relative risk estimates

Topic: Methods - Others

Presenter: Trine Nøhr Winding

Trine Nøhr Winding 1); Johan Hviid Andersen 1); Merete Labriola 1), 2); Ellen Aagaard Nøhr 3)

1) Danish Ramazzini Centre, Department of Occupational Medicine, Regional Hospital Herning, Denmark; 2) Department of Clinical Social Medicine, Public Health and Quality Management, Central Denmark Region and Section of Clinical Social Med, and Rehabilitation, School of Public Health, Aarhus University, Denmark; 3) Department of Public Health, Section for Epidemiology, Aarhus University, Denmark
Background: Initial non-participation and loss to follow-up in the Danish youth cohort Vestliv could introduce selection bias of the measured risk estimates.

Objectives: To investigate the impact of initial non-participation and loss to follow-up on the validity of descriptive measures and selected estimates of relative risk.

Methods: Of the 3681 young people defining the source population, $83 \%$ answered a questionnaire at baseline in 2004. At follow-up in 2007 and 2010, the response rates were 71 and $64 \%$, respectively. Register information was retrieved from Statistics Denmark. Relative odds ratios (ROR) were used to examine the impact of initial nonparticipation and loss to follow-up on the association between socioeconomic or personal risk factors at age 14/15 and educational attainment at age 20/21. RORs were calculated as OR (baseline population)/OR (source population) or OR (follow-up population)/OR (baseline population).

Results: The participants tended to have better school abilities and came more often from homes with two adults, higher income, or higher educational level. This tendency increased at subsequent follow-ups. The effect of initial non-participation on the ORs was negligible with most RORs being close to one. Loss to follow-up led to larger variations in the RORs, especially for rare outcomes, but for most estimates, the bias was minor and did not exceed $23 \%$. Also, none of the measured RORs were statistically different from one indicating no significant bias.

Conclusions: Although certain characteristics were related to those who initially chose to participate in the study and especially to those who participated at follow-ups, it did not have any large influence on the relative risk estimates measured in the study.

\section{P-213}

Meta-analysis of cytokine gene polymorphisms and outcome of heart transplantation

Topic: Methods - Others

Presenter:

Sasitorn Yongcharoen 1); Sasivimol Rattanasiri 1);

D Olga McDaniel 2); Chukiat Viwatwongkaseam 3);

Piangchan Rojanavipart 3); Ammarin Thakkinstian 1)

1) Section for Clinical Epidemiology and Biostatistics, Faculty of Medicine, Ramathibodi Hospital, Mahidol University, Bangkok, Thailand; 2) Department of Surgery, the University of Mississippi Medical Center, Jackson, Mississippi, USA; 3) Department of Biostatistics, Faculty of Public Health, Mahidol University, Bangkok, Thailand

Background: It has been reported that cytokine gene polymorphisms were associated with graft rejection after heart transplantation. However, gene effects were controversial, i.e., some studies found positive associations while others did not.

Objectives: To perform a systematic review and meta-analysis with the aim at assessing effects of cytokine polymorphisms on graft rejection in heart transplantation.

Methods: We identified relevant studies from Medline and Embase since inception to February 2013. Data extraction and risk of bias assessment were independently performed by two reviewers. Allele frequencies were pooled, genotypic effects were assessed using a mixed-logit model. Heterogeneity and publication bias were assessed. Results: Four to five studies were included in the pooling of TNFa308, TGFb1-c10, and TGFb1-c25 polymorphisms. The pooled prevalence of the minor $\mathrm{A}, \mathrm{C}$, and $\mathrm{C}$ alleles in the control groups for TNFa-308, TGFb1-c10, and TGFb1-c25 were respectively 0.166 (95\% CI 0.129, 0.203), 0.413 (95\% CI 0.363, 0.462), and 0.082 
(95\% CI 0.054, 0.111). Carrying the A allele for the TNFa-308 had $18 \%(95 \%$ CI of OR $0.46,3.01)$ increased risk but not significant for graft rejection than the $\mathrm{G}$ allele. For the genotype effects, carrying AA and GA genotypes were respectively 1.98 (95\% CI $0.30,13.12)$ and $1.11(95 \%$ CI $0.61,2.02)$ times non-significantly higher odds of graft rejection than those carrying the GG genotype. The effect of TGFb1 at $\mathrm{c} 10$ and $\mathrm{c} 25$ polymorphisms were conversely; carrying the $\mathrm{C}$ alleles for both TGFb1 at c10 and c25 were non-significantly lower odds of graft rejection with the pooled ORs of $0.87(95 \%$ CI $0.65,1.18)$ and 0.70 (95\% CI $0.40,1.23)$, than those carrying the $\mathrm{T}$ and $\mathrm{G}$ alleles, respectively. The $\mathrm{CC}$ and $\mathrm{TC}$ genotypes for TGFb1-c10 were 0.76 $(95 \%$ CI $0.40,1.46)$ and 0.84 (95\% CI 0.53, 1.33) times non-significantly lower odds of graft rejection than those carrying the TT genotype, respectively. The OR for TGFb1-c25 was 0.63 (95\% CI $0.35,1.14$ ), that is carrying $\mathrm{CC} / \mathrm{GC}$ were approximately at $37 \%$ nonsignificance lower risk of graft rejection than those with GG genotype. The genotypic effects of the 3 polymorphisms were mild to moderately heterogeneous. There was no evidence of publication bias for all poolings.

Conclusions: Although none of these 3 polymorphisms were significantly associated with graft rejection in heart transplantation, this review suggested signals of associations. An updated meta-analysis is required when more studies have been published to increase the power of detection for the association between these polymorphisms and allograft rejection.

\section{P-214}

Gender-sensitive research in epidemiology: a survey among German epidemiologists

Topic: Methods - Others

Presenter: Ingeborg Jahn

\section{Dirk Gansefort 1); Johann Frick 1); Ingeborg Jahn 1)}

1) Leibniz-Institut for Prevention Research and Epidemiology - BIPS $\mathrm{GmbH}$

Background: Good epidemiological practice guidelines such as those developed by the German Society for Epidemiology provide guidance for sex/gender-sensitive research. What is currently missing is the integration into routine research practice. The Epi goes Gender project promotes the adoption of sex/gender-sensitive research approaches, e.g. through organising a training program, based on actual needs and requirements of epidemiologists.

Objectives: To investigate needs, requirements, attitudes and experiences of German epidemiologists regarding gender-sensitive research.

Methods: A cross-sectional study was carried out among members of the German epidemiological scientific associations, who were accessed via the societies' mailing lists. The questionnaire was developed and piloted in close cooperation with representatives of the scientific associations. Data were collected from July till August 2012 using an online survey tool.

Results: Overall 276 persons $(175 \mathrm{w}, 101 \mathrm{~m})$ took part in the survey. The response rate was estimated as $25-35 \%$, as the exact number of the society members included in the mailing could not be determined. Female participants tended to be younger than male participants. Correspondingly, the professional experience in women was 11.3 years $( \pm 9.6$; median 8.0$)$ and in men 18.1 years $( \pm 12.0$; median 17.0). The most frequent discipline was Public Health $(33.1 \%$ women; $22.8 \%$ men), followed by mathematics/statistics $(16.6 \%$ women; $33.7 \%$ men). $49.1 \%$ of the women and $31.7 \%$ of the men reported having interest in gender-sensitive research in epidemiology. While $27.7 \%$ of the female participants and $22.8 \%$ of the male participants judged the gender-based recommendations of the GEP Guidelines as being relevant for them, $15.1 \%$ of the women and $26.1 \%$ of the men were not aware of these guidelines. Many of the respondents indicated that specific training in gender-sensitive research was missing (42.4\% male $/ 45.2 \%$ female agreement). Nearly $90.0 \%$ of women and $70.0 \%$ of men reported to be interested in an engagement in gender-sensitive research in future.

Conclusions: Although selection bias due to the relatively low response rate and information bias due to aspects of social desirability have to be taken into account, participating epidemiologists in Germany showed some experience as well as interest to deal with sex/ gender based topics in their field of research. The fact that a large majority of the participants indicated interest in the field topic is a good basis for further strengthening of sex/gender based research approaches in epidemiology. Professional associations can play an important role in ensuring this.

\section{P-215}

Incidence of venous thromboembolism in and around pregnancy using linked primary and secondary care data: a cohort study and meta-analysis

Topic: Methods - Others

Presenter: Alyshah Abdul Sultan

Alyshah Abdul Sultan 1); Laila Tata 1); Matthew Grainge 1); Joe West 1)

1) Division of Epidemiology and Public Health, University of Nottingham

Background: Recent linkage between primary and secondary care data within England has enabled us to quantify how the measurement of incidence in and around pregnancy can be affected by the definition of venous thromboembolism (VTE) and source of data used.

Objective: Using these data we have precisely measured the risk among pregnant women and compared it systematically to the existing literature on the subject.

Methods: We used primary care data from the Clinical Practice Research Datalink (CPRD) which incorporates linkages to secondary care contained within Hospital Episode Statistics (HES) between 1997 and 2010 to estimate absolute and relative rates of VTE in the antepartum and postpartum compared with women's time outside pregnancy. We systematically searched the literature on the incidence of VTE during antepartum and postpartum period and performed a meta-analysis.

Findings: Using combined primary and secondary care data and a restrictive VTE definition, the absolute rate during the antepartum and postpartum were calculated in our study to be 101 and $274 / 100,000$ person-years respectively. These rates were broadly comparable to the pooled estimates from our meta-analysis (of 21 studies) during the antepartum (80/100,000 person-years) and postpartum (272/100,000 person-years) period. When we used only secondary care data to identify VTE events, incidence was lower during the postpartum (171/100,000 person-years) and time not associated with pregnancy $(21 / 100,000$ person-years) whereas relying only on primary care data lead to lower incidence during the time around delivery but higher rates during the early postpartum period.

Conclusion: Use of linked primary and secondary care data gives precise estimates of VTE risk in and around pregnancy that are comparable to the existing literature. This methodology allows risk stratification by time period in pregnancy and will provide better ascertainment of maternal risk factors for VTE, information on hospitalisation, lifestyle related factors, primary care prescriptions, and co-morbidities in future studies. 


\section{P-216}

Completeness of congenital anomaly diagnoses in children's primary care records by age at registration

Topic: Methods - Others

Presenter: Rachel Sokal

Rachel Sokal 1); Kate Fleming 1); Linda Fiaschi 1); Laila Tata 1)

1) Division of Epidemiology and Public Health, University of Nottingham, Nottingham, United Kingdom

Background: General practice data provide large population-based cohorts that can be used to study the causes and consequences of congenital anomalies (CAs). For some children with a CA, corrective treatment in early life may lead to relatively low subsequent on-going morbidity and therefore little need for later medical monitoring or intervention. Whilst important medical history should be included in all individuals' medical records we carried out an assessment of whether recording of major CA diagnoses were likely to be complete for children registered with their general practice at older ages compared with those registered in the first months of life.

Method: Using all children in The Health Improvement Network (THIN) primary care database born between 1990 and 2009 (with follow-up data until September 2011), we identified those with major CAs, classified into anatomical groups according to the European Surveillance of Congenital Anomalies network (EUROCAT). The prevalence of any CA was calculated per 10,000 live births for the whole population and for those registered with their general practice within 1, 3, 6 and 12 months of their date of birth.

Results: Of 1,403,015 children included in the study population, 37,557 had a CA diagnosis, resulting in a birth prevalence of 268 per 10,000 (95\% confidence interval 265.0-270.4). $30 \%(\mathrm{n}=427,655)$ of the study population was registered at less than 1 month old and $57 \%(\mathrm{n}=794,215)$ by 12 months. For both overall prevalence measures and 16 anatomical subgroups the $95 \%$ confidence intervals of CA prevalence estimates overlapped amongst the populations restricted by time of registration. Prevalence estimates for the unrestricted population i.e. those registered with their general practice at any age, however, were lower and did not overlap with those from the populations registered within 1, 3, 6 or 12 months of birth.

Conclusion: The prevalence of CAs within the THIN database does not differ in those registered by 1, 3, 6 and 12 months of diagnosis thus the size of study populations can be maximised by including children registered by 12 months of their date of birth. The lower prevalence estimates for children registered at older ages may be due to under recording of historical CA diagnoses including those which may have spontaneously resolved or been surgically corrected at an earlier stage of life.

\section{P-217}

\section{Comparative datasets and the 'turn to parenting' in children's health}

Topic: Methods - Others

Presenter: Carolyn Stolberg

\section{Carolyn Stolberg 1)}

1) Georg-August-University Goettingen, Institute of Sociology

Health inequality already starts within childhood. Poor health in the early years is strongly related to a lack of parental resources. In order to raise equal access to a healthy life from the beginning, parental resources on the one hand and children's participation in health on the other need to be uncoupled from one another. Thus, to raise 'fairness' and 'equity' still remains to be solved. As a consequence, healthy upbringing is not only a parental — private-duty but also a statepublic-responsibility.

Within welfare states that pursue a social investment strategy, there is wide consensus about state intervention that has to increase. According to the principle the sooner the better, children represent a key element and the future of one's society. Aiming on raising human capital, the social investment strategy comprises the objective future investment through investment at the very beginning of life (Morel et al. 2012). In France, Germany, Great Britain, the Netherlands and Sweden, preventive measures in general have become important instruments by the state. In the last decades, more and more parenting support measures as well as parenting programmes have been established. Children Centres and family midwives are just two examples of services aiming at supporting parents in raising their children 'properly'. But not only preventive measures in general have increased-in the last decades, also the term 'positive parenting' in children's health has become more important.

However, it still remains to be solved whether France, Germany, Great Britain, the Netherlands and Sweden follow identical-rather contrasting?-Intervention aspects with respect to parenting support in general and children's participation in health in particular. Thus, the question is, whether it is generally possible to analyse the 'turn to parenting' in children's health by means of existing quantitative datasets. Which cross national datasets can be used for analysing a states' intervention in the sphere of children's health? How could the turn to parenting in health be measured? In sum, this contribution holds a comparison on datasets including France, Germany, Great Britain, the Netherlands and Sweden.

\section{P-218}

Evaluation of sensitivity and predictive value of Crimean-Congo hemorrhagic fever by surveillance system in Iran

Topic: Diseases - Infections

Presenter: Tayebeh Shafieezadeh

Fariba Farnoosh 1); Tayebeh Shafieezadeh 1); Mohammad reza Sarbazi 1); Ali Golmohammadi 1); Farzaneh Farbakhsh 1)

1) Department of prevention and control of diseases Shahid Beheshti University of medical sciences

Background and Objectives: Crimean-Congo hemorrhagic fever (CCHF) is an acute infectious disease that is included in emergency call reporting system since 1999 in Iran. The objective of this study is to determine the sensitivity, specificity and positive predictive value (PVP) as the most important parts of surveillance system.

Methods: A cross sectional approach was used in 87 cases with $\mathrm{CCHF}$ clinical signs that were reported to surveillance system. Suspect case was defined as cases with fever and hemorrhagic manifestation (petechiae, rash, nose and mouth mucous bleeding, hematemesis or melena, hematuria) associated with one of the epidemiological characteristics: History of tick bite or Crushing the tick with hand, direct contact with fresh blood or other animal tissue, direct contact with definite or probable patients discharge, Residence in or traveling to the rural area where there is a probable contact with animals but no specific contact can be mentioned. Clinical criteria were compared to laboratory results. Therefore, gold standard was genomic segment of virus by RT-PCR and suspect definition was considered as positive clinical criteria. Serologic test were carried out for all cases reported. Data were analyzed using the software SPSS18. Results: A total of 87 cases reported to surveillance system, age range were of 9 months to 83 years and median 29 years. Classification of reported cases was shown that 47 cases $(54 \%)$ were having the 
suspect criteria. Three $(3.4 \%)$ cases became laboratory definite. The ratio of death among the cases reported were $20 \%$ and among suspect cases were $27.7 \%$.Three $(3.4 \%)$ of the reported cases did not have fever and hemorrhagic manifestations simultaneously. One of the laboratory definite cases was a 63 year old afghan who has not fever at the time of reporting and he has hemorrhagic manifestations with contact history to live animal. Sensitivity, specificity, and positive predictive value were estimated $66.7,46.42$, and $4.25 \%$ respectively. Conclusions: Approximately, $50 \%$ of reported cases do not have criteria for suspect definition. The low sensitivity in this study refers to low sensitivity in criteria definition. Since, positive predictive value depends on by the prevalence, the low positive predictive value in this study conforms to the low prevalence rate in population.

Key words: sensitivity, predictive value, $\mathrm{CCHF}$

\section{P-219}

Epidemiological aspects of meningococcal disease in the Distrito Federal, Brazil, from 2000 to 2011

Topic: Diseases - Infections

Presenter: Marcia Cantuaria Tauil

\section{Marcia Cantuaria Tauil 1); Eliseu Alves Waldman 1)}

1) Public Health School of University of São Paulo, Brazil

Background: Meningococcal disease (MD) is a serious public health concern, associated with high case fatality rates (10-20\%) and substantial morbidity.

Objectives: To analyze epidemiological aspects of MD in the Distrito Federal (DF), Brazil, from 2000 to 2011 and to assess the impact of the introduction of the conjugate vaccine against meningococcus $\mathrm{C}$ in 2010.

Methods: Descriptive study involving the DF residents who had had MD. Confirmed cases of MD recorded in the national information systems of notification, mortality, and/or laboratory were included. The following variables were analyzed: socio-demographic, clinical and related to the agent (serogroup, serotype and serosubtype). Incidence rates of pre and post-vaccine period, by age group, was compared to assess the impact of conjugate vaccine against serogroup $\mathrm{C}$ introduction.

Results: We analyzed 490 confirmed cases of MD in the period. The strain B:4, 7:P1.19, 15 predominated before 2005 with $67.8 \%(61 / 90)$ and the strains C:23:P1.14-6 with $40.9 \%(61 / 149)$ and C:2a:P1.5, 2 with $12.8 \%$ (19/149) were more prevalent after 2005. The average annual rates of incidence and mortality of MD in the period were, respectively, 1.7 and 0.4/100.000 inhabitants/year and the average fatality rate was $21 \%$. The incidence rate of MD was higher in children under 1 year, ranging from 13.1 in 2011 to 38.7/100.000 inhabitants/ year in 2000 . Fatality rate was higher in the group of 40 years old and more $(40 \%)$ and among children with 2 years old (32\%). There was a statistically significant reduction $(p$ value $=0.02)$ in the incidence of MD in children under 2 years old from 21.3/100.000 inhabitants/year in 2009 , to $6.6 / 100.000$ inhabitants/year in 2011.

Conclusions: This study enabled us to verify the age groups most affected by MD, the circulating phenotypes and to make a preliminary assessment of the impact of conjugate vaccine against serogroup $\mathrm{C}$, after the first year of its introduction in the routine immunization program.

\section{P-220}

A cross-sectional study on anti-hepatitis B immune status in patients vaccinated before surgery with a two-dose regimen in Poland

Topic: Diseases - Infections

\section{Presenter: Maria Ganczak}

\section{Maria Ganczak 1); Zbigniew Szych 2)}

1) Dept of Public Health, Pomeranian Medical University; 2) Dept of Computer Science and Education Quality Research, Pomeranian Medical University

A two-dose vaccination schedule against HBV before surgery is widely accepted in Poland. An Anti-HBs level between 0 and $10 \mathrm{~m} \mathrm{IU} / \mathrm{ml}$ after $\mathrm{HBV}$ vaccination is considered as none response.

Objective: To assess the fraction of patients vaccinated against HBV before surgery with 2 doses of vaccine and presenting a protective level of anti-HBs.

Methods: Consecutive patients from surgical/gynecologic wards of 10 randomly selected hospitals in West Pomerania, Poland, hospitalized between November 2010-February 2013 vaccinated against HBV only with a two-dose regimen were asked to complete an anonymous questionnaire. Patients admitted to the same wards, vaccinated with a 3-dose regimen served as controls. Serum samples were assayed for anti-HBs with the use of third-generation testing methods.

Results: There were 185 participants $(59 \%$ women, with a mean age of 50.2 years); $76.8 \%$ were immunized at the request of referring surgeons. Regarding anti-HBs titer after taking 2 doses of HBV vaccine-in $17.8 \%$ of the subjects it was $0.0 \mathrm{~m} \mathrm{IU} / \mathrm{ml}$, in $31.4 \%$ between 0.1 and $10 \mathrm{~m} \mathrm{IU} / \mathrm{ml}$, in $23.8 \%$ between 10.1 and $100 \mathrm{~m} \mathrm{IU/}$ $\mathrm{ml}$, in $27.0 \%>100 \mathrm{~m} \mathrm{IU} / \mathrm{ml} .46 .0 \%$ of the patients were operated on within 0-60 days of taking the second vaccine dose, $16.2 \%-61$ to 180 days after, and $37.8 \%>180$ days after. In those vaccinated 0-60 days before surgery, $61.2 \%$ had anti-HBs titer below the protective level, in those vaccinated 61-180 days before surgery$23.3 \%(p=0.0008)$, in those vaccinated $>180$ days before surgery $-45.7 \%(p=0.08)$. In the group of 42 controls vaccinated with a 3 -dose regimen all were operated on $>180$ days after taking the third vaccine dose. Only $11.9 \%$ of them presented anti-HBs titer below the protective level, significantly less $(p<0.0001)$ than in the group vaccinated with a 2 -dose regimen. In patients vaccinated $>180$ days before surgery, the fraction of those presenting anti-HBs titer below the protective level was significantly $(p=0.0005)$ higher in the group vaccinated with a 2-dose regimen compared to the group vaccinated with a 3 -dose regimen.

Conclusions: 1. Current recommendations regarding a two-dose vaccination schedule against HBV before surgery should be revised, as it does not protect almost half of operated patients against HBV infection.

2. Special attention should be paid to the group vaccinated with a twodose regimen less than 2 months before surgery due to the highest percentage of those unprotected observed in this group.

3. In more than a third of cases the standard three-dose regimen could have been implemented, as participants had time to complete a third dose.

\section{P-221}

Factors associated with the immune response to hepatitis A vaccination in HIV-infected patients in the era of highly active antiretroviral therapy

Topic: Diseases - Infections

Presenter: Guillermo Mena

Guillermo Mena 1), 2); Anna Llupià 1), 2); Alberto L. GarcíaBasteiro 2); Consolación Díez 1); Josep Costa 3); Josep M. Gatell 4); Felipe García 4); José M Bayas 1), 2)

1) Preventive Medicine and Epidemiology Unit, Hospital Clinic, Barcelona, Spain; 2) Barcelona Centre for International Health 
Research (CRESIB, Hospital Clinic-Universitat de Barcelona), Barcelona, Spain; 3) Clinical Microbiology and Parasitology Unit, Barcelona, Spain; 4) Infectious Diseases Unit, Hospital Clinic, Barcelona, Spain

Background: HIV seropositivity is considered a risk factor for complications in hepatitis A virus (HAV) infection. HAV vaccination schedules are widely implemented in HIV-infected patients, but the immune response remains impaired

Objectives: The aim of this study was to analyze the proportion of responders among HIV-infected patients receiving the primary HAV standard or rapidly accelerated vaccination schedule and identify the factors associated with seroprotection rates.

Methods: We analyzed the response to vaccination (antiHAV titers $\geq 20$ IU/L) in $282 \mathrm{HIV}$-infected patients included in a standard (1440 EIU at 0, 6 months) or rapidly-accelerated schedule (720 EIU at 0,7,21 days and 6 months) between 1997 and 2009. Factors associated with the response to vaccination were analyzed using logistic regression.

Results: The overall response rate was $73.4 \%$. Male sex (OR 0.16, $95 \%$ CI 0.05-0.51) and hepatitis C virus co-infection (OR 0.30, $95 \%$ CI 0.14-0.74) were associated with a lower probability of response. Protective antibody response was associated with a higher CD4/CD8 ratio (OR 3.69, $95 \%$ CI 1.3-10.5) and having received two doses of standard schedule (compared with patients receiving only one dose of the same schedule (OR 2.51, $95 \%$ CI 1.22-5.15). Three doses of the rapidly-accelerated schedule were not more effective than a single dose of 1440 EIU (OR 1.32, $95 \%$ CI 0.48-3.63).

Conclusion: The low responses observed in patients receiving a single dose suggest the need to emphasize adhesion to vaccination protocols to avoid failure. The CD4/CD8 ratio may be considered as an immune status marker which could help to better choose the moment of vaccination. Our findings underscore the importance of identifying strategies that optimize the timing and effectiveness of hepatitis A vaccination in HIV-infected patients and of the need for further studies on individual factors such as sex and hepatitis $\mathrm{C}$ coinfection that may affect the response to vaccination. Likewise, the sub-optimal effectiveness of three doses of $720 \mathrm{U}$ in the rapidlyaccelerated schedule if confirmed in future studies, might lead to a revision of the current schedule recommended for HIV-infected travellers.

\section{P-222}

Prediction of hospital admissions associated with $\mathrm{PM10}$ and $\mathrm{O}_{3}$ pollution in metropolitan areas in portugal using ARIMA models

Topic: Diseases - Infections

Presenter: Mónica Alexandra Rodrigues

\section{Mónica Alexandra Rodrigues 1); Paula Santana 1), 2)}

1) Departamento de Geografia, Universidade de Coimbra, Coimbra, Portugal; 2) Centro de Estudos Geográficos e de Ordenamento do Territorio (CEGOT), Departamento de Geografia da Universidade of Coimbra, 3030, Coimbra, Portugal

Background: Air pollution has become one main environmental concern because of its known impact on human health. Early severe air pollution episodes, characterized by high levels of particulate pollution, have been associated with considerable hospital admissions.

Objectives: This study examined the effects of concentrations of pollutants in ambient air on hospital admissions for respiratory diseases in Metropolitan Areas in Portugal (Lisbon and Porto).

Methods: Data on hospital admissions in persons aged less than 14 year (children), 14-35 year (adults) and 64 year and older were obtained from records for the years 2000 through 2010. Daily counts of admissions, by admit date, were computed for pneumonia and influenza (ICD9 480-489), chronic obstructive pulmonary disease (COPD) (ICD9 490-499), asthma (ICD9 493), emphysema (ICD9 492), chronic bronchitis (ICD9 491), respiratory diseases (ICD9 460-519). Daily air pollution data from all monitoring stations for ozone and PM10 in Lisbon and Porto were obtained and was computed. In the same time, the autoregressive integrated moving average (ARIMA) model was used to make real-time on the number of Hospital Admissions for respiratory diseases (HARD) in Metropolitan Areas and, a statistical model will be proposed by the use of using Box \& Jenkins methodology. To the HARD series the used data were the month average, from January 2000 to December 2010. For modeling by autocorrelation function (ACF) and partial autocorrelation (PACF) methods, examination of values relative to auto regression and moving average were made and at last, an appropriate model for estimation of HARD values were found. To prevent from excessive fitting errors, AIC statistics was used. In comparison of the schemes obtained considering the least AIC and BIC value, the final model with the best fitting of data using the method of maximum likelihood.

Results: The results indicate that the seasonal ARIMA models provide reliable and satisfactory predictions for HARD parameters on monthly scale.

Conclusions: Exposure to PM10 and $\mathrm{O}_{3}$ increases the risk for hospital admission for respiratory diseases and the implementation of forecasting service to general practices in Portugal (to the Metropolitan Areas) contributed to reduce the respiratory diseases admissions. The reduction in air pollution is necessary to protect the health of the population that live in metropolitan areas.

\section{P-223}

\section{Tuberculosis in indigenous children: Guarani-Kaiowá}

Topic: Diseases - Infections

Presenter: Naise Lara Tontini

Vania Paula Stolte 1); Everton Ferreira Lemos 2); Naise Lara Tontini 3); Cynthia Mitie Nakagawa 4)

1) UNIGRAN; 2) Health Sciences Federal University of Grande Dourados; 3) Pavol Josef Safárik University; 4) Federal University of Mato Grosso do Sul/Prof Hélio Mandetta Faculty of Medicine.

Introduction: Tuberculosis (TB) remains one of the most prevalent and worrying diseases in the world, especially in indigenous populations who face socioeconomic, environmental and territorial difficulties, making them vulnerable to diseases and worsening health conditions. When it appears in children is considered a sentinel event. Objective: estimate the incidence of tuberculosis in children of indigenous ethnic Guarani-Kaiowá that have less than 5 years old, from 2001 to 2009, living in Indigenous Lands (IL) located on the municipalities of Amambai and Dourados/MS, Brazil.

Methods: this is a descriptive and retrospective epidemiological study, where we used data reported by TB on children that have less than 5 years between the period 2001-2009. The data are part of the Livro de Registros e Controle de Tuberculose do Distrito Sanitário Especial Indígena de Mato Grosso do Sul (DSEI/MS)" (Book of Records and Tuberculosis Control of Special Indigenous Sanitary District of Mato Grosso do Sul).

Results: We found that in 2001-2003 the IL belonging to Dourados showed high incidence rate, an average of 1,240 cases per 100,000 inhabitants. The IL Amambai had in the 2004-2007 higher incidence rate recorded in the period, reaching Limão Verde villgade with 630/100.000 and 225/100.000 on Amambai village. The analysis from 
2001 to 2009 showed that, despite the reduction of TB cases along the years, the incidence rate is far above national targets presented to Amambai values between Dourados and 167-321/100.000 respectively.

Conclusions: Despite the implementation of affirmative action to control the disease in indigenous populations, incidence rates among children and indigenous Guarani Kaiowá still remain high, representing a sentinel event for disease control and justifying the need for special attention to this population.

\section{P-224}

Assessment of the epidemiological surveillance system for viral hepatitides in João Pessoa, Brazil

Topic: Diseases - Infections

Presenter: Rudgy Pinto De Figueiredo

Magda Cecilia Cardoso Ferreira 1); Alessandro Pecego Martins Romano 2); Rudgy Pinto De Figueiredo 3); Patricia Carla Dos Santos 3)

1) Municipal Health Secretariat; 2) Ministry of Health; 3) University of São Paulo

Background: Viral hepatitides are infectious diseases, of endemicepidemic behavior, great magnitude and worldwide distribution. It is estimated that two billion people have been contaminated with the hepatitis B virus and that about 600 millions die each year in the world as a result of its consequences (WHO 2012). In Brazil, where hepatitis A is highly endemic, hepatitides B and D are markedly prevalent, hepatitis $\mathrm{C}$ is moderately endemic, viral hepatitides constitute a grave public health problem and a challenge to the epidemiological surveillance system.

Objective: To assess the epidemiological surveillance system for the viral hepatitides during from 2009 to 2011 in the municipality of João Pessoa, Brazil, using the methodology proposed by the directives of the Centers of Disease Control and Prevention (CDC).

Methods: The study has been based on information from the National System of Registration of Diseases. The CDC methodology was used for the assessment of the epidemiological surveillance of viral hepatitides, by means of the appraisal of the following attributes: simplicity (structure and ease of operation), acceptability (rate of participation), representativeness (precision and distribution of the occurrence of the event concerned), opportunity (rapidity between the steps of the surveillance system) and data quality (completeness and validity).

Results: The results revealed a system of low acceptability and high percentages of sub-notification at the health units (5.8 and $11.6 \%$ ). The various flows of entry and the difficulties involved in the interpretation of the serum markers were responsible for the complexity of the surveillance system. Representativeness was considered positive, due to an increase (of 2.3 times) in the number of cases notified between 2009 and 2011. The attribute opportunity was considered reasonable (an average number of 9.3 days for the conclusion of cases). On the other hand, the high number of spaces left blank and ignored contributed to the low data quality, compromising the trustworthiness of the system of epidemiological surveillance for the viral hepatitides and consequently the adoption of effective actions and strategies for the surveillance and control of this disease.

Conclusions: If the quality and trustworthiness of the epidemiological survey system of the viral hepatitides are to be perfected, it is fundamental that investment be made in the training of the personnel responsible for the filling up of the records of notification/investigation, as well as in greater integration between the sectors responsible for the flow of information, so that the quality of the registration of notifications be improved.

\section{P-225}

Seroprevalence of measles and rubella in immigrant workers at the Republic of Korea

Topic: Diseases - Infections

Presenter: Yu-Mi Kim

Yu-Mi Kim 1); Byoung-Gwon Kim 1); Young-Seoub Hong 1); Hyunsuk Hong 2); Hyun Jin Son 3)

1) Dong-A University, Busan, Korea; 2) National University Hospital, Seoul, Korea; 3) Korea Center of Disease Control

This study aims to collect sera of immigrant workers in Busan city and Gyeongsangnamdo province, Korea and to analyze seroprevalence for measles and rubella.

The voluntary foreign workers who have agreed informed consents with a translated format into the native language, participated in this study. IgG antibodies for measles and rubella were examined by ELISA using the automation equipment (BEP-ELISA). This study obtained the approval of Dong-A University Clinical Research Ethics Review Committee.

Total of 404 people were recruited. 364 people of foreign workers participated in the study, and 40 women who immigrated for the international marriage participated. Regional distribution for study participants was composed of 78 people in Vietnam $(19.3 \%)$, Uzbekistan 73 people $(18.1 \%)$, Thailand 70 people $(17.3 \%)$, China 68 people $(16.8 \%)$, Philippines 41 people (10.2\%), and Indonesia 32 people $(7.9 \%)$.

Those surveyed 404 people showed $97.3 \%$ positivity $(95 \% \mathrm{CI}$ : 95.7-98.9\%) for measles antibody and $89.4 \%$ positivity $(95 \%$ CI 86.4-92.4) for rubella. Considering herd immunity, the subjects of the measles, rubella seropositivity was considered the recommended level on. The seroprevalence of rubella was different depending on the purpose of the immigrant. The positive rates of rubella antibody were $72.5 \%$ of foreign workers to $91.2 \%$ of marital immigrant women $(P$ value by Fisher's exact test $=0.0013)$.

In the survey, marital immigrant women were more susceptible to rubella, which was estimated to be due to younger age at immigration. As the goal of rubella immunization is prevention of congenital rubella syndrome during pregnancy, a concern for marital immigrant women is needed. Many of those surveyed represents a hope for immunization services and health services for foreign migration. The support systems in health policy for immigrant workers, which can increase the accessibility should be planned.

\section{P-226}

Trends in pandemic H1N1 and other viral influenza types in Japan from 2010 to 2012

Topic: Diseases - Infections Presenter: Yoshitaka Murakami

Yoshitaka Murakami 1); Shuji Hashimoto 2); Miyuki Kawado 2); Akiko Ohta 3); Kiyosu Taniguchi 5); Yuki Tada 4); Mika Shigematsu 4); Masaki Nagai 3)

1) Department of Medical Statistics, Shiga University of Medical Science; 2) Department of Hygiene, Fujita Health University; 3) Department of Public Health, Saitama Medical University; 4) Infectious Disease Surveillance Center, National Institute of Infectious Diseases; 5) Department of Pediatrics, National Mie Hospital

Background: Quantitative information on influenza from the infectious disease surveillance system in Japan provides an important 
resource for helping to predict and prevent future influenza epidemics. However, so far, few have been reported with regard to estimating numbers of cases or identifying trends of influenza types.

Objective: To estimate the numbers of cases and identified type specific trends of pandemic influenza $\mathrm{H} 1 \mathrm{~N} 1(\mathrm{~A}(\mathrm{H} 1) \mathrm{pdm})$ and other virological-types (A(H1), A(H3) and B) in Japan from 2010 to 2012, by using data from the infectious disease surveillance system.

Methods: Two sources of weekly information from the infectious disease surveillance system are available; the reported influenza cases from sentinels in each prefecture and the proportions of different virological-types of influenza that were identified. Our study period was from week 36 (September 6) of 2010 to week 18 (May 6) of 2012. The weekly numbers of influenza cases were estimated, assuming that the sentinel samples in each prefecture had been selected randomly. The estimated numbers of influenza cases were multiplied by the weekly fraction of each virological-type identified, to calculate the numbers of pandemic influenza $\mathrm{H} 1 \mathrm{~N} 1$ and other virological-type cases. Results include the estimated numbers and their $95 \%$ confidence intervals $(95 \% \mathrm{CI})$.

Results: The estimated number of cases of influenza types during September 2010 to May 2011 (in thousands) is as follows; A(H1)pdm: 6484 and 28, $\mathrm{A}(\mathrm{H} 1): 1$ and 38, $\mathrm{A}(\mathrm{H} 3): 4126$ and 10888, and $\mathrm{B}: 3009$ and 5336 respectively. We found that different peak weeks were observed in the pandemic in 2010: $\mathrm{A}(\mathrm{H} 1)$ pdm:1405 (95\% CI 1332-1477) (in thousands) in week 4), seasonal influenzas A(H3):364 (95 \% CI: 319-409) in week 5 and B:357 (95 \% CI 307-407) in week 11). Different peak patterns were also observed in seasonal influenza in season 2011: (A(H3):1744 (95\% CI 1644-1844) in week 5 and B:559 (95\% CI 493-624) in week 10). Overall, the weekly trends showed that the pandemic influenza H1N1 was dominant in the 2010 season and the seasonal influenza types, $\mathrm{A}(\mathrm{H} 3)$ and $\mathrm{B}$ were dominant in the 2011 season.

Conclusion: We used the routinely collected data by the influenza surveillance system in Japan to estimate the numbers of pandemic influenza $\mathrm{H} 1 \mathrm{~N} 1 \mathrm{~A}(\mathrm{H} 1) \mathrm{pdm})$ and other virological-type cases in 2010/2011 and analyzed the type-specific trends. This type of analysis will be useful for predicting the future outbreaks of influenza in Japan.

\section{P-228}

Ongoing hepatitis A outbreak in Bijeljina, Bosnia and Herzegovina, August 2012-April 2013

Topic: Diseases - Infections

Presenter: Zoran Dakic

\section{Zoran Dakic 1); Sanjin Musa 2); Marjena Dakic 1);} Sladjana Tesic 1)

1) Health center Bijeljina, Bijeljina; 2) Institute for Publice Health of Federation of Bosnia and Herzegovina, Sarajevo

Background: On 17 January 2013 Epidemiological service of Bijeljina (ES) declared hepatitis A outbreak. Bijeljina is a town and municipality with about 120,000 inhabitants, located in the north-east of Bosnia and Herzegovina, on a borderline with Croatia and Serbia. In the past 20 years, the epidemiological situation of infectious intestinal diseases in this area was generally stable. Despite numerous challenges for public health, since 1992 there were no cases of hepatitis A.

Objectives: Aim of this report is to describe hepatitis A outbreak in Bijeljina and the measures taken in order to suppress it.

Methods: In the Bosnian governing entity Republic of Srpska (RS) hepatitis A is notifiable disease in accordance with Law on protection of population against infectious diseases. Case definition of communicable diseases in this Law corresponds to EU case definitions.
However, specific guidelines for handling cases of hepatitis A do not exist.

Results: Epidemiological service of the Health center Bijeljina reported to the RS Institute of Public Health hepatitis A outbreak in Bijeljina, on 17 January 2013. A total of 28 cases ( 16 females and 12 males) were reported until 02 April 2013. Age distribution: the youngest case was 7 years old, the oldest one 70 years old. The most affected age group was of 0-14 years with $29.7 \%$ number of cases. The first notified case was a child from the Roma population, who got sick in August 15, 2012. After this, 5 more hepatitis A cases were registered from the same population ( $21 \%$ of total number). ES performed disinfection of houses and the immediate environment of patients also as disinfection of kindergartens and school facilities and other collective accommodation buildings. Monitoring of drinking water quality from the waterworks and affected households was intensified. Vaccines and immunoglobulin against hepatitis A are not available in RS.

Conclusions: According to epidemiological data -most probable routes of transmission were indirect contact. Lessons learned from this outbreak demonstrated the weak points for possible future outbreaks in this area: unresolved infrastructure issues, poor hygiene practices of certain parts of the population, a very modest level of hygienic conditions in the most of public institutions, restaurants and often passive work of inspection services. Considering fact that one of the countries in the region (Croatia) this year will become EU member state, there is an obvious need for the establishment and improvement of institutional relations in public health at the regional and interstate level.

\section{P-229}

Treatment outcomes in Pulmonary Tuberculosis and associated factors worldwide: a systematic review and meta-analysis

Topic: Diseases - Infections

Presenter: Ana Costa Veiga

Ana Costa Veiga 1); Cristiana Areias 2); Teodoro Briz 2); Carla Nunes 3)

1) National School of Public Health (ENSP)/NOVA University of Lisbon and Lisbon School of Health Technology (ESTeSL)/ Polytechnic Institute of Lisbon; 2) National School of Public Health (ENSP)/NOVA University of Lisbon; 3) National School of Public Health (ENSP) and CMDT LA/NOVA University of Lisbon

Background: Tuberculosis (TB) is the second leading cause of death from an infectious disease in the world. The Global Plan to Stop TB 2011-2015 recommends a treatment success rate of $87 \%$ by 2015 as a target. Monitoring treatment success and its factors is therefore a key issue in TB control programmes.

Objectives: To describe treatment success rates in pulmonary $\mathrm{TB}$ cases and to identify factors associated with unsuccessful treatment outcomes, according to ad-hoc studies.

Methods: A systematic review of articles published between 2000 and 2012 was made, following the MOOSE guidelines (Meta-analysis of Observational Studies in Epidemiology Group). Online bibliographic databases PUBMED and WEB OF KNOWLEDGE were searched to identify relevant papers. Treatment outcomes corresponded to WHO categories. Factors associated with unsuccessful outcomes explored were divided into: sociodemographic (age, sex), disease-related (co-morbidities), behavioral (drug and alcohol abuse) and treatment-related (directly observed therapy-DOT). Randomeffect meta-analysis was used to estimate a combined success rate and pooled odds ratios $(\mathrm{OR})$. 
Results: From the 225 articles initially identified, 24 were considered for full-text review on the basis of the inclusion criteria. 17 of these approached determinants for successful outcomes and 7, for unsuccess. Successful outcomes included cured patients ( 8 articles) and cured plus completed treatment cases (16 articles).

Success rates reported ranged from 49.6 to $92.8 \%$ and their pooled estimate was of $79.0 \%$ (95\% CI 76.0-81.8\%). 7 showed success rates above $85 \%$, and only 5 presented a success above $87 \%$, 3 of which were institution-based. Studies with the highest rates $(>87 \%)$ included new pulmonary patients only.

A random-effect model was used, as studies did not share a common effect size $\left(\mathrm{Q}=1550.81 ; p=0.000 ; \mathrm{I}^{2}=98.5 \%\right)$. No possible effect of publication bias was found (Begg's test, $p=0.206$; Egger's tests, $p=0.744$ ). None of the studies was removed, based on a sensitivity analysis.

Meta-analysis was conducted only for age and sex, due to the limited number of studies focusing on other factors. Unsuccesses were significantly associated with male sex (OR 1.22, $95 \%$ CI 1.06-1.40). Age did not appear as a relevant factor.

Conclusions: Treatment success varied widely and only $1 / 5$ of the studies evinced success rates above the $87 \%$ WHO recommended threshold for pulmonary TB. Diversity of the studies regarding risk factors identified made it difficult to analyze their association with unsuccessful outcomes; male sex was the most commonly identified factor. Most programs performance need to be closely followed and improved.

\section{P-230}

\section{Are vets at zoonotic risk for hepatitis $\mathrm{E}$ virus from pets?}

Topic: Diseases - Infections

Presenter: João Rodrigo Mesquita

João Rodrigo Mesquita 1), 2); Nádia Conceição-Neto 3); Gregório Valente-Gomes 3); Maria São José Nascimento 2), 3)

1) Escola Superior Agrária, Instituto Politécnico de Viseu. Quinta da Alagoa - Estrada de Nelas, Ranhados, 3500-606 Viseu, Portugal; 2) CIBIO/UP, Centro de Investigação em Biodiversidade e Recursos Genéticos/Universidade do Porto, Campus Agrário de Vairão, Vairão, Portugal; 3) Laboratório de Microbiologia, Departamento de Ciências Biológicas, Faculdade de Farmácia, Universidade do Porto. Rua de Jorge Viterbo Ferreira, 228, 4050-313 Porto, Portugal

Background: The discovery of autochthonous hepatitis E in industrialized countries has substantially changed our understanding about this viral infection. Nowadays it is recognized that hepatitis E virus (HEV) infection has two distinct epidemiological profiles, one of large outbreaks and epidemics observed in developing countries mainly transmitted via faecally-contaminated water and caused by genotype 1 , the other seen in industrialized countries, characterized by sporadic cases of hepatitis, associated to zoonotic transmission and mainly due to genotype 3 . Autochthonous infections have been linked to the consumption of undercooked pork or game meat and also to the direct contact to infected swine. Although the full range of species that are reservoirs for HEV is still unknown, serologic studies have shown the presence of antibodies against HEV (anti-HEV) in dogs and cats suggesting HEV circulation in these animals. However, until now, no concern on the potential zoonotic risk of pets have been seen. Objectives: Hence, the aim of the present work was to evaluate the potential zoonotic transmission of HEV from dogs and cats by studying the HEV seroprevalence in the occupationally exposed pet veterinarians and matched general population.

Methods: A total of 483 sera (363 from vets and 120 from general population) were studied for the presence of IgG anti-HEV using a commercial ELISA kit (Wantai Biological Pharmacy Co., Beijing,
China). A Chi square test for homogeneity of proportions (SPSS 13.0, SPSS Inc., Chicago, IL, USA) was used to compare the differences between the two studied populations.

Results: The HEV seropositivity in veterinarians and general population was $9.92 \%(36 / 363)$ and $13.33 \%(16 / 120)$, respectively. No statistically significant difference $(p=0.2311)$ was found between these two seroprevalences. Within the veterinarians group, history of owning dogs (adjusted odds ratio [OR] 15.34, $95 \%$ confidence interval [95\% CI] 4.15-56.7, $p<0.001)$ and experiencing needlestick injury with sharps contaminated with dog blood (OR 12.99, $95 \%$ CI 3.42-49.36, $P<0.001)$ were found to be risk factors for HEV seropositivity. No statistical association was found with any of the other variables (age, sex, district, years in practice, history of owning cats and needlestick injury with sharps contaminated with cat blood).

Conclusions: Overall, this study shows that veterinarians are not at higher risk for zoonotic transmission of HEV from pets when comparing to general population. To our knowledge this is the first work studying the occupational risk of pet veterinarians to the potential zoonotic transmission of HEV from dogs and cats.

\section{P-231}

Seroepidemiological survey of Toxoplasma gondii in wild boar, Portugal, 2012: a Public Health risk

Topic: Diseases - Infections

Presenter: João Rodrigo Mesquita

Catarina Coelho 1), 2); João Rodrigo Mesquita 1), 3); Ana Patrícia Lopes 2), 4); Madalena Vieira-Pinto 2), 4)

1) Escola Superior Agrária, Instituto Politécnico de Viseu. Quinta da Alagoa - Estrada de Nelas, Ranhados, 3500-606 Viseu, Portugal; 2) Centro de Ciência Animal e Veterinária (CECAV), Universidade de Trás-os-Montes e Alto Douro (UTAD), Vila Real, Portugal; 3) CIBIO/UP, Centro de Investigação em Biodiversidade e Recursos Genéticos/Universidade do Porto, Campus Agrário de Vairão, Vairão, Portugal; 4) Departamento de Ciências Veterinárias, Escola de Ciências Agrárias e Veterinárias Universidade de Trás-os-Montes e Alto Douro (UTAD), Vila Real. Portugal

Background: Toxoplasma gondii is an important zoonotic pathogen with worldwide distribution that is known to cause abnormalities in the newborn or even abortion after primary infection during pregnancy. Human foodborne infection occurs by ingestion of food or water contaminated with sporulated Toxoplasma oocysts or tissue cysts, upon consumption of raw or undercooked meat. Although $T$. gondii infection is of limited clinical importance in wild boar, when infected, these animals pose as a source of infection for people if their meat is eaten undercooked.

Objective: The purpose of the present work was to access the risk of human infection via meat consumption by estimating the seroprevalence of T. gondii in wild boars hunted in Portugal.

Methods: Blood (97) samples were taken from wild boars killed during the hunting season 2011/2012 from four sampling sites of the north of Portugal. Data regarding collection site, animal gender and age was collected. Sera diluted at 1:20 were tested for immunoglobulin $\mathrm{G}$ antibodies to $\mathrm{T}$. gondii, using the modified agglutination test (MAT). A cut-off titre of 20 was chosen to maximize both sensitivity and specificity of the test. Multivariate logistic regression models were performed for risk factor analysis using "Epicalc" package in the R software ( $\mathrm{R}$ 2.13.1).

Results: Overall, 20 (20.6\%) out of the 97 animals were seropositive for $T$. gondii $\mathrm{IgG}$ antibodies. Adult age (adjusted odds ratio $[\mathrm{aOR}]=18.48,95 \%$ confidence interval $[\mathrm{CI}] 13.89,180.44)$ and the 
male gender $(\mathrm{aOR}=3.75,95 \% \mathrm{CI}: 1.09,12.88)$ were found to be risk factors for $T$. gondii $\mathrm{IgG}$ seropositivity in wild boars. Increased probability for seropositivity in the older animals is likely to be linked to more chances for exposures occurring during the years, while males might be at risk due to their increased exploratory behaviour. Conclusions: These results reveal that $T$. gondii infection occurs frequently in the wild boar population from Portugal. Wild boar is widely used to make homemade sausages in an uncontrolled traditional process that does not undergo any heat stage, thus being consumed raw. The consumption of raw or undercooked meat or meat products reveals a potential risk for Public Health. T. gondii naive pregnant women should take special care and avoid consuming meat from older and male wild boars from Portugal.

\section{P-232}

Relationship between tobacco and bacterial load, caga and vaca i1 in patients infected by helicobacter pylori

Topic: Diseases - Infections

Presenter: María Paz

María Paz 1); Miguel Santibáñez 1), 2); Estefanía Aguirre 3); Nuria Aragones 4); Sofía Belda 3); Jesús Saez 5); J. C. Rodríguez 3); J. Sola-Vera 5); Montserrat Ruíz-García 3); Gloria Royo 3), 6)

1) Universidad de Cantabria, Santander, Spain; 2) IFIMAV, Santander, Spain; 3) S. Microbiología. Hospital General Universitario de Elche, Alicante, Spain; 4) Área de Epidemiología Ambiental y Cáncer, Centro Nacional de Epidemiología, ISCIII, Madrid, Spain; 5) S. Medicina Digestiva. Hospital General Universitario de Elche, Elche (Alicante), Spain; 6) Universidad Miguel Hernández, Elche, Spain

Background: Our aim was to study the relationship between smoking and $H$. pylori infection, focusing on the cagA and vacA il virulence factors and bacterial load.

Methods: Biopsies of the gastric corpus and antrum from 155 consecutive patients in whom there was clinical suspicion of infection by H. pylori were processed. Classical and molecular methods were used to quantify the number of microorganisms by Real-Time PCR and presence of cagA and vacA il genes. A standardized questionnaire was used to obtain patients' clinical data and information on demographic and lifestyle variables, including tobacco and alcohol consumption. Unconditional logistic regression was used to estimate the association between tobacco smoking and $H$. pylori infection, bacterial load, and cagA and vacA i1 virulence factors.

Results: There was a statistically significant higher prevalence of $H$. pylori infection among active-smokers at endoscope according to both Classic Gold standard and Real-Time PCR. Likewise, cagA gene was significantly associated with smoking: adjusted Odd Ratio (ORa) 4.52 (95\% CI 1.28-15.98) and evidence of association was found for vacA i1, Crude OR 2.69 (95\% CI 0.92-7.92). Bacterial load was higher in active-smokers, although these differences were not statistically significant (median of 262.2 vs. 79.4 copies of H.Pylori per cell). Positive associations without yielding statistical significance were found between active smoking when the bacterial load was divided into ordinal categories (scarce, moderate and abundant), ORa abundant 2.64 (95 \% CI 0.72-9.65).

Conclusions: The associations between smoking, colonization of the mucosa by a virulent bacterial population and bacterial load, support a complex interaction between the microorganism and gastric mucosa in which synergistic effects could increase gastric pathology.

\section{P-233}

Epidemiology of extrapulmonary tuberculosis in Brazil: use of the hierarchical model

Topic: Diseases - Infections

Presenter: Ethel Leonor Noia Maciel

Teresa Gomes 1); Ethel Leonor Noia Maciel 1); Bárbara Reis-Santos 1)

1) Laboratório de epidemiologia - UFES

Background: Extrapulmonary tuberculosis (EPTB) is less frequent and less ddressed by programs of control of tuberculosis (TB), but with the advent of the human immunodeficiency virus (HIV), the EPTB is gaining importance on the world stage.

Objectives: This study aimed to analyse the epidemiology of EPTB in Brazil in the period 2007 to 2011.

Methods: It's a cross-sectional study with all TB cases reported in the National Surveillance System Information in Brazil between 2007 and 2011. It was made a descriptive analysis of EPTB and pulmonary TB (PTB) cases followed by the comparison between the clinical forms regarding socioemographic and clinical aspects. Chi square test was conducted, the variables with $p$ value $<0.05$ followed for a hierarchical regression model, and variables with $p<0.05$ in each level remained in the model.

Results: The study had 427,548 TB cases, including 356.341 (83.35\% IC $95 \%$ 83.23-83.45 \%) PTB cases, 53.853 (12.60\% IC $95 \%$ 12.49-12.69 \%) EPTB cases, 11.586 (2.70 \% IC $95 \% 2.66$ $2.75 \%$ ) concomitant pulmonary and extrapulmonary TB cases (PTB + EPTB) and 5.768 (1.35\% IC $95 \%$ 1.31-1.38 \%) miliary TB cases. The trend of PTB and EPTB had no variation in the period ( $p$ : $0.635)$. The main age group was $18-39$ years $(47.0 \%)$ but the proportion of children aged 0-7 years was significant when compared to PTB (OR $1.9195 \%$ CI 1.73-2.10). EPTB cases were mostly white skin $(45.2 \%)$ and most had 5-8 years of education (28.0 \%). Among the comorbidities stood out HIV (OR $2.2495 \%$ CI 2.18-2.31) and high proportion of HIV testing in progress or not performed $(39.9 \%)$. Alcohol use (OR $0.4595 \%$ CI 0.43-0.47), diabetes (OR: $0.5495 \%$ CI $0.51-0.57$ ) and mental illness (OR 0.88 CI $95 \% 0.82-0.95$ ) were related to PTB. The EPTB group had a higher proportion of smearnegative result $(21.2 \% p<0,001)$. The Directly Observed Treatment (DOT) was not performed in $65 \%$ of EPTB cases. About outcome, there was a large number of transfers compared to PTB (OR 1.23 $95 \%$ CI 1.18-1.29).

Conclusions: The trend of PTB and EPTB in the country in 05 years showed no increase or decline striking, and it showed that TB control measures should be more effective to decrease the number of cases. Results demonstrated that characteristics related to EPTB often differ from those related to PTB, and differ from those reported in countries with a low burden of $\mathrm{TB}$, so this study helps in understanding the magnitude of EPTB in Brazil - a high burden TB country.

\section{P-234}

Does marriage protect against hospitalisation with pneumonia? A population-based case-control study

Topic: Diseases - Infections

Presenter: Anil Mor

Anil Mor 1); Elisabeth Svensson 1); Sinna P. Ulrichsen 1); Henrik Toft Sørensen 1); Reimar Wernich Thomsen 1) 
1) Department of Clinical Epidemiology, Aarhus University Hospital, Aarhus, Denmark

Objectives: Being unmarried may affect health behaviours, economic resources, and immune response perhaps due to physiological and psychological distress, leading to increased risk of infections. This study examines the association between marital status and risk of hospitalization with pneumonia.

Methods: In this large population-based case-control study we identified adult patients with a first-time pneumonia-related hospitalisation between 1994 and 2008, using health care databases in Northern Denmark. For each case, 10 sex- and age-matched population controls were selected from Denmark's Civil Registration System. We estimated odds ratios (ORs) for pneumonia hospitalisation among persons who were divorced/widowed or never married, as compared to married persons. We used regression analysis to control for a wide range of confounding factors.

Results: The study included 67,162 patients with a pneumonia-related hospitalisation and 671,620 population controls. Among pneumonia cases, $47 \%$ were married, $40 \%$ were divorced/widowed, and $13 \%$ had never been married. As compared with married persons, the unadjusted ORs for pneumonia were 1.34 in divorced/ widowed individuals and 1.30 in never married persons, respectively. After adjustment for age, gender, 19 different comorbidities, alcoholism, immunosuppressive treatment, urbanization, and living with small children, the adjusted OR for pneumonia-related hospitalisation among patients who were divorced/widowed was 1.20 (95\% CI $1.17-1.22$ ), as compared to patients who were presently married. Among never married patients, the adjusted OR was 1.33 (95\% CI 1.29-1.37).

Conclusions: Married patients have a clearly decreased risk of being hospitalised with pneumonia, possibly related to more social support, healthier lifestyle or higher education.

\section{P-235}

Pulmonary Tuberculosis in Continental Portugal 2000-2010: temporal trends clustering as a tool for control evaluation

Topic: Diseases - Infections

Presenter: Cristiana Areias

Cristiana Areias 1); Carla Nunes 1), 2); Ana Veiga 1), 3); Teodoro Briz 1), 2)

1) National School of Public Health/University NOVA of Lisbon; 2) Centre of Public Health Studies and Research; 3) Lisbon School of Health Technology/Polytechnic Institute of Lisbon

Background: Clustering analysis is a useful tool to detect and monitor disease patterns and, consequently, to contribute for an effective population disease management. Portugal has the highest incidence of Tuberculosis in the European Union (in 2012, 21.6 cases per 100000 inhabitants), although it has been decreasing consistently. Two critical PTB (Pulmonary Tuberculosis) areas, metropolitan Oporto and metropolitan Lisbon regions, were previously identified through spatial and space-time clustering for PTB incidence rate and risk factors. Identifying clusters of temporal trends can further elucidate policy makers about municipalities showing a faster or a slower TB control improvement.

Objectives: To identify clusters of municipalities with high and low temporal trends, in continental Portugal.

Methods: Clustering analysis of spatial variation in temporal trends adjusted for sex (2000-2010) for all Portuguese continental municipalities $(n=278)$ was undertaken using the SaTScan Software. Data were obtained from National Tuberculosis Surveillance System (number of cases of PTB) and from Statistics Portugal (estimated population). Results were interpreted considering critical areas previously identified.

Results: A global annual decrease of $5.8 \%$ PTB cases was observed. Two clusters of municipalities with increasing trends were identified, one $(\mathrm{n}=3)$ including Grândola, Alcácer-do-Sal and Santiago do Cacém, with an annual increase trend of $35.3 \%(p=0.001)$; other $(\mathrm{n}=2)$, including Alfândega-da-Fé and Vila Flor, showing an annual increase of $86.9 \%(p=0.001)$.

Four clusters with decreasing trends were identified: a cluster $(n=29)$ comprising all municipalities of the previously identified metropolitan Oporto critical area, with an annual decrease of $7.6 \%$ $(p=0.001)$; a larger cluster $(\mathrm{n}=113)$, that included three municipalities of metropolitan Lisbon critical area (Lisbon, Odivelas, Amadora), presenting an annual decrease of $4.2 \%(p=0.001)$; a cluster with two municipalities previously identified in the critical area of metropolitan Lisbon (Almada, Oeiras) with an annual decrease of $5.6 \%(p=0.01)$; and finally a cluster $(\mathrm{n}=9)$, comprising Algarve region with an annual decrease of $0.8 \%(p=0.012)$.

Conclusions: Although a global decreasing trend was identified, clusters for both increasing and decreasing trends were found. Clusters of increasing trends showed much pronounced increases, but none of these municipalities were identified as part of PTB critical areas previously identified, and should be cautiously interpreted considering the low magnitude of the number of PTB cases. All municipalities previously identified as part of PTB critical areas were included in clusters with decreasing trends sharper than the national one, with the exception of Lisbon, Odivelas and Amadora. Algarve region cluster showed a very slow decreasing trend. These results may be explained by differences in trends of PTB risk factors or/and the impact of health policies management.

\section{P-236}

Introduction of pneumococcal vaccine in children aged 0-5 years in Mangistau, Kazakhstan in 2010-2012

Topic: Diseases - Infections

Presenter: Indira Karibayeva

Indira Karibayeva 1); Aikan Akanov 1); Rune Andersson 2); Saken Amireev 1); Andrej Grjibovski 3), 4)

1) School of Public health, Kazakh National Medical University named after S.D. Asfendiyarov, Almaty, Kazakhstan; 2) Institute of Biomedicine, Sahlgrenska Academy at Gothenburg University, Gothenburg, Sweden; 3) Department of International Public Health, Norwegian Institute of Public Health, Oslo, Norway; 4) International School of Public Health, Northern State Medical University, Arkhangelsk, Russia

Background: Respiratory diseases among children in Kazakhstan, and pneumonia in particular, are among the leading causes of death in children under 5 years. Altogether, there were 33774 cases of pneumonia in children under 5 years in Kazakhstan in 2008 alone. Total number of deaths in children under 5 years in 2008 was 8225 with 1.5 thousand deaths from pneumonia. In 2010, Kazakhstan initiated a vaccination programme for pneumococcal diseases in two of the 14 regions: East Kazakhstan and Mangistau, but the effectiveness of the vaccination has not been studied.

Objective: to assess the effectiveness of the vaccination for pneumococcal diseases in the Mangistau region, Kazakhstan.

Methods: The data was collected from medical cards of children under 5 years hospitalized in Regional Hospital for Infectious Diseases in the Mangistau region with diagnosis pneumonia from 2010 to 2012 years.

Results: Pneumococcal vaccine is given to children at ages 2, 4, and 12-15 months. Comparative analysis of the morbidity among 
vaccinated children showed that the overwhelming majority of the children hospitalized for pneumonia were not vaccinated. Altogether, 10411 children $(65.3 \%)$ in the Mangistau region were vaccinated in 2010-2012. At the moment, we can judge the epidemiological effectiveness of the vaccine in reducing the incidence of pneumonia. According to official data from the "Health of the population of the Republic of Kazakhstan and activity of the organizations of Health care in 2011' for 2010-2011 years incidence pneumonia of children under 5 years decreased by $20 \%$-from 12.3 in 2010 to 9.9 in 2011 on 1000 children of this age.

Conclusion: These preliminary results suggest that most of the cases of pneumonia in 2010-2012 among children in the age-group $0-5$ years occurred among non-vaccinated children. Moreover, a we observed a reduction on the incidence of pneumonia by $20 \%$ during the first year of implementation of pneumococcal vaccine. Further studies are warranted to study the effectiveness of vaccination of children in Kazakhstan.

\section{P-237}

Was it possible to anticipate the intensity of the Madeira's dengue epidemic for the year 2013?

Topic: Diseases - Infections

Presenter: Andreia Costa

\section{Andreia Costa 1); Matilde Rosa 1); Paulo Nogueira 1)}

\section{1) Directorate General for Health}

As dengue viruses infect millions of people each year, this disease is considered to be one of the most important infectious diseases in many tropical and subtropical countries. Studies indicate that about $40 \%$ of the world's population lives in countries at risk of dengue infection [1]. Although cyclic, incidences of this disease are increasing and spreading geographically [2]. The dengue virus has been detrimental for the inhabitants of many tropical areas, infecting millions of people worldwide. The dengue epidemic in the Autonomous Region of Madeira, a Portuguese archipelago, was officially declared by the Portuguese Health authorities on October 3rd, 2012.

The goals of this study were: (1) to better understand the evolution of the dengue fever in Madeira and (2) to anticipate its trend/intensity for the year of 2013. This study was challenged by the inexistence of past information; because Madeira is experiencing its first dengue epidemic. Thus, past information regarding outbreaks in other countries was obtained to better understand the behavior of the dengue fever itself. Collection criteria were: outbreak information available (year, serotype, duration, frequency, cases, attack rate, urban attack rate); and information related to infant mortality rate, urban population percentage and population density indicators.

Therefore, 6 regions of the globe were chosen according to the defined criteria aiming at obtaining similar conditions to Madeira in what would concern the dengue spread like climate, temperature and rainy season.

The 6 countries selected were: Cuba, The Bahamas, Guadeloupe, Vietnam (in particular South Vietnam), Australia (in particular Queensland) and Puerto Rico. They all have tropical or sub-tropical climates and their minimum and maximum temperatures do not exceed the interval from 15 to $34^{\circ} \mathrm{C}$.

The dengue attack rates in Madeira were expected to be approximately from 0.8 to $1.3 \%$ (relative to urban population), for clinical cases, and from around $0.4 \%$ (relative to urban population) to $0.7 \%$, for confirmed cases. Applying this interval attack rate to the population of 0.27 million living in Madeira Island, and taking into account that 0.19 million is urban population, after studying dengue fever trends in these selected countries in previous decades, it was concluded that, this year (2013), between 2160 and 2470 dengue clinical cases can be expected in Madeira. Up until February 3rd, 2013 there were 2164 diagnosed cases.

This analysis is subjective because information about the dengue trend in other countries is being used to anticipate the trend/intensity in Madeira. Each country reacts to an outbreak in a different way. However, taking into account that the goal of this study was not to have exact numbers for this year for dengue cases (2013), but to find some boundaries for them this study fulfills it and feels adequate. In fact, the prediction of the exact number of cases is really improbable; the presentation of a sound estimate is the most useful tool for the Portuguese health institutions' planning and action.

\section{P-238}

Infectious diseases mortality among migrants and host population in five European countries

Topic: Diseases - Infections

Presenter: Rafael Mikolajczyk

Rafael Mikolajczyk 1); Stefanie Helmer 2); Tanja Wörmann 3); Raj Bhopal 4); Grégorie Rey 5); Knud Juel 6); Enrique Regidor 7); Patrick Deboosere 8); Johan Mackenbach 9); Anton Kunst 10)

1) Department of Epidemiology, Helmholtz Centre for Infection Research, Braunschweig, Germany; 2) Leibniz Institute for Prevention Research and Epidemiology; 3) School of Public Health, Bielefeld University; 4) Edinburgh Ethnicity and Health Research Group, Centre for Population Health Sciences, University of Edinburgh, UK; 5) INSERM, CepiDc, Paris, France; 6) National Institute of Public Health, University of Southern Denmark, Copenhage, Denmark; 7) Department of Preventive Medicine and Public Health School of Medicine Universidad Complutense de Madrid Ciudad Universitaria 28040 Madrid, Spain; 8) Department of Social Research, Vrije Universiteit, Brussel, Brussel, Belgium; 9) Department of Public Health, Erasmus Medical Center, Rotterdam, Netherlands; 10) Department of Public Health, Academic Medical Center (AMC), University of Amsterdam, Amsterdam, Netherlands

Background: Infectious diseases mortality might differ between migrants and the host population, due to for example a higher prevalence in countries of origin or unequal access to medical care in the host country. Differences in infectious diseases mortality in the native population across the host countries can further influence mortality of migrants living in different countries. Cross country analysis can reveal whether it matters where migrants migrated to. We aimed to describe variation in infectious diseases mortality among migrant groups in comparison to the native population in five European countries.

Methods: We obtained national death and population data from Denmark, France, the Netherlands, Scotland and Spain. In each of the countries, we identified tuberculosis, HIV and other infections deaths (excluding pneumonia). We applied Poisson regression to calculate mortality rate ratios (MRRs) for native population and migrants by host country and sex, adjusting for age in 5-years age groups from 0 to $80+$. Migrants were defined on the basis of country of birth and restricted to those with country of birth outside of Western Europe.

Results: Percentage of migrants (defined as above) among the total population in the study samples varied between $1.7 \%$ in Spain and $8.3 \%$ in the Netherlands. The differences between migrants and host population were largest for tuberculosis (without any sex differences) and HIV (stronger differences among women). For other infectious diseases, MMRs for migrant versus host population varied for males between 0.98 (95 \% CI 0.82-1.16) in Spain and 1.2-2.2 in Denmark, France, Scotland and the Netherlands. For females the differences 
between migrants and native population were similar: 1.00 (0.76-1.33) in Spain, 1.2-1.7 in the remaining countries.

Conclusions: While mortality difference between migrants and host populations were largest for tuberculosis and HIV, in most of the studied countries also other infectious diseases mortality was elevated among migrants. Future analyses should distinguish between different groups of migrants according to the country of birth.

\section{P-239}

Different methods for evaluation of non-adherence to antibiotics, different risk factors?

Topic: Diseases - Infections

Presenter: Andreia Leite

Milene Fernandes 1), 2); Andreia Leite 1); Maria Basto 3); Miguel Nobre 1); Nuno Vieira 4); Paulo Nogueira 1), 5); Paulo Nicola 1)

1) Institute of Preventive Medicine, Faculty of Medicine, University of Lisbon, Portugal; 2) Socio-Pharmacy Department, Faculty of Pharmacy, University of Lisbon, Portugal; 3) Medical Department, Rottapharm Madaus, Portugal; 4) Internal Medicine Department, Centro Hospitalar do Barlavento Algarvio, Portimão, Portugal; 5) General-Directorate of Health (DGS), Lisbon, Portugal

Background: Bacteria resistance is associated to patient non-adherence to antibiotics. There is no gold-standard for the evaluation of non-adherence to medication. Regarding short-term treatments, measuring this behaviour often relies on self-report measures, such as validated scales (e.g. Morisky-Green test) or through the comparison of the prescribed intake instructions with the one reported by the patient.

Objectives: To compare two measures of patient non-adherence to short-term antibiotic treatment, regarding the concordance level and associated factors.

Methods: We conducted an observational longitudinal study during 2009's first semester. Patients attending to pharmacies from Lisbon region, aged $\geq 18$ years and with a prescription of one oral $A B$ were invited to participate. Prescriptions' data were collected at the pharmacy. After the expected date for the treatment conclusion, participants were contacted for a phone interview regarding adherence and related factors. Participants were classified as non-adherents if they (1) answered positively or didn't answer to at least one question from the Morisky scale or (2) if they reported an intake regimen-regarding number of pills, intake frequency or duration of treatment-different from the one prescribed. Concordance and Cohen's kappa statistics were calculated and logistic regression models were constructed to identify factors associated to nonadherence.

Results: In 312 eligible patients who accepted to participate, 243 $(78 \%)$ were included, with a mean age of $46.5 \pm 16.6$ years-old, $74.9 \%$ female and $59.9 \%$ had secondary or higher educational level. Non-adherence frequency was $108 / 243(44.8 \%)$ when using the Morisky scale, and 39/195 (20\%) through the comparison between reported versus prescribed intake regimen. For the participants with both measures, the concordance was $56.2 \%$, Kappa $=0.065$ (poor agreement). The logistic regression models identified the following independently and significant associated factors: Morisky scaleyounger age, perceived economical difficulty for the acquisition of the antibiotic, longer treatment, difficulty of intake and poor satisfaction with the information received from the prescriber; reported/prescribed comparison-younger age, professional status other than employed, intake frequency $\geq 2 \mathrm{x} /$ day, pharmacological group, longer treatment, difficulty of intake, total number of medicines and knowledge about antibiotics.

Conclusions: The agreement between measures of non-adherence is poor, which may be related to the assessment of different behaviours and different patients at risk. The comparison reported/prescribed regimens seem to be more affected by the pharmacological characteristics of the regimen than the Morisky scale. The use of multiple measures should be preferred when evaluating patient non-adherence to short-term treatments, in order to enable a comprehensive identification of misuse dimensions and its further prevention.

\section{P-240}

\section{Longitudinal analysis of antiretroviral therapy switch in HIV patients}

Topic: Diseases - Infections

Presenter: Andreia Leite

Andreia Leite 1); Milene Fernandes 1); Paulo Jorge Nicola 1); Luís Caldeira 2); Ana Paula Martins 3); Vasco Maria 1); Valeska Andreozzi 4)

1) Institute of Preventive Medicine, Faculty of Medicine, University of Lisbon; 2) Infectious Disease Department, Hospital de Santa Maria; 3) Research Institute for Medicines and Pharmaceutical Sciences (iMed.UL), Faculty of Pharmacy, University of Lisbon, Lisbon, Portugal; 4) Faculty of Sciences, University of Lisbon

Background: To decide whether or not to change or not an antiretroviral regimen is a difficult decision for a physician. Even though some international studies had described factors associated with treatment switch, the results are not consensual. Besides, most studies have only investigated factors associated to first change.

Objectives: To describe factors associated with antiretroviral switching, s according to time-to-change.

Methods: A retrospective cohort study was conducted with HIV-1 patients followed at Hospital de Santa Maria (Lisbon, Portugal). Data from clinical records was collected for a random sample of 320 patients with at least two pharmacy refills between 2005 and 2008 . Switches were considered if individuals changed at least one drug. Subjects were censored if they had stopped therapy. Cox regression was used to describe factors associated with first therapy switch and Prentice-Williams-Peterson model to describe factors associated with switches until the fourth. To fit models, covariates were selected using stepwise method, for an inclusion p-value of 0.20 and an exclusion $\mathrm{p}$-value of 0.25 . All models included a variable adjusting for year of antiretroviral therapy (ART) beginning (before 2000, between 2000 and 2004 and after 2004).

Results: A total of 194 subjects were included. Sample was mainly formed by men $(65.9 \%)$, and presented a mean age of $36.1 \pm 10.0$ years at ART beginning. $29.4 \%$ of subjects started ART before 2000, $40.2 \%$ between 2000 and 2004, and $30.4 \%$ after 2004 . Almost all individuals had therapy started with 2 nucleoside reverse transcriptase inhibitors plus a nonnucleoside reverse transcriptase inhibitors (NNRTI) $(49.0 \%)$ or a protease inhibitors (PI) $(46.9 \%)$. Initial regimen was associated to the first switch-Hazard Ratio (HR) of PT versus NNRTI: 1.777 [95 \% Confidence Interval (95\% CI) 1.121-2.816]. CD4+ cells count was independently associated with switches until the fourth (HR: 0.999; $95 \%$ CI 0.998-0.9997).

Conclusions: Factors associated with first switch differ from the ones associated with the switches until the fourth. Although a relatively small effect was observed, patients with lower CD4 + counts should be carefully monitored as they have a higher risk of switching and exhausting therapy options. 


\section{P-241}

Wistar rats exposed to toluene diisocyanate vapor secretes preferentially IL-4 and IL-5 in their airways

Topic: Exposure - Enviromental factors

Presenter: Kouame Kouadio

Kouame Kouadio 1); Zheng Kui-Cheng 1); Abdoulaye Abba Toure 2); Mireille Dosso 2); Todoriki hidemi 1)

1) Department of Environmental and Preventive Medicine, Faculty of Medicine, University of the Ryukyus, 207 Uehara, Nishihara, Okinawa 903-0215, Japan; 2) Eco-epidemiology Unit, Department of Environmental Health, Institut Pasteur of Côte d'Ivoire, 01 BP 490 Abidjan 01, Côte d'Ivoire, West Africa

Background: Asthma is a chronic respiratory disease characterized by the presence of reversible airway constriction and airway hyperresponsiveness to various stimuli. Nearly nine million individuals are exposed to agent at their workplace associated with asthma, and isocyanates such as toluene diisocyanate (TDI) are the most common cause of occupational asthma. TDI induced asthma has been difficult to diagnose and control, in part because the biological mechanisms responsible for the disease and the determinants of exposure have not been yet defined and validated animal models do not yet.

Objectives: The objectives were to establish a Wistar rat model of asthma caused by TDI exposure, and investigate the relationship between TDI exposure concentrations and respiratory hypersensitivity, airway inflammation, and cytokine secretions in animals in order to better understand the mechanism of toluene diisocyanate (TDI) induced occupational asthma.

Methods: Wistar rats were exposed to two different concentrations of TDI vapor $4 \mathrm{~h}$ a day for five consecutive days. Bronchoalveolar lavage (BAL) was performed, and differential leucocytes from the BAL fluid were analyzed. Lung histopathological examination was carried out to investigate the inflammatory status in the airways. Cytokines IL- 4 and IL-5 productions in the BAL fluid in vivo were determined with ELISA kits.

Results: The TDI exposed rats exhibited airways hypersensitivity symptoms as compared to the control rats. BAL differential cell count and lung histopathological examination demonstrated that inflammation reactions were present in both the central and peripheral airways, characterized with marked infiltration of eosinophils in the TDI exposed rats. The cytokine assay showed that IL-4 and IL-5 were predominantly produced in the BAL fluid in vivo.

Conclusion: These findings imply that TDI exposure concentrations may greatly affect the occurrence and extent of inflammatory events and that Th2 type cytokines may play an important role in the immunopathogenesis of TDI induced occupational respiratory hypersensitivity.

Key words: TDI- Wistar Rat- BAL- Cytokines- Eosinophils- Airway

\section{P-242}

Association of ambient air pollution with emergency department visits for health outcomes

Topic: Exposure - Enviromental factors

Presenter: Mieczyslaw Szyszkowicz

Mieczyslaw Szyszkowicz 1); Errol Thomson 1); Brian Rowe 2)

1) Health Canada; 2) University of Alberta

Background: Some recent studies suggest that adverse health effects of air pollutants may extend beyond the respiratory and cardiovascular systems. I While there is considerable evidence supporting a causal relationship between exposure to air pollution and cardiopulmonary morbidity and mortality, little is known about other potential health effects. Given recent findings of novel health impacts (e.g. abdominal pain, appendicitis, headache, suicide) associated with air pollutants, an approach that assesses effects of air pollutants on a range of health effects is warranted.

Objectives: In this work the authors used International Classification of Diseases, 9th Revision (ICD-9 code) coding to determine 18 groups of health conditions. Ten years of emergency department (ED) data $(2,951,878$ visits $)$ were classified into 18 groups and linked to levels of ambient air pollution: carbon monoxide $(\mathrm{CO})$, nitrogen dioxide $\left(\mathrm{NO}_{2}\right)$, ozone $\left(\mathrm{O}_{3}\right)$, sulphur dioxide $\left(\mathrm{SO}_{2}\right)$, and particulate matter of median aerodynamic diameter less than 10 and 2.5 microns (PM10 and PM2.5, respectively). We examined associations between air pollutants and 18 groups of health conditions.

Methods: A time-stratified case-crossover technique was used. Temperature and relative humidity were adjusted in the constructed models using natural splines with three degrees of freedom.

Results: The results for diseases of the respiratory system (ICD-9: 460-519) confirmed a strong impact of ozone. A number of other ICD-9 groups were significantly associated with pollutant exposure, including diseases of the skin and sense organs (ozone) and mental disorder and diseases of the nervous system (carbon monoxide). The most striking relations were for injury and poisoning (ICD-9: 800-999 with 827,084 cases), with strong associations with air pollution (CO, $\mathrm{NO}_{2}, \mathrm{SO}_{2}$, and $\left.\mathrm{PM}\right)$. For neoplasms (cancers), complications of pregnancy, diseases of the musculoskeletal system, and congenital anomalies (ICD-9: 140-239, 630-679, 710-139 and 740-759, respectively), there were no observed associations.

Conclusions: The results confirm associations for cardiorespiratory diseases, and in addition suggest associations with a number of other health conditions. The results for injury and poisoning agree with known relations between depression or personal disorders and injury.If substantiated, these findings imply that the extent of adverse health effects of air pollutants is greater than generally appreciated.

\section{P-243}

\section{Systematic review of air pollution and risk of diabetes}

Topic: Exposure - Enviromental factors

Presenter: Mohsen Janghorbani

\section{Mohsen Janghorbani 1); Fatemeh Moneni 1)}

\section{1) Isfahan University of Medical Sciences}

Background: A systematic review and meta-analysis of the observational studies addressing the relationship of air pollution and diabetes is lacking.

Objectives: The authors conducted a systematic review and metaanalysis of published observational studies on the association between air pollution and diabetes risk.

Methods: The authors searched online databases through January 2013, and examined the reference lists of pertinent articles (limited to studies in humans). Pooled relative risks (RR) and $95 \%$ confidence intervals $(\mathrm{CI})$ were calculated with a random-effects model. Twentynine effect estimates from 17 studies (five cross-sectional, six cohort and six case-crossover) were included, with 4,381,503 participants and 98,537 cases of diabetes.

Results: Air pollution was associated with slightly increased risk of diabetes (summary RR (95\% CI) 1.04 (1.02, 1.05)). These results were consistent between cross-sectional, cohort and case-crossover studies and between studies conducted in the North America and in Europe. The association between air pollution and diabetes was stronger for gaseous pollutants (summary RR (95\% CI) 1.05 (1.03, 
1.07)) than for particulate matter (summary RR (95\% CI) 1.01 $(1.005,1.02))$.

Conclusions: This meta-analysis suggested that air pollution may be a risk factor for diabetes.

\section{P-244}

Gestational diabetes and preeclampsia in association with air pollution at levels below current air quality guidelines

Topic: Exposure - Enviromental factors

Presenter: Ebba Malmqvist

Ebba Malmqvist 1); Kristina Jakobsson 1); Håkan Tinnerberg 1); Anna Rignell-Hydbom 1); Lars Rylander 1)

\section{1) Occupational and Environmental Medicine Lund University}

Background: Several studies have estimated associations between air pollution and birth outcomes, but few have evaluated potential effects on pregnancy complications.

Objective: To investigate whether low-level exposure to air pollution is associated with gestational diabetes and preeclampsia.

Method: High quality registry information on 81,110 non-selected singleton pregnancy outcomes in southern Sweden during 1999-2005 was linked to individual-level exposure estimates with high spatial resolution. Modeled exposure to nitrogen oxides (NOx), expressed as mean concentrations per trimester, and proximity to roads of different traffic densities, were used as proxy indicators of exposure to combustion-related air pollution. The data were analyzed by logistic regression, with and without adjusting for potential confounders.

Results: The prevalence of gestational diabetes increased with each NOx quartile, with an adjusted odds ratio (OR) of 1.69 (95\% CI 1.41, $2.03)$ for the highest $\left(>22.7 \mathrm{~g} / \mathrm{m}^{3}\right)$ compared to the lowest quartile $\left(2.5-8.9 \mathrm{~g} / \mathrm{m}^{3}\right)$ of exposure during the second trimester. The adjusted OR for acquiring preeclampsia after exposure during the third trimester, was $1.51(1.32,1.73)$ in the highest quartile of NOx compared to the lowest. Both outcomes were associated with high traffic density, but ORs were significant for gestational diabetes only.

Conclusion: NOx exposure during pregnancy was associated with gestational diabetes and preeclampsia in an area with air pollution levels below current air quality guidelines.

\section{P-245}

Childhood and adult socioeconomic position and social mobility as determinants of low back pain outcomes

Topic: Exposure - Enviromental factors

Presenter: Tea Lallukka

Tea Lallukka 1), 2); Eira Viikari-Juntura 1); Olli Raitakari 3), 4); Mika Kähönen 5); Terho Lehtimäki 6); Jorma Viikari 7); Svetlana Solovieva 1)

1) Finnish Institute of Occupational Health, Helsinki, Finland; 2) Hjelt Institute, Department of Public Health, University of Helsinki, Finland; 3) Research Centre of Applied and Preventive Cardiovascular Medicine, University of Turku, Finland; 4) Department of Clinical Physiology and Nuclear Medicine, Turku University Hospital, Turku, Finland; 5) Department of Clinical Physiology, University of Tampere and Tampere University Hospital, Tampere, Finland; 6) Department of Clinical Chemistry, Fimlab Laboratories, Tampere University Hospital and School of Medicine, University of Tampere; 7) Department of Medicine, University of Turku, Finland; Turku University Hospital, Turku, Finland
Background: Low back pain is a prevalent public health problem and it tends to be socioeconomically patterned. Relatively little is known about lifecourse socioeconomic circumstances as determinants of different types of low back pain in adulthood.

Objectives: This study aimed to examine whether multiple indicators of childhood and adult socioeconomic position, and social mobility are associated with radiating and non-specific low back pain and sciatica among women and men. An additional aim was to examine whether the associations remain after considering own socioeconomic position and covariates.

Methods: Data were derived from the prospective Young Finns Study $(n=2231)$. Childhood socioeconomic position was measured by parental education, occupational class, and family income at baseline in 1980. Data on completed own education and low back pain outcomes were collected in the end of follow-up in 2007. Social mobility was based on years of parental and own education. Covariates comprised age, parental body mass index and smoking.

Results: Childhood and own socioeconomic position were associated with radiating low back pain and sciatica after adjustments, but the associations varied by socioeconomic indicator and gender. Stable lower socioeconomic position and downward mobility were strongly associated with radiating low back pain

Conclusions: Childhood socioeconomic circumstances affect risk of radiating low back pain and sciatica in adulthood among both women and men. To prevent low back disorders, early socioeconomic circumstances need to be considered alongside own socioeconomic position

\section{P-246}

Exposure to polybrominated diphenyl ethers and male reproductive function in Arctic and European populations

Topic: Exposure - Enviromental factors

Presenter: Gunnar Toft

Gunnar Toft 1); Virissa Lenters 2); Dick Heederik 2); Cathrine Thomsen 3); Aleksander Giwercman 4); Lars Rylander 5); Henning S Pedersen 6); Pawel Strucinski 7); Valentyna Zviezdai 8); Jens Peter Bonde 9)

1) Danish Ramazzini Center, Department of Occupational Medicine, Aarhus University Hospital, Aarhus, Denmark; 2) Institute for Risk Assessment Sciences, Utrecht University, Utrecht, The Netherlands; 3) Division of Environmental Medicine, Norwegian Institute of Public Health, Oslo, Norway; 4) Reproductive Medicine Centre, Skåne University Hospital, Lund University, Malmö, Sweden; 5) Division of Occupational and Environmental Medicine, Lund University, Lund University Hospital, Lund, Sweden; 6) Centre for Arctic Environmental Medicine, Nuuk, Greenland; 7) Department of Toxicology and Risk Assessment, National Institute of Public Health - National Institute of Hygiene, Warsaw, Poland; 8) Departmet of Social Medicine and Organization of Public Health, Kharkiv National Medical University, Kharkiv, Ukraine; 9) Department of Occupational and Environmental Medicine, Bispebjerg University Hospital, Copenhagen, Denmark

Background: Animal and a few human studies suggest that polybrominated diphenyl ethers (PBDEs) may affect male reproductive function.

Objectives: The aim of the present study was to evaluate if male reproductive function was associated to serum level of PBDEs.

Methods: We evaluated the effects of environmental exposure to BDE-28, BDE-47 and BDE-153 on reproductive hormones and semen quality including markers of DNA damage and apoptosis in 297 men from Greenland, Poland and Ukraine. 
Results: Adjusted linear regression models indicated a positive association between serum follicle stimulating hormone (FSH) and lipid adjusted BDE- 153 concentration with $0.16 \%$ confidence interval (CI) 0.05 to $0.26 \%$ higher FSH for each percentage increase in BDE-153. Similarly serum luteinizing hormone (LH) was $0.09 \%$ (0.01-0.18\%) higher for each percentage increase in BDE-153 exposure.

Conclusions: In the largest study to date PBDE exposure was not associated to altered semen characteristics and gonadal hormones. However, the pituitary hormones LH and FSH may be affected by BDE-153 exposure, but not BDE-28 or BDE-47.

\section{P-247}

Motor development following prenatal exposure to $\mathbf{p}, \mathbf{p}^{\prime}$-DDE and CB-153: a follow-up study of Greenlandic and European children

Topic: Exposure - Enviromental factors

Presenter: Birgit Bjerre Hoyer

Birgit Bjerre Høyer 1); Cecilia Høst Ramlau-Hansen 2); Henning Sloth Pedersen 3); Jens Peter Bonde 4); Gunnar Toft 1)

1) The Danish Ramazzini Centre, Department of Occupational Medicine, Aarhus University Hospital, Aarhus, Denmark, birghoey@rm.dk; 2) Department of Public Health, Section for Epidemiology, Aarhus University, Aarhus, Denmark; 3) Primary Health Care Clinic, Nuuk, Greenland; 4) Department of Environmental and Occupational Medicine, Copenhagen University Hospital Bispebjerg, Copenhagen, Denmark

Background: Both polychlorinated biphenyls (PCBs) and dichlorodiphenyldichloroethylene (DDE) are lipophilic compounds which bio-accumulate in adipose tissue and cross the placental barrier. Prior studies of the association between prenatal exposure to PCBs and DDE and child motor development have found contradicting results. Objectives: The aim of this follow-up study was to examine the association between prenatal exposure to DDE and PCBs and motor development and developmental milestones; crawling, standing-up and walking in children in Greenland, Ukraine and Poland.

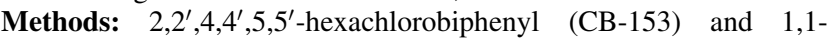
dichloro-2,2-bis(p-chlorophenyl)ethylene ( $\mathrm{p}, \mathrm{p}^{\prime}$-DDE) were measured in maternal blood in 2 nd or 3rd trimester of pregnancy as a biomarker of the child's prenatal exposure to the compounds. A total of 1,103 children aged 5-9 years were followed up in 2010-2012. Motor development were measured in terms of the parentally assessed screening tool Developmental Coordination Disorder Questionnaire 2007 (DCDQ'07) and developmental milestones were assessed via parental reports of child age at the first time of crawling, standing-up and walking. The association between PCBs/DDE and motor skills and milestones were analyzed by means of multiple linear regression analyses using tertiles of exposure and stratified by country. Both complete case analyses and multiple imputation based analyses were executed. Adjustment were performed for the co-variates; maternal age, maternal smoking during pregnancy, maternal alcohol before pregnancy, maternal education, parity, gestational age at blood sampling, preterm birth, breastfeeding, child sex and child age at interview.

Results: We found no associations between prenatal PCBs and DDE exposure and developmental milestones or motor skills. Complete case- and multiple imputation based analyses showed adjusted mean differences in motor skills and age at milestones around null, in all three countries.

Conclusions: These results on 1.103 mother-children-pairs from the INUENDO cohort in Greenland, Warsaw (Poland) and Kharkiv
(Ukraine), indicate no association between in utero PCBs/DDE exposure and developmental milestones and motor skills.

\section{P-248}

Menstrual cycle characteristics in European and Greenlandic women exposed to perfluorinated chemicals

Topic: Exposure - Enviromental factors

Presenter: Julie Lyngs $\phi$

Julie Lyngsø 1); Cecilia Ramlau-Hansen 2); Birgit Høyer 1); Henrik Støvring 3); Jens Peter Bonde 4); Gunnar Toft 1)

1) The Danish Ramazzini Centre, Department of Occupational Medicine, Aarhus University Hospital, Aarhus, Denmark; 2) Department of Public Health, Section for Epidemiology, Aarhus University, Aarhus, Denmark; 3) Department of Public Health, Section for Biostatistics, Aarhus University, Aarhus, Denmark; 4) Department of Environmental and Occupational Medicine, Copenhagen University Hospital Bispebjerg, Copenhagen, Denmark

Background: Perfluorooctane sulfonate (PFOS) and perfluorooctanate (PFOA) are persistent man-made chemicals. Studies suggest that they are possible hormonal disruptors, but findings are inconsistent. Objectives: We examined the association between measured PFOS and PFOA exposure and menstrual cycle length and irregularities in European and Greenlandic women.

Methods: This cross-sectional study included 1,623 pregnant women from the INUENDO cohort, enrolled during antenatal care visits between June 2002 and May 2004 in Greenland, Poland and Ukraine. Information on menstrual cycle characteristics were obtained by questionnaires and the woman had a blood sample drawn. Serum concentrations of PFOS and PFOA were measured by liquid chromatography tandem mass spectrometry (LC/MS/MS). Multiple imputations was performed due to missing data. The association between PFOS/PFOA and menstrual cycle length and irregularities were analyzed using logistic regression with tertiles of exposure.

Results: Longer menstrual cycles were associated with higher exposure levels of PFOA in pooled estimates of all three countries. Compared with women in the lowest exposure tertile, the adjusted odds ratio (OR) of long cycle was 2.0 (95\% confidence intervals (CI) 1.2;3.3) among women in the highest tertile of PFOA exposure. No significant associations were observed between menstrual cycle characteristics and PFOS exposure. However, we observed a tendency towards more irregular cycles with higher exposure to PFOS (OR 1.7 (95\% CI $0.8 ; 3.8)$. Results based on multiple imputation of missing values were similar to those based on complete case analysis.

Conclusions: These findings on 1,623 pregnant women from the INUENDO cohort imply that the female reproductive system may be sensitive to background PFOA exposure. Exposure to PFOS is unlikely to be a main cause of menstrual disturbances in humans.

\section{P-249}

Occupational lifting during pregnancy and fetal growth measures in a large national cohort study

Topic: Exposure - Enviromental factors

Presenter: Mette Juhl

Mette Juhl 1); Anne-Marie Nybo Andersen 2); Pernille Stemann Larsen 2); Per Kragh Andersen 2); Susanne Wulff Svendsen 3); Jens Peter Bonde 4); Katrine Strandberg-Larsen 2)

1) Copenhagen University Hospital, Copenhagen, Denmark; 2) University of Copenhagen, Copenhagen, Denmark; 3) Herning 
Regional Hospital, Herning, Denmark; 4) Bispebjerg Hospital, Copenhagen, Denmark

Background: Physically strenuous work is generally discouraged during pregnancy, but still $13 \%$ of the women in the Danish National Birth Cohort lifted more than 100 kilos per day while pregnant in a well-regulated welfare state as Denmark. Occupational lifting may affect the growth of the fetus, and reassignment to a safer job or withdrawal from work has been found to reduce the risk of small-forgestational-age in the offspring of women with occupational lifting. Methods: We estimated the association between number of kilos lifted and frequency of lifts per day and birth weight, length, ponderal index, abdominal circumference, head circumference, and placental weight in linear regression models, and odds ratios for small-forgestational-age in logistic regression analysis. We analyzed 66,693 live born children in the Danish National Birth Cohort. Recruitment took place at first antenatal visit at the general practitioner during 1996-2002. Exposure data came from telephone interviews around week 16 of pregnancy and were linked to national register-based information on pregnancy outcome.

Results: After confounder adjustment, we found a slight tendency toward decreasing offspring birth size with increasing number of kilos lifted per day during pregnancy, but the differences were very small. There was no association between maternal occupational lifting and SGA in the offspring among women in jobs with no person lifting, but women in jobs that included person lifting, the likely of having an SGA-child was increased among women who lifted over 500 kilos per day compared to no lifting.

Conclusions: Overall, we did not find maternal occupational lifting associated with impaired fetal growth. Only, we did observe increased odds of SGA in the children of women who lifted over 500 kilos per day in jobs with person lifting. Thus, the association may vary according to the character of lifting or other factors related to the occupational environment.

\section{P-250}

A prospective study of long-term effects of prenatal exposure to persistent organochlorine pollutants on female reproduction

Topic: Exposure - Enviromental factors

Presenter: Susanne Lund Kristensen

Susanne Lund Kristensen 1); Cecilia Høst Ramlau-Hansen 2); Erik Ernst 3); Sjurdur Frodi Olsen 4); Jens Peter Bonde 5); Anne Vested 1); Thorhallur Hallsorsson 6); Gunnar Toft 1)

1) Danish Ramazzini Centre, Department of Occupational Medicine, Aarhus University Hospital, Denmark; 2) Department of Public Health, Section for Epidemiology, Aarhus University, Denmark; 3) Department of Gynecology and Obstetrics, Aarhus University Hospital, and Institute of Anatomy, Aarhus University, Denmark; 4) Centre for Fetal Programming, Statens Serum Institut, Copenhagen, Denmark; 5) Department of Occupational and Environmental Medicine, Bispebjerg Hospital of Copenhagen University, Denmark; 6) Faculty of Food Science and Nutrition, University of Iceland, Reykjavik, Iceland

Background: Concerns exist regarding prenatal exposure to persistent organochlorine pollutants since the foetus is highly susceptible to toxic exposures due to the developing organs. Foetal developmental disruptions may have health implications later in life. The long-term effects on in utero exposure to persistent organochlorines on human female reproduction have only been investigated sparsely.

Objectives: The aim of this prospective population-based study was to evaluate the long-term effects of prenatal exposure to polychlorinated biphenyls (PCBs) and dichlorodiphenyldichloroethylene (DDE) on female reproductive function.

Methods: We used data from a Danish pregnancy cohort established in 1988-89. Among 1212 eligible pregnant women, 965 (80\%) participated. Follow-up on female offspring was initiated in 2008. 367 $(84 \%)$ daughters answered a questionnaire and 267 (61\%) women attended the clinical examinations conducted in 2008-2009.

Concentrations of DDE and 6 PCBs in maternal serum from pregnancy week 30 were used as measures of prenatal exposure. The reproductive function of the daughters was evaluated by means of age at first menstrual bleeding, cycle length, levels of reproductive hormones and number of ovarian follicles.

Results: The final study population consisted of 343 daughters. At the time of clinical examination, the women had a mean (SD) age of 20.1 (0.3) years. Exposure levels were analysed in $95 \%$ of the participants. At the EuroEpi2013 congress, results including estimates from regression analyses adjusted for relevant covariates will be presented. Conclusions: With this prospective study, we have a unique opportunity to evaluate the possible long-term effects of prenatal exposure to PCBs and DDE on several measures of female reproductive function in women from the general population.

\section{P-251}

Prenatal stress following maternal bereavement during pregnancy and obesity in early adulthood: a cohort study in $\mathbf{1 6 8 , 4 6 5}$ Danish conscripts

Topic: Exposure - Enviromental factors

Presenter: Lena Hohwü

Lena Hohwü 1); Jiong Li 2); Beibei Xu 2); Jørn Olsen 2); Thorkild I.A. Sørensen 3), 4); Carsten Obel 1)

1) Section for General Practice, Department of Public Health, Aarhus University, Denmark; 2) Section for Epidemiology, Department of Public Health, Aarhus University, Denmark; 3) Institute of Preventive Medicine, Frederiksbjerg and Bispebjerg Univesity Hospital, Copenhagen, Denmark; 4) Novo Nordisk Foundation Center for Basic Metabolic Research, Faculty of Health and Medical Sciences, University of Copenhagen, Denmark

Background: It has been suggested that exposure to prenatal stress may be a causal factor for developing obesity later in life. This hypothesis has been supported by animal studies and some studies following children into school age.

Objective: We aimed to examine the association between exposure to prenatal stress by maternal bereavement during pregnancy and the risk of obesity in early adulthood.

Methods: A population-based cohort study was conducted including 168,465 Danish young male conscripts (including 1,750 full siblings) born in 1983-1991 and presented for conscription in 2006-2011. Linking the conscription file and national registers, we identified 4,943 conscripts (including 859 siblings) exposed to prenatal stress, defined by being born to mothers who were bereaved by death of a close family member from 1 year before pregnancy until birth of the child. Mean, median and mean differences in body mass index (BMI) were estimated. Multiple and conditional logistic regression was performed to estimate adjusted odds ratio (OR) of obesity $\left(\mathrm{BMI}>30 \mathrm{~kg} / \mathrm{m}^{2}\right)$ between the exposed and unexposed.

Results: The mean difference in BMI was statistically significantly higher in exposed children than children born after those exposed $\left(1.24 \mathrm{~kg} / \mathrm{m}^{2}\right.$ [95\% confidence interval $\left.\left.0.33 ; 2.15\right]\right)$, and the odds for obesity was $45 \%$ higher (OR 1.45 [95\% confidence interval 1.16; 1.81]). Children born to mothers who experienced the death of her partner/spouse or a child during pregnancy had a higher mean BMI 
$\left(24.4 \mathrm{~kg} / \mathrm{m}^{2}\right)$ than siblings born before $\left(23.8 \mathrm{~kg} / \mathrm{m}^{2}\right)$ or after $(23.3 \mathrm{~kg} /$ $\mathrm{m}^{2}$ ) the exposed child. This pattern was also seen in the sibling cohort. The odds ratio for obesity was 5.86 [95\% confidence interval: $1.32 ; 25.03$ ] in siblings born to mothers who were bereaved due to death of her partner/spouse or a child than to siblings born of unexposed mothers.

Conclusions: Our data support that severe stress during prenatal period is associated with an increased risk of obesity in early adulthood.

\section{P-252}

Developmental delays in children of international migrants: Family profiles

Topic: Exposure - Enviromental factors

Presenter: Anita Gagnon

\section{Anita Gagnon 1); Stephanie Bouris 2); Lisa Merry 1) \\ 1) McGill University; 2) McGill University Health Centre}

Background and Objectives: This study aims to describe the families of children of migrant women new to Montreal, Quebec, Canada with developmental delays at 12-36 months post-birth.

Methodology: 258 international migrant women were recruited postbirth from 6 Montreal-area hospitals and followed to 12-36 months. The Nipissing Developmental Screen was administered by project nurses at 12, 18, 24, 30, and/or 36 months of age, as part of a series of assessments of the health of migrant families. Family characteristics of children with $>2$ signs of developmental delay were descriptively analyzed and compared to those without delays.

Results: $26 \%$ had $>2$ signs of developmental delay during at least 1 project nurse visit. Few statistically significant differences between groups were found for individual factors although their overall profile suggests differences: mothers were more often: asylum-seekers, in Canada for $<2$ years, originated in Africa/Latin America, had either IFHP or no health insurance, began prenatal care late, did not attend prenatal education, had experienced physical abuse, and had symptoms of PPD at 2 weeks post-birth. Fathers were more often: unemployed, asylum-seekers, in Canada $<2$ years, and originated in Africa/Latin America. Families more often: had a low income and smoked in the home. The majority $(65 \%)$ of the combined sample did not speak English or French at home.

Conclusions: Early childhood developmental delays are relatively common in international migrant families. Certain indicators may help to identify children at greatest risk.

\section{P-253}

Responses of international migrant women to abuse associated with pregnancy

Topic: Exposure - Enviromental factors

Presenter: Anita Gagnon

\section{Anita Gagnon 1); Praem Mehta 1); Lisa Merry 1)}

\section{1) McGill University}

Background and Objectives: Abuse associated with pregnancy is a major public health concern. Victims experience several physical and mental health conditions including depression, anxiety, headaches, sleep disorders and post-traumatic stress disorder. International migrant women may be at particular risk of suffering from these conditions. The aim of this study was to describe the profile of international migrant women who have experienced abuse associated with pregnancy and their responses in the early months post-birth.

Methodology: Line-by-line thematic analyses were performed of textual data extracted from the records of international migrant women living in Montreal, Toronto or Vancouver who had participated in a study examining their health and care around the time of birth and reported having been abused. Recurring topics, words, phrases, feelings, and activities related to abuse were identified, coded, and interpreted.

Results: Of the 1025 migrant women participating in the original study, $59(5.8 \%)$ reported having been abused. Nearly $50 \%$ had not completed high school and had a low household income. The majority were not living with partners, were asylum-seekers from Africa or Latin America and had been in Canada $<2$ years. Nearly $50 \%$ were at risk of postpartum depression and were experiencing symptoms of anxiety and depression; $25 \%$ showed symptoms of PTSD. The abuser was identified as the partner by more than $50 \%$. Responses to abuse included: geographical separation; emotional/psychological responses; isolation; silence; lifestyle changes; reporting; and legal processes.

Conclusions: Migrant women who have experienced abuse associated with pregnancy have a high psycho-social risk profile and demonstrate a range of responses to the abuse.

\section{P-254}

Developmental delay at 2-3 years: relative importance of gestational age and social risk factors

Topic: Exposure - Enviromental factors

Presenter: Hilary K. Brown

Hilary K. Brown 1); Kathy Speechley 1), 2); Jennifer Macnab 1); Renato Natale 3); M. Karen Campbell 1), 3)

1) Department of Epidemiology and Biostatistics, The University of Western Ontario; 2) Department of Paediatrics, The University of Western Ontario; 3) Department of Obstetrics and Gynaecology, The University of Western Ontario

Background: Preterm birth is traditionally defined as delivery prior to 37 weeks gestation. Outcomes of very preterm birth ( $<34$ weeks) are well-documented. Research is beginning to show that children born late preterm (34-36 weeks) and early term (37-38 weeks) are also at increased risk for poor developmental outcomes. However, most studies only controlled for proxies of socioeconomic status rather than considering processes in the social environment, such as parenting, that directly affect child development.

Objectives: The objective of this analysis was to determine the relative importance of late preterm and early term birth and social risk factors (social context variables and proximal social processes) as determinants of developmental delay at 2-3 years.

Methods: Data were obtained from the National Longitudinal Survey of Children and Youth (conducted by Statistics Canada). Children who were 0-1 years in Early Child Development Cycles 2-6 were pooled and followed to Cycles 3-7. The study sample included singleton births delivered at 34-41 weeks $(\mathrm{N}=15,099)$. Gestational age was determined by maternal report; implausible values (birth weight for gestational age \pm 4 standard deviations from the median) were excluded. Developmental delay was assessed using the Motor and Social Development Scale. Social factors included social context variables (maternal marital status, number of siblings, family income adequacy, maternal education, maternal age, maternal health and mental health, family functioning) and proximal social processes (parenting interactions, parenting effectiveness, parenting consistency). Multivariable modified Poisson regression, with chunkwise entry of variables, was used to directly estimate adjusted relative 
risks. Analyses were weighted using normalized longitudinal weights, and design variables were controlled for.

Results: In the final model, the association between late preterm birth and developmental delay ( $\mathrm{aRR}=1.21,95 \%$ CI $0.95,1.53)$ and the association between early term birth and developmental delay $(\mathrm{aRR}=1.10,95 \% \mathrm{CI} 0.95,1.26)$ were non-significant. Of the social factors, transitions in family income from adequate to inadequate $(\mathrm{aRR}=1.41,95 \%$ CI $1.08,1.84)$, maternal education of secondary school or less $(\mathrm{aRR}=1.30,95 \%$ CI $1.12,1.51)$, negative parenting interactions $(\mathrm{aRR}=1.35,95 \% \mathrm{CI} 1.13,1.62)$, and inconsistent parenting $(\mathrm{aRR}=1.32,1.07,1.63)$ were significantly associated with developmental delay.

Conclusions: Gestational age was of less importance than social risk factors in determining risk of developmental delay at 2-3 years. Increased surveillance of families with social risk factors may help to identify children in need of early intervention, and parenting may be an aspect of the child's social environment which could be targeted for intervention.

\section{P-255}

Prevalence of diabetes mellitus in the Seveso cohort

Topic: Exposure - Enviromental factors

Presenter: Dario Consonni

Luca Cavalieri D'Oro 1); Magda Rognoni 1); Dario Consonni 2); Angela Cecilia Pesatori 2), 3); Pier Alberto Bertazzi 2), 3)

1) Epidemiology Unit, ASL della Provincia di Monza e Brianza, Monza, Italy; 2) Epidemiology Unit, Fondazione IRCCS Ca' Granda Ospedale Maggiore Policlinico, Milan, Italy; 3) Department of Clinical Sciences and Community Health, Università degli Studi di Milano, Milan, Italy

Background: The 1976 Seveso, Italy accident caused the contamination of an area by 2,3,7,8-tetrachlorodibenzodioxin (TCDD). Three zones with decreasing soil TCDD levels were delimited: A (highest), $\mathrm{B}$ (high) and R (low). The cohort of residents in the contaminated zones and in a surrounding non-contaminated (non-ABR) referent area are followed-up for mortality and cancer incidence. A relationship between TCDD and diabetes mellitus (DM) has been postulated based on animal and some epidemiology studies, including ours (a DM mortality increase among zone B women as of 2001). Recently the Lombardy Region implemented an integrated system (Banca Dati Assisititi, BDA) to identify subjects with selected chronic diseases by exploiting several sources (hospital inpatient discharge, ambulatory care, drug prescription, and exemption from payment records). In preliminary analyses, using BDA data for the period 2006-2008, we had found a prevalence excess ( $40 \%$ in women and $28 \%$ in men) of DM among Zone $B$ residents compared with Zone non-ABR residents.

Objectives: To estimate the prevalence of DM in the Seveso cohort in the period 2006-2011.

Methods: Using unique personal identifiers, we performed a recordlinkage between the Seveso cohort and the local BDA database (years 2006-2011, one million people covered) to identify prevalent cases of DM. We restricted the analysis to residents in the area at the time of the accident. With logistic regression we calculated prevalence odds ratios $(\mathrm{OR})$ and $95 \%$ confidence intervals $(\mathrm{CI})$ for each polluted zone (reference: non-ABR Zone), controlling for gender and age at the accident.

Results: The linkage was $91 \%$ successful. We found increased DM prevalence in zone B among both genders: women, OR $1.21(95 \% \mathrm{CI}$ $1.01-1.45,147$ cases); men, OR 1.23 (95\% CI 1.03-1.45, 184 cases). These results were confirmed after adjusting for arterial hypertension, cardiovascular diseases, and hypercholesterolemia (as surrogate markers for body mass index). There were few DM cases (20 women and 24 men) in zone A, with no increased risks.

Conclusions: These results add some evidence in favour to the association between TCDD exposure and risk of diabetes. In interpreting the findings one should take into account the following limitations or inconsistencies: incomplete BDA coverage (the local BDA covers only one province and not the whole region); BDA is only available for recent years, therefore only cohort survivors 30 years after the accident could be studied; we had no information on potential confounders; no increased risk was found in the most polluted zone.

\section{P-256}

How farming exposure affects lung development in young adults: a 15 year follow-up study

Topic: Exposure - Enviromental factors

Presenter: Anneli C. S. Bolund

Anneli C. S. Bolund 1); Ioannis Basinas 1); Grethe Elholm 1); Martin R. Miller 2); Øyvind Omland 3); Vivi Schlünssen 1); Torben Sigsgaard 1)

1) Department of Public Health, Section of Environment, Occupation and Health, University of Aarhus, Aarhus C, Denmark, DK-8000; 2) Institute of Occupational and Environmental Medicine, University of Birmingham, Birmingham, United Kingdom, B15 2TT; 3)

Department of Occupational Medicine, Aalborg University Hospital, Aalborg, Denmark, DK-9100

Background: Organic dust exposure in agricultural work has been shown to have a negative effect on lung function. Especially the forced expiratory volume in the first second (FEV1) among swine confinement workers seems to be affected. Longitudinal studies on lung function decline in young agricultural workers are few.

Objectives: To explore if farming exposure suppresses lung function development in young adults of a large follow-up study of Danish farmers.

Methods: We studied 1,964 farming students and 407 controls in 1992-1994, inclusive, and 963 and 172 of these again at follow-up in 2007-2008. Spirometry, skin prick test, bronchial hyperresponsiveness, height, and weight were measured, and questionnaires concerning health, smoking and occupational history was collected at both baseline and follow-up. Cumulative dust and endotoxin exposures were estimated from job-exposure matrices based on personal dust measurements from a random selection of participants in the study. Lung function was expressed as change in z-scores during follow-up calculated by the LMS equations (Stanojevic S, et al. AJRCCM 2008; 177: 253-60).

Results: In 1135 subjects with longitudinal data and aged under 25 years at baseline the FEV1 z-score change (zFEV1) was positive (i.e. increased) for female farmers (mean $\pm \mathrm{SD}$ of $0.08 \pm 0.78$ ), male farmers $(0.09 \pm 0.69)$ and controls $(0.17 \pm 0.68)$. For the forced vital capacity $\mathrm{z}$-score change $(\mathrm{zFVC})$ the increase was even higher and significantly different between groups (female farmers $(0.18 \pm 0.80)$, male farmers $(0.39 \pm 0.76)$, and controls $(0.54 \pm 0.67), p<0.001$ ANOVA). This confirms that longitudinal lung function change is less than that estimated from cross sectionally derived equations such as LMS. In multivariate linear regression controlling for age, height, smoking, weight, farm upbringing, bronchial hyperresponsiveness, and atopy current farming had an adverse effect on $\mathrm{zFVC}$ versus never farming (mean of $-0.19,95 \% \mathrm{CI}$ of -0.33 to -0.06 ). The effect of $3 \mathrm{rd}$ quartile dust and 3rd quartile endotoxin exposure versus 1st quartile exposures was in both cases also adverse for $\mathrm{zFVC}(-0.15,-0.27$ to $-0.03)$ and $(-0.19,-0.31$ to -0.07$)$, respectively. This effect was not 
seen for the 4th quartiles in either the dust or the endotoxin variable. There was no effect modification by smoking or sex.

Conclusion: We conclude that moderate farming exposures had a significant effect on lung development, primarily on zFVC. This was not seen in those most heavily exposed which could reflect a healthy workers selection effect.

\section{P-257}

Effects of interventions to improve school readiness on social inequalities in educational achievement

Topic: Exposure - Enviromental factors

Presenter: Catherine Chittleborough

Catherine Chittleborough 1); Debbie Lawlor 2), 3); Murthy Mittinty 1); John Lynch 1), 3 )

1) School of Population Health, University of Adelaide; 2) MRC Centre for Causal Analysis in Translational Epidemiology, University of Bristol; 3) School of Social and Community Medicine, University of Bristol

Background: Inequalities in early childhood development are important because they influence social inequalities in health later in life. Socioeconomic disadvantage in childhood is associated with reduced readiness to learn at school, and poorer later educational, health and labour market outcomes. Evidence from randomized controlled trials shows that early intervention improves readiness for school and later educational success.

Objectives: To quantify the effects of improving school readiness at age 5 on subsequent educational success, and how this would affect social inequality in educational achievement at age 16. Methods: Using marginal structural models with data from the Avon Longitudinal Study of Parents and Children in the United Kingdom $(n=11764)$ we first examined the causal effects of early life socioeconomic disadvantage and school readiness (teacher-rated assessment of language, reading, writing, maths) on poor educational achievement (not achieving at least five GCSEs with grades of $\mathrm{A}^{*}$ to $\mathrm{C}$, including English and Maths). We then simulated improvements in school readiness among the population and disadvantaged groups through universal and targeted intervention scenarios.

Results: Overall, $49.5 \%$ (95\% CI 48.6-50.4) of children had poor educational achievement at age 16. Children of low socioeconomic position had increased risk of poor educational achievement (RR 2.37, $95 \%$ CI 2.11-2.65) than children of high socioeconomic position. Children with low school readiness scores were also at increased risk of poor educational achievement (RR 2.06, 95 \% CI 1.87-2.27). Progressive universal interventions to improve school readiness, providing universal services for all children, with more intensive support programs that have a greater effect on school readiness for children with greater need, could reduce population levels of poor educational achievement by around $5 \%$ and reduce absolute social inequality in poor educational achievement by approximately $15 \%$

Conclusions: Socioeconomic disadvantage in early childhood has an enduring effect on later educational outcomes. Interventions to improve school readiness have the potential to reduce both overall population levels and some of the social inequalities in poor educational achievement

\section{P-258}

Three decades of work ability and its main determinants: alternate explanations or similarities?

Topic: Exposure - Enviromental factors

Presenter: Risto Kaikkonen
Risto Kaikkonen 1); Tommi Härkänen 1); Ossi Rahkonen 2); Raija Gould 3); Seppo Koskinen 1)

1) National Institute for Health and Welfare; 2) Hjelt Institute, Department of Public Health; 3) Finnish Centre for Pensions

Background: Limitations in functional capacity and more detailed in work ability have decreased substantially in Finland and also in other developed countries. There is a marked socioeconomic gradient in work ability but the possible changes of the causes of this gradient are poorly known.

Objectives: Aim was to analyze population attributable fractions and explain educational differences in work ability during the past three decades. Moreover aim was to find explanations and their possible changes an similarities in 30-year period. Socioeconomic position (education), general health, health behaviour and functional capacity as well as work-related factors were expected to be the main determinants of changes in work ability and changes in them were observed. Our hypothesis was that educational differences can partly be explained by different main determinants during the 30-year period.

Methods: The study was based on three nationally representative surveys from three decades 1980-2010: (1) Mini-Finland, a comprehensive cross-sectional health interview, through health examination and complementary questionnaire, collected 1978-1980 from 8000 Finns aged 30-64 years with $96 \%$ participation. (2) Health 2000 Survey, based on Mini-Finland, collected 2000-2001 with 5835 Finns aged 30-64 years, of whom 5,171 (89\%) participated. (3) Health 2011 Survey, based on Health 2000 with 7964 Finns and participation of $73 \%$. Analyzes were done using Stata to produce Population Attributable Fraction (PAF) and predicted margins (PM). Results: Clear socioeconomic gradient was found in this 30-year period. Preliminary PAF-results show that the significance and association to limited work ability of different health status, health behavior (ex. smoking PAF 4-11\%), risk-factors (ex. obesity PAF $7-12 \%$ ) and the nature of job (physically and psychosocially demanding work) varied during the period. Moreover the similarities and differences with these factors were pointed out when explaining the gradient during this long period (PAF 62-54 \%).

Conclusions: There is evidence that socioeconomic differences (by education) varies in time and moreover the contribution of these factors varies in time. Population attributable fraction shows that these differences are not the law of nature and justifies the potential that could be achieved when reducing these differences in work ability.

\section{P-259}

The association between parental occupational pesticide exposure and the risk of childhood leukaemia: analyses of CLIC data

Topic: Exposure - Enviromental factors

Presenter: Helen Bailey

Helen Bailey 1); Tracy Lightfoot 2); Jacqueline Clavel 3); Lucia Miligi 4); Claire Infante-Rivard 5); Debbie Glass 6); Lin Fritschi 7); John Dockerty 8); Joachim Schuz 1) Childhood Leukemia International Consortium)

1) International Agency for Research on Cancer, Section of Environment and Radiation, 150 Cours Albert Thomas, 69372 Lyon Cedex 08, France; 2) Department of Health Sciences, University of York, Seebohm Rowntree Building, Heslington, York YO10 5DD, United Kingdom; 3) nserm, CESP Centre for Research in Epidemiology and Population Health, Environmental Epidemiology of Cancer Team, Université Paris-Sud, UMRS 1018, 16 Avenue Paul Vaillant-Couturier, F-94807 Villejuif Cedex, France; 4) ISPO-Cancer 
Prevention and Research Institute, Occupational and Environmental Epidemiology Unit, Ponte Nuovo - Padiglione Mario Fiori, Via delle Oblate 2, 50141 Florence, Italy; 5) Department of Epidemiology, Biostatistics, and Occupational Health, Faculty of Medicine, McGill University, 1110 Pine Avenue West, Montréal, Province of Québec H3A 1A3, Canada; 6) Monash Centre for Occupational and Environmental Epidemiology, Monash University; 7) University of Western Australia, Western Australian Institute for Medical Research, Perth, Western Australia, Australia; 8) Dean's Department and Department of Preventive and Social Medicine, Dunedin School of Medicine, University of Otago, PO Box 913, Dunedin, New Zealand

Background: Leukaemia is the commonest childhood cancer. Little is known about the aetiology, although it is likely that both genetic and environmental factors play a role. Some of the most common chromosomal translocations seen in childhood leukaemia are present at birth and some studies have found an association with pre-natal parental occupational pesticide exposure. Because of the heterogeneity of childhood leukaemia, it is important to investigate risk by leukaemia subtype, but previous studies have had insufficient power. The Childhood Leukemia International Consortium (CLIC) is an international consortium of case-control studies of childhood leukaemia that aims to pool comparable data, in order to increase statistical power.

Objectives: To investigate the association between pre-natal parental occupational pesticide exposure and the risk of acute lymphoblastic leukaemia (ALL) and acute myeloid leukaemia (AML) in the offspring.

Methods: Data were collected from 13 case control studies conducted in Europe, North America, and Oceania over about a 30 year period. Data from the time periods of interest, namely time around conception for fathers, and time around conception and during pregnancy for mothers, were extracted from parental occupational histories and converted to a standardized format for pooling. The original data were based on expert assessment or coded job titles. Final exposure categories were based on the comparison of job codes and expert assessment in studies that had both. For maternal exposures the pooled dataset consisted of approximately 8,200 cases of ALL (6500 B-cell and 840 T-cell progenitors), 1300 cases of AML and 15,500 controls, with slightly smaller numbers available for paternal exposure analyses. Analysis by unconditional logistic regression is being used to calculate odds ratios, with adjustment for study matching factors, potential confounders and study centre.

Results: Overall, 362 of 24,257 (1.5\%) mothers and 1,196 of 24,241 (4.9\%) fathers were classified as having been occupationally exposed to pesticides during the time periods of interest. The analyses are currently in progress and will be presented for ALL (B- and T-cell), AML and specific cytogenetic subtypes.

Conclusions: To our knowledge, this is the first study of parental occupational exposures and the risk of childhood leukaemia by subtype using pooled data. Because of the large sample size, it will have more power than any previous individual study. Thus, this study may be able to help clarify whether maternal and/or paternal occupational exposures to pesticides in the prenatal period play a role in the development of leukaemia in the offspring.

\section{P-260}

Cold temperatures and mortality from diseases of circulatory system in Astana, Kazakhstan

Topic: Exposure - Enviromental factors

Presenter: Andrej Grjibovski

Andrej Grjibovski 1), 2); Nassikhat Nurgaliyeva 3), 4); Altay Sharbakov 3); Telman Seysembekov 3); Aliya Kosbayeva 4); Bettina Menne 5)
1) Department of International Public Health, Norwegian Institute of Public Health, Oslo, Norway; 2) International School of Public Health, Northern State Medical University, Arkhangelsk, Russia; 3) Medical University of Astana, Astana, Kazakhstan; 4) WHO office in Kazakhstan, Astana, Kazakhstan; 5) WHO European Centre for Environment and Health, Bonn Office, Bonn, Germany

Background: Several European and North American studies have reported associations between cold temperatures and mortality from diseases of the circulatory system. However, the effects of cold vary between the settings warranting further research in other parts of the world.

Objectives: To study associations between minimum apparent temperature and mortality from selected diseases of circulatory system in Astana, Kazakhstan-the second coldest capital in the world.

Methods: Daily counts of deaths from hypertensive diseases (ICD-10 codes: I10-I15), ischemic heart disease (I20-I25) and cerebrovascular diseases (I60-I69) among adults 18 years and older in Astana, Kazakhstan during cold periods (October-March) in 2000-2001 and 2006-2010, were collected from the City Registry Office. Associations between mortality and minimum apparent temperature (average for lags 0-15) were studied using Poisson regression controlling for wind speed and effects of month, year, weekends and holidays. Percent changes in daily mortality counts per $1{ }^{\circ} \mathrm{C}$ increase in temperature were calculated with $95 \%$ confidence intervals (CI).

Results: Altogether, there were 320, 4,468 and 2,364 deaths from hypertensive disorders, ischemic heart disease and cerebrovascular diseases, respectively. No significant associations between minimum apparent temperature and either deaths from hypertensive disorders $(-2.8,95 \% \mathrm{CI}-8.2 ; 2.7)$ or ischemic health disease $(-0.3 \%, 95 \%$ $\mathrm{CI}-1.7 ; 1.1)$ or cerebrovascular diseases $(0.2 \%, 95 \% \mathrm{CI}-2.4 ; 2.8)$ were found. Gender-stratified analyses yielded similar results.

Conclusions: Contrary to expectations, we did not find inverse associations between apparent temperatures and mortality from diseases of circulatory system in Astana. This can be partly explained by the fact that Astana is a rapidly developing capital of the oil rich Republic of Kazakhstan with well established warning system, nearly universal central heating and good public transport infrastructure, combined with a local culture of putting massive cloths when going outdoors. Further research on sensitivity of population in Kazakhstan and its adaptive capacity to climate change is needed.

\section{P-261}

Investigating the interaction between cognition and speech-in-noise hearing using data from UK Biobank

Topic: Exposure - Enviromental factors

Presenter: Mark Edmondson-Jones

Mark Edmondson-Jones 1); Abby McCormack 1); Heather Fortnum 1); David R Moore 2)

1) National Institute for Health Research Nottingham Hearing Biomedical Research Unit, Nottingham, UK; 2) Cincinnati Children's Hospital Medical Center, Cincinnati, USA

Background: Hearing in real-life situations, particularly noisy ones, is a cognitively demanding task that has been associated particularly with working memory, processing time and IQ. However the interaction between aging, cognition and speech-in-noise hearing has not been widely studied in large-scale studies. In May 2012 UK Biobank ( http://www.ukbiobank.ac.uk/) was opened as a resource to investigate the 40-70 year old UK population, an age range in which declining function becomes increasingly apparent. The prevalence of clinical hearing loss is particularly affected, increasing from around $5 \%$ at age 40 to around $50 \%$ at age 70 . 
Objective: We investigated the relationship between cognition and speech-in-noise hearing ( $\mathrm{SiN})$, and how this relationship is mediated by age. We also explored the effects of gender and socioeconomic status ('deprivation'), and the relationship between age and selfreported difficulty with hearing.

Methods: UK Biobank is a large observational study, recruiting $>500,000$ adults between 40 and 70 years old. SiN was tested using a 'digit triplet test', which presented triplets of monosyllabic digits against a background speech-shaped noise. Signal level varied adaptively with performance. Participants were also asked whether they had difficulty hearing, and whether they had difficulty hearing in noise. Additionally, a number of cognitive tests were performed which tested fluid intelligence, processing speed, and visual, numerical and prospective memory. Cognitive scores were derived using principal components analysis. Interactions between cognition and age with regard to speech-in-noise, and self-reported hearing difficulty were analysed using general and generalised linear modelling while controlling for sex and deprivation.

Results: Although a linear decline in pure tone audiometry with age over this range has been observed elsewhere, we observed a significant quadratic accelerating decline of $\mathrm{SiN}$ with age $(p<0.001)$ with SiN worsening by $0.009 \mathrm{~dB} /$ year at age 40 and $0.055 \mathrm{~dB} /$ year by age 70. We independently observed declining SiN with declining cognition, over and above this apparent effect of aging $(p<0.001$ in each domain) with some mediation between processing speed and age. This pattern may indicate that individuals are able to use cognitive function to compensate for some age-related decline in hearing function. Both sex and deprivation were significantly associated with SiN. Self-report difficulty increased with age $(p<0.001)$ but was not clearly associated with cognition.

Conclusion: Within this population reduced cognition contributed almost as much to differences in speech-in-noise hearing as age.

\section{P-262}

Use of UK Biobank data to identify spatial variation in hearing impairment and treatment

Topic: Exposure - Enviromental factors

Presenter: Mark Edmondson-Jones

Mark Edmondson-Jones 1); Abby McCormack 1); Heather Fortnum 1); David R Moore 2)

1) National Institute for Health Research Nottingham Hearing Biomedical Research Unit, Nottingham, UK; 2) Cincinnati Children's Hospital Medical Center, Cincinnati, USA

Background: In the 1980s hearing impairment was estimated to affect approximately $16 \%$ of the adult UK population, with prevalence increasing with age and affecting a majority of the population by about 70 years of age. A number of factors are understood to influence individuals' hearing sensitivity, including various biological and genetic factors and a range of current and historic exposures, for example through residence, employment and leisure. Although clinical hearing impairment is relatively common treatment through use of hearing aids is underutilised. Only $2-3 \%$ of the UK adult population are estimated to use a hearing aid

Objective: We set out to identify characteristics of areas with relatively high prevalence of hearing impairment. We also report areas of relatively high unmet need for use of hearing aids. Ecological factors associated with relatively high levels in either case are also identified. Methods: UK Biobank (http://www.ukbiobank.ac.uk/) is a large study in which over 500,000 adults aged between 40 and 70 completed a battery of baseline tests and questionnaires between 2006 and 2010. In addition to the taking of biological samples (blood, saliva and urine) and physiological measures, various tests were performed, including a speech-in-noise hearing test. An extensive questionnaire was completed. Invitations were issued to residents living within a catchment area centred on each of 22 assessment centres throughout the UK, and so coverage of the UK was incomplete. In addition to presenting raw, indirectly age-standardised prevalence rates of hearing impairment at local authority district level, we analysed these data using a spatial Bayesian ecological regression model incorporating deprivation (Index of Multiple Deprivation) and area classification (Output Area Classification).

Results: We present here details of geographical variation in prevalence of hearing impairment and uptake of hearing aids. Estimated effects of ecological factors of deprivation and area classification illustrate an increase in prevalence with deprivation. Industrialised areas have amongst the highest prevalence of hearing impairment and London amongst the lowest. Overall, hearing aid use was around $3 \%$ within this study, with $10 \%$ of those reporting difficulty with their hearing using a hearing aid. This proportion varies significantly between districts. Model estimates were used to predict variations in relative prevalence and uptake nationally.

Conclusion: Analysis of spatial and ecological variability in health conditions and treatment uptake can be facilitated through use of data from large national observational studies, and used to identify health inequalities, demand for treatment and areas of unmet need.

\section{P-263}

Effects of in utero exposure to persistent organochlorine pollutants on human semen quality and reproductive hormones

Topic: Exposure - Enviromental factors

Presenter: Anne Vested

Anne Vested 1); Cecilia Høst Ramlau-Hansen 2); Sjurdur Frodi Olsen 3); Jens Peter Bonde 4); Susanne Lund Kristensen 1); Thorhallur Ingi Halldorsson 5); Panu Rantakokko 6); Hannu Kiviranta 6); Emil Hagen Ernst 7); Gunnar Toft 1)

1) Danish Ramazzini centre, Department of Occupational Medicine, Aarhus University Hospital, Denmark; 2) Department of Public Health, Section for Epidemiology, University of Aarhus, DK-8000 Aarhus C, Denmark; 3) Centre for Fetal Programming, Statens Serum Institut, Copenhagen, DK-2300, Denmark; 4) Department of Occupational and Environmental Medicine, Bispebjerg Hospital of Copenhagen University, DK-2400 Copenhagen NV, Denmark; 5) Faculty of Food Science and Nutrition, University of Iceland, Reykjavik, Iceland; 6) National Institute for Health and Welfare (THL), Neulanen Research Centre, Kuopio, Finland; 7) Reproductive Laboratory, Institute of Anatomy, University of Aarhus, DK-8000 Aarhus C, Denmark

Background: Persistent organochlorine pollutants (POPs) are ubiquitous, persistent organic compounds with potential endocrine disrupting effects. They have long half-lives, accumulate in adipose tissue, and bioaccumulate in the food chain. Hence, humans are mainly exposed to POPs through diet and the compounds can be traced in humans worldwide. Hypotheses speculating whether exposure to endocrine modulating compounds during fetal life could affect normal development of male reproductive organs and cause long-term effects on semen quality have been put forward.

Objectives: The aim of the present study was to investigate potential effects of in utero exposure to background levels of PCBs and p, $\mathrm{p}^{\prime}$ DDE on semen quality and reproductive hormone levels.

Methods: We recruited 176 young men (median age 20 years) who were offspring from a Danish pregnancy cohort. Semen samples were analysed for sperm concentration, total count, motility, volume, and 
morphology, and blood samples were assessed for reproductive hormone levels. Maternal blood samples from pregnancy week 30 were analysed for concentrations of 6 PCBs (PCB-118, -138, -153, -156, 170 , and -180) and p,p'-DDE as a proxy for prenatal exposure. Potential associations between in utero exposure to PCBs, nondioxin-like (non-DL) PCBs (PCB-138, -153, -170, and -180), dioxin like-(DL) PCBs (PCB-118 and -156), and p,p'-DDE and sperm quality and reproductive hormone levels were investigated using multiple regression analysis adjusted for potential confounders.

Results: PCBs, Non-DL-PCBs, and DL-PCBs were highly correlated and adjusted, preliminary results from complete-case analyses suggested that higher PCB levels in utero were associated with lower testosterone, estradiol, and sex hormone binding globulin (SHBG) levels and higher follicular stimulating hormone (FSH) levels ( $p$ for trend $=0.01,<0.01,<0.01$, and 0.04 , respectively). These trends were not corroborated by analyses where in utero exposure levels were categorized into tertiles.

Surprisingly, results on in utero exposure to p,p'-DDE suggested a protective effect of $\mathrm{p}, \mathrm{p}$ '-DDE on sperm concentration (adjusted $p$ for trend $=0.03$ )

Conclusions: Preliminary adjusted complete-case analyses do not suggest an effect of in utero exposure to background levels of PCBs on semen quality but suggest inverse associations between PCBs and testosterone, estradiol, and SHBG, and a positive association between PCB and FSH, whereas p,p'-DDE appears to be positively associated with sperm concentration.

\section{P-264}

Maternal environmental exposures and children's development: China-Anhui birth cohort study

Topic: Exposure - Enviromental factors

Presenter: Shilu Tong

Fangbiao Tao 1); Jiahu Hao 1); Kun Huang 1); Shilu Tong 2)

1) Department of Maternal, Child \& Adolescent Health, School of Public Health, Anhui Medical University, Hefei, China; 2) School of Public Health and Institute of Health and Biomedical Innovation, Queensland University of Technology, Kelvin Grove, Qld. 4059, Australia

The China-Anhui Birth Cohort Study (C-ABCS) was conducted to examine the delayed, cumulative and interactive effects of maternal environmental exposures on birth outcomes and children's development. The C-ABCS recruited pregnant women from six major cities of Anhui province, China, between 2008 and 2010. A range of data (including demographic, obstetric, occupational, nutritional and psychosocial factors) were collected by both interviews and laboratory tests. In each trimester, women's blood samples were drawn, and pregnancy complications were abstracted from physician's medical records. By the end of 2012, birth outcomes/birth defects were observed/identified by clinicians within 12 months after the delivery of over 13,000 singleton live births of six cities. In addition, 4,668 children from Ma'anshan city will be further followed up during the pre-school period till they reach adolescence to obtain the data on familial environmental exposures as well as children's physical, psychological, behavioural and sexual development. The impact of maternal environmental exposures on birth outcomes and early childhood development is currently being analysed.

\section{P-265}

Food security in Iran in 2011-2012

Topic: Exposure - Enviromental factors
Presenter: Farid Najafi

\section{Farid Najafi 1); Fariba Kolahdoz 2)}

1) School of Public Health, Kermanshah University of Medical Sciences, Kermanshah, Iran; 2) Department of Medicine, University of Alberta

Background: At the World Food Summit in 1996, most countries, including Iran committed to reducing hunger by $50 \%$ in 2015 . In the year 2007 and after recommendation by Food and Agriculture Organization and approval of Iranian government cabinet, the Iranian Health government were assigned to set up the Food Insecurity and Vulnerability Information and Mapping System. Responsibility for this project in 2010 was transferred to the authors of this report.

Objectives: To measure the food insecurity and vulnerability in different provinces in Iran

Methods: Data related to food and nutrition security status was provided from following sources: Statistical Center of Iran, Ministry of Health and Medical Education, Ministry of Agriculture, Ministry of Welfare, Nutrition and Food Research Institute and other relevant organizations. Data using principal factor analysis and human development index methods were analyzed, and the provinces were divided into different regions based on the state of food and nutrition insecurity.

Results: Based on principal factor analysis, the country was classified to six regions of very secure, secure, relatively secure, relatively insecure, insecure, and very insecure food division. The general pattern shows higher food and nutrition security in central areas and higher insecurity in borders and marginal areas. While the provinces of Tehran, Qom, Isfahan, Yazd and Semnan were classified as very secure areas, provinces of Hormozgan, Sistan and Baluchestan and Kohkyloyeh and Boyer Ahmad were very food insecure. Using human development index, the classification of provinces according to three areas of food supply, health care and economic power was similar to the previous model.

Conclusion: Given the state of food insecurity in the country and the basic reasons for it, such as cultural, economic, and social infrastructures, implementation of multi-sectoral interventions with effective participation of all sectors of the national and provincial level is essential.

\section{P-266}

Discomfort index as a predictor of increased mortality risk: time series analysis of population daily records in beersheba region, Israel

Topic: Exposure - Enviromental factors

Presenter: Michael Friger

Michael Friger 1); Michela Baccini 2), 3); Arkady Bolotin 1); Liliana Tzivian 1); Annibale Biggeri 2), 3)

1) Ben-Gurion University of the Negev, Beersheba, Israel; 2) Department of statistics, informatics and applications \&quot;G. Parenti\&quot; (DiSIA), University of Florence, Italy; 3) Biostatistics Unit, ISPO Cancer Prevention and Research Institute, Florence, Italy

Background: The discomfort index (DI) is the measure of discomfort during the warm season of the year (defined by the sum of the drybulb and wet-bulb thermometers). Theoretically, DI can evaluate health danger in the population during very hot periods better than standalone measurements of temperature and relative humidity. It is also reasonable to believe that variations in DI around the year can affect the mortality rate, especially in sub- and tropical geographical areas. Obviously, the DI effect may be confounded by environmental airborne pollutants such as nitrogen oxides mixture NOx, sulphur 
dioxide $\mathrm{SO}_{2}$, particle matter $10 \mathrm{~m} \mathrm{~km} \mathrm{PM10,} \mathrm{etc.} \mathrm{And} \mathrm{yet,} \mathrm{realistic} \mathrm{as}$ such assumptions might seem at first glance, they need a thorough examination before been declared correct.

Objectives: To examine associations between DI and all mortality causes for different age and ethnic groups in the city of Beersheba, Israel, and the suburb area.

Methods: Daily rates of all-natural-cause mortality (DRM) in 65and $65+$ age groups were analyzed for Jewish and Bedouin populations through the period of 1997-2010 years. The study dataset also included daily DI readings, the maximum difference in daily temperature (DmaxT), daily values of relative humidity, and daily concentrations of PM10, NOx, and $\mathrm{SO}_{2}$. A multivariate time seriesbased Poisson regression controlled for seasonal factors and pollutants with the broken line linear spline of DI at $24{ }^{\circ} \mathrm{C}$ was used to estimate the rate ratio of death incidences.

Results: Any DI effects have been found virtually absent in 65- age groups. In contrast, in 65+ age groups, increased DRM were found associated with DI for Jewish (IRR $=1.0195 \%$ CI 1.001-1.021, $p<0.02$ ) and Bedouin populations (IRR $=1.0495 \%$ CI 1.03-1.05, $p<0.01)$. A significant interaction between DI and DmaxT in Bedouin population aged $65+$ was observed. In both ethnicity groups aged $65+$, significant DI effects on DRM were found with the delay of 1,2 and 3 days.

Conclusion: We have found evidence that higher DI is associated with increased mortality risk (both immediate and delayed), worsened by age and ethnicity factors.

\section{P-267}

\section{Associated factors to women urinary incontinence in Botucatu, Brazil}

Topic: Exposure - Enviromental factors

Presenter: Adriano Dias

Luana Schneider Vianna 1); Ana Carolina Monteiro Santini 1); Angélica Pascon Barbosa 2); Liamara Cavalcante Assis 1); Gladys Dias 1); Adriano Dias 1)

1) Graduate Program in Gynecology, Obstetrics and Mastology Botucatu Medical School; 2) UNESP - Marilia Campus

Background: The urinary incontinence (UI) is defined by the International Continence Society like any loss of urine, regarded to a health public problem that leads to social, physical, psychological, economic and hygienic disorders to the population.

Objectives: To determine the sociodemographic and anthropometric profile of women from Botucatu, Brazil, and to seek the association among various risk factors mentioned in the literature and the occurrence of UI.

Methods: This is a transversal epidemiological study made in 2 hospitals with maternity in operation in the city of Botucatu, Brazil. 299 women were interviewed between April, 2011 and August, 2012 during hospitalization for childbirth, when responded the structured survey with sociodemographic and risk factors issues for UI, according the relevant literature. Were adjusted univariated logistic regression model, where the dichotomic variable was occurrence of UI and predictive variables were: smoking, physical activity, use of alcohol and illicit drugs, coffee, soda, tea and chocolate consumption, urinary tract infection, hypertension, diabetes mellitus type 1 and type 2, gestational diabetes mellitus, respiratory diseases, use of oral contraceptives and abortion occurrence. Thereafter, was adjusted a conditional multiple regression model, where in the dichotomic variable answer was the UI occurrence and the predictors variables were those which in the univariated model have had estimates of OR with $p<0.25$.

Results: The 299 women that composed the study were most of white race, married, family income of two minimum wages, share the residence with three or four individuals, home ownership, independently of being continent or incontinent. The women of the continent group predominantly work out of home and concluded the high school, while the women incontinent group, work at home and didn't conclude the basic education. It was observed that among several covariates, the risk factors associated with the occurrence of UI were parity and gestational diabetes mellitus (GDM) with OR values equal to $1.170(p<0.0001,95 \%$ CI $1.170-1.752)$ and $13.215(p=0.003$, $95 \%$ CI 2.402-72.695), respectively.

Conclusions: The parity and GDM appeared as associated factors to the increased prevalence of UI. Owing to the few studies about the GDM effect in the end of the pregnancy and its connection with UI, the study presents a large contribution, showing the DMG how an important risk factor for female UI.

\section{P-268}

Psychosocial work environment and ambulatory blood pressure: Independent and combined effect of demand-control and effortreward imbalance models

Topic: Exposure - Enviromental factors

Presenter: Xavier Trudel

Xavier Trudel 1); Chantal Brisson 2); Alain Milot 3); Benoit Masse 4); Michel Vézina 5)

1) Santé des populations: URESP, Centre de recherche du CHU de Quebec; 2) Département de médecine sociale et préventive,

Université Laval; 3) Département de médecine, Université Laval; 4) Département de médecine sociale et préventive, Université de Montréal; 5) CSSS de la Vieille Capitale, Quebec City, Québec, Canada

Background: Two major theoretical models have been used to assess the impact of psychosocial work factors on blood pressure (BP): the demand-control model (DC) and the effort-reward imbalance (ERI) model. Little is known on their independent and combined effect.

Objective: To examine the independent and combined effect of the DC and the ERI models on ambulatory BP.

Method: Data were collected three times during a 7-year period, among 3395 white-collar women and men. At each time, psychosocial work factors were measured using validated scales. Ambulatory BP was measured every 15 min during a working day. Systolic and diastolic BP means were examined, according to contemporaneous and past exposure. Both models were mutually adjusted. A combined exposure variable was computed.

Results: High strain, active, and ERI exposure were associated with BP, in men, using contemporaneous exposure. However, the high strain/BP association was not significant after adjustment for ERI. In women, no association was found with the DC model while women exposed to ERI had higher BP. Using past exposure showed a stronger association between BP and DC intermediate groups (active and passive) in men, while ERI associations were attenuated. Combined exposure to active jobs and to ERI was associated with BP, in both genders.

Conclusion: High strain effect on BP was not independent of ERI exposure. However, both DC intermediate groups were independently associated with BP. ERI exposure was independently associated with $\mathrm{BP}$ using contemporaneous exposure, but not using past exposure. Combined active and ERI exposure was also associated with BP. 


\section{P-269}

Population attributable risk of unintentional childhood poisoning in Karachi Pakistan

Topic: Exposure - Enviromental factors Presenter: Bilal Ahmed

\section{Bilal Ahmed 1); zafar Fatmi 1); Rehana Siddiqui 1)}

1) The Aga Khan university

Introduction: The percentage of unintentional childhood poisoning cases in a given population attributable to specific risk factors (i.e., the population attributable risk) was calculated as they are necessary to focus on the prevention strategies.

Methods: We calculated PARs, using 120 cases with unintentional poisoning and 360 controls in a hospital based matched case- control study. The risk factors were accessibility to hazardous chemicals and medicines due to unsafe storage, child behavior reported as hyperactive, storage of kerosene and petroleum in soft drink bottles, low socioeconomic class, less education of the mother and the history of previous poisoning.

Results: The following attributed risks were observed: $12 \%$ (95\% confidence interval [CI] 8-16\%) for both chemicals and medicines stored unsafe, $19 \%(15-23 \%)$ for child reported as hyperactive, $40 \%(38-42 \%)$ for storage of kerosene and petroleum in soft drink bottles, $48 \%(42-54 \%)$ for low socioeconomic status, $38 \%$ (32-42\%) for no formal mothers education and $5.8 \%(2-10 \%)$ for history of previous poisoning. $48 \%$ of cases for overall study population which could be attributed to at least one of the six risk factors. Among girls, this proportion was 23 and $43 \%$ among boys. About half of the unintentional childhood poisoning cases in this Pakistani population could be avoided.

Conclusion: Exposure to potentially modifiable risk indicators explained about half of the cases of unintentional poisoning among children under 5 years of age in this Pakistani population, indicating the theoretical scope for prevention of the disease.

\section{P-270}

Serum levels of perfluorinated chemicals in pregnant women and fetal growth: Preliminary results

Topic: Exposure - Enviromental factors

Presenter: Cathrine Carlsen Bach

Cathrine Carlsen Bach 1), 2); Bodil Hammer Bech 2); Ellen Aagaard Nøhr 2); Niels Uldbjerg 3); Rossana Bossi 4); Eva Cecilie Bonefeld-Jørgensen 5); Jørn Olsen 2); Tine Brink Henriksen 6)

1) Perinatal Epidemiology Research Unit, Aarhus University Hospital, Denmark; 2) Section for Epidemiology, Department for Public Health, Aarhus University, Denmark; 3) Department of Obstetrics and Gynecology, Aarhus University Hospital; 4) Department of Environmental Science, Aarhus University; 5) Centre for Arctic Health, Department of Public Health, Aarhus University; 6) Pediatric Department, Aarhus University Hospital

Background: Perfluorinated chemicals (PFCs) are widespread environmental toxicants that accumulate in the human organism. In pregnant women, PFCs cross the placenta thereby exposing the fetus. Based on previous animal and human studies, fetal exposure to PFCs is suspected to cause impaired fetal growth, but studies have shown conflicting results and have not included all PFC compounds.

Objectives: The main objective of this study is to investigate the association between all measurable maternal PFC levels and fetal growth.
Methods: 1350 participants (250 per year in 2008-2010 and 200 per year in 2011-2013) will be randomly selected from the Aarhus Birth Cohort Biobank from pregnant women who gave a blood sample before 14 weeks of gestation, were nulliparous and provided a time to pregnancy if the pregnancy was planned. A total of 17 PFCs are measured by high performance liquid chromatography - tandem mass spectrometry. Data on gestational age, birth weight and length are provided by the Aarhus Birth Cohort Database. Information on various covariates is available in the Aarhus Birth Cohort.

Results: Preliminary results from 499 participants (2008 through 2009) are presented. The median serum concentration of perfluorooctanoate $(\mathrm{PFOA})$ was $2.4 \mathrm{ng} / \mathrm{ml}$ (interquartile range $(\mathrm{IQR})=$ $1.9-3.0 \mathrm{ng} / \mathrm{ml}$ ), while the median serum concentration of perfluorooctane sulfonate (PFOS) was $9.7 \mathrm{ng} / \mathrm{ml}$ (IQR $=7.3-12.8 \mathrm{ng} / \mathrm{ml}$ ). Linear regression analyses will be used to estimate the association between levels of PFCs, particularly perfluorooctane sulfonate (PFOS) and perfluorooctanoate (PFOA), and proxy estimates of fetal growth while adjusting for covariates (Maternal age, prepregnancy BMI, maternal smoking and alcohol intake during pregnancy, maternal education, maternal diseases, parity, infant sex, and gestational age at birth).

Conclusions: This study investigates the association between fetal exposure to PFCs and impaired fetal growth. Levels of PFOS and PFOA are within the expected range. The data analyses are ongoing and results will be presented.

\section{P-271}

Vegetable, fruit and nitrate intake in relation to the risk of Barrett's esophagus in a large Dutch cohort

Topic: Exposure - Enviromental factors

Presenter: András Keszei

András Keszei 1); Leo Schouten 1); Ann Driessen 2); Clément Huysentruyt 2); Yolande Keulemans 3); R Alexandra Goldbohm 4); Piet van den Brandt 1)

1) Department of Epidemiology, School for Oncology and Developmental Biology, Maastricht Univerisity; 2) Department of Pathology, School for Oncology and Developmental Biology, Maastricht University; 3) Department of Gastroenterology, Maastricht University Medical Centre; 4) Department of Prevention and Health, TNO Quality of Life, Leiden

Background: The incidence of esophageal adenocarcinoma and its precursor, Barrett's esophagus has been increasing in several developed countries. There are few prospective epidemiological data about dietary risk factors of Barrett's esophagus.

Objective: This prospective study investigated the associations between vegetable, fruit, and nitrate intake and the risk of Barrett's esophagus in a large prospective cohort.

Methods: The Netherlands Cohort Study recruited 120,852 individuals aged 55-69 years in 1986. Vegetable and fruit intake was assessed using a 150-item food frequency questionnaire, and nitrate intake was measured from dietary sources and drinking water. During 16.3 years of follow-up incident Barrett's cases were detected by linkage to the nationwide network and registry of histopathology and cytopathology in the Netherlands (PALGA). Diagnosis of intestinal metaplasia was abstracted from PALGA-summaries and from full pathology reports collected through PALGA. 433 cases of Barrett's esophagus with specialized intestinal metaplasia and 3717 subcohort members were analyzed in a case-cohort design using Cox proportional hazards models while adjusting for potential confounders.

Results: Men showed a lower risk of Barrett's esophagus in the highest compared to the lowest quintile of total (multivariable 
adjusted hazard ratio (HR):0.66; $95 \%$ confidence interval (CI) $0.43,1.01$ ), raw (HR: 0.63; CI 0.40, 0.99), raw leafy (HR: 0.55; CI 0.36, 0.86), and Brassica vegetables (HR: 0.64; CI 0.41,1.00). No association was found for other vegetable groups and fruits. Among women, the strongest inverse association was found with the intake of Brassica vegetables, but the estimate was not significant (HR: 0.65; CI $0.40,1.07)$. Total nitrate intake was inversely associated with Barrett's disease in men (HR: 0.50; CI 0.25, 0.99), and positively in women (HR: 3.77; CI 1.68, 8.45) ( $p$ for interaction 0.04).

Conclusions These results suggest that vegetable intake may contribute to the prevention of Barrett's esophagus. The possible differential effect of nitrate in men and women should be further evaluated.

\section{P-272}

Optimal summer temperature among elderly persons with chronic disease: a study of Northern and Mediterranean Europe.

Topic: Exposure - Enviromental factors

Presenter: Daniel Oudin Åström

Daniel Oudin Åström 1); Christoffer Åström 1); Patrizia Scifano 2); Adele Lallo 2); Paola Michelozzi 2); Joacim Rocklöv 3); Bertil Forsberg 1)

1) Occupational and Environmental Medicine, Umeå University, Sweden; 2) Department of Epidemiology Lazio Region, Italy; 3) Epidemiology and Global Health, Umeå University, Sweden

Background: The on-going climate change is projected to increase the number, as well as the intensity, of extreme weather events. Elevated temperatures are well known to cause significant adverse health consequences with the elderly and chronically ill among the most susceptible groups.

Objectives: We aim to investigate the temperature-mortality relationship among the population above 50 as well as certain cohorts of persons with chronic disease according to health care registers in Rome, Italy and Stockholm, Sweden during the summers of 2000-2008. We investigated the following diseases; Chronic Obstructive Pulmonary Disease (COPD); Congestive Heart Failure (CHF); Survival to Myocardial Infarction (MI); Diabetes and Psychiatric disease.

Methods: For the 50+ population and our cohorts we collected daily information on the deaths occurring between 15th May and 15th September each year. We investigated the relationship between allcause mortality and the 2 day moving average (lag01) of maximum apparent temperature using Generalised Additive Models, adjusting for time trends, day of week and public holiday. The optimal temperatures were, for each of the vulnerable cohorts, extracted as the temperature with the minimum mortality.

Results: The optimal temperature for the above 50 population was 18.3 and $26.6{ }^{\circ} \mathrm{C}$ in Stockholm and Rome respectively. In Stockholm the range of optimal temperatures was from $15.1{ }^{\circ} \mathrm{C}$ for the MI cohort to $19.6{ }^{\circ} \mathrm{C}$ for the $\mathrm{CHF}$ cohort. The range of optimal temperatures in Rome was from $20.1{ }^{\circ} \mathrm{C}$ diabetes cohort to for the $28.0^{\circ} \mathrm{C}$ for the $\mathrm{CHF}$ cohort. Conclusions: Optimal temperatures differ during the summertime between certain vulnerable cohorts in both Northern and Mediterranean Europe. With an expected increase of elderly and other potentially vulnerable groups in society it is of importance to identify the temperature-mortality relationships in order to be able to target vulnerable groups and improve future heat warning systems.
P-273

Perfluorinated chemicals in maternal serum and the risk of autism, attention-deficit/hyperactivity disorder and cerebral palsy in children

Topic: Exposure - Enviromental factors

Presenter: Zeyan Liew

Zeyan Liew 1); Beate Ritz 1); Eva Cecilie Bonefeld-Jørgensen 2); Tine Brink Henriksen 3); Ellen Aagaard Nøhr 4); Bodil Hammer Bech 4); Chunyuan Fei 5); Ondine von Ehrenstein 6); Jørn Olsen 4), 1)

1) Department of Epidemiology, Fielding School of Public Health, University of California at Los Angeles; 2) Centre of Arctic Environmental Medicine (CAM), Department of Public Health, Aarhus University; 3) The Department of Paediatrics, Institute of Clinical Medicine, Aarhus University; 4) Unit of Epidemiology, Department of Public Health, Aarhus University; 5) Biostatistics Branch, National Institute of Environmental Health Science (NIEHS) North Carolina USA; 6) Department of Community Health Sciences, Fielding School of Public Health, University of California at Los Angeles

Background: Perfluorinated Compounds (PFCs) are persistent organic pollutants that are used extensively in industry and commercial products, and are found throughout the environment and in humans. Potential neurotoxic effects of PFCs have been repeatedly observed in highly exposed animals, but results from human studies are still limited and inconclusive. We investigated for the first time whether prenatal exposures to PFCs affect fetal neurodevelopment and increase the risk of autism, Attention-Deficit/Hyperactivity Disorder (ADHD) or Cerebral Palsy (CP) in children.

Method: We used data from the Danish National Birth Cohort (DNBC) with a source population of 83,389 liveborn singleton children and mothers first enrolled in the DNBC during 1997-2003. By using a case-cohort study design, in 2011 we randomly selected 220 children with ADHD, 220 with autism, 156 with CP and a common control group of 550 children drawn from the entire cohort at baseline. We then measured blood levels of the four most prevalent PFCs, including perfluorooctanote acid (PFOA), perfluorooctane sulfonate (PFOS), perfluorohexane sulfonate (PFHxS), and perfluorononanoic acid (PFNA) in specimen drawn from mothers approximately during 8 weeks of gestation. Diagnoses of ADHD and autism were identified from the Danish National Hospital Registry and the Danish Psychiatric Central Registry, while children with a CP diagnosis were identified from the Danish National Cerebral Palsy Register. Odds ratios (ORs) for each outcome of interest were estimated using unconditional logistic regression with PFC concentrations modeled as continuous variables or categorized into quartiles according to the distribution in controls group.

Results: Higher odds of $\mathrm{CP}$ was estimated among children born to women with the highest quartile of PFOS (OR $1.9295 \%$ CI 1.10 3.34) and PFOA (OR $1.7595 \%$ CI 0.99-3.09) in their serum compared to the lowest after adjusting for potential confounders. The adjusted ORs per $1 \mathrm{ng} / \mathrm{mL}$ increases in PFOS (continuous) and CP was 1.01 (95\% CI 1.00-1.03), and per $1 \mathrm{ng} / \mathrm{mL}$ increases in PFOA and $\mathrm{CP}$ it was 1.06 (95\% CI 0.96-1.18). No associations were found between maternal PFHxS and PFNA levels with CP, and for all four PFCs with ADHD and autism.

Conclusion: Higher plasma levels of some PFCs in pregnancy may increase the risk of having $\mathrm{CP}$, but not ADHD or autism in children. 


\section{P-274}

School environment and childrens' fruit and vegetable intake levels in Greece: a multilevel logistic modelling approach

Topic: Exposure - Enviromental factors

Presenter: Joanna Moschandreas

Joanna Moschandreas 1); Angeliki Papadaki 2); Agneta Yngve 3); Inga órsdóttir 4); Christel Lynch 3); Anthony Kafatos 1)

1) University of Crete, Greece; 2) University of Bristol, U.K.; 3) Karolinska Institutet, Sweden; 4) University of Iceland, Iceland

Background:To our knowledge, the extent to which fruit and vegetable $(\mathrm{F} \& \mathrm{~V})$ consumption may be affected by school environment has not previously been investigated in Greece.

Objectives: The present study explores between-school variation in $\mathrm{F} \& \mathrm{~V}$ intakes and possible differences in effects of individual characteristics, using baseline data from primary school pupils participating in the European ProGreens project (2009-2011).

Methods: A detailed questionnaire incorporating a $24 \mathrm{~h}$ recall was completed by 913 pupils at 36 schools (70\% school response rate). Fruit/vegetable intakes were dichotomized at the 75 th percentile ( $300 \mathrm{~g} /$ days fruit and $143 \mathrm{~g} /$ days vegetables). Multilevel logistic regression analyses were undertaken using subject-level (gender, parental influence) and school-level (school food policy) covariates. Parental influence" was derived from 2 ordinal items regarding the frequency with which parents asked their offspring to consume fruit/ vegetables and dichotomized (daily basis vs other). Variance partition coefficients (VPC) and Median Odds Ratios (MOR) were calculated to quantify between-school variation. Likelihood ratio (LR) test statistics were used a) to assess the null hypothesis of zero betweenschool variability and $b$ ) to test for the presence of random slopes for parental influence. Stata 11 was used throughout (xtmelogit).

Results: Between-school variability in fruit intake was estimated to be $6.2 \%$ of total variability using a 2-level random intercept model without covariates (LR statistic 10.97, $p=0.0005$ ). The estimated MOR was 1.55 . In a multivariable model, the odds of being a "high" fruit consumer were increased where parental influence was present (OR 1.7, $95 \%$ CI 1.2-2.4) but not according to gender (OR 0.8, $95 \%$ CI $0.6-1.1$ ) or the existence of a school food policy (OR 1.2, $95 \%$ CI $0.8-1.9)$. There was no evidence that the effects of parental influence on fruit intake level differed across schools (LR statistic 0.86 on 2df, $p=0.650$ ). Minimal between-school variance in vegetable intake was detected, using a 2-level random intercept model without covariates (MOR 1.00). The odds of being a "high" vegetable consumer appeared higher where parental influence was present (OR 2.0, $95 \%$ CI 1.4-2.7) and lower for boys (OR 0.8, $95 \%$ CI 0.6-1.0), but the existence of a school food policy did not affect intake level (OR $1.0,95 \%$ CI $0.7-1.3$ ).

Conclusions: Most of the variability in fruit intake, and practically all the variability in vegetable intake categories, appears to occur at the subject level. The small school-level effects are findings in line with other European studies.

\section{P-275}

Place of upbringing in early childhood and occurrence of Inflammatory Bowel Disease in adulthood: a longitudinal study in Northern Europe

Topic: Exposure - Enviromental factors

Presenter: Signe Timm

Signe Timm 1); Christer Janson 2); Cecilie Svanes 3); Torben Sigsgaard 1); Ane Johannesen 3); Thorainn Gislason 4); Rain Jogi 5); Kjell Torén 6); Vivi Schlünssen 1)
1) Department of Public Health, Aarhus University, Denmark; 2) Institute of Clinical Science, Uppsala University, Sweden; 3) Department of Pulmonary Medicine, Tartu University, Estonia; 4) Institute of Medicine, University of Bergen, Norway; 5) Pulmonary and sleep medicine, Iceland University, Reykjavik, Iceland; 6) Department of Allergology, University of Gothenburg, Sweden

Background: A rapid increase of the two inflammatory bowel diseases (IBD), ulcerative colitis and Crohn's disease, has been observed during the twentieth century. A broad range of potential exposure variables have been investigated, generally in case-control studies, but the aetiology is still poorly understood. The "Hygiene Hypothesis", proposing impaired immunological competence with low microbial stimulation, has been stated as one possible explanation for the increasing incidence. There is a need for more longitudinal studies to understand how modern lifestyle may influence the risk of IBD.

Objectives: The aim of this study was to investigate the role of place of upbringing in early childhood on the prevalence of IBD in adulthood. Place of upbringing was used as a proxy variable for the level and diversity of microbial exposure.

Methods: RHINE III (Respiratory Health In Northern Europe) is a postal questionnaire follow-up of the ECRHS cohorts established in 1990-1994. The study population were 10,864 subjects born 1945-1971 in Denmark, Norway, Sweden, Iceland and Estonia, who responded to questionnaires in 2000-2002 (age, sex, smoking, BMI) and 2010-2012, concerning IBD and place of childhood upbringing (response categories: farm with livestock (livestock farm living), farm without livestock, village in rural area, small town, suburb of city (village living) and inner city (city living)).

Analyses included multiple logistic regression models adjusting for age, sex, smoking and BMI.

Results: The prevalence of IBD was $1.3,1.6$ and $2.3 \%$ among participants growing up on a livestock farm, in a village or in a city, respectively. Growing up at least the first 5 years of life on a livestock farm was significantly associated with a lower risk of IBD compared with city living (OR $0.54,95 \%$ CI $0.31 ; 0.94)$. Furthermore, a test for trend comparing livestock farm living, village living and city living was statistically significant $(p<0.05)$.

Sub-analysis showed that the protective effect of livestock farming was only present among subjects below the 75th age-percentile (subjects born after 1952).

Conclusion: In a population study from Northern Europe, subjects who grew up on a farm with livestock had less inflammatory bowel diseases, particularly among younger cohorts. We speculate that lower microbial diversity could be an explanation for the findings.

\section{P-276}

Daily variations in air pollution and primary health care visits of asthma

Topic: Exposure - Enviromental factors

Presenter: Anna Oudin

\section{Tahir Taj 1); Anna Oudin 2); Kristina Jakobsson 1)}

1) Occupational and Environmental Medicine, Lund University; 2) Occupational and Environmental Medicine, Umeå University

Background: Air pollution has a rather well-documented association with daily variations in asthma morbidity, most often studied with inpatient admissions or emergency-room visits. Data on primary health care has seldom been available in such studies, but are now available in Scania, Southern Sweden.

Objectives: To study different metrics of daily levels air pollution and primary health care visits of asthma in Scania.

Methods: Hourly readings of ambient Particulate Matter (PM10, $\mathrm{PM} 2.5)$, nitrogen dioxide (NO2), nitrogen oxide (NO), ozone (O3) 
and sulphur dioxide (SO2) from the Swedish Meteorological and Hydrological Institute (SMHI) from 2005 to 2010 were used. Six different PM metrics were created from these hourly readings: (1) daily 1-h maximum; (2) 24-h average; (3) commuting-period average; (4) daytime average; (5) night-time average; and (6) the daily 8-h maximum. Outcome data were obtained from a regional primary health care database, covering approximately half a million people living in Malmö and neighbouring municipalities. Poisson generalized linear models were used to examine the relations between daily primary health care (PHC) visits due to asthma and the various metrics of PM.

Results: Air pollutant levels throughout the study period remained well within the WHO air quality guidelines. The PM10 daily mean was $16.4 \mathrm{~g} / \mathrm{m} 3$. The strongest association was observed between PHC visits and daily mean PM10 lag0, with an increase of $12.5 \%(95 \%$ Confidence Interval (CI) 8.3-16.8\%) associated with every 10-unit increase in pollutant. The other metrics were also associated with an increased number of PHC visits, but not as strongly.

Conclusions: The results suggest that air pollution has adverse effects on visits to the primary health care for respiratory symptoms, even at low concentrations. The effects are generally stronger than what has previously been observed for in-patient care or emergency room visits, implying a larger public health impact than has previously been suggested.

\section{P-277}

Independent effects of traffic, sandstorm and volcanic particles on emergency hospital visits in Reykjavík, Iceland?

Topic: Exposure - Enviromental factors

Presenter: Hanne Krage Carlsen

\section{Hanne Krage Carlsen 1), 2); Thorarinn Gíslason 1), 3); Bertil} Forsberg 2); Kadri Meister 2); Anna Oudin 2)

1) School of Health Sciences, University of Iceland; 2) Department of Public Health and Epidemiology, Umeå University; 3) Department of Allergy and Sleep medicine, Landspítali University Hospital

Background: Following the spring 2010 Eyjafjallajökull volcanic eruption volcanic ash contributed largely to particulate matter (PM) levels in Iceland. The aim of the present study was to analyze the association between sources of high PM levels and emergency hospital visits in the capital area.

Materials and methods: The outcomes were acute admissions and emergency department visits for cardiopulmonary, stroke and respiratory causes to Landspitali University Hospital in Reykjavík (pop. 200.000) from 2007 to 2010. Air pollution data and the estimated source of particles of each event where the PM10 health limit $(50 \mu \mathrm{g} /$ $\mathrm{m}^{3}$ ) was exceeded were obtained from authorities. Two additional years of data have been collected and will be presented at the conference.

The data were analyzed using generalized additive regression, with indicator variables for each particle source (traffic, sandstorms, or volcanic ash) the current and two previous days (lag02), adjusted for other pollutants, weather and time trend.

Results: During the study period the average number daily emergency hospital visits was 10 . During the 4-year study period, high PM10 levels occurred 91 days, the source was traffic 37 days, sandstorms 11 days, and volcanic ash 9 days. The remaining days, the high levels were due to firework and other causes.

In preliminary analysis, we found that each day with high PM10 levels from traffic were associated with an increase in the number of emergency hospital visits of $9 \%$ (95\% confidence interval (CI) 1-17\%), adjusting for other pollutants and weather.
High PM levels due to sandstorms and volcanic ash were not significantly associated with emergency hospital visits when adjusting for other pollutants. In unadjusted models, high PM10 levels due to sandstorms and volcanic ash were nearly significantly $(\mathrm{p}<0.10)$ associated with emergency hospital visits, increasing $13 \%(95 \% \mathrm{CI}$ $0-27 \%)$ for volcanic ash and $9 \%(95 \% \mathrm{CI}-1$ to $21 \%)$ for sandstorms.

Conclusion: In this study of particle sources we identified independent effects of particles originating in traffic, and near-significant effects of even a few events of volcanic dust and sandstorms.

\section{P-279}

Indoor air at an urban nursery in Porto, Portugal-weekday/ weekend behaviour

Topic: Exposure - Enviromental factors

Presenter: Sofia Sousa

Sofia Sousa 1); Pedro Branco 1); Maria Alvim-Ferraz 1); Fernando Martins 1)

1) LEPAE, Departamento de Engenharia Química, Faculdade de Engenharia, Universidade do Porto

Background: Time spent indoor is usually higher than time spent outdoor, thus indoor air pollution (IAP) can have equal or even greater effects in health than outdoor air pollution. Exposure to IAP has been linked to a variety of health effects, including respiratory health problems and exacerbation of childhood asthma. Children are highly vulnerable to air pollution effects for a variety of reasons, being considered a risk group.

Objectives: This study aimed to evaluate the concentrations of carbon dioxide $(\mathrm{CO} 2)$, carbon monoxide $(\mathrm{CO})$, ozone $(\mathrm{O} 3)$, nitrogen dioxide (NO2), total volatile organic compounds (VOC), formaldehyde and PM10, at an urban nursery influenced by traffic emissions. Methods: The study was made based on data measured at an urban nursery influenced by traffic emissions in Porto. Indoor concentrations were measured at the classroom with children aged 2 years-old, with continuous monitors during 1 week, being the measurements taken each minute. A Haz-Scanner monitor was used with the following detection methods: (i) $\mathrm{CO}_{2}$-nondispersive infrared (NDIR) detection; (ii) $\mathrm{O} 3, \mathrm{NO} 2$ and formaldehyde-electrochemical detection; iii) VOC - photoionization detection (PID); and iv) PM10 — infrared light scattering. Relative humidity (RH) and temperature $(\mathrm{T})$ in the classroom were also measured using Haz-Scanner monitor with electrochemical sensors. All concentrations were corrected for T.

Results: $\mathrm{CO} 2$ concentrations were generally exceeding the Portuguese legislation standard $\left(1,800 \mathrm{mg} \mathrm{m}^{-3}\right)$ during occupancy (9-19 h, weekdays) and until 2 a.m. (maximum mean concentration: $6,498 \mathrm{mg} \mathrm{m}^{-3}$ ), reducing during the night. During the weekend CO2 concentrations were always below detection limit (maximum mean concentration: $1,279 \mathrm{mg} \mathrm{m}^{-3}$ ). Formaldehyde concentrations exceeded the Portuguese legislation standard $\left(100 \mathrm{~g} \mathrm{~m}^{-3}\right)$ during occupancy (12-18 h, maximum mean concentration: $260 \mathrm{~g} \mathrm{~m}^{-3}$ ). During the night formaldehyde concentration decreased to a minimum of $0 \mathrm{~g} \mathrm{~m}^{-3}$, maintaining that value over the weekend. Total VOC showed the same behaviour. Interestingly, PM10 concentrations were higher during the night and weekend, always exceeding the Portuguese legislation standard $\left(150 \mathrm{~g} \mathrm{~m}^{-3}\right.$ for TSP) during those periods (maximum mean concentration: night- $251 \mathrm{~g} \mathrm{~m}^{-3}$; weekend$469 \mathrm{~g} \mathrm{~m}^{-3}$ ). Mean RH and $\mathrm{T}$ values were, respectively $73 \%$ and $16{ }^{\circ} \mathrm{C}$.

Conclusions: Occupancy seemed to increase $\mathrm{CO}_{2}$, formaldehyde and PM10 concentrations. Considering the well-known effects of air pollution in the health of children, it seems important, according to 
the results achieved, to implement strategies to reduce these effects since infancy, which could be achieved by involving directly the parents and nurseries responsible on environmental education.

\section{P-280}

Why should we focus in cold extremes health negative outcomes in Mediterranean climate like Porto, Portugal?

Topic: Exposure - Enviromental factors

Presenter: Ana Monteiro

\section{Ana Monteiro 1); Sara Velho 1); Carlos Sousa 1)}

\section{1) University of Porto; ISPUP; CITTA}

Background: Scientific research and media refer persistently the fatal effects of heat waves due to global climate change and diminish the importance of the cold in Mediterranean areas. However, the climate adaptation of human beings, their lifestyles and the architectural features gathered with the greater economic differences in southern Europe, show a high vulnerability of the poorer, older and less informed to sporadic and unexpected episodes of low temperatures during winters. Eurowinter Group (1997) or Healy (2003) are some examples of important research done that suggest the inadequacy of the existent warning systems.

Objectives: Our ambition is to understand the importance of cold episodes in a mild winter climate like Porto through the comparison of Physiological Equivalent Temperature (PET) and morbidity and mortality in Porto (2000-2007).

Methods: First we collect the daily minimum and maximum temperature of Porto (1900-2007) to define the 10th and 5th percentiles and define the extreme cold events (duration and intensity) considering the importance of adaptation to climate context. Then, using Solweig software, we calculate the PET of Porto to differentiate the climate data recorded and the perceived one. Afterwards, we gather the daily admissions at the 4 main hospitals (2002-2007) with the corresponding cause. We assemble also the daily mortality (20022007). Finally, we estimate the excess of morbidity and mortality and compare the observed values during the predefined extreme cold events with the homologous periods.

Results: From 2002 to 2007, the common period with mortality, morbidity and climatological data, emerge 9 extreme cold events. During the majority of these extreme cold events we observe an increase of mortality from 12 to $34 \%$. The excess of morbidity showed a considerably increase also if we analyse either the respiratory and the circulatory diseases (all causes), or some diseases separately. Bronchitis and asthma admissions reach an increase of $76 \%$, COPD above $40 \%$, pneumonia and pleurisies above $50 \%$, myocardial infarction had more than $50 \%$ and stroke overpass in some events $100 \%$.

Conclusions: In climates with mild winters, the low temperature thresholds considered as a risk to human beings health should be redesigned and adapted to a complex equation where climate and socioeconomic vulnerability need to be included with equal weight. The impulsive behaviour of climate system maintains anomalies of either extreme cold or heat even during warmer times. Moreover, the seasonality of Mediterranean climates added with the greatest socioeconomic differences among society, create a lack of comfort efficiency of housing to protect people from the cold and damp particularly acute among the poorer social classes who, in most cases, do not have the economic resources to bear the costs of artificial heating in their homes. The elderly, who in many cases live alone, and the increased number of homeless people, comprise the more vulnerable groups during these cold episodes. Since they are less supervised, aware and prepared, they take much longer to take the necessary steps to protect themselves.

\section{P-281}

Environmental determinants for overweight and obesity in an urban context, Brazil

Topic: Exposure - Enviromental factors

Presenter: Larissa Loures Mendes

Larissa Loures Mendes 1); Milene Cristine Pessoa 2); Cristina Maria Proença Padez 3); Gustavo Velásquez-Meléndez 2); Deborah Carvalho Malta 4)

1) Universsidade Federal de Juiz de Fora; 2) Universidade Federal de Minas Gerais; 3) Universidade de Coimbra; 4) Secretaria de Vigilância em Saúde, Ministério da Saúde,

Background: Over the past decade there has been a growing recognition of the role of the environment in influencing health and health behavior. For nutrition and physical activity behaviors, the emergence of obesity pandemic has brought this issue into sharp focus.

Objective: The aim of this study was to evaluate variables of the built and social environment and their association with overweight in an urban Brazilian population.

Methods: Participants were selected from the Surveillance of Risk Factors for Chronic Diseases through telephone interview organized by the Ministry of Health. The study was conducted with data from the city of Belo Horizonte, 3,425 interviews for the years 2008 and 2009. Georeferenced information of parks, public squares, places for physical activity, population density and food stores were used as the built environment. To characterize the social environment, we used neighborhood income and homicide rate. The results of multilevel regression showed that the data had no structure in two levels. Thus, we used adjusted Poisson regression models in a single level.

Results: The population density, presence of parks, squares and places for physical activity and the self-reported presence of places for physical activity were independently associated with overweight. Conclusion: The evidence from this study shows that characteristics of the places that people live are associated with overweight in urban Brazilian context.

\section{P-282}

Food environment, neighborhood income and consumption of fruit and vegetables in an urban context

Topic: Exposure - Enviromental factors

Presenter:

Milene Cristine Pessoa 1); Larissa Loures Mendes 2); Crizian Saar Gomes 1); Gustavo Velásquez-Meléndez 1); Deborah Carvalho Malta 3)

1) Universidade Federal de Minas Gerais; 2) Universsidade Federal de Juiz de Fora; 3) Secretaria de Vigilância em Saúde, Ministério da Saúde,

Background: People with higher incomes and greater educational attaiment tent to consume more fruit and vegetables than those who are less affluent and less educated. In addition, recent published studies have suggested that closer proximity to a chain supermarket is positively associated with fruit and vegetable intake or overall dietary quality. 
Objective: To estimate associations between environmental and individual factors and the consumption of FV among adults in a Brazilian urban context.

Methods: Data from the surveillance system for risk factors for chronic diseases of Brazilian Ministry of Health was used (VIGITEL-2008/2009/2010). A cross-sectional telephone survey was carried out with 5826 adults $(45.8 \%$ men and $54.2 \%$ women, mean age 39.5 years) in the urban area of Belo Horizonte. Individual variables was collected by telephone interviews. The frequency of FV consumption was assessed from number of servings, weekly frequency and a FV intake score. Georeferenced variables was used to characterize the food environment. The number of stores selling FV (supermarkets, hypermarkets, shops and markets specialized in selling FV) and the neighborhood quartiles of family income were investigated and combined to score FV intake. Weighted multilevel linear regression was used to evaluate the associations between the food environment and the FV intake score.

Results: We observed that the food enviroment was not associated with FV intake score and higher FV intake score in women and physically active people during leisure time and lower scores in smokers. It was also observed increase in FV intake score with increasing age as well as schooling. The neighborhood income joined the scores of FV intake, showing that the score of consumption increases as the total income of the neighborhood increases.

Conclusion: The food environment was not associated with FV consumption, showing that in this case, family income explains much of the context variables associated with FV intake.

\section{P-283}

Fruit and vegetable consumption and food environment in a Brazilian capital: a multilevel analysis

Topic: Exposure - Enviromental factors

Presenter: Larissa Loures Mendes

Milene Cristine Pessoa 1); Larissa Loures Mendes 2); Crizian Saar Gomes 1); Gustavo Velásquez-Meléndez 1); Deborah Carvalho Malta 3)

1) Universidade Federal de Minas Gerais; 2) Universsidade Federal de Juiz de Fora; 3) Secretaria de Vigilância em Saúde, Ministério da Saúde,

Background: Environmental, social and individual factors influence eating patterns, which in turn affect the risk of many chronic diseases. Fruit and vegetable (FV) consumption plays a protective role in the onset of non-communicable diseases.

Objective: To test associations between environmental factors and the consumption of FV among adults in a Brazilian urban context.

Methods: Data from the surveillance system for risk factors for chronic diseases of Brazilian Ministry of Health was used (VIGITEL-2008/2009/2010). A cross-sectional telephone survey was carried out with 5826 adults $(45.8 \%$ men and $54.2 \%$ women, mean age 39.5 years) in the urban area of Belo Horizonte. Individual variables was collected by telephone interviews. The frequency of FV consumption was assessed from number of servings, weekly frequency and a FV intake score. Georeferenced variables was used to characterize the food environment. Weighted multilevel linear regression was used to evaluate the associations between the food environment and the FV intake score.

Results: The results showed higher FV intake scores in women and people physically active and lower scores in smokers. We observed a positive relationship between the number of grocery stores in the neighborhood and FV intake score and a negative relationship between worse health vulnerability index (HVI) and the score of FV consumption.

Conclusion: Characteristics of the neighbourhood that people live are associated with FV in urban Brazilian adults. Surveillance systems as VIGITEL are interesting tools in this type of research and with the information based on GIS, are important tools for planning actions to promote fruit and vegetable consumption.

\section{P-284}

Evaluation of the iodine situation on the background of USI in Kazakhstan

Topic: Exposure - Enviromental factors

Presenter: F Ospanova

F Ospanova 1); A Beisbekova 1); Zh Tolysbayeva 1); A Nurgabylova 1); Sh Tazhibayev 1); T Sharmanov 1); Andrej Grjibovski 2), 3)

1) School of Public health, Kazakh National Medical University named after S.D. Asfendiyarov, Almaty, Kazakhstan; 2) Department of International Public Health, Norwegian Institute of Public Health, Oslo, Norway; 3) International School of Public Health, Northern State Medical University, Arkhangelsk, Russia

Background: In 2003 the Republic of Kazakhstan (RK) was one of the first countries of CIS region adopted the Law on mandatory iodization of table and cattle salt. Based on the results of the national Multiple Indicator Cluster Survey (MICS) of 2006 and the subsequent application, the country was certified in 2010 as reached universal salt iodization (USI). After this time, researches on iodine deficiency at the national level were not held in Kazakhstan.

Objectives: The evaluation of the situation on iodine intake at the national level by urine iodine excretion, revelation of salt iodization quality and the awareness level of the population on iodine deficiency, comparison of indicators overtime in RK.

Methods: A cross-sectional survey of 2011 included 64 clusters on 22 women in each cluster. The women of reproductive age (15-49 years) were interviewed and 1,145 salt samples were collected from their households for quantitative determination of iodine, and 1,296 their urine samples were analyzed on content of iodine by ammonium persulfate digestion method, based on the Sandell-Kolthoff reaction. It was conducted a comparative analysis of the results with data from national studies before adopting USI strategy (1999) and MICS (2006).

Results: The urinary iodine median at women in 2011 was $181.1 \mathrm{mg} /$ 1, in 2006-235.9 mg/l, in 1999-93.9 mg/l. The prevalence of iodine deficiency of women was $22.8 \%$ in 2011, it was $15.9 \%$ in 2006, and $54.2 \%$-in 1999. In 2011 the percentages of salt samples with adequately iodized salt $(\geq 15 \mathrm{ppm})$ were $95.9 \%$, in $2006-91.4 \%$, in 1999-29\%. In 2011 among interviewed women $92.7 \%, \mathrm{n}=1303$ knew that salt is iodized, in 2004 these were $93.3 \%, \mathrm{n}=1500$, and only $58.6 \%$ were aware of the need to use iodized salt, $\mathrm{n}=4800$ in 1999.

Conclusion: Since the adoption of the Law on mandatory salt iodization consumption percentage of adequately iodized salt has increased, which in turn affected the rising of urinary iodine levels at the population. After 8 years of USI median of urinary iodine excretion decreased slightly comparing with 2006 , but remained in the normal range on the background of adequately iodized salt coverage more than $90 \%$. It indicates the necessity of periodic biological monitoring and continuing communication activity among population on long-term and constantly basis. 


\section{P-286}

Psychosocial work factors, personality traits, and risk of insomnia: results from the Danish PRISME study

Topic: Exposure - Enviromental factors Presenter: Linda Kaerlev

Linda Kaerlev 1), 2); Anne Helene Garde 3); Henrik Albert Kolstad 4); Jens Peter Bonde 5); Jane Frølund Thomsen 5); Anette Kærgaard 6); Sigurd Mikkelsen 5); Matias B. Grynderup 4); Reiner Rugulies 3); Ase Marie Hansen 7)

1) Research Unit of Clinical Epidemiology, Institute of Clinical Research, University of Southern Denmark, Odense, Denmark; 2) Center for Clinical Epidemiology, Odense University Hospital, Odense, Denmark; 3) National Research Centre for the Working Environment, Copenhagen, Denmark; 4) Department of Occupational Medicine, Danish Ramazzini Centre, Aarhus University Hospital, Denmark; 5) Department of Occupational and Environmental Medicine, Bispebjerg University Hospital, Denmark; 6) Department of Occupational Medicine, Danish Ramazzini Centre, Regional Hospital Herning, Denmark; 7) Department of Public Health, University of Copenhagen, Denmark

Objectives: Several studies indicate strong associations between perceived psychosocial job stress and insomnia. However, these associations may be biased by response style and psychological reactivity, and personality traits may also play a role. We measured the association between job stressors according to three models and insomnia both at baseline and with a secondary aim to explore the importance of personality traits.

Methods: The study base was a sample of Danish public service workers in Aarhus, Denmark. In January 2007 a total of 4,491 participants filled-in a mailed questionnaire assessing psychological job demand (Karasek), Effort-reward imbalance (ERI)-10 (Siegrist), Procedural and relational justice (Kivimäki), depressive symptoms (CMDQ-scale), and neuroticism (Eysenck Personality Questionnaire Revised-A). Subjective sleep quality during the previous 4 weeks (disturbed sleep, awakening problems) and overall sleep quality were measured by Karolinska sleep questionnaire (KSQ). Psychosocial responses were scored on Likert five-point frequency scales and sleep quality on a dichotomised scale. A random sample of the study population and cases with high score on the questionnaire burn out, perceived stress, and depression scale went through a diagnostic SCAN interview. Similar data were collected in 2009.

We calculated odds ratios (OR) with $95 \%$ CIs for the $20 \%$ highest psychosocial exposures adjusted for a) individual factors (age, gender, education) and b) adding neuroticism and the interaction product to the model.

Results: The prevalence of poor overall sleep quality was $25 \%$ $(\mathrm{n}=1,122)$. Neuroticism OR $=4.0(2.8-5.7)$, high job demands $\mathrm{OR}=2.4(1.9-3.0)$, high ERI OR $=5.2(4.1-6.2)$, low procedural justice $\mathrm{OR}=2.0(1.5-2.7)$ and relational justice $\mathrm{OR}=2.0(1.6-2.6)$ showed increased odds ratios of poor sleep at baseline. For effortreward imbalances an exposure-effect pattern were seen. After adjustment for neuroticism and the interaction product, the ORs for poor sleep did not decline.

Conclusions: we found statistically significant associations between psychosocial work factors and insomnia in civil servants at baseline which were not modified when adjusted for personality traits.

\section{P-289}

Early sexual intercourse: the importance of family, school and their surroundings

Topic: Exposure - Lifestyle factors
Presenter: Maryane Oliveira-Campos

Maryane Oliveira-Campos 1); Luana Giatti 2); Deborah Malta 1); Sandhi Barreto 3)

1) University of Minas Gerais-UFMG, Brazil; 2) University of Ouro Preto-UFOP, Brazil; 3) General Management of the National Subsystem for Surveillance of Noncommunicable Diseases and Injuries- Ministry of Health, Brazil

Background: Early sexual intercourse is associated with an increased risk of multiple sexual partners, inconsistent use of contraceptives, sexually transmitted diseases and unplanned pregnancy. The present study investigated the influence of contextual factors related to family and school and their surroundings on sexual initiation among adolescents aged 14 years old, who study in public and private Brazilian schools. Methods: Participated in this study 27.243 adolescents, with the age of 14 years and included in the National Survey of School Health (PeNSE), carried out in 2009. After determining the incidence of sexual intercourse (yes/no), we investigated its association with sociodemographic characteristics, the use of psychoactive substances, involvement in fights and factors from the family and school contexts by means of logistical multiple regression.

Results: Approximately one-fourth of the students reported one or more sexual relationships. The chance of having had sexual intercourse was lower among private school students and adolescents who reported having received information about the prevention of pregnancy and/or STDs at school. In the family context, living with only one or none of the parents, low parental supervision and having suffered family abuse were associated with higher chances of early sexual intercourse. There is an upward dose-response in the associations between the number of absences from school without parental permission, and absences due to lack of safety at school and/or on the way from home to school, and the chance of early sexual intercourse. Conclusion: Sexual education, promoting greater family involvement in the student's activities and preventing violence at school and in its surroundings seem to contribute to prevent early sexual initiation among adolescents.

\section{P-290}

Contextual factors associated with sexual behavior among Brazilian adolescents

Topic: Exposure - Lifestyle factors Presenter: Maryane Oliveira-Campos

\section{Maryane Oliveira-Campos 1); Luana Giatti 2);} Deborah Malta 1); sandhi Barreto 3)

1) University of Minas Gerais-UFMG, Brazil; 2) University of Ouro Preto-UFOP, Brazil; 3) General Management of the National Subsystem for Surveillance of Noncommunicable Diseases and Injuries, Ministry of Health, Brazil.

Background: There are few studies about the influence of the context on sexual behavior among adolescents in developing countries, such as Brazil. The object of this study is to investigate if factors from family and school contexts are associated with sexual behavior among Brazilian adolescents.

Method: This study used data from 60.973 adolescents. The response variable was sexual behavior, described in three categories (never had sexual intercourse, had protected sexual intercourse, had unprotected sexual intercourse). The variables associated with having protected and unprotected sexual relations in each context were identified by means of multinomial logistic regression.

Results: approximately one-fourth of the adolescents have already had sexual intercourse. Family and school context factors are 
associated with sexual behavior. These associations are generally stronger for unprotected sex.

Conclusion: Information about the prevention of pregnancy and STDs/AIDS has to be disseminated very early due to the young age of sexual initiation.

\section{P-291}

Association between the risk of metabolic syndrome and TV viewing among a sample of 11-17 years old girls

Topic: Exposure - Lifestyle factors

Presenter: Aristides Machado-Rodrigues

Aristides Machado-Rodrigues 1); Manuel Coelho-e-Silva 1); Cristina Padez 1); Luis Mascarenhas 2); Margaret Boguszewski 2); Neiva Leite 2)

1) University of Coimbra, Portugal; 2) Federal University of Paraná, Brazil

Background: The prevalence of metabolic syndrome (MetS) has increased over the last few decades in children and adolescents and has become an important health challenge worldwide. Contemporary lifestyles have been blamed for the epidemic of diseases of Western civilization", since young people are seen as particularly vulnerable to the influence of electronic media.

Objectives: The present study examined the relationships between TV viewing and the MetS in a sample of female children and adolescents. Methods: A cross-sectional school-based study was conducted in 522 female youth aged 11-17 years. Height, weight, waist circumference, fasting glucose, HDL-cholesterol, triglycerides, and blood pressure were measured. A sum of Metabolic Risk Factors (MRF) was computed, and participants were classified into two groups: less than three MRF, and three or more MRF, using age- and sex-specific values for some of the criteria. TV viewing and moderate-to-vigorous physical activity (MVPA) were assessed by a three-day diary and cardiorespiratory fitness (CRF) was assessed using the 20-m shuttle run test. Logistic regression models were used.

Results: Prevalence of MetS was significantly associated with TV viewing in female adolescents, and the association was not altered with adjustment for the potential confounding factors included in the regression model (i.e., age, body fat, BMI, CRF, MVPA, and parental education). Results showed that female children and adolescents with MetS were $67 \%$ more likely to be classified as sedentary (with more than $2 \mathrm{~h} /$ day of TV viewing) than adolescents classified with less than three MRF. Inspection of the final regression model also indicated that youth classified as having three or more MRF were $132 \%$ more likely to be classified as inactive ( $<60 \mathrm{~min} /$ day of MVPA) compared to their counterparts classified with less than three MRF (odds ratio $2.32,95 \%$ CI 1.02-5.26, $p<0.05$ ). Furthermore, adolescents with metabolic syndrome were more likely to have higher waist circumference than adolescents with less than three MRF (odds ratio 1.05, $95 \%$ CI 1.02 to $1.09, p<0.05$ ).

Conclusion: TV viewing was independently associated with an increased prevalence risk of MetS in children and adolescents. Our findings emphasize the importance of decreasing of TV viewing and promoting regular PA to reduce the public health burden of chronic diseases associated with a sedentary lifestyle.

\section{P-292}

Association between single-person households and metabolic disease in Japan: report from the comprehensive survey of living conditions

Topic: Exposure - Lifestyle factors
Presenter: Yoshitaka Murakami

\section{Yoshitaka Murakami 1)}

1) Department of Medical Statistics, Shiga University of Medical Science, Otsu, Shiga, Japan

Background: Factors associated with the prevalence of metabolic disease, such as sex and single-person households, have not been sufficiently investigated in Asian populations.

Objective: We examined the association between single-person households and persons with metabolic disease in a Japanese population using random sample data from the Comprehensive Survey of Living Conditions survey in 2003.

Methods: The study included 11,928 participants aged 20 years and above living in the community, and excluded inpatients and nursing home residents. The household status was categorized into two groups (singe-person household or not). Metabolic disease was defined as diabetes, obesity, hyperlipidemia, and thyroid diseases. Men and women were analyzed separately. Logistic regression models were used to estimate the odds ratios (ORs) for metabolic disease after adjusting for age, smoking, impaired activities of daily living, and employment status.

Results: There were 443 males and 529 females with metabolic disease living in the community, of whom 35 men and 67 women lived in single-person households. In both men and women, the ORs for metabolic disease increased with age, and the increase was more apparent in subjects aged 65 years and old and older. In men, the ORs for metabolic disease in single-person households were higher than in households with two or more persons across all age groups [20-49 years old: single-person household, 1.42 (95\% confidence intervals: $0.60-3.35)$ versus two or more persons household, 1.00 (reference group); 50-64 years old: 5.69 (2.98-10.85) versus 4.31 (3.16-5.88); $\geq 65$ years: 6.69 (3.57-12.53) versus $5.51(2.98-10.85)]$. However, there was no significant association between single-person households and metabolic disease in women.

Conclusions: The data from the national survey suggest that Japanese men of all age groups with metabolic disease are more likely to live in single-person households. We conclude that outpatient management of metabolic disease should focus particularly on men living alone, especially those aged 65 years and older. Our findings provide useful evidence that indicates the need for targeted management of metabolic disease in the community.

\section{P-294}

Maternal pre-pregnancy obesity and pubertal development among sons

Topic: Exposure - Lifestyle factors

Presenter: Cecilia Høst Ramlau-Hansen

Marie Louise Hounsgaard 1); Linn Berger Håkonsen 1); Anne Vested 1); Ane Marie Thulstrup 1); Jørn Olsen 2); Jens Peter Bonde 3); Ellen A Nohr 2);

Cecilia Høst Ramlau-Hansen 2)

1) Department of Occupational Medicine, Aarhus University Hospital, 8000 Aarhus C, Denmark; 2) Department of Public Health, Section for Epidemiology, Aarhus University, 8000 Aarhus C, Denmark; 3) Department of Occupational and Envirnomental Medicine, Bispebjerg Hispital of Copenhagen University, 2400 Copenhagen NVm Denmark

Background: Maternal overweight in pregnancy has been associated with earlier age of mearche in daughters as well as reduced semen quality in sons. Whether maternal overweight in pregnancy is associagted with timing of sexual maturation in sons has not been investigated previously. 
Objectives: To compare timing of pubertal development among sons born of mothers who were obese during pregnancy with sons of normal weight mothers.

Methods: Participants $(\mathrm{N}=2,522)$ were sons of mothers that during pregnancy in 1984-87 were enrolled in a mother-child cohort and gave information on their pre-pregnancy height and weight from which we calculated their body mass index (BMI). Information om sons' pubertal development was obtained from web-based questionnaires in 2005. Outcome variables are age at the following markers of pubertal development: Regular shaving, voice break, acne and first nocturnal emission.

Results: Multiple linear regression analyses showed that sons of obese mothers on average started to shave 8.3 (95\% confidence interval (CI) 2.5-14.0) months earlier than sons of normal weight mothers. For the three other indicators of pubertal development, results also indicated erlier onset for sons of obese mothers, but none reached statistical significance in the mail analyses. After exclusing sons of underweight mothers, we observed a statistically significant inverse trend between maternal pre-pregnancy BMI and age at regular shaving, acne and first nocturnal emission.

Conclusion: Maternal pre-pregnancy obesity may be related to earlier onset of puberty among sons. More evidence, preferable based on information on pubertal development collected with shorter recall time, is needed to draw firm conclusions.

\section{P-295}

Physical inactivity in Chilean youth: suboptimal strategies for making health related choices

Topic: Exposure - Lifestyle factors

Presenter: Paulina Correa-Burrows

\section{Paulina Correa-Burrows 1); Raquel Burrows 2)}

1) Rey Juan Carlos University, Dept of applied economics II (Spain); 2) University of Chile, Inst. of nutrition and food technology (Chile)

Background: A recent economic approach suggests self-control problems, procrastination and the fact that people do not account for the long term implications of unhealthy behaviours, prevent individuals from performing a fully rational trade-off between current benefits and future costs. This usually leads to negative health outcomes, which have been referred to as the internal costs of individual health decision-making or internalities.

Aim: We examined whether the current allocation of time to physical activity (PA) among adolescents leads to outcomes that may affect their well being as youth and their life prospect. In doing so, we will be providing insights on the magnitude of internalities from physical inactivity.

Data and method: Random sample of 1,692 high-school students $(14.8 \pm 0.7$ years old $)$ from urban Santiago attending private and non-private schools. We measured PA habits accounting for: time spent in class, studying and screen-based entertainment; hours of daily recreational activity; hours of weekly scheduled exercise; blocks walked daily to get to and from school; and daily sleeping hours. Obesity and abdominal obesity were diagnosed according to $\mathrm{CDC} /$ NCHS 2000, NHANES III and IDF criteria, respectively. Bivariate and multivariate regression analyses were used to assess the relation between current PA habits, confounders, and the odds of obesity and abdominal obesity. Models considered three different exposures: active play, scheduled exercise and overall PA.

Results: $15.7 \%$ of adolescents showed high allocation of time of overall PA while $53.1 \%$ and $31.2 \%$ showed moderate and poor allocation. Odds of obesity were significantly higher in adolescents reporting the lowest allocation of time to PA (OR 2.0 CI 1.2-3.3), scheduled exercise (OR 2.1 CI 1.4-3.0) and active play (OR $1.6 \mathrm{CI}$ 1.1-2.34). Odds of abdominal obesity were significantly higher in individuals reporting the lowest allocation of time to PA (OR $4.4 \mathrm{CI}$ 2.8-7.1), scheduled exercise (OR 3.6 CI 2.6-4.9) and active play (OR 2.8 CI 2.1-3.9). Those reporting moderate allocation of time to PA, scheduled exercise and active play showed higher odds of abdominal obesity as defined by IDF.

Conclusions: In our sample, adolescents appear not to be performing optimal strategies for making health related choices. Reduced PA, obesity and central obesity are proximate causes of metabolic and cardiovascular disorders.

Discussion: This new framework to understand health-related choices has important policy implications. The appropriate role of public institutions may be beyond the external costs of unhealthy behaviours. They should also address their internal costs.

\section{P-296}

Economic stress in childhood and adulthood, and self-rated health: a population-based study concerning risk accumulation, critical period and social mobility

Topic: Exposure - Lifestyle factors

Presenter: Kristina Hansen

Martin Lindström 1); Kristina Hansen 1); Maria Rosvall 1)

1) Department of clinical sciences, Social medicine and health policy, Malmö University Hospital, Lund University.

Economic stress in childhood and adulthood, and self-rated health: a population-based study concerning risk accumulation, critical period and social mobility

Background: Research in recent decades increasingly indicates the importance of conditions in early life for health in adulthood. Only few studies have investigated socioeconomic conditions in both childhood and adulthood in relation to health testing the risk accumulation, critical period, and social mobility hypotheses within the same setting.

Objectives: This study investigates the associations between economic stress in childhood and adulthood, and self-rated health with reference to the accumulation, critical period and social mobility hypotheses in life course epidemiology, taking demographic, social support, trust and lifestyle factors into account.

Methods: The public health survey in Skåne (southern Sweden) in 2008 is a cross-sectional postal questionnaire study based on a random sample, in which 28,198 persons aged $18-80$ years participated (55\% participation). Logistic regression models were used to investigate associations between economic stress in childhood and adulthood, and self-rated health.

Results: Three life-course socioeconomic models concerning the association between economic stress and self-rated health (SRH) were investigated. The results showed a graded association between the combined effect of childhood and adulthood economic stress and poor SRH in accordance with the accumulation hypothesis. Furthermore, upward social mobility showed a protecting effect and downward mobility increased odds ratios of poor SRH in accordance with the social mobility hypothesis. High/severe economic stress exposures in both stages of life were independently associated with poor SRH in adulthood. Furthermore, stratifying the study population into six age groups showed similar odds ratios of poor SRH regarding economic stress exposure in childhood and adulthood in all age groups among both men and women.

Conclusions: The accumulation and social mobility hypotheses were confirmed. The critical period model was confirmed in the sense that both economic stress in childhood and adulthood had independent 
effects on poor SRH. However, it was not confirmed in the sense that a particular window in time (in childhood or adulthood) had a specifically high impact on self-rated health.

\section{P-297}

Nutritional status among adults in a post-conflict area, northern Uganda: are humanitarian assistance programmes creating disparities in health?

Topic: Exposure - Lifestyle factors

Presenter: Stine Schramm

Stine Schramm 1); Felix Ocaka Kaducu 2); Ceaser Law 2); Emilio Ovuga 2); Morten Sodemann 1)

1) University of Southern Denmark, Denmark; 2) Gulu University, Uganda

Background: Two decades of armed conflict in northern Uganda forced 2 million people to move into internally displaced peoples' camps, where they were reliant on food rations from United Nations World Food Programme (WFP). Distributions of food aid may have long-term health implications, but are rarely studied. Population and health data is highly needed in the current post-conflict vacuum of northern Uganda, as well as for humanitarian food aid in future emergencies.

Objectives: To determine the prevalence of malnutrition and associated risk factors including exposure to WFP rations and displacement into camps among former internally displaced adults in northern Uganda.

Methods: A population-based cross sectional study was performed from September 2011 to August 2012. All residents in Gulu Health and Demographic Surveillance System (HDSS) aged 15 years and above were considered eligible. Trained field assistants collected anthropometric measurement (weight and height) and administered questionnaires with information on socio-demographic characteristics, food security, smoking, alcohol, household exposure to receiving food rations from WFP, and displacement to camps. Nutritional status was classified by body mass index (BMI).

Results: 1,486 men and 2,095 women participated in the study (participation rate of 50 and $67 \%$ respectively). The prevalence of underweight was $25 \%$ for men and $18 \%$ for women, whereas, the prevalence for overweight was 1 and $7 \%$ respectively. For men, underweight was associated with younger (15-17 years) and older age $(>55$ years $)(P<0.001)$, being divorced/separated $(P=0.018)$ and daily smoking $(P<0.001)$. There was no association with years receiving WFP rations $(P=0.213)$ or displacement $(P=0.137)$. For women, underweight was associated with the rainy season $(P=$ 0.023 ) and having received WFP rations $<5$ years (OR 2.23, $95 \% \mathrm{CI}$ $1.20 ; 4.17)$. No effect was observed for having received WFP rations $>5$ years. Displacement to a camp more than once was associated with a protective effect on underweight $(P=0.004)$.

Conclusion: The findings from the present study suggest that malnutrition differed markedly between genders and age groups. The high prevalence of underweight among men, especially young and elderly, does not match with the focus from humanitarian assistance programmes on children and women. Targeted food aid and humanitarian assistance, with global standards not adjusted to local context, may create unintended disparities in health. Needs assessments prior to humanitarian assistance and continuous monitoring of all population groups could help mitigate such disparities and improve health in post-conflict areas.

\section{P-298}

Maternal cigarette smoking during pregnancy and pubertal development in sons: a follow-up study of a birth cohort

Topic: Exposure - Lifestyle factors

Presenter: Linn Berger Håkonsen

Linn Berger Håkonsen 1); Jørn Olsen 2); Henrik Støvring 3); Andreas Ernst 1); Ane Marie Thulstrup 1); Jin Liang Zhu 2); Anshu Shrestha 4); Cecilia Høst Ramlau-Hansen 2)

1) Danish Ramazzini Center, Department of Occupational Medicine, Aarhus University Hospital, Denmark; 2) Department of Public Health, Section of Epidemiology, University of Aarhus, Denmark; 3) Department of Public Health, Section of Biostatistics, University of Aarhus, Denmark; 4) Department of Epidemiology, School of Public Health, UCLA, Los Angeles, USA; 6)

Background: Epidemiological studies have raised concern about the reproductive consequences of prenatal cigarette smoke exposure, possibly affecting semen quality and pubertal development of the offspring.

Objectives: To further investigate pubertal development in young men exposed to cigarette smoking in foetal life.

Methods: In the Danish Healthy Habits for Two" cohort, information on maternal smoking during pregnancy was available from questionnaires administered in 1984-1987, and information on pubertal development, assessed by age at first nocturnal emission, acne, voice break and regular shaving, was obtained from a follow-up questionnaire administered in 2005 to the young men (age: 18-21). We performed multiple imputations and multiple regression analyses adjusted for several potential confounders.

Results: The study population constituted 2,522 men. The crude mean $(95 \% \mathrm{CI})$ age at the four indicators of pubertal development among all participants were: acne: $14.6(14.5 ; 14.7)$ years, voice break: $14.5(14.5 ; 14.6)$ years, regular shaving: $17.2(17.2 ; 17.3)$ years and first nocturnal emission: $14.8(14.7 ; 14.9)$ years. Higher prenatal tobacco exposure was associated with a statistically non-significant tendency toward earlier age of first nocturnal emission, acne and voice break, indicating an accelerated age of pubertal development. Men exposed to $\geq 15$ cigarettes/day had 3.1 months (95\% CI -6.4 ; $0.2)$ earlier age at acne and, 2.2 months earlier $(95 \% \mathrm{CI}-7.3 ; 3.0)$ age at first nocturnal emission, 1.2 months earlier $(95 \% \mathrm{CI}-4.6 ; 2.2)$ age at voice break, however, 1.3 months later (95\% CI -1.6; 4.3) age at regular shaving, compared to unexposed young men.

Conclusions: Prenatal cigarette smoking exposure may induce an earlier pubertal development in young men, but larger studies with prospectively collected puberty data are needed to explore this hypothesis further.

\section{P-299}

Dietary intake distribution over a week among preschool children in a rural area of Japan

Topic: Exposure - Lifestyle factors

Presenter: Yuki Sato

Yuki Sato 1); Motoyuki Yuasa 2); Hirohide Yokokawa 2); Reiko Suzuki 3)

1) Center for Environmental Health Sciences, National Institute for Environmental Studies, Japan; 2) Juntendo University School of Medicine; 3) Tokyo Healthcare University 
Background: The influence of dietary factors during childhood is a global concern because they affect health outcomes in early life and have long-lasting physical effects in later life. Several dietary assessment methods have been developed to address this issue in adult; however, appropriate methods to assess Japanese children are scarce.

Objectives: To collect baseline data on dietary intake for preschool children on weekdays and weekend days, and identify variations and the number of days for which dietary recording is needed to assess usual food intake and nutritional status.

Methods: We used 7-day weighted dietary records and questionnaire surveys to collect background information on children from the Shinhidaka area, Hokkaido Prefecture. The study period comprised 5 consecutive weekdays and 2 weekend days. Mothers and/or guardians (the person who prepared the child's diet) of children aged 2-6 years who attended private preschools and the local public health centre were enrolled in this study. The inclusion criteria were: one child per one family. The dietary data were software processed and coded according to the Standard Tables of Food Composition in Japan (2010 version). We calculated and compared the dietary intake distribution between weekdays and weekend days and its inter- and intraindividual differences for 45 nutrient items. Data collected at the end of June 2012 were used in this study. All analyses were performed using the IBM SPSS Statistics version 19.0.

Results: This study included 36 volunteers ( 21 boys and 15 girls). Participant characteristics were as follows: $40 \%$ of the children attended half-day preschool, $33 \%$ attended full-day preschool, and $11 \%$ had food allergies and restrictions. The mean \pm standard deviation of total energy intake on weekend days was $945 \pm 309 \mathrm{kcal}$ and on weekdays was $996 \pm 281 \mathrm{kcal} ; p$ value difference by $t$ test between weekday and weekend day dietary intake was 0.54 . The ratio of intra- to interindividual variance was 1.43 for dietary intake on weekend days and 0.42 on weekdays. Correspondingly, protein intake was $30 \pm 12 \mathrm{~g}$ on weekend days and $31 \pm 13 \mathrm{mg}$ on weekdays, with a $p$ value of 0.7 and variance of 0.22 for weekend days and 0.29 for weekdays.

Conclusions: We did not observe any substantial difference between nutrient intake on weekend days and weekdays in this study subjects. The intraindividual variance was highest for weekend dietary intake.

\section{P-300}

Folic acid or multivitamin supplement use and menstrual cycle patterns: an internet-based cross-sectional study

Topic: Exposure - Lifestyle factors

Presenter: Heidi Cueto

Heidi Cueto 1); Anders Riis 1); Elizabeth Hatch 2);

Lauren Wise 2), 4); Kenneth Rothman 1), 2), 3);

Henrik Sørensen 1); Ellen Mikkelsen 1)

1) Department of Clinical Epidemiology, Aarhus University Hospital;

2) Department of Epidemiology, Boston University School of Public;

3) RTI Health Solutions, Research Triangle Park, North Carolina; 4)

Slone Epidemiology Center, Boston University

Background: Folate status may play an essential role in hormonal balance and follicular development, enhancing menstrual cycle function, but only few studies have examined the association between folic acid (FA) or multivitamin (MV) supplement use and menstrual patterns.

Objectives: We examined the association between preconceptional FA or MV supplement use and menstrual cycle regularity, cycle length, and duration and intensity of menstrual flow.
Methods: We examined the association between FA or MV supplement use and menstrual cycle characteristics in a cross-sectional study of 5,386 women aged 18-40 years, who were enrolled in an Internetbased study of Danish women attempting pregnancy during 2007-2011. For the analysis of cycle length and duration and intensity of menstrual flow, we restricted the analysis to women with regular cycles $(n=4,041)$. We used polytomous logistic regression to estimate odds ratios (ORs) and $95 \%$ confidence intervals (CIs) for the associations of FA or MV use with menstrual cycle regularity, short ( $<27$ days) and long ( $\geq 30$ days) cycle length, and duration and intensity of menstrual flow. The multivariate analyses were adjusted for age, parity, previous spontaneous abortions, BMI, pack-years of ever smoking, alcohol and caffeine intake, physical activity, last method of contraception, and all other menstrual cycle characteristics. Results: Relative to non-use, use of FA or MV supplements was associated with reduced odds of both short cycle length [adjusted OR $0.75,95 \%$ CI $0.62-0.90$ ] and long cycle length [adjusted OR 0.91, $95 \%$ CI 0.79-1.06] compared with cycle length of 27-29 days. The associations were stronger among 18-30 year-old women [adjusted OR $0.65,95 \%$ CI $0.51-0.83$ for short cycle length and adjusted OR $0.80,95 \%$ CI $0.67-0.95$ for long cycle length]. We found no association between FA or MV use and cycle regularity or duration and intensity of menstrual flow.

Conclusion: Preconceptional FA or MV supplement use was inversely associated with both short and long menstrual cycle length.

\section{P-301}

Maternal smoking during pregnancy and reproductive health of daughters: a follow-up study spanning two decades

Topic: Exposure - Lifestyle factors

Presenter: Andreas Ernst

Andreas Ernst 1); Susanne Lund Kristensen 1); Gunnar Toft 1); Ane Marie Thulstrup 1); Linn Berger Håkonsen 2); Sjurdur Frodi Olsen 3); Cecilia Høst Ramlau-Hansen 2)

1) Department of Occupational Medicine, Aarhus University Hospital; 2) Department of Public Health, Section of Epidemiology, University of Aarhus; 3) Department of Epidemiology Research, The Centre for Fetal Programming, Statens Serum Institute

Background: Studies observe potential effects of in utero exposure to constituents of cigarette smoke on the intrauterine formation of female gonads, but the consequences on long-term reproductive health in daughters remain unclear.

Objectives: The aim of this study was to investigate the associations between in utero exposure to constituents of cigarette smoke and age of menarche as well as other markers of reproductive health in young women.

Material and methods: Around 30th pregnancy week, 965 women from a Danish pregnancy cohort established in 1988-1989 provided information on lifestyle factors during pregnancy, including smoking habits. In 2008, 438 daughters were invited to complete a web-based questionnaire on reproductive health and attend a clinical examination. A total of $367(85 \%)$ answered the questionnaire of whom 267 $(61 \%)$ agreed on further examination. At examination, blood samples were drawn to analyse for level of reproductive hormones, interviews on menstrual pattern were carried out and number of follicles between 2 and $9 \mathrm{~mm}$ were determined by transvaginal ultrasound. The daughters were divided into three exposure groups according to the level of maternal smoking during first trimester (non, $>0-9$ cigarettes/ day or $\geq 10$ cigarettes/day). Data were analyzed by multiple regression analyses in which we adjusted for potential confounders. 
Results: We found an inverse association between prenatal exposure to cigarette smoke and age of menarche $(p=0.001)$. Daughters exposed to $\geq 10$ cigarettes/day debuted with $-4.1(95 \% \mathrm{CI}-6.6 ;-1.5)$ percentage earlier age of menarche, corresponding to 6.5 (95\% CI -10.7 to -2.2 ) months. There was a tendency towards lower levels of testosterone and dehydroepiandrosterone sulfate (DHEAS) with increasing in utero exposure to constituents of cigarette smoke, but the results were not statistically significant. Maternal cigarette smoking during pregnancy was not associated with follicle number, cycle length, luteinizing hormone $(\mathrm{LH})$, follicle stimulating hormone (FSH), estradiol, sex hormone-binding globulin (SHBG), anti-müllerian hormone $(\mathrm{AMH})$, free testosterone and free estradiol.

Conclusion: In utero exposure to maternal cigarette smoke may lead to earlier age of menarche and altered testosterone profile in young women.

\section{P-302}

Carbohydrate-rich foods and risk of cardiovascular disease in the Malmö Diet and Cancer cohort

Topic: Exposure - Lifestyle factors

Presenter: Emily Sonestedt

\section{Emily Sonestedt 1); Sophie Hellstrand 1);} Marju Orho-Melander 1)

1) Lund university, Sweden

Background: It is unclear whether carbohydrate intakes are associated with risk of cardiovascular disease (CVD). Evidence suggests that carbohydrate quality is more important than carbohydrate quantity.

Objectives: The objective of this project was to examine the association between intake of different carbohydrate-rich foods and CVD risk.

Methods: In the Malmö Diet and Cancer cohort, 26,445 individuals (44-74 years; $62 \%$ females) had no history of myocardial infarction, stroke and diabetes at baseline. Blood pressure, fasting blood glucose, plasma insulin, and serum lipids were measured in 4,535 individuals. During a mean follow-up time of 14 year, 2,921 CVD cases (coronary and stroke events) were identified through registers. Dietary data was collected using a modified diet history method including a 7-day registration of lunch and dinner meals.

Results: After adjusting for several lifestyle factors, total intake of carbohydrates was not associated with CVD risk $(P$ trend $=0.60)$. The highest intake quintile of whole grains was associated with $13 \%$ (95\% CI 3-23\%; $P$ trend: 0.002) lower risk of CVD compared to the lowest quintile. None of the other foods (vegetables, fruit and berries, juice, refined grains, potatoes, sugar-sweetened beverages, sugar and sweets, and cookies and cakes) showed a significant association with CVD risk. Only foods rich in added sugar (sugar and sweets, and sugar-sweetened beverages) showed a significant cross-sectional association at baseline with adverse levels of triglycerides and HDLC. Moreover, while intakes of sugar and sweets, and sugar-sweetened beverages were associated with higher insulin resistance (measured by homeostatic model assessment), vegetables, fruit and berries and cookies and cakes were associated with lower insulin resistance.

Conclusion: In this prospective cohort study examining food sources of carbohydrates, individuals with high intake of whole grains had a decreased risk of CVD. None of the other foods showed a significant association with CVD risk.

\section{P-303}

Individual and contextual characteristics as determinants of sagittal standing posture: a population-based study of adults

Topic: Exposure - Lifestyle factors
Presenter: Fábio Miguel Azevedo Araújo

Fábio Miguel Azevedo Araújo 1), 2); Raquel Lucas 1), 2); Nuno Alegrete 3), 4); Ana Azevedo 1), 2); Henrique Barros 1), 2)

1) Department of Clinical Epidemiology, Predictive Medicine and Public Health, University of Porto Medical School, Porto, Portugal; 2) Institute of Public Health - University of Porto (ISPUP), Porto, Portugal; 3) Centro Hospitalar São João, Porto, Portugal; 4) Department of Surgery, University of Porto Medical School, Porto, Portugal

Background: Sagittal standing posture was shown to be associated with musculoskeletal symptoms and quality of life in patients of heterogeneous clinical settings. However, the frequency and determinants of suboptimal sagittal alignment outside the clinical context remain to be clarified.

Objectives: To estimate the association of sociodemographic, anthropometric and behavioral characteristics with sagittal standing posture among adults from the general population.

Methods: As part of the EPIPorto study, 489 adults were assessed during 2005-2008. Individual spino-pelvic parameters were recorded from 36-inch sagittal radiographs obtained in free-standing posture. Additionally, participants were classified into 1 of 4 types of sagittal postural patterns as proposed by Roussouly (types 1, 2 and 4 corresponding to non-neutral postures and type 3 to a neutral one). Data regarding age, sex, education, occupation, body mass index (BMI), waist circumference, total physical activity, leisure-time physical activity, time spent in sitting position, smoking status and tobacco cumulative exposure were collected. Individual parameters and patterns of sagittal posture were compared across categories of participants' characteristics.

Results: Older age, lower formal education, blue collar occupation, and overall and central obesity were associated with increased sagittal vertical axis and pelvic-tilt/pelvic-incidence ratio. Taking the neutral postural pattern (type 3 ) as reference for the outcome in a multinomial regression model, independently of age, sex, education, total physical activity and smoking status, overweight adults had higher odds of type 2 (odds ratio [OR] 1.92) and type 4 (OR 2.13) postural patterns, in comparison to normal weight subjects. Overall and central obesity were positively related with type 1 postural pattern (OR 6.10 and OR 3.54 , respectively). There was also a weak direct association between female sex and a type 1 postural pattern. Regarding behavioral factors, subjects with total physical activity above the first tertile exhibited all non-neutral postural patterns less frequently, and current smokers were more likely to present a type 4 postural pattern.

Conclusions: Higher BMI and central obesity were important potential determinants of non-neutral posture among adults from the general population. Strategies focused on reducing adiposity are expected to prevent all non-neutral pathologic standing postures at a population level.

\section{P-305}

Self-reported sleep patterning in the EPIC-Norfolk prospective population study: a closer look at sleep quantitative measures

Topic: Exposure - Lifestyle factors

Presenter: Yue Leng

Yue Leng 1); Nick Wainwright 1); Francesco Cappuccio 2);

Paul Surtees 1); Robert Luben 1); Nick Wareham 3); Carol Brayne 1); Kay-Tee Khaw 1)

1) Department of Public Health and Primary Care, University of Cambridge, Cambridge, UK; 2) Division of Mental Health \& Wellbeing, Warwick Medical School, University of Warwick, UK; 3) 
Medical Research Council Epidemiology Unit, Institute of Metabolic Science, Cambridge, UK

Background: Sleep patterns have been linked to various health outcomes in epidemiological studies, but sleep patterns in the British population have not been extensively reported.

Objectives: We aimed to describe the sleep characteristics reported by the EPIC-Norfolk participants, with a particular emphasis on the comparison of different sleep quantitative measures.

Methods: During 2006-2007, a total of 8,480 participants aged 45-90 years reported sleep timing, night-time sleep duration and sleep difficulties. Time spent in bed was calculated from the difference between rise time and bedtime, and sleep efficiency was defined as the ratio of sleep duration and time spent in bed.

Results: On average, the reported time in bed was more than $1.5 \mathrm{~h}$ longer than sleep durations. Compared to men, women spent $15 \mathrm{~min}$ longer in bed, but slept for 11 min less and reported more sleep difficulties. In multivariable analysis sleep duration and time in bed varied with socioeconomic factors, but sleep efficiency was consistently lower among women, older people, those who were widowed, separated or divorced, those who were not working, and those who had lower education, poor general health or depression and among those who had reported sleep difficulties.

Conclusions: Self reported sleep duration and time spent in bed have different meanings and implications for health. Sleep efficiency may be a useful indicator of sleep patterns in the general population.

\section{P-306}

Are low birth weight babies more often smokers as adults? Results from a generational prospective, population based cohort study

Topic: Exposure - Lifestyle factors

Presenter: Liv Grimstvedt Kvalvik

Liv Grimstvedt Kvalvik 1); Rolv Skjærven 1), 2); Kari Klungsøyr 1), 2); Stein Emi Vollset 1), 2); Kjell Haug 1)

1) Department of Global Public Helath and Primary Care, University of Bergen; 2) Medical Brith Registry of Norway, National institute of Public Health, Bergen

Background: Low birth weight has been postulated to lead to, through early programming, cardiovascular disease in adult life. Numerous of studies have focused on the association between factors in early life and later disease. This relation may however, also be explained by common genetic factors related to both low birth weight and adult cardiovascular disease or common environmental factors related to both low birth weight and cardiovascular disease, such as socioeconomic factors and smoking; or both of these in addition to or instead of early programming. Smoking is well known to increase the risk of both low birth weight and cardiovascular disease. We wanted to explore the possibility of common environmental factors as important in the relation between infant low birth weight and adult cardiovascular disease by studying the relation between infant low birth weight and adult smoking habits. We suggest that low birth weight also is a marker for maternal smoking during pregnancy, and that these smoking habits will be passed on to next generation.

Objectives: To study whether women born with low birth weight more often smoke as adults compared to women born with normal birth weight.

Methods: We used data from the Medical Birth Registry of Norway (MBRN), which has national coverage of all births since 1967. We studied birth weight of term, singleton women $(n=220781)$ in 1967-1995, and linked these to their own infants delivered in 1999-2009, where maternal smoking habits during pregnancy were registered. Z-score of birth weight by gestational age in the first generation was exposure and maternal smoking habits of the adult women or the partners of the adult men were outcomes.

Results: $20.3 \%$ of mothers with Z-score -3 were daily smokers, compared to $11.7 \%$ of women with Z-score 1 (RR 1.74 (1.58-1.91)). The relation between maternal birth weight and later smoking habits persisted when adjusting for grandmothers' education (mothers of the first generation infants).

Conclusions: Our findings indicate that being born low birth weight is associated with smoking in adulthood.

\section{P-307}

A community based study on determinants of circulating markers of cellular immune activation and kynurenines: The Hordaland Health Study

Topic: Exposure - Lifestyle factors

Presenter: Despoina Theofylaktopoulou

Despoina Theofylaktopoulou 1), 2); Øivind Midttun 3); Arve Ulvik 3); Per Magne Ueland 1), 4); Grethe Tell 2); Stein Emil Vollset 2); Ottar Nygård 5), 6); Simone Eussen 127)

1) Section for Pharmacology, department of Clinical Science, University og Bergen; 2) Department of Global Public Health and Primary Care, University of Bergen; 3) Bevital AS, Bergen, Norway; 4) Laboratory of Clinical Biochemistry, Haukeland University Hospital, Bergen, Norway; 5) Section for Cardiology, at Department of Clinical Science, University of Bergen; 6) Department of Heart Disease, at Haukeland University Hospital, Bergen, Norway; 7) Department of Epidemiology, School for Public Health and Primary Care - CAPHRI, Maastricht University, Maastricht, The Netherlands

Background: Circulating neopterin and kynurenine/tryptophan ratio are markers of cellular immune activation. The increased degradation of tryptophan to kynurenine during inflammation, reflected by the kynurenine/tryptophan ratio, also leads to higher plasma concentrations of metabolites of the kynurenine pathway, collectively known as kynurenines. Several kynurenines have distinct immunoregulatory and/or neurocative properties, and may be associated with the development of chronic diseases. However, to date data on determinants of those markers and metabolites are sparse.

Objectives: To investigate the role of age, gender, renal function and lifestyle factors as determinants of neopterin, kynurenine/tryptophan ratio, and kynurenines.

Methods: We measured neopterin, tryptophan, and the kynurenines: kynurenine, anthranilic acid, kynurenic acid, 3-hydroxykynurenine, 3-hydroxyanthranilic acid and xanthurenic acid in plasma in two age groups, $45-46$ years $(n=3,723)$ and $70-72$ years $(n=3,329)$. Differences across categories of the potential determinants, including age, gender, renal function, body mass index (BMI), smoking and physical activity, were tested by Mann-Whitney $U$ test and multiple linear regression including age group, gender, renal function and lifestyle factors.

Results: In this multivariate model, neopterin, kynurenine/tryptophan ratio and most kynurenines were $20-30 \%$ higher in the older group, whereas tryptophan was $7 \%$ lower. Men had 6-19\% higher concentrations of tryptophan and most kynurenines than women of the same age. Compared to the fourth age-specific estimated glomerular filtration rate quartile, the first quartile was associated with higher concentrations of neopterin $(25 \%)$ and kynurenine/tryptophan ratio (24\%) and 18-36\% higher concentrations of kynurenines, except 3-hydroxyanthranilic acid. Additionally, kynurenine/tryptophan ratio, tryptophan and all kynurenines, except anthranilic acid, were 2-8\% higher in overweight and 3-17\% higher in obese, than in normal- 
weight individuals. Heavy smokers had 4-14\% lower levels of tryptophan and most kynurenines than non-smokers.

Conclusions: Age and renal function were the strongest determinants of plasma neopterin, kynurenine/tryptophan ratio and most kynurenines. These findings are relevant for the design and interpretation of studies investigating the role of plasma neopterin, kynurenine/tryptophan ratio and kynurenines in chronic diseases.

\section{P-308}

Trajectories of infant and childhood weight change and risk of non-alcoholic fatty liver disease in adolescence

Topic: Exposure - Lifestyle factors

Presenter: Emma L Anderson

Emma L Anderson 1), 2); Laura D Howe 1), 2); Abigail Fraser 1), 2); Mark Callaway 3); Naveed Sattar 4); Chris Day 5); Kate Tilling 2); Debbie A Lawlor 1), 2)

1) MRC CAiTE Centre, University of Bristol, Bristol, UK; 2) School of Social and Community Medicine, University of Bristol, Bristol, UK; 3) University Hospitals Bristol NHS Foundation Trust; 4) Institute of Cardiovascular \& Medical Sciences, BHF Glasgow Cardiovascular Research Centre, Faculty of Medicine, University of Glasgow, UK; 5) Institute of Cellular Medicine, Faculty of Medical Sciences, Newcastle University, UK

Background: Although adiposity is a key risk factor for NAFLD, few studies have examined prospective associations of change in adiposity throughout infancy and childhood with later risk of NAFLD.

Objectives: To examine associations of trajectories of weight-forheight (our measure of adiposity) from birth to age 120 months with liver outcomes in adolescence, and the extent to which associations are mediated through fat mass at the time of outcome assessment.

Methods: We used data from the Avon Longitudinal Study of Parents and Children. Individual trajectories of weight and height were estimated using random-effects linear-spline models. We explored associations of birthweight (adjusted for birth length) and weight change (adjusted for length/height change) from $0-3,3-12,12-36$, 36-84 and 84-120 months with ultrasound scan (USS) determined liver fat, stiffness and volume, and serum alanine aminotransferase (ALT), aspartate aminotransferase (AST), gamma-glutamyl transferase (GGT) and haptoglobin at mean age 17.8 year, using multivariable linear and logistic regression. Potential mediation of associations by fat mass at the time of outcome assessment was assessed with additional adjustment for total body fatness at mean age 17.8 year.

Results: Birth weight was positively associated with liver fibrosis and volume but not with USS liver fat; it was negatively associated with ALT and AST. Weight change from birth to age 12 months was not associated with any liver outcomes. Weight change from 12-36, 36-84 and 84-120 months was consistently positively associated with USS and blood-based liver outcomes (e.g. with adjustment for potential confounders, odds of USS liver fat per one standard deviation $[0.31 \mathrm{~kg} / \mathrm{month}]$ increase in weight change per month between ages 84 and 120 months is 1.89 [95\% CI 1.11-3.20]). Adjusting for total body fatness at the time of outcome assessment generally attenuated associations toward the null (e.g. the odds of USS liver fat per one standard deviation $[0.31 \mathrm{~kg} / \mathrm{month}]$ increase in weight change per month between ages 84 and 120 months after additional adjustment for total body fatness is 1.33 [95 \% CI 0.77-2.29]), suggesting that associations between growth and liver outcomes are largely mediated by concurrent body fatness.

Conclusions: Greater rates of weight-for-height change between ages 12 and 120 months are consistently associated with greater risk of having USS determined liver fat and more adverse levels of biomarkers of liver health in adolescence, and these associations are largely mediated through fatness at the time liver outcomes are assessed.

\section{P-309}

\section{2}

Topic: Exposure - Lifestyle factors Presenter: Roy M. Nilsen

Roy M. Nilsen 1); Pierpaolo Mastroiacovo 2); Nina Gunnes 3); Elin R. Alsaker 3); Anne Lise Bjørke-Monsen 4); Simone J. Eussen 5); Margaretha Haugen 3); Ane Johannessen 6); Helle Margrete Meltzer 3); Camilla Stoltenberg 3)

1) Department of Global Public Health and Primary Care, University of Bergen, Bergen, Norway; 2) Alessandra Lisi International Centre on Birth Defects and Prematurity, Rome, Italy; 3) Norwegian Institute of Public Health, Oslo, Norway; 4) Laboratory of Clinical Biochemistry, Haukeland University Hospital, Bergen, Norway; 5) Department of Epidemiology, School for Public Health and Primary Care - CAPHRI, Maastricht University, Maastricht, The Netherlands; 6) Centre for Clinical Research, Haukeland University Hospital, Bergen, Norway; 7) Section for Pharmacology, Institute of Medicine, University of Bergen, Bergen, Norway

Objective: Women who become pregnant shortly after a previous delivery may be at increased risk of low folate status. The present study aimed to investigate if maternal preconception folic acid supplement use was associated with the interval length between subsequent pregnancies.

Methods: The study was based on 44,940 pairs of pregnancies with the second pregnancy recorded in the Norwegian Mother and Child Cohort Study in 1999-2009. Inter-pregnancy interval was defined as the time between the birth of a child and the estimated conception of the subsequent child of the same mother. Preconception folic acid supplement use was defined as any use of folic acid supplements within the last 4 weeks before pregnancy.

Results: The overall prevalence of preconception folic acid supplement use was $32 \%$. Compared to women with inter-pregnancy intervals of 13-24 months, those with intervals $\leq 6$ months had a crude relative risk of 0.65 (95\% CI $0.58,0.73)$ of supplement use, whereas mothers with intervals $\geq 49$ months had a crude relative risk of 0.68 (95\% CI $0.66,0.71)$ of supplement use. Women with interpregnancy intervals $\leq 6$ months were also associated with a substantially lower proportion of planned pregnancies and a lower proportion of women with higher education. After adjustment for these variables, only marginal associations between short intervals and supplement use were seen.

Conclusions: Preconception folic acid supplement use varies with the interval length between subsequent pregnancies. The low intake in closely spaced pregnancy intervals is mainly attributable to a low proportion of planned pregnancies and fewer women with higher education.

\section{P-310}

Cost-effectiveness of lifestyle counselling in primary prevention of gestational diabetes

Topic: Exposure - Lifestyle factors

Presenter: Riitta Luoto 
Riitta Luoto 1); Päivi Kolu 1); Jani Raitanen 1), 2); Pekka Rissanen 2)

1) UKK Institute for Health Promotion; 2) Department of Health Sciences, University of Tampere

Background: Health-promoting interventions during pregnancy are effective in preventing newborns' perinatal complications and improving the quality of life of women with GDM. Only two earlier cost-effectiveness studies related to GDM have been published, although GDM is known to increase health care costs.

Objectives: The aim of this study was to evaluate the cost-effectiveness of primary prevention of gestational diabetes mellitus (GDM) through intensified counselling on physical activity, diet, and appropriate weight gain.

Methods: The cost-effectiveness analysis was based on data from a cluster-randomised controlled GDM prevention trial carried out in primary health-care maternity clinics in Finland. Women (=399) with at least one risk factor for GDM were included. The incremental costeffectiveness ratio (ICER) was calculated in terms of birth weight, 15D, and perceived health as measured with a visual analogue scale (VAS). A bootstrap technique for cluster-randomised samples was used to estimate uncertainty around a cost-effectiveness acceptability curve.

Results: The mean total cost in the intervention group was $€ 7,763$ (standard deviation (SD): $€ 4,511)$ and in the usual-care group was $€ 6,994$ (SD: $€ 4,326,=0.14$ ). The mean intervention cost was $€ 141$. The difference for costs in the birth-weight group was $€ 753$ (95\% CI 250 to 1,818$)$ and in effects for birth weight was $115 \mathrm{~g} \mathrm{(95 \%} \mathrm{CI} 15$ to 222). The ICER for birth weight was almost $€ 7$, with $86.7 \%$ of bootstrap pairs located in the north-east quadrant, indicating that the intervention was more effective and more expensive in birth weight terms than the usual care was. The data show an $86.7 \%$ probability that each gram of birth weight avoided requires an additional cost of $€ 7$.

Conclusions: Intervention was effective for birth weight but was not cost-effective for birth weight, 15D, or VAS when compared to the usual care.

TRIAL REGISTRATION: ISRCTN 33885819.

\section{P-311}

Genetic variation in FADS modifies the association between intake ratio of alpha-linolenic acid/linoleic acid and incidence of cardiovascular disease

Topic: Exposure - Lifestyle factors

Presenter: Sophie Hellstrand

Sophie Hellstrand 1); Ulrika Ericson 1); Bo Gullberg 2); Bo Hedblad 3); Marju Orho-Melander 1); Emily Sonestedt 1)

1) Diabetes and Cardiovascular Disease - genetic epidemiology;

2) Nutrition Epidemiology; 3) Cardiovascular Epidemiology

Background: The link between intake of polyunsaturated fatty acids (PUFA) and risk of cardiovascular disease (CVD) is unclear and might be clarified by taking into account the genetic variation between individuals. Minor alleles of single nucleotide polymorphisms in the $\Delta-5$ fatty acid desaturase 1 gene (FADS1) have been associated with lower blood concentrations of long-chain PUFA, indicating an associated loss of function effect.

Objectives: To examine if genetic variation in FADS1 modifies the association between intakes of 7 PUFA variables; alpha-linolenic acid (ALA), long-chain n-3 PUFA, total n-3 PUFA, linoleic acid (LA), total n-6 PUFA, ALA:LA ratio (ALA/LA), and n-3:n-6 PUFA ratio $(n-3 / n-6)$ and the risk of CVD.

Methods: We included 24,032 participants $(62 \%$ females, 44-74 years) from the Malmö Diet and Cancer cohort. During a mean follow-up time of 14 years, 2,648 CVD cases, defined by coronary event and ischemic stroke as the first event, were identified. Information about CVD was taken from the national Swedish Hospital Discharge register, Cause-of-death register and the local stroke register in Malmö (STROMA). All participants were genotyped for the FADS1 polymorphism rs 174546 (45 \% CC, $44 \%$ CT and $11 \%$ TT). Dietary intake was assessed by a modified diet history method combining a 168-item dietary questionnaire, a 7-day menu book (including cooked meals and cold beverages) and a 1-h diet history interview. The PUFA intakes were energy-adjusted and divided into quintiles. The risk of CVD was assessed by Cox proportional hazard regression, adjusted for potential confounders.

Results: We did not observe any statistically significant association between intake of any of the PUFA variables and incident CVD and neither was the rs174546 associated with CVD. However, a borderline significant interaction was observed between the intake ratio of ALA/LA and FADS genotype on CVD incidence $(P=0.06)$. ALA/ LA was inversely associated with CVD risk only among individuals homozygote for the minor T-allele of rs 174546 (HR for highest vs lowest quintile $=0.72,95 \%$ CI $0.50-1.04, P$ trend $=0.049$ ).

Conclusions: In this observational prospective study we found no clear effect modification by genetic variation in FADS on the association between PUFA intake and CVD risk. However, for the $11 \%$ of the population homozygous for the allele that associates with lower $\Delta$-5 FADS activity, a high ALA/LA may be preferable in prevention of CVD.

\section{P-312}

In-utero exposure to maternal smoking is not linked to smoking in adulthood after controlling for genetic and family influences

Topic: Exposure - Lifestyle factors

Presenter: Mina Rydell

Mina Rydell 1); Fredrik Granath 2); Sven Cnattingius 2); Cecilia Magnusson 1), 3); Maria Rosaria Galanti 1), 3)

1) Department of Public Health Sciences, Karolinska Institutet, Stockholm, Sweden; 2) Department of Medicine, Karolinska Institutet, Stockholm, Sweden; 3) Centre for Epidemiology and Community Medicine, Stockholm County Council, Stockholm, Sweden

Background: Prenatal exposure to maternal smoking during pregnancy has been linked with later tobacco use in previous epidemiological studies. However, intergenerational transmission of tobacco use may be influenced by both genetic and early-life environmental factors not taken into account by previous studies. The causal effect of maternal smoking during pregnancy can be better assessed by comparing exposure-discordant siblings, since this comparison of siblings adjusts for confounding from shared genetic and environmental factors.

Objectives: The objective was to investigate the association between maternal smoking during pregnancy and the risk of regular smoking or smokeless tobacco use in offspring, using a matched cohort study of exposure-discordant siblings.

Methods: A cohort of 1,538 exposure-discordant sibling pairs, 19-27 years old and living in Sweden 2010, was identified by using population-based Swedish registers. Lifetime and current history of tobacco use (including both smoking and use of snus) was selfreported in a questionnaire, and information about maternal smoking during pregnancy was retrieved from the Medical Birth Register. Odds ratios were calculated by conditional logistic regression and hazard ratios by stratified Cox proportional hazards regression, with precision estimated by $95 \%$ confidence intervals. 
Results: Analyses of exposure-discordant siblings did not reveal any statistically significant associations between prenatal exposure to maternal smoking and offspring's lifetime or current daily tobacco use, intensity of use, or progression from experimental use to regular use.

Conclusions: We found no evidence of an association between prenatal exposure to maternal smoking and tobacco use in adult exposure-discordant siblings. These findings suggest that the higher risks of tobacco use previously found in offspring of mothers who smoked during pregnancy were confounded by genetic or environmental factors.

\section{P-313}

A group-based modeling strategy to evaluate the association between body shape throughout life and mortality risk in the $\mathbf{E} 3 \mathrm{~N}$ cohort

Topic: Exposure - Lifestyle factors

Presenter: Guy Fagherazzi

\section{Aude Lasfargues 1); Françoise Clavel-Chapelon 1); Guy Fagherazzi 1)}

1) Inserm U1018 "Nutrition, Hormones and Women's Health"; unit, Villejuif, France

Background: To our knowledge, all existing studies have only evaluated adiposity at a specific time in life with the risk of mortality at adulthood but none has analyzed the association between patterns of changes of body shape throughout life in relation with all-cause and cause specific mortality risk at adulthood.

Objectives: To study the evolution of body size during life, in relation to all-cause and cause-specific mortality risk using a group-based modeling strategy.

Methods: The population was composed of 85,190 women from the E3 N cohort study. Lifetime body shape trajectories based on Sørensen's body shapes at different ages, considered as longitudinal data, were constructed using Nagin's approach to group-based trajectory modeling. This method is an application of finite mixture modeling that enables to define clusters of women with similar body shape evolution over age. Trajectories were constructed using the censored normal model (CNORM) of the SAS PROC TRAJ. As these variables were hypothesized to influence body shape trajectories, models were adjusted for birth weight and birth length. The optimal number of groups and trajectories were selected to best fit the data, as evaluated by change in the Bayesian Information Criterion (BIC) and the percentage of individuals attributed to each category. Estimation of body shape trajectory throughout life was performed using a cubic function of age for five trajectories and a quadratic function for one trajectory. Cox models were then used to estimate the risk of mortality associated with body shape throughout life.

Results: During 1,364,924 person-years of follow-up, 4,197 deaths occurred. Women who were thin at age 8 , and experienced a strong increase in body shape along the years, were at significant increased risk of all-cause mortality ( $\mathrm{RR}=1.59 \mathrm{CI} 95 \%$ [1.36-1.85]), compared to women with constantly thin body shape. This association was driven by cancer and cardiovascular disease mortality. A decreased risk of breast cancer mortality for women who experienced a large increase in body shape at puberty was found. No other trajectory was associated with mortality risk.

Conclusions: A large increase in body shape throughout life was associated with the highest mortality risk at adulthood. From a public health perspective, knowing the history of body shape evolution-not only the current level—would help clinicians to elaborate adequate recommendations targeted to women of various age.

\section{P-314}

Prescribing of nicotine replacement therapy in and around pregnancy in the UK: a population based study using primary care data

Topic: Exposure - Lifestyle factors

Presenter: Nafeesa Dhalwani, Laila Tata

Nafeesa Dhalwani 1), 2); Laila Tata 1); Tim Coleman 2); Lisa Szatkowski 1)

1) Division of Epidemiology and Public Health, University of Nottingham; 2) Division of Primary Care, University of Nottingham

Background: Smoking in pregnancy increases the risk of foetal and maternal problems, therefore reducing smoking in pregnancy is a public health priority. In 2005, licensing arrangements for nicotine replacement therapy (NRT) in the United Kingdom were broadened such that NRT could be prescribed to pregnant smokers. However, little is known about trends and uptake of NRT by pregnant women. Objectives: We aimed to quantify annual trends of NRT uptake in and around pregnancy and variation in prescribing by maternal characteristics.

Methods: In The Health Improvement Network primary care database we identified all pregnancies from 2000 to 2010 in women aged 15-49 years and used READ codes to determine women's smoking status. NRT prescriptions were identified in all women during three time periods: up to 9 months before conception, during pregnancy, and up to 9 months after delivery. Annual proportions of pregnancies where NRT was prescribed were calculated and variations by age and socio-economic deprivation were assessed using logistic regression.

Results: Women were classified as smokers in $19 \%$ of 227,552 pregnancies identified in 215,703 women. NRT was prescribed in 4,827 of all pregnancies which represented a prescribing prevalence of $9 \%$ of pregnancies in smokers and $2 \%$ of pregnancies overall. The rate of NRT prescribing was approximately $50 \%$ lower 9 months before and after pregnancy, compared to during pregnancy. There was a gradual increase in the annual rate of prescribing until 2005, after which the rate became stable. The rate of prescribing during pregnancy in smokers was higher in older age groups such that the rate in 30-35 years age group was $17 \%$ higher (95\% CI 1.05-1.31) and the rate in 15-20 year age group was $4 \%$ lower (95 \% CI $0.85-1.08$ ) than the rate of prescribing in the 25-30 year age group. The rate of prescribing during pregnancy in smokers also increased with deprivation such that it was $34 \%$ (95\% CI 1.16-1.54) higher in the most deprived group than in the least deprived group. Pregnant smokers with asthma and mental illness were $22 \%$ (95\% CI 1.11-1.34) and $47 \%$ (95\% CI 1.38-1.58) more likely to receive a prescription for NRT respectively as compared to pregnant smokers without these comorbidities.

Conclusion: Uptake of NRT is higher during pregnancy compared to time periods outside pregnancy and is higher in smokers from older and more socio-economically deprived groups.

\section{P-315}

Can primary care data be used to monitor the prevalence of current smoking during pregnancy?

Topic: Exposure - Lifestyle factors

Presenter: Nafeesa Dhalwani, Laila Tata

Nafeesa Dhalwani 1), 2); Laila Tata 1); Tim Coleman 2); Linda Fiaschi 1); Lisa Szatkowski 1) 
1) Division of Epidemiology and Public Health, University of Nottingham; 2) Division of Primary Care, University of Nottingham

Background: Collecting timely data on maternal smoking during pregnancy is important to monitor progress towards national prevalence goals and to evaluate whether current interventions are effective in reducing smoking during pregnancy.

Objectives: We aimed to assess the potential of primary care data to provide these estimates at a national level by comparing the estimates for maternal smoking in pregnancy from primary care data with those obtained from the other available data sources.

Methods: From the pregnancies recorded in The Health Improvement Network (THIN) between 2000 and 2009, resulting in live birth or stillbirth, we identified women who were current smokers at some point during their pregnancy using three different methodologies. Firstly, we used only gestational records to identify current smokers. Secondly, we used smoking records within 27 months before conception to identify smokers, where no gestational recording was present. Finally, we used smoking records from any time before delivery to identify whether they have ever been a smoker. Annual smoking prevalence in THIN was then calculated, based on these three methodologies and comparisons were made to nationally available maternal smoking data i.e. Child Health Systems Programme (CHSP), Scotland, Scottish Morbidity Record (SMR) and the Smoking at Time of Delivery (SATOD) data, using indirect standardisation where appropriate.

Preliminary results: Using data on 227,552 pregnancies in THIN between 2000 and 2009, we found that the annual estimates of current smoking in THIN, using only gestational smoking records were in good agreement with the SATOD estimates. When comparing to SMR and CHSP, THIN estimates for smoking were not comparable to these data sources. However, restricting the analysis to only Scottish THIN practices we found that THIN estimates were fairly similar to the CHSP and SMR and showed a converging pattern with the estimates from these data sources.

Conclusion: The prevalence estimates from only gestational recording in primary care are in good agreement with the other national data sources, especially after 2004

\section{P-316}

Sleep impairment and prognosis of Acute Myocardial Infarction: a prospective cohort study

Topic: Exposure - Lifestyle factors

Presenter: Alice Clark

Alice Clark 1), 2); Theis Lange 3); Johan Hallqvist 4), 5); Poul Jennum 6), 7); Naja Hulvej Rod 1), 2)

1) Section of Social Medicine, University of Copenhagen; 2) The Copenhagen Stress Research Center, Copenhagen, Denmark; 3) Section of Biostatistics, University of Copenhagen; 4) Division of Social Medicine, Karolinska Institute; 5) Department of Public Health and Caring Sciences, Uppsala University; 6) Danish Center for Sleep Medicine, Department of Clinical Neurophysiology, Glostrup Hospital; 7) Center for Healthy Aging, Faculty of Health Sciences, University of Copenhagen

Background: Impaired sleep is an established risk factor for development of cardiovascular disease, while less is known about how impaired sleep affects cardiovascular prognosis.

Objective: The aim of the current study is to determine how different aspects of impaired sleep affect the risk of case fatality and subsequent cardiovascular events in women and men with a first-time acute myocardial infarction (AMI).
Methods: The Stockholm Heart Epidemiology Program included 2,246 women and men with first-time AMI. Recall of sleep impairment was assessed by the Karolina Sleep Questionnaire, which covers various aspects of impaired sleep: disturbed sleep, impaired awakening, daytime sleepiness, and nightmares. Case fatality, defined as death within 28 days of initial AMI, and new cardiovascular events were identified through national registries within a 10-year follow-up. Information from a physical examination and a comprehensive questionnaire enabled thorough adjustment for confounders.

Results: In women, disturbed sleep showed consistent effects on long-term cardiovascular events, with a higher risk of all assessed cardiovascular events: AMI (hazard ratio $=1.69 ; 95 \%$ confidence interval $0.95-3.00)$, stroke ( $\mathrm{HR}=2.61 ; 95 \%$ CI 1.19-5.76), and heart failure $(\mathrm{HR}=2.43 ; 95 \%$ CI $1.18-4.97)$, while no clear effect of impaired sleep on case fatality was found in women. In men, a strong short-term effect on case fatality (odds ratio 3.27; $95 \% \mathrm{CI}$ 1.76-6.06) was observed in regards to the experience of impaired awakening. While the index of daytime sleepiness was not associated with the risk of case fatality, two of the composite items i.e. feeling sleepy during work/leisure time and involuntary sleep episodes during leisure time were associated with twice the risk of case fatality in men. Only small, if any, effects were indicated between impaired sleep and long-term cardiovascular prognosis among men.

Conclusion: Results suggest gender-specific effects of impaired sleep that differ by short and long-term prognosis. Sleep complaints are frequent, easily recognizable, and potentially manageable for most patients and evaluation of sleep complaints may thus, even if they represent prognostic markers rather than risk factors, provide additional information in clinical risk assessment that could benefit secondary cardiovascular prevention.

\section{P-317}

Serum uric acid concentrations in meat eaters, fish eaters, vegetarians and vegans: a cross-sectional analysis in the EPIC-Oxford cohort

Topic: Exposure - Lifestyle factors Presenter: Julie Andersen Schmidt

Julie Andersen Schmidt 1); Francesca Crowe 1); Paul Appleby 1); Timothy Key 1); Ruth Travis 1)

1) Cancer Epidemiology Unit, Nuffield Department of Medicine, University of Oxford, Oxford, United Kingdom

Background: High circulating concentrations of uric acid can lead to gout. Associations between uric acid concentrations and chronic kidney disease, cardiovascular disease and cancer have also been observed, although the causal nature of these associations is not clear. Uric acid concentrations may be affected by dietary components such as meat, fish and dairy products, but only a few studies have compared uric acid concentrations among individuals who exclude some or all of these foods from their diet.

Objectives: The aim of this study was to investigate differences in serum uric acid concentrations between meat eaters, fish eaters, vegetarians and vegans.

Methods: A sample of 670 men and 1,023 women (424 meat eaters, 425 fish eaters, 422 vegetarians and 422 vegans, matched on age and sex) from the European Prospective Investigation into Cancer and Nutrition Oxford cohort were included in this cross-sectional analysis. Diet was assessed using a semi-quantitative food frequency questionnaire and serum concentrations of uric acid were measured. Mean concentrations of uric acid by diet group were calculated after adjusting for age, body mass index, calcium and alcohol intake. 
Results: In both men and women, serum uric acid concentrations differed significantly by diet group $(p<0.0001$ and $p=0.01$, respectively). The differences between diet groups were most pronounced in men; vegans had the highest concentration $(340,95 \%$ confidence interval 329-351 $\mu \mathrm{mol} / \mathrm{l})$, followed by meat eaters $(315$, 306-324 $\mu \mathrm{mol} / \mathrm{l})$, fish eaters $(309,300-318 \mu \mathrm{mol} / \mathrm{l})$ and vegetarians (303, 294-312 $\mu \mathrm{mol} / \mathrm{l})$. In women, serum uric acid concentrations were slightly higher in vegans $(241,234-247 \mu \mathrm{mol} / 1)$ than in meat eaters $(237,231-242 \mu \mathrm{mol} / \mathrm{l})$ and lower in vegetarians $(230,224$ $236 \mu \mathrm{mol} / \mathrm{l})$ and fish eaters $(227,221-233 \mu \mathrm{mol} / \mathrm{l})$.

Conclusions: Individuals consuming a vegan diet had the highest serum concentrations of uric acid compared to meat eaters, fish eaters and vegetarians, especially in men. Vegetarians and individuals who eat fish but not meat had the lowest concentrations of serum uric acid.

\section{P-319}

Feasibility study concerning an intervention to reduce overweight among 10 to 13 year old children

Topic: Exposure - Lifestyle factors

Presenter: Anne Marie Moeller

\section{Anne Marie Moeller 1); Gabriele Berg-Beckhoff 1) \\ 1) University of Southern Denmark, Unit for Health Promotion Research, Esbjerg}

Background: Overweight among children is a rising problem, and is among other things caused by a decrease in physical activity and an increase in sedentary screen time. To increase the physical activity levels, some of the sedentary screen time could be used on interactive video gaming like dance mats.

Objectives: The aim of this study was to assess the feasibility of an intervention study investigating whether interactive video gaming with dance mats at home decreases overweight in Danish children aged $10-13$ years.

Methods: To assess the feasibility, first a thorough literature review on similar intervention studies was conducted. The purpose was to assess the potential health effect and feasibility of interactive video gaming with dance mats. Second, a questionnaire survey among 117 6th and 7th grade students in the municipality of Esbjerg was developed and carried out. The purpose was to estimate the children's computer related infrastructure at home, their possibility to use dance mats, and to find factors potentially motivating children to participate in this intervention.

Results: The intensity of physical activity when using interactive video gaming with dance mats is moderate at beginner's level, and contributes to the $60 \mathrm{~min}$ of recommended daily activity for children. Previous intervention studies are few. The survey showed that nearly no family had the dance mats at home, but more than $79 \%$ had technical equipment and space to use the dance mats. With regard to participation motivation, a successful intervention must include the ability for the children to play with playmates and that new updates for the game must occur to avoid declining participation. Furthermore, it seemed as if the overweight children were motivated by health promoting activities, which therefore must be incorporated in the recruitment material. The results were used to create a study protocol on the intervention.

Conclusions: The results of this feasibility study will improve the effectiveness of the proposed intervention. It has identified potential issues and secured that a sufficiently large amount of children will be recruited to identify a potential effect. Even though the feasibility study was limited by the fact, that no pilot test of the intervention was carried out, it was concluded that the intervention study to reduce overweight among 10-13 year old children as proposed by the study protocol, is feasible.

\section{P-320}

Social determinants of healthy habits of the Japanese children: individual factors and family circumstances factors

Topic: Exposure - Lifestyle factors

Presenter: Tomoo Nakata

\section{Tomoo Nakata 1)}

\section{1) Hokusei Gakuen University}

The aims of this study are to examine the impact of exposure of parents and family circumstances of the Japanese home on their children's lifestyle concerning health using latent class analysis. Previous researches concerning influences of place of origin on healthy habits are mostly analyzed using retrospective answers of the respondents. However, it is sometimes pointed out the unreliability of the retrospective data Therefore, the impacts of the respondents' generation on their children's healthy behaviors will be examined this study.

This study employed 'Japanese Study of Stratification, Health, Income, and Neighborhood (J-SHINE)', which was a large-scale dataset to examine social stratification and health in 2009. The sample included people who is from 25 to 50 years old male and female $(\mathrm{n}=4381)$. Respondents resided in 23 wards and neighboring cities in the Metropolis of Tokyo, Japan. And interview was also conducted for the respondents' spouses and children in this survey. The independent variables in this study were four children's health habits, which were 'brushing teeth', 'eating breakfast', 'not eating a snack between meals' and "defecating regularly" These items were often asked to explore children's healthy behaviors by the school in Japan. And dependent variables for identification of the factors were age, gender for children and age, income, self-rated social status, social capital and occupational status for their parents.

At first, latent class analysis was conducted for the dependent variables. As the results of the analysis, three latent groups were extracted using from the goodness of fit test; the first group was the group selecting of health behaviors but eating a snack between meals, the second group was the group that have a good habit for the dietary, breakfast and snacks, and the third one was the group that have every healthy habits consistently. Secondly, multiple logistic regression analysis was conducted to investigate the factors on the latent groups. The results of this analysis were as follow; (1) Individual factors like children's gender, age and number of siblings were the positive significant effects for the healthy habits. (2) Social stratification factors, like fathers' education level and social capital of the native household also had positive effects on the healthy behaviors of the children.

These results show that individual factors are stronger effects for the healthy life style for the children than their parents, but some factors, for example, social capital of the native home place have impacts on the healthy habits.

\section{P-321}

Prenatal smoking exposure and children's motor development: a follow-up study

Topic: Exposure - Lifestyle factors

Presenter: Line Høgenhof Christensen

Line Høgenhof Christensen 1); Birgit Bjerre Hoeyer 1); Jens Peter Bonde 2); Gunnar Toft 1)

1) The Danish Ramazzini Centre, Department of Occupational Medicine, Aarhus University Hospital, Aarhus, Denmark; 2) Department of Occupational and Enviromental Medicine, Copenhagen University Hospital, Bispebjerg, Copenhagen, Denmark 
Background: Some cohort studies indicate an association between prenatal smoking exposure and children's motor difficulties. However, the results are inconsistent, the outcome measures are different between studies and exposure is most often self reported. Studies indicate that measurement of serum cotinine can result in a more accurate status of smoking exposure in comparison with self report. Objectives: This follow-up study aimed to investigate whether prenatal exposure to smoking measured as maternal serum cotinine affects children's age at developmental milestones; crawling, standing-up and walking and motor skills at age 5-9 years.

Method: In 2002-2004, 1,250 pregnant women from Greenland and Ukraine were included in the INUENDO cohort. The participating women filled in questionnaires and 1,177 provided blood samples, which were analysed for serum cotinine. Smokers were defined as women with $>10 \mathrm{ng} / \mathrm{ml}$ serum cotinine. At follow-up in 2010-2012, 1,018 of the mothers or fathers from Greenland and Ukraine participated in the CLEAR project. They completed an interview-based questionnaire including questions about age at developmental milestones of their children and child motor development was assessed using the validated questionnaire Developmental Coordination Disorder Questionnaire 2007. Linear regression analyses were performed and adjusted for the co-variates; age of the mother and child, sex, parity, breastfeeding, alcohol and education.

Results: Preliminary analyses did not indicate major effects of in utero smoking on child motor development. However, further analyses are being performed and results will be presented on the conference.

Conclusion: The present study will provide new evidence-based knowledge concerning child development and adverse effects of exposure to cigarette smoking during pregnancy, which may contribute to improve the scientific basis of recommendations concerning precautions to be taken by pregnant women.

\section{P-322}

Impact of five lifestyle practices on cancer risk: results from the $\mathrm{E} 3 \mathrm{~N}$ cohort

Topic: Exposure - Lifestyle factors

Presenter: Laureen Dartois

\section{Laureen Dartois 1); Fagherazzi Guy 1); Françoise Clavel-Chapelon 1)}

1) Inserm (Institut National de la Santé et de la Recherche Médical), Centre for Research in Epidemiology and Population Health (CESP), U1018, Team 9, Institut Gustave Roussy, Villejuif, France.

Paris-South University, UMRS1018, Villejuif, France

Background: Lifestyle characteristics are known to individually decrease the risk of cancer. However little is known about their combined effect and about the proportion of cancer cases that could be prevented by improving lifestyle behaviors.

Objectives: To quantify the impact of five lifestyle characteristics on all-site and site-specific cancer risk in middle-aged women.

Material: The study included 64732 women from the French E3 N prospective cohort, aged 42-72 years in 1993. During 15 years of follow-up, 6938 cases of invasive cancer were diagnosed.

Methods: Five lifestyle characteristics were aggregated into a health index based on smoking, body mass index, alcohol drinking, fruits and vegetables consumption and physical activity assessed at baseline. Proportional hazard Cox regressions were performed in order to evaluate their association with cancer risk and estimate multivariate hazard ratios (HR) and their $95 \%$ confidence intervals (CI). In addition, population attributable fractions were estimated to quantify the proportion of cancer cases that would have been prevented if exposure had been minimal.
Results: A significant decrease in all-site cancer risk was observed, associated with a healthy lifestyle $(\mathrm{HR}=0.77(0.70 ; 0.85)$ when comparing the highest to the lowest health index category; Ptrend across categories $<0.01$ ). If smoking exposition had been modified (if current and former smokers were all never smokers), $1.3 \%$ of cancers of any-site, $0.3 \%$ of premenopausal breast cancer, $2.3 \%$ of postmenopausal breast cancer and $6.9 \%$ of colorectal cancer cases would have been prevented, whatever the 4 others characteristics. These percentages were respectively $1.3,-3.1,0.4$ and $1.2 \%$ for BMI (if overweight and underweight women were all within the normal range $[18.5 ; 25]), 3.3,2.4,6.9$ and $6.6 \%$ for alcohol (if moderate and heavy alcohol consumers were all light consumers), 1.5, 1.4, 3.0 and $4.9 \%$ for fruits and vegetables consumption (if light and moderate fruits and vegetables consumers were all heavy consumers), and 1.3, 1.1, 2.2 and $1.7 \%$ for physical activity (if lightly and moderately active women were all highly active). Combining all five healthy practices would have prevented $8.5 \%(3.0 ; 14.0)$ of cancers of any-site cancer, $0.6 \%(-12.4 ; 13.5)$ of premenopausal breast cancer, $16.5 \%(7.0$; $25.8)$ of post-menopausal breast cancer and $7.1 \%(-13.8 ; 27.4)$ of colorectal cancer cases.

Conclusion: Adhering to one single health recommendation has moderate impact on cancer risk, whereas adhering to a whole healthy lifestyle may significantly decrease the number of diagnosed cancer cases.

\section{P-323}

Factors associated with smoking relapse in the postpartum period: an analysis of the child health surveillance system data in southeast England

Topic: Exposure - Lifestyle factors

Presenter: Anjum Memon

\section{Clare Harmer 1); Anjum Memon 2)}

1) Public Health Directorate, NHS East Sussex and East Sussex County Council, Lewes, UK; 2) Brighton and Sussex Medical School; and Public Health Directorate, NHS Sussex (Brighton and Hove), and Brighton and Hove City Council, Brighton, UK

Background: There is increasing evidence that a high proportion (47-63\%) of women who quit smoking during pregnancy relapse during the postpartum period. Because of the serious health consequences of smoking for mother and child, preventing postpartum relapse is an important public health issue.

Objective: The purpose of this population-based study (which is probably the first such study in the UK) was to examine the association between selected lifestyle/demographic factors and smoking relapse in the early postpartum period (within the first 6 weeks) in women who had successfully quit smoking during the pregnancy. Methods: The study included 512 women resident in southeast England, who had quit smoking during the pregnancy. Information on the prevalence of smoking and selected lifestyle/demographic factors at the 6-weeks postpartum review by health visitor was obtained from the Child Health Surveillance System, which records and monitors the health and development of children from birth until school entry.

Results: Of the 512 women who had quit smoking during the pregnancy, about half $(\mathrm{n}=238,46.5 \%)$ relapsed in the early postpartum period. In the bivariate analysis, there was an association between deprivation and smoking relapse in the early postpartum period $(\mathrm{OR}=5.3,95 \%$ CI 2.5-11.4), with a significant trend in increasing risk of relapse with increasing level of deprivation $(p<0.01)$. Stepwise logistic regression analysis showed that women who lived in deprived urban areas $(\mathrm{OR}=2.3,95 \%$ CI 1.2-4.2), had $\geq 3$ children $(\mathrm{OR}=3.8,95 \% \mathrm{CI} 2.2-6.4)$, and had other smokers in the 
household $(\mathrm{OR}=5.6,95 \%$ CI 3.6-8.8) were significantly more likely to relapse in the early postpartum period. On the other hand, women who were breast feeding were significantly less likely to relapse $(\mathrm{OR}=0.6,95 \%$ CI $0.4-0.9)$.

Conclusions: Factors associated with early postpartum smoking relapse identified in this study, particularly breast feeding, high parity, and concurrent smoking by partner/other household member(s), may contribute to the development of effective and targeted interventions to maintain smoking cessation in women and their household. These interventions may be more effective if they include strategies aimed at increasing breast feeding rates and assisting household members to stop smoking. Women who are most vulnerable to relapse can be identified in the antenatal period as requiring extra support/counselling to maintain smoke-free status after delivery.

\section{P-325}

Serum 25(OH)D status and the risk of cognitive decline in the very old: the newcastle $85+$ study

Topic: Exposure - Lifestyle factors

Presenter: Antoneta Granic

\section{Antoneta Granic 1); Tom Hill 2); Thomas Kirkwood 1); Karen Davies 1); Joanna Collerton 1); John Mathers 1), 2); Carol Jagger 1)}

1) Institute for Ageing and Health, Newcastle University; 2) Human Nutrition Research Centre, Campus for Ageing and Vitality, Newcastle University

Background: Several population-based studies have reported the adverse effect of low or high levels of serum 25-hydroxyvitamin D $[25(\mathrm{OH}) \mathrm{D}]$ on cognitive functioning in older adults but there is a lack of studies in the very old, where rates of cognitive impairment and reduction in cutaneous production of vitamin $\mathrm{D}$ are the highest.

Objectives: To evaluate the association between serum $25(\mathrm{OH}) \mathrm{D}$ and prevalent cognitive functioning at baseline and decline over 3 years in the population-based study of 845 older adults living in Newcastle and North Tyneside, United Kingdom aged 85 years at baseline interview. Methods: Cognitive functioning and decline at 3 years was assessed globally by the Mini-Mental State Examination (MMSE), and more in-depth by the Attention Battery of the Cognitive Drug Research (CDR) computerized sub-test at baseline, 1.5 and 3 years. We examined the association between 25(OH)D and MMSE at baseline and 3 years by cross-sectional and prospective multivariate logistic regression modelling. Changes in attention tasks over 3 years (Simple Reaction Time [SRT], Choice Reaction Time [CRT], Digit Vigilance [DVT], Power of Attention [PoA], and Cognitive Reaction Time [CogRT]) were examined by multilevel linear modelling. All models were adjusted for putative risk factors for cognitive decline/dementia and variables associated with $25(\mathrm{OH}) \mathrm{D}$ levels. For analysis $25(\mathrm{OH}) \mathrm{D}$ was categorised into quartiles with the middle two quartiles combined and taken as reference.

Results: After controlling for potential confounders, prevalent global cognitive impairment was significantly increased in the lowest quartile of serum $25(\mathrm{OH}) \mathrm{D}(\mathrm{OR}=1.63,95 \%$ CI 1.07-2.48, $p=0.02)$ and the highest quartile $(\mathrm{OR}=1.74,95 \% \mathrm{CI} 1.15-2.63, p=0.009)$ compared with the middle quartile. We found limited evidence of a relationship between $25(\mathrm{OH}) \mathrm{D}$ and incident global cognitive impairment or cognitive decline in the very old. However when changes in attention were analysed we found that participants belonging to highest 25(OH)D quartile declined by $11.7 \mathrm{~ms}$ $(\mathrm{SE}=5.8 \mathrm{~ms}, p=0.05)$ more per month over study period in the DVT attention task compared with those in middle quartiles after adjusting for potential confounders.
Conclusion: Although serum 25(OH)D does not appear to be associated with global cognitive decline or incident cognitive impairment in the very old this may be due to learning effects with the MMSE. However objectively measured attention tasks showed significantly slower performance in DVT attention-specific tasks over 3 years in those with the highest levels of $25(\mathrm{OH}) \mathrm{D}$. Further investigation is needed to determine whether $25(\mathrm{OH}) \mathrm{D}$ affects other domain-specific cognitive functions in this age group.

\section{P-326}

Smoking behaviour during pregnancy and foetal loss: a study in the Danish National Birth Cohort (DNBC)

Topic: Exposure - Lifestyle factors

Presenter: Maria M. Morales-Suárez-Varela

Maria M. Morales-Suárez-Varela 1); Ellen A. Nohr 1); Bodil Hammer Bech 1); Jørn Olsen 2)

1) 1 Unit of Public Health and Environmental Care, Department of Preventive Medicine, University of Valencia, Valencia, Spain 2 CIBER Epidemiology and Public Health (CIBERESP), Spain 3 Center for Public Health Research (CSISP), Valencia, Spain; 2) The Danish Epidemiology Science Centre, University of Aarhus, Aarhus, Denmark

Introduction: Tobacco use increases the risk of developing several diseases. Therefore, tobacco use is one of the main preventable causes of chronic disease and death in developed countries, and is the second leading cause of death worldwide. The high prevalence of smoking among young women highlights smoking-related risks of adverse pregnancy outcomes, such as spontaneous abortions, stillbirth, preterm birth and foetal growth restriction. Given that pregnant women are usually concerned about foetal well-being, young adulthood and pregnancy may be an ideal time to quit smoking

Methods: The study was performed in the DNBC, a nationwide prospective cohort study with long-term follow-up. The cohort consisted in 98,282 pregnancies recruited in Denmark in 1996-2002. Enrolment criteria were intention to carry to term, ability to fill in questionnaires and to take part in interviews in Danish. Women were enrolled at the first antenatal visit to their general practitioners. Information on the lifestyle and socio-economic status of the pregnant women was obtained from a recruitment form, and four telephone interviews (see the timing of the activities in the cohort follow-up, which is still ongoing) with 22,674 (23\%) smoking women and 75,608 pregnancies among non-smoking women and women not exposed to tobacco behaviour, performed in the DNBC at the beginning of the pregnancy. Pregnancy outcomes were ascertained and information about lifestyle, occupational, medical, and obstetric factors was obtained. Logistic regression was used to calculate the relative risk ratios (RR) for foetal loss (spontaneous abortions, stillborn) by adjusting for lifestyle, mother's age, socioeconomic status, parity, alcohol consumption and mother's educational factors.

Results: In a cohort study of 98,282 pregnancies, $5.7 \%(\mathrm{n}=5,606)$ lost their baby (spontaneous abortion $(\mathrm{n}=5,303)$ and stillborn $(\mathrm{n}=303))$. When analysing the risk of exposure to the mother's (RRc: 2.5, 95 CI 2.4-2.6, RRa: 1.4, $95 \%$ CI 0.8-2.7) and father's (RRc:1.1, 95CI 0.8-1.3, RRa: 1.1, $95 \%$ CI 0.8-1.4) Smoking. When we analysed the interaction between mother and father's smoking habits, we observed that the greater the intensity of smoke exposure, the higher the risk of spontaneous abortion. The father's strong smoking habit and the mother consuming more than 20 cigarettes per day have an RRc:9.7 (95\% CI 13.6-69,5; $p=0.023$ ). 
Conclusion: Our findings suggest that smoking is a risk for loss of babies, and pregnant women and their partners should be informed about it.

\section{P-327}

Exogenous hormone use and all-cause mortality: results from the HAPIEE study

Topic: Exposure - Lifestyle factors

Presenter: Bernardine Stegeman

Bernardine Stegeman 1); Ruzena Kubinova 2); Sofia Malyutina 3); Andrzej Pajak 4); Abdonas Tamosiunas 5); Anne Peasey 1); Martin Bobak 1)

1) Department of Epidemiology and Public Health, University College London, London, United Kingdom; 2) Centre for Environmental Health, National Institute of Public Health, Prague, Czech Republic; 3) Institute of Internal Medicine, Russian Academy of Medical Sciences, Novosibirsk, Russia; 4) Department of Epidemiology and Population Studies, Institute of Public Health, Faculty of Health Care, Jagiellonian University Medical College, Krakow, Poland; 5) Department of Population Studies, Institute of Cardiology, Academy of Medicine, Lithuanian University of Health Sciences, Kaunas, Lithuania

Background: Previous studies have suggested that the risk of death was similar in hormonal contraceptive (HC) users and non-users. Data from randomized controlled trials and observational studies have shown that hormone replacement therapy (HRT) was inversely associated with all-cause mortality, in particular in women who were younger than 60 years when starting HRT. None of the studies assessed the combined effect of HC and HRT use on all-cause mortality.

Objectives: To assess the effect of HC, HRT and their combined use on all-cause mortality.

Methods: Postmenopausal women from the HAPIEE cohort study in Czech Republic, Poland and Russia (2002-2005) and in Lithuania (2006-2008), were followed for all-cause mortality up to December 2011. Women with cancer, cardiovascular diseases or chronic obstructive pulmonary diseases at baseline, or with missing data on reproductive history questions, were excluded. HC use was categorised as never and past use and HRT use was classified as never, past and current. Cox proportional hazards models were used to estimate hazard ratios (HR) with $95 \%$ confidence intervals (CI), adjusted for age, BMI, country, education, smoking, and age at menopause and only in the analysis of HRT use, for time since menopause. Multiplicative interaction was assessed with the likelihood ratio test.

Results: In total, 8687 women were included with a total of 53,748 years of follow-up. Over a median of 5.9 years, 307 deaths from all-causes were observed resulting in a mortality rate (MR) of 57.1 per 10,000 woman-years (95\% CI 51.1-63.9). 36 deaths were found among women who were ever exposed to exogenous hormones (MR 25.4, $95 \%$ CI 18.3-35.2). The risk of death among past HC users was about half compared with never HC users (HR 0.50, $95 \% \mathrm{CI}$ $0.30-0.83$ ). Current and past HRT use was associated with a decreased mortality (HRcurrent $0.69,95 \%$ CI $0.36-1.32$ and HRpast $0.34,95 \%$ CI 0.17-0.66). Having ever used both HC and HRT was negatively associated with the risk of death (HR 0.17, $95 \%$ CI 0.04-0.70). No evidence was found for multiplicative interaction $(p=0.80)$.

Conclusions: HC and HRT use were independently associated with a reduced risk of all-cause mortality. Having ever used HC and HRT did not decrease the risk of death more than could be expected from their separate effects. The magnitude of the effect is larger than previously reported. In Eastern Europe women using HC and HRT may be a special subgroup and the low mortality in these women may reflect other factors than HRT or HC.

\section{P-328}

The contribution of changes in diet and other risk factors to recent favourable trends in blood pressure in middle-aged Irish adults

Topic: Exposure - Lifestyle factors

Presenter: Janas $M$ Harrington

Janas M Harrington 1); Ivan J Perry 1); Zubir Kabir 1); Gemma Browne 1); Patricia M Kearney 1)

1) Department of Epidemiology \& Public Health, University College Cork

Background: Recent declines in CVD mortality in Ireland and other developed countries are partially attributable to favourable secular trends in blood pressure (BP). However the underlying causes of these $\mathrm{BP}$ trends are not well defined. In particular, the contribution of changes in the dietary and other lifestyle determinants of population $\mathrm{BP}$ levels is unclear.

Objectives: To examine changes in distribution and determinants of systolic blood pressure (SBP) in middle-aged Irish adults over a 12 year period.

Methods: Data are from two cross-sectional studies of men and women aged 47-73 years recruited from 17 General Practices in Cork and Kerry $(\mathrm{N}=1018$, response rate $70 \%)$ and from one large General Practice in Cork ( $\mathrm{N}=2047$, response rate $67 \%)$ in 1998 and 2010 respectively. Similar procedures were used at both time points, including recruitment methods, exclusion criteria, health and lifestyle questionnaire, dietary assessment (food frequency questionnaire [FFQ]) and physical examination (height, weight and blood pressure). Dietary quality was assessed using the Dietary Approaches to Stop Hypertension (DASH) score constructed from the FFQ: higher scores indicating healthier diets. Changes in mean SBP were examined in linear regression models and are presented as means (95\% C.I.).

Results: Mean SBP fell by $7.1 \mathrm{mmHG}(138.0-130.9)$ in men and 4.7 mmHG (133.0-128.3) in women between 1998 and 2010. Adjusted for age, gender and education mean SBP, was $5.8 \mathrm{mmHG}$ ( -7.3 to -4.5$)$ lower in 2010 than in 1998. Average BMI increased from 27.8 (4.0) to 29.1 (4.1) in men and from 27.4 (4.6) to 28.0 (5.1) in women. By contrast diet quality improved over the period in men and women from 22.5 (4.4) to 27.6 (5.6) and from 23.5 (4.7) to 30.1 (5.6) respectively. Significant trends in physical activity (favourable), smoking (favourable), and alcohol intake (unfavourable) were observed. The proportion of the sample with doctor diagnosed hypertension increased from $25 \%$ to $29 \%$ over the 12 year period. Adjusting for age, gender, education, BMI, smoking and alcohol the change in mean SBP remained relatively unchanged. Adjusting for DASH diet score the mean SBP change it was reduced to $4.1 \mathrm{mmHG}$ $(-6.5$ to -1.7$)$ and on further adjustment for doctor diagnosed hypertension it was reduced to $3.9 \mathrm{mmHG}(-6.3$ to -1.6$)$.

Conclusions: The findings suggest that average blood pressure levels in the population are falling, despite the increasing prevalence of obesity, and much of the change is due to improvements in diet.

\section{P-329}

Positive mental health and well-being among a third level student population

Topic: Exposure - Lifestyle factors 
Presenter: Martin P. Davoren

\section{Martin P. Davoren 1); Eimear Fitzgerald 1); Frances Shiely 1); Ivan J. Perry 1)}

1) Department of Epidemiology and Public Health, University College Cork, Ireland

Background: Much research on the health and well-being of third level students is focused on risky behaviours and poor mental health. More recently public health is increasingly addressing the distribution and determinants of positive mental health and well-being in the population. There is a dearth of information on positive mental health and well-being in the student population. Recently, the Warwick Edinburgh Mental Well-being scale (WEMWBS) was developed as a measurement of positive mental health. The aim of this research is to investigate the distribution and determinants of positive mental health and well-being in a large, broadly representative sample of Irish third level students.

Methods: Undergraduate students from one large third level institution were randomly sampled using probability proportional to size sampling. Questionnaires were distributed to students attending lectures on randomly chosen days in the randomly selected modules. A total of 2,332 self-completed questionnaires were obtained, yielding a response rate of $51 \%$ based on the list of students registered to relevant modules. The response rate for those in attendance was $84 \%$. Positive mental health and well-being was measured using WEMWBS. 2,044 undergraduates completed the full fourteen questions involved in WEMWBS and are the subject of this analysis. One-way ANOVAs and multivariate logistic regression were utilised to investigate factors associated with positive mental health and wellbeing.

Results: The sample was predominantly female $(62.66 \%)$, in first year $(46.9 \%)$ and living in their parents' house $(42.4 \%)$ or in a rented house or flat $(40.8 \%)$. The median age of participants was 20 years. Average WEMWBS scores were higher in men than women. No significant differences were noted by area of study, alcohol or smoking status. In multivariate analysis adjusted for age and gender, WEMWBS scores were higher among students with low levels of physical activity $(p=0.04)$ and a higher number of sexual partners $(p<0.001)$. WEMWBS scores were also higher for those reporting the use of recreational drugs in the previous 12 months $(p=0.04)$.

Conclusions: This is one of the first studies to examine positive mental health and well-being scores in a third level student sample. The findings suggest that students with a relatively adverse health and lifestyle profile have higher than average mental health and wellbeing. However, although the response rate is high for a third level student survey, the issue of response bias may be a cause of concern. To confirm these results, this work needs to be replicated in other third level institutions.

\section{P-330}

Estimated 24-hour sodium excretion among Danish adults and the association with hypertension

Topic: Exposure - Lifestyle factors

Presenter: Betina Thuesen

Betina Thuesen 1); Ulla Toft 1); Allan Linneberg 1); Torben Jørgensen 1)

1) Research Centre for Prevention and Health, The Capital Region of Denmark, Denmark

Background: A simple and valid method for estimating 24-hour urinary sodium excretion is crucial if salt intake is to be monitored in large populations. Recently we developed a model to estimate 24-hour urinary sodium excretion on a population level based on sodium and creatinine measurements in a casual spot urine. However, the model has not yet been applied in a population different from the population in which it was developed.

Objectives: The objective of this study was to use our newly developed model to estimate the 24-h sodium excretion and thus the daily salt intake in a population of nearly 3300 Danish adults. Since a positive association between salt intake and increasing blood pressure is well described, we also aimed to evaluate this association to further validate the model of estimating salt intake.

Methods: We included 3,294 participants (1,477 men and 1,817 women) aged 18-69 years from the population based Danish Health2006 cohort. Estimated 24-hour sodium excretion was calculated from measurements of creatinine and sodium in samples of spot urine. The relation between 24-h sodium excretion and blood pressure was evaluated by linear and logistic regression models.

Results: The mean estimated 24-h sodium excretion among men was $4.32 \mathrm{~g} \mathrm{(5 \% /95 \%} \mathrm{percentiles:} 3.06 \mathrm{~g} / 5.73 \mathrm{~g}$ ) and $2.43 \mathrm{~g}(5 \% / 95 \%$ percentiles: $2.43 \mathrm{~g} / 3.57 \mathrm{~g}$ ) among women, corresponding to daily salt intakes of $10.80 \mathrm{~g}$ and $7.52 \mathrm{~g}$, respectively. High salt intake was significantly associated with high blood pressure $(p<0.0001$, linear regression adjusted for confounding by sex and age). The effect of salt intake on blood pressure was markedly attenuated when adjusting for BMI. However, BMI is both highly positively correlated with blood pressure and salt intake and thus BMI may be considered a mediator more than a confounder when evaluating the association between salt and hypertension.

Conclusions: The daily mean intake of salt estimated from measurements of creatinine and sodium in spot urine was $10.80 \mathrm{~g}$ among men and $7.52 \mathrm{~g}$ among women. As expected, a high daily salt intake was significantly associated with increased blood pressure. Thus, this study support the validity of our newly developed model for estimating daily salt intake from measurements of sodium and creatinine in single samples of spot urine on a population level. However, the association between salt intake and blood pressure is markedly attenuated when adjusting for BMI, and thus this study raises an important question about how to handle BMI when evaluating associations between salt intake and cardiovascular outcomes.

\section{P-331}

Socioeconomic inequalities in health risk factors in Korea

Topic: Exposure - Lifestyle factors

Presenter: $\mathrm{Yu}$-Mi Kim

\section{Yu-Mi Kim 1)}

Tackling socioeconomic inequalities in health risk factors is an important pathway for alleviating health inequalities. The aim of this study was to analyze the current state of inequality in health risk factors by socioeconomic status in Korea through a literature review of recently published studies and description of secondary data from the KNHANES. We evaluated the extent and trends of socioeconomic inequalities in health behavior (smoking, high-risk alcohol drinking, moderate exercise, and nutritional deficiency) and clinical risk factors (hypertension, diabetes mellitus, hypercholesterolemia, and obesity) with 1998-2010 KNHANES data based on socioeconomic status. Furthermore, we summarized the impact of several distal health determinants like income, education and occupation, and childhood period on health inequalities in Korea. The results showed that a wide range of health risk factors including more distal causes were socioeconomically patterned to varying degrees.

In order to reduce health inequalities by socioeconomic status, more comprehensive monitoring and measures, and well-designed 
studies are required for promoting the understanding of the causal pathway and developing interventional strategies.

\section{P-332}

Quality of life, physical activity and digital literacy protects against impairment in instrumental activities of daily living: results from ELSA

Topic: Exposure - Lifestyle factors

Presenter: Eleonora d'Orsi

Eleonora d'Orsi 1); Andre Junqueira Xavier 2); Andrew Steptoe 3); Cesar de Oliveira 3); Luiz Ramos 4); Martin Orrell 5); Panayotes Demakakos 3); Michael Marmot 3)

1) Department of Public Health, Federal University of Santa Catarina, Florianopolis, Brazil; 2) University of South of Santa Catarina, Florianopolis, Brazil; 3) Department of Epidemiology and Public Health, University College London, London, UK; 4) Department of Preventive Medicine, Federal University of São Paulo, São Paulo, Brazil; 5) Mental Health Sciences Unit, Faculty of Brain Sciences, University College London, London, UK

Background: Limitations in Instrumental Activities of the Daily Living (IADL) are major predictors of cognitive impairment and dementia. They are also preventable and reversible and thus identifying the determinants of IADL dynamics could contribute to more successful health promotion and ageing policies.

Objective: To investigate the factors related to IADLs dynamics (decline, recovery and time elapsed with limitation).

Methods: Data from the English Longitudinal Study of Ageing (ELSA); a biennial, longitudinal, panel study of people living in England aged 50 years or over. We used data from waves 1-5 collected in 2002-2003, 2004-2005, 2006-2007, 2008-2009, and 2010-2011. From the initial 11,391 participants, we selected a sample of 8,154 who reported not having any difficulty in performing Activities of Daily Living (ADL) or IADL. The first outcome was impairment in at least one IADL between any two consecutive waves, and the second was total recovery. The main predictor was socioeconomic position measured using wealth quartiles at baseline. Unadjusted and adjusted incidence rate ratios (IRR) and their $95 \%$ confidence intervals were calculated through Generalized Estimating Equations, controlling for socio-demographic, lifestyle, quality of life, and health variables.

Results: On average 1656 (20.31\%) of the population reported some IADL impairment between any two consecutive waves. The adjusted IRR showed a social gradient in IADL impairment. Lifestyle variables included in the fully adjusted model accounted for about $12 \%$ of the effect of wealth, which decreases from a 34-21\% lower risk comparing the best-off with the worst-off. Those who reported high quality of life, vigorous physical activity, paid work, digital literacy (use of internet/email) and leisure activities had significantly lower risk of IADL impairment. Those who reported smoking, cardiovascular diseases, arthritis, fair/poor memory and low delayed recall had significantly higher risk of the outcome. Diabetes and depressive symptoms were independent risk factors for reporting IADL and for a slower recovery from IADL impairment. On average $50.29 \%$ of the population who reported some IADL impairment had a total recovery. The time elapsed with IADL impairment was $38.47 \%$ of the followup time. High quality of life and vigorous physical activity were the only variables associated to IADL impairment and recovery.

Conclusions: High quality of life (control, autonomy, self-realization and pleasure), vigorous physical activity, no smoking, paid work, leisure and digital literacy can mitigate to a large extent wealth inequalities in IADL impairment. Wealth nevertheless remained a significant independent determinant of IADL impairment.

\section{P-333}

Does 5 a day keep the Tau away?

Topic: Exposure - Lifestyle factors

Presenter:

Peter Elwood 1); Tony Bayer 1); Mark Fish 2); Janet Pickering 1); Yoav Ben-Shlomo 3)

1) Cardiff University; 2) Taunton Hospital Trust; 3) Bristol University

Background: Healthy lifestyles based on non-smoking, low BMI, fruit and vegetable rich diet, regular physical activity and low alcohol consumption, are associated with reductions in incidence of several chronic diseases and death, but there is little evidence in relation to cognitive function and dementia

Objectives: To investigate the association of healthy lifestyle and incident dementia

Method: Lifestyle data were recorded in a representative general population sample of 2,235 men aged 45-59 years, in South Wales, of whom 1,027 were alive 29 years later and underwent cognitive assessment.

Results: After adjustment for crystallised intelligence (used as a proxy for pre-morbid cognitive function), the odds ratio for dementia associated with adopting 4-5 healthy behaviours was $0.32(95 \% \mathrm{CI}$ 0.11-0.97) for cognitive impairment and $0.30(0.05,1.11)$ for dementia. There were reducing trends in the odds ratio for diabetes (0.47: $0.18,1.24)$, vascular disease $(0.47: 0.28,0.79)$ and death $(0.42$ : $0.25,0.71)$. No association of cancer with lifestyle was detected. The time until a cognitive related outcome was detected was delayed by a healthy lifestyle by up to 7 years for impairment and 5 years for dementia. Death was delayed by 6 years.

Conclusions: A healthy lifestyle reduces risk of dementia and increases disease free survival.

\section{P-334}

Relationship between risk behaviour and awareness of HIV status among injecting drug users: a multisite study

Topic: Exposure - Lifestyle factors

Presenter: Mait Raag

Mait Raag 1); Anneli Uusküla 1); Don Des Jarlais 2); Lucas Wiessing 3)

1) Department of Public Health, University of Tartu, Tartu, Estonia; 2) Edmond de Rothschild Chemical Dependency Institute, Beth Israel Medical Center,New York, NY, USA; 3) European Monitoring Centre for Drugs and Drug Addiction (EMCDDA), Lisboa, Portugal

Background: Inadequate uptake of HIV testing remains a primary bottleneck towards universal access to treatment and care, and is an obstacle to realizing the potential of interventions for preventing HIV infection. Further, HIV serostatus awareness may directly affect risk behaviour, and is thus an independent target for interventions.

Objectives: To assess the relationships between HIV risk behaviour and HIV-testing among current injection drug users (IDU) in Europe. Methods: Data from 10 cross-sectional studies (sites), conducted in Europe (Denmark, Estonia, Latvia, Netherlands, Poland, Portugal, Russia) between 2001 and 2010, were used. Within each study, multiple logistic regression was used to estimate the magnitude of associations between high risk injection/sexual behavioural and HIV-serostatus adjusting for age, injecting career length, and gender. Results across the countries were pooled using meta-analytic techniques.

Results: The proportion of those never tested ranged between $0 \%$ in Portugal, 2010, and $42 \%$ in Omsk, Russia, 2009; those who had 
tested HIV + before the studies made up from $5 \%$ (Omsk) to $94 \%$ (Oryol, Russia, 2008) from those ever tested for HIV. Prevalence of HIV varied from $9 \%$ in Omsk to $62 \%$ in St. Petersburg and Leningradskaya region, Russia, 2009. IDUs who had tested for HIV were more likely to be sexually active (OR $1.50,95 \%$ CI 1.24-1.81; I2 $0 \%$ ) and more likely to inject daily (OR $1.34,95 \%$ CI 1.04-1.72; I2 $58 \%$ ) than those who had never tested. In comparison to those never tested, IDU tested HIV- were more likely to be sexually active (OR 1.72, $95 \%$ CI 1.41-2.11; I2 $0 \%$ ), and IDU tested HIV + were more likely to inject at least daily (OR 1.45, $95 \%$ CI 1.07-1.97; I2 $40 \%$ ). IDUs who had tested HIV negative were more likely to be sexually active (OR 1.66, $95 \%$ CI 1.34-2.05; I2 $13 \%$ ), but less likely to use condom if sexually active (OR 0.61 , CI $0.38-0.99$, I2 $82 \%$ ), and less likely to share syringes/needles (OR $0.56,95 \%$ CI $0.46-0.67$, I2 $0 \%$ ), compared to those tested HIV positive.

Conclusions: Our results indicate complex relationship between risk behaviour and awareness of one's HIV status among IDUs. As awareness of HIV+ status is associated with lower levels of selfreported high risk sexual behaviour, better access to HIV testing may decrease HIV burden attributable to sexual risk behaviour among IDUs.

\section{P-335}

Body mass index and subsequent psychotropic medication: a register linkage study

Topic: Exposure - Lifestyle factors

Presenter: Anna Svärd

\section{Anna Svärd 1); Jouni Lahti 1); Ossi Rahkonen 1); Eero Lahelma 1)}

1) Hjelt Institute, Department of Public Health, University of Helsinki

Background: Obesity and mental health problems are growing public health issues worldwide. Cross-sectional studies have shown an association between obesity and mental health problems. Follow-up studies examining the association have shown that mental health problems may lead to obesity, but whether obesity leads to mental illhealth is less clear. However, some studies suggest that obesity may lead to mental ill-health, especially among women.

Objectives: The main aim of this study was to examine whether obesity and overweight among employed women and men are associated with subsequent mental ill-health measured by prescribed psychotropic medication. A further aim was to examine the contribution of sociodemographic factors, health behaviours, physical functioning, diagnosed illnesses, previous psychotropic medication and strenuousness of work explain these associations.

Methods: 40-60-year-old employees of the City of Helsinki, Finland, participated in the baseline survey in 2000-2002 $(\mathrm{N}=8960$, response rate $67 \%$ ). Information on prescribed reimbursed psychotropic medication purchases from 1995 to 2009 and was derived from the registers of the Social Insurance Institution of Finland (74\% gave permission to the register linkages). The analysis included 4,892 women and 1,354 men. Hazard ratios (HR) for first psychotropic medication purchase were calculated using Cox proportional hazard models. Antidepressants and sedatives were examined separately.

Results: Psychotropic medication was more common among women (35\%) than men $(25 \%)$. Obese men were more likely to have psychotropic medication (age adjusted $\mathrm{HR}=1.77$; CI 1.32-2.37) than men with normal weight $(\mathrm{HR}=1.00)$. After adjusting for previous medication the associations remained for both antidepressants $(\mathrm{HR}=1.48$; CI 1.04-2.11) and sedatives (HR = 1.97; CI 1.38-2.81). After further adjusting for other covariates the associations attenuated for antidepressants $(\mathrm{HR}=1.39$; CI $0.96-2.00)$ but remained for sedatives $(\mathrm{HR}=1.90 ; \mathrm{CI} 1.31-2.77)$. The strongest factors affecting the association were previous psychotropic medication, physical functioning and health behaviours such as alcohol consumption, smoking and physical activity. Among women the association between obesity and subsequent psychotropic medication was weak. However, an association was found for antidepressants (OR 1.26; CI 1.08-1.47), but adjusting for previous antidepressive medication abolished the association (OR 1.04; CI 0.89-1.22).

Conclusion: Obesity was associated with subsequent sedative medication among men. For preventing antidepressive medication among obese men, improving physical functioning and enhancing healthy behaviours may be beneficial. Among women obesity as such is unlikely to lead to mental ill-health.

\section{P-336}

Maternal vegetarianism during pregnancy and risk impaired motor-, neuro- and cognitive development in the offspring

Topic: Exposure - Lifestyle factors

Presenter: Pernille Stemann Larsen

Pernille Stemann Larsen 1); Anne-Marie Nybo Andersen 1); Peter Uldall 2); Bodil Hammer Bech 3); Jørn Olsen 2); Anne Vinkel Hansen 1); Katrine Strandberg-Larsen 1)

1) Section of Social Medicine, Department of Public Health, University of Copenhagen, Denmark; 2) Paediatric Clinic, The Juliane Marie Centre, Rigshospitalet, Copenhagen University Hospital, Co-penhagen, Denmark; 3) Department of Public Health, Institute of Epidemiology and Social Medicine, Aarhus University, Denmark

Background: It has been reported, based on observations from casestudies, that vitamin B-12 defi-ciency during pregnancy lead to impaired development in infants. Offspring of vegans may be at increased risk of vitamin B-12 deficiency, since animal products is the only dietary source of vitamin B-12.

Objective: To examine, in a large-scale cohort study, the associations between sub-types of maternal vegetarianism and the risk of impaired motor-, neuro-, and cognitive development in the offspring.

Method: The study was based on data from the Danish National Birth Cohort in which 100,418 pregnancies were enrolled in the period from 1996 to 2002. A total of 80,743 pregnant wom-en, who gave birth to a live-born singleton with gestational age $>154$ days, provided information on vegetarianism and relevant confounding factors through an interview taking palace around week 12-16 of gestation. Diagnoses of severe impaired motor-, neuro- and cognitive development in the off-spring were obtained from the national health registers. Information on achievement of gross mile-stones was obtained in an interview with the mother at child age 18 months. At child age 7-years parent-reported information on problem behaviors was obtained, as well as information on devel-opment coordination disorder. For the women with complete information on vegetarianism, we cal-culated the distributions of maternal and offspring characteristic according to sub-types of vegetari-anism. Also, distributions of outcomes of impaired motor-, neuro- and cognitive development were calculated according to the sub-types of maternal vegetarianism. The number of exposed cases was low and did not allow for further statistical analyses.

Results: A total of 986 women reported vege-tarianism, and of these $21(2.1 \%)$ reported to be vegans. The vegetarians were older (35+), living alone, nulliparous, unemployed, had a healthy pre-pregnancy body mass index $\left(18.5-24.9 \mathrm{~kg} / \mathrm{m}^{2}\right)$, were physically active (>60 min/week), non-smokers, and non-drinkers. Of the vegetarians, the ve-gans were of young age, underweight, heavy-smokers, did take 
vitamin supplements, and suffered from anemia and eating disorders. Only few register-based cases were observed, and there was no indication of increased risk among offspring of vegetarians. Based on parent-reported achievement of gross motor milestones, no increased risk of impairment was observed. Problem behavior scores slightly increased according to the restrictiveness of vegetarianism. No increased risk of develop-mental coordination disorder was observed for offspring of vegetarians.

Conclusion: Findings from one of the largest birth cohorts with prospective collected data on maternal vegetarianism did not indicate any signs of impaired motor-, neuro- and cognitive development in the offspring.

\section{P-337}

FTO gene association with coronary heart disease risk is not reduced by physical activity

Topic: Exposure - Lifestyle factors

Presenter: Jaana Gustavsson

Jaana Gustavsson 1); Kirsten Mehlig 2); Karin Leander 3); Lauren Lissner 2); Lena Björck 4); Annika Rosengren 4); Fredrik Nyberg 1), 5)

1) Occupational and Environmetal medicine, Department of Public Health and Community Medicine, Sahlgrenska Academy, University of Gothenburg, Sweden; 2) Public Health Epidemiology, Department of Public Health and Community Medicine, Sahlgrenska Academy, University of Gothenburg, Sweden; 3) Division of Cardiovascular Epidemiology, Institute of Environmental Medicine, Karolinska Institute, Stockholm, Sweden; 4) Department of Emergency and Cardiovascular Medicine, Sahlgrenska Academy, University of Gothenburg, Sweden; 5) AstraZeneca R\&D Mölndal, Sweden

Background: Variation in the Fat mass and obesity related (FTO) gene predisposes to obesity and diabetes, and association with cardiovascular disease has also been demonstrated in later years. Interaction studies have suggested that increased physical activity may attenuate the FTO gene effect on obesity, but it is less studied whether the association with cardiovascular disease is modified by level of physical activity.

Objectives: To study the association between the FTO rs9939609 $(\mathrm{T}>\mathrm{A})$ variant and coronary heart disease $(\mathrm{CHD})$, and whether leisure time physical activity modifies this association.

Methods: Two Swedish population-based case-control studies: INTERGENE (INTERplay between GENEtic susceptibility and environmental factors for the risk of chronic diseases in West Sweden) and SHEEP (Stockholm Heart Epidemiology Program) were combined to a total sample of 1743 cases with CHD (myocardial infarction, angina pectoris or exacerbations of chronic CHD) and 4,402 population controls, aged $25-75$ years, including $41 \%$ women and genotyped for FTO rs 9939609 . Leisure time physical activity data were collected in self-reported questionnaires and we used 3 categories for the analysis: low (mainly sitting/very little activity), medium (moderate exercise now and then), high (regular exercise at least once/week). The main effect of FTO genotype (TA/AA versus TT) and its interaction with physical activity were estimated as relative risks expressed as odds ratios (OR) with $95 \%$ confidence intervals (CI) from logistic regression models, adjusted for age, sex and original study.

Results: The FTO risk genotype (TA/AA) was associated with higher risk of CHD (OR 1.25, $95 \%$ CI 1.10-1.42), and additional adjustment for body mass index (BMI) did not change this association markedly (OR 1.20, $95 \%$ CI 1.06-1.37), which indicates that the FTO association with CHD may not be entirely mediated by BMI. We found no statistical evidence of interaction between FTO genotype and physical activity on CHD risk ( $p$ for interaction $=0.3$ ), while the effect estimates suggested that subjects with low physical activity had no significantly increased CHD risk associated with FTO genotype (OR $1.10,95 \%$ CI 0.83-1.44), in contrast to subjects with medium (OR $1.23,95 \%$ CI 1.08-1.40), or high physical activity (OR 1.38, $95 \%$ CI 1.09-1.73). As expected, the FTO risk (A) allele was associated with higher BMI in population control subjects with a per allele effect of $+0.35 \mathrm{~kg} / \mathrm{m}^{2}, p<0.0001$, adjusted for age, sex and study.

Conclusions: Variation in the FTO gene is associated with increased risk of $\mathrm{CHD}$, which is not reduced by leisure time physical activity.

\section{P-338}

Noise exposure, lifestyle factors and the risk of hearing loss

Topic: Exposure - Lifestyle factors

Presenter: Thomas Winther Frederiksen

Thomas Winther Frederiksen 1), 2); Cecilia Ramlau-Hansen 3); Zara Stokholm 1); Jesper Vestergaard 1); Jens Peter Bonde 4); Søren Peter Lund 5); Henriette Buttenschön 6); Randi Wetke 7); Henrik Kolstad 1)

1) Department of Occupational Medicine, Danish Rammazzini Centre, Aarhus University Hospital; 2) Department of Otology, Hospitalsenheden Vest; 3) Department of Public Health, Section for Epidemiology, Aarhus University; 4) Department of Occupational and Environmental Medicine, Copenhagen University, Bispebjerg Hospital; 5) National Research Centre for the Working Environment, Copenhagen; 6) Institute for Clinical Medicine, Aarhus University;

7) Department of Audiology, Odense University Hospital

Background: The association between noise exposure and hearing loss has been known since the beginning of industrialisation, but individual hearing thresholds vary considerably following identical levels of noise exposure. Whether this is mainly due to variation in individual noise susceptibility or the influence of life style or other environmental factors is still uncertain.

Objective: The objective of this study is to clarify whether life style and individual factors such as smoking habits, blood pressure, plasma cholesterol levels, glycosylated haemoglobin, alcohol intake, BMI and physical activity modify the effect of noise exposure on hearing loss or if they act as independent risk factors.

Methods: The study population comprises 665 workers selected as a random sample from 12 industrial trades in Aarhus, Denmark, between 2009 and 2010. In all, 10 of these trades had high notification levels of noise induced hearing loss. The remaining 2 trades represented children day care units (due to recent concerns of high noise levels) and the financial sector, the latter selected as a reference sample with low noise exposure. The study database contains information on individual noise exposure levels, possible confounders and the previous mentioned lifestyle and individual factors. Based on 1200 individual noise dosimetries from 2001 to 2010, and employment histories since 1970 from a national pension fund, a noise exposure matrix is under construction, enabling retrospective estimation of cumulative noise exposure levels. Data will be analysed by use of multiple linear regression analyses.

Results: Data management and systematization is still in progress but preliminary results show that of the 665 participants, $73 \%$ were males, $30 \%$ had a hearing handicap (ISO-definition), $61 \%$ had total cholesterol levels above $5 \mathrm{mmol} / \mathrm{L}, 30 \%$ were current smokers, $63 \%$ had a BMI above $25 \mathrm{~kg} / \mathrm{m}^{2}, 18 \%$ were physically inactive during leisure time, $12 \%$ had glycosylated haemoglobin levels above 6 , $14 \%$ consumed more than 14 drinks of alcohol per week, and $28 \%$ were hypertensive according to ambulatory blood pressure recordings. 
Preliminary results for cholesterol so far show no significant association with hearing threshold level $(p=0.3)$ when adjusted for age and sex.

Conclusions: Individual hearing thresholds vary with identical noise exposure levels. Whether this is partly explained by the contribution of individual life style factors is uncertain. Preliminary results from our study show no significant association between cholesterol levels and hearing thresholds, but further analyses on other lifestyle factors are in progress and will be presented.

\section{P-339}

Varicose veins in lower extremities in relation to lifestyle factors: a cohort study based on the Danish Musculoskeletal Research Database

Topic: Exposure - Lifestyle factors

Presenter: Sorosh Tabatabaeifar

Sorosh Tabatabaeifar 1); Poul Frost 1); Johan Hvid Andersen 3); Lone Donbæk Jensen 1); Jane Frølund Thomsen 2); Susanne Wulff Svendsen 3)

1) Danish Ramazzini Centre, Department of Occupational Medicine, Aarhus University Hospital, Denmark; 2) Department of Occupational and Environmental Medicine, Bispebjerg Hospital, Denmark; 3) Danish Ramazzini Centre, University Department of Occupational Medicine, Herning Regional Hospital, Denmark

Background: Varicose veins (VV) in the lower extremities are dilated, palpable, subcutaneous veins. The influences of age, gender, and pregnancy on development of VV are well known, whereas the influences of lifestyle factors remain unclear.

Objectives: The aim of this study was to evaluate the influences of lifestyle factors in terms of body mass index (BMI), smoking, and physical activity on risk of surgery for VV.

Methods: The study was based on the Danish Musculoskeletal Research Database, which comprises data from nine previous questionnaire studies on musculoskeletal symptoms in the working population. We limited this study to participants aged $18-65$ at baseline. Participants were followed from baseline until the first operation for VV according to the Danish National Patient Register or until December 31, 2011, whichever came first. We categorized age as $<36$ years (reference), $36-45, \geq 45-56$ and $\geq 56$; BMI as $<25 \mathrm{~kg} / \mathrm{m}^{2}$ (reference), 25-30 and $\geq 30$; physical activity as $<2 \mathrm{~h} /$ week, $2-4$ (reference), and $>4$ h or 2-4 and $>4 \mathrm{~h}$ of strenuous activity; smoking status as never smoker (reference), ex-smoker, and smoker. We used the Danish Medical Birth Register to identify number of childbirths. Subjects were excluded if they had surgery for VV before baseline $(n=959)$ or did not report a job title $(n=261)$. We used Cox regression analysis adjusting for occupational exposures (time spent standing/walking, total daily load lifted).

Results: The study cohort comprised 38,030 individuals, $43 \%$ men and $57 \%$ women. Total follow-up time was 416,261 person-years. Mean age at baseline was 42.9 years. During follow-up 851 cases occurred, $28 \%$ male and $72 \%$ female. For men, age groups $\geq 45-56$ (HR 1.98 (CI 1.26-3.13)) and $>56$ (HR 2.18 (1.25-3.80)) were associated with and increased risk. For women, $<2 \mathrm{~h}$ of physical activity/week was associated with a decreased risk (HR 0.68 (CI 0.51-0.90)) as was smoking (HR 0.61 ((CI 0.49-0.76)). Childbirth was associated with risk of VV surgery in an exposure-response manner with HRs increasing from 1.38 to $1.65,2.15$, and 2.58 for 1 to $\geq 4$ children ( $p$ for trend $<10-3$ ); age did not affect risk significantly. For both genders BMI did not increase risk of VV.

Conclusions: The results add to the evidence that increasing age is a risk factor for $\mathrm{VV}$ among men, while childbirths are a risk factor among women. The examined lifestyle factors were not associated with an increased risk of VV. Actually physical activity $<2 \mathrm{~h} /$ week and smoking seemed protective among women.

\section{P-340}

Adipose tissue trans fatty acids and changes in body weight and waist circumference

Topic: Exposure - Lifestyle factors

Presenter: Camilla Plambeck Hansen

Camilla Plambeck Hansen 1), 2), 3); Tina L. Berentzen 3); Jane N. Østergaard 1), 2); Christina C. Dahm 1); Lars I. Hellgren 4); Erik B. Schmidt 2); Anne Tjønneland 5); Thorkild I.A. Sørensen 3), 6); Kim Overvad 1), 2); Marianne U. Jakobsen 1)

1) Section for Epidemiology, Department of Public Health, Aarhus University, Aarhus, Denmark; 2) Department of Cardiology, Center for Cardiovascular Research, Aalborg University Hospital, Aalborg, Denmark; 3) Institute of Preventive Medicine, Bispebjerg and Frederiksberg Hospitals - a part of Copenhagen University Hospital, Copenhagen, Denmark; 4) Center for Biological Sequence Analysis, Department of Systems Biology, Technical University of Denmark, Lyngby, Denmark; 5) Danish Cancer Society Research Center, Copenhagen, Denmark; 6) Novo Nordisk Foundation Center for Basic Metabolic Research, University of Copenhagen, Copenhagen, Denmark

Background: Previous studies have suggested that intake of trans fatty acids plays a role in the development of obesity and that the individual trans fatty acids may have divergent effects. For fatty acids not synthesized endogenously in humans, such as trans fatty acids, the proportions in adipose tissue usually correlate well with the dietary intake. Hence, using these biomarkers may provide a more accurate measure of habitual trans fatty acids intake than obtained with dietary questionnaires.

Objectives: Our objective was to investigate the associations between specific trans fatty acids in adipose tissue and subsequent changes in body weight and waist circumference.

Methods: The relative content of fatty acids in adipose tissue biopsies from a random sample of 996 men and women aged 50-64 years drawn from a Danish cohort study were determined by gas chromatography. Baseline data on body weight, waist circumference and potential confounders were available together with information on body weight and waist circumference 5 years after enrolment. The exposure measures were total trans octadecenoic acids (18:1t), 18:1 6-10t, vaccenic acid $(18: 111 \mathrm{t})$ and rumenic acid $(18: 29 \mathrm{c}, 11 \mathrm{t})$. The data were analysed using multiple regression with cubic spline modelling.

Results: The median proportion of total adipose tissue 18:1t was $1.17 \%$ (90\% central range: $0.72,1.74)$ in men and $1.13 \%(0.74$, 1.71 ) in women. No significant associations were observed between total 18:1t, 18:1 6-10t, vaccenic acid or rumenic acid and changes in body weight or waist circumference.

Conclusions: The present study does not suggest that the proportions of specific trans fatty acids in adipose tissue are associated with subsequent changes in body weight or waist circumference within the exposure range observed in this population.

\section{P-342}

The association between marital status and Body mass Index Trajectories.

Topic: Exposure - Lifestyle factors 


\section{Presenter: Tuba Mazhari}

\section{Tuba Mazhari 1); Mai Stafford 2); Anne Mcmunn 1)}

1) University College London, Department of Epidemiology and Public Health; 2) Medical Research Council National Survey of Health and Development

Background: The existing literature suggests that body mass index (BMI) tends to be higher for married compared with unmarried people. To date, few studies have investigated the influence of marital status on BMI trajectories.

Objective: The objective of this study was to investigate the association between marital status and BMI trajectories throughout adulthood.

Methods: Data were obtained from the MRC National Survey for Health and Development (1946 birth cohort). Measured heights and weights spanning 27 years at 4 times points since mid-adulthood (at ages 36, 43, 53 and 60-64) were used to create BMI trajectories. Multi-level models were used to estimate initial and age related changes in BMI for married compared with single, divorced and widowed respondents, stratified by sex. Age was centred at 43 . Marital status was defined as a time-varying covariate. Both men $(\mathrm{N}=1844)$ and women $(\mathrm{N}=1824)$ contributed data for an average of 3 occasions.

Results: Each 1 year increase in age was associated with an increase in BMI for married men $0.13 \mathrm{~kg} / \mathrm{m}^{2}$ (95\% CI 0.13, 0.14) and women $0.20 \mathrm{~kg} / \mathrm{m}^{2}(0.19,0.21)$. Compared with married men BMI gain for a divorced man was $0.02 \mathrm{~kg} / \mathrm{m}^{2}$ more per year. Being divorced was associated with lower initial BMI for women, by $0.43 \mathrm{~kg} / \mathrm{m}^{2}(-0.71$, -0.15 ). Compared with married women BMI gain for widows was $0.06 \mathrm{~kg} / \mathrm{m}^{2}$ lower each year

Conclusions: This study provides some longitudinal evidence for differences in weight change by marital status, pointing to the importance of marital status in shaping one's social environment in ways that can potentially affect trajectories of BMI. However, the magnitude of this effect is small, compared with the effect of socioeconomic factors for example, and is likely to represent at most modest, differences in the risk of developing cardiovascular disease.

\section{P-343}

Maternal antibiotic use during pregnancy and child development in the Danish National Birth Cohort

Topic: Exposure - Lifestyle factors

Presenter:

\section{Jessica Miller 1); Lars Henning Pedersen 2); Jørn Olsen 3)}

1) Department of Epidemiology, Fielding School of Public Health, University of California, Los Angeles, California; 2) Department of Obstetrics and Gynecology, Institute of Clinical Medicine, Aarhus University, Aarhus, Denmark; 3) Department of Public Health, Section for Epidemiology, Aarhus University, Aarhus, Denmark

Background: Specific maternal infections during pregnancy have been suggested to adversely affect fetal brain development. Our study examines maternal use of antibiotics during pregnancy, as a marker of infection, and child development, focusing on the achievement of developmental milestones at 6 and 18 months of age and on behavioral problems at 7 years of age, in the Danish National Birth Cohort. Objectives: To assess the association between the maternal use of antibiotics during pregnancy, as a marker of maternal infection, and the achievement of developmental milestones and behavioral problems in the child.

Methods: Participants were selected from the Danish National Birth Cohort, a nation-wide follow-up study of pregnant women and their offspring in Denmark. Information on antibiotic exposure was selfreported during two prenatal and one postnatal interview. Achievement of specific developmental milestones was based on mothers' self-reports in two postnatal interviews. Information on behavioral development, evaluated by the Strengths and Difficulties Questionnaire (SDQ), was self-reported by mothers during the follow-up interview when the child was 7 years old. Odds ratios (OR) and $95 \%$ confidence intervals $(95 \% \mathrm{CI}$ ) were estimated by logistic regression models for developmental milestones and by ordinal logistic regression models for the SDQ score.

Results: Self-reported maternal use of general antibiotics during pregnancy was not associated with achievement of developmental milestones but was associated with the SDQ score for behavioral problems (OR 1.23, $95 \%$ CI 1.08-1.39).

Conclusion: We found little evidence to support the influence of maternal antibiotic use in general on the achievement of developmental milestones in early childhood but maternal antibiotic use may be associated with behavioral development in early childhood.

\section{P-344}

Maternal infections during pregnancy and cerebral palsy: a population-based cohort study

Topic: Exposure - Lifestyle factors

Presenter: Elani Streja

Jessica Miller 1); Lars Henning Pedersen 2); Elani Streja 1); Bodil Hammer Bech 3); Marshalyn Yeargin-Allsopp 4); Kim Van Naarden Braun 4); Diana E. Schendel 4); Deborah Christensen 4); Peter Uldall 5); Jørn Olsen 3)

1) Department of Epidemiology, Fielding School of Public Health, University of California, Los Angeles, California; 2) Department of Obstetrics and Gynecology, Institute of Clinical Medicine, Aarhus University, Aarhus, Denmark; 3) Department of Public Health, Section for Epidemiology, Aarhus University, Aarhus, Denmark; 4) National Center on Birth Defects and Developmental Disabilities, Centers for Disease Control and Prevention, Atlanta, Georgia; 5) The Danish Cerebral Palsy Registry, National Institute of Public Health, Southern University, Denmark

Background: Cerebral palsy $(\mathrm{CP})$ is one of the most common motor disabilities in childhood with prevalence estimates close to 2 per 1000 live births in Denmark. Fetal exposures during gestation, such as maternal infections, have been associated with an increased risk for $\mathrm{CP}$ and other neurological disorders in the child. Neurological outcome studies often focus on in-patient hospital diagnosed infections with less attention given to infections diagnosed by primary care doctors outside of hospitals, or in outpatient clinical settings. Prescription data, as a marker of infection, provides the ability to study infections not requiring hospitalization otherwise excluded from hospital-based studies.

Objectives: To assess the association between maternal infections during pregnancy and the risk of congenital cerebral palsy in the child. Methods: Live-born singletons in Denmark between 1997 and 2003 were identified from the Danish National Birth Registry and followed from 1 year of life until 2008. Redemption of antibiotics from the National Register of Medicinal Product Statistics and maternal infections reported by the National Hospital Register were used as markers of infection during pregnancy. CP diagnoses were obtained from the Danish Cerebral Palsy Registry. Adjusted hazard ratios (aHR) and $95 \%$ confidence intervals $(95 \% \mathrm{CI})$ were estimated by Cox proportional hazard models.

Results: We followed 440,564 singletons and identified 840 children with congenital CP. Maternal genitourinary tract infections (aHR: 2.14, 
$95 \%$ CI 1.42-3.22) were associated with CP in all births, in term births (aHR: 1.88, $95 \%$ CI 1.10-3.20), in children with spastic CP (aHR:2.12, $95 \%$ CI 1.36-3.29), and among first born children (aHR:1.92, $95 \%$ CI 1.36-3.29). Overall, we found associations between maternal chorioamnionitis (aHR:4.03, $95 \%$ CI 1.01-16.1) and nitrofurantoin redemption (aHR:1.67, $95 \%$ CI 1.05-2.75) with CP. Among trimesterspecific exposures, risk for $\mathrm{CP}$ was associated with prescriptions redeemed in the first trimester for any antibacterials, beta-lactam antibacterials, and nitrofurantoin, an antibiotic commonly used for lower urinary tract infection; chorioamnionitis in the second trimester; and genitourinary tract infections in the third trimester.

Conclusion: Genitourinary tract infections, chorioamnionitis and antibiotic use during pregnancy were associated with increased risks of $\mathrm{CP}$, indicating some infections, correlates, or causes of infections present in prenatal life may be part of a causal pathway leading to $\mathrm{CP}$.

\section{P-345}

Predictors of sickness absence in pregnancy: preliminary results from a Danish cohort

Topic: Exposure - Lifestyle factors

Presenter: Mette Lausten Hansen

Mette Lausten Hansen 1); Ane Marie Thulstrup 1); Mette Juhl 2); Jette Kolding Kristensen 3); Cecilia Høst Ramlau-Hansen 4)

1) Danish Ramazzini Centre, Department of Occupational Medicine, Aarhus University Hospital, Denmark; 2) The Research Unit Women's and Children's Health, The Juliane Marie Centre, Rigshospitalet, Copenhagen, Denmark; 3) Section of General Practice, Department of Public Health, Aarhus University, Denmark; 4) Section of Epidemiology, Department of Public Health, Aarhus University, Denmark

Background: Increasing sickness absence among gainfully employed people is of rising concern in several European countries. Some Scandinavian studies indicate that sickness absence in pregnancy is going up, although there are no well-established medical grounds for this increase. Research in pregnancy-related sickness absence, its consequences and related risk-factors is very sparse.

Objectives: To investigate whether the following person-related factors are associated with sickness absence during pregnancy; parity (nulliparous/multiparous), age ( $<20$ years, $\geq 20$ to $<30$ years, $\geq 30$ to $<40$ years, $\leq 40$ years), fertility treatment (yes/no), body mass index (BMI $\left(\mathrm{kg} / \mathrm{m}^{2}\right) ;<18.5, \geq 18.5$ to $\left.<25, \geq 25-30, \geq 30\right)$, time to pregnancy ((TTP); 0 months, 1-2 months, 3-5 months, 6-12 months, $>12$ months) and engagement in physical exercise (yes/no).

Methods: We used data from The Danish National Birth Cohort (DNBC), which contains information on 100,418 pregnancies. The women were interviewed around pregnancy week 12-16 and 30, and 6 months postpartum from 1997 until 2004. We analyzed 38,551 pregnancies, in which the mother had participated in all 3 interviews, was employed during pregnancy, was singleton pregnant, and had applicable data on the person-related factors. The outcome measure was self-reported sickness absence during pregnancy for more than 3 consecutive days (yes/no). Data were analysed by an adjusted logistic regression model with mutual adjustment of the independent variables. Results: A number of factors were associated with increased odds of sickness absence in pregnancy; multiparity (OR $1.54(95 \%$ CI 1.47-1.61)), age younger than 20 years (OR $3.73 \quad(95 \%$ CI 1.90-7.35)), receiving fertility treatment (OR $1.30 \quad(95 \%$ CI $1.18-1.43)$ ), obesity (OR 1.70 (95\% CI 1.57-1.84)), underweight (OR 1.18 (95\% CI 1.06-1.31) and TTP of $>12$ month (OR 1.16 (95\% CI 1.08-1.26)). Age above 30 years and engagement in physical exercise were associated with reduced odds of sickness absence (OR > 30 years: 0.66 (95\% CI 0.63-0.69)) and (ORphysical exercise: 0.70 (95\% CI 0.67-0.73)).

Conclusions: These results suggest that parity, age, fertility treatment, BMI, TTP, and physical exercise are predictors of sickness absence in pregnancy. However, the results should be interpreted cautiously, as important risk factors such as chronic diseases, socioeconomic status, and field of occupation not yet have been adjusted for. In future analyses, we will further apply a continuous measure of the outcome sickness absence.

\section{P-346}

\section{Binge drinking in Spain}

Topic: Exposure - Lifestyle factors

Presenter: Hosanna Soler-Vila

Hosanna Soler-Vila 1), 2); Luz M. León-Muñoz 1), 3); Iñaki Galán 4); Pilar Guallar-Castillón 1), 3); José Valencia-Martín 1), 3); Fernando Rodríguez-Artalejo 1), 3)

1) Department of Preventive Medicine and Public Health, School of Medicine, Universidad Autónoma de Madrid/IdiPAZ, Madrid, Spain; 2) Department of Epidemiology and Public Health, Miller School of Medicine, University of Miami, Miami, FL, USA; 3) CIBER of Epidemiology and Public Health (CIBERESP), Madrid, Spain; 4) National Centre for Epidemiology. Instituto de Salud, Carlos III. Madrid, Spain

Background: Eastern Europe's alcohol intake, the highest in the world, is characterized by consumption of spirits and binge drinking (BD). Data on BD in Mediterranean countries are still scarce but existing evidence points to patterns approaching those of Eastern Europe. This is the first nationally representative study to describe the prevalence and distribution of BD in Spain. Additionally, we investigate the number and characteristics of $\mathrm{BD}$ episodes and the joint distribution of $\mathrm{BD}$ with heavy regular drinking and problem-drinking (PD).

Methods: Cross-sectional study conducted in 2008-2010 among 9,130 individuals representative of the population aged 18-64 years in Spain. We measured alcohol consumption with a validated dietary history. BD was defined as intake of $\geq 80 \mathrm{~g}$ of alcohol in men $(\geq 60 \mathrm{~g}$ in women) during any drinking occasion in the previous month, with $\geq 3 \mathrm{BD}$ episodes discriminating between frequent and sporadic BD. The threshold between average moderate and heavy alcohol intake in the previous year was set at $\geq 40 \mathrm{~g}$ of alcohol/day in men $(\geq 24 \mathrm{~g}$ in women). We defined PD as a CAGE questionnaire score $\geq 2$. Statistical analyses included multivariate logistic regression to adjust for main potential confounders.

Results: BD prevalence was $7.2 \%(95 \%$ confidence interval 6.5-7.9\%). Prevalence was higher in men $(10.0 \%)$ than women (4.2\%) and highest among 18-24 year old men and women (19.5 and $10.3 \%$, respectively). During the latest BD episode, men consumed an average of $114 \mathrm{~g}$ of alcohol versus $85.3 \mathrm{~g}$ in women, with spirits accounting for 65.2 and $66.2 \%$ of total intake, respectively. The average number of monthly BD episodes was 2.3 in men and 2.0 in women, and increased with age. Over $80 \%$ of binge drinkers reported average moderate drinking; even as $25 \%$ of binge drinkers reported frequent $\mathrm{BD}$ and $22.8 \%$ reported $\mathrm{PD}$. As expected, male frequent binge drinkers were more likely than sporadic ones to report average heavy drinking and PD; nevertheless, both frequent and sporadic BD were strongly associated with PD regardless of average alcohol intake. Of all problem-drinkers $80 \%$ were male, $50 \%$ were 25-44 year-olds, $82 \%$ reported moderate regular consumption, and $48.8 \%$ practiced BD.

Conclusions: Binge drinking prevalence in Spain is moderately high, especially among younger males, male average heavy drinkers, and 
problem drinkers of both sexes. However, prevention interventions should consider that the majority of binge drinkers are 18-34 yearolds with average moderate intake and no problem-drinking traits. Funding: Grant 06/2010 from the Plan Nacional sobre Drogas, Ministry of Health (Spain).

\section{P-347}

Folic acid knowledge and adequate intake in the Sint Vincentius Hospital in Suriname: preliminary results from the Perisur project

Topic: Exposure - Lifestyle factors

Presenter: Ashna Mohangoo

Ashna Mohangoo 1), 2); Lynn Leermakers 1); Manodj Hindori 3)

1) TNO, Department Child Health, Leiden, The Netherlands; 2) Anton de Kom University of Suriname, Faculty of Medical Sciences, Paramaribo, Suriname; 3) Sint Vincentius Hospital, Paramaribo, Suriname

Background: Adequate folic acid intake during the periconceptional period prevents development of congenital anomalies, including neural tube defects and congenital heart defects. Folate deficiency during pregnancy may also increase the risk of preterm delivery, fetal growth retardation, as well as pregnancy complications, such as preeclampsia.

Objectives: To study determinants of knowledge about folic acid and adequate use of folic acid among pregnant women in the Sint Vincentius Hospital in Suriname and identify target groups for primary prevention.

Methods: Pregnant women who visited the Sint Vincentius Hospital for routine antenatal care and women at the maternity ward were asked to complete a questionnaire on folic acid knowledge and use and social demographic characteristics between January and March 2013. Multivariate logistic regression analyses were conducted to study independent determinants of folic acid knowledge expressed as odds ratios (OR) with $95 \%$ confidence intervals [95\% CI].

Results: 282 women responded to the survey (99\%). Of them, 208 had knowledge about folic acid (74\%) and $61 \%$ were informed by their general practitioner. In total 176 used folic acid (62\%), but only 42 women $(14 \%)$ used folic acid during the periconceptional period. Women without knowledge of folic acid were more often non-native Surinamese and not insured. They more often had a Chinese or Indigenous ethnicity and a lower educational level. Multivariate logistic regression analyses revealed that being insured [OR 3.7; $95 \% \mathrm{CI}$ 1.7-8.1] and having a higher educational level [OR 3.2; $95 \%$ CI 1.5-6.7] were independently and positively correlated with folic acid knowledge. Conclusions: Although three-quarters of women had some knowledge about folic acid in the Sint Vincentius Hospital, periconceptional intake of folic acid was very low. Non-insured and less educated women were at higher risk of having insufficient knowledge and are therefore important target groups for educational campaigns.

\section{P-348}

The beginning of professional life as a potential key timing to act on work-related psychosocial factors that influence musculoskeletal pain

Topic: Exposure - Lifestyle factors

Presenter: Sara Lourenço

Sara Lourenço 1), 2); Daniela Simões 1), 2); Raquel Lucas 1), 2); Henrique Barros 1), 2)
1) Department of Clinical Epidemiology, Predictive Medicine and Public Health of the University of Porto Medical School; 2) Institute of Public Health of the University of Porto

Background: The impact of work-related psychosocial contexts on the onset of musculoskeletal pain has been widely explored. Workrelated mechanical factors are related to the onset of musculoskeletal acute pain, whereas work psychosocial characteristics have a particular impact on maintaining musculoskeletal pain. However, most studies have been performed in long-term workers, where musculoskeletal pain is already established, leaving little room for preventive strategies. It is of great relevance to explore the role of work-related psychosocial factors on the onset of musculoskeletal pain in samples of young adults where work is a recent exposure and therefore may be more amenable to intervention.

Objectives: To assess the early influence of work-related psychosocial factors on the report of musculoskeletal pain in a populationbased cohort of 21-year-old adults.

Methods: The EPITeen cohort was assembled during the 2003/2004 school year in public and private schools of the city of Porto (Portugal) providing teaching to adolescents born in 1990. The 21-year-old followup is ongoing since August $2011(\mathrm{n}=1509,51.8 \%$ women). Participants who had worked in the previous 12 months were asked to complete the Job Content Questionnaire (JCQ). This instrument evaluates the subjective perception of the main occupational psychosocial factors: skill discretion, decision authority, decision latitude and psychological demands required at work. Musculoskeletal pain was evaluated using the Nordic Musculoskeletal Questionnaire that gathers data on pain in nine body regions (neck, shoulders, elbows, wrists/ hands, upper back, lower back, hips/thighs/buttocks, knees and ankles/ feet) in the preceding 12 months. Subjects were categorized according to the presence of pain in each anatomical region. Mean scores of workrelated psychosocial factors of JCQ and the corresponding $95 \%$ confidence intervals were obtained using ANOVA.

Results: From 1509 participants, $33.5 \%(\mathrm{n}=506)$ had an occupation during at least 1 month at full- or part-time ( $>15 \mathrm{~h} /$ week). Mean scores (standard-deviation) of skill discretion, decision authority, decision latitude and psychological demands related to work were, respectively, 32.2 (6.8), 33.6 (6.8), 28.5 (4.7) and 65.9 (12.3). The most prevalent anatomical regions reported as painful were lower back $(52.6 \%)$ and neck (37.3\%). Mean scores of JCQ were not significantly different according to the presence of pain in each anatomical region, except for low back, where individuals with low back pain have higher psychological job demands perception when compare to those without low back pain (29.0 vs. $28.8, p=0.009)$. Conclusions: At the beginning of professional life, adverse workrelated psychosocial factors seem not to interfere substantially with the onset of musculoskeletal pain, even though an association with low back pain is apparent. These findings suggest a wide scope for the primary prevention of negative effects of work-related factors on the onset of chronic musculoskeletal pain in early adulthood.

\section{P-349}

Is the association between child's screen time and cardiometabolic risk mediated by snacking and physical activity?

Topic: Exposure - Lifestyle factors

Presenter: NE Berentzen

NE Berentzen 1); H Smit 2); L van Rossem 2); M Kerkhof 3); GH Koppelman 4); Bert Brunekreef 2), 5); HC Boshuizen 6), 7); AH Wijga 1)

1) Center for Nutrition, Prevention, and Health Services, National Institute for Public Health and the Environment (RIVM), Bilthoven, 
The Netherlands; 2) Julius Center for Health Sciences and Primary Care, University Medical Center Utrecht (JC/UMCU), The Netherlands; 3) Department of Epidemiology, UMCG, GRIAC Research Institute, University of Groningen, The Netherlands; 4) Department of Pediatric Pulmonology and Pediatric Allergology, Beatrix Children's Hospital, Groningen Research Institute for Asthma and COPD, University Medical Center Groningen, University of Groningen, The Netherlands; 5) Institute for Risk Assessment Sciences (IRAS), Division of Environmental Epidemiology, Utrecht University, The Netherlands; 6) Center for Information Technology and Methodology Consultancy, National Institute for Public Health and the Environment, Bilthoven, The Netherlands; 7) Division of Human Nutrition, Wageningen University, The Netherlands

Background: Screen time, which refers to time spent in front of a TV or computer, is a common sedentary behavior in adolescents, and is positively related to weight status and metabolic syndrome. The extent to which this relation is mediated by snacking and lack of physical activity is yet unknown.

Objectives: We investigated mediation by snacking and moderate physical activity (PA) in the association between screen time and cholesterol ratio, via waist circumference. For this aim, we used structural equation modeling (SEM), a statistical approach where hypothesized multivariate relationships can be drawn in a path diagram and subsequently estimated and tested.

Methods: We analysed data of 797 children who completed a questionnaire at age 11 years and underwent a medical examination at age 12 years. Screen time (hours/week), snacking (portions/day), moderate PA (days/wk active for at least $1 \mathrm{~h}$ ) and pubertal status were self-reported. During the medical examination, height and waist circumference (WC) were measured (in $\mathrm{cm}$ ) and serum total-to-highdensity-lipid-protein cholesterol (TC/HDLC) ratio was determined.

Results: Mean screen time was 12.9 (standard deviation 8.1) hours/ week. The structural equation model with screen time mediated by snacking and PA showed acceptable fit on three measures, Chi square (0.077, $d f=1, p=0.78)$, Comparative Fit Index (1.00), RMSEA $(0.00, p=0.92)$. Increase of $1 \mathrm{~h} /$ week screen time was significantly associated with $0.09 \mathrm{~cm}(95 \%$ CI 0.03-0.15) larger WC. Screen time was significantly associated with higher snacking but not with PA, and these variables were not mediators in the relation between screen time and WC. Increase of $1 \mathrm{~cm} \mathrm{WC}$ was significantly associated with 0.05 (95 \% CI 0.04-0.06) higher TC/HDLC ratio. No association with TC/HDLC ratio was found for screen time, snacking or PA, although reducing screen time may improve TC/HDLC ratio indirectly by reducing WC. After adjusting the model for gender, age, height, puberty and maternal education, the association of screen time with WC was lowered to $0.04 \mathrm{~cm}(95 \% \mathrm{CI}-0.01$ to 0.10$)$ larger WC per $1 \mathrm{~h} /$ wk screen time, and was no longer significant.

Conclusions: Structural equation analyses showed that snacking or PA was not mediating the association between increased screen time and larger WC; and suggested that screen time may affect TC/HDLC ratio indirectly by first influencing WC. SEM provides insight in the role of mediators in relationships between several lifestyle factors.

\section{P-351}

Characteristics of dietary patterns behind change in waist circumference and body weight

Topic: Exposure - Lifestyle factors

Presenter: Jane Nautrup Østergaard

Jane Nautrup Østergaard 1), 2); Christina Catherine Dahm 2); Anders Gorst-Rasmussen 1); Tina L. Berentzen 3); Lotte Maxild Mortensen 1), 2); Anne Tjønneland 4); Marianne Uhre Jakobsen 2); Thorkild IA Sørensen 3); Kim Overvad 1), 2)
1) Department of Cardiology, Centre for Cardiovascular Research, Aalborg University Hospital, Aalborg, Denmark; 2) Section for Epidemiology, Department of Public Health, Aarhus University, Aarhus, Denmark; 3) Institute of Preventive Medicine, Bispebjerg and Frederiksberg Hospitals - a part of Copenhagen University Hospital, Copenhagen, Denmark; 4) Danish Cancer Society Research Centre, Copenhagen, Denmark

Background: Although weight loss in most observational studies has been associated with an increased risk of morbidity and premature death, a recent study has found that reducing waist circumference was associated with reduced mortality.

Objective: The aim of our study was to investigate whether specific dietary patterns could characterize changes in anthropometry, especially reductions in waist circumference and body weight.

Methods: Data on 26,491 healthy men and women from the Danish Diet, Cancer and Health study was descriptively analyzed. Waist circumference and weight were assessed in 1993-1997 and in 1999-2002. Dietary information was collected through a food frequency questionnaire in 1993-1997. A multi-dimensional graphic representation (so-called spider plots) of relative food intakes was used to illustrate differences in dietary patterns by waist change and weight change group within each sex.

Results: No consistent findings were observed between food choices and changes in waist circumference or weight loss. However, some food choice tendencies were seen with regard to weight gain among overweight men and women: a higher reported intake of sugar, chocolate and confectionary, snack and chips, and soft drinks and juices appeared to characterize those who gained in weight compared with the overall sex specific mean among the overweight.

Conclusions: We did not find food patterns characterizing participants who lost in waist circumference or body weight, but we did find that those overweight who gained in weight reported a higher consumption of the usual suspects such as sugar, chocolate and confectionary, snack and chips, and soft drinks and juices, than the overall sex specific mean intake among overweight participants.

\section{P-352}

Maternal alcohol intake prior to and during pregnancy and risk of adverse birth outcomes: evidence from a British cohort

Topic: Exposure - Lifestyle factors

Presenter: Camilla Nykjaer

Camilla Nykjaer 1); Nisreen Alwan 1); Darren Greenwood 2); Nigel Simpson 3); Janet Cade 1)

1) Nutritional Epidemiology Group, School of Food Science and Nutrition, University of Leeds, Leeds, LS2 9JT, UK; 2) Division of Biostatistics, Centre for Epidemiology and Biostatistics, University of Leeds, Leeds, LS2 9JT, UK; 3) Department of Obstetrics and Gynaecology, University of Leeds, Leeds, UK

Background: The evidence linking low maternal alcohol consumption and birth outcomes is inconsistent. This is reflected in the different country-level guidelines regarding alcohol consumption during pregnancy.

Objectives: To investigate the association between alcohol intake prior to and during pregnancy with both birth weight and preterm birth, and to examine their association with the timing of exposure. Methods: Analysis of prospective data from the Caffeine \& Reproductive Health Study (CARE), a birth cohort including 1303 pregnant women from Leeds, UK aged 18-45 years. Three self-administered questionnaires assessed alcohol consumption prior to pregnancy and for each trimester separately. Frequency of weekly alcohol consumption was split into categories of intake ( $\leq 2$ and $>2$ units/week) 
including a non-drinking category as the referent. This was related to birth size and preterm delivery in multivariable regression models, adjusting for confounders including salivary cotinine as an objective biomarker of smoking status.

Results: 1,153, 1,135, 793 and 377 deliveries had data available on birth outcomes and alcohol consumption in the period up to pregnancy and for the 3 trimesters respectively. Nearly $2 / 3$ of women prior to pregnancy and over half in the 1st trimester reported alcohol intakes above the Department of Health (UK) recommendation of $\leq 2$ units/week. Compared to non-drinkers, the adjusted change in customised birth centile was -7.7 (95\% confidence interval (CI) $-12.8,-2.6),-8.2$ (95\% CI $-12.6,-3.7),-6.4$ (95\% CI -11.8, $-1.1)$ and $-1.8(95 \% \mathrm{CI}-9.3,5.7)$ for intakes above 2 units/week in the period before pregnancy, trimester 1,2 and 3 respectively. A similar association could be observed for intakes below 2 units/week. Compared to non-drinkers, the adjusted change in birth weight were $-106 \mathrm{~g}(95 \% \mathrm{CI}-184,-28),-100 \mathrm{~g}(95 \%$ CI $-166,-35)$, $-100 \mathrm{~g}(95 \% \mathrm{CI}-176,-22)$ and $-50 \mathrm{~g}(95 \% \mathrm{CI}-161,-60)$ for intakes above 2 units/week in the period before pregnancy, trimester 1,2 and 3 respectively. The association with small for gestational age $(<10$ th centile) and preterm birth was strongest in trimester 1 , with adjusted odds ratios of 2.0 (95\% CI 1.2, 3.4) and 3.5 (95\% CI 1.1, 11.2) respectively for intakes above 2 units/week.

Conclusion: Maternal alcohol intake before and during pregnancy was associated with adverse birth outcomes, with the first trimester and prior to pregnancy being the most vulnerable periods of exposure.

\section{P-353}

\section{ADH1B and ADH1C genotype, alcohol consumption and biomarkers of liver function: Findings from a Mendelian Randomization study in 58,313 Danes}

Topic: Exposure - Lifestyle factors

Presenter: N Maneka De Silva

Debbie Lawlor 1); Marianne Benn 2); Luisa Zuccolo 1); N Maneka De Silva 1); Anne Tybjaerg-Hansen 2); George Davey Smith 1); Børge Nordestgaard 2)

1) MRC Centre for Causal Analyses in Translational Epidemiology, School of Social and Community of Medicine, University of Bristol, UK; 2) The Copenhagen General Population Study, Herlev Hospital, Copenhagen, Denmark

Background: Alcoholic liver disease is a major global health problem. The effects of lifetime differences in alcohol consumption in the general population are unclear, and is difficult to determine because of reporting bias and potential residual confounding.

Objectives: To determine this effect using a Mendelian randomization study

Methods: We used variants in $\mathrm{ADH} 1 \mathrm{~B}$ and $\mathrm{ADH} 1 \mathrm{C}$ genes as instrumental variables (IV) to estimate the causal effect of long-term alcohol consumption, across the whole of its distribution, on alanine aminotransferase (ALT), -glutamyl transpeptidase (-GT), alkaline phosphatase (ALP), bilirubin and prothrombin action. Analyses were undertaken on 58,313 Danes (mean age 56).

Results: In both confounder adjusted multivariable and IV analyses greater alcohol consumption, amongst those who drank any alcohol, resulted in higher ALT [mean difference per doubling of alcohol consumption: $3.4 \%(95 \%$ CI 3.1, 3.7) from multivariable analyses and $3.7 \%(-4.5,11.9)$ from IV analyses; $p$ value for difference in these results $=0.62]$ and $-\mathrm{GT}[8.2 \%(7.8,8.5)$ and $6.8 \%(-2.8$, 16.5); Pdifference $=0.30]$. Results from the multivariable analyses suggested a weak inverse association with ALP $[-1.5 \%(-1.7$,
$-1.3)$ ], which differed from the strong positive association with IV analyses $[11.6 \%(6.8,16.4) ; P$ difference $<0.0001]$. For bilirubin and prothrombin action there were very weak positive multivariable associations that were statistically consistent with the IV results.

Conclusions: As ADH1B and ADH1C will influence alcohol consumption across the life-course from the time that someone first starts to drink, these results suggest that greater lifetime consumption of alcohol, in a dose-response way, adversely affects the liver as indicated by higher ALT, -GT and ALP.

\section{P-354}

Family sociodemographic factors at birth as determinants of body mass index development of the offspring until 7 years of age

Topic: Exposure - Lifestyle factors

Presenter: Susanna Lehtinen-Jacks

Susanna Lehtinen-Jacks 1), 2); Liisa Uusitalo 2); Hanna-Mari Takkinen 1); Jaakko Nevalainen 3); Mike G Kenward 4); Riitta Veijola 5); Olli Simell 6); Jorma Ilonen 7), 8); Mikael Knip 9), 1); Suvi Virtanen 1), 2)

1) School of Health Sciences, University of Tampere, Tampere, Finland; 2) Nutrition Unit, National Institute for Health and Welfare, Helsinki, Finland; 3) Department of Mathematics and Statistics, University of Turku, Turku, Finland; 4) London School of Hygiene and Tropical Medicine, London, UK; 5) Department of Pediatrics, Institute of Clinical Medicine, University of Oulu, Oulu, Finland; 6) Department of Pediatrics, University of Turku, Turku, Finland; 7) Immunogenetics Laboratory, University of Turku, Turku, Finland; 8) Department of Clinical Microbiology, University of Eastern Finland, Kuopio, Finland; 9) Children's Hospital, University of Helsinki and Helsinki University Central Hospital, Helsinki, Finland; 10) Department of Pediatrics, Tampere University Hospital, Tampere, Finland

Background: Associations between sociodemographic factors and obesity are less well known in childhood than in adulthood. Particularly little is known about when sociodemographic disparities evolve.

Objectives: To assess associations of parental education, socioeconomic status (SES) and place of residence with BMI development of the offspring from birth to 7 years.

Methods: Subjects in this birth cohort were born in 1997-2004 in Pirkanmaa, Finland, have HLA-conferred susceptibility to type 1 diabetes (14\% of the total population) and participate in the Type 1 Diabetes Prediction and Prevention Study. Anthropometrics were measured every 3-12 months. Subject-specific BMI-for-age curves $(\mathrm{n}=3,911)$ were estimated using mixed model regression splines. Information on maternal and paternal education, occupation and home municipality came from parental reports. The Statistical Grouping of Municipalities and the Classification of Occupations by Statistics Finland were used to determine the degree of urbanisation of the home municipality and parental SES.

Results: In the univariate analyses, sons of parents living in rural areas had higher BMI than other boys. The difference to boys with an urban origin was $+0.3 \mathrm{~kg} / \mathrm{m}^{2}$ at the age of 1 year, and $+0.5 \mathrm{~kg} / \mathrm{m}^{2}$ at 6 years. A similar, although not statistically significant, association was observed in girls. Parental education and SES were not associated with BMI development of the offspring.

Conclusions: Rural place of residence at the time of birth had a solid association with higher offspring BMI, especially in boys. Socioeconomic factors were not associated with childhood BMI development from birth to 7 years. 


\section{P-356}

Determinants of quality-of-life among a Portuguese sample of adolescents

Topic: Exposure - Lifestyle factors

Presenter: Odete Amaral

Odete Amaral 1); Carlos Pereira 1); Nélio Veiga 1), 2); Claudia Chaves 1); Marco Baptista 1); Paula Nelas 1); Manuela Ferreira 1); Inês Coelho 3)

1) CI\&DETS - Polytechnic Institute of Viseu; 2) Department of Health Sciences - Universidade Católica Portuguesa; 3) USF Grão Vasco-Viseu

Background: Adolescents' health has become an increasingly important issue and the perception of their health related quality-oflife (HRQoL) are influenced by socio-demographic factors and lifestyle habits.

Objectives: The aim of this study was to analyze the determinants of HRQoL in a Portuguese sample of adolescents.

Methods: A sample of 309 adolescents (66.9 \% female gender) aged 11-19 years old, attending a public school in Sátão, Portugal, was enrolled in this cross-sectional study. A self-administered questionnaire was used to assess social and demographic variables that included the 12-Item Short Form of Medical Outcomes Study (SF12) to assess HRQoL grouped in physical and mental dimensions (lower scores, better quality-of-life). We used the qui-square test to compare proportions and the Kruskal-Wallis test to compare continuous variables.

Results: The total scores of HRQoL were associated with depressive symptoms (yes $=30.80 \pm 2.60$ vs. no $=33.42 \pm 2.97, p<0.01$ ). The total scores of HRQoL were not associated with gender (female $=33.58 \pm 2.81$ vs. $33.39 \pm 3.26, p=0.62$ ), neither were the mental dimensions (female $=131.35 \pm 13.37$ vs. $130.42 \pm$ $15.53, p=0.61$ ) and physical dimensions (female $=183.89 \pm 18.72$ vs. $182.59 \pm 21.74, p=0.61)$. Total scores of HRQoL were also not associated with age $(11-13$ years $=34.00 \pm 3.41 ; \quad 14-16$ years $=$ $33.59 \pm 3.22 ; 17-20$ years $=33.25 \pm 2.60, \quad p=0.27)$, residence area (rural $=33.66 \pm 3.01$ vs. urban $=33.24 \pm 2.74, p=0.26$ ); alcohol consumption (yes $=33.03 \pm 2.68$ vs. no $=33.76 \pm 3.13$, $p=0.06$ ), coffee consumption (yes $=33.15 \pm 3.11$ vs. no $=$ $33.77 \pm 2.88, p=0.10$ ); smoking habits (yes $=33.01 \pm 2.56$ vs. no $=33.72 \pm 3.12, p=0.08$ ); sports practice (yes $=33.53 \pm 3.17$ vs. $33.60 \pm 2.60, p=0.84$ ) and insomnia (yes $=32.44 \pm 2.59$ vs. $33.08 \pm 2.38, p=0.24)$.

Conclusions: The impairment of quality-of-life in adolescents was not associated with socio-demographic variables, but highly associated with depressive symptomatology.

\section{P-357}

Physical inactivity and burden metabolic syndrome diseases in Brazilian adults

Topic: Exposure - Lifestyle factors

Presenter: Larissa Loures Mendes

Fernanda Penido Matozinhos 1); Crizian Saar Gomes 1); Alexandra Dias Moreira 1); Larissa Loures Mendes 2); Milene Cristine Pessoa 1); Gustavo Velásquez-Meléndez 1); Débora Carvalho Malta 3)

1) Universidade Federal de Minas Gerais; 2) Universidade Federal de Juiz de Fora; 3) Secretaria de Vigilância em Saúde, Ministério da Saúde,
Background: The prevalence of the metabolic syndrome (MS), a cluster of central obesity, hyper/dyslipidemia, hyperglycemia, and hypertension is constantly increasing worldwide. Physical inactivity, unhealthy nutrition and obesity, characteristics related to modern lifestyle, are factors considered responsible for MS, although the exact mechanisms of its development are not completely understood. Objective: This study was conducted to identify the association between physical inactivity and burden metabolic syndrome diseases (BMSD) in an urban Brazilian population.

Methods: A cross-sectional telephone survey was conducted with 3,419 adults $(46.03 \%$ men and $53.97 \%$ women, mean age 41.7 years) in the urban area of Belo Horizonte, Brazil. Data from the telephone-base Brazilian surveillance system for chronic diseases (VIGITEL-2008/2010) was used. Burden metabolic syndrome diseases (BMSD) was defined as the self-reported of at least two of the following factors: diabetes, dyslipidemia, hypertension and obesity. Physical activity (PA) indicators were analyzed in free time (leisure), work, home and transportation and inactivity indicators considered all PA domains. Sociodemographic, health status and lifestyle habits were also used. Odds ratios (OR) and $95 \%$ confidence intervals were estimated by multivariate logistic regression.

Results: In this sample, $13.53 \%$ of participants are physically inactive and the prevalence of BMSD in the sample was $15.61 \%$. Physical inactivity was independently associated with BMSD $(\mathrm{OR}=1.37 ; 95 \% \mathrm{CI}: 1.04$ to 1.80$)$, adjusted to age, schooling, bad health behaviors and gender.

Conclusion: In this sample, BMSD is a significant public health problem. The evidence from this study shows that physical inactivity was independently associated with metabolic syndrome.

\section{P-358}

Comparison of seventh day adventist mortality with a general population in the Espírito Santo state, Brazil

Topic: Exposure - Lifestyle factors

Presenter: Elizabete Regina Araújo Oliveira

Elizabete Regina Araújo Oliveira 1); Ana Paula Costa Velten 1); Nágela Valadão Cade 1); Cintya Schultz de Oliveira 1)

\section{1) Federal University of Espírito Santo, Brazil}

Noncommunicable diseases are the leading cause of death in Brazil and worldwide, mainly cardiovascular diseases, cancer, diabetes and respiratory diseases. Studies have found that a healthy lifestyle is associated with reduced mortality from noncommunicable diseases. The Seventh-day Adventists have been researched for their recommendations about lifestyle: having a healthy diet, preferably vegetarian, avoid caffeine consumption, physical activities regularly, and abstain from tobacco, alcohol and other drugs. Surveys in many countries show that the Seventh-day Adventists have lower mortality and greater longevity when compared to the general population. This study aimed to compare the mortality profile of Seventh-day Adventists with general population in the period 2003-2009 in the state of Espírito Santo, Brazil. This was a descriptive study that studied all deaths in the state during the period covered by separating them into two groups: Adventist and the general population.The separation was performed by searching the names of the deceased Adventists, provided by the administration of the church, in the state bank of Mortality Informations System. Groups of deaths were reported and distributed by the $10^{\circ}$ chapter of the International Classification of Diseases. Was also checked the average years lived in groups and Standardized Mortality Ratios (SMR) and confidence intervals of $95 \%$. The results show that Adventists die more often in older age groups relative to general population. The main causes of death for the Adventists were the circulatory diseases $(34.45 \%)$, 
neoplasms $(20.06 \%)$ and external causes $(9.87 \%)$. For the general population the circulatory diseases were also the leading cause of death $(31.70 \%)$, followed by external causes $(18.57 \%)$ and neoplasms $(15 \%)$. Adventists had 4.57 years on average over the years lived in the general population. The SMR of Adventists were 55.87 (40.99-74.38) for the circulatory diseases, 48.59 (24.3-86.84) for the respiratory diseases, $15.48(7.31-28.76)$ for the digestive diseases, and 41.3 (20.91-73.18) for the external causes. It was concluded that although no one knows the extent of health recommendations adoption by the Adventist who died there is evidence that the recommendations have beneficially influenced the mortality profile of the same relative the general population.

\section{P-359}

Consumption of sugar-sweetened beverages and the burden metabolic syndrome diseases in adults

Topic: Exposure - Lifestyle factors

Presenter: Larissa Loures Mendes

Crizian Saar Gomes 1); Alexandra Dias Moreira 1); Fernanda Penido Matozinhos 1); Milene Crsitine Pessoa 1); Larissa Loures Mendes 2); Gustavo Velásquez-Meléndez 1); Déborah Carvalho Malta 3)

1) Universidade Federal de Minas Gerais; 2) Universidade Federal de Juiz de Fora; 3) Secretaria de Vigilância em Saúde, Ministério da Saúde,

Background: The soft drink intake has increased worldwide and its high consumption is associated with the development of metabolic syndrome.

Objective: To estimate the association between the consumption of sugar-sweetened beverages (SSB) and the burden metabolic syndrome diseases (BMSD).

Methods: A cross-sectional telephone survey was conducted with 3419 adults $(46.03 \%$ men and $53.97 \%$ women, mean age 41.7 years) in the urban area of Belo Horizonte, Brazil. Data from the telephone-base Brazilian surveillance system for chronic diseases was used (VIGITEL - 2008/2010). Burden Metabolic syndrome diseases were defined as the self-reported of at least two of the following factors: diabetes, dyslipidemia, hypertension and obesity. The intake of $\geq 5$ days/week soft drinks and artificial juices was assessed. Sociodemographic, self-reported health status and lifestyle habits were also used. Odds ratios (OR) and $95 \%$ confidence intervals were estimated by multivariate logistic regression.

Results: In this sample, $24.07 \%$ of participants consumed $\geq 5$ days/ week sugar-sweetened beverages (SSB). The prevalence of BMSD in the sample was $15.61 \%$. The prevalence of BMSD in people who consumed SSB ( $\geq 5$ days/week) was lower than people didn't consume the beverages (OR 0.53 ; $95 \%$ CI $0.39-0.71$ ) 5 or more days per week. The final model was also adjusted for physical activity, education, marital status, poor delf-reported health and poor consumption of fruits and vegetables.

Conclusion: These results indicate possibly reverse causality, since people with MS, especially obese individuals, may modify SSB consumption due to nutritional status or heath professional advice.

\section{P-360}

Changes in smoking habits in Brazilian elderly: SABE Cohort Study, São Paulo, Brazil

Topic: Exposure - Lifestyle factors
Presenter: Gabriela Arantes Wagner

Gabriela Arantes Wagner 1); Maria Lúcia Lebrão 1);

Yeda Aparecida de Oliveira Duarte 2);

Dirce Maria Trevisan Zanetta 1)

1) Department of Epidemiology, School of Public Health, University of São Paulo, Brazil; 2) Medical-Surgical Nursing Department, School of Nursing, University of São Paulo, Brazil

Background: Smoking stands out among the habits that offer higher health risk because it is the main cause of preventable death in the world, especially in relation to cardiovascular disease. Among the elderly the effects of smoking may be related to longer exposures to cigarettes without filter and with high levels of nicotine than cohorts of later generations, which makes this population more susceptible to damage caused by cigarette.

Objectives: To verify changes in smoking habits in an elderly population-based sample followed for 10 years and possible relations with the incidence of chronic diseases and changes of sociodemographic characteristics.

Methods: A prospective Saúde, Bem-Estar e Envelhecimento (SABE [Health, Wellbeing and Aging]) cohort study conducted in 2000, 2006 and 2011 in the City of São Paulo, Brazil. 2,143 individuals aged 60 years old or older were selected through multi-stage sampling in the year 2000 (baseline), 1,115 individuals belonging to the follow-up cohort were evaluated in 2006 (second wave). The third wave was carried out in 2011, when 746 elders were re-interviewed. The smoking habits was obtained through self-reports of interviewees. After the 10-year follow-up period, changes in smoking habits were evaluated by estimating the frequency of individuals who were stable non-smoker, stable ex-smoker, stable smoker and transitional smoker (individuals who transited between cessation and restarting smoking habit in the three evaluations).

Results: Among elderly followed in 10 years, it was verified that $59.3 \%$ were stable non-smoker, $27.9 \%$ were stable ex-smoker, $5.6 \%$ were stable smoker and $7.2 \%$ were transitional smoker. Among women, $83.7 \%$ were stable non-smoker, $15.3 \%$ were stable ex-smoker, $5.8 \%$ were stable smoker and only $4.8 \%$ were transitional smoker A higher proportion of transition was observed only among those with osteoporosis incidence. For the men, $29.4 \%$ were stable non-smoker, $53.5 \%$ were stable ex-smoker, $5.1 \%$ were stable smoker and $12.0 \%$ were transitional smoker. A higher proportion of transition was observed among those with incidence hypertension and with increased number of chronic diseases in the 10 years of monitoring. For both sexes, the elderly who had reduced household income and marital status changed over time tended to restart smoking.

Conclusions: With aging, the highest proportion of elderly remained with the same smoking behavior. However, the incidence of chronic disease, change in marital status and income were the variables related with the transition of habits between smokers and ex-smokers in this population-based sample of the city of São Paulo, Brazil.

\section{P-361}

Factors associated with alcohol use in elderly: SABE Cohort Study, São Paulo, Brazil

Topic: Exposure - Lifestyle factors

Presenter: Dirce Maria Trevisan Zanetta

Dirce Maria Trevisan Zanetta 1); Maria Lúcia Lebrão 1); Yeda Aparecida de Oliveira Duarte 2); Gabriela Arantes Wagner 1)

1) Department of Epidemiology, School of Public Health, University of São Paulo, Brazil; 2) Medical-Surgical Nursing Department, School of Nursing, University of São Paulo, Brazil 
Background: Health problems associated with the use of alcohol and other drugs among older adults are generally related to physiological changes stemming from the ageing process, such as increased sensitivity to such substances, an increase in the number of comorbidities and the concomitant use of various medications. Little is known regarding the frequency of alcohol consumption among the elderly in Brazil and associated factors.

Objectives: To examine the prevalence and associated factors with mean daily alcohol consumption in the previous 3 months in an elderly population-based sample.

Methods: A cross-sectional household-based epidemiological study, part of the prospective Saúde, Bem-Estar e Envelhecimento (SABE [Health, Wellbeing and Aging]) cohort study. Was conducted in 2011 (third wave) in São Paulo City, Brazil. Mean daily alcohol consumption in the previous 3 months was calculated, and respondents were categorized as nondrinkers, those drinking up to one drink per day, one to two drinks per day, and more than two drinks per day. Descriptive analysis was carried out for the sociodemographic and health characteristics of the sample. Comparisons were made using Pearson's Chi square test with the Rao\&Scott correction. Polynomial regression was used for the analysis of factors associated with alcohol consumption, with mean daily alcohol consumption as the dependent variable.

Results: In 2011, $70.1 \%$ was nondrinkers, $9.5 \%$ were drinking up to one drink per day, $14.4 \%$ were drinking one to two drinks per day and $6.0 \%$ were drinking more than two drinks per day. The higher drinks consumption was more frequent among the male as well as those with greater schooling and income, married and with good selfrated health $(p<0.05)$. Drinking more than two drinks per day was positively associated with single/divorced marital status and male gender. Smoking habits were not associated with this type of consumption. Drinking one to two drinks per day was positively associated with current smoking habits, male gender and good selfrated. Drinking up to one drink per day was only associated with good self-rated health.

Conclusions: In the present population-based sample, mean daily alcohol consumption was low and, like other populations, the major drinks consumption was more frequent among the male as well as those with greater schooling and income, married and with good selfrated health. The present study can assist understanding the alcohol consumption among older adults throughout the ageing process.

\section{P-362}

Perceived barriers to quitting smoking among women from deprived communities: a qualitative study in Southeast England

Topic: Exposure - Lifestyle factors

Presenter: Anjum Memon

John Barber 1); Emma Rumsby 1); Samantha Parker 1); Lisa Mohebati 1); Susan Venables 2), 3); Kate Lawson 2), 3); Tom Scanlon 2), 3); Anjum Memon 1), 2), 3)

1) Division of Primary Care and Public Health, Brighton and Sussex Medical School; 2) Public Health Directorate, NHS Brighton and Hove; 3) Brighton and Hove City Council

The prevalence of smoking continues to remain disparately high amongst people from low socio-economic classes and deprived communities. It is well established that women start to smoke earlier, are more susceptible to smoking-related disease and find it more difficult to quit than men. However, little is known about the perceived barriers to quitting smoking among women - particularly those from deprived communities.
We conducted a qualitative study in Southeast England to elicit perceived barriers to quitting smoking among women from deprived communities.

Eleven women who had used the NHS Stop Smoking Service were engaged in focus group discussions. Data were subjected to manual and NVIVO software-based thematic analysis.

Women confirmed that it is more difficult for them to quit smoking than men. Women felt that post cessation weight gain was inevitable and acted as a barrier to quitting. Menstrual fluctuations and greater levels of stress were perceived as obstacles to quitting and reasons for relapse. They highlighted the importance of continuity of care, capacity for peer support, flexibility of time and location and free cessation aids in increasing their engagement with the Service. Social networking was seen as a way to facilitate peer support. Women felt that tailoring of the Service to their needs would improve quit rates. They felt the Service was poorly advertised, that access was not universally good and that women would be more likely to access and engage with the Service if female-only groups and drop-in clinics were available. Women were most affected by adverts focusing on appearance and the effect of smoke on children. They found other shock tactics ineffective, and suggested positive messaging may improve the impact of advertising.

These findings support consideration of issues around weight gain, menstrual fluctuations, stress, appearance and guilt about exposing children to passive smoke in the design of public health campaigns and interventions. Female-specific policy/programmes have been shown to facilitate cessation in women. More research is needed on how to limit the impact of post-cessation weight gain, stress and menstrual fluctuations on cessation. Women value service flexibility in terms of group size, location and timing. Improved advertising, more universal access and more female-centred options such as female-only groups may help to better meet women's needs. Low socioeconomic status is the strongest predictor of smoking in women, and more research is needed into how the Service and interventions can be best designed to impact on women living in deprived communities.

\section{P-363}

The effect of education on mortality: evidence from a completely deceased Cohort of Danish Twins

Topic: Exposure - Lifestyle factors Presenter: Lena Janys

Gerard van den Berg 1); Lena Janys 1); Kaare Christensen 2)

1) University of Mannheim, Department of Economics; 2) The Danish Twin Registry, and The Danish Aging Research Center, University of Southern Denmark, Odense, Denmark

Background: Education and its effect on health and mortality have been studied extensively in the economic literature, as well as in the epidemiological literature. The effect of schooling on mortality is of particular interest, but also the most difficult to study empirically, since mortality records from completely deceased cohorts rarely contain individual level information on schooling or education levels. Objectives: In this paper we examine the effect of schooling on the mortality on completely deceased cohorts of Danish twins, born between 1888 and 1897, each cohort comprising around 500 individuals.

Methods: We exploit an exogenous variation in the level of schooling caused by a large scale schooling reform in the beginning of the 20th century to identify a causal effect of education on mortality. We have a unique data set that includes individual level information on schooling that we collected from paper records from the Danish 
Ministry of Education, the exact lifetime duration of both twins and important individual characteristics at birth from the Danish twin registry. We estimate a mixed proportional hazard model and control for unobserved heterogeneity by exploiting the statistical dependence in the lifetime duration of twins and model the unobserved effect as a correlated frailty model, where the individual random effect is a combination of an individual effect and a term that is shared between the two twins.

Results: We find weakly statistically significant evidence that education has a protective effect on the longevity of males, but not on females when using the standard individual frailty model, but not when correctly controlling for unobserved heterogeneity with the correlated frailty model. This result holds whether we use actual schooling or eligibility for the reform as an exogenous proxy for schooling.

Conclusions: We find no evidence that schooling significantly and causally decreases adult mortality, although there is some weak evidence that there could be a numerically small effect for males. These results suggest that if there is an effect of schooling on mortality, the channel through which education influences mortality in these old cohorts is not information capital, but rather for example, the choice of less hazardous occupations.

\section{P-364}

Sociodemographic factors associated with tobacco consumption and cessation in Portugal

Topic: Exposure - Lifestyle factors

Presenter: Andreia Leite

\section{Andreia Leite 1); Ausenda Machado 1); Carlos Matias Dias 1)}

1) National Institute of Health Doutor Ricardo Jorge, Department of Epidemiology

Background: Smoking is a significant risk factor for several diseases. Social inequalities have been described for tobacco consumption and though to a lesser extend for tobacco cessation.

Objectives: Describe sociodemographic factors associated with tobacco consumption and cessation.

Methods: Data from the 2005/2006 Portuguese National Health Interview Survey was analysed. A multinomial log-linear model was fitted considering 3 groups: present smokers, former smokers and never smokers. Relative Risk Ratios (RRR) were obtained. Men and women were analysed separately. RRR adjusted for age and comparatively with reference categories (Centre for regions, married for marital status, student for professional situation and higher education for education) are presented. RRR of present smokers compare to never smokers and RRR of former smokers to present smokers.

Results: Men from groups that have higher RRR of present smoking also have lower RRR of stopping. The lowest risk of cessation and higher of consumption was observed in Azores [RRR (95\% Confidence Interval (CI 95)) $0.53(0.46,0.61)$ and 1.89 (1.64, 2.18), respectively]. Divorcees, unemployed, and men with lower secondary education all had lower risk of cessation and higher of consumption, [respectively RRR (IC 95): $0.50(0.41,0.61)$ and $2.01(1.64,2.46)$; RRR (IC 95): $0.16(0.13,0.20)$ and $6.29(5.00,7.91)$; RRR (IC 95): $0.65(0.56,0.77)$ and $1.53(1.30,1.79)]$.

Similar results were observed in women (higher RRR of smoking and lower RRR of stop). Exceptions were found in the non-unemployed women and those with less that upper secondary. Taking region into consideration women in Madeira had a lower RRR of stop smoking and in Lisbon they had the higher risk of smoking [RRR (IC 95) $0.40(0.27,0.58)$ and $2.11(1.74,2.57)$, respectively]. The same was observed for divorced women [RRR (IC 95) $0.54(0.41,0.69)$ and
$3.21(2.69,3.84)$, respectively]. Unemployed had the lower risk of quitting smoking and the highest of smoking [RRR (IC 95) 0.83 (0.51, $1.38)$ and $4.23(3.20,5.58)$, respectively], while the other groups had highest RRR of stop smoking and smoking, comparatively with reference group. Less educated women (lower secondary or less) had lower risk of cessation but also lower risk of smoking.

Conclusions: Unfavourable sociodemographic characteristics are associated with higher risks of being a current smoker and lower risks of being an ex-smoker. Different/further cessation measures are needed in these less prone to quit group of individuals, namely men from Azores, divorced, unemployed and with lower secondary education and for divorced and unemployed women.

\section{P-365}

Strong and graded association between weight status in the first and seventh year of life: consistent patterns across subgroups

Topic: Exposure - Lifestyle factors

Presenter: Sanne Ellegaard Jorgensen

Sanne Ellegaard Jørgensen 1); Maria Svendsen 1); Signe Boe Rayce 1); Carina Sjöberg Brixval 1); Bjørn E. Holstein 1)

1) National Institute of Public Health, University of Southern Denmark

Background: Overweight and obesity among children is a public health problem, which has increased greatly over the past 50 years. Previous reviews have shown that rapid weight gain and high weight status in infancy is positively associated with childhood obesity. Only few studies have addressed whether the association between weight in the first year of life and later in childhood is different among children with social and biological vulnerability.

Objectives: This study investigates the association between weight status in infancy and overweight at 6-7 years and whether this association is modified by indicators of vulnerability in childhood: socioeconomic background, gestational age, birth weight, duration of breastfeeding, exposure to passive smoking and motor development in infancy. Methods: The study population comprises 1,675 children, born between 2003 and 2006, from 11 municipalities in the metropolitan area of Copenhagen, Denmark. Data was collected by health visitors and origin from the Danish Health Visitor's Child Health Database. The Database Steering Committee provided the data. The 6-10month-old infants were measured lying on a table and weighed wearing light clothes while the 6-7-year-old children were measured without shoes and weighed wearing light clothes. Based on the health visitors records of weight, length/height and specific age, the WHO growth references were used to calculate BMI Z-scores. The Z-scores were then used to assess the weight status of the children. Prediction analyses were conducted using stratified multivariate logistics regression. Modifications by indicators of vulnerability were tested by interactions terms.

Results: The OR (95\% CI) for overweight at age 6-7 years was 3.18 (2.33-4.33) among children who were at risk of overweight in infancy (age 6-10 months) and 7.52 (4.36-12.96) among children who were overweight or obese in infancy. The analyses were adjusted for parental education level, parental occupational status, duration of breastfeeding, and birth weight. The stratified analyses revealed no differences across sub-groups and the $p$ values of all interaction terms exceeded 0.1

Conclusions: There was a significant prediction pattern in overweight from infancy to age 6-7 year. This prediction pattern was independent of socio-economic background, gestational age, birth weight, duration of breastfeeding, exposure to passive smoking, and motor development in infancy. 


\section{P-366}

Identification of clusters of body composition and time changes from 4 to 7 years in children from Generation XXI

Topic: Exposure - Lifestyle factors

Presenter: Susana Santos

Susana Santos 1); Milton Severo 1), 2); Ana Cristina Santos 1), 2); Carla Lopes 1), 2); Henrique Barros 1), 2); Andreia Oliveira 1), 2)

1) Institute of Public Health, University of Porto, Portugal; 2) Department of Clinical Epidemiology, Predictive Medicine and Public Health and Cardiovascular Research \& Development Unit, University of Porto Medical School, Portugal

Background: Changes in single measures of obesity during childhood have been extensively addressed, but changes in clusters combining general and regional measures of fat location, could provide a new perspective to understand their effects on health outcomes. Objectives: To identify and compare clusters of body composition at 4 and 7 years.

Methods: Generation XXI is a prospective population-based birth cohort of 8,647 children assembled during 2005-2006 at 5 public maternity units of Porto, Portugal. These analyses include children reevaluated at $4-5$ (86.2\% of participation) and 7 years (ongoing since 2012), after excluding potential outliers ( $\pm 3 *$ interquartile range) in anthropometrics $(\mathrm{n}=2012,48.4 \%$ girls). Body mass index (BMI), waist-to-hip ratio (WHR) and fat mass index (FMI = fat mass from tetra-polar bioelectric impedance/height ${ }^{2}$ ) were ascertained by trained personnel. Patterns were identified by model-based clustering. Measures were standardized separately at 4 and 7 years and z-scores were compared (Z4 vs. Z7) using paired samples $T$ test. Sex-stratification was not considered, since clusters were similar by sex.

Results: The Bayesian information criterion supported a model with 4 clusters: $26.8 \%$ of children were included in cluster $1,25.4 \%$ in cluster $2,15.7 \%$ in cluster 3 and $32.1 \%$ in cluster 4 . In clusters 1,2 and 4, children at 4 and 7 years were characterized by an adequate BMI, FMI and WHR (less than $1 \mathrm{SD}$ above/below the population mean). However, from 4 to 7 years they embraced different changes: in cluster 1, children increased BMI $(\mathrm{Z} 4=0.29, \mathrm{Z7}=0.42 ; \quad p<0.001)$ and $\mathrm{FMI}$ $(\mathrm{Z} 4=0.27, \mathrm{Z7}=0.36 ; p=0.01)$, but decreased WHR $(\mathrm{Z} 4=0.10$, $\mathrm{Z7}=-0.23 ; p<0.001) ;$ in cluster 2 , children decreased BMI $(\mathrm{Z} 4=-0.27, \mathrm{Z} 7=-0.32 ; p<0.001)$, but did not change their FMI $(\mathrm{Z} 4=-0.52, \mathrm{Z} 7=-0.56 ; p=0.23)$ and WHR $(\mathrm{Z} 4=-0.01$, $\mathrm{Z7}=0.04 ; p=0.13)$; and in cluster 4 , children decreased BMI $(\mathrm{Z} 4=-0.65, \mathrm{Z7}=-0.83 ; p<0.001)$, FMI $(\mathrm{Z} 4=-0.44, \mathrm{Z7}=$ $-0.60 ; p<0.001)$ and WHR $(\mathrm{Z} 4=-0.14, \mathrm{Z} 7=-0.35 ; p<0.001)$. In cluster 3 , children at 4 years were characterized by a high BMI and FMI (more than 1 SD above the population mean) but by an adequate WHR. BMI $(\mathrm{Z} 4=1.25, \mathrm{Z} 7=1.50 ; p<0.001)$, FMI $(\mathrm{Z} 4=1.28$, $\mathrm{Z7}=1.54 ; p<0.001)$ and WHR $(\mathrm{Z} 4=0.13, \mathrm{Z7}=1.04 ; p<0.001)$ increased from 4 to 7 years.

Conclusions: Although most children (clusters 1, 2 and 4) maintained their anthropometrics under normal ranges from 4 to 7 years, they experienced different changes, which can have different effects on cardio-metabolic health. An increase of BMI and FMI with time was observed in children already presenting values above normal ranges at 4 years, who also changed from an adequate to a high WHR (cluster 3 ).

\section{P-367}

Survival of Estonian native and foreign origin population by chronical health problems: Linkage of survey data and death certificates

Topic: Exposure - Lifestyle factors
Presenter: Kati Karelson, Luule Sakkeus

\section{Kati Karelson 1); Luule Sakkeus 2)}

1) National Institute for Health Development/Tallinn University; 2) Tallinn University

Estonia has been among the forerunners of the demographic transition. This implies that the so-called epidemiological transition follows the trends of that of Northern and Western Europe. However, the occupation of Estonia by the Soviet Union, which began in the 1940s and lasted for almost 50 years, brought along the stagnation in the health status of the population. In addition, the composition of the population changed significantly during the second half of the 20th century. Estonian society is characterized by one of the largest and oldest immigrant populations in Europe. This immigrant population comes mostly from the former territories of the Soviet Union.

Previous studies have revealed a persistent gap in LE between the immigrant and native populations in Estonia. To look for the explanations for that phenomenon we focus on remaining life expectancy related to specific chronic diseases. We analyse separately foreignorigin and native population groups. The interest is to find out whether people who live in the same society and have similar socioeconomic backgrounds experience different remaining life expectancies from the time point when chronic diseases were diagnosed.

The hypothesis is that the length of life after the diagnosis is related to nativity and gender. This heavily relies on the different habits of the health behavior. In case of observed diagnosis we expect to see that the life-span is remarkable shorter for the immigrant and male population compared to the native or female population.

Our focus is on cancer and the diseases of circulatory system. To explain the results, additional covariates will be included to the model that describes health care utilization and risky health behavior.

We will use three data sets for this analysis: Estonian Health Interview Survey 1996 (Est HIS 1996), Estonian Health Interview Survey 2006 (Est HIS 2006) and death certificates information from the Causes of Death Registry. Survey data and death information will be linked in the Causes of Death Registry. The sample sizes of Est HIS 1996 and Est HIS 2006 were 4,711 and 6,434 respectively. Both surveys involved partly life-course approach. Therefore, information about health behavior and chronic conditions are available from the retrospective point of view.

Preliminary results: both men and women of foreign-origin were older at the time of the condition diagnosed. In case of cancer, the foreign-origin population survives longer than native population. Among those with chronic diseases of circulatory system the trend is the opposite to that of cancer.

\section{P-368}

Perceived barriers to quitting smoking and seeking smoking cessation counselling amongst pregnant women: a qualitative study in Southeast England

Topic: Exposure - Lifestyle factors Presenter: Anjum Memon

Reeanne Jones 1); Anjum Memon 2); Marjan Arabizedah 3); Anna Fairhurst 3); Susan Venables 3); Kate Lawson 3); Tom Scanlon 3)

1) Brighton and Sussex Medical School; 2) Brighton and Sussex Medical School and NHS Brighton and Hove; 3) NHS Brighton and Hove and Brighton and Hove City Council

Background: Smoking during pregnancy is strongly associated with infant morbidity and mortality and health inequalities, and is the single most modifiable risk factor for adverse outcomes in pregnancy. 
The UK government has set a target for decreasing the percentage of pregnant women who smoke from $23 \%$ (in 1998) to $11 \%$ by the year 2015. While many women stop smoking before becoming pregnant or soon after, 1 in 4 women smoke for part of pregnancy and 1 in 8 smoke throughout; and only $5 \%$ of pregnant women who smoke attend the (free) NHS Stop Smoking Service.

Objectives: We conducted a qualitative study in Southeast England to elicit perceived barriers to quitting smoking and seeking smoking cessation counselling amongst pregnant women.

Methods: Pregnant women $(n=25)$ who were attending the NHS Stop Smoking Service were engaged in semi-structured interviews/ discussions. Quantitative data were analyzed using Microsoft Excel and qualitative data were subjected to manual thematic analysis.

Results: Overall, the pregnant women had poor knowledge of the adverse effects of smoking during pregnancy. An evaluation of the Stop Smoking Service records suggested that about $60 \%$ of the women, referred by their midwife/general practitioner, decline support from the Service; and $60 \%$ do not attend after making an appointment with the smoking cessation counsellor. The perceived barriers to quitting smoking and/or seeking smoking cessation counselling included fear of attending the Service, no desire to quit, denial, lack of motivation, fear of being judged, feeling of pressure (from society/family), time restraints, lack of information, addiction, embarrassment, counsellors' attitude, lack of privacy. About $80 \%$ of the women had not received any advice about remaining smoke free after delivery.

Conclusions: The perceived barriers to quitting smoking and seeking smoking cessation counselling highlighted by pregnant women need to be considered in the design and delivery of public health campaigns/interventions and stop smoking services. To decrease the prevalence of smoking during pregnancy, the stop smoking services need to adopt a more innovative and tailored approach to improve women's knowledge about adverse effects of smoking during pregnancy, referral system, and attendance rates. There is also a need for continued support after delivery to prevent relapse to smoking.

\section{P-369}

Effect on weight loss of food and lifestyles intervention in treated hypertensive natives and African immigrants in Lisbon (PRAdA Study)

Topic: Exposure - Lifestyle factors

Presenter: Violeta Alarcão

Catarina Peixoto 1); Gisela Carrilho 1); Beatriz Goulão 1); Violeta Alarcão 1); Rui Simões 1); Filipa Guerra 1); Isabel Cardoso 1); Milene Fernandes 1); Paulo Nicola 1); Sofia Guiomar 1), 2)

1) Institut of Preventive Medicine, Faculty of Medicine of Lisbon; 2) National Health Institute Doutor Ricardo Jorge (INSA)

Background: One of the risk factors identified in the development of hypertension (HTA) is overweight. International studies have shown that Blacks are more vulnerable to the effects of HTA. Furthermore, behavioral interventions for weight loss seem to be less successful in Blacks than in Caucasians. In a Portuguese study DIMATCH-HTA with hypertensive African immigrants and Portuguese natives, there was a high prevalence of obesity, higher in immigrants.

Objectives: To assess adherence to recommendations of the intervention cohorts (hypertensive African immigrants and natives) and to compare the proportion of weight loss and associated factors in both groups.

Methods: Intervention study to decrease blood pressure and weight loss through diet and lifestyle changes in medicated hypertensive immigrants from African Countries of Portuguese Official Language and Portuguese natives, followed in Primary Health Care in the Lisbon Region. The sample was selected from the observational study DIMATCH-HTA and the intervention lasted 6 months. To compare the populations, we performed the 22 test, $t$ student and linear regression models. To evaluate the success of the intervention, we compared the participants' weight change before the intervention (between DIMATCH-HTA and the beginning of intervention PRAdA) with the change between the beginning and end of the intervention. Analyses were done with SPSS.v20 using a $p$ value $<0.05$.

Results: Of 152 participants, 87 were immigrants and 65 natives, with an average age of 58.2 and 66.2 years $(p<0.01)$ and mean BMI of 30.3 and $30.0 \mathrm{~kg} / \mathrm{m}^{2}(p=0.25)$, respectively, $80.3 \%$ of participants were overweight. The number of dietary recommendations known and complied with at the end of the intervention was significantly greater than baseline $(p<0.01)$ in both groups, however at the end of the study the native performed a greater number of recommendations $(p<0.01)$, without significant sex differences. Weight loss during the intervention period was greater than before intervention $(0.20$ vs. $0.06 \%$ of total body weight/month $p=0.053$ ). During the intervention weight loss was verified as significant only in natives $(p<0.05)(-1.15$ vs. $-0.67 \mathrm{~kg}, p=0.265)$. In stratified multiple linear regression analysis, intervention was a determinant of successful weight loss in males and natives $(p<0.001$ and $p=0.012$; $\mathrm{R}=0.21$ and $\mathrm{R}=0.10$ ), independently of age, ethnicity and HTA control.

Conclusions: The natives fulfilled a greater number of recommendations and lost more weight, which is similar to what has been reported by international studies. Future interventions should provide adherence motivational techniques to diet and lifestyles and cultural component to improve weight management and reduce health disparities.

\section{P-370}

The role of social position and quality of life on 10 years survival of the English Longitudinal Study of Ageing

Topic: Exposure - Lifestyle factors

Presenter: Eleonora Dorsi

Eleonora Dorsi 1); Andre Xavier 2); Cesar Oliveira 3); Andrew Steptoe 3); Panayotes Demakakos 3); Michael Marmot 3)

1) Department of Public Health, Federal University of Santa Catarina, Florianópolis, Santa Catarina, Brazil; 2) University of South of Santa Catarina, Florianópolis, Santa Catarina, Brazil; 3) Department of Epidemiology and Public Health, University College London, London, UK

Objective: To investigate the influence of socioeconomic inequalities and the role of quality of life on 10 years survival of older adults living in England.

Method: Data were from the English Longitudinal Study of Ageing (ELSA); which is a biennial, longitudinal, panel study of men and women living in England aged 50 years or over. We used data from Waves 1-5 collected in 2002-2003, 2004-2005, 2006-2007, 2008-2009, and 2010-2011. Data on mortality were collected until 2012. The outcome was survival time, measured as the interval between date of wave 1 and date of death or last contact with the participant. Our main predictor was socioeconomic position measured as wealth quartiles at baseline. The covariates were sociodemographic variables, cognitive function, physical function, lifestyle variables, self-reported morbidity and quality of life measured by CASP-19 (control, autonomy, self-realization and pleasure). Statistical analysis 
includes Kaplan-Meier curves and log-rank test used for comparing stratified curves. Unadjusted and adjusted hazard ratios (HR) and their $95 \%$ confidence intervals were calculated through Cox model, considering time varying variables, in Stata 11.0.

Results: The adjusted HR results showed a social gradient in 10 years survival: the higher on the wealth quartile (best-off), the lower the HR. Those who reported high quality of life, vigorous physical activity, paid work and leisure activities (going to the cinema) had significantly lower HR. Those who reported smoking, cardiovascular diseases, arthritis, disability and low delayed recall had significantly higher HR of mortality.

Conclusions: High quality of life (control, autonomy, self-realization and pleasure), vigorous physical activity, no smoking, paid work and leisure can reduce the effect of wealth on mortality. Wealth still determines survival when comparing the best-off with the worst-off.

\section{P-371}

Maternal atopic dermatitis is associated with decreased risk of preterm birth

Topic: Exposure - Other

Presenter: Håvard Trønnes

Håvard Trønnes 1); Allen Wilcox 2); Rolv Terje Lie 1); Trond Markestad 3); Dag Moster 1), 3)

1) Department of Global Public Health and Primary Health Care, University of Bergen, Norway; 2) Epidemiology Branch, National Institute of Environmental Health Sciences, NIH, Durham, USA; 3) Department of Clinical Medicine, University of Bergen, Norway

Background: The association between maternal atopic dermatitis and preterm birth has not been thoroughly examined.

Objective: To explore if maternal atopic dermatitis is associated with preterm birth.

Methods: By linking the Medical Birth Registry of Norway to other national registries, we were able to follow all live births in Norway from 1967 through 2003. We included mother-child dyads when both mother and child were registered in the MBRN, had complete data on gestational age at birth, and were Norwegian residents. The study cohort was followed through 2007. The registries provided information on pregnancy and birth in mother and child, maternal health, parental education, and migration.

Results: Of 2,160,251 live births, 358,269 mother-child dyads met the inclusion criteria. Among these, 22,571 (6.3\%) children were born preterm and 13,430 mothers $(3.7 \%)$ had a record of atopic dermatitis. Mothers with atopic dermatitis were at decreased risk for delivery at 23-31 weeks' gestation (RR 0.88 (95\% CI 0.73-1.07)), and for delivery at 32-36 weeks' gestation (RR 0.87 (95\% CI 0.81-0.94)), compared to mothers without atopic dermatitis. Adjustment for maternal asthma strengthened the association, while further adjustment for socio-demographic factors and maternal gestational age at birth did not change the risk estimates substantially.

Conclusions: Mothers with atopic dermatitis are at decreased risk of preterm birth. Shared immunological features of pregnancy and atopic dermatitis may explain this association.

\section{P-372}

Impact of censoring at or truncating risk time of hospitalizations on the association between antiepileptic drugs and suicide attempt

Topic: Exposure - Other

Presenter: Aske Astrup
Aske Astrup 1); Christiane Gasse 1); Liselotte Petersen 1); Henrik Støvring 2)

1) National Centre for Register-based Research, Aarhus University, Aarhus, Denmark; 2) Department of Public Health - Biostatistics, Aarhus University, Aarhus, Denmark

Background: In observational pharmacoepidemiological studies prescription data is frequently used, which generally do not include inpatient medication information. This case of missing data is only seldom considered and censoring patients at first hospitalization requires this to be an independent censoring event.

Objectives: To evaluate the scope of the problem of hospitalizations on exposure status and the impact of censoring on estimates of the association between antiepileptic drug (AED) use and suicide attempts.

Methods: We performed a register based cohort study including $25 \%$ of the Danish population with all incident users of AEDs between 1/7-1996 and 16/12-2006. Further we used Cox analysis of suicide attempt comparing treatment status of AEDs, with adjustment for treatment indications and other treatment-related variables.

Results: We identified 26,916 hospitalizations (unrelated to outcome) among 43,069 incident users of AEDs. 5.16, 11.49 or $21.33 \%$ of patients were hospitalized within 10,30 or 90 days, respectively, after the index date (the first AED prescription). Time spent in hospital accounted for 1.77 and $46.65 \%$, respectively, of 159,065 person years. Comparing treatment status, the hazard rate ratios (HRR) estimate of suicide attempt was increased by $28 \%$ and by $31 \%$ when censoring after one overnight stay or 3 days at the hospital, respectively. The HRR increased by $5 \%$ if the periods of hospitalization were omitted, while keeping the follow-up time after discharge.

Conclusions: Censoring at first hospitalization changed estimates of increased risk of suicide among AED patients. This indicates that hospitalizations are not independent censoring events, and hence they should not simply be ignored when studying the risk of suicide in these patients.

\section{P-373}

Paternal age and the risk of congenital malformations of the musculoskeletal system in offspring: a population-based study of Denmark 1978-2009.

Topic: Exposure - Other

Presenter: Stine Kjar Urhøj

Anne-Marie Nybo Andersen 1); Laust Hvas Mortensen 1); Stine Kjær Urhøj 1)

1) Section of Social Medicine, Department of Public Health, University of Copenhagen

Background: The relationship between paternal age and the risk of congenital malformations in general has not been well examined, and evidence from population data associating paternal age and the risk of congenital musculoskeletal malformations specifically have been limited and the results inconsistent. However, a plausible biological mechanism linking advanced paternal age and congenital malformations exists.

Objectives: A population-based register study of 1,917,689 live births born from 1978 through 2009 was conducted to examine the association between paternal age and risk of congenital musculoskeletal malformations in offspring.

Methods: The study was conducted based on information obtained from several nation-wide registers. 20 categories of congenital musculoskeletal malformations were investigated, including several pooled categories, i.a. total musculoskeletal malformations. Logistic 
regressions with cluster correction were used to estimate the association between paternal age and specific malformation subgroups after adjustment for maternal age, year of birth, ethnicity, education, parity, previous miscarriages, previous stillbirths, and maternal diabetes or epilepsy prior to the birth. Odds ratios (ORs) and $95 \%$ confidence intervals (CIs) were calculated.

Results: There was little evidence of higher risk for advanced paternal age for every malformation subgroups except congenital malformation syndromes overall. In this subgroup, statistically significantly higher risk was seen for fathers aged 35-39 years (OR 1.38 [CI 1.07-1.77]), 40-44 years (OR 1.60 [CI 1.14-2.24]), and 45-49 years (OR 1.67 [CI 1.01-2.75]) compared to fathers 25-29 years of age. For total musculoskeletal malformations, adjusted analysis showed similar tendency in estimates, though statistically insignificant for all paternal age groups except 35-39 years of age (OR 1.07 [CI 1.02-1.12]. A pattern of moderately elevated, but statistically insignificant, risk with higher paternal age was seen for several malformation subgroups; limb reduction, upper limb reduction, lower limb reduction, complete absence of limb, and craniosynostosis. For the subgroups polydactyly, syndactyly, and skeletal dysplasias a $\mathrm{J}$-shaped relation was seen in the risk estimates, with elevated estimates among younger fathers and older fathers, but none of these associations were statistically significant. No association was apparent in the risk estimates in the subgroups club foot and other musculoskeletal malformations.

Conclusions: The present study did not show consistent evidence that paternal age was associated with the risk of congenital musculoskeletal malformations in offspring other than congenital malformation syndromes overall. Results suggested a positive association between advanced paternal age and several malformation subgroups as well as elevated risk among younger paternal age in some malformation subgroups, but the associations were not statistically significant. Results of the present study indicated that paternal age appeared to play a small role in the etiology of congenital musculoskeletal malformations, if any.

\section{P-374}

Age of menarche and time to pregnancy: a study within the Danish National Birth Cohort

Topic: Exposure - Other

Presenter: Karen Guldbrandsen

Karen Guldbrandsen 1); Linn Berger Håkonsen 1); Andreas Ernst 1); Gunnar Toft 2); Julie Lyngsø 2); Jørn Olsen 1); Cecilie Høst Ramlau-Hansen 1)

1) Department of Public Health, Section for Epidemiology, Aarhus University, Denmark; 2) Danish Ramazzini Centre, Department of Occupational Medicine, Aarhus University Hospital, Denmark

Background: An early age of menarche (AOM) has been associated with several health risks such as breast cancer and cardiovascular disease, but whether AOM affects the long term biological ability to conceive, fecundity is poorly investigated. To the best of our knowledge, only one published study has examined this relation, and it did not find an association between AOM and waiting time to pregnancy (TTP). Objectives: The main objective of this study is to estimate the association between AOM and TTP in a large population-based sample of Danish pregnant women.

Methods: A total of 92,891 pregnant women enrolled in the Danish National Birth Cohort between 1996 and 2002 and were interviewed around the 16th pregnancy week and gave information on AOM in years and TTP. After exclusion of women with "unplanned pregnancies" and women with reported AOM > 24 years there was information available on AOM and TTP for 82,839 (89\%) and $76,221(82 \%)$ women, respectively. Information on both AO M and TTP was available for 69,892 (75\%) women. Furthermore, information on potential confounders is available from the interview and the National Patient Register. Here we report descriptive results on AOM and TTP as well as preliminary results. Results adjusted for potential confounding factors such as e.g. chronic diseases in childhood, socioeconomic status, age and lifestyle factors will be presented at the conference.

Results: The mean (SD) AOM was 13.3 (1.4) years and $16 \%$ had a TTP $>12$ months. With AOM as exposure we found estimated frequencies of TTP $>12$ months in different age groups of menarche. There was no difference in mean (SD) AOM between TTP $<12$ months and TTP $>12$ months. Compared to women who were 13 years old at menarche, we found that women with $\mathrm{AOM}<12$ and AOM $>15$ years were slightly more likely to have a TTP $>$ 12 months (odds ratio (OR) 1.07 (95\% confidence interval (CI) 0.99; $1.15)$ ) and OR 1.07 (95\% CI 0.98; 1.17)).

Conclusions: These preliminary crude res ults show a weak tendency toward a higher risk of subfecundity among women with an $\mathrm{AOM}<12$ years as well as AOM $>15$ years. Fully adjusted results will be presented at the conference.

P-375

\section{Prevalence of carpal tunnel syndrome in pregnancy}

Topic: Exposure - Other

Presenter: Adriano Dias

Gladys Dias 1); Ana Carolina Santini 1); Luana Vianna 1); Adriano Dias 1)

1) Graduate Program in Gynecology, Obstetrics and Mastology Botucatu Medical School

Introduction: Carpal Tunnel Syndrome (CTS) is one of the complications that may occur during pregnancy and puerperium, most commonly in the third trimester of pregnancy. This dysfunction is due to the median nerve compression in the carpal tunnel and the symptoms usually presented are tingling, pain and paresthesia in the hands and wrist regions.

Objective: Estimate the prevalence of CTS in pregnancy in post-natal women and those who have recently given birth, in Botucatu/SP city, and identify factors associated to CTS occurrence in pregnancy.

Methods: Populational cross sectional study carried out in the two maternity hospitals in Botucatu, Brazil, with 203 post-natal women. It was used an assessment protocol containing patient's personal data, the Tinnel and Phalen tests, sensitiveness evaluation through estesiometria and the Boston Questionnaire.

Results: Out of the individuals interviewed, $81.8 \%$ (166) presented no CTS clinical symptom (CTS-), but $18.2 \%$ (37) presented (CTS+). At maternal age, CTS- group presented higher percentage of women at age 16-25 (57.2\%) and CTS+ from 26 to 31 years old $(32.4 \%)$. As for the number of children, $41 \%$ (68) of women in CTS- were primiparous, while $37.8 \%$ (14) of women with CTS+ were pregnant for the second time. $31.9 \%$ of women with CTS- gained from 10 to $15 \mathrm{~kg}$, while in the CTS+ group, $35.1 \%$ gained from 5 to $10 \mathrm{~kg}$, but this difference was statistically non-significant. The CTS+ group presented positive Tinnel in the right hand in $35.1 \%$ of cases and $24.3 \%$ in the left hand. The Phalen test was positive in $40.5 \%$ in the right hand and $37.8 \%$ in the left hand, a statistically non-significant difference. The beginning of CTS symptoms in the 8th month of pregnancy was reported by $43.2 \%$ of women. In the sensitive evaluation, most women CTS+ $(74 \%)$ presented normal sensitiveness in the assessed dermatomes. In the Boston Questionnaire, the CTS+ 
group presented normal responses to the questions related to pain $(78 \%)$ and to the daily life activities (38\%), results that did not differ significantly from those in the CTS - group. However, concerning the questions that quantified tingling and worse of symptoms at night, in the group CTS+ $30 \%$ reported intense increase in them.

Conclusions: In this study, the prevalence of CTS was $18.2 \%$, with higher onset of the symptoms from the 8th month of pregnancy.

\section{P-376}

\section{Associated factors to carpal tunnel syndrome in pregnancy}

Topic: Exposure - Other

Presenter: Adriano Dias

Gladys Dias 1); Ana Carolina Santini 1); Luana Vianna 1); Adriano Dias 1)

1) Graduate Program in Gynecology, Obstetrics and Mastology Botucatu Medical School

Introduction: Carpal Tunnel Syndrome (CTS) is defined as a dysfunction occurred due to the median nerve compression within the carpal tunnel frequently found during pregnancy which is associated with the maternal age, excessive weight gain, increase of peripheral edema due to hormonal factors and metabolic changes during pregnancy.

Objective: Identify possible predictor factors associated to CTS occurred during pregnancy.

Methods: Populational cross sectional study carried out in the two maternity hospitals of Botucatu city, Brazil, with 203 post-natal women. The instruments used were an assessment protocol containing the pregnant obstetric data: age, number of children, weight gain, comorbidities such as arterial hypertension, gestational diabetes mellitus and cramps. In the analysis, it was initially adjusted univariated models of simple logistic regression, having as variable dichotomy response the occurrence of CTS and as predictor variables each variable described. Later, a model of multiple conditional logistic regression was adjusted, where the variable dichotomy response was the occurrence of CTS and the predictor variables were those which in the univariated models produced estimates of OR with value $p \leq 0.25$.

Results: Out of the 203 individuals interviewed, $81.8 \%$ (166) presented no CTS clinical symptom, but $18.2 \%$ (37) presented. The factors associated with CTS occurrence in pregnancy were gestational diabetes mellitus and maternal age, with OR (IC $95 \%$ ) of, respectively, 3.81 (1.52-9.58) and 1.09 (1.03-1.16).

Conclusion: In the present study, the factors associated with CTS were maternal age and gestational diabetes.

\section{P-377}

A prospective cohort study of time-to-pregnancy and adverse pregnancy outcomes

Topic: Exposure - Other

Presenter: Elizabeth Hatch

Lauren Wise 1), 4); Ellen Mikkelsen 2); Henrik Sorensen 2), 4); Anders Riis 2); Kenneth Rothman 3), 4); Elizabeth Hatch 4)

1) Slone Epidemiology Center, Boston University, Boston, MA, USA; 2) Dept of Clinical Epidemiology, Aarhus University; 3) RTI International, North Carolina, USA; 4) Department of Epidemiology, Boston University School of Public Health, Boston, MA
Background: A growing body of evidence indicates that longer timeto-pregnancy (TTP) or infertility (TTP $\geq 12$ months), independent of fertility treatment use, is associated with increased risk of adverse pregnancy outcomes.

Objectives: We used data from the Snart Gravid Study, an internetbased prospective cohort study of Danish pregnancy planners (2007-2011), to examine the association between TTP and adverse pregnancy outcomes.

Methods: TTP was ascertained from self-reported questionnaire data. Outcome data were derived from the Danish Birth Registry and the Danish Hospital Registry. Prospectively-reported TTP was classified into 4 categories $(<3,3-5,6-11, \geq 12$ cycles) and adverse pregnancy outcomes included preterm birth $(<37$ completed weeks of gestation), low birth weight $(<2,500 \mathrm{~g})$, preeclampsia, placental disorders (placenta previa, abruptio placenta, placenta accreta, or placental insufficiency), and caesarean delivery. The cohort was restricted to women with singleton births who had complete follow-up $(\mathrm{N}=2,344)$. We used log-binomial regression models to estimate risk ratios (RRs) and $95 \%$ confidence intervals for each association, adjusting for maternal age, paternal age, maternal body mass index $\left(\mathrm{kg} / \mathrm{m}^{2}\right)$, maternal smoking, infant sex, and parity.

Results: We found a small positive association between TTP and preterm birth risk. The RRs for TTPs of 3-5, 6-11, and $\geq 12$ versus $<3$ cycles were: 1.65 (95 \% CI 0.96-2.82), 0.93 (95\% CI 0.50-1.74), and 1.72 (95\% CI 0.93-3.16). When preterm birth was defined as $<36$ weeks, the respective associations were stronger: $1.64(95 \% \mathrm{CI}$ 0.77-3.48), 1.56 (95\% CI 0.72-3.39), and 2.35 (95\% CI 1.04-5.28). Longer TTP was also positively associated with placental disorders (TTP $\geq 12$ vs. $<3: \quad \mathrm{RR}=2.87,95 \%$ CI $1.15-7.17)$, caesarean delivery (TTP $\geq 12$ vs. $<3$ : RR $=2.02,95 \%$ CI 1.35-3.02), and low birth weight after further adjustment for preterm birth (TTP $\geq 12$ vs. $<3: \mathrm{RR}=2.04,95 \%$ CI 1.06-3.93). TTP was not appreciably associated with preeclampsia. The exclusion of women with prepregnancy hypertension or diabetes made little difference in the effect estimates.

Conclusions: These data suggest that delayed conception is a risk factor for some adverse pregnancy outcomes.

\section{P-378}

Prevalence of women urinary incontinence in Botucatu, Brazil

Topic: Exposure - Other

Presenter: Adriano Dias

Luana Schneider Vianna 1); Ana Carolina Monteiro Santini 1); Angélica Pascon Barbosa 2); Liamara Cavalcante Assis 1); Gladys Dias 1); Adriano Dias 1)

1) Graduate Program in Gynecology, Obstetrics and Mastology Botucatu Medical School; 2) UNESP - Marilia Campus

Background: The International Continence Society define Urinary Incontinence (UI) like any loss of urine with can lead damages physical, psychological and social that affect negatively on the quality of life, femininity and sexuality in the women life. The prevalence of UI among women of different ages is between 8 and $71 \%$. For the reason that Urinary incontinence be a health public problem and represents social, physical, psychological and economic disorders, a health survey is the object of interesting from this study, a view to improve the knowledge about this condition, generating evidences that will help in the establishment of prevention and treatment.

Objectives: The aim of this study was to determine the prevalence and the presence of bother with the UI and to observe the most common at the moment of the urinary loss from the city of Botucatu, Brazil. 
Methods: This is a transversal epidemiological study made in 2 hospitals with maternity in operation in the city of Botucatu, Brazil. 299 women were interviewed between April, 2011 and August, 2012. During hospitalization for childbirth, when they responded the structured survey with questions about the presence of UI and it types, presence of bother with the UI and the main moments in which occurred, as: coughing, sneezing, laught, during sleep and sexual relations, when making mild exertion and carring weight, while having strong urge to urinate, when hearing the sound of water or having contact with it; and even, when losing without realizing it.

Results: It was interviewed 299 women, of these 49 (16.4\%) were incontinent and among the incontinent $27(55.1 \%)$ had mixed urinary incontinence (MUI), $16(32.7 \%)$ had Stress Urinary Incontinence (SUI) and $6(12.2 \%)$ had Urge Urinary Incontinence. $23(46.9 \%)$ women reported discomfort related to UI. Among the most common activities during the loss of urine were found (highest to lowest occurrence) sneeze, cough, laugh, and when they were strong urge to urinate.

Conclusions: The UI was highly prevalent among the women studied, and the most frequent type was MUI. Despite of the magnitude of occurrences and the common moments, day by day routine, where this happen, most of the part of women didn't report discomfort with UI. These findings highlight the importance of research on the prevalence of UI as well as approach and care for women with this symptom.

\section{P-380}

Gestational age, birth weight, and infantile colic

Topic: Diseases - Others

Presenter: Ioanna Milidou

Ioanna Milidou 1); Charlotte Søndergaard 2); Morten S Jensen 1); Jørn Olsen 3); Tine Brink Henriksen 1)

1) Perinatal Research Unit, Department of Pediatrics, Aarhus University Hospital Skejby, Denmark; 2) Department of Pediatrics, Herning Regional Hospital, Denmark; 3) Department of Epidemiology, School of Public Health, Aarhus Univwrsity, Denmark

Background: Preterm and growth restricted infants may have developmental delays or deviant organ function related to the central nervous system and the gastrointestinal tract. Since both organ systems are hypothesized to be involved in the pathogenesis of infantile colic, a condition characterized by excessive crying during the first months of life, impaired fetal growth and preterm birth may be risk factors for infantile colic.

Objectives: To investigate the association between gestational age, birth weight, and infantile colic.

Methods: We used maternal interviews from the Danish National Birth Cohort (1996-2002) conducted at gestational week 17 and at 6 months post partum. We included 62761 live-born singletons with complete information on birth weight, gestational age, and crying symptoms recorded at 6 months of age. Infantile colic was defined according to Wessel's modified criteria: crying for more than $3 \mathrm{~h}$ per day for more than 3 days per week.

Results: A total of 2,605 (4.2\%) infants were born preterm, 54441 $(86.7 \%)$ at term, and $5715(9.1 \%)$ post-term. A total of 4,353 (7.7 \%) infants fulfilled Wessel's modified criteria. The risk for infantile colic increased with lower gestational age and birth weight after adjustment for several possible confounders. The highest risk (odds ratio [95\% confidence interval]) was observed for infants born before 32 complete gestational weeks (1.5 [1.0-2.2; $p<0.05]$, reference: 40 gestational weeks) or with birth weight less than 2,000 g (1.7 [1.3-2.2], reference: 3,500-3,999 g). Small for gestational age infants (birth weight below 10th percentile) had higher risk for infantile colic in all gestational ages. This was statistically significant for infants born between 37 and 41 complete gestational weeks (1.2 [1.1-1.3]).

Conclusions: We found an association between infantile colic, and gestational age and birth weight in a large cohort study. This indicates that the aetiology of infantile colic may be found in the prenatal time period.

\section{P-381}

Immigrant women and maternal mortality in Western Europe: a meta-analysis of observational studies

Topic: Exposure - Other

Presenter: Grete Skфtt Pedersen

Grete Skøtt Pedersen 1); Anders Grøntved 2); Laust Hvas Mortensen 3); Anne-Marie Nybo Andersen 3); Janet Wilson RichEdwards 4)

1) Department of Epidemiology, Institute Public Health, University of Southern Denmark, Odense, Denmark; 2) Institute of Sports Science and Clinical Biomechanics, Centra of Research in Childhood Health, University of Southern Denmark, Odense, Denmark; 3) Section of Social Medicine, Department of Public Health, University of Copenhagen, Copenhagen, Denmark; 4) Connors Center of Women's Health and Gender Biology, Brigham and Women's Hospital and Harvard Medical School, Boston, MA, US.

Background: Western Europe have some of the lowest maternal mortality rates in the World, yet not all women have benefitted equally from obstetrical progresses, and research has suggested a less favorable decline for especially immigrant women.

Objectives: Therefore we conducted a systematic quantitative assessment to examine whether an excess risk of maternal mortality exists among immigrant women in Western Europe.

Methods: We searched the epidemiologic literature for eligible studies published 1970 through 2012, and included all observational studies comparing maternal mortality between the majority- and a defined immigrant population. Results were derived from a randomeffects meta-analysis, and statistical heterogeneity assessed by the I2statistics. In sub-analysis we also pooled data stratified by direct and indirect death causes.

Results: We included 13 studies with more than 42 million women and 4995 maternal deaths. Compared with indigenous born women, the pooled risk estimate (RR) was 2.00 with $95 \%$ confidence interval (CI) of $(1.72,2.33)$. Immigrant women had a higher risk of dying from direct than indirect death causes; pooled RRs of $2.65 \mathrm{CI}$ (1.88-3.74) and 1.83 CI (1.37-2.45).

Conclusion: This meta-analysis provides evidence that immigrant women in Western European countries have an excess risk of maternal mortality especially from direct death causes.

\section{P-382}

Association between exposure to family violence in childhood and self-reported lifetime suicide attempts in Latvian youth

Topic: Exposure - Other

Presenter: Lauma Springe

Lauma Springe 1); Anita Villerusa 1); Toms Pulmanis 2); Iveta Pudule 2); Daiga Grinberga 2); Biruta Velika 2)

1) Riga Stradins university, Department of Public Health and Epidemiology; 2) Centre for Disease Prevention and Control of Latvia, Department of Research, Statistics and Health Promotion 
Background: According to WHO Detailed Mortality database suicide rate in Latvia is 3rd highest in European Union and leading cause of death in age group 15-19 years highlighting suicide prevention as an important public health issue in Latvia. There are studies suggesting that exposure to family violence in childhood is considered as a risk factor for devastating consequences in later lives of the individuals by increasing stress level in children that can manifest as adverse behaviour in later life.

Objective: To assess relationship between exposure to emotional, physical and sexual family violence in childhood and lifetime suicide attempts in young adults in Latvia.

Methods: Data source for the research was representative crosssectional population survey about adverse childhood experiences (ACE). The survey was conducted in Latvia in 2011. In total 1,223 young adults (615 males and 608 females) aged 18-25 years were included in analysis. In survey methodology recommended by WHO was used. In study dependent variable was self-reported lifetime suicide attempt. Independent variables were: exposure to physical, emotional and sexual violence in family, gender, family history of suicide, parental divorce or separation, addictive substance abuse in family and family income level. Logistic regression analysis was performed - one model for each type of violence. Statistical significance was based on $95 \%$ confidence intervals.

Results: Those respondents who had experienced family violence in childhood had higher odds of lifetime suicide attempt-emotional violence OR 2.3 (95\% CI 1.4-3.9), physical violence OR 4.1 (95\% CI 2.4-6.8) and sexual violence OR 1.2 (95\% CI 0.6-2.4) adjusted for gender, suicide history in family, parental divorce or separation, addictive substance abuse in family and family income level. Female had higher odds of lifetime suicide attempt-OR $2.4(95 \% \mathrm{CI}$ 1.4-4.3) when adjusted for emotional violence, OR $2.5(95 \%$ CI 1.4-4.4) when adjusted for physical violence and OR 2.3 (95\% CI 1.3-4.2) when adjusted for sexual violence and other family related factors. Important factor regarding suicide attempts was history of suicide in family - adjusting for emotional violence OR $3.8(95 \% \mathrm{CI}$ 2.1-7.2), adjusted for physical violence OR 3.5 (95\% CI 1.8-6.6) and OR 3.9 (95\% CI 2.5-7.6) when adjusted for sexual violence and other family related factors.

Conclusions: After controlling for gender and family related factors in childhood there remained association between emotional and physical violence exposure in childhood and higher odds of selfreported lifetime suicide attempt. Female had higher odds of lifetime suicide attempts after controlling for violence exposure and family related factors. Family history of suicide was associated with higher odds of lifetime suicide attempts in Latvian youth.

\section{P-383}

Parental feeding practices and their association with growth trajectories in a Portuguese Birth Cohort during the first years of life

Topic: Exposure - Other

Presenter: Milton Severo

Milton Severo 1), 2); Hélio Alves 2); Andreia Oliveira 1), 2); Ana Cristina Santos 1), 2); Henrique Barros 1), 2); Carla Lopes 1), 2)

1) Public Health Institute, University of Porto, Portugal; 2) Department of Clinical Epidemiology, Predictive Medicine and Public Health, University of Porto Medical School, Portugal

Background: Studies have shown that children growing under different parental control practices have different BMI scores, however the age at onset of these influences are unknown.
Objective: Determine the relationship between maternal control feeding attitudes and growth trajectories, from birth to 4-5 years of age.

Methods: Weight and height measures were extracted from the child's health booklets of 4,388 children from the Generation XXI birth cohort, during the 4-5 years follow-up evaluation (2009-2010). As BMI trajectories across infancy follow a complex pattern, a local polynomial regression model was used to find the best-fitting average growth trajectory and the regression model predictions were used to derive a piecewise linear model. Linear mixed effect models were used to estimate individual growth trajectories (mean observations per child of 18). Random effects allow each individual to have different intercepts and slopes for each period.

From 4,388 children, 3,604 mothers completed a combined version of the Child Feeding Questionnaire and the over/covert control dimensions ( 38 items). The association between the growth trajectories and five dimensions of parental control (restriction, pressure to eat, monitoring, overt control and covert control) were adjusted for child's sex, maternal years of education and age.

Results: The BMI average pattern showed 3 distinct periods of growth (birth to 5 months, 5-34 months and 34-67 months). The velocity of growth $\left(\mathrm{kg} / \mathrm{m}^{2}\right.$ per month) at $0-5$ months was 0.834 ; at 5-34 months we found a difference in velocity from the previous period of -0.890 and of 0.045 when considering the 34-67 months. Pressure to eat was negatively significantly associated with the velocity of growth at $0-5$ months $(=-0.03, p<0.001)$.

An interaction effect was found between maternal education and the cover and overt control on the association with the velocity of growth at $0-5$ months $(p<0.001)$ and the velocity of growth at 34-67 months $(p=0.019)$, respectively. In more educated mothers, the covert control showed a positive association with the velocity of growth $(=0.024, p=0.009)$ while the overt control showed a negative association in less educated mothers $(=-0.007, p=0.046)$. Restriction and monitoring did not show a significant association with growth trajectories.

Conclusions: Pressure to eat and covert control promoted by more educated mothers differentiate the velocity of BMI growth at early childhood, while the overt control in less educated mothers differentiates growth velocity in later stages of childhood.

(The project was supported by FCT-PTDC/SAU-ESA/108577/ 2008 grant)

\section{P-384}

Interactions between maternal diabetes mellitus, periconceptional folic acid use, and MTHFR $677 \mathrm{C}>\mathrm{T}$ in the risk of congenital anorectal malformations

Topic: Exposure - Other

Presenter: Charlotte HW Wijers

Charlotte HW Wijers 1); Ivo de Blaauw 2), 3); Nadine Zwink 4); Loes FM van der Zanden 1); Alice S Brooks 5); Paul MA Broens 6); Ekkehart Jenetzky 3); Heiko Reutter 7), 8); Nel Roeleveld 1); Iris ALM van Rooij 1)

1) Department for Health Evidence, Radboud University Nijmegen Medical Centre, Nijmegen, The Netherlands; 2) Department of Pediatric Surgery, Radboud University Nijmegen Medical Centre, Nijmegen, The Netherlands; 3) Department of Pediatric Surgery, Sophia Children's Hospital-Erasmus MC, Rotterdam, The Netherlands; 4) Division of Clinical Epidemiology and Aging Research, German Cancer Research Centre, Heidelberg, Germany; 5) Department of Clinical Genetics, Erasmus MC, Rotterdam, The 
Netherlands; 6) Department of Surgery, University Medical Center Groningen, Groningen, The Netherlands; 7) Institute of Human Genetics, University of Bonn, Bonn, Germany; 8) Department of Neonatology, Children's Hospital, University of Bonn, Bonn, Germany

Background: Congenital anorectal malformations (ARM) occur in 2-6 cases per 10,000 births worldwide. The etiology of ARM is still largely unknown, but the involvement of both genetic and exogenous risk factors is suggested. A few studies found an association between ARM and maternal preexisting diabetes mellitus. As periconceptional use of supplements containing folic acid was found to reduce the risk of neural tube defects and orofacial clefts, with even larger effects in combination with the $677 \mathrm{C}>\mathrm{T}$ polymorphism in the MTHFR (methylenetetrahydrofolate reductase) gene, similar effects may exist for ARM. However, the results for folic acid supplementation are inconsistent and the role of interactions in the etiology of ARM has hardly been studied.

Objectives: To investigate interactions between maternal diabetes mellitus, periconceptional folic acid supplementation, the MTHFR $677 \mathrm{C}>\mathrm{T}$ polymorphism, and the risk of ARM.

Methods: We conducted a case-control study among 522 nonsyndromic ARM cases and 2,139 population-based controls within the AGORA biobank. Maternal questionnaires were used to obtain information on preexisting diabetes and any use of supplements containing folic acid during 4 weeks before through 10 weeks after conception. DNA samples of mothers and children were genotyped for the MTHFR 677C $>$ T polymorphism in a subset of 367 cases and 697 controls plus 166 additional German ARM cases. Odds ratios for independent and joint effects were estimated.

Results: No independent association was found between ARM and a lack of periconceptional folic acid use (OR 0.9; $95 \%$ CI 0.7-1.2), whereas preexisting diabetes seemed to increase the risk of ARM (OR 2.8; $95 \%$ CI 0.9-8.1). A joint association between ARM and diabetes plus a lack of folic acid use was not observed. Independent associations between ARM and child and maternal MTHFR 677CT/TT genotypes were identified in the German population (OR 1.5; $95 \% \mathrm{CI}$ 1.1-2.2 and OR 1.4; $95 \%$ CI 1.0-2.0, respectively), whereas these associations did not exist in the Dutch population. However, both Dutch child and maternal MTHFR 677CT/TT genotypes in combination with a lack of periconceptional folic acid use seemed to be associated with a small increased risk of ARM (OR 1.3; $95 \% \mathrm{CI}$ $0.9-2.0$ and OR $1.2 ; 95 \%$ CI $0.8-1.9$, respectively). The relative excess risks suggest interactions on an additive scale (RERIs $>0$ ). Interactions will also be studied in the German study population shortly.

Conclusions: These preliminary results suggest a role for maternal preexisting diabetes in the etiology of ARM and may point towards a gene-environment interaction between a lack of folic acid use and the MTHFR $677 \mathrm{C}>\mathrm{T}$ polymorphism.

\section{P-385}

Risk factors for VTE during the postpartum period using linked primary and secondary care data: a population based cohort study from England

Topic: Exposure - Other

Presenter: Alyshah Abdul Sultan

Alyshah Abdul Sultan 1); Matthew Grainge 1); Joe West 1); Kate Fleming 2); Catherine Nelson-Piercy 2); Laila Tata 1)

1) Division of Epidemiology and Public Health, University of Nottingham; 2) Women's Health Academic Centre, Guy's \& St Thomas' Foundation Trust, St Thomas' Hospital
Background: Venous thromboembolism (VTE) is a serious maternal complication and one of the leading causes of maternal mortality in developed countries. Better understanding of maternal risk factors and their impact on postpartum VTE risk can help target women who are most likely to benefit from thromborprophylaxis.

Objective: To determine the the risk factors for VTE during the postpartum period while adequately controlling for other confounding and mediatory factors.

Methods: We used linked primary and secondary care data to analyse 222,407 pregnancies resulting in live or stillbirth among women aged 15-44 years between 1997 and 2010 with postpartum follow-up. The risk factors for VTE during the postpartum period were assessed in terms of absolute and relative risk using a conceptual hierarchical analysis as well as their impact on the timing of VTE during specific postpartum periods.

Findings: Pregnancies of women resulting in stillbirths are at sixfold (95\% CI 3.17-14.6) increased risk of VTE during the postpartum period. We also found that those with caesarean delivery (elective or emergency), pre-term birth or postpartum haemorrhage have at least a twofold or higher risk of VTE during postpartum compared to their respective baseline. These factors are not confounded by pregnancy related characteristics and complications, pre-existing medical comorbidities or demographic or life style related characteristics. Finally we have demonstrated that the risk of VTE remains consistently high up to 6 weeks postpartum ( $>700 / 100,000$ person-years) in women with BMI $>30 \mathrm{~kg} / \mathrm{m}^{2}$ or caesarean delivery whereas the risk of VTE is only increased up until the first 3 weeks postpartum (>1300/100,000 person-years) in those with pre-term birth or postpartum haemorrhage.

Conclusion: Delivery associated characteristics and complications including placental disorder, stillbirth, caesarean delivery, postpartum haemorrhage are important risk factors for VTE particularly during the first 3 weeks postpartum. The rate of VTE remains high up to 6 weeks after delivery in women with high BMI and those who had a caesarean section delivery. These findings should be carefully considered when planning thromboprophylaxis.

\section{P-386}

Trends in medicine use during pregnancy in Finland 1996-2010

Topic: Exposure - Other

Presenter: Mika Gissler

Mika Gissler 1); Miia Artama 2); Anna-Maria LahesmaaKorpinen 1); Heli Malm 3); Marja-Leena Nurminen 4); Annukka Ritvanen 1); Leena Saastamoinen 5)

1) National Institute for Health and Welfare, Helsinki, Finland; 2) Cancer Society of Finland, Helsinki, Finland; 3) Teratology Information, HUSLAB and Helsinki University Central Hospital, Helsinki, Finland; 4) Finnish Medicines Agency Fimea, Helsinki, Finland; 5) The Social Insurance Institution of Finland, Helsinki, Finland

Background: The use of medicines during pregnancy is common, but drug safety studies in pregnant women cannot be conducted due to ethical reasons. Pregnancy-related safety data is mostly based on animal testing, which sometimes poorly applies to humans. Epidemiological studies on large nation-wide data are needed for identifying drug-related risks to pregnancy and the fetus.

Objectives: The aim of this study was to identify the trends and proportions of medicine use during pregnancy and the degree of safety of the medicine use during pregnancy. Additionally, we evaluated how many pregnant women have chronic diseases and what drugs are used to treat these diseases during pregnancy. 
Methods: Data on births $(\mathrm{n}=859$ 359) in Finland during 1996-2010 was obtained from the Medical Birth Register. This data was linked to information on maternal reimbursed drug purchases and maternal eligibility for higher drug reimbursement for certain chronic diseases from the databases of the Social Insurance Institution.

Results: Medicine use was common in pregnant women, with $51 \%$ having at least one drug purchase at some point during the pregnancy, including 1 month prior to pregnancy. About $30 \%$ purchased at least one drug during the first month of pregnancy or one month prior to pregnancy. Almost one-third (29\%) of women purchased three or more drugs during the study period. The percentage of pregnant women purchasing medicines increased from $49 \%$ in 1996 to $59 \%$ in 2010. The number of women with chronic diseases increased from $5.6 \%$ in 1996 to $7.7 \%$ in 2010. There was also an increase in drug use, for example selective serotonin reuptake inhibitors (a tenfold increase from $0.43 \%$ in 1996 to $4.5 \%$ in 2010) and the antiepileptic lamotrigine (from $<0.01 \%$ at its start of licensing in 1996 to $0.23 \%$ in 2010, the most used antiepileptic). Of the purchased medicines, $34 \%$ were known to be harmful to the fetus according to the pregnancy safety classification (FASS) and clearly harmful in $4 \%$. The number of harmful drug purchases decreased with advancing gestation.

Conclusions: Monitoring of drug use during pregnancy and lactation, drug safety and possible harmful effects on the fetus are important, and these data show that this can be done efficiently and reliably by combining national registers. Continuing the surveillance in the future will increase knowledge about newer drugs and will play an important role in monitoring drug safety.

\section{P-387}

\section{Disparities in self-rated health by social factors in Kazakhstan}

Topic: Exposure - Other

Presenter: Andrej Grjibovski

Akmaral Abikulova 1); Kazbek Tulebaev 1); Aigulsum Izekenova 1); Ainur Kumar 1); Manshuk Ramazanova 1); Alfiya Igissenova 1); Bolat Zhanturyiev 1); Zarina Sagyndykova 1); Elmira Abdraimova 2); Andrej Grjibovski 3), 4)

1) School of Public Health, Kazakh National Medical University, Almaty, Kazakhstan; 2) Kazakh - Russian Medical University, Almaty, Kazakhstan; 3) Norwegian Institute of Public Health, Oslo, Norway; 4) International School of Public Health, Northern State Medical University, Arkhangelsk, Russia

Background: Self-rated health (SRH) has been widely studied to assess health inequalities in both developed and developing countries. However, no studies have been performed in Central Asia. The aim of the study was to assess gender-, ethnic-, and social inequalities in SRH in Almaty, Kazakhstan.

Methods: Altogether, 1,500 randomly selected adults aged 45 years or older were invited to participate in a cross-sectional study and 1,199 agreed (response rate $80 \%$ ). SRH was classified as poor, satisfactory, good and excellent. Multinomial logistic regression was applied to study associations between SRH and socio-demographic characteristics. Crude and adjusted odds ratios (OR) for poor versus good and for satisfactory versus good health were calculated with $95 \%$ confidence intervals $(\mathrm{CI})$.

Results: Altogether, poor, satisfactory, good and excellent health was reported by $11.8,53.7,31.0$ and $3.2 \%$ of the responders, respectively. Clear gradients in SRH were observed by age, education and income in both crude and adjusted analyses. Women were more likely to report poor (OR 1.9, $95 \%$ CI 1.2-3.1) or satisfactory (OR 1.6, $95 \%$ CI 1.2-2.1) than good health. Ethnic Russians and unmarried participants had greater odds for poor versus good health (OR 2.3, $95 \%$ CI 1.5-3.7 and OR 4.0, $95 \%$ CI 2.7-6.1, respectively) and for satisfactory versus good health (OR 1.4, $95 \%$ CI 1.1-1.9 and OR 1.9, $95 \%$ CI 1.4-2.5, respectively) in crude analysis, but the estimates were reduced to non-significant levels after adjustment. Unemployed and pensioners were less likely to report good health than white-collar workers while no difference in SRH was observed between white- and blue-collar workers.

Conclusion: Considerable levels of inequalities in SRH by age, gender, education and particularly income, but not by ethnicity or marital status were found in Almaty, Kazakhstan. Further research is warranted to identify the factors behind the observed associations in Kazakhstan.

\section{P-388}

The socioeconomic determinants of myocardial infarction incidence, fatality and mortality

Topic: Exposure - Other

Presenter: Fanny Kilpi

Fanny Kilpi 1); Hanna Konttinen 1); Karri Silventoinen 1); Pekka Martikainen 1)

\section{1) Department of Social Research, University of Helsinki}

Background: Lower socioeconomic position (SEP) has been associated with an increased risk of myocardial infarction (MI) incidence, mortality and fatality. However, different dimensions of SEP may have varying impacts at different stages of the disease.

Objectives: To use Finnish register-based data to compare the independent effects of education, occupation, income and wealth in MI incidence, mortality and fatality. We tested a hypothesis that education is particularly influential for MI incidence, whereas income becomes a stronger determinant of subsequent survival.

Methods: A large nationally representative sample of 40-60 year old men and women with no prior CHD and with information on level of education, occupational class, household disposable income and taxable wealth in 1995 was followed up for MI incidence and mortality 1996-2007 $(\mathrm{n}=302,885)$. Data linkages with the cause of death register and hospital discharge register were used to identify MI (ICD-10 I21-22). The sample for the analysis of fatality included only those with MI incidence in the hospital discharge register ( $n=6,251$ ), with the baseline set at the date of admission. The associations of different SEP measures with all three MI outcomes were analyzed with Cox's regression method separately in men and women. To identify the independent effects of education, occupation, income and wealth, models were adjusted for age, region of residence and all of the SEP measures.

Results: Each of the indicators of SEP had significant independent effects in MI mortality and for men also in incidence, with some indications of gradient effects. In women, only income and wealth had statistically significant effects in incidence. In both genders only income and wealth retained significant associations with fatality. Wealth had the most consistent relationship with all of the MI outcomes. The greatest hazard ratios in incidence and mortality were found for the lowest wealth quintile compared to the highest quintile (in incidence: men $\mathrm{HR}=1.77,95 \%$ CI $(1.59$, 1.97), women $\mathrm{HR}=2.03,95 \% \mathrm{CI}(1.67,2.47)$, and in mortality: men $\mathrm{HR}=2.36$, $95 \%$ CI $(2.14,2.60)$, women HR $=2.94,95 \%$ CI $(2.41,3.58))$.

Conclusions: Wealth was a particularly important determinant of MI at all stages in both men and women. Also, in men education was a stronger determinant of incidence than income, but income showed a stronger association with post-MI survival. By disentangling the 
effects of different SEP measures on MI, we highlight the multidimensionality of social position and its effect on health.

\section{P-389}

Effect of neonatal weight change on child adiposity at 4-5 years of age: generation XXI birth cohort study

Topic: Exposure - Other

Presenter: Henrique Barros

Maria João Fonseca 1), 2); Sofia Correia 1), 2); Henrique Barros 1), 2); Ana Cristina Santos 1), 2)

1) Department of Clinical Epidemiology, Predictive Medicine and Public Health - University of Porto Medical School; 2) Institute of Public Health - University of Porto

Excessive or insufficient neonatal weight losses (NWL) have been associated with health problems in the neonatal period, but little is known about its long term consequences. We aim to evaluate the effect of weight change in the first $96 \mathrm{~h}$ of life on child adiposity, measured by body mass (BMI), fat mass and fat-free mass indexes, waist circumference, and waist-to-height ratio, measured at 4-5 years of age.

Generation XXI included 8647 newborns recruited between 2005/2006 at the public units providing obstetrical and neonatal care in Porto. Information was gathered by face to face interviews and additional data was abstracted from clinical records, including birth weight. Neonatal anthropometrics were obtained by trained interviewers and we restricted the analysis to those weighed up to $96 \mathrm{~h}$ of life. Neonatal weight change was estimated as (weight-birth weight)/birth weight 100 , adjusted for newborn age in hours. We categorized as excessive NWL (bellow 10th percentile of the distribution of weight change: $\leq-9.6 \%$ ), normal NWL (between 10th and 90th percentiles: -9.5 to $-4.3 \%$ ) and insufficient NWL (above 90th percentile: $\geq-4.2 \%$ ). At $4-5$ years of age, children were reevaluated according to standard procedures, including weight, height, waist circumference, and body composition. We present life course data for 875 normal term singletons. Adjusted regression coefficients and $95 \%$ confidence intervals [ $(95 \% \mathrm{CI})]$ were computed using generalized linear models.

Children with excessive NWL had a reduction in BMI $[-0.471$ $(-0.878 ;-0.064)]$, fat-free mass index $[-0.294(-0.556 ;-0.033)]$ and waist-to-height ratio $[-0.011(-0.020 ;-0.002)]$ and children with insufficient NWL had a reduction in waist-to-height ratio $[-0.010(-0.019 ;-0.002)]$, both compared with normal NWL children.

This study provides evidence for a long term effect of weigh changes in the first few days of life on the amount and distribution of adiposity.

\section{P-390}

Socioeconomic determinants of parental discipline in school-aged children

Topic: Exposure - Other

Presenter: Isabel Moreira

Isabel Moreira 1); Diogo Costa 1); Henrique Barros 1)

1) Institute of Public Health, University of Porto, Portugal

Background: Abusive parental disciplinary practices compromise children's physical and psychosocial healthy development. Therefore, exploring the determinants of these behavioural profiles is important to depict at-risk groups and provide insights to prevention strategies.
Objective: To analyse the association between socioeconomic characteristics and parental disciplinary practices, used in Portuguese school-aged children.

Methods: We evaluated 1666 parent-child dyads (1196 mothers and 470 fathers) of 7 years-old children as part of the follow-up of Generation XXI birth cohort $(\mathrm{n}=8647)$. Parental characteristics, including socioeconomic and health features, were obtained through face-to-face interviews performed by trained interviewers using standardized questionnaires. Weight and height were measured according to standard procedures. The Conflict Tactics Scale ParentChild version (CTSPC) was self-completed independently by mothers and fathers. Items were rated according to the original dimensions of the scale into nonviolent discipline (NVD), corporal punishment (CP), psychological aggression (PsyA) and physical assault (PA). Past-year prevalence was computed for each dimension. Associations were estimated using logistic regression [odds ratio (OR) and $95 \%$ confidence intervals $(95 \% \mathrm{CI})$ ] and adjusted for parent education, child's gender and, for mothers only, victimization before child's birth.

Results: Mothers reported $99 \%$ of NVD, $93 \%$ of CP, $97 \%$ of PsyA and $7 \%$ of PA toward their child in the previous year. In fathers, prevalence was $99 \%$ for NVD, $88 \%$ for CP, $96 \%$ for PsyA and $4 \%$ for PA. In mothers, CP was significantly associated with age (OR 0.94 $95 \%$ CI $0.90 ; 0.99)$ while PsyA was associated with both age (OR $0.9195 \%$ CI $0.85 ; 0.98$ ) and education (OR $1.1895 \%$ CI 1.07 ; 1.29). In fathers, $\mathrm{CP}$ was significantly associated with family structure (OR $0.2895 \%$ CI $0.09 ; 0.84$ ) whereas PsyA was associated with fathers' education (OR $1.2295 \%$ CI 1.05; 1.42), employment status (OR $0.2695 \%$ CI 0.09;0.78) and smoking habits $(\mathrm{OR}=0.3695 \%$ CI $0.13 ; 0.98)$. No significant association were found between NVD and PA practices and parents answers.

Conclusion: Younger mothers are more prone to use CP and PsyA when compared to older mothers. A positive association was also found for educational level and PsyA among mothers and fathers. Smoking and non-employed fathers tend to use less PsyA when compared with non-smoking and employed fathers, respectively. Fathers of children that do not live with both parents tend to report less CP. These findings, in the context of the Portuguese culture, are important steps to further explorations on the impact of parent-child interactions on children development.

Funding: FCT [PTDC/SAU-SAP/122904/2010]

\section{P-391}

Contribution of maternal age to preterm birth rates in Denmark and Québec, 1981-2008

Topic: Exposure - Other

Presenter: LH Mortensen

\section{N Auger 1); AL Park 2); AV Hansen 3); LH Mortensen 4)}

1) Institut national de santé publique du Québec, Montréal, Québec; 2) Institut national de santé publique du Québec, Montréal, Québec; 3) Univeristy of Copenhagen, Denmark; 4) Univeristy of Copenhagen, Denmark

Background, objectives: Advancing maternal age is supposedly causing a rise in preterm birth (PTB) rates, but evidence supporting this hypothesis is lacking for high income countries.

Methods: We assessed maternal age-specific trends in PTB using all singleton live births in Denmark $(\mathrm{N}=1,674,308)$ and Québec, Canada $(\mathrm{N}=2,291,253)$ from 1981 to 2008 . We decomposed the country-specific contributions of age-specific PTB rates and maternal age distribution to overall PTB rates over time.

Results: PTB rates increased over time from 4.4 to $5.0 \%$ in Denmark and 5.1-6.0\% in Québec. Rates increased the most in women aged 
20-29 years, while rates decreased or remained stable in women $\geq 35$ years. The overall increase over time was driven by age-specific PTB rates, although the contribution of younger women was countered by fewer births at this age in both Denmark and Québec.

Conclusions: PTB rates increased among women aged 20-29 years, but their contribution to the overall PTB rates was offset by older maternal age over time. Women aged 20-29 years should be targeted to reduce PTB rates, as potential for prevention may be greater in this age group

\section{P-392}

\section{Risk for latent tuberculosis infection among children}

Topic: Exposure - Other

Presenter: António Madureira Dias

António Madureira Dias 1); Regina Saraiva 2); Madalena Cunha 3); Carlos Pereira 4); Carlos Albuquerque 5)

1) CI\&DETS and FCT (PEst-OE/CED/UI4016/2011) - Superior Health School, Polytechnic Institute of Viseu, Portugal; 2) CI\&DETS and FCT (PEst-OE/CED/UI4016/2011) - Superior Health School, Polytechnic Institute of Viseu, Portugal; 3) CI\&DETS and FCT (PEst-OE/CED/UI4016/2011) - Superior Health School, Polytechnic Institute of Viseu, Portugal; 4) CI\&DETS and FCT (PEst-OE/CED/ UI4016/2011) - Superior Health School, Polytechnic Institute of Viseu, Portugal; 5) CI\&DETS and FCT (PEst-OE/CED/UI4016/ 2011) - Superior Health School, Polytechnic Institute of Viseu, Portugal

Background: Tuberculosis is a serious public health problem. In the pediatric group measures and control policies of infection have been limited by the difficulty in diagnosis and in the absence of a consensual definition.

Objective: To determine the prevalence of latent tuberculosis infection (LTI) in people who contacts with tuberculosis patients.

Methods: It is a retrospective, descriptive and cross study to patients followed in CDP Viseu in the program "Following people who contacts with tuberculosis patients". The study sample consisted of 77 individuals with 0-18 years-old (exclusive) from 2010-2012 who were in contact with pulmonary tuberculosis patients. To collect data were analyzed all records computerized (SAM and SAPE).

The majority $(54.0 \%)$ were male, mean age of $9.25 \pm 4.95$ years. The males' age were superior than the females (10.0 vs. 8.31; $t=1.567, p=0.121)$. The majority $(62.2 \%)$ belonged to the age group of 5-15 years and $23.2 \%$ to $1-4$ years. $97.4 \%$ were Portuguese. As for the relationship with the index case, $59.7 \%$ were relatives and $32.5 \%$ lived with the patient. Of the 77 individuals only $5.2 \%$ ( 4 cases) had symptoms " $t=1.567, p=0.121$ ). The majority $(62.2 \%)$ belonged to the age group of 5-15 years and $23.2 \%$ to 1-4 years. $97.4 \%$ were Portuguese. As for the relationship with the index case, $59.7 \%$ were relatives and $32.5 \%$ lived with the patient. Of the 77 individuals only $5.2 \%$ (4 cases) had symptoms ("cough and phlegm") and $2.6 \%$ had comorbidities (asthma). Disadvantaged socio-economic conditions were registered in $14.3 \%$. We used the Chi square test in SPSS 20.0.

Results: $24.7 \%$ did Interferon Gamma Release Assays (IGRA), which was positive in $84.2 \%$ (16 cases). $24.7 \%$ did chemoprophylaxis and $26.3 \%$ stopped this treatment.

We found a relationship between socio-economic disadvantage and positive Quantiferon $(p=0.047)$. The IGRA had a sensitivity of $100 \%$ and specificity of $75 \%$ with an accuracy of $94.7 \%$ (Kappa $=0.826 ; p=0.000)$.

Conclusions: IGRA tests are a first breakthrough in search of better diagnostic tests representing a more effective control of the disease.

\section{P-393}

30 years of continued success: gestational age and infant mortality in Denmark 1980-2009

Topic: Exposure - Other

Presenter: LH Mortensen

LH Mortensen 1); AM Krogh Thrane 2); L Billeschou 2); TB Henriksen 3); AMN Andersen 1)

1) University of Copenhagen; 2) University of Southern Denmark; 3) University of Aarhus

Background, objective: Infant mortality has dropped dramatically over the last century. In the last decades the mortality among infant born preterm has declined substantially. This paper examines the gestational age-specific decline in Denmark 1980-2009.

Methods: Using the Danish Medical Birth Registry, we examined the association between gestational age and infant mortality over time. Two-dimensional beta-splines and Kitagawa decomposition was used to examine the association.

Results: As expected a remarkably strong decline was observed for all infant born preterm. The yearly relative decline was close to constant over the 30-year period. In comparison the decline in mortality among infants born at term was weak. The declines in the overall infant mortality is driven by the strong decline in the gestational age-specific mortality, but the contribution from the decline in risk is partly counteracted by an increase in the number of infants born preterm.

Conclusion: As in many other populations, the infant mortality in Denmark has declined, particularly due to the decrease in risk among infants born preterm. This continuing success does not appear to be leveling off.

\section{P-395}

\section{Risk factors of caregiver fatigue}

Topic: Other - Health promotion Presenter: Ricardo Casas Fischer

\section{Ricardo Casas Fischer 2); Belen Herrero Cembellin 1);} Carmen Muñoz Simon 3); Begoña Sahagún Salcedo 4)

1) Nurse of the Epidemiology Section AVILA of the Ministry of Health, Castile and Leon; 2) Chief of Epidemiology Section SS.TT. Health of Avila; 3) Nurse of the Epidemiology Section AVILA of the Ministry of Health, Castile and Leon; 4) Medical doctor of the Epidemiology Section AVILA of the Ministry of Health, Castile and Leon, Spain

Background: The increase of life expectancy carries an increase of the population that needs daily help for basic activities, which is provided by $80 \%$ of informal supports (family/relatives), and $20 \%$ of formal caregivers (administrative support) and home employees, . This activity of aid produces physical and emotional overload, in addition to own health problems and conflicts with the cared relative. In contrast, there exists positive reinforcement .

One of the programs of the Sentinel Network of Castilla y León (RCSCYL) is 'Risk Factors of caregiver fatigue'.

Objectives: We measure the overload felt by caregivers of none autonomous people and its impact in the physical, psychological and social area. We also describe the characteristics of the cared person. Materials and methods: We carry out a prevalence study in caregivers of non autonomous people, captured by sentinel nursery staff during 2011 in scheduled consultation, on demand or on home visits. A weekly register is performed, collecting the variables included in 
the RCSCYL registration form (Appendix 1). The relatives ("principal informal caregiver ') of non autonomous participate with the following inclusion criteria: 'Cares for a dependent individual ', 'Does not get financial reward', 'does not belong to any healthcareor social institution', 'Assumes this commitment most of his time'.

For caregivers we compute the variables included in the RCSCYL registration form and the overload level with the Zarit scale, and for cared people the level of dependence is computed by the Barthel Index (Appendix 2). The data are processed with Excel (Windows 2000).

Results: From the 38 completed registers, $71 \%$ are women $(70 \%$ of 35-64 years), $60 \%$ with primary education. The relationship between caregiver and care is 'son/daughter' by $50 \%$ (70 \% females); $87 \%$ are cohabiting and the attention is daily in $100 \%$ ). A $68 \%$ have 'some kind' of overload (Zarit), $34 \%$ of such 'moderate or greater'. Musculoskeletal and sleep problems are present in 71 and $42 \%$, respectively. All non-autonomous people present 'some kind' of dependence (Barthel), only $23 \%$ with mild level.

Conclusions: The predominant caregiver profile is that of the 'sandwich' generation (daughter cohabiting with a dependent person with moderate/severe dependence, housewife aged 35-64 years with basic education level and children, presenting physical and emotional overload.

Recommendations: The results make necessary the implementation of multidisciplinary preventive actions in order to improve the working conditions of caregivers.

Keywords: Caregiver, Overload, Quality of life

Annex 1. Registration form for the Sentinel Network of Castilla y León

Annex 2: Zarit scale and Barthel index

\section{P-396}

Epidemiology of the use of complementary and alternative medicine in central area of São Paulo

Topic: Other - Health promotion

Presenter: Braian Castro

\section{Oziris Simoes 1); Braian Castro 1)}

1) Faculdade de Ciências Médicas da Santa Casa de São Paulo

Background: Alternative medicine is used to describe practice in health independently or in place of the conventional one such as acupuncture, chiropractic, and herbal medicine. The constant and growing use in the world, makes the epidemiological study of these practices very important.

Objectives: Establish the profile of São Paulo citizen regarding alternative practices, based on the literature and on the assumption that the use of CAM (Complementary and Alternative Medicine) is mediated by factors such as socioeconomic status, gender, age and dissatisfaction with official medicine. Also, determining the usage frequency and amount, types, costs and reasons. Furthermore, it aims to contribute to the health actions of the local Health Unit.

Methods: Cross-sectional study, population-based, through interviews with adults above 18 years old, living in Barra Funda in the influence area of the Health Centre, which was selected from a systematic sample, by homogeneous cluster, in areas covered by the PSF (Programa Saúde da Família). Were excluded from the sample those who could not respond to the interview, otherwise we requested the help of other family member. The sample contains 380 individuals considering the frequency of use, the difference found between the proportions of users or not, according to personal characteristics. The sample size considered the effect of the study design, losses due to refusal, lack of locals (after two visits to residences), failure in filling the questionnaire. The analysis was made with the Chi square test. Results: Significant relations were found on the use of these therapies with family income, gender and education. Also, it was determined the frequency, motivation, costs and the types of CAM most used by respondents. The use of CAM was more prevalent in people with income greater than the median household income of the sample. Furthermore, individuals with college degrees use complementary therapies more than others. It was also found that users spend little with CAM, considering the fact that about $60 \%$ of the users spent nothing on this medicine. Finally, we had acupuncture, prayer, tea, home remedy and homeopathic therapies as known by most respondents.

Conclusions: These findings allow us to identify the need for greater closeness between health professionals and alternative therapies. Also, it was found a high frequency and diversification of knowledge and use of CAM, and the need to expand the use of complementary therapies to assist in the restoration of health problems, and on preventive health.

\section{P-397}

Compositional and contextual predictors for emotional problems among adolescents

Topic: Other - Health promotion

Presenter: Charlotte Meilstrup

Charlotte Meilstrup 1); Annette Kjær Ersbøll 1); Line Nielsen 1); Pernille Due 1); Vibeke Koushede 1); Bjørn Holstein 1)

1) National Institute of Public health, University of Southern Denmark

Background: A large proportion of adolescents suffer from emotional problems and great variation is observed across schools. To what degree these variations are caused by compositional or contextual predictors is unknown.

Objectives: The objectives of this presentation is to identify factors and conditions at the individual-, classroom- and school level that are associated with students' experiences of emotional problems.

Methods: Data come from the Danish contribution to the international Health Behaviour in School-aged Children (HBSC) study in 2010 and include 4,922 students aged 11,13 and 15 years from a random sample of Danish schools. Students answered a questionnaire including mental health and the classroom environment. The outcome measure emotional problems was defined as daily presence of at least one of four symptoms: feeling low; irritable or bad tempered; feeling nervous and having difficulties falling asleep. Further, schooladministrators answered a questionnaire on school environment and characteristics, e.g. policies against bullying. We applied multilevel multivariate logistic regression analyses to identify and quantify explanatory factors at individual, classroom and school levels.

Results: Initially a large variation in emotional problems was observed from one school to another. The proportion of students with daily experience of emotional problems varied between $7 \%$ and $32 \%$ across schools. Individual level variables such as socio-economic position and family composition explained much of the variation across schools. Students from low (OR 1.74 (CI 1.37; 2.22)) and medium (OR 1.53 (CI 1.24; 1.87)) social class, girls (OR 1.37 (CI $1.15 ; 1.63$ )) and students exposed to bullying (OR 3.20 (CI 2.17; 4.72)), had increased odds of experiencing emotional problems. At the classroom level, a high proportion of students who reported a negative classroom climate was significantly associated with emotional problems (OR 1.43 (CI 1.07; 1.92)). Further, bullying seems to reach beyond the individual. In classes with a high prevalence of 
bullying, students have significantly higher odds (OR 1.33 (CI 1.05; 1.70)) of experiencing emotional problems compared to classes where bullying didn't appear. These findings suggest that contextual exposure affects emotional problems.

Conclusions: We expected compositional factors to explain much of the variation in prevalence of emotional problems between schools. This study suggests that contextual factors at the school and class level are important to take into account in studies of emotional problems among children and adolescents.

\section{P-398}

Identifying the most effective public health nutrition policies: the views of policy-makers and thought-leaders across $14 \mathrm{EU}$ countries

Topic: Other - Health promotion

Presenter: Ffion Lloyd-Williams

Ffion Lloyd-Williams 1); Bromley Bromley 1); Lois Orton 1); Rory McGill 1); May Moonan 1); David Taylor-Robinson 1); Martin O'Flaherty 1); Nicola Calder 1); Mike Rayner 2); Simon Capewell 1)

\section{1) University of Liverpool; 2) University of Oxford}

Background: EuroHeart 2 is a European research programme led by the European Heart Network and European Society of Cardiology. ( http://www.ehnheart.org/euroheart-ii.html). One aspect of the project aimed to identify the most effective public health nutrition policies, in order to inform future evidence-based strategies to promote cardiovascular health.

Objectives: We interviewed senior policy-makers and thought-leaders in 14 diverse EU countries to elicit their views on a very wide range of possible nutrition strategies covering the entire public health policy spectrum aimed at improving approaches to public health nutrition.

Methods: We first mapped national nutrition policies across 30 European countries. We then identified, contacted and recruited potential participants in 14 diverse countries. Policy-makers, thoughtleaders and others active in the field of public health nutrition at the national level were interviewed. Questions were first developed and piloted with senior stakeholders in the UK. The interviews were conducted in English, either by person, telephone or Skype. The interviews typically lasted between 45 and $60 \mathrm{~min}$. The interviews were transcribed and entered into NVIVO software. The Framework approach was used to analyse the transcripts.

Results: We conducted 66 interviews in 14 countries across Europe. The interviews enabled more up to date and accurate information than was provided on websites or in reports. Responses revealed important differences between official lists of food policies and their actual implementation on the ground".

European countries are at very different stages of addressing public health nutrition issues. Most are promoting dialogue, recommendations and guidelines (often considered an early part of the policy process). Voluntary reformulation of foods is also common, especially for salt, sugar and total fat. However, legislation regulation or fiscal interventions targeting salt, sugar, fat or fruit and vegetable consumption are still uncommon. Many interviewees expressed a preference for regulation and fiscal interventions and generally believed they were more effective, albeit politically more challenging. Conversely, information-based interventions were often seen as being more politically feasible.
Conclusions: Public health nutrition policies in Europe represent a complex, dynamic and rapidly changing arena. Encouragingly, the majority of countries are engaged in activities intended to increase consumption of health food, and decrease the intake of junk food and sugary drinks. Exemplar countries demonstrating notable progress might include Finland, Norway, Iceland, Hungary, the UK and Portugal. However, most countries fall well short of optimal activities. Implementation of potentially powerful nutrition policies remains frustratingly patchy across Europe.

\section{P-399}

The effect of a pedometer-based physical activity intervention on health outcomes: a 3-month randomised controlled trial

Topic: Other - Health promotion

Presenter: Christina Bjørk Petersen

Christina Bjørk Petersen 1); Morten Grønbæk 1); Janne Schurmann Tolstrup 1)

1) National Institute of Public Health, University of Southern Denmark, Øster Farimagsgade 5A, 2. floor, 1399 Copenhagen, Denmark

Background: Walking is a popular and simple form of exercise and for this reason pedometer-based intervention program have the potential of reaching a large part of the population. In a recent study we found that receiving a pedometer and a goal-setting training program increased total walking time in older individuals $(65+$ years $)$ who were physically inactive or had low aerobic fitness. In younger individuals, no effect was observed.

Objectives: The aim of this study was to examine if the pedometerbased physical activity intervention was related to improved health outcomes.

Methods: The intervention was nested in the Danish Health Examination Survey (DANHES) and carried out in 2008. Participants were randomly assigned to either a pedometer group $(\mathrm{n}=326)$ or a control group $(\mathrm{n}=329)$. Outcome measures were assessed at baseline and at 3-monts follow up, and included blood pressure, body fat percentage, waist circumference, body mass index, self-rated health, daily stress and sleep quality. Differences were tested by Wilcoxons-signed rank tests and Chi squared tests.

Results: At follow-up, no overall differences in health outcomes were found. However, age-stratified results showed a significantly lower prevalence of restless sleep among the oldest participants (65+ years) in the pedometer group compared to the controls (controls $=34 \%$, pedometer group $=13 \%, p=0.03$ ). Also, although not significant, a higher proportion of older participants in the pedometer group had an optimal self-rated health compared to participants in the control group (controls $=76 \%$, pedometer group $=92 \%, p=0.06$ ). In younger individuals, no differences were observed. When stratifying results by gender, significantly more women reported an optimal health at follow-up in the pedometer group compared to the control group (controls $=83 \%$, pedometer group $=68 \%, p<0.01$ ).

Conclusions: In general, health outcomes were not improved following the pedometer-based physical activity intervention. However, the results suggest that receiving a pedometer and a goal-setting training program have a positive effect on health and well-being in terms of better sleep quality and improved self-rated health in older individuals as it increase physical activity. Thus, these results contribute to the understanding of differences in health effects of physical activity interventions.

Trial registration number: NCT01071811 


\section{P-401}

International migrant women and risk factors for not following infant health and safety recommendations

Topic: Other - Health promotion

Presenter: Anita Gagnon

Lisa Merry 1); Anita Gagnon 1)

1) McGill University

Background: $20 \%$ of births in Canada are to international migrant women. Recommended infant health and safety practices post-birth include administering vitamin $\mathrm{D}$ to breastfed infants and sleeping infants on their backs to prevent SIDS.

Objectives: To determine the rate of international migrant women not administering vitamin D or sleeping their infant prone and to identify risk factors for not following recommended practices.

Methods: 1,025 migrant women were recruited in 1 of 12 hospitals in the 3 largest migrant-receiving Canadian cities and followed 4 months post-birth to collect data on maternal and infant health concerns. Logistic regression was used to model social, migration, and health service factors predictive of not administering vitamin D or sleeping an infant prone.

Results: At 4 months post-birth $11.3 \%$ of migrant women were sleeping their infant prone; $10.5 \%$ were not administering vitamin D to their infant. Risk factors for not administering vitamin D/sleeping an infant prone included: education $\leq 12$ years [OR $1.4(95 \%$ CI 0.99 , 2.0)], maternal age $<25$ years [OR $1.6(95 \% \mathrm{CI} 1.03,2.56)$ ], unsure/ never had a pap smear [OR $1.4(95 \%$ CI $0.96,2.11)]$, did not attend prenatal education [OR 1.4(95\% CI 1.02, 2.1)] and giving birth in Montreal (vs. Vancouver) [OR 1.8(95\% CI 1.0,3.3)]. Being primiparous was protective [OR $0.73(95 \% \mathrm{CI} 0.50,1.05)]$.

Conclusions: Certain social and health service factors are important indicators of migrant women who might not follow recommended infant health and safety practices and could alert public health nurses, obstetricians and midwives to women who might need additional information/teaching. Differences in following recommended practices by location of birth warrant further investigation.

\section{P-402}

Comparison between foreign and Italian workers in work-related emergency department injury admissions

Topic: Other - Health promotion

Presenter: Anteo Di Napoli

Anteo Di Napoli 1); Aurora Marchetti 1); Veruscka Leso 1); Domenico Di Lallo 1); Gabriella Guasticchi 1)

1) Comparison between foreign and Italian workers in work-related emergency department injury admissions; 2) Agency for Public Health of Lazio Region

Background: The promotion of occupational safety and health in workplaces is a relevant target for public health. According to Italian National Institute of Statistics (ISTAT), about $15 \%$ of recorded work-related accidents in Italy involved foreign workers (more than $2,000,000$ ), employed in production sectors such as building trade, agriculture, tourism, manufacturing industry and housework. Therefore, the evaluation of the characteristics of work-related injuries among foreign workers represents a relevant issue.

Objectives: To analyze some characteristics of work-related injury and to evaluate factors potentially associated with a hospitalization in the population of foreign workers compared with Italians.
Methods: Retrospective study conducted in 2008 in Lazio, a region of central Italy, on 51,075 Emergency Departments (ED) admissions for work-related injuries, among 16-65 years old workers. We measured differences for proportions and frequency distributions between Italian and foreign injured workers, calculating Chi squared and Wilcoxon rank sum tests, respectively. We performed multivariate logistic regression to evaluate the probability of hospitalization after the work-related injury ED admission of foreign workers compared with Italians.

Results: We observed that $12.7 \%$ of all work-related injury ED admissions referred to foreign workers ( $46 \%$ from Romania). Compared with Italians, foreign injured workers were younger $(35.6+9.7$ vs. $39.6+11.0$ years old, $p<0.01)$ and more often $(p<0.01)$ were males ( 81.7 vs. $70.0 \%$ ), arrived to Emergency Department without an ambulance (95.2 vs. $93.9 \%$ ), had a triage code classified as red/yellow $\operatorname{tag}(5.3$ vs. $4.4 \%)$, a diagnosis of "Open wounds" (16.1 vs. $14.6 \%)$, "Penetrating injury with foreign body" (18.1 vs. $5.5 \%)$, "Disorders of the eye" (6.5 vs. $3.2 \%)$, "Burns" (2.6 vs. $1.3 \%)$ and a higher frequency of hospitalization as consequence of a work-related ED admission (3.5 vs. $2.5 \%$ ). We observed a higher probability of hospitalization for foreigner workers compared with Italians (OR 1.55; $95 \%$ CI 1.31-1.82).

Conclusions: We found a higher probability of more severe triage codes, of diagnoses related to more traumatic accidents and of hospital admission among foreign workers compared with Italians. We confirm more frequent and serious work-related injuries among foreign workers, probably because more often employed in dangerous activities, without an adequate professional training, also for the difficulty related to the language. These results are even more worrying, considering that an under-reporting of serious injuries could be more frequent among foreigners. Specific public health interventions of prevention should be projected and carried out, in particular towards foreign workers, a group of people generally more disadvantaged than Italians.

\section{P-403}

Perceived health status and related factors in elderly people

Topic: Other - Health promotion

Presenter: Itziar Vergara

Isabel Larrañaga 1); Monica Machon 2); Miren Dorronsoro 3); Nerea Larrañaga 2); Kalliopi Vrotsou 1); Bakarne Aguirre 1); Itziar Vergara 1)

1) Public Health Department, Basque Government; 2) Primary Care Research Unit Gipuzkoa, Osakidetza; 3) Health Department, Basque Government

Background: self health-perception is a complex construction that may be affected and determined by various factors. To explore such factors and characteristics in elderly subjects may provide valuable information regarding the assessment of this population group, healthrelated needs.

Objectives: to assess health and living conditions related factors and its relation with self perceived health status in elderly population.

Methods: a sample of 816 community dwelling individuals, aged 65 or more was studied. Integrity of intellectual functions was required as inclusion criteria. This sample proceeds from a research study where a multistage sampling was performed considering the municipality size, the district deprivation index, and afterwards, settling an age and sex share. A newly developed tool aimed to the characterization of health status and living conditions of elderly population (an abstract describing the construction of this tool has been submitted to this conference) was used in order to recall individual information. 
This tool includes questions related to health, autonomy, healthy living habits, social network and community conditions, among others. 2 and t-test were used for univariate analysis and binary logistic regression models were derived to perform multivariate analysis.

Results: $50 \%$ of subjects were women with a mean age of 77 (SD 7.6). A $5.4 \%$ described its own health status as excellent, the $21.4 \%$ as very good, $53 \%$ as good, 17.9 regular and $2.5 \%$ as bad: This variable was recoded in two categories: good health $(79.7 \%)$ and bad health $(20.3 \%)$. Some of the factors related to a bad perception of health were sex, with women referring worst health $(p=0.037)$, low family income $(p=0.033)$, multi-morbidity, more than 3 prescription, sense disabilities and depression symptoms $(p<0.0001)$. Factors related with a good perception of health were having an adequate level of physical activity $(p=0.006)$, moderate drinking $(p=0.008)$ and an adequate sleeping pattern $(p<0.0001)$. Also were found to be related with a positive perception of health status the absence of physical barriers at home $(p=0,001)$, taking care of someone $(p=0.002)$, being satisfied with their social life $(p<$ $0.0001)$ and the consideration that the services and conditions of their community were adequate $(p=0.013)$. Considering all these variables in a multivariate analysis, the relation with self perceived health status is maintained for depression symptoms, an adequate sleeping pattern, taking care of someone, being satisfied with their social life and a good consideration of their community.

Conclusions: this study allows for an integrated and comprehensive assessment of the existing associations between health status, living conditions and self perceived health status of elderly subjects. It is remarkable the observed relevance of social satisfaction and community conditions.

\section{P-404}

Use of social and health services in a cohort of pationts with dementia

Topic: Other - Health promotion

Presenter: Silvia Scalmana

Silvia Scalmana 1); Anteo Di Napoli 1); Nicola Vanacore 2); Francesco Franco 1); Domenico Di Lallo 1); Gabriella Guasticchi 1)

1) Agency for Public Health of Lazio Region, Rome, Italy; 2) National Centre of Epidemiology, Surveillance and Health Promotion, National Institute of Health, Rome, Italy

Background: In high and middle income countries, dementia is one of the main causes of disability and death in persons aged 65 or more. Dementia interferes with daily life activities and social relationships, with a strong impact on the quality of life. The global estimated prevalence aged 60 or more years ranged between 5 and $7 \%$, with a prevalence of $6.9 \%$ in Western Europe; the EURODEM study found a prevalence of $6.4 \%$ (all form of dementia) and $4.4 \%$ (Alzheimer Disease), aged 65 or more. Caring dementia patients requires a network of social and health services in different stages of the disease. A multidisciplinary management improves clinical conditions and caregivers' burden, increases use of community services reducing institutionalization and reduces direct and indirect costs. Despite the need for community services, findings of literature indicate low rates of use.

Objectives: The aim of the study was to describe the frequency of use of health and social services in a cohort of patients with dementia, who previously had never used any health or social care services, and to evaluate the social and clinical characteristics associated with the use, observed over a 12-months period.

Methods: We enrolled in a cohort study (2008-2009) 712 patients with dementia, diagnosed at five specialized evaluation units in Lazio
(Italy), who previously had never used any health or social care services, with a 12 month follow-up. Socio-demographic and clinical data were collected at baseline by clinicians with validated instruments, while the use of services through a questionnaire, administered to the caregiver. We calculated crude odds ratios through logistic regression models to evaluate the association between relevant demographic, clinical and social variables and the use of any health or social service. A multiple logistic regression model was used to investigate the factors associated with the use of any service.

Results: We found that only $11.9 \%$ of patients used health or social services. The most widespread diagnoses were: Alzheimer Disease (72.1\%), Mixed Dementia (20.5\%), Vascular Dementia (9.7\%). A higher probability of services' use was observed in patients with more than 5 years of schooling (OR 1.79; $95 \%$ CI 1.08-2.96), with one or more comorbidity (OR 4.87; $95 \%$ CI 2.05-11.57), with severe (OR $4.78 ; 95 \%$ CI 1.75-13.06) or moderate dementia (OR 2.08; $95 \%$ CI $0.98-4.40)$.

Conclusions: The low social and health services'use among dementia patients could be explained by the shortage of services' availability, not always close to patient's needs. Public health authorities should plan an adequate network of services, considering both patients and caregivers' needs.

\section{P-405}

Social inequalities in post-cesarean complication rates in Brazil: a hierarchical analysis

Topic: Other - Health promotion

Presenter: Paulo Freitas

Paulo Freitas 1), 2); Eduardo Savi 2)

1) Universidade Federal de Santa Catarina; 2) Universidade do Sul de Santa Catarina

Background: Medically indicated caesarean sections are those directed to safeguard mother and children's health. Brazil presents one of the highest rates of caesarean section in the world. Caesarean sections without definite medical indications can lead to both higher maternal mortality and post-partum maternal morbidity.

Objectives: To investigate the association between social factors and complications following cesarean sections.

Methods: A sample of 604 women delivering in two main maternity hospitals in southern Brazil, were interviewed $24 \mathrm{~h}$ after delivery and, 2 weeks postpartum, using in-hospital and home interviews. Cox regression was applied, using a hierarchical framework of factors associated with post-cesarean complications.

Results: Post-partum complications were twice as frequent after cesareans as compared to vaginal delivery, independently of socioeconomic conditions. However, the increased risk of complications associated with cesarean section proved to be mediated by socioeconomic circumstances, as represented by prenatal and childbirth care in the public health system, not having the same physician throughout prenatal care and delivery, and having the decision made for the cesarean while the patient was already in labor.

Conclusions: The study's results show that post-cesarean complications can adversely impact women, especially those living in the worst social and health conditions, that is, precisely those that lack support when returning home with a surgical wound.

\section{P-406}

Relationship between profile and procedures to abortion in public maternity in Natal, Brazil

Topic: Other - Health promotion 
Presenter: Severina Alice da Costa Uchoa, Julia Gondim da Costa

Severina Alice da Costa Uchoa 1); Julia Gondim da Costa 2); Julia Serafim Fernandes 1); Marcelo Amorim Araújo 1); Maria Célia de Carvalho Formiga 1); Maria Helena Constantino Spyrides 1); Marize Barros de Souza Araújo 1); Nilma Dias Leão Costa 1); Paulo de Medeiros Rocha 1); Renata Melo Maroto 1)

1) Universidade Federal do Rio Grande do Norte; 2) Secretaria Municipal da Saúde de Maceió

Background: Unsafe abortion is considered a serious health problem worldwide. According to estimates from the World Health Organization (WHO), occur each year, 19-22 million unsafe abortions. In hospitals, the treatment traditionally is done by uterine curettage (D\&C).Another method is the manual vacuum aspiration (MVA), which allows the use of instrumental easy handling and his technique is simple to implement. The WHO recognizes this procedure as the most appropriate method for treating incomplete abortion, assessing it as less aggressive to the endometrium and with less risk of uterine perforation and infection after the procedure.

Objectives: To analyze the relationship between social-demographic, reproductive and obstetric profiles and procedures for abortion among adolescents and young women in public maternity.

Methods: The study was descriptive, retrospective cross-sectional one and the data was collected between July and November 2011. It included 1463 teenagers and young women who were attended in a public maternity hospitals in Natal, Rio Grande do Norte (RN), who were diagnosed as having had an abortion in the period between 2006 and 2007 and came from RN. Statistical analysis was descriptive and Pearson test and logistic regression, assuming a significance level of $5 \%$, were used to measure associations.

Results: Most of teenagers and young women were single with a low education level and average age of 20.2 years. There was a prevalence of incomplete abortion (74.4\%), uterine curettage was the method most used $(59.1 \%)$ and the intercurrence tax after the procedure was of $6.6 \%$. There was no significant association between procedures and marital status $(p=0.323)$, schooling $(p=0.120)$, the usage of abortive medication $(p=0.773)$ and intercurrences $(p=$ 0.948 ). In all reasons for procedures there was predominance of curettage with the exception of ultrasonography indication. The usage of curettage increased with the mother's age $(p=0.001)$, gestacional age $(p<0.001)$, and was the method more used in all kinds of abortion except in retained ones $(p=0.001)$. Women who were submitted to MVA had the less amount of hospital hours $(p=0)$.

Conclusion: There was a predominance of curettage over manual aspiration no matter which profile the respondent women had in despite of MVA be the most recommended method by National Public Health Department of Brazil.

\section{P-407}

Neighborhood green qualities, social capital and safety in relation to well-being and a healthy lifestyle: a longitudinal study.

Topic: Other - Health promotion

Presenter: Hanna Weimann

Hanna Weimann 1); Lars Rylander 1); Maria Albin 1); Erik Skärbäck 2); Patrik Grahn 3); Per-Olof Östergren 4); Jonas Björk 1)

1) Division of Occupational and Environmental Medicine, Lund University; 2) Department of Landscape Architecture, Planning and Management, Swedish University of Agricultural Sciences; 3) Department of Work Science, Business Economics and Environmental Psychology, Swedish University of Agricultural
Sciences; 4) Division of Social Medicine and Global Health, Lund University

Background: Health status has been associated with availability of green or natural areas in nearby neighborhood environments. Previous studies have mainly used cross-sectional designs. Such studies, recently performed in Sweden, have used a validated index, Scania Green Score, to assess health effects using aggregated area units as an appealing compromise between objective measures and individual self-assessments of the environment and have found positive associations. Previous studies have also identified clear inequalities with respect to economic difficulties, country of origin and type of residence in the distribution of both self-reported health and access to green qualities. Studies further assessing the causality between neighborhood characteristics (Scania Green Score, social capital and safety) and health outcomes in longitudinal settings are needed.

Objectives: In this study, based on longitudinal survey data collected in $2000(\mathrm{n}=13604), 2005(\mathrm{n}=10475)$, and $2010(\mathrm{n}=9031)$ from Scania, southern Sweden, we will assess how distinct qualities within the Scania Green Score are associated with self-reported indicators of well-being such as neighborhood satisfaction and physical activity, general health (GHQ-12) and quality of life (SF-36). Furthermore, we will seek to identify individual factors and life-situations where neighborhood characteristics are especially important for general health and well-being.

Methods: Characteristics of green neighborhood quality and a variety of variables related to neighborhood socioeconomic status, social capital and safety were aggregated in defined area units of $1,000 \mathrm{~m}^{2}$ based on information obtained from external survey data collected in the same study area in Scania in $2008(\mathrm{n}=24847)$. Coordinates of residence for the respondents of the longitudinal survey were obtained and geocoded for each year 2000-2010. Analyses will be guided by causal diagrams and performed using mixed effects logistic regression while controlling for confounding factors.

Results: About $20 \%$ who responded in both 2000 and 2010 reported a change in general health status by 2 units or more on a 7 degree scale. The proportions who had increased versus decreased their general health were approximately the same. These figures indicate a good possibility to detect health changes related to changes in neighborhood characteristics over time. The statistical analyses are in progress and results will be presented at the conference.

Conclusions: Studies assessing the causality between health outcome and neighborhood characteristics using longitudinal data are warranted. The present study will hopefully contribute to increase the understanding regarding the relationship between neighborhood characteristics and human well-being.

\section{P-408}

Social risk factors, lack of prenatal care, and neonatal morbidity in african immigrant women and native-born in Northern Spain

Topic: Other - Health promotion

Presenter: Maria Paz.

Maria Paz 1); Javier Llorca 1), 2); Abraham Delgado 3); Luis Rioja 3); Miguel Santibáñez 1), 2)

1) University of Cantabria, Santander, Spain; 2) IFIMAV, Santander, Spain; CIBERESP, Spain; 3) Gerencia de Atención Primaria, Servicio Cántabro de Salud, Spain

Background: The principal factor associated with the lack of prenatal care in African immigrant women and native-born is social risk factors. The main objectives of a prenatal care program are reducing the number of abortions, maternal morbidity from obstetric causes, perinatal morbidity and promoting breastfeeding. Studies show that 
lack of prenatal care in African immigrant women increases the risk of inequality of the social determinants of health, compared to the native population.

Objectives: To compare the association between social risk factors, lack of prenatal care and neonatal morbidity in African immigrant women and native-born.

Methodology: Retrospective cohort study. We identified 231 pregnant African immigrant women from 2007 to 2010. The native-born population sample was obtained by simple random sampling in a ratio1:3. The degree of the pregnancy control was estimated by Kessner Index (KI), being categorized in proper compliance versus noncompliance. Low birth weight $(<2,500 \mathrm{~g}$. at birth) and preterm birth deliveries (less than 37 weeks in gestation) were collected as neonatal morbidity variables. Odds ratios (OR) were estimated by non-conditional logistic regression with their Confidence Intervals at $95 \%(95 \% \mathrm{CI})$.

Results: The prevalence of the lack of adherence to prenatal and Social Risk factors were higher in African immigrant women compared to native-born $(p<0.001)$. In both groups, positive associations were found between noncompliance of KI and preterm (OR 8.74 African women, native-born OR 3.00) and low weight (OR 6.81 African women, native-born OR 3.00). The findings held when adjusted for age and social risk detection by the midwife. By classifying the lack of adherence to prenatal care as intermediate and inadequate, statistically significant dose response patterns were obtained $(p<0.01)$. Social risk factors was the main independent factor associated with low weight (Adjusted OR 10.20; $95 \%$ CI [1.77-58.86]).

Conclusions: Our results suggest that both the lack of prenatal care and the existence of social risk factors are associated with an increased risk of neonatal morbidity. The effect of the lack of prenatal care on neonatal morbidity appears to be higher in African immigrant women than in native-born. As a consequence, this could be a source of health inequalities.

\section{P-409}

Factors associated with the lack of adherence to prenatal care in African immigrant women and Spanish women in Northern Spain

Topic: Other - Health promotion

Presenter: María Paz

\section{María Paz 1); Javier Llorca 1), 2); Miguel Santibáñez 1), 2)}

1) University of Cantabria, Santander, Spain; 2) IFIMAV, Santander, Spain; CIBERESP, Spain

Background: Lack of knowledge of language has been postulated as the main barrier to prenatal care in African immigrant women whereas social risk factors traditionally has been associated with worse prenatal care in native-born.

Objectives: To examine the association and the interaction between language, social risk factors and the lack of adherence to prenatal care.

Methodology: Retrospective cohort study. 231 African immigrant women with delivery dates between January 1, 2007 and December 31, 2010 were identified, collecting data about their knowledge of Spanish, referral to a social worker because of social risk factors, and adequacy of prenatal care through the Kessner Index (KI) and an Own Index (OI). The native-born population sample was obtained by simple random sampling in a ratio1:3. Odds ratios (OR) were estimated by non-conditional logistic regression. To study interaction, the term of interaction [language* ${ }^{*}$ referral to social worker] was included in the logistic models.
Results: $84 \%$ of pregnant African immigrant women did not know how to speak Spanish. $47 \%$ were referred to a Social Worker. African immigrant women who were not referred to a social worker, the association between their insufficient knowledge of Spanish and inadequacy of prenatal care was weak and not significant (OR for $\mathrm{KI}=1.31$; OR for $\mathrm{OI}=1.66)$. On the contrary, African immigrant women who were referred to a social worker, the association was strengthened and yielded statistical significance $(\mathrm{OR}$ for $\mathrm{KI}=8.98$; OR for OI $=6.94$ ) and the term of interaction was statistically significant $(p$ interaction $=0.026$ ). Social Risk factors were the main independent factor associated to inadequacy of prenatal care in native-born (Adjusted OR 3.17; $95 \%$ CI [1.42-7.06]).

Conclusions: Our results support that the principal factor associated with the lack of prenatal care in African immigrant women is insufficient language proficiency, but solely in the presence of social risk factors, which have been also associated to worse prenatal care also in Spanish women.

\section{P-410}

Differences in adherence to prenatal care between Spanish and African immigrant women, as a source of health inequalities

Topic: Other - Health promotion

Presenter: María Paz.

\section{María Paz 1); Miguel Santibáñez 1), 2); Javier Llorca 1), 2)}

1) University of Cantabria, Santander, Spain; 2) IFIMAV, Santander, Spain; CIBERESP, Spain

Background: The main objectives of a prenatal care program are reducing the number of abortions, maternal morbidity from obstetric causes, perinatal morbidity and promotion of breastfeeding. In pregnant African immigrants, lack of knowledge of language has been postulated as the main barrier to access to health services with the consequent lack of prenatal care. Studies show that lack of prenatal care increases the risk of inequality of the social determinants of health compared to the native population.

Objectives: To compare the lack of prenatal care between African immigrant women and native-born, as a source of Health Inequalities. Methodology: Retrospective cohort study. We identified 231 pregnant African immigrant women from 2007 to 2010. The native-born population sample was obtained by simple random sampling in a ratio1:3. The degree of the pregnancy control was estimated by Kessner Index (KI) and an Own Index (OI) based on the seven quality indicators according to WHO and NICE guidelines, being categorized in proper compliance versus noncompliance. Odds ratios $(\mathrm{OR})$ were estimated by non-conditional logistic regression with their Confidence Intervals at $95 \%(95 \% \mathrm{CI})$.

Results: In 73-78 \% of African immigrant women, prenatal care was inadequate analyzed based on the index (KI or OI). Only $61.5 \%$ of prenatal care beginning in the first trimester of pregnancy and only $34.6 \%$ folic acid prophylaxis started before week 8 . The likelihood (odds) of lack of prenatal care was multiplied by $11.41,95 \% \mathrm{CI}$ [7.94-16.40] and 9.67, $95 \%$ CI [6.82-13.69] in African immigrant compared to native-born, according to the index used (KI or OI). These associations remained, and dose-response patterns, adjusting for age, referral to the social worker, obstetric history and number of pregnancies (Adjusted OR for $\mathrm{KI}=6.44 \mathrm{KI}$, Adjusted OR for OI $6.37 \mathrm{OI})$.

Conclusions: Being an immigrant has been identified as a major independent risk factor associated with lack of prenatal care in our sample. The negative impact that this lack of prenatal care could have on maternal and neonatal morbidity outcomes, should be fully studied as it constitutes a source of social inequalities in health. 


\section{P-411}

Determinants of adolescent attitudes towards sexuality

Topic: Other - Health promotion

Presenter: Paula Nelas, Carlos Albuquerque

Manuela Ferreira 1); Paula Nelas 1); João Duarte 1); Carlos Albuquerque 1)

1) School of Health Viseu - Polytechnic Institute of Viseu (CI\&DETS-FCT)

Background: Sexuality is build and learned and is an integral part of personality development, which may interfere with the learning process, the mental and physical health of the individual. Parents and educators should respect the developmental stages of children and adolescents.

Objectives: To analyze the influence of sociodemographic variables and sexual and familiar context variables, knowledge of STI and contraception in the attitudes of adolescents towards sexuality.

Methods: A descriptive, non-experimental and cross-sectional study. The non-probabilistic convenience sample consists of 1216 adolescents attending the 9th grade in Portuguese Public Schools and is a part of the project PTDC/CPE-CED/103313/2008.

Results: $15.1 \%$ of boys and $10.5 \%$ of girls had sexual intercourse. Teenagers aged 14 have more inadequate attitudes $(21.3 \%)$ towards condoms. Most teenagers who believe it is important to use condoms has inappropriate attitudes against the pill $(48.5 \%)$. Age $(p=0.242)$ and residence $(p=0.719)$ did not influence attitudes towards condoms, sexual intercourse $(p=0.038)$ influences the attitudes towards condom and the pill. Adolescents who have their father $(p=0.030)$, friends $(p=0.039)$ and boyfriend or girlfriend $(p=0.023)$ as interlocutors about sexuality have less culpability against the use of condoms. The sexual context variables have no significant effect on attitudes towards the pill $(p>0.05)$. Those who did not had sexual intercourse $\left(\mathrm{x}^{2}=9.17, p=0.01\right)$, with ages between 14 and 15 years $\left(\mathrm{x}^{2}=19.69 p=0.00\right)$, also demonstrate greater knowledge about sexually transmitted infections.

They belong mostly ( $55.8 \%$ boys and $49.5 \%$ girls) to a family with poor interpersonal relationships culture. The majority of males $(51.8 \%)$ and females $(58.9 \%)$ have a family with a moderate heuristic culture. Boys and girls (33.6 \% and $36.9 \%$ ) both demonstrate to have a family with a predominantly moderate hierarchal culture and a culture of moderate social objectives as well. Adolescents who have a bad attitude towards sexuality, mostly ( $43.2 \%$ ) have a family with a culture of weak interpersonal relationships with statistical significance $\left(X^{2}=32,092\right.$, $p=0.000)$ and have a moderate hierarchal culture and also a culture of moderate social objectives, without statistical significance.

Conclusion: Based on our results, supported by the referenced authors, we conclude that the adolescents' sexual education must be a priority to parents, professionals of health and education as they are the fundamental pillars of education for sexual and reproductive health of adolescents, promote empowerment, making them safer, healthier and happier.

\section{P-412}

Process evaluation of a curricular component in the schoolrandomized Boost trial targeting fruit and vegetable intake among Danish adolescents

Topic: Other - Health promotion

Presenter: Thea Suldrup Jørgensen

Thea Suldrup Jørgensen 1); Mette Rasmussen 1); Pernille Due 1); Anne Kristine Aarestrup 1); Sanne Ellegaard Jørgensen 1); Rikke Krølner 1)
1) University of Southern Denmark, National Institute of Public Health

Background: School-based interventions which combine educational and environmental strategies seem most effective in increasing fruit and vegetable (FV) intake among adolescents, but are often not fully implemented. Studies of implementation of intervention strategies are required to develop interventions which can be easily implemented by teachers. Furthermore, knowledge on implementation may guide the interpretation of intervention effects.

Objectives: To evaluate the implementation of the curricular component in the multi-component school-randomized Boost trial designed to increase 13-year-old's FV intake.

Methods: Post-intervention questionnaire data from students $(\mathrm{N}=1,078,98.3 \%)$ and teachers from all 20 intervention schools were analyzed descriptively. Process measures: Dose delivered: number of Boost activities implemented by teachers (Teacher manuals included 16 compulsory and 10 optional activities); Dose received: number of activities received by students; Fidelity: adherence to teacher manuals; Reach: dose received stratified by gender and parental occupation; Teachers' appreciation of teaching material; Perceived barriers for implementation; Contamination: FV curriculum in control schools.

Results: Dose delivered: On average, 5.2 activities were implemented per school [range: 0 ( 2 schools $)$-10.7 (1 school)]. Most frequent reasons for low implementation: "The Boost curriculum did not fit the regular curriculum" (21.9\%), "It was difficult to maintain motivation for Boost throughout the school year" (17.5\%). $95.6 \%$ of teachers had distributed the Boost student workbook; $58.7 \%$ had used it. Dose received: On average, 7.9 assignments per school [range: $3.6(2$ schools) -12.3 (1school)]. In 16 schools, students reported higher dose than teachers. $41 \%$ of students had used the student workbook for Boost activities (almost) every time. Fidelity: $87.5 \%$ of teachers had read and/or used teacher manuals. $28.9 \%$ adapted Boost activities to some extent. Reach: Average number of activities received: Girls: 8.4 versus boys: $7.3(P<.0001)$; high social class: 7.9 versus low: $8.0(P=0.0872)$. Appreciation: $65 \%$ of teachers found the manuals useful. $37.5 \%$ of students liked the curricula $r$ activities. The activities were perceived as fun by $24 \%$ of students, as difficult by $10 \%$, and as providing new knowledge by $40 \%$. Contamination: $27.9 \%$ of students at control schools had been exposed to curricular activities on FV during the intervention period.

Conclusions: On average, teachers only implemented one-third of the assignments. One-third of students at control schools were exposed to FV curricular activities. This may compromise the effect of the intervention. The dose received by students was larger compared to the dose delivered by teachers. This disagreement is an area for future research.

\section{P-413}

Study on status of tobacco use and its associated factors among adolescent students in Beijing

Topic: Other - Health promotion

Presenter: Wenjie $\mathrm{Xu}$

\section{Wenjie Xu 1); Henghui Liu 1); Zheng Liu 1); Xin Guo 1)}

1) School Health Institute, Beijing Center for Disease Control and Prevention

Objective: To know the current condition of tobacco use among adolescent students and its associated factors, and to provide the basis for tobacco control among adolescents in the future.

Methods: The research ivestigated 5182 primary school students and 12012 middle school students in 160 schools. Questionnaire survey 
was conducted to collect related information on adolescent smoking and passive smoking as well as adolescents' knowledge,attitudes and practice about tobacco use.

Results: 1.The rate of students experience of tobacco use in primary,junior,senior high school was $6.2,14.5,26.8,49.6 \%$ respectively, and the rate of current smoking was $0.9,3.3,7.9,23.4 \%$ per respectively. Boys were higher than girls, there was significance statistical between two groups. (1)The rate of passive smoking is $35.4 \%$ in primary school students, $39.6 \%$ in middle school, there was no significant difference between boys and girls. (2) The rate of awareness of the knowledge and the positive atti tude to the tobbaco of female students and the ones who don't smoke is higher respectively than the two rates of the male students and the current smokers. (3) the multi-factor Logistic regression analysis indicated that the "male", "parents smoking", "having more pocket money", "having some friends who smoke" were risk factors, and "perform well in school", "refuse the cigarettes given by the friends", "when friends smoke, advicing them to give up smoking or avoid"were the protective factors.

Conclusion: The rate of experience of tobacco use and the passive smoking of The students from elementary school to senior high school in Beijing was declining respectively than the former years, and the current smoking was still high.Tobacco control should be strengthened; We propose keep strengthenning the publicity of the smoking control among schools, working out more explicit rules of smoking control, focusing on the smoking control among family and classmates.

\section{P-414}

Voluntary counseling and HIV testing aimed at preventing risky behavior and HIV infection among students

Topic: Other - Health promotion

Presenter: Biljana Kocic

\section{Biljana Kocic 1); Branislav Petrovic 1)}

1) University of Nis, Faculty of Medicine Nis, Department of Epidemiology, Nis, Serbia

Background: Several models of HIV prevention counseling in conjuction with HIV testing have been developed. Client-centered HIV prevention counseling should focus on the client's own unique circumstances and risk and should help the client set and reach an explicit behavior-change goal to reduce the chance of acquiring or transmitting HIV. Students and young people, are very vulnerable to HIV. Voluntary counseling and HIV testing (VCCT) services, which meet the needs of young people, offer number of opportunities for changing HIV-related risk behavior. At the end of 2004 voluntary counseling and testing service was established in Institute for Public Health Nis, Serbia. Objectives: The aim of this paper was to present some demographic and epidemiological characteristics of students tested for HIV at the VCT Centre in Nis, and to assess their needs, HIV-related risky behavior and feasibility of VCCT intervention aimed at preventing their risky behavior and HIV infection.

Methods: We conducted a descriptive study of epidemiological characteristics of students tested for HIV in VCT Centre in Nis between 2004 and 2010. The data were obtained from the Register of the clients attended VCT Centre and anonymous questionnaires of the clients. The data was analyzed descriptively. Pearson Chi square test for statistical significance was performed.

Results: Within the observed period, among 1942 clients who attended VCT Centre in Nis, students accounted for $39 \%$. Boys accounted for $58 \%$ while girls accounted for $42 \%$. The mean age at the first sexual intercourse was of 17.1 years for girls and 16.7 for boys. Most of students were unmarried $(94.7 \%)$, practiced unprotected sex (85.6\% hetero and $0.7 \%$ homo-bi), didn't use condom during the last sexual intercourse $(56 \%)$, had more than two sexual partners during the past year, reported one risk seksual behavior in the past $(78 \%)$, defined their sexual orientation as heterosexual $(82 \%)$, changed the place of residence due to studying $(61 \%)$ and didn't perceive risk of acquiring HIV infection during their life (58\%).

Conclusion: VCCT has been accepted in developed and in majority of developing countries, and became part of a standard package of prevention strategies. People, who are offered personalized VCCT intervention are likely to reduce their practice of risky sexual and other HIV-related behaviours, and prevent HIV infection. Services must be developed to respond to these needs including a stimulation to attend VCCT center and a motivation for testing.

\section{$\mathbf{P}-415$}

Prevalence of dental caries and fluorosis among a sample of adolescents living in a fluoridated and a non-fluoridated water region

Topic: Other - Health promotion

Presenter: Nélio Veiga

Nélio Veiga 1); Odete Amaral 2); Carlos Pereira 2); Claudia Ribeiro 1); Ana Arrimar 1); Inês Coelho 3)

1) Department of Health Sciences - Universidade Católica Portuguesa; 2) CI\&DETS - Polytechnic Institute of Viseu; 3) USF Grão Vasco-Viseu

Background: The application of fluoride has been a efficient method used for dental caries prevention. However, its use has been associated with a higher risk of dental fluorosis

Objectives: Quantify the prevalence of dental caries and dental fluorosis among a sample of students from two different portuguese regions (Ponta Delgada and Viseu).

Methods: A cross-sectional study was conducted to assess a sample of 157 adolescents from Ponta Delgada (fluoridated water region) and 98 from Viseu (non-fluoridated water region), aged 12-17 years old. An intra-oral examination to identify the decayed, missing and filled teeth index (DMFT index) and Dean's Fluorosis Index was performed in every adolescent, whom was also asked to fill in a questionnaire to assess oral health behaviours. Prevalence was expressed in proportions and statistical analysis was completed using the Qui-square test and Fisher's exact test to identify dependence among the variables included in the study.

Results: The DMFT index was significantly higher among adolescents of Viseu, comparatively to adolescents of Ponta Delgada (DMFT index: 2.20 vs.1.60; $p>0.05$ ). The results revealed a lower prevalence of dental caries and a higher prevalence of adolescents free from dental caries in Ponta Delgada (58.0 vs. $48.0 \%, p>0.05$ ). The prevalence of dental fluorosis in the sample of adolescents of Ponta Delgada is also higher in comparison with the adolescents of Viseu (15.3 vs. $4.1 \%, p<0.05)$. Toothbrushing at least twice a day (76.6 \% vs. $46.9 \%, p<0.05)$ and daily use of dental floss $(88.5 \%$ vs. $56.0 \%, p<0.05$ ) was higher among the adolescents of Ponta Delgada.

Conclusion: Higher prevalence of fluorosis and lower prevalence of dental caries can be associated with fluoridated water consumption, considering that the concentration of fluoride in the public water in Viseu is $<0.1 \mathrm{ppm}$ and in Ponta Delgada $1.5 \mathrm{ppm}$. However, adolescents of Ponta Delgada also have better knowledge about oral health behaviours. 


\section{P-416}

Therapeutic adherence in a sample of adults with chronic disease

Topic: Other - Health promotion

Presenter: Odete Amaral

Odete Amaral 1); Claudia Chaves 1); Nélio Veiga 1), 2); Rui Dionísio 1)

1) CI\&DETS - Polytechnic Institute of Viseu; 2) Department of Health Sciences - Universidade Católica Portuguesa

Background: Therapeutic adherence is one of the main causes of treatment failure.

Objectives: The objective of this study was to assess the pharmacological therapeutic adherence among adults with chronic disease.

Methods: A cross-sectional study was carried out with a sample of 537 adults (55.3\% female) with ages between 23 and 95 years (mean $=65.3 \pm 12.2$ ). Data collection was accomplished in the central region of Portugal, using a self-administered questionnaire. Therapeutic adherence was assessed using the Measure Treatment Adherence (MAT) developed by Morisky, Green and Levine with 7 items. The prevalences were expressed in proportions with $95 \%$ confidence intervals (CI) and the magnitude of association between variables was determined using the odds ratio (OR).

Results: In the final sample, 320 adults presented chronic disease $(59.6 \%)$. The prevalence of chronic disease was not associated with gender (male OR 0.94; CI $95 \%$ 0.66-1.33), lower among adults $\leq 65$ years old (OR 0.45; CI $95 \% 0.31-0.63$ ), adults with lower education levels ( $\leq 4$ years OR $2.71 \mathrm{CI} 95 \% 1.45-5.09$ and $5-12$ years OR $1.05 \mathrm{CI} 95 \% 0.55-2.00)$. The prevalence of therapeutic adherence was $66.1 \%$. The prevalence of mild adherence was $44.3 \%$; moderate adherence $14.9 \%$ and high adherence $40.8 \%$.

Therapeutic adherence was associated with residence area (rural OR 0.59 CI $95 \% 0.41-0.85)$ and not associated with gender $(p=0.50)$, age $(p=0.19)$, level of education $(p=0.14)$ and marital status $(p=0.61)$. Conclusion: Beyond the studies of adherence interventions, is the need to further examine the predictors of adherence connected with the life context of the patients. We need to develop more efforts on the intervention/motivation field with chronic disease patients.

\section{P-417}

Promoting fruit and vegetable consumption among teenagers: first year results from the multi-component cluster-randomised Boost study

Topic: Other - Health promotion

Presenter: Rikke Krølner

Rikke Krølner 1); Lau Caspar Thygesen 1); Anette Andersen 1); Sanne Ellegaard Jørgensen 1); Anne Kristine Aarestrup 1); Thea Suldrup Jørgensen 1); Pernille Due 1)

1) The National Institute of Public Health, University of Southern Denmark, Copenhagen, Denmark

Background: Large proportion of children and adolescents do not meet the World Health Organization's recommendations of eating at least 400 grams (g) of fruit and vegetables (FV) a day. Intake of FV decreases from age 11 to 15 , but few intervention studies have targeted teenagers. Among children younger than 13, multicomponent interventions which combine environmental and educational strategies in schools and homes tend to result in larger improvements of FV consumption than single component interventions.

Objectives: To examine the effect of a school-based, multicomponent intervention on 13-year-olds' FV intake at the end of intervention (first follow-up).
Methods: We used data from the Danish Boost study. The intervention included curricular activities and free daily provision of $\mathrm{FV}$ at school, newsletters to parents, and fact sheets to sports- and youth clubs. All components focused on increasing adolescents' access to $\mathrm{FV}$ at school, home and at leisure time activities. The intervention was tested in a cluster-randomised trial among 2,289 year 7 pupils (age 13) from 40 randomly selected schools randomized into 20 intervention- and 20 control schools. The intervention was implemented from September 2010-May 2011 (9 months). Prior to intervention start (August 2010), 2,156 pupils completed a baseline questionnaire (response rate: $94.2 \%$ ), among them 1,906 pupils $(88.4 \%)$ completed a first follow-up questionnaire at the end of intervention (May/June 2011). Primary outcome measure: Daily FV intake assessed by $24-\mathrm{h}$ recall questionnaires. Intervention effects on fruit-, vegetable- and FV intake were analysed by multilevel linear regression analyses adjusted for baseline intake of $\mathrm{FV}$, family social class, and gender. We performed available case analyses including only pupils with complete information $(\mathrm{N}=1,681)$. Intention-to-treat (ITT) analyses included all students $(\mathrm{N}=2,156)$ after imputing missing values by multiple imputation.

Results: The intervention had significant effects on intake of FV and fruit, but not on vegetable intake alone. Available case analysis: Pupils at intervention schools ate 89.3 gram (SE: 29.0) more FV a day at first follow-up compared to pupils at control schools. ITT analyses: Pupils at intervention schools ate 81.0 gram (SE: 26.9) more FV a day at first follow-up compared to pupils at control schools.

Conclusions: Both available case analyses and ITT analyses showed that the Boost intervention was effective in increasing 13-year-olds' FV intake, but mainly fruit intake, at the end of intervention. Second followup data will reveal if effects can be maintained. Effective strategies to promote teenagers' vegetable intake need to be developed.

P-421

The regional disparities in provincial maternal mortality surveillance system and maternal mortality in China: an analytical review

Topic: Other - Others

Presenter: Xiaoling Gan, Wei-Hong Zhang

Xiaoling Gan 1), 2); Xiaojing Dong 2); Sophie Alexander 1); Michèle Wilmet-Dramaix 1); Lina Hu 2); Wei-Hong Zhang 1)

1) Université Libre de Bruxelles, School of Public Health; 2) The Second Affiliated Hospital of Chongqing Medical University

Background: Provincial maternal mortality surveillance (MMS) systems were set up in all the provinces in China to monitor local maternal mortality and provide scientific evidence for maternal health interventions suited to local conditions, which covered a much larger population than national maternal and child mortality surveillance (NMCMS) system. However, till now little is known outside of China about the characteristics of provincial MMS systems.

Objective: This study was to give an overview on provincial level maternal mortality in China.

Methods: A systematic review of the scientific literature contained in PubMed, EMBASE, Medline and China Academic Journal Network Publishing database was carried out, searching for studies published between 1988 and January 2013 and reporting on provincial maternal mortality in China. The current situation and regional disparities of provincial MM surveillance systems were described, trends and regional disparities in the MMR in China were pooled analyzed using the different data source.

Results: A total of 361 articles were retrieved and 45 of them met the inclusion criteria for narrative analysis, 28 studies were finally 
included in the pooled quantitative analysis, of which 25 were published in Chinese. Complete registrations of maternal deaths were implemented in nearly all provinces in coast regions, by contrast, stratified random sampling surveillance were adopted in remote areas. The MMR in coastal regions, inner lands and remote areas all declined during the 1996-2009 period. The absolute difference in MMR between the remote and coast regions declined from 108.54 deaths per 100000 live births in 1996 to 31.5 per 100000 live births in 2009.

Conclusions: Great regional disparities in maternal mortality surveillance system and maternal mortality do exist within the country, but there is a trend of narrowing disparities across socio-economic regions over time. More emphasis should be placed on improving provincial maternal mortality surveillance systems, particularly in the remote regions.

\section{$\mathbf{P}-422$}

Prevalence and factors affecting needlesticks and sharp injuries among operating room nursing personnel in Thailand

Topic: Other - Others

Presenter: Nongyao Kasatpibal

Nongyao Kasatpibal 1); Sadubporn Katechanok 2); Sukanya Ngamsakulrat 2); Benjawan Malairungsakul 2); Pinyo Sirikulsathean 3); Chutatip Nuntawinit 4); Thanisara Muangnart 5); On behalf of the Thai Perioperative Nurses Association 6)

1) Faculty of Nursing, Chiang Mai University, Chiang Mai, Thailand; 2) Operating Theater and Recovery Room Service, Maharaj Nakorn Chiang Mai Hospital, Thailand; 3) Operating Theater, Rajavithi Hospital, Bangkok, Thailand; 4) Operating Theater, Siriraj Hospital, Bangkok, Thailand; 5) Operating Theater, Police General Hospital, Bangkok, Thailand; 6) The Thai Perioperative Nurses Association, Bangkok, Thailand

Background: Perioperative nurses are at high risk for exposure to bloodborne pathogens such as hepatitis $\mathrm{B}$ virus, hepatitis $\mathrm{C}$ virus, and HIV from needlesticks, sharp injures, and blood and body fluid exposure. However, national data on needlesticks and sharp injuries among perioperative nurses are not currently available in Thailand. Objectives: This analytical cross-sectional study was conducted to examine the situation and risk factors of needlesticks, sharp injuries, and blood and body fluid exposure among perioperative nurses in Thailand.

Methods: Data were collected from June 2011 to May 2012. The study sample consisted of 2,031 perioperative nurses in 247 hospitals throughout Thailand. The research instruments used for data collection developed by the researchers consisted of a demographic questionnaire, an occurrence of needlesticks and sharp injuries questionnaire, and a risk factors for needlesticks and sharp injuries questionnaire. Data were analyzed using descriptive statistics and multiple logistic regression analysis.

Results: The study revealed that the majority of study participants sample were female $(92.32 \%)$. The mean age was 38.40 years (range $=18-65$ years) and $78.58 \%$ were registered nurses. The average working experience in the operating theatre of those sampled was 13 years 7 months (range 1 month-39 years). The prevalence of needlesticks and sharp injuries were 23.68 and $9.80 \%$, respectively. Most injuries occurred during the day shift, which accounted for $45.15 \%$ of injuries reported. Of these, $63.03 \%$ of puncture wounds were exposed to non-intact skin. The four most common devices causing percutaneous injuries were suture needles $(36.11 \%)$, hollowbore needles $(23.25 \%)$, scalpel blades $(15.33 \%)$, and wires $(10.90 \%)$. Post-exposure, $59.89 \%$ of operating room nurses did not report the incident and $62.56 \%$ did not seek medical treatment. Of those injured, $57.68 \%$ reported feelings of anxiety. Risk factors for needlesticks were age 30-34 years ( $\mathrm{RR}=0.46$; $95 \%$ CI $0.24-0.87)$, 35-39 years $(\mathrm{RR}=0.46 ; 95 \%$ CI $0.22-0.93)$, incomplete training $(\mathrm{RR}=1.67 ; 95 \% \mathrm{CI} 1.29-2.17)$, sense of being rushed $(\mathrm{RR}=4.81$; $95 \%$ CI 3.41-6.79), personal habits $(\mathrm{RR}=1.67 ; 95 \% \mathrm{CI}$ 1.04-1.77), inadequate personnel $(\mathrm{RR}=1.60 ; 95 \% \mathrm{CI} 1.21-2.11)$, and out of date guidelines $(\mathrm{RR}=1.69 ; 95 \% \mathrm{CI}$ 1.04-2.74). Risk factors for sharp injuries was sense of being rushed $(\mathrm{RR}=2.43$; $95 \%$ CI 1.57).

Conclusions: The prevalence of needlesticks and sharp injuries in Thailand is quite high. The potential risk factors were personal factors, practical factors, and organizational factors. Clear policy, appropriate prevention guidelines, education, and providing adequate personnel are ways to reduce the incidence of needlesticks and sharp injuries.

\section{P-423}

Do Saudi medical students know enough about tobacco dependence? Findings from three medical schools

Topic: Other - Others

Presenter: Hoda Jradi, Ali Alshehri

Hoda Jradi 1); Ali Alshehri 1)

\section{1) King Saud Bin ABdulaziz University}

Background: Tobacco smoking is the leading cause of preventable death worldwide. Educating and training medical students about tobacco dependence prevention and treatment will prepare them as future physicians for the task of helping smokers quit. To date, in Saudi Arabia, little is known about medical students' knowledge on this topic.

Methods: This study was conducted among 237 medical students from three medical schools. Students were asked to complete a 55-item questionnaire about the knowledge of smoking epidemiology, practice of smoking cessation, knowledge of treatment of nicotine addiction, and knowledge of the benefits of smoking cessation.

Results: The majority of the surveyed students do not have adequate knowledge about the epidemiology of smoking ( $8.6 \%$ estimated within the acceptable range the prevalence of smoking among adults in Saudi Arabia). Students demonstrated a below average knowledge of the health risks associated with tobacco use (average score $53 \%$; $\mathrm{SD}=11.6$ ), a fair understanding of the benefits of smoking cessation (76.3\% knew that stopping smoking reduced risk of premature death), and insufficient information about treatment of nicotine addiction (only $52.8 \%$ knew that a provider should ask about smoking status at every visit and $81.6 \%$ falsely thought that NRT is contraindicated for cardiovascular disease). No more than $20.9 \%$ of the respondents thought they were adequately prepared to counsel/ advise their patients to quit smoking

Conclusions: These findings suggest that medical students in Saudi Arabia are not well informed and trained in tobacco dependence and treatment. It is necessary to address this deficit by prioritizing these topics in medical education curricula.

\section{P-424}

Social determinants of adolescent pregnancy in Brazil

Topic: Other - Others

Presenter: Paulo Freitas, Marina Domimgues

Paulo Freitas 1), 2); Marina Domimgues 2) 
1) Universidade Federal de Santa Catarina; 2) Universidade do Sul de Santa Catarina

Background: About 16 million adolescent girls give birth every year - most in low- and middle-income countries. Brazilian data from the Family Welfare Survey showed that $51 \%$ of 15-19-year-old girls with no schooling were already mothers. Objectives: To investigate how socioeconomic indicators and individual variables from woman, pregnancy and delivery, can influence rates of adolescent pregnancy in South Brazil.

Methods: A study combining ecological and cross-sectional methods used data, concerning 419,361 births, from the Live Birth National Information System (SINASC) for the period from 2003 to 2007. Correlations between socioeconomic indicators and rates of adolescent pregnancy were obtained. Annual rates and crude and adjusted rates, using Cox Regression, were calculated for literacy, ethnicity, duration of pregnancy and frequency to antenatal care consultations. Results: Rates of adolescent pregnancy in the study period was $18.26 \%$. Rates showed to be inversely associated to socioeconomic and demographic indicators. Negative correlations were found for the Human Development Index (HDI), Educational Development Index (EDI), Literacy, and Income Rate. The categories with higher proportions of adolescent mothers were low level of literacy, lower frequency to antenatal care, lower gestational age and non-white ethnicity. For extremes in the period, the Prevalence Ratios increased for low literacy, lower frequency to antenatal care, and for non-white women.

Conclusions: Adolescent pregnancy in Brazil is an important Public Health problem with socioeconomic determinants whose effects will impact mainly those women living in the less privileged groups

\section{$\mathbf{P}-425$}

Population aging in Brazil: socio-demographic and spatial profile of lifelong elderly

Topic: Other - Others

Presenter: Paulo Cesar Ramos

Paulo Cesar Ramos 1); Maria Célia Formiga 1); Nilma Costa 2); Kalline Silveira 3)

1) Department of Demography and Actuarial Science/UFRN; 2) Department of Collective Health/UFRN; 3) Graduate Program in Demography/UFRN

Background: The population aging has been observed in all countries worldwide, regardless the level of development. This is considered a natural and irreversible global phenomenon, which involves many political, economic and social challenges. The ageing process of the Brazilian population has been quite accelerated, due to the sharp reduction in fertility rates and the decrease in infant mortality, with a consequent increase in life expectancy at birth. The speed of this process is observed by the 2010 Census, revealing an elderly population (age 60 years and older) of 20,590,599 people, representing $10.8 \%$ of the Brazilian population, with 2,935,585 (14.3\%) of which being lifelong/octogenarians elderly ( 80 years and over).

Objectives: To analyze the spatial concentration of the population of octogenarians in the Northeast region of Brazil establishing a demographic profile, according to the units of the Federation (UF), correlating with socio-economic factors.

Methods: The Census and socio-economic data from the Brazilian Institute of Geography and Statistics (IBGE), 2010, was used. An exploratory descriptive analysis and demographic correlation, regression and nonparametric tests of Mann-Whitney, were performed with the UF 27 , in groups of regions, adopting a $5 \%$ confidence level.
Results: The highest proportion of the elderly, by 100 young people, is found in the State of Rio de Janeiro (RJ) (66.9 \%), followed by the State of Rio Grande do Sul (RS) (66.5\%), in the South and Southeast, respectively, the most developed regions in Brazil, while the lowest rates are in the Northeast, in the UF with higher socio-economic vulnerabilities. However, the highest proportion of elderly octogenarians under the total population, as well as under the total of elderly population, are in the UF of the Northeast region. In the states of Paraíba and Rio Grande do Norte, for example, 17.7 and $17.6 \%$ of the elderly are octogenarians, respectively, while in Rio de Janeiro and Rio Grande do Sul, this proportion is only 14.7 and $13.8 \%$, respectively. Octogenarian women are in absolute majority in Brazil, with gender ratio of $159 \%$, reaching $202 \%$ in RJ and $198 \%$ in RS. Significant differences were detected $(p$ value $<0.05)$ between indicators of ageing and socio-economic conditions among regions.

Conclusions: Despite the South and Southeast regions being the more aged and more developed in the country, the relative concentration of lifelong elderly people is in the Northeast, the most socio-economically vulnerable, pointing out to the implementation of effective public policies of care and attention to this population group.

\section{P-426}

A randomised trial of high-dose vitamin $A$ at vaccination contacts after 6 months of age: Sex-differential effects on mortality

Topic: Other - Others

Presenter: Ane B Fisker

Ane B Fisker 1); Carlito Bale 1); Amabelia Rodrigues 1); Mathias J Jørgensen 2); Niels Danneskiold-Sørensen 2); Linda Hornshøj 1); Julie Rasmussen 1); Bo M Bibby 3); Peter Aaby 1); Christine S. Benn 2)

1) Bandim Health Project, INDEPTH Network, Apartado 861, Bissau, Guinea-Bissau; 2) Research Center for Vitamins and Vaccines (CVIVA), Bandim Health Project, Statens Serum Institut, 2300 Copenhagen S, Denmark; 3) Department of Biostatistics, Institute of Public Health, University of Aarhus, Bartholins Alle 2, 8000 Århus C, Denmark

Background: WHO recommends vitamin A supplementation (VAS) at routine vaccination contacts after 6 months of age. The policy has never been evaluated for its effect on mortality.

Objective: We aimed to evaluate the effect of VAS at vaccination contacts on all-cause mortality overall and by sex and vaccine at enrolment in a randomised controlled trial (RCT) in Guinea-Bissau. Methods: Children aged 6-23 months were randomised 1:1 to VAS (100,000 IU if aged 6-11 months, 200,000 IU if aged 12-23 months) or placebo at vaccination contacts and followed to migration, death or subsequent VAS for up to 12 months. Mortality rates were compared in Cox proportional hazards models overall, and by sex and vaccine. Results: Between August 2007 and November 2010, 7587 children were enrolled. There were 99 non-accident deaths (VAS: 50; placebo: 49). The mortality rate ratio (MRR) comparing VAS versus placebo recipients was $1.02(95 \% \mathrm{CI} 0.69 ; 1.51)$. The effect differed significantly between boys (MRR $=1.71$ (95\% CI 0.96; 3.06)) and girls $(\mathrm{MRR}=0.62(95 \%$ CI $0.35 ; 1.09)) \quad(p=0.01$ for interaction between VAS and sex). Among the $42 \%(3161 / 7587)$ who received a live measles vaccine (with or without yellow fever vaccine) at enrolment the MRR was $1.32(95 \%$ CI $0.60 ; 2.90)$. Among the $28 \%$ (2154/7587) who received inactivated DTP/pentavalent (DTP combined with Hepatitis B and $H$. influenzae type B) vaccine the MRR was 1.18 (95\% CI $0.59 ; 2.34)$ and among the $21 \%$ (1607/7587) who received both live and inactivated vaccines the MRR was 0.62 (95\% CI $0.29 ; 1.32)$. 
Conclusion: This is the first evaluation of the effect of VAS at routine vaccination contacts on mortality. VAS had no overall effect, but the effect differed significantly by sex. There is a need for more RCTs to ensure an optimal evidence-based VAS policy.

P-427

Reference of placenta weight and determinants of placenta weight: a population-based study

Topic: Other - Others

Presenter: Chunsen $W u$

\section{Chunsen Wu 1); Yuelian Sun 1); Olsen Jørn 1)}

1) Section for Epidemiology, Department of Public Health, Aarhus University; 2) Department of Epidemiology, School of Public Health, UCLA, USA

Background: Placenta is essential for the maintenance of pregnancy. Placenta growth and function may not only play a role for the survival of the fetus but also have long-term health consequence for the offspring. Abnormal placenta weights or placenta weight/birth weight ratio have been associated with cardiovascular and metabolic outcomes such as an increased risk of hypertension, coronary heart disease, cardiovascular mortality, and impaired glucose tolerance in the offspring. Placenta may play a key role in what has been labeled fetal programming".

A better understanding of what guides placenta growth and development may thus add important information in studies on fetal programming. Placenta weight can be influenced by maternal diseases such as diabetes mellitus and it is influenced also by maternal body composition and parity. However, population-based reference stratified by different factors has been lacking.

Methods: In this presentation, we identified live-born singletons of women giving birth in Denmark between January 12004 and December 312008 from the Danish Medical Birth Register. After excluding children due to including criteria or missing vital information $(\mathrm{N}=51,715)$, a total of 258,307 children left in the study population.

We explored distribution of placenta weight by taking maternal diseases, maternal age, maternal BMI (body mass index), maternal smoking, cesarean section, parity, fetal gender, and siblings into consideration.

Results: Placenta weight increases almost linearly through gestational age, however, ratio of placenta weight/birth increases and peak at about 24th week of gestation and then continuously decrease. Placental weights influenced by maternal age, maternal diseases, maternal BMI, parity, fetal gender. Placental weight increases in pregnancies with diabetes, higher BMI and decreases if pregnant women are smokers or older. Sibling comparisons indicated interaction between parity and fetal gender.

\section{P-428}

Drastic state of health research in Kazakhstan as reported by researchers, health managers and practicing physicians in Almaty

Topic: Other - Others

Presenter: Aigulsum Izekenova

Botagoz Turdalieva 1); Aigulsum Izekenova 1); Ainur Kumar 1); Akmaral Abikulova 1); Manshuk Ramazanova 1); Elmira Abdraimova 2); Alma-Gul Ryskulova 2); Bolat Zhanturyiev 1); Alfiya Igissenova 1); Andrej Grjibovski 3), 4)
1) School of Public Health, Kazakh National Medical University, Almaty, Kazakhstan; 2) Kazakh - Russian Medical University, Almaty, Kazakhstan; 3) Department of International Public Health, Norwegian Institute of Public Health, Oslo, Norway; 4) International School of Public Health, Northern State Medical University, Arkhangelsk, Russia

Background: The state of health research in the countries of the Commonwealth of Independent States has been called as drastic in a recent evaluation. Several efforts have been made to break isolation of Kazakhstan from international medical research, education and practice. The aim of this study was to assess the opinion of researchers, health managers and practicing physicians about the current status of health research in Kazakhstan.

Methods: A cross-sectional survey was performed at 3 research institutes, 3 research centers and 2 randomly selected clinics in Almaty (former Alma-Ata). Altogether, 207 (99\%) of 209 employees involved in research participated in the study. Researchers, health managers and physicians comprised $63.8,16.4$ and $19.8 \%$ of the sample, respectively. Proportions were compared using Chi squared and Fisher's exact tests.

Results: Although $38 \%$ of the total sample had the highest research degrees, less than $1 \%$ of the sample has international publications. More than three-thirds of researchers reported unsatisfactory degree of transparency of financing health research comparing to health managers and physicians $(p<0.001)$. Considerable variability between the groups was observed in the perception of the agreement between the research conducted and health needs of the vulnerable groups $(p<0.001)$. Career opportunities for researchers were also perceived differently with the highest proportion of those who unsatisfactory among researchers themselves $(p=0.025)$. A greater proportion of physicians $(39.0 \%)$ compared to the other groups considered the existing conditions for dissemination and implementation of research results as unsatisfactory $(p=0.008)$.

Conclusions: The perception of our responders of the current state of health research in Kazakhstan raise concerns about criteria for awarding $\mathrm{PhD}$ degrees without international publications, transparency of funding and agreement between the research conducted and real health needs in the country as well existing conditions for disseminating and implementation of the results of health research in practice. Given self-reported nature of the data, the findings should be interpreted with caution.

\section{P-429}

Satisfaction by the quality of medical care among elderly in Almaty, Kazakhstan: a cross-sectional study

Topic: Other - Others

Presenter: Venera Baisugurova

Gulzhakhan Kashafutdinova 1); Venera Baisugurova 1); Botagoz Turdalieva 1); Gulshara Aimbetova 1); Manshuk Ramazanova 1); Bakhit Mussaeva 1); Zhanar Buribayeva 1); Murat Tulegenov 1); Zhanna Kalmataeva 2); Andrej Grjibovski 3), 4)

1) School of Public health, Kazakh National Medical University named after S.D. Asfendiyarov, Almaty, Kazakhstan; 2) Kazakhstan School of Public Health, Almaty, Kazakhstan; 3) Department of International Public Health, Norwegian Institute of Public Health, Oslo, Norway; 4) International School of Public Health, Northern State Medical University, Arkhangelsk, Russia

Background: The number of elderly people in the European WHO region is steadily increasing posing additional demands to the health care system. Data on the quality of medical care and patients' 
satisfaction, particularly for the elderly, in the former Soviet Republics of Central Asia is almost non-existent.

Objectives: To study satisfaction of the quality of medical care by $60+$ year olds in Kazakhstan.

Methods: This study is a part of the population-based cross-sectional study performed in Almaty (former Alma-Ata) in 2012, which included 1200 randomly selected individuals aged 45 years or older. Trained interviewers visited study participants in their homes and filled out a 160-items questionnaire on health-related issues. We studied the opinion of 589 individuals aged 60+ years about their satisfaction of the quality of medical care. Self-reported satisfaction of quality of care was assessed by a direct question with a dichotomous answer. The frequency of medical visits was classified as: less than once a year, once a year, twice a year, once a quarter, once a month, once a week or more often. Self-rated health was classified as poor, satisfactory and good. Associations between self-reported satisfaction by the medical care and other variables were assessed by Pearson's Chi squared tests.

Results: Altogether, $57.5 \%$ of the responders were not satisfied by the quality of care. Twelve percent $(12.4 \%)$ did not visit health care facilities during the previous year. Corresponding proportions for other variables reflecting frequency of visits were 20.7, 21.8, 22.8, 19.3 and $3.0 \%$, respectively. The three main reasons for dissatisfaction were inattentiveness of medical personnel $(29.3 \%)$, long waiting time $(37.7 \%)$, dissatisfaction with treatment $(13.3 \%)$. Poor, satisfactory and god health were reported by $17.9,60.6$ and $21.4 \%$, respectively. Individuals with better health were less likely to be dissatisfied with medical services $(p=0.001)$. No association was observed between the frequency of visits and satisfaction $(p=0.578)$.

Conclusions: More than half of elderly in Almaty, Kazakhstan are not satisfied by the quality of medical care. Dissatisfaction by the quality of care was not associated with the frequency of use of care, but was inversely associated with self-rated health. Further studies on factors that influence satisfaction of medical care quality are needed.

\section{$\mathbf{P}-430$}

Incidence of and mortality rates of cervical cancer in the Semey region, East Kazakhstan, 2008-2012: a registry-based study

Topic: Other - Others

Presenter: Gulnar Shalgumbayeva, Andrej Grjibovski

Gulnar Shalgumbayeva 1); Yuliia emenova 1); Aigul Baibussinova 1); Marzhan Dauletyarova 1); Aidos Tlemisov 1); Andrej Grjibovski 2), 3)

1) Semey State Medical University, Kazakhstan; 2) International School of Public Health, Northern State Medical University, Arkhangelsk, Russia; 3) Department of International Public Health, Norwegian Institute of Public Health, Oslo, Norway

Background: Cancer cervix is the second most common cancer among women. Association between radiation and cancerogenesis is well-established. Semey (former Semipalatinsk) is a town adjacent to the Soviet nuclear tests site in Kazakstan. However, little is known about epidemiology of cancer in this region.

Objective: To study the incidence of and mortality from cervical cancer in Semey, Kazakhsan in 2008-2012.

Methods: Data on all cases of cervical cancer that occurred in the town of Semey during the study period were obtained from the Semey Regional Cancer Registry.

Population size was obtained from the Regional Bureau of Statistics. Standard World Population was used for calculation of the age standardized mortality and incidence rates.
Results: Altogether, 365 primary cases of cervical cancer were registered during the study period. The incidence increased from 10.0 per 100,000 in 2008 to 13.2 per 100,000 in 2012. Most cases occurred in age-group $40-44$ years $(19.5 \%)$ followed by $35-39$ years $(16.2 \%)$ and $50-54$ years old $(13.2 \%)$.

Mortality rate increased from 4.7 in 2008 to 7.2 in 2012. Most women who died from cervical cancer belonged to age-group 70-74 years $(12.4 \%)$ followed by age-groups $40-44$ years $(11.1 \%)$ and $50-54$ years $(11.1 \%)$

Conclusions: Both incidence of and mortality from cervical cancer in Semey, East Kazakhstan increased. Both indicators exceed the average for Eastern Kazakhstan region and the whole country. This might be partly explained by either delayed effects of nuclear tests performed at Semipalatinsk test site or by other factors, which will be discussed.

\section{$\mathbf{P}-431$}

Preliminary results of the implementation of the WHO programme on safe motherhood in Eastern Kazakhstan

Topic: Other - Others

Presenter: Marzhan Dauletyarova

Marzhan Dauletyarova 1); Yuliia Semenova 1); Galiya Kaylyubaeva 1); Gulnar Shalgumbayeva 1); Aidos Tlemisov 1); Gulshat Manabaeva 1); Andrej Grjibovski 2), 3)

1) Semey State Medical University, Kazakhstan; 2) International School of Public Health, Northern State Medical University, Arkhangelsk, Russia; 3) Department of International Public Health, Norwegian Institute of Public Health

Background: Since 2008 Kazakhstan implements the WHO program on safe motherhood and child health with the activities related to safe pregnancy, family planning and safe abortion services, introduction of youth friendly clinics, support, promotion of breastfeeding, and integrated management of childhood diseases.

Objectives: To assess the current status of selected routines recommended by the WHO in the maternity clinics in Eastern Kazakhstan. Methods: This cross-sectional study was performed in the town of Semey, the second largest city of Eastern Kazakhstan region. The 'Tool to assess the quality of hospital care for mothers and newborns,' developed by the WHO Regional Office for Europe was used to assess the status of the selected outcomes during 2011-2012, i.e. at least 3 years after the implementation of the programme began. Altogether, 546 women in postnatal wards in all maternity clinics of Semey comprised the sample.

Results: The mean age of women was 27.4 years. Forty-two percent were primiparas. Mean birthweight was 3455.4 g. All $100 \%$ of infants had 'skin to skin' contact with the mothers immediately after birth. Mean number of antenatal visits to family clinics was 8.6. Five hundred and four women $(92.4 \%)$ reported that they did not get full information related to the childbirth and $515(94.4 \%)$ women were not informed on the principles of optimal breastfeeding during prenatal care. Only $42.1 \%$ of the respondents used contraceptives while the rest were not aware of contraception, never applied it and could not distinguish between different methods and devices. Nearly a third of the responders $(29.8 \%)$ had medical abortions prior to the index pregnancy. Only $9.6 \%$ of respondents reported that they received post-partum counseling on contraception.

Conclusions: Counseling on breastfeeding and postpartum contraception should be improved in clinics of Semey. The results should be generalized with due caution because Semey is a more favorable place for childbirth compared to most other towns in Eastern 
Kazakhstan. The reasons for slow implementation of the WHO programme on safe motherhood in the region will be discussed.

\section{P-432}

Maternal socio-demographic factors associated with abandonment of infants in Kazakhstan

Topic: Other - Others

Presenter: Nurgul Yelissinova, Andrej Grjibovski

Nurgul Yelissinova 1); Tolebai Rakhypbekov 1); Yuliya Semenova 1); Ainur Yelissinova 1); Tamara Rymbayeva 1); Akbayan Markabayeva 1); Andrej Grjibovski 1), 2)

1) Semey State Medical University, Kazakhstan; 2) Department of International Public Health, Norwegian Institute of Public Health, Oslo, Norway

Background: The proportion of abandoned children in the countries of the former Soviet Union is among the highest in the world. The proportion of institutionalized children in Kazakhstan is the highest in the European WHO region (1.59\% in 2010) representing a considerable public health problem in the country. However, the information on the circumstances surrounding infant abandonment specific to Kazakhstan remains unknown.

Objectives: To study socio-demographic determinants of infant abandonment in Kazakhstan.

Methods: A case-control study. We included all 17 women who abandoned their infants in maternity units in a town of Semey, East Kazakhstan from September 2011 through September 2012 (cases). A total of 140 randomly selected women gave birth in the same maternity units during the study period and who did not abandon their infants served as controls. Socio-demographic factors included in this study were maternal age, ethnicity (Kazakh, Russian or other), place of residence (urban or rural), marital status, parity, education, occupation, availability of a stable income, and infant sex. Comparisons between cases and controls were performed using Pearson's Chi squared tests for categorical variables and Mann-Whitney tests for continuous variables.

Results: Median age of women who abandoned their children was lower ( 24 vs. 27 years, $p=0.005$ ). Cases were less likely to be married $(29.4$ vs. $91.4 \%, p<0.001)$, but more likely to be cohabiting, single or divorced. They were also less likely to have higher education ( 58.8 vs. $80.0 \%, p=0.047)$, more likely to be unskilled workers (64.7 vs. $22.1 \%, p=0.004)$, although the proportion of unemployed women did not differ between the groups. Women who abandoned their child were more than twice as likely to have no stable income as controls (58.8 vs. $27.1 \%, p=0.006)$. Maternal ethnic background, place of residence, parity and infant sex were not associated with abandonment.

Conclusions: Women who abandon their infants in Kazakhstan are more likely to be younger, unmarried, unskilled workers, have less education and no stable income thus representing a marginalized group of the society similar to what has been reported from other countries. The results should be used with caution given that the study was performed only in one town. More data are needed to study independent association between these and other factors and the outcome. The reasons for high abandonment rate in Kazakhstan and current activities to reduce it will be presented.

\section{P-434}

Socio-demographic differences between families with children with cerebral palsy and families with children without disabilities in Kazakhstan

Topic: Other - Others
Presenter: Ainur Yelissinova, Andrej Grjibovski

Ainur Yelissinova 1); Tolebai Rakhypbekov 1); Yuliya Semenova 1); Nurgul Yelissinova 1); Sholpan Kulzhanova 1); Akbayan Markabayeva 1); Andrej Grjibovski 1), 2)

1) Semey State Medical University, Kazakhstan; 2) Department of International Public Health, Norwegian Institute of Public Health, Oslo, Norway

Background: According to UNICEF, there are about 150 million children with disabilities worldwide. Families with children particularly in developing countries, where social support is limited, have many problems directly associated with child's disability including poverty, divorce, unemployment etc. In Kazakhstan, 62081 or $1.2 \%$ of all children are disabled. However, there have been no publications about the main problems in these families or about the differences between families with children with and without disabilities in Kazakhstan.

Objective: To study the main problems in families with children with cerebral palsy and to assess whether these families differ from other families by socio-demographic characteristics in Kazakhstan.

Methods: A cross-sectional study. All 192 families residing in a town of Semey (former Semipalatinsk) were invited and 175 of them participated. Their socio-demographic charactoeristics were compared to those of 180 families selected at random in the same town. Moreover, both types of families were asked about the main problems (they could select $0-3$ out of 9). Categorical data were compared using Chi squared tests. Continuous data were analyzed using Mann-Whitney tests. The study was approved by the local ethical committee.

Results: The main problems reported by the families with a child with cerebral palsy were child disability $(71.4 \%)$ followed by material deprivation $(38.8 \%)$ and hopelessness $(12.6 \%)$ while in families with children without disabilities the three most common answers were 'we do not have problems (58.9\%), material deprivation $(21.1 \%)$ and lack of free time $(19.4 \%)$. Differences between groups were significant $(p<0.001)$. Families with a child with cerebral palsy were more likely to be single-parent families (20.6 vs. $5.6 \%, p<0.001)$. Mothers in families with disabled child were less likely to have higher education ( 30.3 vs. $40.0 \%, p=0.007)$ and be a housewife $(68.0$ vs. $36.1 \%, p<0.001)$. Fathers in these families were also less likely to have higher education (31.7 vs. $45.9 \%$, $p=0.026)$ and be a white-collar worker (36.0 vs. $55.3 \%$, $p=0.006$ ). No differences in parental age, ethnic background and the number of children in the family between the groups were observed. Conclusions: We observed significant differences between families with children with and without disabilities by marital status, education and occupation of both parents. Given that many families with children with disabilities report poverty and hopelessness as their main problems, social and psychological support should be provided to these families should be improved. Further research is needed on the effectiveness of the services provided to these families.

\section{P-435}

Sociodemographic, religious and ethnic influences on willingness to donate organs: a Portuguese perspective with Portuguese natives and immigrants

Topic: Other - Others

Presenter: Violeta Alarcão

Violeta Alarcão 1); Filipe Leão Miranda 1); Rui Simões 1); Elisa Lopes 1); Marta Godinho 1); Mário Carreira 1); Irene Rodrigues 2); José Mapril 3); Clara Saraiva 3)

1) Institut of Preventive Medicine, Faculty of Medicine of Lisbon; 2) School of Social and Political Sciences (ISCSP); 3) Network Center for Research in Anthropology (CRIA) 
Background: Willingness to commit to posthumous organ donation has been shown to be related to common death anxiety defenses, and to differ between cultures and socio-demographic variables. Studies indicate that Chinese people are less likely to donate organs than the Westerners. In Portugal organs donation after death is presumed, meaning that any national or foreign citizen resident in Portuguese territory is considered a donor since birth. Nevertheless death' attitudes haven't been much surveyed and even less among immigrants. Objectives: To investigate death anxiety influence on willingness to donate organs and explore differences between Portuguese and Chinese, considering age, gender, educational and religious factors. Methods: Multi-ethnic cross-sectional population-based study among adults from Bangladesh, Brazil, Cape Verde, Guinea-Bissau, China, and Portuguese natives living in Lisbon. Trained interviewers from the communities in study collected qualitative and quantitative data through face-to-face interviews, namely socio-demographic, death attitudes, and the Death Anxiety Scale (DAS). Logistic regression was performed to identify factors associated to willingness to donate organs in multivariate-adjusted models for each population $(=0.05$, SPSSv21).

Results: The 99 Portuguese had a mean age of $36.2 \pm 14.6$ years and $13.1 \pm 4.9$ years of education; $60.6 \%$ were female; $78.8 \%$ were religious (96.2\% Catholics). The 97 Chinese had a mean age of $35.8 \pm 10.4$ and $10.3 \pm 3.6$ years of education; $58.8 \%$ were female; $57.3 \%$ were religious ( $74.5 \%$ Buddhists); and their mean residence time in Portugal was $9.4 \pm 6.6$ years.

Willingness to donate organs was $77.8 \%$ among the Portuguese and $25.8 \%$ among the Chinese $(p<0.001)$. Higher educational level was associated with organ donation willingness in both groups; age, sex, religion and marital status were not. DAS mean was higher among the Portuguese $(48.6 \pm 9.3)$ compared to the Chinese $(42.3 \pm 7.3)(p<0.01)$. No differences were found between DAS and willingness to organ donation.

Multiple regression analysis showed an association between education level and compliance with organs donation among Portuguese male (OR 1.64, $p=0.05$ ) and Chinese female (OR 1.31, $p=0.02$ ), independently of age, religious commitment and death anxiety, plus residence time in Portugal for the Chinese.

Conclusions: Similar to other studies, education was found to be a predictor of organ donation willingness, but DAS was not. Further analyses will be performed with the others immigrant groups. Since organ transplantation is a medical therapy that offers the potential to enhance and save life, factors that could influence the supply of organs should be better understood. Death education could reduce the stigma and negative emotions related to death and organ donation.

\section{P-436}

Elderly population health status and living conditions

Topic: Other - Politics

Presenter: Itziar Vergara

Itziar Vergara 1); Monica Machon 1); Miren Dorronsoro 2); Nerea Larrañaga 3); Kalliopi Vrotsou 1); Bakarne Aguirre 1); Isabel Larrañaga 3)

1) Primary Care Research Unit Gipuzkoa, Osakidetza; 2) Health Department, Basque Government; 3) Public Health Department Gipuzkoa, Basqeu Government

Background: comprehensive evaluations of the wellbeing of elderly individuals should include the assessment of their health status but also their living conditions. These issues are often studied separately, but the overwhelming evidence of the role of the social determinants of health, highlights the necessity to assess, as a whole, those key aspects of the wellbeing.
Objectives: to describe the health status and the living conditions of elderly individuals considering the deprivation index of their residence area. Methods: a sample of 1,000 community dwelling individuals, 65 or more years old, was studied in a defined geographic area. A multistage sampling was performed considering the municipality size, the district deprivation index, and afterwards, settling an age and sex share. A newly developed tool aimed to the characterization of health status and living conditions of elderly population (an abstract describing the construction of this tool has been submitted to this conference) was used in order to recall individual information. Data regarding the municipalities size and privation index was provided by the Statistical Agency and the Health Department of the area.

Results: 3 deprivation index stages were defined, $33.33 \%$ of subjects included in each one. $50 \%$ were women and the mean age was 77 years (SD 7.6). For each described variable, the range of values of the three index categories is provided. The $94-97 \%$ is autonomous or mildly dependent. A 74-77\% considers that their health status is good. The $35-41 \%$ is receiving 3 or more prescriptions and the $3-43 \%$ suffers of 3 or more chronic conditions. The $16-20 \%$ presents active depression symptoms. The 74-79\% maintains an adequate level of physical activity and an 11-18\% performs a healthy eating habit. A $13-19 \%$ considers that its community provides with adequate services and only the 5-7\% considers that lives in an elderly friendly community, according to the criteria proposed by the WHO in the same name program me. The $88-92 \%$ finds their social live satisfactory. Significant differences are found regarding the privation index in: the prevalence of multi-morbidity, some healthy living habits, the community consideration or the social live satisfaction. Nevertheless, in order to properly evaluate these findings, additional analysis is required. These relevant results will be presented at the conference.

Conclusions: Some of these findings, like the good perception of the health status or the performance of healthy habits are relevant to tackle the needs of this population groups. And other findings, like the poor consideration of the community environments should generate a reflection and action process regarding the living conditions that are being provided to our elderly.

\section{P-437}

Obstetrician profile and organization of obstetrics practice associate with individual factors driving decisions towards caesarean sections in Brazil

Topic: Other - Politics Presenter: Paulo Freitas

\section{Paulo Freitas 1), 2); Maria de Lourdes Drachler 3)}

1) Universidade Federal de Santa Catarina; 2) Universidade do Sul de Santa Catarina; 3) Faculdade Católica de Medicina

Background: Caesarean section is widespread as a means of delivery in Brazil, to the extent that it is used well beyond the level indicated by strict medical need. This excess has been mentioned as an aspect of the increasing medicalisation of childbirth and, caesarean delivery often perceived by the medical profession and their female patients as safe, painless, modern, and potentially ideal for any pregnant woman Objectives: To investigate factors contributing to decisions towards caesarean section from a multifactorial perspective.

Methods: The study used qualitative and quantitative methods of data collection and analysis. Qualitative methods consisted of participant observations in the labour and delivery wards, supported by in-depth interviews with on-duty obstetricians. These were followed by a quantitative survey of 660 delivering women. Logistic Regression 
was applied based on a Hierarchical framework of factors associated caesarean delivery.

Results: The results show that factors pertaining to the women, the obstetricians and to the maternity environment were all relevant to the excess use of caesarean delivery. Important factors identified in both phases of the study were previous experience for herself or her social network favouring caesarean, antenatal care with the doctor who ultimately performed the delivery, arriving in the maternity before labour is fully established and being in the care of a senior obstetrician who was assessed to be more interventionist on the basis of the in-depth interviews.

Conclusions: The overall environment and organisation of the obstetric unit in the maternity was assessed as contributory to many of these factors. Interventions to reduce the excessive rates should focus on maternity staff, service organisation and women approaching delivery.

\section{P-438}

Analysis of the HIV/AIDS research productivity in the European Union member countries

Topic: Other - Politics

Presenter: Karolin Toompere

Karolin Toompere 1); Kaja-Triin Laisaar 1); Marika Rosenthal 2); Don Des Jarlais 3); Anneli Uusküla 1); Mari-Liis Pürjer 1)

1) Department of Public Health, University of Tartu, Tartu, Estonia; 2) Centre for Continuing Medical Education, University of Tartu, Tartu, Estonia; 3) Edmond de Rothschild Chemical Dependency Institute, Beth Israel Medical Center,New York, NY, USA

Background: Research on HIV/AIDS is a top priority for the European Commission. Bibliometric assessment of research presence, performance, and impact is based on a core assumption that scientists with important information actively seek to publish their findings in open, international journals.

Objectives: To assess of HIV/AIDS research productivity and quality in the 27 member states (countries) of the European Union.

Methods: A bibliometric analysis HIV/AIDS research was conducted for the period of January 1, 2002 to December 31, 2011 (search databases: Ovid MEDLINE, PubMed, Ebsco and Embase; no language restrictions). The number of HIV research publications (per 1 000000 population) was used as surrogate for total HIV research productivity, and the number of publications (per 1000000 population) in very high (IF > 16) 5-year impact factor journals as a marker of research quality. For each country, data abstracted from different electronic databases were merged and duplicate entries were deleted.

Results: The median number of HIV/AIDS research articles published in a country $(\mathrm{n}=27)$ at the 10 year period was 319 (mean 1468, SD 2466, range 7-9128) and the median number of articles per million of population was 44.9 (mean 58.8, SD 49.3, range 4.7 [Lithuania] - 150.0 [United Kingdom]). The median number of articles per million of population published in journals with very high impact factor was 0.2 (mean 0.6, SD 0.9, range 0 [Bulgaria, Cyprus, Czech Republic, Hungary, Lithuania, Luxembourg, Malta, Poland, Portugal, Slovakia, Slovenia] - 4.5 [United Kingdom]). The proportion of articles published in very high impact journals varied from $0 \%$ [Bulgaria, Cyprus, Czech Republic, Hungary, Lithuania, Luxembourg, Malta, Poland, Portugal, Slovakia, Slovenia] to $6.2 \%$ [Latvia] of all the publications.

Conclusions: The research productivity varies noticeably across the EU countries. Bibliometric analyses can be a critically important source of objective information about the quantity and quality of scientific work.

\section{P-439}

Analysis of the use of protocols for primary care teams in Brazil

Topic: Other - Politics

Presenter: Severina Alice da Costa Uchôa

Severina Alice da Costa Uchôa 1); Paulo de Medeiros Rocha 1); Claudia Santos Martiniano 2); Ardigleusa Alves Coelho 2); Anna Ferla Monteiro Silva 3); Adrianna Ribeiro Lacerda 4); Marize Barros de Souza Araújo 1); Alan Charles Dantas Emiliando 1); Marcia Cunha da Silva Pellense 1); Marsilene Gomes Freitas 1)

1) Universidade Federal do Rio Grande do Norte; 2) Universidade Estadual da Paraíba; 3) Universidade Federal da Paraíba; 4) Faculdade de Ciências Médicas

Background: The Brazilian Ministry of Health in 2011 launched the National Program for Improving Access and Quality of Primary Care (PMAQ) in order to expand access and improve the quality of the offered shares, ushering in a culture of evaluation in health services. One of the dimensions assessed by the program is the use of protocols by Family Health teams. The protocols have been widely used throughout the network of health services, including all levels of health care. Specifically, the protocols used in Primary Health Care are intended to regulate the practices of the Family Health Team, with guidance for managers and professionals in an effort to implement a uniform range of actions that signal good health practices that level of attention.

Objective: To determine the prevalence of the use of health care protocols between teams of Primary Care in Brazil.

Methods: Cross-sectional, multicenter 16,823 questionnaires that were applied in Basic Health Units who joined the PMAQ in all Brazilian municipalities between May to September 2012. For statistical analysis, we built a database in Statistical Package for Social Sciences version 17.0.0, and performed the frequency distribution of the use of protocols.

Results: Among the protocols used by the teams observed that $37.9 \%$ use protocols to define therapeutic guidelines for hosting the spontaneous demand/urgency; $42.5 \%$ employ protocols that guide the prioritization of cases that need referral and 41.9 use protocol definition of priority situations for home visits.

Conclusion: Family health teams have not prioritized the use of protocols. It is suggested that others will be performed to assess the impact of using protocol or not the team assisted in population health

\section{P-440}

Recent development of the health care system in Kazakhstan

Topic: Other - Politics

Presenter: Ainur Kumar

Ainur Kumar 1); Aizhan Kyzayeva 1); Aigulsum Izekenova 1); Akmaral Abikulova 1); Gulzhan Abdakimova 1); Tlekbek Meimanaliev 1); Aikan Akanov 1); Andrej Grjibovski 2), 3)

1) School of Public health, Kazakh National Medical University named after S.D. Asfendiyarov, Almaty, Kazakhstan; 2) Department of International Public Health, Norwegian Institute of Public Health, Oslo, Norway; 3) International School of Public Health, Northern State Medical University, Arkhangelsk, Russia 
Background: Internal diseases rank first according to the mortality and disability level constituting an immediate problem not only for the public health system by reason of a high cost of treatment procedures, clinical course duration, and a high invalidization of the population, but also for the social \& economic systems of the branch, as consequently labour resources decrease and are lost, social allocations increase, and there occurs loss of human labor resources and material labour resources.

Methods: We used Information-analytical, content-analysis and comparative analysis of statistical data on health system in Kazakhstan over the past 10 years.

Results: A share of internal diseases in the total morbidity patterns in 2012 is $46.3 \%$ (48.2 \% in 2001). Over the last 10 years, the highest mortality in the RK population mortality patterns was caused by internal diseases (in 2007-635,0 cases per 100000) and in its course it had a constant tendency to some increase while the total mortality rate in RK is 8.71 per 1000 population.

The circulatory, respiratory and digestive diseases are the principal ones among all internal diseases with respect to the population morbidity, mortality and invalidization rates. Over the last 10 years, the total morbidity rate per 100000 population in view of the said diseases changed as follows: there was a slight decline in the respiratory diseases rate from 31050.3 in 2001 to 29840.1 in 2011, in the digestive diseases rate-from 10144.4 in 2001 to 9189.5 in 2011, and a multiple increase in the circulatory diseases rate from 2605.2 in 2001 to 11751.1 in 2011.

The dynamics of the total morbidity rate from cardiovascular diseases in RK showed a steady increase over 2001-2011. The statistically average data on the total morbidity rate from circulatory diseases increased by $451 \%$ (or 4.5 times more). The total number of registered patients in RK by 2011 was 1.945822 .

According to the World Health Organization, the standardized mortality rate for the population of the Republic of Kazakhstan subject to circulatory diseases is twice as high as the same in the countries of the European region (867.9 against 448.0 per 100,000 population, respectively). Besides that, the absolute number of patients, who died from circulatory diseases, is 81.1 ths. of persons. More than 80 ths. of persons die from circulatory diseases in the country annually. In the nosological spectrum of the causes of the population disability, circulatory diseases ranked first.

Conclusion: The above-mentioned statistical data cannot fully reflect the existing situation as morbidity and disability cases are registered after references to doctors, there is no disease intelligence at an early stage, a lot of patients just do not visit doctors and remain untreated and unobserved, and there is even no fact of registration of a disease. The problems of development and further improvement of therapeutic service are always pressing in the health-care reform generally. An emphasis laid on the socially significant diseases, in particular, on the circulatory ones, should be of considerable importance in the health system basis.

\section{P-441}

Contribution of different chronic conditions to objective and subjective health outcomes: results from a National Health Survey

Topic: Other - Politics

Presenter: Ana Daniela Gomes Araújo Simões

Ana Daniela Gomes Araújo Simões 1), 2); Teresa Monjardino 1), 2); Raquel Lucas 1), 2); Henrique Barros 1), 2)

1) Institute of Public Health of the University of Porto, Portugal; 2) Department of Clinical Epidemiology, Predictive Medicine \& Public Health, University of Porto Medical School, Portugal
Background: Representative national health data allow us to estimate the impact of chronic conditions on adverse outcomes useful to define politics and public health strategies. Population-attributable fraction (PAF) is a valuable measure for quantifying the potential impact of preventing a disease on a particular adverse health outcome.

Objective: To examine the population-level impact of chronic conditions on objective and subjective health outcomes in the Portuguese population.

Methods: We analysed data of 23,841 individuals from the 4th Portuguese National Health Survey (2005/2006), a representative sample of the Portuguese population. The presence of chronic conditions (diabetes, hypertension, rheumatic disease, chronic obstructive pulmonary disease (COPD), stroke, depression, myocardial infarction) was self-reported. The adverse health outcomes considered were negative self-rated health (subjective perception of poor or very poor health), frequent health care utilization (two or more medical appointments in the previous three months), short-term disability (at least 1 day of impairment at work, home or leisure activities for health reasons, in the previous 2 weeks) and long-term disability (severe limitation in function or restriction in at least one daily activity). For each chronic condition, we estimated the risk of having an adverse outcome using Log-Poisson regression to calculate prevalence ratios adjusted for age, sex, education and other diseases (adjPR). We estimated PAF for each condition on the outcomes $(\mathrm{PAF}=$ proportion of those with the adverse outcome reporting the condition [(adjPR-1)/adjPR)].

Results: Prevalence of diabetes, hypertension, rheumatic disease, COPD, stroke, depression, myocardial infarction was 9.0, 27.4, 20.8, $4.4,2.0,10.8$ and $1.7 \%$, respectively. Depression had the strongest association with negative self-rated health $(\operatorname{adjPR}=1.846,95 \% \mathrm{CI}$ $1.838 ; 1.854)$, frequent health care utilization $(\operatorname{adjPR}=1.550,95 \%$ CI $1.544 ; 1.556)$, and short-term disability (adjPR $=1.842,95 \% \mathrm{CI}$ $1.831 ; 1.852)$. Stroke had the strongest association with long-term disability (adjPR $=1.639,95 \%$ CI 1.627; 1.652). However, because of their high frequency, rheumatic diseases had the greatest population impact on negative self-rated health $(\mathrm{PAF}=16.9 \%)$ and shortand long-term disability ( $\mathrm{PAF}=15.0$ and $14.2 \%$, respectively), while hypertension had the greatest impact on frequent health care utilization $(\mathrm{PAF}=10.9 \%)$.

Conclusions: Differences in the ranking of individual risks and PAF for different chronic conditions and outcomes were considerable. To decrease the burden of adverse health outcomes in rheumatic diseases, hypertension and depression are potential priority prevention targets.

\section{P-442}

\section{Embryo disposition in Northern Portugal}

Topic: Other - Politics

Presenter: Catarina Samorinha

Catarina Samorinha 1), 2); Elisabete Alves 1), 2);

Sandra Sousa 2); Helena Machado 3); Teresa Rodrigues 1), 2); Susana Silva 1), 2)

1) Department of Clinical Epidemiology, Predictive Medicine and Public Health, University of Porto Medical School, Porto, Portugal; 2) Institute of Public Health of the University of Porto (ISPUP), Porto, Portugal; 3) Department of Sociology, University of Minho, Braga, Portugal

Background: Human embryonic stem cell research has been central to molecular epidemiology and public health genomics, raising social expectations towards the health sciences. The suppliers of embryos have been rarely studied.

Objectives: To estimate the prevalence of in vitro fertilization (IVF) patients' decisions about embryo disposition according to social and obstetric characteristics of women and men. 
Methods: Within a hospital-based study, 221 heterosexual couples and 93 women were interviewed at Hospital de São João (Porto, Portugal), between August 2011 and August 2012. After blood sample collection that aim to diagnose pregnancy, a structured questionnaire was administered to gather data on embryo disposition, sociodemographics, obstetrics and the importance attributed to research on human embryos. The present analysis is based on 204 cases of IVF couples with available data for all the variables included. Multinomial logistic regression was used to compute odds ratios (OR) and $95 \%$ confidence interval $(95 \% \mathrm{CI})$. The final model comprised the statistically significant variables and possible confounders.

Results: Almost two-thirds (64.2\%) accepted to donate embryos for research and for other couples, $11.8 \%$ refused it, and one quarter $(24.0 \%)$ donated embryos only for research. Refusal to donate was more frequent when couples considered research not very important ( 25.0 vs. $6.8 \% ; p=0.001)$ and when at least one member of the couple was non-Catholic (23.7 vs $9.0 \% ; p=0.027)$. Donating embryos only for research was less likely among women aged above 34 years old (13.1 vs. $28.7 \% ; p=0.011)$ and couples trying to conceive for more than 36 months (18.4 vs. $32.9 \% ; p=0.061)$. After adjustment, couples who consider research not very important (multivariate OR 4.03; $95 \%$ CI 1.60-10.36) as well as those with at least one non-Catholic member (multivariate OR 3.83; $95 \%$ CI $1.38-10.60)$ were more likely to refuse donation simultaneously to research and to other couples. Donating embryos exclusively for research was inversely associated with trying to conceive for more than 36 months (multivariate-adjusted OR 0.49; 95 \% CI 0.26-0.99). Conclusions: The proportion of IVF couples who accepted to donate embryos for scientific research and to other couples in northern Portugal was higher among Catholic than non-Catholic and among those who considered embryonic research very important.

Willingness to donate embryos for scientific research in other European countries is lower than the observed in this study. A focus on comparative analysis of embryo disposition according to sociodemographic and obstetric characteristics of women and men is needed in reproductive epidemiology studies.

\section{P-443}

Long-living population in the region of Halland, Sweden: impact of emigration on the risk of death

Topic: NordicEpi - Nordic studies

Presenter: Amir Baigi, Anders Holmén

\section{Amir Baigi 1), 2); Anders Holmén 1)}

1) Department of Research, Development and Education, Region Halland, Sweden; 2) Department of Primary Healthcare, Sahlgrenska academy, Gothenburg University

Background: The Region of Halland has about 300000 inhabitants and is situated in the southwest of Sweden. The population is living in towns, villages, and in the countryside. Data from the National Bureau of Statistics (SCB) have shown that the population of Halland has the highest life expectancy in Sweden. In previous studies we were able to trace the beginning of this long life expectancy to the early twentieth century. During the 19th century, the population of Halland doubled despite considerable internal (i.e., within Sweden) and external emigration. Given the computerized population databases available in Sweden, we found it possible to investigate the impact of internal emigration on the risk of death in the long living population of Halland.

Objectives: The aim of this study was to examine, the impact of internal emigration on the risk of death among individuals born in the region but emigrated to other parts of Sweden.
Methods: The Swedish inhabitants made up the study population and comprised 1,654,744 men and 1,592,467 women in Sweden as a whole for the age group under study. The corresponding figures for the province of Halland were 45,394 men and 43,403 women. The study comprised the 25-55 year age interval, in order to cover the population of working age with socioeconomic background variables. All deaths listed in the international classification of diseases (ICD) during the 10-year period from 1980 to 1990 were included. The study had a historical prospective design. The risk of death was estimated by Poisson regression as a function of Birth place, age, marital status, educational level and socio-economic classification. Separate analyses were done regarding men and women. For the hazard rate (HR), $95 \%$ confidence intervals were calculated.

Results: The results revealed that those natives of Halland who had moved to another part of the country retained their advantage compared to Swedes born outside the province and living in other parts of Sweden. This difference was statistically significant among men (Men: HR $=0.90 ;$ CI 0.81-0.99; Women: HR $=0.92 ;$ CI $0.81-$ 1.05).

Conclusion: An important aspect in the study is the fact that the protective factor against death is maintained when natives of Halland move to other parts of Sweden. This protective factor seems to be strong enough to slow the rate of emigration as a significant risk factor.

Data about the duration of residence are lacking, and therefore not accounted for.

\section{P-444}

Childhood bereavement and pediatric asthma hospitalization-a population-based cohort study in two Nordic countries

Topic: NordicEpi - Nordic studies

Presenter:

Jørn Olsen 1); Esben Agerbo 2); Wei Yuan 3); Jiong Li 1)

1) Department of Public Health,Aarhus University, Denmark; 2) Department of Economics and Business, Aarhus University, Denmark; 3) Department of Reproductive Epidemiology and Social Science, Shanghai Institute of Planned Parenthood Research, Shanghai, China

Background: Asthma is one of the leading causes of pediatric hospitalization. Exposure to psychological stress in early life may result in functional changes in immune reactivity, which could influence asthma development.

Objective: To examine whether bereavement in childhood is associated with an increased risk of pediatric asthma hospitalization.

Methods: We undertook a cohort study based on data from several national population-based registers in Denmark and Sweden. All singleton live births during 1977-2008 in Denmark and during 1973-2006 in Sweden were included in the study. The children were followed from birth to the first hospitalization due to asthma, emigration, death, their 18th birthday, the end of 2007 in Sweden, or the end of 2008 in Denmark, whichever came first. All the children were assigned to the non-bereaved group until they lost a close relative (loss of a mother, a father or a sibling), when they were then included in the bereaved group. First hospitalization due to asthma was monitored in these two groups. We evaluated the hazards ratio (HR) of asthma hospitalization in bereaved children using Cox proportional hazards regression models.

Results: The study population consisted of 5,202,576 children. A total of 168,649 (3.2\%) children lost a close relative during followup. We identified 147,829 children who were hospitalized due to asthma. After adjustment for potential confounders, the overall 
adjusted HR of asthma hospitalization in bereaved children was 1.10 (95\% confidence interval (CI) 1.04-1.16), compared to non-bereaved children. The relative risk of asthma hospitalization was higher when time elapsed since bereavement" was less than 1 year following bereavement $(\mathrm{HR}=2.02,95 \% \mathrm{CI} 1.79-2.28)$. The loss of a close relative was not associated with an increased risk of asthma hospitalization 1 year after bereavement.

Conclusions: Bereavement in childhood was associated with a shortterm increased risk of asthma hospitalization.

Key words: Bereavement, childhood, psychological stress, pediatric asthma hospitalization

\section{P-445}

Association between sense of coherence in adolescence and social benefits. A 12-year follow-up study

Topic: NordicEpi - Nordic studies

Presenter: Else Toft Würtz.

\section{Else Toft Würtz 1); Jens Tølbøll Mortensen 1);} Kirsten Fonager 1), 2)

1) Department of Social Medicine, Aalborg University Hospital, Aalborg, Denmark; 2) Department of Health Science and Technology, Aalborg University, Aalborg, Denmark

Background: Local government concern over expenditure on social and health care is growing, and the need for new strategies and initiatives for prevention is an obvious issue.

Objectives: The aim of the present study was to explore the association between a low 'sense of coherence' (SOC) in teens and their subsequent risk of receiving social and health care benefits during young adulthood, and to monitor how SOC developed during this period.

Methods: The teenagers' SOC were determined by a questionnaire and included seven items of the original SOC-13 questionnaire (denoted SOC-7). The questionnaire was distributed to 7th and 8th grade pupils from 15 Danish public schools in 1998. Follow-up SOC13 was assessed in 2010. Low SOC was defined as the lowest 1 st quartile. Data $(\mathrm{N}=773)$ were linked to the Danish Rational Economic Agents Model (DREAM) database embracing disbursed public social benefits.

Results: 722 had answered the SOC-7 items in 1998. Girls with a low SOC-7 in 1998 had a significantly increased risk of receiving unemployment benefits (RR 1.3 (1.1-1.6)), social assistance (RR 1.8 (1.3-2.5)) and sickness benefits (RR 1.5 (1.2-2.0)) compared with girls with a high SOC-7. For boys, only minor protective and nonsignificant differences were found. The SOC answers from 1998 and 2010 were compared $(n=394)$. SOC increased significantly and mostly in the girls.

Conclusion: SOC-7 may serve as a predictor for social life event outcomes and may hence facilitate an early identification and a selective approach to support teenage girls with a low SOC. From adolescence to young adulthood SOC-7 was of a relatively unstable nature.

\section{P-446}

The nordic prescription databases as a resource for pharmacoepidemiological research: a literature review

Topic: NordicEpi - Nordic studies

Presenter: Björn Wettermark

Björn Wettermark 1); Helga Zoega 2); Kari Furu 3); Maarit Korhonen 4); Jesper Hallas 5); Mette Nørgaard 6);
Morten Andersen 1), 5); Marie Mortensen 6); Helle Wallach Kildemoes 7); Henrik Toft Sørensen 6)

1) Centre for Pharmacoepidemiology, Department of Medicine, Karolinska Institutet, Stockholm, Sweden; 2) Faculty of Medicine \& Faculty of Pharmacy, Centre of Public Health Sciences, University of Iceland, Reykjavík, Iceland; 3) Department of Pharmacoepidemiology, Division of Epidemiology, Norwegian Institute of Public Health, Oslo, Norway; 4) Department of Pharmacology, Drug Development and Therapeutics, University of Turku, Turku, Finland; 5) Department of Clinical Pharmacology, University of Southern Denmark, Odense, Denmark; 6) Department of Clinical Epidemiology, Institute of Clinical Medicine, Aarhus University Hospital, Aarhus, Denmark; 7) Center for Healthy Aging, Faculty of Health and Medical Sciences, University of Copenhagen, Copenhagen, Denmark

Background: Pharmacoepidemiological studies are needed to study the effectiveness and safety of drug therapy in real-life settings. They are also essential to assess the quality of prescribing and to evaluate the effectiveness of interventions aimed at promoting rational use of drugs. All five Nordic countries have nationwide prescription databases covering all dispensed drugs, with potential for linkage to outcomes. In most Nordic countries the notion of a national prescription drug database was vigorously debated before its creation, with public and political discussions about the usefulness and integrity of collecting and storing personal data on such a large scale. Given this history, it is important to evaluate the extent to which these databases have contributed to scientific knowledge.

Objectives: To present an overview of therapeutic areas and methods of pharmacoepidemiologic studies published between 2005 and 2010 that used data from the Nordic prescription databases.

Methods: The study consists of a Medline-based structured literature review of scientific papers published during 2005-2010 using data from the prescription databases in Denmark, Finland, Iceland, Norway, and Sweden, covering 25 million inhabitants. Relevant studies were analyzed in terms of pharmacological group, study population, outcomes examined, type of study (drug utilization vs. effect of drug therapy), country of origin, and extent of cross-national collaboration. Results: A total of 515 studies were identified. Of these, 262 were conducted in Denmark, 97 in Finland, 4 in Iceland, 87 in Norway, and 61 in Sweden. Four studies used data from more than one Nordic country. The most commonly studied drugs were those acting on the nervous system, followed by cardiovascular drugs and gastrointestinal/endocrine drugs. A total of 228 studies examined drug utilization and 263 focused on the effects and safety of drug therapy. Pregnant women were the most commonly studied population in safety studies, while prescribers' adherence to guidelines was the most frequent topic of drug utilization studies.

Conclusions: The Nordic prescription databases, with their possibility of record-linkage, represent an outstanding resource for assessing the beneficial and adverse effects of drug use in large populations, under routine care conditions, and with the potential for long-term follow-up.

\section{$\mathbf{P}-447$}

\section{The effect of chronic stresses on the structure of skin}

Topic: NordicEpi - Others

Presenter: Mahdi Akhbardeh

\section{Mahdi Akhbardeh 1); Alireza Sadegi 2)}

1) Tehran Medical University; 2) iran.boston medical university.usa

Objectives: The scientist study of the psychic stresses effects on the destruction and premature senility in the structure of skin cells directly and indirectly. 
Method: During the fetal period. Skin and nerves have a common origin causing by the fetal texture called ectoderm a this closeness causes that many diseases of the skin and nerve are related. Considering the nerves and skin (dermal) diseases, one of the most important points can be the relationship between stress and its role on the destruction of dermal cells. Stress influences the skin from several aspects.

These effects can be as follows.

1. Directly: contraction of the face skin muscles upon the nervous disorders and in long-term the spasmodic. Contractions creates some lines around the lips and the eyes. In the stressful conditions, the people resort to some materials like alcohol and cigarette for comforting them from the emotional and psychic problems which these toxic materials are the main source of free radicals. The negative effect of these toxic materials on the skin cells has been proved for several years.

2. Indirectly: In this stressful state, the biologic factors cause the most damage on the skin. For example when the body lies in the stressful conditions, a material called cortisol is released in the body. Increasing the plasma level of this material is concordant with:

a. Catabolism of the face muscles, collagen and of the skin and consequently the thinness, wrinkles and subsidence of the skin.

b. Upon increasing the cortisol plasma level, the inflammatory like prostaglandins increase in the skin and then the skin capillaries are destroyed and the rate of receiving oxygen is reduced.

c. The recent studies indicate that the enhancement of the cortical is concordant with the cell telomere. This reduction then reduces the cell division and the rate of cell density in the skin textures.

Clearly there is a relationship between the beginning of the dermal diseases like psoriasis eczema and albinism mostly in the stressful situation. All of the three mentioned diseases are the autoimmune deceases caused mostly by the negative function of the body immune system. There is a close relationship between the negative function of the body immune system and the secretion of cortisol.

In this paper, it has been tried that the physical and chemical effects of the stress and its role in the destruction of the stress dermal cells is discussed.

In other works, stress is one of the factors of premature senility in people.

Conclusion: The mental stresses have a direct effect on the dermal cells physically and chemically. Undoubtedly, the various studies performed in the valid medical centers indicate the direct relationship between the chronic stresses and the premature senility of the skin.

\section{P-448}

Low birth weight, small-for-gestational age and preterm births before and after the economic collapse in Iceland

Topic: NordicEpi - Register-based epidemiology

Presenter: Unnur Anna Valdimarsdóttir

Védís Helga Eiríksdóttir 1); Tinna Laufey Ásgeirsdóttir 2); Ragnhei ur Ingibjörg Bjarnadóttir 3); Robert Kaestner 4); Sven Cnattingius 5); Unnur Anna Valdimarsdóttir 1)

1) Centre of Public Health Sciences, University of Iceland; 2) Department of Economics, University of Iceland; 3) LandspítaliUniversity Hospital; 4) The Institute of Government \& Public Affairs,
University of Illinois; 5) Department of Medicine, Unit of Clinical Epidemiology, Karolinska Institutet

Background: Infants born small for gestational age (SGA) or preterm have increased rates of perinatal morbidity and mortality. Stressful events have been suggested as potential contributors to preterm birth (PB) and low birth weight (LBW).

Objectives: Using the nationwide Medical Birth Registry, we examined the effect of the 2008 national economic collapse in Iceland on infant health, as measured by LBW, SGA and PB.

Methods: The study population constituted all Icelandic women giving birth to live-born singletons from January 1st 2006 to December 31st 2009. LBW infants were defined as those weighing $<2500$ grams at birth, PB infants as those born before 37 weeks of gestation and SGA as those with a birth weight for gestational age more than 2 standard deviations (SD's) below the mean according to the Swedish fetal growth curve. We used unadjusted and adjusted logistic regression analysis to estimate odds ratios [OR] and corresponding $95 \%$ confidence intervals $[95 \% \mathrm{CI}]$ of adverse birth outcomes by exposure to calendar time of the economic crisis, i.e. after October 6th 2008.

Results: Compared to the preceding period, we observed an adjusted increased odds in LBW deliveries following the economic collapse $(\mathrm{aOR}=1.24,95 \%$ CI $[1.02,1.52])$, particularly among infants born to mothers younger than 25 years $(\mathrm{aOR}=1.85,95 \% \mathrm{CI}[1.25,2.72])$ and not working mothers $(\mathrm{aOR}=1.61,95 \% \mathrm{CI}[1.10,2.35])$. Similarly, following the economic collapse, we found a tendency towards higher incidence of SGA births $(\mathrm{aOR}=1.14,95 \% \mathrm{CI}[0.86,1.51])$ particularly among children born to mothers younger than 25 years $(\mathrm{aOR}=1.87,95 \% \mathrm{CI}[1.09,3.23])$ and to not working mothers $(\mathrm{aOR}=1.86,95 \% \mathrm{CI}[1.09,3.17])$. No change in risk of PB was observed. The increase of LBW deliveries was most distinct in the time period April-June 2009 and diminished towards the end of 2009. Conclusions: The results suggest an increase in risk of low birth weight shortly after the dramatic collapse of the Icelandic national economy. The increase in LBW seems to be driven by reduced fetal growth rate rather than shorter gestation.

\section{P-449}

Joint contribution of pain and insomnia to sickness absence and disability retirement: a register-linkage study among Norwegian and Finnish employees

Topic: NordicEpi - Register-based epidemiology Presenter: Tea Lallukka

Tea Lallukka 1), 2); Simon Øverland 3), 4); Peija Haaramo 1); Peppiina Saastamoinen 1); Bjørn Bjorvatn 5), 6); Børge Sivertsen 3), 4)

1) Hjelt Institute, Department of Public Health, University of Helsinki, Finland; 2) Finnish Institute of Occupational Health, Helsinki, Finland; 3) Faculty of Psychology, University of Bergen, Bergen, Norway; 4) Division of Mental Health, Norwegian Institute of Public Health, Bergen, Norway; 5) Department of Public Health and Primary Health Care, University of Bergen, Bergen, Norway; 6) Norwegian Competence Center for Sleep Disorders, Haukeland University Hospital, Bergen, Norway

Background: Pain and insomnia are both independently associated with work disability. Although pain and insomnia often co-occur, their joint associations with subsequent sickness absence and disability retirement have not been studied.

Objectives: We aimed to examine the joint associations of pain and insomnia with subsequent work disability in two prospective occupational cohorts while considering key covariates. 
Methods: Norwegian Hordaland Health Study $(\mathrm{n}=6,892,59 \%$ women) and Finnish Helsinki Health Study $(\mathrm{n}=6,060,78 \%$ women) baseline questionnaire survey and register data were used. Those with only pain, only insomnia, or both conditions at baseline were compared to those with no pain or insomnia. Work disability outcomes were derived from national and employer's register data. Medically certified sickness absence spells lasting 2 weeks or more and all-cause disability retirement were examined. Covariates were sex, age, marital status, education, smoking, alcohol use, body mass index, and blood pressure. Poisson (number of sickness absence spells) and Cox (disability retirement events) regression models were fitted.

Results: Both pain and insomnia were associated with subsequent sickness absence and disability retirement, but the associations were stronger for those reporting comorbid pain and insomnia with support for a synergistic interaction effect, particularly regarding disability retirement. The associations were largely similar in both cohorts, and remained after full adjustments.

Conclusions: This study is the first to report the separate and combined effects of pain and insomnia on objective health outcomes. Common patterns observed in two separate cohorts suggest that the combination of pain and insomnia might be particularly relevant for subsequent disability retirement.

\section{$\mathbf{P - 4 5 0}$}

Symptoms of anxiety and depression measured once or twice as risk factors for first future AMI. The HUNT 2 study

Topic: NordicEpi - Register-based epidemiology

Presenter: Lise Gustad

Lise Gustad 1), 2); Lars Erik Laugsand 3), 4); Imre Janszky 3), 5); Håvard Dalen 1), 6); Ottar Bjerkeset 2), 7)

1) Dept. Internal Medicine, Sykehuset Levanger, Nord-Trøndelag Health Trust, Norway; 2) Dept. of Neuromedicine, Norwegian University of Science and Technology, Trondheim, Norway; 3) Dept. of Public Health and General Practice Norwegian University of Science and Technology, Trondheim, Norway; 4) Dept. Internal Medicine, StOlav University Hospital, Trondheim, Norway; 5) Department of Public Health Sciences, Karolinska Institutet, Stockholm, Sweden; 6) Department of Circulation and Medical Imaging, NTNU, Trondheim, Norway; 7) Department of Research and Development, Nord-Trøndelag Hospital Trust, Norway.

Background: Depression and anxiety are associated with elevated risk for acute myocardial infarction (AMI). However, the nature of this association remains controversial.

Objectives: We investigated the associations between mixed depression and anxiety symptoms (MSAD) with risk for hospitalization or death due to first AMI in a large population-based Norwegian cohort free of cardiovascular disease, taking previous symptoms of MSAD into account.

Methods: A total of 57,953 individuals that participated in the NordTrøndelag Health Study (HUNT 2, 1995-1997) gave self-report on the Hospital Anxiety and Depression Scale (HADS). Previous MSAD was available from the first HUNT Study (1984-1986) for 32,963 persons. Incident AMI was identified at the two county hospitals or by the National Cause of Death Registry. Multivariate Cox hazard models was used to assess the risk of first AMI related to MSAD.

Results: During 11.4 years of follow up, 2111 incidents of AMI occurred. Age and sex adjusted hazard for future first AMI related to one episode of MSAD in HUNT 2 was 1.26 (95 \% CI 1.04-1.51).
Taking MSAD from HUNT 1 into consideration, those who had symptoms of MSAD at both HUNT waves had a $63 \%$ higher risk for future AMI (95\% CI 1.24-2.19). These results were not substantially altered after adjustments for psychosocial and traditional cardiovascular risk factors. However, common chronic disorders in the population attenuated the hazards for repeated MSAD (HR 1.38, $95 \%$ CI $0.79-2.40)$.

Conclusions: Common chronic disorders seem to partly explain the association between MSAD and risk for future AMI.

\section{P-451}

Early life exposure to psychological stress and risk of childhood cancer: A population-based study

Topic: NordicEpi - Register-based epidemiology Presenter: Natalie Momen

Natalie Momen 1); Jørn Olsen 1), 2); Mika Gissler 3), 4); Sven Cnattingius 5); Jiong Li 1)

1) Section for Epidemiology, Department of Public Health, Aarhus University; 2) Department of Epidemiology, University of California; 3) THL National institute for Health and Welfare, Helsinki; 4) Nordic School of Public Health NHV, Gothenburg; 5) Department of Medical Epidemiology and Biostatistics, Karolinska Institute

Background: Despite childhood cancer being a leading cause of child death in developed countries, relatively little is known about its etiology. Almost half are diagnosed in the first 5 years of life. A better understanding of the causal role of early life factors is required in order to develop effective prevention strategies. Psychological stress has been linked to the development of cancers in adults. Subsequent hormonal dysfunction has been suggested to affect immune function, DNA repair and epigenetic silencing. However stress is likely to affect children differently to adults, due to their physiological and psychological development.

Objectives: The study aimed to investigate the association between bereavement due to death of a close relative, as an indicator of early life stress during childhood, and childhood cancer, using the national registers of Denmark and Sweden.

Methods: All live-born children born in Denmark between 1968 and $2007(\mathrm{~N}=2,729,308)$ and in Sweden between 1973 and 2006 $(\mathrm{N}=3,395,166)$ were included in the study. Children were 'unexposed' until they experienced a bereavement, at which point they became 'exposed' and contributed observation time for the exposed group. Cancer rates were compared between these two groups using Cox regression.

Results: $1,505,938(24.5 \%)$ children experienced bereavement and 9,823 of the children were diagnosed with cancer during the follow up period. A $10 \%$ increased risk of childhood cancer was seen among children who experienced the death of a relative (hazard ratio 1.10, $95 \%$ confidence interval 1.04-1.17). Considering specific cancer types, a significant association was seen only for central nervous system tumours (hazard ratio 1.14, $95 \%$ confidence interval $1.02-1.28$ ).

Conclusions: The data suggests there is a small increased risk of childhood cancers in children exposed to psychological stress, through death of a close relative, in early life. However, the case numbers were too few to provide sufficient information for specific types of childhood cancer. Some childhood cancers may be initiated in utero and bereavement may provide the second or subsequent hit" required for cancer to develop. 


\section{P-452}

Mortality after parental death in childhood: a nationwide cohort study from 3 Nordic countries

Topic: NordicEpi - Register-based epidemiology

Presenter: Jiong $\mathrm{Li}$

Jiong Li 1); Mogens Vestergaard 1); Sven Cnattingus 2); Mika Gissler 3), 4); Bodil Hammer Bech 1); Carsten Obel 1); Jørn Olsen 1), 5)

1) Department of Public Health, Aarhus University, Aarhus, Denmark; 2) Department of Medicine, Karolinska Institute, Stockholm, Sweden; 3) Nordic School of Public Health (NHV), Gothenburg, Sweden; 4) National Institute for Health and Welfare (THL), Helsinki, Finland; 5) Department of Epidemiology, School of Public Health, University of California, Los Angles, USA

Background: Death of a parent in childhood is a serious life event, but its impact on mortality has received little attention.

Objectives: We aimed to examine the association between parental death in childhood and subsequent mortality risk from childhood until adulthood.

Methods: We studied a nationwide cohort involving all children born in Denmark from 1968 to 2008 ( $N=2,809,393)$, all children born in Sweden from 1973 to 2006 ( $N=3,400,212)$, and a random sample of $89.3 \%$ of all children born in Finland from 1987 to 2007 $(\mathrm{N}=1,136,409)$. The study population was followed for up to 42 years from 6 months after birth. We used Log-linear Poisson Regression to compare mortality rates between subjects who lost a parent before 18 years and those who did not experience such a loss. Results: In the population of 7,3020,33 children, 189,194 (2.6 \%) lost a parent before 18 years of age $(57,281$ lost a mother and 131,913 lost a father). Exposure to parental death in childhood was associated with $50 \%$ higher all-cause mortality (mortality risk ratio (MRR), 1.50; $95 \%$ confidence interval $[\mathrm{CI}] 1.43$ to 1.58 ). Those who lost a parent by death from external causes had a higher all-cause mortality (MRR, $1.84 ; 95 \%$ CI 1.71 to 2.00 ) than those who lost a parent from diseases (MRR, $1.33 ; 95 \%$ CI 1.24 to 1.41 ). The MRRs were particularly prominent for some specific causes of death, such as digestive diseases, circulatory diseases, and suicide. The MRRs for external causes were the highest were increased from the start of follow-up and remained elevated until adulthood.

Conclusions: Parental death in childhood was associated with a longterm increased mortality from both diseases and external causes.

\section{$\mathbf{P}-453$}

Pre-eclampsia and development of breast cancer

Topic: NordicEpi - Register-based epidemiology Presenter: Mads Kamper-Jorgensen

Mads Kamper-Jørgensen 1); Nadja Pacheco 1); Anne-Marie Nybo Andersen 1)

1) Department of Public Health, University of Copenhagen, Denmark

Background: Women with a pregnancy complicated by preeclampsia have been reported to be less likely to develop breast cancer, compared with women without pre-eclampsia.

Objectives: To investigate the association between pre-eclampsia and breast cancer overall, and according to offspring sex and preeclampsia severity.

Methods: In the Danish Medical Birth Register, we identified all registered live and still births in Denmark during 1978-2010. For each birth, we identified maternal hospitalizations with pre-eclampsia during pregnancy by linkage to the Danish National Patient Registry. Also maternal breast cancer hospitalizations were identified in the Danish National Patient Registry. Emigrations and deaths were identified in the Danish Civil Registration System. Time at risk was counted from 2 years after giving birth until breast cancer diagnosis, death, emigration, or end of follow-up ultimo 2010, whichever occurred first. We evaluated the association between pre-eclampsia and breast cancer based on incidence rate ratios (IRR) with $95 \%$ confidence intervals (CI) estimated in Poisson regression models adjusted for attained age, period and pregnancy number.

Results: During 1978-2010, 904,923 women gave birth to $1,558,481$ children, of which $99.8 \%$ were live births. Women were followed for a total of $13,565,115$ person-years at risk, during which 22,534 were diagnosed with pre-eclampsia and 16,177 developed breast cancer. 201 women were diagnosed with pre-eclampsia as well as developed breast cancer. Overall, women diagnosed with pre-eclampsia were at $22 \%$ significantly reduced risk of developing breast cancer $(\mathrm{IRR}=0.78 \quad[95 \%$ CI 0.68-0.90]). Stratifying on offspring sex revealed a significantly reduced risk of breast cancer among preeclamptic women giving birth to boys (IRR $=0.66[95 \% \mathrm{CI}$ 0.53-0.81], but none so among women giving birth to girls $(\mathrm{IRR}=0.91 \quad[95 \%$ CI 0.76-1.09]. Analyses according to preeclampsia severity showed that risk of breast cancer was reduced among women diagnosed with less severe pre-eclampsia (IRR $=0.69$ [95\% CI 0.58-0.82]), but not among women diagnosed with severe pre-eclampsia (IRR $=1.04$ [95\% CI 0.82-1.31]).

Conclusions: Although the biological mechanism underlying the association is not clear at present, women with a pregnancy complicated by pre-eclampsia are at reduced risk of developing breast cancer. Indeed, the present analysis points towards reduced risk of breast cancer in pre-eclamptic women giving birth to boys and women diagnosed with less severe pre-eclampsia.

\section{P-454}

Weight change and subsequent sickness absence: a register-linkage prospective study

Topic: NordicEpi - Register-based epidemiology Presenter: Eira Roos

Eira Roos 1); Mikko Laaksonen 1), 2); Ossi Rahkonen 1); Eero Lahelma 1); Tea Lallukka 1), 3)

1) University of Helsinki; 2) Finnish Centre for Pensions; 3) Finnish Institute of Occupational Health

Background: Obesity is prevalent among middle aged employees and it is associated with increased risk of sickness absence. There is evidence that not only obesity but weight gain itself is detrimental to health, however the consequences of weight change on health are less known, particularly in an occupational setting.

Objectives: The aim of this study was to examine whether weight change is associated with subsequent sickness absence among middleaged women and men.

Methods: The data were derived from the Helsinki Health Study cohort. The baseline questionnaire surveys were conducted during 2000-2002 among 40-60 year old employees of the City of Helsinki, Finland $(\mathrm{n}=8960$, response rate $67 \%)$. Follow-up survey was carried out in 2007 among the respondents of the baseline survey $(\mathrm{n}=7332$, response rate $83 \%)$. Weight change was defined as the percent of weight change during the follow-up period and classified in 5 groups: weight loss (weight loss $>2 \%$ ), stable weight (weight change $\mp 2 \%)$, minor weight gain $(2.1-5 \%)$, intermediate weight gain $(5.1-10 \%)$ and major weight gain $(+10 \%)$. Self-certified short (1-3 days), medically certified intermediate (4-14 days) and long 
(+15 days) sickness absence spells were derived from employee's registers from those with written informed consent for such linkage $(\mathrm{n}=6988,78 \%)$. The associations of weight change with subsequent sickness absence spells were analysed with Poisson regression models.

Results: Women who gained weight $10 \%$ or more had persistently increased risk of short (RR 1.24, CI $95 \% 1.11-1.39$ ), intermediate (RR 1.32, CI $95 \% 1.14-1.53$ ) and long (RR 1.42, CI $95 \%$ 1.09-1.84) sickness absence spells when adjusting for age only, as compared to those whose weight remained stable. The associations were not affected by adjustment for baseline body mass index, socioeconomic status, obesity related illnesses, health functioning, or working conditions. Minor weight gain and weight loss were unassociated with subsequent sickness absence. Among men, no statistically significant associations were found.

Conclusions: Major weight gain was associated with increased risk of sickness absence among women. More attention should be devoted to prevention of weight gain in order to promote work ability and curb sickness absence.

\section{$\mathbf{P}-455$}

28 days survival in the Norwegian Cardiovascular Disease Registry 2012

Topic: NordicEpi - Register-based epidemiology

Presenter: Rupali Akerkar

Rupali Akerkar 1); Grace M Egeland 1), 2); Jannicke Igland 1), 2); Vernar Sundvor 1); Ingvei Seliussen 1); Yngve Klakegg 1); Stein Emil Vollset 1), 2); Grethe S Tell 1), 2); Marta Ebbing 1)

1) Norwegian Institute of Public Health (NIPH), Bergen, Norway; 2) Department of Global Health and Primary Health Care, University of Bergen, Norway

The number of people living with chronic diseases, such as cardiovascular disease (CVD) is increasing in Norway, and CVD is one of the major causes of death in Norway. However, the current burden of CVD is not known in Norway, given the lack of a comprehensive cardiovascular disease registry in the country up to 2012 when the Norwegian Cardiovascular Disease Registry (NCVDR) was established. The data collection started in January 2012. The registry consists of a core registry housed and maintained by the Norwegian Institute of Public Health that is supplemented with information from the medical quality registries housed and maintained by the hospital trusts.

In the core registry, all patients hospitalized for CVD (defined according to relevant ICD-10 codes) are registered in NCVDR. From the Norwegian Patient Registry (NPR), one main diagnosis and a maximum of 19 secondary diagnoses plus all medical and surgical procedure codes are recorded during each admission of a patient. By linking data from NPR with data from the Central Population Registry and the Cause of Death Registry through the unique 11 digit personal ID number, the NCVD core registry also contains information on survival among patients who have been hospitalized with CVD.

In the core registry, data from the first 8 months for year 2012 consists of information of 251,976 patients with median (mean) age of 66 (62) years of whom $52 \%$ are male. Of the total patient population $55 \%$ were hospitalized with CVD as the main diagnosis, the rest with one or several secondary diagnoses indicating CVD. NCVDR will soon receive complete data for year 2012.

The aim of the current analysis is to study major causes of morbidity and mortality among patients with different manifestations of CVD conditions leading to hospitalizations and outpatient visits. The conditions studied are ischemic heart disease (IHD), atrial fibrillation, heart failure and stroke, and the study population will be patients $>18$ years diagnosed with these conditions according to the NCVDR for year 2012.

Patients without a Norwegian unique ID number are excluded. Short term survival (28 days) following a cardiovascular event is to be presented with respect to age and gender, leading co-morbidities and region of residence. The current study will provide epidemiological knowledge that can be used for primary and secondary prevention and health care planning.

\section{P-456}

The tuberculosis incidence among immigrants in Denmark from 1992 to 2011: is it time to screen?

Topic: NordicEpi - Register-based epidemiology Presenter: Mikael Andersson

Bolette Soborg 1), 2); Mikael Andersson 3); Steen Ethelberg 1); Peter Andersen 1); Jan Wohlfahrt 3); Kaare Molbak 1)

1) Department of Infectious Disease Epidemiology, Statens Serum Institut, Copenhagen, Denmark; 2) European Programme for Intervention Epidemiology Training (EPIET), European Centre for Disease Prevention and Control, (ECDC), Stockholm, Sweden; 3) Department of Epidemiology Research

Background and objectives: More than $60 \%$ of annual Danish tuberculosis (TB) cases are diagnosed among immigrants who contribute $8 \%$ of the Danish population. $60 \%$ of Danish immigrants derive from high TB incidence countries and two of the ten largest immigrant nationalities in 2011 were countries with high rates of multi-drug resistant TB. At present, no national TB screening of immigrants exist in Denmark. In this study the risk of TB among immigrants arriving in Denmark from 1992 to 2011 was estimated to assess whether post entry TB screening should be considered.

Methods: The cohort was based on immigrants entering Denmark from 1992 to 2011 as identified in the Danish civil registration system (CRS). TB was identified by linkage with the Danish National Tuberculosis surveillance database using the unique CRS identifier. Time of entry and continent of origin were identified in the CRS and immigration status (data from 1997 to 2011) in the Danish Immigration Register, Ministry of Justice. Incidence rate ratios (RRs) were estimated by log-linear Poisson regression.

Results: 621.850 immigrants entered Denmark during the period. The median age was 23 years (Interquartile range; 19-29 years), $50 \%$ were male. A total of $2442 \mathrm{~TB}$ cases were identified. The overall TB incidence rate for the period were 607 per 100.000 person years for Sub- Saharan African immigrants and 124 per 100.000 for Asians. The incidence of TB was associated with time since entry (RR (first 2 year vs. $>14$ years in Denmark): $2.7295 \%$ confidence interval (CI) 1.76-4.21), continent of origin (RR (Sub-Saharan Africans vs. EUEuropeans): $10395 \%$ CI 77.3-139) and immigrant status (RR (asylum seekers vs. EU-applicants): $2.2495 \%$ CI 1.50-3.34).

Conclusions: The study documents a high TB incidence among immigrants from high endemic countries. Implementing TB post entry screening could accelerate a decline in TB incidence and special attention should be given to asylum seeks and immigrants from high TB endemic countries.

\section{P-457}

Indicators of fetal growth measured at birth in children born by parents with rheumatoid arthritis

Topic: NordicEpi - Register-based epidemiology

Presenter: Ane Lilleøre Rom 
Ane Lilleøre Rom 1); Hanne Kjaergaard 1); Lina Steinrud Moerch 1); Jørn Olsen 2); Chun Sen Wu 2); Damini Jawaheer 3); Merete Lund Hetland 4); Mogens Vestergaard 5)

1) The Research Unit Women's and Children's Health, The Juliane Marie Centre, Copenhagen University Hospital Rigshospitalet, Denmark; 2) The Department of Epidemiology, Department of Public Health, University of Aarhus, Aarhus, Denmark; 3) The Children's Hospital Oakland Research Institute, Oakland, CA, USA; 4) The Copenhagen Center for Arthritis Research, Glostrup Hospital, Glostrup, Denmark; 5) The Research Unit for General Practice, Department of Public Health, University of Aarhus, Aarhus, Denmark

Background: Previous studies have shown an association between rheumatoid arthritis (RA) and a range of adverse pregnancy outcome e.g. reduced birth weight. The majority of these studies addressed low birth weight or small for gestational age. Low weight or short length at birth may indicate poor fetal growth during pregnancy, which by some is believed to be a mediating factor for impaired health later in life. Small abdominal circumference has been associated with impaired liver growth, and small head circumference with reduced brain size, lower IQ, high blood pressure, higher risk of cardiovascular disease, and impaired glucose tolerance. These indicators of fetal growth have not yet been investigated in the context of mothers with RA specifically. We hypothesized that RA could reduce fetal growth through changes in the intrauterine environment induced by the disease, through genetic factors or through unmeasured confounding. In an effort to disentangle these options, we included children born by mothers diagnosed with RA after birth and children of fathers diagnosed with RA prior to birth.

Objective: To assess whether maternal and paternal RA correlate with indicators of fetal growth measured at birth among offspring. Methods: We conducted a population-based cohort study of 1917 723 singletons born in Denmark from 1977 to 2008. We used general linear models to estimate the associations between parental RA and indicators of fetal growth measured at birth.

Results: From 1977 to 2008, $2101(0.11 \%)$ children were born by mothers diagnosed with RA prior to birth. The exposed children had lower birth weight (mean difference $-87.04 \mathrm{~g}$; $95 \%$ CI -111.23 ; -62.84 ), birth length (mean difference $-0.38 \mathrm{~cm}$; $95 \%$ CI -0.49 ; -0.26), placenta weight $(-13.45 \mathrm{~g} ; 95 \% \mathrm{CI}-21.46 ;-5.43)$ and a slightly lower head circumference (mean difference $-0.13 \mathrm{~cm} ; 95 \%$ CI - 0.23 ; - 0.03), abdominal circumference (mean difference $-0.20 \mathrm{~cm} ; 95 \% \mathrm{CI}-0.32 ;-0.07$ ), and Ponderal Index (mean difference $\left.-0.014 \mathrm{~g} / \mathrm{cm}^{3} ; 95 \% \mathrm{CI}-0.026 ;-0.002\right)$. Even children born by mothers, who were diagnosed with RA after birth $(n=11455)$ had lower mean differences in birth weight and birth length and a slightly lower mean difference in head circumference and abdominal circumference. Paternal RA was not associated with any statistically significant reduction in indicators of fetal growth measured at birth.

Conclusions: This study showed that maternal RA was associated with decreased indicators of fetal growth measured at birth and that even preclinical RA seems associated with reduced indicators of fetal growth.

\section{P-458}

Thirty-six years of celiac disease surveillance: an update on recent changes in the childhood incidence in Sweden

Topic: NordicEpi - Register-based epidemiology

Presenter: Fredinah Namatovu

Fredinah Namatovu 1); Olof Sandström 2); Cecilia Olsson 3); Marie Lindkvist 1); Anneli Ivarsson 1)

1) Department of Public Health and Clinical Medicine, Epidemiology and Global Health, Umeå University, Umeå, Sweden; 2) Department of Clinical Sciences, Paediatrics, Umeå University, Umeå, Sweden; 3) Department of Food and Nutrition, Umeå University, Umeå, Sweden

Background: Worldwide, celiac disease (CD) has been considered rare, but over the last decades it has emerged as a public health problem in many parts of the world. Sweden experienced a unique CD epidemic among young children in 1985-1995, which was partly explained by changes over time in infant feeding. Despite these changes Sweden is still among those with the highest CD incidence in the world. A recent population based screening study revealed $3 \%$ prevalence among 12 -year-olds, out of which $2 / 3$ were previously undiagnosed.

Objectives: To examine the CD incidence among children in Sweden over a 36-year-period, to assess any changes over time with respect to calendar time, age groups, and birth cohorts and to estimate the clinical impact of these changes.

Methods: Using the National Swedish Childhood CD register, a cohort follow-up study was conducted among children aged $0-14.9$ years for the period of 1973-2009. Reporting was retrospective for cases diagnosed in 1973-1990 and prospective for 1991-2009. All cases had diagnosis confirmed through a small intestinal biopsy (current gold standard for diagnosis). We estimated the annual incidence rates for different age groups and cumulative incidence for different birth cohorts. Also we estimated the clinical impact of these changes.

Results: A total of 9107 cases were identified during 1973-2009. Comparing the periods of 1973-1984, 1985-1997, 1998-2009 the average incidence rates in the age group of $0-14.9$ years were 9.6, 30.7, and 36.4 per 100000 person-years. Over time there has been an upward shift in median age of diagnosis from 1.0 year (interquartile range [IQR]; 0.8-1.5 years), to 1.5 years (IQR; 0.9-2.6 years), and finally 6.2 years (IQR; $2.5-10.4$ years). Over the same time periods the number of cases per year has increased from 146, to 465 , and to 548 during the last period.

Conclusion: The CD epidemic in Sweden among children 0-1.9 years of age, without comparison in any other part of the world, has abated and also thereafter CD has become even rarer in the youngest children. During the same period the disease has become increasingly diagnosed in older children (2-14.9 years). Overall, the increased CD incidence has both clinical and public health implications. Intensified research is needed on reasons for these changes in that preventive strategies may be developed and disseminated.

\section{P-459}

Maternal folic acid and multivitamin supplementation and risk of neural tube defects: a population-based registry study

Topic: NordicEpi - Register-based epidemiology Presenter: Trude Gildestad

Trude Gildestad 1); Tone I Nordtveit 3); Roy M Nilsen 4); Anne Kjersti Daltveit 1); Kari Klungsøyr 2); Nina Øyen 1); Stein Emil Vollset 1)

1) Department of Global Public Health an Primary Care; 2) Medical Birth Registry of Norway, Norwegian Institute of Public Health, Bergen, Norway; 3) Centre for Medical Genetics and Molecular Medicine, Haukeland University Hospital, Bergen, Norway; 4) Centre for Clinical Research, Haukeland University Hospital, Bergen, Norway

Background: Several countries worldwide have implemented food fortification with folic acid to prevent neural tube defects. This health promotion strategy targets all fertile women and contributes to remove inequalities in maternal nutritional status. There are on-going debates about the benefits and risks of this strategy. Norway has no 
fortification with folic acid in flour, however, women are advised to take folic acid supplements prior to conception and in the beginning of their pregnancy to reduce neural tube defects.

Objectives: The authors investigated the association between preconceptional use of folic acid and multivitamin supplementation on the risk of neural tube defects in non-terminated pregnancies in Norway.

Methods: The study was based on data from 713,502 non-terminated pregnancies recorded in the population based medical birth registry of Norway during 1999-2010. Odds ratios adjusted by maternal age, marital status, maternal education level, parity, pregestational diabetes and epilepsy were calculated by logistic regression analyses.

Results: From 1999 to 2010, use of folic acid supplements before pregnancy was reported for $17.5 \%$ of the women, while use of multivitamin supplements before pregnancy was reported by $12.7 \%$. During the study period, there was an increase in reported use of folic acid and multivitamin supplements both before and during pregnancy. The increase in supplement use was much higher during pregnancy than before conception. Supplement use were generally more frequent among older women, mothers who had lower parity, who were married or cohabiting, women with a higher educational level and women who did not smoke at the time of their pregnancy. Mothers who used multivitamin supplements alone or in combination with folic acid before pregnancy, had a statistically significant risk reduction of having a child with neural tube defects compared with non-users (adjusted odds ratio $=0.62,95 \%$ confidence interval: $0.39,0.98)$. Further, women who used folic acid supplements before pregnancy had an adjusted odds ratio of 0.74 (95\% confidence interval: $0.51,1.09$ ), relative to supplement non-users.

Conclusion: In conclusion, the authors found that preconceptional use of multivitamins showed a statistically significant reduction on the occurrence of neural tube defects in this population based study of non-terminated pregnancies. Use of preconceptional folic acid was associated with a non-significant reduction in neural tube defects.

\section{$\mathbf{P}-460$}

\section{A SAS macro for stratifying follow-up data by time-varying covariates}

Topic: NordicEpi - Register-based epidemiology

Presenter: Klaus Rostgaard

\section{Klaus Rostgaard 1)}

\section{1) Statens Serum Institut, Copenhagen, Denmark}

Background: In survival analysis we often model a hazard function $\mathrm{h}$ (' $\mathrm{Z}(\mathrm{t})$ ) where $\mathrm{Z}(\mathrm{t})$ is a time-dependent vector of covariates. The standard epidemiological models for this take $\mathrm{h}($.$) to be the expo-$ nential function and fit the model using Cox regression or Poisson regression. This may most practically, and in some cases only be achieved after having stratified each record of follow-up time into several records, such that $\mathrm{Z}(\mathrm{t})$ does not vary within the subinterval of follow-up described by that record, perhaps with the exception of predictable variation with $t$ in some components of $Z(t)$ such as age and calendar period. Some components of $\mathrm{Z}(\mathrm{t})$ will require such type stratification in order to be predictable, such as time since last birth. In other situations $\mathrm{Z}(\mathrm{t})$ must be categorized to be used in Poisson regression, SIRs and SMRs.

Objectives: The stratification of individual follow-up time by timevarying variables is often the most difficult aspect of analyzing rates, potentially involving much programming. Furthermore, post-processing may be preferable, e.g. some aggregation of the stratified data. Therefore, there is a desire for a methodology and a user interface that enable fast, bug-free programming, as well as for easily readable programs and documentation. A SAS macro would contribute to this goal in a SAS environment by performing many of the tasks involved and by forcing a certain order and logic upon the whole process and program.

Methods: The SAS macro \%stratify uses input data sets as needed in specific formats representing potential time intervals at risk, outcome events, exposure events and background rates. It generates an output data set suitable for either Cox-regression, Poisson regression or SIR analysis, the latter two by default suitably aggregated. Common types of time-varying covariates can be generated and their values grouped by $\%$ stratify. $\%$ stratify can simultaneously produce similar output for different outcomes. The user interface and documentation of \%stratify closely resembles that of a SAS procedure.

Results: Users of \% stratify produce shorter, more readable programs in less time than they would have done without $\%$ stratify. To date the macro and documentation have been downloaded $1900+$ times. Feedback from a wide-spread user community attests to the usability of the software and ensures that bugs are found and fixed.

Conclusions: $\%$ stratify is a mature state-of-the-art but still evolving tool for handling some difficult and error-prone aspects of analyzing rates. It is freely available on the internet, together with a manual and a tutorial.

\section{P-461}

Socioeconomic disparities in stroke case fatality: observations from Riks-Stroke, the Swedish stroke register

Topic: NordicEpi - Register-based epidemiology

Presenter: Anita Lindmark

Anita Lindmark 1); Eva-Lotta Glader 2); Kjell Asplund 2); Bo Norrving 3); Marie Eriksson 1)

1) Department of Statistics, Umeå School of Business and Economics, Umeå University, Sweden; 2) Department of Public Health and Clinical Medicine, Umeå University, Sweden; 3) Department of Clinical Sciences, Section of Neurology, Lund University, Sweden

Background: Low socioeconomic status (low education and income level) has been found to be associated with increased stroke mortality. However, findings from previous studies on the association between socioeconomic status and case fatality (survival) after stroke have been inconsistent.

Objectives: To explore the association between socioeconomic status and survival after stroke, using Riks-Stroke, the Swedish Stroke Register with emphasis on changes in survival (in)equality with time after stroke .

Methods: All 76 hospitals in Sweden admitting acute stroke patients participate in Riks-Stroke. Riks-Stroke data on 18-74 year old patients with onset of first stroke during the years 2001-2009 were combined with data from other official Swedish registers. Case fatality was analyzed by socioeconomic status (education, income, country of birth and cohabitation) and other patient characteristics.

Results: Of the 62497 patients in the study, a total of 6094 (9.8\%) died within the first year after stroke. Low income, primary school education and living alone were independently associated with higher case fatality after the acute phase. Differences related to income and cohabitation were present already early, at 8-28 days after stroke, with the gaps expanding thereafter. The association between education and case fatality was not present until 29 days-1 year after stroke. Dissimilarities in secondary preventative medications prescribed on discharge from hospital had only a minor impact on these differences.

Conclusions: Socioeconomic status had only a limited effect on acute phase case fatality, indicating minor disparities in acute stroke 
treatment. The survival inequality, present already in the subacute phase, increased markedly over time since the stroke event. The socioeconomic differences could not be explained by differences in secondary prevention at discharge from hospital. Large socioeconomic differences in long-term survival after stroke may exist also in a country with limited income inequity.

\section{P-462}

Association between maternal age and attention-deficit/ hyperactivity disorder among children and adolescents

Topic: NordicEpi - Register-based epidemiology

Presenter: Susanne Hvolgaard Mikkelsen, Carsten Obel, Jørn Olsen, Bodil Hammer Bech

\section{Susanne Hvolgaard Mikkelsen 1); Carsten Obel 1); Jørn Olsen 2); Bodil Hammer Bech 2)}

1) The Institute of Public Health, department of general medical practice; 2) The Institute of Public Health, department of epidemiology

Background: One of the most common childhoods psychiatric disorder is attention-deficit/hyperactivity disorder [ADHD] with an estimated prevalence of $3-5 \%$ in a general population. ADHD frequently co-occurs with other emotional, developmental and learning problems such as conduct disorder, depression, anxiety and learning disabilities. More than $85 \%$ of the children have at least one comorbid condition and it has become known that 50-75\% continue to have problems into adulthood with increased risk of antisocial behaviour, substance abuse and poor educational attainment.

Twin and adoption studies suggest that genetic influences contribute substantially to its etiology, but environmental factors may also play a substantial role.

A few previous studies have reported that parents of children with ADHD are younger than other parents, but to our knowledge no studies have attempted to explore the cause of this difference.

Objectives: The aim of this study was to explore the association between maternal age and ADHD among children and adolescents in Denmark.

Methods: A study cohort of 968,080 children was identified in the Danish Medical Birth Registry as all singleton children born in the period January 1, 1991 through December 31, 2006. By using the unique personal identification number children with ADHD were identified by using a combination of the ICD-10 Hyperkinetic Disorder [HKD] diagnosis and prescription of ADHD medicine. Children with pervasive developmental disorders, including autism and mental retardation were excluded. Data on maternal age were obtained from the Danish Central Population Register.

Results: A total of 18,315 children (1.89\%) had the HKD diagnoses. The risk of ADHD in the offspring increased with decreased maternal age: $\mathrm{RR}=3.36(3.11-3.63) ; 2.15(2.03-2.28) ; 1.36(1.29-1.44)$ and $1.03(0.97-1.09)$ in the maternal age categories: $<21 ; 21-25 ; 26-30$; $31-35$, respectively when compared to the oldest category of maternal age $>36$ years. More complex analyses will be presented at the EuroEpi Congress 2013.

Conclusions: Decreasing maternal age is associated with increasing risk of ADHD in the offspring. We plan to include paternal age and to do sibling analysis, to study if this association can be accounted for by common genetic, social and environmental factors.

\section{P-463}

The use of anitmalarial drugs among patients with systemic lupus erythematosus in Denmark, A register based cohort study

Topic: NordicEpi - Register-based epidemiology

\section{Presenter: Jens Christian Nørgaard}

Jens Christian Nørgaard 1); Annette de Thurah 1), 2); Kristian Stengaard-Pedersen 1); Mette Nørgaard 3)

1) Department of Rheumatology, Aarhus University Hospital; 2) Institute of Public health \& Institute of Clinical medicine; 3) Department of Clinical Epidemiology, Aarhus University Hospital

Background: Antimalarial (AM) drugs is today considered the anchor drug in treating systemic lupus erythematosus (SLE) since these drugs have shown to reduce both SLE activity and mortality, and furthermoreare generally well tolerated. Hence, today it is recommended that AM's should be given to most SLE patients, irrespective of disease severity. However, little is known about the actual use of antimalarial drugs among Danish SLE patient.

Objectives: To describe patterns of AM treatment initiation among newly diagnosed SLE patients in Denmark in 2000-2011.

Methods: Using data from the Danish National Patient Registry (NPR) we conducted a large cohort study including all patients with a first time diagnosis of SLE. Information on AM treatment was retrieved from the Danish Register of Medicinal Product Statistics (RMPS).

Kaplan-Meier estimates were used to compute the cumulative probability of AM treatment start.

Cox regression analysis was used to compare the time to treatment between the patient groups among those patients who started AM during the follow-up time, by estimating the hazard ratios (HR) and associated $95 \%$ confidence intervals $(\mathrm{CI})$

Results: A total of 2211 newly diagnosed SLE patients were identified during the study period corresponding to an incidence of 3.4 per 100,000 person-years at risk. Of these, $50.2 \%(n=1110)$ started AM treatment. Compared to users, non-users tended to be older, were more often men, had no comorbidity, were not receiving prednisolone treatment, and diagnosed with SLE before 01-01-2005. Regarding time to AM treatment we found that women compared to men had an adjusted HR of 1.26(95\% CI 1.06-1.50). Compared to younger patients, those older than 44 had an adjusted HR of 0.88 (95\% CI 0.78-0.99). Patients diagnosed with SLE after 01-01-2005 had an adjusted HR of 1.33 (95\% CI 1.17-1.51) compared with patients diagnosed with SLE before this date. Patients with nephritis had an adjusted HR of 0.67 (95\% CI 0.5556-0.83) compared with patients with no nephritis. Both current users of prednisolone and of mycophenalate or azathioprine were more likely to start AM treatment than non-users ((adjusted HR 1.93 (95\% CI 1.68-2.23) and adjusted HR (1.28 (95\% CI 1.11-1.47)).

Conclusion: Treatment with AM's among Danish SLE patients could be optimized, especially among patients with more severe symptoms as nephritis. However, our study suggest that treatment practice in recent years has changed towards increased AM treatment.

\section{P-464}

Maternal use of SSRIs and Risk of spontaneous abortions: assessing potential biases

Topic: NordicEpi - Register-based epidemiology

Presenter: Rie Laurine Rosenthal Johansen

Rie Laurine Rosenthal Johansen 1); Laust Hvas Mortensen 1); Anne-Marie Nybo Andersen 1); Anne Vinkel Hansen 1); Katrine Strandberg-Larsen 1)

1) Section of Social Medicine, Department of Public Health, University of Copenhagen, Denmark

Background: Use of Selective Serotonin Reuptake Inhibitors (SSRIs) in pregnancy has been associated with an increased risk of spontaneous abortions. However, an uncertainty about the risk still remains. 
Objective: The objective of this study was to address how maternal mental illness, life style factors, and misclassification of exposure influence the association between use of SSRIs in pregnancy and spontaneous abortion.

Methods: We identified all women with a pregnancy-related diagnosis in either the Danish Medical Birth Registry or the Danish National Patient Register in the period between 1996 and 2010. Linkage with national registries, including the Register of Medicinal Product Statistics, and the Danish National Birth Cohort (DNBC) was made. In the DNBC information on self-reported use of SSRIs, mental illness, and health behaviors in pregnancy was available for a subset of the identified women. We examined the exchangeability of women exposed and unexposed to SSRIs in pregnancy in regard to indicators of use, and a verity of health behaviors. Techniques of probability weighting were used to standardize for age, parity, and socioeconomic factors. We assessed the agreement between redeemed prescriptions and self reported use of SSRIs in pregnancy. We further plan to conduct a multiple imputation analysis.

Results: A total of 1.010 .352 pregnancies were included in the study, and of these 97.878 pregnancies also participated in the DNBC. After standardization, women exposed and unexposed to SSRIs differed with respect to diagnosis of depression or anxiety, self reported mental illness, and self-reported overall health. Furthermore, they were not exchangeable in regard to alcohol consumed per week, body mass index, smoking, and whether the pregnancy was planned. In regard to exercise the groups were exchangeable. The proportionate positive agreement between redeemed prescriptions and self-reported information was $60.4 \%$ (95\% CI 60.16-67.78).

Conclusion: A large proportion of women who redeemed a prescription did not report use of SSRIs, and may have been misclassified. Thus, the risk of SSRIs in pregnancy may have been underestimated in register based studies. Oppositely, the risk may have been overestimates, as the analyses may have been confounded by differences in mental illness, and health behaviors in pregnancy. Latter will be further explored in the multiple imputation analysis.

\section{P-465}

Overweight and obesity in expectant parents: socio-demographic patterns and within-couple associations: a population-based, cross-sectional study.

Topic: NordicEpi - Register-based epidemiology

Presenter: Eva Eurenius

Kristina Edvardsson 1), 2); Marie Lindkvist 1), 3); Eva Eurenius 1); Ingrid Mogren 4); Rhonda Small 2); Anneli Ivarsson 1)

1) Department of Public Health and Clinical Medicine, Epidemiology and Global Health, Umeå University, Umeå, Sweden; 2) Mother and Child Health Research, La Trobe University, Melbourne, Australia; 3) Department of Statistics, Umeå University, Umeå, Sweden; 4) Department of Clinical Sciences, Obstetrics and Gynecology, Umeå University, Umeå, Sweden

Background: Overweight and obesity in pregnancy increases the risk of several adverse pregnancy outcomes. However, both mothers' and fathers' health plays an important role for long-term health outcomes in offspring. While aspects of health and lifestyle of pregnant women have been reported, the health of expectant fathers and correlations of health variables within couples have received less attention.

Objectives: This study aimed to explore the prevalence and sociodemographic patterns of overweight and obesity in Swedish expectant parents, and to assess within-couple associations.

Methods: This population-based, cross-sectional study investigated self-reported data from 4352 pregnant women and 3,949 expectant fathers, comprising 3,356 identified couples. Data were collected in antenatal care clinics between January 2008 and December 2011. Descriptive, correlation and logistic regression analyses were performed.

Results: The prevalence of overweight (BMI 25.00-29.99) and obesity (BMI $\geq 30.00$ ) was $29 \%$ among women (pre-pregnancy) and $53 \%$ of expectant fathers. In a majority of couples $(62 \%)$, at least one partner was overweight or obese. The likelihood of being overweight or obese increased relative to partner's overweight or obesity, with women and expectant fathers being more than 6 times more likely to be obese if their partners were obese, compared to if their partners had normal weight (OR 6.24, CI 4.21-9.25). A sociodemographic gradient was found in both genders in relation to education, occupation and area of residence, with higher likelihood of being obese further down the social ladder. The cumulative influence of these factors showed a sevenfold increase in likelihood for obesity for the least compared to the most privileged (OR 7.16, CI 4.12-12.44).

Conclusions: The prevalence of overweight and obesity in expectant parents was high, with a clear social gradient, and a minority of couples reported both partners with a healthy weight at the onset of pregnancy. Partner influence on health and health behaviours, and the role both mothers and fathers play in health outcomes of their offspring, underpins the need for a more holistic and gender inclusive approach to the delivery of pregnancy care and postnatal and child health services, with active measures employed to involve fathers.

\section{P-466}

Nationwide trends in in-hospital and overall hip fractures in Denmark from 1996 to 2009: a Danish cohort study

Topic: NordicEpi - Register-based epidemiology

Presenter: Terese Sara Høj Jørgensen

Terese Sara Høj Jørgensen 1); Annette Højmann 2); Gunnar Gislason 3); Christian Torp-Pedersen 4); Ellen Holm 1)

1) Department of geriatric medicine, Nykøbing-Falster Hospital, Denmark; 2) Department of geriatric medicine, Slagelse Hospital, Denmark; 3) Department of Cardiology, Gentofte Hospital, Denmark; 4) Institute of Health, Science and Technology, Aalborg University, Denmark

Background: Recent Danish studies on yearly incidence of hip fractures are based on the number of individuals with at least one hip fracture the given year, therefore the real incidence of hip fractures may be even higher than previously reported. Furthermore, due to patient safety, the fraction of hip fractures in-hospital is important, but remains unknown in Denmark.

Objectives: To investigate (1) the overall pattern of hip fractures and (2) the pattern of in-hospital hip fractures during the period 1996-2009.

Methods: The Danish National Patient Registry and the National Population Registry were used to identify both the number of hip fractures and the number of discharges from non-orthopedic departments among individuals aged 65 years or above. When multiple hip fracture admissions occurred in a period of 30 days, only the first admission was included. In-hospital hip fractures were identified when the date of the first admission in an orthopedic department was the same as the date of discharge from a non-orthopedic department. We calculated yearly incidence of hip fractures per 100,000 individuals, and the numbers of hip fractures occurring during hospital admission per 1,000 hip fractures. Multiple logistic regression model was applied to test for trend of hip fractures across years.

Results: In the period 1996-2009 the yearly incidence of hip fractures per 100,000 individuals aged 65 years or above increased from 890 to 970 overall (OR 1.00, confidence interval (CI) 1.00-1.00), 
from 420 to 590 for males (OR 1.01, CI 1.01-1.02), and from 1,220 to 1,280 for females (OR 1.00, CI 1-00-1.00). However, since a peak in year 2000, the incidence of hip fractures per 100,000 individuals aged 65 years or above has declined with 380 (OR 0.97, CI 0.96-0.97) overall, 199 (OR 0.97, CI 0.97-0.98) among males and 483 (OR 0.97, CI 0.97-0.97) among females in year 2009. In the period 1996-2009 the fraction of in-hospital hip fractures increased from 19 to 40 per 1,000 hip fractures (OR 1.04, CI 1.04-1.05). However, since the fraction of in-hospital hip fractures peaked in 2004 with 61 per 1,000 hip fractures, the fraction has declined with 19 (OR 0.92, CI $0.90-0.94)$ in year 2009.

Conclusions: In a nationwide cohort of individuals aged 65 years or above both the incidence of hip fractures and the fraction of inhospital hip fractures has increased from year 1996 to 2009

\section{P-467}

Nutritional status among Northern Swedish school children 1929-1931: a unique baseline for future multigenerational epidemiology

Topic: NordicEpi - Register-based epidemiology

Presenter: Lena Maria Nilsson

Lena Maria Nilsson 1); Magnus Domellöf 2); Mats Eliasson 3); Elisabeth Engberg 4); Lars-Göran Nilsson 5); Bethany Van Guelpen 6)

1) Public health and clinical medicine, Nutritional research, Umeå university, Sweden, Arctic Research Centre, Umeå university, Sweden; 2) Clinical science, Pediatrics, Umeå university, Sweden; 3) Public health and clinical medicine, Medicine, Umeå university, Sweden, Sunderby hospital, Luleå, Sweden; 4) Demographic Data Base, Umeå university, Sweden; 5) Department of Psychology, Stockholm University, Sweden; 6) Medical Biosciences, Pathology, Umeå University, Sweden.

Background: In 1929-1931, physicians in northernmost Sweden collected social and health information and dietary intake questionnaires from approximately 4000 school children as part of a largescale health investigation, Norrlandsundersökningen (the NU cohort). Health information included measures such as hemoglobin status and a physician's estimate of general health and nutritional status. From these data, it was concluded that the northern Swedish population had a diet very low in both quality and in quantity, and obesity was essentially nonexistent. To date, no systematic, long-time follow up of the NU participants has been done.

Objectives: As a first step toward evaluating the feasibility of a longitudinal follow up of the NU cohort, this study compared anthropometric NU measures among school children at the time of survey with present-day WHO guidelines for children.

Methods: NU questionnaires from 3999 school children 7-17 years of age were digitalized. After excluding children aged 15-17 years $(\mathrm{n}=50)$, children with missing data $(\mathrm{n}=76)$, and outliers $(\mathrm{n}=32)$, data from 3,841 school children (1,916 boys and 1,925 girls) were included in the analysis. Body height, body weight and BMI were compared with present-day WHO guidelines by the LMS model. The data presented are preliminary.

Results: In the 1930's, mean height, weight and BMI among school children 7-14 years old in northernmost Sweden were lower than present-day WHO standards, but largely within the recommended interval, i.e. LMS z-score -1.0 to 1.0. Z-scores were lowest for height in both boys and girls. In 10-year-old boys, weight and BMI were below WHO standard means [mean weight $30 \mathrm{~kg}$, SD 4, LMS $\mathrm{z}$-score $=-0.24$, mean BMI $16.1 \mathrm{~kg} / \mathrm{m}^{2}$, SD 1.3, LMS z-score $=-$ 0.34 ], whereas height approached the criterion for under height [mean
$134 \mathrm{~cm}$, SD 6, LMS z-score $=-0.98$ ]. In contrast, 10 year old girls were approximating criteria for underweight [mean $28 \mathrm{~kg}$, SD 4, LMS $\mathrm{z}$-score $=-0.78$ ], fulfilling criteria of under height [mean $134 \mathrm{~cm}$, SD 6, LMS z-score $=-1.2$ ], and had a correspondingly low BMI [mean $15.9 \mathrm{~kg} / \mathrm{m}^{2}$, SD 1.6, LMS z-score $=-0.52$ ].

Conclusion: The relatively short stature of school children in northernmost Sweden in the 1930s suggests persistent low energy intake. A longitudinal follow up of these results could contribute to the growing base of knowledge concerning the importance of early nutrition for health later in life. The variability in these anthropometric measures appears to be of sufficient magnitude to allow longtime follow up of the NU cohort, addressing health-related endpoints from both individual and multigenerational perspectives.

\section{P-468}

The higher proportion of men with psoriasis treated with biologics may be explained by more severe disease in men.

Topic: NordicEpi - Register-based epidemiology

Presenter: David Hägg

\section{David Hägg 1); Marie Eriksson 2); Anders Sundström 3);} Marcus Schmitt-Egenolf 1)

1) Dermatology and Venereology, Public Health and Clinical Medicine, Umea University, SE- -901 85 Umea, Sweden; 2) Umeå School of Business and Economics (USBE), Department of Statistics, Umea University, SE- -901 87 Umea, Sweden; 3) Centre for Pharmacoepidemiology (CPE), Karolinska Institutet, Karolinska University Hospital, T2, S- - 17176 Stockholm, Sweden

Background: Moderate to severe psoriasis, once regarded as merely a skin disease, is today seen as an inflammatory systemic disease. The sex ratio of the prevalence of psoriasis is balanced. In recent years several reports have documented that men receive more systemic or UV treatment than women, and different hypotheses were made. In PsoReg, the national registry for systemic treatment of psoriasis in Sweden, we have, like other European registries, observed a predominance of men $(59 \%)$, especially of men treated with biologics $(63 \%)$. Biologics are a relatively new group of very effective but high-priced drugs.

Objectives: The objective of this study was to analyse if women are discriminated by not having the same access to the high-priced biologics.

Design: Population based cohort study using data from a nationwide quality register of psoriasis patients.

Population: 2,294 patients with moderate to severe psoriasis receiving systemic treatment from a specialist in dermatology.

Main outcome measures: Time to initiation of biologic treatment. A multiple Cox proportional hazard's regression was performed, with time to initiating a biologic treatment as the outcome in order to assess the independent role of the patient's sex in initiating such therapy. The psoriasis severity was defined as a time-varying variable. Results: Men had more severe psoriasis than women according to the Psoriasis Area and Severity Index (PASI), regardless of age at enrolment, and throughout the study period. The analysis in the multiple Cox regression show that age, psoriasis severity and psoriasis arthropathy were relevant factors for initiating biologic therapy, whereas sex is not.

Conclusions: Although as many women as men are believed to suffer from psoriasis, men seem to be more severely affected by psoriasis. The asymmetry in allocation of biologic therapy thereby probably reflects the differing disease activity between the sexes, and is not a discrimination against women per se. 


\section{P-469}

Maternal bereavement and the risk of placental abruption: a cohort study from Denmark and Sweden

Topic: NordicEpi - Register-based epidemiology Presenter: Krisztina László

Krisztina László 1); Cande Ananth 2), 3); Anna-Karin Wikström 1), 4); Tobias Svensson 1); Jiong Li 5); Jørn Olsen 5), 6); Mogens Vestergaard 7), 8); Carsten Obel 7), 8), 9); Sven Cnattingius 1)

1) Clinical Epidemiology Unit, Department of Medicine, Karolinska University Hospital and Karolinska Institute, Stockholm, Sweden; 2) Department of Obstetrics and Gynecology, College of Physicians and Surgeons, Columbia University, New York, USA; 3) Section for Epidemiology, Department of Public Health, Aarhus University, Aarhus, Denmark; 4) Department of Epidemiology, Joseph L. Mailman School of Public Health, Columbia University, New York, USA; 5) Department of Women's and Children's Health, Uppsala University, Uppsala, Sweden; 6) Department of Epidemiology, School of Public Health, University of California, Los Angeles, California, USA; 7) Research Unit for General Practice, Department of Public Health, University of Aarhus, Aarhus, Denmark; 8) Section for General Practice, Department of Public Health, University of Aarhus, Aarhus, Denmark; 9) Research Program for Mental Child Health, Department of Public Health, University of Aarhus and Institute of Handicap and Communication, The Central Region, Aarhus, Denmark

Background: Maternal stress during pregnancy is associated with a modestly increased risk of foetal growth restriction and preeclampsia. Placental abruption, foetal growth restriction and preeclampsia, conditions that constitute the syndrome of "ischaemic placental disease" share similar pathophysiology and risk factors, suggesting a relation between stress during pregnancy and abruption risk is plausible. Nevertheless, this has not been studied much.

Objectives: We analyzed whether maternal bereavement during pregnancy, a major life event that is likely to cause stress for almost everyone, is associated with an increased risk of placental abruption. Methods: We studied singleton births in Denmark (1978-1908) and Sweden $(1973-1906)(n=5,103,272)$. In nationwide registries, we obtained data on deaths of women's close family members (older children, siblings, parents, and partners), placental abruption and potential confounders. Analyses were conducted using logistic regression.

Results: A total of 30,312 (6 per 1,000) pregnancies in the cohort had a recorded diagnosis of abruption. Among normotensive women, loss of a child was associated with a $54 \%$ increased odds of abruption (95\% confidence interval: $1.30-1.82$ )

\section{$\mathbf{P}-470$}

Is low socioeconomic position reducing the odds of male birth? A registry based study of the Danish population 1995-2008

Topic: NordicEpi - Register-based epidemiology

Presenter: Maria Morandi Thomsen

\section{Maria Morandi Thomsen 1); Mads H. Dahl 2);} Jens Peter Bonde 1)

1) Copenhagen University Hospital Bispebjerg, Department of Occupational and Environmental Medicine, Copenhagen, Denmark; 2) PensionDanmark, Copenhagen, Denmark

Background: During the last 60 years the proportion of male to female births has declined steadily in several European countries, including Denmark. Occupational, environmental and medical causes have been subjected to research to identify explanatory determinants. Socioeconomic position (SEP) is known to have a significant impact on lifestyle, chronic diseases, birth weight etc. and has been proposed to also have an impact on the birth sex ratio.

Objective: To examine, if low SEP of the mother is related to fewer male births.

Methods: Data was compiled from six national registries. All singleton births $(\mathrm{N}=875,435)$ in Denmark in the period 1st January 1995 to 31 st December 2008, their mother and their legal father were included in the study population.

Results: We observed a significantly lower proportion of male births among mothers with the lowest SEP (OR $=0.960,0.932-0.98995 \%$ CI) compared to mothers with the highest SEP, with an overall $p$ value $=0.0079$ of the trend. For the Danish population this results in a total loss of ten males for each thousand births across the five socio economic classes.

Conclusion: The mother's SEP is associated with lower ratio of male birth, indicating that material or social conditions may influence the secondary sex ratio. The association was weak and only detectable because of the large study population.

\section{P-471}

Maternal pre-pregnancy BMI and offspring ADHD: a population based cohort study using a sibling-comparison design

Topic: NordicEpi - Register-based epidemiology Presenter: Qi Chen

Qi Chen 1); Arvid Sjölander 1); Niklas Långström 1); Alina Rodriguez 2), 3), 4); Eva Serlachius 5); Brian M. D'Onofrio 6); Paul Lichtenstein 1); Henrik Larsson 1)

1) Department of Medical Epidemiology and Biostatistics, Karolinska Institutet, Stockholm, Sweden; 2) Department of Social Sciences Psychology, Mid Sweden University, Sweden; 3) Department of Epidemiology and Biostatistics, Imperial College London, London, United Kingdom; 4) Medical Research Council Social Genetic Developmental Psychiatry Centre, King's College London, London, United Kingdom; 5) Department of Clinical Neuroscience, Karolinska Institutet, Stockholm, Sweden; 6) Department of Psychological and Brain Sciences, Indiana University, Bloomington, IN, USA

Background: High maternal pre-pregnancy body mass index (BMI) is associated with increased risk of offspring Attention-Deficit/ Hyperactivity Disorder (ADHD). However, the role of unmeasured familial confounding for this association remains unclear.

Objectives: To investigate the association between maternal prepregnancy BMI and offspring $\mathrm{ADHD}$, and to examine whether the association can be affected by unmeasured familial confounding.

Methods: We conducted a population based cohort study via linkage of Swedish national and regional registers to investigate maternal prepregnancy BMI (underweight: $\mathrm{BMI}<18.5$; overweight: $25 \leq \mathrm{BMI}$ $<30$; obesity: BMI $\geq 30$ ) in relation to offspring ADHD in 673632 individuals born in Sweden between 1992 and 2000, with prospectively collected information on maternal pre-pregnancy BMI and offspring ADHD diagnosis. Hazard Ratios (HRs) were estimated by using Cox proportional hazards models. Stratified Cox models were applied to data on full siblings to control for unmeasured familial confounding.

Results: At the population level, high pre-pregnancy BMI was associated with increased risk of offspring ADHD (HRoverweight, 1.23 ; $95 \%$ CI 1.18-1.27; HRobesity $1.64 ; 95 \%$ CI 1.57-1.73), after adjustment for measured covariates. When comparing differentially 
exposed full siblings, however, the observed associations were no longer significant (HRoverweight, 0.98; $95 \%$ CI 0.83-1.16; HRobesity 1.15 ; $95 \%$ CI $0.85-1.56$ ).

Conclusions: The results suggested that the association between high maternal pre-pregnancy BMI and offspring ADHD can partly be ascribed to unmeasured familial confounding.

\section{P-473}

Academic performance of opposite-sex versus same-sex twins in adolescence: a Danish national cohort study

Topic: NordicEpi - Register-based epidemiology Presenter: Linda Juel Ahrenfeldt

Linda Juel Ahrenfeldt 1); Inge Petersen 1); Kaare Christensen 1)

1) The Danish Twin Registry, Epidemiology, Institute of Public Health, University of Southern Denmark, Odense, J.B. Winsløwsvej 9B, Odense 5000, Denmark

Background: Developmental differences between opposite sex (OS) co-twin and same sex (SS) co-twin could be expected for two reasons. Firstly, studies have demonstrated prenatal hormonal interaction between opposite sexed animal fetuses and in humans evidence of higher testosterone levels among OS compared to SS female twins. Secondly, OS and SS twins may have different psycho-social environment based on the sex of their co-twin. We hypothesized that OS females may approach a more boyish pattern and vice versa for OS males.

Objectives: To compare OS and SS twins as well as singletons with regard to ninth grade test scores and teacher evaluations in mathematics, physics/chemistry, Danish, and English, taking into account sex differences and gender of co-twin.

Methods: A cohort study of 5,920 twins and 13,943 singletons born 1986-1990 (age 15-16 years at test) in Denmark, based on information from four Danish databases (civil registration, demographic, labour market, and school assessments and test scores) and the Danish Twin Registry, linked through Statistics Denmark. All results were based on linear regression models adjusting for birth weight percentile, parents' age, and education.

Results: Similar performance was found for twins and singletons except from significantly higher test scores in English for singleton boys compared to twin boys (0.1-0.2 SD) and slightly higher scores of singleton than twin girls in written Danish (0.1 SD). Sex differences were dependent on topics. Significantly higher test scores in mathematics were demonstrated for boys compared to girls (0.1$0.3 \mathrm{SD}$ ), whereas girls performed significantly better in Danish, English (0.3-0.8 SD), and with regard to marks for neatness (0.7-0.8 SD). Similar performance was found between boys and girls in physics/ chemistry. Contrary to expectation, OS girls had significantly lower test scores and teacher evaluations than SS girls in mathematics and physics/chemistry $(0.2 \mathrm{SD})$, but similar performance was found in Danish, English and with regard to marks for neatness. Scores of OS versus SS twin boys were similar in all topics.

Conclusions: Overall results showed similar academic performance in adolescence for twins and singletons, whereas a negative influence of being an OS twin girl was found in mathematics and physics/ chemistry compared to SS twin girls. The performance of OS and SS twin boys were similar.

\section{P-474}

Cardiovascular disease risk and subsequent survival in multiple sclerosis: a Swedish register study

Topic: NordicEpi - Register-based epidemiology
Presenter: Homayoun Roshanisefat

Homayoun Roshanisefat 1); Scott Montgomery 2); Shahram Bahmanyar 2); Jan Hillert 1); Tomas Olsson 1)

1) Neuroimmunology Unit, Department of Clinical Neuroscience and Center for Molecular Medicine, Karolinska Institute at Karolinska University Hospital, Solna, Sweden; 2) Clinical Epidemiology Unit and Centre for Pharmacoepidemiology, Department of Medicine, Karolinska University Hospital, Karolinska Institute, Sweden;

Background: Cardiovascular disease (CVD) risk among multiple sclerosis (MS) patients is raised, possibly due to disease-related factors such as immobility and treatment. Therefore MS phenotype, describing disease severity and degree of immobility, may be relevant to CVD risk. Cautious medical management of MS patients may have inflated previously reported associations between MS and CVD among inpatients: survival following hospital admission for CVD could indicate severity of CVD associated with MS among inpatients. Objectives: To assess if there is variation in CVD risk by MS phenotype and whether mortality following hospital admission for CVD differs in those with and without MS.

Methods: To examine associations with phenotype, 8,985 patients with MS identified through the Swedish MS Register were matched with 89,101 members of the general population. The Patient Register identified 5,050 individuals with MS admitted to hospital for CVD and 44,010 without MS also admitted for CVD. Poisson regression was used to examine associations with MS after adjustment for age, sex region and socioeconomic index.

Results: MS patients had increased adjusted relative risks (with $95 \%$ confidence intervals) of CVD compared with the general population cohort of overall $1.52(1.42-1.63)$, and with some variation by phenotype: relapsing-remitting $1.50(1.28-1.76)$, secondary progressive 1.53 (1.38-1.69), and primary progressive 1.25 (1.02-1.53). The relative risk of CVD among MS patients with secondary progressive phenotype and primary progressive phenotype were 1.21 (1.01-1.45) and $1.10(0.86-1.39)$ compared with MS patients with relapsingremitting phenotype.

Among inpatients admitted for a CVD diagnosis, those with MS had higher one-month mortality, with an adjusted relative risk of 1.30 (1.23-1.38).

Conclusions: A raised risk for CVD is present in all MS phenotypes, but higher among those with more aggressive disease, consistent with a causal role for MS disease sequelae. Among individuals admitted to hospital due to CVD, the higher mortality among MS patients is consistent both with a raised CVD risk and with worse prognosis among MS patients.

\section{P-475}

\section{The prevalence of hypospadias in Denmark}

Topic: NordicEpi - Register-based epidemiology Presenter: Jan Wohlfahrt

Tine H Schnack 1); Nete Munk Nielsen 1); Charlotte Myrup 1); Jan Wohlfahrt 1); Mads Melbye 1)

1) Department of Epidemiology Research, Statens Serum Institut

Background and objectives: To analyse the register based prevalence of hypospadias in Denmark taking non-causative factors such as alterations in registration practices and recommendations for treatment of hypospadias into account.

Methods: Based on information from the Danish Hospital Discharge Register we identified 5167 Danish born males with a diagnosis of hypospadias (1980-2007). We calculated the annual cumulative risk (CR) of being registered with a diagnosis or a surgery code for 
hypospadias before the age of one month and one, six and 20 years of age, respectively.

Results: When considering all hospital contacts for males $<20$ years there appeared to be an increase in the annual CR of hypospadias during 1980-2007. CR for inpatients only, 1980-2001 however, seemed to be stable, but a steep increase in the age group 1-6 years during 2002-2003 was still present. Furthermore we found an increased $\mathrm{CR}$ of having confirmatory surgery in the period 1990-2007, again most pronounced 2002-2003. Interestingly the proportion of inpatients that also had surgery performed increased from 35 to $67 \%$ in the period 1980-2007

Conclusions: The CR of being diagnosed with hypospadias in Denmark did not change during 1980-2001. However, a steep increase was observed during 2002-2003 in the age group 1-6 years. This increase is most likely caused by changes in registration and surgical activity. Thus we found no convincing evidence for an increase in the occurrence of hypospadias in Denmark.

\section{P-476}

Short look-back periods in pharmacoepidemiologic studies of new users of asthma and antibiotic medications introduce severe misclassification

Topic: NordicEpi - Register-based epidemiology Presenter: Anders Hammerich Riis

\section{Anders Hammerich Riis 1); Martin Berg Johansen 1); Jacob Bonde Jacobsen 1); M Alan Brookhart 2); Til Stürmer 2); Henrik Støvring 3)}

1) Department of Clinical Epidemiology, Aarhus University, 8200 Aarhus N, Denmark; 2) Department of Epidemiology, University of North Carolina at Chapel Hill, NC 27599, USA; 3) School of Public Health, Biostatistics, Aarhus University, 8000 Aarhus C, Denmark

Background: The ability to separate new users from prevalent users is essential in pharmacoepidemiology. Most studies only have prescription data for a limited time period with no information on drug use before the start of the study.

Objectives: We identified two cohorts of true new users of antibiotics (AB) and asthma medication (AM), respectively, and compared them to sub-cohorts of defined new users according to various lengths of the look-back periods. We quantified the relative misclassification (RM) of drug use as a function of the length of the look-back period. Methods: We conducted a nationwide register-based cohort study of all children born in Denmark from 1995 through 2006. From the Danish Register of Medicinal Product Statistics we retrieved all prescriptions of $A B$ and $A M$ from 1995 through 2011. We used the 5 year period from 2007 through 2011 as the accrual period. Individuals redeeming their first prescription in this period were categorised as true new users. Defined new users were individuals without a prescription in the look-back period but with a prescription in the accrual period. The look-back periods ranged from 30 days up to 12 years prior to the accrual period. We estimated the RM as the number of defined new users divided by the number of true new users. We calculated the RM for each look-back period. Analyses were stratified on gender and age at start of the accrual period.

Results: We identified 763,357 children. For AM there were 69,557 $(9.1 \%)$ with a prescription during the accrual period. The RM decreased from 1.89 for a look-back period of 30 days to 1.10 for 3 year look-back. For AB we found 470,819 (61.7\%) with a prescription in the accrual period. The RM decreased from 4.75 for a look-back period of 30 days to 1.86 for 3 year look-back. At 8 years of look-back the RM was 1.07 . We found no substantial differences between girls and boys, however higher age was as expected associated with a dramatic increase in RM.

Conclusions: Studies applying the new user design are strongly dependent on the available information on prescriptions. Short lookback periods and advanced age at start of the accrual period threaten the ability and validity of separating new users from prevalent users. The misclassification is greater for drugs like $\mathrm{AB}$ that are dispensed as multiple short durations.

\section{$\mathbf{P}-477$}

The effect of oral contraceptives on the risk of venous thromboembolism is influenced by the length of run-in period

Topic: NordicEpi - Register-based epidemiology Presenter: Martin Berg Johansen

Martin Berg Johansen 1); Anders Hammerich Riis 1); Jacob Bonde Jacobsen 1); M Alan Brookhart 2); Til Stürmer 2); Henrik Støvring 3)

1) Department of Clinical Epidemiology, Aarhus University, 8200 Aarhus N, Denmark; 2) Department of Epidemiology, University of North Carolina at Chapel Hill, NC 27599, USA; 3) School of Public Health, Biostatistics, Aarhus University, 8000 Aarhus C, Denmark

Background: The new-user design is a key element in pharmacoepidemiological studies of drug safety. Misclassification of prevalent users as new users can severely bias the effect estimates and it is therefore common to introduce a look-back period to differentiate between prevalent users with a previous prescription and new users. A prominent example of this is the use of combined oral contraceptives (COCs) among women, where the risk of venous thromboembolism (VTE) is increased in the first few months after initiation of COC use.

Objectives: The aim of this study is to investigate how the estimated effect of initiating COC treatment on the risk of VTE depends on using either a true or defined new user definition with look-back periods of varying length.

Methods: We included all women resident in Denmark on their thirteenth birthday if it occurred in the period from 1 January 1995 until 31 December 2011. We excluded women with a history of any disease of the circulatory system.

We assessed recent initiation of COCs in the cohort using lookback periods ranging from 1 to 10 years. We defined recent initiation as the period from first prescription to 6 months after this date.

To assess the effect of being a recent initiator of COCs according to the different look-back periods we followed patients from their entry into the study cohort until either their first hospital admission with a diagnosis of VTE or censoring. Hence, we used the age of the women as the natural time-scale in our analyses in a Cox proportional hazards regression model.

Results: We included 523,710 women. Among these, 1,294 were diagnosed with VTE during follow-up. Defining recent initiation of COCs using a 1-year look-back period, $15.1 \%$ of these were categorized as recent initiators at the time of their VTE diagnosis. Increasing the length of the look-back period to 10 years, this proportion decreased to $10.1 \%$. Using the true new user definition, the proportion was $10.0 \%$.

The hazard ratio (HR) for developing VTE comparing recent initiators to non-users correspondingly increased from 3.6 using 1 year of look-back to 8.4 using 10 years. The HR using the true new user definition was 8.8 .

Conclusions: The estimated effect of recent initiation of COCs depends on the length of look-back period used to define recent 
initiation, and is underestimated with realistic lengths of the lookback period $(<5$ years $)$.

\section{P-478}

Prevalence and characteristics of accidents in the Icelandic population 2012

Topic: NordicEpi - Register-based epidemiology

Presenter: Edda Bjork Thordardottir

Edda Bjork Thordardottir 1), 2), 3); Sigridur Haraldsdottir 1), 2)

1) Directorate of Health, Reykjavik, Iceland; 2) Centre of Public Health Sciences, University of Iceland, Reykjavik, Iceland; 3)

Department of Psychology, University of Iceland, Reykjavik, Iceland

Injuries resulting from accidents are a major public health concern that need hightened attention. Accidents are a major cause of death and disability and are the leading cause of death in children and young adults. Greater emphasis should be placed on researching the characteristics of accidents since the majority of them are preventable. The main objective of this study is to assess the prevalence of accidents reported in Iceland in 2012. In addition, possible risk factors for accidents are assessed, including accident type (i.e. traffic, occupational, home and leisure), age and gender of the injured individual, activity when injured, geographic location and timing of accident. Data for the study is aquired through The Icelandic Accident Register. The register is a centralized databank maintained by the Directorate of Health which receives information from various institutions in the country (i.a., hospitals, health care centers, the police, Administration of Occupational Safety and Health). Currently, data cleansing is underway. Data processing and analyses will be finished in April. This study aims to provide information on what risk groups future prevention strategies in Iceland need to target.

\section{P-479}

Impact of hospitalisation on antepartum VTE using primary and secondary care data: a population based cohort study from England

Topic: NordicEpi - Register-based epidemiology Presenter: Alyshah Abdul Sultan

\section{Alyshah Abdul Sultan 1); Joe West 1); Laila Tata 1); Kate Fleming 1); Catherine Nelson-Pierct 2); Matthew Grainge 1)}

1) Division of Epidemiology and Public Health, University of Nottingham; 2) Women's Health Academic Centre, Guy's \& St Thomas' Foundation Trust, St Thomas' Hospital

Background: Hospitalisation is known to increase the risk of venous thromboembolism (VTE) around 100-fold in the general population. Objective: To address the potential for preventing venous thromboembolism (VTE) during and after antepartum hospital admissions occurring among pregnant women.

Methods: We utilized linked primary and secondary care data to analyse 245,250 pregnancies resulting in live birth or stillbirth among women aged 15-44 years between 1997 and 2010. We assessed the impact of hospitalisation by calculating the absolute rate (AR) of VTE and compared these rates to those outside hospital using a Poisson regression model in terms of the incidence rate ratio (IRR).

Findings: Overall, antepartum hospitalisation was associated with a high risk of VTE $(\mathrm{AR}=1760 / 100,000$ person-years $)$ corresponding to a 17-fold increase (IRR $=17.795 \%$ CI 7.7-39.6) compared to time outside hospital. The rate of VTE was also high during the
28 days post-discharge $(\mathrm{IRR}=5.9 ; 95 \%$ CI 3.5-10.0; $\mathrm{AR}=646$ ). The rate of VTE during hospitalisation/post-hospitalisation periods combined was highest in the third trimester (IRR $=5.7 ; 95 \% \mathrm{CI}$ 3.4-9.6; $\mathrm{AR}=998)$ and those aged $>35(\mathrm{IRR}=18.5 ; 95 \% \mathrm{CI}$ 5.69-39.4). While the absolute rate in the combined period was highest for those with 3 or more days of hospital stay (IRR $=14.5$; $95 \%$ CI 7.8-27.0; $\mathrm{AR}=1525)$, there was also a fivefold $(95 \% \mathrm{CI}$ $2.5-8.7 ; \mathrm{AR}=521$ ) increase in the risk of VTE for those admitted to hospital for less than 3 days.

Interpretation: The overall rate of antepartum VTE is substantially increased during non-delivery related hospitalisations and this increase is sustained in the 28 days post-discharge. Pharmacological thromboprophylaxis during that period may be justified particularly in women with a prolonged hospital stay.

\section{P-480}

Determinants of return to work after temporary disability retirement due to different medical reasons

Topic: NordicEpi - Register-based epidemiology

Presenter: Mikko Laaksonen

Mikko Laaksonen 1); Raija Gould 1)

1) Finnish Centre for Pensions

Background: Premature exit from working life due to disability causes significant costs to societies. In Finland, there are two forms of disability pension, a permanent disability pen-sion and a temporary disability pension (formally cash rehabilitation benefit") which can be granted if the employee's work capacity is expected to be restored through treatment or rehabilitation. Currently nearly half of all disability pensions are first granted as temporary.

Objectives: To identify prognostic factors for return to work after temporary disability re-tirement.

Methods: Data on employment and different forms of retirement were derived from the registers of the Finnish Centre for Pensions. The study included all Finnish residents whose temporary disability pension started in $2007(\mathrm{~N}=10.837)$. Competing risk regression models were applied to examine factors predicting return to work.

Results: The cumulative incidence of return to work by the end of 2011 was 27.5 percent.

Return to work was more common after temporary disability retirement due to musculoskeletal diseases (age-adjusted subhazard ratio (SHR) 2.26 (95\% confidence interval (95\% CI) 2.07-2.47)) and other physical diseases (SHR 1.65 (95\% CI 1.51-1.81)) than due to mental disorders. Return to work was more common among women, younger employees, white-collar employees, more highly educated, and the married. There were no differences between private and public sector employees or between residents of more urban and rural areas. Having a valid employment contract (SHR 2.45 (95 \% CI 2.28-2.64)) and participating in vocational rehabilitation (SHR 2.09 (95\% CI 1.91-2.29)) increased the probability of return to work.

Conclusions: Return to work was more common after temporary disability retirement due to physical diseases than due to mental disorders. Job security and active rehabilitation measures promote return to work after temporary disability retirement.

\section{P-481}

Reason for the increasing use of vacuum extraction in Sweden: a population-based study

Topic: Other - Others

Presenter: Charlotte Elvander 
Charlotte Elvander 1); Cecilia Ekéus 2); Kristina Gemzell 3); Sven Cnattingius 1)

1) Department of Medicine, Division of Clinical Epidemiology, Karolinska Institutet, Stockholm; 2) Department of Women's and Children's Health, Division of Reproductive Health, Karolinska Institutet, Stockholm; 3) Department of Women's and Children's Health, Division of Obstetrics and Gynecology, Karolinska Institutet/ Karolinska University Hospital, Stockholm, Sweden

Objective: To explain the increasing rates of vacuum extraction in Sweden.

Design: Population based register study

Setting: Nationwide study in Sweden

Population: 585904 primiparous women with singleton, term live births in 1992-2010.

Methods: Odds rations with $95 \%$ confidence intervals were estimated for potential risk factors for vacuum extraction and emergency cesarean. To explain the increase in vacuum extraction over time, we successively adjusted for maternal and infant characteristics in four different models.

Main outcome measures: Vacuum extraction

Results: Rates of vacuum extraction increased from $11.3 \%$ in 1992 to $14.4 \%$ in 2010 . The risk of vacuum extraction increased with maternal age and gestational length, but decreased with increasing maternal height. The increased use of vacuum extraction over time was partly explained by increasing maternal age and increased use of epidural anaesthesia. Both among women without and with epidural anaesthesia, the increase in vacuum extraction over time was confined to vacuum extraction due to signs of fetal distress.

Conclusions: Depending on risk factors, the odds of being delivered by vacuum extraction can vary immensely from one woman to another. Increasing maternal age explains a substantial fraction of the increase in vacuum extraction use since 1992. Whether the increase in vacuum extractions due to fetal distress reflects a true increase in fetal distress during labor remains to be explained.

\section{P-482}

The ecological association between self-harm, deprivation, social fragmentation, area type and distance to hospital: a national registry based study

Topic: NordicEpi - Register-based epidemiology Presenter: Irene O'Farrell

\section{Irene O'Farrell 1); Paul Corcoran 2); Ivan Perry 1)}

1) Department of Epidemiology and Public Health, Fourth Floor, Western Gateway Building, Western Road, Cork City; 2) National Suicide Foundation,Fourth Floor, Western Gateway Building, Western Road, Cork City

Background: Previous research has shown an inconsistent relationship between the spatial distribution of hospital treated deliberate selfharm and area level factors such as deprivation and social fragmentation. However, many of these studies have been confined to urban centres, with few focusing on rural settings and even less studies being carried out at a national level. To our knowledge, this is the first study to examine how geographical distance to the nearest hospital emergency department influences area level incidence rates of selfharm.

Objectives: This study aims to investigate the area level relationship between hospital treated deliberate self-harm, and the following latent area constructs: deprivation, social fragmentation, distance to the nearest hospital emergency department and geographical area type (rurality/urbanicity), in the Republic of Ireland.
Methods: From 2009 to 2011, the Irish National Registry of Deliberate Self Harm collected data on self-harm presentations to all hospital emergency departments in the Republic of Ireland. The Registry uses standard methods of case ascertainment and also geocodes patient addresses to small area geography level. Negative binomial regression was used to explore the relationship between area level self-harm rates and the various area level factors.

Results: From 2009 to 2011, an estimated 23652 persons aged 15-64 years presented with self-harm. The total, male and female incidence rates were 257, 245 and 268 per 100 000, respectively. Across both genders, self-harm was significantly and positively associated with deprivation and fragmentation. In particular, the most striking finding was the association between self-harm and deprivation. Self-harm rates in the most deprived areas were two fold higher than self-harm rates in the most affluent areas. In testing for effect modification by gender, it was shown that the effect of deprivation and fragmentation was more marked in males than females. Urban areas were found to have increased rates of self-harm compared to rural areas. In general, areas that had a shorter journey time to hospital had higher area level incidence rates of self-harm.

Conclusion: Deprived urban areas were shown to have the highest rates of self-harm, and this effect was more pronounced in males than females. In terms, of resource allocation for strategies aimed at tackling self-harm, priority should be given to deprived urban areas with a particular focus on males.

\section{P-483}

Risk of childhood injuries after prenatal exposure to maternal bereavement, a Danish national cohort study

Topic: NordicEpi - Register-based epidemiology Presenter: Jasveer Virk

\section{Jasveer Virk 1); Jiong Li 2); Jens Lauritsen 3); Jørn Olsen 4)}

1) UCLA; 2) Aarhus University; 3) University of Southern Denmark; 4) Aarhus University

Background: Developmental plasticity related to early life exposures leading to disease programming in offspring is a theory with substantial theoretical and empirical support. Prenatal stress exposure has been linked to neurological outcomes, such as temperament, behavioral problems, cognitive function, and affective disorders. If exposure modifies risk seeking behavior, perceived danger and reaction time, it is also expected to modify injury risk.

Objectives: The aim of this study is to assess the risk of injuries among children exposed to a stressful life exposure (defined as bereavement) before conception or during fetal life.

Design: Population based cohort study.

Setting: Denmark.

Participants: All singleton births in Denmark between January 1st 1995 and December 31st 2006 were identified. These newborns were then linked to mothers, fathers, grandparents and siblings using individually assigned civil personal registration numbers (CPR).

Primary and secondary outcome measures: We identified Data on childhood injuries were obtained from the Danish National Patient Registry, which contains data on all hospital stays and outpatient visits. Incidence rate ratios (IRRs) were estimated from birth using log-linear poisson regression models, person-years were used as the offset variable. Age, residence, calendar period, maternal education, maternal income and parental cohabition status are treated as timedependent variables (records were extracted from offspring's birth year).

Results: Exposure to maternal bereavement due to a fathers' death had the strongest association with childhood injuries, especially when 
cause of death was due to a traumatic event (aIRR: $1.25,95 \% \mathrm{CL}$ $0.99,1.58)$. We did not find an association for childhood injuries and maternal bereavement due to grandparents' death, and we only found an association for sibling death when restricting to deaths due to traumatic events (aIRR: 1.20, $95 \%$ CL 1.03,1.39).

Conclusions: The etiology of childhood injuries is complex and may be related to events that take place during prenatal life. This study suggests that exposure to a stressful life event during gestation may be linked to injury susceptibility in childhood. However, changes in postnatal family conditions related to the loss or genetic factors may also play a role

\section{P-484}

Geographic variation in the prevalence of ADHD_GIS for a better understanding of the aetiology of ADHD

Topic: NordicEpi - Register-based epidemiology

Presenter: Kathrine Bang Laursen

\section{Kathrine Bang Laursen 1); Carsten Obel 1); Marianne Simonsen} 2); Jørn Olsen 1)

1) Aarhus University, Department of Health; 2) Aarhus University, Department of Economics and Business

Background: Attention Deficit Hyperactivity Disorder (ADHD) is probably the most common childhood psychiatric disorder with an estimated prevalence of 3-5\%. In Denmark as well as in several other countries the incidence of ADHD has been increasing for the last decades. However, little is known about the aetiology or the causes that could explain the rise in ADHD. Although studies suggest a strong heritability factor genetic factors cannot explain the epidemic occurrence and only changes in interaction with environmental risk factors can explain the rise in the incidence of the disorder. Other factors such as changes in the child up growth environment as well as more attention to the problem may be alternative explanations. The Geographic Information System (GIS) can be used to show the geographic variation in the occurrence of $\mathrm{ADHD}$ and by combining different indicators of ADHD including psychometrics, medication and diagnosis we expect to detect patterns that develop our understanding of this disorder.

Objectives: We aim to explore the use of GIS in detecting geographic patterns of ADHD distribution and relating those to different layers of information. We wish to examine the hypotheses that the diagnosis of ADHD will cluster in urban regions with easy access to diagnostic facilities whereas prescriptions of ADHD medication will vary more across the country showing a higher prevalence.

Methods: Our study population include all children born in Denmark between 1 January 1980 and 31 December 2005. We combine the use of register data from the Medical Birth Registry, the National Patient Registry on ICD-10 diagnosis of Hyperkinetic disorder (HKD), the National Prescription Database on ADHD medication and psychometric data from the National Birth Cohort and www.schoolhealth.eu. Results: Preliminary results based on the ICD-10 diagnosis HKD alone suggested that there was a large geographic variation. There were 19,591 diagnosed with HKD of a total of 1,613,393 persons born in the period resulting in an overall prevalence of 12 per mille. However, the prevalence ranged from 0.4 to 40 per mille in towns of medium size in the eastern and northern part respectively. In the capital area the prevalence was higher in areas with lower social level. We will present a number of GIS maps to support the understanding of the geographical variation.

Conclusions: GIS mapping may be a promising tool in developing new causal models and may contribute to the development of specific hypotheses to be tested in other epidemiological designs.

\section{P-485}

Increase of home and leisure accidents in Iceland 2003-2011

Topic: NordicEpi - Register-based epidemiology

Presenter: Edda Bjork Thordardottir

Edda Bjork Thordardottir 1), 2), 3); Sigridur Haraldsdottir 1), 2); Thordis Katrin Thorsteinsdottir 4); Brynjolfur Mogensen 4), 5)

1) Directorate of Health, Reykjavik, Iceland; 2) Centre of Public Health Sciences, University of Iceland, Reykjavik, Iceland; 3) Department of Psychology, University of Iceland, Reykjavik, Iceland; 4) Research Institute of Emergency Care, The University Hospital of Iceland; 5) Faculty of Medicine, University of Iceland, Reykjavik, Iceland

Background: Home and leisure accidents have been the most common type of accidents since the Icelandic Accident Register was founded in 2002. In the year 2011, home and leisure accidents accounted for an alarming $51 \%$ of all registered accidents. These results are in line with previous research which indicates that these types of accidents are the most predominant cause of injury related to hospitalization and emergency care in Europe. In Iceland, population based information is available on the characteristics of this category of injuries since the Icelandic Accident Register has information on all accidental caused admissions to the Emergency Department at the University Hospital.

Objectives: The main objective of this study was to assess the prevalence of home and leisure accidents in Iceland reported at the University Hospital between 2003 and 2011. Possible risk factors for these types of accidents were also assessed, including age and gender. Methods: Data on home and leisure accidents recorded by the Emergency Department of the University Hospital during the years 2003-2011 was collected from the Icelandic Accident Register. Prevalence by year as well as age and gender was calculated and compared. Results: There is a yearly increase in the prevalence of home and leisure accidents from 2003 to 2011, from 38 to 43 accidents per 1.000 inhabitants. The prevalence of accidents fluctuated between years. The greatest increase in accidents was between 2004-2005 and 2007-2008. Accidents increased from 37 to 40 per 1.000 inhabitants in both time periods. Home and leisure accidents were more prevalent in the summer months than at other times of the year. Males had higher prevalence of accidents than women at all time periods, independent of age ( 38 vs. 42 accidents per 1.000 inhabitants on average). Children and the elderly had higher prevalence of accidents at all time periods than other age groups. Accidents in the age group 0-19 have increased from 54 to 64 per 1.000 inhabitants from 2003 to 2011. In the age group 75 years and older accidents increased from 50 to 59 cases per 1.000 inhabitants during this time period.

Conclusions: Males, children and the elderly were at higher risk for home and leisure accidents than other groups during 2003-2011 in Iceland. Prevention strategies could place special emphasis on interventions targeting these groups.

\section{P-486}

Fertility treatment and the risk of childhood and adolescent mental disorders: a register-based cohort study

Topic: NordicEpi - Register-based epidemiology

Presenter: Bjфrn Bay

Bjørn Bay 1), 2); Erik Lykke Mortensen 3); Dorte Hvidtjørn 4); Ulrik Schiøler Kesmodel 1), 2)

1) School of Public Health, Department of Epidemiology, Aarhus University, Denmark; 2) The Fertility Clinic, Dept. Of Obstetrics and 
Gynaecology, Institute of clinical medicine, Aarhus University, Denmark; 3) Institute of Public Health, University of Copenhagen, Denmark; 4) Epidemiology, Institute of Public Health, University of Southern Denmark

Background: Several follow up studies have been investigating potentially adverse effects of fertility treatment, mostly focusing on perinatal outcomes. In contrast, much fewer and smaller studies have been conducted on the long-term development, and further, the comparison of the previous studies is restricted by methodological shortcomings.

Objectives: To study whether children conceived after fertility treatment have higher, comparable or lower risk of mental disorders in childhood or adolescence compared with children born after spontaneous conception?

Methods: We compared the risk of mental disorders among 33,139 children conceived after fertility treatment and 555,828 children born after spontaneous conception when the children were 8-18 years old. We assessed information on exposure to in vitro fertilization/ intracytoplasmic sperm injection (IVF/ICSI) and ovulation induction (OI) and intrauterine insemination (IUI) and information on mental disorders through the Danish national health registers. We estimated the risk of mental disorders while adjusting for potential confounding variables. Further, we estimated the association between subtypes of procedures, hormonal treatment, gamete types and cause of infertility on the one hand and the risk of mental disorders on the other.

Results: The risk of mental disorder in children born after IVF/ICSI compared to spontaneous conceived children were not increased, except for a borderline significant increased risk of tic disorders (HR $1.4(1.0-1.9))$. In contrast, children born after OI/IUI had significantly increased risks of any mental disorder (HR 1.21 (1.11-1.31)), disorders of psychological development (HR 1.2 (1.1-1.3)) and behavioural, emotional or social disorders (HR 1.2 (1.1-1.4)).

Conclusion: We found an increased risk of mental disorders in children born after OI/IUI, while children born after IVF/ICSI were found to have overall comparable risk with children conceived spontaneously. 
Authors' Index

Aaby, Peter

Aadland, Eivind

Aakvaag, Helene

Aarestrup, Anne Kristine

Abdakimova, Gulzhan

Abdraimova, Elmira

Abhari, Sara Ahmadi

Abikulova, Akmaral

Abraira, Víctor

Abubakar, Ibrahim

Adilbekova, Bibigul

Adler, Carolin

Adolfsson, Jan

Adroher, Núria D.

Affa, Rita

Agabiti, Nera

Agerbo, Esben

Agergaard, Peter

Aguirre, Bakarne

Aguirre, Estefanía

Aguirre, Urko

Ahern, Thomas

Ahlbom, Anders

Ahmed, Bilal

Ahmed, Faryal

Ahola, Kirsi

Ahrenfeldt, Linda Juel

Ahrens, Wolfgang

Aiassa, Elisa

Aimbetova, Gulshara

Aitsi-Selmi, Amina

Akanov, Aikan

Akerkar, Rupali

Akhbardeh, Mahdi

Alarcão, Violeta

Albar, Salwa

Albertsen, Ida Ehlers

Albin, Maria

Albini, Laura

Albuquerque, Carlos

Aldwairji, Maryam

Alegrete, Nuno

Alexander, Sophie

Alexanderson, Kristina

Alm, Ragnar

Almeida, Mário

Almond, Douglas

Alonso, Jordi

Alpin, Shan

Alsaker, Elin R.

Alshehri, Ali

Álvarez-Pedrerol, Mar

Alves, Elisabete

Alves, Hélio

Alvim-Ferraz, Maria

Alwan, Nisreen

Amaral, Odete

Amireev, Saken

Andersen, Anette

Andersen, Anne-Marie Nybo

Andersen, Charlotte Brasch

Andersen, Elisabeth W.
O-019, O-025, O-076, P-426

O-095

O-037

P-412, P-417

P-440

P-387, P-428

O-005, O-006, P-020, P-048, P-111

P-387, P-428, P-440

P-203

$\mathrm{O}-026$

P-019, P-106, P-141

O-050

P-081

$\mathrm{O}-072$

O-024

$\mathrm{P}-055$

O-131, P-147, P-148, P-444

O-049

P-188, P-403, P-436

$\mathrm{P}-232$

P-067, P-069

P-077

$\mathrm{O}-042$

P-269

P-092

P-154

P-473

P-082

O-085

P-429

O-075

P-236, P-440

P-455

P-447

P-369, P-435

O-110

P-039

P-407

P-084

P-392, P-411

$\mathrm{O}-047$

P-095, P-303

P-421

O-036

P-035

P-021

O-029

O-072, P-145

O-061

P-309

P-423

P-125

P-442

P-383

P-279

O-110, P-352

P-356, P-415, P-416

P-236

P-417

O-031, P-249, P-336, P-373, P-381, P-393, P-453, P-464

P-004

P-129
Andersen, Grethe

P-100

Andersen, Ingelise

Andersen, Johan Hvid

Andersen, Jon Traerup

Andersen, Lea Kjær

Andersen, Morten

Andersen, Niels Trolle

Andersen, Per Kragh

Andersen, Peter

Andersen, Stine Linding

Anderson, Emma L

Anderssen, Sigmund Alfred

Andersson, Mikael

Andersson, Rune

Andersson, Tomas

Andreozzi, Valeska

Ankarfeldt, Mikkel Z

Anothaisintawee, Thunyarat

Antonsen, Sussie

Antunes, Luis

Anwar, Elspeth

Appleby, Louis

Appleby, Paul

Aquilina, Lucy

Arabizedah, Marjan

Aragones, Nuria

Arah, Onyebuchi

Araújo, Fábio Miguel Azevedo

Araújo, Marcelo Amorim

Araújo, Marize Barros de Souza

Arcos, Estela

Areias, Cristiana

Argyropoulos, Konstantinos

Ariansen, Inger

Armstrong, Miranda

Arrimar, Ana

Artama, Miia

Artmann, Elizabeth

Asakhin, Sergey

Ásgeirsdóttir, Tinna Laufey

Asplund, Kjell

Astrup, Arne

Astrup, Aske

Ata, Baris

Atladottir, Hjördis Osk

Auger, N

Autrup, Herman

Avery, Pascale

Ayansine, Dolapo

Aynabekova, Bayan

Azevedo, Ana

Azimi, Aziza

Baccini, Michela

Bach, Cathrine Carlsen

Bahmanyar, Shahram

Baibussinova, Aigul

Baigi, Amir

Bailey, Helen

Baisugurova, Venera

Bale, Carlito

Balkwill, Angela

Bamia, Christina

Ban, Lu

Baptista, Marco
O-078, O-087

O-125, P-212, P-339

O-135

P-117

P-446

P-005

P-176, P-249

P-456

O-133

P-308

O-095

P-151, P-456

P-236

O-042, P-182

P-240

O-111, P-174

P-071

O-013, P-075

$\mathrm{P}-090$

P-033, P-034

$\mathrm{P}-134$

P-317

P-083

P-368

P-232

P-012

P-095, P-303

P-406

P-406, P-439

P-068, P-070

P-229, P-235

P-128, P-130

O-091

O-044

P-415

P-386

P-061

O-058, P-087

P-448

P-461

O-111, P-174

P-372

O-067

P-158

P-391

P-077

P-091

O-139

P-019

P-095, P-303

O-094

P-266

P-270

P-081, P-474

P-121, P-430

P-443

P-259

P-429

P-426

O-117

O-015

O-033, O-034, P-099

P-356 
Barbaglia, Gabriela

Barber, John

Baré, Marisa

Bargagli, Anna Maria

Barreto, Sandhi

Barros, Henrique

Bartlett, Jonathan

Bartsokas, Christos

Bashir, Saghir

Basinas, Ioannis

Basso, Olga

Bastiaannet, Esther

Basto, Maria

Batty, David

Bauld, Linda

Baumeister, Sebastian

Baumert, Jens

Bayas, José M

Bayer, Tony

Bech, Bodil Hammer

Becher, Heiko

Becker, Ulrik

Behrens, Thomas

Beisbekova, A

Belda, Sofía

Bell, Ruth

Belleudi, Valeria

Ben Ali, Doha

Ben-Shlomo, Yoav

Benjamin, Emelia J

Benlarabi, Sanae

Benn, Christine S.

Benn, Marianne

Bento, Maria Jose

Beral, Valerie

Beranger, Rémi

Berentzen, NE

Berentzen, Tina L.

Berg Johansen, Martin

Berg-Beckhoff, Gabriele

Berg, Christina

Berger Håkonsen, Linn

Bertazzi, Pier Alberto

Berti de Azevedo Barros, Marilisa

Berti, Alessandra

Best, Kate

Bhaniani, Amit

Bhaskaran, Krishnan

Bhattacharya, Sohinee

Bhopal, Raj

Bibby, Bo M

Biddulph, Jane

Bidstrup, Pernille Envold

Biering, Karin

Biggeri, Annibale

Billeschou, L

Bilous, Rudy

Bjarnadottir, Gudrun Dora

Bjarnadóttir, Ragnhei ur Ingibjörg

Bjerkeset, Ottar

Bjerre Hoeyer, Birgit

Bjorvatn, Bjørn

Björck, Lena

Björckelund, Cecilia
O-072, P-145

P-362

P-067, P-069

P-055

P-289, P-290

P-095, P-303, P-348, P-366, P-383, P-389, P-390, P-441

O-007

P-128, P-130

$\mathrm{O}-085$

O-074, P-256

O-067, P-179

O-057

P-239

O-051, O-077, O-115

O-107

P-043

P-008, P-135

$\mathrm{P}-221$

P-333

O-023, O-064, P-270, P-273, P-326, P-336, P-344, P-452, P-462

O-046, O-108, P-060, P-062

O-109

P-082

P-284

P-232

P-053

P-055

P-193

P-333

P-029

P-193

O-019, O-025, O-076, P-426

P-353

P-090

O-044, O-048, O-117, P-066

P-063

P-349

P-340, P-351

P-476, P-477

P-162, P-319

P-051

P-301

P-255

P-046

$\mathrm{P}-032$

P-054, P-112

O-006

O-020, O-088, P-009, P-073, P-186

O-139

P-238

P-426

O-066

P-016

O-089, P-100, P-181

P-266

P-393

P-053

P-153

P-448

P-450

P-321

P-449

O-045, P-051, P-337

O-004
Bjørge, Tone

Björk, Jonas

Bjørke-Monsen, Anne Lise

Black, Corri

Blasco, Juan Antonio

Blasi, Alessandra

Blettner, Maria

Bliddal, Mette

Blondel, Béatrice

Bobak, Martin

Bochud, Murielle

Boeing, Heiner

Bof de Andrade, Fabiola

Boffetta, Paolo

P-006, P-079

$\mathrm{P}-407$

P-309

O-139, P-047, P-189, P-199

P-067

P-032

P-162

O-060

P-110

P-327

$\mathrm{O}-114$

P-194

P-123

O-122

P-291

P-196

P-266

P-256

P-056

Bolund, Anneli C. S.

Bond, Tom

Bonde Jacobsen, Jacob

Bonde, Jens Peter

$\mathrm{P}-476, \mathrm{P}-477$

O-125, O-128, P-172, P-246, P-247, P-248, P-249, P-250, P-263, P-286, P-294,P-321, P-338, P-470

Bonefeld-Jørgensen, Eva Cecilie

P-270, P-273

Bontempi, Katia

Bora, Elcin

Bora, Seymen

P-032, P-164

P-085

P-085

Borre, Michael

Boshuizen, HC

O-013, O-055

P-349

P-270

P-168

P-252

O-115

P-129

P-208

P-143

P-153

P-279

Bragadottir, Helena

O-023, P-344

Braun, Kim Van Naarden

Bray, Freddie

Brayne, Carol

O-010, P-063

$\mathrm{P}-305$

Breckkamp, Juergen

Breinholt, Finn

P-162

Breitbart, Eckhard W.

Brenu, Ekua

Briffa, Tom

Brink Henriksen, Tine

P-159, P-202

Briones, Eduardo

Brisson, Chantal

Brito, Maila

Brixval, Carina Sjöberg

Briz, Teodoro

Broens, Paul MA

Bromley, Bromley

Bromley, Helen

Brookhart, M Alan

Brooks, Alice S

Brown, Hilary K.

Brown, Morven

Browne, Gemma

Brunekreef, Bert

Bruno Nunes Izídio, Wesley

O-097, P-014, P-050

$\mathrm{O}-010$

$\mathrm{O}-100$

O-064

P-067

P-268

P-096

P-365

P-229, P-235

P-384

P-398

O-071, P-033, P-034

P-476, P-477

P-384

P-254

O-039

P-328

O-028, P-349

P-115

P-194

$\begin{array}{ll}\text { Buck, Katharina } & \text { O-056 } \\ \text { Buggle, Florian } & \text { O-046 }\end{array}$

Buggle, Florian

P-041 
Buribayeva, Zhanar

Burley, Victoria

Burrows, Raquel

Burstyn, Igor

Busch, Markus

Buttenschön, Henriette

Byass, Peter

Byberg, Stine

Büchele, Gisela

Byrne, Michael

Baatout, Sarah

Cable, Noriko

Caccamo, Giovanni

Cade, Janet

Cade, Nágela Valadão

Cairns, Benjamin J.

Caldeira, Luís

Calder, Nicola

Callaway, Mark

Campbell, M. Karen

Campbell, Oona

Cannegieter, Suzanne

Cantarero Arévalo, Lourdes

Capewell, Simon

Cappelen, Inger

Cappuccio, Francesco

Carandina, Luana

Cardis, Elisabeth

Cardoso, Isabel

Carla Dos Santos, Patricia

Carlsen, Hanne Krage

Carreira, Mário

Carrilho, Gisela

Carstensen, Bendix

Carter, Michelle

Carvalho, Vânia

Casas Fischer, Ricardo

Cascini, Silvia

Castro, Braian

Cavalcante Assis, Liamara

Cavalieri D'Oro, Luca

Celotti, Andrea

Cerqueira, Charlotte

Chailurkit, Laor

Chaix, Basile

Chandacham, Anchalee

Chandola, Tarani

Chang-Claude, Jenny

Chang, Zheng

Chanprasertyothin, Suwannee

Chaves, Claudia

Chen, Qi

Chini, Francesco

Chittleborough, Catherine

Chlouverakis, Gregory

Christensen, Deborah

Christensen, Jane

Christensen, Kent Lodberg

Christensen, Kaare

Christensen, Mariann

Christensen, Ulla

Christensenb, Jakob

Christiansen, Christian

Christiansen, Christian Fynbo
P-429

O-047

P-295

O-123

P-155, P-197

O-125, O-128, P-338

O-141

O-025

P-122

P-192

P-074

O-104

P-089

O-047, O-110, P-180, P-352

P-358

O-044, P-066. O-117

P-240

P-398

P-308

P-254

P-007

O-124

O-148

O-071, P-033, P-034, P-398

$\mathrm{O}-026$

P-305

P-046

P-074

P-369

P-224

P-152, P-277

P-435

P-369

O-103

O-110

P-021

P-395

P-055

P-396

P-267, P-378

P-255

P-089

O-096

P-024

$\mathrm{O}-073$

P-065

O-075

O-053, O-056, O-116

P-132

$\mathrm{P}-024$

P-356, P-416

P-471

P-164

P-257

P-078

O-023, P-344

O-041

O-128

O-016, O-119, P-363, P-473

P-077

O-113

O-032

O-126

P-166
Christiansen, Christine Benn

Christiansen, Peer

O-130

Chungkham, Holendro Singh

P-200

Ciccolallo, Laura

Cilingiroglu, Nesrin

-127

O-085

P-210

Cipière, Sébastien $\quad$ P-196

Clark, Alice

Cláudia Helena

Clavel-Chapelon, Françoise

P-316

$\mathrm{P}-173$

P-313, P-322

$\mathrm{P}-259$

Clavel, Jacqueline

Cnattingius, Sven

Cnattingus, Sven

Coelho-e-Silva, Manuel

Coelho, Ardigleusa Alves

P-081, P-312, P-448, P-451, P-481

P-452

P-291

P-439

P-231

Coelho, Catarina

Coelho, Inês

Cohen, Arieh

P-356, P-415

P-129

$\mathrm{O}-063$

Colitz, Paul

Coleman, Tim

Collerton, Joanna

P-314, P-315

$\mathrm{P}-325$

P-230

O-121

P-089

P-255

P-115

P-195

P-482

P-295

P-389

P-229

P-237

O-084

P-390

P-221

P-052, P-425

P-406

P-189

O-122

Crilly, Mike

Crispo, Anna

Cristine Pessoa, Milene

Cronin-Fenton, Deirdre

Croudace, Tim

Crowe, Francesca

Crsitine Pessoa, Milene

Crump, Casey

Cruz, Rita

Cueto, Heidi

Culliford, David

Cunha da Silva Pellense, Marcia

Cunha, Madalena

D'Onofrio, Brian M.

d'Orsi, Eleonora

da Costa Uchoa, Alice

da Costa Uchoa, Severina Alice

Dahl, Mads H.

Dahlin, Marie

Dahm, Christina C

Dakic, Marjena

Dakic, Zoran

Dalen, Håvard

Dalton, Susanne Oksbjerg

Daltveit, Anne Kjersti

Dalum, Peter

Damkier, Per

Damsgaard, Mogens Trab
P-281, P-282, P-283, P-357

O-013, P-075, P-076, P-077

$\mathrm{P}-183$

P-317

P-359

O-138

O-084

P-300

P-175

P-439

P-040, P-184, P-392

P-132, P-471

P-332

P-115

P-406, P-439

P-470

$\mathrm{O}-142$

P-037, P-340, P-351

P-228

P-228

P-450

O-041

O-132, P-459

O-105

P-076

O-070 
Danneskiold-Sørensen, Niels

Dartois, Laureen

Data, Tadesse

Dauletyarova, Marzhan

Davey Smith, George

Davidson, Melissa

Davies, Karen

Davis, Adrian

Davoli, Marina

Davoren, Martin P.

Day, Chris

Daysal, Meltem

de Araújo Torres, Lidiane

de Blaauw, Ivo

de Bruijne, Martine

de Carvalho Formiga, Maria Célia

de Craen, Ton

de Glas, Nienke

de Graaf, Ron

de Medeiros Rocha, Paulo

de Oliveira, Cesar

De Silva, N Maneka

de Thurah, Annette

De Vogli, Roberto

Deboosere, Patrick

Debost, Jean-Christophe

Philippe Goldtsche

Deepak, Desh

Delgado, Abraham

Delnord, Marie

Demakakos, Panayotes

Demarest, Stefaan

Demin, Denis

Denaxas, Spiros

Denholm, Rachel

DeRoos, AJ

Des Jarlais, Don

Dethlefsen, Claus

Dhalwani, Nafeesa

Di Lallo, Domenica

Di Lallo, Domenico

Di Martino, Mirko

Di Napoli, Anteo

Di Turi, Roberta

Dias Leão Costa, Nilma

Dias Moreira, Alexandra

Dias, Adriano

Dias, Gladys

Dias, Joana

Diderichsen, Finn

Dietz, Andreas

Díez, Consolación

Dimopoulou, Maria

Dionísio, Rui

Dockerty, John

Domellöf, Magnus

Domimgues, Marina

Donato, Francesco

Dong, Xiaojing

Dorkenoo, Efua

Dorronsoro, Miren

Dorsi, Eleonora

Dosso, Mireille

Dossus, Laure

Douglas, Ian
P-426

P-322

P-190

P-121, P-430, P-431

O-151, P-353

P-170

P-325

P-109

P-055, P-059

P-192, P-329

P-308

O-027

P-115

P-384

O-083

P-115, P-406

O-057

O-057

P-145

P-406, P-439

P-332

P-353

P-463

O-068

P-238

O-035

O-012

P-408

P-110

O-066, P-332, P-370

P-187

P-057, P-156, P-157

P-009

O-012

$\mathrm{O}-123$

P-334, P-438

P-098

P-314, P-315

O-090

P-195, P-404 $\mathrm{P}-055$

O-090, P-404

P-032

P-115

P-357, P-359

P-096, P-267, P-375, P-376, P-378

P-267, P-375, P-376, P-378

P-035

O-078, O-087

P-060

$\mathrm{P}-221$

P-130

P-416

P-259

P-467

P-424

P-084, P-089

P-421

O-101

P-188, P-403, P-436

O-066, P-017, P-370

P-241

P-016

O-088, P-073, P-186
Doyle, Pat

Drachler, Maria de Lourdes

Draper, Elizabeth

Drefahl, Sven

Dregan, Alex

Drewniak, Nicolas

Driessen, Ann

Duarte-Salles, Talita

Duarte, João

Duarte, Yeda

Duarte, Yeda Aparecida

de Oliveira

Due, Karen M

Due, Pernille

Dyachenko, A

Dörr, Marcus

Easton, Doug

Ebbesen, Finn

Ebbing, Marta

Eberlein-Gonska, Maria

Edmondson-Jones, Mark

Edvardsson, Kristina

Egeland, Grace

Egeland, Grace M

Egeland, Grace M.

Ehlers Albertsen, Ida

Ehrenstein, Ondine von

Ehrenstein, Vera

Eichelberger, Kacey

Eiríksdóttir, Védís Helga

Ekéus, Cecilia

Elholm, Grethe

Eliasen, Marie

Eliasson, Mats

Ellegaard Jørgensen, Sanne

Ellert, Ute

Ellison-Loschmann, Lis

Elvander, Charlotte

Elwood, Peter

Emenova, Yuliia

Emeny, Rebecca Thwing

Emiliando, Alan Charles Dantas

Engberg, Elisabeth

Engdahl, Johan

Engels, Charla

Engström, Gunnar

Engström, Karin

Erazo, Natalia

Erdmann, Friederike

Ergor, Gul

Erichsen, Rune

Ericson, Ulrika

Eriksen, Hanne-Lise

Eriksson, Marie

Ernst, Andreas

Ernst, Emil Hagen

Ernst, Erik

Ersbøll, Annette Kjær

Escobar, Antonio

Esnaola, Mikel

Essink-Bot, Marie-Louise

Esteves, Fernando

Ethelberg, Steen

Eurenius, Eva
O-033

P-437

P-054, P-208

$\mathrm{O}-042$

P-104

P-110

P-271

O-015

P-411

P-123

P-360, P-361

P-098

P-397, P-412, P-417

O-058

P-043

O-053

$\mathrm{P}-185$

O-043, P-028, P-030, P-031, P-455

P-094

P-261, P-262

P-465

O-043, P-028

P-455

P-030, P-146

P-002

$\mathrm{P}-273$

O-055, O-146, P-167

O-064

P-448

P-481

P-256

O-109

P-467

P-412, P-417

$\mathrm{O}-050$

P-083

P-481

P-333

P-430

P-008, P-135

P-439

P-467

O-080

O-057

P-035

P-205

$\mathrm{P}-135$

O-010, P-064

P-085

O-146, P-093, P-117

P-035, P-311

$\mathrm{O}-060$

P-461, P-468

P-298, P-301, P-374

P-263

P-250

P-397

P-027, P-067

P-125

O-083

O-084

P-456

P-465 
Eussen, Simone

Eussen, Simone J.

Evans, Charlotte

Evans, Jillian

Evans, Stephen

Evdokushkina, Galina

Ewertz, Marianne

Fagherazzi, Guy

Fairhurst, Anna

Falk Hvidbergb, Michael

Fall, Caroline

Fall, Katja

Fang, Fang

Fano, Valeria

Faraggiana, Tulio

Farbakhsh, Farzaneh

Farkas, Dóra Körmendiné

Farnoosh, Fariba

Fatmi, zafar

Fazel, Seena

Fedirko, Veronika

Fei, Chunyuan

Fenger-Grøn, Morten

Fernandes, Manuel

Fernandes, Milene

Fernandez-de-Larrea, Nerea

Ferreira Lemos, Everton

Ferreira, Magda Cecilia Cardoso

Ferreira, Manuela

Fervers, Béatrice

Fiaschi, Linda

Field, David

Filipi, Kozeta

Filogna, Marco

Filskov Overvad, Thure

Firestone, Ridvan

Fish, Mark

Fisker, Ane B.

Fitzgerald, Eimear

Fleming, Kate

Flesch-Janys, Dieter

Flinterman, Linda

Floud, Sarah

Fluck, Nick

Fonager, Kirsten

Fonseca, Luis

Fonseca, Maria João

Forastiere, Francesco

Forbes, Harriet

Forman, David

Formiga, Maria Célia

Fornander, Tommy

Forns, Joan

Forouhi, Nita

Forsberg, Bertil

Forsell, Yvonne

Fortes, Cristina

Fortnum, Heather

Franceschi, Silvia

Franco, Francesco

Fraser, Abigail

Frederiksen, Birgitte

Frederiksen, Thomas Winther

Fredrikson, Gunilla
P-307

P-309

O-110

P-199

O-038

P-023

P-077

P-313

P-368

O-097

P-056

O-144

O-144

P-032, P-164

P-059

P-218

O-129

P-218

P-269

P-144

O-015

P-273

P-029

$\mathrm{O}-025$

P-239, P-240, P-369

P-069

P-223

$\mathrm{P}-224$

P-356, P-411

P-063

O-033, O-034, P-011, P-116, P-216, P-315

P-208

P-080

P-032

P-002

P-083

P-333

O-025, O-076, P-426

P-329

P-011, P-116, P-216, P-385, P-479

O-056, O-116

0-124

O-117

P-047

P-445

P-201

P-389

$\mathrm{P}-122$

O-020, P-073

P-063

P-052, P-425

P-081

$\mathrm{P}-125$

O-006

P-272, P-277

P-205

P-059

P-261, P-262

$\mathrm{O}-122$

P-404

P-308

O-078

O-128, P-338

P-035
Freitas, Marsilene Gomes

Freitas, Paulo

Frick, Johann

Friel, Sharon

Friger, Michael

Fritschi, Lin

Frodi Olsen, Sjurdur

Frost, Gary

Frost, Lars

Frost, Poul

Frydenberg, Morten

Frøslev, Trine

Fuchs, Judith

Fuglsang-Frederiksen, Anders

Funch Lassen, Christina

Fung, Glenn

Furu, Kari

Fusco, Danilo

Føge Jensen, Per

G. Aasland, Olaf

Gaertner, Beate

Gagnon, Anita

Gagnon, Robert

Galán, Iñaki

Galanti, Maria Rosaria

Gallardo, Maria Soledad

Gammelager, Henrik

Gan, Xiaoling

Ganczak, Maria

Gansefort, Dirk

García-Basteiro, Alberto L.

Garcia-Closas, Montserrat

Garcia-Gutierrez, Susana

García, Felipe

Garcia, Lorena

Garde, Anne Helene

Garne, Jens Peter

Gasparrini, Antonio

Gasse, Christiane

Gatell, Josep M.

Gavrilova, Nataly

Gaynor, James William

Gehring, Ulrike

Gemzell, Kristina

Geoffroy, Dominique

Gerber, Jan J

Gerster, Mette

Ghaderi, Sara

Giannopoulou, Dimitra

Giatti, Luana

Gibson, Jack Edward

Gibson, John

Gildestad, Trude

Gimeno, David

Giorgis-Allemand, Lise

Gislason, Gunnar

Gíslason, Thorarinn

Gissler, Mika

Giwercman, Aleksander

Glader, Eva-Lotta

Glass, Debbie

Glinianaia, Svetlana

Glover, Vivette

Glukhareva, Natalia
P-439

P-405, P-424, P-437

O-082, P-214

O-075

P-266

P-259

P-301

O-110

P-029

O-143, P-113, P-339

O-089, O-102, P-181

O-146

P-197

P-113

O-118, P-013

$\mathrm{P}-043$

P-446

P-055

O-094

P-133

P-155, P-197

P-252, P-253, P-401

$\mathrm{O}-067$

P-346

P-205, P-312 $\mathrm{P}-027$

O-126, P-117

P-421

P-220

O-082, P-214

P-221

$\mathrm{O}-053$

P-027, P-069

P-221

$\mathrm{O}-123$

P-200, P-286

P-001, P-077

O-093

O-079, P-131, P-372

P-221

P-023

O-049

O-028

P-481

P-146

P-107

P-176

P-006

P-130

P-289, P-290

O-033, O-034

O-092

P-459

O-068

O-028

O-130, P-105, P-466

P-275, P-277

O-040, P-386, P-451, P-452

P-246

P-461

P-259

P-053

P-149

O-058, P-086, P-088, P-119 
Glümer, Charlotte

Godinho, Marta

Goi Porto Alves, Maria Cecília

Goldbaum, Moisés

Goldberg, David

Goldbohm, R Alexandra

Gollhofer, Sandra

Golmohammadi, Ali

Gomes Araújo Simões, Ana Daniela

Gomes de Oliveira, Mailza

Gomes, Teresa

Gondim da Costa, Julia

González, Judith

Gonzalez, Nerea

González, Nerea

Gorbatova, Lyubov

Gorbatova, Maria

Gordon, Sharon

Gorst-Rasmussen, Anders

Gotti, Daria

Gottliebsen, Kristian

Goulão, Beatriz

Gould, Raija

Gourzis, Philippas

Gradus, Jaimie

Graff-Iversen, Sidsel

Grahn, Patrik

Grainge, Matthew

Granath, Fredrik

Granic, Antoneta

Grau, Armin

Graversen, Lise

Gray, Michelle

Green, Jane

Greene, Naomi

Greene, Richard A

Greenwood, Darren

Greinert, Rüdiger

Grinberga, Daiga

Grjibovski, Andrej

O-058, P-010, P-019, P-036, P-057, P-086,

P-087, P-088, P-106, P-119, P-120,

P-141, P-156, P-157, P-236, P-260,

P-284, P-387, P-428, P-429, P-430,

P-431, P-432, P-434, P-440

Grond-Ginsbach, Caspar

Grove, Jakob

Gruer, Laurence

Grynderup, Matias B.

Grønbæk, Morten

Grøntved, Anders

Gualandi, Lucia

Guallar-Castillón, Pilar

Guasticchi, Gabriella

Guerra, Filipa

Guimarães de Amorim, Mário

Guiomar, Sofia

Guldbrandsen, Karen

Gullberg, Bo

Gulliford, Martin

Gulmez, Ezgi

Gunnes, Maria Winther

Gunnes, Nina

Gunter, Marc

Guo, Xin

Gustad, Lise

Gustavsson, Jaana
O-046

O-097, O-150

P-435

P-046

P-046

$\mathrm{O}-022$

P-271

P-191

P-218

P-441

P-173

P-233

P-406

P-125

P-027

P-067, P-069

P-120

P-120

P-199

P-351

P-084

O-111

P-369

P-258, P-480

P-128

P-143

O-091, O-095

P-407

P-215, P-385, P-479

P-312

P-325

O-046

O-003

P-083

O-044, O-048, O-117

O-118, O-149, P-013

O-131

O-047, O-110, P-352

O-010

P-382

Hashimoto, Shuji

Hatch, Elizabeth

Hatzis, Christos

Haug, Kjell

Haugen, Margaretha

Haukka, Eija

Hauptmann, Michael

O-107

O-074, O-125, P-172, P-286

O-109, P-399

P-381

P-032

P-346

O-090, P-404

P-369

P-115

P-369

P-374

P-035, P-311

P-104

P-101, P-102, P-103

P-006

P-309

P-016

P-413

P-450

P-337

Guttmann, Aline

Guy, Fagherazzi

H. Gislason, Gunnar

Hadrya, Fatine

Heli

Hall, Stephannie

Hallqvist, Johan

mi, Hinde

Hannaford, Phil

Hansen, AV

Hansen, John-B

Hansen, Johnni

Hansen, Kristina

Hao, Jiahu

apke, Ulfert

, Sigridu

Harmer, Clare

Harrington, Janas M

Harrison, Stephanie

Hart, Carole

Hawkes, Corinna

Hedblad, Bo

Heederik, Dick

Heitmann, Berit L

Heldal, Einar

Heliövaara, Markku

Hellgren, Lars I.

Hellstrand, Sophie

Helmer, Stefanie

Hemingway, Harry
Hajiebrahimi, Mohammadhossein

Halldorsson, Thorhallur Ingi

Hallsorsson, Thorhallur

Stephen

Hansen, Anne Vinkel

, Camilla Plambeck

Mette Lausten

P-196

P-322

P-033

O-094

P-193

P-081

P-209

P-098

P-199

P-446

P-263

P-182, P-316

$\mathrm{P}-250$

P-058, P-193

P-077

O-150

P-476, P-477

O-110

P-199

P-138, P-336, P-464

P-391

P-340

P-004, P-143

$\mathrm{O}-124$

P-200

P-296

P-345

$\mathrm{P}-172$

O-125, O-128, P-200, P-286

O-127

P-264

$\mathrm{P}-155$

P-478, P-485

P-153

P-323

P-328

O-086

O-107

O-037

$\mathrm{P}-226$

O-065, P-300, P-377

P-078

P-306

P-309

P-003, P-154

P-074

$\mathrm{O}-071$

Heinsbæk Thuesen, Betina

Heinz, Judith

Helin-Salmivaara, Arja

Helles Carlsen, Anders

Helligs $\emptyset$ de Leon, Emilie

Helmer, Stefanie Maria

Helms Andreasen, Anne

Henriksen, Tine Brink
P-311

P-246

O-096

O-056, O-116

$\mathrm{O}-111$

O-026

$\mathrm{P}-044$

P-003, P-154

P-037

P-340

O-031

P-035, P-302, P-311

$\mathrm{P}-238$

$\mathrm{O}-112$

O-096

P-009

O-035, O-049, O-131, P-185, P-270, P-273, P-380, P-393 
Henschke, Nicholas

Herrero Cembellin, Belen

Herrett, Emily

Hetland, Merete Lund

hidemi, Todoriki

Hill, Tom

Hillert, Jan

Hindori, Manodj

Hirunpat, Siriporn

Hjelmborg, Jacob

Hjern, Anders

Hjollund, Niels Henrik

Hjortdal, Vibeke

Hjøllund, Niels Henrik

Hohwü, Lena

Holleczek, Bernd

Hollegaard, Mads V.

Hollegaard, Mads Vilhelm

Holm, Ellen

Holme, Ingar Morten

Holmén, Anders

Holstein, Bjørn E.

Holzhausen, Martin

Hong, Hyunsuk

Hong, Young-Seoub

Hornshøj, Linda

Horsbøl, Trine Allerslev

Horváth-Puhó, Erzsébet

Hotopf, Matthew

Hougaard, David M.

Hounsgaard, Marie Louise

Howe, Laura D

Hsu, Paul

$\mathrm{Hu}$, Lina

Huang, Kun

Hubbard, Richard

Hudson, Lee

Hulme, Claire

Hultin, Hanna

Hulvej Rod, Naja

Husemoen, Lotte

Hutchinson, Sharon

Huupponen, Risto

Huysentruyt, Clément

Hvas Mortensen, Laust

Hvelplund, Carolina

Hvilsted Rasmussen, Lars

Hvolgaard Mikkelsen, Susanne

Hägg, David

Hägg, Sara

Häggström, Christel

Härkänen, Tommi

Hærvig, Katia Buch

Högberg, Ulf

Høgenhof Christensen, Line

Højmann, Annette

Høst Ramlau-Hansen, Cecilia

Høyer, Birgit Bjerre

Håkonsen, Linn Berger

Haaland, Oystein Aziansen

Haaramo, Peija

Igissenova, Alfiya

Igland, Jannicke

Ilonen, Jorma
O-046

P-395

P-009

P-457

P-241

P-325

P-474

P-347

P-071

O-017

O-134

P-198

O-049, O-126

O-089, P-100, P-181

P-251

P-062

P-148

P-185

P-105, P-466

O-095

O-080, P-443

O-070, P-365, P-397

P-197

P-225

P-225

P-426

P-005

O-055

O-037

P-129, P-148, P-185

P-294

P-308

O-137

P-421

P-264

O-033

O-008

P-180

P-139

O-113, O-123, P-316

P-025

O-022

P-044, P-209

P-271

P-373

P-151

P-002

P-462

P-468

P-045

P-079

P-258

P-138

O-141

P-321

P-105, P-466

P-298, P-301

P-247, P-248

P-294, P-298, P-374

O-132

P-449

P-387, P-428

P-028, P-030, P-031, P-455

P-354
Infante-Rivard, Claire

P-259

Ingsathit, Atiporn

Innes, Hamish

Intaraphet, Suthida

Ittermann, Till

Ivarsson, Anneli

Izekenova, Aigulsum

Izmailov, Timur

Izzo, Francesco

Jagger, Carol

Jahn, Ingeborg

Jahnen, Andreas

Jakobsen, Marianne Uhre

Jakobsson, Kristina

Janghorbani, Mohsen

Janson, Christer

Janszky, Imre

Janys, Lena

Jarvelin, Marjo-Riitta

Jawaheer, Damini

Jeffreys, Mona

Jelastopulu, Eleni

Jenab, Mazda

Jenetzky, Ekkehart

Jenkner, Carolin

Jennum, Poul

Jensen, Lone Donbæk

Jensen, Morten S

Jeppesen, Jørgen

Jeune, Bernard

Jimenez-Solem, Espen

Jogi, Rain

Joglekar, Charu

Johannesen, Ane

Johannessen, Ane

Johannsson, Magnus

Johansen, Christoffer

Johansen, Martin Berg

Johansen, Nanna B

Johansen, Rie Laurine Rosenthal

Johnsen, Birger

Johnsen, Søren Paaske

Johnson, Samantha

Johnson, Theron

Johnston, Marjorie

Johnston, Samantha

Jones, Reeanne

Jonsson, Håkan

José Segri, Neuber

Joubert, Rianda

Jradi, Hoda

Juárez, Sol

Juárez, Sol Pía

Juel, Knud

Juhl, Mette

Junqueira Xavier, Andre

Juul Nilsson, Charlotte

Järvelin, Marjo-Riitta

Jöckel, Karl-Heinz

Jørgensen, Mathias J

Jørgensen, Sanne Ellegaard

Jørgensen, Terese Sara Høj

Jørgensen, Torben

Jørn, Olsen
P-042

O-022

P-065

$\mathrm{P}-043$

$\mathrm{P}-458, \mathrm{P}-465$

P-387, P-428, P-440

$\mathrm{O}-058$

O-122

P-325

O-082, P-214

P-074

P-098, P-174, P-340, P-351

P-244, P-276

P-243

P-275

P-450

P-363

O-003

$\mathrm{P}-457$

P-083

P-128, P-130

O-015

P-384

O-108

P-316

P-339

P-380

P-025

O-119

O-135

P-275

$\mathrm{P}-056$

P-275

P-309

P-153

O-041

P-166

O-009

P-464

P-113

O-126, P-100

P-208

O-056

P-189

P-159, P-202

P-368

P-079

P-046

P-049

P-423

P-204

O-148

O-109, P-238

P-249, P-345

P-332

O-113

P-149

O-012

P-426

P-365

P-105, P-466

O-096, P-025, P-043, P-330

P-427 
K, Kristiansen

K, Overvad

Kabir, Zubir

Kadi, Mai

Kaducu, Felix Ocaka

Kaerlev, Linda

Kaestner, Robert

Kafatos, Anthony

Kaijser, Magnus

Kaikkonen, Risto

Kaila-Kangas, Leena

Kalmataeva, Zhanna

Kamper-Jørgensen, Mads

Kantomaa, Marko

Kaprio, Jaakko

Kaptoge, Stephen

Kapur, Navneet

Karelson, Kati

Karibayeva, Indira

Karppinen, Jaro

Karusisi, Noëlla

Kasamesup, Vijj

Kasatpibal, Nongyao

Kashafutdinova, Gulzhakhan

Katballe, Niels

Katechanok, Sadubporn

Katikireddi, Srinivasa

Kausto, Johanna

Kawado, Miyuki

Kaylyubaeva, Galiya

Kearney, Patricia M

Kenny, Louise c

Kenward, Mike G

Kerkhof, M

Kesminiene, Ausra

Kesmodel, Ulrik

Keszei, András

Keulemans, Yolande

Key, Timothy

Khalife, Natasha

Khan, Anzalee

Khanolkar, Amal

Khashan, Ali S

Khaw, Kay-Tee

Kiderlen, Mandy

Kilpi, Fanny

Kim, Byoung-Gwon

Kim, Yu-Mi

King, Ronja

Kirchmayer, Ursula

Kirkwood, Thomas

Kirmayer, Laurence J.

Kistorp, Caroline

Kivekäs, Teija

Kivimäki, Mika

Kiviranta, Hannu

Kjaergaard, Hanne

Kjeldgård, Linnea

Klakegg, Yngve

Klassen, Oliver

Kleiner, Andrea

Klenk, Jochen

Klungsøyr, Kari

Knight, Julia

Knip, Mikael

Knudsen, Ann Kristin
P-098

P-098

P-328

P-016

P-297

P-286

P-448

P-078, P-274

P-074

P-258

P-003, P-154

P-429

P-453

P-149

O-017

P-020

O-142

P-367

P-236

P-003

O-073

P-071

P-065, P-422

P-429

O-011

P-422

O-120

O-140

P-226

P-431

O-131, P-328

O-131

P-354

P-349

P-074

O-060

P-271

P-271

P-317

P-149

O-145

O-007

O-131

O-005, O-006, P-020, P-048, P-111, P-305

O-057

P-388

P-225

P-225, P-331

P-107

P-055

P-325

P-146

P-025

$\mathrm{P}-154$

O-051, O-077, O-115, O-127, P-209

P-263

P-457

O-036

P-455

P-191

P-122

O-121

O-043, P-306, P-459

$\mathrm{O}-053$

P-354

O-037
Koch, Susanne V.

Kocic, Biljana

P-151

Koenig, Wolfgang

Kogevinas, Manolis

Kolahdoz, Fariba

Kolstad, Henrik A.

Kolu, Päivi

Konttinen, Hanna

Koppelman, GH

Korhonen, Maarit Jaana

Korhonen, Tellervo

Korotov, Dmitri

Kosbayeva, Aliya

Koskinen, Seppo

Kouadio, Kouame

Koupil, Ilona

Koushede, Vibeke

Kouvonen, Anne

Kovacs, Francisco M.

Kowal, Bernd

Kozhakhmetova, Gulmira

Krage Carlsen, Hanne

Krasilnikov, Andrey

Krause, Tyra

Kretzschmar, Mirjam

Krewski, Daniel

Kriegbaum, Margit

Kristensen, Jette Kolding

Kristensen, Susanne Lund

Kristiansen, Ivar Sønbø

Kristiansen, Jesper

Krivonogova, Elena

Kroemer, Heyo

Kroenke, Candyce

Krogh Thrane, AM

Kroll, Lars Eric

Kroll, Mary

Kronborg, Mette Lise

Kruse, Johannes

Krølner, Rikke

Kubinova, Ruzena

Kuh, Diana

Kui-Cheng, Zheng

Kulzhanova, Sholpan

Kumar, Ainur

Kumaran, Kalyanaraman

Kumari, Meena

Kunst, Anton

Kurinczuk, Jenny

Kvalvik, Liv Grimstvedt

Kyzayeva, Aizhan

Kähönen, Mika

Kærgaard, Anette

Kærlev, Linda

Kærslund Hansen, Malene

Kääriä, Sanna

Köhler, Ole

Kaakinen, Marika

Kaaks, Rudolf

Kaatsch, Peter

La Vecchia, Carlo

Labriola, Merete

Lacerda, Adrianna Ribeiro

Ladwig, Karl-Heinz
P-414

O-059

$\mathrm{O}-028$

P-265

O-074, O-125, O-128, P-172, P-200, P-286, P-338

P-310

P-388

P-349

P-044, P-209, P-446

$\mathrm{O}-017$

O-058

P-019, P-036, P-106, P-141, P-260

P-258

P-241

O-007, P-045

P-397

O-068

P-203

P-162

P-019, P-106, P-141

$\mathrm{O}-045$

O-058, P-087, P-088, P-119

O-019

O-021

P-170

O-113

O-002, O-136, P-345

P-250, P-263, P-301

$\mathrm{P}-015$

O-128

P-057, P-156, P-157

P-043

O-123

P-393

P-060

O-048

P-015

P-008

O-106, P-412, P-417

P-327

O-081

P-241

P-434

P-387, P-428, P-440

P-056

O-115

P-238

P-054

P-306

P-440

P-245

P-172, P-286

O-125, P-172

O-126

P-154

O-079

O-003

O-056, P-194

P-064

O-122

P-212

P-439

P-008, P-135 
Lafuente, Iratxe

Lahelma, Eero

Lahesmaa-Korpinen, Anna-Maria

Lahti, Jouni

Laide Nigro, Erika

Laisaar, Kaja-Triin

Laitinen, Jaana

Lallo, Adele

Lallukka, Tea

Lambe, Mats

Lambert, Paul

Lamberts, Morten

Lamera, Rossella

Lamiraud, Karine

Landen, Mikael

Lane, Deirdre

Langan, Sinead

Lange, Theis

Langner, Ingo

Lapucci, Enrica

Lara Tontini, Naise

Larrañaga, Isabel

Larrañaga, Nerea

Larsen, Inger Kristin

Larsen, Louise Pape

Larsen, Pernille Stemann

Larsen, Torben Bjerregaard

Larsson, Henrik

Lasfargues, Aude

Lash, Timothy

Laugsand, Lars Erik

Laurberg, Peter

Lauritsen, Jens

Lauritzen, Torsten

Laursen, Kathrine Bang

Lautrup, Charlotte K

Law, Catherine

Law, Ceaser

Lawlor, Debbie

Lawson, Kate

Laxy, Michael

Layton, Bradley

Lazaro, Santiago

Le Cornet, Charlotte

Leander, Karin

Leão Miranda, Filipe

Lebedeva, Ludmila

Lebrão, Maria Lúcia

Lee, Pei-chen

Leermakers, Lynn

Lehtimäki, Terho

Lehtinen-Jacks, Susanna

Leino-Arjas, Päivi

Leitão, José

Leite, Andreia

Leite, Neiva

Leng, Yue

Lenters, Virissa

León-Muñoz, Luz M.

Leonor Noia Maciel, Ethel

Lerdsitthichai, Panuwat

Levinsson, Anna

$\mathrm{Li}$, Jiong

LI, Xinran
P-027

O-069, P-335, P-454

P-386

P-335

P-046

P-438

O-003, P-149

$\mathrm{P}-272$

O-069, P-245, P-449, P-454

P-081

P-045

O-130

P-089

O-114

P-144

P-002, P-039

$\mathrm{O}-020$

O-078, O-087, P-316

P-082

O-090, P-195

P-223

P-188, P-403, P-436

P-188, P-403, P-436

O-018

P-198

O-031, P-249, P-336

P-002, P-039

P-132, P-471

P-313

O-011, O-013, O-055 P-001, P-075, P-076, P-077, P-143

P-450

O-096, O-133

P-483

O-009

P-484

O-011

O-008

P-297

P-257, P-308, P-353

P-362, P-368

O-059

O-126

P-067

P-063

P-051, P-182, P-337

P-435

P-088

P-123, P-360, P-361

$\mathrm{O}-030$

P-347

P-245

P-354

P-003, P-154

P-201

P-239, P-240, P-364

P-291

P-305

P-246

P-346

P-233

P-071

O-045

O-136, O-147, P-251, P-444, P-451,

P-452, P-483

P-196
Lichtenstein, Paul

Lie, Rolv Terje

Liefers, Gerrit-Jan

Liew, Zeyan

Lightfoot, Tracy

Limina, Rosa Maria

Lind, Caroline

Lindahl-Jacobsen, Rune

Lindbohm, Marja-Liisa

Lindkvist, Marie

Lindmark, Anita

Lindström, Martin

Lindtjørn, Bernt

Linkohr, Birgit

Linneberg, Allan

Linseisen, Jakob

Lip, Gregory

Lissner, Lauren

Liu, Henghui

Liu, Simin

Liu, Zheng

LLopis-Gonzalez, Agustin

Llorca, Javier

Lloyd-Williams, Ffion

Llupià, Anna

Lopes, Ana Patrícia

Lopes, Carla

Lopes, Elisa

López-Vicente, Monica

López, Fanny

Lorenz, Eva

Lortet-Tieulent, Joannie

Lourenço Cassemiro, Lucélia

Lourenço, Sara

Loures Mendes, Larissa

Lowbrigde, Pam

Lubbe, Martie

Lubbe, Martie S

Luben, Robert

Lubree, Himangi

Lucas, Raquel

Lucena Pereira, Ivoneide

Ludvigsson, Jonas

Luiz Galvão Cesar, Chester

Luiz Oliveira Alves, Ricardo

Lukaschek, Karoline

Lund Kristensen, Soeren

Lund Vinding, Anker

Lund, Rikke

Lund, Søren Peter

Lundberg, Michael

Lundin, Andreas

Lundqvist, Marika

Luoto, Riitta

Luukkonen, Ritva

Luund, Søren Peter

Lydiana, Lynna

Lynch, Christel

Lynch, John

Lyngs $\varnothing$, Julie

Laaksonen, Mikko

Långström, Niklas

Maccia, Carlo

Macfarlane, Alison

Machado-Rodrigues, Aristides
$-101$

O-142, P-132, P-144, P-471

O-132, P-006, P-371

O-057

O-030, P-273

P-064, P-259

P-089

O-124

O-119

$\mathrm{O}-145$

P-458, P-465

P-461

P-296

P-190

O-059

O-150, P-025, P-043, P-330

$\mathrm{O}-056$

P-002, P-039

O-004, P-051, P-337

$\mathrm{P}-413$

O-123, O-149

P-413

$\mathrm{P}-140$

P-408, P-409, P-410

O-071, P-033, P-034, P-398

$\mathrm{P}-221$

$\mathrm{P}-231$

P-366, P-383

P-435

P-125

O-108

O-010, P-063

$\mathrm{P}-173$

P-348

P-281, P-282, P-283, P-357, P-359

P-199

P-041, P-049

P-107

O-005, O-006, P-020, P-048, P-111, P-305

P-056

P-095, P-303, P-348, P-441

P-173

O-142

P-046

$\mathrm{P}-115$

P-008, P-135

$\mathrm{O}-130$

O-097

0-113, O-123

P-338

P-139

P-139

P-129

P-310

P-154

O-128

$\mathrm{P}-124$

P-274

P-257

P-248, P-374

P-454, P-480

P-132, P-471

P-074

P-291
P-068, P-070 
Machado, Ausenda

Machado, Helena

Machon, Monica

Mackenbach, Johan

Macnab, Jennifer

Madsen, Lise

Madsen, Mia

Madsen, Morten

Madureira Dias, António

Magliano, Dianna

Magnusson, Andres

Magnusson, Cecilia

Magtengaard Robinson, Kirstine

Mahamadou Zaki, Harouna

Mahande, Michael Johnson

Maigne, Lydia

Maisyarah, Tiara Aulia

Malairungsakul, Benjawan

Malaspina, Dolores

Malki, Ninoa

Malling, Tine

Malm, Heli

Malmqvist, Ebba

Malta, Deborah Carvalho

Malyutina, Sofia

Mamoudou Garba, Salamatou

Manabaeva, Gulshat

Mangtani, Punam

Manjer, Jonas

Manktelow, Bradley

Mann, Kay

Manongi, Rachel

Manooranparanpampil, Thomas

Mapril, José

Marchetti, Aurora

Mari-Bauset, Salvador

Maria Proença Padez, Cristina

Maria, Vasco

Marino, Claudia

Markabayeva, Akbayan

Markestad, Trond

Marks, Angharad

Marley-Zagar, Ella

Marmot, Michael

Maroto, Renata Melo

Marques-Vidal, Pedro

Marshall-Gradisnik, Sonya

Martikainen, Pekka

Martiniano, Claudia Santos

Martino, Laura

Martins, Ana Paula

Martins, Cesario

Martins, Fernando

Martinussen, Torben

Martus, Peter

Mascarenhas, Luis

Masenga, Gileard

Maske, Ulrike

Masse, Benoit

Masters Pedersen, Jolene

Mastroeni, Simona

Mastroiacovo, Pierpaolo

Mathers, John

Matias Dias, Carlos

Matthiesen, Niels
P-364

P-442

P-188, P-403, P-436

P-238

P-254

P-098

P-176

P-167

P-040, P-392

O-100

P-152, P-153

P-139, P-312

O-097

P-058

O-132

P-196

P-124

P-422

O-145

P-045

P-004

P-386

P-244

P-281, P-282, P-283, P-289,

P-290, P-357, P-359

P-327

P-058

P-431

O-026

P-079

P-208

O-039, O-086, O-092, P-026, P-109

$\mathrm{O}-132$

P-059

P-435

O-090

P-140

P-281

P-240

P-059

P-432, P-434

P-371

P-047, P-189

P-056

O-066, O-075, O-115, P-332, P-370

P-406

$\mathrm{O}-114$

P-159, P-202

P-388

P-439

O-085

P-240

$\mathrm{O}-025$

P-279

P-025

P-197

P-291

O-132

P-155

P-268

O-123

P-059

P-309

P-325

P-364

O-049
Mattison, Donald

Maughan, Barbara

Mauramo, Elina

Mayer, Flavia

Mazhari, Tuba

Mazumder, Bhashkar

McAlaney, John

McCormack, Abby

McCormack, Valerie

McDaniel, D Olga

McGill, Rory

McGivern, Mark

McGrath, John

McGue, Matt

McKenzie, Fiona

Mcmunn, Anne

McNair, Douglas

Mega, Cristina

Mehlig, Kirsten

Mehta, Praem

Meilstrup, Charlotte

Meimanaliev, Tlekbek

Meisinger, Christa

Meister, Kadri

Melbye, Mads

Meltzer, Helle Margrete

Memon, Anjum

Mena, Guillermo

Mendonça, Denisa

Menne, Bettina

Merikukka, Marko

Merlo, Juan

Merry, Lisa

Mesquita, João Rodrigo

Messerlian, Carmen

Mester, Birte

Mette Gitz Charlot, Mette

Miao Jonasson, Junmei

Micali, Nadia

Miceli, Maria

Michael, Yvonne

Michelozzi, Paola

Midttun, Øivind

Mikkelsen, Ellen

Mikkelsen, Martin

Mikkelsen, Sigurd

Mikolajczyk, Rafael

Milidou, Ioanna

Miligi, Lucia

Miller, Jessica

Miller, Martin R.

Milne, Roger

Milot, Alain

Minassian, Caroline

Miranda, Helena

Mitchell, Richard

Mitie Nakagawa, Cynthia

Mittinty, Murthy

Mmbaga, Blandina Theophil

Modig, Karin

Modig, Lars

Moeller, Anne Marie

Moerch, Lina Steinrud

Mogensen, Brynjolfur

Mogren, Ingrid

Mohangoo, Ashna
P-170

O-081

O-069

P-055

P-342

O-029

$\mathrm{O}-112$

P-261, P-262

$\mathrm{O}-054$

P-213

P-033, P-034, P-398

P-112

P-147, P-148

O-119

P-083

P-342

P-170

O-084

O-004, P-051, P-337

P-253

O-070, P-397

P-440

P-008, P-135

P-277

P-129, P-475

P-309

P-091, P-323, P-362, P-368

P-221

P-090, P-178

P-019, P-036, P-106, P-141, P-260

$\mathrm{O}-040$

O-148, P-177, P-204, P-206, P-207

P-252, P-253, P-401

P-230, P-231

O-067

P-082

O-094

O-004

O-031

P-032

$\mathrm{O}-123$

P-059, P-272

P-307

O-011, O-065, P-300, P-377

O-126

O-125, P-172, P-286

O-021, O-112, P-238

P-380

P-259

O-023, P-343, P-344

P-256

$\mathrm{O}-053$

P-268

P-007

P-003, P-154

$\mathrm{O}-120$

$\mathrm{P}-223$

P-257

O-132

O-042

O-045

P-319

P-457

P-485

P-465

O-099, P-110, P-211, P-347 
Mohebati, Lisa

Mohsin, M.Nadeem

Mohsin, M.Naeem

Molbak, Kaare

Molfino, Sarah

Momen, Natalie

Moneni, Fatemeh

Monjardino, Teresa

Monteiro Fernandes, Kallil

Monteiro Santini, Ana Carolina

Monteiro, Ana

Monteiro, Maria João

Montella, Maurizio

Montenegro, Luís

Montgomery, Scott

Montomoli, Jonathan

Moonan, May

Moore, David R

Mor, Anil

Moradinazar, Mehdi

Morales-Suárez-Varela, Maria M.

Moreira, Isabel

Mors, Ole

Mortensen, Jens Tølbøll

Mortensen, Laust Hvas

Mortensen, Lotte Maxild

Mortensen, Marie

Mortensen, Preben Bo

Moschandreas, Joanna

Moster, Dag

Mott, Susan

Muangnart, Thanisara

Mucci, Lorelei

Muñoz Simon, Carmen

Murakami, Yoshitaka

Muriel, Alfonso

Musa, Sanjin

Mussaeva, Bakhit

Mykletun, Arnstein

Myrup, Charlotte

Møller, Bjørn

Møller, Henrik

Möller, Jette

Månsdotter, Anna

Nagai, Masaki

Nagel, Gabriele

Najafi, Farid

Nakata, Tomoo

Namatovu, Fredinah

Natale, Renato

Nazareth, Irwin

Nedkoff, Lee

Nelas, Paula

Nelson-Piercy, Catherine

Neuhauser, Hannelore

Nevalainen, Jaakko

$\mathrm{Ng}$ Fat, Linda

Ngamsakulrat, Sukanya

Nguipdop Djomo, Patrick

Nguyen-Nielsen, Mary

Nickels, Stefan

Nicod, Edouard

Nicola, Paulo

Nicola, Paulo Jorge

Niedzwiedz, Claire
P-362

P-126

P-126

P-456

P-089

P-451

P-243

P-441

P-115

P-267, P-378

P-021, P-201, P-280

P-040

O-122

O-084

O-144, P-474

P-093

O-071, P-398

P-261, P-262

P-234

P-142

P-140, P-326

P-390

O-079, P-148, P-172

$\mathrm{P}-445$

P-138, P-381, P-391, P-393, P-464

P-351

P-446

O-035, O-131, O-143, P-134, P-148

P-274

O-064, P-006, P-371

P-026

P-422

O-017

P-395

P-226, P-292

P-203

P-228

P-429

O-037

P-475

$\mathrm{P}-015$

O-052

P-205

P-205

$\mathrm{P}-226$

O-121, P-079, P-122

P-142, P-265

P-320

P-458

P-254

O-038, O-098

O-100

P-356, P-411

P-385, P-479

O-050

P-354

O-104

P-422

O-026

O-055, P-117

O-053

O-114

P-239, P-369

P-240

O-120
Nielsen, Bendt

Nielsen, Claus Vinther

Nielsen, Line

Nielsen, Nete Munk

Nielsen, Nina Odgaard

Nielsen, Rikke Bech

Nieuwenhuijsen, Mark J.

Nikitina, Svetlana

Nilsen, Roy M

Nilsen, Roy M.

Nilsson, Jan

Nilsson, Lars-Göran

Nilsson, Lena Maria

Nilsson, Tove

Nobre, Miguel

Nóbrega, Cármen

Nogueira, Paulo

Norat, Teresa

Nordahl, Helene

Nordestgaard, Børge

Nordtveit, Tone I

Norrving, Bo

Nouhou, Hassan

Nouraei, Reza

Nugraha, Gaga Irawan

Nunes, Carla

Nuntawinit, Chutatip

Nurgabylova, A

Nurgaliyeva, Nassikhat

Nurminen, Marja-Leena

Nyberg, Fredrik

Nyegaard, Mette

Nygård, Ottar

Nykjaer, Camilla

Nyström, Petra

Næss, Øyvind

$\mathrm{N} ø$ hr, Ellen Aagaard

Nørgaard-Pedersen, Bent

Nørgaard, Jens Christian

Nørgaard, Mette

O Neill, Sinead M

O'Farrell, Irene

O'Flaherty, Martin

Obel, Carsten

Obi, Nadia

Obure, Joseph

Ohta, Akiko

Ojajärvi, Anneli

Oldereid, Nan

Olesen, Frede

Olesen, Jonas Bjerring

Olin, Anna-Carin

Oliveira-Campos, Maryane

Oliveira, Andreia

Oliveira, Cesar

Oliveira, Cintya Schultz de

Oliveira, Elizabete Regina Araújo

Olsen, Jørn

O-023, O-030, O-032, O-060, O-063, O-133, O-136, O-137, O-147, O-149, P-012, P-137, P-251, P-270, P-273, P-294, P-298, P-326, P-336, P-343, P-344, P-374, P-380, P-444, P-451, P-452, P-457, P-462, P-483, P-484 
Olsen, Morten

Olsen, Sjurdur Frodi

Olsson, Ann

Olsson, Cecilia

Olsson, Lisa

Olsson, Tomas

Omland, Øyvind

Ongphiphadhanakul, Boonsong

Opler, Mark G.A

Ording, Anne

Orho-Melander, Marju

Orrel, Martin

Orrell, Martin

Orsini, Nicola

Orton, Lois

Osler, Merete

Ospanova, Dinara

Ospanova, F

Ouchchane, Lemlih

Oudin Åström, Daniel

Oudin, Anna

Overvad, Kim

Overvad, Thure Filskov

Ovesen, Lars

Ovuga, Emilio

Oyanedel, Juan Carlos

Paccaud, Fred

Pacheco, Nadja

Padez, Cristina

Pagnozzi, Eugenia

Pajak, Andrzej

Palm, Frederick

Papadaki, Angeliki

Papandreou, Christopher

Pape, Louise

Park, AL

Parker, Louise

Parker, Samantha

Pascon Barbosa, Angélica

Passarelli, Francesca

Pastbin, Mikhail

Pastbina, Irina

Patumanond, Jayanton

Paula Stolte, Vania

Paz, María

Pearce, Anna

Pearce, Mark

Pearson, Fiona

Peasey, Anne

Pecego Martins Romano, Alessandro

Pedersen, Asger

Pedersen, Carsten Bøcker

Pedersen, Erling B

Pedersen, Grete Skøtt

Pedersen, Henning S

Pedersen, Henning Sloth

Pedersen, Jacob Krabbe

Pedersen, Lars

Pedersen, Lars H.

Pedersen, Lars Henning

Pedersen, Marie

Pedersen, Michael S.

Peeters, Anna
O-147, P-014, P-050

P-250, P-263

$\mathrm{O}-012$

P-458

O-134

P-474

P-004, P-256

P-024

$\mathrm{O}-145$

P-001

P-302, P-311

O-066

P-332

P-168

O-071, P-033, P-034, P-398

O-078

O-058, P-086, P-087, P-088

P-284

P-196

P-272

P-276, P-277

O-124, P-002, P-029, P-037, P-039, P-174, P-340, P-351

P-039

O-096

P-297

P-068

O-114

P-453

P-291

P-032

P-327

O-046

P-274

P-078

P-100

P-391

O-039

P-362

P-267, P-378

P-059

P-120

P-120

P-065

P-223

P-232, P-408, P-409, P-410

O-008

O-039, O-086, O-092, P-026, P-074, P-109

P-109

P-327

P-224

P-029

P-147, P-148

O-009

P-381

P-246

P-247

O-016

P-077, P-093, P-166

O-149

O-023, P-343, P-344

O-025, O-028

P-148

O-100
Peeters, Petra

P-016

Peixoto, Catarina

P-369

Pell, Jill

Pelucchi, Claudio

Penido Matozinhos, Fernanda

Pentti, Jaana

Pereira, Carlos

Perez-Vicente, Raquel

Perrild, Hans

Perry, Ivan

Perry, Ivan J

Perry, Ivan J.

Pershagen, Göran

Persson, Margaretha

Pesatori, Angela Cecilia

Peters, Annette

Petersen, Christina Bjørk

Petersen, Inge

Petersen, Irene

Petersen, Jesper Padkær

Petersen, Kasper Meidahl

Petersen, Liselotte

Petherick, Emily

Petkeviciene, Janina

Petrovic, Branislav

Petticrew, Mark

Petzold, Thomas

Pezzotti, Patrizio

Pickering, Janet

Pickett, Kate

Pilegaard Hansen, Rikke

Pinnarelli, Luigi

Pinto De Figueiredo, Rudgy

Pistolla, Georgia

Platt, Robert

Ploner, Alex

Polesel, Jerry

Portielje, Johanneke

Portolani, Nazario

Poskotinova, Liliya

Potthoff, Karin

Poulsen, Anja

Poulsen, Henrik Enghusen

Powell, Natasha

Prady, Stephanie

Prescott, Eva

Prescott, Gordon

Prins, Maria

Pudule, Iveta

Puhó, Erzsébet

Pulido, Esther

Pulmanis, Toms

Pürjer, Mari-Liis

Paananen, Reija

Qin, Ping

Quezada, Orlando

Quintana, Hedley

Quintana, José M.

Quintana, Jose Maria

Quiros-Roldan, Eugenia

Quyou, Ali

Rachet, Bernard

Rafaelle Andrade Gurgel, Michelle
O-120

O-122

P-357, P-359

P-209

P-040, P-356, P-392, P-415

$\mathrm{O}-148$

O-096

P-482

P-328

P-192, P-329

$\mathrm{O}-028$

P-035

P-255

O-059

P-399

P-473

O-038, O-098

P-185

O-135

O-003, O-035, O-079, P-372

P-183

O-112

P-414

P-034

P-094

P-164

P-333

P-183

O-102

P-055

P-224

P-018, P-169

O-067

P-045

O-122

O-057

P-089

P-057, P-156, P-157

P-191

O-019

O-135

O-006

P-183

O-087

P-047, P-189

$\mathrm{O}-021$

P-382

P-001

P-027

P-382

P-438

O-040

P-134

P-068, P-070

P-182

P-067, P-069

P-027

P-084

P-058

P-090

P-115 
Raffetti, Elena

Rafnar, Bjarni Ossurarson

Rahkonen, Ossi

Raitakari, Olli

Raitanen, Jani

Rakhypbekov, Tolebai

Ramarokoto, Charles-Emile

Ramazanova, Manshuk

Ramlau-Hansen, Cecilia Høst

Ramos, Luiz

Ramos, Paulo Cesar

Ramroth, Heribert

Randremanana, Rindra

Randrianirina, Frédérique

Rankin, Judith

Rantakokko, Panu

Rapp, Kilian

Rasmussen, Julie

Rasmussen, Lars Hvilsted

Rasmussen, Mette

Rasmussen, Signe $\mathrm{S}$

Ratanachaiwong, Wipa

Rattanasiri, Sasivimol

Ravn, Henrik

Rayce, Signe Boe

Rayner, Mike

Razafindratsimandresy, Richter

Rebordosa, Cristina

Redondo, Maximino

Rees, Adrian

Reeves, Gillian, K.

Regidor, Enrique

Reigstad, Marte Myhre

Reis-Santos, Bárbara

Resaland, Geir Kåre

Resick, Patricia

Reutter, Heiko

Rey, Grégorie

Ribeiro, Claudia

Riboli, Elio

Rich-Edwards, Janet Wilson

Richard, Vincent

Rignell-Hydbom, Anna

Riis Hansen, Peter

Riis, Anders H.

Rikala, Maria

Rioja, Luis

Rissanen, Pekka

Ristikari, Tiina

Ritvanen, Annukka

Ritz, Beate

Rizzato Stopa, Sheila

Robertson, Lynn

Robshamn, Trude Eid

Rocklöv, Joacim

Rod, Naja Hulvej

Rodger, Joanne

Rodrigo Mesquita, João

Rodrigues Galvão, Maria Helena

Rodrigues, Amabelia

Rodrigues, Anabela

Rodrigues, Irene

Rodrigues, Laura

Rodrigues, Mónica Alexandra

Rodrigues, Teresa
P-084, P-089

P-153

O-069, P-258, P-335, P-454

P-245

P-310

$\mathrm{P}-432, \mathrm{P}-434$

$\mathrm{O}-024$

P-387, P-428, P-429

P-247, P-248, P-250, P-263,

P-294, P-345, P-374 P-338

P-332

P-052, P-425

$\mathrm{P}-060$

O-024

$\mathrm{O}-024$

P-053, P-054, P-112

P-263

O-121

P-426

P-039

P-412

O-009

P-024

P-213

O-019, O-025, O-076

P-365

O-071, P-033, P-398

$\mathrm{O}-024$

O-030

P-069

P-109

O-044, O-048, O-117, P-066

P-238

O-018

P-233

O-095

P-143

P-384

P-238

P-415

P-016

P-381

O-024

P-244

O-094

O-065, P-300, P-377

P-044

P-408

P-310

O-040

P-386

O-030, O-118, P-013, P-273

P-046

P-047, P-189

O-018

$\mathrm{O}-141, \mathrm{P}-272$

O-036, O-078, O-087

P-199

O-084

P-173

O-025, P-426

P-178

P-435

O-026

P-222

P-442
Rodríguez-Artalejo, Fernando

Rodriguez, Alina

Rodríguez, J.C.

Roeleveld, Nel

Rognoni, Magda

Rojanavipart, Piangchan

Rom, Ane Lilleøre

Roman, Eve

Romieu, Isabelle

Ronning, Karin

Roos, Eira

Rosa, Matilde

Rosengren, Annika

Rosenqvist, Mårten

Rosenthal, Marika

Roshanisefat, Homayoun

Rossi, Alessandra

Rosta, Judith

Rostgaard, Klaus

Rostila, Mikael

Rosvall, Maria

Rothman, Kenneth J.

Rowe, Brian

Royo, Gloria

Royuela, Ana

Rudolph, Anja

Rugulies, Reiner

Ruíz-García, Montserrat

Rumsby, Emma

Runeson, Bo

Russ, Tom

Rückert, Ina-Maria

Rydell, Mina

Ryl, Livia

Rylander, Lars

Rymbayeva, Tamara

Ryskulova, Alma-Gul

Raag, Mait

Sabgayda, Tamara

Sadegi, Alireza

Saez, Jesús

Safer, Anton

Sagyndykova, Zarina

Sahagún Salcedo, Begoña

Sakkeus, Luule

Sallmén, Markku

Salo, Paula

Samorinha, Catarina

Sanchez, Mikel

Sandbæk, Annelli

Sandström, Olof

Sannikov, Anatoliy

Santana, Paula

Santi, Irene

Santibáñez, Miguel

Santini, Ana Carolina

Santos, Ana Cristina

Santos, Carla

Santos, Célia

Santos, Eduardo

Santos, Jair

Santos, Nuno

Santos, Susana

Santosa, Ailiana

São José Nacimento, Maria
P-346

P-149, P-471

$\mathrm{P}-232$

P-384

P-255

P-213

P-457

P-064

O-015

O-026

P-454

P-237

O-045, P-337

O-080

P-438

P-474

P-032, P-164

P-133

P-460

O-134

P-296

O-065, P-300, P-377

P-242

P-232

P-203

O-053

O-125, P-172, P-286

$\mathrm{P}-232$

P-362

O-142

O-051, O-077

P-008

P-312

P-155

P-244, P-246, P-407

$\mathrm{P}-432$

P-428

P-334

P-023

P-447

P-232

O-046

P-387

P-395

P-367

O-145

O-036

P-442

P-027

O-002, O-003, O-009

P-458

P-010

P-222

P-060

P-232, P-408, P-409, P-410

P-375, P-376

P-366, P-383, P-389

O-084

P-040

P-184

$\mathrm{P}-123$

P-040

P-366

O-141

O-084 
São José Nascimento, Maria

Saraiva, Clara

Saraiva, Regina

Saraiva, Regina Célia

Sarasqueta, Cristina

Sarbazi, Mohammad reza

Sarink, Danja

Saris, Wim HM

Sato, Yuki

Sattar, Naveed

Sauerbrei, Willi

Savi, Eduardo

Savoglidou, Ilektra

Sayers, Susan

Scalmana, Silvia

Scanlon, Tom

Scheidt-Nave, Christa

Scheike, Thomas

Schendel, Diana

Schlehofer, Brigitte

Schlünssen, Vivi

Schmeißer, Nils

Schmidt, Erik B.

Schmidt, Julie Andersen

Schmidt, Marjanka

Schmidt, Martina

Schmidt, Morten

Schmitt-Egenolf, Marcus

Schmitt, Jochen

Schnack, Tine $\mathrm{H}$

Schneider Vianna, Luana

Schneider, Barbara

Schoeps, Anja

Schonfeld, Sara J.

Schougaard, Liv Marit

Schouten, Leo

Schramm, Stine

Schuz, Joachim

Schüz, Joachim

Scifano, Patrizia

Seaton, Sarah

Sebastian-Galles, Núria

Sebena, Rene

Seibold, Petra

Seitz, Ina

Seliussen, Ingvei

Semenova, Yuliia

Semenova, Yuliya

Semyonova, Viktoria

Serafim Fernandes, Julia

Serfontein, Jan

Serlachius, Eva

Severinsen, Marianne

Severo, Milton

Seysembekov, Telman

Shafieezadeh, Tayebeh

Shah, Imran

Shalgumbayeva, Gulnar

Sharbakov, Altay

Sharmanov, T

Shaw, Jenny

Shaw, Jonathan

Shelton, Nicola

Shiely, Frances

Shigematsu, Mika

Shipley, Martin
P-230

P-435

P-392

P-040

P-067

P-218

O-100

P-174

P-299

P-308

O-108

P-405

P-130

P-026

P-404

P-362, P-368

P-197

O-017

O-023, P-344

P-162

O-074, P-004, P-256, P-275

P-082

P-340

P-317

O-053

O-116, P-191, P-194

P-014, P-050

P-468

P-094

P-475

P-267, P-378

P-135

O-053

O-054

P-198

P-271

P-297

P-063, P-259

O-010, O-012, O-054, P-064

P-272

P-208

P-125

O-112

O-056, O-116

P-197

P-455

$\mathrm{P}-121, \mathrm{P}-431$

P-432, P-434

$\mathrm{P}-023$

P-406

P-049

P-471

O-124

P-366, P-383

P-036, P-106, P-260

P-218

O-081

P-121, P-430, P-431

P-036, P-106, P-260

P-284

P-134

O-100

O-104

P-192, P-329

P-226

O-075, O-115
Shiri, Rahman

P-154

Shrestha, Anshu

Siddiqui, Rehana

Sifaki-Pistolla, Dimitra

Sigfusson, Sigmundur

Sigsgaard, Torben

Sigurdsson, Engilbert

Sigurdsson, Martin Ingi

Silva, Anna Ferla Monteiro

Silva, Susana

Silveira, Daniele

Silveira, Kalline

Silventoinen, Karri

Simell, Olli

Simões, Daniela

Simoes, Oziris

Simões, Rui

Simonsen, Marianne

Simpson, Nigel

Simpson, William

Singh, Gurmeet

Sipetic, Sandra

Siriaunkgul, Sumalee

Sirikulsathean, Pinyo

Sivertsen, Børge

Sjölander, Arvid

Skjærven, Rolv

Skjøth, Flemming

Skogli, Hans-Ragnar Skogli

Skoog, Ingmar

Skov-Ettrup, Lise Skrubbeltrang

Skovgaard, Anne Mette

Skytthe, Axel

Skärbäck, Erik

Skaaby, Tea

Slama, Rémy

Small, Rhonda

Smeeth, Liam

Smit, $\mathrm{H}$

Smith, Cairns

Smith, Samuel

Smith, WCS

Soares de Morais Freitas, Cláudia Helena

Soborg, Bolette

Sodemann, Morten

Soja, Anne

Sokal, Rachel

Sola-Vera, J.

Solbraa, Ane

Soler-Vila, Hosanna

Solli, Henrik

Solovieva, Svetlana

Son, Hyun Jin

Sonestedt, Emily

Soulaymani-Bencheikh, Rachida

Soulaymani, Abdelmajid

Sousa, Carlos

Sousa, José

Sousa, Sandra

Sousa, Sofia

Sovio, Ulla

Sparen, Par

Speechley, Kathy

Springe, Lauma

Spyrides, Maria Helena Constantino
P-298

P-269

P-018, P-078, P-169

$\mathrm{P}-152$

P-256, P-275

P-153

$\mathrm{P}-152$

P-439

P-442

P-061

P-425

P-388

P-354

P-348

P-396

P-369, P-435

P-484

P-352

P-047

P-026

P-038

P-065

P-422

P-449

P-471

O-043, P-306

P-002, P-039

P-146

O-004

O-105

P-151

O-016, O-017

P-407

P-025

$\mathrm{O}-028$

P-465

O-020, O-033, O-088, P-007, P-009, P-073, P-186

P-349

O-139

$\mathrm{P}-017$

P-047

P-173

P-456

P-297

O-061

O-033, P-011, P-116, P-216

$\mathrm{P}-232$

O-095

P-346

P-014, P-050

O-140, P-245

$\mathrm{P}-225$

P-302, P-311

P-193

P-058, P-193

$\mathrm{P}-280$

P-201

P-442

P-279

O-003, O-007

P-045

P-254

P-382

P-406 
Srinakarin, Jiraporn

Sritara, Piyamitr

Stafford, Mai

Staines, Don

Stamatakis, Manos

Starr, John

Stattin, Pär

Stegeman, Bernardine

Stegmaier, Christa

Steindorf, Karen

Steingrimsson, Steinn

Stengaard-Pedersen, Kristian

Stepien, Magdalena

Steptoe, Andrew

Stevens, Hanne

Stigum, Hein

Stocks, Tanja

Stokholm, Zara

Stokholm, Zara A.

Stokholm, Zara Ann

Stolberg, Carolyn

Stoltenberg, Camilla

Storeng, Ritsa

Strachan, David

Straif, Kurt

Strand, Bjørn Heine

Strandberg-Larsen, Katrine

Strandhagen, Elisabeth

Strate, Lisa Lynn

Streja, Elani

Stringhini, Silvia

Stronks, Karien

Strucinski, Pawel

Strøm, Marin

Strömberg, Ulf

Stücker, Isabelle

Stürmer, Til

Støvring, Henrik

Subbotina, Anna

Sudlow, Cathie

Sukpan, Kornkanok

Suldrup Jørgensen, Thea

Sulo, Gerhard

Sultan, Alyshah Abdul

Sun, Yuelian

Sunde, Lone

Sundquist, Jan

Sundquist, Kristina

Sundström, Anders

Sundvor, Vernar

Sunyer, Jordi

Suppli, Nis Palm

Surtees, Paul

Suvisaari, Jaana

Suzuki, Reiko

Svanes, Cecilie

Svendsen, Maria

Svendsen, Susanne Wulff

Svensson, Elisabeth

Svärd, Anna

Sværke, Claus

Szamotulska, Katarzyna

Szatkowski, Lisa

Szych, Zbigniew

Szyszkowicz, Mieczyslaw
P-071

P-024

O-081, P-342

P-159, P-202

O-051, O-077

O-077

P-079

P-327

$\mathrm{P}-062$

O-116, P-191, P-194

P-152, P-153

P-463

O-015

P-332, P-370

P-134

O-091

P-079, P-174

P-338

O-074

O-128

P-217

P-309

O-018

P-122

$\mathrm{O}-012$

O-091

O-064, P-138, P-249, P-336, P-464

P-051

P-093

O-023, P-012, P-344

O-114, O-115

O-083

P-246

P-129

O-080

$\mathrm{O}-012$

P-476, P-477

O-002, O-060, P-015, P-131, P-248,

P-298, P-372, P-476, P-477

$\mathrm{O}-048$

P-065

P-412, P-417

P-028, P-030, P-031

P-215, P-385, P-479

O-032, P-427

O-011

O-138

O-138

P-468

P-455

P-125

O-041

P-305

O-145

P-299

P-275

P-365

O-143, P-249, P-339

P-143, P-234

P-335

O-014

P-211

P-314, P-315

P-220

P-242
Søgaard, Kirstine Kobberøe

Søndergaard, Charlotte

Sørensen, Henrik Toft

Sørensen, Thorkild IA

Sørup, Signe

Saar Gomes, Crizian

Saastamoinen, Leena

Saastamoinen, Peppiina

Tabak, Adam

Tabatabaeifar, Sorosh

Tada, Yuki

Tafforeau, Jean

Taj, Tahir

Takkinen, Hanna-Mari

Talamini, Renato

Talarmin, Antoine

Tammelin, Tuija

Tamosiunas, Abdonas

Tan, Seang-Lin

Taniguchi, Kiyosu

Tao, Fangbiao

Tarp, Maja

Tata, Laila

Tata, Laila Jal

Tauil, Marcia Cantuaria

Taylor-Robinson, David

Tazhibayev, Sh

Teerawattananon, Yot

Teixeira, Laetitia

Telén Andersen, Ane Birgitte

Tell, Grethe

Tell, Grethe S

Tell, Grethe S.

ten Have, Margreet

Tennant, Peter

Tesic, Sladjana

Teucher, Birgit

Thakkinstian, Ammarin

Thamm, Michael

Thayssen, Per

Thelle, Dag

Theofylaktopoulou, Despoina

Thierry-Chef, Isabelle

Thinggaard, Mikael

Thomas, Sara

Thomas, Sara L

Thompson, Rosie

Thomsen, Cathrine

Thomsen, Jane F.

Thomsen, Jane Frølund

Thomsen, Maria Morandi

Thomsen, Reimar Wernich

Thomsen, Simon Francis

Thomson, Errol

Thorand, Barbara

Thordardottir, Edda Bjork

Thorlacius-Ussing, Ole

Thorsteinsdottir, Thordis Katrin

Threapleton, Diane

Thuesen, Betina
O-014, O-129

P-380

O-014, O-055, O-065, O-129, O-146,

O-147, P-001, P-014, P-050, P-077, P-093, P-117, P-143, P-166, P-167,

P-234, P-300, P-377, P-446

O-003, O-111, O-060, P-037, P-098, P-174, P-251, P-340, P-351

O-019

P-282, P-283, P-357, P-359

P-386

P-449

O-115

P-339

P-226

P-187

P-276

P-354

$\mathrm{O}-122$

$\mathrm{O}-024$

P-149

P-327

O-067

P-226

P-264

P-117

P-011, P-116, P-215, P-216, P-314, P-315, P-385, P-479

O-033, O-034, P-099

P-219

O-071, P-033, P-034, P-398

P-284

P-071

P-178

O-146

O-043, P-028, P-307

P-455

P-030, P-031

$\mathrm{P}-145$

P-053

P-228

P-194

P-024, P-042, P-071, P-213

O- 050

O-094

P-051

P-307

P-074

O-119

O-020

$\mathrm{P}-007$

P-054

$\mathrm{P}-246$

O-125, P-172

P-286, P-339

P-470

O-014, O-129, P-234

O-150

P-242

O-059

P-478, P-485

P-185

P-485

O-047

P-025, P-330 
Thuesen, Leif

Thulstrup, Ane Marie

Thurah, Annette Ladefoged de

Thygesen, Lau

Thygesen, Lau Caspar

Thysen, Sanne

Thyssen, Jacob

Tiikkaja, Sanna

Tilling, Kate

Tilsted, Hans-Henrik

Tim, Card

Timm, Signe

Timmins, Kate

Timmis, Adam

Tinnerberg, Håkan

Tjønneland, Anne

Tlemisov, Aidos

Toft, Gunnar

Toft, Ulla

Tognon, Gianluca

Tollånes, Mette

Tolstrup, Janne Schurmann

Tolysbayeva, Zh

Tong, Shilu

Toompere, Karolin

Torén, Kjell

Torp-Pedersen, Christian

Toure, Abdoulaye Abba

Trab Damsgaard, Mogens

Trandafir, Mircea

Travis, Ruth

Trichopoulou, Antonia

Trudel, Xavier

Truong, Thérèse

Truter, Ilse

Trønnes, Håvard

Tucker, David

Tulebaev, Kazbek

Tulegenov, Murat

Turati, Federica

Turdalieva, Botagoz

Tybjaerg-Hansen, Anne

Tzanakis, Nikolaos

Tzivian, Liliana

Tønder Nielsen, Anita

Tørring, Marie Louise

Taanila, Anja

Udumyan, Ruzan

Ueland, Per Magne

Uldall, Peder

Uldall, Peter

Uldbjerg, Niels

Ulgenalp, Ayfer

Ulmer, Hanno

Ulrich, Cornelia

Ulrichsen, Sinna P.

Ulvik, Arve

Unzurrunzaga, Anette

Urbàn, Robert

Urhøj, Stine Kjær

Uusitalo, Liisa

Uusküla, Anneli

\begin{tabular}{|c|c|}
\hline O-094 & Vahtera, Jussi \\
\hline P-294, P-298, P-301, P-345 & Vala, Helena \\
\hline P-005 & Valdimarsdóttir, Unnur \\
\hline O-061 & Valdimarsdóttir, Unnur Anna \\
\hline P-417 & Valencia-Martín, José \\
\hline O-025 & Valente Guimarães, Vanessa \\
\hline P-025 & Valente-Gomes, Gregório \\
\hline $\mathrm{P}-045$ & Valkov, Mikhail \\
\hline P-308 & Valkova, Lyudmila \\
\hline O-094 & van Boven, Frank \\
\hline P-099 & van de Velde, Cornelis \\
\hline P-275 & van de Water, Willemien \\
\hline P-180 & van den Berg, Gerard \\
\hline P-009 & van den Berg, Saskia \\
\hline P-244 & van den Brandt, Piet \\
\hline P-016, P-029, P-037, P-340, P-351 & Van der Heyden, Johan \\
\hline P-121, P-430, P-431 & van der Zanden, Loes FM \\
\hline P-246, P-247, P-248, P-250, P-263, & van Dorsselaer, Saskia \\
\hline P-301, P-321, P-374 & Van Ewijk, Reyn \\
\hline O-096, O-097, P-330 & Van Guelpen, Bethany \\
\hline $\mathrm{P}-051$ & van Rooij, Iris ALM \\
\hline O-064 & van Rosse, Floor \\
\hline O-105, O-109, P-399 & van Rossem, L \\
\hline $\mathrm{P}-284$ & van Staa, Tjeerd-Pieter \\
\hline O-063, P-264 & Vanacore, Nicola \\
\hline P-438 & Vandborg, Pernille Kure \\
\hline P-275 & Vangen, Siri \\
\hline $\mathrm{O}-094, \mathrm{O}-130, \mathrm{O}-135, \mathrm{P}-105, \mathrm{P}-466$ & Varakina, Zhanna \\
\hline P-241 & Vassos, Evangelos \\
\hline O-106 & Vedsted, Peter \\
\hline $\mathrm{O}-027$ & Veiga, Ana \\
\hline $\mathrm{P}-317$ & Veiga, Nélio \\
\hline O-015 & Veijola, Riitta \\
\hline P-268 & Vejakama, Phisitt \\
\hline O-053 & Velásquez-Meléndez, Gustavo \\
\hline \multicolumn{2}{|l|}{ O-062, P-136 } \\
\hline P-371 & Velho, Sara \\
\hline $\mathrm{P}-054$ & Velika, Biruta \\
\hline P-387 & Velten, Ana Paula Costa \\
\hline P-429 & Venables, Susan \\
\hline O-122 & Vergara, Itziar \\
\hline P-428, P-429 & Vested, Anne \\
\hline P-353 & Vestergaard, Jesper \\
\hline P-078 & Vestergaard, Jesper Meedom \\
\hline P-266 & Vestergaard, Mogens \\
\hline P-137 & Vézina, Michel \\
\hline 0-102 & Vianna, Luana \\
\hline O-003 & Vieira-Pinto, Madalena \\
\hline & Vieira, Nuno \\
\hline 0-144 & Vigl, Matthäus \\
\hline P-307 & Viikari-Juntura, Eira \\
\hline $\mathrm{P}-012$ & Viikari, Jorma \\
\hline $\mathrm{O}-023, \mathrm{P}-336, \mathrm{P}-344$ & Vikström, Max \\
\hline P-270 & Vilagut, Gemma \\
\hline P-085 & Villerusa, Anita \\
\hline O-121, P-079 & Viner, Russell \\
\hline P-191 & Vinther Skriver, Mette \\
\hline $\mathrm{P}-234$ & Vinther-Larsen, Mathilde \\
\hline P-307 & Virk, Jasveer \\
\hline $\mathrm{P}-027$ & Virta, Lauri J \\
\hline $\mathrm{O}-112$ & Virtanen, Suvi \\
\hline P-373 & Vistisen, Helene Tilma \\
\hline P-354 & Viwatwongkaseam, Chukiat \\
\hline P-334, P-438 & Vogt, Susanne \\
\hline
\end{tabular}

Vahtera, Jussi

P-209

O-084

O-144

P-448

P-346

P-046

P-230

O-058, P-086, P-087, P-088, P-119

P-087, P-119

P-097

O-057

$\mathrm{O}-057$

P-363

O-001

P-271

P-187

P-384

$\mathrm{P}-145$

O-027, O-029

$\mathrm{P}-467$

P-384

O-083

P-349

P-186

P-404

P-185

$\mathrm{O}-018$

$\mathrm{P}-010$

P-147

O-102

P-235

P-356, P-415, P-416

P-354

P-042

P-281, P-282, P-283,

P-357, P-359

P-021, P-201, P-280

P-382

P-358

P-362, P-368

P-188, P-403, P-436

P-250, P-263, P-294

P-338

O-128

O-136, O-147, P-452, P-457 P-268

P-375, P-376

P-231

P-239

P-194

O-140, P-003, P-245

P-245

P-182

O-072, P-145

P-382

$\mathrm{O}-008$

O-002

O-106

O-136, O-137, P-483

O-140

P-354

P-200

P-213

O-059 
Vollenweider, Peter

Vollset, Stein Emil

Von Wagner, Christian

Vrieling, Alina

Vrotsou, Kalliopi

Vujcic, Isidora

Vupa, Ozgul

Vyazmin, Alexander

Waeber, Gerard

Waern, Margda

Wagner, Cordula

Wagner, Gabriela Arantes

Wagner, Philippe

Wainwright, Nick

Waldman, Eliseu Alves

Wallach Kildemoes, Helle

Wallby, Thomas

Waltoft, Berit Lindum

Wang, Jiajia

Wardel, Jane

Wareham, Nicholas

Wareham, Nick

Wark, Petra

Webb, Roger

Weimann, Hanna

Weinberg, Clarice R

Weinmayr, Gudrun

Wellesley, Diana

West, Joe

Westerlund, Hugo

Wetke, Randi

Wettermark, Björn

Whitaker, Heather

Whitehead, Margaret

Wientzek, Angelika

Wiesing, Lucas

Wiessing, Lucas

Wiinberg, Niels

Wijers, Charlotte HW

Wijga, AH

Wijlaars, Linda

Wilcox, Allen

Wilde, Katie

Wildman, John

Willert, Morten Vejs

Williams, David J

Williams, Gail

Wilmet-Dramaix, Michèle

Wimberley, Theresa

Winding, Trine Nøhr

Wing, Kevin

Winkleby, Marilyn

Winkler, Volker

Wiratkapun, Cholatip

Wise, Lauren

Wise, Lauren A.

Wiskemann, Joachim
O-114

P-030, P-031, P-306, P-307, P-455, P-459

P-017

O-056, O-116

P-188, P-403, P-436

P-038

P-085

P-010

O-114

O-004

O-083

P-360, P-361

P-177, P-204, P-206, P-207

P-305

P-219

P-446

O-007

O-143

O-063

P-017

O-005, O-006, P-020, P-048, P-111 $\mathrm{P}-305$

O-110, P-016

O-142, P-134, P-144

P-407

P-179

P-122

P-054

O-033, O-034, P-215, P-385, P-479

O-127

P-338

P-446

O-038

P-034

P-194

O-021

P-334

O-009

P-384

P-349

O-038, O-098

O-064, P-179, P-371

P-199

O-039

O-074

P-007

O-063

P-421

P-131

P-212

P-186

O-138

P-062

P-071

P-300, P-377

O-065

P-191
Witte, Daniel R

Wiuf, Carsten

Wohlfahrt, Jan

Wongwaisayawan, Sansanee

Woodtichartpreecha, Piyanoot

Wright, John

$\mathrm{Wu}$, Chunsen

Wulff Svendsen, Susanne

Würtz, Else Toft

Wärnberg, Fredrik

Wörmann, Tanja

Xavier, Andre

Xhani, Anila

$\mathrm{Xu}$, Beibei

$\mathrm{Xu}$, Wenjie

Yajnik, Chittaranjan

Yamwong, Sukit

Yang, TienYu Owen

Yardim, Mahmut Sadi

Yaya, Yaliso

Yeargin-Allsopp, Marshalyn

Yelissinova, Ainur

Yelissinova, Nurgul

Yngve, Agneta

Yokokawa, Hirohide

Yongcharoen, Sasitorn

You, Yuko

Yuan, Wei

Yuasa, Motoyuki

Zanetta, Dirce Maria Trevisan

Zappalà, Alba

Zeeb, Hajo

Zeitlin, Jennifer

Zetterberg, Henrik

Zhang, Wei-Hong

Zhanturyiev, Bolat

Zhu, Jin Liang

Zierer, Astrid

Zoega, Helga

Zubillaga, Elena

Zuccolo, Luisa

Zviezdai, Valentyna

Zwink, Nadine

Zwisler, Ann-Dorthe

Ängquist, Lars

Östergren, Per-Olof

Østergaard, Jane N.

Østergaard, Jane Nautrup

Østergaard, John Rosendahl

Östling, Svante

$\emptyset$ verland, Simon

$\varnothing$ yen, Nina

Åkerstedt, Torbjörn

Åström, Christoffer

órsdóttir, Inga
O-009

P-148

P-129, P-456, P-475

P-071

P-071

P-183

O-133, P-012, P-427, P-457

$\mathrm{P}-113$

P-004, P-445

P-081

P-238

O-066, P-017, P-370

P-080

P-251

P-413

P-056

$\mathrm{P}-024$

P-066

P-210

P-190

O-023, P-344

P-432, P-434

P-432, P-434

P-274

P-299

$\mathrm{P}-213$

O-123

P-444

P-299

P-360, P-361

P-059

O-010, P-064

O-099, P-110, P-211

P-051

P-421

P-387, P-428

O-147, P-137, P-298

O-059

P-446

P-027

P-353

P-246

P-384

O-061

O-111, P-174

P-407

P-340

P-351

O-049

O-004

O-037, P-449

O-043, P-459

O-036, O-127

P-272

P-274 